\title{
MEETING ABSTRACTS
}

\section{3rd International Symposium on Intensive Care and Emergency Medicine}

\author{
Brussels, Belgium, 19-22 March 2013
}

Published: 19 March 2013

P1

Protective role of autophagy in mouse cecal ligation and puncture-induced sepsis model

WTakahashi', H Hatano², H Hirasawa', S Oda

'Graduate School of Medicine, Chiba University, Chiba, Japan;' 2 Biomedical

Research Center, Chiba University, Chiba, Japan

Critical Care 2013, 17(Suppl 2):P1 (doi: 10.1186/cc11939)

Introduction Autophagy is well known as one of the biogenic responses against various stresses, which possesses the beneficial roles for survival, but little is known about the dynamics and its significance during the septic condition. We hypothesized that autophagy is induced during the septic condition, and contributes to protect from tissue damage which subsequently leads to organ dysfunction. We confirm whether the autophagic process is accelerated or sustained in an acute phase of sepsis and we also determine its physiological role. Methods Sepsis was induced by cecal ligation and puncture (CLP) in mice. We examined the kinetics of autophagosome and autolysosome formation which may explain the status of autophagy by western blotting, immunohistochemistry, and electron microscopy. To investigate a precise role of autophagy in CLP-induced sepsis, chloroquine, an autophagy inhibitor, was administered to the CLP-operated mice, and blood chemistry, pathology of the liver and survival were evaluated. Results Autophagy demonstrated by the ratio of LC3-II/LC3-I was induced over the time course up to 24 hours after CLP. The ratio was particularly increased in the liver, heart and spleen. Autophagosome formation became maximal at 6 hours and declined by 24 hours after CLP. Autolysosome formation as evaluated by both fusion of GFP-LC3 dots with LAMP1 immunohistochemistry and electron microscopy was also increased after the procedure. Furthermore, inhibition of autophagy by chloroquine during the CLP procedure resulted in elevation of serum AST levels, and significantly increased mortality in mice.

Conclusion Autophagy was induced in several organs over the time course of the CLP sepsis model and then the process was gradually completed to degradation of the components. Our data suggest autophagy plays a protective role in organ dysfunction in sepsis.

\section{P2}

Reversible depressive effect of TNFa on a model of isolated perfused rat heart

BV Nguyen', M Guillouet², MA Giroux-Metges², G Gueret ${ }^{3}$,

M Ould-Ahmed', JP Pennec ${ }^{2}$

'Hôpital d'Instruction des Armées Clermont Tonnerre, Brest, France;

2Laboratory of Physiology, Faculty of Medicine, Brest, France; ${ }^{3}$ University

Hospital, Brest, France

Critical Care 2013, 17(Suppl 2):P2 (doi: 10.1186/cc11940)

Introduction Acute myocardial depression in septic shock is common [1]. Myocardial depression is mediated by circulating depressant substances, which until now have been incompletely characterized [2].
The aim of our study was to observe the effects of TNFa on the model of perfused rat heart.

Methods After profound anesthesia with pentothal, the Wistar rats were killed by exsanguination. After sternotomy, the heart was taken and connected to the Langendorf column. The apex of the heart was hooked to a strength sensor. Biopac student laboratory software was used to record and analyse heart contractions. Contractions were recorded every 5 minutes during periods of 20 minutes. Control measurements were first recorded. We measured four parameters: heart rate, contraction force, speeds of contraction and relaxation for control, during TNFa $(20 \mathrm{ng} / \mathrm{ml})$ exposure and after removal of TNFa. We express the variations of parameters as percentage of the control \pm SEM. A paired $t$ test was used to compare heart rate, contraction amplitude, speeds of contraction and relaxation with TNFa and control measurements and after removal of TNFa.

Results Eight rat hearts Wistar (weight $=325 \pm 23 \mathrm{~g}$ ) were studied. See Table 1.

Table 1 (abstract P2)

\begin{tabular}{lcc}
\hline & TNFa & Removal of TNFa \\
\hline Heart rate & $78 \pm 6^{*}$ & $91 \pm 5$ \\
Contractile & $62 \pm 8^{*}$ & $91 \pm 4$ \\
Speed of contraction & $72 \pm 6^{*}$ & $93 \pm 2$ \\
Speed of relaxation & $53 \pm 10^{*}$ & $89 \pm 4$ \\
\hline
\end{tabular}

Results expressed as percentage of control \pm SEM. ${ }^{*} P<0.05$.

Conclusion TNFa decreases significantly the heart rate, contractile force, speeds of contraction and relaxation on isolated perfused rat heart. TNFa probably plays a role in the pathophysiology of cardiomyopathy during septic shock. The partial reversibility of these effects could explain why left ventricular hypokinesia in patients with septic shock is reversible.

References

1. Vieillard-Baron A, et al: Actual incidence of global left ventricular hypokinesia in adult septic shock. Crit Care Med 2008, 36:1701-1706.

2. Hunter JD, et al: Sepsis and the heart. Br J Anaesth 2010, 104:3-11.

P3

Effect of cdp-choline on microcirculatory alterations during endotoxemia

K Schmidt', M Doerr' ${ }^{1}$, T Brenner', S Hofer ${ }^{1}$, A Walther ${ }^{2}$

'Universitätsklinikum Heidelberg, Germany; ${ }^{2}$ Klinikum Stuttgart, Klinik für

Anästhesiologie u. operative Intensivmedizin, Stuttgart, Germany

Critical Care 2013, 17(Suppl 2):P3 (doi: 10.1186/cc11941)

Introduction The cholinergic anti-inflammatory pathway (CAP) is a physiological mechanism that inhibits cytokine production and minimizes tissue injury during inflammation. CAP-mediated antiinflammatory signals in vagal efferent nerve fibers result in the release of acetylcholine, which interacts with innate immune cells that express the nicotinic acetylcholine receptor subunit $a 7$ ( $a 7 n A C h R)$. 
Endothelial dysfunction during sepsis is responsible for increased endothelial permeability, leukocyte-endothelial interaction and functional breakdown of microvascular perfusion. Endotoxemiainduced endothelial dysfunction can be reduced by cholinergic CAP activation [1]. The aim of this study was to determine the effects of the a7nAChR-agonist cdp-choline on microcirculatory alterations during experimental endotoxemia.

Methods Using fluorescent intravital microscopy, we determined venular wall shear rate, macromolecular efflux and leukocyte adhesion in mesenteric postcapillary venules of male Wistar rats. Endotoxemia was induced over 120 minutes by intravenous infusion of lipopolysaccharide (LPS). Control groups received an equivalent volume of saline. Cdp-choline was applied as an i.v. bolus in treatment groups. Animals received either (i) saline alone, (ii) cdp-choline 10 minutes prior to saline administration, (iii) cdp-choline 10 minutes prior to LPS administration, (iv) cdp-choline 30 minutes after LPS administration or (v) LPS alone.

Results There were no significant differences in venular wall shear rate between the groups after 120 minutes. There was no significant difference in the number of adhering leukocytes between the cdpcholine/LPS groups (iii, iv) and the LPS group after 120 minutes. Macromolecular efflux significantly increased in all groups over 120 minutes. All groups (i, ii, iii, iv) showed a significantly reduced macromolecular efflux compared with the LPS group after 120 minutes. Conclusion Cdp-choline has no effect on leukocyte-endothelial interaction and microhemodynamic alterations during endotoxemia. By activating the CAP, cdp-choline reduces capillary leakage. Thus cdpcholine might have a prophylactic and therapeutic anti-inflammatory effect on LPS-induced endothelial permeability. These findings identify the endothelium as a target of anti-inflammatory cholinergic mediators and cdp-choline as a potential therapeutic substance in sepsis treatment.

Reference

1. Peter C, et al: Shock 2010, 33:405-411.

P4

Immune response after stimulation with wall components of Gram-positive bacteria

S Aloizos', ETsigou², P Myrianthefs'2, S Gourgiotis', A Tsakris³, G Baltopoulos² ${ }^{\prime}$ NIMTS Hospital, Athens, Greece; ${ }^{2}$ A. Anargiroi Hospital, Athens, Greece;

${ }^{3}$ Medical School, Athens, Greece

Critical Care 2013, 17(Suppl 2):P4 (doi: 10.1186/cc11942)

Introduction The purpose of this study was to evaluate the immune response of patients susceptible to infection by Gram-positive bacteria after ex vivo provocation with lipoteichoic acid (LTA) and to compare the reaction with the one of healthy adults.

Methods Blood sample was obtained from 10 healthy volunteers, 10 hemodialysis patients with end-stage chronic renal failure (CRF), 10 patients with type II diabetes mellitus (DM) and $10 \mathrm{ICU}$ patients on the second day of hospitalization, who suffered nonseptic SIRS and had an APACHE II score $>25$. After suitable treatment the samples were incubated with $1 \mathrm{mg} \mathrm{LTA}$ for 8 hours and maintained at $-20^{\circ} \mathrm{C}$ until the measurement of cytokines TNFa, IL-6, IL-1 $\beta$, and IL-10, using the ELISA method. The results are presented as mean values \pm SEM. Graph Pad 4.0 was used, applying a $t$ test to test the variation of each cytokine in each group, and ANOVA to assess the differences between the four groups. Results Baseline cytokine values in the three groups were increased compared with the control group, but the difference was significant only for the ICU group (Table 1, data only for IL-6 and IL-10). The quotient IL-10/IL-6 of baseline values was between 0.23 and 0.96 among healthy, ESRD and DM persons, and 1.32 among ICU patients. In all examined groups the levels of cytokines increased significantly after stimulation with LTA, although ICU patients showed a differential response (a fivefold to ninefold rise compared with other groups who had an increase of 14 -fold to 36 -fold).

Conclusion Severely ill patients and secondarily hemodialysis and diabetic patients are in a proinflammatory state. The response of all examined groups to provocation by LTA was sufficient, with a differential expression of severely ill patients, a fact that reflects their different immunologic status.

P5

Correlation of the oxygen radical activity and antioxidants and severity in critically ill surgical patients: preliminary report

J Lee', H Shim², JY Jang'

${ }^{\prime}$ Yonsei University College of Medicine, Seoul, South Korea; ${ }^{2}$ Wonju Severance Christian Hospital, Yonsei University Wonju College of Medicine, Wonju, Korea Critical Care 2013, 17(Suppl 2):P5 (doi: 10.1186/cc11943)

Introduction In septic patients, the oxygen radical (OR) showed toxic effect to induce inflammation and antioxidant activity could affect organ dysfunction. This study was designed to determine the relationship between antioxidant level and severity of organ dysfunction.

Methods The medical records of adult patients managed in a surgical ICU from August 2012 to December 2012 were reviewed prospectively. Abstracted data included age, body weight (with BMI), APACHE II scores, SOFA scores, MODS scores, fluid intake, fluid output, nutritional support, shock, antioxidant levels, OR activities, zinc and selenium levels, complication and mortality. In addition, length of stay (LOS) in the ICU and in hospital, and in-hospital mortality were collected. These data were investigated on the first, the third and the seventh day, respectively.

Results A total of 13 patients were enrolled. The in-hospital mortality rate was $7.7 \%$ and mean LOS in the ICU and hospital was 6.5 and 27.6, respectively. Mean APACHE II score was 20.2. On the first day of ICU, the mean antioxidant level and OR were $1.5( \pm 0.5) \mathrm{mmol} / \mathrm{l}$ and $1.6( \pm 0.5)$ $\mathrm{mmol} / \mathrm{l}$, respectively. At the same time, SOFA and MODS scores were 7.3 and 5.0, respectively, and zinc and selenium were $32.6 \mu \mathrm{g} / \mathrm{dl}$ and $68.4 \mathrm{ng} / \mathrm{ml}$. On the third day, mean antioxidant and OR were $1.5( \pm 0.4)$ and $1.8( \pm 0.7)$ respectively (SOFA 6.6 , MODS 4.9 , zinc 50.0 , selenium 70.7). On the seventh day, mean antioxidant and OR were $1.4( \pm 0.5)$ and $1.9( \pm 0.7)$, respectively (SOFA 4.3 , MODS 3.1 , zinc 62.8 , selenium 77.3). In the correlation analysis, MODS scores and antioxidant level had significant correlations on the first and seventh days of ICU $(P=$ $0.001, P=0.009$ ).

Conclusion Antioxidant level had a correlation with organ dysfunction which might be used as a prognostic factor in critically septic patients. To prove this, large-scale data collection is required.

\section{References}

1. Noveanu M, Mebazaa A, Mueller C: Cardiovascular biomarkers in the ICU. Curr Opin Crit Care 2009, 15:377-383.

2. Piechota M, Banach M, Irzmanski R, Barylski M, Piechota-Urbanska M, Kowalski J, et al:: Plasma endothelin-1 levels in septic patients. J Intensive Care Med 2007, 22:232-239.

3. Kotsovolis $G$, Kallaras $K$ : The role of endothelium and endogenous vasoactive substances in sepsis. Hippokratia 2010, 14:88-93.

P6

Simultaneous analysis of the expression of CD64 and HLA-DR in the peripheral blood and bronchoalveolar lavage fluid in sepsis

T Skirecki', M Mikaszewska-Sokolewicz'2, G Hoser', U Zielińska-Borkowska'

'The Centre of Postgraduate Medical Education, Warsaw, Poland;' ${ }^{2}$ Medical University of Warsaw, Poland

Critical Care 2013, 17(Suppl 2):P6 (doi: 10.1186/cc1 1944)

Introduction The core pathophysiological changes in sepsis involve systemic activation of the immune system followed by the

Table 1 (abstract P4). Levels of cytokines before and after stimulation with LTA

\begin{tabular}{lcccc}
\hline & Control baseline LTA & ESRD baseline LTA & DM baseline LTA & ICU baseline LTA \\
\hline IL-6 & $8.90 \pm 0.76,245.30 \pm 26.68$ & $86.60 \pm 45.55,1,310.00 \pm 154.80$ & $15.90 \pm 1.89,252.00 \pm 35.52$ & $372.40 \pm 120.60,3,659.00 \pm 485.20$ \\
IL-10 & $3.00 \pm 1.08,40.90 \pm 7.45$ & $19.20 \pm 7.14,273.10 \pm 126.50$ & $15.30 \pm 2.08,350.50 \pm 89.42$ & $492.60 \pm 66.72,2,822.00 \pm 432.70$ \\
\hline
\end{tabular}

Values in $\mathrm{pg} / \mathrm{ml}$. 
anti-inflammatory compensatory response. However, controversies exist regarding the status of the immune system in local tissue compartments during sepsis. The aim of this study was to compare selected markers of activation between the systemic circulation and local lung environment.

Methods Twenty patients with severe sepsis were included into this study. Peripheral blood (PB) samples and bronchoalveolar lavage fluid (BALF) samples were obtained on the day of diagnosis (D1). BALF was collected from 11 patients. Samples were stained with antibodies: CD15/CD64 and CD3/CD14/HLA-DR and isotypic control. Cells were analysed by flow cytometry. Expression of markers of activation was analysed as the geometric median of fluorescence (GMF). All values are expressed as median values. Comparisons between groups were performed using Mann-Whitney and Wilcoxon tests.

Results The mortality of sepsis reached $70 \%$. Nonsurvivors had significantly $(P=0.001)$ elevated expression of CD64 on neutrophils. Expression of HLA-DR was higher in monocytes from BAL than PB GMF $(1,032$ vs. $342 ; P=0.02)$ and this tendency was present in sepsis originating from both pneumonia and peritonitis. Percentage of HLADR-positive T cells was lower in PB than in BAL ( $2.9 \%$ vs. $6.5 \% ; P=0.07$ ), but the GMF values for HLA-DR were higher in the circulating $T$ cells $(1,904$ vs. 1,$346 ; P=0.004)$. The expression of CD64 on neutrophils was not significantly different in PB and BAL, but there was a trend towards its higher expression in BAL from patients with pneumonia while its expression was higher in PB of patients with peritonitis.

Conclusion In this study we noticed that during sepsis some significant differences in the status of activation of immune cells exist between peripheral blood and lung resident cells. The lung milieu seems to promote activation of monocytes while neutrophil activation is more dependent on the site of infection. However, these observations require further studies in a larger group of patients.

Acknowledgements This study was supported by the Centre of Postgraduate Medical Education grant no 501-01-02-012 and by the sources of the Medical University of Warsaw.

\section{P7}

Anti-inflammatory effects of Kupffer cells through a7-nicotinic acetylcholine receptors

Y Li, X Shi

Changzheng Hospital, Second Military Medical University, Shanghai, China Critical Care 2013, 17(Suppl 2):P7 (doi: 10.1186/cc141945)

Introduction Nicotine exerts anti-inflammatory effects in several cell types. a7-nicotinic acetylcholine receptor (a7-nAChR), which has high permeability to calcium, is believed to contribute significantly to nicotinic anti-inflammatory effects. However, the molecular mechanism is largely unknown. Kupffer cells in the liver play an important role in inflammatory response to pathogens invading, but whether there is a7-nAChR expression in Kupffer cells or cholinergic anti-inflammatory pathway involved in this process remains unclear.

Methods (1) Kupffer cells, isolated by collagenase digestion and differential centrifugation from mice and labeled with FITC-aBGT, were observed under laser scanning confocal microscope to test the expression of a7-nAChR. Protein level was also tested by western blotting, with RAW264.7 as positive control; (2) $100 \mathrm{nM}$ LPS was given to Kupffer cells, with or without $1 \mathrm{mM}$ nicotine. TNFa, IL-10 and HMGB-1 were tested at 4 hours, 12 hours or 24 hours, respectively; (3) $100 \mathrm{BALB} / \mathrm{c}$ mice were randomly divided into four group: Group I (only lethal dose of LPS was given), Group II (nicotine and LPS were given), Group III (LPS, nicotine and GdCl3 were given), and Group IV (LPS and nicotine were given and the left cervical vagus nerve was cut off). The mortality of mice was observed for 72 hours.

Results (1) Expression of a7-nAChR in Kupffer cells was confirmed by confocal microscope and western blotting; (2) after nicotine was administered, the level of TNFa and HMGB-1 increased and the level of IL-10 decreased. Given left cervical vagus nerve cut off or aBGT, the effect of nicotine was weakened; (3) Group I had the highest mortality rate, while in Group II nicotine did reduce the mortality rate dramatically. After the left cervical vagus nerve was cut off or aBGT was given, the effects of nicotine were weakened. Difference for the mortality rate between Group III and Group IV was not significant.
Conclusion Kupffer cells played a crucial rule in modulating inflammation and the anti-inflammatory effect of nicotine was partially weakened after left cervical vagus nerve cut off or aBGT was given. It was verified that left cervical vagus nerve was essential for the antiinflammatory effect of nicotine and a7 acetylcholine receptors might play a critical role.

References

1. Wang $\mathrm{H}$, et al: Nicotinic acetylcholine receptor a7 subunit is an essential regulator of inflammation. Nature 2003, 421:384-388.

2. Wang $\mathrm{H}$, et al:: Cholinergic agonists inhibit HMGB1 release and improve survival in experimental sepsis. Nat Med 2004, 10:1216-1221.

\section{P8}

Prevention of sepsis by correcting apoptosis

M Puhtinskaya, V Estrin

Research Institute of Obstetrics and Pediatrics, Rostov-on-Don, Russia Critical Care 2013, 17(Suppl 2):P8 (doi: 10.1186/cc11946)

Introduction Activation of apoptosis in lymphocytes determines the development of neutropenia and of sepsis $[1,2]$. We investigated prevention of sepsis and correction of lymphocyte apoptosis by recombinant human granulocyte colony-stimulating factor (hr-GCSF, filgrastim) $[1,2]$.

Methods With the permission of the ethics committee, a controlled, randomized, blind clinical trial included 69 term newborns on mechanical ventilation, without neutropenia and clinical signs of infection, with a content of lymphocytes in early apoptosis (AnnexinVFITC+PI-) of $>9.59 \%$, and in late (AnnexinV-FITC+PI+) of $0.56 \%$. Lymphocytes in apoptosis were detected using antibodies to AnnexinV and propidium iodide staining method of immunophenotyping (flow cytometry; Beckman Coulter Epics XL, USA). The survey was conducted at admission, at 3 to 5 days, and 20 days. The method of random numbers in Group I included 39 newborns who on admission (with written parental consent) received an intravenous infusion of hr-GCSF dose of $10 \mu \mathrm{g} / \mathrm{kg}, 3$ days. Newborns of Group II $(n=30)$ did not receive hr-GCSF. Power of the study was $80 \%(a \leq 0.05)$.

Results For 3 to 5 days, Group I significantly decreased apoptosis of lymphocytes in the early from $16.1 \%$ to $7.8 \%$, and in late from $1.3 \%$ to $0.1 \%$. The development of sepsis and neutropenia have been reported. We observed no clinical or laboratory signs of adverse effects of the drug. Fatal outcomes $(n=4)$ are not associated with hr-GCSF, which was confirmed postmortem. Decreased duration of mechanical ventilation $(P<0.05)$. In Group II, 27 patients at 3 to 5 days developed neutropenia and increased lymphocytes in apoptosis $(P<0.05)$. Sepsis was diagnosed in 19 children; eight fatal outcomes.

Conclusion hr-GCSF reduces the incidence of septic complications and one of the mechanisms of its clinical effectiveness is the reduction of apoptotic factors affecting the development of neutropenia.

References

1. Gillan ER, Christensen RD, Suen Y, et al:: A randomized, placebo-controlled trial of recombinant human granulocyte colony-stimulating factor administration in newborn infants with presumed sepsis: significant induction of peripheral and bone marrow neutrophilia. Blood 1994, 84:1427-1433.

2. Pukhtinskaya MG, Estrin W, Gulova ES: Clinical and diagnostic value of apoptosis markers in the pathogenesis of neutropenia and bacterial complications in newborns with respiratory distress syndrome. Cytokines Inflamm 2011, 10:66-69.

\section{P9} Immune paralysis in trauma patients; implications for prehospital intervention

M Kox, KTimmermans, M Vaneker, GJ Scheffer, P Pickkers

Radboud University Nijmegen Medical Center, Nijmegen, the Netherlands Critical Care 2013, 17(Suppl 2):P9 (doi: 10.1186/cc11947)

Introduction Multi-trauma is one of the major indications for intensive care admission. Recovery is frequently complicated by post-injury immunological complications, caused by a dysfunctional immune system; for example, sepsis and multiple organ failure. In order to treat 
or prevent this immune paralysis, knowledge on the time course of immune paralysis in vivo and the pathophysiological mechanisms of immune paralysis is essential. The aim of this study is to determine factors that could predict and/or induce immunological complications in these patients to ultimately find a suitable target and timeframe for intervention.

Methods Blood was drawn from adult multi-trauma patients $(n=94)$ admitted to the emergency room (ER) of the Radboud University Nijmegen Medical Center. Blood was drawn at the trauma scene by the helicopter emergency medical services (HEMS), at arrival in the ER and at days $1,3,5,7,10$ and 14 after trauma. Plasma concentrations of TNFa, IL-6, IL-10, IFNY, IL-8 and MCP-1 were determined by Luminex. Ex vivo 24-hour whole blood stimulations with LPS or pam3cys were performed and produced TNFa, IL- 6 and IL-10 was measured using ELISA to determine the level of immune paralysis. Clinical data - for example, Injury Severity Scores, trauma mechanism, medication and survival - were collected from electronic patient files.

Results The plasma IL-10 concentration at ER was 16.5 -fold increased in comparison with time-point HEMS $(P<0.01)$. Similar but less pronounced effects were found for IL- 8 and MCP-1. A significant correlation ( $P=0.03, R=0.53$ ) was found between injury severity scores and IL-10 plasma concentration at time-point ER. Time-courses of ex vivo produced cytokines suggest that LPS-induced IL-6 and TNFa production is already decreased in the first few hours after trauma and recovering from day 5. Ex vivo IL-10 production shows an inverse pattern.

Conclusion Immune paralysis can be established within hours after trauma. Production of anti-inflammatory IL-10 in the prehospital phase could play a crucial role in the pathogenesis. Patients with a higher injury severity score are more prone to produce excessive IL-10 in this phase. Immune stimulatory strategies applied by the HEMS or early after hospital admission could form a potential future approach to prevent immune paralysis in multitrauma patients in the intensive care ward.

\section{P10}

Is hemoglobin concentration affected by sepsis in the acute phase?

G Jansma, H Buter, RT Gerritsen, EC Boerma

Medical Centre Leeuwarden, the Netherlands

Critical Care 2013, 17(Suppl 2):P10 (doi: 10.1186/cc11948)

Introduction In the acute phase of sepsis several potential mechanisms may change the hemoglobin $(\mathrm{Hb})$ concentration. On the one hand, endothelial activation may lead to increased vascular permeability and fluid sequestration to the interstitium, leading to hemoconcentration. On the other hand, degradation of the glycocalyx has been reported [1]. Shedding of this carbohydrate-rich layer with an estimated thickness of 0.2 to $0.5 \mu \mathrm{m}$ may lead to a substantial increase of the intravascular space, and thus to decrease of $\mathrm{Hb}$ concentration [2]. The aim of this study is to determine whether there is a decrease in $\mathrm{Hb}$ in the acute phase of sepsis.

Methods In this single-center retrospective analysis we identified patients with sepsis as the primary reason for non-elective ICU admission from a standard patient database. Patients who fulfilled the international criteria of sepsis and organ failure during ICU admission were included in the sepsis group (S-group). The control group was formed by patients with other non-elective reasons for ICU admission (C-group). Exclusion criteria were (recent) bleeding, surgery in the last 6 weeks, chronic renal failure (creat $>177 \mu \mathrm{mol} / \mathrm{l}$, or hemodialysis), untreated chronic anemia, pregnancy, polytrauma, age $<18$, hematologic or metastasized malignancies, cardiac arrest, and use of bone marrow suppressive drugs. Laboratory data were collected from blood samples, prior to in-hospital i.v. fluid therapy. In order to detect a difference in $\mathrm{Hb}$ concentration of $0.2 \mathrm{mmol} / \mathrm{l}$, we anticipated a sample size of 283 per group, based on a standard deviation (SD) of 1.2, $a=0.05$ and $\beta=0.8$. Data are expressed as mean \pm SD.

Results We included 296 patients in the S-group and 320 in the $\mathrm{C}$-group. The difference in $\mathrm{Hb}$ between the S-group and $\mathrm{C}$-group was not significant $(8.76 \pm 1.18 \mathrm{mmol} / \mathrm{lvs} .8 .93 \pm 1.16 \mathrm{mmol} / \mathrm{l}, P=0.07)$. After correction for a number of confounders, using a multivariate regression analysis, we observed a significant difference in $\mathrm{Hb}$ of $-0.23 \mathrm{mmol} / \mathrm{l}$ in the $\mathrm{S}$-group in comparison with the $\mathrm{C}$-group $(P=0.01)$.

Conclusion At first presentation, prior to in-hospital i.v. fluid therapy, $\mathrm{Hb}$ concentration in patients with sepsis is significantly lower in comparison with controls; however, the difference is very small, without the existence of anemia.

References

1. Steppan J, et al: Sepsis and major abdominal surgery lead to flaking of the endothelial glycocalyx. J Surg Res 2011, 165:136-141.

2. van den Berg BM, et al:: The endothelial glycocalyx protects against myocardial edema. Circ Res 2003, 92:592-594.

P11

Do changes in red blood cell deformability in patients with septic shock correlate with changes in SOFA scores?

T Clark' S Jewell ${ }^{2}$, M Sair 1 , P Petrov ${ }^{2}$. P Winlove ${ }^{2}$

'Derriford Hospital, Plymouth, UK; '2University of Exeter, UK Critical Care 2013, 17(Suppl 2):P11 (doi: 10.1186/cc11949)

Introduction Traditional whole blood experiments suggest that sepsis causes abnormal red blood cell (RBC) deformability. To investigate this at the cellular level, we employed a novel biophysical method to observe individual RBC membrane mechanics in patients with septic shock.

Methods We collected blood samples from patients with septic shock until either death or day 5 of admission. Thermal fluctuations of individual RBCs were recorded allowing a complete analysis of RBC shape variation over time. Mean elasticity of the cell membrane was then quantified for each sample collected.

Results We recruited nine patients with septic shock. Table 1 shows mean RBC thermal fluctuation and SOFA scores.

Conclusion RBC thermal fluctuation analysis allows variations in RBC elasticity during sepsis to be quantified at a cellular level. We could not identify any specific trend between sepsis severity and erythrocyte elasticity. Cells demonstrated both increases and decreases in fluctuation independent of SOFA score. This is contrary to current evidence that suggests RBC deformability is reduced during sepsis.

Reference

1. Piagnerelli M, et al:: Intensive Care Med 2003, 29:1052-1061.

P12

Do erythrocytes subjected to cardiopulmonary bypass exhibit changes in their membrane mechanical properties?

T Clark', S Jewell ${ }^{2}$, M Sair', P Petrov'2, P Winlove ${ }^{2}$

'Derriford Hospital, Plymouth, UK; ${ }^{2}$ University of Exeter, UK

Critical Care 2013, 17(Suppl 2):P12 (doi: 10.1186/cc11950)

Introduction Whole blood experiments suggest that cardiopulmonary bypass (CPB) causes red blood cell (RBC) trauma and changes in deformability that may contribute to postoperative microcirculatory

Table 1 (abstract P11). Mean RBC fluctuation (daily SOFA score)

\begin{tabular}{|c|c|c|c|c|c|c|c|c|c|}
\hline Day & A & B & $C$ & D & E & $F$ & G & $\mathrm{H}$ & 1 \\
\hline 1 & $4.8(10)$ & $5.2(9)$ & - & $4.8(12)$ & $4.6(16)$ & $4.9(13)$ & $5.1(16)$ & $4.6(18)$ & $5.1(15)$ \\
\hline 2 & $4.9(9)$ & $5.0(10)$ & $4.6(13)$ & $5.1(11)$ & $4.8(17)$ & $5.1(13)$ & $5.0(16)$ & $4.9(19)$ & $-(16)$ \\
\hline 3 & $4.0(6)$ & $5.1(9)$ & $4.7(12)$ & $4.8(11)$ & $4.9(18)$ & $4.8(13)$ & $5.0(16)$ & $4.7(21)$ & $5.3(16)$ \\
\hline 4 & - & $4.8(7)$ & $4.6(11)$ & $5.0(9)$ & $5.9(19)$ & - & $-(18)$ & - & $5.0(15)$ \\
\hline 5 & - & - & - & $5.1(6)$ & $5.2(18)$ & - & $-(19)$ & - & $5.0(10)$ \\
\hline
\end{tabular}


Table 1 (abstract P12). Change in RBC thermal fluctuation relative to baseline: two distinct groups seen

\begin{tabular}{|c|c|c|c|c|c|c|c|c|c|c|c|c|c|c|c|}
\hline Patient & $\mathrm{A} 1$ & $\mathrm{~A} 2$ & A3 & A4 & A5 & A6 & A7 & B8 & B9 & B10 & B11 & B12 & B13 & B14 & B15 \\
\hline Post CPB & +0.1 & -0.4 & -0.1 & -0.5 & -0.1 & -0.2 & -0.1 & +0.5 & 0 & +0.4 & +0.1 & +0.2 & 0 & +0.2 & +0.1 \\
\hline Day 1 & 0 & 0 & +0.1 & -0.1 & -0.1 & +0.1 & +0.1 & 0 & +0.5 & +0.4 & +0.7 & +0.4 & +0.6 & +0.5 & +0.7 \\
\hline Day 2 & -0.2 & +0.1 & 0 & NA & NA & 0 & NA & +0.5 & -0.1 & +0.4 & NA & +0.1 & NA & NA & NA \\
\hline
\end{tabular}

dysfunction. We used a novel fluctuation microscopy technique to quantify the effects of CPB on RBC elasticity at a cellular level.

Methods We collected blood samples from elective cardiac surgery patients pre (at induction) and post (immediately, each day until CICU discharge) CPB. Thermal fluctuations of individual RBCs were recorded using a high-frame-rate camera allowing a complete analysis of RBC shape variation over time. Mean elasticity of the cell membrane was then quantified for each sample collected.

Results Fifteen patients were recruited. Table 1 displays the results. $\mathrm{RBC}$ thermal fluctuation is measured relative to pre-bypass values. An increase in RBC fluctuation marks a decrease in stiffness. CPB caused two distinct changes in RBC elasticity; pre-fix $A$ indicates samples where stiffness increases or shows no change, $B$ those where stiffness decreases. Data on day 2 were not collected in patients discharged from the CICU. CPB type or time had no apparent impact on RBC response to $C P B$.

Conclusion RBC thermal fluctuation analysis quantifies the impact of CPB on erythrocyte membrane elasticity. We clearly identified two separate RBC elasticity responses to CPB. This finding is contrary to traditional flow measurement techniques that suggest CPB impairs whole blood flow and reduces RBC deformability.

Reference

1. Lindmark et al.: J Thoracic Cardiovasc Surg 2002, 123:381-383.

P13

Platelet-associated oxidative stress and ADAMTS-13 levels are inversely associated with a poor prognosis in septic shock L Montini', G De Pascale', MA Pennisi', ES Tanzarella', SL Cutuli', A Occhionero', R De Cristofaro², M Antonelli'

'Catholic University School of Medicine, Rome, Italy; 'Haemostasis and Thrombosis Center, Catholic University School of Medicine, Rome, Italy Critical Care 2013, 17(Suppl 2):P13 (doi: 10.1186/cc11951)

Introduction Sepsis causes widespread microvascular injury and thrombosis. Some hemostatic factors mediate the mechanisms involved in sepsis-related organ ischemia and failure. Oxidative stress is also increased in sepsis and reactive oxygen species (ROS) favor secretion of von Willebrand factor (vWF) multimers from endothelium and inhibit vWF proteolysis by ADAMTS-13. Moreover, the enzyme indoleamine-2,3-dioxygenase, an important immune regulator, is activated in sepsis and, through generation of kynurenins, promotes antioxidative and anti-infective activities. This study evaluated the relative role of ADAMTS-13, vWF and fibrinogen in the morbidity and mortality of patients with septic shock (SS). The above hemostatic factors were measured together with kynurenine and plasma protein carbonyls, marker of oxidative stress.

Methods One group of 12 patients with SS, defined using standard criteria, was enrolled in the ICU of the 'A. Gemelli' Hospital (Rome, Italy). Biochemical, hematologic and hemodynamic parameters were measured on days 1 to 4, 7, 14 and 21. A group of 12 age-matched and gender-matched healthy subjects was used as controls.

Results Low ADAMTS-13 activity was observed in SS patients (268 $\pm 123 \mathrm{ng} / \mathrm{ml}$ vs. $760 \pm 80 \mathrm{ng} / \mathrm{ml}$ in controls). vWF levels (antigen and activity) were increased $\sim 3$-fold compared with controls. Likewise, plasma protein carbonyls and kynurenine were globally increased in patients $(2.1 \pm 1.5 \mathrm{nmol} / \mathrm{mg}$ vs. $0.3 \pm 02 \mathrm{nmol} / \mathrm{mg}$ and $14.4 \pm 9.7 \mu \mathrm{M}$ vs. $2.3 \pm 1.3 \mu \mathrm{M}$, respectively). Intra-ICU mortality ( 3 of 15 ) was strongly and inversely correlated with carbonyl levels $(P=0.04)$ and platelets $(P=0.022)$.

Conclusion Hence, we hypothesize that, in the SS setting, platelets contribute to oxidative stress that counteracts the organ failureassociated mortality. Thus, low platelet count, irrespective of bleedings, may favor mortality in SS patients by generating lower ROS amounts.

\section{Reference}

1. Strauss $R$, et al:: Thrombocytopenia in patients in the medical intensive care unit: bleeding prevalence, transfusion requirements, and outcome. Crit Care Med 2002, 30:1765-1771.

P14

Neutrophil gelatinase-associated lipocalin/lipocalin2, derived from gut crypt cells, exerts intestinal antimicrobial effect via bacterial stimulation of Toll-like receptor 4 and 9

K Mori, T Igarashi, K Inoue, T Suzuki, H Morisaki, JTakeda

Keio University School of Medicine, Tokyo, Japan

Critical Care 2013, 17(Suppl 2):P14 (doi: 10.1186/cc11952)

Introduction Neutrophil gelatinase-associated lipocalin (NGAL)/ lipocalin2, known as a sensitive biomarker of acute kidney injury, prevents bacterial iron uptake, resulting in the inhibition of its overgrowth [1]. We previously demonstrated that this protein was discharged into gut lumen from crypt cells in septic conditions, and inhibited the growth of Escherichia coli [2]. However, it remains unclear which pathway is associated with the upregulation of NGAL. We therefore designed the present study to reveal whether the patternrecognition receptor of bacteria, the Toll-like receptor (TLR) family, plays a pivotal role for NGAL secretion from gut crypt cells.

Methods With our institutional approval, the ileum and colon of male C57BL/6J mice ( 6 to 7 weeks) were everted and washed by $\mathrm{Ca}^{2+}$ and $\mathrm{Mg}^{2+}$ free PBS buffer five times. Tissues were incubated with $\mathrm{Ca}^{2+}$ and $\mathrm{Mg}^{2+}$ free PBS containing $30 \mathrm{mM}$ EDTA for 1 hour to isolate crypt cells of gut. The cell suspension was filtered through a cell strainer $(40 \mu \mathrm{m})$ twice, and deposited the crypt cells by centrifugation at 700 $\mathrm{xg}$. The isolated crypt cells were resuspended in PBS and stained with $0.25 \%$ amido black for labeling paneth cells. The $5 \times 10^{5}$ crypt cells were resuspended in $50 \mathrm{ml} \mathrm{HBSS}$ containing $2.5 \%$ fetal bovine serum and $1 \%$ penicillin-streptomycin. The crypt cells were incubated at $37^{\circ} \mathrm{C}$ with or without TLR ligands: lipopolysaccharide (TLR4 ligand, $10 \mu \mathrm{g} / \mathrm{ml}$ ) and CpG-DNA (TLR9 ligand, $8 \mu \mathrm{g} / \mathrm{ml}$ ). After a 2-hour incubation period, the crypt cells were deposited and eluted mRNA to measure the expression of both NGAL and TLR mRNA using real-time PCR.

Results More than 70 to $80 \%$ of collected cells were stained by amido black. LPS significantly upregulated the expression of NGAL and TLR4 mRNA in ileum and colon crypt cells $(P<0.05)$. Although the CpG-DNA did not upregulate NGAL and TLR9 mRNA in ileum crypt cells, the apparent expression of NGAL and TLR9 mRNA was found in colon crypt cells $(P<0.05)$. Conclusion Bacterial stimulation of TLR4 and TLR9 pathways plays a pivotal role in the expression of NGAL mRNA in gut, suggesting that NGAL, derived from gut crypt cells, could contribute to the regulation of the intraluminal microflora in the critically ill.

References

1. Nature $2004,432: 917$

2. Crit Care Med 2011, 39:46

P15

Lethal influenza virus $A \mathrm{H} 1 \mathrm{~N} 1$ infection in two relatives with autosomal dominant GATA-2 deficiency

J Sole-Violan', I Sologuren', E Betancor', S Zhang ', C Pérez',

E Herrera-Ramos', M Martínez-Saavedra', M López-Rodríguez', J Pestano², J Ruiz-Hernández', J Ferrer', F Rodríguez de Castro', J Casanova³,

C Rodríguez-Gallego

'Hospital GC Dr Negrín, Las Palmas de GC, Spain; 'Universidad Las Palmas de GC, Spain; ${ }^{3}$ The Rockefeller University, New York, NY, USA

Critical Care 2013, 17(Suppl 2):P15 (doi: 10.1186/cc11953)

Introduction Most individuals infected with the 2009 pandemic H1N1 influenza $A$ virus (IAV) (H1N1pdm) experienced uncomplicated flu. 
However, in a small subset of patients the infection rapidly progressed to primary viral pneumonia (PVP) and a minority of them developed ARDS. Inherited and acquired variability in host immune responses may influence susceptibility and outcome of IAV infection. However, the molecular nature of such human factors remains largely elusive.

Methods We report three adult relatives with the autosomal dominant GATA-2 deficiency. P1 and his son P2 had a history of myelodysplastic syndrome and a few episodes of mild respiratory infections. They developed PVP by H1N1 pdm which rapidly evolved to ARDS. They died at the age of 54 and 31 , respectively.

Results Patients were heterozygous for a novel R396L mutation in GATA2. Like other patients with GATA-2 deficiency, the three relatives had absence of peripheral NK and B cells and monocytopenia. However a high number of plasma cells, which were found to be pauciclonal, were observed in peripheral blood from $\mathrm{P} 1$ during $\mathrm{H} 1 \mathrm{~N} 1 \mathrm{pdm}$ infection. $\mathrm{P} 1$ and P2 had normal levels of immunoglobulins and IgG antibodies against common viruses. Microneutralization test showed that $\mathrm{P} 1$ produced normal titers of neutralizing antibodies against $\mathrm{H} 1 \mathrm{~N} 1 \mathrm{pdm}$ and against the previous annual $\mathrm{H} 1 \mathrm{~N} 1$ strain. Our results suggest that a few clones of long-living memory B cells against IAV expanded in $\mathrm{P} 1$; and that these cells produced cross-reactive antibodies against H1N1pdm, similar to those recently described. During the flu episode $\mathrm{P} 1$ had a strong increase of IFNY-producing $\mathrm{T}$ cells and of IFNY production. The Th1-related chemokines CXCL10 and CXCL9, as well as IFN $\gamma$, MCP-1 and IL-8, were strongly elevated in serum from P1 and P2 in the course of $\mathrm{H} 1 \mathrm{~N} 1 \mathrm{pdm}$ infection.

Conclusion GATA-2 deficiency is the first described Mendelian inborn error of immunity underlying severe IAV infection. Primary immunodeficiencies predisposing to severe IAV infections may debut, even in adults without a history of previous severe infections. The massive IFNY-mediated cytokine storm may explain the fatal course of H1N1pdm infection in our patients.

P16

Bacterial translocation primes proinflammatory responses and is connected to early death in an experimental model of lethal injury N Baxevanos, TTsaganos, A Pistiki, D Droggiti, A Spyridaki,

E Giamarellos-Bourboulis

University of Athens, Medical School, Athens, Greece

Critical Care 2013, 17(Suppl 2):P16 (doi: 10.1186/cc11954)

Introduction Some cases of multiple trauma are rapidly deteriorating; the mechanism was investigated.

Methods Forty-one rabbits were assigned into two groups; shamoperated and subject to crush of the right femur. Survival was recorded; peripheral blood was sampled for LPS measurement by the kinetic QCL-1000 LAL assay; quantitative tissue growth was assessed after death. Some rabbits were sacrificed at 48 hours; blood was sampled from the portal vein for LPS measurement; splenocytes were

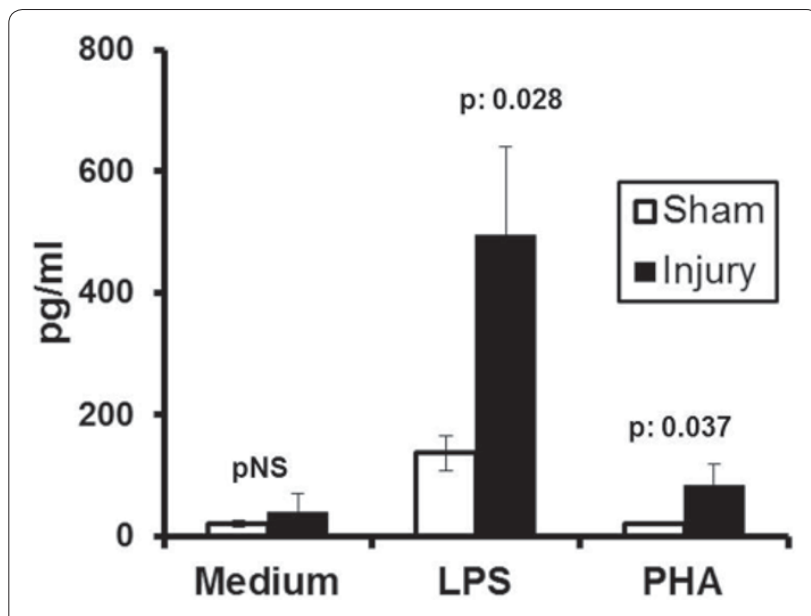

Figure 1 (abstract P16). Stimulation of TNFa from isolated splenocytes. isolated and incubated for 24 hours in the presence of $10 \mathrm{ng} / \mathrm{gl}$ LPS of Escherichia coli O55:B5 and of $5 \mu \mathrm{g} / \mathrm{ml}$ phytohemagglutin (PHA); TNFa was measured in supernatants by a bioassay on L929 fibroblasts.

Results Fifty percent of rabbits died early; that is, within the first 48 hours. Mean \pm SE $\log _{10}$ bacteria in the liver and lung of animals that died early was $2.27 \pm 0.62$ and $3.16 \pm 0.78 \mathrm{cfu} / \mathrm{g}$; respective values of rabbits that started dying late (that is, after 72 hours) were below the limit of detection. Mean circulating LPS at 24 hours was 2.09 EU/ $\mathrm{ml}$ and $1.99 \mathrm{EU} / \mathrm{ml}$ respectively $(P=\mathrm{NS})$. Mean LPS of the portal vein of the sham and of the injury groups were 1.25 and $5.62 \mathrm{EU} / \mathrm{ml}(P=$ 0.047). Concentrations of TNFa in splenocyte supernatants are shown in Figure 1.

Conclusion Early death after injury is not related to peripheral endotoxemia and sepsis; bacterial translocation priming for enhanced proinflammatory responses is a likely explanation.

\section{P17}

Effects of the common 34C $>$ T variant of the AMPD1 gene on immune function, multiorgan dysfunction and mortality in patients with sepsis

B Ramakers', E Giamarellos-Bourboulis², M Coenen'1, M Kox',

J Van der Hoeven', C Routsi ${ }^{2}$, A Savva ${ }^{2}$, I Perdios ${ }^{3}$, F Diamantea ${ }^{4}$

D Sinapidis' ${ }^{5}$, S Smits' 1 , N Riksen'1, P Pickkers ${ }^{1}$

'Radboud University Nijmegen Medical Centre, Nijmegen, the Netherlands; 2University of Athens, Medical School, Athens, Greece; ${ }^{3}$ Nafplion General

Hospital, Nafplio, Greece; ${ }^{4}$ G. Gennimatas' General Hospital, Athens, Greece; ${ }^{5}$ Alexandra General Hospital, Athens, Greece

Critical Care 2013, 17(Suppl 2):P17 (doi: 10.1186/cc11955)

Introduction Adenosine exerts anti-inflammatory and tissue protective effects during systemic inflammation. While the anti-inflammatory properties may induce immunoparalysis and impede bacterial clearance, the tissue protective effects might limit organ damage. The effects of a common loss-of-function variant of the adenosine monophosphate deaminase 1 gene (AMPD1), which is associated with increased adenosine formation, in patients with sepsis are unknown.

Methods In a prospective cohort, genetic-association study, the effects of the presence of the AMPD1 gene on immune function, multiorgan dysfunction and mortality in septic patients was studied. Pneumosepsis patients $(n=402)$ and controls without infection $(n=$ 101) were enrolled.

Results In pneumosepsis patients and controls, a similar prevalence of the 34C>T (rs17602729) mutation in the AMPD1 gene was found. Univariate logistic regression analysis showed a tendency of increased mortality in patients with the CT genotype, compared with patients with the CC genotype (OR $1.53 ; 95 \% \mathrm{Cl} 0.95$ to 2.5 ). Moreover, carriers of the CT genotype tended to suffer more from multiorgan dysfunction, OR 1.4 (0.84 to 2.3 ) and 3.0 (0.66 to 13.8), for CT and TT, respectively $(P=0.07)$. In septic carriers of the CT genotype, the ex vivo production of TNFa by LPS-stimulated monocytes was attenuated $(P=0.005)$,

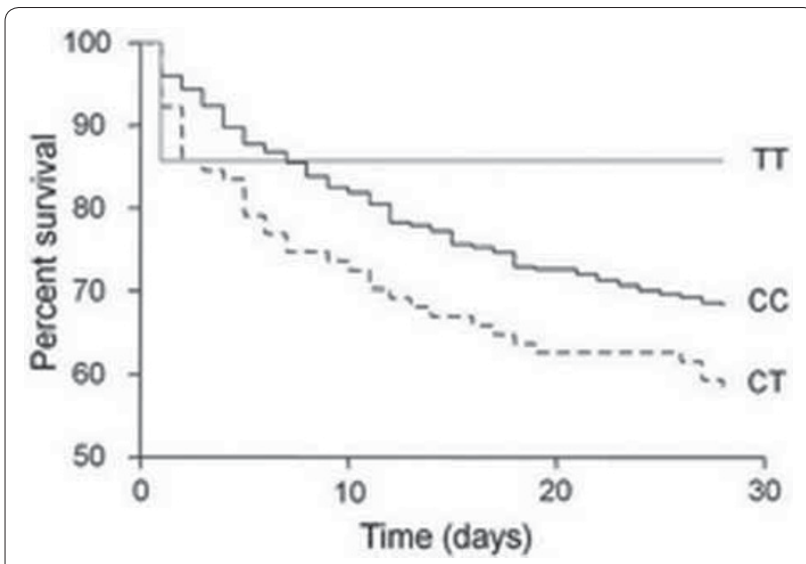

Figure 1 (abstract P17). Kaplan-Meier curve for the 402 sepsis patients. 
indicative for more pronounced immunoparalysis in these patients. See Figure 1.

Conclusion The presence the $34 \mathrm{C}>\mathrm{T}$ variant of the AMPD1gene is not related to infection susceptibility; however, it is associated with more pronounced immunoparalysis in patients with sepsis, and shows a tendency towards increased mortality. Mechanistically, the antiinflammatory effects of adenosine may account for this and apparently overrule its tissue protective effects.

P18

Exploring the translational disconnect between the murine and human inflammatory response: in vitro analysis of the doseresponse relationship of LPS and NFKB activation in murine and human immune cells

EP McCarron, I Welters, D Williams, D Antoine, A Kipar

University of Liverpool, UK

Critical Care 2013, 17(Suppl 2):P18 (doi: 10.1186/cc11956)

Introduction Inflammation, as seen in sepsis and systemic inflammatory response, is dependent on the activation of the NFKB pathway through Toll-like receptors (TLRs) [1]. Recreating an inflammatory response using lipopolysaccharide (LPS) can provide results that are different to clinical sepsis [2]. By examining NFKB activation in murine and human cells, a species comparison can be made to investigate differences at the cell level that may contribute to the translational disconnect seen in vivo.

Methods THP1 human monocytes (passages 9 to 11) and RAW 264.7 murine macrophages (passages 15 to 20) were cultured in RPMI-1640 and DMEM respectively and then challenged with LPS. After settling for 24 hours, cells were dosed with six or seven doses of LPS. After 1 hour, nuclear extraction and proteins were separated by acrylamide gel electrophoresis. Membranes where then immunoblotted for actin and $\mathrm{p} 65$, followed by densitometric analysis in order to quantify the amount of p65 that had translocated from the cytoplasm to the nucleus (by subtraction from consistent nuclear actin).

Results Murine cells required higher doses of LPS compared with human cells in order to detect p65 (human, $1 \mathrm{pg} / \mathrm{ml}$ to $100 \mathrm{ng} / \mathrm{ml}$; murine, $30 \mathrm{pg} / \mathrm{ml}$ to $1,000 \mathrm{ng} / \mathrm{ml}$ ). THP1 cells showed a greater fold increase in the p65:actin ratio compared with RAW 264.7 cells. Human cells responded to lower concentrations of LPS. Murine cells appeared to show a molecular resistance to lower doses, but their response was very sensitive at higher doses. A dose-response relationship of LPS dosing and NFKB activation was observed in both cell lines.

Conclusion Immunoblotting for p65 is a reliable and reproducible method to determine NFKB activation in cultured cells. Macrophages are more responsive to LPS than monocytes [3] so differences between cell lines would have been expected to be the reverse of what was observed. The species difference in response to LPS may contribute to the apparent disconnect between human and murine responses to LPS and may partially explain the difficulties of translating therapeutic interventions into clinical human sepsis.

References

1. Bonizzi G, Karin M: Trends Immuno/ 2004, 25:280-288

2. Remick DG, Ward PA: Shock 2005, 24(Suppl 1):7-11.

3. Takashiba S, et al: Infect Immun 1999, 67(11):5573-5578.
P19

Hypogammaglobulinemia in sepsis is not correlated to high circulating angiopoietin-2 levels

U Kovačič ', F Starič̌2, M Kmet², M Godnič̌2, R Kapš2

'Faculty of Medicine, University of Ljubljana, Slovenia; ${ }^{2}$ General Hospital Novo Mesto, Slovenia

Critical Care 2013, 17(Suppl 2):P19 (doi: 10.1186/cc11957)

Introduction Hypogammaglobulinemia has been frequently found in adult patients with severe sepsis and septic shock. Furthermore, it seems that at least a low serum level of IgM is correlated with higher mortality in sepsis. The mechanisms of hypogammaglobulinemia in septic shock have not yet been explained. It has been hypothesized that outflow of immunoglobulins into the extravascular space due to increased capillary permeability could reduce immunoglobulin serum concentrations. Angiopoietin-2, which directly disrupts the endothelial barrier, is markedly elevated in sepsis and other inflammatory states and its serum level has been correlated with microvascular leakage, end-organ dysfunction and death in sepsis.

Methods In the prospective, noninterventional study, we assessed the correlation between the capillary leakage marker angiopoetin- 2 and serum levels of IgG and IgM in 41 patients with community-acquired severe sepsis or septic shock on admission. Blood samples were obtained during the first 12 hours after admission to hospital.

Results Mean age of patients (17 females) was 70 years. Median APACHE II and SOFA scores at admission were 24 and 11, respectively. The mortality rate was $45 \%$. Thirty-four percent of all patients had level of $\operatorname{lgG}<650 \mathrm{mg} / \mathrm{dl}$. The median concentration of angiopoietin-2 in the hypo-lgG group was $11,958 \mathrm{pg} / \mathrm{ml}$, which was not statistically different (Mann-Whitney; $P>0.05$ ) than in the rest of patients with normal levels of $\operatorname{lgG}(15,688 \mathrm{pg} / \mathrm{ml})$. The concentration of $\mathrm{lgM}<40 \mathrm{mg} / \mathrm{dl}$ was found in only four patients (10\%) and all died. Pearson's correlation test showed that the correlation between the concentrations of angiopoietin-2 and IgG (correlation coefficient 0.191 ) or IgM (correlation coefficient $0.0408)$, respectively, were not statistically significant $(P<0.05)$.

Conclusion At present the hypothesis that increased microvascular leakage is responsible for hypogammaglobulinemia in septic patients could not be accepted. Studies on larger number of patients are needed. In addition, it is necessary to further explore other possible mechanisms, such as increased catabolism and consumption of antibodies or inadequate synthesis of immunoglobulins, which could also be responsible for hypogammaglobulinemia in sepsis.

References

1. Taccone FS, et al: Gamma-globulin levels in patients with communityacquired septic shock. Shock 2009, 32:379-385.

2. Werdan $\mathrm{K}$, et al: Score-based immunoglobulin $\mathrm{G}$ therapy of patients with sepsis: the SBITS study. Crit Care Med 2007, 35:2693-2701.

P20

TREM-1 levels are elevated in patients with liver cirrhosis

SD Gurney', CR Graham², P Kelleher², N Soni', M Foxton', S Singh

'Chelsea and Westminster Hospital, London, UK; 2 Imperial College, London, UK Critical Care 2013, 17(Suppl 2):P20 (doi: 10.1186/cc11958)

Introduction Sepsis and spontaneous bacterial peritonitis (SBP) are common sequelae in patients with cirrhosis. Cirrhotics admitted
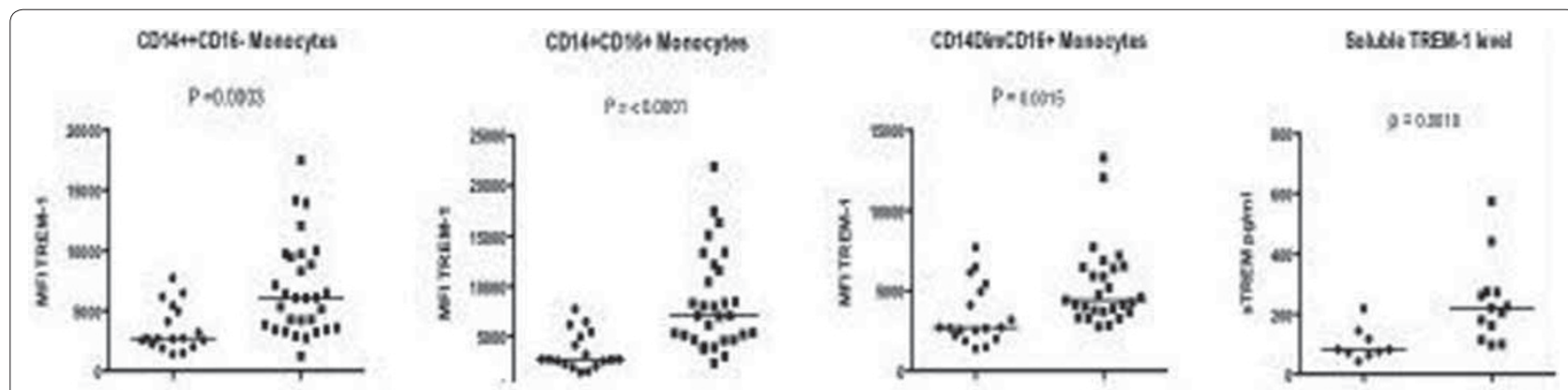

Figure 1 (abstract P20). TREM-1 expression in healthy controls compared with cirrhotic patients. 
to the ICU have an in-hospital mortality of up to 50\% [1]. Microbial translocation (MT) is the pathogenic mechanism implicated in SBP. The triggering receptor expressed by myelocytes-1 (TREM-1) modulates the immune response with resultant production of proinflammatory cytokines and has been used as a biomarker in the diagnosis of bacterial infection. We wish to evaluate the role of TREM-1 as a biomarker in cirrhosis.

Methods Blood samples were obtained from 18 healthy controls $(\mathrm{HC})$ and 29 cirrhotic patients (CA) as defined by clinicoradiological criteria. Disease severity was graded according to Child-Pugh class (median 10 , range 5 to 13) and modified end-stage liver disease (MELD) score (median 14, range 6 to 21). Simultaneous ascitic fluid samples were taken from 10 patients in the CA group. Soluble TREM-1 and CD14 levels (a surrogate marker of MT) were measured by ELISA. Flow cytometry was used to quantify the expression of TREM-1 on monocytes and neutrophils in blood and ascitic fluid.

Results TREM-1 expression is significantly higher in the CA group compared with $\mathrm{HC}$ across all monocyte subsets but not neutrophils, even in the absence of sepsis (see Figure 1). There is no correlation between cell surface TREM- 1 expression and severity of cirrhosis by Child-Pugh or MELD score. sTREM and sCD14 levels were elevated in the CA group compared with HC ( $P=0.0010$ and 0.0016 respectively). In addition, plasma sTREM-1 levels correlated with disease severity according to MELD score $(R=0.71, \mathrm{Cl}=0.22$ to $0.92 P=0.012)$ and serum bilirubin $(R=0.78, \mathrm{Cl}=0.36$ to $0.94, P=0.004)$. There was no correlation with either form of TREM-1 with sCD14 levels. There was no difference in cell surface or soluble TREM-1 expression between blood and ascitic fluid monocytes in culture-negative, non-neutrophilic ascites.

Conclusion Blood monocyte and soluble TREM-1 are elevated in cirrhotic patients even in the absence of sepsis. Soluble TREM- 1 levels correlate with disease severity in cirrhosis. Further studies are ongoing to ascertain the utility of TREM-1 as a biomarker in cirrhosis.

Reference

1. Olson JC, et al:: Intensive care of the patient with cirrhosis. Hepatology 2011, 54:1864-1872

P21

Effects of different doses and serotypes of LPS on blood-brain barrier permeability in Sprague-Dawley rats

E Senturk, F Esen, P Ergin Ozcan, G Orhun, N Orhan, N Arican, M Kucuk, M Kaya

University of Istanbul, Turkey

Critical Care 2013, 17(Suppl 2):P21 (doi: 10.1186/cc1 1959)

Introduction The blood-brain barrier (BBB) is highly restrictive of the transport of substances between blood and the central nervous system. Lipopolysaccharide (LPS) from Gram-negative bacteria was reported to affect the permeability of the BBB. BBB disruption using a LPS is used as a model of septic encephalopathy in mice. The present study was designed to investigate the effects of different doses and serotypes of LPS on BBB integrity in Sprague-Dawley rats.

Methods Male Sprague-Dawley rats weighing 200 to $250 \mathrm{~g}$ were used in the study. Rats were given two different types of LPS (026:B6L5543 and 026:B6-L2762) in different doses (3, 5, and $10 \mathrm{mg} / \mathrm{kg}$; i.v.). Rectal temperature and arterial blood pressure measurements were recorded for sepsis severity. The changes in the BBB permeability were measured using the Evans blue (EB) and sodium fluorescein (NaFl) dye extravasation techniques 24 hours after LPS administration.

Results Both LPS serotypes showed comparable arterial blood pressure and rectal temperature recordings and the severity of the disease increased with the increasing doses ( $5 \mathrm{mg} / \mathrm{kg}$ and $10 \mathrm{mg} / \mathrm{kg}$ ) of LPS and the mortality rates were found to be $29 \%$ and $63 \%$ respectively. The extravasated contents of EB and NaFI tracers did not significantly increase in brain parenchyma following the administration of different doses of LPS with different serotypes.

Conclusion Our results showed no disruption to BBB by two different serotypes of LPS even administered in increasing doses. These result indicate that the BBB integrity of Sprague-Dawley rats are resistant to the effects of two different serotypes of LPS.
P22

Bioenergetic imbalance and oxidative stress in the pathophysiology of septic encephalopathy

J D'Avila', R Rodrigues' ${ }^{2}$, H Castro-Faria-Neto' ${ }^{1}$, M Oliveira ${ }^{3}$, F Bozza ${ }^{4}$

'Oswaldo Cruz Foundation - FIOCRUZ, Rio de Janeiro, Brazil;'2Federal

University of Rio de Janeiro (UFRJ) and D'Or Institute for Research and

Education (IDOR), Rio de Janeiro, Brazil; ${ }^{U}$ UFRJ, Rio de Janeiro, Brazil; ${ }^{4}$ Oswaldo

Cruz Foundation - FIOCRUZ and IDOR, Rio de Janeiro, Brazil

Critical Care 2013, 17(Suppl 2):P22 (doi: 10.1186/cc11960)

Introduction Septic encephalopathy is a frequent complication in severe sepsis but its pathogenesis and mechanisms are not fully understood. Oxygen supply and utilization are critical for organ function, especially for the brain, a tissue extremely dependent on oxygen and glucose. Disturbances in oxygen utilization are common in sepsis and a number of mitochondrial dysfunctions have been described in different tissues in septic animals as well as in septic patients. Our group described mitochondrial dysfunctions in the brain during experimental sepsis.

Methods Experimental sepsis was induced by endotoxemia (LPS $10 \mathrm{mg} /$ kg i.p.) in Sprague-Dawley rats and by polymicrobial fecal peritonitis in Swiss mice. Brain glucose uptake was observed in vivo in endotoxemic rats using positron emission tomography with $\left[{ }^{18} \mathrm{~F}\right]$ fluorodeoxyglucose and autoradiography with 2-deoxy- ${ }^{14} \mathrm{C}$-glucose.

Results Mice with polymicrobial sepsis present hypoglycemia, hyperlactatemia and long-term cognitive impairment. We observed a rapid increase in the uptake of fluorescent glucose analog 2-deoxy2-((7-nitro-2,1,3-benzoxadiazol-4-yl)amino)-D-glucose in brain slices from septic mice in vitro. A similar increase in brain glucose uptake was observed in vivo in endotoxemic rats. Remarkably, the increase in glucose uptake started 2 hours after LPS injection, earlier than other organs. The brains of mice with experimental sepsis presented neuroinflammation, mitochondrial dysfunctions and oxidative stress, but mitochondria isolated from septic brains generated less ROS in vitro in the first 24 hours. This led us to investigate the role of NADPH oxidase, an enzyme induced during innate immune response, as a potential source of reactive oxygen species in experimental sepsis. Inhibiting NADPH oxidase with apocynin acutely after sepsis prevented cognitive impairment in mice.

Conclusion Our data indicate that a bioenergetic imbalance and oxidative stress is associated with the pathophysiology of septic encephalopathy. We are observing a new metabolic phenotype in the brain during sepsis, characterized by a rapid increase in glucose uptake and mitochondrial dysfunctions that may be secondary to inflammation and hypoxia.

\section{P23}

Pathophysiology of sepsis-associated brain dysfunction: an experimental study of cerebral microdialysis and mitochondrial function

P Kurtz'1 , C Vargas-Lopes', C Madeira', I Mello', R Panizzutti', L C Azevedo², F A Bozza

'Fiocruz, Rio de Janeiro, Brazil; ${ }^{2}$ Hospital Sirio e Libanes, São Paulo, Brazil Critical Care 2013, 17(Suppl 2):P23 (doi: 10.1186/cc11961)

Introduction Pathophysiology of brain dysfunction associated with sepsis is still poorly understood. Potential mechanisms involve oxidative stress, neuroinflammation and blood-brain barrier alterations. Our purpose was to study the metabolic alterations and markers of mitochondrial dysfunction in a clinically relevant model of septic shock.

Methods Twelve anesthetized (midazolam/fentanyl/pancuronium), invasively monitored, and mechanically ventilated pigs were allocated to a sham procedure $(n=5)$ or sepsis $(n=7)$, in which peritonitis was induced by intra-abdominal injection of autologous feces. Animals were studied until spontaneous death or for a maximum of 24 hours. In addition to global hemodynamic and laboratory assessment, intracranial pressure and cerebral microdialysis were assessed at baseline, 6, 12, 18 and 24 hours after sepsis induction. After death, brains were removed and brain homogenates were studied to assess markers of mitochondrial dysfunction. 


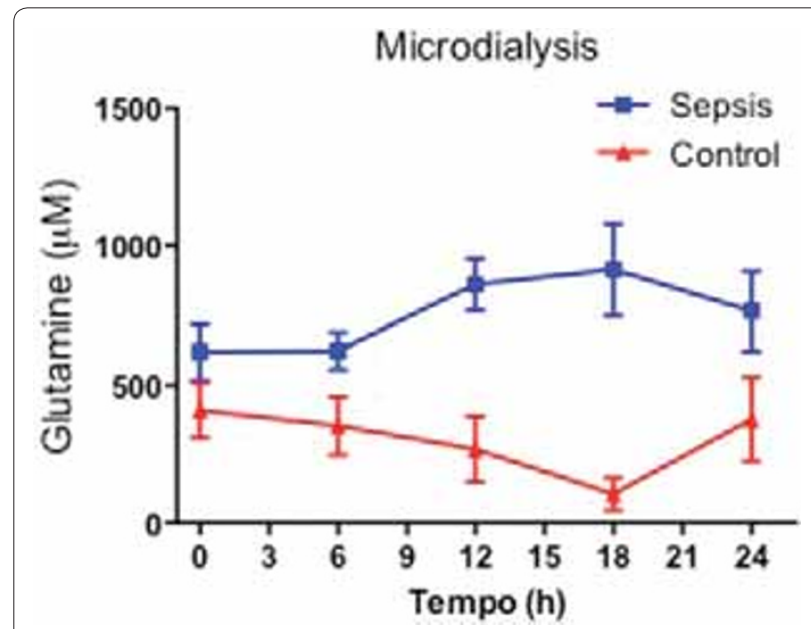

Figure 1 (abstract P23).

Results All septic animals developed a hyperdynamic state associated with lower arterial pressure, fever and organ dysfunction in comparison with control animals. In the septic animals, we observed increased brain dialysate glutamin levels at 12, 18 and 24 hours after sepsis induction, as compared with control animals. Moreover, after analyzing homogenates from the frontal cortex, we found higher concentrations of glutamin and glutamate in septic as compared with control animals $(85.67 \pm 14.98$ vs. $28.77 \pm 7.0 ; P=0.01$ and $132.1 \pm 19.72$ vs. $53.33 \pm 16.83 ; P=0.02$, respectively). See Figure 1 .

Conclusion We found higher concentrations of glutamate and glutamin in brain tissues of septic animals as compared with control. Furthermore, glutamin concentrations increased over time in the extracellular space as measured by cerebral microdialysis. These findings suggest an increased excitatory state that is potentially associated with high energy expenditure. However, associations with neuronal injury need further study.

P24

Cholinergic modulation of hippocampal activity during septic encephalopathy

A Zivkovic', CP Bengtson², O Sedlaczek', R Von Haken', H Bading'2, S Hofer ${ }^{1}$ 'Universitätsklinikum Heidelberg, Germany; ${ }^{2}$ Universität Heidelberg, Germany Critical Care 2013, 17(Suppl 2):P24 (doi: 10.1186/cc11962)

Introduction Septic encephalopathy is a sepsis-related brain dysfunction with a deterioration of cortical functions. The experimental studies in the rat brain revealed a deranged neurotransmitter profile during septic encephalopathy. Glutamatergic synapses, essential in learning and memory, undergo use-dependent changes in synaptic strength, referred to as plasticity. Permanent strengthening of synapses after a brief stimulus, termed long-term potentiation (LTP), was discovered in the hippocampus, and here it has been most thoroughly studied. Cholinergic neurotransmission plays an important role in regulating the cognitive functions of the brain. It acts as a signal-tonoise ratio modulator of sensory and cognitive inputs. The irregularities in brain functions give rise to the symptoms of delirium, including disorganized thinking and disturbances of attention and consciousness, which in turn might affect learning and memory. Possible mechanisms for cholinergic deficiency include impairment of synaptic functions of acetylcholine. Imbalances in the cholinergic system during sepsis might therefore play an extensive role in the septic delirium.

Methods By using MRI imaging we identified functional changes in the hippocampal region of patients with severe sepsis. This finding was further supported by the experimental recordings in the rat brains of lipopolysaccharide (LPS)-treated rats using the electrophysiological patch clamp technique.
Results Critically ill ICU patients diagnosed with septic delirium using the CAM-ICU method underwent diagnostic MRI scans. The serial MRI analysis revealed increased signal intensity in the hippocampal region in diffusion-weighted MRI (DWI). We used endotoxemia model to induce sepsis in the rats. Electrophysiological analysis of the hippocampal neurons in LPS-treated rats showed impaired LTP in the excitatory synapses, as compared with controls. Application of physostigmine, a blood-brain barrier permeable cholinesterase inhibitor, resulted in a partial recovery of LTP in the hippocampal synapses of LPS-treated rats. Conclusion The patients with septic delirium show functional changes in the hippocampus. Furthermore, we show that endotoxemia affects synaptic plasticity in the rat hippocampus, suggesting the involvement of this brain region in the pathophysiology of septic delirium. Moreover, the effect of the cholinergic neurotransmission onto the induction and maintenance of synaptic plasticity in the rat hippocampus during endotoxemia suggests that cholinergic neurotransmission might play a critical role in septic encephalopathy.

P25

Neutrophil-lymphocyte count ratio as a biomarker of severe sepsis in Escherichia coli infections in adults

LR Ljungstrom', G Jacobsson'1, R Andersson²

'Skaraborgs Sjukhus, Skövde, Sweden; '2Gothenburg University, Gothenburg,

Sweden

Critical Care 2013, 17(Suppl 2):P25 (doi: 10.1186/cc11963)

Introduction The neutrophil-lymphocyte count ratio (NLCR) is an easy to analyse biomarker reacting very early in the course of acute inflammation. It has previously been reported to correspond to bacteremia and recently to disease severity in community-acquired pneumonia [1]. We have looked at 205 consecutive patients with Escherichia coli infections (ECI) and found the same to be true for $\mathrm{ECls}$. This may be of great clinical importance since $E$. coli is the most frequently isolated pathogen in patients with infections requiring in hospital care.

Methods This study is part of a 9-month consecutive study of community-acquired severe sepsis and septic shock in adults at Skaraborg Hospital in the western region of Sweden. The hospital serves a population of 256,000 inhabitants and has approximately 60,000 annual visits to the ED. All patients admitted to the hospital receiving intravenous antibiotic treatment within the first 48 hours of admission were evaluated for severe sepsis and septic shock. Upon admission, two sets of blood cultures and other relevant cultures were obtained from each patient as well as sampling for NLCR and venous plasma lactate. The patient records were evaluated by one infectious diseases specialist. Approximately 2,300 patients were diagnosed as having a bacterial infection. From those, an informed consent to participate in the study could be obtained from approximately 1,600 patients.

Results Of the 1,600 patients who gave consent to participate in the study, 205 had an ECl. Sixty-four had a positive blood culture for E. coli. Fifty of the patients met one or more criteria for severe sepsis or septic shock. The NLCR was significantly higher $(P<0.001)$ within the severe sepsis group (median $=21.1$ with quartiles 11.1 to 42.4 ) compared with the group with no severe sepsis (median $=11.6$ with quartiles 7.6 to 18.9).

Conclusion The NLCR can be used as a biomarker of disease severity even in ECls. The biomarker reacts rapidly, is cheap and needs no extra sampling. The higher the value, the higher the probability for severe sepsis. A high value can even precede the development of severe sepsis or septic shock. However, a low value never excludes neither bacteremia nor severe sepsis. The method cannot be used in patients with disturbances in neutrophil or lymphocyte levels due to other causes than sepsis.

Reference

1. de Jager $C$, et al:: The neutrophil-lymphocyte count ratio in patients with community acquired pneumonia. PLOS ONE 2012, 7:e46561. 
P26

Obesity and inflammatory markers in severe sepsis

P Simon', D Thomas-Rüddel'2, T Nemes' ', K Reinhart ${ }^{2}$, F Bloos², UX Kaisers' 'University Hospital of Leipzig, Germany; ${ }^{2}$ Center for Sepsis Control and Care, Jena, Germany

Critical Care 2013, 17(Suppl 2):P26 (doi: 10.1186/cc11964)

Introduction Chronic inflammation has recently been recognized as an important factor in the pathophysiology of obesity and associated morbidities [1]. In this clinical study we aimed at identifying possible effects of obesity on inflammatory markers in severe sepsis.

Methods With institutional ethical committee approval, 243 consecutive patients treated for severe sepsis or septic shock in the ICUs of two university hospitals over a period of 5 months were studied. Six patients were excluded due to cachexia, syndromal disorders or missing clinical data. Diagnosis of sepsis was made according to SCCM criteria. Serum levels of C-reactive protein (CRP, $\mathrm{mg} / \mathrm{l}$ ) and procalcitonin (PCT, ng/ml) on day 1 of sepsis were compared among five body mass index (BMI) strata according to WHO definitions. Two groups (BMI $<30$, normal weight, and $\mathrm{BMI} \geq 30$, obesity) were formed for further analysis, and PCT was logarithmically transformed (LogPCT), resulting in normal distribution. Statistical analysis was performed using a $t$ test.

Results Patients with BMI $\geq 30$ had higher values of PCT and CRP (Table 1). The difference in LogPCT was of borderline significance $(P=$ 0.052). However, patients with positive blood cultures had significantly higher LogPCT values $(P=0.017)$ (Figure 1). Difference in CRP was not significant $(P=0.09)$. The trends over all five BMl strata (Table 1$)$ were not significant.

Conclusion Obesity with BMI $\geq 30$ seems to be associated with an increase in inflammatory markers in patients with severe sepsis, particularly in bacteraemia. The role of adipose tissue in severe sepsis should therefore be studied in more detail.

Reference

1. Wellen K, et al:: Inflammation, stress, and diabetes. J Clin Invest 2005, 115:1111-1119.

Table 1 (abstract P26). PCT and CRP as median (IQR)

\begin{tabular}{lccc}
\hline BMI & $\boldsymbol{n}$ & PCT $(\mathbf{n g} / \mathbf{m l})$ & CRP $(\mathbf{m g} / \mathbf{l})$ \\
\hline 18.5 to 24.9 & 196 & $4.8(11.9)$ & $153(189)$ \\
$\geq 30.0$ & 47 & $7.3(24.6)$ & $228(254)$ \\
18.5 to 24.9 & 101 & $4.9(11.7)$ & $147(175)$ \\
25 to 29.9 & 95 & $4.4(12.0)$ & $157(218)$ \\
30 to 34.9 & 32 & $6.9(22.6)$ & $212(118)$ \\
35 to 39.9 & 7 & $7.4(38.3)$ & $241(264)$ \\
$\geq 40.0$ & 8 & $11.4(32)$ & $278(272)$ \\
\hline
\end{tabular}

A

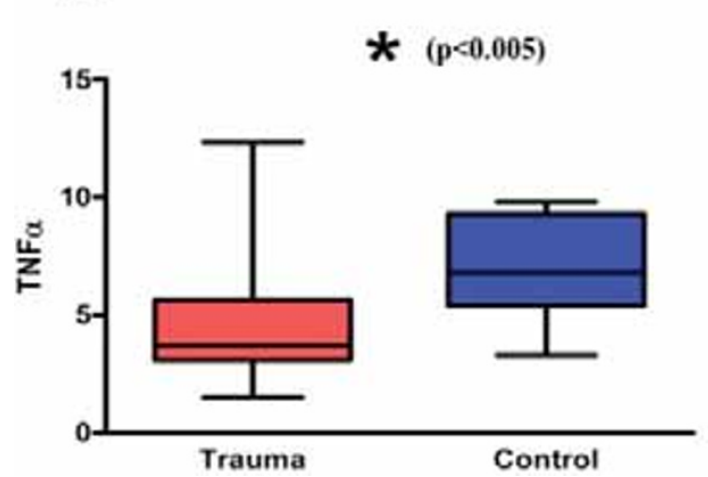

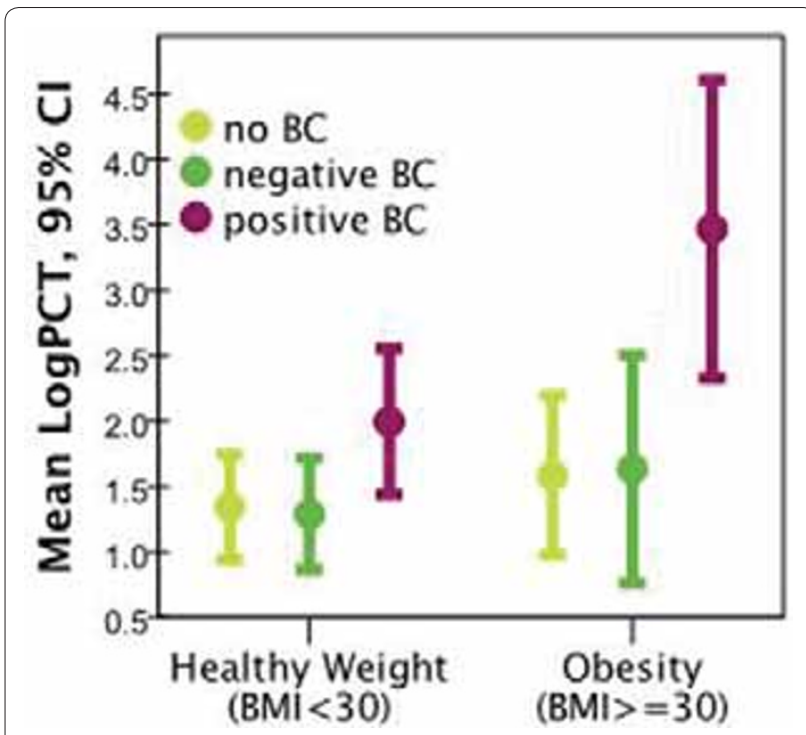

Figure 1 (abstract P26).

\section{P27}

Cytokine gene expression can predict infectious complications following severe trauma

HD Torrance, K Brohi, CJ Hinds, MJ O'Dwyer

Bart's Health NHS Trust, London, UK

Critical Care 2013, 17(Suppl 2):P27 (doi: 10.1186/cc11965)

Introduction Identifying a group of patients at high risk of developing infectious complications is the first step in the introduction of effective pre-emptive therapies in specific patient groups. Quantifying cytokine gene expression also furthers our understanding of trauma-induced immunosuppression. Our group has already demonstrated that a predictive immunological signature derived from mRNA expression in elective thoracic surgical patients accurately predicts pneumonia risk [1]. Methods In total, 121 ventilated polytrauma patients were recruited. mRNA was extracted from PaxGene tubes collected within 2 hours of the initial insult, at 24 and 72 hours. T-helper cell subtype specific cytokines and transcription factors mRNA was quantified using qPCR. Ten healthy controls served as a comparator.

Results The Median Injury Severity Score (ISS) was 29. Time 0 bloods demonstrated a reduction in $\mathrm{TNFa}^{\dagger}, \mathrm{IL}-12^{\S}, \mathrm{IL}^{\mathrm{L}}-23^{\ddagger}, \mathrm{ROR}^{\mathrm{T}}{ }^{*}$ and $\mathrm{T}$ bet ${ }^{\S}$, and an increase in IL-10* and IL-4 $4^{+}$mRNA levels in comparison with the control group ( ${ }^{*} P<0.0001,{ }^{\dagger} P<0.001$ to $0.0001,{ }^{\ddagger} P<0.01$ to $0.001,{ }^{\circledR} P<0.05$ to 0.01 ). There was a positive correlation between ISS and IL-10 $0^{\ddagger}$ whilst both IL-23 $3^{\S}$

Figure 1 (abstract P27). Cytokine mRNA levels in trauma (time 0) and control groups.

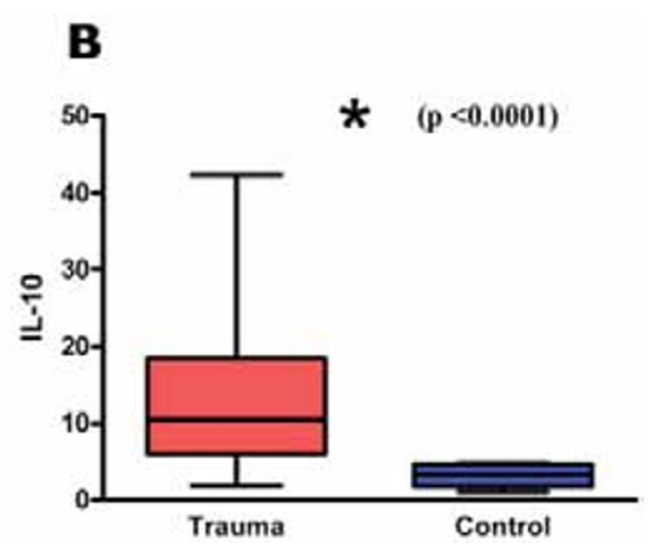


and $\mathrm{ROR} \mathrm{T}^{\ddagger}$ were negatively correlated at time $0 . \mathrm{TNFa}^{\dagger}, \mathrm{IL}-10^{*}$ and $\mathrm{IL}-27^{\ddagger}$ increased and IFNY', IL-12*, IL-17A ${ }^{\S}$, RORYT* and T bet* mRNA levels decreased over the initial 24 hours. Subsequent bacteraemia (18/121 patients) was associated with a lower TNFa/lL-10 ratio ${ }^{\ddagger}$ at baseline. Similarly, higher IL-10 $0^{\ddagger}$ and lower $\mathrm{T}$ bet $^{\ddagger} \mathrm{mRNA}$ at 24 hours also predicted later bacteraemic episodes. Development of pneumonia followed a similar pattern. A multivariate logistical regression model proved highly accurate in predicting infectious complications from mRNA analysis of early blood samples. See Figure 1.

Conclusion Cytokine gene expression patterns indicate an immediate and sustained impairment in Th1, Th17 and innate immunity with concurrent upregulation of the Th2 response following major trauma. The magnitude of this response predicts subsequent infectious complications.

Reference

1. White M, et al:: Chest 2011, 139:626-632.

P28

A cohort study of routinely used sepsis biomarkers and 28-day mortality

M De La Torre-Prados', A Garcia-De la Torre², C Trujillano-Férnández' 'Hospital Virgen de la Victoria, Málaga, Spain; ${ }^{2}$ Hospital Puerto Real, Cádiz, Spain

Critical Care 2013, 17(Suppl 2):P28 (doi: 10.1186/cc11966)

Introduction The evaluation of sepsis severity is complicated by the highly variable and nonspecific nature of clinical signs and symptoms. We studied routinely used biomarkers together with clinical parameters to compare their prognostic value for severe sepsis and evaluate their usefulness.

Methods A cohort study of 150 patients $>18$ years with severe sepsis according to the Surviving Sepsis Campaign, in an ICU of a university hospital. Demographic, clinical parameters and coagulation, infection and inflammation parameters during the first 24 hours from severe sepsis or septic shock onset were studied. Descriptive and comparative statistical analysis was performed using SPSS version 15.0 (SPSS Inc., Chicago, IL, USA).

Results We analyzed 150 consecutive episodes of severe sepsis (16\%) or septic shock (84\%) in the ICU. The median age of the patients was 64 (interquartile range, 48.7 to 71 ) years; the main sources of infection were intra-abdomen (45\%) and respiratory $(38 \%) ; 70.7 \%$ had medical diseases. The 28 -day mortality was $22.7 \%$. The profile of death patients were men $(64.7 \%, n=22)$, with significantly higher average age (63 vs. 57 years; $P=0.049$ ), as well as clinical severity scores, APACHE II (29.8 vs. $24.1 ; P<0.001)$ and SOFA $(12.1$ vs. 8.9; $P<0.001)$ and major dysfunction organ number ( 4.6 vs. $3.6 ; P<0.001)$. Bilirubin was the best predictor of 28-day mortality with the largest AUC (0.71), followed by hemoglobin $(0.69)$ and C3 (0.67). The multivariate logistic regression was adjusted for three risk parameters, hemoglobin (OR: $0.68 ; 95 \% \mathrm{Cl}: 0.51$ to 0.94 ), bilirubin (OR:1.63; $95 \%$ Cl: 1.08 to 2.45 ) and white blood cells (OR:1.04; $95 \% \mathrm{Cl}: 1.01$ to 1.08 ) and with these parameters a ROC analysis was performed, giving an AUC of 0.77 (0.69 to 0.84).

Conclusion The assessment of routine biomarkers (bilirubin, white blood cells and hemoglobin) may be a helpful tool in the decisionmaking process at the bedside, for the evaluation of early ICU admission of recoverable patients, as indicators of inflammatory response, organ dysfunction or catabolism level, and their significant predictive value on mortality.

Reference

1. Glickman SW, Cairns CB, Otero RM, et al:: Disease progression in hemodynamically stable patients presenting to the emergency department with sepsis. Acad Emerg Med 2010, 17:383-390.

P29

Procalcitonin as prognostic marker of mortality

S Zampieri', P Bettonte'2, M Ortolani', G Frison'1, V Schweiger1, L Gottin³,

E Polati'

'Policlinico G.B. Rossi, Verona, Italy: 2 Santa Chiara Hospital, Trento, Italy;

${ }^{3}$ Ospedale Maggiore Civile, Verona, Italy

Critical Care 2013, 17(Suppl 2):P29 (doi: 10.1186/cc11967)

Introduction We analyze procalcitonin (PCT) as a prognostic marker, in order to assess the clinical impact of a daily PCT measure.
Methods From November 2010 to November 2011 we collected clinical data, drug administration, scores and PCT values of 420 consecutive patients during hospitalization. Statistical analysis was made using SPSS software. We calculated ICU mortality, 1-month mortality and 1-year mortality. Median percentage daily variation was calculated as: (PCT day after - PCT of the date value) / PCT of the date valuex 100 . PCT variation in the last 48 hours of hospitalization was calculated as: (PCT at discharge - PCT at 48 hours before discharge) / PCT 48 hours before discharge $\times 100$. We compared peak values in dead patients versus alive patients. A logistic regression was performed in order to assess mortality odds ratio.

Results Of the 420 patients, 63 (15\%) died in the ICU, 12 (2.86\%) died 1 month after ICU discharge and $16(3.80 \%)$ died 1 year after ICU discharge. PCT values were higher during the last day of hospitalization in dead patients versus alive patients. PCT percentage variation during the last 48 hours of hospitalization had a slower trend in patients who died than in those who survived; these differences are even more marked in patients who had a septic event. A slower descending trend of daily PCT values was found in patients who died than in those who survived. PCT peak levels during the ICU stay were higher in dead patients with respect to alive ones. At logistic regression analysis PCT decrease in the last 48 hours $<-30 \%$ (OR 3.71), PCT peak higher than $10 \mathrm{ng} / \mathrm{ml}$ (OR 2.38), and PCT last day/PCT peak ratio $>50 \%$ (OR 2.064) were ICU mortality risk factors. PCT values were a higher predictive ICU mortality risk factor than SOFA and APACHE II scores. Other prognostic factors were age and lactate values. Only age was a risk factor in 1 -month and 1-year mortality.

Conclusion PCT is a good prognostic marker and is strongly correlated to the clinical status and gravity of the patients, so PCT seems to be a useful marker in an intensive care scenario.

References

1. Jensen JU, Heslet L, Jensen TH, et al.: Procalcitonin increase in early identification of critically ill patients at high risk of mortality. Crit Care Med 2006, 34:2596-2602.

2. Fritz HG, Brandes H, Bredle DL, et al: Post-operative hypoalbuminaemia and procalcitonin elevation for prediction of outcome in cardiopulmonary bypass surgery. Acta Anaesthesiol Scand 2003, 47:1276-1283.

P30

Changes in circulating procalcitonin versus C-reactive protein in predicting evolution of infectious disease in febrile, critically ill patients

S Hoeboer, 1,2 J Groeneveld 1,2

'VU University Medical Center, Amsterdam, the Netherlands; ${ }^{2}$ Erasmus

Medical Center, Rotterdam, the Netherlands

Critical Care 2013, 17(Suppl 2):P30 (doi: 10.1186/cc11968)

Introduction Although absolute values for C-reactive protein (CRP) and procalcitonin (PCT) are well known to predict sepsis in the critically ill, it remains unclear if and how changes in CRP and PCT predict evolution of infectious disease and how they compare in this respect.

Methods In 72 critically ill patients with new-onset fever, CRP and PCT were measured on day 0, 1,2 and 7 after inclusion, and their clinical course was documented over 1 week with follow-up to day 28. Infection was microbiologically defined, as was bloodstream infection; septic shock was defined as infection plus shock.

Results From peak at day 0 to 2 to day 7, CRP decreases most when (bloodstream) infection and septic shock (day 0 to 2 ) resolve and increases most when complications such as a new (bloodstream) infection or septic shock (day 3 to 7 ) supervene (area under the receiver operating characteristic curve 0.70 or higher, $P=0.04$ or lower). PCT decreases most when septic shock resolves (AUC 0.72, $P=0.007$ ) and increases most when a new bloodstream infection or septic shock supervenes (AUC 0.82 or higher, $P<0.001$ ). The day 7 value of $P C T$ rather than of CRP was predictive for 28-day outcome (AUC 0.70, $P=0.005$ ). Conclusion The data, obtained during ICU-acquired fever and infections, suggest that CRP and PCT changes predict the course of infectious disease and its complications. CRP may be favoured over PCT courses in decisions on appropriateness and duration of antibiotic treatment, whereas PCT rather than CRP courses may help predicting complications such as bloodstream infection, septic shock and mortality. 
P31

Procalcitonin-guided antibiotic therapy in patients with congestive heart failure and suspicion of lower respiratory tract infection: results from a randomized trial

P Schuetz, E Grolimund, A Kutz, S Haubitz, B Mueller

Kantonsspital Aarau, Switzerland

Critical Care 2013, 17(Suppl 2):P31 (doi: 10.1186/cc11969)

Introduction Differentiation of acute heart failure from infection in patients with respiratory symptoms and a history of congestive heart failure (CHF) is challenging due to overlap of clinical symptoms and $\mathrm{X}$-ray findings. The $\mathrm{BACH}$ study found higher mortality rates if patients presenting with dyspnea were treated with antibiotics and their procalcitonin (PCT) levels were low indicating absence of bacterial infection. Yet the BACH study was observational and causal inference cannot be drawn. Herein, we analyzed the effects of PCT-guided antibiotic stewardship in CHF patients from a previous trial (ProHOSP). Methods This is a secondary analysis of a previous randomized trial of adult ED patients with respiratory symptoms and a history of CHF. Patients were randomized to administration of antibiotics based on a PCT algorithm (PCT group) or standard guidelines without knowledge of PCT levels (control group). The primary endpoint of this analysis is the risk of adverse outcome defined as death or ICU admission within 30 days after ED admission.

Results A total of 233 patients met the inclusion criteria, with 116 in the PCT-guided group and 117 in the control group. In the subgroup of patients with low initial PCT levels $<0.25 \mathrm{ng} / \mathrm{l}(n=110)$, PCT-guided patients had a significant reduction in antibiotic exposure (mean 3.7 vs. 6.5 days, difference $-2.8(95 \% \mathrm{Cl}-4.4,-1.2), P<0.001)$. Furthermore, PCT-guided patients had a significant lower risk for death and ICU admission (4\% vs. $20 \%$, odds ratio $6.0(1.3,28.2), P=0.02)$. See Figure 1. Conclusion In CHF patients with suspicion of respiratory infection, use of a PCT protocol resulted in a significant decrease of antibiotic exposure and significantly improved outcomes in patients with low PCT levels indicating absence of bacterial infection. Whether inadequate antibiotic therapy in these CHF patients requiring diuretic treatment explains this difference in clinical outcomes needs verification.

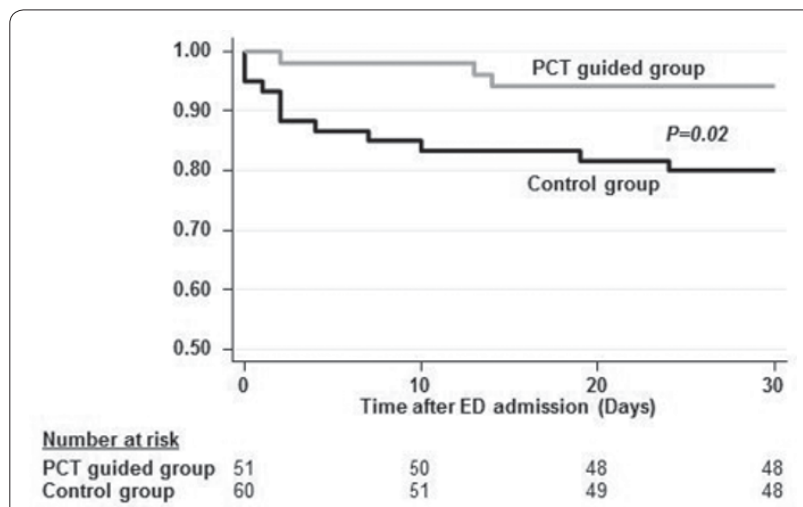

Figure 1 (abstract P31). Time to adverse outcome according to group allocation

P32

Pro-adrenomedullin as a clinical predictor after cardiac surgery

J Van Fessem, F De Graaf, J Van Paassen, S Arbous

LUMC, Leiden, the Netherlands

Critical Care 2013, 17(Suppl 2):P32 (doi: 10.1186/cc11970)

Introduction Pulmonary complications after cardiac surgery like ARDS are frequent and linked to high mortality [1]. Pro-adrenomedullin (pro-ADM) has a possible role in the development of ARDS [2] and a positive correlation between levels of pro-ADM and inflammation was found [3]. In this study we investigated whether intraoperative and postoperative pro-ADM transpulmonary gradient could predict postoperative morbidity.

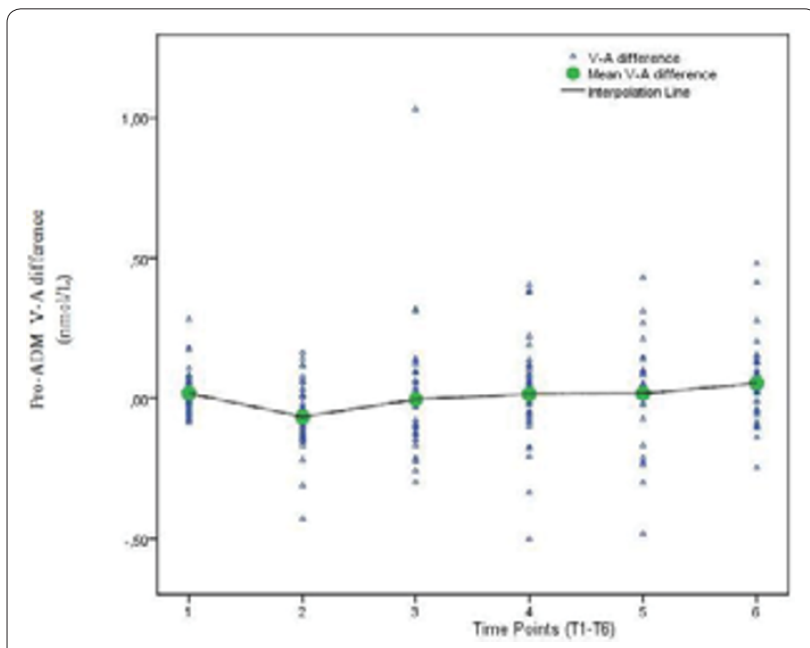

Figure 1 (abstract P32). Pro-ADM transpulmonary gradient at different time points.

Methods In this prospective cohort study, 39 patients undergoing cardiac surgery using CPB were included. Blood was collected before surgery (T0), after induction of anesthesia (T1), after termination of CPB (T2), at ICU arrival (T3) and 3 hours (T4), 6 hours (T5) and 18 hours (T6) after arrival. Pro-ADM was measured with a sandwich immunoassay. Primary endpoints were length of ICU and hospital stay (ICU-LOS, hospital-LOS).

Results An increase of arterial and venous pro-ADM plasma concentrations was observed after surgery. Immediately after termination of CPB the venous concentration was significantly lower than arterial proADM concentration, but at T6 the venous concentration was significantly higher, indicating a switch from a negative to positive transpulmonary gradient (Figure 1). The pro-ADM venous-arterial difference at T5 was a significant predictor of ICU-LOS $(P=0.032)$ and the difference at T3 was a significant predictor of hospital-LOS $(P=0.001)$.

Conclusion We found that the transpulmonary gradient of pro-ADM was a predictor for ICU-LOS and hospital-LOS at T3 and T5, respectively. Pro-ADM might be a promising marker for prediction on outcome of patients undergoing cardiac surgery on CPB. The transpulmonary shift of pro-ADM might be caused by an inflammatory response.

References

1. Apostolakis E, et al.: J Card Surg 2010, 25:47-55.

2. Kamei M, et al:: Acta Anaesthesiol Scand 2004, 48:980-985

3. Ueda S, et al:: Am J Respir Crit Care Med 1999, 160:132-136.

P33

Pro-adrenomedullin as prognostic biomarker in the sepsis

M De La Torre-Prados, A Garcia-De la Torre, A Enguix, M Mayor,

N Zamboschi, CTrujillano-Fernández, A Garcia-Alcantara

Hospital Virgen de la Victoria, Málaga, Spain

Critical Care 2013, 17(Suppl 2):P33 (doi: 10.1186/cc11971)

Introduction Measurement of biomarkers is a potential approach to early assessment and prediction of mortality in septic patients. The purpose of this study was to ascertain the prognostic value of proadrenomedullin (pADM), measured in all patients admitted to the ICU of our hospital with a diagnosis of severe sepsis or septic shock during 1 year.

Methods A cohort study of 117 patients $>18$ years with severe sepsis according to the Surviving Sepsis Campaign, in an ICU of a university hospital. Demographic, clinical parameters and pADM, C-reactive protein and procalcitonin were studied during 1 year. Descriptive and comparative statistical analysis was performed using the statistical software packages Statistica Stat Soft Inc 7.1 and MedCalc 9.2.1.0.

Results We analyzed 117 consecutive episodes of severe sepsis (15\%) or septic shock (85\%) in the ICU. The median age of the patients was 
64 (interquartile range, 53 to 72) years; the main sources of infection were respiratory tract $(46 \%)$ and intra-abdomen $(21 \%)$. The 28 -day mortality was $32.5 \%$. The profile of death patients had a significantly higher average age (64.7 vs. 57.6 years; $P=0.024)$, as well as clinical severity scores, APACHE II (26.6 vs. $23 ; P=0.006)$ and SOFA (11.6 vs. 89.2; $P<0.001)$. Kaplan-Meier survival analysis was significant. $P=0.0017$ for patients with $\mathrm{pADM}<1.2 \mathrm{nmol} / \mathrm{l}$. Cox regression analysis also showed statistical significance $(P=0.0033)$ and a likelihood ratio $=1.18$ per each $1 \mathrm{nmol} / \mathrm{l}$ increase in pADM.

Conclusion The protein PADM is an important prognostic biomarker of survival when measured on admission of septic patients to the ICU. References

1. Pezzilli R, Barassi A, Pigna A, et al:: Time course of proadrenomedullin in the early phase of septic shock. A comparative study with other proinflammatory proteins. Panminerva Med 2012, 54:211-217.

2. Wang RL, Kang FX: Prediction about severity and outcome of sepsis by proatrial natriuretic peptide and pro-adrenomedullin. Chin J Traumatol 2010, 13:152-157.

P34

Early IL-6 response in sepsis is correlated with mortality and severity score

P Srisangthong, A Wongsa, P Kittiworawitkul, A Wattanathum

Phramongkutklao Hospital, Bangkok, Thailand

Critical Care 2013, 17(Suppl 2):P34 (doi: 10.1186/cc11972)

Introduction IL-6, a proinflammatory cytokine, is synthesized from fibroblasts, T lymphocytes, endothelial cells and monocytes. It serves as an important mediator during the acute phase response to inflammation in sepsis. We hypothesized that the plasma IL-6 is correlated with mortality and severity scores in critically ill patients with sepsis.

Methods We conducted a prospective study of plasma IL- 6 level at the initial phase of sepsis and the risk of mortality. A total of 203 patients with sepsis, who were admitted to the medical ICU at Phramongkutklao Hospital, Bangkok during January to December 2011, were analyzed. Serum IL-6, C-reactive protein (CRP), and lactate were measured within the first 24 hours of ICU admission. Severity scores (APACHE II, SAP II, and SOFA scores) were measured. The primary outcome variable was 28-day all-cause mortality.

Results We found that the overall 28-day mortality was 46\% (93 out of 203 patients). There was a significantly positive correlation between mortality rate and plasma IL-6 (survivors vs. nonsurvivors; 74 (4.4 to 1,718) vs. 206 $(19$ to 5,000$) \mathrm{pg} / \mathrm{ml}, P<0.05)$, lactate (survivors vs. nonsurvivors; $1.65(0.7$ to 11.61$)$ vs. $2.47(0.94$ to 19.13$) \mathrm{mmol} / \mathrm{l}, P<0.05)$, but not CRP levels $(P=$ 0.24). Compared with the patients with plasma IL- $6<100 \mathrm{pg} / \mathrm{ml}$, septic patients with IL- 6 levels $\geq 100$ were associated with an increased 28-day mortality with the odd ratio of $2.99(95 \% \mathrm{Cl} 1.42$ to $6.29, P<0.05)$. We also found that plasma IL-6 levels were well correlated with APACHE II $(P<0.05)$, SAPS II $(P<0.05)$, and SOFA $(P<0.05)$ scores.

Conclusion The initial phase plasma IL-6 levels were correlated with severity and mortality in critically ill patients with sepsis.

\section{References}

1. Pathan N, et al:: Crit Care Med 2005, 33:1839-1844.

2. Harbarth S, et al:: Am J Respir Crit Care Med 2001, 164:396-402.

P35

Compared values of presepsin (sCD14-ST) and procalcitonin as early markers of outcome in severe sepsis and septic shock: a preliminary report from the Albumin Italian Outcome Sepsis (ALBIOS) study P Caironi', S Masson², ESpanuth ${ }^{3}$, R Thomae ${ }^{4}$, R Fumagalli', A Pesenti, M Romero' ${ }^{6}$, G Tognoni ${ }^{6}$, R Latini', L Gattinoni ${ }^{1}$

'Fondazione IRCCS Ca' Granda - Ospedale Maggiore Policlinico, Università degli Studi di Milano, Milan, Italy; 'Istituto di Ricerche Farmacologiche Mario Negri, Milan, Italy; ${ }^{3}$ Diagnostic Engineering \& Research GmbH, Heildelberg, Germany; ${ }^{4}$ Mitsubishi Chemical Europe $\mathrm{GmbH}$, Munich, Germany; ${ }^{5}$ Ospedale San Gerardo, Monza, Italy; ${ }^{6}$ Consorzio Mario Negri Sud, Santa Maria Imbaro, Italy

Critical Care 2013, 17(Suppl 2):P35 (doi: 10.1186/cc11973)

Introduction Sepsis results from complex interactions between infecting microorganisms and host responses, often leading to multiple organ failures and death. Over the years, its treatment has been standardized in early goal-oriented therapies, which may benefit from circulating biomarkers for early risk stratification. We aimed to evaluate the prognostic value of presepsin (sCD14-ST), a novel marker of bacterial infection.

Methods We performed a nested case-control study from the randomized controlled Albumin Italian Outcome Sepsis (ALBIOS) trial, enrolling patients with severe sepsis or septic shock from 100 ICUs in Italy. Fifty survivors and 50 nonsurvivors at ICU discharge were selected, matched for age, sex, center and time of enrollment after inclusion criteria were present. EDTA-plasma samples were collected at days 1, 2 and 7 after enrolment for presepsin (immunechemiluminescence assay PATHFAST Presepsin, URL 320 pg/ml, CV 5\%; Mitsubishi Chemicals) and procalcitonin assay (PCT, Elecsys BRAHMS Cobas $^{\oplus}$ PCT, URL $0.046 \mathrm{ng} / \mathrm{ml}$, CV 8.8\%; Roche Diagnostics).

Results Clinical characteristics were similar between the two groups, except for a worse SOFA score at day 1 in decedents. Presepsin at day 1 was significantly higher in decedents $(2,268(1,145$ to 4,305$) \mathrm{pg} /$ $\mathrm{ml}$, median (Q1 to Q3)) than in survivors $(1,184(855$ to 2,158$) \mathrm{pg} / \mathrm{ml}$, $P=0.001$ ), while PCT did not differ (18.5 (3.3 to 45.7 ) vs. 10.8 ( 2.6 to 46.4) $\mathrm{ng} / \mathrm{ml}, P=0.31)$. Presepsin decreased over time in survivors, but remained elevated in decedents (974 (674 to 1,927$)$ vs. $2,551(1,438$ to 5,624$) \mathrm{pg} / \mathrm{ml}$ at day $7, P=0.02$ for time-survival interaction); PCT decreased similarly in the two groups $(P=0.19)$. Patients with early elevated presepsin had worse SOFA score, higher number of MOFs, hemodynamic instability (lower mean arterial pressure at baseline and after 6 hours), and mortality rate at 90 days (75\% vs. $42 \%$, logrank $P<0.001$ ). The association between presepsin and outcome was more marked in patients with late enrollment (6 to 24 hours), and in septic shock. Early presepsin had better prognostic accuracy than PCT (AUROC 0.69 vs. $0.56, P=0.07$ ), and improved discrimination over SOFA score, especially in septic shock.

Conclusion Early presepsin measurements may provide important prognostic information in patients with severe sepsis or septic shock, and may be of crucial importance for early risk stratification.

P36

Usefulness of presepsin in the diagnosis of sepsis in acute kidney injury patients

Y Nakamura, H Ishikura, T Nishida, Y Kawanno, R Yuge, R Ichiki, A Murai Fukuoka University, Fukuoka, Japan

Critical Care 2013, 17(Suppl 2):P36 (doi: 10.1186/cc11974)

Introduction The level of presepsin is useful for differentiating sepsis from noninfectious systemic inflammatory response syndrome. It has been reported that the presepsin levels in patients with chronic renal failure are abnormally high. However, there are no studies investigating the usefulness of presepsin for diagnosis of sepsis in patients with acute kidney injury (AKI). Our purpose of this study is to clarify the diagnostic accuracy of presepsin in patients with AKI and the relationship between presepsin level and AKI severity.

Methods This study was conducted as a single-center retrospective study. Blood samples were collected from patients admitted to the emergency room at Fukuoka University Hospital between June 2010 and October 2012. We enrolled 254 patients with suspected sepsis and other disease patients. We classified the patients into an AKI group according to the RIFLE criteria (Risk $n=52$, Injury $n=39$, Failure $n=41$, Loss of kidney function and End-stage kidney disease $n=7$ ) and a nonAKI group $(n=115)$. The AKI patient group was further classified into a sepsis group and a nonsepsis group in each AKI stage and we analyzed the diagnostic accuracy of presepsin in patients with sepsis.

Results For the non-AKI patients, the median of presepsin in patients with nonsepsis $(n=78)$ and the sepsis group $(n=37)$ were $406 \mathrm{pg} / \mathrm{ml}$ (range: 86 to 4,374 ) and $1,065 \mathrm{pg} / \mathrm{ml}$ (range 86 to 9,960 ), respectively $(P<0.0001)$. For the Risk patients, the median of presepsin in patients with nonsepsis $(n=25)$ and the sepsis group $(n=27)$ were $299 \mathrm{pg} / \mathrm{ml}$ (range: 71.2 to 3,361 ) and $831 \mathrm{pg} / \mathrm{ml}$ (range: 233 to 16,759 ), respectively $(P<0.01)$. For the Injury patients, the median of presepsin in patients with nonsepsis $(n=12)$ and the sepsis group $(n=27)$ were $463 \mathrm{pg} / \mathrm{ml}$ (range: 122 to 1,197$)$ and $1,451 \mathrm{pg} / \mathrm{ml}$ (range: 237 to 4,200 ), respectively $(P<0.001)$. For the Failure patients, the median of presepsin in patients 
with nonsepsis $(n=14)$ and the sepsis group $(n=27)$ were $1,607 \mathrm{pg} / \mathrm{ml}$ (range: 454 to 8,516 ) and $1,523 \mathrm{pg} / \mathrm{ml}$ (range: 293 to 16,764 ), respectively $(P=0.175)$. The diagnostic accuracy of presepsin in patients with sepsis was determined by ROC analysis, the area under the curve was 0.789 for the non-AKI patient group, 0.735 for the Risk patient group, 0.855 for the Injury patient group and 0.593 for the Failure patient group.

Conclusion In Failure and more progressed AKI patients, the diagnostic accuracy of the presepsin level was lower than the other groups.

References

1. Endo S, et al:: I Infect Chemother 2012, 18:891-897.

doi:10.1007/s10156-012-0435-2

2. Bellomo R, et al.: Crit Care 2004, 8:R204-R212. doi:10.1186/cc2872

P37

Serum levels of presepsin reflects the APACHE II and SOFA scores in patients with sepsis

R Sato, Y Suzuki, M Sato, G Takahashi, M Kojika, Y Inoue, S Endo

I wate Medical University, Morioka, Japan

Critical Care 2013, 17(Suppl 2):P37 (doi: 10.1186/cc1 1975)

Introduction As a method for the diagnosis of sepsis, we previously reported an ELISA method for measuring the serum levels of presepsin. That method, however, took approximately 2 hours to yield results.

Methods To resolve this problem, we later developed a simplified assay kit making use of immunochromatography (Point of Care test; POC test), and are currently evaluating the usefulness of this kit for diagnosis of sepsis and evaluation of its severity. In 21 septic patients with sepsis, we analyzed the serum levels of presepsin in relation to APACHE II and SOFA scores.

Results APACHE II and SOFA scores at baseline were $28.7 \pm 7.5$ and $10.8 \pm 3.9$, respectively, in these patients. These were significant correlations between the serum presepsin levels and the APACHE II score, and also between the serum presepsin levels and the SOFA score. Conclusion Furthermore, there was also a significant correlation between the results of the POC test and the serum presepsin levels. These results indicate that measurement of the serum presepsin might be useful for evaluating the severity of sepsis.

P38

Relationship between serum nitrite/nitrate levels in the early phase of septic acute lung injury and prognosis

M Sato, Y Suzuki, T Masuda, G Takahashi, M Kojika, Y Inoue, S Endo

I wate Medical University, Morioka, Japan

Critical Care 2013, 17(Suppl 2):P38 (doi: 10.1186/cc11976)

Introduction Serum nitrite/nitrate (NOx) levels in the early phase of septic acute lung injury (ALI)/acute respiratory distress syndrome (ARDS) have recently been reported to possibly play a key role in pathogenesis of ALI/ARDS.

Methods NOx levels in the early phase of septic ALI/ARDS were measured by autoanalyzer (TCI-NOX 1000; Tokyo Kasei Kogyo Co., Ltd, Tokyo, Japan). Cytokine was measured by ELISA (Medogenix, Fleurus, Belgium).

Results Both NOx and TNFa levels were significantly higher in the ARDS group than in the ALI group. A negative correlation was found between the $\mathrm{PaO} / \mathrm{FIO}_{2}$ (P/F) ratio and serum NOx levels. In addition, a positive correlation was found between the TNFa and serum NOx levels. The 30-day, 60-day and 90-day mortality rates were $8.7 \%, 15.2 \%$ and $19.6 \%$, respectively, in the patients with ALI/ARDS. There were no differences in the P/F ratio, serum NOx levels or TNFa levels in the early phase of ALI/ARDS between the 30-day survival and death groups. On the other hand, the P/F ratio, serum NOx levels and TNFa levels in the early phase of ALI/ARDS were significantly higher in the 60-day and 90-day death groups than in the corresponding survival groups. There were no significant differences in the 90 -day mortality rates between the ALI and ARDS groups.

Conclusion Our findings suggested that NOx may be involved in the pathogenesis of ALI/ARDS.
P39

Simple and fast prediction of Legionella sp. in community-acquired pneumonia: validation of a prediction rule

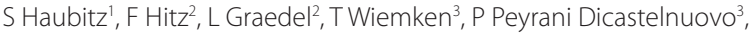

J Ramirez', M Batschwaroff', C Fux', B Mueller', P Schütz

'Kantonsspital Aarau, Switzerland; ' University of Basel, Switzerland;

${ }^{3}$ University of Louisville School of Medicine, Louisville, KY, USA

Critical Care 2013, 17(Suppl 2):P39 (doi: 10.1186/cc11977)

Introduction Ruling out Legionella $\mathrm{sp}$. in patients presenting with community-acquired pneumonia (CAP) is important due to differences in treatment regimens. Yet antigen tests as well as blood cultures have low sensitivity and an important time delay, making empirical broad spectrum coverage necessary particularly in severe cases. Fiumefreddo and colleagues recently proposed a clinical score based on six clinical and laboratory variables (fever, cough, sodium, lactate-dehydrogenase, C-reactive protein, platelet count) which allowed assessing the likelihood of Legionella [1]. Yet these variables need validation in an independent patient cohort before implementation into clinical routine.

Methods We analyzed data from a large multinational database of patients with CAP (CAPO) [2] between 2001 and 2012. We performed logistic regression analysis and the area under the receiver operating characteristics (AUC) curve to study the association of these variables with the diagnosis of Legionella.

Results Data for 8,278 CAP patients were analysed; the infectious organism was known in 2,321 cases (28\%), including a total of 101 patients with urinary antigen-confirmed Legionnaires' disease and 983 patients with confirmed pneumococcal disease. All variables were predictors for Legionella with odds ratios ranging from 1.002 to 5.767 . Combining the variables in a joint logistic regression model showed a high predictive accuracy with an AUC of 0.86 .

Conclusion This analysis validates the Legionella score in an independent sample and shows high diagnostic accuracy. Interventional trials with adapted antibiotic regimes for non-inferiority in a real live population are warranted.

References

1. Fiumefreddo R, Zaborsky R, Haeuptle J, et al:: Clinical predictors for Legionella in patients presenting with community-acquired pneumonia to the emergency department. BMC Pulm Med 2009, 9:4.

2. Ramirez JA: Fostering international multicenter collaborative research: the CAPO Project. Int J Tuberc Lung Dis 2007, 11:1062-1065.

P40

Host genetic variants associated with community-acquired pneumonia

LE Salnikova', TV Smelaya', WV Moroz', A Golubev', AV Rubanovich²

'V.A. Negovsky Research Institute of General Reanimatology, Moscow, Russia; ${ }^{2}$ N.I. Vavilov Institute of General Genetics, Russian Academy of Sciences,

Moscow, Russia

Critical Care 2013, 17(Suppl 2):P40 (doi: 10.1186/cc11978)

Introduction It has been shown that polymorphic variants at some host genes can modify risk of community-acquired pneumonia (CAP), including those critical for the host response to CAP - innate immune system, the lung's defense against inhaled microorganisms, and inhibition of fibrinolysis and the renin-angiotensin system. The aim of the study was to analyze polymorphisms in genes potentially relevant to CAP pathogenesis mechanisms to reveal novel and confirm reported genetic risk factors in the general Russian population.

Methods Patients with CAP $(n=334)$, volunteers without a previous history of CAP, control group A $(n=141)$ and a second control group $\mathrm{B}(n=314)$ were included in the study. Using allele-specific tetraprimer $\mathrm{PCR}$, all subjects were genotyped for 13 polymorphic variants in the genes of xenobiotic detoxification CYP1A1 (rs2606345, rs4646903, rs1048943), GSTM1 (Ins/Del), GSTT1 (Ins/Del), ABCB1 (rs1045642); immune and inflammation response IL-6 (rs1800795), TNFa (rs1800629), MBL2 (rs7096206), CCR5 (rs333), NOS3 (rs1799983), angiotensin-converting enzyme (ACE; rs4340), and occlusive vascular disease/hyperhomocysteinemia MTHFR (rs1801133). 
Results Seven genes CYP1A1 rs2606345T/T, GSTM1 Ins/*, ABCB1 C/C, IL-6 C/C-G/G, NOS3 T/T-G/G, CCR5 Ins/Ins, and ACE Del/Del were associated with CAP. The highest effect was detected for the CYP1A1 rs2606345: in comparison with the control $A, P=3.9 \times 10^{-5}, O R=$ $2.40,95 \% \mathrm{Cl}: 1.59$ to 3.64 ; and in comparison with the control $\mathrm{B}, P=$ $1.4 \times 10^{-5}, \mathrm{OR}=2.0,95 \% \mathrm{Cl}: 1.46$ to 2.74 . For the two genes $\mathrm{CYP} 1 \mathrm{~A} 1$ and GSTM1, associations remained significant after correction for multiple comparisons. Multiple analysis by the number of all risk genotypes showed a highly significant association with CAP $\left(P=2.4 \times 10^{-7}, \mathrm{OR}=\right.$ $3.03,95 \% \mathrm{Cl} 1.98$ to 4.64 ) with the threshold for three risk genotypes. Using the ROC analysis, the AUC value for multi-locus model was estimated as 68.38, which is rather high for genetic markers.

Conclusion We have provided the first experimental evidence for the associations of genes coding detoxification enzymes with the risk of CAP. Our results also demonstrate that predisposition to CAP is strongly attributed to the effects of a number of genes with low penetrance and therefore imply that inter-locus interactions may be regarded as an important component of polygenic and multifactorial factors of susceptibility to CAP.

\section{P41}

Outcome of severe community-acquired pneumonia: the impact of comorbidities

JM Pereira', JA Paiva', F Froes², JP Baptista ${ }^{3}$ J Gonçalves-Pereira'

'Centro Hospitalar S. João, Porto, Portugal; ${ }^{2}$ Hospital Santa Maria-CHLN,

Lisboa, Portugal; ${ }^{3}$ Hospitais da Universidade de Coimbra - CHUC, Coimbra,

Portugal; ${ }^{4}$ Hospital São Francisco Xavier, Lisboa, Portugal

Critical Care 2013, 17(Suppl 2):P41 (doi: 10.1186/cc11979)

Introduction Several comorbidities have been independently associated with both predisposition to community-acquired pneumonia and a worse outcome. The goal of this study was to evaluate the impact of comorbidities on the outcome of patients with severe communityacquired pneumonia (SCAP).

Methods A prospective, multicentre, observational cohort study of all patients with SCAP consecutively admitted to 14 Portuguese ICUs during a 12-month period. Several comorbidities were evaluated: congestive heart failure, cancer, chronic renal failure, chronic respiratory failure, chronic hepatic disease, alcoholism, diabetes mellitus, neurologic disease, immunosuppression, HIV infection. To evaluate the impact of comorbidities associated with hospital mortality in univariate analysis, a logistic regression analysis adjusted to other variables (clinical relevant or statistically significant in univariate analysis) was performed.

Results A total of $536(14 \%)$ of the 3,766 enrolled patients had SCAP. They were mostly male $(66 \%)$ with median age 59 (29 to 82$)$ years, median SAPS II 44 ( 21 to 80 ) and total SOFA score 8 (3 to 16). Thirtyseven per cent of the cases were microbiologically documented (St. pneumoniae - 24\%; Enterobacteriaceae - 20\%; influenza A (H1N1) virus $-18 \%$ ) and $45 \%$ had septic shock. Antibiotic combination was used in $76 \%$ of the patients and $61 \%$ received a macrolide. Median hospital length of stay was 19 (3 to 70) days and hospital mortality was $35 \%$. Comorbidities were present in $70 \%$ of the patients. The most frequent were: diabetes mellitus $(21 \%)$, chronic respiratory failure (18\%) and alcoholism (15\%). Median Charlson's comorbidity index (CCI) was 4 (0 to 13). In univariate analysis, the presence of at least one comorbidity (odds ratio (OR) $2.29 ; 95 \% \mathrm{Cl} 1.49$ to 3.52 ), namely cancer (OR 3.80; $95 \% \mathrm{Cl} 2.14$ to $6.74 ; P<0.001$ ), chronic renal failure (OR 3.23 ; $95 \% \mathrm{Cl} 1.53$ to $6.82 ; P=0.001)$, immunosuppression (OR $2.12 ; 95 \% \mathrm{Cl}$ 1.15 to $3.92 ; P=0.014)$ and neurologic disease (OR $1.87 ; 95 \% \mathrm{Cl} 1.10$ to 3.17; $P=0.02$ ), increased the chances of dying in the hospital. Median $\mathrm{CCl}$ was also significantly higher in nonsurvivors ( 5 vs. $3 ; P<0.001$; OR per point 1.10 (95\% Cl: 1.05 to 1.15)). The only independent risk factor for hospital mortality was the presence of at least one comorbidity (OR 2.09; $95 \% \mathrm{Cl} 1.13$ to 3.85 ).

Conclusion In SCAP, the presence of at least one comorbidity doubles the chances of dying in the hospital and is an independent risk factor for hospital mortality.
P42

Prognostic scores and infective endocarditis

P Fernandez Ugidos'1, R Gomez Lopez'1, P Vidal Cortes',

A Ceniceros Barros', L Seoane Quiroga', JM Lopez Perez²

${ }^{1}$ Complejo Hospitalario Universitario Ourense, Spain; ${ }^{2}$ Complejo Hospitalario Universitario A Coruña, Spain

Critical Care 2013, 17(Suppl 2):P42 (doi: 10.1186/cc11980)

Introduction Infective endocarditis (IE) is a high-mortality disease, especially in the early surgery subgroup. The aim of this study was try to identify prognostic factors of IE that will require surgery during the same admission of their diagnosis, including evaluation of surgical severity scores.

Methods A retrospective study (5 years) of all patients admitted to a tertiary hospital in northern Spain with diagnosis of IE (modified Duke criteria) who required early surgery. Demographic, clinical and microbiology data were collected. Chi-square and Student $t$ tests. Significance: $P<0.05$. SPSS17.

Results We had 73 patients, $79.5 \%$ men, age 65 years. Eighty-two percent had positive blood cultures. Forty-one percent of cases required previous ICU admission. Surgery was urgent in 35\%. Fifty-six per cent of patients had postoperative shock and $58 \%$ suffered postsurgery ARF. Hospital mortality was $31.5 \%$. Regarding prognostic scales: the mean EuroSCORE was $11.38 \pm 3.93$ points. No patient was placed in the low-risk group. Ninety-four per cent of cases were at high risk. The Parsonnet mean score was $27.7 \pm 11.6$. The mean Beth Israel Medical Center was $37.6 \pm 10$ points. The mean Ontario scale was $7.09 \pm 2.7$. The mean Surgical risk scale of Roques was 6.69. And the mean by Pons Scale was 24.02. Dead patients are older, with previous heart disease, require urgent surgery and have previous ICU stay; usually they have MOF and they had received less than 7 days of antibiotic treatment. The etiology did not worsen the prognosis.

Conclusion In early surgery IE, it appears to be associated with mortality: age $>70$ years, previous heart disease, emergency surgery, antibiotics within 7 days before surgery, preoperative MOF, and high scores on scales of Pons and Ontario. The causal agent and echocardiography have no relation with worse prognosis. The general syndrome debut and the Streptococcus spp. etiology seem to have lower mortality. IE has pathologies with high . preoperative severity scores but these are not sufficient to guide therapeutic decisions.

P43

Effectiveness of early ureteric stenting for urosepsis associated with urinary tract calculi

S Nishiguchi', Y Tokuda²

'Shonan Kamakura General Hospital, Kamakura, Japan: ${ }^{2}$ Mito Kyodo General Hospital, Mito, Japan

Critical Care 2013, 17(Suppl 2):P43 (doi: 10.1186/cc11981)

Introduction Urosepsis associated with urinary tract calculi is a critical disease. Patients with this condition occasionally require drainage, mostly ureteric stent placement and these patients need longer hospitalization. However, indications for timely ureteric stenting for urosepsis associated with urinary tract calculi have not been clearly determined. The objective of the study was to evaluate whether earlier stent placement might lead to shorter length of stay (LOS) in hospitals. Methods For patients who required ureteric stent procedures for urosepsis associated with urinary tract calculi in our hospital from July 2008 to July 2012, we compared the LOS in our hospital between patients with earlier stenting and those without it. A linear regression model was used for multivariable-adjusted comparison.

Results In a total of 30 patients (mean age, $72 ; 13$ males), the mean days from emergency room admission to ureteric stenting was 3.5 days (range, 1 to 14 days) and the overall mean LOS was 36 days (range, 8 to 102 days). The early-stenting group (mean LOS, 21 days) had significantly shorter LOS than the delayed-stenting group (mean LOS, 50 days) with adjusted $\beta$ coefficient of -26 days $(95 \% \mathrm{Cl},-46,-6)$.

Conclusion In patients with urosepsis associated with urinary tract calculi, earlier stenting within 2 days of admission may reduce the hospital LOS.

References

1. Yamamoto Y, et al:: BMC Urol 2012, 12:4

2. Yoshimura K, et al:: J Urol 2005, 173:458-462. 
P44

Outcomes in urinary sepsis

C Joya-Montosa, ETrujillo-Garcia, MJ Delgado-Amaya, E Curiel-Balsera

Hospital Regional Carlos Haya, Málaga, Spain

Critical Care 2013, 17(Suppl 2):P44 (doi: 10.1186/cc11982)

Introduction Analysis of mortality-related factors in urinary sepsis patients.

Methods A retrospective descriptive study of urologic sepsis patients in the ICU from 2008 to 2010. Clinical, epidemiological and outcome variables were analysed. Quantitative variables are expressed as either mean and standard deviation or as median and interquartile range for asymmetric variables. Qualitative variables are expressed as percentages and absolute values. Mann-Whitney's $U$ test and Fisher's exact test were applied (a error was 5\% in both cases), as well as binary logistic regression for multivariate analysis.

Results There was a total number of 44 patients (aged $59.39 \pm 17.71$; $63.8 \%$ females). APACHE II score upon admission was $18 \pm 6$. Out of these patients, $27.3 \%$ showed no underlying disorder and $18.2 \%$ (no = 8) showed chronic renal failure; $25 \%$ were immunodepressed patients; $31 \%$ underwent urinary instrumentation in the previous 15 days, yet only three of them had undergone permanent urine catheterization. Observed mortality was $25 \%$, while sepsis-related mortality was $22.7 \%$. The patients who died were, on average, older that those who survived $(67.9 \pm 710.2$ vs. $56.8 \pm 18.7 ; P=0.02)$. Besides, the former also reported greater delay in turning to the hospital after symptom onset (13.4 \pm 6.6 vs. $6.2 \pm 4.7$ days; $P=0.0001)$. Immunodepressed patients presented higher mortality rate: OR $8.7(95 \% \mathrm{Cl} 1.7$ to 42.3$)$, as well as those who underwent inappropriate initial antibiotic treatment: OR 10.8 (95\% Cl 2.1 to 54.7). No relation was observed between germ typology or resistance to $\beta$-lactam antibiotics and mortality. After adjustment of mortality due to APACHE II score upon admission, delay in the onset of appropriate antibiotic treatment was an independent predictor of mortality in our patients: OR $1.2,95 \% \mathrm{Cl}(1.02$ to 1.42$)$.

Conclusion Urinary sepsis mortality is associated with late-onset and/ or inappropriate antibiotic use, as well as with immunodepression and advanced age.

References

1. Clec'h C, Schwebel C, Français A: Does catheter-associated urinary tract infection increase mortality in critically ill patients. Infect Control Hosp Epidemiol 2007, 28:1367-1373.

2. Marx G, Reinhart K: Urosepsis: from the intensive care viewpoint. Int J Antimicrob Agents 2008, 315:579-S84.

P45

Nosocomial pneumonia in the postoperative period after heart transplantation

R Gómez López'1, P Fernández Ugidos', P Vidal Cortes', M Bouza Vieiro², J Miñiz², S Fojon Polanco², M Paniagua Martin², R Marzoa Rivas², E Barge Caballero², M Crespo Leiro ${ }^{2}$

${ }^{1}$ Complexo Hospitalario Universitario de Ourense, Spain; ${ }^{2}$ Complexo Hospitalaro Universitario de A Coruña, Spain

Critical Care 2013, 17(Suppl 2):P45 (doi: 10.1186/cc1 1983)

Introduction Infections are a major complication during the postoperative period after heart transplantation (HT). In our hospital, nosocomial pneumonia is the most frequent infection in this period. The objective of this study is to determine the epidemiological and microbiological characteristics of this disease in our centre.

Methods A descriptive retrospective study of all medical records of HT performed in a single institution from 1991 to 2009 followed until June 2010. Clinical and microbiological variables were considered. Centre for Diseases Control (CDC) criteria were used to define nosocomial infections. Invasive aspergillosis was considered if there were criteria for probable aspergillosis according to IDSA criteria.

Results In $594 \mathrm{HTs}$ there were 97 infectious episodes in 75 patients (12.6\%). Eighty-five patients (14.3\%) died during hospitalization. Infection is the second cause of mortality during the postoperative period $(17.9 \%$ of dead patients). The most common locations of infections were pneumonia ( $n=31,31.9 \%$ of infection episodes), bloodstream ( $n=24,24.7 \%)$, urinary tract $(n=14,14.4 \%)$, surgical site $(n=13,13.4 \%)$ and intraabdominal infections ( $n=13,13.4 \%)$. Patients with pneumonia were treated according to knowledge in a specific moment, thus different antibiotics were used. The duration of antibiotic therapy was $20 \pm 15.5$ days. In nine episodes of pneumonia according to the CDC no germ was isolated in the cultures. Six of the episodes were polymicrobial infections. The most frequent microbes isolated were E. coli $(n=7,22.5 \%$ of pneumonia cases), A. fumigatus ( $n=7$, $22.5 \%)$, S. aureus $(n=3,9.68 \%)$, P. aeruginosa $(n=3,9.68 \%)$, P. mirabilis, K. pneumoniae, E. cloacae, E. faecalis, C. glabrata, and S. marcescens (one case each, 3.22\%). Pneumonia was suspected but not confirmed in 75 patients. Despite this, antibiotic treatment was maintained for a media of $17.35 \pm 7.01$ days: 56 wide-spectrum treatments and 18 targeted therapy after knowing the antibiogram. The length of ICU stay was $38.4 \pm 70.8$ ( 3 to 264 ) days, of hospital stay was $66.2 \pm 80.5$ ( 3 to 304 ) days and of mechanical ventilation was $27.3 \pm 50.2$ ( 3 to 264 ) days. The mortality of patients with pneumonia was $32.3 \%$.

Conclusion Nosocomial pneumonia is the most frequent infection in our series. Despite when infection was not confirmed, antibiotic therapy was maintained in suspect cases. We found a high incidence of aspergillosis. Limitations because of wide duration of this study should be considered.

P46

Abdominal infection plays a role in the incidence of ventilatorassociated pneumonia

GB Bouroche', S Ruckly², B Misset ${ }^{3}$, JF Timsit', F Philippart ${ }^{3}$

'Institut Gustave Roussy, Villejuif, France; 'Institut Albert Bonniot, La Tronche,

France; ${ }^{3}$ Hôpital Saint Joseph, Paris, France; ${ }^{4} \mathrm{CHU}$ Grenoble, France

Critical Care 2013, 17(Suppl 2):P46 (doi: 10.1186/cc11984)

Introduction Despite many therapeutic interventions, ventilatoracquired pneumonias (VAP) are frequent in the ICU and are associated with major morbidity and mortality. Sepsis causes a time-dependent modification of the inflammatory response. This reprogramming could promote the occurrence of a secondary infection and worsen the prognosis. In animals, peritonitis is associated with an alteration of pulmonary immunity and an increasing mortality from secondary pneumonia.

Methods To investigate, in humans, the potential involvement of previous intra-abdominal infection (IIA) in preventing or promoting VAP, we realized a prospective observational study using data from a multicenter database (OUTCOMEREA), including all patients admitted

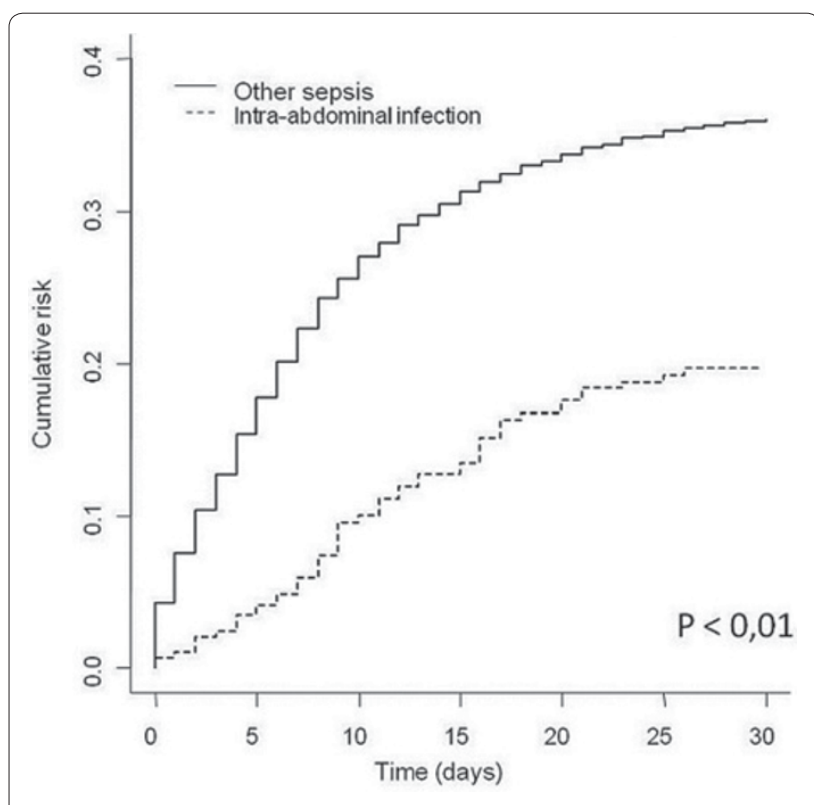

Figure 1 (abstract P46). Cumulative incidence of VAP. 


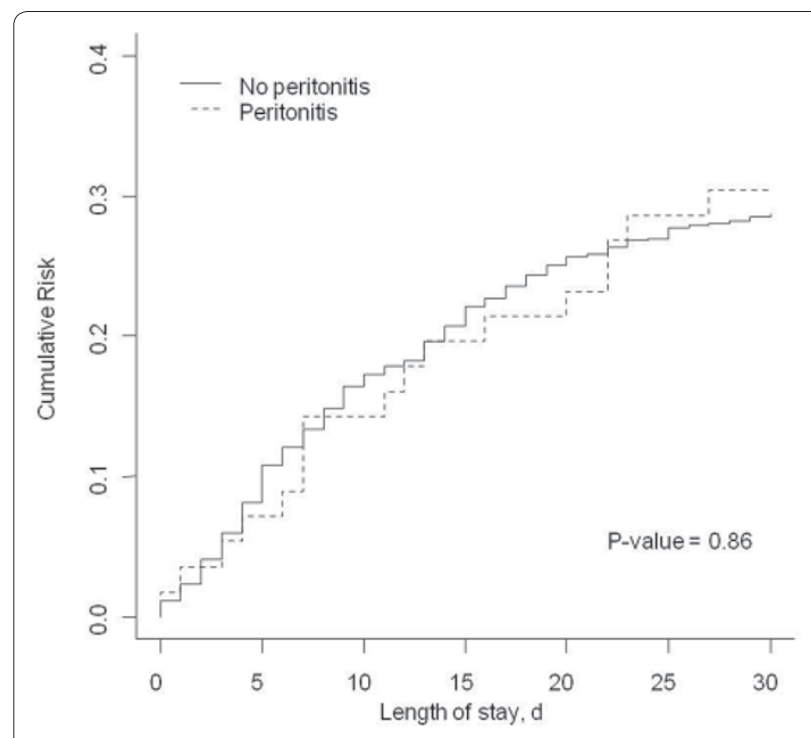

Figure 2 (abstract P46). Cumulative occurrence of death.

to the ICU for severe sepsis or septic shock who required mechanical ventilation for at least 72 hours.

Results In total, 2,623 patients were included, of which 290 had an IIA. A total of 862 patients (33\%) developed a VAP, $56(19 \%)$ in the IIA group and $806(34 \%)$ in the non-IIA group $(P<0.01)$. VAP, after sepsis, occurred less frequently and later in patients with IIA. The occurrence of IIA, in comparison with another sepsis, is a protective factor against VAP ( $\mathrm{HR}=0.643$ (0.478 to 0.863$), P=0.003$ ). There is, however, no significant difference between the groups in terms of ICU mortality ( $28 \%$ vs. $32 \%$, $P=0.16)$. See Figures 1 and 2 .

Conclusion In this study, the presence of an abdominal sepsis, in a context of severe sepsis or septic shock, was associated with a lower incidence of later VAP. These results have to be confirmed in other studies, especially prospective. They open interesting new research directions.

\section{P47}

Incidence and risk factors for ventilator-associated pneumonia in Siriraj Hospital

O Chaiwat, S Nakaviroj

Siriraj Hospital Mahidol University, Bangkok, Thailand

Critical Care 2013, 17(Suppl 2):P47 (doi: 10.1186/cc11985)

Introduction Ventilator-associated pneumonia (VAP) is a serious illness with substantial morbidity and mortality and increases the cost of hospital care. Even when bundles of care to prevent VAP have been implemented, the incidence of VAP was not dramatically improved. This study aims to determine the incidence and risk factors of VAP in the SICU of Siriraj Hospital.

Methods During a 1-year period, 228 patients admitted to the SICU were enrolled. All patients required ventilatory support longer than 48 hours. Data were collected by reviewing patient medical records including demographic data, onset of VAP, type of organisms, medication used, number of central venous catheters (CVC) used and blood transfusion. VAP outcomes were also reported.

Results VAP occurred in 21 patients $(9.21 \%)$ or 8.21 per 1,000 ventilatordays. The onset of VAP was late in the majority of patients. The most common organism was A. baumannii (66\%), followed by P. aeuruginosa (19\%). Compared with non-VAP groups, patients in the VAP group had higher APACHE II score (18 vs. $13, P<0.001)$, blood transfusion ( $95 \%$ vs. $75 \%, P=0.04$ ), numbers of CVC used ( 3 vs. $1, P<0.001$ ), muscle relaxant used $(43 \%$ vs. $3 \%, P<0.001)$ and steroid used ( $33 \%$ vs. $4 \%, P<0.001)$. The VAP group also had a significantly higher number of intubation, reintubation and self-extubation. Multiple logistic regression showed that numbers of CVC, intubation and surgery, the use of muscle relaxant and steroid were independent risk factors for developing VAP. Ventilator days and ICU length of stay were longer in the VAP group ( 25 vs. 6 and 25 vs. 7 days, respectively). Lastly, the hospital mortality rate was significantly higher in the VAP group (33\% vs. $12 \%, P=0.008)$. Conclusion The incidence of VAP was $9.2 \%$ in the SICU of Siriraj Hospital, which was comparable with previous reports. Bundles of care to prevent VAP should include weaning from a ventilator. Muscle relaxant and steroid should be administered according to strong indication. Meticulous care of the airway should be implemented as protocol in order to prevent complications that can result in the development of VAP.

Reference

1. Werarak $P$, Kiratisin $P$, Thamlikitkul V: Hospital acquired pneumonia and ventilator associated pneumonia in adults at Siriraj hospital, etiology, clinical outcomes and impact of antimicrobial resistance. J Med Assoc Thai 2010, 93(Suppl 1):126-138.

P48

Hospital-acquired bloodstream infection: Indian perspective

R Agrawal, A Varma

FEHI, New Delhi, India

Critical Care 2013, 17(Suppl 2):P48 (doi: 10.1186/cc11986)

Introduction This is a 1-year prospective study to determine the incidence, source and etiology of hospital-acquired bloodstream infection (HABSI) in the Indian context. The resistance pattern was also reviewed.

Methods A single-centre prospective study in a 35-bed ICU. HABSI was defined according to current CDC guidelines. HCAP, catheterassociated UTI (CAUTI) and skin-related infections causing BSI was also defined according to recent guidelines and analysed.

Results Out of 332 positive samples, 90 samples $(n=45)$ were HABSI. The microbiological analysis showed $60 \%$ were Gram-negative, $6 \%$ were candida and $27 \%$ were Gram-positive. The commonest isolate was klebsiella and MRSA was commonest in Gram-positive. The source of HABSI showed CRBSI was the commonest cause at $69 \%$, which correlates with international data. Ventilator-associated pneumonia and CAUTI caused $9.5 \% \mathrm{BSI}$ respectively. The resistance pattern among Gram-negative bacteria showed multidrug-resistant (MDR) and extreme drug-resistant (XDR) isolates were highest. See Tables 1 and 2.

Table 1 (abstract P48). Source

\begin{tabular}{lc}
\hline Source & Total (\%) \\
\hline CRBSI & 69 \\
HCAP & 9.5 \\
CAUTI & 9.5 \\
Devices & 7 \\
Skin & 5 \\
\hline
\end{tabular}

Table 2 (abstract P48). Resistance pattern

\begin{tabular}{lc}
\hline Resistance & Total (\%) \\
\hline Non-MDR & 15 \\
MDR & 44 \\
XDR & 33 \\
PDR & 8 \\
\hline
\end{tabular}

Conclusion The incidence of HABSI is $27 \%$. Of this, CRBSI cause $70 \%$ and Gram-negative bacteria were commonest with high resistance. This is in contrast to western data where Gram-positive infections are common. Our study highlights need for stringent guidelines for CRBSI prevention.

References

1. Richard et al:: Crit Care Med 1999, 27:887-892.

2. Valles et al: : Infect 2008, 56:27-34. 
P49

Risk factors for catheter-related bloodstream infections in a surgical ICU

A Kundakci, O Ozkalayci, P Zeyneloglu, H Arslan, A Pirat

Baskent University Hospital, Ankara, Turkey

Critical Care 2013, 17(Suppl 2):P49 (doi: 10.1186/cc11987)

Introduction Preventing catheter-related bloodstream infections (CR-BSI) can reduce the duration of hospital stay, healthcare costs, and mortality rates. Identifying the risk factors and correction of modifiable factors should be one of the main objectives of infection control measures. The aim of this study is to determine the risk factors of CR-BSI in a cohort of surgical ICU patients admitted to Baskent University Hospital.

Methods Following Institutional Review Board approval, data for 876 patients admitted to the surgical ICU between January 2009 and July 2012 were reviewed retrospectively. After completing the review, 25 patients diagnosed with CR-BSI were compared with 66 appropriate matches who did not have CR-BSI. Demographical features, underlying diseases, APACHE II (Acute Physiology and Chronic Health Evaluation) and SOFA (Sequential Organ Failure Assessment) scores, length of stay, organ dysfunctions, laboratory values, use of vasopressors, mechanical ventilation, nutrition, antibiotics, transfusions, and features related to central venous catheterization were recorded. Patients who did not have a central venous catheter and were discharged within 2 days of ICU stay were excluded.

Results Out of 91 patients included in the final analysis, 25 (27\%) patients had CR-BSI. When compared with patients who did not have CR-BSI, those who did were older $(P=0.029)$, required more blood product transfusions during the first 3 days of ICU $(P=0.016)$, had a longer duration of catheter stay $(P=0.019)$, and were more frequently catheterized via the internal jugular vein (IJV) $(P=0.022)$. A logistic regression model revealed that advanced age (OR: 1.037; $95 \% \mathrm{Cl}: 1.001$ to $1.073 ; P=0.042$ ) was an independent risk factor for CR-BSI. Fourteenday and 28-day mortality rates for CR-BSI were $12 \%(P=0.749)$ and $28 \%$ $(P=0.406)$, respectively.

Conclusion Although age, blood product transfusion, duration of catheter stay, and use of IJV were different between patients who did and did not have CRBSI, advanced age was the only independent risk factor for CR-BSI. Early suspicion of CR-BSI by the other well-known risk factors has a substantial effect on the treatment of CR-BSI.

References

1. O'Grady NP: Guidelines for the prevention of intravascular catheter-related infections. Clin Infect Dis 2011, 52:e162-e193.

2. Bouza E: Catheter-related infections: diagnosis and intravascular treatment. Clin Microbiol Infect 2002, 8:265-274.

P50

Analysis of risk factors for catheter-related bloodstream infection in a parenteral nutrition population

I Conrick-Martin', M McGovern², K Boner', J Bourke', E Fitzgerald', R Hone', M Lynch', D Phelan' ${ }^{1}$, C Walshe $^{3}$

'Mater Misericordiae University Hospital, Dublin, Ireland; ${ }^{2}$ Harvard School of Public Health, Cambridge, MA, USA; ${ }^{3}$ Beaumont Hospital, Dublin, Ireland Critical Care 2013, 17(Suppl 2):P50 (doi: 10.1186/cc11988)

Introduction Catheter-related bloodstream infection (CRBSI) is a complication of central venous catheters (CVCs) with an attributable morbidity, mortality and cost [1]. We examined patient risk factors for CRBSI in an adult parenteral nutrition (PN) population.

Methods The study was carried out in a 525-bed tertiary-referral teaching hospital over a 14-year study period (1997 to 2010). All inpatients referred for PN via CVCs were included. Prospectively collected data were recorded in a specific PN record. The CRBSI audit group met quarterly to review all sepsis episodes, assigning a diagnostic category (CRBSI or non-CRBSI). Patient risk factors for development of CRBSI were examined using a logistic regression model to take account of the dichotomous nature of the outcome. Odds ratios from a model incorporating demographic and clinical data were tested for statistical significance.
Results The study population was 1,961 patients in whom 3,213 CVCs were utilised over 19,511 CVC days. There were 256 CRBSI episodes in 216 patients. Median (IQR) patient age was 62 (23), and $58 \%$ were male. The incidence of CRBSI decreased significantly $(P<0.001)$ during the study period from $16 \%$ of patients in the period 1997 to 2003 to $7 \%$ in 2004 to 2010. The corresponding rate of CRBSI infection (per 1,000 CVC days) decreased from 18 to 10 . There was a significant decrease $(P<0.001)$ in numbers of CVCs inserted per patient (from 1.87 to 1.49). Each extra CVC PN day was associated with an increased risk of developing CRBSI of 3.4\% (OR 1.034, $P<0.05$ ). The number of PN CVCs was associated with developing CRBSI (OR 1.218, $P<0.10$ ). Patient factors significantly associated with CRBSI included perioperative PN use (compared with medical patients) (OR 2.414, $P<0.01)$, and male sex (OR 1.952, $P<0.01$ ).

Conclusion This prospective study demonstrated that perioperative $\mathrm{PN}$ use was associated with increased risk of CRBSI. The association between CRBSI and CVC PN days is consistent with the theory suggesting benefit to limiting CVC duration and changing from PN to enteral nutrition as soon as appropriate.

Reference

1. Blot Sl, et al: Clinical and economic outcomes in critically ill patients with nosocomial catheter-related bloodstream infection. Clin Infect Dis 2005; 41:1591-1598.

P51

Can we predict the postoperative infections?

T Mózes', K Gornicsar², A Grosz³, ZS Domján², I Buzogány²

'Ministry of Defense Health Centre, Budapest, Hungary;'Péterfy Hospital,

Budapest, Hungary; ${ }^{3}$ University of Szeged, Hungary

Critical Care 2013, 17(Suppl 2):P51 (doi: 10.1186/cc11989)

Introduction Many patients develop infections following operations. Decreased immune competence has been demonstrated in acute neurological conditions. A strong cytokine-mediated antiinflammatory response was observed in stroke patients at infection, although infection due to the decreased proinflammatory mediators can be expected as well. To investigate this question the following experiment was performed.

Methods Twenty-two urinary bladder cancer patients with radical cystectomy and lymphadenectomy were studied. Blood samples were taken on day 0 (before) and days 1, 3, 6, 9 and 14 after operation as well as on days $30,60,90$ and 270 during follow-up. TNFa, soluble TNFa receptor I and IL-6 levels in sera were determined by HS ELISA and/or ELISA. Plasma ACTH and cortisol values were measured by RIA kits. Results From 22 patients, eight deep wound and urine infections were found in 14 days and six urine and wound infections in 30 days after surgery, all survived. All patients were bacterially contaminated, as wound samples taken at the end of operation demonstrated. On day 0 the circulating TNFa values were lower in infected patients. TNF started to increase from day 3 to day 9 , never reaching values of the uneventful healing group. Soluble TNF receptor I, IL-6, ACTH, and cortisol concentrations did not demonstrate any difference on day 0 but from day 1 started to increase transiently, reaching higher levels in septic patients.

Conclusion A low proinflammatory response is a key facilitating factor for the development of infection. Measuring serum TNFa levels before and after operations can thus predict the outcome.

P52

Results for introduction of a new hand hygiene program in the ICU

P Vos, A Harts-Laurijsen, R Snoeren, R Ramnarain, JA Van Oers

St Elisabeth Hospital, Tilburg, the Netherlands

Critical Care 2013, 17(Suppl 2):P52 (doi: 10.1186/cc11990)

Introduction Although hand hygiene is known as a core element in prevention of healthcare-associated infections, compliance to its program is not high. With a new campaign we tried to enlarge awareness on hand hygiene.

Methods We performed an experimental, before and after, study design on our 30-bed, mixed medical-surgical ICU. We conducted a baseline 
evaluation during 3 days in May 2012 including direct observation of hand hygiene compliance by control nurses and hand cultures of 50 healthcare workers (HCW). Based on the WHO Guidelines on Hand Hygiene in Health Care [1], cleaning of hands with alcohol-based hand rubs (Sterillium) was prescribed before touching a patient and before aseptic procedures, after body fluid exposure risk and after touching a patient and touching his/her surroundings. Promotion of the hand hygiene program consisted of lectures and web-based self-learning, posters located near points of care and verbal reminders by control nurses. New observations of hand hygiene by control nurses during 3 days and hand cultures of 50 healthcare providers were performed in September 2012. Consumption of alcohol-based hand rub (product volume use per patient-days) was used as a surrogate marker of hand hygiene over time. The difference in hand hygiene compliance during the two periods was examined using a chi-squared test. Differences in hand cultures were examined using a Student's $t$ test. Time trends in the consumption of alcohol-based hand rub were examined using linear correlation. $P<0.05$ was considered statistically significant. The study was approved by the institutional Ethics Review Board.

Results During the survey, in May 158 opportunities to observe hand hygiene were presented and 286 in September. Overall compliance improved from $34.2 \%(54 / 158)$ to $51 \%(146 / 286), X^{2}=11.7(P<0.001)$. In May, $50 \mathrm{HCW}$ had a mean of $63.20 \pm 39.37$ colony-forming units (CFU) on their hands compared with $43.0 \pm 40.19$ CFU on the hands of 50 HCW in September $(P=0.024)$. We also observed an initial increased use of alcohol-based hand rubs from $21 \mathrm{ml}$ per patient-day in May to a maximum $72 \mathrm{ml}$ per patient-day in June, but a decline to $44 \mathrm{ml}$ per patient-day in September, Pearson correlation coefficient $=0.31(P=$ 0.61).

Conclusion Implementation of a new hand hygiene program at our ICU resulted in improved hand hygiene compliance and less CFU on the hands of HCW. There was no significant increased use of alcohol-based hand rubs over time. The results indicate that constant awareness is vital for success.

Reference

1. Pittet D, et al:: Infect Control Hosp Epidemiol 2009, 30:611-622.

P53

Improving hand hygiene performance in a surgical ICU: a novel method for data collection to change physician behavior

SA Nasraway

Tufts Medical Center, Boston, MA, USA

Critical Care 2013, 17(Suppl 2):P53 (doi: 10.1186/cc11991)

Introduction ICU-acquired infection is directly related to hospital mortality. Hand hygiene is an effective, low-cost intervention that can prevent the spread of bacterial pathogens, including multidrugresistant organisms. Historical compliance with hand hygiene guidelines by physicians, nurses and other care providers is poor. Methods Present expectations by the Infection Control Committee are to 'pump in, pump out' of every room, using $63 \%$ isopropyl alcohol. We performed 17,622 observations of hand hygiene in the surgical ICU

\section{$\%$ Hand Hygiene}

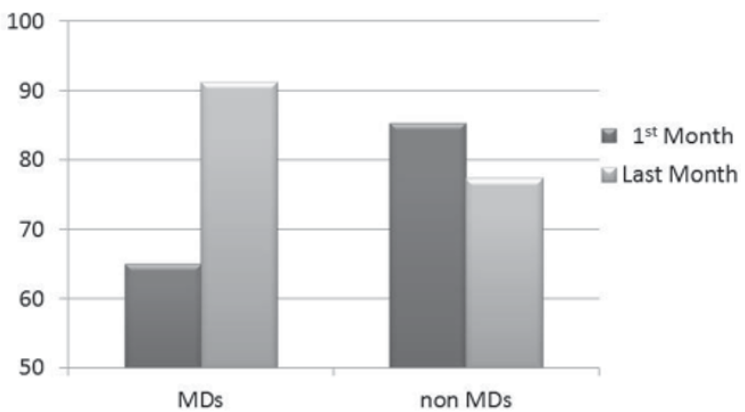

Figure 1 (abstract P53) from March through October 2012, and intervened to change behavior by providing monthly feedback to specific provider groups and services. We made use of the Unit Coordinator to measure compliance of all individuals in the ICU.

Results Overall compliance by physicians was $82.1 \%$, for nonphysicians was $84.8 \%$. Feedback to physicians, individually and by service, dramatically increased hand hygiene compliance, defined as both on entry and exit from the patient room, over the study period. See Figure 1.

Conclusion Physician behavior is responsive to monthly feedback that is specific to the individual or surgical service. Use of the Unit Coordinator was very effective at gathering a very large sample size in a short period of time.

P54

Compliance with the implementation of an ICU cluster-randomized trial assessing the benefits and potential harms of universal glove and gowning

D Kett', DJ Morgan², L Pineles², MJ Zervos ${ }^{3}$, LS Munoz-Price', AD Harris², BUGG Investigators 2

'University of Miami/Jackson Memorial Hospital, Miami, FL, USA; '2 University of Maryland/NA Maryland Healthcare System, Baltimore, MD, USA; ${ }^{3}$ Henry Ford Health System, Detroit, MI, USA

Critical Care 2013, 17(Suppl 2):P54 (doi: 10.1186/cc11992)

Introduction The Benefits of Universal Glove and Gowning (BUGG) study is a cluster-randomized trial to evaluate the use of wearing gloves and gowns for all patient contact in the ICU. The primary outcome is VRE and MRSA acquisitions; secondary outcomes include frequency of healthcare worker visits, infection rates, hand hygiene compliance and adverse events.

Methods We enrolled 20 ICUs in 15 states. ICUs collected nasal and perianal swabs on all patients at admission and discharge/transfer. After a 3-month baseline period, 10 units were randomized to the intervention arm and required to wear gloves and gowns for all patient contact. An intervention toolkit was created based on site feedback and compliance reports. Swab collection compliance was fed back and discussed during site conference calls on a weekly basis. Site coordinators monitored compliance with gloves and gowns, hand hygiene and frequency of HCW visits and reviewed patient charts for adverse events.

Results During the 12-month study period, 100,210 swabs were collected. After the baseline period, we were able to achieve and maintain swab compliance rates between 85 and $97 \%$. Monthly discharge compliance increased by $21 \%$ by the beginning of the intervention period (Figure 1). Observers found $86 \%$ compliance with universal glove and gowning over 1,242 30-minute observation periods (Figure 1). Ninety charts at each site were reviewed for adverse events. Conclusion Over a diverse group of US hospitals, we achieved high compliance with surveillance cultures and implementing universal gloving and gowning was achieved quickly with high compliance.

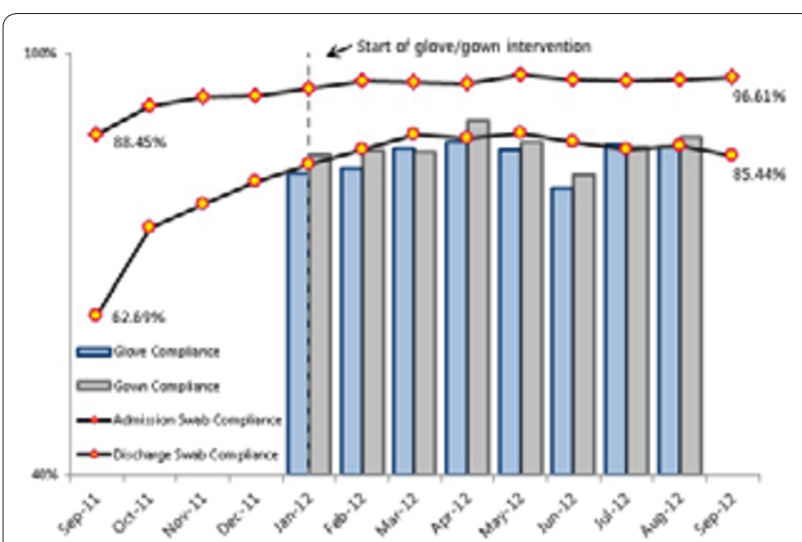

Figure 1 (abstract P54). Swab collection and gown/glove compliance. 
P55

Fighting hospital sepsis

AR Calini, S Vesconi, R Fumagalli, S Marchesi, L Ghezzi, G Monti

Ospedale Niguarda Ca' Granda, Milan, Italy

Critical Care 2013, 17(Suppl 2):P55 (doi: 10.1186/cc11993)

Introduction Sepsis accounts for a very high mortality. The Surviving Sepsis Campaign recommends a first 6 hours resuscitative bundle to improve patient outcome. Despite this, the bundle is poorly performed because of several organizational and cultural barriers. In recognition of this, we guess that an Educational and Organizational Intervention out of the ICUs could impact on septic patient outcome. In order to test our hypothesis we carried out, in 12 hospitals, a pre-intervention survey of the human and organizational resources (HOR) available in the management of septic patients. The aim is to seek any barrier potentially affecting correct Guidelines implementation.

Methods Thirty-nine medical wards (MW) and 12 emergency departments (ED) were enrolled. Every unit was asked to fill in a pre-agreed HOR Checklist focused on the main requirements suggested by the Guidelines.

Results Analysing the human resources available, we see that the bedto-doctor ratio significantly $(P<0.01)$ increases from the day to the night shift: from 6 to 43 beds per doctor on the MW (median). Otherwise, the ED staff remains roughly the same: from 3.5 to 2.5 doctors on duty (median). The analysis of the organizational tools (Table 1) points out

Table 1 (abstract P55)

\begin{tabular}{lc}
\hline Resource & $\begin{array}{c}\text { Availability } \\
\text { (\%) }\end{array}$ \\
\hline Diagnostic and therapeutic protocol for septic patient management & 8.3 \\
Use of early warning score for diagnosis and management & 8.3 \\
Sepsis team & 25 \\
Microbiology laboratory: open 7 days a week & 66.6 \\
Lactate dosage: 24 hours a day availability & 83.3 \\
Central venous catheter insertion (CVC) available 24 hours a day & 91.6 \\
Hospital empirical antibiotic therapy guidelines & 0 \\
Infectious diseases team advice in any case of severe sepsis septic shock & 25 \\
Infective source eradication & 8.3 \\
Infective source eradication protocols & 50 \\
Intervention radiology available 24 hours a day & 58.3 \\
Operating room available 24 hours a day & 58.3 \\
Specific infection management protocol (that is, CVC infection) & 41.6 \\
\hline Imaging reporting service available 7 days a week &
\end{tabular}

a low percentage of hospitals having: a Diagnostic and Therapeutic Protocol for sepsis management (8.3\%), some Hospital Empirical Antibiotic Therapy Guidelines (0\%) and an Infective Source Eradication Protocol (8.3\%). Moreover, just $25 \%$ of hospitals involve an infectious diseases expert in every case of severe sepsis or septic shock.

Conclusion We guess that the poor availability of HOR showed by the hospitals could have a role in the Guidelines implementation and in the patient's outcome. Only a comparison between these results and data collected from a Clinical Checklist, focused on sepsis bundle compliance, and from a patient's outcome summary could confirm our hypothesis. This is the aim for our next part of the study.

\section{Reference}

1. Dellinger RP, et al: Intensive Care Med 2008, 34:17-60.

\section{P56}

Sinks as a correctable source of ESBL contamination for patients in the ICU

I Wolf', P Bergervoet ${ }^{1}$, W Van der Zwet', H Van den Oever', P Savelkoul',

F Sebens ${ }^{1}$

'Deventer Hospital, Deventer, the Netherlands; ${ }^{2}$ VU Medical Centre,

Amsterdam, the Netherlands

Critical Care 2013, 17(Suppl 2):P56 (doi: 10.1186/cc11994)

Introduction The incidence of patients carrying ESBL-positive bacteria in our ICU (12 in 780 admissions in 2011) was not considered problematic. However, routine cultures had identified ESBL-negative patients who had become colonized with ESBL strains during their ICU stay. Self-disinfecting siphons, preventing bacterial growth by antibacterial coating and intermittent heating, and biofilm formation by electromechanical vibration, were placed in all sinks in the ICU. The aim of the present study was to evaluate the effect of this intervention. Methods An intervention study in a 12-bed ICU. The intervention involved placement of 19 self-disinfecting siphons (Biorec). All patients with an expected ICU stay of 2 days or more between January 2011 and December 2012 were studied. Samples of throat, sputum and rectum were taken at admission and twice weekly, and cultured for ESBLs. Between June 2011 and October 2011, sinks in patient rooms were cultured regularly for ESBLs. After the intervention in April 2012, multiple repeat cultures were taken. Whenever the species and antibiogram of bacteria cultured from patients and sinks matched, they were typed by AFLP.

Results Before intervention Multiple ESBL-forming strains were found in sinks of all patient rooms. Eighteen patients who were ESBL-negative on ICU admission became colonized with 11 different ESBL strains, that were present in sinks of their admission rooms (Figure 1). Four contaminations were proven by AFLP-tying. One patient died of ESBLpositive E. cloacae pneumonia. After intervention All sinks were negative for ESBL strains. No further patients became ESBL colonized during the ICU stay.

Conclusion Wastewater sinks were the likely source of ESBL colonization for $18 \mathrm{ICU}$ patients. After placing self-disinfecting siphons

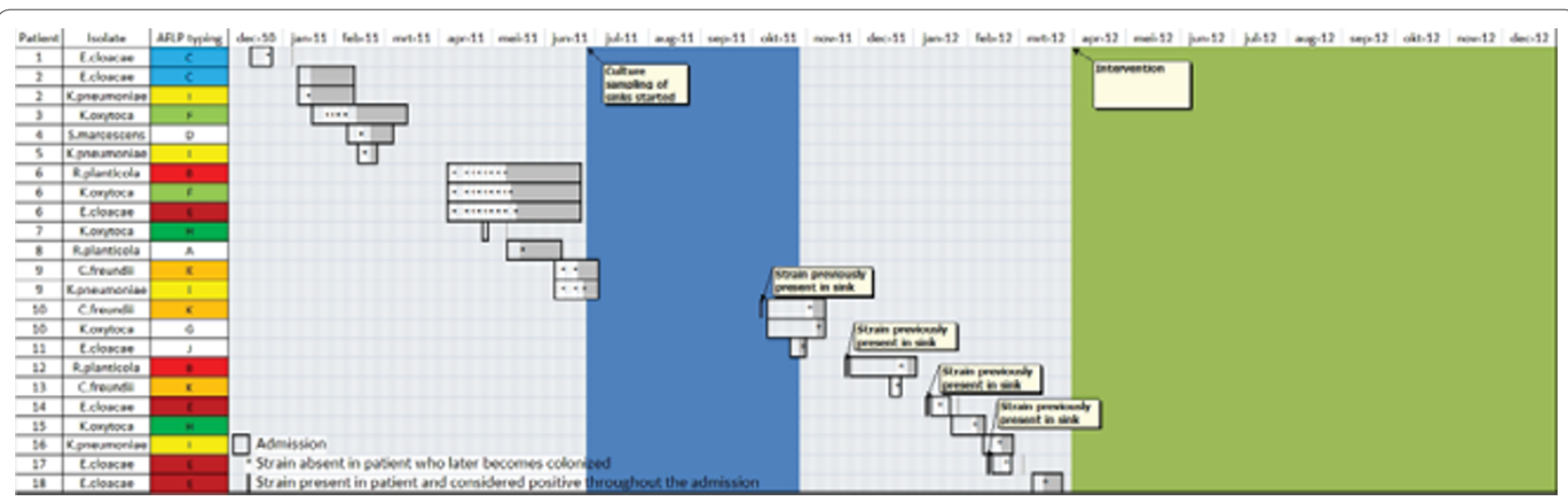

Figure 1 (abstract P56). Design and results of the intervention. 
Table 1 (abstract P57). Hemodynamic variables, among times and groups

\begin{tabular}{|c|c|c|c|c|c|c|c|}
\hline & $B L$ & Roh & $\mathrm{R} 12 \mathrm{~h}$ & R24h & R36h & $\mathrm{R} 48 \mathrm{~h}$ & $\begin{array}{c}P \\
\text { (timexgroup) }\end{array}$ \\
\hline MAP (ENL/MH/ML) & $\begin{array}{c}66(9) / 72(15) / \\
65(9)\end{array}$ & $\begin{array}{c}65(11) / 73(10) / \\
81(16)\end{array}$ & $\begin{array}{c}57(4) / 82(6) / \\
72(13)\end{array}$ & $\begin{array}{c}58(8) / 78(6) / \\
67(12)\end{array}$ & $\begin{array}{c}56(12) / 80(5) / \\
61(6)\end{array}$ & $\begin{array}{c}56(11) / 79(9) / \\
56(13)\end{array}$ & 0.003 \\
\hline $\mathrm{CO}$ (ENL/MH/ML) & $\begin{array}{c}106(13) / 89(7) / \\
105(13)\end{array}$ & $\begin{array}{c}92(17) / 67(11) / \\
88(22)\end{array}$ & $\begin{array}{c}169(16) / 95(19) / \\
104(13)\end{array}$ & $\begin{array}{c}187(22) / 129(28) / \\
143(31)\end{array}$ & $\begin{array}{c}161(25) / 139(32) / \\
145(13)\end{array}$ & $\begin{array}{c}157(37) / 140(21) / \\
150(18)\end{array}$ & $<0.0001$ \\
\hline Carotid flow (ENL/MH/ML) & $6(2) / 7(2) / 7(2)$ & $5(1) / 4(1) / 5(2)$ & $10(3) / 5(1) / 6(2)$ & $10(3) / 8(1) / 9(2)$ & $8(2) / 8(2) / 8(2)$ & $8(3) / 8(2) / 8(2)$ & $<0.0001$ \\
\hline Femoral flow (ENL/MH/ML) & $4(1) / 3(1) / 4(2)$ & $2(1) / 2(0) / 2(1)$ & $4(1) / 2(1) / 2(0)$ & $4(1) / 3(1) / 3(1)$ & $3(1) / 3(1) / 4(1)$ & $3(2) / 3(1) / 4(1)$ & 0.004 \\
\hline
\end{tabular}

there were no new ESBL colonizations in patients. This coincided with the disappearance of ESBL strains from all sinks.

P57

Hemodynamic and mitochondrial effects of enalapril in experimental sepsis

AJ Pereira, V Jeger, T Corrêa, M Vuda, S Djafarzadeh, J Takala, S Jakob

University Hospital (Inselspital)/University of Bern, Switzerland

Critical Care 2013, 17(Suppl 2):P57 (doi: 10.1186/cc11995)

Introduction Sepsis may cause mitochondrial dysfunction. The renin-angiotensin system (RAS) activity is increased in sepsis, and can interfere with the mitochondrial function either directly or by modifying hemodynamics. We studied the effects of ACE inhibition by enalapril on hemodynamics and hepatic mitochondrial function in sepsis. Two septic groups without enalapril and with different blood pressure targets served as controls.

Methods Sepsis (fecal peritonitis) was induced in 24 anesthetized, mechanically ventilated pigs $(40.1 \pm 2.1 \mathrm{~kg})$, divided into three groups ( $n=8$ for each): ENL (enalapril pretreatment for 7 days and at 0.005 to $0.02 \mathrm{mg} / \mathrm{kg} /$ hour during the study; MAP target 75 to $85 \mathrm{mmHg}$ ), ML (target MAP 50 to $60 \mathrm{mmHg}$ ), and MH (target MAP 75 to $85 \mathrm{mmHg}$ ). Hemodynamic support with fluids, norepinephrine (NE; maximum dose $5,000 \mu \mathrm{g} /$ hour), and antibiotics were started after 12 hours of peritonitis and continued for 48 hours.

Results All enalapril pigs received the maximum NE dose without reaching the target MAP. Enalapril resulted in lower MAP, higher $\mathrm{CO}$, and transiently increased regional blood flows (Table 1). Fluid administration and urinary output were similar among groups. Liver mitochondrial respiration was reduced (State 3, State 4) in the enalapril group. One animal died in each ML and enalapril group.

Conclusion Enalapril enhances cardiac output and early regional blood flows in sepsis despite lower MAP, and reduces liver mitochondrial respiration, suggesting that RAS is involved in the hemodynamic and metabolic changes in sepsis.

P58

Effects of intraperitoneal immunoglobulin therapy on behavior and cognitive functions in CLP-induced sepsis model in rats F Esen', E Senturk', P Ergin Ozcan', G Orhun', S Gümrü², M Kaya',

N Orhan', N Arıcan', F Arıcıoglu²

'University of Istanbul, Turkey; ${ }^{2}$ Marmara University Faculty of Pharmacy, Istanbul, Turkey

Critical Care 2013, 17(Suppl 2):P58 (doi: 10.1186/cc11996)

Introduction The present study investigated the effects of a single dose of intraperitoneal (i.p.) IgG and lgGAM administration on various behavioral alterations in a cecal ligation perforation (CLP)-induced sepsis model in rats.

Methods Female Wistar albino rats (200 to $250 \mathrm{~g}$ ) were divided into five groups $(n=8)$ : a naive Control group, a Sham operated group receiving conventional antibiotic treatment, a CLP group receiving CLP procedure and conventional antibiotic treatment, and IgG and IgGAM groups which were also applied $1 \mathrm{~g} / \mathrm{kg}$, i.p. IgG and IGAM therapy 5 minutes after the CLP procedure. Ten, 30 and 60 days after the surgery, animals underwent three behavioral tasks: an open field test to evaluate the locomotor activity, an elevated plus maze test to measure the level of anxiety, and a forced swim test to assess the possible depressive state. The results acquired from these tests were used to estimate the effect of immunoglobulin therapy on behavioral changes in CLP-induced sepsis in rats.

Results In the open field test, the CLP group showed a significant decrease in total squares passed on days 10 and 30. Similarly, total numbers of rearing and grooming were dramatically decreased in the CLP group in comparison with control and sham groups $(P<0.005)$. In the elevated plus maze test, the number of entries to open arms decreased in the CLP group. In the forced swim test, there was a tendency for increase in immobility time in the CLP group, although the data were statistically insignificant. All of these values which were indicating the importance of behavioral alterations were improved on day 60 . Immunoglobulin therapy prevented the occurrence of these behavioral changes. Especially, animals in the IgGAM group conserved the values quite near to those of the control group in measured parameters.

Conclusion Sepsis, even though it has been treated with conventional antibiotics, caused a negative effect on behavioral parameters. In this study, IgG and IgGAM treated animals in the presence of CLP did not show these behavioral changes. Therefore our results suggest that a single dose of i.p. IgG and IgGAM treatment, which was applied immediately after the sepsis procedure, prevents behavioral defects observed following sepsis.

P59

Clinical efficacy of recombinant human soluble thrombomodulin in patients with septic disseminated intravascular coagulation

Y Suzuki, R Sato, M Sato, S Endo

Iwate Medical University, Morioka, Japan

Critical Care 2013, 17(Suppl 2):P59 (doi: 10.1186/cc11997)

Introduction Thrombomodulin is an endothelial cell cofactor and glycoprotein for thrombin-catalyzed activation of protein C. A recombinant human soluble thrombomodulin (rhsTM) has been recently developed, and this new agent has a unique amino-terminal structure exhibiting anti-inflammatory activity including sequestraction and cleavage of high-mobility group box 1(HMGB-1).

Methods In this study, 13 patients with septic disseminated intravascular coagulation (DIC) were treated with rhsTM, which is Recomodulin ${ }^{\circledR}$ Inj. 12800 (Asahi Kasei Pharma Co., Tokyo, Japan). Patients with septic DIC were treated with 130 to $380 \mathrm{U} / \mathrm{kg} /$ day.

Results There were significant results for improvement of APACHE II score and DIC diagnostic criteria score for critically ill patients after treatment using rhsTM $(P<0.01)$. Improvement for platelet count and D-dimer level were also observed in this study $(P<0.05)$. Activation of antithrombin (AT) also was significantly increased after treatment $(P<0.05)$. Hospital mortality was $15.4 \%$ in this study.

Conclusion The rhsTM might be one of most important endogenous regulators of coagulation, acting as the major inhibitor of thrombin as well as AT III. This new agent may play an important role in treatment for septic DIC.

P60

Antithrombin III concentrate therapy may have an efficacy in sepsis N Saito, M Takeda, T Harada, R Moroi, M Namiki, A Yaguchi

Tokyo Women's Medical University, Tokyo, Japan

Critical Care 2013, 17(Suppl 2):P60 (doi: 10.1186/cc11998)

Introduction Antithrombin III (AT III) has been known to contribute to anti-inflammatory response as well as its anticoagulation. Our previous 
study showed AT III deficiency happened in the early stage of sepsis with no relation to DIC status. However, whether AT III concentrate is a beneficial therapy or not for septic patients is controversial. Our hypothesis is that AT III concentrate may have efficacy as antiinflammation for sepsis.

Methods From January 2009 to December 2011, adult septic patients, whom were given AT III concentrate in our medico-surgical ICU, were included in this study. AT III concentrate was administered 30 to $60 \mathrm{U} / \mathrm{kg}$ intravenously every 24 hours for 3 days in the patients with DIC status and AT III deficiency. Between before and after the AT III concentrate therapy, WBC $\left(/ \mathrm{mm}^{3}\right)$, CRP $(\mathrm{mg} / \mathrm{dl})$, platelet $\left(\times 10^{4} / \mu \mathrm{l}\right)$, PT (seconds), fibrinogen $(\mathrm{mg} / \mathrm{dl})$, FDP $(\mu \mathrm{g} / \mathrm{ml})$, SOFA score and DIC score were evaluated. Values are expressed as mean $\pm \mathrm{SD}$. Data were analyzed by Wilcoxon signed-rank test. $P<0.05$ was considered significant.

Results There were 85 patients ( 52 men, 33 women; age range 19 to 92 years (mean $69.0 \pm 16.9)$ ), and the mortality rate was $27.1 \%$ and APACHE II score was $20.1 \pm 7.6$. WBC, CRP, PT and SOFA score were significantly improved after AT III concentrate therapy $(13,176 \pm 9,400$ vs. $11,693 \pm 7,089, P=0.011,15.9$ vs. $13.3, P=0.0015,16.5 \pm 4.4$ vs. $15.9 \pm 5.9, P=0.0045$, and $9.7 \pm 3.8$ vs. $8.6 \pm 4.6, P=0.01$, respectively). Platelet was significantly decreased (11.7 \pm 9.7 vs. $10.6 \pm 9.0, P=0.028)$, while there were no significant differences in fibrinogen, FDP and DIC score $(387.3 \pm 202.6$ vs. $381.8 \pm 163.2, P=0.088,36.9 \pm 52.7$ vs. $28.8 \pm 39.9, P=0.059$, and $2.9 \pm 1.6$ vs. $2.6 \pm 1.8, P=0.25$, respectively) after the therapy. One week after the therapy, platelet and DIC score were significantly improved compared with before the therapy $(15.6 \pm 10.0, P=0.0036$ and $1.8 \pm 1.9, P=0.0041)$.

Conclusion In the patients with septic DIC, WBC, CRP and SOFA score were immediately improved after the AT III concentrate therapy, while platelet and DIC score were improved later. AT III concentrate may also contribute to anti-inflammatory for septic DIC with anticoagulation.

P61

A clinical randomized trial on the use of atorvastatin in patients with sepsis or septic shock: effects on endothelial function

K Prado, C Ribeiro, T Furian, R Pinto Ribeiro, D Silvello, L Rohde, N Clausell,

L Becker

Hospital de Clinicas de Porto Alegre, Brazil

Critical Care 2013, 17(Suppl 2):P61 (doi: 10.1186/cc11999)

Introduction Sepsis and septic shock are complex inflammatory syndromes. Multiple cellular activation processes are involved, and many humoral cascades are triggered. Presumably, endothelial cells play a pivotal rule in the pathogenesis of sepsis, not only because they may influence the inflammatory cascade but also because, upon interaction with excessive amounts of inflammatory mediators, the function of these cells may become impaired. It is likely that a general dysfunction of the endothelium is a key event in the pathogenesis of sepsis [1]. HMG-CoA-reductase inhibitors have been shown to exhibit pronounced immunomodulatory effects independent of lipid lowering. Most of these beneficial effects of statins appear to involve restoring or improving endothelial function [2]. We hypothesize that statins can improve endothelial dysfunction in septic patients.

Methods A double-blinded, placebo-controlled, randomized trial was undertaken. We enrolled adult patients within 24 hours of severe sepsis or septic shock diagnosis and randomized them to placebo or atorvastatin $80 \mathrm{mg} /$ day for a short term. Endothelial dysfunction was assessed measuring plasmatic levels of IL-6, ET-1, VCAM- 1 by ELISA and measuring flow-mediated vasodilatation of the brachial artery at basal, 24 and 72 hours after randomization.

Results We studied 47 patients, 24 in the placebo group (mean age $52 \pm 20$ years, $29.1 \%$ male; APACHE II risk score $23.5 \pm 7.3$ ) and 23 in the statin group (mean age $49.5 \pm 18$ years, $53.4 \%$ male; APACHE II risk score $23 \pm 6.9$ ). The baseline characteristics of the placebo group were similar to statin patients as well as the mean length of stay in the ICU ( $8.6 \pm 7.4$ and $9.1 \pm 8$ days, respectively) and the time on vasopressors ( $49.3 \pm 47.1$ and $59 \pm 91.1$ hours, respectively). No significant difference was observed on the temporal variation of biomarker levels (IL-6, VCAM-1, ET-1) between treatment and control groups. The intrahospital mortality rate was $26 \%$ in the statin group and $45 \%$ in the placebo group $(P=0.17)$.
Conclusion Our data showed no benefit with the use of a potent statin acutely in patients with sepsis or septic shock with regards to improvement in endothelial function.

References

1. Hack EC, et al:: The endothelium in sepsis: source of and a target for inflammation. Crit Care Med 2001, 29:S21-S27.

2. Davignon J: Beneficial cardiovascular pleiotropic effects of statins. Circulation 2004, 109(Suppl III):|II-39-|II-43.

P62

A multicenter randomized controlled study of an extracorporeal cytokine hemoadsorption device in septic patients

D Schädler', C Porzelius', A Jörres ${ }^{3}$, G Marx ${ }^{4}$, A Meier-Hellmann ${ }^{5}$, C Putensen ${ }^{6}$, M Quintel $^{7}$, C Spies $^{8}$, C Engel $^{2}$, N Weiler ${ }^{1}$, M Kuhlmann $^{9}$ 'University Medical Center Schleswig-Holstein, Campus Kiel, Germany; ${ }^{2}$ University of Leipzig, Germany; ${ }^{3}$ Charité University Hospital Campus VirchowKlinikum, Berlin, Germany: “RWTH University Hospital Aachen, Germany; ${ }^{5}$ HELIOS Klinikum, Erfurt, Germany; ${ }^{6}$ University of Bonn, Germany; ${ }^{7}$ University Hospital Göttingen, Germany: ${ }^{8}$ Campus Charité Mitte and Campus Charité Virchow-Klinikum, Charité-University Medicine Berlin, Germany; ${ }^{9}$ Vivantes Klinikum im Friedrichshain, Berlin, Germany

Critical Care 2013, 17(Suppl 2):P62 (doi: 10.1186/cc12000)

Introduction A novel sorbent hemoadsorption device for cytokine removal (CytoSorbents, USA) was developed and successfully tested in animal models of sepsis. The experience in the clinical setting is still limited to case reports. In this first clinical trial, we tested the hypothesis that treatment with sorbent hemoadsorption could safely and effectively reduce cytokines in septic patients with acute lung injury (ALI).

Methods Ventilated patients fulfilling the criteria for severe sepsis and ALI were enrolled in this multicenter randomized, controlled, openlabel study comparing standard of care with or without hemoperfusion treatment. Primary endpoints were safety and IL- 6 reduction. Treated patients underwent hemoperfusion at flow rates of $\sim 200$ to $300 \mathrm{ml} /$ minute for 6 hours per day for 7 consecutive days. The overall mean reduction in individual plasma cytokines for the control and treatment groups during the treatment period was calculated using a generalized linear model.

Results Forty-three patients (18 treated, 25 control) completed the study and were further analyzed. Incidence of organ dysfunction at enrollment (treatment vs. control) was: septic shock ( $94 \%$ vs. $100 \%$, $P=0.42)$, acute respiratory distress syndrome (67\% vs. $56 \%, P=0.33)$, and renal failure (39\% vs. $24 \%, P=0.54)$. During 115 treatments no serious device-related adverse events occurred. On average, there were no changes in hematology and other blood parameters except for a modest reduction in platelet count $(<10 \%)$ and albumin $(<5 \%)$ with treatment. Hemoperfusion decreased IL-6 blood concentration significantly $(-49.1 \%, P=0.01)$, with similar reductions of MCP-1 $(-49.5 \%, P=0.002)$, IL-1ra $(-36.5 \%, P=0.001)$, and IL-8 $(-30.2 \%, P=$ 0.002 ). The 28 -day mortality ( $28 \%$ vs. $24 \%$ control, $P=0.84$ ) and 60 day mortality ( $39 \%$ vs. $32 \%$ control, $P=0.75$ ) did not differ significantly between the two studied groups.

Conclusion In this first clinical study of a novel sorbent hemoadsorption device in patients with severe sepsis and ALI, the device appeared to be safe and decreased the blood concentration of several cytokines. Further research is needed to study the effect of the device on the clinical outcome of septic patients.

P63

Continuous renal replacement therapy with the adsorbent membrane oXiris in septic patients: a clinical experience

F Turani, F Candidi, R Barchetta, E Grilli, A Belli, E Papi, A di Marzio, M Falco Aurelia Hospital/European Hospital, Rome, Italy

Critical Care 2013, 17(Suppl 2):P63 (doi: 10.1186/cc12001)

Introduction Renal failure is an important complication of sepsis and CRRT with adsorbing membranes may be useful in this clinical setting [1]. The aims of the study in septic/septic shock patients are to evaluate: the safety of a new hemofilter membrane oxiris with adsorbing properties and anti-endotoxin activity; the renal and hemodynamic 
Table 1 (abstract P63)

\begin{tabular}{lccc}
\hline Parameter & Units & T0 & T1 \\
\hline Creatinine & $\mathrm{mg} / \mathrm{dl}$ & $1.9 \pm 0.1$ & $1.18 \pm 0.1^{*}$ \\
Diuresis & $\mathrm{ml} / 24$ hours & $1,284 \pm 78$ & $1,573 \pm 98$ \\
Norepinephrine & $\mu \mathrm{g} / \mathrm{kg} / \mathrm{minute}$ & $0.17 \pm 0.2$ & $0.06 \pm 0.1^{*}$ \\
$\mathrm{IL}-6$ & $\mathrm{pg} / \mathrm{ml}$ & $572 \pm 78$ & $278 \pm 57^{*}$ \\
Procalcitonin & $\mathrm{ng} / \mathrm{ml}$ & $35 \pm 7$ & $15 \pm 2^{*}$ \\
Endotoxin & $\mathrm{Level} / \mathrm{U}$ & $0.64 \pm 0.2$ & $0.49 \pm 0.1$ \\
\hline
\end{tabular}

${ }^{*} P<0.05$ vs. T0

response; and the changes of endotoxin and proinflammatory molecules.

Methods Forty septic/septic shock patients with renal failure were enrolled in the study. All patients had preoperative endotoxin $>0.6$ level/units (EAA Spectral D) and were submitted to high-volume hemodiafiltration (50 ml/kg/hour, Prismaflex; Gambro) with a new treated heparin-coated membrane (oXiris; Gambro). At T0 (pretreatment) and T1 (24 hours) the main clinical and biochemical data were evaluated. All data are expressed as mean \pm SD. One-way ANOVA test with Bonferroni correction was used to evaluate the data changes. $P<0.05$ was considered significant.

Results Table 1 presents the main results of this study.

Conclusion In septic/septic shock patients with renal failure, CRRT with a new treated heparin-coated membrane (oXiris; Gambro) is clinically feasible, and has a positive effect on renal function and hemodynamics. An adsorbing effect on proinflammatory mediators may have a role in these results. These data and the trend toward a decrease of endotoxin during the treatment warrant further investigation.

Reference

1. Rimmelé T, et al:: Nephrol Dial Transplant 2009, 24:421-427.

\section{P64}

Combined use of pumpless extracorporeal lung assist system and continuous renal replacement therapy with citrate anticoagulation in polytrauma patients

HK Atalan, M Dumantepe, TB Denizalti, IA Tarhan, A Ozler

Atasehir Memorial Hospital, Istanbul, Turkey

Critical Care 2013, 17(Suppl 2):P64 (doi: 10.1186/cc12002)

Introduction The usefulness of a pumpless extracorporeal lung assist system ( $p E C L A)$ and continuous renal replacement therapy (CRRT) in critically ill patients has been demonstrated in previous studies [1,2]. The aim of this report was to examine combined use of pECLA and CRRT to improve carbon dioxide and inflammatory mediator removal, which allows for lung protective ventilation strategies.

Methods In our 10 patients with ARDS due to polytrauma and sepsis, pECLA was established by insertion of cannulae to the femoral artery and vein. CRRT cannulae were introduced by venous line of the same vascular access (Figure 1). We preferred regional anticoagulation with trisodium citrate for both CRRT and ILA.

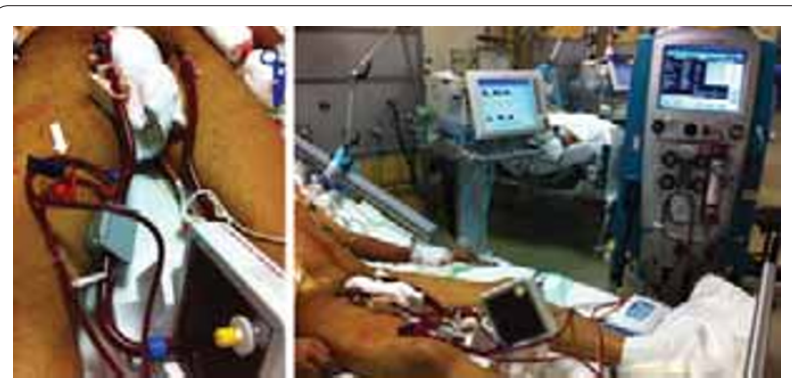

Figure 1 (abstract P64). ILA and CRRT connected to the patient.
Results Mean SAPS II and APACHE II scores were 55 and 23 respectively. Mean time on mechanical ventilation was 22 days. Mean ICU stay was 30 days for survivors and 38 days for nonsurvivors. When compared with baseline values most relevant parameters were the improvement in tidal volumes, plateau pressures, $\mathrm{PaCO}$ levels and $\mathrm{pH}$ (Figure 2). Four patients survived while six patients died from sepsis-MOF.

Conclusion We concluded that PECLA can effectively address the impaired gas exchange in ARDS and CRRT is a safe procedure with potential therapeutic value for treating MOF. Citrate anticoagulation was well tolerated and filter life was appropriate. The use of the same vascular access for ILA and CRRT may minimize invasive procedures and related side effects.

References

1. Bein T, et al:: Int J Emerg Med 2010, 3:177-179.

2. Demetrios J, et al.: Kidney Int 2005, 67:2361-2367.

P65

Abstract withdrawn

P66

Use of extracorporeal endotoxin elimination therapy for septic shock

BA Adamik, JS Smiechowicz, SZ Zielinski, AK Kübler

Medical University, Wroclaw, Poland

Critical Care 2013, 17(Suppl 2):P66 (doi: 10.1186/cc12004)

Introduction Endotoxin, a component of the outer membrane of Gramnegative bacteria, is considered an important factor in pathogenesis of septic shock [1]. The aim of our study was to determine whether endotoxin elimination treatment added to the standard treatment would improve organ function in patients with septic shock.

Methods Adult patients with septic shock who required renal replacement therapy (RRT), with a confirmed endotoxemia, and suspected Gram-negative infection were consecutively added to the study within the first 24 hours after diagnosis. All patients received full standard treatment for septic shock. Endotoxin elimination was performed using the membrane oXiris (Gambro, Sweden), a medical device for continued RRT with the unique feature of endotoxin adsorbtion. An endotoxin activity assay was used to monitor endotoxin elimination therapy at baseline (T0), 3 hours (T1), 12 hours (T2), 24 hours (T3), 48 hours (T4), and 72 hours (T5). Our key indicators were the improvement in hemodynamics and organ function, and decrease of endotoxin activity (EA) in blood. Continuous variables are presented as mean values with standard deviations.

Results High EA level at baseline $(0.74 \pm 0.14$ endotoxin activity units (EAU)) significantly decreased during RRT with oXiris membrane to $0.46 \pm 0.02$ (T1), $0.34 \pm 0.01$ (T2), $0.4 \pm 0.02$ (T3), $0.46 \pm 0.04$ (T4), $0.35 \pm 0.07$ (T5) EAU $(P<0.05)$. MAP increased from baseline $72 \pm 14$ to $81 \pm 18,76 \pm 6,77 \pm 7,83 \pm 13,87 \pm 10 \mathrm{mmHg}(P<0.05)$, and the mean norepinephrine use decreased from $0.23 \pm 0.04$ to $0.19 \pm 0.02$, $0.11 \pm 0.01,0.09 \pm 0.01,0.04 \pm 0.01,0.0 \mu \mathrm{g} / \mathrm{kg} / \mathrm{minute}(P<0.05)$ at T0, T1, $\mathrm{T} 2, \mathrm{~T} 3, \mathrm{~T} 4, \mathrm{~T} 5$, respectively. The SOFA score had decreased from $14 \pm 4$ to $12 \pm 2,9 \pm 3,7 \pm 3$ points $(P<0.05)$, and the procalcitonin level declined from $107 \pm 123$ to $45 \pm 41,29 \pm 30,17 \pm 157 \pm 1 \mathrm{ng} / \mathrm{ml}(P<0.05)$ at T0, T3, T4, T5

Conclusion RRT with oXiris membrane resulted in the effective elimination of endotoxins from the blood. The therapy was associated with an increase in blood pressure, a reduction of vasopressor requirements, and an improvement of organ function. The application of the endotoxin activity assay was useful for bedside monitoring of endotoxemia in ICU patients.

Reference

1. Marshall JC, Foster D, Vincent JL, Cook DJ, Cohen J, Dellinger RP, Opal S, Abraham E, Brett SJ, Smith T, Mehta S, Derzko A: Diagnostic and prognostic implications of endotoxemia in critical illness: results of the MEDIC Study. $J$ Infect Dis 2004, 190:527-534. 


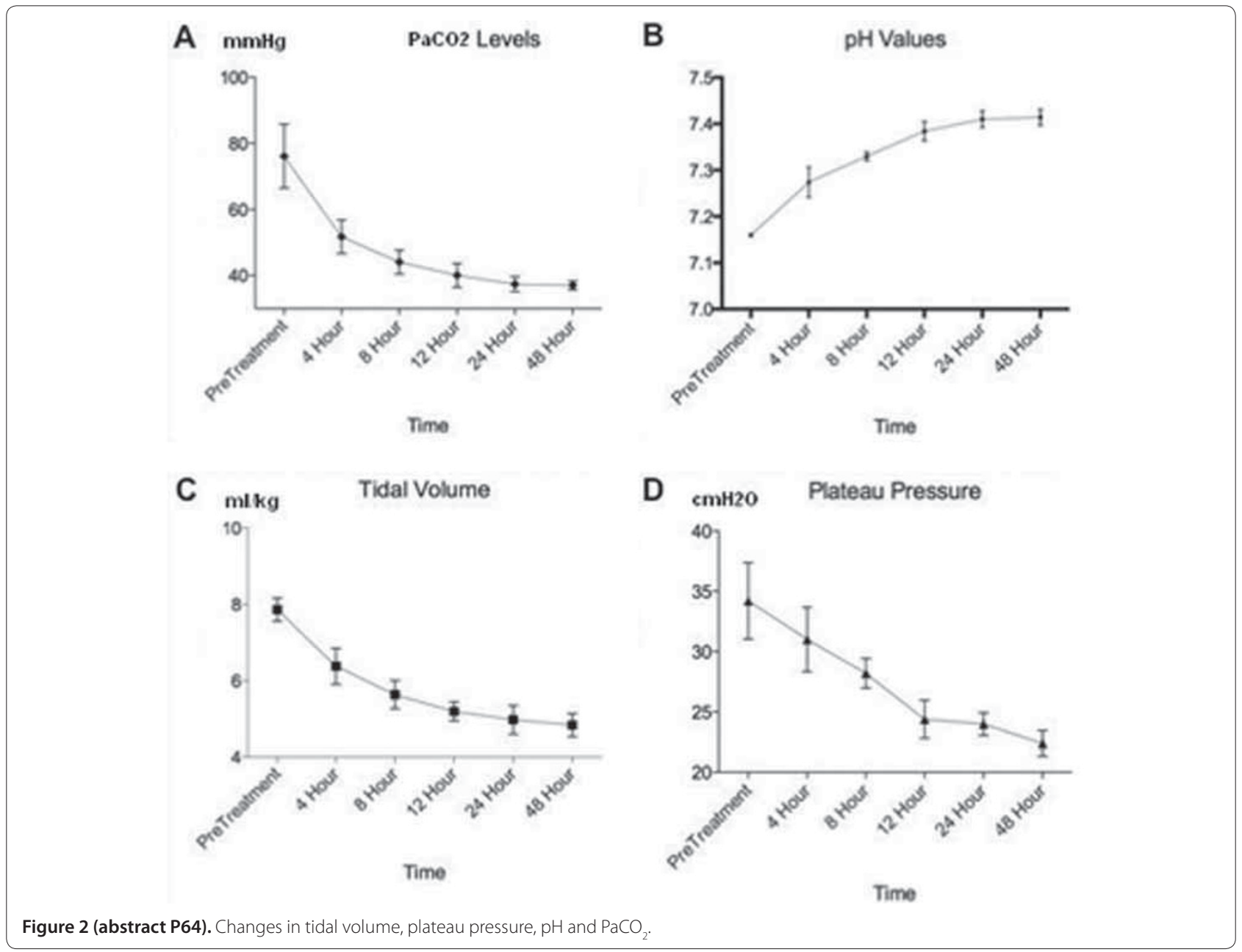

P67

Selective adsorption of lipopolysaccharide in the complex treatment of patients with severe sepsis

S Rei, I Aleksandrova, V Kiselev, M Ilynskiy, G Berdnikov, L Marchenkova, G Bulava, N Borovkova

Hospital Research Institute for Emergency Medicine named after N.V.

Sklifosovsky, Moscow, Russia

Critical Care 2013, 17(Suppl 2):P67 (doi: 10.1186/cc12005)

Introduction Severe sepsis and septic shock remain the most serious problem of critical care medicine with a mortality rate of 30 to $55 \%$ [1]. Several studies have demonstrated positive effects of selective adsorption of LPS on blood pressure, $\mathrm{PaO}_{2} / \mathrm{FiO}_{2}$ ratio, endotoxin removal and mortality [2,3]. The purpose of the study was to evaluate the efficiency of using the selective adsorption of LPS, Toraymyxin PMX-F (Toray, Japan) and Alteco ${ }^{\circledR}$ LPS Adsorber (Alteco Medical AB, Sweden), in the complex treatment of patients with severe sepsis. Methods Forty-six patients with Gram-negative sepsis in the postoperative period were enrolled into the study. Toraymyxin - PMX-F was used in the PMX-F group $(n=14)$, while Alteco LPS adsorption was used in the Alteco LPS group $(n=32)$. The clinical characteristics are listed in Table 1. The SOFA score, $\mathrm{PaO}_{2} / \mathrm{FiO}_{2}$, procalcitonin (PCT), C-reactive protein (CRP), endotoxin activity assay (EAA) was noted before, 24 and 48 hours after the selective adsorption of LPS.

Results At 48 hours after PMX-F, significantly decreased PCT from 17.5 $(5.0 ; 40.9)$ to $7.1(4.8 ; 13.0) \mathrm{ng} / \mathrm{ml}, P=0.028$, decreased CRP from 180
$(133 ; 286)$ to $132(68 ; 155) \mathrm{mg} / \mathrm{l}, P=0.015$ and SOFA score from $7.0(3,0$; $8.0)$ to $6.0(3,0 ; 7.0), P=0.007$. At 24 hours after Alteco LPS, significantly decreased PCT from $8.7(3.0 ; 25.9)$ to $4.8(2.1 ; 10.0) \mathrm{ng} / \mathrm{ml}$. The 28-day mortality rate was $14.2 \%(n=2)$ in the PMX-F group and $31.3 \%(n=10)$ in the Alteco LPS group.

Table 1 (abstract P67). Clinical characteristics of the groups

\begin{tabular}{lcc}
\hline Characteristic & PMX-F & Alteco LPS \\
\hline Sex M/F & $9 / 5$ & $17 / 15$ \\
Age (years) & $41.6 \pm 17.1$ & $42.1 \pm 13.5$ \\
Shock & $6(42.9 \%)$ & $13(40.6 \%)$ \\
APACHE II & $21(17 ; 25)$ & $21(16 ; 24)$ \\
SOFA & $7(3.0 ; 8)$ & $7(3.5 ; 11)$ \\
\hline
\end{tabular}

Conclusion The use of the LPS-selective adsorption (particularly PMX-F) in patients with severe sepsis leads to improvement of systemic inflammation and organ dysfunction.

\section{References}

1. Vincent JL, et al:: Crit Care Med 2006, 34:344-353.

2. Cruz DN, et al:: JAMA 2009, 301:2445-2452.

3. Yaroustovsky M et al.: Blood Purif 2009, 28:227-233. 
P68

Alteco endotoxin hemoadsorption in Gram-negative septic shock patients

HP Shum, KC Chan, WW Yan

Pamela Youde Nethersole Eastern Hospital, Hong Kong

Critical Care 2013, 17(Suppl 2):P68 (doi: 10.1186/cc12006)

Introduction Septic shock is a common cause of mortality and morbidity in the ICU. Endotoxin hemoadsorption using a Polymyxin B fiber column can improve patient outcome [1]. This study investigated the therapeutic effect of a new endotoxin hemoadsorption device in Gram-negative septic shock patients.

Methods An open, controlled, prospective, randomized, single-centre trial conducted between February 2010 and June 2012. Patients with septic shock due to intra-abdominal sepsis were recruited and randomized to either standard therapy (ST, $n=8)$ or standard therapy plus two 2 hourly sessions of Alteco endotoxin hemoperfusion (AT, $n=$ 7). Standard therapy included infective source control, appropriate early antibiotic, low-dose steroid, early continuous renal replacement therapy in the presence of acute kidney injury (RIFLE Injury class or more), hemodynamic optimization and lung-protected ventilatory support. Primary outcome was change in organ dysfunction at 48 hours measured by Sequential Organ Failure Assessment (SOFA) scores. Secondary outcomes were change in vasopressor requirement (measured by vasopressor score), $\mathrm{PaO}_{2} / \mathrm{FiO}_{2}$ (fraction of inspired oxygen) ratio, total urine output at 72 hours and 28 -day mortality. Results This study was terminated early as interim analysis identified no significant clinical benefit. Baseline characteristics (age/APACHE IV score) were similar between two groups of patients. No significant difference was noted between two groups with respect to change in total SOFA score ( +1 vs. -5.5 for AT vs. ST, $P=0.382$ ), vasopressor score ( -29 vs. $-46.6, P=0.775), \mathrm{PaO}_{2} / \mathrm{FiO}_{2}$ ratio $(-26$ vs. $+163, P=0.199)$, total urine output from 0 to 72 hours $(3,850 \mathrm{ml}$ vs. $4,570 \mathrm{ml}, P=0.355)$ and 28 -day mortality ( $14.3 \%$ vs. $37.5 \%, P=0.569)$. No significant side effect was noted when using this new hemoadsorption device.

Conclusion This small study cannot identify any extra clinical benefit on addition of Alteco endotoxin hemoadsorption to standard therapy in patients suffering from intra-abdominal sepsis with shock due to Gram-negative bacterial infection.

Reference

1. Cruz DN, et al.: JAMA 2009, 301:2445-2452.

\section{P69}

Steroid therapy in septic shock: survey of practice amongst UK critical care physicians

G Rajendran, K Dasari, A Dhrampal

Norfolk \& Norwich University Hospital, Norwich, UK

Critical Care 2013, 17(Suppl 2):P69 (doi: 10.1186/cc12007)

Introduction Corticosteroid (CS) therapy in sepsis remains controversial and was first introduced in sepsis management for its antiinflammatory property. CS has found a role in septic shock amelioration with inconsistent outcomes. The Surviving Sepsis Campaign (SSC) includes CS as a Level 2C recommendation in septic shock [1]. Adapting and practicing SSC guidelines vary between critical care units. Accordingly, a survey was conducted to elucidate the usage of CS for septic shock by UK critical care physicians (CCPs).

Methods Following approval by the UK Intensive Care Society (ICS), the survey was publicised on the ICS website and its newsletter.

Results A total of 81 intensivists responded to this online survey. Seventy-four (92.5\%) CCPs prescribed CS only if the septic shock is poorly responsive to fluid resuscitation and vasopressor therapy. Six $(7.5 \%)$ initiated CS at the same time as vasopressor therapy. None initiated CS for patients with severe sepsis. No CS other than hydrocortisone is being used. The most commonly used intravenous regimen is $50 \mathrm{mg} 6$ hourly (65\%) followed by $50 \mathrm{mg} 8$ hourly (11\%). Only $10 \%$ of CCPs would prescribe it by infusion. Less commonly used regimens were $100 \mathrm{mg} 8$ hourly (6\%) and $100 \mathrm{mg} 6$ hourly (5\%). Only $5 \%$ would consider adding fludrocortisone. Prior to initiating CS, $5 \%$ of CCPs would perform a short synacthen test, while $94 \%$ would not. The majority (89\%) of CCPs would stop CS after resolution of shock state or when vasopressor infusion is terminated whilst $11 \%$ after a fixed duration. Withdrawal of CS also differed, in that 25\% tapered/weaned steroids, $31 \%$ stopped it abruptly and $44 \%$ of CCPs would base their CS cessation pattern on the clinical context. Only $46 \%$ of CCPs believe that CS is beneficial whereas $44 \%$ were unsure of the benefits in septic shock. Only $29(36 \%)$ responders indicated that their critical care unit had a written protocol for CS in septic shock.

Conclusion The perceptions, usage and cessation of CS in septic shock vary but do appear to have shifted in the last decade. A UK survey in 2003 identified that only $60 \%$ of ICUs used CS for septic shock and over $22 \%$ perform a short synacthen test [2]. It appears that many intensivists are using CS for septic shock, despite conflicting outcome data. We all strive to practice evidence-based medicine but until we have a robust, reliable and methodical randomised control trial that attempts to resolve the CS debate, practice will remain diverse on this subject, as reflected by our survey.

References

1. Dellinger et al:: Crit Care Med 2008, 36:296-327.

2. Bourne et al: Anaesthesia 2003, 58:591-596.

\section{P70}

Need for restoration of cortisol serum levels for successful antimicrobial therapy in experimental sepsis

T Doulias', A Pistiki², P Christopoulos', V Papaziogas'1,

E Giamarellos-Bourboulis', I Koutelidakis'

'University of Thessaloniki, Medical School, Thessaloniki, Greece: '2University of Athens, Medical School, Athens, Greece

Critical Care 2013, 17(Suppl 2):P70 (doi: 10.1186/cc12008)

Introduction It is postulated that clinical benefit of low-dose hydrocortisone in septic shock is related to reversal of relative adrenal insufficiency [1]. This was proved in an animal model of sepsis.

Methods Sixty-nine Wistar male rats were assigned to the following groups: A, sham-operation; B, sepsis; C, bilateral adrenalectomy and sepsis; D, bilateral adrenalectomy, sepsis and hydrocortisone treatment; $\mathrm{E}$, bilateral adrenalectomy, sepsis and ertapenem treatment; and $\mathrm{F}$, bilateral adrenalectomy, sepsis, hydrocortisone and ertapenem treatment. Sepsis was induced by the i.p. infusion of $1 \times 10^{6} \mathrm{cfu} / \mathrm{ml}$ of Escherichia coli after adrenalectomy. Hydrocortisone $10 \mathrm{mg} / \mathrm{kg}$ was infused i.v. bid starting 1 hour after bacterial challenge. Ertapenem $5 \mathrm{mg} / \mathrm{kg}$ was infused i.v. once daily starting 1 hour after bacterial challenge. Survival was recorded. In a separate set of experiments in 18 rats, animal sacrifice was performed to measure the free cortisol concentration.

Results Survival is shown in Figure 1. Experiments in each animal were starting at 7:00 am. At 8:00 am, respective mean free cortisol of groups $\mathrm{A}, \mathrm{C}$ and $\mathrm{D}$ was $1.81,0.55$ and $2.05 \mu \mathrm{g} / \mathrm{dl} ;$ at $1: 00 \mathrm{pm}$ they were $0.92,0.47$ and $1.40 \mu \mathrm{g} / \mathrm{dl}$.

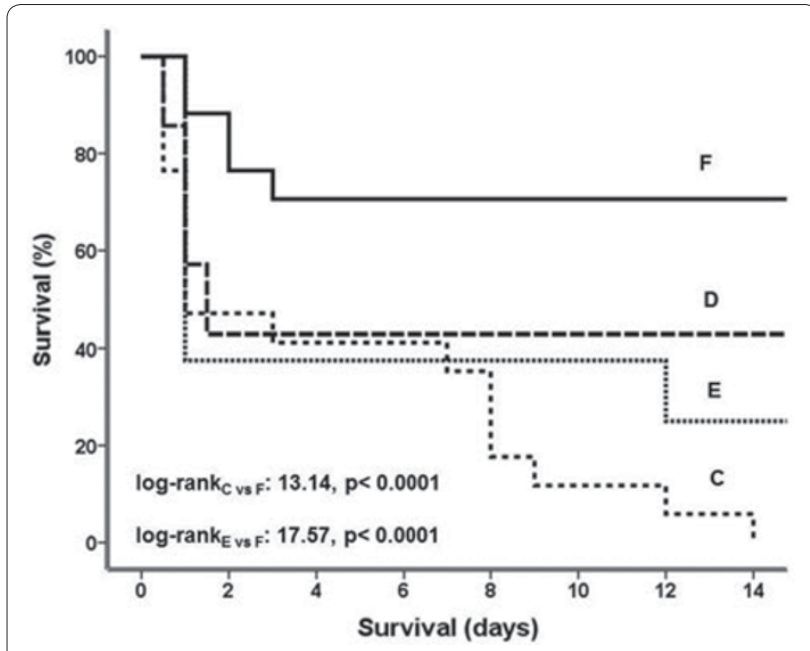

Figure 1 (abstract P70). Survival of rats. 
Conclusion Even when effective antimicrobial treatment is administered, administration of hydrocortisone at a regimen restoring normal secretion is mandatory for survival.

Reference

1. Annane D, et al:: JAMA 2002, 288:862-871.

P71

Impact of time to initiation of appropriate antibiotic therapy on early mortality of patients with septic shock

JP Quenot', C Binquet ${ }^{2}$, S Vinault ${ }^{2}$, A Pavon'

'University Hospital Dijon, France; ${ }^{2} \mathrm{CHU}$ de Dijon, Centre d'Investigation

Clinique - Epidémiologie Clinique, Dijon, France

Critical Care 2013, 17(Suppl 2):P71 (doi: 10.1186/cc12009)

Introduction The impact of appropriate antibiotic therapy on prognosis of patients with septic shock is well established. However, the prognostic influence of time to initiation of antibiotics remains debated. We evaluated the effect on 7-day mortality of time to initiation of appropriate antibiotic therapy in patients hospitalised in critical care for septic shock.

Methods Secondary analysis from the EPISS cohort. We included only patients admitted to the University Hospital Dijon. Septic shock was defined as initiation of vasopressors in a patient with suspected or documented infection with at least one criterion of hypoperfusion (metabolic acidosis and/or renal insufficiency and/or hepatic dysfunction). We excluded patients with no available bacteriological data. Antibiotherapy was considered appropriate if at least one of the antibiotics prescribed was active against the germ identified. Bivariate and multivariate logistic regression analysis was used to assess the impact of time to initiation of appropriate antibiotherapy on 7-day mortality.

Results In total, 383 patients were admitted with septic shock, of whom 253 (66\%) were included; 231(92\%) had appropriate antibiotic therapy, of whom 52 (22.5\%) died at 7 days. Average time to initiation of appropriate antibiotic therapy was $9 \pm 23$ hours. By bivariate analysis, body mass index (BMI) $<20$, SAPS $\| \geq 56$, SOFA score $\geq 11$ and bacteremia were significantly associated with 7-day mortality. Urinary tract infection (UTI) was a protective factor. Age, sex, comorbidities (particularly immunosuppression), Knaus score, nosocomial infection and type of germ had no influence on 7-day mortality. By multivariate logistic regression, $\mathrm{BMI}<20(\mathrm{OR}=4.87,95 \% \mathrm{Cl}=1.36$ to $17.43, P=0.01)$ and SOFA score $\geq 11$ (OR $=7.99,95 \% \mathrm{Cl}=3.11$ to $20.5, P<0.001)$ were the only factors significantly associated with 7-day mortality. UTI was a significant protective factor $(\mathrm{OR}=0.30,95 \% \mathrm{Cl}=0.10$ to $0.88, P=0.03)$. Time to initiation of appropriate antibiotherapy was not associated with 7-day mortality $(\mathrm{OR}=0.99,95 \% \mathrm{Cl}=0.99$ to $1.00, P=0.48)$.

Conclusion Prognosis at 7 days of patients with septic shock is largely related to the number of failing organs. The majority received appropriate antibiotic therapy although time to initiation is longer than recommended. Further efforts are warranted to reduce mortality in patients with septic shock.

\section{P72}

Comparison of survivors and nonsurvivors in 27 confirmed injectional anthrax cases from the $\mathbf{2 0 0 9}$ outbreak in Scotland M Booth', L Donaldson', C Xizhong', S Junfeng', P Eichacker ${ }^{2}$ 'Glasgow Royal Infirmary, Glasgow, UK: '2 National Institutes for Health Bethesda, MD, USA

Critical Care 2013, 17(Suppl 2):P72 (doi: 10.1186/cc12010)

Introduction From December 2009 to December 2010, 47 patients in Scotland presented with confirmed anthrax infection manifested by soft tissue disease related to heroin injection. These cases represent the first known outbreak of a recently recognized form of anthrax, termed injectional anthrax, which appears to be associated with a high mortality rate ( $28 \%$ in confirmed cases from the UK outbreak). While epidemiologic data from this outbreak have been published, no report has systematically described findings in patients at presentation or compared these findings in nonsurvivors and survivors.

Methods To better describe injectional anthrax, we developed a questionnaire and sent it to clinicians who had cared for confirmed cases during the outbreak. Completed questionnaires describing 27 patients, 11 nonsurvivors and 16 survivors, were returned.

Results In preliminary analysis of categorical data, a significantly (Fisher exact test) greater proportion of patients with compared with without the following findings did not survive; history of alcohol use $(P=0.05)$; the presence of lethargy $(P=0.01)$, confusion $(P=$ $0.03)$, nausea $(P=0.04)$, abdominal pain $(P=0.02)$, or the need for vasopressors $(P=0.002)$, oxygen, mechanical ventilation, or steroids (all $P=0.004)$ at presentation; and excessive bleeding at surgery $(P=$ 0.01 ). Initial analysis of continuous data demonstrated that, compared with survivors at presentation, nonsurvivors had significantly (one-way ANOVA) increased respiratory rate, percent neutrophils on complete blood count, hemoglobin, INR, C-reactive protein, and bilirubin and significantly decreased temperature, systolic blood pressure, platelets, sodium, albumin, calcium (corrected for albumin), base excess and bicarbonate (all $P \leq 0.05$ ).

Conclusion The implications of the apparent differences noted between nonsurvivors and survivors in this survey of cases from the first known outbreak of injectional anthrax require further study. However, these differences might inform the design of research during future outbreaks or of methods to identify patients most in need of anthrax-specific therapies such as toxin-directed antibodies.

\section{P73}

Efficacy and utility of preemptive anti-Gr(+) coverage in selected critically ill patients

VT Todorova, SM Milanov, G Georgiev, M Milanov

Emergency Hospital 'Pirogov', Sofia, Bulgaria

Critical Care 2013, 17(Suppl 2):P73 (doi: 10.1186/cc12011)

Introduction Based on the results of our previous studies [1] we have identified clinical risk factors for the emergence of $\mathrm{Gr}(+)$ infections in our ICU and we have developed a new algorithm for combating them. The choice of the particular antibiotic drug is guided by additional risk factors for severity of illness and data on the infectious focus. The response to therapy and its duration are also stated. The aim of the current study was to evaluate the efficacy and safety of this preemptive approach.

Methods A randomized prospective controlled trial was carried out from September 2010 to September 2012. Patients were submitted to block randomization and stratified on the basis of their initial SAPS II exp score. Antibiotic therapy was started on the day of inclusion in the treatment group and only with proven $\mathrm{Gr}(+)$ pathogen in the control group. Initial data were gathered on demographics, diagnosis, proven risk factors for sepsis-related mortality, severity of inflammatory response, ventilator-associated pneumonia and organ dysfunction. Dynamics of SIRS, CPIS and SOFA scores, subsequent infectious isolates, ventilator-free days, length of ICU stay and outcome were followed for each patient.

Results A total of 170 patients were enrolled. No statistically significant differences in their basal characteristics were found. The subsequent score values, length of ICU stay and the number of ventilator-free days were also comparable between groups. The majority of $\mathrm{Gr}(+)$ pathogens were isolated between 6 and 10 days of inclusion. No differences were found regarding the concomitant $\mathrm{Gr}(-)$ flora and the related antibiotic therapy. The new organ dysfunction severity was similar in both groups $(P=0.37)$. The in-hospital mortality was $26.2 \%$ in the treatment group versus $18.6 \%$ in the control group $(P=0.56)$. Significant differences between the Kaplan-Meier estimates of survival were also not found (log-rank test $P=0.81$ ). No major adverse reactions were observed.

Conclusion The implementation of this new policy failed to reduce the degree of organ dysfunction severity and was not associated with significant survival benefit. Moreover, even though it did not reach statistical significance, a second peak of $\mathrm{Gr}(+)$ isolates was observed as a possible complication of the preemptive therapy. Whether this approach could lead to vancomycin MIC creep or there could still be a niche for it later in the course of treatment and/or in nontrauma patients remains to be further explored.

Reference

1. Milanov S, et al:: Gram positive nosocomial infections in a general ICU emerging new clues. Crit Care 2011, 15(Suppl 1):P224. 
P74

Gram-negative resistance and need for ICU among urinary tract infections in the United States

M Zilberberg ${ }^{1}$, A Shorr ${ }^{2}$

'EviMed Research Group, LLC, Goshen, MA, USA; 'Washington Hospital Center, Washington, DC, USA

Critical Care 2013, 17(Suppl 2):P74 (doi: 10.1186/cc12012)

Introduction Urinary tract infection (UTI) can lead to both hospitalization and severe sepsis. We theorized that UTI due to Gramnegative (GN) multidrug-resistant $P$. aeruginosa (MDR-PA), extendedspectrum $\beta$-lactamase (ESBL), E. coli (EC) and Klebsiella sp. (KP), and carbapenemase-producing Enterobacteriaceae (CPE) would be frequently isolated in the ICU.

Methods We analyzed a large US-based microbiology database, Eurofins TSN, between the years 2000 and 2009. We determined the proportion of isolates caused by MDR-PA, ESBL-EC, ESBL-KP, and CPE relative to their susceptible counterparts. We defined MDR-PA as any isolate resistant to $\geq 3$ drug classes. ESBL organisms were defined as E. coli and K. pneumoniae resistant to a third-generation cephalosporin. Enterobacteriaceae were considered CPE if resistant to both a thirdgeneration cephalosporin and a carbapenem. We further examined the evolution of the frequency of resistance among GN UTIs over time. Results We identified 115,201 PA (13.7\% MDR-PA), 359,090 EC $(5.6 \%$ ESBL), 97,419 KP (12.9\% ESBL), and 176,110 Enterobacteriaceae $(0.6 \%$ CPE) UTI specimens. The prevalence of resistance rose for each organism of interest from 2000 through 2009: MDR-PA, 11.6 to 12.3\%; ESBL-EC, 3.3 to $8.0 \%$; ESBL-KP, 9.1 to $18.6 \%$; CPE 0 to $2.3 \%$. For each organism the proportion of resistant pathogens was consistently higher among ICU specimens than among specimens from other hospital locations, reaching nearly $20 \%$ for MDR-PA (Figure 1 ).

Conclusion The microbiology of GN UTI hospitalizations has shifted over the last decade and greater antimicrobial resistance is evident. The prevalence of MDR-PA, ESBL-EC, ESBL-KP, and CPE is higher in the ICU than in other hospital locations.

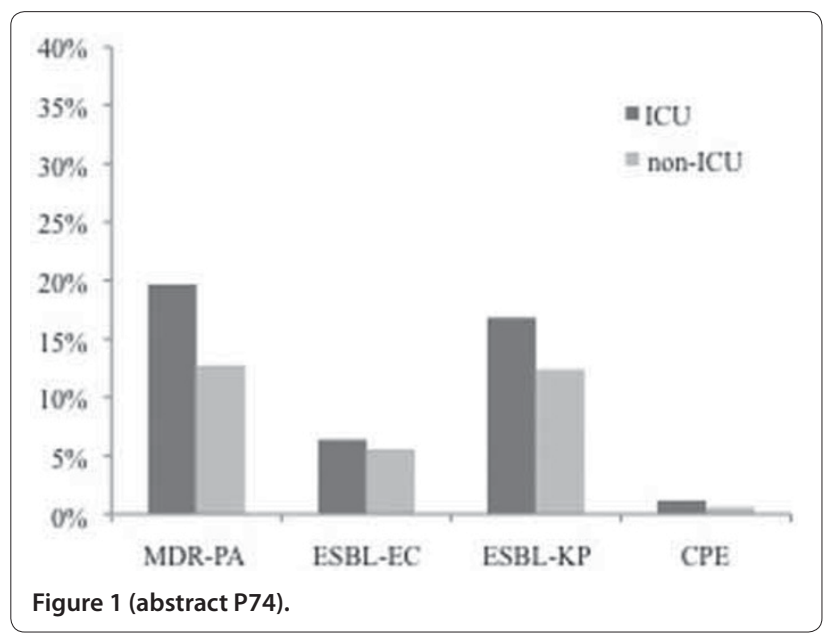

P75

Multidrug resistance among $P$. aeruginos $a$ and Enterobacteriaceae in US hospitals, 2000 to 2009

M Zilberberg', A Shorr ${ }^{2}$

'EviMed Research Group, LLC, Goshen, MA, USA; 'Washington Hospital Center, Washington, DC, USA

Critical Care 2013, 17(Suppl 2):P75 (doi: 10.1186/cc12013)

Introduction Gram-negative resistance remains a major challenge in the care of critically ill patients. Traditionally, P. aeruginosa (PA) has represented the most concerning pathogen. However, carbapenemaseproducing Enterobacteriaceae (CPE) have emerged as a challenge, but the epidemiology of this pathogen is poorly understood.
Methods We analyzed a large US-based microbiology database, Eurofins TSN, between the years 2000 and 2009. We aimed to describe the prevalence of infection with either multidrug-resistant (MDR) PA or CPE. We defined MDR-PA as any PA isolate resistant to $\geq 3$ drug classes. Enterobacteriaceae were classified as CPE if resistant to both a thirdgeneration cephalosporin and a carbapenem. We evaluated specimens from respiratory, bloodstream, urinary tract (UTI) and complicated intra-abdominal infections.

Results We identified 327,912 PA (60,695 (18.5\%) MDR-PA) and 279,600 Enterobacteriaceae $(2,558(0.9 \%) \mathrm{CPE})$ specimens. More than onequarter (26.1\%) of all PA were recovered from ICU patients as compared with $17.9 \%$ of all Enterobacteriaceae specimens. Of those specimens originating in the ICU, MDR-PA represented $21.9 \%$ of all PA organisms, while CPE represented $1.6 \%$ of all Enterobacteriaceae. Pneumonia and UTI accounted for $92.4 \%$ of all PA and $84.0 \%$ of all Enterobacteriaceae specimens. The proportion of both MDR-PA and CPE was highest in pneumonia (22.0\% and $1.6 \%$, respectively) and lowest in UTI $(13.7 \%$ and $0.6 \%$, respectively). Over the time frame of the study, CPEs emerged and stabilized at approximately $2.8 \%$ of all Enterobacteriaceae, while MDR-PA increased slightly from $16.0 \%$ of all PA in 2000 to $17.8 \%$ in 2009. Conclusion Although CPE organisms have emerged as an important pathogen, MDR-PA remains an order of magnitude more prevalent in the United States. Pneumonia patients and those in the ICU are at an increased risk for both MDR-PA and CPE infections compared with those outside the ICU.

\section{P76}

Prevalence of multidrug resistance, extensive drug resistance and pandrug resistance among multiple Gram-negative isolates: experience in a tertiary-care hospital ICU in North India

S Dewan, T Sahoo, N Chandra, A Varma

Fortis Escorts Heart Institute, New Delhi, India

Critical Care 2013, 17(Suppl 2):P76 (doi: 10.1186/cc12014)

Introduction Antibiotic resistance is a major worldwide problem in the ICU [1]. The situation in developing countries like India is particularly serious. Since the presence of drug-resistant bacteria in the environment is a threat for public health, up-to-date information on local pathogens and the drug sensitivity pattern is very crucial to treat patients. This study was carried out to evaluate the prevalence of multidrug resistance (MDR), extensive drug resistance (XDR) and pandrug resistance (PDR) among multiple Gram-negative isolates in a medical-surgical ICU in a tertiary care hospital in North India.

Methods We conducted a prospective observational study. All data were analysed using descriptive statistics. All Gram-negative culture isolates over a period of 13 months (October 2011 to October 2012) were included in this study. Isolation and identification were performed using the bact alert system and VITEK2, respectively. Sensitivities were determined by Kirby Bauer disc diffusion and broth dilution using VITEK2-AST cards and interpreted according to Clinical and Laboratory Standards Institute criteria. For the purpose of this study, we used MDR to denote isolates resistant to representatives three or more classes of antimicrobial agents, XDR as those resistant to all but one or two classes and PDR as those resistant to all classes of antimicrobial agents available $[2,3]$.

Results Out of a total 2,796 culture specimens sent over 13 months, 250 isolates were Gram-negative (8.9\%). Among these 250 (n) Gramnegative isolates, $195(78 \%)$ were extended-spectrum $\beta$-lactamase (ESBL) producers and the remaining $55(22 \%)$ were non-ESBL producers. Among the ESBL producers, PDR, XDR and MDR isolates were 14 (5.6\%), $113(45.2 \%)$ and $68(27.2 \%)$, respectively (Figure 1). Among the XDR-positive organisms, seven (6.1\%) organisms were New Delhi metallo- $\beta$-lactamase-1 (NDM-1) producers and five $(4.4 \%)$ organisms were NDM-2 producers. Among ESBL-positive isolates, the most predominant isolate was Klebsiella pneumoniae (29.7\%) followed by Acinetobacter aeruginosa (22.5\%) and Escherichia coli (20.5\%) (Figure 2). Among non-ESBL-positive isolates, the most predominant isolate was Escherichia coli (34.5\%) followed by Klebsiella pneumoniae (21.8\%) and Pseudomonas aeruginosa (14.5\%) (Figure 2).

Conclusion ESBL producers were the most frequently isolated Gramnegative bacterial isolates in this tertiary-care hospital in north India. 


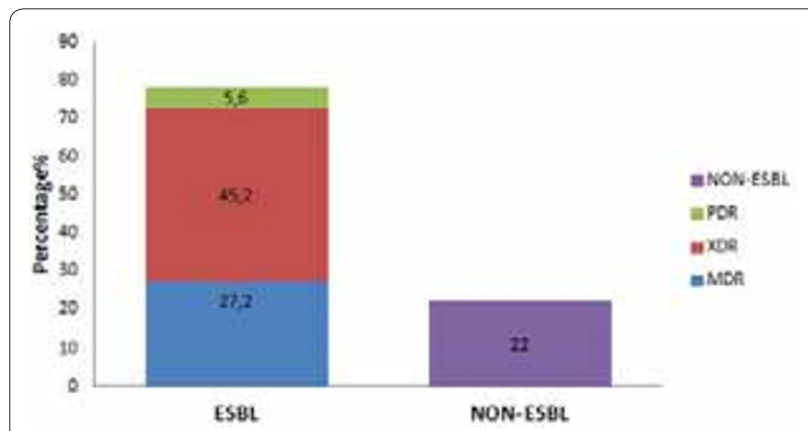

Figure 1 (abstract P76). Prevalence of drug-resistant Gram-negative isolates.

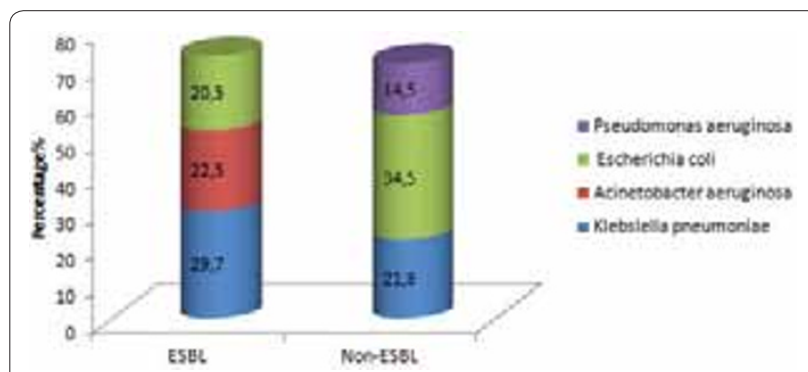

Figure 2 (abstract P76). Prevalence of Gram-negative isolates.

Among ESBL producers, XDR organisms were most frequent, followed by MDR and PDR organisms. Few of the XDR isolates were NDM producers, which have propensity to spread to other bacteria. In view of significant prevalence of multidrug resistance amongst Gram-negative organisms in the ICU, regular surveillance of antibiotic susceptibility patterns plays a crucial role for setting orders to guide the clinician in choosing empirical or directed therapy of infected patients.

\section{References}

1. Radji M, et al:Asian Pac J Trop Biomed 2011, 1:39-42.

2. Falagas ME, et al: Clin Infect Dis 2008, 46:1121-1122.

3. Souli M, et al:: Euro Surveill 2008, 13:30-40.

P77

Risk factors associated with Acinetobacter baumannii septicemia and its mortality rates in critically ill patients

K Kontopoulou, KTsepanis, I Sgouropoulos, A Triantafyllidou, D Socratous, PTassioudis, E Chasou, E Antypa, F Renta, E Antoniadou, K Mandraveli

General Hospital G. Gennimatas, Thessaloniki, Greece

Critical Care 2013, 17(Suppl 2):P77 (doi: 10.1186/cc12015)

Introduction Acinetobacter baumannii (A. baum) is a leading cause of septicemia of patients hospitalized in the ICU with high mortality rates. The aim of our study is to investigate the risk factors associated with A. baum bacteremia and its mortality rates.

Methods A total of 937 patients (457 women and 480 men, median age 59) admitted to the ICU during the period 1 January 2009 to 30 September 2012 were enrolled in our retrospective study. Blood cultures were obtained from all patients. The identification and the antimicrobial susceptibility testing were performed by the automated system VITEK2 (Bio Merieux, France). Data collected included underlying diseases, malignancies and immune suppression (MIS), prognostic factors (APACHE score, adjusted mortality), age, sex, length of ICU stay (LS), recent administration of broad-spectrum $\beta$-lactam antibiotics (especially carbapenems; $A B L$ ), mechanical ventilation (MV), implementation of invasive procedures (central venous catheter and urine catheter; INV.PR) and outcome. At first a univariate statistical model was used with significance level set at $P=0.05$. For the multivariate statistical analysis model we used all variables with
$P<0.05$ from the previous model and those mentioned at recent medical literature as significantly related with A. baum septicemia and its mortality.

Results A total of 101 patients (10.78\%) developed bloodstream infection caused by $A$. baum and the mortality rate due to $A$. baum septicemia was estimated as $49.5 \%(50 / 101)$. Multiple regression analysis revealed adjusted mortality $>55 \%(\operatorname{Exp}(B)=2.01, P=0.013)$, MIS $(\operatorname{Exp}(B)=1.97, P=0.017), A B L(\operatorname{Exp}(B)=2.34, P=0.009), L S>14$ days $(\operatorname{Exp}(B)=1.34, P=0.034), M V(\operatorname{Exp}(B)=2.67, P=0.005)$ and INV.PR $(\operatorname{Exp}(B)=3.27, P=0.001)$ as independent risk factors associated with A. baum septicemia.

Conclusion The fact that the probability of $A$. baum bacteremia increases in immunocompromised patients reflects the opportunistic characteristic of these infections. MV accelerates respiratory A. baum colonization, which is a risk factor for $A B$ bacteremia. Recent INV. PR increased the incidence of $A$. baum bacteremia and this result is probably related to the severe status in patients with central venous catheter. The administration of carbapenems inhibits the growth of other more susceptible bacteria, allowing the growth of multidrugresistant A. baum.

References

1. Wu HS, et al:: Clin Microbiol Infect 2012, 18:E373-E376

2. Huang ST, et al:: J Microbiol Immunol Infect 2012, 45:356-362.

P78

Prevalence of colonization by multiresistant bacteria on admission to the ICU in the French military hospital in Kabul, Afghanistan JV Schaal, P Pasquier, H Delacour, A Salvadori, A Jarrassier, JR Renner, SM Mérat

Military Teaching Hospital Bégin, Saint-Mandé, France Critical Care 2013, 17(Suppl 2):P78 (doi: 10.1186/cc12016)

Introduction The French military hospital at the Kaboul International Airport (KalA) base provides surgical care for International Force and Afghan National Army soldiers, and also local patients. The development of multiresistant bacteria (MRB) nosocomial infections has raised a major problem complicating the care of combat casualties [1]. The aim of this study is to assess the prevalence of MRB carriage on admission to the ICU in this combat support hospital.

Methods We used a prospective observation study on patients admitted to the French military ICU in KalA over 3 months (July to September 2012). All hospitalized patients were assessed for the presence of colonization with MRB: nasal and rectal swabs were performed to identify, respectively, methicillin-resistant Staphylococcus aureus (MRSA) and extended-spectrum $\beta$-lactamases bacteria (ESBLB). The following data were recorded for each patient on admission: demographic characteristics, bacteriological results, length of stay, type of previous hospitalization.

Results Sixty-three patients were admitted. The mean length of stay (MLS) was $3 \pm 3$ days, and the mean age was $25 \pm 14$ ( 13 patients $<15$ years). Patients were hospitalized for combat-related trauma (74\%), noncombat-related trauma, medical pathologies (10\%), and postoperative care (8\%). They were Afghans (92\%) or westerners (8\%). Swabs were not realized for eight patients. Forty-three percent revealed an ESBLB colonization: Escherichia coli (22 patients), Klebsiella pneumoniae (one patient), Acinetobacter baumanii (one patient). No patients were colonized with MRSA. Ten patients $(16 \%)$ were directly admitted to the ICU, 12 (19\%) had been hospitalized before admission, $39(62 \%)$ were transferred after resuscitative and stabilization care in a level 2 unit. For the two last categories, the MLS (for previous hospitalization) was respectively $14 \pm 28$ days and $8 \pm 6$ hours. Among patients transferred after care in a level 2 unit, MLS was no different between colonized and noncolonized patients: $8 \pm 7$ versus $9 \pm 6$ hours $(P=0.5$, Mann-Whitney test).

Conclusion In this study, prevalence of colonization with ESBLB at admission is very high, suggesting a high prevalence of MDR colonization in the local population in Afghanistan. It remains important to intensify the prevention policy against MRB cross-transmission in the deployed ICU.

Reference

1. Whitman TJ: Infection control challenges related to war wound infections in the ICU setting. J Trauma 2007, 62:S53. 
P79

Extended-spectrum $\beta$-lactamase Providencia stuartii in a general ICU

P Myrianthefs' ${ }^{1}$ E Evodia², G Fildissis' ${ }^{1}, \mathrm{G}$ Baltopoulos ${ }^{1}$

'Athens University, Athens, Greece; 'latriko Kentro Athinon, Athens, Greece

Critical Care 2013, 17(Suppl 2):P79 (doi: 10.1186/cc12017)

Introduction Providencia stuartii, a member of the family of Enterobacteriacea, is a Gram-negative pathogen causing colonization and opportunistic infections in ICU patients.

Methods We retrospectively recorded the characteristics of $P$. stuartii infections in our ICU in a total period of 1 year (six-bed general ICU).

Results A total of 116 patients ( 80 males, $68.9 \%$ ) were hospitalized in our ICU of mean age $58.5 \pm 1.8$, mean ICU stay: $23.2 \pm 3.3$ days, APACHE II: $19.3 \pm 0.7$, SAPS II: $45.7 \pm 1.6$, SOFA: $7.9 \pm 0.4$ and mortality: $18.9 \%$. Admission diagnosis was multiple trauma (29.3\%), emergency surgery (37.1\%), and medical (33.6\%). Of them 21 (18.1\%) developed P. stuartii infection that was related to multiple trauma $(P=0.0289)$, length of ICU stay $69.8 \pm 12.6$ (median 51.0$)$ versus $12.6 \pm 1.7$ (median 6.0) $(P<0.0001)$ and illness severity APACHE II (21.7 \pm 1.3 vs. $17.5 \pm 0.8 ; P=0.0056)$, SAPS II $(54.9 \pm 2.9$ vs. $43.6 \pm 1.8 ; P=0.0296)$ and SOFA $(10.6 \pm 0.5$ vs. $7.2 \pm 0.4$; $P<0.0001)$. There was no statistically significant difference regarding sex, age or mortality $(P=0.3789)$. Mean day of first isolation was $31.1 \pm 2.1$ (median 28.0). The number of isolations per site were - blood: 11 (median day: 25.0), tracheal aspirates: 9 (median: 28.5), catheter tip: 15 (median: 31.0), urine: 6 (median: 32.0), wound: 12 (median: 25.0), biological fluids: 4 (median: 29.5), other catheters: 1 (median: 28.0), middle ear: 2 (median: 32.5), and nose: 1 (median: 25.0). Six patients had only one site isolation and the remaining 15 had multiple sites of $P$. stuartii isolation. We totally recorded 63 isolates of which $82.5 \%$ were second-generation and third-generation cephalosporin-resistant, $80.3 \%$ aztreonam-resistant and $81 \%$ carbapenem-resistant strains expressing an extended-spectrum $\beta$-lactamase phenotype. All patients had previously received colistin or meropenem or tygecycline for a median period of $18.5,15$ and 10.5 days, respectively.

Conclusion P. stuartii infection/colonization may develop in critically ill patients, especially those with multiple trauma and prolonged ICU stay, and maybe isolated in blood after the 25th day of ICU stay. Also, infections due to extended-spectrum $\beta$-lactamase-producing multidrug-resistant $P$. stuartii are an emerging problem.

P80

High-dose tigecycline use in severe infections

G De Pascale', L Montini', T Spanu², V Bernini', A Occhionero', DL Grieco', M Biancone', P De Santis ${ }^{1}$, ES Tanzarella', SL Cutuli', MA Pennisi',

MA Antonelli

'Sacro Cuore Catholic University, Rome, Italy; 'Institute of Microbiology, Sacro Cuore Catholic University, Rome, Italy

Critical Care 2013, 17(Suppl 2):P80 (doi: 10.1186/cc12018)

Introduction The aim of this study is to describe the clinical and epidemiological profile of ICU patients receiving tigecycline (TGC) and to evaluate the potential benefits of TGC higher doses.

Methods All patients admitted to our ICU between 1 June 2009 and 31 May 2012 who received TGC were evaluated. Cases were excluded when infections were not microbiologically confirmed.

Results Over the study period, 100 patients fulfilled the inclusion criteria: 54 in the SD group (50 mg every 12 hours) and 46 in the HD group (100 mg every 12 hours). The SD group and the HD group were not significantly different in terms of age, severity of disease, duration of TGC therapy, rate of concomitant other active antibiotic use and of inadequate empirical antimicrobial therapy (IIAT) $(P=N S)$. MDR $A$. baumannii and $K$. pneumoniae were the main pathogens isolated. The percentage of germs other than A. baumannii and $K$. pneumoniae was higher in the SD TGC group $(P<0.01)$. Otherwise infections due to less susceptible germs (TGC MIC value $\geq 1 \mu \mathrm{g} / \mathrm{ml}$ ) were mainly treated with TGC higher doses $(P<0.01)$. No significant differences were found in terms of ICU mortality $(P=0.8)$. The rate of abnormal laboratory measures during TGC treatment was similar between the two groups $(P=\mathrm{NS})$. No patients required TGC discontinuation or dose reduction because of suspected adverse events. In the VAP subpopulation
(63 patients: 30 received SD and $33 \mathrm{HD}$ ), the clinical cure rate and microbiological eradication percentage were higher when TGC was used at higher doses $(57.6 \%$ vs. $33.3 \% ; P=0.08$ and $57.1 \%$ vs. $30.4 \%$; $P=0.1$ ). Table 1 shows multivariate analysis of clinical cure predictors in the VAP subgroup.

Table 1 (abstract P80). Predictors of clinical cure in patients with VAP (multivariate analysis)

\begin{tabular}{lcc}
\hline & OR $(\mathbf{9 5} \% \mathrm{Cl})$ & $P$ value \\
\hline HDTGC & $5.93(1.38$ to 25.6$)$ & 0.02 \\
IAT & $0.23(0.06$ to 0.85$)$ & 0.03 \\
SOFA score & $0.62(0.45$ to 0.86$)$ & $<0.01$ \\
\hline
\end{tabular}

Conclusion In critically ill patients, HD TGC use seems to be safe and, combined with other active antibiotics, may increase the rate of MDR germ VAP clinical success. IIAT and the severity degree of patients' clinical condition still remain major determinants of VAP treatment failure.

Reference

1. Tasina E, et al:: Efficacy and safety of tigecycline for the treatment of infectious diseases: a meta-analysis. Lancet Infect Dis 2011, 11:834-844.

P81

In vitro efficiency of Amikacin Inhale, a novel drug-device delivery system

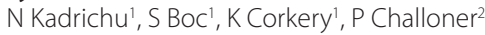

${ }^{1}$ Novartis Pharmaceuticals, San Carlos, CA, USA; ${ }^{2}$ Nektar Therapeutics, San Francisco, CA, USA

Critical Care 2013, 17(Suppl 2):P81 (doi: 10.1186/cc12019)

Introduction Amikacin Inhale (NKTR-061, BAY41-6551) is a drugdevice combination in clinical development for adjunctive treatment of intubated and mechanically ventilated patients with Gram-negative pneumonia. The product uses a proprietary vibrating mesh nebulizer system (PDDS Clinical) with amikacin sulfate formulated for inhalation ( $3.2 \mathrm{ml}$ of $125 \mathrm{mg} / \mathrm{ml}$ amikacin solution) for a 10-day twice-daily course of therapy. It is designed for use with two delivery systems: one system for intubated patients (On-vent; Figure 1), and a second Handheld (HH) system for patients who are extubated before completing the course of therapy (Figure 2). We investigated in vitro the amikacin lung dose delivered by PDDS Clinical.

Methods An estimated lung dose (ELD) for On-vent setting was measured in vitro after collecting aerosolized amikacin from a filter at the end of an endotracheal tube during ventilation. The ELD for the HH device was calculated from the fine particle fraction (FPF $<5 \mu \mathrm{m}$ ) postmouthpiece, multiplied by the in vitro delivered dose post-mouthpiece. FPF $<5 \mu \mathrm{m}$ reflects lung deposition observed during phase 2 clinical trials [1]. Eighty-one nebulizers with volume median diameter (VMD)

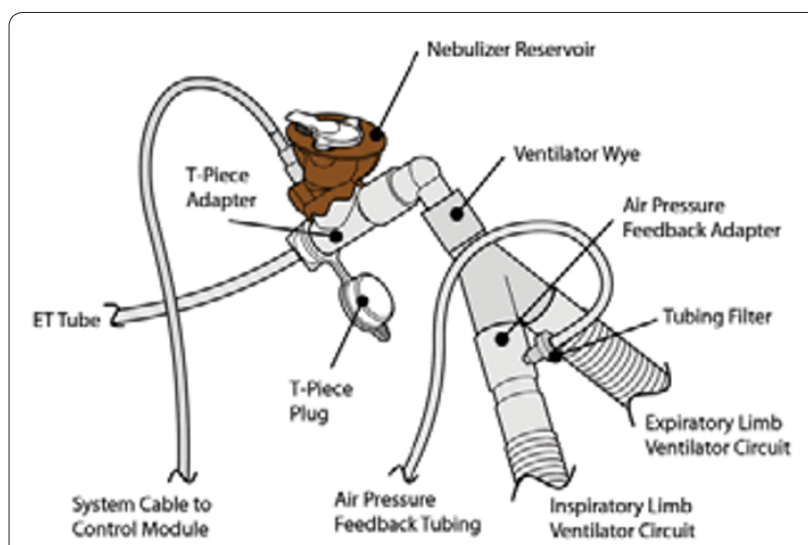

Figure 1 (abstract P81). On-vent system with brown blinder shell for clinical trials. 


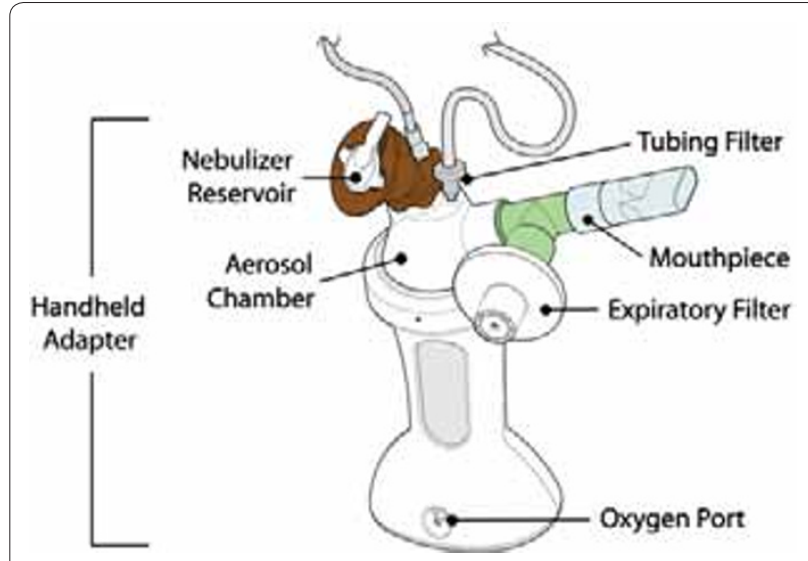

Figure 2 (abstract P81). Handheld system with brown blinder shell for clinical trials.

of $4.4 \pm 0.5 \mu \mathrm{m}$ and output rates of $0.23 \pm 0.10 \mathrm{ml} /$ minute were tested for each system. Delivered dose data were fit to the independent variables (that is, VMD and output rates) using a least-squares fit with $95 \%$ confidence limits.

Results Total percentage recoveries for On-vent and $\mathrm{HH}$ test runs were between $85 \%$ and $115 \%$ of the nominal dose. The mean ELDs were $50 \pm 9 \%$ (On-vent) and $49 \pm 11 \%(\mathrm{HH})$ of the nominal dose. Nebulizers with longer dosing times and lower VMDs had higher ELD values for both delivery systems.

Conclusion The results support the use of the PDDS Clinical with either system to administer aerosolized amikacin with high efficiency and no dose adjustment is required when switching from the On-vent to the $\mathrm{HH}$ system for extubated patients.

Reference

1. Fink J, et al: High in vivo amikacin lung deposition after NKTR-061 dosing correlates with in vitro aerosol characterization. Am J Respir Crit Care Med 2008, 177:A530

P82

Analysis of parameters in critically ill patients influencing amikacin peak levels

IM Hollevoet', SJ Toye'2, J De Waele

'UZ Gent, Belgium:'25tijn Toye, Gent, Belgium

Critical Care 2013, 17(Suppl 2):P82 (doi: 10.1186/cc12020)

Introduction Recent studies demonstrate that a loading dose of $25 \mathrm{mg} / \mathrm{kg}$ (total body weight) of amikacin in septic patients is required to reach a sufficient peak concentration. This study examines parameters influencing the relation between amikacin dose and peak concentration.

Methods In this retrospective study we looked at 47 patients (128 peak levels) between 2003 and 2012. Multivariate linear regression analysis was done for several parameters: administered dose calculated with total body weight, ideal body weight, adjusted body weight, type of intensive care patient, BMI, daily fluid balance, SOFA score and APACHE score, and patient characteristics were analyzed.

Results A linear correlation between dose and amikacin peak level was confirmed (Figure 1). A total $54.69 \%$ of all amikacin administrations did not result in a therapeutic peak level. The multivariate linear regression analysis showed the best linear correlation with adjusted body weight and SOFA score. The comparison of variables between four patient groups, based on the deviation between measured peak level and predicted peak level (according the linear correlation), showed new variables that may influence peak level.

Conclusion This confirms that low doses $(<18 \mathrm{mg} / \mathrm{kg})$ of amikacin in intensive care patients seldom result in a therapeutic peak level. The proposed loading dose of $25 \mathrm{mg} / \mathrm{kg}$ is good for reaching a therapeutic level, although $29.6 \%$ remains subtherapeutic. Due to the linear correlation, more therapeutic levels may be reached with higher doses ( 25 to $30 \mathrm{mg} / \mathrm{kg}$ ). New variables need further investigation to explain the high variability in achieved peak level.

References

1. Delattre IK, et al: Ther Drug Monit 2010, 32:749-756.

2. Taccone FS, et al:: Crit Care 2010, 14:R53.

3. Gálvez R, et al: Int J Antimicrobial Agents 2011, 38:146-151.

4. Avent ML, et al: Internal Med J 2011, 41:441-449.

5. Fernández de Gatta MM, et al.: I Clin Pharm Ther 1996, 21:417-421.

\section{P83}

Cost implication of antibiotic-associated diarrhoea and the financial impact of probiotic use in its prevention

CEbm, M Cecconi, C Moran, A Rhodes, T Rahman

St Georges Healthcare Trust, London, UK

Critical Care 2013, 17(Suppl 2):P83 (doi: 10.1186/cc12021)

Introduction Antibiotic-associated diarrhoea (AAD) occurs in as many as $30 \%$ of patients receiving antibiotics, often leading to increased morbidity, prolonged in-hospital stay and additional healthcare resource utilisation. Age, antibiotics and prolonged postoperative ward and ICU stay have been suggested to be independent risk factors. In such patient populations, probiotics may be used to prevent antibioticassociated diarrhoea, yet they are not routinely recommended as a component of perioperative care. The aim of this study was to model the long-term costs associated with AAD and to assess the effectiveness of probiotics as a preventive strategy.

Methods We developed a simulation model to determine clinical costs and outcomes attributable to AAD. To assess the cost-effectiveness of probiotics, as part of a perioperative regime, we constructed a decision
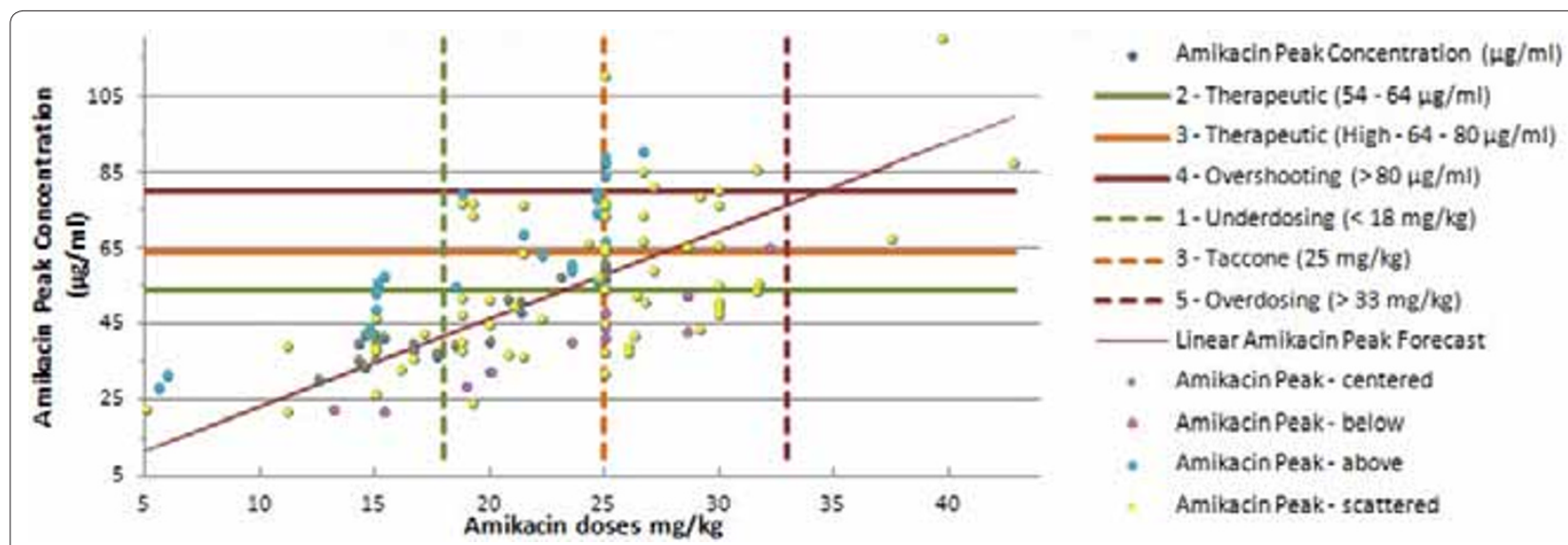

Figure 1 (abstract P82). Correlation between dose of amikacin and peak concentration. 
tree. The model observes long-term costs and outcomes of probiotics as compared with conventional therapy, from a societal perspective. Input parameters, extracted from meta-analysis, clinical trials and national databases, include incidence numbers, costs and qualityadjusted health states for the remaining life (QALYs). Outcomes assessed were overall costs attributable to ADD and the cost-effectiveness of probiotics, described as costs/QALY.

Results Our results indicate an estimated incremental lifetime cost of $£ 13,272.53$ per ADD patient, largely driven by increased ICU length of stay and readmission rates. The addition of probiotics to the standard perioperative regime is associated with a small survival benefit of 1.2 months, yet a cost reduction of $£ 917.3 /$ ADD patient. The main cost was increased duration of ICU stay and readmissions, which contribute to $85 \%$ of total expenses.

Conclusion AAD is associated with a significant increase in costs from a societal perspective. The provision of probiotics can achieve substantial cost savings and can be recommended as a cost-effective regime in the perioperative setting. Preventing ADD offers a potentially significant reduction of in-hospital costs and resource expenditures.

P84

Empirical versus preemptive antimycotic therapy in terms of outcome benefit

S Milanov, VT Todorova, G Georgiev, M Milanov

Emergency Hospital 'Pirogov', Sofia, Bulgaria

Critical Care 2013, 17(Suppl 2):P84 (doi: 10.1186/cc12022)

Introduction Novel treatment strategies for invasive candidiasis (IC) are constantly emerging. Nevertheless, difficulties in diagnosis pose a challenge on their reliability, efficacy and safety. We have previously developed and approbated in our ICU an algorithm for empirical antimycotic therapy, combining the most significant risk factors for IC with three major clinical criteria for persistent nonbacterial sepsis [1]. On the other hand, preemptive therapy, based on identification of mycotic antigens and/or anti-mycotic antibodies in serum, is regarded as more reliable, even though it is known for its low sensitivity. The aim of the current study was to compare and evaluate the possible outcome benefit of our protocol implementation versus detection of galactomanan in patient's serum as a trigger for antimycotic treatment initiation.

Methods A randomized prospective controlled trial was carried out from September 2010 to September 2012. After the implication of the inclusion and exclusion criteria, patients were submitted to block randomization and stratified on the basis of their initial SAPS II exp score. Antimycotic therapy was started on the day of inclusion in the control group and only with positive galactomanan serum test in the preemptive therapy group. Initial data were gathered on demographics, proven risk factors for IC-related mortality, severity of inflammatory response and organ dysfunction. Dynamics of SIRS and SOFA values, Candida colonization index, ventilator-free days, length of ICU stay and outcome were followed for each patient.

Results A total of 106 patients were enrolled. No statistically significant differences in their basal characteristics were found. The subsequent SIRS and SOFA scores showed firm dynamics in the control group, although the new organ dysfunction severity was insignificantly lower. The length of ICU stay and the number of ventilator-free days were comparable. The in-hospital mortality was $47.1 \%$ in the preemptive therapy group versus $31.3 \%$ in the control group $(P=0.94)$. A total of seven adverse reactions were observed among treated patients, yet not associated with higher mortality risk.

Conclusion The choice of empirical versus preemptive therapy led to earlier and more stable reduction in the degree of organ dysfunction severity. It showed to be at least not inferior if not equal; in terms of survival benefit and expediency of treatment. Moreover, galactomanan detection fails to guide the choice of the individual antimycotic, based on the expected Candida spp.

Reference

1. S Milanov, et al: Efficacy and utility of a protocol for pre-emptive antimycotic therapy. Crit Care 2010, 14(Suppl 1):P70.
P85

Impact of echinocandins as first-choice therapy in cardiothoracic high-risk patients with candidemia

G Langebartels, Y Choi, J Catteleans, T Wahlers

University of Cologne, Germany

Critical Care 2013, 17(Suppl 2):P85 (doi: 10.1186/cc12023)

Introduction Invasive candidemia is a major cause of increased mortality among ICU patients. Antifungal agents like liposomale amphotericin B and azoles could not accomplish the claim to be first choice in the treatment of invasive fungal infection (IFI) because of side effects and effectiveness. Especially, cardiothoracic surgery patients as a group of high-risk patients are in a focus for new strategies and agents. A new class of antimycotic agents, the echinocandins, with a low profile of side effects, low interactive potential and high effectiveness in the treatment of candidemia, is a powerful option in the treatment of IFI. We report our single-center experience with a modified clinical treatment approach based on clinical score of Leon and using echinocandins as first-line therapy for proven and suspected fungal infection.

Methods From May 2011 to October 2012, 2,844 patients were treated on our cardiothoracic ICU. We evaluated 37 cardiothoracic postoperative patients with proven or suspected IFI or prophylaxis (Figure 1). The records were evaluated for cardiothoracic procedures, microbiological and yeast date, cardiothoracic surgery score (CASUS), ICU and clinical data.

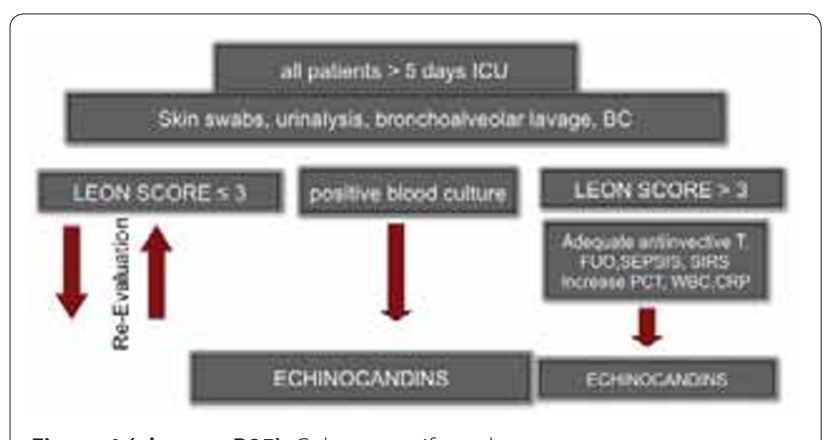

Figure 1 (abstract P85). Cologne antifungal strategy.

Results Mean age was 67.4 years with $64 \%$ male patients. Most patients had combined CABG and valve procedure $(n=20)$, other groups were HTX and LTX $(n=4)$, assist therapy $(n=4)$, TAVI $(n=3)$ and other procedures. Mean predicted mortality using the logarithmic CASUS score at the onset of IFI was 59\%. C. albicans was isolated in 73\%, C. glabrata in $21 \%$. Length of antifungal treatment using micafungin in 30 cases was $14 \pm 5$ days. Eradication of yeast was successful in $79 \%$ but mortality of all patients remains high at $36.8 \%$ but was lower than predicted in the CASUS score. Mortality was not yeast related.

Conclusion Our described treatment approach shows encouraging results for the treatment of IFI especially in high-risk cardiothoracic patients.

References

1. Eggiman et al.: Invasive Candida infections in the ICU. Mycoses 2012, 55(Suppl):65-72.

2. Andes et al.: Impact of treatment strategy on outcomes in patients with candidemia and other forms of invasive candidiasis: a patient-level quantitative review of randomized trials. Clin Infect Dis 2012, 54:1110-1122.

P86

Fungal ventricular assist device infections occur in colonized patients and are associated with high mortality rate P Gaudard, J Eliet, N Zeroual, G Culas, P Colson

CHRU Montpellier, France

Critical Care 2013, 17(Suppl 2):P86 (doi: 10.1186/cc12024)

Introduction Infection is a common complication of the ventricular assist device (VAD) and is associated with poor outcome especially 
with fungi [1]. The relationship between colonization and invasive fungal infection (IFI) in severely ill ICU patients with a VAD support is not described. This study analyzes the incidence and outcome of fungal infection and colonization in VAD patients in bridge to transplantation or in destination therapy.

Methods We conducted a retrospective review of all VAD implantations in our surgical ICU between 2007 and 2012. The incidence of fungal colonization, antifungal prophylaxis, bacterial sepsis and the mortality of IFI versus no IFI patients were compared.

Results In the study period, 34 patients with severe heart failure or cardiogenic shock were selected for a VAD implantation (nine in destination therapy). The overall mortality rate was $50 \%$ during mechanical assistance. Confirmed $(n=8)$ and highly suspected $(n=2)$ IFI occurred during the ICU stay in $29 \%$ of patients who were treated with echinocandins, voriconazole and/or liposomal amphotericin B. The isolated fungi were: six Candida albicans, two parapsilosis, one glabrata and one invasive pulmonary aspergillosis. Antifungal prophylaxis with fluconazole was administered to $18 \%$ of patients at mean for 5 days mainly in the more recent implantations. In the no IFI population, $54 \%(n=13)$ had a systemic or VAD bacterial sepsis with a mortality rate about $54 \%$. The mortality without any sepsis was reduced to $18 \%$. Fungal colonization was significantly more present (90\% vs. $50 \%)$ before IFI in VAD patients. The mortality rate was dramatically higher with IFI ( $80 \%$ vs. $38 \%)$ in accordance with the literature [1]. See Table 1.

Table 1 (abstract P86). Fungal colonization (FC), infection and outcome during VAD support

\begin{tabular}{lccc}
\hline & $\boldsymbol{n}$ & FC & Mortality \\
\hline No IFI & 24 & 12 & 9 \\
$\mid \mathrm{FI}$ & 10 & 9 & 8 \\
$P$ value & & 0.029 & 0.024
\end{tabular}

Conclusion In our center, we observed a high incidence of IFI in ICU patients with VAD that was associated with a mortality rate of $80 \%$. Screening of fungal colonization appears to be very important during the ICU stay for VAD patients. Trials are needed for investigating the use, the drug choice and the timing of antifungal prophylaxis for such high-risk patients.

Reference

1. Aslam S, et al.: Clin Infect Dis 2010, 50:664-671.

\section{P87}

Cost-effectiveness analysis of anidulafungin in the treatment of candidaemia

G Auzinger', G Playford ${ }^{2}$, C Graham 3 , H Narula 3 , C Charbonneau 4 , D Weinstein ${ }^{4}$, M Kantecki $^{4}$, H Schlamm ${ }^{5}$, M Ruhnke

'King's College Hospital, London, UK; ${ }^{2}$ Princess Alexandra Hospital, Brisbane, Australia: ${ }^{3}$ RTI Health Solutions, Durham, NC, USA. 4 Pfizer International

Operations, Paris, France; ${ }^{5}$ fizer Inc., New York, NY, USA; ${ }^{6}$ Charité University Medicine, Berlin, Germany

Critical Care 2013, 17(Suppl 2):P87 (doi: 10.1186/cc12025)

Introduction Echinocandins are recommended first-line treatment for candidaemia [1]. A cost-effectiveness model developed from a UK perspective examined costs and outcomes of antifungal treatment for candidaemia and other forms of invasive candidiasis based on European clinical guidelines [1].

Methods Costs and treatment outcomes with the echinocandin anidulafungin were compared with caspofungin, micafungin, fluconazole, voriconazole and amphotericin B. The model included non-neutropenic patients aged $\geq 16$ years with confirmed candidaemia/ another form of invasive candidiasis receiving intravenous first-line treatment [2]. Patients were categorised as a clinical success or failure (patients with persistent/breakthrough infection); frequency data for each outcome were taken from a mixed-treatment comparison [3]. Successfully treated patients switched to oral therapy. Clinical failures switched to a different antifungal class. It was assumed that second-line treatment duration was equivalent to that of first-line treatment and only two lines of therapy were required to treat infection. Other inputs were all-cause 6-week mortality, cost of treatment-related adverse events (AEs) and other medical resource use costs. Life-years were calculated using a published model [4]. Antifungal agent-related AEs were taken from the product label/literature. Resource use was derived from the literature and discussion with clinical experts. Drug acquisition/ administration costs were taken from standard UK costing sources.

Results First-line anidulafungin for treatment of candidaemia was cost-effective per life-year gained versus fluconazole (incremental cost-effectiveness ratio $£ 813$ ). Anidulafungin was cost saving versus caspofungin and micafungin in terms of life-years gained due to lower ICU costs and a higher rate of survival combined with a higher probability of clinical success.

Conclusion Anidulafungin was cost-effective compared with fluconazole for treatment of candidaemia and was cost saving versus other echinocandins in the UK. European guidelines recommend echinocandins as first-line treatments for candidaemia [1]; this model indicates that anidulafungin marries clinical effectiveness and cost-effectiveness.

\section{References}

1. Cornely OA: Clin Microbiol Infect 2012, 18:19-37.

2. Reboli AC, et al:: N Engl J Med 2007, 356:2472-2482.

3. Mills EJ, et al:: Ann Clin Microbiol Antimicrob 2009, 8:23.

4. Sidhu MK, et al:: Curr Med Res Opin 2009, 25:2049-2059

P88

Prevalence and impact of invasive fungal infections in intensive care

JM Patel, K Couper, T Melody, R O'Brien, D Parekh

Heart of England NHS Foundation Trust, Birmingham, UK

Critical Care 2013, 17(Suppl 2):P88 (doi: 10.1186/cc12026)

Introduction Invasive fungal infections (IFI) affect $1 \%$ of ICU patients and are increasing in incidence. IFIs are associated with a poor prognosis, which is further complicated by difficulties in identification of fungal organisms by traditional culture methods and the emergence of Candida species resistant to triazole therapy $[1,2]$. This study aimed to assess the prevalence of IFIs, the organisms responsible and outcomes of patients affected.

Methods Patients admitted to the Heart of England NHS Foundation Trust ICUs who acquired an IFI were identified through the hospital Fungal Infection Risk Evaluation (FIRE) study database. All ICU patients admitted between October 2009 and March 2011 were used as a comparative cohort. Data collected included: baseline demographics, length of stay, ICU and hospital mortality, and nature of IFI. Data were analysed using Student's $t$ test for continuous data and Fischer's exact test for categorical data.

Results A total of 2,426 patients were admitted to Heart of England NHS Foundation Trusts ICUs during the study period. Of these, 31 patients were identified as having an IFI (1.3\%). Baseline demographic data were similar between groups. Patients with IFI had significantly longer ICU length of stay ( 19 days vs. 5 days, $P<0.0001$ ) and required more days of advanced organ support (12 days vs. 3 days, $P<0.0001$ ). A trend towards higher hospital mortality $(41 \%$ vs. $27 \%, P=0.08)$ was observed. Candida albicans was the predominant organism cultured (64\%), followed by Candida glabrata (23\%), with other Candida species accounting for the remaining $13 \%$ of IFIs. Sites of IFIs were blood (45\%), intra-abdominal (39\%), and pleural (16\%). Most patients (52\%) who acquired an IFI had had intra-abdominal surgery prior to ICU admission. The majority of patients (71\%) were treated with echinocandins, whilst of the nine patients who were initially treated with flucanazole, six (67\%) required therapy escalation to an echinocandin.

Conclusion The results of our study are consistent with other published data, in that whilst IFI prevalence is low, they are associated with increased morbidity in critically ill patients. This study has led to a change in hospital policy regarding antifungal use in the ICU, with echinocandins being first-line in the pre-emptive treatment of IFI. We keenly await the results of the FIRE study, which will provide important insights to identification of patients at risk of IFIs and optimal drug therapy.

References

1. Allou N, et al:: Curr Infect Dis Rep 2011, 13:426-432.

2. Zaragosa R, et al: Adv Sepsis 2008, 6:90-98. 
P89

Beliefs and actual practice of oxygen therapy in the ICU

HJ Helmerhorst ${ }^{1}$, MJ Schultz², PH Van der Voort ${ }^{3}$, E De Jonge ${ }^{1}$

DJ Van Westerloo'

'Leiden University Medical Center, Leiden, the Netherlands; ${ }^{2}$ Academic

Medical Center, Amsterdam, the Netherlands; ${ }^{3}$ Onze Lieve Vrouwe Gasthuis,

Amsterdam, the Netherlands

Critical Care 2013, 17(Suppl 2):P89 (doi: 10.1186/cc12027)

Introduction The aim of this study was to compare self-reported beliefs with actual clinical practice of oxygen therapy in the ICU. Hyperoxia is frequently encountered in ventilated patients and prolonged exposure has repeatedly been shown to induce lung injury and (systemic) toxicity.

Methods An online questionnaire for ICU clinicians was conducted to investigate beliefs and motives regarding oxygen therapy for critically ill patients. Furthermore, arterial blood gas (ABG) samples and corresponding ventilator settings were retrieved to retrospectively assess objective oxygenation between 1 April 2011 and 31 March 2012 in the ICUs of three teaching hospitals in the Netherlands.

Results Analyzable questionnaire responses were received from 200 ICU physicians and nurses. The majority of respondents believed that oxygen-induced lung injury is a concern, although barotrauma and volutrauma are generally considered to impose a greater risk in mechanical ventilation. Frequently allowed minimal saturation ranges in the questionnaire were 85 to $95 \%$ and 7 to $10 \mathrm{kPa}$ (Figure 1). Selfreported $\mathrm{FiO}_{2}$ adjustment in hypothetical patient cases with variable saturation levels was moderately impacted by the underlying clinical condition. To study actual clinical practice, a total of 107,888 ABG samples with corresponding ventilator settings, covering 5,565 patient admissions, were retrieved. Analysis showed a median (IQR) $\mathrm{PaO}_{2}$ of $11.7 \mathrm{kPa}$ (9.9 to 14.3), median $\mathrm{FiO}_{2}$ was 0.4 (0.4 to 0.5), median PEEP was 5 (5 to 8 ). A total $63.5 \%$ of all $\mathrm{PaO}_{2}$ registries were higher than previously suggested oxygenation goals ( 7.3 to $10.7 \mathrm{kPa}$ ) [1]. In $56.8 \%$ of cases with $\mathrm{PaO}_{2}$ higher than the target range, neither $\mathrm{FiO}_{2}$ nor PEEP levels had been lowered when the next ABG sample was taken.

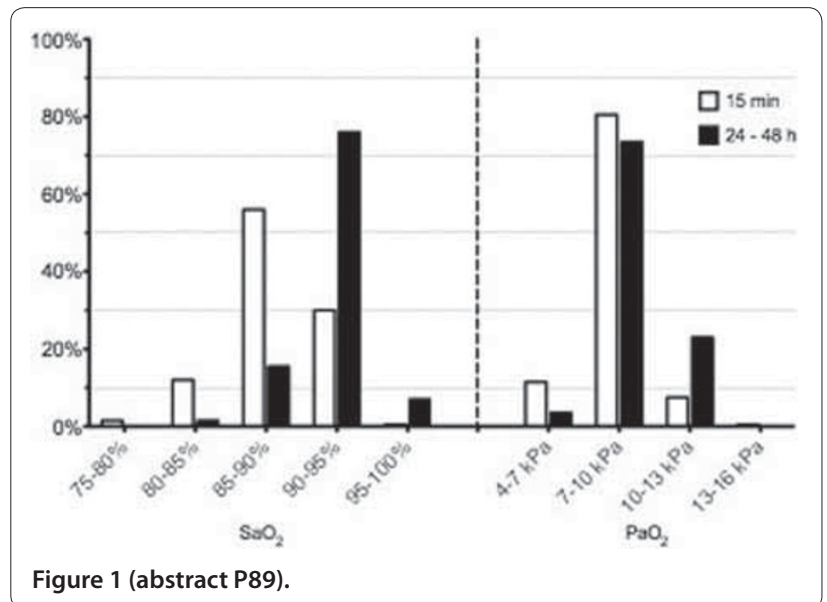

Conclusion Most clinicians acknowledge the detrimental effects of prolonged exposure to hyperoxia in the ICU and report a low tolerance for high saturation levels. However, the self-reported intention for conservative oxygen therapy is not consistently expressed in our objective data of actual clinical practice and a large proportion of patients was exposed to high and potentially toxic oxygen levels. Reference

1. Brower RG, et al:: N Engl J Med 2004, 351:327-336.
P90

$\mathrm{FiO}_{2} / \mathrm{PEEP}$ index: a simple tool for opitimizing ventilator settings

DTrasy, M Nemeth, K Kiss, ZTill, Z Molnar

University of Szeged, Hungary

Critical Care 2013, 17(Suppl 2):P90 (doi: 10.1186/cc12028)

Introduction During mechanical ventilation, oxygenation can be influenced by adjusting $\mathrm{FiO}_{2}$ and positive end-expiratory pressure (PEEP). There have been recommendations for how the $\mathrm{FiO}_{2}$ and PEEP should be set [1]. However, in a recent audit we found that the compliance of doctors of these recommendations is very low [2]. Therefore we invented a simple parameter called the $\mathrm{FiO}_{2} / \mathrm{PEEP}$ index ( $\mathrm{FPi}$ ) of which the physiologic value is $\leq 7$ (that is, $\mathrm{FiO}_{2}=21 \% / \mathrm{PEEP}=$ $3 \mathrm{cmH}_{2} \mathrm{O}$ ), which corresponds to the ARDSNet trial's minimum $\mathrm{FiO}_{2}$ / PEEP settings: $35 \% / 5 \mathrm{cmH}_{2} \mathrm{O}$ [2]. The aim of this case-control study was to investigate the impact of an $\mathrm{FPi} \leq 7$ targeted protocol on clinical practice.

Methods A prospective observational study in 2010 and 2012. Every mechanically ventilated patient was recruited. Demographics, outcome data and Lung Injury Score (LIS) were recorded after admission. Respiratory settings, oxygenation and ventilation parameters were recorded after mechanical ventilation was commenced and the first arterial blood gas sample was taken (T0). Measurements were repeated in 24 hours (T1). Data are presented as mean \pm SD, paired-sample and independent-sample $t$ test and chi-square tests were used for statistics. Results In 2010, 75 patients, and in 2012, 130 patients were included. There was no difference in demographics, disease severity, but LIS was higher in 2012: $1.34 \pm 1.13$ versus $0.84 \pm 0.98, P=0.001$. There was no significant difference in FPi between the two groups at T0: $10.91 \pm 4.25$ versus $10.26 \pm 5.01$ ( 2012 vs. 2010 , respectively). At T24 the FPi was significantly lower in 2012 as compared with 2010: $7.28 \pm 2.58$ versus $8.17 \pm 3.3, P=0.001$; which was due to the higher PEEP applied: $7.08 \pm 2.87$ versus $6.63 \pm 2.91, P=0.014$. Although in 2012 significantly more patients, $112(86 \%)$, were ventilated with $\mathrm{FiO}_{2} \geq 50 \%$ at TO as compared with $2010,44(58 \%)(P=0.001)$, by T24 significantly less patients received $\mathrm{FiO}_{2} \geq 50 \%, 46(35 \%)$ vs. $34(45 \%)(P=0.011)$. There was no significant difference between the two groups regarding $\mathrm{FiO}_{2^{\prime}}$ $\mathrm{PaO}_{2}$ and $\mathrm{PaCO}_{2}$ at $\mathrm{T} 0$ and $\mathrm{T} 24$.

Conclusion Implementing an $\mathrm{FPi} \leq 7$-based algorithm significantly reduced the $\mathrm{FiO}_{2}$ and increased the PEEP applied in mechanically ventilated within the first 24 hours. Whether this has any impact on earlier weaning due to reaching the weaning criteria of $\mathrm{FiO}_{2}$ sooner, and as a result shortening the duration of mechanical ventilation, has to be investigated in the future.

References

1. ARDSNet: N Eng/ J Med 2000, 342:1301.

2. Kiss K, et al:: Intensive Care Med 2011, 37(Suppl 1):S195.

P91

Use of high-flow nasal canulae: effect on alveolar pressure and its limitation

H Hayami, K Mizutani, M Shioda, STakaki, H Maejima, K Ueno,

Y Yamaguchi, T Kariya, T Gotoh

Yokohama City University Hospital, Yokohama, Japan

Critical Care 2013, 17(Suppl 2):P91 (doi: 10.1186/cc12029)

Introduction High-flow nasal canulae (HFNC) deliver high-flow humidified gas at up to $60 \mathrm{l} /$ minute. There are two types of respiratory circuit to generate mix gas flow, Blender type (typeB) and Venturi type (typeV). The therapy is well established in the pediatric population and HFNC use has been described in the adult population. It has been reported that HFNC provide higher $\mathrm{FIO}_{2}$ compared with low-flow canulae, and also create mild positive pharyngeal airway pressure, but the effect on alveolar pressure is unknown. We aimed to investigate the effect of HFNC on alveolar pressure, by measuring intratracheal pressure in patients with a cricothyrotomy catheter (CTC). At the same time, we measured the actual gas flow rate (AGFR) by flowmeter and compared it with assumed flow.

Methods Seven patients with a CTC were participated. A tube was connected to the CTC and the tube was then connected to a pressure transducer to measure intratracheal pressure. The HFNC (Optiflow 
system) were applied with the humidifier to optimize humidication. TypeB was used in three patients and typeV in four patients. The flow was started at $10 \mathrm{l} /$ minute. This flow rate was titrated upwards to a maximum of $60 \mathrm{l} /$ minute $(10,25,30,40,50,60 \mathrm{l} /$ minute) and the AGFR was measured. Intratracheal pressure tracing was done over 1 minute. Airway pressure measurement was repeated and the maximal expiratory pressure was measured in $\mathrm{mmHg}$.

Results The AGFR in the respiratory circuit was almost same in typeB, but there was obvious decrease in the AGFR in typeV (7.1 \pm 1.0 , $17.7 \pm 0.8,21.9 \pm 0.9,29.9 \pm 3.6,36.9 \pm 2.7,45.0 \pm 5.5 \mathrm{l} /$ minute at assumed flow, 10, 25, 30, 40,50, $60 \mathrm{l} /$ minute, respectively). HFNC significantly increased maximal expiratory pressure in both groups, $1.5 \pm 2.1$, $2.0 \pm 1.0,3.0 \pm 2.8,4.5 \pm 3.5 \mathrm{mmHg}$ for typeV and $2.5 \pm 0.7,5.8 \pm 2.4$, $6.0 \pm 2.8,8.0 \pm 2.8 \mathrm{mmHg}$ (maximum $10 \mathrm{mmHg}$ ) for typeB, when AGFR was set at $30,40,50,60 \mathrm{l} /$ minute. Higher AGFRs were found to result in larger increase in maximum expiratory pressure. The data indicate that HFNC are associated with an increase in intratracheal expiratory pressure. Because it was difficult to determine end-expiratory pressure, we chose maximal expiratory pressure for a substitute. The reason why AGFR in typeV was lower than assumed flow may be the resistance generated by NC. The larger increase in expiratory pressure in our study than previously reported may be due to the effect of high respiratory resistance of Japanese who have relatively small airway structure compared with western people.

Conclusion HFNC are effective in providing higher expiratory pressure. It is important to know the flow rate is lower than expected when the Venturi type is used.

\section{P92}

Relation between $\mathrm{PaO}_{2} / \mathrm{FiO}_{2}$ ratio, $\mathrm{SpO}_{2} / \mathrm{FiO}_{2}$ ratio, oxygenation

index and ventilation ratio in critically ill patients

T Aslanidis, A Myrou, E Chytas, E Anastasiou, E Geka, E Efthimiou,

V Ourailoglou, I Soultati, S Primikiri, M Giannakou-Peftoulidou

A.H.E.P.A. University Hospital, Thessaloniki, Greece

Critical Care 2013, 17(Suppl 2):P92 (doi: 10.1186/cc12030)

Introduction Many authors have proposed less invasive ways to measure oxygenation in patients with ALI. The aim of the present study is to compare the already popular oxygenation index $(\mathrm{OI})$ and $\mathrm{PaO}_{2} /$ $\mathrm{FiO}_{2}$ ratio $(\mathrm{PFr})$ with other recently proposed indices such as $\mathrm{SpO}_{2} / \mathrm{FiO}_{2}$ ratio (SFr) and ventilation ratio (VR) in a mixed ICU population.

Methods During a 6-month prospective observational study carried out in a polyvalent 10-bed adult ICU, ABGs were obtained from 145 patients. Two independent measurements were taken from each patient under the same mode of ventilation (SIMVPSV). PFr, SFr, VR and OI were calculated. Demographic data (APACHE II score, age, sex, diagnosis, body weight) were also recorded. Kolmogorov-Smirnov test for normality was calculated followed by bivariate nonparametric analysis (statistical significance: $P<0.05$ ).

Results A total of 290 measurements were included for further analysis. Mean \pm SD values for age and APACHE II score were $61.2 \pm 16$ years and $15.4 \pm 1.8$, respectively, while median \pm SD values for PFr are $257 \pm 135$, for SFr $211 \pm 58$, for Ol $4.8 \pm 4.46$ and for VR $1.19 \pm 0.31$. Relations between PFr, SFr and OI are displayed in Table 1.

Table 1 (abstract P92). Correlations (Spearman $\rho$ ) between VR, PFr, SFr and OI

\begin{tabular}{lccc}
\hline & $\mathrm{PFr}$ & $\mathrm{SFr}$ & Ol \\
\hline $\mathrm{VR}$ & $-0.326^{*}$ & $-0.325^{*}$ & $0.373^{*}$ \\
$\mathrm{PFr}$ & - & $0.759^{*}$ & $-0.939^{*}$ \\
$\mathrm{SFr}$ & - & - & $-0.773^{*}$ \\
\hline
\end{tabular}

${ }^{*} P<0.01$.

Conclusion Our study identified a strong relation between PFr, SFr and Ol but not VR. Thus, these markers may be used interchangeably as bedside indices of oxygenation in critically ill patients. Yet larger studies are needed to come to a safer conclusion.

References

1. Rice TW, et al: Chest 2007, 132:410-417.

2. Sinha P, et al:: Br J Anaesth 2009, 102:692-697.

P93

Decreased respiratory complexity during apnea in acute respiratory distress syndrome

A Batchinsky', C Necsoiu', T Langer², V Vecchi², W Baker', J Salinas', L Cancio ${ }^{1}$

'US Army Institute of Surgical Research, Fort Sam Houston, TX, USA; 2University of Milan, Italy

Critical Care 2013, 17(Suppl 2):P93 (doi: 10.1186/cc12031)

Introduction Respiratory complexity (RC) as assessed by sample entropy (SampEn) is lower in patients failing spontaneous breathing trials. We evaluated the role of RC in monitoring subjects with severe acute respiratory distress syndrome (ARDS) treated with the Cardiohelp (Maquet, Rastatt, Germany) extracorporeal life-support system (ECLS). We hypothesized that $\mathrm{RC}$ is reduced during apnea.

Methods Six sheep sedated with midazolam were connected to the Cardiohelp via a 23F Avalon catheter. Blood flow was $\sim 2 \mathrm{l} /$ minute and $\mathrm{FiO}_{2}$ was 0.5 . Sheep were on CPAP of $8 \mathrm{cmH}_{2} \mathrm{O}$ with $\mathrm{FiO}_{2}$ of 1 via tracheostomy. After $\sim 6$ hours of ECLS, ARDS was induced by injection

Table 1 (abstract P93)

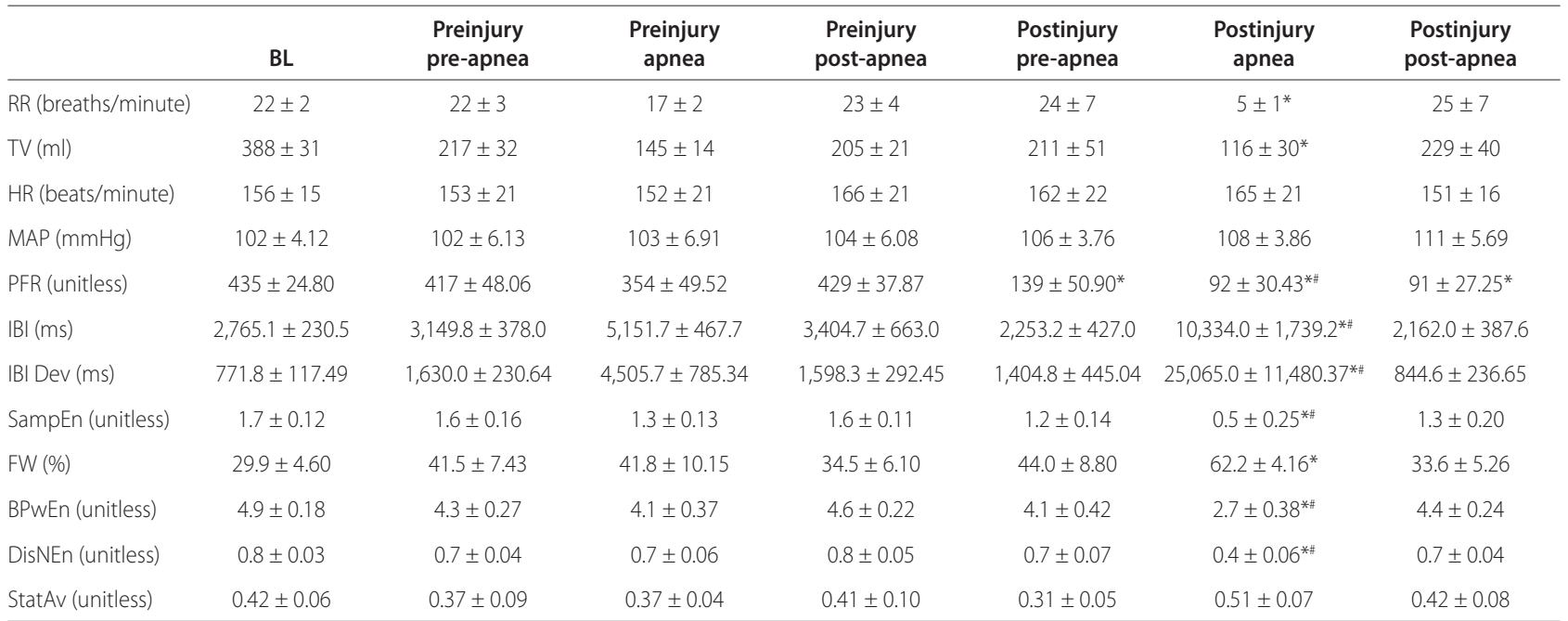

*Significant difference versus Baseline. "Significant difference between Preinjury and Postinjury apnea. 
of $0.1 \mathrm{ml} / \mathrm{kg}$ oleic acid. Apneas (defined as $>60 \%$ reduction in minute ventilation) developed both before (Preinjury) and after ARDS (Postinjury). Heart rate (HR), mean arterial pressure (MAP), respiratory rate (RR) and tidal volume (TV) were recorded, and the $\mathrm{PaO}_{2}: \mathrm{FiO}_{2}$ ratio (PFR) calculated corresponding to sections of respiratory pressure waveforms containing 200 breaths during Baseline, during Preinjury and Postinjury apnea periods. Similar periods were selected before and after apneas. Inter-breath interval (IBI) and its deviation, SampEn, percentage of forbidden words (FW), bit per-word entropy (BPWEn), symbol distribution entropy (DisNEn), and stationarity (StatAv) were calculated using software. Statistics by one-way ANOVA with adjustment for multiple comparisons.

Results See Table 1. SampEn was associated with PFR $\left(r^{2}=0.31\right.$, $P<0.001$ ).

Conclusion Respiratory complexity during apnea and ECLS remains unchanged in a healthy state but decreases after ARDS. It has a significant association with PFR and may serve as an index of injury severity.

\section{P94}

Acute respiratory distress syndrome - the Berlin definition: impact on an ICU of a university hospital

A Agrifoglio, J Lopez, J Fiqueira, M Hernández, L Fernández, M Irazabal,

S Yus, M Jiménez

Hospital Universitario La Paz, Madrid, Spain

Critical Care 2013, 17(Suppl 2):P94 (doi: 10.1186/cc12032)

Introduction The current definition of ARDS was established in 1994 by the AECC. In 2011 emerged the Berlin definition. The aim of this study was to find a new approach from the impact of ARDS in an ICU and to analyze APACHE II score, days of mechanical ventilation (MV), duration of ARDS and mortality.

Methods We analyzed the database of our ICU collected prospectively from October 2008 to January 2011 and regarded all patients undergoing MV for 48 hours or more and who met the AECC criteria. All patients were ventilated according to the ARDSNet protocol.

Results During this period 1,900 patients were admitted, 607 were under MV for at least 48 hours and 104 had ARDS criteria. Moderate ARDS 60.6\%: 60\% male, median age 53 years, APACHE II mean 22.49 ( $95 \% \mathrm{Cl}=20.71$ to 24.27$)$, mean duration MV $23.84(95 \% \mathrm{Cl}=18.08$ to 29.60), ARDS duration $12.87(95 \% \mathrm{Cl}=9.83$ to 15.90$)$, average stay 29.22 , mortality $52.4 \%$. Severe ARDS 39.4\%: 63\% male, median age 45 years, APACHE II mean score $23.19(95 \% \mathrm{Cl}=20.62$ to 25.75$)$, mean duration MV $18.52(95 \% \mathrm{Cl}=13.01$ to 24.03$)$, ARDS duration $12.15(95 \% \mathrm{Cl}=$ 8.72 to 15.58 ), average stay 22.65 , mortality $48.8 \%$ with no statistically significant difference with moderate ARDS $(P=0.72)$. Leading cause of death in both groups: multiorgan dysfunction (60\%). See Tables 1 and 2. Conclusion In this new approach of our ARDS patients we found no statistically significant differences in relation to the variables of interest to analyze in the two groups. Patients with severe ARDS who survive

Table 1 (abstract P94). Subgroup analysis: moderate ARDS

\begin{tabular}{lccc}
\hline Variable & Survivors & Nonsurvivors & $P$ value \\
\hline APACHE II & $20.06 \pm 6.96$ & $24.83 \pm 6.17$ & 0.006 \\
MV (days) & $25.63 \pm 29.13$ & $22.21 \pm 15.43$ & 0.557 \\
ARDS (days) & $10.29 \pm 9.07$ & $15.20 \pm 12.92$ & 0.107 \\
ICU stay & $34.33 \pm 33.26$ & $24.57 \pm 16.91$ & 0.142 \\
\hline
\end{tabular}

Table 2 (abstract P94). Subgroup analysis: severe ARDS

\begin{tabular}{lccc}
\hline Variable & Survivors & Nonsurvivors & $P$ value \\
\hline APACHE II & $20.82 \pm 8.89$ & $25.31 \pm 5.63$ & 0.761 \\
MV (days) & $26.95 \pm 18.03$ & $10.10 \pm 11.64$ & 0.001 \\
ARDS (days) & $15.8 \pm 11.60$ & $8.5 \pm 8.6$ & 0.031 \\
ICU stay & $33.52 \pm 18.25$ & $11.25 \pm 11.80$ & 0.001 \\
\hline
\end{tabular}

ICU admission have a longer duration of ARDS, which means more days on MV and therefore increased ICU stay.

Reference

1. Ranieri M, et al:: JAMA 2012, 307:2526-2533.

P95

Extravascular lung water, B-type natriuretic peptide and blood volume contraction for diagnosing weaning-induced pulmonary edema

M Dres, JL Teboul, N Anguel, C Richard, X Monnet

Service de réanimation médicale, Hôpital de Bicêtre, Le Kremlin Bicêtre, France Critical Care 2013, 17(Suppl 2):P95 (doi: 10.1186/cc12033)

Introduction We compared three different methods for diagnosing pulmonary edema induced by weaning from mechanical ventilation: the increase in extravascular lung water index (EVLWI), the increase in B-type natriuretic peptide (BNP) and blood volume contraction, reflected by increases in plasma protein and in hemoglobin concentrations, all observed during a spontaneous breathing trial (SBT).

Methods We included 12 difficult-to-wean patients (22 recordings). Before and at the end of a SBT (T tube), we measured pulmonary occlusion arterial pressure (PAOP), EVLWI (PiCCO device), BNP, plasma protein and hemoglobin concentrations. Weaning-induced pulmonary edema was confirmed if a clinical intolerance to SBT was associated with an increase of PAOP $>18 \mathrm{mmHg}$ at the end of SBT.

Results A weaning-induced pulmonary edema was diagnosed in 12 instances (PAOP significantly increased from $15.6 \pm 0.6$ to $25.8 \pm 0.9$ in these cases). EVLWI, BNP, plasma protein and hemoglobin concentrations significantly increased in these instances $(28.3 \pm 5.7 \%$, $20.2 \pm 7.8 \%, 9.6 \pm 0.8 \%$ and $9.3 \pm 1.3 \%$, respectively) while they did not significantly changed in cases without weaning-induced pulmonary edema. The increase of EVLWI $\geq 8.5 \%(+1.5 \mathrm{ml} / \mathrm{kg})$, an increase in BNP $\geq 6.7 \%(+23 \mathrm{pg} / \mathrm{ml})$, an increase in plasma protein concentration $\geq 5 \%$ and in hemoglobin concentration $\geq 5 \%$ exhibited good areas under the ROC curves to predict weaning-induced pulmonary edema $(0.97 \pm 0.03,0.80 \pm 0.11,1.0 \pm 0.00$ and $0.92 \pm 0.05$, respectively). These areas under the ROC curves were not statistically different. The baseline values of EVLWI, BNP, plasma protein and hemoglobin concentrations did not predict weaning-induced pulmonary edema.

Conclusion The increases in EVLWI, in plasma protein and hemoglobin concentration and in BNP are valuable alternatives to the pulmonary artery catheter for diagnosing weaning-induced pulmonary edema.

P96

Pressure support ventilation with minimal sedation as the main ventilatory mode in critically ill patients with lung injury: effect on mortality and incidence of complications

A Gomez', A Leon', G Fernandez', G Montenegro', H Gomez²

'Clinica Palermo, Bogota, Colombia; 'University of Pittsburgh, PA, USA

Critical Care 2013, 17(Suppl 2):P96 (doi: 10.1186/cc12034)

Introduction The primary aim of this study is to assess the impact of pressure support ventilation (PSV) on the rate of pneumothorax and mortality in critically ill patients with lung injury. The secondary aim is to evaluate pressure-volume $(\mathrm{P}-\mathrm{V})$ relationships. Spontaneous modes of ventilation have been associated with lower rates of atelectasis, less muscle atrophy, better airflow distribution and importantly lower sedation requirements, which relates to lower mortality. Accordingly, we hypothesized that the use of PSV in patients with moderate/severe lung injury would have rates of pneumothorax and mortality within the standard of care. We further hypothesized that given its spontaneous nature, set pressures (PEEP and PS) but not tidal volume (Vt) would be related to airway pressures.

Methods All adult patients admitted to two surgical/medical ICUs subjected to invasive mechanical ventilation (MV) were enrolled. Patients were stratified by Lung Injury Score (LIS) in two groups: $<2.5$ (LISL); $\geq 2.5$ (LISH). Exclusion criteria included pneumothorax on admission, use of other ventilatory strategies, and inability to trigger ventilation. Patients were ventilated with PSV, and treated only with pro re nata haldol, morphine and clozapine. Airway pressures and 
ventilatory mechanics were measured twice daily. Data are presented as mean $\pm 95 \% \mathrm{Cl}$. ${ }^{*} P>0.05$.

Results A total of 166 patients with mean age 55 (52 to 58) years, LIS of 2.27 (2.16 to 2.37) and MODS of 3.13 (2.9 to 3.3) were enrolled and stratified by LISL (65.8\%) and LISH (34.2\%). Mortality, and incidence of pneumothorax and atelectasis were $21 \%$ (14.9 to 28.2$), 1.6 \%(0.8$ to 3.1 ) and 6.4 (3.2 to 11.2). Duration of MV was 6 days (4.83 to 7.16). Pneumothorax in LISL and LISH was $1 \%$ (0 to 5.2 ) versus $2.4 \%(0.7$ to 6.1$)^{*}$, and mortality $11.4 \%$ (3.8 to 24.6 ) versus $25 \%$ (9.8 to 46.7$)^{*}$. Mean parameters were: $\mathrm{Vt} 10.3 \mathrm{ml} / \mathrm{kg}$ (10.1 to 10.6); PEEP 10.6 (9.9 to 11); PS 16.6 (15.9 to 17.1); plateau pressure (Ppl) 25.7 (25.1 to 26.2). Ppl was $>26.2$ only in $2.5 \%$. PEEP and PS $(P<0.0001)$, and MODS were associated with Pplt, but not $\mathrm{Vt}^{*}$ or LIS. Only lower Vt was associated with barotrauma $(\mathrm{OR}=0.996, P=0.02)$.

Conclusion We demonstrate that PSV in minimally sedated patients with severe lung injury is safe as it is associated with low incidence of barotrauma, atelectasis and mortality, and with Ppl and duration of MV within standard of care. We also demonstrate in PSV that P-V relationships may differ and that in this setting higher $\mathrm{Vt}$ may not be deleterious.

P97

Comparison of a fully automatic ARDSNet protocol and a

feedback-controlled open lung management concept

A Pomprapa', D Schwaiberger'2, B Lachmann², S Leonhardt

'RWTH Aachen University, Aachen, Germany; ${ }^{2}$ Charite Berlin, Germany

Critical Care 2013, 17(Suppl 2):P97 (doi: 10.1186/cc12035)

Introduction The aim of this study is to compare two ventilation strategies, the ARDSNet protocol and open lung management, using computer control for 6 hours. The standard therapy for patients with ARDS does typically apply a mechanical ventilator to support breathing. The cost of therapy is high and it requires much attention from physicians to adjust the proper ventilation settings in a timely manner. A closed-loop ventilation concept has therefore been developed and tested with two induced ARDS pigs.

Methods The hardware system is composed of a ventilator (Servo 300), a spectrophotometry (CEVOX), a capnography device (CO2SMO+), an electrical impedance tomography device (GOE MF II) and a patient monitor (Sirecust). The software is developed with Labview 7.1. With approval from the ethical committee, two $27 \mathrm{~kg}$ pigs were exposed to surfactant depletion with a warm saline washout to induce ARDS ( $\mathrm{PaO}$ $\mathrm{FiO}_{2}<200 \mathrm{mmHg}$ ). One pig model was ventilated with an automatic ARDSNet protocol and another was automatically ventilated with open lung management. Blood gas analysis (BGA) was carried out every half an hour.

Results Artificial ventilation using the auto ARDSNet protocol successfully stabilized oxygenation, minimized plateau pressure $\left(<30 \mathrm{cmH}_{2} \mathrm{O}\right)$, and controlled the $\mathrm{pH}$ value for acidosis and alkalosis management. On the other hand, auto open lung management offers a distinctive result of ventilation. A significant improvement of oxygenation and lung compliance was observed within a few breaths after the recruitment maneuvers. Both subjects were ventilated at the same tidal volume of $6 \mathrm{ml} / \mathrm{kg}$ and the comparative results of automatic ventilation settings and BGA are provided in Table 1 for every 2 hours.

Conclusion The auto open lung management concept gave much better gas exchange than the auto ARDSNet protocol. These preliminary results showed a necessity to evaluate the two different ventilation strategies. Therefore, further experiments with pig models will be implemented in the near future to obtain results with statistical significance and to ensure the safety of automation in a mechanical ventilation system.

P98

Comparison of an entirely automated ventilation mode, Intellivent-ASV, with conventional ventilation in ARDS patients: a 48-hour study

E Bialais', L Vignaux², X Wittebole1, D Novotni ${ }^{2}$, J Meyer ${ }^{2}$, M Wysocki², T Sottiaux³, G Reychler', J Roeseler', P Laterre', P Hantson'

'Cliniques Universitaires Saint Luc, Brusells, Belgium; ${ }^{2}$ Hamilton Medical AG,

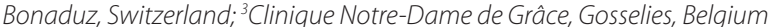
Critical Care 2013, 17(Suppl 2):P98 (doi: 10.1186/cc120236)

Introduction Intellivent-ASV has been developed to provide fully closed loop mechanical ventilation using a ventilation controller keeping $\mathrm{EtCO}_{2}$ and $\mathrm{SpO}_{2}$ within expert-based ranges. Ventilation of ARDS patients focuses on delivering adequate oxygenation and allowing elimination of $\mathrm{CO}_{2}$ while protecting the lung. The objectives were to compare Intellivent-ASV with conventional ventilation on safety and efficacy, and to compare the number of manual adjustments between the two ventilatory modalities.

Methods A randomized, controlled study including all consecutive patients receiving mechanical ventilation for at least 48 hours. Patients were randomly ventilated either with Intellivent-ASV or conventional ventilation, with a S1 (Hamilton, Bonaduz, Switzerland). Parameters were adjusted by the clinician in charge of the patient. Ventilatory and oxygenation parameters were recorded cycle by cycle during 48 hours and blood gases were performed every 6 hours.

Results Twenty-four patients with ARDS were included, 10 female, 14 male, median age 58 (46 to 63) years, APACHE II score 22 (17 to 29), $\mathrm{PaO}_{2} / \mathrm{FiO}_{2}$ at inclusion 136 (107 to 154). Eleven were ventilated in the conventional group and 13 in the Intellivent-ASV group. The study was stopped for one patient from the Intellivent-ASV group because of a pneumothorax not caused by ventilation. The delivered $\mathrm{Vt}$ was slightly higher during Intellivent-ASV (7.9 (7.5 to 8.5$)$ vs. 7.2 (6.8 to 7.8$) \mathrm{ml} / \mathrm{kg}$, $P=0.013$ ). The time spent by the various parameters in the suboptimal zone (safety) is the same for the two ventilation modes. The time spent in the optimal zone (efficacy) is the same for the two ventilation modes,

Table 1 (abstract P97). Comparative results between auto ARDSNet protocol and auto open lung management

\begin{tabular}{|c|c|c|c|c|c|c|c|c|}
\hline & \multicolumn{2}{|c|}{ Before auto ventilation } & \multicolumn{2}{|c|}{ After 2 hours } & \multicolumn{2}{|c|}{ After 4 hours } & \multicolumn{2}{|c|}{ After 6 hours } \\
\hline & Auto ARDSNet & Auto OLM & Auto ARDSNet & Auto OLM & Auto ARDSNet & Auto OLM & Auto ARDSNet & Auto OLM \\
\hline $\mathrm{FiO}_{2}$ & 1.0 & 1.0 & 0.40 & 0.25 & 0.40 & 0.25 & 0.50 & 0.25 \\
\hline RR (bpm) & 22 & 40 & 23 & 38 & 23 & 39 & 23 & 35 \\
\hline I:E ratio & $1: 2$ & 1:1 & $1: 2$ & 1:1 & $1: 2$ & 1:1 & $1: 2$ & 1:1 \\
\hline$V_{T}(\mathrm{ml} / \mathrm{kg})$ & 6 & 6 & 6 & 6 & 6 & 6 & 6 & 6 \\
\hline $\mathrm{P}_{\text {plat }} / \mathrm{PIP}(\mathrm{mmHg})$ & 22 & 22 & 18 & 20.6 & 15.8 & 17.7 & 14.6 & 15.4 \\
\hline $\operatorname{PEEP}\left(\mathrm{cmH}_{2} \mathrm{O}\right)$ & 5 & 10 & 8 & 15 & 2 & 13 & 10 & 11 \\
\hline $\mathrm{pH}$ & 7.34 & 7.30 & 7.32 & 7.52 & 7.37 & 7.60 & 7.38 & 7.51 \\
\hline $\mathrm{pO}_{2} / \mathrm{FiO}_{2}(\mathrm{mmHg})$ & 59 & 59 & 180 & 380.8 & 155 & 387.2 & 122 & 404 \\
\hline $\mathrm{pCO}_{2}(\mathrm{mmHg})$ & 52 & 64 & 56 & 39.6 & 58 & 31.5 & 54 & 41 \\
\hline
\end{tabular}

OLM, open lung management. 
expect for $\mathrm{SpO}_{2}$ (78\% (75 to 89 ) with Intellivent-ASV vs. $44 \%$ (41 to 58 ), $P=0.001)$. Intellivent-ASV required less manual adjustments during 48 hours (2 ( 0 to 7$)$ vs. 17 (10 to 27), $P<0.001$ ). The total number of adjustments during 48 hours, including automatic regulation, was higher in the Intellivent-ASV group (379 (274 to 493 ) vs. 17 (10 to 27), $P<0.001$ ).

Conclusion Over 48 hours, Intellivent-ASV allowed safe ventilation for ARDS patients, with better oxygenation and an identical ventilation efficacy when compared with conventional ventilation, with less manual adjustments.

\section{P99}

Difference in pulmonary permeability between indirect and direct acute respiratory distress syndrome assessed by the transpulmonary thermodilution technique

K Morisawa' Y Yaira', M Yanai'1 Y Takamatu', S Kushimoto² S Fujitani ${ }^{3}$

'St Marianna University, Kawasaki-Shi, Japan; ${ }^{2}$ Tohoku University Graduate School of Medicine, Sendai-shi, Japan; ${ }^{3}$ Tokyo Bay Urayasu/Ichikawa Medical

Center, Urayasu-shi, Japan

Critical Care 2013, 17(Suppl 2):P99 (doi: 10.1186/cc12037)

Introduction ARDS is characterized by increased pulmonary capillary permeability secondary to diffuse alveolar inflammation and injury. Common risk factors can be classified into two groups: extrapulmonary causes (indirect etiologies: ARDSexp) or pulmonary causes (direct etiologies: ARDSp). There are few quantitative methods to distinguish between the differences in these two ARDS categories.

Methods A subanalysis of the trial by the PiCCO Pulmonary Edema Study (prospective, observational, multi-institutional study) in 23 ICUs of academic tertiary referral hospitals in Japan. All consecutive adult patients requiring mechanical ventilation with the diagnosis of ARDS were monitored by the transpulmonary thermodilution technique system (PiCCO; Pulsion Medical Systems, Munich, Germany) for 3 days: day 0 , day 1 and day 2 . The pulmonary vascular permeability index (PVPI), extravascular lung water index and intrathoracic blood volume index were measured concurrently. Three experts retrospectively determined the pathophysiological mechanism causing ARDS by patient history, clinical presentation, chest computed tomography and radiography. Patients were classified into two groups: patients with ARDS triggered by ARDSexp and ARDSp.

Results During the study period from March 2009 to August 2011, a total of 173 patients were assessed including 56 ARDSexp patients and 117 ARDSp patients, with the most common cause of ARDSexp secondary to sepsis (71\%) and of ARDSp pneumonia (80\%). The measurement of PVPI was significantly elevated in the ARDSp group on all days. There were no significant differences in mortality at 28 days, mechanical ventilation days, and hospital length-of-stay between the two groups, while the ARDSexp group seemed to be associated with prolonged mechanical ventilation days and hospital length-of-stay. Conclusion This study suggests the existence of several differences in pathogenetic pathways and the degree of pulmonary permeability between patients with ARDSexp and ARDSp. We therefore believe that using the PVPI may provide us with timely quantitative information to make clinical decisions.

\section{P100}

Biomarkers for early stage of acute respiratory distress syndrome in septic patients: surfactant protein $D$ and Clara cell protein

A Kuzovlev', V Moroz', A Goloubev', S Polovnikov²

${ }^{\prime} V$. A. Negovsky Scientific Research Insitute of General Reanimatology RAMS,

Moscow, Russia; ${ }^{2}$ N.N. Burdenko Main Military Hospital, Moscow, Russia

Critical Care 2013, 17(Suppl 2):P100 (doi: 10.1186/cc12038)

Introduction The injury of alveolar epithelium and endothelium is the basis of pathogenesis for the early stage of acute respiratory distress syndrome (ARDS). A prompt detection of this injury will provide us with a possibility for early ARDS diagnosis and treatment. Currently no biomarkers of alveolar epithelial injury are clinically available. The purpose of our study was to investigate the role of surfactant protein $D$ (SPD) and Clara cell protein (CCP) as biomarkers of early ARDS.
Methods This observational study in ICU mechanically ventilated septic patients with intra-abdominal surgical infections was carried out at the V.A. Negovsky Research Institute of General Reanimatology in 2010 to 2012. ARDS was diagnosed and staged according to the research institute criteria [1] and the American-European criteria. Plasma concentrations of SPD and CCP were measured on ARDS diagnosis (day 0 ) and days 3, 5, and 7 by immunoenzyme essay (Bio Vendor, USA). Data were statistically analyzed by STATISTICA 7.0, ANOVA method, and presented as mean $\pm \sigma, \mathrm{ng} / \mathrm{ml}$. $P<0.05$ was considered statistically significant. Areas under the receiver operating curves (ROC) were calculated.

Results Fifty-five patients (out of 250 screened) were enrolled according to the inclusion/exclusion criteria. Patients were assigned into groups: ARDS $(n=30$; subgroups - ARDS stage $1(n=15)$, stage $2(n=15))$ and noARDS $(n=25)$. In the ARDS group SPD was higher at all points than in the noARDS group (day $0-352.5 \pm 287.8$ vs. $141.0 \pm 103.4$; day $3-$ $278.6 \pm 204.8$ vs. $151.8 \pm 125.9$; day $5-339.9 \pm 331.4$ vs. $162.3 \pm 138.8$; day $7-339.9 \pm 300.1$ vs. $169.8 \pm 154.5 ; P<0.05$ ). SPD levels were lower at ARDS stage 1 in comparison with stage 2 (day $0-154.6 \pm 125.2$ vs. $451.8 \pm 299.2$; day $3-129.3 \pm 74.9$ vs. $362.9 \pm 202.0$; day $5-167.8 \pm 120.4$ vs. $428.5 \pm 372.4$; day $7-186.2 \pm 127.1$ vs. $415.5 \pm 337.0 ; P<0.05)$. Plasma SPD had a good diagnostic capacity for stage 1 ARDS: SPD on day $0 \leq 253.0 \mathrm{ng} / \mathrm{ml}$ yielded a sensitivity of $90 \%$ and specificity of $74 \%$ (AUC $=0.83 ; 95 \% \mathrm{Cl}=0.631$ to $0.951 ; P=0.0001$ ). . No such differences for CCP were detected.

Conclusion Plasma SPD level $\leq 253.0 \mathrm{ng} / \mathrm{ml}$ is a sensitive and specific biomarker of the early stage of ARDS in septic patients.

Reference

1. Kuzovlev A, et al: Semin Cardiothorac Vasc Anesth 2010, 14:231-241.

P101

Intravenous perfluorocarbons for prevention of

ventilator-associated ARDS

A Scultetus', A Haque', F Arnaud', G McNamee², C Auker', R McCarron'1, P McKay', R Mahon

'Naval Medical Research Center, Silver Spring, MD, USA; ${ }^{2}$ Uniformed Services

University of the Health Sciences, Bethesda, MD, USA

Critical Care 2013, 17(Suppl 2):P101 (doi: 10.1186/cc12039)

Introduction Emulsified perfluorocarbons (PFC) are synthetic hydrocarbons that can carry 50 times more oxygen than human plasma. Their properties may be advantageous in applications requiring preservation of tissue viability in oxygen-deprived states [1,2], making them a potential candidate for combat and civilian prehospital resuscitation. Our hypothesis is that an intravenous dose of PFC increases vital organ tissue oxygenation, improves survival after hemorrhagic shock (HS) and may reduce or prevent the development of ventilator-associated ARDS. Here we report data from the first part (HS only) of a multiphase swine study to study the benefits of PFC in treating HS and preventing ARDS. This initial study was designed to ensure safe use of PFC in traumatized animals.

Methods Anesthetized Yorkshire swine were hemorrhaged $55 \%$ of their estimated blood volume (Time 0 (T0)) over 15 minutes. At T15 minutes, pigs received a bolus of the PFC Oxycyte or $10 \%$ hydroxyethyl starch (HES). At T60 animals underwent continuous hemorrhage $\left(0.5 \mathrm{~cm}^{3} / \mathrm{kg} /\right.$ minute) until death. Time to death and physiological parameters were primary endpoints.

Results Average survival time after onset of second hemorrhage was 60 minutes in PFC-treated animals versus 65 minutes in the HES group. Mean arterial pressure (MAP) was similar between T0 and T60, thereafter PFC-treated animals had lower MAP, mean pulmonary artery pressure (MPAP), heart rate and cardiac output $(P>0.05)$.

Conclusion There was no significant difference in survival time, MAP and MPAP in the PFC group compared with HES control. These data suggest that it is safe to administer in this HS model. Regulatory approval of hemoglobin-based oxygen carriers has been halted due to possible side effects related to vasoconstriction. In this model, and with this class of oxygen-carrying drugs (PFCs), we did not observe evidence of vasoconstriction. Using PFCs did not result in a survival advantage here; however, there was also no observation of adverse events. Based 
on these data we will continue to the next phase of this project and test PFC in the prevention of ARDS alone, and in combination with HS. References

1. Keipert $P E$, et al: Enhanced oxygen delivery by perflubron emulsion during acute hemodilution. ArtifCells Blood Substit Immobil Biotechnol 1994, 22:1161-1167.

2. Habler $\mathrm{O}$, et al: IV perflubron emulsion versus autologous transfusion in severe normovolemic anemia: effects on left ventricular perfusion and function. Res Exp Med (Berl) 1998, 197:301-318.

P102

Effect of angiotensin-converting enzyme gene I/D polymorphism and its expression on clinical outcome in acute respiratory distress syndrome

I Tsangaris, A Tsantes, P Kopterides, G Tsaknis, S Kokkori, I Karampela, D Konstantonis, S Karabi, EVrigkou, A Pappas, S Orfanos, A Armaganidis University Hospital 'Attikon', University of Athens Medical School, Athens,

Greece

Critical Care 2013, 17(Suppl 2):P102 (doi: 10.1186/cc12040)

Introduction The role of the $D$ allele of the angiotensin-converting enzyme (ACE) gene I/D polymorphism in the clinical outcomes of patients with acute lung injury and acute respiratory distress syndrome (ALI/ARDS) remains controversial. We assessed simultaneously the effect of the ACE I/D polymorphisms as well as the serum and BALF ACE levels on prognosis of ARDS patients.

Methods We recruited 69 mechanically ventilated ALI/ARDS patients. ACE activity levels in serum and BALF were assessed by chemical methods. Patients were genotyped for ACE I/D polymorphisms. Timeto-event analysis evaluated the variables associated with the 28-day and 90-day mortality.

Results In the multivariable model, age, lung compliance, serum lactate and serum ACE levels were significantly associated with both 28-day and 90-day mortality. No significant correlation was found between serum and BALF ACE levels (Spearman's $\rho=0.054 ; P=0.66$ ). Serum ACE concentrations were significantly higher $(P=0.046)$ in patients with $D / D$ genotype versus the two other groups combined (I/D and $\mathrm{I} / \mathrm{I}$ genotypes). A meta-analysis of six studies (including ours) provided evidence that the $D$ allele is significantly associated with increased mortality in ALI/ARDS patients, yielding a per-allele odds ratio of 1.76 (95\% Cl: 1.19 to 2.59). See Figure 1 and Table 1.

Conclusion Serum ACE levels appear to be affected by the I/D polymorphism and are correlated with prognosis in patients with ALI/ARDS, indicating that further investigation of the clinical significance of the ACE in ARDS might be of value.

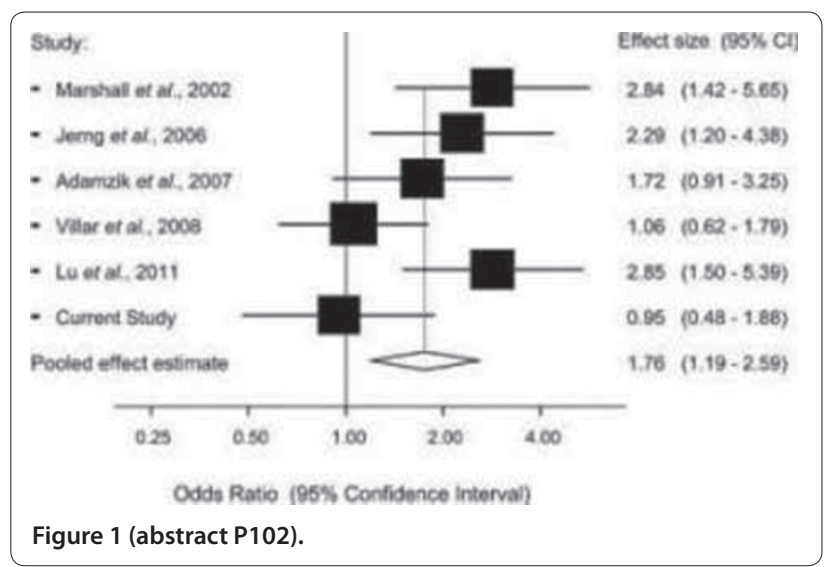

Table 1 (abstract P102). Characteristics of the study population $(n=69)$

\begin{tabular}{|c|c|}
\hline Age (years) & $64.4 \pm 17.9 ; 69$ (28 to 89$)$ \\
\hline Sex (males, \%) & $43(62.3 \%)$ \\
\hline APACHE II score & $22.1 \pm 6.2 ; 21$ (14 to 35$)$ \\
\hline SOFA score & $8.4 \pm 3.2 ; 8$ (4 to 16$)$ \\
\hline Lung compliance $\left(\mathrm{ml} / \mathrm{cmH}_{2} \mathrm{O}\right)$ & $29.4 \pm 7.2 ; 30(17$ to 40$)$ \\
\hline $\mathrm{PaO}_{2} / \mathrm{FiO}_{2}$ & $139,1 \pm 47.1 ; 140(72$ to 223$)$ \\
\hline PEEP & $8.1 \pm 2.9 ; 8(5$ to 15$)$ \\
\hline Lung Injury Score & $2.5 \pm 0.6 ; 2.5$ (1.75 to 3.50$)$ \\
\hline Blood lactate (mmol/l) & $1.7 \pm 1.7 ; 1.3(0.5$ to 5.2$)$ \\
\hline Septic status & $39(56.5 \%)$ \\
\hline Serum ACE (U/I) & $16.5 \pm 10.8 ; 13.6$ (4.8 to 39.8$)$ \\
\hline $\operatorname{BALF}(U / I)$ & $2.3 \pm 1.4 ; 2.0(0.3$ to 4.7$)$ \\
\hline \multicolumn{2}{|l|}{ ACE I/D polymorphism: } \\
\hline$D / D$ & $27(39.1 \%)$ \\
\hline $1 / D$ & $28(40.6 \%)$ \\
\hline $1 / 1$ & $14(20.3 \%)$ \\
\hline 28-day mortality & $34 / 69(49.3 \%)$ \\
\hline 90-day mortality & $43 / 69(62.3 \%)$ \\
\hline Ventilator-free days & $5.1 \pm 7.8 ;(0$ to 23$)$ \\
\hline Days w/o cardiovascular failure & $14.0 \pm 10.2 ; 16(0$ to 27$)$ \\
\hline Days w/o renal failure & $15.4 \pm 10.6 ; 16(0$ to 28$)$ \\
\hline
\end{tabular}

\section{P103}

End-expiratory esophageal pressure versus lower inflection point in acute lung injury

A Yaroshetskiy ${ }^{1}$, D Protsenko'1, E Larin², O Ignatenko', B Gelfand ${ }^{1}$

${ }^{1}$ Russian National Research Medical University, Moscow, Russia; ${ }^{2}$ City

Hospital \#7, Moscow, Russia

Critical Care 2013, 17(Suppl 2):P103 (doi: 10.1186/cc12041)

Introduction No recommendations available concerning protocols of static PV loop and esophageal pressure measurements use set positive end-expiratory pressure (PEEP). The aim of the study was evaluation of the significance of the lower inflection point (LIP) and esophageal pressure monitoring for PEEP adjustment in ALI and ARDS.

Methods A prospective study performed in one general ICU. We include 72 patients who received mechanical ventilation before evaluation and met ARDS criteria by AECC (1994) - acute onset, $\mathrm{PaO}_{2} / \mathrm{FiO}_{2}$ lower than 250 Torr, bilateral infiltrates on chest X-ray. Exclusion criteria were age $<15$ years and pregnancy. We drew a static pressure-volume loop with sustained inflation $40 \times 30$ (PV loop) for all patients using a lowflow technique (Hamilton G5) and measured the esophageal pressure (Avea) in 36 of 72 patients. After that PEEP was set according to zero end-expiratory transpulmonary pressure. We compare PV loop data with esophageal pressure measurements.

Results The low inflection point median was $8(95 \% \mathrm{Cl}=5$ to 10.5$)$ mbar, which does not correspond to the empirically set optimal PEEP of $13(95 \% \mathrm{Cl}=12$ to 15$)$ mbar $(P<0.001$, Wilcoxon test). End-expiratory esophageal pressure (EEEP) median was $14(95 \% \mathrm{Cl}=12$ to 18$) \mathrm{mbar}$, the correlation between LIP and EEEP was poor $(\rho=0.279, P=0.049)$. We find significant correlation between static compliance and EEEP ( $\rho=-0.421, P=0.005$ ). Sustained inflation did not lead to improved oxygenation $(P>0.05)$. PEEP adjustment by EEEP led to an increase in $\mathrm{PaO}_{2} / \mathrm{FiO}_{2}-$ median $107 \mathrm{mmHg}(95 \% \mathrm{Cl}=18$ to $147, P<0.001)$. EEEP was similar to empirically set PEEP $(P>0.05)$.

Conclusion LIP has poor correlation with EEEP. PEEP adjustment by esophageal pressure was close to empirically set PEEP and can improve oxygenation. 
P104

Lung recruitment induced by Sigh in hypoxemic intubated critically ill patients

TM Mauri', G Bellani', A Coppadoro', P Tagliabue², A Barletta', V Meroni², M Teggia Droghi', N Patroniti', A Pesenti'

'University of Milan-Bicocca, Monza, Italy; '2San Gerardo Hospital, Monza, Italy Critical Care 2013, 17(Suppl 2):P104 (doi: 10.1186/cc12042)

Introduction In intubated critically ill patients, cyclic short recruitment manoeuvres (Sigh) introduced during pressure support ventilation (PSV) improve oxygenation, probably by increasing end-expiratory lung volume (EELV). We assessed Sigh effects on regional distribution of EELV by electrical impedance tomography (EIT): a bedside, radiationfree lung imaging technique. Moreover, we investigated baseline characteristics correlated with response to Sigh.

Methods We enrolled 20 intubated critically ill patients undergoing PSV with $\mathrm{PaO}_{2} / \mathrm{FiO}_{2} \leq 300 \mathrm{mmHg}$ and PEEP $\geq 5 \mathrm{cmH}_{2} \mathrm{O}$. We applied on each patient's thorax a 16-electrode belt connected to an EIT monitor (PulmoVista $500^{\circledR}$; Dräger Medical GmbH, Lübeck, Germany). Sigh was introduced as $35 \mathrm{cmH}_{2} \mathrm{O}$ continuous positive airway pressure phase lasting 3 to 4 seconds at different rates $(0,0.5,1,2$ per minute, random order) for 20 minutes. From raw EIT data, we calculated global changes in EELV $(\triangle E E L V g l)$, calculated as changes in end-expiratory lung impedance calibrated versus tidal volume to estimate changes in $\triangle E E L V$ and considering $0 \mathrm{Sigh} /$ minute as baseline; and $\triangle \mathrm{EELV}$ of nondependent and dependent lung regions ( $\triangle E E L V$ nondep and $\Delta E E L V d e p)$. Together, we collected ventilation parameters, hemodynamics and arterial blood gases.

Results Patients were $65 \pm 13$ years old, ventilation days were $9 \pm 10$ and $\mathrm{PaO}_{2} / \mathrm{FiO}_{2}$ was $194 \pm 49 \mathrm{mmHg}$ at PEEP $8 \pm 2 \mathrm{cmH}_{2} \mathrm{O}$. Introduction of Sigh improved $\mathrm{PaO}_{2} / \mathrm{FiO}_{2}(P<0.001)$ and $\triangle \mathrm{EELVgl}(P<0.001)$. The increase in EELV was diffuse across all lung regions as both $\triangle$ EELVnondep and $\triangle$ EELVdep changed $(P<0.01$ and $P=0.06)$. minute ventilation, arterial blood $\mathrm{pH}$ and hemodynamics were not significantly affected by introduction of Sigh, while mean airway pressure increased $(P<0.001)$, albeit only at the highest Sigh rate and by a limited extent $\left(\sim 2 \mathrm{cmH}_{2} \mathrm{O}\right)$. Patients with mean $\triangle \mathrm{EELVgl}$ over the three Sigh phases $>200 \mathrm{ml}$ (that is, Sigh-responders) had significantly lower $\mathrm{PaO}_{2} /$ $\mathrm{FiO}_{2}$ values at baseline (that is, at Sigh rate $=0$ /minute) than Sighnonresponders $(163 \pm 25 \mathrm{mmHg}$ vs. $222 \pm 39 \mathrm{mmHg}, P<0.01)$.

Conclusion Introduction of Sigh in intubated critically ill patients undergoing PSV improves gas exchange by inducing alveolar recruitment across all lung regions with minimal increase of airway pressure. Patients with more severe gas exchange impairment present the highest degree of potentially recruitable lung.

\section{P105}

\section{Quantification of stress raisers in ARDS}

M Cressoni', M Amini', P Cadringher², C Chiurazzi', D Febres', E Gallazzi' A Marino', M Brioni', F Menga', I Cigada', E Carlesso', D Chiumello², $L$ Gattinoni²

'Università degli Studi di Milano, Milan, Italy; ${ }^{2}$ Fondazione IRCCS Ca' GrandaOspedale Maggiore Policlinico, Milan, Italy

Critical Care 2013, 17(Suppl 2):P105 (doi: 10.1186/cc12043)

Introduction Ventilator-induced lung injury (VILI) is a well-known side effect of mechanical ventilation. The pressures and volumes needed to induce VILI in healthy animals are far greater than pressure and volumes applied in clinical practice [1]. A possible explanation may be the presence of local pressure multipliers (stress raisers).

Methods We retrospectively analyzed CT scans of 147 patients with ARDS and CT scans of 100 healthy subjects. A homogeneous lung would have the same gas/tissue ratio in all its regions. If a lung region expands less than the neighbour regions these will be more strained to vicariate the non/less expanding region. We measured the stress raisers by computing the ratio between the gas fraction of the region of interest and the neighbouring regions: if the inflation would be the same (homogeneity), the ratio will be equal to one; if the inflation of the surrounding regions would be greater than the region of interest (that is, more strained), the ratio between the two will be greater than one and was taken as a measure of stress raiser. We considered pathological stress raisers as the regions showing inflation ratio greater than the 95th percentile of the control group (1.61) and defined as the extent of the stress raisers the fraction of lung volume above this threshold. Results The extent of stress raisers increased with the severity of ARDS $(14 \pm 5,18 \pm 8,23 \pm 1 \%$ of lung parenchyma in mild, moderate and severe ARDS, $P<0.0001)$. The extent of stress raisers correlated with the dead space fraction $\left(r^{2}=0.34, P<0.001\right)$, with the fraction of poorly aerated tissue $\left(r^{2}=0.36, P<0.0001\right)$ and also has a negative correlation with the fraction of well inflated tissue $\left(r^{2}=0.47, P<0.0001\right)$. The response to PEEP, passing from 5 to $45 \mathrm{cmH}_{2} \mathrm{O}$ is minimal (average decrease of stress raiser extent $6 \pm 5 \%$ ) and inter-individual variability is great (in 11 patients, stress raisers increased passing from PEEP 5 to PEEP 45). Stress raisers turn out to be greater in nonsurvivor patients than in survivor patients ( $17 \pm 7$ vs. $20 \pm 9 \%$ of lung volume, $P=0.03)$.

Conclusion Stress raisers correlate with the severity of disease; lungs with greater stress raisers are subjected to higher local pressures and probably those patients are more susceptible to VILI. The application of PEEP has little effect on stress raisers.

Reference

1. Protti A: Am J Respir Crit Care Med 2011, 183:1354-1362.

\section{P106}

Recruited lung tissue does not resume normal mechanical properties

M Cressoni', C Chiurazzi', M Amini', D Febres', E Gallazzi', E Carlesso',

P Cadringher', T Langer', D Chiumello', L Gattinoni²

'Università degli Studi di Milano, Milan, Italy; 'Fondazione IRCCS Ca' GrandaOspedale Maggiore Policlinico, Milan, Italy

Critical Care 2013, 17(Suppl 2):P106 (doi: 10.1186/cc12044)

Introduction Positive end-expiratory pressure (PEEP) is commonly used in patients with ARDS to prevent end-expiratory collapse. The increase in gas volume and/or change in mechanical properties of the lung are used at the bedside to estimate lung recruitment and individualize PEEP selection.

Methods Retrospective analysis of patients who underwent wholelung $C T$ scan at 5 and $15 \mathrm{cmH}_{2} \mathrm{O}$ PEEP. Every chest $\mathrm{CT}$ was divided both into 10 apex-base levels of equal height and into 10 sterno-vertebral levels, yielding 100 units per scanned lung (200 units per patient); each unit was classified according to its average CT number $(<-100$ not inflated, -100 to -500 poorly inflated, -500 to -900 well inflated and -900 overinflated). Lung regions defined as not inflated at PEEP $5 \mathrm{cmH}_{2} \mathrm{O}$ and inflated (poorly, well or over) at PEEP $15 \mathrm{cmH}_{2} \mathrm{O}$ were classified as recruited. Moreover, a surrogate specific lung compliance (CLsp) was determined on the expiratory limb of the pressure-volume curve (15 to $5 \mathrm{cmH}_{2} \mathrm{O}$ PEEP at CT) and was defined as $\Delta \mathrm{gas} / \mathrm{cmH}_{2} \mathrm{O}$ $\mathrm{PEEP} / \mathrm{gram}$ of tissue.

Results We included in our study the CT scans of 89 patients (24 mild, 55 moderate and 10 severe ARDS, age $58 \pm 17$ years, BMI $25.6 \pm 5.4$, $\mathrm{PaO}_{2} / \mathrm{FiO}_{2} 165 \pm 66, \mathrm{PaCO}_{2} 42 \pm 9,60$ discharged alive from ICU $(67 \%))$. At PEEP $5 \mathrm{cmH}_{2} \mathrm{O} 39 \pm 16 \%$ of the lung parenchyma was not inflated, $33 \pm 13 \%$ poorly inflated, $28 \pm 15 \%$ well inflated and $0 \pm 1 \%$ overinflated. The median lung recruitability between 5 and $15 \mathrm{cmH}_{2} \mathrm{O}$ PEEP was $6 \%$ (3 to $10 \%$ ) of lung parenchyma ( $71 \mathrm{~g} \mathrm{(26} \mathrm{to} 182 \mathrm{~g})$ ). Mean EELV at PEEP $5 \mathrm{cmH}_{2} \mathrm{O}$ was $1,233 \pm 709$ while it increased to $1,784 \pm 848$ at PEEP $15 \mathrm{cmH}_{2} \mathrm{O}(\Delta \mathrm{EELV}=551 \pm 407 \mathrm{ml})$. More than one-half ( $52 \%$ (31 to $75 \%)$ ) of the increase in end-expiratory lung volume (EELV) was due to an increase in inflation of the already well-aerated tissue; $38 \%$ (23 to $43 \%$ ) was due to the inflation of poorly aerated tissue, while only $4 \%$ (1 to $8 \%$ ) to the recruitment of lung tissue. The CLsp of the well, poorly and recruited tissue was 0.07 (0.031 to 0.13$), 0.03(0.01$ to 0.06$)$ and 0.02 (0.01 to 0.04$) \mathrm{ml} / \mathrm{cmH}_{2} \mathrm{O} / \mathrm{g}$, respectively $(P<0.0001)$.

Conclusion Most of the PEEP-related EELV increase is due to an increased inflation of already well aerated lung tissue. In agreement with the baby lung theory [1], the well-aerated tissue showed near to normal mechanical properties.

Reference

1. Gattinoni L, et al:: Int Care Med 2005. 31:776-784. 
P107

Intraoperative management of hypoxemia with recruitment maneuvers: are the benefits worth the costs?

V Grosomanidis, B Fyntanidou, K Karakoulas, K Kotzampasi, E Oloktsidou, C Nouris, C Skourtis

Aristotle Medical School, Thessaloniki, Greece

Critical Care 2013, 17(Suppl 2):P107 (doi: 10.1186/cc12045)

Introduction Several strategies can be applied for the prevention and management of anesthesia-related hypoxemia. The aim of our study was to investigate the cardiovascular effects of a specific recruitment maneuver (RM) used for the management of intraoperative hypoxemia. Methods Forty-nine patients (29 male/20 female) of mean age $57.4 \pm 9.9$ years, mean weight $80.2 \pm 13.6 \mathrm{~kg}$ and ASA-PS classification I to IV (I: 2, II: 27, III: 16, IV: 6), undergoing general surgery procedures, who developed intraoperative hypoxemia $\left(\mathrm{PaO}_{2} / \mathrm{FiO}_{2}<200\right)$, were enrolled in our study. For the management of hypoxemia a total of $67 \mathrm{RMs}$ have been applied, which consisted of a manual increase of the airway pressure to $40 \mathrm{cmH}_{2} \mathrm{O}$. This rise has been maintained for 10 seconds and was followed by an increase of positive end-expiratory pressure from 5 to $10 \mathrm{cmH}_{2} \mathrm{O}$. Before RM application an oesophageal Doppler monitor probe was inserted into the patients for measuring stroke volume (SV), cardiac output (CO), peak velocity (PV) and flow corrected time (FTC). Standard monitoring also included ECG, IBP, ETCO and $\mathrm{SpO}_{2}$. Heart rate $(\mathrm{HR})$, systemic arterial pressure (SAP), SV, CO, PV and FTc were recorded directly before, during, right after and 5 minutes after RM application (T1 to T4). Kolmogorov-Smirnoff was used to test normal distribution of data and ANOVA was used for the statistical analysis. $P<0.05$ was considered statistically significant.

Results HR, SAP, SV, CO, PV and FTc showed a statistically significant decrease during and right after RM compared with the baseline values but they were gradually restored to control values after RM discontinuation (Table 1).

Table 1 (abstract P107)

\begin{tabular}{lcccc}
\hline & T1 & T2 & T3 & T3 \\
\hline HR (beats/minute) & $69.8 \pm 7.1$ & $60.2 \pm 10.6^{*}$ & $67.1 \pm 7.4^{*} \wedge$ & $68.4 \pm 6.9 \wedge$ \\
SAP mean (mmHg) & $89.8 \pm 7.7$ & $53.4 \pm 5.7^{*}$ & $81.5 \pm 7.7^{*} \wedge$ & $87.6 \pm 6.7 \wedge$ \\
SV $(\mathrm{ml})$ & $82.2 \pm 21.2$ & $22.5 \pm 15.5^{*}$ & $75.4 \pm 21.7^{*} \wedge$ & $79.6 \pm 6.4 \wedge$ \\
CO (I/minute) & $5.75 \pm 1.7$ & $1.4 \pm 0.9^{*}$ & $5.1 \pm 1.7^{*} \wedge$ & $5.5 \pm 1.5 \wedge$ \\
PV (cm/second) & $72.6 \pm 17.1$ & $26 \pm 15.9^{*}$ & $68 \pm 16.8^{*} \wedge$ & $70.7 \pm 15.9 \wedge$ \\
FTc (seconds) & $0.4 \pm 0.06$ & $0.19 \pm 0.08^{*}$ & $0.36 \pm 0.06^{*} \wedge$ & $0.36 \pm 0.06 \wedge$ \\
\hline
\end{tabular}

${ }^{*} P<0.05$ versus $\mathrm{T} 1 . \wedge P<0.05$ versus $\mathrm{T} 2$.

Conclusion According to our results, RM application causes a profound impairment of the cardiovascular system. This can be attributed to the increase of the airway pressures, which results in preload decrease and afterload increase of the right ventricle and cardiac contractility attenuation. Nevertheless, these effects are transient and reversible and RM application is a safe technique for the management of intraoperative hypoxemia, provided that adequate cardiac preload is ensured and the anesthesiologist is alert to discontinue the RM if it exceeds cardiovascular reserves.

\section{P108}

Haemodynamic changes during alveolar recruitment manoeuvre in patients with diastolic dysfunction

A Rubino, E Marini, B Ferro, M Collareta, F Forfori, F Guarracino, F Giunta

Azienda Ospedaliero Universitaria Pisana, Pisa, Italy

Critical Care 2013, 17(Suppl 2):P108 (doi: 10.1186/cc12046)

Introduction To investigate the haemodynamic effects of an alveolar recruitment manoeuvre in intubated patients with diastolic dysfunction compared with patients with normal diastolic function in a mixed ICU. Methods Between October 2011 and February 2012, 16 mechanically ventilated patients admitted to the ICU with normal systolic function and without inotropic support were enrolled. A transthoracic echocardiography was performed to assess diastolic function: $\mathrm{E} / \mathrm{A}$, isovolumic relaxation time (IVRT), deceleration time (DT), E/e' were assessed. Eight patients (five with poor relaxation pattern, three with decreased left ventricle compliance) were identified in diastolic dysfunction group (Group 1) and eight were included in the normal diastolic function group (Group 2). A recruitment manoeuvre was performed by applying a PEEP of $40 \mathrm{cmH}_{2} \mathrm{O}$ for 30 seconds. Haemodynamic parameters of cardiac output (CO), stroke volume (SV), heart rate (HR), pulse pressure variation (PPV), and cardiac cycle efficiency (CCE) were continuously recorded during the manoeuvre using the MostCare pulse contour method.

Results No significant haemodynamic changes happened in the first part of the recruitment (t0 to $\mathrm{t} 15$ seconds). A significant decrease in CO $(P<0.05)$, SV $(P<0.01)$, systolic pressure $(P<0.04)$ and PPV $(P<0.04)$ occurred in Group 1 during the remaining part of the manoeuvre. No significant decrease in the same haemodynamic parameters were noted when only E/A was used to discriminate the diastolic dysfunction. See Table 1.

Table 1 (abstract P108). CO difference associated with diastolic dysfunction parameters

\begin{tabular}{lccc}
\hline & E/A $<1$ & E/A $>1$ & $P$ value \\
\hline CO 15 to 30 & 3.9 & 4.8 & 0.20 \\
E 15 to 30 & 3.92 & E $/ \mathrm{e}^{\prime}>1$ & \\
\hline
\end{tabular}

Conclusion The recruitment manoeuvre can compromise left diastolic filling by increasing the transpulmonary pressure. In patients with diastolic dysfunction a significant decrease in SV can be observed during the recruitment manoeuvre with no compensatory mechanisms evoked determining a significant decrease in $\mathrm{CO}$. The E/A ratio is not able to discriminate between the two groups, confirming the power of tissue Doppler imaging to recognize the correct diastolic pattern.

Reference

1. Gernoth C, et al:: Respiratory and haemodynamic changes during decremental open lung positive end-expiratory pressure titration in patients with acute respiratory distress syndrome. Crit Care 2009, 13:R59.

\section{P109}

Alveolar Recruitment for ARDS Trial: preliminary results

AB Cavalcanti', EA Suzumura', M Abreu', GF Ribeiro', A Kodama', F Moreira', HP Guimarães', E Romano', MB Amato², O Berwanger', CR Carvalho², ART Investigators

'Hospital do Coração - HCor, São Paulo, Brazil;' 'Universidade de São Paulo, Brazil

Critical Care 2013, 17(Suppl 2):P109 (doi: 10.1186/cc12047)

Introduction The aim of the ongoing Alveolar Recruitment for ARDS Trial (ART) is to evaluate whether a maximum stepwise alveolar recruitment maneuver (MSARM) followed by ventilation at optimal PEEP may decrease 28-day mortality in patients with moderate to severe ARDS compared with ventilation with the ARDSNet strategy (NCT01374022). Here we report the results of a planned a priori preliminary analysis involving 100 patients to assess feasibility, physiologic variables and safety outcomes.

Methods ART is an event-driven multicenter randomized controlled trial planned to last until 520 deaths within 28 days are observed. Patients assigned to the experimental group receive a MSARM achieving PEEP of $45 \mathrm{cmH}_{2} \mathrm{O}$ and plateau pressure of $60 \mathrm{cmH}_{2} \mathrm{O}$ plus PEEP titrated according to the static compliance of the respiratory system (ART strategy). The target tidal volume is 4 to $6 \mathrm{ml} / \mathrm{kg}$ predicted body weight (PBW) and plateau pressure $\leq 30 \mathrm{cmH}_{2} \mathrm{O}$ in both groups. Results We randomized 101 patients between November 2011 and October 2012 in 51 centers. Considering 124 active sites in July 2013 and the current recruitment rate, we will finish enrollment by November 2014. MSARM was complete in $34 / 47$ (72\%) of patients allocated to the 
ART group. The main reason for MSARM interruption was hypotension (8/9 (90\%)). Mean titrated PEEP in the ART group was $16.1 \pm 3.5$ versus $12.9 \pm 3.4 \mathrm{cmH}_{2} \mathrm{O}$ in ARDSNet $(P<0.001)$. One hour after randomization, tidal volume was similar between the ART and ARDSNet groups $(5.1 \pm 0.8$ vs. $5.3 \pm 0.7 \mathrm{ml} / \mathrm{kg}$ PBW, respectively; $P=0.08)$. Mean values remained below $6 \mathrm{ml} / \mathrm{kg}$ PBW up to day 3 . Few patients had plateau pressure $>30 \mathrm{CmH}_{2} \mathrm{O} 1$ hour after randomization in the ART and ARDSNet groups (3/43 (7.0\%) vs. $4 / 47(8.5 \%)$, respectively; $P=1.00)$ and on subsequent days. $\mathrm{PaO}_{2} / \mathrm{FiO}_{2}$ was significant higher in the ART group $(179.5 \pm 84.5$ vs. $143.3 \pm 46.8, P=0.01)$ and increased over time up to 7 days after randomization $(272.3 \pm 136.5$ vs. $192.6 \pm 72.3, P=0.003)$. The ART strategy did not increase the risk of barotrauma (relative risk $(\mathrm{RR})=0.78,95 \% \mathrm{Cl}=0.19$ to 3.30 ) in the first 7 days after randomization or the need to initiate or increase vasopressors or mean arterial pressure $<65 \mathrm{mmHg}$ ( $\mathrm{RR}=1.14,95 \% \mathrm{Cl}=0.65$ to $2.02, P=0.67) 1$ hour after randomization. However, the ART strategy increased the risk for severe acidosis $(\mathrm{pH}<7.10) 1$ hour after randomization $(\mathrm{RR}=3.20,95 \%$ $\mathrm{Cl}=1.12$ to $9.20, P=0.03$ )

Conclusion ART is feasible. The incidence of adverse events was similar between groups except for severe acidosis 1 hour after randomization. Hence we adjusted the study protocol, increasing the respiratory rate (from 10 to 15/minute) during MSARM.

P110

Intensive alveolar recruitment protocol reduces pulmonary complications and intensive care permanence after cardiac surgery A Leme', L Hajjar', E Nozawa', C Hashizume', J Almeida', J Fukushima', J Auler Jr', R Santiago', R lanotti', M Amato², E Osawa', M Feltrim', F Galas 'Heart Institute, São Paulo, Brazil;'2Faculdade de Medicina da Universidade de São Paulo, Brazil

Critical Care 2013, 17(Suppl 2):P1 10 (doi: 10.1186/cc12048)

Introduction Cardiac surgical procedures are associated with a high incidence of postoperative complications, increasing costs and mortality. The purpose of this study is to evaluate prospectively the impact of two protective mechanical ventilation strategies, both using low-tidal volume ventilation $(6 \mathrm{ml} / \mathrm{kg} / \mathrm{ibw})$ after cardiac surgery.

Methods We prospectively evaluated 270 patients immediately after cardiac surgery, presenting hypoxemia and $\mathrm{PaO}_{2} / \mathrm{FiO}_{2}<250$. Patients were randomized to an intensive alveolar recruitment maneuver (ARM) or a moderate ARM strategy. Intensive ARM group: recruitment with an inspiratory pressure amplitude of $15 \mathrm{cmH}_{2} \mathrm{O}$ and PEEP of $30 \mathrm{cmH}_{2} \mathrm{O}$, followed by ventilation with PEEP $=13 \mathrm{cmH}_{2} \mathrm{O}$, during 4 hours of protective mechanical ventilation with $\mathrm{VT}=6^{2} \mathrm{ml} / \mathrm{kg} / \mathrm{pbw}$. Moderate ARM group: recruitment with opening pressures of $20 \mathrm{cmH}_{2} \mathrm{O}$ in the airways, followed by ventilation with PEEP $=8 \mathrm{cmH}_{2} \mathrm{O}$, during 4 hours of protective mechanical ventilation with $V T=6 \mathrm{ml} / \mathrm{kg} / \mathrm{pbw}$. The primary outcome was a composite endpoint of severe pulmonary complications in the postoperative period defined as intra-hospital death, need for mechanical ventilation for more than 48 hours after surgery, pulmonary infection or after reintubation within 28 days after randomization. The secondary outcome was the incidence of nonpulmonary complications as postoperative myocardial ischemia, acute renal failure (RIFLE-R), respiratory mechanics and blood gas analysis after ARM, ICU length of stay, hospital length of stay and 30-day mortality.

Results The intensive ARM group compared with the moderate ARM group had lower incidence of the primary outcome, mainly due to the reduced rate of pulmonary infection $(2.3 \%$ vs. $10.1 \%, P=0.009)$. Moreover, the intensive ARM group presented higher lung compliance $\left(68 \pm 19\right.$ vs. $\left.51 \pm 17 \mathrm{ml} / \mathrm{cmH}_{2} \mathrm{O}, P<0.001\right)$ and $\mathrm{PaO}_{2} / \mathrm{FiO}_{2}$ ratio $(360 \pm 68$ vs. $240 \pm 74, P<0.001$ ) after intervention when compared with the moderate group. Also, the intensive ARM presented a lower length of ICU stay ( 3 days vs. 4 days, $P=0.027$ ) than the moderate ARM. There are no differences regarding severe nonpulmonary complications and 30day mortality between groups.

Conclusion An intensive ARM strategy reduces postoperative pulmonary complications, reduces hypoxemia, increases lung compliances and decreases the length of ICU stay after cardiac surgery.

Reference

1. Amato MB, et al:: NEngl J Med 1998, 338:347-354.
P111

Reliability of pressure measurements via balloon catheters is high: an evaluation of six esophageal catheters

S Walterspacher, L Isaak, HJ Kabitz, J Guttmann, S Schumann

University Medical Center Freiburg, Germany

Critical Care 2013, 17(Suppl 2):P111 (doi: 10.1186/cc12049)

Introduction Reliable measurement of esophageal pressure is a prerequisite for analysis of respiratory system mechanics in spontaneous breathing patients. For that purpose, various types of balloon catheters exist that differ in material, size and shape. In physical models we studied the quality of pressure measurement via six different balloon catheters, three of them containing a second balloon for measurement of gastric pressure.

Methods Nine balloons of six esophageal catheters were investigated in three conditions: measurement of balloon pressure during initial inflations immediately after unpacking; measurement of static pressures at different filling volumes; and compliance estimation in physical models $\left(27,54,90 \mathrm{ml} / \mathrm{cmH}_{2} \mathrm{O}\right)$ at different levels of superimposed pressure.

Results During the initial inflation most catheters showed pressure artifacts resulting from material adhesion. Those artifacts disappeared during following inflations. Static pressure measurements could be performed with an error below $1 \mathrm{CmH}_{2} \mathrm{O}$ if the balloon was filled appropriately. Overfilling of the balloon resulted in larger errors only in two catheters. Compliance estimations resulted in errors below $1 \mathrm{ml} /$ $\mathrm{cmH}_{2} \mathrm{O}$. Superimposed pressure had no relevant effect on compliance estimation.

Conclusion The reliability of pressure measurements and also of compliance estimation via the tested catheters is high. Only in two catheters was the filling volume a critical point for a precise measurement of pressure or for estimation of compliance. Immediately after unpacking, adhesion of the balloon material might prevent reliable pressure measurement, therefore before the first measurement overfilling of the balloon and retention of the excess gas seems strongly recommended.

P112

Effect of protective ventilation on organ-specific cytokine production in an experimental postoperative sepsis model J Sperber', M Lipcsey², A Larsson 33, A Larsson², J Sjölin33, M Castegren 'Centre for Clinical Research Sörmland, Uppsala University, Uppsala, Sweden; ${ }^{2}$ Surgical Sciences, Uppsala University, Uppsala, Sweden; ${ }^{3}$ Medical Sciences, Uppsala University, Uppsala, Sweden Critical Care 2013, 17(Suppl 2):P112 (doi: 10.1186/cc12050)

Introduction Low tidal volume (VT) ventilation in intensive care patients without lung injury attenuates the systemic inflammatory response [1]. The contribution of the specific organ inflammatory responses to the systemic picture remains to be elucidated. We investigated the effect of low VT ventilation compared with medium high VT on hepatic, splanchnic and cerebral cytokine responses in an experimental large animal postoperative sepsis model.

Methods Twenty pigs, group Protective Ventilation (PV), were ventilated with low VT $(6 \mathrm{ml} / \mathrm{kg})$ and PEEP $10 \mathrm{cmH}_{2} \mathrm{O}$ while 10 pigs, group Control (C), were ventilated with a VT of $10 \mathrm{ml} / \mathrm{kg}$ and PEEP $5 \mathrm{cmH}_{2} \mathrm{O}$. Catheters were introduced into an artery, the jugular bulb, the hepatic vein and the portal vein. Laparotomy for 2 hours simulated a surgical procedure after which baseline ensued and a continuous endotoxin infusion was started at $0.25 \mu \mathrm{g} / \mathrm{kg} / \mathrm{hour}$ for 5 hours. Differences were analyzed with ANOVA for repeated measures.

Results TNFa levels were higher in the hepatic vein than in the artery, the jugular bulb and the portal vein. IL-6 levels were higher in the artery and the jugular bulb compared with the portal and hepatic veins. IL-10 levels were higher in the portal vein compared with the jugular bulb and hepatic vein. The organ-specific IL-10 concentrations were all higher than the arterial concentration. Comparison between the ventilation groups showed that TNFa, IL- 6 and IL-10 in the hepatic vein were higher in group $\mathrm{C}$ compared with group PV at the end of the experiment. Peak concentrations of TNFa and IL- 6 in the portal vein were higher in group $\mathrm{C}$ compared with group PV. 
Conclusion In this experiment TNFa was mainly generated in the liver while the results point to significant nonhepatic IL-6 and IL-10 production. Ventilation with low VT and medium-high PEEP attenuated hepatic and splanchnic cytokine production compared with mediumhigh VT and lower PEEP.

Reference

1. Determann et al.: Crit Care 2010, 14:R1. doi:10.1186/cc8230

P113

Time required for gas exchange equilibration after a change of positive end-expiratory pressure in acute respiratory distress syndrome

S Coppola', D Chiumello', F Menga², M Brioni², I Cigada², S Froio'

'Fondazione IRCCS Ca' Granda - Ospedale Maggiore Policlinico, Milan, Italy;

¿Università degli Studi di Milano, Milan, Italy

Critical Care 2013, 17(Suppl 2):P113 (doi: 10.1186/cc12051)

Introduction Positive end-expiratory pressure (PEEP) is fundamental to prevent lung collapse in ARDS patients. A common method to titrate PEEP is to perform a PEEP test, recording the variation of cardiorespiratory parameters after a PEEP change [1]. The aim of this study was to evaluate the time-course changes of gas exchanges following a PEEP test.

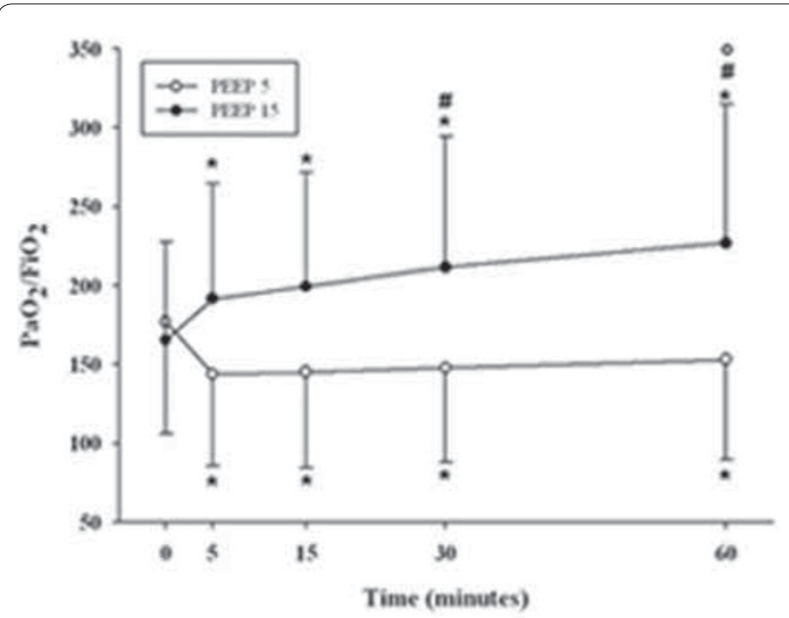

Figure 1 (abstract P113). Two-way ANOVA RM. P $<0.05$, *versus Time 0, \#versus 5 minutes, ${ }^{\circ}$ versus 15 minutes.

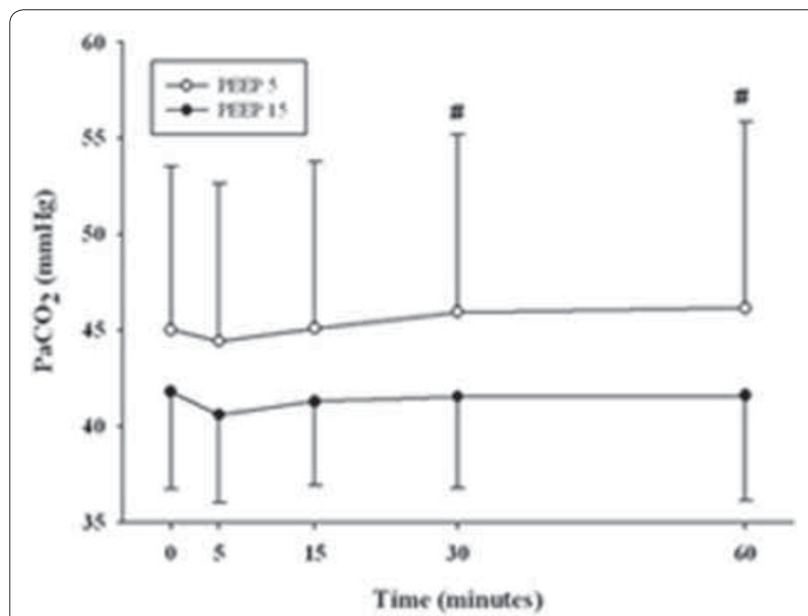

Figure 2 (abstract P113). Two-way ANOVA RM. $P<0.05$, \#versus 5 minutes.
Methods Mechanically ventilated patients (PEEP $10 \mathrm{cmH}_{2} \mathrm{O}$ and TV $7 \mathrm{ml} / \mathrm{kg}$ - Baseline) were randomized to two groups: in the PEEP 15 group, PEEP was increased from 10 to $15 \mathrm{cmH}_{2} \mathrm{O}$; while in the PEEP 5 group, PEEP was decreased from 10 to $5 \mathrm{cmH}_{2} \mathrm{O}$. Arterial gas analyses were performed in both groups after 5, 15, 30 and 60 minutes from the change of PEEP.

Results We enrolled 44 ARDS patients: 23 in the PEEP 15 group and 21 in the PEEP 5 group. At Baseline, $\mathrm{PaO}_{2} / \mathrm{FiO}_{2}(\mathrm{P} / \mathrm{F})$ and $\mathrm{PaCO}_{2}$ were similar in both groups (P/F $169.5 \pm 78.8$ vs. $165.4 \pm 80.6 ; \mathrm{PaCO}_{2} 45.6 \pm 8.5$ vs. $41.7 \pm 5.0 \mathrm{mmHg}$, PEEP 15 vs. PEEP 5, respectively). In the PEEP 15 group, P/F significantly continuously increased over time. In PEEP 5 , $\mathrm{P} / \mathrm{F}$ significantly decreased after 5 minutes and remained stable over time. In the PEEP 15 group, $\mathrm{PaCO}_{2}$ did not change within 60 minutes after PEEP increase. When PEEP was reduced (PEEP 5) $\mathrm{PaCO}_{2}$ remained stable for the first two steps, while at 30 and 60 minutes $\mathrm{PaCO}_{2}$ was significantly higher than at Baseline (Figures 1 and 2).

Conclusion Our data indicate that it is important in critically ill patients to allow sufficient time for the full effect of PEEP increase on oxygenation and to prevent excessive delay when P/F decrease occurs following the application of a lower level of PEEP.

Reference

1. NEngl J Med 2006, 354:1775-1786.

P114

Comparison between the standard and low-dose chest CT scans on the lung quantitative analysis in critically ill patients.

S Froio', D Chiumello', I Cigada², M Brioni², S Coppola', F Menga², L Gattinoni ${ }^{2}$

'Fondazione IRCCS Ca' Granda - Ospedale Maggiore Policlinico, Milan, Italy; 2 Università degli Studi di Milano, Milan, Italy

Critical Care 2013, 17(Suppl 2):P114 (doi: 10.1186/cc12052)

Introduction Quantitative analysis of a lung CT scan is considered the reference method to study lung recruitability to optimize the ventilatory strategy in ARDS patients. However, CT implies a risk of radiation exposure, especially when $\mathrm{CT}$ analysis is necessary to monitor the evolution of ARDS. The aim of this study was to evaluate the impact of a lower radiation dose on lung CT quantitative analysis compared with analysis computed by standard CT.

Methods Sedated and paralyzed patients underwent two chest CT scans: a Standard CT (120 kV, $110 \mathrm{mAs}$, pitch 1.2, collimation $0.6 \mathrm{~mm}$; Care Dose Technology) and a CT performed by a lower radiation dose (Lowdose: $120 \mathrm{kV}, 60 \mathrm{mAs}$ ). CTs were performed during the same inspiratory or expiratory hold, for different values of airway pressure. Each CT image was manually delineated excluding pleural effusions and mediastinal structures. We analyzed Standard and Lowdose CT images with dedicated software to quantify the tissue weight of lung regions with different degrees of inflation. Lung quantitative data computed by Standard and by Lowdose scans were compared according to Bland-Altmann analysis.

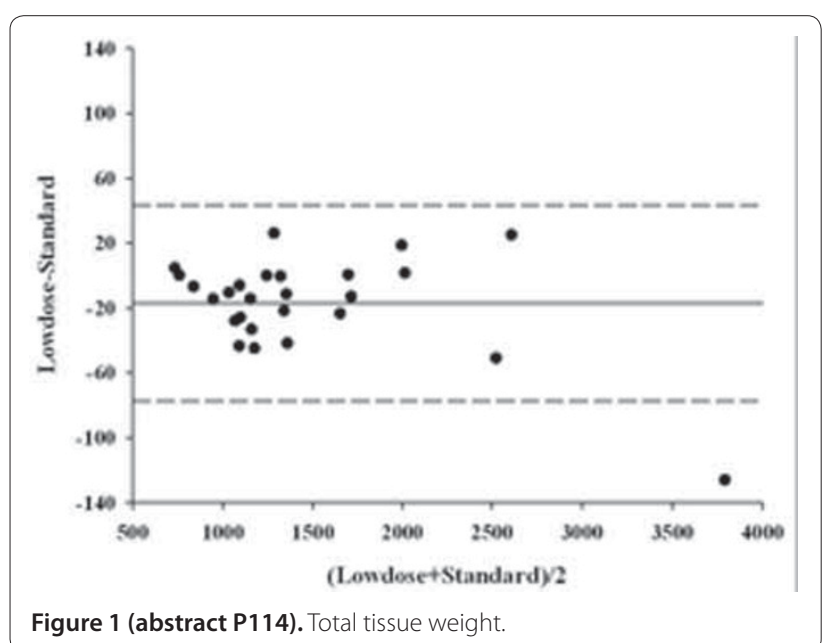




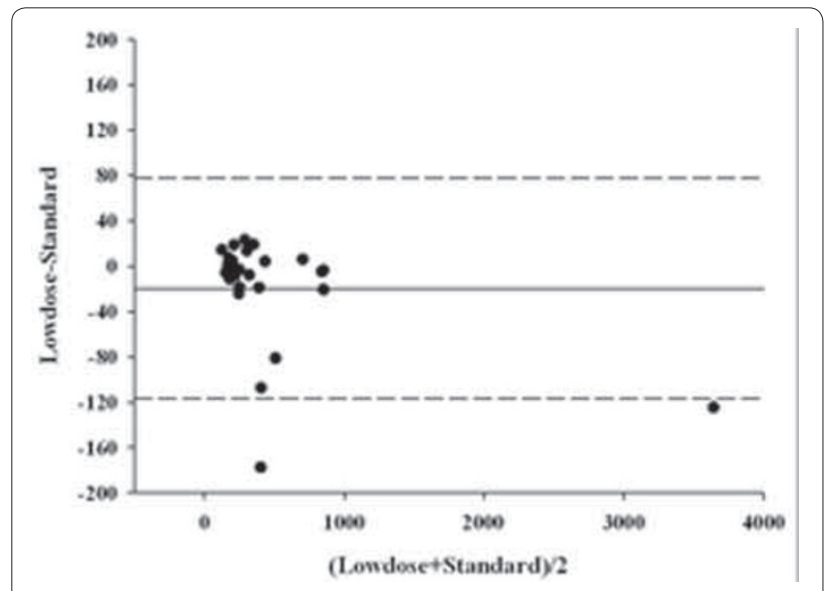

Figure 2 (abstract P114). Not inflated tissue weight.

Results We enrolled 13 patients admitted to our ICU. In the BlandAltman analysis of the lung total tissue and not inflated tissue, the bias and agreement bands for Standard CT scan and Lowdose CT quantitative analysis were $-16.97 \mathrm{~g}(-77.43$ to $+43.49 \mathrm{~g})$ and $-9.65 \mathrm{~g}$ $(-116.81$ to $77.51 \mathrm{~g})$, respectively (Figures 1 and 2).

Conclusion Lung CT quantitative analysis computed by Lowdose CT scans could be a useful tool for monitoring and ventilatory management of ARDS patients.

Reference

1. NEngl J Med 2006, 354:1775-1786.

P115

Preliminary experience with airway pressure release ventilation on hemodynamics in patients with septic shock in a medical/surgical

ICU

A Hussain, H Lababidi, A Mir, A AlHamoud, A Al Oheli, A Al Enezi

King Fahd Medical City, Riyadh, Saudi Arabia

Critical Care 2013, 17(Suppl 2):P115 (doi: 10.1186/cc12053)

Introduction Airway pressure release ventilation (APRV) allows spontaneous breathing throughout the ventilation cycle. It increases venous return and cardiac index, which will significantly improve organ perfusion. This is important in septic shock patients to prevent extrathoracic organ system failure secondary to poor perfusion. Benefits of APRV with cardiovascular changes are noticed in patients with acute lung injury and acute respiratory distress syndrome. It is not well established whether applying APRV will improve the survival outcome for septic shock patients. The primary outcome is whether the use of APRV in septic shock patients restores hemodynamic stability earlier than the CMV mode. The secondary hypothesis is whether the use of APRV in septic shock patients improves their survival in the ICU. Methods After Institutional Review Board approval, we retrospectively analyzed the clinical data of 129 septic shock patients who received ventilator support between January and December 2011 at a tertiary care hospital. The Cox proportional hazards model was used in adjusting potential confounding factors. The nonparametric Wilcoxon rank sum test was used to assess significant outcome differences between groups. Time to event/survival data will be analyzed using Kaplan-Meier methods. These analyses were accomplished using SAS, version 9.3.

Results Among the 187 patients, 58 were excluded as per the exclusion criteria: incomplete data $(n=28)$, do not resuscitate $(n=16)$, ICU readmission $(n=12)$ and head injury $(n=4)$. Finally, 129 patients were included, from these 91 received CMV and 38 received APRV. At the beginning of the study, there were no differences between the groups in relation to hemodynamic parameters. Reversal of shock achieved in less than 72 hours was statistically significant between the groups (APRV, $n=16(42 \%)$ and CMV $n=8(9 \%), P=0.0101)$. The proportion of patients recovering from septic shock after initiation of ventilator therapy was higher in APRV than the CMV group (72\% vs. $49 \%$, respectively, $P<0.0001)$. The mortality rate was significantly higher in CMV $(n=46,51 \%)$ as compared with APRV $(n=11,29 \%)(P=0.022)$.

Conclusion The use of APRV in septic shock patients restores hemodynamic stability earlier than the CMV mode. There was a significant improvement in ICU survival using APRV over CMV. Early initiation of APRV in ventilated septic shock patients was associated with a decrease in ICU mortality.

\section{P116}

Feasibility and effectiveness of prone position in morbidly obese

ARDS patients: a case-control clinical study

A De Jong, N Molinari, M Sebbane, A Prades, S Jaber

Montpellier University Hospital, Montpellier, France

Critical Care 2013, 17(Suppl 2):P116 (doi: 10.1186/cc12054)

Introduction Obese patients are at risk of developing atelectasis and acute respiratory distress syndrome (ARDS) [1]. The prone position (PP) may reduce atelectasis, and improves oxygenation and outcome in severe hypoxemic patients in ARDS [2], but little is known about its effect in obese ARDS patients.

Methods Morbidly obese patients (body mass index (BMI) $\geq 35 \mathrm{~kg} / \mathrm{m}^{2}$ ) in $\mathrm{PP}$ with ARDS $\left(\mathrm{PaO}_{2} / \mathrm{FiO}_{2}\right.$ ratio $\left.\leq 200 \mathrm{mmHg}\right)$ were matched to nonobese $\left(\mathrm{BMI}<30 \mathrm{~kg} / \mathrm{m}^{2}\right)$ ARDS patients in a case-control clinical study. The primary endpoints were safety and complications of PP; the second endpoints were the effect on oxygenation $\left(\mathrm{PaO}_{2} / \mathrm{FiO}_{2}\right.$ ratio at the end of PP), length of mechanical ventilation and ICU stay, nosocomial infections and mortality.

Results Between January 2005 and December 2009, 149 patients were admitted for ARDS. Thirty-three obese patients were matched with 33 nonobese patients. Median PP duration was 9 (6 to 11) hours in obese patients and 8 ( 7 to 12$)$ hours in nonobese patients $(P=0.28)$. We collected 51 complications, of which 25 in obese patients and 26 in nonobese patients. The number of patients with at least one complication was similar across groups $(n=10,30 \%)$. The $\mathrm{PaO}_{2} / \mathrm{FiO}$ ratio (Figure 1) increased significantly more in obese patients (from $118 \pm 43$ to $222 \pm 84 \mathrm{mmHg}$ ) than in nonobese patients (from $113 \pm 43 \mathrm{mmHg}$ to $174 \pm 80 \mathrm{mmHg}, P=0.03$ ). Length of mechanical ventilation, ICU stay and nosocomial infections did not differ significantly, but mortality at 90 days was significantly lower in obese patients ( 27 vs. $48 \%, P<0.05$ ).

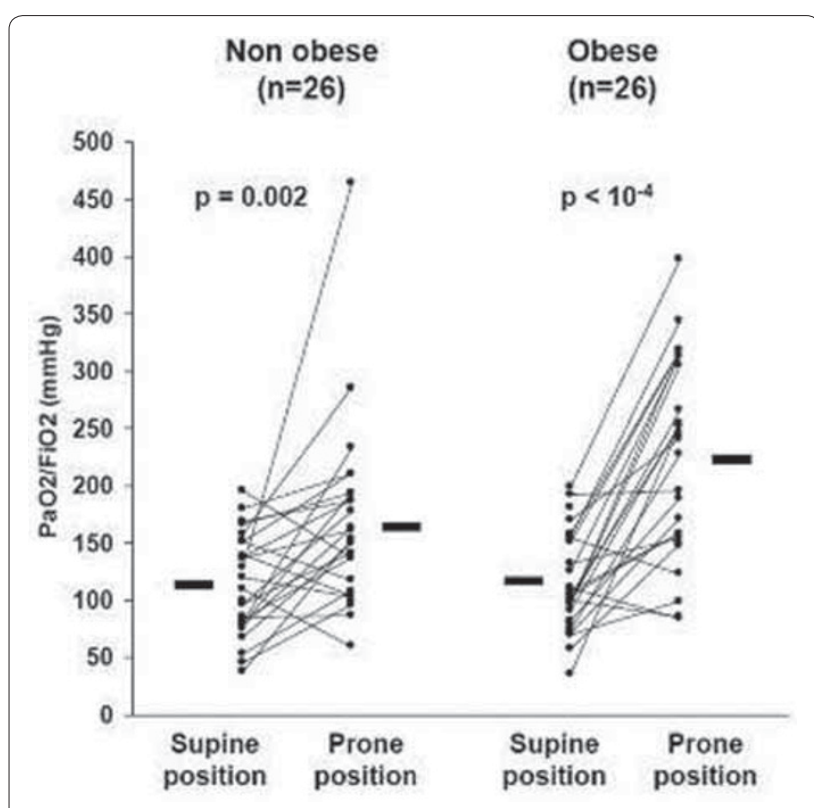

Figure 1 (abstract $\mathrm{P}$ 116). Individual variations of $\mathrm{PaO}_{2} / \mathrm{FiO}_{2}$ ratio between supine and prone positions in obese and nonobese patients. 
Conclusion PP seems safe in obese patients and may improve oxygenation more than in nonobese patients. Obese patients could be a subgroup of ARDS patients who may benefit most from PP.

References

1. Gong MN, et al:: Thorax 2010, 65:44-50

2. Charron C, et al: Intensive Care Med 2011, 37:785-790.

P117

Provision of mechanical ventilation to pregnant/postpartum women with $\mathrm{H} 1 \mathrm{~N} 1$ influenza: a case-control study

WE Pollock', R Bellomo², S Webb³, I Seppelt', A Davies ${ }^{5}$, E Sullivan',

S Morrison? $\mathrm{BHowe}^{7}$

'Mercy Hospital for Women, Heidelburg, Australia; '2Austin Health, Heidelberg, Australia; ${ }^{3}$ Royal Perth Hospital, Perth, Australia; ${ }^{4}$ Nepean Hospital, Penrith,

Australia; ${ }^{5}$ Alfred Hospital, Prahran, Australia; ${ }^{6}$ University of New South Wales,

Randwick, Australia; 'Monash University, Prahran, Australia

Critical Care 2013, 17(Suppl 2):P117 (doi: 10.1186/cc12055)

Introduction During the influenza pandemic of 2009, clinicians delivered mechanical ventilation to pregnant women with little evidence to guide practice. The objective of this study was to compare the provision of mechanical ventilation to pregnant/postpartum women and a nonpregnant matched control group admitted to the ICU with H1N1 influenza.

Methods A case-control study was conducted following ethics approval. A case was defined as a ventilated pregnant/postpartum woman reported to the Australian and New Zealand INFINITE H1N1 09 study from 1 June 2009 to 31 August 2009. Controls were ventilated nonpregnant women (15 to 49 years) reported to the INFINITE H1N1 09 study during the same time frame. Data were entered into SPSS and analysed using nonparametric statistics; two-tailed $P<0.05$ was considered significant.

Results We studied 36 index cases and 38 controls. Index cases were more likely to have a single arterial blood gas (ABG) taken prior to intubation $(P<0.05)$. Similar reasons were given for the trigger to intubate (high respiratory rate, low $\mathrm{PaO}_{2}$, increased work of breathing) apart from a high $\mathrm{PaCO}_{2}$, which was a trigger in the control group only $(P<0.05)$. There were no differences in the pre-intubation and postintubation $\mathrm{ABG}$ s apart from a lower $\mathrm{PaCO}_{2}(P<0.05)$ and lower $\mathrm{HCO}_{3}$ $(P<0.05)$ in cases, and cases presented with a lower haemoglobin $(P<0.05)$. There were six difficult intubations documented with no differences between groups. Initial ventilator settings including mode, tidal volume, minute volume and respiratory rate demonstrated no differences. Both groups showed increases in $\mathrm{PaO}_{2}$ and $\mathrm{PaCO}_{2}$, and a decrease in $\mathrm{pH}$ from the pre-intubation to post-intubation ${ }^{2} \mathrm{ABGs}$ $(P<0.05)$.

Conclusion There were physiological differences between the two groups with pregnant/postpartum women showing lower $\mathrm{PaCO}_{2}$ and $\mathrm{HCO}_{3}$. However, initial ventilator support was not significantly different for pregnant/postpartum women compared with controls.

P118

Inhalation injury in critical burn patients

L Cachafeiro, M Sanchez, E Herrero, L Fernández, M Irazabal, M Hernandez, A Agrifoglio, A Garcia de Lorenzo, M Lendinez

Hospital La Paz, Madrid, Spain

Critical Care 2013, 17(Suppl 2):P118 (doi: 10.1186/cc12056)

Introduction The objective is to analyze the epidemiology and mortality of critical burn patients with inhalation injury.

Methods A prospective, observational and descriptive study was carried out in all patients admitted to an ICU from October 2008 to June 2011. Inhalation injury was defined with two or more of the following criteria: history of injury in an enclosed space, facial burns with singed nasal hair, carbonaceus sputum or stridor. If they were intubated it was diagnosed by bronchoscopy. Demographic data, length of stay, ABSI, APACHE II, duration of mechanical ventilation, hospital course and mortality data were collected. Data are presented as number and percentage or as median and interquartile range and were analyzed with the Fisher exact test and Mann-Whitney test.

Results Of the 362 patients admitted, 84 (23.2\%) had inhalation injury. Seventy-six percent were male and the average age was $52( \pm 17.5)$ years. The mean total burn surface area (TBSA) was $28 \%( \pm 25)$. Fortyfive patients (53.5\%) had TBSA $>20 \%$. These patients had higher severity scores: ABSI $8( \pm 2.8)$ versus $6( \pm 2.2)(P=0.0001)$, APACHE II $13( \pm 6.9)$ versus $7( \pm 6.7)(P=0.0001)$. They also had a higher need for mechanical ventilation $(88.1 \%$ vs. $34.9 \%, P=0.001)$ and shock $(59.5 \%$ vs. $27.0 \%, P=$ 0.001). The $\mathrm{PaO}_{2} / \mathrm{FiO}_{2}$ ratio at admission with and without inhalation was 245 versus $359(P=0.003)$, and at 72 hours was 207 versus 306 $(P=0.002)$. There were no significant differences in the appearance of sepsis, ARDS and renal failure. The length of stay with and without inhalation was 25.2 ( 1 to 95 ) days versus 22.5 ( 1 to 92 ) days $(P=0.4$ ). The inhalation injury group showed a significantly higher mortality $(9.0 \%$ vs. $28.9 \%, P=0.001)$.

Conclusion In this study, inhalation injury is common in burn patients. They had higher severity scores (APACHE II, ABSI) and higher mortality. These patients had a higher need for mechanical ventilation and lower $\mathrm{PaO}_{2} / \mathrm{FiO}_{2}$ ratio, but there was no significant increase in ARDS or respiratory sepsis.

P119

Beneficial effects of intrapulmonary percussive ventilation in

patients with respiratory insufficiency in the ICU

I Blum, R Janssen-Dean, A Overdijk van, B Speelberg

St Anna Hospital Geldrop, Geldrop, the Netherlands

Critical Care 2013, 17(Suppl 2):P119 (doi: 10.1186/cc12057)

Introduction Intrapulmonary percussive ventilation (IPV) is a therapy that is used to clear endobronchial secretions. The IPV ventilator was designed and developed by FM Bird in 1979. The ventilator consists of a phasitron that delivers rapid, high-flow, mini bursts of oxygen, mixed with air. The potential aims of this mechanism are pulmonary recruitment, improved mucus clearance with a direct high-frequency oscillatory effect. We investigated whether IPV has a positive effect on ventilatory values in adult patients on the ICU.

Methods All patients presenting during a 4-month period in 2011 with respiratory insufficiency on our mixed adult ICU were included in this study. Patients were monitored before, directly after and 15 minutes after therapy with IPV using a Bird Intrapulmonary Ventilator Model IPV-2C. All patients received IPV for a period of 20 minutes consisting of two cycles of 10 minutes. Peripheral oxygen saturation $\left(\mathrm{SpO}_{2}\right)$, tidal volume $(\mathrm{Vt})$, respiratory rate, end-tidal $\mathrm{CO}_{2}\left(\mathrm{ET}_{-}-\mathrm{CO}_{2}\right)$, dynamic lung compliance (C-dyn) and work of breathing (WOB) were monitored at the different time points. Paired Student $t$ tests were performed in order to compare the values immediately before IPV, with directly after therapy and 15 minutes later. $P<0.05$ was considered significant.

Results Eighty-three patients were examined. $\mathrm{SpO}_{2}$ improved significantly from $93.7 \pm 3.7$ before IPV to $95.7 \pm 2.8$ after IPV $(P<0.001)$ and 15 minutes later to $95.2 \pm 2.8(P<0.001)$. Vt improved from $418 \pm 111$ before IPV to $476 \pm 102$ directly after $(P<0.001)$ and to $480 \pm 131$ 15 minutes later $(P<0.01)$. Respiratory rate improved from $24 \pm 6$ to $23 \pm 6$ only after 15 minutes significantly $(P<0.01)$. WOB and $C$-dyn did not change. $\mathrm{ET}-\mathrm{CO}_{2}$ decreased from $34.9 \pm 14.8$ to $33.3 \pm 13.3(P<0.05)$ directly after IPV and to $32.1 \pm 12.8(P<0.01) 15$ minutes later.

Conclusion In this study we demonstrated a beneficial effect of IPV on oxygen saturation, tidal volume and end-tidal $\mathrm{CO}_{2}$. IPV has this effect in addition to the mobilization of bronchial secretions.

Reference

1. Salim A, Martin M: High-frequency percussive ventilation. Crit Care Med 2005, 33:S241-S245.

\section{P120}

High-frequency ventilation for acute traumatic and nontraumatic lung injury

RVarutti', R Bigai', M Fiorillo', D Tomasello', W Mercante', G Trillò2

'Azienda Ospedaliera Santa Maria degli Angeli, Pordenone, Italy; ${ }^{2}$ Udine University Hospital, Udine, Italy

Critical Care 2013, 17(Suppl 2):P120 (doi: 10.1186/cc12058)

Introduction ARDS is commonly observed in trauma patients. In some instances the severity of the clinical presentation is such that all conventional ventilatory support mode fails. In this setting, 


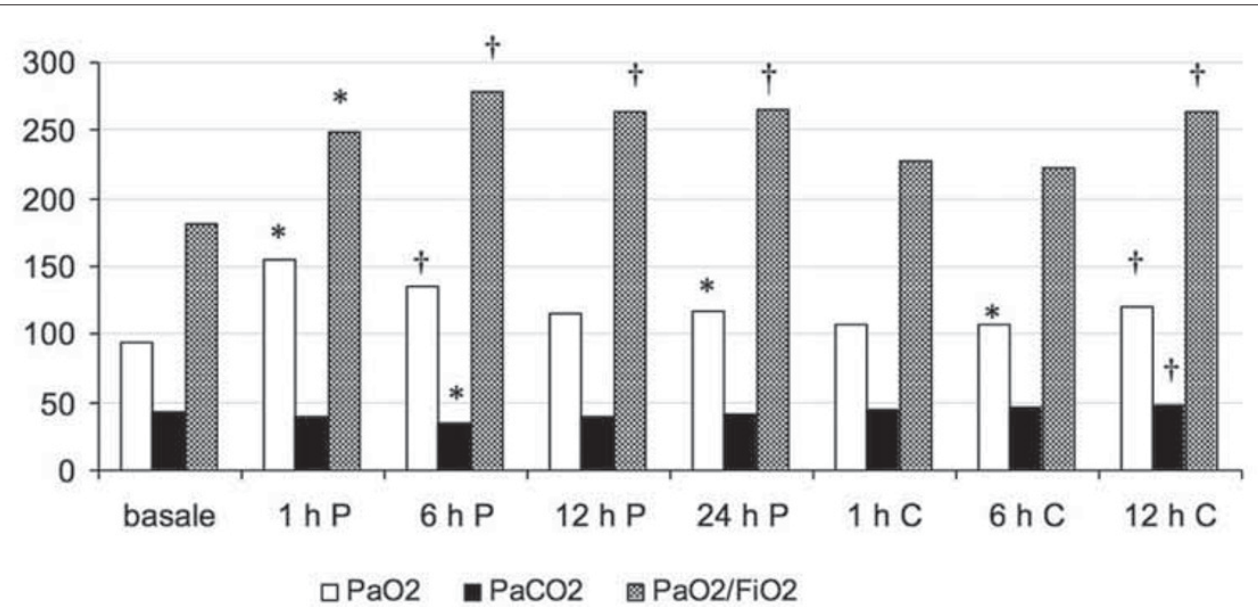

Figure 1 (abstract $\mathrm{P} 120$ ). Mean $\mathrm{PaO}_{2}, \mathrm{PaCO}_{2}, \mathrm{PaO}_{2} / \mathrm{FiO}_{2}$ at baseline and during $\mathrm{HFOV}\left(\mathrm{P}\right.$, percussionator; $\mathrm{C}$, conventional). ${ }^{*} P<0.05$ versus baseline, ${ }^{\dagger} P<0.01$ versus baseline.

Table 1 (abstract P120). Clinical and demographic data

\begin{tabular}{lc}
\hline & Mean \\
\hline Age (years) & 64 \\
Male/female & $11 / 4$ \\
Dead & 4 \\
Trauma & 3 \\
SAPS II & $45 / 40$ \\
APACHE & $23 / 8$ \\
\hline
\end{tabular}

high-frequency oscillatory ventilation (HFOV) was considered mostly a rescue therapy.

Methods Fifteen adult patients admitted to our ICU for acute traumatic and nontraumatic lung injury were submitted to HFOV when conventional mechanical ventilation failed.

Results Clinical and demographic data are shown in Table 1. Figure 1 shows the trend of gas parameters during the recovery. At baseline $\mathrm{PaO}_{2}$ was $94 \pm 28 \mathrm{mmHg}$; after 6 hours of HFOV: $135 \pm 41 \mathrm{mmHg}$, $P<0.01$. At baseline $\mathrm{PaO}_{2} / \mathrm{FiO}_{2}$ was $182 \pm 97 \mathrm{mmHg}$; after 6 hours of HFOV: $264 \pm 101 \mathrm{mmHg}, P<0.01$. The benefits are maintained when returned to conventional ventilation.

Conclusion HFOV may therefore be anticipated to improve endorgan perfusion and gas exchange; it should be considered in severe traumatic and nontraumatic respiratory failure [1].

Reference

1. BMJ 2010, 340:C2327.

\section{P121}

Improvement in oxygenation with high-frequency oscillatory ventilation combined with tracheal gas insufflation is correlated to the extravascular lung water index

CS Vrettou, S Malachias, SG Zakynthinos, SD Mentzelopoulos

Evaggelismos General Hospital, Athens, Greece

Critical Care 2013, 17(Suppl 2):P121 (doi: 10.1186/cc12059)

Introduction High-frequency oscillatory ventilation combined with tracheal gas insufflation (HFO-TGI) can significantly improve oxygenation in patients with ARDS. It has been demonstrated that oxygenation in patients with ARDS has a better response to HFO when extravascular lung water is $>15 \mathrm{ml} / \mathrm{kg}$ body weight (BW). Our aim is to examine whether the extravascular lung water index (ELWI) correlates with changes in $\mathrm{PaO}_{2} / \mathrm{FiO}_{2}$ in patients ventilated with $\mathrm{HFO}-\mathrm{TGI}$.

Methods Data from 18 sessions of HFO-TGI in six patients were included in the analysis. HFO frequency, oscillatory pressure amplitude, and bias flow were $3.5 \mathrm{~Hz}, 85$ to $95 \mathrm{cmH}_{2} \mathrm{O}$, and $40 \mathrm{l} /$ minute, respectively; a 2.5 to $3.5 \mathrm{cmH}_{2} \mathrm{O}$ tracheal tube cuff leak was used. HFO mean airway pressure ( $\mathrm{mPaw}$ ) exceeded preceding conventional ventilation (CV)-mPaw by 7 to $13 \mathrm{cmH}_{2} \mathrm{O}$. $\mathrm{PaO} / \mathrm{FiO}_{2}$ l lung mechanics, and hemodynamics were documented during lung-protective CV (baseline) and 1 hour following the initiation of HFO-TGI ventilation. PULSION PICCOplus v7.0 was used for hemodynamic measurements including the ELWI.

Results Oxygenation $\left(\mathrm{PaO}_{2} / \mathrm{FiO}_{2}\right)$ improved significantly with HFOTGl compared with CMV (125.5 \pm 54.7 vs. $195.6 \pm 108.7, P<0.001)$. Changes in $\mathrm{PaO}_{2} / \mathrm{FiO}_{2}$ were positively correlated with ELWI at baseline (Spearman's $\rho=0.56, P=0.016$ ). See Figure 1. There were no significant changes in patients' fluid balance and hemodynamics including the ELWI.

Conclusion Estimation of the ELWI can help to predict the oxygenation response of ARDS patients considered for HFO-TGI ventilation. The possibility that HFO-TGl exerts an effect on pulmonary oedema needs further investigation.

Acknowledgement This research has been cofinanced by the European Union and Greek national funds through the operating programme 'Education and Lifelong Learning' - Research Funding Programme Heracleitus II.

\section{Reference}

1. Zeravik J, et al:: Acta Anaesthesiol Scand 1989, 33(Suppl 90):149-152.

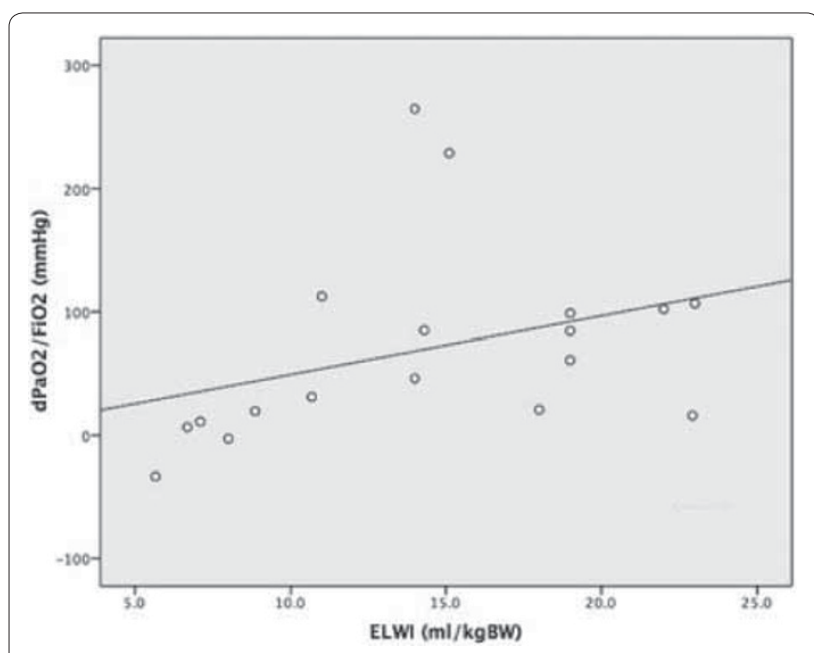

Figure 1 (abstract P121). Scatter plot and fit line of ELWI versus $\mathrm{dPaO}_{2} / \mathrm{FiO}_{2}$. 
P122

Physiologic effects and regional ventilation of high-frequency positive-pressure ventilation using a conventional ventilator in a severe ARDS animal model associated with an inspiratory pause or recruitment maneuvers

RL Cordioli', M Park², M Amato³, S Gomes 3 , E Leite'2, L Azevedo²

${ }^{1}$ Hopitaux Universitaire de Geneve, Geneva, Switzerland; ${ }^{2}$ Hospital Sirio Libanes IEP, São Paulo, Brazil; ${ }^{3}$ Hospital das Clinicas LIM 09, São Paulo, Brazil Critical Care 2013, 17(Suppl 2):P122 (doi: 10.1186/cc12060)

Introduction Protective mechanical ventilation (MV) in ARDS is based on reduced stretch of pulmonary tissue, sometimes resulting in severe hypoventilation that can be avoided when using high respiratory rate. High-frequency positive-pressure ventilation (HFPPV) has not been fully explored, especially when associated with other strategies aiming to avoid hypercapnia.

Methods We induced ARDS in eight pigs by lung lavage with saline plus 3 hours of injurious MV with low PEEP and high driving pressure (DP). We then performed a recruitment maneuver (RM) followed by PEEP titration using the amount of alveolar collapse in electrical impedance tomography (EIT). Then stabilization during 1 hours with tidal volume (VT) at $6 \mathrm{ml} / \mathrm{kg}$, respiratory rate (RR) 35 breaths/minute and PEEP selected with the PEEP-FiO table (ARMA study), which was kept constant during two steps of HFPPV with a RR 60: one without an inspiratory pause (HFPPV-60), and one with a pause of $30 \%$ of inspiratory time (HFPPV-60 w/P30\%). In another HFPPV step, we used PEEP titrated with EIT after RM (HFPPV-60 w/RM). During each HFPPV step, VT was set to reach a $\mathrm{PaCO}_{2}$ of $60 \pm 3 \mathrm{mmHg}$. Distribution of regional ventilation was analyzed using EIT. Equilibrium was considered if $\mathrm{PaCO}_{2}$ was stable ( $<5 \%$ of variation) for $>30$ minutes.

Results HFPPV allowed reduction in $\mathrm{PaCO}_{2}$ levels: $81(77,94)$ versus $60(58,61), 59(58,60), 60(58,61) \mathrm{mmHg}$, besides using lower VT: $5.2(5.0,5.9), 5.1(4.5,6.0), 4.7(4.2,5.7)$ and $4.8(4.5,5.6) \mathrm{ml} / \mathrm{kg}$ during stabilization, HFPPV-60, HFPPV-60 w/P30\% and HFPPV-60 w/RM, respectively. It had no significant different results comparing HFPPV-60 with and without an inspiratory pause. HFPPV-60 w/RM allowed a better alveolar homogenization and improvement in oxygenation, shunt, dead space and DP compared with the other steps. See Table 1.

Table 1 (abstract P122). Physiological variables

\begin{tabular}{lccc}
\hline Variable & VT $=\mathbf{6}$, stabilization & HFPPV-60 & HFPPV-60 w/RM \\
\hline $\mathrm{DP}\left(\mathrm{CmH}_{2} \mathrm{O}\right)$ & $16 \pm 2$ & $16 \pm 3$ & $12 \pm 2^{*}$ \\
$\mathrm{P} / \mathrm{F}$ ratio & $95 \pm 13$ & $149 \pm 60$ & $246 \pm 99^{*}$ \\
\hline
\end{tabular}

*Tukey's post-hoc analysis, $P<0.05$ versus others.

Conclusion HFPPV with a conventional mechanical ventilator is able to maintain stable $\mathrm{PaCO}$ in clinically acceptable values, allowing reductions in VT. HFPPV- $60 \mathrm{w} / \mathrm{RM}$ and PEEP titration using EIT allowed further physiologic benefits in a severe ARDS model.

\section{P123}

High-frequency percussive ventilation at altitude: study in a hypobaric chamber with a mechanical test lung AC Cirodde 1 , S Montmerle 2 , ND Donat 1 , CB Bourhillon², P Jault', L Bargues ${ }^{1}$, T Leclerc $^{1}$ Military Hospital Percy, Clamart, France:2 IRBA, Brétigny, France Critical Care 2013, 17(Suppl 2):P123 (doi: 10.1186/cc12061)

Introduction High-frequency percussive ventilation (HFPV) is a rescue technique for most severe acute lung injury/acute respiratory distress syndrome (ARDS) patients [1], especially with smoke inhalation or respiratory burns [2]. This study aimed at characterizing HFPV as delivered by Percussionnaire VDR $4^{\circledR}$ and at evaluating how hypobarism interferes with HFPV, in order to assess its usability at altitude. Methods Using a mechanical test lung mimicking ARDS (compliance $17 \mathrm{ml} / \mathrm{cmH}_{2} \mathrm{O}$ ) with two resistance levels (5 and $15 \mathrm{cmH}_{2} \mathrm{O} / \mathrm{l} / \mathrm{second}$ ) and ventilated with VDR4 ${ }^{\circledR}$ in a hypobaric chamber, ascents/descents between 0 and 5,000 and then 0 and 8,000 ft were performed. Adjustable
VDR4 ${ }^{\circledR}$ parameters were modified one at a time at each altitude. Besides these parameters (cross-measured with standalone hardware), oxygen consumption of the respirator and three calculated parameters were studied: low-frequency tidal volume (Vt, integrated from instantaneous flows measured with a Fleisch pneumotachograph), end-inspiratory $(\mathrm{PmEl})$ and end-expiratory (PmEE) mean pressures. PmEl and PmEE in HFPV reflect plateau pressure and positive end-expiratory pressure in conventional ventilation. The correction of altitude-induced offset with the modification of working pressure was also tested.

Results Data displayed by VDR $4^{\circledR}$ overestimated pulmonary pressures by more than $10 \%$, but were reliable for other parameters. During ascent, an offset appeared for all respiratory parameters: Vt increased by $59 \%$ and PmEl by $53 \%$ between 0 and $8,000 \mathrm{ft}$. During descent, the offset was reversely directed with a $39 \%$ decrease in Vt and a $28 \%$ decrease in PmEE between 8,000 and $0 \mathrm{ft}$. Modifying working pressure adequately corrected PmEl and PmEE, but not Vt. In all cases, manually correcting VDR4 ${ }^{\circledR}$ parameters to their $0 \mathrm{ft}$ level also corrected these offsets. Multivariate analysis further established that, adjusting for other parameters, $\mathrm{Vt}, \mathrm{PmEl}$ and PmEE did practically not depend on altitude. Oxygen consumption of the respirator was high, $25 \mathrm{I} /$ minute at $0 \mathrm{ft}$, and stable with altitude. It was reduced with percussive rate and with $\mathrm{FiO}_{2}$.

Conclusion HFPV can be safely used at altitude, provided that VDR4 ${ }^{\circledR}$ displayed parameters are used to manually adjust settings in order to avoid exposing patients to volutrauma or barotrauma during ascent, and to major hypoventilation and alveolar collapse during descent. The high oxygen consumption is currently the main limit to its use for longrange aeromedical evacuations.

References

1. Velmahos GC, et al:: Chest 1999, 116:440-446.

2. Chung KK, et al:: Crit Care Med 2010, 38:1970-1977.

\section{P124}

Recruitable volume is comparable in acute respiratory distress syndrome and in healthy lungs

CA Stahl' ${ }^{1}$, K Moeller $^{2}$, D Steinmann 1 , D Henzler ${ }^{3}$, S Lundin $^{4}$, O Stenqvist ${ }^{4}$

University Medical Center Freiburg, Germany; ${ }^{2}$ Biomedical Engineering

Villingen-Schwenningen, Germany; ${ }^{3}$ Klinikum Herford, Germany;

${ }^{4}$ Sahlgrenska University Hospital, Gothenburg, Sweden

Critical Care 2013, 17(Suppl 2):P124 (doi: 10.1186/cc12062)

Introduction The application of PEEP is commonly used in acute respiratory distress syndrome (ARDS) and has been shown to improve oxygenation. To identify patients that most benefit from the application of PEEP, the discrimination of recruiters and nonrecruiters has been postulated by Gattinoni and colleagues [1]. Recently, Dellamonica and colleagues [2] presented a method to predict alveolar recruitment. We hypothesised that the amount of recruitable volume allows the discrimination between ARDS patients and patients with healthy lungs (HL).

Methods We recalculated the recruited volume (RV) in 25 patients with ARDS [3] according to the method proposed by Dellamonica and colleagues during an incremental PEEP manoeuvre (PEEP increased until the plateau pressure reached $45 \mathrm{cmH}_{2} \mathrm{O}$ ). $\mathrm{RV}$ was calculated as the change in end-expiratory lung volume minus total respiratory system compliance times the PEEP change (RV $=\triangle E E L V-C T O T \times \triangle P E E P$ ). For comparison, 12 patients with $\mathrm{HL}$ undergoing elective surgery in general anaesthesia were measured using the same protocol.

Results Both ARDS and HL patients exhibited typical P-V curves and stepwise recruitment (Figure 1). By raising PEEP from 0 to $12 \mathrm{cmH}_{2} \mathrm{O}_{\text {, }}$ ARDS patients recruited $331 \pm 195 \mathrm{ml}$ (mean \pm SD) and $\mathrm{HL}$ patients $435 \pm 43 \mathrm{ml}$. There was a strong correlation $\left(R^{2}=0.88\right)$ of the total RV with the end-inspiratory volume at a plateau pressure of $45 \mathrm{cmH}_{2} \mathrm{O}$ in both groups; that is, recruitment was found to the same extent in both groups (Figure 2).

Conclusion The relative contribution of $\mathrm{RV}$ to lung volume gain is similar in ARDS and in patients with healthy lungs. Our results question the relevance of recruitability as defined by Dellamonica and colleagues as a typical phenomenon of ARDS, but support the baby lung concept, as the recruited volume was closely related to the size of the lung. 


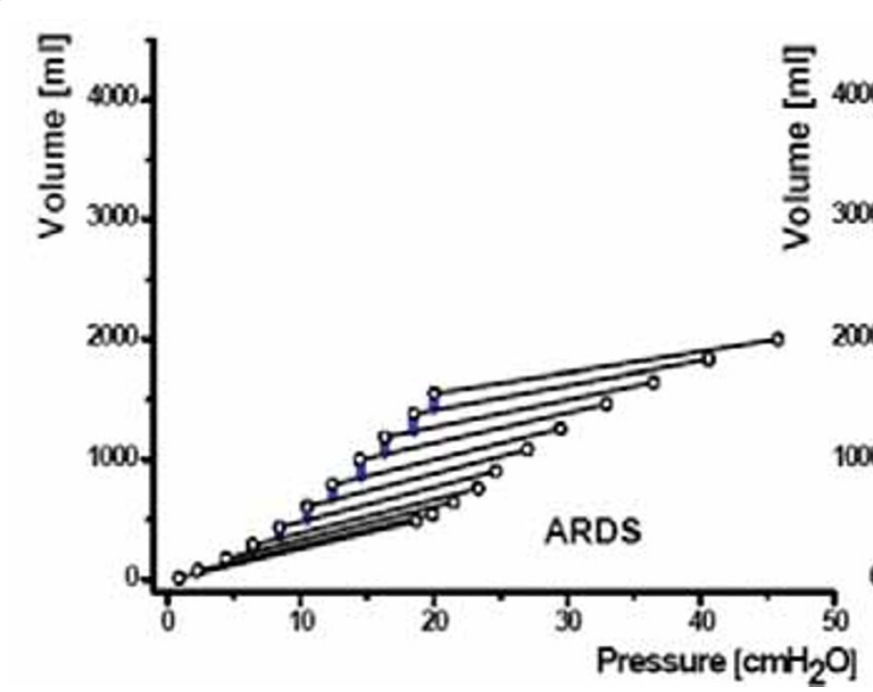

Figure 1 (abstract P124).

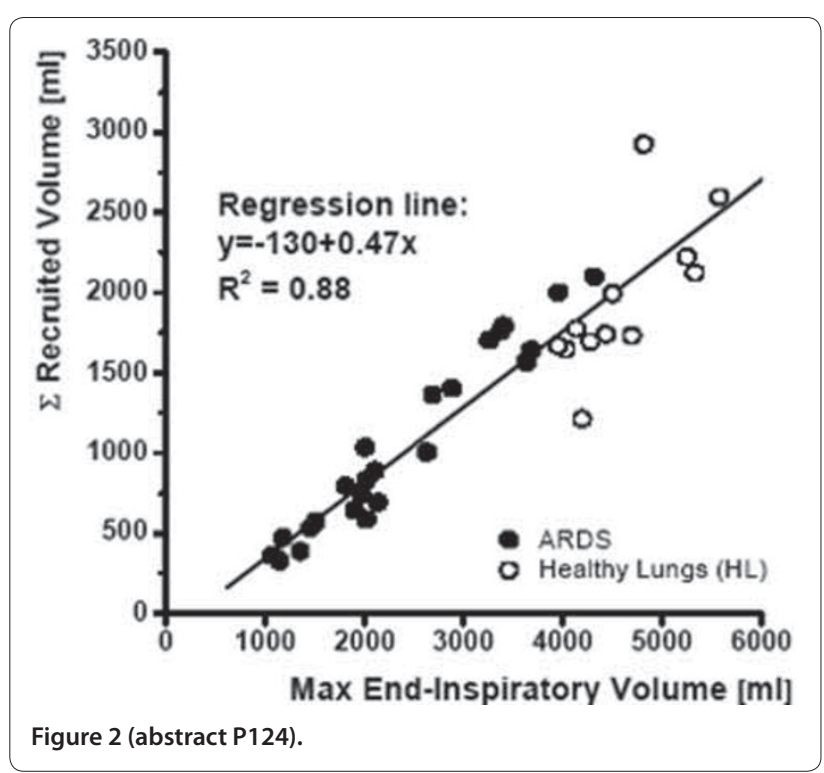

\section{References}

1. Gattinoni et al.: N Engl J Med 2006, 354:1775.
2. Dellamonica et al.: Intensive Care Med 2011, 37:1595.
3. Stahl et al.: Crit Care Med 2006, 34:2090.

P125

Nosocomial bloodstream infection and venovenous extracorporeal membrane oxygenation: a retrospective cohort study

B Serra de Oliveira, S Mendes Fernandes, C França

Hospital de Santa Maria CHLN, Lisboa, Portugal

Critical Care 2013, 17(Suppl 2):P125 (doi: 10.1186/cc12063)

Introduction Venovenous extracorporeal membrane oxygenation (VV-ECMO) for respiratory failure in the ICU is used in a variety of clinical situations and has been demonstrated to significantly improve survival without disability in adult respiratory distress syndrome [1]. ECMO has been presented as a risk factor for bloodstream infection although recently published data do not support this view or the use of antibiotic prophylaxis [2]. We aimed to examine VV-ECMO as a risk factor for nosocomial bloodstream infection.
Methods A retrospective cohort study from patients admitted to our ICU between April 2009 and June 2012. We compared incidence rates of nosocomial bacteremia using the Hospitals in Europe Link for Infection Control through Surveillance (HELICS) between general ICU and VV-ECMO patients and used multiple logistic regression analysis to control for possible confounders.

Results During the study period 1,146 patients were admitted and 16 received VV-ECMO. The incidence of bloodstream infection in patients with ECMO was 19/1,000 exposure-days versus 4,9/1,000 exposuredays in general ICU patients (incidence rate ratio of 3.82; 95\% Cl: 2.0 to $7.3 ; P<0.001)$. Bacteremia was mostly due to Gram-negative agents (65\%). The patients with bloodstream infections under ECMO $(n=$ 10) had a nonsignificant younger age $(P=0.08)$ and a lower SAPS II score $(P=0.03)$ compared with non-ECMO patients $(n=25)$. VV-ECMO patients had a significantly higher risk of primary bacteremia than nonECMO patients $(P=0.04)$. Patients with bloodstream infections in the VV-ECMO group had a lower crude mortality rate (OR: $0.1, P=0.04)$, not confirmed in the adjusted analysis. There were no crude or adjusted differences in the time to bacteremia or infections due to multiple drug-resistant microorganisms (OR: $0.26 ; P=0.085$ ) between groups. Conclusion This study suggests that VV-ECMO patients have a significantly higher risk for primary nosocomial bloodstream infection. A larger study is needed to confirm such findings and to assess the need for specific intervention, namely routine antibiotic prophylaxis. References

1. Peek GJ, et al: Efficacy and economic assessment of conventional ventilatory support versus extracorporeal membrane oxygenation for severe adult respiratory failure (CESAR): a multicentre randomised controlled trial. Lancet 2009, 374:1351-1363.

2. Conrick-Martin I, et al:: Nosocomial infections in a cohort of extracorporeal life support patients. Crit Care Resusc 2012, 14:198-201.

P126

Monitoring anticoagulation during extracorporeal membrane oxygenation in patients with acute respiratory failure

M Panigada', C Mietto², F Pagan², L Bogno ${ }^{2}$, V Berto², L Gattinoni² 'Fondazione IRCCS Ca' Granda Ospedale Maggiore Policlinico, Milan, Italy; 2IRCCS Ca' Granda Maggiore Policlinico Hospital Foundation, Milan, Italy Critical Care 2013, 17(Suppl 2):P126 (doi: 10.1186/cc12064)

Introduction aPTT is a common tool for anticoagulation monitoring during extracorporeal membrane oxygenation (ECMO). Thromboelastography (TEG) is another available option in this setting.

Methods A prospective observational study on 12 consecutive patients during venovenous ECMO. Anticoagulation was provided 
with unfractioned heparin titrated to an aPTT ratio target of 1.5 to 2 . Kaolin-activated TEG (K-TEG) was contemporarily measured but did not guide heparin infusion. Baseline K-TEG reaction time $(\mathrm{R})>20$ minutes is accepted for anticoagulation but when it exceeds 90 minutes anticoagulation may be too great [1].

Results Mean ECMO duration was $9 \pm 4$ days. A total of $152 \mathrm{~K}$-TEGs were collected. Comparison between aPTT and K-TEG R is reported in Table 1. Four patients (33\%) had hemorrhagic complications. Neither aPTT nor K-TEG R were significantly different in patients with hemorrhagic events compared with patients without hemorrhagic events but the latter received a significantly lower total heparin dose $(P=0.0097)$.

Table 1 (abstract P126). Comparison between aPTT and K-TEG R

\begin{tabular}{lcccc}
\hline & \multicolumn{3}{c}{ aPTT range (ratio) } & \\
\cline { 2 - 4 } $\begin{array}{l}\text { K-TEG R range } \\
\text { (minutes) }\end{array}$ & $\begin{array}{c}\text { Low } \\
(<1.5)\end{array}$ & $\begin{array}{c}\text { Therapeutic } \\
(\mathbf{1 . 5} \text { to } 2)\end{array}$ & $\begin{array}{c}\text { High } \\
(>\mathbf{2})\end{array}$ & \\
\hline Low (<20) & $13(9 \%)$ & $8(5 \%)$ & 0 & $21(14 \%)$ \\
Therapeutic (20 to 90) & $10(7 \%)$ & $25(17 \%)$ & $11(7 \%)$ & $46(30 \%)$ \\
High (>90) & $15(10 \%)$ & $55(36 \%)$ & $15(10 \%)$ & $85(56 \%)$ \\
& $38(25 \%)$ & $88(58 \%)$ & $26(17 \%)$ & 152 total \\
& & & & samples \\
\hline
\end{tabular}

Conclusion Anticoagulation was excessive in more than one-half of the samples according to TEG monitoring, while negligible based on aPTT. Reference

1. Oliver WC: Semin Cardiothorac Vasc Anesth 2009, 13:154-175.

P127

Extracorporeal membrane oxygenation: the MOTOR of cytokine production?

Y Hara', O Nishida', T Nakamura', S Uchiyama', J Shibata', C Yamashita' M Yumoto', Y Shimomura', N Kuriyama', N Yasuoka', M Ito', K Kawata',

S Hayakawa', S Yamada², T Miyasho³, K Moriyama'

'Fujita Health University School of Medicine, Toyoake, Japan; 'Shino

Corporation, Tokyo, Japan; ${ }^{3}$ Rakuno Gakuen University, Ebetsu, Japan

Critical Care 2013, 17(Suppl 2):P127 (doi: 10.1186/cc12065)

Introduction The usefulness of extracorporeal membrane oxygenation (ECMO) is being rediscovered in the wake of the pandemic of $\mathrm{H} 1 \mathrm{~N} 1$ influenza. However, it has been reported that patients who received ECMO often developed virus-associated hemophagocytic syndrome (VAHS), compared with those without ECMO support. Although there is ample evidence that extensive cytokine activation is a key factor in VAHS, ECMO itself could be a potential trigger to exacerbate the pathology by amplifying cytokine activation. In this study, we investigated whether mediators such as cytokines may be produced by ECMO.

Methods Patients with severe respiratory failure who were placed on ECMO were enrolled between June and July 2012. This study was approved by the ethics committee. Blood specimens were drawn from the blood circuit at the inlet of the centrifugal pump (before) and outlet of the hollow fiber oxygenator (after) at a frequency of three to four times per day. Blood IL-1 $\beta$, IL-2, IL-4, IL-5, IL-6, IL-7, IL-8, IL-10, IL-12(p70), IL-13, IL-17, G-CSF, GM-CSF, IFN $\gamma$, MCP-1, MIP-1 $\beta$, and TNFa were measured globally using a multiplex cytokine bead array system (Bio-Plex; Bio-Rad, Tokyo, Japan). HMGB1 was measured using an ELISA kit (Shino-Test, Tokyo, Japan).

Results Two patients with interstitial pneumonia were studied. The ECMO system consisted of a Rotaflow Centrifugal Pump (Maquet Japan, Tokyo, Japan), a Biocube TNC coating 6000 (NIPRO, Osaka, Japan), and a percutaneous cardiopulmonary support system (Capiox EBS; Terumo, Tokyo, Japan). The blood flow rate was $2.0 \pm 4.0 \mathrm{l} /$ minute. A total of 34 blood sets were collected. In most cases, blood levels of IL-1 $\beta$, IL-2, IL4, IL-5, IL-12 (p70), IL-13, IL-17, GM-CSF, IFNy, and TNFa were below the detection limit and did not increase during ECMO. The other mediators were detected at the inlet (before), but no significant increase was observed at the outlet (after) (HMGB1, $P=0.33$; IL-6, $P=0.12 ; \mathrm{IL}-7, P=$
$0.22 ; \mathrm{IL}-8, P=0.43 ; \mathrm{IL}-10, P=0.84 ; \mathrm{MCP}-1, P=0.10$; and MIP-1 $\beta, P=0.65$; Wilcoxon signed-rank test).

Conclusion The use of ECMO in patients with severe respiratory failure did not induce systemic inflammatory changes. These observations are preliminary, but may nevertheless have important implications for the future management of patients with severe infections.

P128

Effect of extracorporeal $\mathrm{CO}_{2}$ removal on respiratory rate in spontaneously breathing patients with chronic obstructive pulmonary disease exacerbation

E Spinelli, S Crotti, L Zacchetti, N Bottino, V Berto, R Russo, M Chierichetti, A Protti, L Gattinoni

Fondazione IRCCS Ca' Granda Ospedale Maggiore Policlinico, Milan, Italy Critical Care 2013, 17(Suppl 2):P128 (doi: 10.1186/cc12066)

Introduction During severe exacerbation of chronic obstructive pulmonary disease (COPD) tachypnea, as a consequence of respiratory acidosis, and airflow limitation, due to small airway obstruction, lead to lung hyperinflation, respiratory distress and gas exchange impairment. Invasive mechanical ventilation could worsen lung hyperinflation and produce a vicious circle. We investigated whether increasing extracorporeal carbon dioxide removal $\left(\mathrm{ECCO}_{2} \mathrm{Cl}\right)$ could reduce the respiratory rate (RR), so prolonging time for lung emptying and allowing resolution of hyperinflation.

Methods Six patients with COPD exacerbation with respiratory acidosis $\left(\mathrm{PaCO}_{2} 83 \pm 27 \mathrm{mmHg}, \mathrm{pH} 7.19 \pm 0.1\right)$ and tachypnea (RR $39 \pm 5$ ) despite maximal non-invasive ventilation underwent venovenous extracorporeal membrane oxygenation (VV-ECMO). All patients were awake and spontaneously breathing an adequate air-oxygen mixture to correct hypoxemia $\left(\mathrm{PaO}_{2} 72 \pm 27 \mathrm{mmHg}\right.$ ). While keeping the blood flow stable (2.9 $\pm 0.5 \mathrm{l} / \mathrm{minute})$, we changed the gas flow of the artificial lung to modify the extracorporeal $\mathrm{CO}_{2}$ clearance as a percentage of total patient $\mathrm{CO}_{2}$ production ( $\% \mathrm{ECCO}_{2} \mathrm{Cl}_{\text {/total }} \mathrm{VCO}_{2}$ ) and we observed the variations of RR. We recorded RR at three levels of gas flow in each patient (Figure 1).

Results In all patients RR decreased with the increase of extracorporeal $\mathrm{CO}_{2}$ removal and a negative correlation was found between $\mathrm{RR}$ and $\mathrm{ECCO}_{2} \mathrm{Cl} /$ total $\mathrm{VCO}_{2}\left(r^{2}=0.42, P<0.01\right)$. In all patients we were able to obtain a reduction of RR below 15 ( $28 \pm 4$ vs. $8 \pm 4$, RR at low gas flow vs. $R R$ at maximal gas flow, $P<0.001)$. The selected maximal gas flow was variable between different patients ( $6.7 \pm 2 \mathrm{l} /$ minute), corresponding to different levels of $\mathrm{ECCO}_{2} \mathrm{Cl} /$ total $\mathrm{VCO}_{2}(83 \pm 17 \%$, range 53 to $100 \%)$ and RR response ( $8 \pm 4$, range 5 to 14$)$.

Conclusion In patients with COPD exacerbation, who failed noninvasive ventilation, VV-ECMO allows one to maintain spontaneous breathing. Titration of extracorporeal $\mathrm{CO}_{2}$ removal leads to control RR. This approach could interrupt the vicious circle of dynamic hyperinflation and allow the deflation of lung parenchyma.

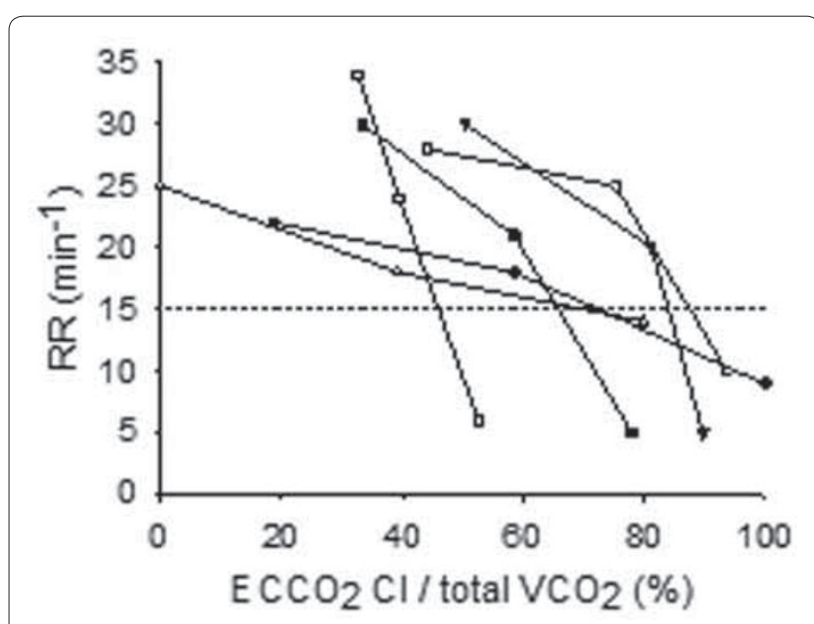

Figure 1 (abstract P128). 
P129

$\mathrm{ECCO}_{2}$ removal with a phosphorylcholine-coated membrane oxygenator in difficult respiratory weaning patients

FTurani, S Martini, A Marinelli, M Falco, R Barchetta, F Candidi, F Gargano

'Aurelia Hospital/European Hospital, Rome, Italy

Critical Care 2013, 17(Suppl 2):P129 (doi: 10.1186/cc12067)

Introduction $\mathrm{ECCO}_{2}$ removal may be a useful support in patients with difficult respiratory weaning. The aim of this study is to evaluate a new phosphorylcholine-coated EECO removal system with no thrombogenic activity to assess the clinical safety of the system, the changes of main cardiorespiratory indices and $\mathrm{CO}_{2}$ removal by the system.

Methods Ten patients were enrolled. Before starting with $\mathrm{ECCO}_{2}$ removal all patients were ventilated with TV $<6 \mathrm{ml} / \mathrm{kg}$, peak pressure $>35 \mathrm{cmH}_{2} \mathrm{O}$ and $\mathrm{pH}<7.25 . \mathrm{ECCO}_{2}$ removal was initiated using a modified continuous venovenous hemofiltration system with a membrane oxygenator (ABYLCAP; Bellco, Mirandola, Italy; membrane surface area: $0.67 \mathrm{~m}^{2}$, blood flow 280 to $350 \mathrm{ml} /$ minute, phosphorylcholine coated). Femoral vein cannulation with a double-lumen catheter was used to connect the patients to the extracorporeal system. Heparin was infused to maintain ACT $<190$ to 240 seconds. All patients had $\mathrm{ECCO}_{2}$ for 4 days. Every 12 hours the $\mathrm{pH}, \mathrm{PaCO}_{2}$, peak pressure and $\mathrm{PaO}_{2}$ were evaluated. $\mathrm{VCO}_{2}$ was determined by indirect calorimetry using a gas analyser equipped on an Engstrom Carestation Ventilator. During the $\mathrm{ECCO}_{2}$ removal the patients were ventilated with $\mathrm{TV} \leq 6 \mathrm{ml} / \mathrm{kg}$ and peak pressure $<30 \mathrm{cmH}_{2} \mathrm{O}$. All data are expressed as mean $\pm \mathrm{SD}$. One-way ANOVA was used to compare the changes of parameters. $P<0.05$ was considered significant.

Results Table 1 presents the main results. The $\mathrm{CO}_{2}$ removal by membrane oxygenator ranged from 56 to $37 \mathrm{ml} /$ minute. All patients survived to the treatment and $7 / 10$ were weaned from the ventilator at the end of $\mathrm{ECCO}_{2}$ removal. Only one oxygenator was used for every patient without clotting of the circuit or any major bleeding problem.

Table 1 (abstract P129)

\begin{tabular}{lccc}
\hline & Day 0 & Day 2 & Day 4 \\
\hline $\mathrm{pH}$ & $7.24 \pm 0.06$ & $7.38 \pm 0.1$ & $7.41 \pm 0.07^{*}$ \\
$\mathrm{PaCO}_{2}(\mathrm{mmHg})$ & $70 \pm 5$ & $57 \pm 8$ & $52 \pm 3^{*}$ \\
$\mathrm{PP}\left(\mathrm{CmH}_{2} \mathrm{O}\right)$ & $48 \pm 4$ & $28 \pm 4^{*}$ & $25 \pm 4$ \\
$\mathrm{VCO}_{2}(\mathrm{ml} /$ minute $)$ & $220 \pm 15$ & $201 \pm 14$ & $180 \pm 13^{*}$ \\
\hline
\end{tabular}

* $P<0.05$ versus $\mathrm{T} 0$.

Conclusion $\mathrm{ECCO}_{2}$ removal with a phosphorylcholine-coated membrane oxygenator is clinically safe, avoids clotting of the oxygenator circuit and allows adequate $\mathrm{CO}_{2}$ removal.

Reference

1. Livigni S, et al:: Crit Care 2006, 10:R151.

\section{P130}

Lung elastance can be determined without esophageal pressure measurements

C Grivans, S Lundin, O Stenqvist

Sahlgrenska University Hospital, Gothenburg, Sweden

Critical Care 2013, 17(Suppl 2):P130 (doi: 10.1186/cc12068)

Introduction We have previously shown, in an ex vivo porcine model, that lung elastance calculated as the PEEP change divided by lung volume increase ( $\triangle \mathrm{PEEP} / \triangle \mathrm{EELV})$ is closely correlated to conventionally measured lung elastance using oesophageal pressure [1]. In this study we hypothesize that the successive change in lung volume during a PEEP-step manoeuvre could be predicted from $\triangle P E E P$ and lung elastance as $\triangle P E E P / E L$. The objective of the study was to validate this hypothesis in patients with acute respiratory failure (ARF).

Methods Thirteen patients with ARF were studied during an incremental PEEP trial, 0-4-8-12-16 $\mathrm{cmH}_{2} \mathrm{O}$. $\triangle \mathrm{EELV}$ was determined as the change in expiratory tidal volume following each PEEP step. Conventional calculation of lung elastance was obtained from tidal variation in airway

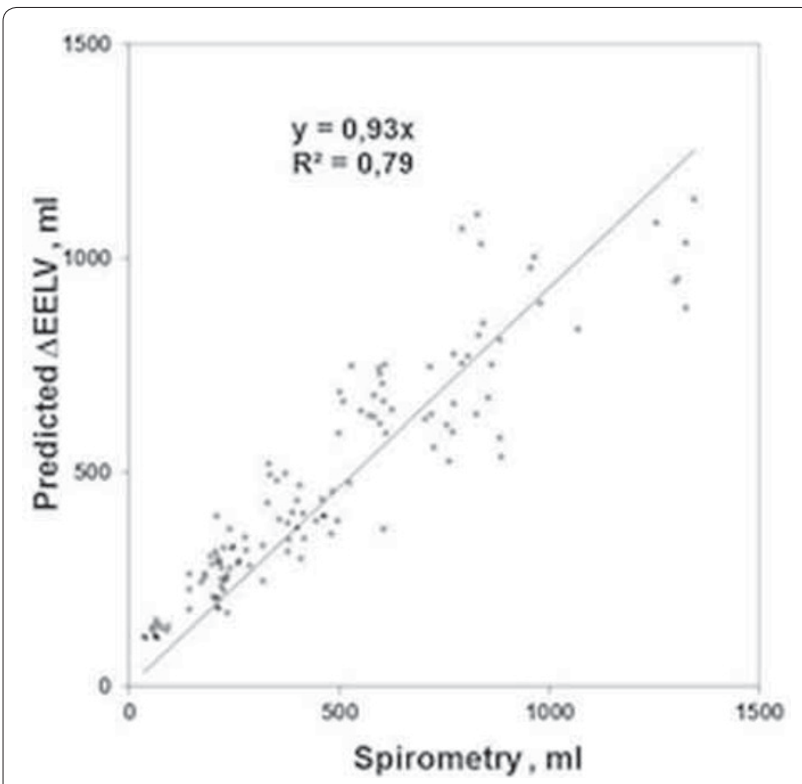

Figure 1 (abstract P130). Correlation between spirometrically measured and calculated increase in EELV.

pressure minus tidal variation in oesophageal pressure divided by tidal volume. Position of the oesophageal catheter was verified according to Baydur [2]. The measured change in end-expiratory lung volume during the PEEP-step manoeuvre using spirometry was compared with the end-expiratory lung volume change calculated from EL and stepwise changes in PEEP as $\triangle \mathrm{PEEP} / \mathrm{EL}$.

Results There was a close correlation between the measured build-up of end-expiratory lung volume during a PEEP-step manoeuvre and $\triangle P E E P / E L$ where EL was conventionally determined using oesophageal pressure measurements (see Figure 1).

Conclusion Esophageal pressure measurements are difficult to perform [3] and rarely used in routine clinical practice. Our findings indicate that a change in PEEP together with measurements of the resulting change in end-expiratory volume by spirometry in the ventilator could be used to determine lung elastance separately, the relation between lung and chest wall elastance as well as the transpulmonary pressure.

References

1. Stenqvist O, et al: Acta Anaesthesiol Scand 2012, 56:738-747.

2. Baydur A: Chest 2002, 121:324-326

3. Hedenstierna G: Minerva Anestesiol 2012, 78:959-966

P131

Feasibility of minimally invasive stimulation of the phrenic nerves for supporting ventilation in fully anesthetized swine

J Bijwadia', M Karamanoglu²

'Regions Hospital, St Paul, MN, USA; ${ }^{2}$ Respithera, Bloomington, MN, USA

Critical Care 2013, 17(Suppl 2):P131 (doi: 10.1186/cc12069)

Introduction Long-term use of mechanical ventilators may lead to ventilator-induced diaphragmatic dysfunction (VIDD) and increase the duration of weaning from MV [1]. It was hypothesized that stimulating the diaphragm during MV may prevent VIDD and may lead to early weaning [2]. In this study, the feasibility of generating coordinated contraction of both diaphragms was investigated using a novel transvenous diaphragmatic pacing system.

Methods Two juvenile pigs were anesthetized with propofol (150 to $250 \mu \mathrm{g} / \mathrm{kg} / \mathrm{minute}$ ) and ventilated (VENT) with an assist control mode MV (Nellcor Puritan Bennett 840). Using fluoroscopy, a novel multipolar neurostimulation catheter (Inspirx RL PICC53; Respithera, Bloomington, MN, USA) was threaded into the left internal jugular vein and advanced to the junction of right atrium and the superior vena cava using a 
modified Seldinger technique. The successful capture of the right and left phrenic nerves was confirmed by fluoroscopic visualization. Peak airway pressures (PAWP) and blood gases were determined after 10 minutes MV (MV), MV and stimulation applied together (MV+STIM) and stimulation only (STIM).

Results No animal-ventilator dyssynchrony during stimulation (MV+STIM) was noted while peak airway pressures were reduced. During STIM there was no discernible paradoxical movement of the diaphragm. In addition, $\mathrm{PCO}_{2}$ and $\mathrm{PO}_{2}$ confirmed that adequate ventilation and oxygenation can be provided by the system, while PAWP could be reduced (Table 1).

Table 1 (abstract P131). Ventilation parameters

\begin{tabular}{lccc}
\hline & MV & MV+STIM & STIM \\
\hline $\operatorname{PAWP}\left(\mathrm{CmH}_{2} \mathrm{O}\right)$ & 20 & 16 & 9 \\
$\mathrm{PO}_{2}(\mathrm{mmHg})$ & 89 & 101 & 95 \\
$\mathrm{PCO}_{2}(\mathrm{mmHg})$ & 32 & 26 & 26 \\
\hline
\end{tabular}

Conclusion It was possible to capture and stimulate both phrenic nerves using a minimally invasive approach to support respiration and sustain blood gases at physiological levels. This development could help wean MV-dependent ICU patients earlier. Further long-term studies are needed to assess the full potential of this novel system.

\section{References}

1. Vassilakopoulos T, Petrof BJ: Am J Respir Crit Care Med 2004, 169:336-341.

2. Sassoon CS, Zhu E, Caiozzo VJ: Am J Respir Crit Care Med 2004, 170:626-632.

\section{P132}

Prehospital management of COPD patients in respiratory failure and short-term outcome

G Campagne, J Cuny, P Gosselin, P Goldstein, N Assez, E Wiel

Lille University Hospital Center, Lille Cedex, France

Critical Care 2013, 17(Suppl 2):P132 (doi: 10.1186/cc12070)

Introduction Respiratory failure in COPD patients is a frequent cal in French emergency dispatching centers. We have evaluated the prehospital management of COPD patients and severity signs, and analyse outcome in the emergency department or ICU.

Methods We conducted an observational, descriptive, retrospective, single-center study during a 4-month period. All COPD patients with respiratory failure and prehospital care were included. Different data were recorded.

Results Ninety patients were included ( $77 \%$ male, $23 \%$ female). Mean age 69 years $( \pm 11.88)$. Fifty-five percent were smokers, $52 \%$ had arterial hypertension, 39\% received long-term oxygenotherapy, 18\% received antibiotics in the 7 days before, $18 \%$ corticosteroids, and $14 \%$ were on long-term NIV support at home. An emergency medical ambulance was immediately sent for $86 \%$ of patients. Ninety-two percent had normal consciousness (Glasgow Coma Scale 15), 78\% had bronchospasm, $71 \%$ had signs of respiratory struggle, and $12 \%$ were unable to speak. The mean respiratory rate was 31.4 cycles/ minute $( \pm 8.18)$, the average cardiac pulse was 103.6 beats/minute $( \pm 23.14)$. Nasal EtCO $44.92 \mathrm{mmHg}( \pm 16.38)$, pulse oximetry with air was $83.48 \%( \pm 12.09)$, and the average flow rate of oxygen delivered was $5.69 \mathrm{l} /$ minute $( \pm 2.93)$. None of the patients had fever. Eighty-five percent were supported on spontaneous ventilation, $22 \%$ received prehospital non-invasive ventilation, they all showed signs of severity and $3 \%$ need tracheal intubation. Seventy-five percent of patients received $\beta_{2}$-agonist and anticholinergic nebulization, $45 \%$ intravenous corticosteroids. Seventy-one percent were admitted to the emergency room, $29 \%$ to the ICU.

Conclusion Most of the patients had signs of severity and bronchospasm. The absence of fever and antibiotics allows us to think that the cause of decompensation is not pneumonia. Although most of them were hypoxic and hypercapnic, they seem to be good candidates for NIV support in prehospital care. Very few studies report the use of NIV in cases of COPD respiratory failure in the first care delivered at home.

\section{References}

1. Antoine G: The epidemiology and outcome of medical emergency team call patients treated with non invasive ventilation. Resuscitation 2011, 82:1218-1223.

2. Schmidbauer W: Early prehospital use of non invasive ventilation improves acute respiratory failure in acute exacerbation of chronic obstructive pulmonary disease. Emerg Med J 2011, 28:626-627.

P133

$\mathrm{N}$-terminal pro-brain natriuretic peptide as a prognostic marker in patients with exacerbation of chronic obstructive pulmonary disease

P Stamatis, H Michalopoulou, D Stamatis

Metaxa Hospital, Athens, Greece

Critical Care 2013, 17(Suppl 2):P133 (doi: 10.1186/cc12071)

Introduction Plasma N-terminal pro-brain natriuretic peptide (NT-proBNP) levels are elevated in patients with pulmonary disease especially in those with concomitant right ventricular dysfunction. The aim of the present study was to investigate the use of plasma NT-pro-BNP levels as a prognostic tool in patients with exacerbation of chronic obstructive pulmonary disease (COPD).

Methods A retrospective medical records analysis of all patients hospitalized between June 2009 and June 2012 with the final diagnosis of acute exacerbation of COPD who had undergone NT-pro-BNP measurements at admission followed by echocardiogram.

Results Seventy-two patients (mean age 69 years, 58\% male) with COPD exacerbation but without clinical or echocardiography-oriented evidence of acute cardiac disease were enrolled. The mean forced expiratory volume in 1 second (FEV 1)/forced vital capacity ratio was $44.2 \%$ and the mean FEV 1 was 0.82 I. Median NT-pro-BNP levels at admission were $424.4 \mathrm{pg} / \mathrm{ml}$ and the tertile limits were 262.1 and $930 \mathrm{pg} / \mathrm{ml}$. NT-pro-BNP levels significantly predicted 30-day mortality (OR: $7.2,95 \% \mathrm{Cl}: 3.9$ to $15.2 ; P<0.001$ ), (OR: $25,95 \% \mathrm{Cl}: 16.3$ to 37.3 ; $P<0.001)$ and (OR: $44,95 \% \mathrm{Cl}: 44.5$ to 76.2$)$ for each tertile. These associations persisted after adjusting for arterial $\mathrm{CO}_{2}$ pressure, body mass index, age, gender and systolic pulmonary artery pressure.

Conclusion Among patients with acute exacerbation of chronic pulmonary disease, NT-pro-BNP could be a useful marker for severity and poor prognosis.

\section{P134}

Novel biomarkers for prediction of mortality after acute exacerbation of chronic obstructive pulmonary disease

H Michalopoulou, H Michalopoulou, P Stamatis, F Kattis, D Stamatis

Metaxa Hospital, Athens, Greece

Critical Care 2013, 17(Suppl 2):P134 (doi: 10.1186/cc12072)

Introduction Retrospective studies suggest that cardiac troponin levels are often elevated in patients with acute exacerbation of chronic obstructive pulmonary disease (AECOPD) indicating a poor survival. Novel high-sensitivity cardiac troponin (hs-cTnT) assays have better analytical precision than standard troponin (cTnT) assays. We elaborated a prospective cohort study to investigate the prognostic value of this novel biomarker in patients with AECOPD.

Methods Fifty-six patients (mean age 64 years, 68\% male) with the final diagnosis of AECOPD were enrolled. Those who were diagnosed with acute coronary syndromes were excluded. We measured cardiac troponin $\mathrm{T}$ with a standard fourth-generation assay and a highsensitivity assay. Clinical, electrocardiographic and echocardiographic data were collected at admission and the primary prognostic endpoint was death during 30 days of follow-up.

Results Mean hs-cTnT levels at admission were $34 \mathrm{ng} / \mathrm{l}$. During the follow-up period seven patients (12\%) died. Thirty-eight percent of patients had hs-cTnT above the range of $14 \mathrm{ng} / \mathrm{l}$. Prognostic accuracy of hs-cTnT for death was significantly higher, with area under the ROC curve (AUC) of $0.83(95 \% \mathrm{Cl}: 0.72$ to 0.86$)$, than that of CTnT (AUC: $0.63,95 \% \mathrm{Cl}: 0.56$ to $0.74 ; P<0.001)$. After adjustment for age, gender, creatinine levels, heart rate, left ventricular ejection fraction, arterial $\mathrm{O}_{2}$ pressure and systolic pulmonary artery pressure, hs-cTnT above the 
99th percentile was associated with a hazard ratio for death of 3.2 (95\% Cl: 1.5 to 6.7$)$.

Conclusion In AECOPD, novel biomarkers such as hs-CTnT appear to be positively associated with COPD severity and could be a determinant of mortality.

\section{P135}

High incidence of respiratory viruses in critically ill adult patients with respiratory failure

M Sietses' ${ }^{1}$, TE Faber ${ }^{1}$, L Bont $^{2}$, H Buter ${ }^{1}$, EC Boerma ${ }^{1}$

${ }^{1}$ Medical Center Leeuwarden, the Netherlands; ${ }^{2}$ University Medical Center

Utrecht, the Netherlands

Critical Care 2013, 17(Suppl 2):P135 (doi: 10.1186/cc12073)

Introduction Laboratory testing for viral infections is not routinely performed in adult patients admitted to the ICU. However, reports indicate that viruses may contribute to significant morbidity and mortality and that viral-bacterial co-infection is associated with poor outcome in this particular patient population. The aim of the study was to document the incidence of respiratory virus infections in critically ill adult patients admitted to the ICU for acute respiratory failure.

Methods The prospective, observational study took place during two consecutive winter seasons in a mixed 16-bed ICU at the Medical Center Leeuwarden, the Netherlands. Adult patients admitted to the ICU, suspected of respiratory failure due to community-acquired pneumonia, were included. After informed consent brushed nasopharyngeal swab (Copan $\left.{ }^{\oplus}\right)$ samples were taken within 24 hours after admission and analyzed by PCR assays for the presence of respiratory syncytial virus (RSV), influenza virus, metapneumovirus (MPV), parainfluenza virus, adenovirus, rhinovirus and coronavirus. Data are presented as median (IQR).

Results Thirty-four patients were enrolled. Median age was 66 (54 to 71) years; $65 \%$ of the patients were male; median APACHE IV score on admission was 76 (61 to 96 ). Median ICU stay was 8 ( 5 to 17 ) days, with an overall hospital mortality of $12 \%$. Thirteen patients (38\%) tested positive for a respiratory virus. The most frequently found virus was influenza (37\%), followed by RSV (15\%), rhinovirus (15\%), MPV (15\%), corona virus (9\%) and parainfluenza virus (9\%). Two of the patients $(6 \%)$ had a bacterial-viral co-infection (blood culture and PCR positive for Streptococcus pneumonia and rhinovirus in one patient and for Haemophilus influenza and influenza virus in the second patient). Length of stay in the ICU was significantly longer in PCR-negative patients, in comparison with PCR-positive patients (11 (6 to 20) vs. 7 (2 to 8 ) days, respectively, $P<0.05$ ).

Conclusion Respiratory viruses, and particularly influenza virus, are frequently found in adult patients with respiratory failure admitted to the ICU.

References

1. Carrat F, et al: Intensive Care Med 2006, 2:156-159.

2. Choi SH, et al:: Am J Respir Crit Care Med 2012, 186:325-332

3. Miggins M, et al:: PLoS One 2011, 6:18890.

\section{P136}

Role of bronchoscopy during non-invasive ventilation in

hypercapnic respiratory failure

W Rady, A Abouelela, A Aly, W Youssef

Alexandria University, Alexandria, Egypt

Critical Care 2013, 17(Suppl 2):P136 (doi: 10.1186/cc12074)

Introduction Non-invasive positive-pressure ventilation (NIPPV) is the first-line treatment for hypercapnic acute respiratory failure (ARF) secondary to COPD exacerbation in selected patients. Limited data exist supporting the use of fiberoptic bronchoscopy (FOB) during this clinical setting. The aim of this study is to assess the role of FOB during NIPPV in patients with decompensated COPD acute exacerbation.

Methods This study is a randomized prospective case-control pilot study carried out on 50 patients admitted to critical care units at Alexandria University Hospital, Egypt suffering from hypercapnic ARF secondary to COPD exacerbation with Kelly Matthay Score from 2 to 4. All patients received NIPPV. Patients were divided randomly into two equal groups: group I (cases, 25 patients) were subjected to additional intervention, early FOB during the first 6 to 12 hours from admission; while group II (control, 25 patients) received the conventional treatment and NIPPV only. Outcome parameters measured were changes in ABG data, duration of NIPPV, rate of its success, ICU stay and mortality as well as the safety of $\mathrm{FOB}$ and possible complications.

Results No significant difference was detected between the two groups regarding the baseline characteristics. No serious complications happened from FOB, oxygen desaturation occurred in 4/25 patients $(16 \%)$, tachycardia in $2 / 25$ patients (8\%). In group I, 23 patients (92\%) were successfully weaned from NIPPV versus 16 patients (64\%) in group II $(P=0.037)$. Total duration of NIPPV was 28.52 hours in group I versus 56.25 hours in group II $(P=0.001)$. Length of ICU stay was 4.84 days in group I versus 8.68 days in group II $(P=0.001)$. Only one patient died in group I versus three patients in group II $(P=0.609)$.

Conclusion The early application of FOB during NIPPV in patients with ARF due to COPD exacerbation was shown to be safe. Significant improvement in the outcome of patients who underwent FOB was noticed in terms of improved ABG data, shorter duration of NIPPV, higher percentage of success and shorter ICU stay while no significant difference was detected in mortality.

References

1. Scala R, et al:: Crit Care 2010, 14:R80.

2. Ambrosino N, et al:: Eur Respir J 2008, 31:874-886

3. Heunks LM, et al:: Intensive Care Med 2010, 36:143-147.

\section{P137}

SMART-COP score for patients admitted with community-acquired pneumonia (CAP) to an ICU in a district general hospital: a smarter way of identifying patients with severe CAP?

M Pachucki', F Kelly², A Padkin²

'Frenchay Hospital, Bristol, UK; 'RUH, Bath, UK

Critical Care 2013, 17(Suppl 2):P137 (doi: 10.1186/cc12075)

Introduction British Thoracic Society guidelines on communityacquired pneumonia (CAP) advocate ICU referral for patients with CURB65 score of 4 and 5. A recently developed scoring system, SMARTCOP, designed to identify patients at need of intensive respiratory or vasopressor support (IRVS), has been validated in a variety of settings. It predicts the need for ICU admission (defined as need for IRVS) with greater accuracy than CURB65, but is not used routinely in our UK institution.

Methods We retrospectively analysed critical care admissions of patients with a diagnosis of CAP in a UK district general hospital ICNARC-coded diagnoses of pneumonia (bacterial, viral, no organisms isolated) over a 7-month period (August 2011 to January 2012). We ascertained the CURB65 and SMART-COP scores on referral to the ICU and matched them in relation to the need for IRVS, length of inotropic and ventilatory support and ICU length of stay.

Results Our search revealed 28 potential matches. Five patients were excluded (not CAP) and the notes for seven patients were not available for analysis. We analysed the notes of 16 patients matching our criteria. In this small sample, there was a strong association between increasing SMART-COP score and the need for IRVS (correlation coefficient $r=$ $0.96)$. There was also a strong correlation with longer inotropic support $(r=0.85)$ and longer ventilatory support $(r=0.96)$ with increasing SMART-COP scores but a weaker correlation with length of ICU stay $(r=$ 0.49). Moreover, none of the patients admitted to the ICU had CURB65 score higher than 3 at the time of ICU referral.

Conclusion In our small sample, higher SMART-COP score was associated with increased likelihood of IRVS. This suggests that a further study with a larger sample size should be performed to investigate whether SMART-COP is an improvement on CURB65 in predicting the need for IRVS in UK intensive care patients.

References

1. Lim WS, et al: BTS guidelines for the management of community acquired pneumonia in adults: update 2009. Thorax 2009, 64(Suppl 3):iii1-iii55. doi:10.1136/thx.2009.121434

2. Charles PG, et al: SMART-COP: a tool for predicting the need for intensive respiratory or vasopressor support in community-acquired pneumonia. $J$ Clin Infect Dis 2008, 47:375-384. 
3. Robins-Browne K, et al: The SMART-COP score performs well for pneumonia risk stratification in Australia's Tropical Northern Territory: a prospective cohort study. Trop Med Int Health 2012, 17:914-919.

4. Fang et al:: Application and comparison of scoring indices to predict outcomes in patients with healthcare associated pneumonia. Crit Care 2011, 15:R32.

P138

Effects of steroid therapies to severe streptococcal pneumoniae that required mechanical ventilation

T Nagura, T Ikeda, K Ikeda, T Ueno, S Suda

Tokyo Medical University, Hachioji Medical Center, Tokyo, Japan

Critical Care 2013, 17(Suppl 2):P138 (doi: 10.1186/cc12076)

Introduction Streptococcal pneumonia remains the most common cause of community-acquired pneumonia (CAP), bacterial meningitis and bacteremia. Severe pneumonia caused by streptococcal pneumonia frequently exists in the emergency room or ICU. We performed this study to evaluate the effect of steroid therapy for severe streptococcal pneumonia patients with mechanical ventilation retrospectively. Methods We enrolled 13 adults of streptococcal pneumonia patients who required mechanical ventilation. Seven of 13 patients (S group) were administered with steroid (hydrocortisone 200 to $300 \mathrm{mg} /$ day), and the remaining six patients received no steroid therapy (NS group). As the conventional therapies, mechanical ventilation was commenced when a patient's $\mathrm{PaO}_{2} / \mathrm{FiO}_{2}$ showed less than 200 or they clinically complained of being short of breath. All patients received appropriate fluid therapies, vasoactive agents and blood transfusion according to the protocol of early goal-directed therapy in the Surviving Sepsis Campaign Guidelines 2008, and also were treated with antibiotics, immunoglobulins ( $5 \mathrm{~g} /$ day for 3 days) and sivelestat sodium hydrate $(4.8 \mathrm{mg} / \mathrm{kg} /$ day for 7 days).

Results The APACHE scores in the S group and NS group were $27 \pm 10$ and $23 \pm 4$, Sequential Organ Failure Assessment scores were $8 \pm 4$ and $7 \pm 3$, respectively. These scores showed no significant difference between the groups. Procalcitonin (PCT) in the $S$ and NS groups was $20.7 \pm 21.7$ and $45.0 \pm 47.7 \mathrm{ng} / \mathrm{ml}$, respectively, and there was no significant difference between the groups. PCT declined significantly in both groups. $\mathrm{PaO}_{2} / \mathrm{FiO}_{2}$ of the NS group was significantly higher than the $S$ group on ICU admission and 4 days after admission, but no significant difference on 7 days after ICU admission. IL-6 of the NS group declined significantly after ICU admission, and the $S$ group also tended to decline.

Conclusion Steroid therapy for severe streptococcal pneumonia patients with mechanical ventilation may have a potential to maintain oxygenation of the lung, but no significant effects on changes of inflammatory markers (IL-6, CRP).

\section{P139}

Risk factors for ventilator-associated pneumonia in a surgical ICU

A Kundakci, O Ozkalaycı, P Zeyneloglu, H Arslan, A Pirat

Baskent University Hospital, Ankara, Turkey

Critical Care 2013, 17(Suppl 2):P139 (doi: 10.1186/cc12077)

Introduction Ventilator-associated pneumonia (VAP) is the most common nosocomial infection in ICU patients who require mechanical ventilation support. The aim of this study was to determine predictors for the development of VAP in surgical ICU patients admitted to Baskent University Hospital.

Methods Following Institutional Review Board approval we performed this retrospective study, including 876 patients admitted to the surgical ICU between January 2009 and July 2012. After completing a review of patient data, 45 patients diagnosed with VAP were compared with 26 appropriate matches who were not. Patients' demographical features (age, sex, body weight), underlying diseases, etiology for ICU admission, APACHE II and Sequential Organ Failure Assessment scores, duration of hospitalization, organ dysfunctions, fluid balances, laboratory values, use of vasopressors, mechanical ventilation, nutrition, antibiotics, transfusions, features related to central venous catheterization, urinary catheterization, and intubation were the recorded parameters. Patients who were not intubated and were discharged or died within 2 days of ICU admission were excluded.

Results Out of 71 patients who were included in the final analysis, 45 patients (63\%) had VAP. Comparing with the control group, patients who developed VAP were more likely to have diabetes mellitus and immunosuppression ( $P=0.020$ and $P=0.014$, respectively). These patients also had higher APACHE II scores $(P=0.020)$ and a longer duration of mechanical ventilation $(P<0.001)$, and more frequently had an open wound $(P=0.001)$. Following regression analysis, presence of diabetes mellitus (OR: 12.048; $95 \% \mathrm{Cl}: 1.157$ to $125.000 ; P=0.037$ ), immunosuppression (OR: $16.949,95 \% \mathrm{Cl}: 2.463$ to $111.111 ; P=0.004$ ), and open wound (OR: $5.714 ; 95 \% \mathrm{Cl}: 1.017$ to $37.258 ; P=0.048$ ), higher APACHE II scores (OR: $1.132 ; 95 \% \mathrm{Cl}: 1.022$ to $1.254 ; P=0.018$ ), and prolonged duration of mechanical ventilation (OR: 1.084; $95 \% \mathrm{Cl}: 1.002$ to $1.171 ; P=0.043$ ) were determined as risk factors for VAP. Fourteenday and 28 -day mortality rates for VAP were $19 \%$ and $29 \%$, respectively $(P=0.760$ and $P=1.000$, respectively).

Conclusion The presence of diabetes mellitus and immunosuppression, higher APACHE II scores, longer mechanical ventilation duration, and presence of open wounds were predictors of VAP.

Reference

1. Guidelines for the management of adults with hospital-acquired ventilator-associated, and healthcare-associated pneumonia. Am J Respir Crit Care Med 2005, 171:388-416.

P140

Ventilation distribution measured with electrical impedance tomography at varying levels of assist during pressure support versus neurally adjusted ventilatory assist in ICU patients

MS Van Mourik', P Blankman', D Hasan², D Gommers

'Erasmus Medical Center, Rotterdam, the Netherlands; 'Maasstad, Rotterdam, the Netherlands

Critical Care 2013, 17(Suppl 2):P140 (doi: 10.1186/cc12078)

Introduction Electrical impedance tomography (EIT) is a non-invasive and nonradiating imaging technique, which can be used to visualize ventilation distribution of the lungs and could distinguish between the dependent (dorsal) and nondependent (ventral) parts.

Methods The aim of this study was to observe ventilation distribution between dependent and nondependent lung regions, for the individual patient, during three different levels of support during pressure support (PS) and neurally adjusted ventilatory assist (NAVA) ventilation. Ten mechanically ventilated patients in the ICU were included.

Results The ratio for dependent/nondependent distribution of ventilation is significantly higher at lower support levels compared with higher support levels in both PS and NAVA. However, during NAVA there was significantly less impedance loss between the different levels of assist compared with PS. Tidal volumes decreased when decreasing assist levels during PS whereas not during NAVA ventilation. The electrical activity of the diaphragm decreased in both PS and NAVA with higher levels of assist. Three patients showed an increase in dependent tidal impedance variation (TIV) after lowering the assist level from 15 to $10 \mathrm{cmH}_{2} \mathrm{O}$. This increase in TIV did not occur during NAVA ventilation. Conclusion There is more ventilation in the dependent part of the lung, compared with the nondependent part, at lower levels of assist. This could indicate that at higher support levels the contribution of the diaphragm is reduced. During NAVA ventilation, there is an autoregulation in which the patient is adjusting his tidal ventilation to maintain homogeneous ventilation distribution.

\section{P141}

Analysis of bilevel positive airway pressure therapy in children based on weight

A Williams, T Abramo

Vanderbilt University Medical Center, Nashville, TN, USA

Critical Care 2013, 17(Suppl 2):P141 (doi: 10.1186/cc12079)

Introduction Obesity as a comorbidity adds a challenge when treating children presenting to the PED in status asthmaticus. Bilevel positive airway pressure (BiPAP) is an accepted treatment modality for children 
Table 1 (abstract P141). Time on BiPAP by weight percentile and severity of asthma exacerbation (hours)

\begin{tabular}{|c|c|c|c|c|c|c|c|c|c|}
\hline & \multicolumn{3}{|c|}{ Moderate exacerbation } & \multicolumn{3}{|c|}{ Severe exacerbation } & \multicolumn{3}{|c|}{ Combined } \\
\hline & $\begin{array}{c}<90 \\
\text { percentile } \\
(n=113)\end{array}$ & $\begin{array}{c}90 \text { to } 97 \\
\text { percentile } \\
(n=35)\end{array}$ & $\begin{array}{c}>97 \\
\text { percentile } \\
(n=36)\end{array}$ & $\begin{array}{c}<90 \\
\text { percentile } \\
(n=109)\end{array}$ & $\begin{array}{c}90 \text { to } 97 \\
\text { percentile } \\
(n=35)\end{array}$ & $\begin{array}{c}>97 \\
\text { percentile } \\
(n=31)\end{array}$ & $\begin{array}{c}<90 \\
\text { percentile } \\
(n=222)\end{array}$ & $\begin{array}{c}90 \text { to } 97 \\
\text { percentile } \\
(n=70)\end{array}$ & $\begin{array}{c}>97 \\
\text { percentile } \\
(n=67)\end{array}$ \\
\hline Mean & 3.53 & 4.03 & 5.01 & 5.67 & 5.56 & 5.88 & 4.58 & 4.85 & 5.41 \\
\hline SD & 3.35 & 3.24 & 4.26 & 4.78 & 4.03 & 7.25 & 4.25 & 3.75 & 5.81 \\
\hline
\end{tabular}

Table 2 (abstract P141). Initial mean BiPAP settings by age and weight percentile

\begin{tabular}{|c|c|c|c|c|c|c|}
\hline \multirow{2}{*}{$\begin{array}{l}\text { Age } \\
\text { (years) }\end{array}$} & \multicolumn{2}{|c|}{$<90$ percentile } & \multicolumn{2}{|c|}{90 to 97 percentile } & \multicolumn{2}{|c|}{ >97 percentile } \\
\hline & IPAP & EPAP & & & IPAP & $\mathrm{P}$ \\
\hline to 4 & & & & & & \\
\hline to & 13.4 & $69+$ & 12.4 & & $14.2=$ & \\
\hline 7 to 9 & $14.2 \pm 2.2$ & & $14.9 \pm 2.7$ & & $14.1 \pm 2.4$ & $7.1 \pm 1.3$ \\
\hline 0 to 12 & $14.1 \pm 2.7$ & $7.3 \pm 1.7$ & $15.4 \pm 4.1$ & $7.9 \pm 2.0$ & $15.1 \pm 3.1$ & $8.0 \pm 1.6$ \\
\hline 13 to 16 & $15.2 \pm 4.2$ & $8.6 \pm 1.8$ & $14.0 \pm 1.8$ & $7.7 \pm 1.5$ & $16.5 \pm 3.9$ & $8.8 \pm 1.8$ \\
\hline
\end{tabular}

in status asthmaticus. Our purpose was to analyze BiPAP use and outcomes for children with status asthmaticus and obesity in our PED. Methods Patients placed on BiPAP in the PED for status asthmaticus from 1 January 2010 to 31 August 2012 were included in the analysis. Subjects were divided into moderate and severe exacerbations and then further subdivided into the following growth curve-based weight subgroups: $<90$ percentile, 90 to 97 percentile and >97 percentile. Subjects received standard asthma therapies in addition to BiPAP. Data were obtained at the bedside by the respiratory therapist or collected retrospectively by study investigators. Data were stored and analyzed using a RedCap database.

Results Three hundred and fifty-nine subjects were analyzed. Table 1 shows the time on BiPAP per visit. Children whose weight was $>97$ percentile revealed trends towards longer treatment times on BiPAP compared with the other two groups. The moderate subjects who weighed $>97$ percentile had statistically significant longer treatment periods $(P<0.006)$ when compared with the $<90$ percentile moderate group. Initial BiPAP settings are listed in Table 2 . When controlling for age, higher BiPAP settings correlated with increasing weight. There were no weight-based trends for admissions to the PICU or overall hospital lengths of stay. No serious complications were noted.

Conclusion This is the first study to analyze the weight effect on BiPAP application in children with status asthmaticus. Subjects who weighed more trended greater mean time on BiPAP and initial BiPAP settings. Weight did affect PICU admissions or overall length of hospital stay.

P142

Determinants of non-invasive ventilation success or failure in morbidly obese patients in acute respiratory failure

M Lemyze, P Taufour, J Mallat, O Nigeon, D Thevenin

Schaffner Hospital, Lens, France

Critical Care 2013, 17(Suppl 2):P142 (doi: 10.1186/cc12080)

Introduction Malignant obesity hypoventilation syndrome (MOHS) refers to the association between morbid obesity (body mass index $\left.>40 \mathrm{~kg} / \mathrm{m}^{2}\right)$, daytime hypercapnia $\left(\mathrm{PCO}_{2}>45 \mathrm{mmHg}\right)$ after other respiratory or neuromuscular causes of alveolar hypoventilation have been excluded, and multiple organ disorders (diabetes mellitus, arterial hypertension, metabolic syndrome, obstructive sleep apnea syndrome) [1]. Acute respiratory failure (ARF) is a common life-threatening complication of MHOS. Non-invasive ventilation (NIV) provides the cornerstone of the therapeutic management of decompensated MOHS [2]. We aimed to identify the determinants of NIV success or failure in this indication.
Methods A monocentric prospective observational study including 48 patients with MOHS and treated by NIV for ARF over a 2-year period. Results NIV failed to reverse ARF in only seven patients. NIV failure was associated with a sixfold increase in in-hospital mortality $(85.7 \%$ vs. $14.6 \% ; P<0.001)$. Factors associated with NIV failure and need for endotracheal intubation included pneumonia ( $n=5,71.4 \%$ vs. $n=8$, $19.5 \% ; P=0.01$ ), higher SOFA (11 vs. $5 ; P<0.001)$ and SAPS 2 (69 vs. 39; $P=0.001$ ) score at admission. The only factor associated with successful response to NIV was idiopathic decompensated MOHS ( $n=19,100 \%$ vs. $n=0,0 \% ; P=0.03)$. In these patients, $\mathrm{pH}$ (7.23 vs. 7.28 vs. 7.44, respectively, at $\mathrm{HO}, \mathrm{H} 2, \mathrm{H} 24 ; P<0.05)$ and $\mathrm{PCO}_{2}(74.5$ vs. 68.5 vs. $57 \mathrm{mmHg}$, respectively, at $\mathrm{H0}, \mathrm{H} 2, \mathrm{H} 24 ; P<0.05)$ dramatically improved with NIV.

Conclusion NIV provides a very efficient therapy to reverse hypercapnic ARF in morbidly obese patients. Multiple organ failure and pneumonia are the main factors associated with NIV failure in patients with MOHS. References

1. Marik PE, et al.: J Intensive Care Med 2012. [Epub ahead of print]

2. Piper AJ, et al:: Am J Respir Crit Care Med 2011, 183:292-298.

\section{P143}

Non-invasive ventilation in patients with a clinical diagnosis of pneumonia: a clinical audit

CJ Wright ${ }^{1}$, J Hornsby ${ }^{2}$, DA Barr ${ }^{3}$, SR Moonesinghe 2

'Glasgow Royal Infirmary, Glasgow, UK; ${ }^{2}$ University College Hospital, London, UK; ${ }^{3}$ Brownlee Institute, Glasgow, UK

Critical Care 2013, 17(Suppl 2):P143 (doi: 10.1186/cc12081)

Introduction The benefits of non-invasive ventilation (NIV) in patients with type 2 respiratory failure secondary to exacerbations of COPD are widely acknowledged [1], but its efficacy in respiratory failure of other aetiologies is less clear. We describe use of NIV (bilevel and/ or continuous positive airway pressure modes) in the subgroup of patients with a clinical diagnosis of pneumonia in a 35-bed adult critical care unit (ACCU).

Methods A retrospective review of data recorded prospectively on an electronic clinical information management system at University College Hospital (UCH), London, UK. Patients requiring NIV acutely at UCH are managed on the ACCU. Electronic records reviewed for patients admitted from 1 November 2010 to 2 December 2011 with clinical diagnosis of pneumonia, and in whom NIV was recorded on $\geq 6$ occasions on the electronic chart. We concede some patients on NIV $<6$ hours may have been missed. We excluded patients in whom NIV was the ceiling of therapy and those using domiciliary NIV. The following data were collected: baseline patient demographics, comorbidities, therapy received in the $A C C U$, respiratory physiological variables, length of stay/unit outcome. Descriptive statistics of the cohort and variables associated on univariate analysis with requirement for intubation were generated using MedCalc Version 12.3.0.0 (MedCalc Software, Mariakerke, Belgium).

Results Fifty-two patients fulfilled the inclusion criteria, 29 (55.8\%) were male, median age was 62.4 (IQR 48.8 to 71.6), median unit stay was 8 days (IQR 4.5 to 16.5), mean duration of NIV use was 2 days (IQR 1 to 3$), 27(51.9 \%)$ required intubation of whom $48 \%$ died. Total mortality was $13(25 \%)$ - all intubated. Patients with recorded comorbidities were more likely to be intubated than those without $(O R=4.3, P=0.0362)$; $\mathrm{pH}$ was significantly lower in those requiring intubation at all recorded time points; and mean $\mathrm{FiO}_{2}$ at 4 to 6 hours was significantly higher in those requiring intubation( 0.72 vs. $0.56, P=0.032)$. There was a trend 
toward patients with COPD requiring intubation more often $(\mathrm{OR}=8.4$, $P=0.0711$ ).

Conclusion NIV was successful in $48.1 \%$ of patients with pneumonia, the remainder requiring intubation. Given the high mortality in those patients who failed NIV we believe its use in a ward setting is hazardous. We conclude that NIV may be useful in some individuals with pneumonia, but they should be managed in the ACCU setting. Further work is required to identify those patients at risk of deterioration on NIV given the possible excess mortality.

Reference

1. Brochard $L$, et al: Noninvasive ventilation for acute exacerbations of chronic obstructive pulmonary disease. N Eng/ J Med 1995, 333:817-822.

P144

Analysis of management of non-invasive ventilation support in prehospital care for COPD patients and short-term outcome

J Cuny, G Campagne, P Gosselin, P Goldstein, N Assez, EWiel

Lille University Hospital Center, Lille Cedex, France

Critical Care 2013, 17(Suppl 2):P144 (doi: 10.1186/cc12082)

Introduction Benefits of the use of NIV in emergency departments are well established. Training and available staff, and choice of respiratory machines are essential criteria for success.

Methods We conducted an observational, descriptive, retrospective, single-center study in a 4-month period. COPD patients with respiratory failure who received prehospital NIV were included. We compared two groups: COPD patients with NIV, and COPD patients without NIV.

Results Forty-two patients were included, mean age 68.86 years $( \pm 11.98), 57.14 \%$ smokers, $64.28 \%$ arterial hypertension, $100 \%$ longterm oxygenotherapy, $23.80 \%$ antibiotics in the 7 days before, $28.57 \%$ corticosteroids. A total of $88.09 \%$ had bronchospasm, $78.26 \%$ had struggle signs, $28.57 \%$ were unable to speak, $14.28 \%$ of patients were sweating. The mean respiratory rate was 30.5 cycles/minute $( \pm 7.17)$, mean pulse rate was 105.76 ( \pm 25.34 ). Nasal EtCO was $47.75 \mathrm{mmHg}$ $( \pm 16.53)$, pulse oxymetry in air was $85 \%$ ( \pm 10.94$)$, oxygen flow rate was $5.45 \mathrm{I} /$ minute $( \pm 2.42)$, temperature was $37.14^{\circ} \mathrm{C}( \pm 8.15)$. Twenty patients received NIV. A total of $61.90 \%$ were admitted to the emergency department, $35.71 \%$ to the ICU, and one patient was left at home. One patient received tracheal intubation in the hosting service. Mortality in the first month was $13.04 \%$. A significant difference $(P<0.05)$ was found for: sweats $(30 \% / 0)$, respiratory rate $(34 \pm 8.23 / 27 \pm 6.11)$, nasal $\mathrm{EtCO}_{2}(55.0 \pm 24.4 / 40.50 \pm 9.03)$, pulse oxymetry in air $(80 \% \pm 8.63 / 90 \% \pm 13.25)$, pulse oxymetry with oxygen $(89.4 \% \pm 4.24 / 87.90 \% \pm 2.55), \beta_{2}$-mimetic and anti-cholinergic nebulization $(60 \% \pm 0.5 / 90 \% \pm 0.29)$, emergency room admission $(35 \% \pm 0.35 / 86 \% \pm 0.48), I C U$ admission $(60 \% \pm 0.5 / 13 \% \pm 0.35)$, arterial blood gases on arrival in the host service $\left(\mathrm{PaCO}_{2}\right.$ $76.6 \pm 18.66 / 43.93 \pm 11.78)$. No difference in mortality at 1 month $(2 / 3)$. Conclusion Non-invasive ventilation has improved the management and prognosis of COPD patients admitted to the emergency room. Very few studies concern the prehospital management. NIV seems to show an effect on prehospital care, especially in patients with signs of severity, hypercapnia, and without fever. Oxygenation and hypercapnia seem to be improved. Also fewer patients are admitted to the ICU. Bronchospasm does not seem to be an obstacle.

\section{Reference}

1. Schmidbauer W, et al.: Early pre hospital use of NIV. Emerg Med J 2011, 28:626-627.

\section{P145}

A window into diaphragm kinetics: feasibility, precision, and physiological meaning of ultrasound measurements of diaphragm thickness

EC Goligher', F Laghi², ND Ferguson'

'University of Toronto, Canada; 'Loyola University, Chicago, IL, USA

Critical Care 2013, 17(Suppl 2):P145 (doi: 10.1186/cc12083)

Introduction Inadequate respiratory muscle activity has been linked to ventilator-induced diaphragm dysfunction. Current techniques for monitoring respiratory muscle activity during mechanical ventilation are specialized and relatively invasive. Visualizing diaphragm thickening during inspiration by ultrasound may permit non-invasive monitoring. We explored the feasibility, reliability and physiological significance of diaphragm thickening on ultrasound.

Methods Five healthy subjects participated. We monitored inspiratory flow, volume, esophageal and gastric pressures, and diaphragm electrical activity (by esophageal and surface electromyography) while subjects performed a series of inspiratory maneuvers: tidal breathing, threshold-loaded breathing, a Muller maneuver, and inspiration to various lung volumes above functional residual capacity. At the end of each inspiratory effort, subjects were instructed to close the glottis and relax the respiratory muscles (so as to maintain lung volume while eliminating diaphragm activation). Sonographic images of diaphragm thickening during these maneuvers were obtained using $\mathrm{M}$-mode with a $13 \mathrm{MHz}$ linear array probe placed in the right ninth, 10th, or 11 th intercostal space between the middle and anterior axillary lines.

Results Diaphragm thickening in the zone of apposition was readily visualized by ultrasound in all five subjects. Mean end-expiratory diaphragm thickness was $2.1 \mathrm{~mm}(\mathrm{SD}=0.3 \mathrm{~mm})$. During tidal breathing, the diaphragm thickened by a mean of $35 \%(S D=31 \%)$. The Bland-Altman coefficient of reproducibility was $0.5 \mathrm{~mm}$; approximately $50 \%$ of measurement variability arose from caliper positioning on the ultrasound machine; diaphragm thickness measurements changed as the probe was placed in different intercostal interspaces. Diaphragm inspiratory thickening increased significantly with increasing inspiratory effort but also varied with lung volume independent of effort. At inspiratory volumes below $40 \%$ of inspiratory capacity, lung volume change contributed minimally to diaphragm thickening.

Conclusion Visualizing diaphragm thickening in the zone of apposition by ultrasound provides a feasible non-invasive technique for monitoring diaphragm activation in healthy subjects. Diaphragm thickening primarily reflects muscular effort rather than altered muscle conformation induced by changes in lung volume, especially at lower inspiratory volumes.

\section{P146}

Retrospective review of utilisation and outcomes of diaphragmatic EMG monitoring and neurally adjusted ventilatory assist in a central London teaching hospital over a 3-year period

A Skorko, D Hadfield, A Vercueil, C Bell, A Feehan, K Peters, P Hopkins King's Health Partners AHSC, London, UK

Critical Care 2013, 17(Suppl 2):P146 (doi: 10.1186/cc12084)

Introduction The theoretical advantages of monitoring the electrical activity of the diaphragm (EAdi) and neural triggering of support breaths (NAVA-Maquet) have not yet been shown to translate into significant clinical benefit [1]. Here we assess the effect of EAdi monitoring, in patients at risk of prolonged weaning, on outcomes.

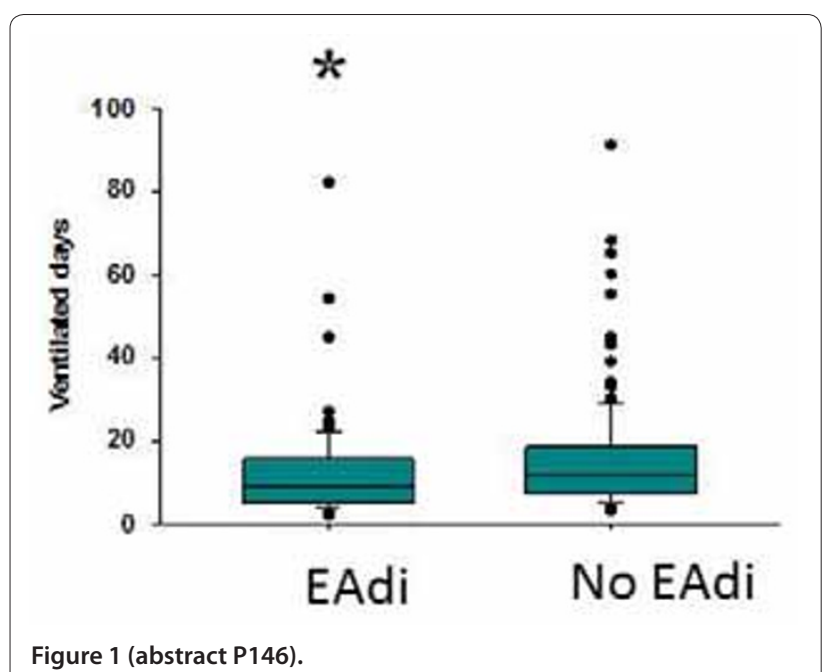


Methods Institutional ethics approval was obtained. The Medtrack clinical information system was searched to identify patients who received $\mathrm{MV}$ for $>48$ hours and who had significant chronic pulmonary disease or left/right ventricular impairment between April 2009 and March 2012. Age, APACHE II score, ventilated days, time in NAVA and outcome were compared between groups who had or had not received EAdi monitoring.

Results In total, 493/2,684 (18.3\%) patients had heart-lung risk factors for prolonged weaning. One hundred and four patients received EAdi monitoring. Ventilated days were significantly reduced (Figure 1) in the EAdi monitored group (median 9) versus the nonmonitored group (median 12); $P=0.024$ (Mann-Whitney U test). ICU mortality was not significantly different and there was no correlation between time spent in NAVA and days ventilated.

Conclusion EAdi catheter insertion was associated with a significant reduction in time spent on MV. A prospective RCT will be needed to confirm benefit and explore mechanisms.

Reference

1. Brander L, et al:: Chest 2009, 135:695-703.

P147

Validation of a lung ultrasound protocol in acute respiratory failure: preliminary results

RV Cremonese', AC Tabajara Raupp', JM Stormovski de Andrade', RS Townsend', FS Neres', AS Machado', MC Prestes', JG Maccari²,

FL Dexheimer Neto ${ }^{1}$

'Ernesto Dornelles, Porto Alegre, Brazil; ${ }^{2}$ Hospital Moinhos de Vento, Porto Alegre, Brazil

Critical Care 2013, 17(Suppl 2):P147 (doi: 10.1186/cc12085)

Introduction Patients with acute respiratory failure demand dynamic evaluation and interventions. Lung ultrasound is a bedside technique, very promising in this setting. Previously, Lichtenstein and colleagues proposed an algorithm approach with a diagnostic accuracy of $90.5 \%$, named Bedside Lung Ultrasound in Emergency (BLUE protocol). However, this approach has never been validated in other populations or ultrasound operators. The aim of this study was to evaluate the diagnostic accuracy of the lung ultrasound algorithm (BLUE protocol) in ICU patients admitted with acute respiratory failure.

Methods A prospective study, single-center consecutive case series evaluating critically ill adult patients in acute respiratory failure, admitted to a 23-bed general ICU between October 2011 and November 2012. The ultrasound examination was performed by an ICU physician until 20 minutes after admission, without interfering with usual care, and the ultrasound operator was blinded to the medical history of the patient. Three items were assessed: artifacts (horizontal A lines or vertical B lines indicating interstitial syndrome), lung sliding, alveolar consolidation and/or pleural effusion. BLUE protocol results were compared with clinical diagnosis by the medical assistant team at the end of the ICU stay.

Results Forty-two patients were evaluated ( $42 \%$ male), mean age was 73 years (SD \pm 15 ) and APACHE II score was 18.77 (median 20). Based upon evaluation of the findings of BLUE protocol, a positive correlation was found in $83.78 \%$ of the sample. For the diagnosis of pulmonary edema $(n=15)$, sensitivity was $85 \%$ and specificity was $86 \%$, while for the diagnosis of pneumonia $(n=17)$, sensitivity was $88 \%$ and specificity was $90 \%$. In this sample there were only four cases of reversible airway obstruction and one case of pulmonary embolism. Five patients were excluded of the analysis because they had rare diagnoses.

Conclusion Preliminary results demonstrate diagnostic accuracy of lung ultrasound examination performed on the admission of patients with acute respiratory failure similar to the original publication. Lung ultrasound, as proposed in the BLUE protocol, has good accuracy, and seems reproducible and useful in this group of patients.

Reference

1. Lichtenstein DA, Mezière GA: Relevance of lung ultrasound in the diagnosis of acute respiratory failure: the BLUE protocol. Chest 2008, 134:117-125.
P148

Risk of weaning failure with PAOP $\geq 15$ versus $P A O P \geq 18$

Y Nassar, A Abdelbary

Cairo University, Giza, Egypt

Critical Care 2013, 17(Suppl 2):P148 (doi: 10.1186/cc12086)

Introduction Despite $18 \mathrm{mmHg}$ being recognized as a classical cutoff value, there is no definite value of PAOP above which cardiogenic oedema develops. We aimed to assess the risks of weaning failure with PAOP $\geq 15$ and with $P A O P \geq 18$.

Methods A prospective enrollment of 30 adult medical ICU patients on invasive mechanical ventilation who were fulfilling the criteria for initiation of weaning from mechanical ventilation according to the Sixth International Conference Statement of Intensive Care Medicine. We followed the two-step weaning strategy, which involves assessment regarding readiness for weaning followed by a spontaneous breathing trial (SBT) as a diagnostic test to determine the likelihood of successful extubation. Invasive right heart catheterization to measure PAOP, and non-invasive transthoraxic echo and tissue Doppler parameters $E / E^{\prime}$, E/A, DT, IVRT were measured before initiation and after termination of the SBT.

Results E/E' was significantly correlated with PAOP $(r=503, P<0.001)$. A cutoff value of septal $E / E^{\prime}>11.3$ predicted $P A O P$ elevation $\geq 18 \mathrm{mmHg}$ with a sensitivity of $90.9 \%$ and specificity of $87.8 \%$, while a value of septal $E / E^{\prime}>10.9$ predicted PAOP elevation $\geq 15 \mathrm{mmHg}$ with a sensitivity of $59.1 \%$ and specificity of $87.8 \%$. The incidence of weaning failure was $23 \%$, and $72 \%$ of patients who failed to be weaned exhibited significantly higher PAOP during the trial. Patients with PAOP $\geq 18$ at the end of the SBT mostly (72.4\%) failed weaning while a minority $(27.6 \%)$ successfully weaned. The majority $(91.3 \%)$ of the patients with PAOP $<18$ had a successful weaning outcome and $8.7 \%$ failed weaning outcome. PAOP $\geq 18$ significantly negatively correlated with weaning success $(P=0.001, r=-0.627)$. On the other hand, PAOP $\geq 15$ did not show a significant correlation $(P>0.05, r=-0.274)$. The PAOP $\geq 18$ weaning failure risk estimate was 8.2 , with $95 \% \mathrm{Cl} 0.004$ to 0.3 . The $\mathrm{PAOP} \geq 15$ weaning failure risk estimate was 1.83 , with a significant $95 \%$ $\mathrm{Cl}$ of 0.913 to 3.648 .

Conclusion E/E' was significantly correlated with PAOP ( $r=503$, $P<0.001)$. E/E' $>11.3$ predicted PAOP elevation $\geq 18 \mathrm{mmHg}$ (sensitivity $90.9 \%$ and specificity $87.8 \%$ ), while $E / E^{\prime}>10.9$ predicted PAOP elevation $\geq 15 \mathrm{mmHg}$ (sensitivity $59.1 \%$ and specificity $87.8 \%$ ). Weaning failure risk is greater ( 8.2 vs. 1.8 ) with $P A O P \geq 18$ versus $P A O P \geq 15$, respectively.

P149

Impact of continuous positive airway pressure on the treatment of acute exacerbation of asthma: a randomised controlled trial

Y Sutherasan, S Kiatboonsri, P Theerawit, C Kiatboonsri, S Trakulsrichai Ramathibothi Hospital, Bangkok, Thailand

Critical Care 2013, 17(Suppl 2):P149 (doi: 10.1186/cc12087)

Introduction Despite the extensive use of continuous positive airway pressure (CPAP) in various respiratory failure conditions, its role in acute asthmatic attack is uncertain [1-3]. This study aims at exploring the efficacy of CPAP when use in addition to the conventional treatment of acute asthma exacerbation in the emergency department (ED).

Methods During May to December 2009, acute asthma patients attending ED were randomly assigned to CPAP and control groups. In addition to the conventional treatment, CPAP ventilation of $8 \mathrm{cmH}_{2} \mathrm{O}$ was applied to patients in the CPAP group. Data collections in both groups included peak expiratory flow rates (PEFR, \%predicted) at each 15-minute interval (T0, T15, ...), the number of short-acting bronchodilators (SABA) used, ED length of stay, relapse rate and admission rate. The primary outcome is the improvement in peak expiratory flow rate during a short period stay in the ED.

Results There were 86 patients enrolled, with 43 patients in each study group. PEFR in the CPAP group showed no difference from the control group at T0 (52.7 \pm 20.33 vs. $47.2 \pm 16.64, P=N S)$ but was significantly better at T15 $(65.58 \pm 21.64$ vs. $56.49 \pm 18.53 \%, P<0.05)$. Such improvement was consistently observed for all subsequent PEFR measurement, along with the fewer SABA used and relapse rate, but the figures did not meet statistical difference. 
Conclusion The addition of CPAP to conventional acute asthma treatment in the ED could accelerate PEFR improvement with a trend showing fewer SABA needed, lower relapse rate and shorter ED length of stay.

\section{References}

1. Shivaram U, et al:: Effects of continuous positive airway pressure in acute asthma. Respiration 1987, 52:157-162.

2. Meduri GU, et al:: Noninvasive positive pressure ventilation in status asthmaticus. Chest 1996, 110:767-774

3. Lin $\mathrm{HC}$, et al:: Effect of nasal continuous positive airway pressure on methacholine-induced bronchoconstriction. Respir Med 1995, 89:121-128.

P150

Novel method to confirm tracheal intubation based on airway pressures: validation in patients with lung disease

A Stemerdink', KG Monsieurs', F Luijmes', W Dieperink', JG Zijlstra', AF Kalmar ${ }^{1}$

'University Medical Center Groningen, the Netherlands; ${ }^{2}$ Antwerp University Hospital, Antwerp, Belgium

Critical Care 2013, 17(Suppl 2):P150 (doi: 10.1186/cc12088)

Introduction Emergency endotracheal intubation results in accidental oesophageal intubation in up to $17 \%$ of patients often with disastrous consequences. We have previously published a highly specific and sensitive novel method to detect endotracheal intubation based on differences in ventilation pressure waveforms in the oesophagus and in the trachea in patients with healthy lungs [1]. A detection algorithm, based on differences in compliance/elasticity between the lung and the oesophagus, generated a $\mathrm{D}$-value indicating tracheal intubation if $D>0.5$ and oesophageal intubation if $D<0.5$. The aim of the current study was to validate the algorithm in patients with lung disease.

Methods After obtaining institutional approval, 20 intubated and ventilated ICU patients were included. Inclusion criteria were controlled mechanical ventilation and at least mild to moderate lung injury according to a Murray lung injury score $>0.1$. A connecting piece was placed between the endotracheal tube and the ventilation bag. This piece comprised a thin air-filled catheter inserted through the tube lumen at $1 \mathrm{~cm}$ from the distal end, and a second catheter located at the proximal end of the tube. We performed three consecutive manual bag ventilations while recording the pressure curves through both catheters. For each ventilation, a D-value was calculated.

Results Mean age (SD) of the patients was 60 (16) years, $60 \%$ were male. The mean (SD) Murray score was 1.4 (0.6). Pathologies included pulmonary oedema, pneumonia, atelectasis and traumatic lung injury. All $60 \mathrm{D}$-values are represented in Figure 1. The median (IQR, range) D-value was 38 (16 to $74,0.8$ to 1,272 ). Our algorithm therefore confirmed a high sensitivity to detect correct endotracheal intubation also in patients with lung disease. Under the hypothesis that oesophageal compliance does not increase significantly in patients with lung disease, the specificity of our algorithm will not be affected. Conclusion The algorithm to detect correct endotracheal intubation performed excellent in patients with lung disease.

\section{Reference}

1. Kalmar AF, et al.: Resuscitation 2012, 83:177-182.
P151

Main indications, diagnostic and therapeutic yield of bronchoscopy in the ICU

I Iglesias, B Gonzalez, O Rajas

La Princesa Hospital, Madrid, Spain

Critical Care 2013, 17(Suppl 2):P151 (doi: 10.1186/cc12089)

Introduction Bronchoscopy (BC) is a frequently used diagnostic and therapeutic tool in our ICU. We planned to review the patients and conditions in which the procedure was performed. The objective was to determine indications, diagnostic and therapeutic yield.

Methods A retrospective, single-center observational study was carried out. All BC performed in a 20-bed polyvalent ICU of a tertiary university hospital over a period of 30 months (from January 2010 to June 2012) were included. The main variables collected were demographic data and clinical data including comorbidities, endobronchial techniques and results.

Results A total of $470 \mathrm{BC}$ were carried out in 240 patients admitted to the ICU (68\% were male and $32 \%$ were female). All of the procedures were performed in mechanically ventilated patients. The mean patient age was $61 \pm 14$ years, with an APACHE II score at admission of $20 \pm 7$. The main cause for ICU admission was respiratory failure (27\%). The most common comorbidities were smoking, chronic obstructive pulmonary disease, cardiovascular risk factors and atrial fibrillation. The main indications for performing the $\mathrm{BC}$ were percutaneous tracheostomy endoscopically guided ( $28 \%$ ), resolution of atelectasis $(27 \%)$, aspiration of secretions (24\%) and hemoptysis (10\%). Bronchial brushing was performed in 435 cases (93\%) obtaining microbiological growth in 64\% of them, mainly Pseudomonas aeruginosa and Acinetobacter baumanii. Bronchoalveolar lavage was performed in 62 cases (13\%) obtaining microbiological growth in $61 \%$ of them, mainly cytomegalovirus and Stenotrophomona maltophilia. Citology was performed in 60 cases (13\%); $7 \%$ showed the presence of microorganism and $13 \%$ revealed precancerous/cancerous tissue changes.

Conclusion $\mathrm{BC}$ can be performed at the bedside, avoiding transfers outside the ICU that may cause clinical worsening. Most frequent indications were bronchoscopy-guided percutaneous tracheostomy, resolution of atelectasis, aspiration of secretions and hemoptysis. Microbiological results obtained revealed the presence of aggressive pathogens. BC in ICU provides valuable diagnostic information and has therapeutic utility.

References

1. Lucena CM, et al:: Med Intensiva 2012, 36:389-395.

2. Estella A, et al: Med Intensiva 2012, 36:396-401.

3. Shennib H, et al:: Chest Surg Clin NAm 1996, 6:349-361.

4. Turner JS, et al:: Crit Care Med 1994, 22:259-264.

P152

Benchtop study comparing leakages across cuffs of three endotracheal tubes

SM Lam, CW Lau, WW Yan

Pamela Youde Nethersole Eastern Hospital, Hong Kong

Critical Care 2013, 17(Suppl 2):P152 (doi: 10.1186/cc12090)

Introduction The aim was to compare two novel endotracheal tubes (ETT), Mallinckrodt TaperGuard (TG, tapered polyvinyl chloride (PVC) cuff) and KimVent Microcuff (MC, cylindrical polyurethrane cuff),

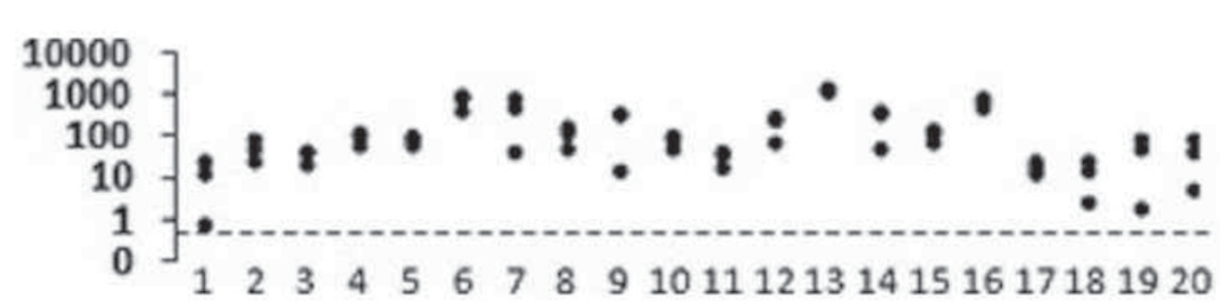

Figure 1 (abstract P150). D-value of individual test ventilations. 


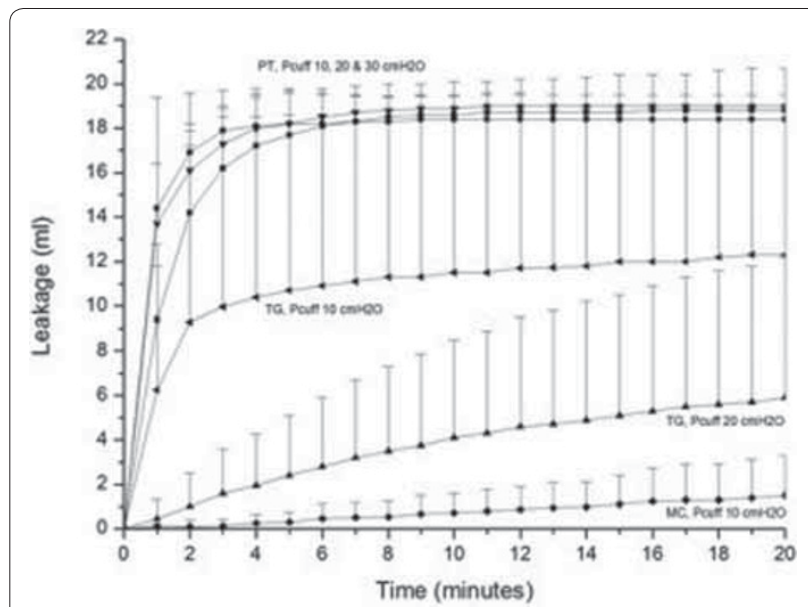

Figure 1 (abstract P152). Downward leak in PEEP $0 \mathrm{cmH}_{2} \mathrm{O}$ without suction. Each point represents the mean and standard deviation of eight repeated measures.

with conventional Portex (PT, globular PVC cuff) in leakages across cuffs (microaspiration) under simulated clinical situations. It has been shown that globular PVC cuffs protect poorly against leakages due to microchannels formed from infolding of redundant cuff material [1]. We hypothesized that TG and MC better prevent microaspiration, which is a major mechanism of ventilator-associated pneumonia (VAP). Methods Each ETT was inserted into a silicone cylinder of $2 \mathrm{~cm}$ wide inclined at $35^{\circ}$. Then $20 \mathrm{ml}$ water was added above the cuff and leakage measured every minute under five different simulated clinical conditions: mechanical ventilation for acute severe asthma (positive end-expiratory pressure (PEEP) $0 \mathrm{cmH}_{2} \mathrm{O}$ ), normal lungs (PEEP $5 \mathrm{cmH}_{2} \mathrm{O}$ ) and acute respiratory distress syndrome (PEEP $10 \mathrm{cmH}_{2} \mathrm{O}$ ), and disconnection from the ventilator with and without spontaneous breathing effort. Spontaneous breathing was simulated with a respiratory gas exchange simulator. Suction was applied at $200 \mathrm{cmH}_{2} \mathrm{O}$ sustained for 3 minutes at the Murphy eye. Each scenario was repeated with cuff pressures (Pcuff) 10, 20 and $30 \mathrm{cmH}_{2} \mathrm{O}$ maintained by a Pcuff maintenance device.

Results PT leaked grossly in all scenarios without PEEP and at PEEP $5 \mathrm{cmH}_{2} \mathrm{O}$ in the presence of suction irrespective of Pcuff (Pcuff $30 \mathrm{cmH}_{2} \mathrm{O}$ : PT $19.7 \pm 0.4, \mathrm{TG} 0.0 \pm 0.1, \mathrm{MC} 0.0 \pm 0.0, P<0.001$; Pcuff $20 \mathrm{cmH}_{2}$ O: PT $19.9 \pm 0.4, \mathrm{TG} 7.4 \pm 6.2$, MC $0.0 \pm 0.0, P<0.001$; Pcuff $10 \mathrm{cmH}_{2} \mathrm{O}$ : PT $20.0 \pm 0.0$, TG $\left.12.7 \pm 5.1, \mathrm{MC} 0.9 \pm 0.8, P<0.001\right)$. Leakage under these scenarios can be reduced in TG and prevented in MC by Pcuff $\geq 20 \mathrm{cmH}_{2} \mathrm{O}$ (Figure 1).

Conclusion Microcuff outperformed the others in preventing microaspiration, while Portex leaked grossly even at a recommended Pcuff of 20 to $30 \mathrm{cmH}_{2} \mathrm{O}$ whenever PEEP was lost. The effect of ETT type on the incidence of VAP warrants further investigation.

Reference

1. Fernandez JF, et al:: Chest 2012, 142:231-238.

P153

Endotracheal tube with tapered-type cuff for preventing ventilator-associated pneumonia: a randomized clinical trial N Saito, T Yagi, Y Hara, H Matsumoto, K Mashiko

Nippon Medical School Chiba Hokusoh Hospital, Inzai, Chiba, Japan Critical Care 2013, 17(Suppl 2):P153 (doi: 10.1186/cc12091)

Introduction The most common cause of ventilator-associated pneumonia (VAP) is aspiration of oral secretion through the endotracheal tube (ET). Subglottic suction drainage (SSD) has been recommended as a safety measure against aspiration due to its high effectiveness. Currently, two types of cuff shape - spindle and tapered - are predominant in high-volume, low-pressure (HVLP) ETs with SSD. However, the shape most suitable for preventing dripping onto the subglottis has not been determined. The purpose of this study was to determine whether an ET with tapered-type cuff can reduce the incidence of VAP.

Methods After approval from the appropriate ethics committee, we conducted a single-institutional prospective randomized clinical trial on the effectiveness of using an ET with a different cuff type. Adult patients ( $n=399 ; \geq 18$ years old) were screened between April 2010 and March 2012, and 289 patients expected to require mechanical ventilation (MV) for at least 48 hours were randomized. Patients were assigned to intubation with one of the following two HVLP ETs with SSD on the experimental tube: tapered type (Taperguard ${ }^{\mathrm{TM}} \mathrm{Evac}^{\oplus}$; Covidien, Dublin, Ireland) or spindle type (Hi-Lo Evac ${ }^{\oplus}$; Covidien). Cuff pressure measurement was carried out every 6 hours, and cuff pressure was maintained between 20 and $30 \mathrm{cmH}_{2} \mathrm{O}$. The primary outcome was VAP incidence based on semiquantitative bronchoalveolar lavage fluid culture of 3+ or phagocytosis on Gram staining in patients intubated for at least 48 hours. The other outcome was the rate of achieving an appropriate cuff pressure ( 20 to $30 \mathrm{cmH}_{2} \mathrm{O}$ ), time to VAP onset, duration of MV, duration of ICU stay, mortality, and adverse events.

Results The rate of microbiologically confirmed VAP was $21.7 \%$ (23/106) for the tapered-type ET patients and $21.7 \%$ (23/106) for the spindle-type ET patients $(P=1.00)$. The rate of achieving appropriate cuff pressure was $83.2 \%(332 / 1,974)$ for the tapered type and $82.4 \%$ $(328 / 1,867)$ for the spindle type $(P=0.549)$. No significant differences between groups were observed for time to VAP onset, duration of MV, ICU stay. The incidence of reintubation due to laryngeal edema after extubation was slightly higher in patients with the tapered-type ET $(11.5 \%, 6 / 52)$ than in patients with the spindle-type ET $(2.0 \%, 1 / 49)$, but the difference was not significant $(P=0.113)$.

Conclusion Differences in cuff type and shape under identical conditions of cuff pressure control have no influence on the incidence of VAP.

Acknowledgement UMIN-CTR UMIN000003371.

P154

Effect of body position changes on endotracheal tube cuff pressure in ventilated patients

C Lizy', W Swinnen², S Labeau³, S Blot

'Gent University, Ghent, Belgium; '² General Hospital Sint Blasius,

Dendermonde, Belgium; ${ }^{3}$ University College Ghent, Belgium

Critical Care 2013, 17(Suppl 2):P154 (doi: 10.1186/cc12092)

Introduction In order to avoid either microaspiration $\left(<20 \mathrm{cmH}_{2} \mathrm{O}\right)$ or tracheal injury $\left(>30 \mathrm{cmH}_{2} \mathrm{O}\right.$ ), the target for endotracheal tube (ETT) cuff pressure (CP) is between 20 and $30 \mathrm{cmH}_{2}$ O. Our objective was to assess the effect of patient positioning changes on $\mathrm{CP}$ in adult patients.

Methods A sample of 12 orally intubated and sedated patients was selected. Patients received neuromuscular blockade and were positioned in a neutral start position (backrest, head of bed elevation ( $\mathrm{HoB})$ $30^{\circ}$, head in neutral position) and $\mathrm{CP}$ set at $25 \mathrm{cmH}_{2} \mathrm{O}$. Subsequently 16 different position changes were performed: anteflexion head, hyperextension head, left and right lateral flexion of head, left and right rotation of the head, semirecumbent position $\left(\mathrm{HoB} 45^{\circ}\right)$, recumbent position $\left(\mathrm{HoB} 10^{\circ}\right)$, horizontal position, trendelenburg $\left(-10^{\circ}\right)$, left and right lateral positioning over $30^{\circ}, 45^{\circ}$ and $90^{\circ}$. Once the patient was correctly positioned, CP was recorded during an end-expiratory ventilatory hold. $\mathrm{CP}$ observed was compared with $\mathrm{CP}$ at start position ( $25 \mathrm{cmH}_{2}$ O). Also the number of values outside the target range (20 to $30 \mathrm{cmH}_{2} \mathrm{O}$ ) was reported as considered clinically relevant.

Results A total of 192 measurements were performed ( 12 subjects $\times 16$ positions). Results are shown in Table 1 (CP reported as median, IQR). In every position a significant deviation in CP was observed. In total, $40.6 \%$ of values exceeded the upper limit of $30 \mathrm{cmH}_{2} \mathrm{O}$. No values beneath the lower target limit of $20 \mathrm{cmH}_{2} \mathrm{O}$ were observed. In each position the upper target limit was exceeded at least once.

Conclusion Simple changes in patient positioning may result in potentially harmful $\mathrm{CP}\left(>30 \mathrm{cmH}_{2} \mathrm{O}\right)$. These observations call for strict $\mathrm{CP}$ monitoring. 
Table 1 (abstract P154). Endotracheal tube cuff pressure values following patient position changes

\begin{tabular}{|c|c|c|c|}
\hline & $\begin{array}{c}\text { Cuff } \\
\text { pressure } \\
\left(\mathrm{cmH}_{2} \mathrm{O}\right)^{\mathrm{a}}\end{array}$ & $\begin{array}{c}P \text { for } \Delta \text { from } \\
\text { start position } \\
\left(25 \mathrm{cmH}_{2} \mathrm{O}\right)\end{array}$ & $\begin{array}{c}\text { Measurements } \\
>30 \mathrm{cmH}_{2} \mathrm{O} \\
(n(\%))\end{array}$ \\
\hline Anteflexion head & 33.5 (27.5 to 42.0$)$ & 0.005 & $9(75)$ \\
\hline Hyperextension head & 30.5 (27.5 to 42.0$)$ & 0.004 & $6(50)$ \\
\hline Lateral flexion head, left & 26.5 (25.3 to 28.8 ) & 0.025 & $1(8)$ \\
\hline Lateral flexion head, right & 27.0 (25.3 to 29.8$)$ & 0.049 & $2(17)$ \\
\hline Rotation head, left & 27.0 (26.3 to 33.8$)$ & 0.003 & $3(25)$ \\
\hline Rotation head, right & 28.0 (26.3 to 38.8$)$ & 0.020 & $4(33)$ \\
\hline Semirecumbent, $45^{\circ} \mathrm{HoB}$ & 28.0 (25.3 to 31.5$)$ & 0.009 & $2(17)$ \\
\hline Recumbent, $10^{\circ} \mathrm{HoB}$ & $28.0(25.3$ to 31.5$)$ & 0.016 & $3(25)$ \\
\hline Horizontal back rest & 28.5 (25.3 to 31.5$)$ & 0.011 & $3(25)$ \\
\hline Trendelenburg, $10^{\circ}$ & 29.0 (25.3 to 32.0$)$ & 0.008 & $5(42)$ \\
\hline Left lateral position, $30^{\circ}$ & 30.5 (25.3 to 32.0$)$ & 0.010 & $6(50)$ \\
\hline Left lateral position, $45^{\circ}$ & 30.5 (27.3 to 32.8$)$ & 0.005 & $6(50)$ \\
\hline Left lateral position, $90^{\circ}$ & 34.0 (27.0 to 36.8$)$ & 0.006 & $7(58)$ \\
\hline Right lateral position, $30^{\circ}$ & 31.0 (27.0 to 34.5$)$ & 0.003 & $6(50)$ \\
\hline Right lateral position, $45^{\circ}$ & 32.5 (28.3 to 35.8$)$ & 0.002 & $8(50)$ \\
\hline Right lateral position, $90^{\circ}$ & $31.0(29.3$ to 35.0$)$ & 0.003 & $7(58)$ \\
\hline Total (192 measurements) & - & - & $78(40.6)$ \\
\hline
\end{tabular}

$\mathrm{HoB}$, head of bed elevation. ${ }^{\mathrm{a} C u f f}$ pressure values reported as median (first to third quartile).

\section{P155}

Towards safer airway management in the critically ill: lessons from National Audit Project 4

N Santana-Vaz, S Tallowin, H Lewis, D Park, R O'Brien, JM Patel

Heart of England NHS Foundation Trust, Birmingham, UK

Critical Care 2013, 17(Suppl 2):P155 (doi: 10.1186/cc12093)

Introduction National Audit Project 4 (NAP4) highlighted the need to improve airway management in ICUs and key recommendations were the continuous use of end-tidal carbon dioxide (ETCO) monitoring, pre-intubation checklists and difficult airway trolleys [1]. This complete cycle audit aimed to quantify the current state of airway management on our ICU and the effectiveness of implementing the NAP4 recommendations.

Methods Data collection was carried out prospectively for both phases and included documentation of intubation, use of $\mathrm{ETCO}_{2}$ and the incidence of serious adverse events (SAEs). The contents of the intubation boxes were compared against the Difficult Airway Society (DAS) guidelines [2]. The re-audit was carried out 6 months following the introduction of a pre-intubation checklist, a documentation sticker, a difficult airway trolley and standardization of the basic bedside airway boxes with a checklist of contents. A training program in airway management for all ICU staff was also introduced.

Results The baseline characteristics of both groups were similar. The initial audit included 45 patients and the re-audit 58 patients. In the initial audit 19\% of patients had accurate documentation of intubation with the use of $\mathrm{ETCO}_{2}$. The continuous use of $\mathrm{ETCO}_{2}$ was $80 \%$. Bedside airway boxes did not contain a checklist of standardized equipment. A difficult airway trolley was not located on the ICU. Following introduction of the changes described, a significant improvement in the use of ETCO to confirm intubation $(46 \%$ vs. $19 \%, P<0.01)$ and continuous use of $\mathrm{ETCO}_{2}$ monitoring $(100 \%$ vs. $80 \% P \leq 0.0004)$ was observed. The use of the preintubation checklist (7\%) and stickers (11\%) was poor. All bedside boxes had a checklist and the required equipment. A difficult airway trolley is located on the ICU with an equipment list and DAS algorithm attached. No SAEs were recorded in either phase.

Conclusion Serious airway complications are rare. Safer airway management is essential in the ICU to prevent morbidity and mortality.
This audit demonstrated that simple measures in conjunction with a structured training program can help to improve the safety of airway management in critically ill patients. Further work is required to improve compliance with the pre-intubation checklist alongside a continued education program.

References

1. The Royal College of Anaesthetists [www.rcoa.ac.uk/nap4]

2. Difficult Airway Society Equipment List

[http://www.das.uk.com/equipmentlistjuly2005.htm]

\section{P156}

Quantitative assessment of endotracheal tube (ETT) biofilm by micro-CT scan: evaluation of the effectiveness of ETT cleaning devices

A Coppadoro', G Bellani', T Mauri', R Borsa', V Meroni', A Barletta', M Teggia Droghi', A Lucchini², R Marcolin², N Patroniti', S Bramati²,

A Pesenti'

'University of Milan-Bicocca, Monza, Italy; ${ }^{2}$ San Gerardo Hospital, Monza, Italy Critical Care 2013, 17(Suppl 2):P156 (doi: 10.1186/cc12094)

Introduction The goal of this study was to assess the amount of biofilm within the endotracheal tube (ETT) by CT scan and to evaluate in a bench test a device aimed at ETT biofilm removal. Biofilm grows within the ETT soon after intubation, resulting in increased resistance to airflow. Biofilm detachment from the ETT can result in bacterial colonization of the distal airways. The use of an ETT cleaning device could effectively reduce the patient's airflow resistance burden. The quantification and distribution of biofilm within the ETT might help to evaluate ETT cleaning device effectiveness.

Methods We collected ETTs of 11 critically ill patients after extubation. Micro-CT scan (SkyScan 1176; Bruker, Belgium) was performed using a resolution of $35 \mu \mathrm{m}$. Axial sections of the $20 \mathrm{~cm}$ above the cuff were reconstructed, and the volume of secretions was assessed by a density criterion. Microbiological cultures of the ETT lavage fluid were then obtained. Patient's demographics and clinical data were recorded. In a different set of bench experiments, we injected $1 \mathrm{ml}$ water-based polymer into new ETTs of different sizes. We measured resistance to airflow before and after using an ETT cleaning device (Airway Medix Closed Suction System; Biovo Technologies, Tel Aviv, Israel). We also obtained resistance values of intact ETTs as controls.

Results The studied ETTs remained in place for a median of 7 days (IQR range 4 to 15). The amount of secretions assessed by CT scan was $0.293 \pm 0.290 \mathrm{ml}$ (range 0.032 to $0.777 \mathrm{ml}$ ). Secretion volumes were not related to patient severity at admission (SAPS 2, P/F ratio) or days of intubation; an inverse correlation with patient's age was present $\left(P=0.032, R^{2}=0.46\right)$. Bacterial growth was present in $9 / 11(82 \%)$ ETT fluids cultures and Candida spp. showed an elevated prevalence $(6 / 11,55 \%)$. In the bench tests, the cleaning device reduced resistance to airflow (difference before and after cleaning $5.5(95 \% \mathrm{Cl}=8.9$ to 1.6) $\left.\mathrm{CmH}_{2} \mathrm{O} / \mathrm{l} / \mathrm{second}, P=0.006\right)$. After cleaning, resistance resulted higher than intact ETTs, although with a clinically negligible difference (difference $0.3\left(95 \% \mathrm{Cl}=0.2\right.$ to $\left.0.6 \mathrm{cmH}_{2} \mathrm{O} / \mathrm{l} / \mathrm{second}\right), P=0.032$ ).

Conclusion Micro-CT scan is a feasible and promising technique to assess secretions volume in ETTs after extubation. The use of an ETT cleaning device decreases resistance to airflow in bench tests; the effectiveness of such a device in the clinical setting could be properly assessed by post-extubation CT scan.

\section{P157}

Early identification of patients at risk of difficult intubation in the ICU: development and validation of the MACOCHA score in a multicenter cohort study

A De Jong', N Molinari', N Terzi², N Mongardon³, B Jung', S Jaber 'Montpellier University Hospital, Montpellier, France; ${ }^{2}$ Caen University Hospital, Caen, France, ${ }^{3}$ Cochin University Hospital, Paris, France Critical Care 2013, 17(Suppl 2):P157 (doi: 10.1186/cc12095)

Introduction Difficult intubation (DI) in the ICU is a challenging issue, associated with severe life-threatening complications $[1,2]$. The objective was to develop and validate a simplified score for identifying patients with DI in the ICU and to report related complications. 
Methods Data collected in a prospective multicenter-study from 1,000 consecutive intubations from 42 ICUs were used to develop a simplified score of $\mathrm{Dl}$, which was then validated externally in 400 consecutive intubation procedures from 18 other ICUs and internally by bootstrap on 1,000 iterations.

Results In multivariate analysis, the main predictors of DI (incidence $=$ $11.3 \%$ ) were related to the patient (Mallampati score III or IV, obstructive sleep apnea syndrome, reduced mobility of cervical spine, limited mouth opening), to pathology (severe hypoxia, coma) and to the operator (non-anesthesiologist). From the $\beta$-parameter, a sevenitem simplified score (MACOCHA score; Table 1) was built, with an area under the curve (AUC) of $0.89(95 \% \mathrm{Cl}=0.85$ to 0.94$)$. In the validation cohort (prevalence of $\mathrm{DI}=8 \%$ ), the AUC was of $0.86(95 \% \mathrm{Cl}=0.76$ to 0.96 ), with a sensitivity of $73 \%$, a specificity of $89 \%$, a negative predictive value of $98 \%$ and a positive predictive value of $36 \%$. After internal validation by bootstrap, the AUC was $0.89(95 \% \mathrm{Cl}=0.86$ to 0.93). Severe life-threatening events (severe hypoxia, collapse, cardiac arrest or death) occurred in $38 \%$ of the 1,000 cases. Patients with DI $(n=113)$ had significantly higher severe life-threatening complications than those who had a non-DI ( $51 \%$ vs. $36 \%, P<0.0001)$.

Table 1 (abstract P157). MACOCHA score calculation worksheet

\begin{tabular}{lc}
\hline & Points \\
\hline Factors related to patient & \\
Mallampati score III or IV & 5 \\
Obstructive apnea syndrome & 2 \\
Reduced mobility of cervical spine & 1 \\
Limited mouth opening $<3 \mathrm{~cm}$ & 1 \\
Factors related to pathology & 1 \\
$\quad$ Coma & 1 \\
Severe hypoxemia (<80\%) & 1 \\
Factor related to operator & \\
$\quad$ Non-anaesthesiologist & 1 \\
Total & 12 \\
\hline
\end{tabular}

Conclusion DI in ICU is strongly associated with severe life-threatening complications. A simple score including seven clinical items discriminates difficult and non-DI in ICU.

References

1. Jaber et al:: Crit Care Med 2006, 34:2355-2361.

2. Jaber et al: Intensive Care Med 2010, 36:248-255.

P158

Incidence of major complications related to endotracheal intubation in ICUs of nonacademic Brazilian public hospitals AB Cavalcanti', K Normilio-da-Silva', JC Acarine Mouro', F Moreira', AA Kodama', R Del-Manto², O Berwanger ${ }^{1}$

'Hospital do Coração - HCor, São Paulo, Brazil:'2Hospital Militar de Área de

São Paulo - HMASP, São Paulo, Brazil

Critical Care 2013, 17(Suppl 2):P158 (doi: 10.1186/cc12096)

Introduction Our objective is to determine the incidence of major complications related to endotracheal intubations (death, cardiac arrest, severe hemodynamic instability or severe hypoxemia) performed in the ICUs of nonacademic Brazilian public hospitals.

Methods This is a report of the baseline phase of a cluster randomized trial with two parallel arms. In this baseline phase, we collected data from sequential patients needing endotracheal intubation in 17 ICUs from Brazil. Patients needing endotracheal intubation after cardiac arrest were excluded. In a second ongoing phase, not reported here, we have randomized ICUs to a multifaceted intervention to prevent complications of endotracheal intubation. Primary outcome was defined as the occurrence of death, cardiac arrest, severe cardiovascular instability (systolic blood pressure $<60 \mathrm{mmHg}$ at least one time, $<90 \mathrm{mmHg}$ and persisting $>30$ minutes or need to initiate vasopressors) or severe hypoxemia $\left(\mathrm{SpO}_{2}<80 \%\right)$ within 1 hour after intubation.

Results We evaluated 246 intubations. The mean age was $49 \pm 21.9$ years; the SAPS 3 admission score was $57.4 \pm 15.4$. The most common indications of intubation were acute respiratory failure $(61.0 \%)$ and coma (17.1\%). The primary outcome occurred in 138 of 246 intubations (56.1\%). Within the first hour, the incidence of severe hypotension was $47.2 \%$, of severe hypoxemia was $20.3 \%$, of cardiac arrest was $5.7 \%$ and of death was $4.5 \%$.

Conclusion Incidence of major complications associated with endotracheal intubation is unacceptably high in Brazilian public ICUs. There is an urgent need for effective measures to increase safety of this common procedure in our environment.

P159

Tracheal intubation for a difficult airway using Airway scope ${ }^{\circledast}$, KingVision ${ }^{\oplus}$ and McGRATH ${ }^{\oplus}$ : a comparative manikin study of inexperienced personnel

J Itai', Y Tanabe ${ }^{2}$, T Nishida', T Inagawa', Y Yorikoshi', Y Kida', TTamura', K Ota', T Otani', T Sadamori', K Une', R Tsumura', Y Iwasaki', N Hirohashi' KTanigawa

'Hiroshima University Hospital, Hiroshima-shi, Japan; ${ }^{2 H i r o s h i m a ~ C i t y ~}$ Hospital, Hiroshima-shi, Japan

Critical Care 2013, 17(Suppl 2):P159 (doi: 10.1186/cc12097)

Introduction The Airway scope ${ }^{\circledast}$ (AWS), the KingVision ${ }^{\circledast}(\mathrm{KV})$ and the McGRATH $^{\otimes}$ (MG) are new indirect video laryngoscopes designed to facilitate effective and safe tracheal intubation under various conditions. However, there are few comparative studies as for performance in tracheal intubation attempted by inexperienced personnel. The purpose of this study was to evaluate success rates, time to intubation with use of these devices by inexperienced personnel in a simulated manikin difficult airway.

Methods Twenty-nine fifth-year medical students with no previous experience in tracheal intubation participated in this study. We used an advanced patient simulator (SimMan ${ }^{\oplus}$; Laerdal Medical, Stavanger, Norway) to simulate normal and difficult airway scenarios including cervical spine rigidity, swollen tongue, and pharyngeal edema. The sequences in selecting devices and scenarios were randomized. Success rate for tracheal intubation, and the time required for visualization of the glottis (T1), tracheal intubation (T2), and inflation of the lungs (T3) were analyzed. Also, numbers of audible dental click during the intubation attempt were recorded. The three different intubation devices were tested in four different scenarios by 29 students.

Results All three devices had very high success rates of tracheal intubation (AWS 100\%; KV 100\%; MG 99\%). In the normal airway, T1, T2 and T3 were AWS: $4.4 \pm 3.3$ seconds, $7.5 \pm 3.8$ seconds, $11.1 \pm 3.8$ seconds; KV: $6.9 \pm 6.9$ seconds, $10.3 \pm 7.9$ seconds, $14.1 \pm 8.3$ seconds; MG: $4.6 \pm 1.3$ seconds, $11.4 \pm 5.2$ seconds, $16.1 \pm 5.4$ seconds, respectively (NS). In the three difficult airway scenarios, $\mathrm{T} 1, \mathrm{~T} 2$ and $\mathrm{T} 3$ were AWS: $5.3 \pm 4.7^{*}$ seconds, $10.4 \pm 6.9^{*}$ seconds, $14.2 \pm 6.9^{*}$ seconds; KV: $10.9 \pm 12.8$ seconds, $21.5 \pm 18.1$ seconds, $25.4 \pm 18.3$ seconds; MG: $13.0 \pm 15.3$ seconds, $26.2 \pm 24.6$ seconds, $31.0 \pm 24.3$ seconds, respectively ( ${ }^{*} P<0.05$ AWS vs. KV and MG). The number of audible dental click sounds with the MG was greater than with the AWS and KV (AWS 5\%*; KV 8\%*; MG 28\%*; ${ }^{*} P<0.05$ AWS and KV vs. MG).

Conclusion The AWS, KV and MG had very high success rates of tracheal intubation and are suitable as intubation devices for inexperienced personnel. In the difficult airway, however, the intubation time with AWS was significantly shorter than with KV and MG. These findings suggested that AWS may be most useful device particularly in difficult situations such as emergency settings. Further studies in a clinical setting are needed to confirm these findings.

\section{P160}

Resistance of neonatal endotracheal tubes: a comparison of four commercially available types

J Spaeth, D Steinmann, J Guttmann, S Schumann

University Medical Center Freiburg, Germany

Critical Care 2013, 17(Suppl 2):P160 (doi: 10.1186/cc12098)

Introduction In mechanically ventilated neonates the flow-dependent resistance of the endotracheal tube (ETT) causes a noticeable pressure difference between airway and tracheal pressure [1]. This may potentially lead to retardation of the passive driven expiration and dynamic lung inflation consecutively but more importantly increases 
the work of breathing. The aim of this study was to compare the resistive pressure drop of four commercially available neonatal ETTs with internal diameter (ID) of $2.0 \mathrm{~mm}$.

Methods The pressure-flow relationship of neonatal ETTs (internal diameter $2.0 \mathrm{~mm}$ ) of four different manufacturers (Mallinckrodt, $\mathrm{Hi}$ Contour, Covidien, Dublin, Ireland; Portex, Cole's Neonatal Tube, Smith Medical, St Paul, MN, USA; Rüsch, Silko Clear, Teleflex Medical, Kernen, Germany; and Vygon, Pediatric Endotracheal Tube, Ecouen, France) was determined in a physical model consisting of a tube connector, an anatomically curved ETT and an artificial trachea. The model was ventilated with a sinusoidal gas flow with an amplitude of $\pm 100 \mathrm{ml} /$ second and a ventilation rate ranging from 40 to 60 cycles/minute. The coefficients of an approximation equation considering ETT resistance were fitted separately to the measured pressure-flow curves for inspiration and expiration.

Results The pressure drop profiles of all ETTs were nonlinearly flow dependent. The expiratory pressure drop $\left(\mathrm{P}_{\text {exp }}\right)$ slightly exceeded the inspiratory one $\left(P_{\text {inss }}\right)$. The ETT of Portex Cole's Neonatal Tube had a significant lower pressure $\operatorname{drop}\left(\mathrm{P}_{1 \mathrm{nsp}} 8.8 \pm 0.3 \mathrm{cmH}_{2} \mathrm{O}\right.$ vs. $\mathrm{P}$ $-11.2 \pm 0.2 \mathrm{cmH}_{2} \mathrm{O}$ at an air flow of $100 \mathrm{ml} / \mathrm{second}$ ) compared with all other ETTs (Mallinckrodt: $P \quad 19.2 \pm 0.4 \mathrm{cmH}_{2}$ Ovs. $P \quad-24.3 \pm 0.7 \mathrm{cmH}_{2} \mathrm{O}$; Rüsch: $P_{\text {insp }} 28.3 \pm 0.8 \mathrm{cmH}_{2} \mathrm{O}$ vs. $\mathrm{P}_{\text {exp }}-33.0 \pm 0.8 \mathrm{cmH}_{2} \mathrm{O}$; Vygon: $\mathrm{P}_{\text {ins }}$ $19.2 \pm 0.4 \mathrm{CmH}_{2} \mathrm{O}$ vs. $P_{\text {exp }}-22.6 \pm 0.6 \mathrm{cmH}_{2} \mathrm{O}$, all $P<0.05$ ) for all flow and ventilation rates, respectively.

Conclusion The ETT resistance highly contributes to the total airway resistance in neonatal ventilation. The flow-dependent pressure drop of shouldered Cole's Neonatal Tube (Portex) was up to $70 \%$ less in inspiration (resp. 67\% in expiration) compared with straight tubes with an internal diameter corresponding to the narrow part of Cole's tube. We conclude that neonatal intubation with a Cole's tube can clearly reduce the resistive load due to the endotracheal tube and thus potentially prevent additionally work of breathing.

Reference

1. Guttmann J, et al:: Crit Care Med 2000, 28:1018-1026

\section{P161}

NT-proBNP and cardiac cycle efficiency changes during extubation process in critically ill patients

F Franchi, P Mongelli, M Cozzolino, B Galgani, C Ragozzino, C Bianchi,

B Biagioli

University of Siena, Italy

Critical Care 2013, 17(Suppl 2):P161 (doi: 10.1186/cc12099)

Introduction Several factors can lead to weaning failure from mechanical ventilation (MV). Among these, cardiac dysfunction is one of the main causes. NT-proBNP has been proposed as a biomarker of cardiovascular function during weaning from MV. Unfortunately it does not provide for a continuous monitoring of cardiac function. Pulse wave analysis may serve as a continuous bedside monitoring tool of cardiovascular performance. Cardiac cycle efficiency (CCE) is an indirect index of left ventricular performance obtained by the pulse contour method MostCare (Vygon, Padova, Italy). The aim of this study was to evaluate the correlation between NT-proBNP and CCE and the potential usefulness of such variables during the weaning process from MV.

Methods Twenty-two long-term ( $>48$ hours) mechanically ventilated patients capable of performing a weaning trial of spontaneous breathing (SBT) were enrolled in the study. Inclusion criteria were: age $>18$ years and equipment with a standard arterial catheter line. Exclusion criteria were: neuromuscular disease, tracheotomy, renal failure, and traumatic brain injury. During the weaning process, NTproBNP plasma levels, CCE, and standard hemodynamic and ventilatory data were collected 30 minutes before extubation (T1), 2 hours (T2) and 12 hours later (T3). After removal of tracheal tube, patients with a history of heart failure received continuous positive airway pressure (CPAP group). Patients with normal cardiac function were maintained with spontaneous breathing (SB group).

Results Sixty-six paired NT-proBNP and CCE values were obtained. Patients in the SB group and in the CPAP group were 10 and 12, respectively. In both groups there was a trend towards an increase in NT-proBNP values after extubation, an opposite trend was observed regarding CCE values $(P<0.05)$. NT-proBNP levels showed an increase after extubation $(\mathrm{T} 2, \mathrm{~T} 3)$ compared with $\mathrm{T} 1$; conversely, CCE showed an inverse trend. Overall, a negative correlation was found between NT-proBNP and CCE values $(R=-0.81, P<0.001)$. Significant inverse correlations were found between NT-proBNP and CCE at T1, T2, and T3 $(R=-0.91,-0.75$ and -0.73 respectively; $P<0.001)$. The overall correlation between NT-proBNP and CCE was -0.74 in the SB group and -0.86 in the CPAP group. Standard hemodynamic and ventilatory data did not show significant changes during the study.

Conclusion NT-proBNP correlated well with CCE. The latter seems to be an additional attractive index of cardiovascular state that, in association with NT-proBNP changes, may provide information about cardiac function on a beat-by-beat basis during weaning process from MV.

P162

Comparison of outcomes between early and late tracheostomy for critically ill patients

K Suzuki', S Kusunoki', TYamanoue', KTanigawa²

'Hiroshima Prefectural Hospital, Hiroshima, Japan; ${ }^{2 H i r o s h i m a ~ U n i v e r s i t y ~}$ Hospital, Hiroshima, Japan

Critical Care 2013, 17(Suppl 2):P162 (doi: 10.1186/cc12100)

Introduction Tracheostomy is one of the more commonly performed procedures in critically ill patients requiring long-term mechanical ventilation. However, the optimal timing or method of performing tracheostomies in this population remains to be established. In the present study, we compared outcomes of early and late tracheostomy in critically adult patients with different clinical conditions.

Methods All patients needing tracheostomy in the Critical Care Medical Center of Hiroshima Prefectural Hospital from January 2009 to December 2011 were surveyed. Patients with tracheostomy who were not indicated for mechanical ventilation were excluded from the subjects. Early tracheostomy (ET) was defined as $<10$ days after tracheal intubation and late tracheostomy (LT) was defined as $\geq 10$ days after intubation. We compared patient characteristics, type of tracheostomy procedure, length of weaning from ventilator and outcomes between the groups. Data are shown as the mean $\pm \mathrm{SD}$, with unpaired $t$ test and Mann-Whitney $U$ test used for statistical analyses. Statistical significance was accepted at $P<0.05$.

Results One hundred patients were surveyed. The ET and LT groups included 49 and 51 patients, respectively. Tracheostomy was performed using a percutaneous procedure in 48 patients (ET: 25, LT: 23) and a surgical procedure in 52 patients (ET: 24, LT: 28). Sixty-two patients (ET: 34, LT: 28) survived to discharge and 16 patients died in the ICU (ET: 7, LT: 9). Fifty-six patients (ET: 31, LT: 25) were weaned from ventilator support and tracheostomy cannula was removed in 20 patients (ET: $11, \mathrm{LT}: 9$ ). There were no significant differences in type of tracheostomy procedure, period from tracheostomy until ICU and hospital discharge, rate of patients who could be weaned from ventilator and removed tracheostomy cannula, and ICU and hospital mortality between the groups. The length of mechanical ventilation and the time to removal of tracheostomy cannula were significantly shorter in the ET group ( $5 \pm 7$ vs. $26 \pm 41$ and $29 \pm 24$ vs. $94 \pm 83$ days, respectively).

Conclusion In this retrospective study, early tracheostomy reduced the length of weaning after tracheostomy and the time to removal of tracheostomy cannula, while there were no differences in the length of ICU stay and patient outcome. In critically ill adult patients who require mechanical ventilation, a tracheostomy performed at an earlier stage may shorten the duration of artificial ventilation. A further randomized clinical trial is essential to determine the effectiveness and safety of early tracheostomy.

Reference

1. Arabi Y, et al:: Crit Care 2004, 8:R347-R352.

P163

Ultrasound scanning for percutaneous dilatational tracheostomy:

a systematic review

R Pugh, A Slater

Glan Clwyd Hospital, Bodelwyddan, UK

Critical Care 2013, 17(Suppl 2):P163 (doi: 10.1186/cc12101)

Introduction Percutaneous dilatational tracheostomy (PDT) remains a frequently performed procedure in the ICU. However, there is great 
variability in the course of blood vessels in the pre-tracheal area. A 5\% risk of clinically relevant bleeding was recently reported for patients undergoing PDT [1]. We conducted a systematic review of reports evaluating clinical outcomes following use of ultrasound scanning (US) for PDT.

Methods Two investigators performed a search of the literature using the following databases: CENTRAL, Embase, MEDLINE and SCOPUS. The following eligibility criteria were used: population including adults $>16$ years managed in the ICU; use of ultrasound to guide decisionmaking pre-PDT or guide PDT performance; report of clinically relevant outcome measures. Nonrandomised controlled trials were classified according to Cochrane Non-Randomised Study Methods Group criteria [2] and evaluated for risk of bias.

Results An initial search identified 2,043 reports, of which 10 studies met eligibility criteria: eight case series, one randomised controlled trial (RCT) and one prospective cohort study, incorporating 488 patients. Two studies specifically reported data on patients with obesity $(n=29$ patients) and one study reported data for a group of patients with spinal cord fixation $(n=6)$. US was used to guide decision to perform PDT or surgical tracheostomy in five studies, with decision to perform surgical tracheostomy ranging from 0 to $27 \%$ of cases. US was used to guide insertion point in seven studies, and used real-time in four studies. Times to perform US-guided PDT were reported in four studies (ranging from 8 to 12 minutes). No studies compared time taken with or without US. Data on complications of procedure were reported in nine studies. Minor bleeding was reported for eight cases $(1.6 \%$ overall). Prolonged bleeding was reported in two cases ( $0.4 \%)$. There were no episodes of catastrophic bleeding among 488 cases. High risk of bias was identified in five studies in terms of patient selection. An intervention protocol was not defined in three reports. No attempt was made at blinding any aspect of the 10 studies.

Conclusion Use of US guidance could theoretically help minimise risk of haemorrhagic complications during PDT and perhaps reduce time taken to perform PDT. However, there is currently inadequate evidence from controlled cohort studies or RCTs to suggest that routine use for PDT in selected or unselected groups improves clinically relevant outcome measure.

\section{References}

1. Delaney et al: Crit Care 2006, 10:R55.

2. Higgins JPT, Green S (Eds): Cochrane Handbook for Systematic Reviews of Interventions. Version 5.1.0. The Cochrane Collaboration; 2011. [www.cochrane-handbook.org]

\section{P164}

Evaluation of a new device for emergency transcricoid ventilation in a manikin model

P Persona, P Diana, A Ballin, F Baratto, M Micaglio, C Ori

Clinica di Anestesia e Medicina Intensiva, Padova, Italy

Critical Care 2013, 17(Suppl 2):P164 (doi: 10.1186/cc12102)

Introduction Failed airway situations are potentially catastrophic events and require a correct approach with appropriate tools. Recently, Ventrain has been presented as a manual device for emergency ventilation through a small-bore cannula, which can provide expiratory assistance by applying the Venturi effect.

Methods We used the SimulARTI Human Patient Simulator to evaluate Ventrain. Initially, we studied the effectiveness and security in ventilating and oxygenating the patient. In a second phase, the Ventrain performance was compared with what is considered to be the present gold standard (Quicktrach II, Portex Mini-Trach II Seldinger Kit, Melker Emergency Cricothyrotomy Catheter Set). Seven anesthesiologists performed an emergency transcricoid ventilation with each device in the same setting.

Results Ventrain provided an average tidal volume of $334 \mathrm{ml}$ and an average minute volume of $2.4 \mathrm{I}$ in the considered situation, with a modification of $\mathrm{PAO}_{2}$ from 32 to $702 \mathrm{mmHg}$ and of $\mathrm{PACO}_{2}$ from 54.5 to $38.8 \mathrm{mmHg}$. In the second phase, the time needed to obtain an effective oxygenation with Ventrain was found to be shorter than other devices (median difference; vs. Minitrach -60 seconds; vs. Melker -35 seconds; vs. Quicktrach -25 seconds) (Figure 1); the ability to remove $\mathrm{CO}_{2}$ resulted bigger (average difference: vs. Minitrach -11.9;

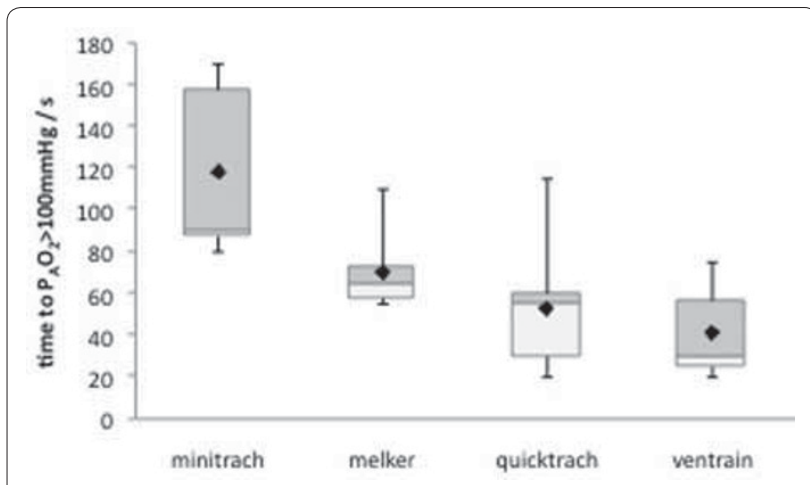

Figure 1 (abstract P164). Oxygenation time to oxygen alveolar pressure $>100 \mathrm{mmHg}$.

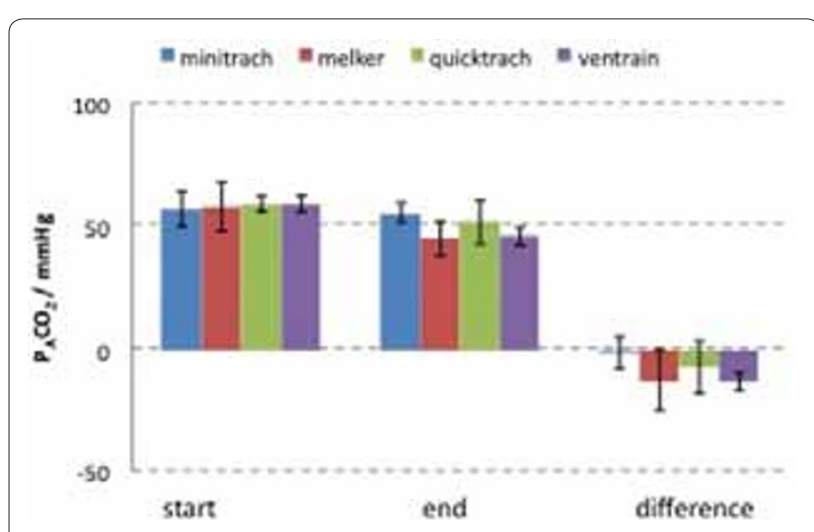

Figure 2 (abstract P164). Mean carbon dioxide alveolar pressure at the start and at the end of the test.

vs. Melker -0.3; vs. Quicktrach -5.9) (Figure 2) and moreover the users judged it more favorably.

Conclusion In this manikin study, Ventrain seemed to be able to appropriately oxygenate and ventilate a patient in a CICV situation. When compared with the best available choices, it has shown not to be inferior.

Reference

1. Cook TM, Nolan JP, Magee PT, Cranshaw JH: Needle cricothyroidotomy. Anaesthesia 2007, 62:289-291.

P165

Device for measuring bronchodilator delivery and response in resource-limited settings

CW Carspecken, D Talmor

Harvard Medical School and Beth Israel Deaconess Medical Center, Boston,

MA, USA

Critical Care 2013, 17(Suppl 2):P165 (doi: 10.1186/cc12103)

Introduction Effective delivery of aerosolized bronchodilators for patients with asthma is crucial for adequate therapy in critical care and emergent settings. Often administered with pressure-metered dose inhalers (pMDIs), bronchodilator delivery depends on the correct patient technique during administration [1] and the ability to measure treatment response, which are difficult to monitor at the point of care and particularly so in resource-poor settings where standard inhospital monitoring is unavailable [2].

Methods A point-of-care device for airflow measurement during bronchodilator delivery was designed and tested for use in resourcelimited settings. The handheld device was constructed from a clinical aerosol delivery tube with a bidirectional sensor for pressure differential detection about the aerosol element (Figure 1). The custom low-cost 

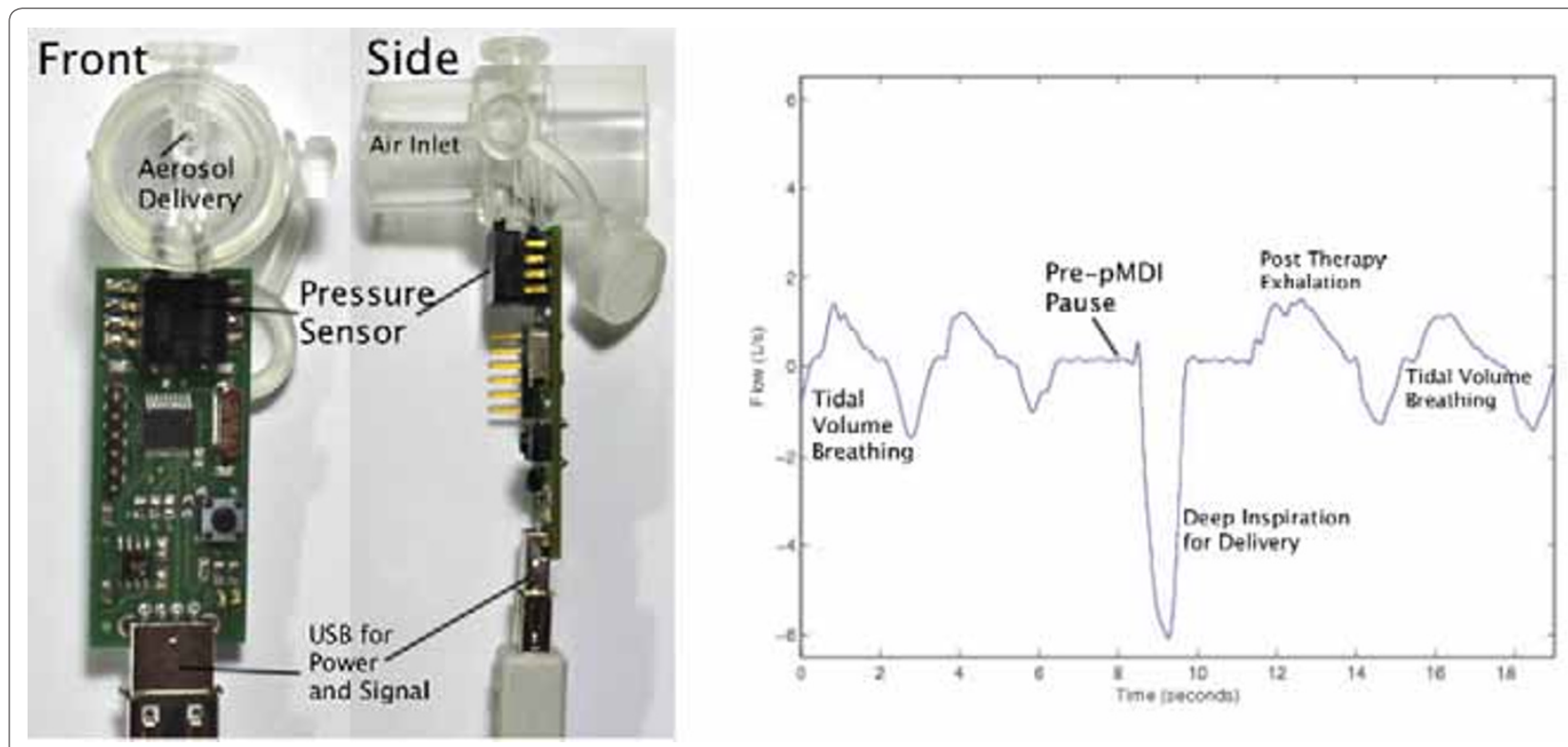

Figure 1 (abstract P165).

$(<\$ 11)$ electronics were designed such that no power supply would be needed apart from a computer or mobile device. Collection of airflow signal and calibration was performed with a standard 3 I syringe with flow volume measurement on one adult subject.

Results Calibration of the assembled device over range of 0.5 to $3 \mathrm{l} /$ second enabled conversion of pressure difference to air flow with a mean measured tube resistance of $0.258\left(\mathrm{kPa}^{*} \mathrm{~second}\right) / \mathrm{l}$. Robust signal responses to an adult subject's continuous respiratory maneuvers on the tube itself were demonstrated. Subject performance of the pMDI technique with subsequent tidal volume breathing was recorded and analyzed (Figure 1).

Conclusion Design and calibration of a novel low-cost monitoring device for bronchodilator delivery monitoring enabled detection and recording of characteristic flow volume respiratory patterns for point-of-care diagnostics in resource-limited settings. Future work will require clinical testing and automated detection of the correct pMDI patient technique.

References

1. Ari A, et al:: Respir Care 2012, 57:613-626.

2. Riviello E, et al:: Crit Care Med 2011, 39:860.

\section{P166}

Evidence-based guidelines and protocols for the management of adult patients with a tracheostomy: a systematic review

J Siddiqui', PB Sherren², MA Birchall ${ }^{3}$

'North West London Hospitals NHS Trust, London, UK; 'Barts and The London NHS Trust, London, UK; ${ }^{3}$ The Royal National Throat Nose and Ear Hospital,

London, UK

Critical Care 2013, 17(Suppl 2):P166 (doi: 10.1186/cc12104)
Introduction Protocol-based care of the tracheostomised patient is important, as adverse events confer a high rate of mortality. Little is known regarding the existence of formal evidence-based guidelines on tracheostomy care. The aim of this study was to perform a systematic review for evidence-based guidelines on adult tracheostomy care.

Methods A systematic search of PubMed, MEDLINE, guideline clearinghouses, centres of evidence-based practice, and professional societies' guidelines relating to care of adult patients with a tracheostomy was performed by two reviewers. In addition, a Google search of publicly available tracheostomy care guidelines was performed. Search terms: (tracheostom* OR tracheotom*) AND (protocol* OR guideline* OR standard* OR management OR consensus $\mathrm{OR}$ algorithm*). Filters: English language, human, from 1 January 1990 to date, adult patients. Guideline appraisal criteria: the quality of guidelines retrieved was assessed using the Appraisal of Guidelines Research and Evaluation II (AGREE II) instrument [1].

Results The search results are summarised in Table 1. A total of 80 guidelines were identified. Five were found to satisfy the AGREE ॥ criteria and only three related to the entire spectrum of tracheostomy management. The majority was informal and was not published or evidence based.

Conclusion Five evidence-based guidelines on adult tracheostomy management were identified. This may represent a paucity of evidence on the subject, suggesting that further clinical trials on the topic are needed to contribute to the evidence base. This also highlights the need for international consensus on the topic, to reduce duplication of efforts, standardise practice, and improve outcomes.

\section{Reference}

1. Appraisal of Guidelines for Research and Evaluation: Instrument. AGREE II [http://www.agreetrust.org/]

Table 1 (abstract P166). Summary of results returned following systematic review

\begin{tabular}{|c|c|c|c|c|c|}
\hline Database & $\begin{array}{c}\text { Total } \\
\text { results }\end{array}$ & $\begin{array}{l}\text { Results with } \\
\text { limits }\end{array}$ & $\begin{array}{l}\text { Full texts } \\
\text { retrieved }\end{array}$ & $\begin{array}{l}\text { Guidelines } \\
\text { found }\end{array}$ & $\begin{array}{l}\text { AGREE instrument } \\
\text { compliant }\end{array}$ \\
\hline PubMed & 4,685 & 1,596 & 33 & & \\
\hline MEDLINE & 3,859 & 1,337 & 17 & 26 & 2 \\
\hline CINAHL & 711 & 172 & 6 & & \\
\hline Medical societies/guideline websites/clearinghouses & N/A & N/A & N/A & 54 & 3 \\
\hline Google & $17,600,000$ & 497 & N/A & & \\
\hline
\end{tabular}


P167

Survey of emergency tracheostomy management in the East of England region

N Lawrence, L Oakley, C Swanavelder, M Palmer

West Suffolk Hospital, Bury St Edmunds, UK

Critical Care 2013, 17(Suppl 2):P167 (doi: 10.1186/cc12105)

Introduction The 4th National Audit Project of the Royal College of Anaesthetists [1] concluded that the majority of airway-related significant complications in ICUs resulted from displaced or blocked tracheostomies and recommended together with the Intensive Care Society and the National Tracheostomy Safety Project that each ICU in the UK should have an emergency airway management plan and guidelines [2]. The aim of this survey was to establish whether such guidelines exist and are familiar to those working within the ICUs of the East of England (EoE), their ease of availability in an emergency and the degree of emergency tracheostomy training within the region.

Methods Data collection was via a telephone survey of 11 ICUs in the EoE training region during July 2012 with one senior ICU nurse and one ICU trainee questioned per hospital. Questions related to the existence and accessibility of guidelines for tracheostomy emergencies, and to the respondent's degree of emergency tracheostomy training and their perceived availability of formal training.

Results All 11 ICUs questioned perform and manage tracheostomies. Of 22 respondents, 10 knew of guidelines covering all of the emergencies described above and their location. Four respondents thought that these guidelines were accessible in an emergency setting, one-half of which were on computer systems requiring a login and search function. With regards to emergency management, 19 respondents felt competent in a tracheostomy emergency; almost exclusively through experience and in-house teaching. No respondents were aware of any formal emergency tracheostomy management courses.

Conclusion Despite national guidance within the UK this survey highlights that implementation and awareness of emergency tracheostomy guidelines in ICUs in the EoE region is poor, and when present they are not readily accessible in an emergency. Emergency training has largely been informal and the availability of formal training courses has not been recognised. In order to improve patient safety there is a need to ensure that emergency tracheostomy management including guidelines, equipment and formalised tracheostomy emergency training are adopted and embraced universally.

References

1. Cook TM, et al:: Results of the 4th National Audit Project of the Royal College of Anaesthetists. Br J Anaesth 2011, 106:632-642.

2. McGrath $B A$, et al: Multidisciplinary guidelines for the management of tracheostomy and laryngectomy airway emergencies. Anaesthesia 2012, 67:1025-1041

P168

Can we keep neck breathers safe? A survey on training adequacy of medical staff caring for tracheostomy/laryngectomy patients

C Tjen, G Rajendran, S Hutchinson

Norfolk and Norwich University Hospital, Norwich, UK

Critical Care 2013, 17(Suppl 2):P168 (doi: 10.1186/cc12106)

Introduction A fatal incident related to a blocked tracheostomy tube prompted a review in our Trust. To provide safe tracheostomy care, changes in staffing, education and operational policies were recommended. Training of potential first responders to tracheostomy or laryngectomy emergencies remains outstanding. We aim to quantify the training deficit. Tracheostomies are common in critical care but these patients require ongoing management of an artificial airway on discharge to the ward and even the community. In 2010 our critical care unit cared for 108 tracheostomy patients, of which 30 were transferred to the wards. The 4th National Audit Project highlighted complications including hypoxic brain injury and death [1] and the National Patient Safety Agency recognised a number of avoidable aspects [2]. Existing guidelines for management of these patients including emergencies are not widely known.

Methods An anonymous online survey was sent to all trainees who may respond to a tracheostomy emergency in our organisation. Trainees in anaesthesia/critical care, general medicine, general surgery,
ENT, thoracics and A\&E were approached. All completed forms were included.

Results We achieved a response rate of 39\% (65/168). Respondents comprised: $33 \%$ anaesthesia/critical care, $47 \%$ medicine and $14 \%$ surgery. Over one-half $(36 / 65)$ had managed tracheostomy/laryngectomy emergencies, with $42 \%(15 / 36)$ of these incidents occurring on the wards and one in an outpatient clinic. Only $20 \%(13 / 65)$ had received any formal training on management of a blocked/misplaced tracheostomy tube and only $18 \%(12 / 65)$ were aware of any guidelines. One-third of responders lacked confidence in management of these emergencies and $88 \%$ felt they would benefit from formal training including simulation.

Conclusion The population of patients with exteriorised tracheas is increasing and represents a high-risk group. Management of airway emergencies in these patients is not part of standard life-support courses. According to our trainees, these scenarios are relatively common and a significant proportion of first responders are poorly equipped to deal with them. Our Trust will be including specific training on the emergency management of neck breathers as part of in-house resuscitation training. We would contend that national resuscitation courses should consider doing the same.

References

1. Cook T, et al.: Br J Anaesth 2011, 106:632-642.

2. McGrath B, et al:: Postgrad Med J 2010, 86:522-525.

\section{P169}

Laryngeal mask as a safe and effective ventilatory device during Blue Dolphin tracheostomy in the ICU

A De Nicola', MJ Sucre', G Donnarumma', A Corcione ${ }^{2}$

'San Leonardo Hospital, Castellammare di Stabia, Italy; ${ }^{2}$ Monaldi Hospital, Naples, Italy

Critical Care 2013, 17(Suppl 2):P169 (doi: 10.1186/cc12107)

Introduction Usually percutaneous tracheostomy is accomplished via the tracheal tube. Some severe complications during percutaneous dilatational tracheostomy (PDT) may be related to poor visualization of tracheal structures. The alternative implies extubation and reinsertion of a laryngeal mask (LMA). An accidental extubation as well as an injuring of the vocal cords (because of the inflated cuff during dislocation) appears impossible in this method. Subjectively, the bronchoscopic view obtained via a LMA seems to be better than that obtained with an endotracheal tube (ET) [1,2].

Methods In this prospective observational study, the bedside PDT was performed using the Ciaglia Blue Dolphin method in 150 critically ill patients. The patient's tracheal tube was exchanged for a LMA Fastrach ${ }^{\text {TM }}$ before undertaking PDT. The insertion of the LMA, the quality of ventilation, the blood gas values, the view of the tracheal puncture site, and the view of the balloon dilatation were rated as follows: very good (1), good (2), barely acceptable (3), poor (4), and very poor (5).

Results PDTs with LMA were successful in $99.3 \%$ of the patients $(n=149)$. The ratings were 1 or 2 in $96 \%$ of cases with regards to ventilation and to blood gas analysis, in $96.6 \%$ for identification of relevant structures and tracheal puncture site, and in $93.3 \%$ for the view inside the trachea during PDT. A rating of 5 was assigned to one patient requiring tracheal reintubation for inadequate ventilation. There were no damages to the bronchoscope or reports of gastric aspiration.

Conclusion The Blue Dolphin PDT using a LMA showed definite advantages regarding inspection of dilation process. This method improves visualization of the trachea and larynx during a video-assisted procedure and prevents the difficulties associated with the use of an ET such as cuff puncture, tube transection by the needle, accidental extubation, and bronchoscope lesions. The LMA results as an effective and successful ventilatory device during PDT. This may be especially relevant in cases of difficult patient anatomy where improved structural visualization optimizes operating conditions. The intensivist performing PDT should be scrupulous when deciding which method to use. In our ICU the Blue Dolphin PDT with LMA has become the procedure of choice. References

1. Linstedt U, et al: Anesth Analg 2010, 110:1076-1082.

2. De Nicola A, Sucre MJ: Abstract 829. Proceedings Book of 40th Congress of the Society of Critical Care Medicine 2011: January 15-192011; San Diego, CA. Mount Prospect, LL: Society of Critical Care Medicine. 
P170

Real-time ultrasound-guided balloon dilatational percutaneous tracheostomy is a safe procedure in critically ill patients: an evaluation study

A Taha, A Shafie, M Mostafa, P Wallen, H Hon, R Marktanner Sheikh Khalifa Medical City, Abu Dhabi, United Arab Emirates Critical Care 2013, 17(Suppl 2):P170 (doi: 10.1186/cc12108)

Introduction Balloon dilatational percutaneous tracheostomy with radial outward dilation minimizes bleeding and injury to tracheal rings [1]. Using the bronchoscope during the puncture of the trachea holds a risk of inaccurate placement, of hypoventilation and of needle puncturing the bronchoscope. This study was conducted in order to evaluate the safety features and feasibility of combining real-time ultrasound-guided puncture of the trachea with visualization of the needle path during tracheal puncture [2]. We provide precise localization of tracheal cartilage and proper site puncture for the stoma by ultrasound, with subsequent performance of balloon dilatational percutaneous tracheostomy using the Blue dolphin technique.

Methods Twenty-five patients including 15 males and 10 females, mean age 61 years, with age range from 23 to 102 years, underwent bedside percutaneous tracheostomy combining real-time ultrasound and the Blue Dolphin technique.

Results The median time for the procedure was 15 (12 to 20) minutes. Targeted placement for the tracheostoma between the second and third or the third and fourth tracheal ring was achieved in $100 \%$. No significant complications (for example, tracheal bleeding, puncture posterior tracheal wall, misplacement of the tracheal cannula) occurred. One fractured tracheal ring was identified using bronchoscopy after the procedure. No conversion into a bronchoscopically guided or into a surgical open technique was necessary.

Conclusion This study demonstrates the feasibility and safeness combining real-time ultrasound guidance and balloon dilatational percutaneous tracheostomy (Blue Dolphin technique) in critically ill patients. In our division, this technique has become the standard bedside tracheostomy procedure because it combines excellent patient safety features with avoidance of intraprocedural tube misplacement, hypoventilation and accidental bronchoscopy damage even in technically difficult cases [3].

References

1. Gromann TW, et al:: Anesth Analg 2009, 108:1862-1866.

2. Venkatakrishna Rajajee et al.: Crit Care 2011, 15:R54.

3. Guinot et al:: Crit Care 2012, 16:R40.

\section{P171}

Right ventricular restriction in interventional lung assist for acute respiratory distress syndrome

G Tavazzi', M Bojan², S Canestrini², M White², S Price²

'University of Pavia Foundation Policlinico San Matteo IRCCS, Pavia, Italy;

${ }^{2}$ Royal Brompton Hospital, London, UK

Critical Care 2013, 17(Suppl 2):P171 (doi: 10.1186/cc12109)

Introduction Acute cor pulmonale (ACP) is associated with increased mortality in patients ventilated for acute respiratory distress syndrome (ARDS). Interventional lung assist (iLA) allows a lung-protective ventilatory strategy, whilst allowing $\mathrm{CO}_{2}$ removal, but requires adequate right ventricular (RV) function. RV restriction (including presystolic pulmonary A wave) [1] is not routinely assessed in ARDS.

Methods A prospective analysis of retrospectively collected data in patients with echo during iLA was performed. Data included epidemiologic and ventilatory factors, LV/RV function, evidence of RV restriction and pulmonary hemodynamics. Data are shown as mean $\pm \mathrm{SD} /$ median (interquartile range).

Results Thirty-two patients ( $45 \pm 17$ years), 22 male (68\%), SOFA score $11.15 \pm 2.38$ were included. Pulmonary hypertension (PHT) was $53 \%$, and hospital mortality $43 \%$. Mortality was not associated with age, days on iLA, length of ICU stay, inotropic support, nitric oxide or level of ventilatory support, but was associated with pressor requirement $(P=0.005)$, a worse $\mathrm{PaO}_{2}: \mathrm{FiO}_{2}$ ratio (9.4 (7.8 to 12.6$)$ vs. 15.2 (10.7 to 23.9), $P=0.009)$ and higher pulmonary artery pressures $(56.5 \mathrm{mmHg}$ (50 to 60 ) vs. 44.5 ( 40.5 to 51.2 ), $P=0.02$ ). No echo features of ACP were

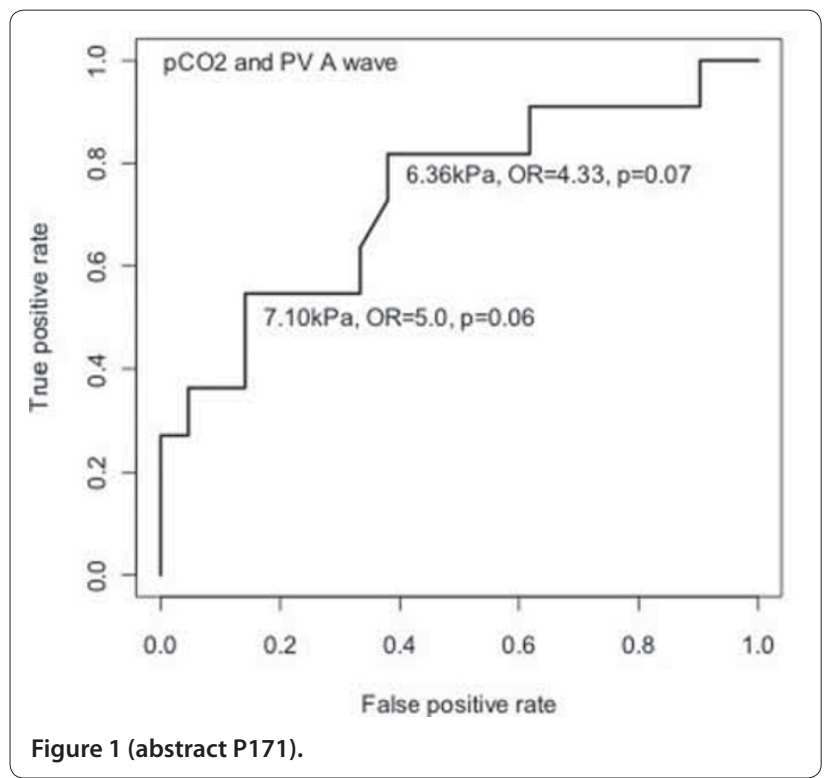

found, with no significant difference between RV systolic function, pulmonary acceleration time and pulmonary velocity time integral between survivors and nonsurvivors. The incidence of RV restriction was high (43\%), and independent of PHT, RV systolic function and level of respiratory support, but correlated with $\mathrm{CO}_{2}$ levels (restrictive $7.1 \mathrm{kPa}$ (7.4 to 8.0) vs. 6.1 (5.8 to 6.8 ), $P=0.03$ ). See Figure 1 .

Conclusion Typical echo features of ACP were not seen in this study, possibly because of the protective ventilatory strategies allowed by use of iLA. The incidence of RV restriction may reflect more subtle abnormalities of RV function. Further studies are required to elucidate RV pathophysiology in critically ill adult patients with ARDS.

Reference

1. Cullen S, et al: Circulation 1995, 91:1782-1789.

P172

Left ventricular electromechanical dyssynchrony and mortality in cardiothoracic intensive care

G Tavazzi', M Bojan², A Duncan³, A Vazir³, S Price

'University of Pavia Foundation Policlinico San Matteo IRCCS, Pavia, Italy;

${ }^{2}$ Necker-Enfants Malades University Hospital, Paris, France; ${ }^{3}$ Royal Brompton

Hospital, London, UK

Critical Care 2013, 17(Suppl 2):P172 (doi: 10.1186/cc12110)

Introduction Global left ventricular electromechanical dyssynchrony (GLVD) is uncoordinated LV contraction that reduces the extent of intrinsic energy transfer from the myocardium to the circulation leading to a reduction in peak LV pressure rise, prolonged total isovolumic time (t-IVT) and fall in stroke volume [1]. This potentially important parameter is not routinely assessed in critically ill cardiothoracic patients.

Methods A prospective analysis of retrospectively collected data in cardiothoracic ICU patients who underwent echocardiography was performed. In addition to epidemiological factors, echo data included comprehensive assessment of LV/RV systolic and diastolic function including Doppler analysis of isovolumic contraction/ relaxation, ejection time (ET) and filling time (FT). t-IVT was calculated as $(60$ - (total ET + total FT)) and the Tei Index as (ICT + IRT) / ET. t-IVT $>14$ second/minute and Tei index $>0.48$ were used to define GLVD [2]. Data are shown as mean $\pm S D /$ median (interquartile range).

Results A total of 103 patients ( $63.5 \pm 18.4$ years), 65 male (63\%), APACHE II score $(14.6 \pm 7.4)$ were included. The prevalence of GLVD was high $(24 / 103,22 \%)$ and associated with significantly increased mortality, $7.5 \%$ vs. $25 \%(P=0.02)$. There was no difference in requirement for cardiorespiratory support between the two populations, but there were significant differences (no GLVD vs. GLVD) in requirement for 
pacing ( $35 \%$ vs. $62 \%, P=0.02)$, atrial fibrillation ( $20 \%$ vs. $41 \%, P=0.03)$, QRS duration (92.0 (80.0 to 120.0) vs. 116.5 (95.0 to 154.0$), P=0.01)$ and QTc (460.0 (416.0 to 498.5$)$ vs. 477.5 (451.2 to 541.0$), P=0.02$ ). There was no significant difference in ejection fraction (no GLVD 43.0 (35.0 to 49.5 ) vs. GLVD 39.6 (29.5 to 49.7$), P=0.43$ ), mitral regurgitation ( $40.5 \%$ vs. $62.5 \%, P=0.06$ ), or any other measures of LV systolic or diastolic function between the two groups. There was good correlation between the two methods used to assess dyssynchrony (LV t-IVT:LV Tei index correlation coefficient $=0.80, P<0.001$ ).

Conclusion GLVD that limits cardiac output is common in the cardiothoracic ICU, and significantly related to mortality. When diagnosed, the underlying cause should be sought and treatment instigated to minimize the t-iVT (pacing optimization/revascularization/ inotrope titration/volaemia optimization).

References

1. Duncan A, et al.: J Am Coll Cardiol 2003, 41:121-128.

2. Tei C, et al:. J Am Coll Cardiol 1996, 28:658-664.

P173

Safety of ultrasound-guided central venous access in critically ill patients with uncorrected coagulopathy

G Reusz', C Langer', G Egervari', P Sarkany', A Csomos²

'Markhot Ferenc Hospital, Eger, Hungary;'2Semmelweis University, Budapest, Hungary

Critical Care 2013, 17(Suppl 2):P173 (doi: 10.1186/cc12111)

Introduction Correction of coagulopathy before central venous catheter (CVC) insertion is a common practice; however, when ultrasound guidance is used this is controversial as mechanical complications are rare. Studies in oncology patients suggest that CVC placement without prior correction of coagulopathy is safe but no studies are available for critically ill patients and guidelines do not give recommendations $[1,2]$. We do not routinely correct coagulopathy, even if severe, when ultrasound guidance is used and the purpose of this retrospective study was to evaluate the safety of this practice. Methods Data for all ultrasound-guided interventions, including complications, are prospectively collected in our department for audit purposes; in this study we involved only CVC insertions in the ICU between February 2011 and November 2012. Electronic medical and laboratory records and paper-based nursing charts were retrospectively studied for all interventions, specifically looking for blood results, coagulation abnormalities and intervention-related complications.

Results In the study period, ultrasound guidance was employed for a total of 291 central line insertions in 220 ICU patients. Coagulopathy was detected in 127 cases at the time of CVC placement (43.6\%). On the day of CVC insertion, coagulation abnormalities were corrected in 20 cases (15.7\%); 33 out of 50 patients with severe coagulopathy (66.0\%) and 74 out of 77 patients with coagulopathy of moderate severity (96.1\%) had no correction at all. Correction was started only after CVC insertion for reasons unrelated to CVC placement in a further eight and two patients with severe and less severe coagulopathy $(16.0 \%$ and $2.6 \%)$, respectively. No bleeding complications were observed.

Conclusion In patients undergoing CVC insertion in our ICU, coagulopathy is common. We observed uncomplicated CVC placement in all 41 patients with severe uncorrected coagulopathy and in a further 76 patients with coagulopathy of moderate severity. When combined with other studies, our data suggest that ultrasound-guided CVC placement without routine correction of coagulation abnormalities may be safe in the ICU.

References

1. Cavanna L, et al:: World J Surg Oncol 2010, 8:91.

2. Lamperti M, et al:: Intensive Care Med 2012; 38:1105-1117.

P174

Cyanoacrylate glue prevents early bleeding of the exit site after CVC or PICC placement

G Scoppettuolo, MG Annetta, C Marano, ETanzarella, M Pittiruti

Catholic University, Rome, Italy

Critical Care 2013, 17(Suppl 2):P174 (doi: 10.1186/cc12112)

Introduction Early bleeding from the exit site after CVC or PICC placement is a very common event that causes difficulties in the patient's care and logistical problems. In our experience, the rate of significant local bleeding after placement of PICCs without reverse tapering may be as high as $40 \%$ at 1 hour and $15 \%$ at 24 hours, while the rate of bleeding after placement of a large-bore dialysis catheter is above $50 \%$ at 1 hour.

Methods The aim of this pilot study was to verify the efficacy of a cyanoacrylate glue in reducing the risk of early bleeding at the exit site after CVC or PICC placement. We studied a group of adult patients consecutively undergoing placement of polyurethane CVCs or PICCs without reverse tapering in a non-intensive ward of our hospital. All lines were inserted according to the same protocol, which included $2 \%$ chlorhexidine antisepsis, maximal sterile barriers, ultrasound guidance, EKG guidance and securement with sutureless device. Two minutes after placement of the glue, the exit site was covered with a temporary gauze dressing, which was replaced by transparent membrane at 24 hours. All patients were assessed at 1 hour and at 24 hours.

Results In 65 consecutive patients (45 PICCs, 11 dialysis catheters and nine (VVs), there was no significant local bleeding at 1 hour or at 24 hours after catheter placement. No local adverse reaction occurred. No damage to the polyurethane of the catheters was detected.

Conclusion Glue is an inexpensive and highly effective tool for avoiding the risk of early bleeding of the exit site after catheter placement. We also suggest that in the next future the glue might prove to have beneficial collateral effects on the risk of extraluminal contamination (by reducing the entrance of bacteria in the space between the catheter and the skin), as well as on the risk of dislocation (by increasing the stability of the catheter inside the skin breach).

P175

Intracardiac ECG for confirmation of correct positioning of central venous catheters is safe and cost-effective

M Eriksson, R Dörenberg

Surgical Sciences, Uppsala, Sweden

Critical Care 2013, 17(Suppl 2):P175 (doi: 10.1186/cc12113)

Introduction About 10 years ago the use of chest radiographs as the golden standard to ensure correct positioning of central venous catheters (CVC) was questioned. The frequent use of CVCs was also challenged. We decided to retrospectively evaluate our routines in a large surgical unit in a Swedish university hospital.

Methods All X-rays were centrally registered. Chest X-ray performed in our unit is almost entirely used to confirm CVC positioning. The Certofix CVC set for the Seldinger technique in combination with Certodyn Universaladapter (B Braun, Germany) is now used as the routine equipment and the right jugular vein is our standard approach.

Results In 2002 the total number of X-rays performed in patients at our unit was 2,306, which corresponds to the approximate number of inserted CVCs at that time, since a confirmatory X-ray was routine. $\mathrm{X}$-rays were rarely performed on other indications in our unit. X-ray costs were at that time approximately $€ 300,000$ ( $€ 130 /$ each). The year after, 1,726 chest X-rays were performed, reflecting both the use of intracardiac confirmation of correct CVC position and also a reduced use of CVCs. This trend has continued over time. In 2011 approximately 600 CVCs were inserted at our unit. X-rays were performed in about $20 \%$ of these cases. The cost for a chest X-ray is today $€ 200$, meaning that $X$-ray costs were approximately $€ 24,000$. We have not experienced any medical problems when intracardiac ECG was used for positioning confirmation. On the contrary, aspiration of venous blood without apparent $\mathrm{p}$-waves in a patient with sinus rhythm may suggest improper placement of the CVC; for example, the right brachial vein.

Conclusion If we had continued to use CVCs at the same frequency as we did 10 years ago, and used X-ray confirmation in practically all cases, we would have paid approximately $€ 460,000$ annually. Reduced use of CVCs, in combination with intracardiac confirmation of CVC positioning, has not only allowed us to reduce costs associated with CVC insertion by more than $€ 400,000$, corresponding to a reduction rate of more than $90 \%$, but also decreased the patient's exposure to $\mathrm{X}$-ray irradiation.

References

1. Stas M, et al: Eur J Surg Oncol 2001, 27:316-320.

2. Joshi A, et al:. Indian Crit Care Med 2008, 12:10-14. 
P176

Is automated brachial cuff measurement of arterial pressure less accurate in cases of arrhythmia?

K Lakhal', S Faiz'2, M Martin', AS Crouzet', F Reminiac², S Ehrmann²,

R Cinotti', X Capdevila 3 , K Asehnoune ${ }^{4}$, Y Blanloeil', B Rozec', T Boulain ${ }^{2}$

${ }^{\prime}$ Centre Hospitalier Universitaire Laennec, Nantes, France; ${ }^{2}$ Centre Hospitalier

Universitaire Bretonneau, Tours, France; ${ }^{3}$ Centre Hospitalier Universitaire

Lapeyronie, Montpellier, France; ${ }^{4}$ Centre Hospitalier Universitaire Hotel-Dieu,

Nantes, France

Critical Care 2013, 17(Suppl 2):P176 (doi: 10.1186/cc12114)

Introduction In cases of arrhythmia, the beat-to-beat variation of arterial pressure (AP) may impair the accuracy of automated cuff measurements. Indeed, this oscillometric device relies on the detection of arterial wall oscillations. Our aim was to determine, in ICU patients, whether brachial cuff measurements are really less reliable during arrhythmia than during regular rhythm.

Methods Patients with arrhythmia and carrying an intra-arterial catheter were prospectively and consecutively included in this multicenter study. After each arrhythmic inclusion, a regular rhythm patient was included. A second inclusion was possible in case of change in the cardiac rhythm. Three pairs of invasive and brachial cuff (Philips ${ }^{\circledast}$ MP70 monitor) measurements of mean arterial pressure (MAP) were respectively averaged. Some patients underwent a second set of measurements, after a cardiovascular intervention (passive leg raising, volume expansion, initiation of/increase in catecholamine infusion) allowing the assessment of MAP changes.

Results In the 111 analyzed inclusions (in 103 patients) there was only one failure in displaying a brachial cuff measurement of MAP. Arrhythmic patients (atrial fibrillation $88 \%$, frequent extrasystoles $7 \%$, flutter $5 \%)$ were similar $(P>0.3)$ to patients in regular rhythm for MAP (median 74 (IQR 67 to 80 ) vs. 75 (69 to 84) $\mathrm{mmHg}$ ), SAPS ॥ score, BMl, arm circumference, norepinephrine administration (36\% vs. $35 \%)$, mechanical ventilation $(80 \%$ vs. $81 \%)$, and site of the intraarterial catheter (radial artery: $80 \%$ vs. $85 \%$ ). Between arrhythmic and regular rhythm patients: the agreement (Bland-Altman analysis) between invasive and brachial cuff measurements of MAP was similar (mean bias $-0.4 \pm 7.2$ (limits of agreement $-14 / 14$ ) $\mathrm{mmHg}$ vs. $3.0 \pm 8.2$ $(-13 / 19) \mathrm{mmHg}$, respectively); the detection of hypotension (invasive MAP $<65 \mathrm{mmHg}$ ) by the brachial cuff was of similar reliability (area under the ROC curve $(A \cup C)=0.91(95 \% \mathrm{Cl}=0.80$ to 0.97$)$ vs. $A U C=0.98$ (0.89 to 1), $P=0.2)$; and the detection of a response ( $>10 \%$ increase in MAP) to therapy was of similar reliability (AUC $=1(0.85$ to 1$)(n=22)$ vs. AUC $=0.99$ (0.78 to 1$)(n=17), P=0.5)$.

Conclusion These preliminary results suggest that arrhythmia does not impair the reliability of automated cuff measurements of MAP.

P177

Cardiac output monitoring in brain-stem-dead potential organ donors: an audit of current UK practice

CJ Wright ${ }^{1}$, A Broderick ${ }^{2}, \mathrm{G}$ Mandersloot ${ }^{3}$

'Glasgow Royal Infirmary, Glasgow, UK; ${ }^{2}$ National Health Service Blood and

Transplant, London, UK: ${ }^{3} T$ The Royal London Hospital, London, UK

Critical Care 2013, 17(Suppl 2):P177 (doi: 10.1186/cc12115)

Introduction Significant changes in haemodynamics occur after brain stem death (BSD) and there is evidence that yield of transplantable organs may be decreased in donors who remain preload responsive prior to donation [1], suggesting that optimisation of the cardiac output (CO) may be beneficial in potential organ donors. We describe current UK practice with regard to CO monitoring in this group.

Methods We reviewed a database of 287 brain-stem-dead potential organ donors collected by specialist nurses in organ donation (SN-OD) over a 6-month period (30 April 2011 to 31 October 2011) across multiple UK centres. The database contained data on donor management in the period from initial SN-OD review to immediately prior to transfer to the operating theatre. We analysed data on CO monitoring and vasopressor/inotrope use. Where information was missing/not recorded in the dataset, the treatment referred to was interpreted as not given/not done.
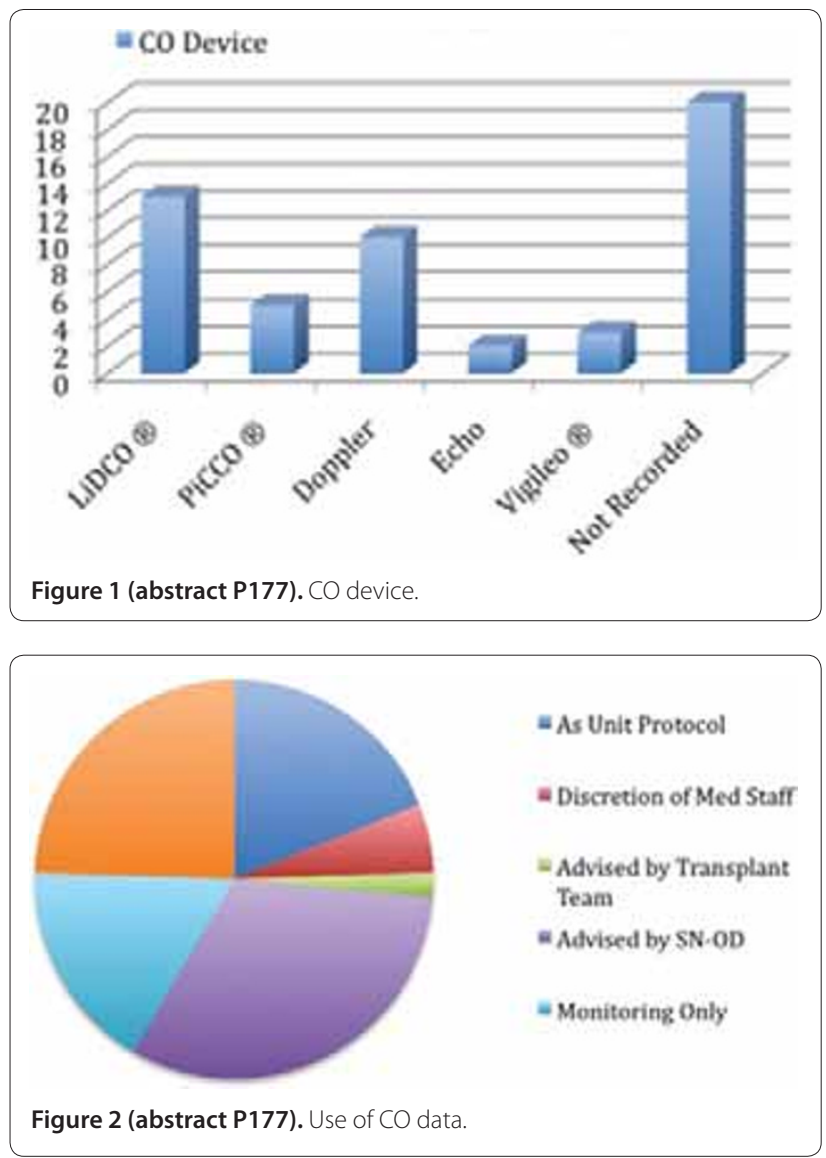

Results Fifty-three patients (18.5\%) had evidence of CO monitoring. LiDCO was the most popular method (Figure 1). A total of $264(94 \%)$ patients received treatment with vasopressors and/or inotropes. CO data were utilised in a variety of ways (Figure 2).

Conclusion The majority of potential donors require vasopressors and/or inotropes post BSD, but it seems only a minority currently have their CO monitored. There is variation in how CO data are utilised to direct haemodynamic management. We welcome the development of standardised bundle-driven donor management.

Reference

1. Murugan R, et al;; HIDonOR Study Investigators: Preload responsiveness is associated with increased interleukin- 6 and lower organ yield from braindead donors. Crit Care Med 2009, 37:2387-2393.

P178

Indocyanine green plasma disappearance rate for assessment of liver function: re-evaluation of normal ranges and impact of biometric data

W Huber, M Kranzmayr, C Schultheiss, W Reindl, A Krug, B Saugel, U Mayr, RM Schmid

Klinikum rechts der Isar, Technical University of Munich, Germany

Critical Care 2013, 17(Suppl 2):P178 (doi: 10.1186/cc12116)

Introduction The indocyanine green plasma disappearance rate (ICG-PDR) is a dynamic liver function test that can be non-invasively measured by pulse densitometry. ICG-PDR is associated with mortality and other markers of outcome. Due to predominant use of ICG-PDR in the ICU setting, the normal range is based on scarce data available outside the ICU and given with 18 to $25 \%$ /minute.

Methods To prospectively re-evaluate the normal range and to analyze the potential impact of biometric data on ICG-PDR, we measured ICG-PDR (i.v. injection of 0.25 mg/kg ICG; LiMON, Pulsion, Munich, 
Germany) in 95 outpatients with ulcerative colitis (UC). Due to a prevalence of primary sclerosing cholangitis (PSC) of $5 \%$ in UC, patients were asked regarding previous diagnosis of PSC. Additionally, serum bilirubin, AST, ALT, INR, AP, YGT and cholinesterase were determined. Association of biometric data (age, gender, height, weight) and diagnosis of PSC on ICG-PDR were evaluated using Spearman correlation, ROC and multivariate analysis (statistics: IBM SPSS 20).

Results A total of 95 patients ( 54 male, 41 female), age $43.2 \pm 15.5$ (21 to 76) years, weight $74 \pm 16 \mathrm{~kg}$, height $175 \pm 10 \mathrm{~cm}, \mathrm{BMI} 23.7 \pm 4$, bilirubin $0.48 \pm 0.27 \mathrm{mg} / \mathrm{dl}$, AST $26.8 \pm 11.4 \mathrm{U} / \mathrm{l}$, ALT $26.8 \pm 14.9 \mathrm{U} / \mathrm{l}$, previous diagnosis of PSC $8 / 95$ (8.4\%). ICG-PDR ranged from 13.8 to 44.0 with a mean of $28.2 \pm 6.8$ and a median of $27.0 \% /$ minute. In univariate analysis ICG-PDR was significantly associated with age $(r=-0.480 ; P<0.001)$, weight $(r=-0.262 ; P=0.011)$ and female gender $(r=0.221 ; P=0.032)$, but not to height $(P=0.681)$. In multivariate analysis $(R=0.459)$ including the variables age, gender, height, weight and diagnosis of PSC, only age was independently $(P<0.001)$ associated with ICG-PDR. With each year in age, ICG-PDR decreased by $0.206 \% /$ minute. In ROC analysis, ICG-PDR above the upper normal range $(>25 \% /$ minute) was significantly associated with young age (AUC $0.746 ; P<0.001$ ).

Conclusion ICG-PDR (normal) values should be corrected for age. With a decrease in ICG-PDR of $0.206 \%$ per year, the range between the upper and lower normal level ( 25 to $18 \%$ /minute) is passed through within about 35 years of life. These findings are in accordance with functional loss of other organ functions (for example, cardiac output, glomerular filtration rate) with increasing age. Normal ranges of innovative markers of organ function mainly derived from ICU populations should be re-evaluated outside the ICU.

\section{P179}

Prediction of 28-day mortality by indocyanine green disappearance rate, other markers of hepatic function and transpulmonary thermodilution parameters: a prospective study in 154 patients W Huber, D Ertekin, T Langer, B Saugel, T Lahmer, M Messer, C Spinner, C Schultheiss, RM Schmid Klinikum rechts der Isar, Technical University of Munich, Germany Critical Care 2013, 17(Suppl 2):P179 (doi: 10.1186/cc12117)

Introduction Hepatic dysfunction has been associated with outcome of ICU patients. However, most scoring systems including APACHE II only marginally reflect acute liver dysfunction on admission. Indocyanine green (ICG) is eliminated by hepatobiliary excretion. Therefore, the ICG plasma disappearance rate (ICG-PDR) is used as a dynamic liver function test. ICG-PDR has been associated with mortality in several studies.

Methods A prospective study to compare prediction of 28-day mortality by ICG-PDR, other markers of liver function and scoring systems (primary endpoint). In the subgroup of patients with transpulmonary thermodilution (TPTD) monitoring (PiCCO device; Pulsion, Munich, Germany), predictive capabilities of ICG-PDR were compared with cardiac index $(\mathrm{Cl})$, extravascular lung water index (EVLWI), global end-diastolic volume index (GEDVI) and pulmonary vascular permeability index (PVPI). ICG-PDR (i.v. bolus of $0.25 \mathrm{mg} / \mathrm{kg}$ ICG; LiMON device, Pulsion) and all other predictors were determined within 48 hours after admission. Statistics: IBM SPSS 20.

Results A total of 154 patients (46 female, 108 male), age $59 \pm 13$ years, APACHE II score $16.0 \pm 5.7$, SOFA score $7.6 \pm 4.2$. Etiology: sepsis $14.4 \%$, cirrhosis $28.8 \%$, Gl bleeding $8.9 \%$, ARDS $17.8 \%$, cardiogenic shock $4.1 \%$, acute renal failure $3.4 \%$, various $22.6 \%$. The 28 -day mortality was significantly predicted by APACHE II (ROC-AUC: $0.762 ; P<0.001)$ and SOFA (AUC: $0.784 ; P<0.001$ ). Among markers of hepatic function on admission, ICG-PDR provided the largest AUC $(0.742 ; P<0.001)$, which was larger than for GOT (AUC: $0.646 ; P=0.019$ ), bilirubin (AUC: 0.641 ; $P=0.023$ ) and INR (AUC: $0.614 ; P=0.067$ ). Among TPTD parameters, only PVPI significantly predicted 28-day mortality (AUC: $0.643 ; P=0.043$ ), whereas $\mathrm{Cl}$, GEDVI and EVLWI were not predictive. Prediction of 28-day mortality by SOFA could not be improved by models including any hepatic parameter. By contrast, ICG-PDR was independently $(P=0.036)$ associated with mortality when included in a model $(R=0.58)$ with APACHE II. This model based on APACHE $\|$ and ICG-PDR provided the largest of all ROC-AUCs (AUC: $0.804 ; P<0.001$ ). ICG-PDR itself was independently associated with age $(P=0.025)$, but not with any other biometric parameter (gender, weight and height).

Conclusion ICG-PDR on admission is an independent predictor of 28-day mortality. Predictive capabilities particularly of APACHE II can be improved by combination with ICG-PDR. Among TPTD-derived parameters, only PVPI provides significant prediction of mortality.

P180

Mixed and central venous oxygen saturation are not interchangeable in patients with cardiogenic shock after cardiac surgery

S Romagnoli, P Balsorano, F Landucci, A De Gaudio

AOUC Careggi, Florence, Italy

Critical Care 2013, 17(Suppl 2):P180 (doi: 10.1186/cc12118)

Introduction Mixed venous oxygen saturation $\left(\mathrm{SVO}_{2}\right)$ represents a well-recognized parameter of oxygen delivery $\left(\mathrm{DO}_{2}\right)$-consumption $\left(\mathrm{VO}_{2}\right)$ mismatch and its use has been advocated in critically ill patients in order to guide hemodynamic resuscitation [1] and oxygen delivery optimization. Nevertheless, the pulmonary artery catheter (PAC) is not readily available and its use is not devoid of risks. Furthermore, its use has been decreasing in recent years in surgical and cardiac surgical patients as the benefit of guiding therapy with this device is unclear [2-4]. Central venous oxygen saturation $\left(\mathrm{SCVO}_{2}\right)$ has been suggested as an alternative to $\mathrm{SVO}_{2}$ monitoring due to its feasibility in several settings. Unfortunately concerns arise from its capability to correlate with $\mathrm{SVO}_{2}$, the relationship being influenced by several factors, such as hemodynamic impairment and pathological process. Hemodynamic instability and shock often complicate cardiac surgery, and the $\mathrm{SVO}_{2}-$ $\mathrm{ScVO}$, relationship has not been specifically investigated in this setting. The aim of this study is to compare $\mathrm{SVO}_{2}$ and $\mathrm{ScVO}_{2}$ values in patients with cardiogenic shock after cardiac surgery.

Methods A prospective observational study was designed and conducted. Inclusion criteria were: patients who had underwent elective or urgent/emergent cardiac surgery, with cardiac index (Cl) $<2.5 \mathrm{l} / \mathrm{minute} / \mathrm{m}^{2}$ estimated by means of a PAC, left ventricle ejection fraction (LVEF) $<40 \%$, lactate $>2 \mathrm{mmol} / \mathrm{l}$, age $>18$ years. A central venous catheter (CVC) and a PAC were inserted for each patient before surgery in the same right internal jugular vein in accordance with standard procedure. Proper position of the PAC was confirmed with pressure tracings and chest X-ray. Mixed and central venous blood samples were collected from the distal ports of the PAC and CVC respectively 30 minutes after ICU admission, and every 6 hours for a total of three samples in a 24-hour period for each patient. All blood samples were analyzed by a co-oximeter (Radiometer ABL800 flex; Radiometer, Copenhagen, Denmark). Statistical analysis was performed by Stats Direct (Ver.2.5.8, Cheshire, UK) and GraphPad (Vers. Prism 4.0; San Diego, CA, USA). All data were tested for normal distribution with the Kolmogorov-Smirnov test. Statistical analysis was performed by linear regression analysis. The agreement between absolute values of $\mathrm{ScvO}_{2}$ and $\mathrm{SvO}_{2}$ were assessed by the mean bias and $95 \%$ limits of agreement (LOA) ((mean bias \pm 1.96$) \times$ standard deviation) according to the method described by Bland and Altman [5].

Results A total of 20 patients were enrolled. In 18 out of 20 cases all three blood samples were collected. In two patients only two blood samples were drawn as they exited the inclusion criteria. Linear regression analysis between the two variables resulted in an $r^{2}$ of 0.708 . Bland-Altman analysis (Figure 1) for the pooled measurements of $\mathrm{SvO}_{2}$ and $\mathrm{ScvO}_{2}$ showed a mean bias and LOA of $6.82 \%$ (SD of bias 5.3 ) and -3.71 to $+17.3 \%$ respectively.

Conclusion $\mathrm{ScVO}_{2}$ has been advocated as an attractive and simple indicator of $\mathrm{DO}_{2}-\mathrm{VO}_{2}$ mismatch [6]. Its role as a surrogate of the wellestablished $\mathrm{SVO}_{2}$ has been investigated in several settings, and it has been purposed in the hemodynamic resuscitation of critically ill septic patients [1]. Nevertheless, the $\mathrm{SVO}_{2}-\mathrm{ScVO}_{2}$ relationship can be influenced by several factors due to $\mathrm{ScVO}_{2}$ dependency from global blood flow redistribution that can occur during hemodynamic impairments. It has been shown previously that in healthy people $\mathrm{ScVO}_{2}$ values tend to underestimate $\mathrm{SVO}_{2}$ values, due to the higher oxygen content from inferior vena cava [7]. During circulatory shock, not homogeneous oxygen extraction and regional blood flow 


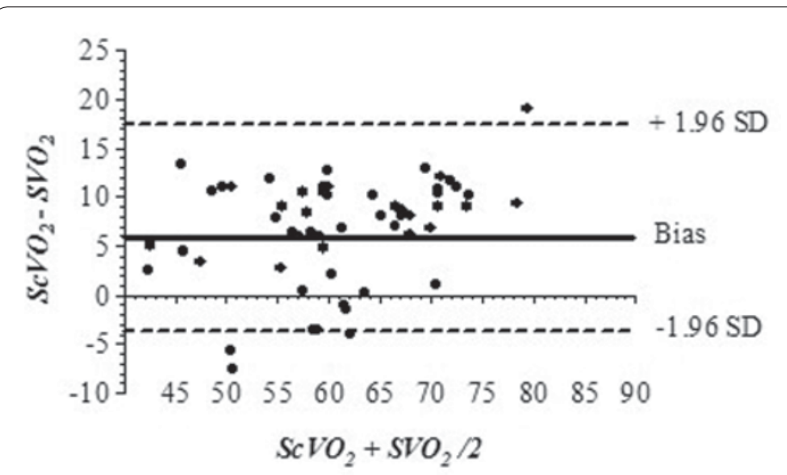

Figure 1 (abstract P180).

redistribution make $\mathrm{SVO}_{2}$ a more reliable parameter suggesting the global adequacy of cardiac output rather than $\mathrm{ScVO}_{2}$. In this study we aimed at evaluating $\mathrm{SvO}_{2}-\mathrm{SCVO}_{2}$ differences in patients with cardiogenic shock, as defined by hyperlactatemia, low $\mathrm{Cl}$, and LVEF $<40 \%$, after cardiac surgery. Our results highlighted a great variability for these two parameters, with a clinically unacceptable mean bias and $\mathrm{LOA}$. As expected, $\mathrm{ScVO}_{2}$ values were consistently higher.

\section{References}

1. Rivers $E$, et al: Early goal-directed therapy in the treatment of severe sepsis and septic shock. N Eng/ J Med 2001, 345:1368-1377.

2. Edwards JD: Oxygen transport in cardiogenic and septic shock. Crit Care Med 1991, 19:658-663.

3. Hadian M, et al: Evidence based of the use of the pulmonary artery catheter: impact data and complications. Crit Care 2006, 10(Suppl 3):S11-S18.

4. Connors $A F$, et al: The effectiveness of right heart catheterization in the initial care of critically ill patients. JAMA 1996, 276:889-897.

5. Bland JM, Altman DG: Statistical methods for assessing agreement between two methods of clinical measurement. Lancet 1986, 1:307-310

6. Walley KR: Use of central venous oxygen saturation to guide therapy. Am J Respir Crit Care Med 2011, 184:514-520

7. Reinhart K, et al:: Comparison of central-venous to mixed-venous oxygen saturation during changes in oxygen supply/demand. Chest 1989, 95:1216-1221

P181

Pulmonary hemodynamic disorder in pediatric sepsis and their correction with L-arginine infusion

M Georgiyants, V Korsunov

Kharkov Medical Academy Post-Graduate Education, Kharkov, Ukraine Critical Care 2013, 17(Suppl 2):P181 (doi: 10.1186/cc12119)

Introduction The acute respiratory distress syndrome and pulmonary hypertension $(\mathrm{PH})$ is one of the factors of septic mortality. Some data demonstrate arginine deficiency as an important pathogenic factor of septic $\mathrm{PH}$. We suppose that intravenous L-arginine infusion improves NO production and reduce $\mathrm{PH}$.

Methods We examined 46 patients with sepsis, severe sepsis and septic shock in accordance with San Antonio Criteria. Organ dysfunction severity was defined according to SOFA. The mean pulmonary artery pressure (MPAP) was estimated by Doppler method, cardiac output (CO), and stroke volume (SV) - by ultrasound $\mathrm{M}$-mode. $\mathrm{SpO}_{2}$, mean arterial pressure (MAP), blood gas, and plasma NO level was evaluated. The patients of Group 1 ( $n=21$, age $22.1 \pm 8.5$ months) had respiratory and hemodynamic support, and antibiotics. Group $2(n=25$, age $27.0 \pm 11.2$ months) had the same treatment with continuous i.v. infusion of $5 \mathrm{ml} / \mathrm{kg}$ body weight $4.2 \%$ L-arginine solution during 24 hours.

Results The patients of Groups 1 and 2 did not have statistical difference of SOFA ( $4.1 \pm 1.0$ vs. $4.4 \pm 0.7)$, need for ventilator support $(57.0 \pm 11.0 \%$ vs. $44.0 \pm 10.0 \%)$, and dose of inotropes $(P>0.05)$. The reduction of MPAP and increase of $\mathrm{SaO}_{2}$ was significantly higher in
Table 1 (abstract P181). Hemodynamic parameters before and after treatment

\begin{tabular}{lcccc}
\hline Value & $\begin{array}{c}\text { Group 1 } \\
\text { before }\end{array}$ & $\begin{array}{c}\text { Group 1 } \\
\text { after }\end{array}$ & $\begin{array}{c}\text { Group 2 } \\
\text { before }\end{array}$ & $\begin{array}{c}\text { Group 2 } \\
\text { after }\end{array}$ \\
\hline $\mathrm{MAP}(\mathrm{mmHg})$ & $66.9 \pm 3.4$ & $68.8 \pm 3.0$ & $72.8 \pm 2.0$ & $71.8 \pm 1.3$ \\
$\mathrm{MPAP}(\mathrm{mmHg})$ & $43.5 \pm 2.6$ & $44.3 \pm 3.4$ & $50.8 \pm 3.0$ & $28.8 \pm 2.1$ \\
$\mathrm{CO}\left(\mathrm{I} / \mathrm{minute} / \mathrm{m}^{2}\right)$ & $4.2 \pm 0.2$ & $5.0 \pm 0.4$ & $4.8 \pm 0.2$ & $4.8 \pm 0.3$ \\
$\mathrm{SpO}_{2}(\%)$ & $93.7 \pm 1.2$ & $93.4 \pm 1.5$ & $94.6 \pm 0.7$ & $98.1 \pm 0.3$ \\
\hline
\end{tabular}

Group 2 compared with Group $1(P<0.001)$. The increase in plasma NO level was significantly higher in Group 2 (Table 1).

Conclusion L-Arginine i.v. infusion $5 \mathrm{ml} / \mathrm{kg}$ during 24 hours increased NO blood level and decreased MPAP in pediatric sepsis, but did not deteriorate hemodynamic system values.

P182

Utility of transesophageal echocardiography in the ICU:

a preliminary US perspective

A Kaynar, D Phillips, H Gomez, M Lischner, S Melhem, K Subramaniam, M Pinsky

University of Pittsburgh, PA, USA

Critical Care 2013, 17(Suppl 2):P182 (doi: 10.1186/cc12120)

Introduction While TEE is providing a direct assessment of the cardiac function and volume status as a diagnostic tool, until recently it has been impractical to be continuously available for monitoring. A new disposable, monoplanar TEE probe (ImaCor) can remain in the patient for up to 72 hours, allowing repeated measures of ventricular function and volume status, parameters needed to monitor response to therapy. Methods We assessed the benefit these TEE data provided in the assessment of five domains: hypovolemia, right ventricular dysfunction, left ventricular dysfunction, sepsis, and valvular abnormality. Bedside practitioners listed their diagnoses before and after seeing primary TEE images perform by trained physicians. We used a 0 to 5 Likert scale to assess differential diagnosis before and after the TEE, comparing changes using a paired $t$ test.

Results All requests for TEE were to access hemodynamic instability. A total of 18 patients were screened and nine were eligible, in which 16 total TEE studies were performed. There were no complications with TEE and all patients tolerated the long-term placement of the probe well. Of the five diagnostic domains studied, right ventricular failure was the most commonly underdiagnosed contributor to the hemodynamic instability among patients prior to TEE $(P=0.054)$ (Figures 1 and 2).

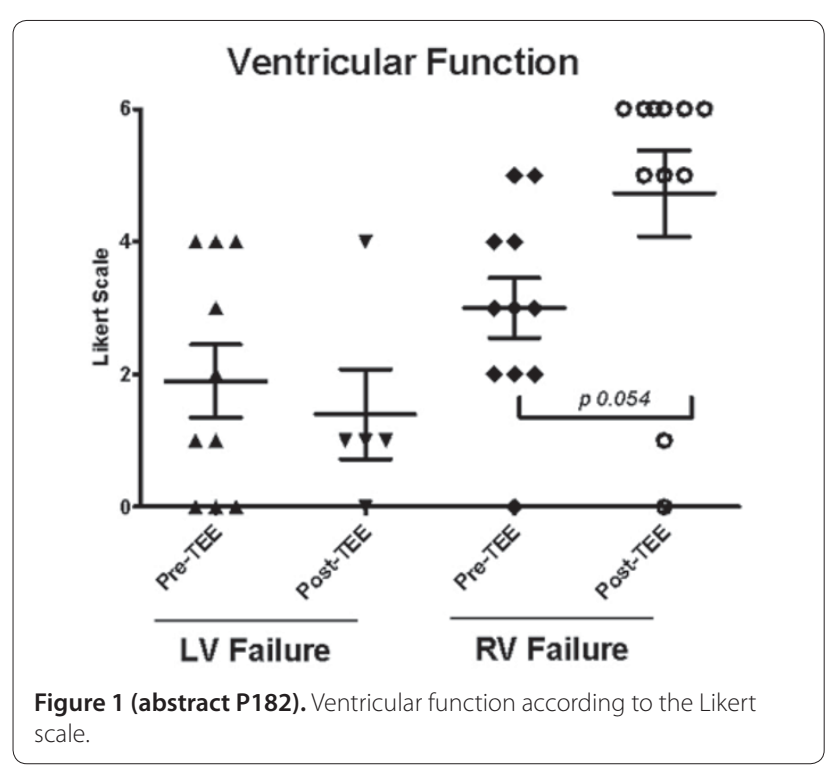




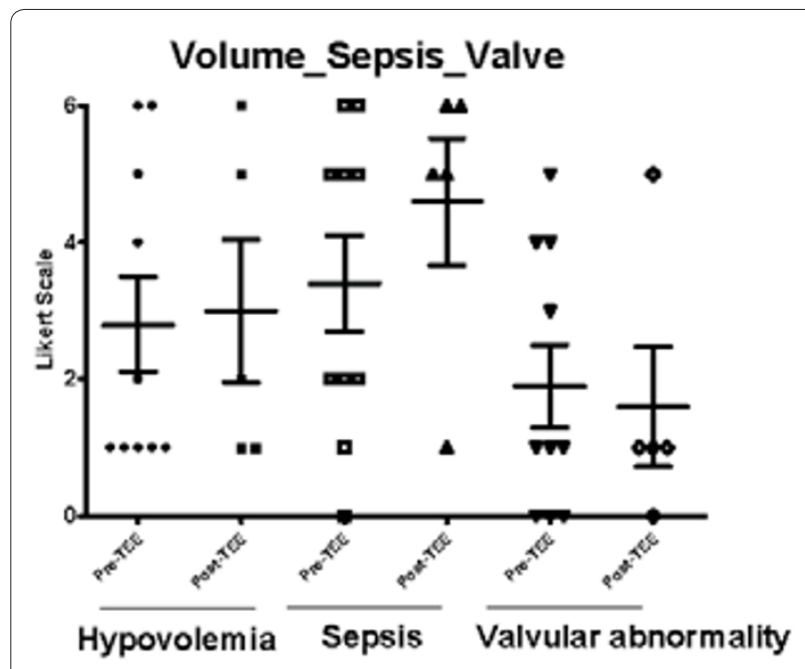

Figure 2 (abstract P182). Volume status, valve, sepsis according to the Likert scale.

Conclusion Our results suggest that having continuously available TEE for monitoring and management of hemodynamically unstable patients increases awareness of right ventricular dysfunction in the ICU. References

1. Vieillard-Baron A, et al: Intensive Care Med 2004, 30:1734-1739.

2. Monnet X, et al:: Intensive Care Med 2005, 31:1195-1201.

P183

Cardiopulmonary monitoring in Thai ICUs: results of ICU-RESOURCE I surveys

K Chittawatanarat', A Wattanatham², D Sathaworn², C Permpikul3,

TSCCM Study Group ${ }^{4}$

'Chiang Mai University, Chiang Mai, Thailand; 'Pramongkudklao Hospital, Bangkok, Thailand; ${ }^{3}$ Siriraj Hospital, Mahidol University, Bangkok, Thailand;

${ }^{4}$ Thai Society of Critical Care Medicine, Bangkok, Thailand

Critical Care 2013, 17(Suppl 2):P183 (doi: 10.1186/cc12121)

Introduction Although rapid progress in ICU monitoring with advanced equipments has been developed, there were limited data on ICU monitoring in Thailand. The objective of this study was to determine the current utilization of monitoring in Thai ICUs.

Methods A self-administered questionnaire was developed by the TSCCM research subcommittee. Data verification was processed by an online medical research tools program (OMERET).

Results A total of 350 questionnaires were sent to ICUs throughout Thailand. In total, 256 questionnaires were confirmed after being received at the end of June 2012. Of these, 140 filled forms (56.9\%) were returned for final analysis. More than $70 \%$ of the ICUs had basic hemodynamic monitoring. Less than $10 \%$ of general and regional hospitals could perform cardiac output monitoring by thermodilution technique compared with $60 \%$ of academic teaching hospitals. New and advanced hemodynamic monitoring techniques such as pulse pressure variation, systolic pressure variation, stroke volume variation, PiCCO, Vigileo-Flo Tract, Pleth variability index device and echocardiography were available only in ICUs of academic teaching hospitals except ultrasound-based techniques including transthoracic and transesophageal echocardiography and USCOM. For respiratory monitoring, all ICUs had a $\mathrm{SpO}_{2}$ monitoring device but only one-half of them had end-tidal $\mathrm{CO}_{2}$ monitoring. Nearly $80 \%$ of ventilator support in participating ICUs was capable of displaying graphic waveform monitoring. Only $43.6 \%$ of participating ICUs had a ventilator machine that could calculate lung mechanics data. Advanced respiratory monitoring such as EIT and esophageal pressure monitoring are available only in ICUs of academic teaching hospitals. There was no
ICU in Thailand that was capable of measuring extravascular lung water. None of the Thai ICUs used transcutaneous $\mathrm{PCO}_{2}$, near-infrared spectroscopy, gut mucosal tonometry and sublingual sidestream darkfield for tissue perfusion monitoring. Only four ICUs had transcutaneous $\mathrm{PO}_{2}$. However, measuring the level of lactate as one of the tissue perfusion markers was routinely performed in about $50 \%$ of the ICUs. Conclusion There were variations in monitoring performance in Thai ICUs. These vary by type of hospital. Academic ICUs had a tendency for advance monitoring in overall aspects. Some advance monitoring used in developed countries is also unavailable in Thailand.

\section{P184}

Establishing a critical care echocardiography laboratory

KYastrebov', A McLean²

'The St George Hospital, Sydney, Australia; ${ }^{2}$ Nepean Hospital, Sydney, Australia Critical Care 2013, 17(Suppl 2):P184 (doi: 10.1186/cc12122)

Introduction Echocardiography is increasingly utilized by intensive care physicians in everyday practice. Standardization of echocardiographic studies and reporting, quality assurance and medicolegal requirements necessitate establishment of a dedicated system within the critical care setting. We describe the process of setting up a critical care echocardiography (CCE) laboratory based on our experience from three separate ICUs.

Methods A retrospective review and analysis of the process involved in establishment of echocardiography laboratories within ICUs.

Results Creating a CCE service involves a number of stages and takes several years to achieve. Major components include staffing, equipment, quality control, study archiving and networking capability. For staffing the objective is to identify and recruit staff with adequate training and expertise in CCE, providing 24/7 specialist cover in addition to supporting and training junior medical and nursing staff. There is further a need to acquire funding for high-quality ultrasound machines and related hardware as well as long-term DICOM-based archiving and reporting systems. This should be based on projections of annual volumes of echo studies and corresponding digital storage. Networking connectivity is highly desirable, including obligatory back-up solutions and site allocations. A business case incorporating all the above should precede any development as identifiable funding sources and administrative approval are essential. The implementation stage requires the presence of a project leader who can organize the trialing of scanners, archiving, reporting and research systems, ensure compatibility with existing hospital and cardiology networks, and who can assist in individualizing archiving and reporting software reflecting institutional and ICU specifics. Coordination with the IT department is very important. Clear contractual vendor obligations for service, maintenance and future upgrades of hardware and software need to be specified. Training and credentialing of staff is best achieved within a systematic framework that includes ongoing competency review, education and QA programs. Partnership with cardiology may benefit both groups. Major pitfalls are associated with poor initial training, lack of expertise and leadership, and bad vendor contracts.

Conclusion Establishment of a CCE laboratory requires careful planning, and allocation of adequate human and financial resources. Many potential problems can be identified and prevented in advance. Strong expert leadership plays an important role.

\section{P185}

Contrast-enhanced ultrasonography in the ICU: promising tool or exciting toy?

I Göcze, R Herzog, BM Graf, A Agha, HJ Schlitt, K Pfister, E Jung, T Bein University Medical Centre Regensburg, Germany Critical Care 2013, 17(Suppl 2):P185 (doi: 10.1186/cc12123)

Introduction Contrast-enhanced ultrasonography (CEUS) is a dynamic digital ultrasound-based imaging technique, which allows quantification of the microvascularisation up to the capillary vessels. As a novel method for assessment of tissue perfusion it is ideally designed for use in the ICU. CEUS is cost-effective and safe and can be repeatedly performed at the bedside without radiation and nephrotoxicity. 
Methods The frequency of CEUS use in the multidisciplinary surgical ICU was retrospectively evaluated for the period from 1 September 2011 to 1 September 2012. Furthermore, contributions of this novel method to the management of critically ill ICU patients as well as its accuracy were assessed.

Results In total, 33 CEUS studies were performed in critically ill ICU patients. The most frequent indications included: assessment of the liver perfusion, assessment of the pancreas and kidney perfusion after pancreas and kidney transplantation, assessment of the renal perfusion in acute kidney injury (AKI), assessment of active bleeding and assessment of the bowel perfusion. In all studies, the correct diagnosis was achieved and the transport of critically ill patients to the radiology department for further diagnostic procedures as well as application of iodinated contrast agents was avoided. In 16 cases significant new findings were detected. Twelve of them were missed by conventional standard Doppler ultrasound prior to CEUS. In assessment of seven cases with AKl, impaired or delayed perfusion and microcirculation of the kidney was observed in six patients. In three patients urgent surgical intervention was performed because of CEUS results. In three cases active bleeding was excluded at the bedside due to absence of contrast agent extravasation into hematoma (thigh and perihepatic) or into abdominal cavity, without need for complementary $\mathrm{CT}$ imaging or angiography. In one case the regular perfusion of intestinal anastomosis was confirmed with no need for surgical exploration. None of patients undergoing CEUS manifested any adverse reactions or developed any complications associated with the imaging technique.

Conclusion Contrast-enhanced ultrasonography clearly improves visualization of the perfusion in various tissues. It is very likely to be superior to standard Doppler ultrasound, and is safe and well tolerated in critically ill patients. Promising indications for the use of CEUS in the ICU may be the assessment of kidney microcirculation and assessment of liver perfusion in liver transplant and liver trauma patients.

\section{P186}

Hemodynamic disclosure of septic shock patients by intensive care ultrasound

P Theerawit, Y Sutherasan, T Hongpanat

Ramathibodi Hospital, Mahidol University, Bangkok, Thailand

Critical Care 2013, 17(Suppl 2):P186 (doi: 10.1186/cc12124)

Introduction Even though invasive hemodynamic devices are usually used for assessment of septic shock victims, they cannot evaluate the heart function. LV dysfunction as well as right heart syndrome are not uncommon in sepsis and critical patients. Intensive care ultrasound discloses these data and leads to appropriate treatment.

Methods The study was a prospective cross-sectional study. The measurement was performed within 24 hours of ICU admission. We excluded patients with history of COPD and pulmonary hypertension from any diseases. Only good-quality images acquired from subjects were included for analysis. The primary objective was to disclose how the hemodynamic changed in septic patients by ICU-US.

Results A total of 133 septic patients were measured by ICU-US. Good image quality was acquired in 115 cases $(86.47 \%)$. The mean ages were $57.48 \pm 17.87$ years. The three major causes of sepsis were pneumonia, unknown source, abdominal infection and bacteremia. Heart failure at admission was found only in $1.79 \%$. Previous history of hypertension, DM, and coronary artery disease was found in $12.17 \%, 11.30 \%$, and $1.74 \%$ of patients. The mean LV ejection fraction (LVEF) was $54 \pm 15.43 \%$. The percentages of patients with LVEF $<35 \%, 35$ to $40 \%, 41$ to $45 \%$ and $>45 \%$ were $13.2 \%, 7.5 \%, 8.5 \%$, and $70.8 \%$ respectively. Diastolic dysfunction defined by E/A ratio $<1$ was observed in $47.5 \%$ of patients. A total $44.6 \%$ of cases had cardiac output under $4 \mathrm{l} /$ minute whereas CO over $6 \mathrm{l} /$ minute was found in $18.1 \%$ of cases. The average mean pulmonary artery pressure was $34.75 \pm 15.13 \mathrm{mmHg}$. The proportion of patients with meanPAP over $25 \mathrm{mmHg}$ was $76.2 \%$. RV to LV ratio $>1$ was found in $42.4 \%$ of septic patients.

Conclusion Cardiac dysfunction, namely left ventricle and probably right ventricle, was not uncommon in septic shock patients. Without intensive care ultrasound, all crucial information was delayed until patient deterioration and initial treatment may be harmful. Thus cardiac ultrasound should be used initially to disclose hemodynamic features before routine resuscitation is initiated.

\section{References}

1. Krishnagopalan S, Kumar A, Parrillo JE, Kumar A: Myocardial dysfunction in the patient with sepsis. Curr Opin Crit Care 2002, 8:376-388.

2. Parker MM, McCarthy KE, Ognibene FP, et al:: Right ventricular dysfunction and dilatation, similar to left ventricular changes, characterize the cardiac depression of septic shock in humans. Chest 1999, 97:126-131.

\section{P187}

Influence of positive end-expiratory pressure on four-chamber longitudinal strain analysis by speckle tracking echocardiography F Franchi, A Faltoni, M Cameli, S Cecchini, M Lisi, M Contorni, S Mondillo, S Scolletta

University of Siena, Italy

Critical Care 2013, 17(Suppl 2):P187 (doi: 10.1186/cc12125)

Introduction Speckle-tracking echocardiography (STE) has emerged as an ultrasound technique for accurately evaluating myocardial function also in critically ill patients. By tracking the displacement of the speckles during the cardiac cycle, the strain rate can be measured offline after adequate image acquisition. The aim of the study was to evaluate the effects of the positive end-expiratory pressure (PEEP) on four-chamber longitudinal strain (LS) analysis in critically ill patients.

Methods We enrolled 20 consecutive patients (mean age $64 \pm 18$ ) who needed mechanical ventilation and were admitted to the ICU due to heterogeneous causes. Inclusion criteria were: hypoxia requiring PEEP titration, invasive arterial pressure monitoring, age $>18$. Exclusion criteria were: myocardial dysfunction, cardiac arrhythmias and valvular pathologies. The same operator performed three standard echocardiography measurements (MyLab 70 Xvision; Esaote), each of them after having increased PEEP at 5, 10, and $15 \mathrm{cmH}_{2} \mathrm{O}(\mathrm{T} 1, \mathrm{~T} 2$, $\mathrm{T} 3$, respectively). Blood gas analysis, respiratory, and hemodynamic parameters provided by a pulse contour method were also recorded. STE analysis was performed offline (XStrain ${ }^{T M}$ MyLab 70 Xvision; Esaote). Results Left peak atrial LS (LA-PALS) was significantly reduced from $\mathrm{T} 1$ to $\mathrm{T} 2$, and from T2 to T3 $(40.2 \pm 12 \%, 35.9 \pm 9 \%, 28.4 \pm 8 \%, \mathrm{~T} 1$, T2, T3, respectively; $P<0.05$ ). Right peak atrial LS (RA-PALS) and right ventricular (RV)-LS showed a significant reduction only at T3 (RA-PALS: $44.7 \pm 48.5 \%$ at T1, $35.9 \pm 11 \%$ at T3; RV-LS: $-20.2 \pm 2 \%$ at $\mathrm{T} 1,-16.3 \pm 1.1 \%$ at T3; $P<0.05$ ). Left ventricular (LV)-LS did not change significantly during titration of PEEP. Cardiac chambers' volumes and cardiac output (CO) showed a significant reduction at higher levels of PEEP. Pulse pressure variation was significantly affected by higher levels of PEEP $(P<0.05)$.

Conclusion In hypoxic patients with normal cardiac function, PEEP titration determined a reduction of LA-LS, RA-LS and RV-LS values. LV-LS values were not influenced by PEEP changes. The fall in CO, observed with higher values of PEEP, seemed to be related to the impairment of preload and not of myocardial contractility. Whenever interpreting data on cardiac function obtained with longitudinal strain analysis, attention of the clinician should be drawn to different levels of PEEP. The higher the PEEP, the more the probability of misleading interpretation of STE data.

\section{P188}

Evaluation of a new calibration index suggesting recalibration of the pulse contour cardiac index by transpulmonary thermodilution: a prospective study

W Huber, KWaldleitner, S Mair, B Saugel, RM Schmid

Klinikum rechts der Isar, Technical University of Munich, Germany

Critical Care 2013, 17(Suppl 2):P188 (doi: 10.1186/cc12126)

Introduction After calibration by thermodilution (TD), the PiCCO device is able to assess cardiac index $(\mathrm{Cl})$ using pulse contour (PC) analysis. The manufacturer suggests recalibration by TD after 8 hours. Recently, we suggested a calibration index indicating a certain probability of a relevant bias and triggering the next calibration. With changes of $\mathrm{Clpc}$ compared with the previous $\mathrm{Cltd}$ being a key predictor of the bias of Clpc compared with the following Cltd, the manufacturer implemented a soft alarm indicating changes in Clpc compared with the last Cltd (thresholds adjustable to $15 \%, 25 \%$ and $35 \%$ ). The aim of 
the study was prospective evaluation of predictive capabilities of the $15 \%$ alarm regarding a bias of Clpc compared with Cltd exceeding critical thresholds of $15 \%, 20 \%$ and $25 \%$.

Methods A prospective analysis of 329 routine TPTD measurements in 70 patients. The $\mathrm{Cl}$-trend alarm was set to $15 \%$, and $\mathrm{Clpc}$, trend alarm (yes/no), relative and absolute changes in Clpc were recorded immediately before TD providing exact measurement of Cltd. Predictive capabilities of the $15 \%$ trend alarm regarding the bias were evaluated using Spearman correlation, chi-square test, ROC analysis and Wilcoxon test (IBM SPSS 20).

Results A total of 70 patients ( 24 female, 46 male), age $62 \pm 14$ years, APACHE II score $19.5 \pm 7.5$, SOFA score $8.9 \pm 4.4$. At $937 \pm 904$ (50 to 5,795 ) minutes after the last Cltd, Clpc provided a bias of $-0.0566 \pm 0.761 \mathrm{I} /$ minute $\mathrm{m}^{2}$ compared with the next Cltd. Percentage error was $34.6 \%$. Absolute and relative bias of Clpc compared with the new Cltd did not correlate to time to last TD, but correlated (all $P<0.001$ ) to Clpc itself $(r=0.333 ; r=0.385)$ and relative $(r=0.531 ; r=0.537)$ and absolute $(r=0.533 ; r=0.529)$ changes in Clpc compared with last Cltd. The $15 \%$ Clpc trend alarm was indicated before 83/329 measurements (25.2\%). The amount of bias exceeded $15 \%$ and $20 \%$ in 101 (30.7\%) and 79 (24\%) of TDs. In TDs with indicated trend alarm ( $\geq 15 \%$ deviation of Clpc to the last $\mathrm{Cltd}$ ), the amount of bias more frequently exceeded $15 \%$ $(P=0.019), 20 \%(P<0.001)$ and $25 \%(P<0.001)$. Time to last calibration $\geq 8$ hours was not associated with bias exceeding 15\% ( $P=0.735), 20 \%$ $(P=0.888)$ or $25 \%(P=281)$.

Conclusion Bias of Clpc compared with the next Cltd does not depend on the time since last Cltd. Changes in Clpc itself compared with the last Cltd are associated with the bias. A 15\% change in Clpc trend alarm provided by the new PiCCO algorithm is significantly associated with bias values exceeding $15 \%, 20 \%$ or $25 \%$ of Cltd, whereas time to last calibration $>8$ hours is not associated with bias exceeding these critical thresholds.

\section{P189}

Validation of less-invasive hemodynamic monitoring with Pulsioflex in critically ill patients: interim results of a multicentre study

KVan de Vijver', C Pigozzi', L Vervliet', V Vanbiervliet', V Brabers', I Vos',

H Maes' ', N Van Regenmortel', I De laet', K Schoonheydt', H Dits', J Belda², Z Molnar ${ }^{3}$, M Malbrain

'Ziekenhuis Netwerk Antwerpen, ZNA Stuivenberg, Antwerp, Belgium; ${ }^{2}$ Hospital Clinico Universitario, Valencia, Spain; ${ }^{3}$ University of Szeged, Hungary Critical Care 2013, 17(Suppl 2):P189 (doi: 10.1186/cc12127)

Introduction Thermodilution (TD) is considered a gold standard for measurement of cardiac index $(\mathrm{Cl})$ in critically ill patients. The aim of this study is to compare intermittent bolus TD Cl with intermittent automatic calibration $\mathrm{Cl}$ (AutoCl) and two continuous Cls obtained by pulse contour analysis with $\mathrm{PiCCO}_{2}(\mathrm{PiCCl})$ and Pulsioflex (PuCCI).

Methods Interim results of an ongoing prospective multicentre study in 53 patients. Age $58.7 \pm 15.4$, SAPS II score $51.4 \pm 14.7$ and SOFA score $10 \pm 3.2$. All patients underwent PiCCO monitoring via a femoral line whilst a radial line was kept in place during four 8-hour time periods (in the first two periods, the Pulsioflex was connected to a radial line; in the last two it was connected to a femoral line). In the first and third periods, the Pulsioflex was calibrated with TDCl, for the second and fourth periods Pulsioflex was calibrated with AutoCl. Simultaneous $\mathrm{PiCCl}$ and $\mathrm{PuCCl}$ measurements were obtained every 2 hours while simultaneous TDCl and AutoCl were obtained every 8 hours. We also looked at the effects of 40 interventions.
Results In total, $940 \mathrm{CCl}$ and $382 \mathrm{TDCl}$ values were obtained: 940 paired $\mathrm{PiCCl}$ and $\mathrm{PuCCl} ; 358$ paired AutoCl-TDCl measurements. TDCl values ranged from 1.5 to $6.9 \mathrm{l} /$ minute $/ \mathrm{m}^{2}$ (mean $3.6 \pm 1.1$ ), AutoCl from 1.8 to $7.2(3.6 \pm 0.9), \mathrm{PiCCl}$ from 1.0 to $7.1(3.5 \pm 1.1)$ and PuCCl from 1.3 to $7.6(3.6 \pm 1)$. Pearson's correlation coefficient comparing mean PuCCI and $\mathrm{PiCCl}$ values per patient had an $R^{2}$ of 0.79 . Comparison between AutoCl and TDCI had an $R^{2}$ of 0.51 . Changes in AutoCl correlated well with changes in TDCI $\left(R^{2}=0.44\right.$, concordance coefficient $\left.=95.7\right)$, as did changes in PuCCl versus changes in PiCCI $\left(R^{2}=0.99, \mathrm{CC}=93.4 \%\right)$. Changes in $\mathrm{PiCCl}$ and $\mathrm{PuCCl}$ induced by an intervention correlated well with each other $\left(R^{2}=0.86, C C=100 \%\right)$. The percentage error (PE) obtained by Bland and Altman analysis and $R^{2}$ for the different comparisons are presented in Table 1.

Conclusion The preliminary results indicate that in unstable critically ill patients, $\mathrm{Cl}$ can be reliably monitored with Pulsioflex technology via a femoral line. Pulsioflex was also able to keep track of changes in $\mathrm{Cl}$.

\section{P190}

Interim results of an ongoing study on the use of non-invasive hemodynamic monitoring with Nexfin in critically ill patients K Van de Vijver, V Brabers, C Pigozzi, L Vervliet, V Vanbiervliet, H Maes, I Vos, M Peetermans, N Van Regenmortel, I De laet, K Schoonheydt, H Dits, M Malbrain

Ziekenhuis Netwerk Antwerpen, ZNA Stuivenberg, Antwerp, Belgium Critical Care 2013, 17(Suppl 2):P190 (doi: 10.1186/cc12128)

Introduction The Nexfin monitor (BMEYE, the Netherlands) enables continuous non-invasive analysis of blood pressure (MAP) as well as cardiac output (CO) measurements. The aim of the present study was to validate the Nexfin in a mixed population of medical ICU patients. Methods Interim results of a prospective ongoing study in 77 patients admitted to the medical ICU (46 patients mechanically ventilated, M/F ratio 1/1). Age $65.6 \pm 15.9$, BMI $25.6 \pm 4.8$, APACHE II score $22.9 \pm 10.9$, SAPS II $48 \pm 20.1$, SOFA score $7.5 \pm 4.5$. A modified outreach score (SOS) was calculated on admission. For all patients, simultaneous recording of arterial pressure by radial line $(n=78)$, PiCCO $(n=44)$ or by NIBP with arm cuff $(n=47)$ was compared with the Nexfin monitor. Statistical analysis was performed with Student's $t$ test, Pearson correlation and Bland-Altman analysis.

Results A total of 103 measurements in 77 patients were performed. In seven patients measurement with Nexfin was not possible. For CO (55 paired measurements), values were $6 \pm 2.1 \mathrm{l} /$ minute (range 2.6 to 12). Pearson's correlation coefficient comparing Nexfin-CO with reference CO showed a good correlation $\left(R^{2}=0.52\right)$. Bland-Altman analysis comparing both $\mathrm{CO}$ techniques revealed a mean bias $\pm 2 \mathrm{SD}$ (LA) of $0.3 \pm 3.6 \mathrm{l} /$ minute (58\% error). The MAP was $84.2 \pm 15.6 \mathrm{mmHg}$ (53 to 131.5 ) and values obtained with the Nexfin correlated well with the reference method with an $R^{2}$ of 0.72 . Bland-Altman analysis comparing both MAP techniques revealed a mean bias $\pm 2 \mathrm{SD}$ (LA) of $-0.3 \pm 18 \mathrm{mmHg}$ (20.9\% error). However, Nexfin-MAP did not correlate well with $\operatorname{NIBP}\left(R^{2}=0.36\right)$. Hemoglobin values obtained with Nexfin Massimo technique did not correlate well with laboratory values $\left(R^{2}=0.26,33 \%\right.$ error). The 26 patients that died in the ICU had higher APACHE II $(P=0.017)$, SAPS II $(P<0.0001)$, SOFA $(P<0.0001)$ and SOS $(P=0.004)$ scores and significantly lower MAP $(P<0.0001)$, hemoglobin $(P=0.01)$ and lower $\mathrm{dp} / \mathrm{dtmax}(P=0.003)$, a marker for contractility. There were no outcome differences with regard to subgroup analysis in patients with either low or high CO or SVR.

Conclusion In unstable critically ill patients, MAP (and CO) can be monitored with the Nexfin. The exact patient population for this

Table 1 (abstract P189). Results of Bland and Altman analysis

\begin{tabular}{|c|c|c|c|c|c|c|c|c|c|c|}
\hline Pulsioflex & AutoCal & PE (CCI) (\%) & $n$ & $R^{2}$ & PE (TD-Pi) (\%) & $n$ & $R^{2}$ & PE (TD-Pu) (\%) & $n$ & $R^{2}$ \\
\hline All & All & 37.9 & 940 & 0.73 & 22.8 & 382 & 0.88 & 38.5 & 382 & 0.66 \\
\hline All & Yes & 43.4 & 510 & 0.50 & 20.4 & 210 & 0.88 & 42.3 & 210 & 0.47 \\
\hline All & No & 27.8 & 430 & 0.83 & 25.6 & 172 & 0.85 & 32.5 & 172 & 0.74 \\
\hline Fem & All & 30.6 & 464 & 0.73 & 20.2 & 192 & 0.88 & 33.0 & 192 & 0.66 \\
\hline Rad & All & 44.2 & 476 & 0.58 & 25.2 & 190 & 0.85 & 43.7 & 190 & 0.56 \\
\hline
\end{tabular}


technology has yet to be defined and more patients are probably needed for pattern recognition, although the results indicate that low MAP and dp/dtmax are associated with poor outcome. In the future, Nexfin data could theoretically be incorporated in a new outreach score.

P191

Cost-effectiveness analysis of stroke volume variation guided perioperative hemodynamic optimization

J Benes, J Zatloukal, A Simanova, I Chytra, E Kasal

The Medical Faculty and Hospital in Plzen - Charles University Prague, Plzen, Czech Republic

Critical Care 2013, 17(Suppl 2):P191 (doi: 10.1186/cc12129)

Introduction Perioperative goal-directed therapy (pGDT) can substantially improve the outcome of high-risk surgical patients [1]. But the approach needs an initial investment and increases the staff workload. Economic factors might participate in the weak adherence to the pGDT concept. Some model studies support pGDT cost-effectiveness, but real economic data based on a recent clinical trial are lacking. We performed an economic analysis of hemodynamic optimization using the stroke volume variation trial [2] in order to elucidate this issue. Methods The hospital care invoices of all 120 patients included in the trial were retrospectively extracted. Due to the nature of the data we have adopted the healthcare payer's perspective. We performed a comparison of induced costs between the Vigileo $(n=60)$ and Control $(n=60)$ groups and constructed a cost tree using the study group and complications occurrence as distributive parameters. The incremental cost-effectiveness ratio per complication avoided was calculated and finally, different reimbursing categories were assessed as potential cost drivers.

Results A decreased rate (18 vs. 35 patients) and number of complications ( 34 vs. 78 ) were observed in the original trials Vigileo group. The mean cost of intervened patient was lower $(€ 2,877 \pm 2,336$ vs. $€ 3,371 \pm 3,238 ; P=0.38$ ). According to the cost-tree analysis, patients with complications $(n=53 ; 44 \%)$ consumed significantly more resources (€235,623; 63\%). A gain of $€ 634$ per avoided complications confirms that the lower complications rate was the most important cost driver. Both the clinical care for patients costs ( $€ 505$ vs. $912 ; P=0.04)$ and ward stay costs ( $€ 244$ vs. $402 ; P=0.03$ ) were decreased by the intervention. On the contrary, the intervention increased anaesthesia costs ( $€ 880$ vs. $688 ; P=0.001$ ).

Conclusion Intraoperative fluid optimization with the use of stroke volume variation and the Vigileo/FloTrac system showed not only a substantial improvement of morbidity, but was also associated with an economic benefit. This observed benefit highly exceed the increased monitoring costs in our trial.

Acknowledgements The study was supported by the MSM0021620819 and the Charles University Research Fund (project number P36). References

1. Hamilton MA, et al: Systematic review and meta-analysis on the use of preemptive hemodynamic intervention to improve postoperative outcomes in moderate and high-risk surgical patients. Anesth Analg 2011, 112:1392-1402

2. Benes J, et al:: Intraoperative fluid optimization using stroke volume variation in high risk surgical patients: results of prospective randomized study. Crit Care 2010, 14:R118.

\section{P192}

EV1000/VolumeView: a new device for a more reliable measurement of extravascular lung water index in patients with lung resections

A Donati, C Melia, V Monaldi, R Domizi, E Damiani, A Carsetti, C Scorcella,

R Castagnani, P Pelaia

Università Politecnica delle Marche, Ancona, Italy

Critical Care 2013, 17(Suppl 2):P192 (doi: 10.1186/cc12130)

Introduction Hemodynamic monitoring is important in high-risk surgical patients in order to detect and correct circulatory instability, thereby improving outcome [1]. The extravascular lung water index

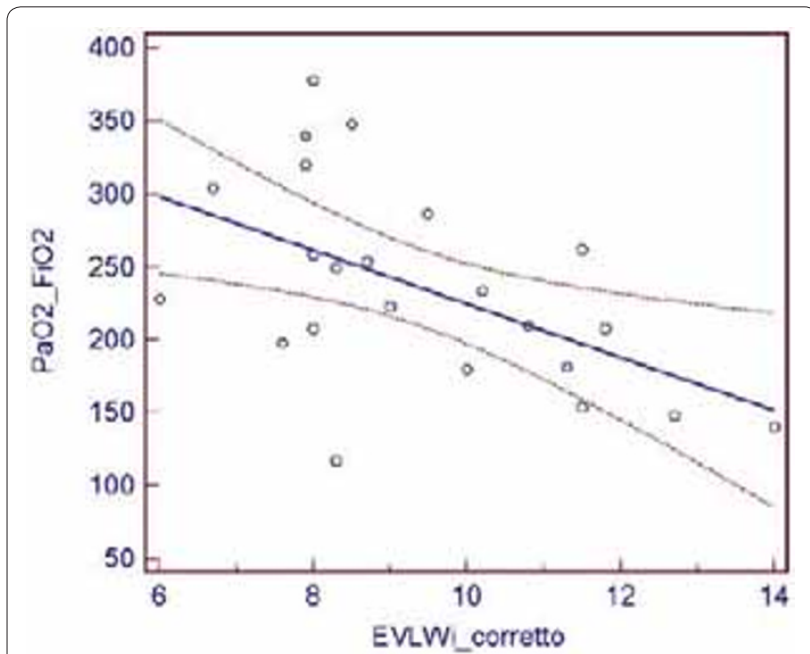

Figure 1 (abstract P192). Correlation between $\mathrm{PaO}_{2} / \mathrm{FiO}_{2}$ and EVLWIC.

(EVLWI) reflects pulmonary edema [2]. The new EV1000/VolumeView (Edwards Lifesciences) can accurately measure EVLWI corrected for the actual volume of lung parenchyma (EVLWIc). The aim of our study is to prove a stronger correlation between EVLWIc and $\mathrm{PaO}_{2} / \mathrm{FiO}_{2}$ compared with EVLWI in patients undergoing pulmonary resection.

Methods A prospective observational study. Seven patients with lung cancer undergoing pulmonary resection were monitored using the EV1000 plathform. EVLWI was assessed by thermodilution at the following time points: after intubation (t1); during single-lung ventilation (t2); after lung resection (t3); after ICU admission (t4); 12 hours ( $\mathrm{t} 5$ ) and 18 hours after ICU admission ( 6 ). EVLWIC values were also collected at $\mathrm{t} 3$ and $\mathrm{t} 4$. $\mathrm{PaO}_{2} / \mathrm{FiO}_{2}$ was measured at the same time points.

Results No significant correlation was found between EVLWI and $\mathrm{PaO}_{2}$ / $\mathrm{FiO}_{2}(r=-0.3124, P>0.05)$, while a significant correlation was seen between EVLWIC and $\mathrm{PaO}_{2} / \mathrm{FiO}_{2}(r=-0.528, P=0.009$; Figure 1$)$.

Conclusion Despite the small sample size, this study shows that in patients undergoing pulmonary resection the EVLWIc is more strongly correlated to $\mathrm{PaO}_{2} / \mathrm{FiO}_{2}$ than EVLWI. Therefore, the EV1000 may be a valuable tool for more reliable hemodynamic monitoring in this subgroup of patients.

References

1. Pinsky MR: Clin Chest Med 2003, 24:549-560

2. Sakka SG, et al: Chest 2002, 122:2080-2086

P193

Goal-directed resuscitation therapy in high-risk patients undergoing cardiac surgery (GRICS study): a randomized controlled trial - preliminary results

E Osawa', A Rhodes'2, JVincent ${ }^{3}$, J Almeida', J Fukushima', B Pileggi', C Park', L Camara', J Auler Jr', R Chan', M Piccioni', M Lima', F Galas', L Hajjar'

'Heart Institute, São Paulo, Brazil; 2 St George's Healthcare NHS Trust and St George's University of London, UK: ${ }^{3}$ Universite Libre de Bruxelles, Brussels, Belgium

Critical Care 2013, 17(Suppl 2):P193 (doi: 10.1186/cc12131)

Introduction A goal-directed resuscitation therapy (GDT) through optimization of cardiac output reduces complications in noncardiac surgeries. We investigated whether the implementation of a GDT protocol in high-risk cardiac surgery with the use of LiDCO Rapid reduces postoperative complications as compared with the standard of care.

Methods We performed a prospective and randomized study whereby consecutive patients fulfilling one high-risk criteria (EuroSCORE $>5$, ejection fraction $<50 \%$, recent myocardial infarction, unstable angina 
or extracardiac arteriopathy) were allocated to GDT or conventional hemodynamic therapy. We excluded patients with endocarditis, previous use of dobutamine, need for IABP, high dose of vasopressors and emergency surgery. The GDT protocol involved hemodynamic resuscitation aimed at a target of a cardiac index $>3 \mathrm{l} / \mathrm{minute} / \mathrm{m}^{2}$ through a three-step approach: fluid therapy of $250 \mathrm{ml}$ lactated Ringer's solution, dobutamine infusion up to a dose of $20 \mu \mathrm{g} / \mathrm{kg} /$ minute, and red blood cell transfusion to reach a hematocrit level above $28 \%$.

Results Twenty patients from the GDT group were compared with 20 control patients. Both groups were comparable concerning baseline characteristics and severity scores, except for a higher prevalence of hypertension and heart failure in the GDT group. Intraoperative data showed no difference regarding length of extracorporeal circulation, fluid balance, transfusion or inotropic requirement. Patients from the GDT group were given more fluids within the first 8 hours as compared with the conventional group $(1,250 \mathrm{ml}$ vs. $500 \mathrm{ml}, P<0.001)$. GDT patients showed a median ICU stay of 3 days ( $95 \% \mathrm{Cl} 3$ to 4$)$ compared with 5 days in control patients ( $95 \% \mathrm{Cl}: 4$ to 7 ). Moreover, hospital stay was less prolonged in GDT patients ( 10 days vs. 14 days, $P=0.043$ ). Inotropic dependence was lower in the GDT group (29 hours vs. 55 hours, $P=0.003$ ) as well as the cumulative dobutamine dosage ( $8 \mathrm{vs}$. $19 \mu \mathrm{g} / \mathrm{kg} /$ day, $P=0.025)$. Also, GDT group presented a lower incidence of infections, tachyarrithmias and acute renal failure (RIFLE R) when compared with the control group.

Conclusion A hemodynamic therapy tailored to an optimized cardiac output reduced the length of ICU stay, vasoactive drug requirement and postoperative complications.

Acknowledgement Grants received from FAPESP 2011/19987-5.

\section{P194}

Perioperative volume management for esophageal cancer surgery

M Karaman Ilic, G Madžarac, J Kogler, D Stančić Rokotov, N Hodoba University Hospital Centre Zagreb, Croatia

Critical Care 2013, 17(Suppl 2):P194 (doi: 10.1186/cc12132)

Introduction Pulmonary complications are the primary reason for extending a patient's stay in the ICU. The aim of this study was to investigate whether perioperative volume management can influence the $\mathrm{PaO}_{2} / \mathrm{FiO}_{2}$ value and total length of stay in the ICU.

Methods Sixteen patients were divided into two groups: one group was treated with a restrictive approach $(\leq 8 \mathrm{ml} / \mathrm{kg} / \mathrm{hour})$, and the other with a liberal approach (>8 ml/kg/hour). Patients were randomly allocated using sealed envelopes. During the thoracic part of the surgical procedure, all patients received one-lung ventilation (OLV).

Results In the group treated with a restrictive volume approach, patients received fluids at the rate of $7.0 \pm 1.0 \mathrm{ml} / \mathrm{kg} / \mathrm{hour}$. $\mathrm{PaO}_{2} / \mathrm{FiO}_{2}$ was $288 \pm 14$ after intubation and $270 \pm 22$ before extubation. In the group treated with a liberal volume approach, fluids were replaced at $11.0 \pm 2.0 \mathrm{ml} / \mathrm{kg} / \mathrm{hour}$. $\mathrm{PaO}_{2} / \mathrm{FiO}_{2}$ was $259 \pm 24$ after intubation and $223 \pm 43$ before extubation. Surgery combined with OLV was found to significantly affect the $\mathrm{PaO}_{2} / \mathrm{FiO}_{2}$ value (ANOVA, $F_{114}=15.85 \mathrm{a}, P=0.001$, partial $\eta^{2}=0.531$ ). The average $\mathrm{PaO}_{2} / \mathrm{FiO}_{2}$ level was significantly higher in the restrictive-replacement group than in the liberal-replacement group (ANOVA, $F_{1,14}=9.66, P=0.008$, partial $\eta^{2}=0.408$ ). There was no interaction between the groups (ANOVA, $F_{114}=1.7 \mathrm{a}, P=0.215$, partial $\eta^{2}=0.108$ ). Mean length of stay in the ICU' was similar between the restrictive-replacement group $(5.2 \pm 2.3$ days $)$ and the liberalreplacement group (6.3 \pm 1.6 days) (ANOVA, $F_{114}=0.814 a, P=0.382$, partial $\eta^{2}=0.055$ )

Conclusion Results from this small sample indicate that esophageal carcinoma surgery by itself had a detrimental effect on the $\mathrm{PaO}_{2} / \mathrm{FiO}_{2}$ value, which restriction of perioperative volume did not significantly affect. Volume restriction also did not affect length of stay in the ICU.

\section{References}

1. Nisanevich V, Felsenstein I, Almogy G, Weissman C, Einav S, Matot I: Effect of intraoperative fluid management on outcome after intraabdominal surgery. Anesthesiology 2005, 103:25-32.

2. Wei S, Tian J, Song X, Chen Y: Association of perioperative fluid balance and adverse surgical outcomes in esophageal cancer and esophagogastric junction cancer. Ann Thorac Surg 2008, 86:266-272.
P195

Treatment-related cardiac complications associated with goal-directed therapy in high-risk surgical patients: a meta-analysis N Arulkumaran, C Corredor, M Hamilton, M Grounds, J Ball, A Rhodes, M Cecconi

St George's Hospital, London, UK

Critical Care 2013, 17(Suppl 2):P195 (doi: 10.1186/cc12133)

Introduction We hypothesized that goal-directed therapy (GDT) is not associated with an increased risk of cardiac complications in high-risk, noncardiac surgical patients. Patients with limited cardiopulmonary reserve are at risk of mortality and morbidity after major surgery [1]. Augmentation of the oxygen delivery index $\left(\mathrm{DO}_{2} \mathrm{l}\right)$ with a combination of intravenous fluids and inotropes (GDT) has been shown to reduce the postoperative mortality and morbidity in high-risk patients [2]. However, concerns regarding cardiac complications associated with fluid challenges and inotropes used to augment cardiac output may deter clinicians from instituting early GDT in the very patients who are more likely to benefit.

Methods Systematic search of MEDLINE, Embase and CENTRAL databases for randomized controlled trials of GDT in high-risk surgical patients. Studies including cardiac surgery, trauma, and pediatric surgery were excluded to minimize heterogeneity. We reviewed the rates of all cardiac complications, arrhythmias, acute myocardial ischemia, and acute pulmonary edema. Meta-analyses were performed and forest plots drawn using RevMan software. Data are presented as odd ratios (ORs) $(95 \% \mathrm{Cls})$, and $P$ values.

Results We identified 23 randomized controlled trials including 2,219 patients, who reported cardiac complications. GDT was associated with a reduction in total cardiovascular complications $(\mathrm{OR}=0.55(0.39$ to 0.78$), P=0.0007$ ), and with a significantly reduced incidence of arrhythmias (OR $=0.59$ (0.38 to 0.91$), P=0.02$ ). GDT was not associated with an increase in acute pulmonary edema $(\mathrm{OR}=0.68(0.42$ to 1.10$)$, $P=0.11$ ) or acute myocardial ischemia ( $O R=0.70$ ( 0.38 to 1.27$), P=0.23$ ). Subgroup analysis of overall cardiovascular complications revealed that the benefit is most pronounced in patients receiving fluid and inotrope therapy ( $\mathrm{OR}=0.55(0.34$ to 0.89$), P=0.01)$ to achieve a supranormal oxygen delivery target $(\mathrm{OR}=0.50(0.32$ to 0.79$), P=0.003)$, guided by the use of minimally invasive cardiac outmonitoring $(\mathrm{OR}=0.50$ ( 0.33 to $0.77), P=0.002$ ).

Conclusion Perioperative, physiologically guided, GDT in high-risk surgical patients is not associated and actually reduces postoperative cardiovascular complications.

References

1. Pearse RM, et al:: Lancet 2012, 380:1059-1065

2. Hamilton MA, et al:: Anesth Analg 2011, 112:1392-1402

P196

Estimation of potential cost-savings related to the implementation of perioperative hemodynamic goal-directed therapy

T Simon, G Marx

RWTH University Hospital Aachen, Germany

Critical Care 2013, 17(Suppl 2):P196 (doi: 10.1186/cc12134)

Introduction Many studies have demonstrated the ability of perioperative hemodynamic goal-directed therapy (pGDT) to decrease postoperative morbidity in patients undergoing medium-to-high-risk surgery [1]. As a result, pGDT may be a cost-saving strategy. Our goal was to provide an estimation of potential cost-savings based on recent literature.

Methods The largest and most recent meta-analysis [1] on pGDT was used to estimate what could be the reduction of postoperative morbidity if pGDT was to be adopted. Costs related to the treatment of patients developing at least one (1+) postoperative complication were obtained from two recent US [2] and Swiss [3] publications. Potential cost-savings related to the adoption of pGDT were calculated according to the actual morbidity rate, assuming 0\% pGDT use so far, and $100 \%$ compliance rate after implementation.

Results The 2011 meta-analysis [1] of 29 RCTs (4,805 patients) showed that pGDT is associated with a reduction in the rate of patients developing $1+$ postoperative complications with odd ratios ranging 
Table 1 (abstract P196)

\begin{tabular}{|c|c|c|c|c|c|c|}
\hline Actual morbidity rate no pGDT (\%) & 10 & 20 & 30 & 40 & 50 & 60 \\
\hline Expected morbidity rate pGDT (\%) & 3.5 to 5.5 & 7 to 11 & 11 to 17 & 14 to 22 & 18 to 28 & 21 to 33 \\
\hline Expected cost reduction/US patient (\$) & 808 to 1,167 & 1,615 to 2,333 & 2,423 to 3,500 & 3,231 to 4,667 & 4,039 to 5,833 & 4,846 to 7,000 \\
\hline Expected cost reduction/CH patient (\$) & 1,550 to 2,239 & 3,100 to 4,478 & 4,478 to 6,545 & 6,200 to 8,956 & 7,578 to 11,023 & 9,300 to 13,434 \\
\hline
\end{tabular}

between 0.35 and 0.55 . Importantly, these odd ratios were not related to the morbidity rates. In the US publication [2], extra costs for treating patients with $1+$ complication were $\$ 17,949$. In the Swiss $(\mathrm{CH})$ publication [3], they were $\$ 34,446$. See Table 1.

Conclusion Depending on the pre-implementation morbidity rate, the degree of pGDT-induced morbidity reduction and the country, cost-savings ranged between $\$ 808$ and $\$ 13,434$ per patient. This large variability suggests that local/hospital estimations are desirable before starting pGDT implementation. These tailored evaluations would also allow more precise cost-saving estimations by taking into account the actual and expected pGDT compliance rates.

\section{References}

1. Hamilton MA, et al: A systematic review and meta-analysis on the use of preemptive hemodynamic intervention to improve postoperative outcomes in moderate and high-risk surgical patients. Anesth Analg 2011, 112:1392-1402.

2. Boltz et al: Synergistic implications of multiple postoperative outcomes. Am J Med Qual 2012, in press.

3. Vonlanthen $\mathrm{R}$, et al:: The impact of complications on costs of major surgical procedures. Ann Surg 2011, 254:907-913.

P197

Cardiac output estimation: Vigileo and Mostcare versus echocardiography

S Romagnoli, D Quattrone, AR De Gaudio

Azienda Ospedaliero Universitaria Careggi, Florence, Italy

Critical Care 2013, 17(Suppl 2):P197 (doi: 10.1186/cc12135)

Introduction In the present study we analyzed the reliability for cardiac output (CO) measurement of Vigileo (Edwards Lifescience, Irvine, CA, USA) and MostCare (pressure recording analytical method; Vygon-Vytech, Padova, Italy) in comparison with transthoracic Doppler echocardiography (as the reference method) in patients undergoing vascular surgery.

Methods Both Vigileo and MostCare were connected to the FloTrac transducer (Edwards Lifescience) for CO calculation. The data from Vigileo and MostCare were registered (COVIG and COMC respectively)
Table 1 (abstract P197)

\begin{tabular}{lccc}
\hline & Pearson $r^{2}$ & Bias & LoA \\
\hline COECHO vs. COVIG (T1) & 0.31 (Cl 0.24 to 1.63) & -0.49 & -3.41 to 2.43 \\
COECHO vs. COMC (T1) & 0.71 (Cl 0.56 to 1.08) & -0.055 & -1.19 to 1.08 \\
COECHO vs. COVIG (T2) & 0.27 (Cl 0.16 to 1.53) & -0.49 & -3.24 to 2.34 \\
COECHO vs. COMC (T2) & 0.79 (Cl 0.66 to 1.107) & -0.023 & -0.94 to 0.9 \\
\hline
\end{tabular}

$\mathrm{Cl}$, confidence interval; LoA, limits of agreement.

and compared with those calculated with the echocardiographic standard formulation (stroke volume $=$ cross-sectional areaxvelocity time integral; $\mathrm{COECHO}=\mathrm{SV} \times$ heart rate). In every patient $\mathrm{CO}$ was measured twice: at baseline (T1) and after volume loading $(500 \mathrm{ml}$ lactate Ringer solution) (T2). Agreements between COVIG, COMC, and COECHO were evaluated by means of simple linear regression $\left(r^{2}\right)$ and Bland-Altman analysis.

Results Twenty patients were enrolled in the study. Values of $r^{2}$, bias and limit of agreement at T1 and T2 are summarized in Table 1. CO values ranged from 3.9 and $8.6 \mathrm{l} /$ minute (echo), from 3.4 to 9.9 (Vigileo) and from 4 to 8.3 (MostCare); the Pearson's and Bland-Altman methods showed poor agreement between $\mathrm{COECHO}$ and $\mathrm{COVIG}$, demonstrating a tendency to overestimation (see Figure 1). The percentage of error (PE) was $51.7 \%$ at T1 and $49.3 \%$ at T2. On the contrary, MostCare measures showed good agreement with echocardiography (see Table 1) with a $\mathrm{PE}$ of $22.4 \%$ at $\mathrm{T} 1$ and of $17 \%$ at $\mathrm{T} 2$.

Conclusion Vigileo did not prove to be a substitute to the reference system; pre-loaded data, necessary for vascular impedance estimation, may be one of the main limitations that made Vigileo measurements less accurate than the MostCare ones. On the contrary, MostCare, an uncalibrated totally independent system, was shown to properly estimate the vascular impedance in these hemodynamically stable patients. Further comparisons in unstable conditions are needed to confirm our observations.

References

1. Romano SM, et al:: Crit Care 2002, 30:1834-1841.

2. Geisen M, et al:: Curr Opin Crit Care 2012, 18:377-384.

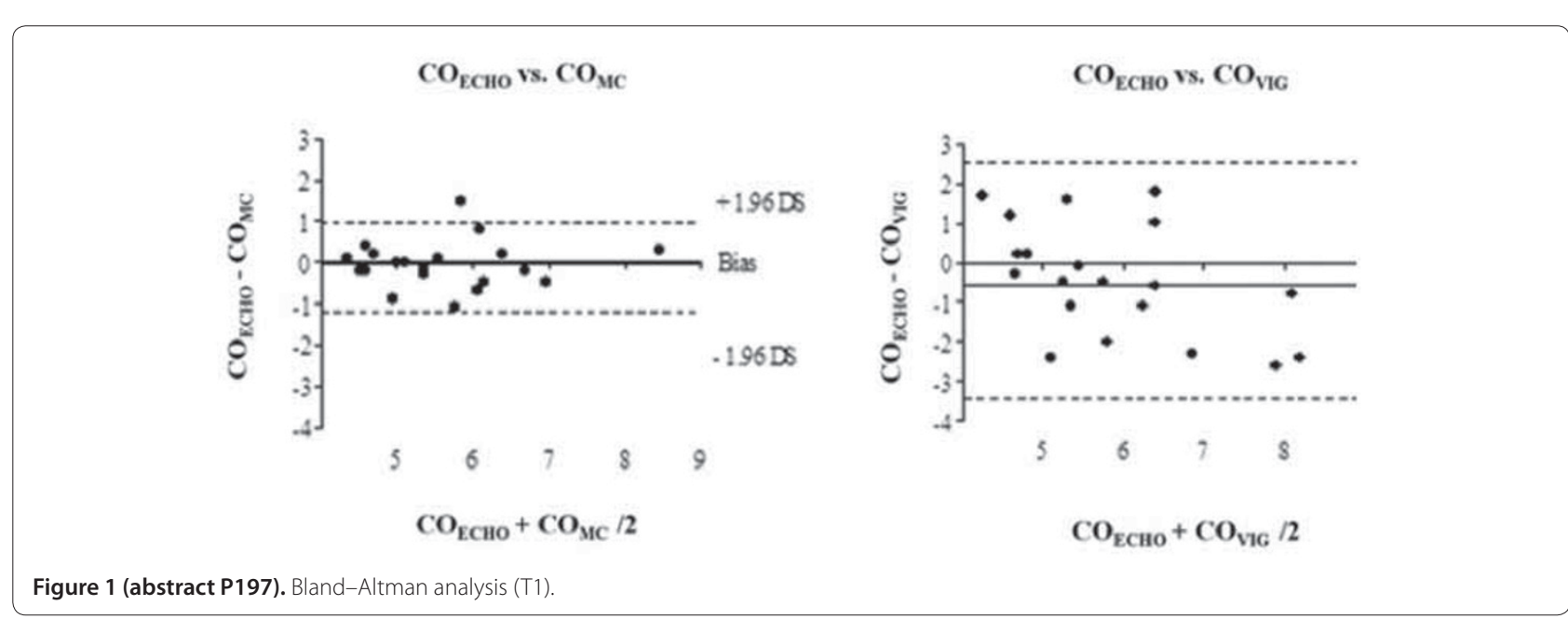


P198

Brachial pulse waveform characteristics predict development of organ failure in septic patients

S Kazune, I Jagmane

Hospital of Traumatology and Orthopaedics, Riga, Latvia

Critical Care 2013, 17(Suppl 2):P198 (doi: 10.1186/cc12136)

Introduction Pulse waveform characteristics (pulse wave transit time and augmentation index) are measures of arterial stiffness. Previous studies have found an association between severity of acute inflammatory states and increased arterial stiffness but it is not known whether non-invasive pulse waveform analysis could predict development of multiple organ failure in septic patients. The purpose of this study was to evaluate the photoplethysmographic brachial artery pulse wave transit time and augmentation index and their changes in response to induced forearm ischemia in septic ICU patients and correlate these indices to the development of subsequent end organ damage.

Methods A prospective observational study in patients with sepsis within 24 hours of admission. Severity of sepsis was assessed with APACHE II score (median 18.5) and SOFA score (median 7.5). Threeminute signal recording was done concurrently from the brachial artery at the elbow and the radial artery at the wrist with an originally designed photoplethysmograph at rest and after 5 minutes of induced forearm ischemia. Recordings were analyzed to obtain the pulse wave transit time and augmentation index at rest and 60 seconds after induced ischemia. The SOFA score was recalculated at 48 hours post recording.

Results We studied 14 consecutive general ICU patients. There was a negative linear relationship between the pulse wave transit time (median $22.6 \mathrm{~ms}$ ) at rest and increase in SOFA score in 48 hours $(P=0.02, r=0.96)$. The postischemic pulse wave transit time increased in all patients (median $25.7 \mathrm{~ms}$ ) but no association was found between the proportion of increase and subsequent change in SOFA. Correlation between rest (median 7.6) and postischemic (median 7.2) augmentation index and 48-hour SOFA scores was not statistically significant $(r=0.57, P=0.46)$.

Conclusion This study indicates that in early sepsis pulse waveform characteristics could predict the risk of developing end organ failure. The pulse wave transit time is more robust than the augmentation index and could be easier to use in patients with poor perfusion. Vascular reactivity indices do not seem to have predictive value in this context.

\section{Reference}

1. Vlachopoulos C, Dima I, Aznaouridis K, Vasiliadou C, loakeimidis N, Aggeli C, Toutouza M, Stefanadis C: Acute systemic inflammation increases arterial stiffness and decreases wave reflections in healthy individuals. Circulation 2005, 112:2193-2200.

P199

Computerized decision support system improves consistency of haemodynamic assessment amongst ICU team members

AE Aneman, R Ranganatha

Liverpool Hospital, Liverpool, Australia

Critical Care 2013, 17(Suppl 2):P199 (doi: 10.1186/cc12137)

Introduction This study evaluated the potential of the NAVIGATOR (Applied Physiology) haemodynamic monitor to improve consistency of cardiovascular assessments amongst ICU members with different levels of expertise and experience.

Methods Patients $(n=20)$ post cardiac surgery were monitored by NAVIGATOR to display heart efficiency (Eh), mean systemic filling pressure (Pms) and vascular resistance (SVR) against targets for mean arterial pressure and cardiac output set by the clinical team. Four categories of staff participated: nine consultants $(C)$, eight senior registrars (SR), nine registrars (R) and 11 nurses $(\mathrm{N})$ (median ICU experience 15, 7, 2 and 10 years, respectively) and were asked to score Eh, Pms, and SVR ranging (discrete steps of one) from -5 (grossly subnormal) to 0 (normal) to 5 (grossly supranormal) first without (BLIND) and then given (OPEN) access to the NAVIGATOR display. Recommendations for therapeutic interventions were noted.
Agreement (maximum two steps deviation for each assessment between staff; number of patients), disagreement (median and interquartile range of steps) and therapeutic agreement (intervention/s to change Eh, Pms and SVR in similar direction/s, number of patients) were recorded and analysed for statistical difference BLIND versus OPEN (Fisher's exact test, Mann-Whitney test, $P<0.05$ ).

Results Eh was commonly overestimated, Pms commonly underestimated with no clear trend for SVR. Agreement amongst all categories of staff increased and disagreement score decreased for Eh, Pms and SVR (Table 1) comparing BLIND versus OPEN assessments. Agreement for therapeutic interventions also increased significantly from $4 / 20$ (BLIND) to $18 / 20$ patients (OPEN).

Table 1 (abstract P199)

\begin{tabular}{lcc}
\hline & \multicolumn{1}{c}{ BLIND } & OPEN \\
\hline Eh & $6 / 20,2$ (1 to 3$)$ & $17 / 20^{*}, 1$ (0 to 1$)^{*}$ \\
Pms & $6 / 20,2.5$ (2 to 4$)$ & $17 / 20^{*}, 2(1 \text { to } 2)^{*}$ \\
SVR & $8 / 20,2(1$ to 2$)$ & $16 / 20^{*}, 1$ (1 to 2$)^{*}$ \\
\hline
\end{tabular}

Patients $(n / 20)$ and score (median and IQR). $* P<0.05$.

Conclusion The assessment of heart function, intravascular filling and resistance state in postoperative cardiac patients was made significantly more consistent amongst different ICU staff members using the NAVIGATOR haemodynamic monitor. Such enhanced consistency could potentially make the haemodynamic management more effective with improved clinical outcomes.

\section{P200}

Volume quantification by contrast-enhanced ultrasound and thermodilution: an in vitro comparison

IH Herold', G Russo², HC Van Assen², HH Korsten', M Mischi²

'Catharina Ziekenhuis Eindhoven, the Netherlands; '2University of Technology, Eindhoven, the Netherlands

Critical Care 2013, 17(Suppl 2):P200 (doi: 10.1186/cc12138)

Introduction In clinical practice, blood volumes (BV) are typically measured by thermodilution. Recently, contrast-enhanced ultrasound (CEUS) has been proposed as an alternative minimally invasive approach for BV assessment [1]. This method measures BV using a single peripheral injection of a small bolus of ultrasound contrast agent (UCA) detected by an ultrasound scanner. By measuring the acoustic backscatter, two indicator dilution curves (IDCs) can be derived from two different sites in the circulatory system. IDC analysis permits deriving the mean transit time (MTT) the bolus takes to travel between the injection site and two measurement sites. Assessment of the BV between these sites is obtained by multiplying the difference in MTT ( $\triangle \mathrm{MTT}$ ) by the blood flow. In this study, we compare different volumes in an in vitro set-up by CEUS with true set-up volumes and thermodilution acquired volumes.

Methods The in vitro set-up consisted of a centrifugal pump, a network of tubes with variable volumes, an electromagnetic flowmeter to measure and adjust the generated flow, heating devices to maintain constant temperature $\left(37^{\circ} \mathrm{C}\right.$ ), two thermistors for thermodilution measurement, an ultrasound transducer and a pressure stabilizer. A small bolus of UCA diluted in cold saline $\left(1 \mathrm{mg}\right.$ SonoVue ${ }^{\oplus}$ in $20 \mathrm{ml}$ saline at $4^{\circ} \mathrm{C}$ ) was injected into the system. The cold UCA passage through a first and a second region of interest (ROI) was measured simultaneously with the ultrasound transducer and the thermistors. The measurements were performed at different flows and volumes. BVs were estimated using the two different approaches, namely CEUS and thermodilution. The IDCs were processed and fitted separately with a dedicated model to estimate the $\triangle$ MTT of the cold UCA bolus between the two ROls and the two thermistors. All the measurements were repeated three times. Results A linear relation between BVs estimated by the two techniques was observed with a correlation coefficient of 0.94 . The bias of CEUS with respect to the true volumes was $-40.1 \mathrm{ml}$; the bias of thermodilution was $84.3 \mathrm{ml}$. The most prominent differences between the two techniques were observed in case of high volume and low flow, possibly due to different transport kinetics between UCAs and heat. 
Conclusion Given the good correlation between BVs estimated with CEUS and thermodilution, CEUS is not inferior to thermodilution with the advantage of being minimally invasive.

Reference

1. Mischi M, et al:: IEEE Trans UFFC 2004, 51:1137-1141.

P201

Successful introduction of cardiac index, fluid responsiveness and oxygen delivery data into the primary survey at a central London major trauma centre and impact on time to $\mathrm{CT}$, fluid resuscitation and disposal

S Helyar, I De Abreu, S Holloway, P Hopkins

King's Health Partners AHSC, London, UK

Critical Care 2013, 17(Suppl 2):P201 (doi: 10.1186/cc12139)

Introduction The use of cardiac output monitoring has been shown to be beneficial in the setting of perioperative medicine and critical illness $[1,2]$. More recently, its application in the setting of major trauma has been described [3]. Here, we describe our preliminary experience of embedding bioreactance flow monitoring within the major trauma primary survey of severely injured patients and the subsequent effect on patient management.

Methods Institutional ethical approval was obtained. Intubated major trauma patients were sequentially enrolled. Exclusions included major thoracic burns and children. Bioreactance flow monitoring (NICOM: Cheetah) was applied at the same time as ECG leads and the calibration step performed during handover from the prehospital team. Time to availability of oxygen delivery data was recorded and trauma team members surveyed regarding for perceived benefits and concerns from this monitoring. The influence of flow monitoring on fluid resuscitation, time to CT and definitive disposal (to OR/ICU) was measured and compared with a control population matched for injury severity score, age and sex.

Results Cardiac index was available at mean 10.6 minutes (median 9 minutes; SD 3.9), fluid responsiveness at mean 35.9 minutes (median 35; SD 11.3) and oxygen delivery calculation at mean 25.3 minutes (median 25; SD 7.7). Passive leg raise was not performed in $63 \%$ of patients due to concerns about pelvic or brain injury. Volume of fluid infused (mean 738 vs. $925 \mathrm{ml} ; P=0.124$ ), time to CT (mean 57.4 vs. 68.8 minutes; $P=0.08$ ), and time to definitive disposal (mean $124.9 \mathrm{vs.}$ 146.1 minute; $P=0.069$ ) were all reduced in the flow monitored group, although not significantly different when compared with a matched control group (Mann-Whitney U rank sum). Eighty-four percent of trauma team members surveyed felt the flow monitoring data to be useful, and only $11 \%$ felt it may impair clinical management.

Conclusion Cardiac index, fluid responsiveness and oxygen delivery data can be obtained inform a primary survey. Rather than introducing delays, the use of flow monitoring was associated with a trend towards decreased time to imaging; less fluid use pre-damage control point and reduced time to definitive disposal. Further research is required to confirm benefits and mechanism.

References

1. Shoemaker WC, et al: Chest 1988, 94:1176-1186.

2. Boyd O, et al:. JAMA 1993, 270:2699-2707.

3. Dunham M, et al:. J Trauma Acute Care Surg 2012, 73:479-485.

P202

Fluid responsiveness in pressure support ventilation: role of asynchrony A Messina, D Colombo, G Cammarota, M De Lucia, F Della Corte,

P Navalesi

Università Maggiore della Carità A. Avogadro, Novara, Italy

Critical Care 2013, 17(Suppl 2):P202 (doi: 10.1186/cc12140)

Introduction Pulse pressure variation (PPV) is a dynamic indicator of fluid responsiveness, which is known to have a low sensibility and specificity in patients ventilated in pressure support (PS) [1]. We aim to investigate patient-ventilator asynchrony as a potential source of hemodynamic interference in PS.

Methods We performed a prospective study including PS ventilated patients who met inclusion criteria for fluid depletion [1]. Patients who showed an asynchrony index (Al) exceeding $10 \%$ were included in the asynchrony group (AG). The remaining patients were included in the synchrony group (SG) [2]. Beat-to-beat hemodynamic variables were recorded through PRAM (Mostcare; Vytech Health srl, Padova, Italy). PPV cutoff of $13 \%$ was used to identify fluid responders/nonresponders. A fluid challenge of $500 \mathrm{ml}$ normal saline was given in 5 minutes. An increase of $15 \%$ of cardiac index after 10 minutes indicated fluid responsiveness.

Results So far, eights patients showed an $\mathrm{Al}>10 \%$ while 16 did not. Overall sensitivity was $28.6 \%$ versus $50 \%$ in SG; overall specificity was $76.5 \%$ versus $91.7 \%$ in AG. Overall Cohen's $k$ was $33.3 \%$ versus $61.2 \%$ in AG (see Figure 1). However, because none of the responders in the AG group was detected by PPV, statistical analysis was not feasible within this subgroup.

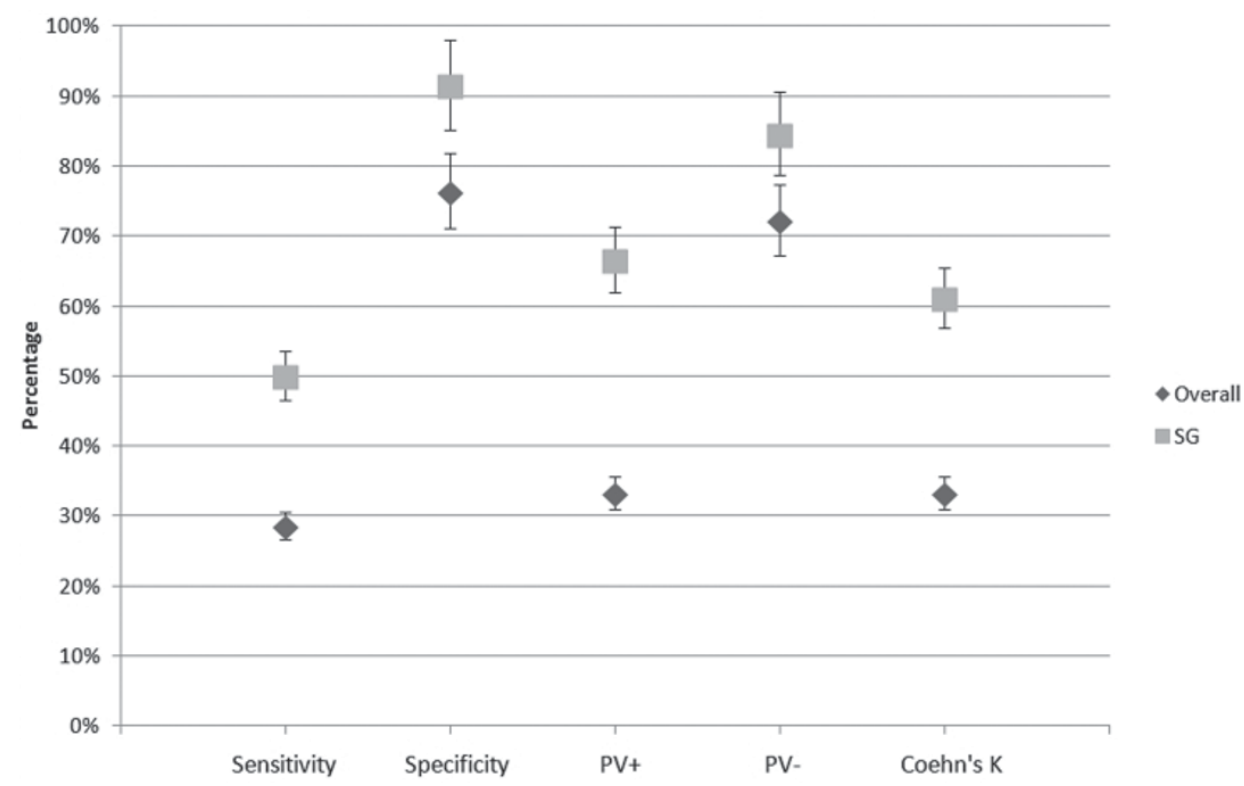

Figure 1 (abstract P202). 
Conclusion The consistency of PPV in predicting fluid responsiveness during PS seems to be more reliable in the patients with better patientventilator synchrony.

References

1. Heenen S, et al: Crit Care 2006, 10:R102.

2. Vitacca M, et al:: Chest 2004, 126:851-859.

P203

Comparison of pulse pressure variation with radial arterial systolic, diastolic and pulse transit time interval variation in pediatric patients undergoing liver transplantation

YJ Moon, IG Jun, WJ Shin, BH Sang, GS Hwang

Asan Medical Center, Seoul, South Korea

Critical Care 2013, 17(Suppl 2):P203 (doi: 10.1186/cc12141)

Introduction In pediatric patients, dynamic preload indices to predict fluid responsiveness have conflicting results in comparison with adults. A recent study demonstrated that pulse pressure variation (PPV) $\geq 16 \%$ has provided an accurate method for predicting fluid responsiveness in pediatric congenital heart surgery. We aimed to compare PPV and respiratory systolic, diastolic and pulse transit time interval variation (STV, DTV and PTTV, respectively) as predictors of fluid responsiveness during pediatric liver transplantation.

Methods A total of 61 data from 16 pediatric patients, median age 5.4 years (range 0.1 to 9 years), were retrospectively evaluated from electrically recorded radial arterial and central venous pressure (CVP) waveform. The time from the onset of systolic upstroke to the dicrotic notch was defined as the systolic time interval (STI), and the time from dicrotic notch to the beginning of systolic upstroke was defined as the diastolic time interval (DTI). The time from peak $R$ wave on electrocardiography to the onset of systolic upstroke was defined as the pulse transit time (PTT) interval. STV was calculated by averaging of three consecutive respiratory cycles with the following: (STI - STI _ ) / STI . The same method was used for calculating DTV, PTTTV and PPV. STV, DTV and PTTV were corrected by cardiac period. Averaged CVP was used as a static preload index. PPV threshold $\geq 16 \%$ was used to discriminate fluid responsiveness. Receiver operating characteristic (ROC) curves and Pearson's correlation analysis were used for the comparison.

Results PPV showed correlations with STV, DTV and PTTV $(r=0.65$, 0.57 and 0.60 , respectively), but less with CVP $(r=-0.30)$. Area under ROC curves (AUC) of STV, DTV, PTTV and CVP were $0.834,0.872,0.832$ and 0.613 , respectively. Cutoff values of STV, DTV, PTTV and CVP were $7.7 \%$ (sensitivity/specificity, 0.80/0.83), 7.7\% (sensitivity/specificity, $0.70 / 0.88$ ), $8.7 \%$ (sensitivity/specificity $0.67 / 1.0$ ) and $3.1 \%$ (sensitivity/ specificity $0.50 / 0.85)$, respectively.

Conclusion This study shows that STV, DTV and PTTV can be used as a surrogate for PPV $\geq 16 \%$, suggesting that this novel method can be used to predict hemodynamic response during pediatric surgery.

Reference

1. Renner J, et al:: Br J Anaesth 2012, 108:108-115.
P204

Mini-fluid challenge: how much fluid and what parameter to use?

B Geerts, RB De Wilde, JJ Maas, LP Aarts, JR Jansen

Leiden University Medical Centre, Leiden, the Netherlands

Critical Care 2013, 17(Suppl 2):P204 (doi: 10.1186/cc12142)

Introduction The mini-fluid challenge is a widely used strategy to manage fluid loading in the ICU and OR. Although it might be a rational strategy, data on the mini-fluid challenge and its reliability are very limited. We investigated the value of changes in pulse contour cardiac output as a result of a mini-fluid challenge of 50 and $100 \mathrm{ml}$ to predict fluid loading responsiveness.

Methods We measured the effects after the administration of 50,100 and $500 \mathrm{ml}$ bolus colloid infusions on CO (Modelflow (COm) and LiDCO (COli)), CVP and MAP in 21 patients on mechanical ventilation after elective cardiothoracic surgery. From the data we analysed the smallest volume that was predictive for the effects of $500 \mathrm{ml}$ on cardiac output. Results COli and COm increased after 50, 100 and $500 \mathrm{ml}$ fluid loading. Best results are observed for changes in COm after $100 \mathrm{ml}$ fluid loading (area under the ROC $0.86,95 \% \mathrm{Cl}$ between 0.65 and 1.00). A change in Modelflow CO of at least $4.3 \%$ has a sensitivity of $67 \%$ and a specificity of $100 \%$ after $100 \mathrm{ml}$ fluid loading. Sensitivity is $60 \%$ and specificity $83 \%$ for a similar cutoff in CO measured with the LiDCO device after $100 \mathrm{ml}$ fluid loading. In our patient population, MAP and COli did not predict responsiveness with more accuracy than mathematical chance. See Figure 1.

Conclusion Changes in pulse contour $\mathrm{CO}$ can be used in a mini-fluid challenge to assess fluid responsiveness in our postcardiac surgery patients.

P205

Efficacy of intraoperatory optimisation of fluids guided with transoesophageal Doppler monitorisation: a multicentre randomised controlled trial

S Maeso', RVillalba', J Ripollés', S Asuero², J Blasco', J Calvo ${ }^{3}$

'Agencia Lain Entralgo, Madrid, Spain; ${ }^{2}$ Hospital Universitario Ramón y Cajal, Madrid, Spain; ${ }^{3}$ Hospital Infanta Leonor, Madrid, Spain

Critical Care 2013, 17(Suppl 2):P205 (doi: 10.1186/cc1243)

Introduction The objective is to compare stay in surgery monitored with oesophageal Doppler with unmonitored.

Methods A randomized trial. We present preliminary results obtained in the first 55 cases. The surgeries were general and urological. ISRCTN93543537.

Results There were no differences in any of the baseline variables. A total of $69.1 \%$ were men. The mean age was 65.80 years. There were $63.6 \%$ general surgery and $36.4 \%$ urologic surgery. There were $81.5 \%$ open surgeries and $18.5 \%$ laparoscopic. The results were favorable to the intervention group for most outcomes; these differences did not
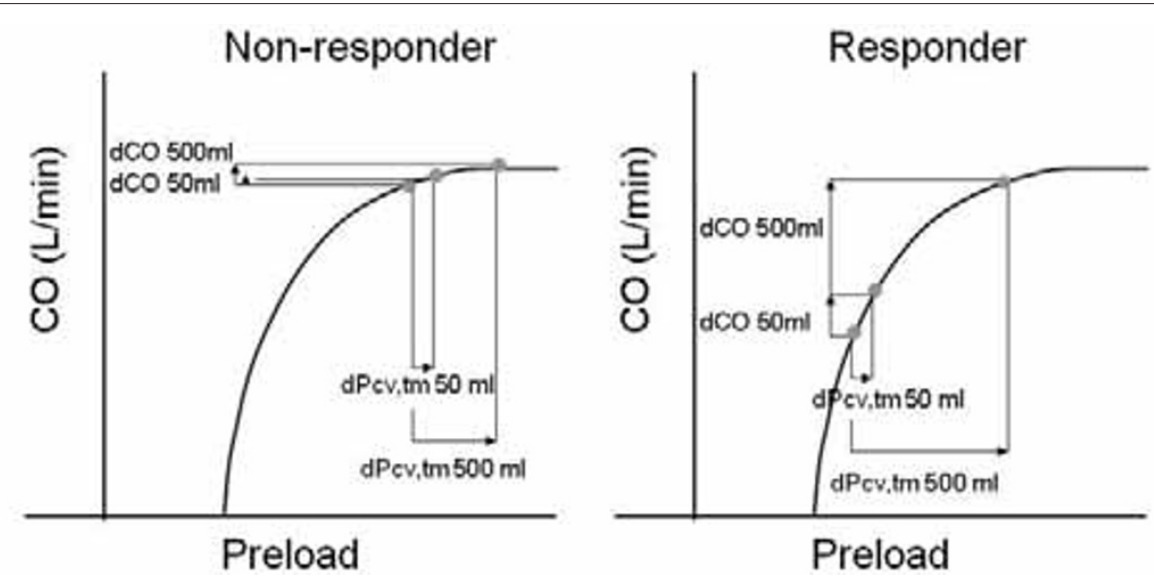

Figure 1 (abstract P204). Cardiac function curve: a fluid challenge of $50 \mathrm{ml}$ in a (non)responder. 
Table 1 (abstract P205). Analysis of the outcome variables

\begin{tabular}{lcccc}
\hline Outcome & Units & $\begin{array}{c}\text { ODM } \\
(\boldsymbol{n}=\mathbf{3 2})\end{array}$ & $\begin{array}{c}\text { Control } \\
(\boldsymbol{n}=\mathbf{2 3})\end{array}$ & $\boldsymbol{P}$ value \\
\hline Surgery time & Minutes & 206.20 & 225.59 & 0.532 \\
Total stay & Days & 13.92 & 18.19 & 0.292 \\
Postsurgery & Days & 10.93 & 14.36 & 0.280 \\
Time to enteral & Days & 2.73 & 3.13 & 0.567 \\
Time to deambulation & Days & 2.52 & 3.00 & 0.359 \\
Complications & \% & 34.4 & 21.7 & 0.377
\end{tabular}

reach statistical significance (Table 1). We emphasize the difference in postoperative stay of 3.4 days. Only complications results were against the intervention.

Conclusion The preliminary results obtained for the postsurgical length of stay, as for most of the outcomes, were favorable to monitoring by oesophageal Doppler.

P206

Looking for the inflexion point of the Frank-Starling curve

HD Aya, M Geisen, C Ebm, N Fletcher, M Grounds, A Rhodes, M Cecconi

St George's Healthcare NHS Trust, London, UK

Critical Care 2013, 17(Suppl 2):P206 (doi: 10.1186/cc12144)

Introduction Fluid responsiveness is defined based on an arbitrary increase of cardiac output (CO) or stroke volume (SV) of 10 to $15 \%$. We hypothesise that the variation of heart efficiency (Eh) and the slope (S) defined by the relative increase of $\mathrm{CO}$ over the relative increase of mean filling pressure (Pmsa) can be used as alternative definitions of fluid responsiveness.

Methods Patients admitted to the ICU were monitored with a calibrated LiDCOplus (LiDCO, UK) and Navigator (Applied Physiology, Australia) to estimate Pmsa and Eh (Pmsa - central venous pressure/Pmsa). A $250 \mathrm{ml}$ fluid challenge was performed over 5 minutes. Categorical data were compared by Pearson chi-square test. Correlation was assessed by Kappa test. The inflexion point of $\mathrm{S}$ to define responders was obtained by ROC curve analysis.

Results A total of 104 fluid challenges were observed in 40 patients. ROC curve analysis reveals an area under the curve of $0.93(95 \% \mathrm{Cl}=0.85$ to $1, P<0.001$ ). The best cutoff for the slope was 0.76 (sensitivity 0.92 , specificity 0.93 ). The proportions of responders identified by the $\Delta \mathrm{Eh}$ (Table 1) and by the slope method (Table 2) are smaller compared with the relative increase of SV method. Significant correlation was found between both methods and the $\Delta \mathrm{SV}(\triangle \mathrm{Eh} \mathrm{K}=0.54, P<0.001 ; \mathrm{S} \mathrm{K}=0.55$, $P<0.001)$. See Figure 1 .

Conclusion Moderate agreement is observed between new and current definitions of fluid responsiveness.

Reference

1. Michard F, et al:: Chest 2002, 121:2000.

Table 1 (abstract P206). Distribution of events according to $\Delta S V$ and $\Delta E h$

\begin{tabular}{lccc}
\hline & Response by $\Delta \mathrm{SV} \geq 10 \%$ & Response by $\Delta$ Eh $\geq 0$ & $P$ value \\
\hline Nonresponder & $62(59.6 \%)$ & $76(73.1 \%)$ & $<0.001$ \\
Responder & $42(40.4 \%)$ & $28(26.9 \%)$ & $<0.001$ \\
\hline
\end{tabular}

Table 2 (abstract P206). Distribution of events according to $\Delta S V$ and the slope (S)

\begin{tabular}{lccr}
\hline & Response by $\Delta \mathrm{SV} \geq \mathbf{1 0 \%}$ & Response by $\mathrm{S} \geq \mathbf{0 . 7 6}$ & $\boldsymbol{P}$ value \\
\hline Nonresponder & $62(59.6 \%)$ & $75(72.1 \%)$ & $<0.001$ \\
Responder & $42(40.4 \%)$ & $29(27.9 \%)$ & $<0.001$ \\
\hline
\end{tabular}

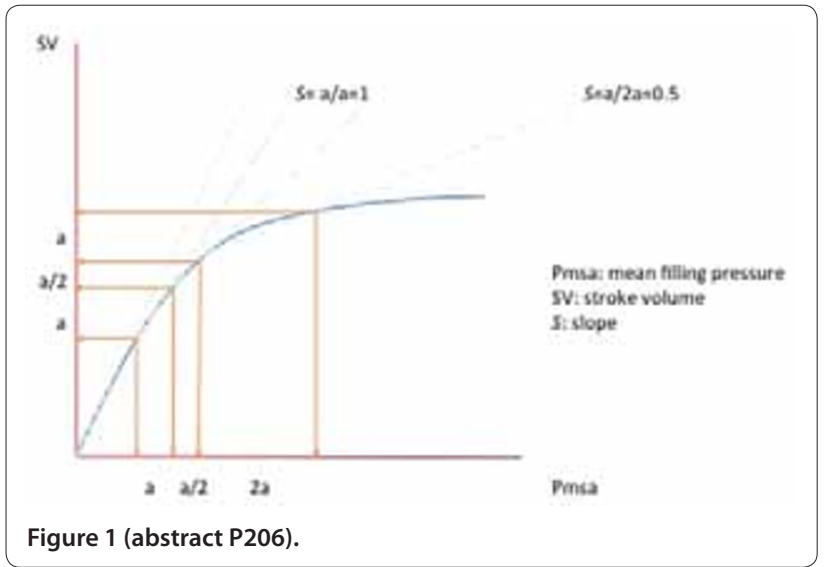

P207

Prediction of stroke volume response to fluid bolus in 100 children

R Saxena, A Durward, I Murdoch, STibby

Guy's \& St Thomas' NHS Trust, London, UK

Critical Care 2013, 17(Suppl 2):P207 (doi: 10.1186/cc12145)

Introduction Fluid overload is associated with poor outcome in the critically ill. Thus, an accurate predictor of a positive haemodynamic response (increase in stroke volume) to fluid challenge is vital.

Methods We studied the predictive value (positive response defined as change in stroke volume $>15 \%$ after $10 \mathrm{ml} / \mathrm{kg}$ fluid bolus) of a range of haemodynamic variables: static (CVP, active circulating volume, central blood volume, total end diastolic volume), dynamic (systolic pressure variation, stroke volume variation) and contactility (dp/dt), in a group of 100 ventilated children (median weight $10 \mathrm{~kg}$ ). Variables were measured using transpulmonary ultrasound dilution and PRAM (an arterial pulse contour method).

Results We performed 168 paired measurements (pre-fluid and postfluid challenge), with a SV response rate of $45 \%$. Overall predictive values were poor, but slightly better for static versus dynamic variables (Table 1). When SV response was analysed as a continuous variable, the two predictive multivariable variables were change in TEDVI and baseline $\mathrm{dp} / \mathrm{dt}\left(r^{2}=0.30\right.$, both $\left.P<0.001\right)$.

Table 1 (abstract P207). ROC areas for haemodynamic variables

\begin{tabular}{llll}
\hline & AUC & \multicolumn{2}{c}{$95 \% \mathrm{Cl}$} \\
\hline CVP & 0.50 & 0.41 & 0.59 \\
ACVI & 0.62 & 0.54 & 0.71 \\
CBVI & 0.59 & 0.50 & 0.68 \\
TEDVI & 0.65 & 0.56 & 0.73 \\
SPV & 0.59 & 0.50 & 0.69 \\
SW & 0.48 & 0.39 & 0.58 \\
$\mathrm{dp} / \mathrm{dt}$ & 0.59 & 0.50 & 0.68 \\
\hline
\end{tabular}

Conclusion The predictive ability for typical static and dynamic haemodynamic variables, when taken in isolation, is poor. However, improved prediction is seen when baseline contractility is taken into account.

P208

Microcirculatory changes during goal-directed or mean arterial pressure-guided fluid therapy in abdominal surgery

SP De Wolf, J Stens, RJ Van der Zwan, N Koning, C Boer

VU University Medical Centre, Amsterdam, the Netherlands

Critical Care 2013, 17(Suppl 2):P208 (doi: 10.1186/cc12146)

Introduction This study compared the effect of pulse pressure variation (PPV) and cardiac index (Cl)-guided fluid therapy versus mean arterial 
pressure (MAP)-guided fluid therapy on microcirculatory perfusion in patients undergoing abdominal surgery.

Methods Patients undergoing elective abdominal surgery were randomized into a PPV/Cl-guided group $(n=11)$ or a MAP-guided $(n=12)$ group. PPV, Cl and MAP were measured using the non-invasive finger arterial blood pressure measurement device ccNexfin (Edwards Lifesciences BMEYE, Amsterdam, the Netherlands). Tidal volumes were $\geq 8 \mathrm{ml} / \mathrm{kg}$ with PEEP $\geq 8 \mathrm{mmHg}$. In both groups, MAP of $70 \mathrm{mmHg}$ was maintained. In the PPV/Cl group, an intraoperative algorithm was used keeping the PPV under $12 \%$ and $\mathrm{Cl}$ above $2.5 \mathrm{l} /$ minute/ $\mathrm{m}^{2}$ using fluid therapy and dobutamine and noradrenaline infusion, respectively. Sublingual microvascular perfusion was measured after anesthesia induction, and every subsequent hour using sidestream dark-field imaging (Microscan; Microvision Medical, Amsterdam, the Netherlands). The perfused small vessel density (PVD) values were offline quantified.

Results The first hour during surgery, the PPV/Cl-guided group tended to receive more fluids than the MAP-guided group $(1,014 \pm 501 \mathrm{ml}$ vs. $629 \pm 463 \mathrm{ml} ; P=0.07)$. At this time point, the PVD was slightly lower in the PPV/Cl-guided group $\left(16.7 \pm 3.1 \mathrm{~mm} / \mathrm{mm}^{2}\right)$ when compared with the MAP-guided group $\left(17.9 \pm 3.9 \mathrm{~mm} / \mathrm{mm}^{2} ; P=0.41\right)$. In both groups the PVD remained stable during the first 2 hours of surgery. However, 2 hours after the start of surgery, the PVD in the PPV/CI group restored and tended to be higher than in the MAP-guided group ( $21.1 \pm 1.9 \mathrm{vs}$. $18.1 \pm 3.4 \mathrm{~mm} / \mathrm{mm}^{2} ; P=0.09$ ). After 1 hour of surgery, the administered fluid volume correlated inversely with PVD $(r=-0.59, P=0.011)$.

Conclusion Goal-directed fluid management resulted in a higher administered fluid volume in the beginning of surgery, and this was associated with a slightly reduced microcirculatory perfusion when compared with MAP-guided fluid management. Microcirculatory perfusion tended to improve as surgery progressed in the goal-directed fluid therapy group. Our findings suggest that goal-directed and MAPguided fluid management are associated with distinct patterns in fluid resuscitation, which may be of consequence for microvascular perfusion.

\section{P209}

Postoperative imaging of the intestinal microcirculation

I Abdo', R Hall', D Henzler', V Cerny², CH Lehmann

'Dalhousie University, Halifax, Canada; ${ }^{2}$ Charles University, Medical Faculty in Hradec Kralove, Czech Republic

Critical Care 2013, 17(Suppl 2):P209 (doi: 10.1186/cc12147)

Introduction Sidestream dark-field (SDF) imaging was introduced recently to study the sublingual microcirculation in humans. Patients with ileostomies offer a unique access to the intestinal microcirculation. To date, no reference ranges for standard microcirculatory parameters of the gut are available. Therefore, the aim of our study was to establish a database for postoperative microcirculatory parameters for ileostomies.

Methods For this observational prospective cohort study 77 patients were screened. In total, 165 SDF measurements could be obtained. All patients included had bowel surgery for chronic inflammatory bowel disease or intestinal malignancies and received an ileostomy. Patients were excluded if they had signs of local infection or bleeding, or if they were admitted to the ICU for sepsis or perioperative complications. The SDF device was gently inserted into the stoma at a depth of 3 to $4 \mathrm{~cm}$, and five real-time images were recorded. All videos recorded were analyzed offline using $\mathrm{AVA}^{\oplus}$ software (Microvision Medical, Amsterdam, the Netherlands). The following parameters were quantified: microvascular flow index (MFI), total vessel density (TVD), perfused vessel density (PVD), and proportion of perfused vessels (PPV). Patients were followed for 3 days post surgery with five images captured every day.

Results We were able to capture clear images of the small intestine microvasculature. Distinct villi were visible with a dense network of microvessels (diameter: 6 to $17 \mu \mathrm{m})$. Mean TVD ( \pm 2SD) was 19.3 $( \pm 1.0) \mathrm{mm} / \mathrm{mm}^{2}$, PVD: $18.5( \pm 1.1) \mathrm{mm} / \mathrm{mm}^{2}$, PPV: $94.5( \pm 5) \%$ and MFI: $2.8( \pm 0.1)$. Patients' age, sex and comorbidity had no significant impact on postoperative microvascular parameters. No significant changes of the microvascular parameters were observed during the first 3 postoperative days.
Conclusion SDF imaging is a feasible, non-invasive bedside method to study the postoperative intestinal microcirculation. The established reference ranges are useful for early detection of postoperative local complications and studies of microcirculatory changes induced by systemic pathologies; for example, in sepsis.

\section{P210}

Monitoring microcirculatory blood flow with a new sublingual tonometer in a porcine model of haemorrhagic shock

P Palagyi, J Kaszaki, Z Molnar

University of Szeged, Hungary

Critical Care 2013, 17(Suppl 2):P210 (doi: 10.1186/cc12148)

Introduction Tissue capnometry has been used to assess organ perfusion but it is not generally available at the bedside [1,2]. Our aim was to test a new sublingual capillary tonometer in haemorrhagic shock.

Methods Thirty-six mini-pigs were anaesthetised, ventilated and divided into sham operated and shock groups. Instrumentation included: intestinal and sublingual tonometry, haemodynamic monitoring (PiCCO; Pulsion, Germany) and orthogonal polarization spectroscopy (OPS). After baseline measurements (TO) haemorrhagic shock was induced and maintained by reducing mean arterial pressure (MAP) to $\sim 40 \mathrm{mmHg}$ for 60 minutes. Measurements were repeated every 30 minutes ( $\mathrm{T} 1$ to $\mathrm{T} 6$ ). Fluid resuscitation started after $\mathrm{T} 2$ aiming to increase MAP to $75 \%$ of the baseline value. OPS imaging was performed at T0, T2 and T6. Data are presented as the median (interquartile range), for statistical analysis Friedmann ANOVA, Mann-Whitney and Spearman tests were used as appropriate.

Results Bleeding resulted in a significant decrease in MAP and cardiac index, and an increase in heart rate. Macrohaemodynamic changes were accompanied by significant changes in red blood cell velocity (RBCV) and a significant increase in the intestinal and sublingual mucosal-to-arterial carbon dioxide partial pressure difference $\left(\mathrm{PCO}_{2}\right.$ gap): from 4 ( 2 to 11 ) to 30 (23 to 37 ) $\mathrm{mmHg}$ in the sublingual, and from 25 (17 to 31 ) to 50 (33 to 64) $\mathrm{mmHg}$ in the ileum. RBCV decreased from $1,075$ (945 to 1,139 ) to 520 (449 to 621$) \mu \mathrm{m} / \mathrm{second}$ in the sublingual area, and from 646 (596 to 712) to 419 (350 to 451) $\mu \mathrm{m} / \mathrm{second}$ in the ileum. There was significant correlation between RBCV and $\mathrm{PCO}_{2}$ gap in sublingual and intestinal regions alike: $r=-0.58 ; r=-0.71, P<0.0001$, respectively.

Conclusion In this model of haemorrhagic shock, sublingual $\mathrm{PCO}_{2}$ gap showed good correlation with RBCV, suggesting that this new sublingual capillary tonometer may be an appropriate tool for monitoring microcirculation at the bedside.

References

1. Creteur et al:: Curr Opin Crit Care 2006, 12:272-277.

2. Cammarata et al: Shock 2009, 31:207-211.

P211

Sublingual microcirculatory changes during transient intra-abdominal hypertension: a study in laparoscopic surgery patients

L Maddison, K Riigor, J Karjagin, J Starkopf

University of Tartu, Tartu University Hospital, Tartu, Estonia

Critical Care 2013, 17(Suppl 2):P211 (doi: 10.1186/cc12149)

Introduction Microvascular alterations play an important role in development of organ failure [1]. It is not known whether increased intra-abdominal pressure (IAP) is associated with microcirculatory perfusion derangements. Our hypothesis was that transiently increased IAP is related to microcirculatory alterations in laparoscopic cholecystectomy patients.

Methods Sixteen patients (14 female, two male) who underwent laparoscopic cholecystectomy were studied. Sublingual orthogonal polarization spectral (OPS) imaging was used to detect microcirculatory function. OPS was done before surgery, at least 15 minutes after initiation of pneumoperitoneum and 1 hour after the end of pneumoperitoneum. The microcirculation cutoff value for vessels was $20 \mu \mathrm{m}$. Data are presented as medians with interquartile ranges. 
Results Patient median age was 54 (39 to 63) years, ASA score 2 (2 to 3 ), BMI 29.7 (24.9 to 34.7), haemoglobin concentration 138 (133 to 142 ) g/l, and hematocrit 42 (39 to 43 ). IAP was held at 12.5 (12 to 13$) \mathrm{mmHg}$, median duration of pneumoperitoneum was 45 (24 to 55) minutes. Median MAP was 86 (69 to 93), abdominal perfusion pressure (APP) 73 (57 to 81$) \mathrm{mmHg}$ during the pneumoperitoneum. Median fluid administration during anesthesia was $1,050(1,000$ to 1,400$) \mathrm{ml}$. Altogether 448 microcirculation videos were taken. Interobserver variability was $24 \%$. The following microcirculatory parameter values describe before, during and after pneumoperitoneum periods. Total vascular density was 19.4 (17.0 to 21.1$) ; 18.5$ (17.0 to 20.9$) ; 19.3$ (16.9 to 20.9) $\mathrm{n} / \mathrm{mm}^{2}$. Perfused vessels density was 13.3 (10.9 to 15.2 ); 13.8 (8.9 to 18.0$) ; 13.1(11.0$ to 16.0$) \mathrm{n} / \mathrm{mm}^{2}$. Proportion of perfused vessels (PPV) was 61 (50 to 69 ); 64 (45 to 76 ); 60 (54 to 67 )\%. Microvascular flow index was 2.4 ( 2.0 to 2.5 ); 2.5 ( 2.0 to 3.0 ); 2.3 (2.0 to 2.9 ) and heterogeneity index was 0.8 (0 to 0.9$) ; 0.6$ ( 0 to 1.0$) ; 0.6$ (0 to 0.8$)$. No significant differences in microcirculatory parameters were observed between time points. PPV was somewhat less than that of described in healthy volunteers (61\% vs. $90 \%)$ [2].

Conclusion Microcirculatory alterations are mild during transient increase of intra-abdominal pressure in laparoscopic surgery patients. References

1. De Backer D, et al:: Crit Care Med 2006, 34:1918-1924.

2. Vellinga NAR, et al.: Crit Care Res Pract 2012. doi:10.1155/2012/121752

P212

Changes in microcirculatory perfusion during cardiac surgery are paralleled by alterations in glycocalyx integrity

C Boer ${ }^{1}$, NJ Koning ${ }^{1}$, J Van Teeffelen ${ }^{2}$, AB Vonk' ${ }^{1}$ H Vink ${ }^{2}$

'VU University Medical Center, Amsterdam, the Netherlands; ${ }^{2}$ Maastricht University, Maastricht, the Netherlands

Critical Care 2013, 17(Suppl 2):P212 (doi: 10.1186/cc12150)

Introduction Previous studies demonstrate that loss of glycocalyx integrity is associated with impaired microvascular function. We investigated whether glycocalyx dimensions are reduced in patients undergoing cardiac surgery with or without cardiopulmonary bypass (CPB), and are paralleled by loss of microcirculatory perfusion using in vivo microcirculation measurements.

Methods Patients undergoing on-pump surgery with nonpulsatile ( $n=11)$ or pulsatile $(n=13)$ CPB or off-pump surgery $(n=13)$ underwent sublingual sidestream dark-field imaging at baseline, during coronary grafting and upon ICU admission to assess perfused microvascular vessel density. Glycocalyx integrity was evaluated using the GlycoCheck Measurement Software, and expressed as the perfused boundary region (PBR). An increase in PBR represents deeper penetration of erythrocytes into the glycocalyx, and is indicative for compromised glycocalyx thickness.

Results The perfused vessel density remained stable during offpump surgery, while the PBR decreased from $2.6 \pm 0.1 \mu \mathrm{m}$ (baseline) to $2.3 \pm 0.1 \mu \mathrm{m}$ (ICU). Nonpulsatile CPB was associated with loss of microcirculatory perfusion during bypass $\left(15.1 \pm 2.7 \mathrm{~mm} / \mathrm{mm}^{2}\right)$ and ICU admission $\left(15.3 \pm 2.6 \mathrm{~mm} / \mathrm{mm}^{2}\right)$ compared with baseline $\left(19.8 \pm 2.8 \mathrm{~mm} / \mathrm{mm}^{2}\right)$. Pulsatile CPB reduced the perfused vessel density from $20.9 \pm 2.4$ to $16.7 \pm 2.6 \mathrm{~mm} / \mathrm{mm}^{2}$, but this was restored towards baseline levels upon ICU admission $\left(20.3 \pm 2.3 \mathrm{~mm} / \mathrm{mm}^{2}\right.$; $P=0.02$ between groups). The PBR increased upon initiation of CPB in both groups, but remained elevated in the nonpulsatile flow group only. In the pulsatile group, the PBR started at $2.4 \pm 0.1 \mu \mathrm{m}$, increased to $2.7 \pm 0.1 \mu \mathrm{m}$ during bypass but restored already during CPB towards a PBR value of $2.3 \pm 0.1 \mu \mathrm{m}$ (ICU). The PBR and perfused vessel density showed a good correlation $(r=-0.65 ; P=0.002)$, demonstrating loss of perfused vascular density when the glycocalyx is damaged.

Conclusion The glycocalyx is damaged after initiation of CPB, and restoration is impaired after exposure to nonpulsatile flow during the use of the heart-lung machine. A reduction in glycocalyx thickness, represented by an increased PBR, correlates with impairment of perfused microvascular vessel density. Our data support the use of intraoperative PBR monitoring as a novel clinical indicator of microcirculatory perfusion.
P213

Microvascular response to cold stress in healthy humans

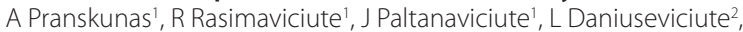
$\checkmark$ Pilvinis'1, M Brazaitis ${ }^{2}$

'Lithuanian University of Health Sciences, Kaunas, Lithuania; '2 Lithuanian Sports University, Kaunas, Lithuania

Critical Care 2013, 17(Suppl 2):P213 (doi: 10.1186/cc12151)

Introduction Cold exposure can be adapted for exercise or therapeutic purposes, but its impact on microcirculation in healthy humans has not been well defined. We hypothesize that whole body cold stress may impair microcirculation.

Methods Seven volunteers were recruited for the water immersion procedure. During the cooling protocol the volunteers every 20 minutes of immersion were asked to step out from the bath and rest for 10 minutes in a room environment and then return to the water bath for the next 20 minutes of immersion. This head-out immersion procedure in bath water at $14^{\circ} \mathrm{C}$ continued until the rectal temperature was dropped to $35.5^{\circ} \mathrm{C}$ or the time of 180 minutes was terminated. Maximum cold water immersion time was 120 minutes. Before, at the end of whole body cooling and 1 hour after cooling was ended, systemic hemodynamics and direct in vivo observation of the sublingual microcirculation were obtained with sidestream dark-field imaging. Assessment of microcirculatory parameters of convective oxygen transport (microvascular flow index (MFI), proportion of perfused vessels (PPV)), and diffusion distance (perfused vessel density (PVD) and total vessel density (TVD)) was done using a semiquantitative method.

Results During cooling and 1 hour after cooling was ended, a significant increase in cardiac output $(P=0.028$ and $P=0.043)$ was observed, but there were no changes in heart rate or mean arterial pressure in comparison with baseline variables. There were no significant changes in PPV, MFI, PVD and TVD of small vessels in comparison with baseline variables during all observational time.

Conclusion Defined cold exposure had no effect on the microcirculation.

P214

Microcirculatory response to experimentally-induced whole body heat stress in healthy humans

A Pranskunas', E Milieskaite', I Maraulaite', L Daniuseviciute².

Z Pranskuniene', V Pilvinis'1, M Brazaitis ${ }^{2}$

'Lithuanian University of Health Sciences, Kaunas, Lithuania: '2 Lithuanian

Sports University, Kaunas, Lithuania

Critical Care 2013, 17(Suppl 2):P214 (doi: 10.1186/cc12152)

Introduction Vasodilation and increased skin blood flow (also sweating) are influential in heat dissipation during heat exposure and exercise. It is unclear how heat stress influences microcirculation. Side dark-field imaging visualizes the blood flow at the capillary level and helps to assess perfusion heterogeneity. Clinical and experimental data show that the sublingual region is clinically relevant for detecting microcirculatory alterations and more represents central microcirculation than cutaneous perfusion. We hypothesize that whole body heat stress may increase capillary density.

Methods Eight healthy men with no history of cold and/or heat injury were recruited to this study. Passive body heating was performed by continuous immersion up to the waist in the water bath at $44^{\circ} \mathrm{C}$ and continued until rectal temperature reached $39.5^{\circ} \mathrm{C}$. Before, at the end of whole body heating and 1 hour after heating was ended, systemic hemodynamics and direct in vivo observation of the sublingual microcirculation were obtained with sidestream dark-field imaging. Assessment of microcirculatory parameters of convective oxygen transport (microvascular flow index (MFI), proportion of perfused vessels (PPV)), and diffusion distance (perfused vessel density (PVD) and total vessel density (TVD)) was done using a semiquantitative method. Vessels were separated into large (mostly venules) and small (mostly capillaries) using a diameter cutoff value of $20 \mu \mathrm{m}$.

Results Whole body heating resulted in significantly increased heart rate $(P=0.012)$ and cardiac output $(P=0.046)$ in comparison with baseline variables. One hour after heating was ended, the heart rate 
remained increased $(P=0.012)$, but cardiac output returned to baseline values. During all observational time the mean arterial pressure remain unaltered. There was no significant difference in MFI and PPV of small vessels at the end of heating and 1 hour after heating in comparison with baseline variables. One hour after heating we observed significant increase in PVD $(P=0.046)$ and TVD $(P=0.028)$ of small vessels regardless of lack of difference at the end of heating $(P=0.753$ and $P=0.075$, respectively) in comparison with baseline variables.

Conclusion Whole body heating induced time-dependent changes in capillary density.

\section{P215}

Observational study of the effects of age, diabetes mellitus, cirrhosis and chronic kidney disease on sublingual microvascular flow

TReynolds' S J Jhanji², A Vivian-Smith', RM Pearse'

'Royal London Hospital, London, UK; 'Royal Marsden Hospital, London, UK Critical Care 2013, 17(Suppl 2):P215 (doi: 10.1186/cc12153)

Introduction Sidestream dark-field (SDF) imaging is an important new technology that has been used to demonstrate microcirculatory abnormalities in a variety of critical illnesses [1]. The microcirculation is also affected by age and chronic comorbidities. However, the effect of these conditions on SDF microcirculatory parameters has not been well described.

Methods Sublingual SDF images were obtained from five groups of 20 participants: healthy volunteers under 25 years, healthy volunteers over 55 years, and stable patients over 55 years with one of diabetes mellitus (DM), cirrhosis and stage 5 chronic kidney disease (CKD). Microcirculatory parameters [1] between the groups were then compared for significance using ANOVA for parametric data and the Kruskal-Wallis test for nonparametric data. This was approved by the local ethics committee.

Results All DM patients were type 2, with mean glycated haemoglobin (HbA1c) of $8.8 \%$ (SD 1.7\%). Seventeen cirrhotic patients were ChildPugh-Turcotte score A and one was score B. For CKD, the mean estimated glomerular filtration rate was $11.5 \mathrm{ml} /$ minute (SD 2.9). Median microvascular flow index (MFI) was 2.85 (IQR 2.75 to 3.0) for participants aged $<25,2.81$ (2.66 to 2.97) for those aged $>55,2.88$ ( 2.75 to 3.0) for those with DM, 3.0 (2.83 to 3.0) for those with cirrhosis and 3.0 (2.78 to 3.0) for those with CKD ( $P$ for difference $=0.14$ ). There were no significant differences in the proportion of perfused vessels and perfused vessel density between the groups. See Figure 1.

Conclusion Older age, diabetes, and chronic kidney and liver disease need not be considered confounding factors for comparison of SDF microcirculatory parameters in the critically ill.

\section{Reference}

1. De Backer D, et al:: How to evaluate the microcirculation: report of a round table conference. Crit Care 2007, 11:R101.

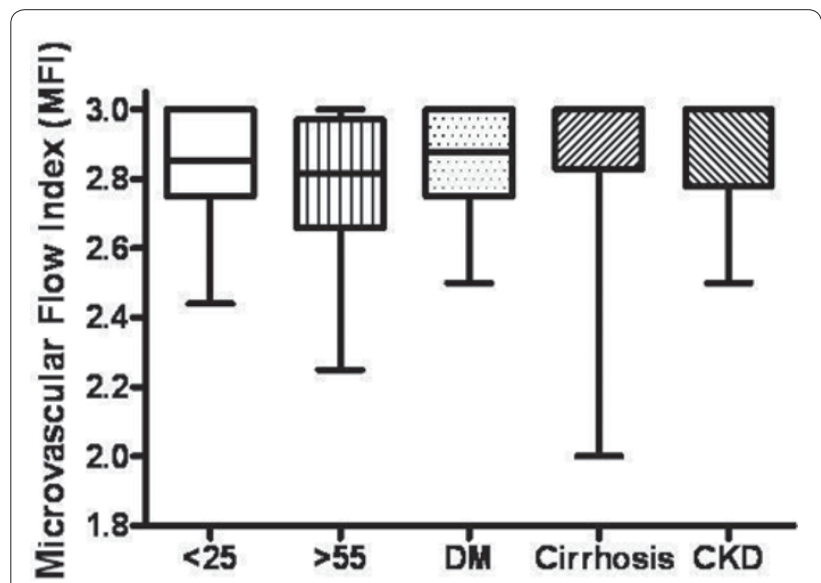

Figure 1 (abstract P215).
P216

Lactate clearance as a determinant of mortality in surgical patients

FF Amorim?', EB Moura², AR Santana', FB Soares', LG Godoy',

TA Rodrigues', LJ Almeida', GM Fllho', TA Silva', AP Amorim³, JA Neto², MO Maia ${ }^{2}$

'Escola Superior de Ciências da Saúde, Brasília, Brazil;'²Unidade de Terapia Intensiva Adulto do Hospital Santa Luzia, Brasília, Brazil; 'iga Academica de Medicina Intensiva do Distrito Federal (LIGAMI), Brasilia, Brazil

Critical Care 2013, 17(Suppl 2):P216 (doi: 10.1186/cc12154)

Introduction Serial measurements of lactate over time may be a better prognosticator than a single lactate concentration [1]. Early lactateguided therapy also reduces ICU length of stay and ICU and hospital mortality [2]. This study aims to assess the prognostic value of the lactate clearance $(\mathrm{LC})$ in the first 24 hours in surgical patients.

Methods In a prospective cohort during 1 year, we followed consecutively enrolled patients admitted immediately postoperative to the surgical ICU of Hospital Santa Luzia, Brasília, Brazil. Patients were assigned to two groups: $\mathrm{LC}>10 \%$ and $\mathrm{LC} \leq 10 \%$. The primary outcome measure was mortality at 7 and 28 days. The secondary outcome included hospital and ICU length of stay (LOS).

Results A total of 417 patients were followed. In total, $50.4 \%$ were male and $83 \%$ underwent elective surgery. The mean age was $59 \pm 16$, APACHE II score $8 \pm 5$, SAPS $226 \pm 11$. The mortality at 7 days was $0.95 \%$ $(n=4)$ and the mortality at 28 days was $2.15 \%(n=9)$, respectively. Hospital mortality was $4.79 \%(n=20)$. Sixty-one percent $(n=255)$ of the patients had $\mathrm{LC}>10 \%$ versus $39 \%(n=162)$ with $\mathrm{LC} \leq 10 \%$. Those who had $\mathrm{LC} \leq 10 \%$ were older ( $62 \pm 16$ vs. $57 \pm 17, P=0.00$ ) and had greater APACHE II score ( $9 \pm 6$ vs. $7 \pm 4, P=0.00)$ and SAPS $2(28 \pm 12$ vs. $25 \pm 10, P=0.02)$. There was no difference in ICU LOS $(5 \pm 12$ vs. $4 \pm 9$ days, $P=0.54)$ and hospital $\operatorname{LOS}(10 \pm 15$ vs. $9 \pm 11$ days, $P=0.48)$. Initial lactate levels were lower in the group with $\mathrm{LC} \leq 10 \%(1.1 \pm 0.9$ vs. $1.9 \pm 1.6, P=0.00)$; however, mean lactate was higher in 24 hours ( $2.0 \pm 1.8$ vs. $1.0 \pm 0.7, P=0.00$ ). All of the patients who died in the first 7 days had $\mathrm{LC} \leq 10 \%(2.46 \%, n=4, P=0.02)$; this group also had a higher mortality at 28 days $(4.32 \%, n=7$ vs. $0.78 \%, n=2 ; P=0.03)$. The relative risk for mortality $\mathrm{LC} \leq 10 \%$ in 7 and 28 days was $1.02(95 \% \mathrm{Cl}$ : 1.00 to 1.05 ) and 5.07 ( $95 \%$ Cl: 1.17 to 27.09 ), respectively. Significant difference was observed in the Kaplan-Meier survival curves for 7 and 28 days ( $P=0.01$ and 0.02 , respectively). The sensibility of $L C \leq 10 \%$ was $100 \%$ (95\% Cl: 51 to 100\%) for 7-day mortality and 78\% (95\% Cl: 45 to 94\%) for 28 -day mortality. The specificity was $62 \%$ (95\% Cl: 57 to $66 \%$ ) for 7 -day mortality and $62 \%(95 \% \mathrm{Cl}: 57$ to $66 \%)$ for 28 -day mortality. Conclusion Despite initial lactate levels, lactate clearance $\leq 10 \%$ proved to be a good predictor of mortality in 7 and 28 days in surgical patients admitted in the postoperative period to the ICU.

References

1. Nguyen HB, et al:: Crit Care Med 2004, 32:1637-1642.

2. Jansen TC, et al:: Am J Respir Crit Care Med 2010, 182:752-756.

\section{P217}

Assessment of the mottling score as a mortality predictor in critically ill patients

E Bastos de Moura, F Ferreira Amorim, C Darwin Silveira, M Oliveira Maia Hospital Santa Luzia, Brasilia, Brazil

Critical Care 2013, 17(Suppl 2):P217 (doi: 10.1186/cc12155)

Introduction The use of peripheral perfusion objective parameters to anticipate successful resuscitation in septic shock has been recently investigated [1]. The mottling score, a perfusion parameter used for decades, has been proposed to correlate with septic shock survival [2], and was tested in this study as a clinical tool in predicting mortality. Methods A prospective observational study was conducted, with patients consecutively admitted to a tertiary hospital ICU in Brasília, Brazil. From July 2011 to May 2012, all patients diagnosed with septic shock were enrolled. Demographic data, diagnoses, shock origin and severity scores were recorded. After initial resuscitation, the score was registered in the first 3 days by the same observer, considering the score on the lower limb without an arterial catheter, or the worst between the lower limbs, and the worst in the 3 days. Exclusion criteria were terminal illness with no intervention decision and incomplete 
data. The scores are pooled in Group 1 (scores 0 and 1), Group 2 (scores 2 and 3 ) and Group 3 (scores 4 and 5) to compare mortality. Statistical analysis was made using the chi-square test.

Results One hundred and seventeen patients were analyzed; 20 were excluded (18 terminal illness, two with incomplete data). Ninety-seven patients were included; the mean age was 72.8 years, mean SAPS II score was 46.8 (SD \pm 15.7 ), mean APACHE II score was 19.2 (SD \pm 8.1 ); mean norepinephrine dose was $1.25 \mu \mathrm{g} / \mathrm{kg} /$ minute; mean length of stay in ICU was 19.2 days (1 to 176); mottling score distribution was: score 0: 41 patients; score 1: 33 patients; score 2: 14 patients; score 3: two patients; score 4: three patients; score 5 : four patients. The sepsis origin was as follows: 65 , pulmonary $(67 \%) ; 18$, abdominal $(18.5 \%)$; nine, urinary (9.5\%); two, osseous (2\%); one, mediastinal; one, skin and soft tissue; and one, central nervous system ( $1 \%$ each). Comparing the mortality in Groups 1, 2 and 3, we found a significant difference $(P=0.042)$, even greater when considering 28 -day mortality $(P=0.004)$. The Kaplan-Meier survival method showed $P=0.000$.

Conclusion The mottling score was an objective reproducible system to bedside use and a good predictor of septic shock mortality.

References

1. Hernandez $G$, et al:: Evolution of peripheral vs metabolic perfusion parameters during septic shock resuscitation. A clinical-physiologic study. J Crit Care 2012, 27:283-288.

2. Ait-Oufella $\mathrm{H}$, et al: Mottling score predicts survival in septic shock. Intensive Care Med 2011, 37:801-807.

P218

Impact of different haemodynamic resuscitation strategies on brain perfusion and tissue oedema markers in a model of severe haemorrhagic shock

KK Ida, DA Otsuki, LU Castro, TR Sanches, MH Shimizu, LC Andrade, JO Auler-Jr, LM Malbouisson

Faculdade de Medicina, Universidade de São Paulo, Brazil

Critical Care 2013, 17(Suppl 2):P218 (doi: 10.1186/cc12156)

Introduction This study aimed to compare the cerebral effects of terlipressin (TERLI) with conventional prehospital fluid resuscitation with lactated Ringer's solution (LR) in a model of haemorrhagic shock (HS).

Methods Pigs (20 to $30 \mathrm{~kg}$ ) were randomized into one of the groups: Sham $(n=2)$, HS $(n=9)$, LR ( $3 \times$ volume bled; $n=9)$ or TERLI $(2$ mg bolus; $n=9$ ). HS induced to target MAP of $40 \mathrm{mmHg}$ was maintained for 30 minutes. Brain tissue oxygen pressure $\left(\mathrm{PbtO}_{2}\right)$, intracranial pressure (ICP), cerebral perfusion pressure (CPP), haemodynamics and blood gas analyses were assessed prior to HS (baseline) up to 120 minutes after treatment. Tissue markers of brain oedema (aquaporin-4 (AQP4) and $\mathrm{Na}-\mathrm{K}-\mathrm{Cl}$ cotransporter-1 (NKCC1)), apoptosis (pre-apoptotic protein (Bax)) and oxidative stress (thiobarbituric acid reactive substances (TBARS)) were also measured.

Results Sham animals had no significant changes in the variables assessed. HS resulted in a significant decrease in CPP (mean varied from 36 to $39 \mathrm{mmHg}$ ), PbtO (from 23.6 to $26.6 \mathrm{mmHg}$ ), ICP (from 1 to $2 \mathrm{mmHg}$ ) and haemodynamics (MAP from 38 to $40 \mathrm{mmHg}$; Cl from 1.8 to $2.1 \mathrm{l} / \mathrm{minute} / \mathrm{m}^{2}$ ), and a significant increase in blood lactate (from 6.7 to $8.9 \mathrm{mmol} / \mathrm{l}$ ) and cerebral AQP4 (mean \pm SE; $167 \pm 54 \%$ of sham), NKCC1 ( $237 \pm 47 \%$ of sham), Bax (167 $\pm 44 \%$ of sham) and TBARS. Fluid resuscitation was followed by an increase in ICP (from 7 to $9 \mathrm{mmHg}$ ) and a decrease in CPP (from 41 to $52 \mathrm{mmHg}$ ), with an increased expression of cerebral AQP4 $(210 \pm 56 \%$ of sham), NKCC1 $(163 \pm 32 \%$ of sham) and Bax (137 $\pm 24 \%$ of sham). Only TERLI restored baseline values of CPP (from 54 to $61 \mathrm{mmHg}$ ) and did not change the cerebral expression of AQP4 (100 $\pm 6 \%$ of sham), NKCC1 (100 $\pm 1 \%$ of sham), Bax (102 $\pm 6 \%$ of sham) and TBARS. Both TERLI and LR recovered baseline levels of $\mathrm{PbtO}_{2}$ (TERLI from 30.0 to $34.2 \mathrm{mmHg}$; LR from 29.4 to $40.7 \mathrm{mmHg}$ ) and MAP (TERLI from 53 to $64 \mathrm{mmHg}$; LR: 48 to $61 \mathrm{mmHg}$ ). Blood lactate levels were not recovered in any group (TERLI from 5.7 to $8.1 \mathrm{mmol} / \mathrm{l}$; LR from 4.5 to $7.7 \mathrm{mmol} / \mathrm{l})$.

Conclusion TERLI recovered cerebral perfusion and oxygenation with no significant changes in ICP and cerebral markers of oedema, apoptosis and oxidative stress. LR did not recover CPP probably due to a significant increase in ICP caused by brain oedema, which may have contributed to the cerebral apoptosis. None of the treatments caused cerebral oxidative stress.

References

1. Cavus E, et al:: Resuscitation 2009, 80:567-572.

2. Bayram B, et al:: Am J Emerg Med 2011, 30:1176-1182

P219

Accuracy of the shock index and various modified shock indexes to predict early mortality in patients suffering from gastrointestinal haemorrhage

J St-Cyr Bourque, J Cliche, J Chauny, R Daoust, J Paquet, É Piette

Hôpital du Sacré-Coeur de Montréal, Canada

Critical Care 2013, 17(Suppl 2):P219 (doi: 10.1186/cc12157)

Introduction The shock index (SI) is an easy-to-use clinical tool that rapidly identifies patients at risk of haemodynamic decompensation. Previous studies focused primarily on patients suffering from pneumonia, pulmonary embolism, ruptured ectopic pregnancy and traumatic haemorrhagic shock. Modified SIs have also been studied. Could the SI be accurate in patients suffering from gastrointestinal (GI) haemorrhage? The aim of this study was to compare the performance of the SI with various modified SIs and conventional vital signs in predicting 30-day mortality in a population of patients with a GI haemorrhage.

Methods A single-center post-hoc analysis was conducted of prospectively collected data from patients diagnosed with a GI haemorrhage episode in an academic emergency department (ED) from March 2008 to December 2011. Data were extracted from two databases used at our ED. The SI (pulse/systolic blood pressure) and nine modified Sls were calculated from the available first documented vital signs. ROC curves were used to determine sensitivity and specificity of the different SIs in predicting 30-day mortality.

Results Of the 770 patients included in the analysis, 52 died within 30 days. The standard $\mathrm{SI}$ at a cutoff point of 0.7 had the highest predictability and sensitivity of 30-day mortality (area under the curve $(A \cup C)=0.7$, sensitivity $=0.79$, specificity $=0.56$ ). In comparison, one of the modified SIs (pulse/diastolic blood pressure) had 0.65 sensitivity and 0.71 specificity (AUC $=0.73$ ). A heart rate $>100 \mathrm{bpm}$ predicted 30 day mortality with 0.40 sensitivity and 0.82 specificity (AUC $=0.63$ ).

Conclusion To our knowledge, this is the first study to examine the relationship between the SI and mortality in patients with a Gl haemorrhage. It appears that the standard SI, when compared with various modified Sls and conventional vital signs, had the highest combined predictability and sensitivity of 30-day mortality in a population of patients suffering from GI haemorrhage. Further prospective studies are needed to confirm these findings.

\section{P220}

Inhaled carbon monoxide or nebulized sodium nitrite protect against hemorrhagic shock-induced mitochondrial dysfunction H Gomez, D Escobar, B Ataya, L Gordon, O Ogundele, M Pinsky, S Shiva,

B Zuckerbraun

University of Pittsburgh, PA, USA

Critical Care 2013, 17(Suppl 2):P220 (doi: 10.1186/cc12158)

Introduction Shock induces mitochondrial damage, which can lead to tissue injury and inflammation. Resuscitative adjuncts to limit mitochondrial injury may be effective to reduce tissue injury and protect against the sequelae of hemorrhagic shock (HS). Others and we have demonstrated the protective effects of inhaled carbon monoxide (CO) or nebulized sodium nitrite $\left(\mathrm{NaNO}_{2}\right)$ in models of $\mathrm{HS}$. Our aim was to test the hypothesis that $\mathrm{CO}$ and $\mathrm{NaNO}_{2}$ protect against hemorrhagic shock-induced tissue injury/inflammation by limiting mitochondrial damage and preventing bioenergetic failure.

Methods Twenty anesthetized female Yorkshire pigs were subjected to severe hemorrhage until unable to compensate or 90 minutes, and were then resuscitated with volume/pressors. Muscle and platelet samples were obtained at baseline (BL) and 2 hours after resuscitation (EndObs). Animals were randomized to: standard of care ( $\mathrm{HSR}, n=5)$; $\mathrm{HSR}+\mathrm{CO}(\mathrm{CO} ; 250 \mathrm{ppm} \times 30$ minutes, $n=6) ;$ or $\mathrm{HSR}+\mathrm{NaNO}_{2}\left(\mathrm{NaNO}_{2}\right.$; 
$11 \mathrm{mg}$ in PBS $\times 30$ minutes, $n=6)$, and sham $(n=3)$. $\mathrm{CO}$ or $\mathrm{NaNO}_{2}$ were initiated $\sim 30$ minutes before resuscitation. Primary endpoints were changes in muscle and platelet mitochondrial respiration between $\mathrm{BL}$ and EndObs, quantified by muscle respiratory control ratio $(\mathrm{RCR}$, traditional respirometry), and by the change in proton-leak respiration (PLR) and mitochondrial reserve capacity in platelets. Secondary endpoint was mortality at EndObs.

Results Skeletal muscle RCR decreased in the HSR group $(P=0.04)$ but not in sham. Decrease in RCR was primarily due to decreased ADPdependent respiration, without change in state 4 respiration. HSR also resulted in platelet mitochondrial dysfunction as demonstrated by increased PLR and decreased reserve capacity. This correlated with increased platelet activation (\%CD62P+ by flow cytometry) in HSR. CO or $\mathrm{NaNO}_{2}$ treatment prevented these deleterious changes in both muscle and platelet mitochondrial respiration, as well as limited HSR-induced platelet activation. CO treatment also improved reserve capacity compared with baseline. Mortality was higher in HSR than in $\mathrm{CO}$ or $\mathrm{NaNO}_{2}$ (80 vs. 33 and 33\%, respectively).

Conclusion In severe $\mathrm{HS}$, mitochondrial injury in platelets and muscle was limited by $\mathrm{CO}$ or $\mathrm{NaNO}_{2}$. Although not powered for a secondary endpoint, mortality was double in HSR versus adjunctive therapies. This suggests that $\mathrm{CO}$ and $\mathrm{NaNO}_{2}$ may protect mitochondrial function by maintaining ATP-coupled respiration and reserve capacity, and that this may confer a survival advantage. However, further investigations are required.

\section{P221}

Is norepinephrine more effective than other vasopressors for septic shock? A systematic review and meta-analysis

F Zhou', Z Peng'2, W Zhang 3 , J Bishop², Q Song

'Chinese People's Liberation Army General Hospital, Beijing, China; ${ }^{2}$ University of Pittsburgh, PA, USA; ${ }^{3}$ University of Pittsburgh School of Medicine, PA, USA

Critical Care 2013, 17(Suppl 2):P221 (doi: 10.1186/cc12159)

Introduction Norepinephrine has been widely used in septic shock. However, its effect remains controversial. We conduct a systematic review and meta-analysis to compare the effect between norepinephrine and other vasopressors.

Methods The PubMed, Embase, and Cochrane Library databases from database inception until October 2012 were searched. We selected randomized controlled trials in adults with septic shock and compared norepinephrine with other vasopressors. The quality of each study included was assessed with Jadad score. After assessing for heterogeneity of treatment effect across trials using the $I^{2}$ statistic, we used a fixed effect model $(P \geq 0.1)$ or random-effects model $(P<0.1)$ and expressed results as the risk ratio (RR) for dichotomous outcomes or the standardized mean difference (SMD) for continuous data with $95 \% \mathrm{Cl}$. Results Eighteen trials $(n=2,715)$ met inclusion criteria, which compared norepinephrine with five different vasopressors (dopamine, vasopressin, epinephrine, terlipressin and phenylephrine). The mean Jadad score was 3.11. Overall, there was no difference in mortality in the comparisons between norepinephrine and vasopressin epinephrine, terlipressin and phenylephrine $(P>0.05$, respectively). However, norepinephrine had a trend in decreasing mortality compared with dopamine (RR, $0.84 ; 95 \% \mathrm{Cl}, 0.68$ to $1.02 ; P=0.08)$. There were a decreased heart rate (HR) (SMD, $-2.10 ; 95 \% \mathrm{Cl},-3.95$ to -0.25 ; $P=0.03)$, cardiac index $(\mathrm{SMD},-0.73 ; 95 \% \mathrm{Cl},-1.14$ to $-0.03 ; P=0.004)$ and an increased systemic vascular resistance index (SVRI) (SMD, 1.03; $95 \% \mathrm{Cl}, 0.61$ to $1.45 ; P<0.0001$ ) with the treatment of norepinephrine compared with dopamine.

Conclusion There is not sufficient evidence to prove that norepinephrine is superior to vasopressin, epinephrine, terlipressin and phenylephrine in terms of mortality. However, norepinephrine is associated with a decreased HR, cardiac index and an increased SVRI, and appears to have a greater effect on decreasing mortality compared with dopamine.

References

1. Jadad AR, et al: Control Clin Trials 1996, 17:1-12

2. Higgins JP, et al.: BMJ 2003, 327:557-560.
P222

Vasopressin Versus Norepinephrine for the Management of Shock After Cardiac Surgery (VaNCS study): a randomized controlled trial L Hajjar', JL Vincent², A Rhodes ${ }^{3}$, D Annane ${ }^{4}$, F Galas', J Almeida', S Zeferino', L Camara', V Santos', J Pereira', E Osawa', E Maciel', A Rodrigues', J Jardim', D Blini', E Araujo', F Bergamin', R Kalil Filho', J Auler J $\mathrm{r}^{1}$

'Heart Institute, São Paulo, Brazil:' 'Universite Libre de Bruxelles, Brussels,

Belgium; ${ }^{3}$ University of London, UK; ${ }^{4}$ Faculte Medicine Paris, France; ${ }^{5}$ Cancer Institute, São Paulo, Brazil

Critical Care 2013, 17(Suppl 2):P222 (doi: 10.1186/cc12160)

Introduction Vasoplegic syndrome is a common complication after cardiac surgery, with negative impact on patient outcomes and hospital costs. Pathogenesis of vasodilatory phenomenon after cardiac surgery remains a matter of controversy. Loss of vascular tone can be partly explained by the depletion of neurohypophyseal arginine vasopressin stores. Vasopressin is commonly used as an adjunct to catecholamines to support blood pressure in refractory septic shock, but its effect on vasoplegic shock is unknown. We hypothesized that the use of vasopressin would be more effective on treatment of shock after cardiac surgery than norepinephrine, decreasing the composite endpoint of mortality and severe morbidity.

Methods In this prospective and randomized, double-blind trial, we assigned patients who had vasoplegic shock to receive either vasopressin (0.01 to $0.06 \mathrm{U} /$ minute) or norepinephrine (0.01 to $1 \mu \mathrm{g} /$ $\mathrm{kg} /$ minute) in addition to open-label vasopressors. All vasopressor infusions were titrated and tapered according to protocols to maintain a target blood pressure. The primary endpoint was major morbidity according to STS (30-day mortality, mechanical ventilation $>48$ hours, mediastinitis, surgical re-exploration, stroke, acute renal failure). Secondary outcomes were time on mechanical ventilation, ICU and hospital stay, new infection, the time to attainment of hemodynamic stability, occurrence of adverse events and safety.

Results A total of 300 patients underwent randomization, were infused with the study drug (148 patients received vasopressin, and 152 norepinephrine), and were included in the analysis. Patients who received vasopressin had a lower rate of morbidity $(23.5 \%$ vs. $34 \%$, $P=0.001)$ as compared with the norepinephrine group. The 30-day mortality rate was $6.1 \%$ in the norepinephrine group and $4.6 \%$ in the vasopressin group $(P=0.570)$. There were no significant differences in the overall rates of serious adverse events $(7.4 \%$ and $6.6 \%$, respectively; $P=0.772$ ).

Conclusion Vasopressin reduces major morbidity after cardiac surgery as compared with norepinephrine among patients with cardiac surgery with vasoplegic shock.

Acknowledgement Clinical Trials number NCT01505231.

\section{P223}

Intraaortic counterpulsation in a second-level institution:

indications, management and outcome

R Gómez López, P Fernández Ugidos, PVidal Cortés, M Lorenzo Lage,

A Tizón Varela, E Rodríguez Álvarez

Complexo Hospitalario Universitario de Ourense, Spain

Critical Care 2013, 17(Suppl 2):P223 (doi: 10.1186/cc12161)

Introduction Counterpulsation is an important support for patients with cardiac diseases. The use of these devices has been limited typically to hospitals with a cardiac surgery service. The aim of this study is to describe the management of patients with an intraaortic balloon pump (IABP) in our second-level institution.

Methods An observational study that includes all patients with IABP in our hospital from January 2010 to September 2012, followed to November 2012. Epidemiological and clinical variables as an indication of $I A B P$, therapeutic management and outcome were collected. Because of the small size sample, statistical analysis was limited to descriptive parameters.

Results Seventeen patients ( $76.5 \%$ men, age $66.6 \pm 11.6$ (43 to 85 ) years, APACHE score $21.5 \pm 14.9$ ( 3 to 46 ) points) were treated with IABP. The reason for ICU admission was STEMI ( $n=6$ patients, 35.3\%), no-STEMI $(n=5,29.4 \%)$, acute heart failure $(n=4,23.5 \%)$ and out-of-hospital 
cardiac arrest $(n=2,11.8 \%)$. The indications of IABP were refractory cardiogenic shock ( $n=12,70.6 \%)$, high-risk percutaneous coronary intervention $(n=2,11.8 \%)$, refractory angina $(n=1,5.9 \%)$, refractory pulmonary edema $(n=1,5.9 \%)$ and electrical storm $(n=1,5.9 \%)$. Five IABPS (27.4\%) were inserted in the ICU and the rest in the catheterization laboratory. Six patients (35.3\%) suffered a cardiac arrest prior to hemodynamic stabilization. Three patients $(17.6 \%$, the electrical storm and the out-of-hospital cardiac arrest) died before coronary catheterization was performed and the other three were treated with mild therapeutic hypothermia. Thirteen patients (76.5\%) needed invasive mechanical ventilation. In six patients (35.3\%) invasive hemodynamic monitoring was performed (one pulmonary artery catheter, five PICCO). Transthoracic echocardiography was performed in all patients and transesophageal in six (35.3\%). Six of the patients $(35.3 \%)$ were transferred to the reference centre for immediate coronary artery bypass grafting (CABG). No complications were reported during the transfer. During the ICU stay, femoral artery pseudoaneurysm was reported in one of the patients and inguinal hematoma after IABP withdrawal in two (no transfusion required). Four patients (27.4\%) died because of refractory cardiogenic shock despite revascularization. The length of mechanical support was $1.67 \pm 1.5$ days (0 to 6 ).

Conclusion In our hospital the IABP was mainly used in refractory cardiogenic shock because of myocardial ischemia, with an all-cause mortality of $41.2 \%$. Low rates of complications were observed. Transfer of patients with IABP was performed safely.

P224

Impact of left ventricular ejection fraction and elevated filling pressures on mortality in mechanically ventilated patients in severe sepsis or septic shock

S Gillespie, J Pulido

Mayo Clinic, Rochester, MN, USA

Critical Care 2013, 17(Suppl 2):P224 (doi: 10.1186/cc12162)

Introduction Myocardial dysfunction in septic shock is common and the presentation is broad. There are conflicting data regarding the prognostic implications of low left ventricular (LV) ejection fraction and elevated $\mathrm{E} / \mathrm{e}^{\prime}$ ratio on mortality in this patient population. We sought to assess the impact of LV dysfunction and elevated E/e' ratio on 30day mortality in mechanically ventilated patients with severe sepsis or septic shock.

Methods Fifty-eight mechanically ventilated patients with severe sepsis or septic shock admitted from 1 August 2007 to 31 January 2009 were prospectively evaluated with transthoracic echocardiogram within 24 hours of admission. Left ventricular ejection fraction was assessed using the modified Simpson method as recommended by the American Society of Echocardiography. Normal LV function was defined as LVEF $40 \%$. Mitral inflow pulsed wave Doppler of peak E waves and tissue Doppler imaging (TDI) of the septal mitral annulus peak velocities were measured, the E/e' ratio was obtained. Elevated LV filling pressures was defined as $\mathrm{E} / \mathrm{e}^{\prime}>15$.

Results All cause 30-day mortality was $50 \%(n=29)$. Forty-six patients (79\%) had normal LV function. Forty-four (76\%) patients had normal LV filling pressures. Patients who survived had lower E/e' ratio, (median $\mathrm{E} / \mathrm{e}^{\prime} 10.6 \pm 4$ vs. $13.6 \pm 7, P=0.1$ ) but this was not statistically significant. However, when defined as E/e' $>15$, the mortality was $71 \%$. On the contrary, patients with low LVEF had a mortality of $41 \%$ and LVEF was no different between survivors and nonsurvivors ( $55 \pm 16$ vs. $58 \pm 14$, $P=0.34$ )

Conclusion Myocardial dysfunction is a well-known entity in patients with septic shock. The clinical spectrum of this entity is broad, including $\mathrm{LV}, \mathrm{RV}$ and diastolic dysfunction. Although the E/e' ratio has been a known prognostic indicator in other cardiac conditions, its role in these patients is less clear. This study demonstrated that when septal mitral annulus $E / e^{\prime}$ was $>15$, it was a better marker for mortality than LVEF in mechanically ventilated patients with severe sepsis or septic shock. Larger studies incorporating diastolic evaluation and TDI should be performed to further clarify this finding.

References

1. J Cardiothorac Vasc Anesth 2011, 25:526-535.

2. Crit Care Med 2009, 37:441-447.

3. Mayo Clin Proc 2012, 87:620-628.
P225

Effect of $\beta$-blockers and calcium channel blockers on shock index predictability in patients suffering from urosepsis

J Cliche, J St-Cyr Bourque, R Daoust, J Chauny, J Paquet, E Piette

Sacré-Coeur Hospital, Montreal, Canada

Critical Care 2013, 17(Suppl 2):P225 (doi: 10.1186/cc12163)

Introduction In our busy emergency departments the identification of patients at risk of rapid hemodynamic decompensation is crucial. The shock index (SI) (pulse/systolic blood pressure) is a non-invasive clinical sign associated with more complications if higher than 0.7. The effect of $\beta$-blockers (BB) and calcium channel blockers (CCB) on the SI has not yet been described. Most studies excluded these patients, owing to the medications' effect on the cardiac pulse. Considering that BB and CCB are commonly prescribed, we studied their effect on the Sl's predictability on 30-day mortality in a urosepsis population.

Methods This single-center post-hoc analysis of prospectively collected data was conducted in an academic Canadian emergency department (ED) between March 2008 and February 2011. Data were extracted from two institutional databases. We included patients with a final diagnosis of urosepsis, sepsis, pyelonephritis and urinary tract infection. Selected patients also had a documented positive urine culture. The SI predictability on 30-day mortality was calculated for patients taking $\mathrm{BB}$ and/or CCB as well as patients taking none of these medications. Sensitivity and specificity were determined using ROC curves. $t$ tests were used to compare mean $\mathrm{SI}$ between both groups.

Results Our urosepsis population contained 364 patients, of which 129 (35.4\%) were either using a BB, a CCB or both before their admission. Mean age was 74.9 years and $48.1 \%$ of the patients were women. A total of 36 patients died $(9.9 \%)$ in a 30-day period. The group taking either BB or CCB had a significantly lower mean SI $(0.76,95 \%$ Cl: 0.72 to 0.81 vs. $0.93,95 \% \mathrm{Cl}: 0.89$ to $0.98, P<0.0001)$. In our urosepsis population, a $\mathrm{SI}$ of 0.7 had a sensitivity of 0.76 and a specificity of 0.27 (area under the curve (AUC): 0.596 ) for patients taking neither BB nor CCB. In the group taking either or both medications, a SI of 0.7 had a sensitivity of 0.57 and a specificity of 0.42 (AUC: 0.578 ). In both groups, lowering the SI to 0.5 increased the sensitivity to more than 0.95 but lowered specificity significantly.

Conclusion To our knowledge this is the only study analysing the effect of BB and CCB on the SI predictability of 30-day mortality. Our results indicate that SI cannot be used to accurately predict mortality with patients suffering from urosepsis. In both our groups, SI performance was poor, as shown by the ROC curves. BB or CCB did not influence these results.

\section{P226}

Levosimendan in critically ill adults: a utilisation review

J Aron', S Harrison², A Milne'2, S Patel2, R Maharaj ${ }^{2}$

'Lewisham NHS Trust, London, UK; 'King's College Hospital NHS Trust, London, UK

Critical Care 2013, 17(Suppl 2):P226 (doi: 10.1186/cc12164)

Introduction Levosimendan improves haemodynamic performance and may have cardioprotective effects. Small trials have demonstrated efficacy in well-defined populations [1]. Given the uncertain benefits we describe contemporaneous patient selection, utilisation of levosimedan, haemodynamic effects and outcomes.

Methods The study was performed at a single centre's three ICUs. Data from 38 consecutive admissions, from 2010 to 2012, treated with levosimendan were collected retrospectively. Demographics, illness severity, comorbidity, haemodynamic, metabolic, biochemical, resource utilisation, organ support and hospital outcomes were analysed.

Results Our cohort had a mean age of $58(95 \% \mathrm{Cl}=53.4$ to 64.4$)$. Only $18 \%$ underwent cardiothoracic surgery: sepsis, pulmonary emboli and myocardial/pericardial diseases were also treated with levosimendan. Admission characteristics included mean $\mathrm{PaO}_{2} / \mathrm{FiO}_{2}$ ratio (PFR) of $186.7 \mathrm{mmHg}$ ( $95 \% \mathrm{Cl}=160$ to 212 ) and mean cardiac index (CI) of 2.3 ( $95 \% \mathrm{Cl} 1.9$ to 2.8 ). The median APACHE II score was 24 (interquartile range 18.5 to 30 ). Levosimendan was initiated at a variable point during ICU treatment (days 0 to 14 ) and was usually the third inotrope 
Table 1 (abstract P226). Treatment outcomes

\begin{tabular}{lcc}
\hline Variable & Mean & $95 \% \mathrm{Cl}$ \\
\hline $\mathrm{Cl} \triangle \mathrm{d} 5-\mathrm{d} 0$ & +1.34 & 0.4 to 2.2 \\
$\mathrm{PFR} \triangle \mathrm{d} 5-\mathrm{d} 0$ & +284 & 223 to 345 \\
$\mathrm{BE} \triangle \mathrm{d} 5-\mathrm{d} 0$ & +2.9 & 0.85 to 5 \\
Lac $\triangle \mathrm{d} 5-\mathrm{d} 0$ & -1 & -2 to 0.2 \\
\hline
\end{tabular}

do, treatment commenced.

(range 0 to 5) commenced. Treatment with levosimendan (Table 1) resulted in improved $\mathrm{Cl}$ and PFR with reductions in $\mathrm{EVLW}, \mathrm{BE}$, lactate and creatinine. The ICU length of stay was 15.4 days $(95 \% \mathrm{Cl}=13.2$ to $17.6)$ and the hospital mortality was $54 \%$. No significant adverse effects were reported.

Conclusion Levosimendan is usually used in patients with cardiogenic shock unresponsive to conventional inotropes or mechanical augmentation. The mortality of this group is high but represents patients with shock refractory to conventional treatment. Its use in sepsis, myocarditis and pulmonary embolism is not well established. In this group levosimendan appears to have a favourable effect on gas exchange, renal function and tissue perfusion. Limitations include retrospective analysis and missing data.

Reference

1. Landoni $G$, et al:: Effects of levosimendan on mortality and hospitilisation. A meta-analysis of RCT. Crit Care Med 2012, 40:634-646.

P227

Levosimendan versus dobutamine in Tako-tsubo cardiomyopathy

FR Righetti, MP Parolini, GC Castellano

St. Boniface Hospital Verona, Italy

Critical Care 2013, 17(Suppl 2):P227 (doi: 10.1186/cc12165)

Introduction Tako-tsubo cardiomyopathy is a clinical entity characterized by a dysfunction of the left ventricle, usually transient, which manifests itself with symptoms that can mimic an acute coronary syndrome. It is characterized by transient ballooning modification of the left ventricular apex, probably due to neurogenic stimuli resulting in low cardiac output syndrome. The aim of this prospective randomized study was to evaluate, by serial transesophageal echocardiography (TEE), what is the best drug treatment between levosimendan and dobutamine to restore a satisfactory cardiac function in the case of low cardiac output.

Methods Twelve adult patients, aged 18 years, were admitted to the ICU with Tako-tsubo cardiomyopathy at entrance. The patients were divided randomly into two groups: levosimendan (six patients) treated with levosimendan and standard treatment, and the control group (six patients) with dobutamine and standard treatment. In all patients, serial TEE was performed studying the systolic function, by ejection fraction of the left ventricle with Simpson's method. The TEEs were performed at the entrance of the patient, after 12 and 24 hours of treatment. The results were expressed as mean with standard deviation. For the comparison between the two groups we used the Fisher test, considered significant with $P<0.05$

Results Patients in the two groups were statistically comparable with respect to sex $(P=0.31)$ and age $(P=0.53)$. The causes of the syndrome of Tako-tsubo were: subarachnoid hemorrhage (six patients) after coronary artery bypass graft (four patients), and polytrauma (two patients). All patients had low cardiac output. In the levosimendan group the ejection fraction at entrance was $25 \pm 6 \%$, after 12 hours $36 \pm 10 \%$, and $47 \pm 5 \%$ after 24 hours. In the control group the ejection fraction at entrance was $24 \pm 7 \%$, after 12 hours $28 \pm 6 \%$ and after 24 hours $33 \pm 4 \%$. Comparing the two groups we reached statistical significance, $P=0.026$.

Conclusion Comparing the two groups, we noticed that both started from a low cardiac output. However, in the group who used the drug therapy based on levosimendan we saw a return of systolic function of the left ventricle to near-normal levels within 24 hours, while in the control group there remains a dysfunction in systolic function. We have shown the drug therapy based on levosimendan contributes to improving the systolic function of the left ventricle compared with treatment with dobutamine despite the initial cardiac stunning. Reference

1. Landoni $G$, et al:: Effects of levosimendan on mortality and hospitalization. A metanalysis of randomized controlled studies. Crit Care Med 2012, 40:634-646.

P228

Combined effects of ivabradine with dobutamine or levosimendan in isolated perfused hearts

D Konrad, R Vicenzi-Moser, M Vicenzi, U Aldenhoff, E Schwarzl, W Toller Medical University Graz, Austria

Critical Care 2013, 17(Suppl 2):P228 (doi: 10.1186/cc12166)

Introduction Dobutamine and levosimendan improve cardiac contractility in patients with heart failure, septic cardiomyopathy or cardiac surgery. Tachycardia in these cases is undesired because of aggravating ischemia. Ivabradine, a selective sinus node inhibitor, does not affect contractility. A combination of ivabradine with positive inotropic drugs might be favorable. Thus, we compared the cardiac effects of dobutamine or levosimendan alone, and combined with ivabradine using the Langendorff method of isolated perfused hearts. Methods Isolated guinea pig hearts $(n=37)$ were perfused with incremental doses of dobutamine $(10 \mathrm{nM}$ to $10 \mu \mathrm{M})$ or levosimendan (30 nM to $10 \mu \mathrm{M}$ ) either alone or combined with $3 \mu \mathrm{M}$ ivabradine. Heart rate $(\mathrm{HR})$, left ventricular pressures, contractility $(+\mathrm{dLVP} / \mathrm{dt})$ and relaxation $(-\mathrm{dLVP} / \mathrm{dt}$ ) were recorded. Data for each drug (dobutamine or levosimendan) were analyzed by two-way ANOVA for repeated measures including the two main effects of ivabradine and drug dose and their interaction. Data are reported as mean \pm standard deviation. Results Ivabradine decreased the HR from $223 \pm 18$ to $196 \pm 15 \mathrm{bpm}$ $(P<0.05)$. Contractility and relaxation remained unchanged. Ivabradine reduced the positive chronotropic effect at all doses of dobutamine ( 10 nM: $232 \pm 37$ vs. $187 \pm 19,100$ nM: $265 \pm 37$ vs. $211 \pm 40,1 \mu \mathrm{M}$ : $316 \pm 35$ vs. $250 \pm 39,10 \mu \mathrm{M}: 320 \pm 33$ vs. $235 \pm 40$ bpm; $P<0.05)$. It shifted the maximum positive inotropic action of dobutamine to lower dose ranges ( $100 \mathrm{nM}: 2,924 \pm 841$ vs. $2,978 \pm 955,300 \mathrm{nM}: 3,743 \pm 925$ vs. $4,795 \pm 1,298,1 \mu \mathrm{M}: 4,138 \pm 935$ vs. $4,896 \pm 1,861 \mathrm{mmHg} / \mathrm{second}$; $P<0.05)$. A comparable shift was seen for relaxation $(100 \mathrm{nM}$ : $-2,178 \pm 686$ vs. $-2,520 \pm 742,300 \mathrm{nM}:-2,615 \pm 726$ vs. $-3,150 \pm 888$, $1 \mu \mathrm{M}:-2,903 \pm 752$ vs. $-2,972 \pm 967 \mathrm{mmHg} / \mathrm{second} ; \quad P<0.05)$. Levosimendan increased the HR only at high doses. With ivabradine, no positive chronotropic effect of levosimendan was observed (100 nM: $185 \pm 30$ vs. $162 \pm 22,1 \mu \mathrm{M}: 208 \pm 28$ vs. $166 \pm 29,10 \mu \mathrm{M}: 242 \pm 27$ vs. $168 \pm 36 \mathrm{bpm} ; P<0.05)$. Ivabradine attenuated the positive inotropic effect of levosimendan (100 nM: 2,303 \pm 303 vs. 1,737 $\pm 262,1 \mu \mathrm{M}$ : $2,977 \pm 481$ vs. $1,940 \pm 449,10 \mu \mathrm{M}: 3,480 \pm 941$ vs. $2,189 \pm 542 \mathrm{mmHg} /$ second; $P<0.05$ ) but did not significantly alter its lusitropic effect.

Conclusion Addition of ivabradine to dobutamine attenuates its chronotropic actions without diminishing its inotropic effects. A combination of levosimendan with ivabradine does not seem to provide benefit. Clinical studies are necessary to confirm these experimental results.

P229

Influence of temperature on the cardiac action of dobutamine and levosimendan in isolated perfused hearts

U Aldenhoff, R Vicenzi-Moser, M Vicenzi, D Konrad, E Schwarzl, W Toller Medical University Graz, Austria

Critical Care 2013, 17(Suppl 2):P229 (doi: 10.1186/cc12167)

Introduction Inotropic agents (catecholamines or calcium-sensitizers) are frequently used in hypothermic as well as hyperthermic patient conditions. Divergent results from animal experiments raise doubt as to whether they act to the same extent at different body temperatures. Thus, we studied the influence of clinically relevant temperatures on the hemodynamic effects of dobutamine and levosimendan using the Langendorff model of isolated perfused hearts. 
Methods Isolated guinea pig hearts $(n=60)$ were perfused with incremental doses $(10 \mathrm{nM}$ to $10 \mu \mathrm{M})$ of dobutamine or levosimendan either at normothermic $\left(37^{\circ} \mathrm{C}\right)$, hyperthermic $\left(40^{\circ} \mathrm{C}\right)$ or hypothermic $\left(34^{\circ} \mathrm{C}\right)$ perfusion conditions. Contractility $(+\mathrm{dLVP} / \mathrm{dt})$, relaxation $(-\mathrm{dLVP} / \mathrm{dt})$, left ventricular pressures and heart rate were recorded. Data with increasing drug dosage were calculated in percent from baseline for each temperature tested. Data for each drug were analysed by twoway ANOVA for repeated measures including the two main effects of temperature and drug dose and their interaction. Data are reported as mean \pm standard deviation.

Results The positive inotropic action of dobutamine was least at $37^{\circ} \mathrm{C}$, more pronounced at $40^{\circ} \mathrm{C}$ and best at $34^{\circ} \mathrm{C}\left(37^{\circ} \mathrm{C}\right.$ vs. $40^{\circ} \mathrm{C}$ vs. $34^{\circ} \mathrm{C}: 300$ nM $280 \pm 71$ vs. $301 \pm 94$ vs. $345 \pm 99 \%, 1 \mu \mathrm{M} 310 \pm 74$ vs. $327 \pm 92$ vs. $389 \pm 144 \%, 10 \mu \mathrm{M} 297 \pm 69$ vs. $339 \pm 96$ vs. $359 \pm 175 \% ; P<0.05)$. Dobutamine's positive lusitropic effect was not significantly altered by temperature. The positive inotropic action of levosimendan was best at $37^{\circ} \mathrm{C}$, in hyperthermia and hypothermia only the three highest doses of levosimendan increased contractility $\left(37^{\circ} \mathrm{C}\right.$ vs. $40^{\circ} \mathrm{C}$ vs. $34^{\circ} \mathrm{C}: 100 \mathrm{nM}$ $121 \pm 21$ vs. $134 \pm 15$ vs. $87 \pm 12 \%, 300$ nM $135 \pm 22$ vs. $137 \pm 17$ vs. $94 \pm 15 \%, 1 \mu \mathrm{M} 153 \pm 22$ vs. $147 \pm 21$ vs. $108 \pm 21 \%, 3 \mu \mathrm{M} 172 \pm 19$ vs. $150 \pm 24$ vs. $115 \pm 23 \%, 10 \mu \mathrm{M} 173 \pm 31$ vs. $156 \pm 28$ vs. $117 \pm 28 \%$; $P<0.05)$. The positive lusitropic effect of levosimendan at $37^{\circ} \mathrm{C}$ was almost absent in hypothermia and hyperthermia $(P<0.05)$.

Conclusion In isolated perfused hearts, dobutamine has its best positive inotropic effect in hypothermia whereas levosimendan increases contractility best at normothermic conditions. Clinical studies are necessary to confirm these experimental results.

P230

Conflicting results between V/Q SPECT and angioscan in pulmonary embolism: what to do?

JM Martel, MN Nadeau, RD Daoust, JP Paquet, JC Chauny

Hôpital du Sacré-Coeur de Montréal, Canada

Critical Care 2013, 17(Suppl 2):P230 (doi: 10.1186/cc12168)

Introduction The objective of this study is to determine the final clinical diagnosis of patients who underwent the double investigation for pulmonary embolism with conflicting results. Pulmonary embolism is a prevalent pathology in the emergency department (ED). A certain proportion of patients undergo a double radiological investigation (V/Q SPECT and angioscan), which incurs higher costs and X-ray doses. However, no study to date has addressed this issue.

Methods This retrospective study included patients who underwent a double investigation in the ED of Hôpital du Sacré-Coeur de Montréal from April 2008 to October 2012. Patients were selected from a computerized database of medical prescriptions (MediaMed Technologie). Data were then extracted from the patient's files: patient characteristics, radiology report, diagnosis and treatment. Descriptive statistics were conducted.

Results In all, 125 patients underwent the double investigation. Our sample had a mean age of 63.1 years (SD \pm 18.6 ) and was composed of $82(65.6 \%)$ women. One hundred and fifteen patients (92\%) underwent the V/Q SPECT first. The result of 66 (52.8\%) SPECT was intermediate or indeterminate. The final diagnosis was pulmonary embolism for 23 patients (18.4\%). A significant proportion of patients (19, 38.0\%) had conflicting results with the two tests. In this subpopulation, four (21.1\%) had a final diagnosis of pulmonary embolism. In the 16 patients with a result of high probability V/Q SPECT and negative angioscan, one (6.3\%) had a final diagnosis of pulmonary embolism, but three (100\%) with low probability SPECT and positive angioscan were given this final diagnosis.

Conclusion Most patients underwent the double investigation because of intermediate or indeterminate V/Q SPECT results. In case of conflicting results, clinicians based their decision on the angioscan. In future studies, it would be useful to identify contributing factors for this discordance.
P231

Intraabdominal hypertension and abdominal compartment syndrome in patients admitted to a medical and surgical ICU at a third referral university hospital

N Chindavech'1, S Yenarkart², S Kongsayreepong ${ }^{2}$

'Siriraj Hospital, Mahidol University, Bangkok, Thailand; '2Division of Critical

Care Medicine, Bangkok, Thailand

Critical Care 2013, 17(Suppl 2):P231 (doi: 10.1186/cc12169)

Introduction Intraabdominal hypertension (IAH), especially abdominal compartment syndrome (ACS), can affect organ function leading to multiple organ failure. Appropriate and prompt management could improve survival. Less recognition of this problem in critically ill patients has been reported. The aim of this study was to study the prevalence, predictive factors and clinical outcome of IAH and ACS in a mixed population of critically ill patients by intermittent measuring bladder pressure during the ICU stay.

Methods This prospective observational study was done in 130 adult patients (age $>18$ years) admitted to a medical and general surgical ICU at a third referral university hospital during June to November 2011. Variables about the patient's profile laboratory data and clinical outcome as ICU and hospital length of stay, and ICU, in-hospital and 28-day mortality were recorded.

Results There were $33(25.4 \%)$ medical and 93 (74.6\%) surgical ICU patients in this study. Surgical patients had higher prevalence of IAH than medical patients $(57.8 \%$ vs. $33.3 \%, P=0.015)$. Medical patients were admitted with severe sepsis/septic shock, AKI, pneumonia and ARDS. Surgical patients were more acutely ill with high ASA (III to IV), severity score, underwent emergency abdominal surgery and received more transfusion but were no different in type of fluid replacement. Significant risk factors of IAH were coagulopathy $(\mathrm{OR}=2.09,95 \%$ $\mathrm{Cl}=1.62$ to 2.69$)$, intraabdominal infection $(\mathrm{OR}=1.87,95 \% \mathrm{Cl}=1.40$ to 2.48 ), retroperitonium hematoma $(\mathrm{OR}=1.82,95 \% \mathrm{Cl}=1.36$ to 2.44$)$, marked ascites ( $\mathrm{OR}=1.76,95 \% \mathrm{Cl}=1.32$ to 2.36 ), acidosis ( $\mathrm{pH}<7.2$ ) $(\mathrm{OR}=1.82,95 \% \mathrm{Cl}=1.37$ to 2.43$)$, severe sepsis/septic shock ( $\mathrm{OR}=1.63$, $95 \% \mathrm{Cl}=1.14$ to 2.33$)$, and massive transfusion $(\mathrm{OR}=1.51,95 \%$ $\mathrm{Cl}=1.10$ to 2.08 ). Patients with $\mathrm{IAH}$ had more reopened surgeries and had higher ICU, hospital and 28-day mortality. Sixteen (12.3\%) patients had ACS, 15 patients underwent emergency surgery, two patients had temporary abdominal closured and one-half of the patients had severe abdominal sepsis and massive transfusion. Fourteen patients died despite temporary abdominal closure. Delayed release abdominal tamponade were most causes of death.

Conclusion The prevalence and morbidity/mortality of IAH and ACS were high in this institute. Early appropriate and prompt management, especially fluid and releasing a tamponade effect, were important.

P232

Red cell distribution width predicts cardiovascular complications after high-risk surgery

M Geisen, HD Aya, C Ebm, MA Hamilton, J Ball, M Grounds, A Rhodes, MCecconi

St George's Healthcare NHS Trust, London, UK

Critical Care 2013, 17(Suppl 2):P232 (doi: 10.1186/cc12170)

Introduction The red-cell distribution width (RDW) is associated with cardiovascular morbidity and mortality and is a predictor of ICU survival. It was the aim of this study to investigate the potential of RDW to predict postoperative cardiovascular complications (new onset of treated arrhythmias, myocardial ischaemia or initiation of vasopressor support) and its association with markers of tissue perfusion (serum lactate $>2.5 \mathrm{mmol} / \mathrm{l})$.

Methods Analysis of prospectively recorded data for a register of patients admitted to an 18-bed ICU in a large teaching hospital after undergoing high-risk surgery. Haemodynamic and laboratory parameters during the first 12 hours of ICU stay were recorded as well as demographic characteristics. Assessment for postoperative complications was performed using the postoperative morbidity survey and the Clavien-Dindo classification. In addition, clinical outcome data (hospital mortality, length of ICU stay, length of hospital stay, readmission to the ICU) were recorded. 


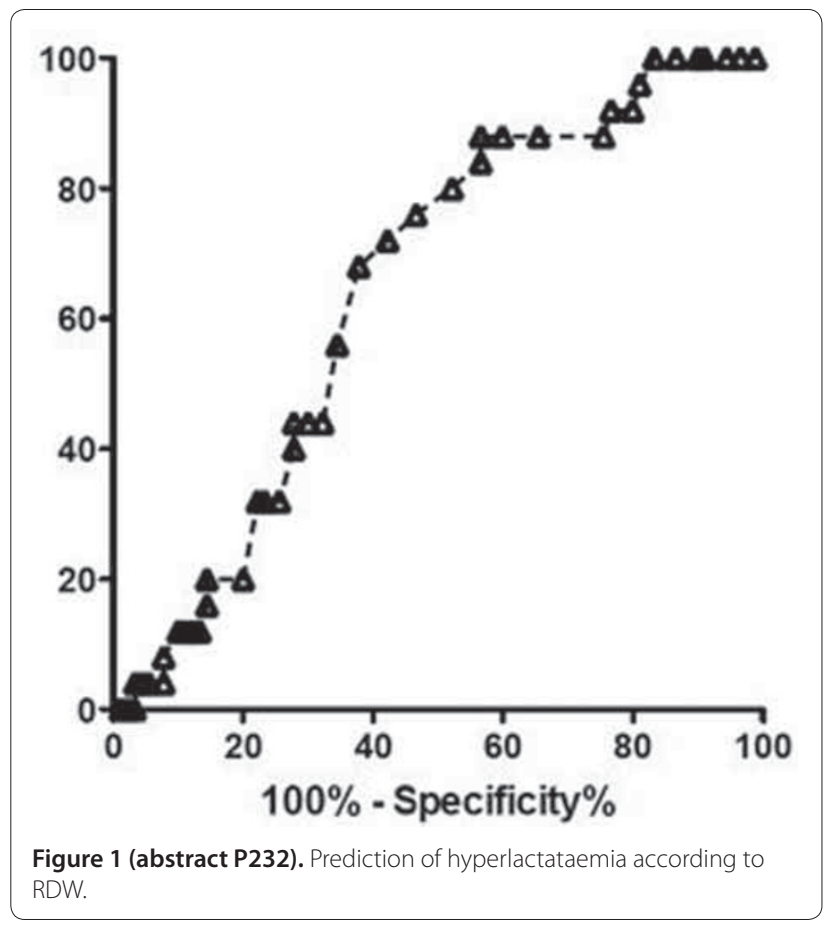

Results A total of 119 patients were included. Seventy-six (63\%) patients developed complications postoperatively. Thirty patients (25.2\%) developed cardiovascular complications. These patients had a higher median RDW than patients without cardiovascular complications ( 14.7 vs. $13.8 \%, P<0.05)$. RDW on ICU admission was associated with serum lactate concentration on ICU admission: receiver operating characteristic analysis showed an area under the curve of $0.68(\mathrm{SE}=0.06$, $P=0.005$; see Figure 1). RDW $>14.35 \%$ predicted hyperlactataemia with a sensitivity of $76.0 \%$ and a specificity of $71.1 \%$.

Conclusion RDW is a potential parameter for perioperative risk stratification. Further studies will have to elucidate the ability of acute elevations in RDW to reflect impaired tissue perfusion.

\section{References}

1. Bazick HS, et al:: Crit Care Med 2011, 39:1913-1921.

2. Hunziker H, et al: Crit Care 2012, 16:R89.

P233

Consequences of obesity in outcomes after cardiac surgery: analysis of the ARIAM registry

EC Curiel-Balsera', J Muñoz-Bono', MJ Delgado-Amaya',

R Hinojosa-Pérez ${ }^{2}$, A Reina-Toral ${ }^{3}$, A Gordillo-Brenes ${ }^{4}$, R Rivera-Fernández

'Hospital Regional Carlos Haya, Málaga, Spain; ${ }^{2}$ Virgen del Rocio Hospital,

Seville, Spain; ${ }^{3}$ Virgen de las Nieves Hospital, Granada, Spain; ${ }^{4}$ Puerta del mar Hospital, Cádiz, Spain

Critical Care 2013, 17(Suppl 2):P233 (doi: 10.1186/cc12171)

Introduction Obesity is a disease that affects a large part of the population and has been associated with worse outcomes after cardiac surgery. The aim of our study is to evaluate the consequences of obesity related to postoperative complications, hospital length of stay and mortality.

Methods An observational, prospective, multicenter study of patients included in the ARIAM registry of adult cardiac surgery between March 2008 and March 2011. We analyzed clinical variables, the surgical procedure, postoperative complications and mortality, comparing the group of patients with body mass index (BMI) greater or less than $30 \mathrm{~kg} / \mathrm{m}^{2}$.

Results The study included 4,712 patients with a mean age of 64.03 (SD \pm 12.08 ) years, BMI 28.53 (SD \pm 4.7 ) and EuroSCORE 5.58 (SD \pm 2.91$)$. In 1,940 patients (35.7\%) BMI was $>30 \mathrm{~kg} / \mathrm{m}^{2}$. There were no differences in the development of overall postoperative complications (33\% in obese and $35.8 \%$ in nonobese, $P=0.07$ ), although less appreciated were reoperation rate or stroke, as well as further development of postoperative renal failure. After adjusting for severity and length of cardio bypass time, obese patients had lower mortality without being statistically significant, $\mathrm{OR}=0.94$ (0.79 to 1.04 ). There were no differences in ICU length of stay, but obese patients had greater ward length of stay, 9.04 (10.43) versus $1.18(9.2)$ days, $P=0.01$.

Conclusion Obese patients undergoing cardiac surgery have mortality, rate of complications and length of stay similar to nonobese patients. Obese patients required less reoperation but developed more frequent postoperative renal failure.

References

1. Jin R, et al:: Circulation 2005, 111:3359-3365.

2. Stamous SC: Ann Thorac Surg 2011, 91:42-48.

P234

Myocardial injury in critically ill patients admitted with noncardiac diagnoses

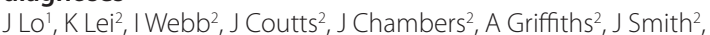

E Connell ${ }^{3}$, P Collinson ${ }^{3}$, J Peacock', DTreacher ${ }^{2}$, M Ostermann ${ }^{2}$

'King's College London, London, UK; 'Guys \& St Thomas Foundation Hospital, London, UK: ${ }^{3}$ St George's Hospital, London, UK

Critical Care 2013, 17(Suppl 2):P234 (doi: 10.1186/cc12172)

Introduction In the critically ill, the incidence of raised cardiac troponin $\mathrm{T}(\mathrm{cTnT})$ levels is high. Although the mechanisms of myocardial injury are not well understood, raised cTnT levels are associated with increased mortality. The aim of our study was to determine the incidence, prevalence and outcome of silent myocardial injury as determined by raised cTnT levels and concomitant ECG changes in critically ill patients admitted for noncardiac reasons.

Methods ECGs were taken and cTnT was measured daily during the first week and on alternate days during the second week until discharge from the ICU or death. After completion of the study, all cTnT levels and ECGs were analysed independently and patients were classified into four groups: definite MI (cTnT $\geq 15 \mathrm{ng} / \mathrm{l}$ and definite ECG changes of MI), possible $\mathrm{MI}$ (cTnT $\geq 15 \mathrm{ng} / \mathrm{l}$ and ischaemic changes on ECG), troponin rise alone (cTnT $\geq 15 \mathrm{ng} / \mathrm{l}$ with no ischaemic ECG changes), or normal. All medical notes were reviewed independently by two ICU clinicians. Results A total of 144 patients were included in the analysis (42\% female; mean age 61.9 (SD 16.9); mean APACHE II score 19.4). In total, 121 patients (84\%) had at least one cTnT level $\geq 15 \mathrm{ng} / \mathrm{l}$ during their stay in the ICU. Twenty patients (14\%) fulfilled criteria for a definite MI, of whom $65 \%$ were septic and $50 \%$ were on noradrenaline at the time (ICU and hospital mortality: $25 \%$ and $30 \%$, respectively). Thirty-nine patients (27\%) had a possible Ml, of whom $69 \%$ were septic and on noradrenaline (ICU and hospital mortality: $31 \%$ and $41 \%$, respectively). Sixty-two patients $(43 \%)$ had a raised troponin without ECG, of whom $69 \%$ were septic and $50.7 \%$ were on noradrenaline (ICU and hospital mortality: $23 \%$ and $31 \%$, respectively). Twenty-three patients had normal CTnT results and serial ECGs, of whom $61 \%$ had sepsis. ICU and hospital mortality was $4 \%$. Only $25 \%$ of definite Mls and $18 \%$ of possible Mls were recognised by the clinical teams at the time.

Conclusion Eighty-four per cent of critically ill patients had a raised cTnT level at some stage during their stay in the ICU. More than $40 \%$ of patients fulfilled criteria for a possible or definite Ml, of whom only $20 \%$ were recognised clinically. ICU and hospital outcome were significantly worse in patients with a cTnT rise. The proportion of patients with sepsis was similar between the patients with a definite, possible or no MI.

P235

Validation of the GRACE score risk in our population

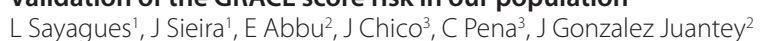
${ }^{1}$ HULA, Lugo, Spain; ${ }^{2}$ CHUS, Santiago de Compostela, Spain; ${ }^{3}$ CHUVI, Vigo,

Spain

Critical Care 2013, 17(Suppl 2):P235 (doi: 10.1186/cc12173)

Introduction The Global Registry of Acute Coronary Events (GRACE) risk score provides an estimation of the probability of death within 
6 months of hospital discharge in patients with acute coronary syndrome (ACS). Our aim was to assess the validity of this risk score in our contemporary cohort of patients admitted to our third-level hospital.

Methods The study involved 1,185 consecutive patients with ACS evaluated between February 2004 and February 2009. Their virtual status was determined 6 months after hospital discharge and the validity of the GRACE risk score was evaluated.

Results In total, 450 (38.8\%) patients were admitted for STEMI and 725 $(61.2 \%)$ for NSTEMI. Percutaneous revascularisation was performed in $846(71.5 \%)$. The median GRACE risk score was 121 (interquartile range $96 / 144)$. Mortality after discharge was $4.4 \%$. The calibration of the GRACE score was Hosmer-Lemeshow $P>0.2$ and its discriminatory capacity was excellent. Area under the ROC curve was $0.86,95 \% \mathrm{Cl}$ 0.807 to 0.916 , in all patients. See Table 1 .

Table 1 (abstract P235)

\begin{tabular}{lccc}
\hline GRACE risk & Total & STEMI & NSTEMI \\
\hline Low & $270(22.7)$ & $177(38.3)$ & $93(12.8)$ \\
Medium & $334(28.2)$ & $141(30.7)$ & $193(26.7)$ \\
High & $580(49)$ & $142(30.9)$ & $438(60.5)$ \\
\hline
\end{tabular}

Conclusion The GRACE risk score for predicting death within 6 months of hospital discharge was validated and can be used in patients with ACS. It would be perfect in the future to include the GRACE risk score in the medical records of this type of patients. Also it would be very interesting to validate this in a multicentric study.

P236

Outcomes of incomplete and complete revascularization in ACS

L Sayagues', J Sieira', E Abbu Assi², J Chico ${ }^{3}$, C Pena ${ }^{3}$, J Gonzalez Juanatey ${ }^{2}$ 'HULA, Lugo, Spain; ${ }^{2}$ CHUS, Santiago de Compostela, Spain; ${ }^{3} \mathrm{CHUVI}$, Vigo,

Spain

Critical Care 2013, 17(Suppl 2):P236 (doi: 10.1186/cc12174)

Introduction This is a prospective study in which all ACS cases attending a level 3 hospital were collected consecutively from February 2004 to 2010, and were clinically followed-up until 2012.

Methods Of a total 4,589 cases, only 2,515 were revascularized with ICP $(1,742$ complete and 907 incomplete; 160 failed). We split the cases into two main groups: STEMI and NSTEMI.

Results We observed a 0.6 higher accumulative survival rate in patients with complete ICP over patients who underwent surgery, incomplete ICP or mixed treatment. Those with conservative measures solely have, obviously, worst prognosis. See Figures 1 and 2.

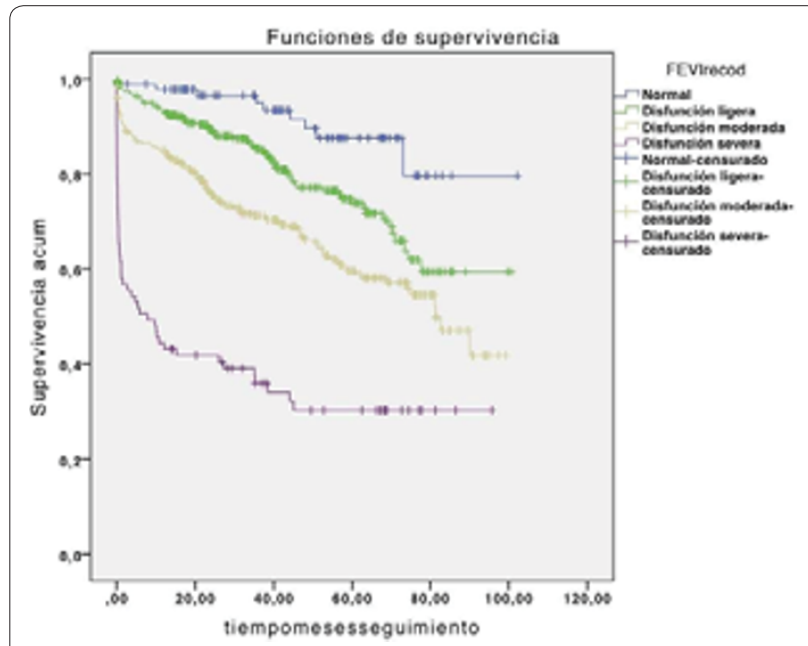

Figure 1 (abstract P236). FEVI and prognosis.

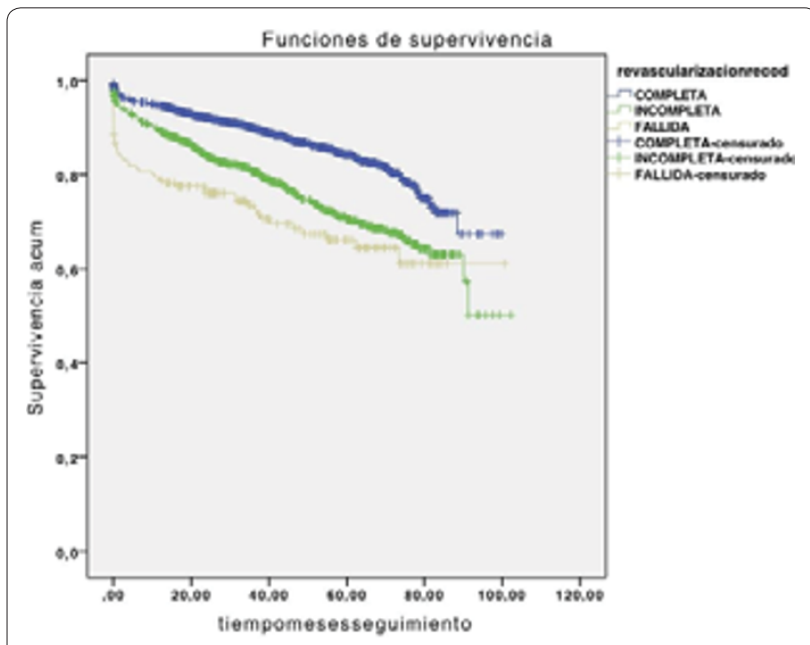

Figure 2 (abstract P236). Incomplete and complete revascularization.

Conclusion In our study patients undergoing ICP have higher survival rates in comparison with cardiac surgery except those $>65$ years old and diabetic groups in which cardiac surgery has higher ratios than ICP. In the rest of the groups, no matter how many coronary arteries were affected, only those with complete ICP present higher survival rates. It would be important to repeat this study in a multicentric cohort.

P237

Non-invasive valve implantation with TAVI versus conventional aortic valve replacement

ETrujillo-García, C Joya-Montosa, MJ Delgado-Amaya, E Curiel-Balsera Hospital Regional Carlos Haya, Málaga, Spain

Critical Care 2013, 17(Suppl 2):P237 (doi: 10.1186/cc12175)

Introduction In patients with severe and inoperable aortic stenosis, some trials have proven that TAVI excels standard procedures and has also been proven less cost-effective. We aim at reviewing short-term results after 2 years since the implementation of this technique in our premises.

Methods A study of a retrospective cohorts of patients who underwent isolated aortic valve replacement (AVR) by either conventional surgery or TAVI (CoreValve device) from June 2010 to December 2011 at the University Hospital Carlos de Haya (Málaga, Spain). Clinical epidemiologic, complication and short-term outcome variables were registered. Qualitative variables are expressed as percentages, while quantitative variables are expressed as means and SD. Fisher's exact test and Mann-Whitney's U-test were used where necessary (5\% maximum error ratio).

Results A total number of 27 TAVI and 154 isolated AVR procedures were completed. Intervention typology was chosen according to the recommendations of scientific societies, apart from patients' fulfillment of the anatomic criteria required for percutaneous implant. Mean age was $67 \pm 11$ years (54\% males) in AVR and $80 \pm 6$ years ( $44 \%$ males) in TAVI $(P<0.05)$. The additive EuroSCORE in AVR was $7 \pm 2$ and $9 \pm 2$ in TAVI $(P<0.05)$. However, $55.6 \%$ of the percutaneous-valve patients presented previous coronary-tree alterations with stent implantation, while only $7 \%$ of AVR patients showed these alterations $(P<0.001)$. ICU mortality in TAVI and AVR patients was $3.7 \%$ and $8.2 \%$, respectively $(P=\mathrm{NS})$. Regarding complications, $48.1 \%$ of TAVI patients showed altered heart rhythm and 33\% required a permanent pacemaker. Electrical disorders were observed in $4 \%$ of AVR patients, while $1.9 \%$ of these patients required a permanent pacemaker $(P<0.001$ for both). Reoperation was necessary in 14.8 and $1.9 \%$ of TAVI and AVR patients, respectively $(P<0.001)$.

Conclusion Even with our limited experience, TAVI patients are observed to be older, to involve higher surgical risk, and to have undergone previous coronary-tree interventions. Although no 
significant differences were found regarding mortality, a higher rate of complications was observed in TAVI patients. With no short-term differences, a significant rate of postsurgical complications, and a costefficiency handicapped technique, analysis of long-term outcomes seems necessary to assess TAVI's advantages over conventional AVR.

P238

Time to recover from shock is determinant of a positive fluid balance in septic shock

S Lobo, AL Cunha

Faculdade de Medicina de São José do Rio Preto, Brazil

Critical Care 2013, 17(Suppl 2):P238 (doi: 10.1186/cc12176)

Introduction Excess fluids may be harmful in critically ill patients. We aimed to evaluate the cumulative fluid balance during 7 days in patients with septic shock after recovery from shock.

Methods A prospective and observational study in septic shock patients. Patients with MAP $>65 \mathrm{mmHg}$ and lactate $<2.0 \mathrm{mEq} / \mathrm{l}$ were included $<12$ hours after weaning from vasopressors. Daily fluid balance was registered during 7 days after the enrollment. Patients were divided into two groups according to the full cohort's median cumulative fluid balance administered during the period of shock (use of vasopressors) calculated on study day 1: Group $1 \leq 4.4$ I $(n=20)$ and Group $2>4.4$ I $(n=20)$.

Results Cumulative fluid balance was $1.6 \pm 1.8 \mathrm{I}$ in Group 1 and $10.2 \pm 4.1 \mathrm{I}$ in Group 2 and $8.5 \pm 5.3 \mathrm{I}$ in Group 1 and $18.5 \pm 7.9 \mathrm{I}$ in Group 2 on study day 8 ( $P<0.001$ for both). Time for recovery from shock was predictive of receiving larger volume of fluids (OR: 1.38, 95\% $\mathrm{Cl}: 1.08$ to $1.75, P=0.009$ ). After zeroing fluid balance on study day 2 , 7 days cumulative fluid balance continues to increase in both groups (Figure 1). Patients in Group 2 had more prolonged length of stay in the ICU and in hospital than patients in Group 1.

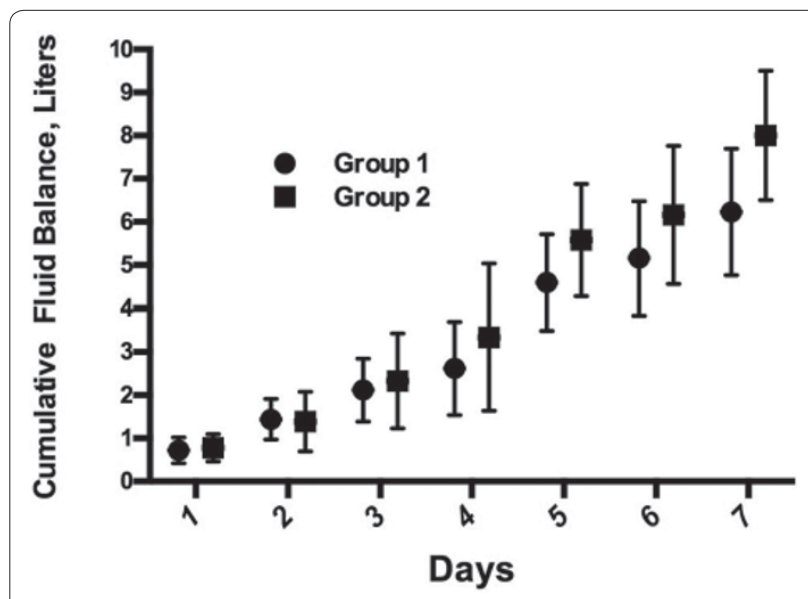

Figure 1 (abstract P238).

Conclusion After recovery from septic shock we notice a huge accumulated fluid balance. A more positive fluid balance was associated with a more prolonged length of stay in the ICU and in the hospital.

P239

Lactate clearance is a predictor of sustained bleeding in emergency room patients with moderate upper gastrointestinal bleeding

TWada', A Hagiwara², N Yahagi', A Kimura²

'University of Tokyo, Japan; ${ }^{2}$ National Center for Global Health and Medicine, Tokyo, Japan

Critical Care 2013, 17(Suppl 2):P239 (doi: 10.1186/cc12177)

Introduction There is no useful predictor of sustained upper gastrointestinal bleeding (UGIB). Glasgow-Blatchford scoring (GBS) is based on simple clinical and laboratory variables and can predict whether a
UGIB patient needs an intervention or not. However, the intervention which the GBS mentions includes not only endoscopy but also blood transfusion. Therefore, we cannot determine whether a UGIB patient needs urgent endoscopy or just blood transfusion by GBS alone. We hypothesized that high lactate clearance (CLac) would decrease the likelihood of sustained UGIB.

Methods This is a retrospective study. UGIB patients, who visited the emergency department (ED) of the National Center for Global Health and Medicine from April 2011 to March 2012 and received urgent endoscopy in the ED, were enrolled. We collected for each patient the GBS, the blood lactate value on arrival in the ED, the blood lactate value after bolus administration of 20 to $40 \mathrm{ml} / \mathrm{kg}$ Ringer's acetate (initial fluid therapy) and the report of urgent endoscopy. We classified the severity of UGIB according to GBS. A score $\leq 12$ was classified as moderate, and a score $\geq 13$ was classified as severe. CLac was defined as the percentage decrease in blood lactate from the time of arrival in the ED to the time when an initial fluid therapy was finished. CLac $<50 \%$ was defined as low, and $C L a c \geq 50 \%$ was defined as high. Whether a patient had sustained bleeding or not was determined based on the report of urgent endoscopy. The relationship between CLac and sustained bleeding was examined by Fisher's exact test, and $P<0.05$ was considered statistically significant.

Results Seventy-nine patients were enrolled. Fifty-one patients were with moderate UGIB, and 28 patients were with severe UGIB. As indicated in Tables 1 and 2, there was a significant relationship between CLac and sustained bleeding in moderate UGIB $(P=0.02)$. On the other hand, there was no significant relationship between CLac and sustained bleeding in severe UGIB $(P=0.58)$.

Table 1 (abstract P239). Relationship between lactate clearance and sustained bleeding in moderate UGIB $(n=51)$

\begin{tabular}{lccc}
\hline & $\begin{array}{c}\text { Sustained bleeding } \\
(\boldsymbol{n}=\mathbf{1 9 )}\end{array}$ & $\begin{array}{c}\text { Nonsustained bleeding } \\
(\boldsymbol{n}=\mathbf{3 2})\end{array}$ & $\boldsymbol{P}$ value \\
\hline $\mathrm{CLac}<0.5$ & $18(95 \%)$ & $21(65 \%)$ & 0.02 \\
$\mathrm{CLac} \geq 0.5$ & $1(5 \%)$ & $11(34 \%)$ & \\
\hline
\end{tabular}

Table 2 (abstract P239). Relationship between lactate clearance and sustained bleeding in severe UGIB $(n=28)$

\begin{tabular}{lccc}
\hline & $\begin{array}{c}\text { Sustained bleeding } \\
(\boldsymbol{n}=\mathbf{6})\end{array}$ & $\begin{array}{c}\text { Nonsustained bleeding } \\
\text { bleeding }(\boldsymbol{n}=\mathbf{2 2})\end{array}$ & $\boldsymbol{P}$ value \\
\hline $\mathrm{CLac}<0.5$ & $4(67 \%)$ & $18(82 \%)$ & 0.58 \\
$\mathrm{CLac} \geq 0.5$ & $2(33 \%)$ & $4(18 \%)$ & \\
\hline
\end{tabular}

Conclusion If an initial fluid therapy for moderate UGIB results in high CLac, the bleeding might already have stopped. For such a patient, we may save urgent endoscopy.

\section{P240}

Muscular glucose assessed by microdialysis and blood glucose can predict mortality in septic shock

A Massoudi, M Belhadj Amor, C Romdhani, A Ben Gabsia, I Labbene,

M Ferjani

Military Hospital of Tunis, Tunisia

Critical Care 2013, 17(Suppl 2):P240 (doi: 10.1186/cc12178)

Introduction The aim of our study was to assess the muscular glucose by microdialysis and its association with mortality in septic shock patients.

Methods We conducted a preliminary prospective study. We included septic shock patients hemodynamically optimized according to international recommendations. A microdialysis catheter was inserted in the femoral quadriceps. Interstitial fluid samples were collected every 6 hours for 5 days. The determination of muscular glucose was performed by the CMA 600 analyzer (CMA/Microdialysis AB, Sweden). We also performed a dosage of concomitant blood glucose. The study population was divided into two groups according to hospital mortality. Statistic analysis: Mann-Whitney test and chi-squared test: 
comparisons between groups. Quantitative variables were expressed as mean \pm standard deviation or median (interquartile range) as appropriate.

Results We included 12 patients with septic shock. The mortality rate was $50 \%$. Demographics were comparable between groups except for age (66 \pm 9 vs. $41 \pm 12$, dead patients vs. survivors, respectively; $P=0.002$ ). Pneumonia was the major cause of septic shock (10 patients). We analysed 167 blood samples and 166 muscular glucose samples. We found a positive association between muscular glucose, blood glucose and mortality. Tissue glucose was significantly higher among dead patients compared with survivors at the 54th hour. Comparing all data, muscular glucose $(P=0.02)$ and blood glucose $(P=0.007)$ were significantly higher in dead patients (Table 1).

Table 1 (abstract P240). Association between muscular glucose, blood glucose and mortality

\begin{tabular}{lccc}
\hline & $\begin{array}{c}\text { Dead patients } \\
(\boldsymbol{n}=\mathbf{6})\end{array}$ & $\begin{array}{c}\text { Survivors } \\
(\boldsymbol{n}=6)\end{array}$ & P value \\
\hline Muscular glucose $(\mathrm{mmol} / \mathrm{l})$ & $9.4(5.66 ; 13.71)$ & $7.87(5.62 ; 10.41)$ & 0.02 \\
Blood glucose $(\mathrm{mmol} / \mathrm{l})$ & $10.9(8.3 ; 15.5)$ & $8.6(6.9 ; 11.4)$ & 0.007 \\
\hline
\end{tabular}

Conclusion Our data suggest that muscular glucose assessed by microdialysis and blood glucose are associated with mortality in septic shock patients. Therefore, muscular glucose may reflect the metabolic alterations and microcirculatory dysfunction induced by septic shock.

\section{P241}

Constipation in critically ill patients and its relationship to feeding and weaning from respiratory support

E Spodniewska, A Guha

Royal Liverpool University Hospital, Liverpool, UK

Critical Care 2013, 17(Suppl 2):P241 (doi: 10.1186/cc12179)

Introduction Constipation can be defined as a failure of the bowels to open for 3 consecutive days. It is a common problem in intensive care settings but not many studies have so far raised the subject.

Methods The audit had the Trust Audit Committee's approval. The existing protocol was used as the benchmark. Patients were studied prospectively to assess compliance with the local bowel protocol, incidence of constipation and relationship to weaning from respiratory support and feeding. All HDU and all mechanically ventilated ICU patients who stayed on the ward for more than 3 days were included, except for patients after bowel surgery and patients with encephalopathy.

Results Among the $24 \mathrm{HDU}$ and 21 ICU patients audited in the Royal Liverpool University Hospital, $67 \%$ and $57 \%$ respectively were constipated. Laxatives were prescribed when patients had not opened their bowels for 3 days in 25\% HDU and 75\% ICU cases. Taking into consideration that the median age, APACHE II score and length of stay for constipated and nonconstipated patients were similar, the relationship to feeding and respiratory support were assessed. Constipated patients required mechanical ventilation for an average of 6.8 days and nonconstipated for 4.3 days. Failure to feed was observed at least once in $58 \%$ constipated and $44 \%$ nonconstipated ICU patients and $19 \%$ constipated and $12.5 \%$ nonconstipated HDU patients.

Conclusion There was a high prevalence of constipation among critical care patients with poor adherence to the bowel protocol, which requires improvements in staff awareness and new recommendations (drug chart amendment, and so forth). Duration of mechanical ventilation and failure to feed were greater in constipated than nonconstipated patients. This may indicate a common correlation and further studies are warranted. Due to the possible significant impact of constipation on patients' recovery, each critical care unit should introduce a bowel protocol or ensure compliance with the existing one before the evidence is clearly established.

References

1. Mostafa SM: Constipation and its implications in the critically ill patient. $\mathrm{Br}$ J Anaesth 2003, 91:815-819.

2. Palácio de Azevedo R: Intestinal constipation in intensive care unit. Rev Bras Ter Intensiva 2009, 21:324-331.
P242

Nasogastric tube dislodgement: a problem on our ICU

B Morton, R Hall, T Ridgway, O Al-Rawi

Liverpool Heart \& Chest, Liverpool, UK

Critical Care 2013, 17(Suppl 2):P242 (doi: 10.1186/cc12180)

Introduction It was noted on our unit that dislodgement of nasogastric tubes occurred commonly. This can lead to an increased risk of aspiration, interruptions in nutritional support, skin breakdown and radiographic exposure [1]. It is recommended that the position of nasogastric tubes should be confirmed by aspiration and $\mathrm{pH}$ testing, with radiographic confirmation used only when this is not possible [2]. Methods We performed a retrospective review of chest X-ray (CXR) requests for the 3-month period June to August 2012 using the trust radiology information system. The proportion of CXR requests for confirmation of position and patient demographics were measured with an estimation of the financial cost performed.

Results There were 541 patients admitted to the critical care area in the study period. In total, 207 out of 2,340 (8.8\%) CXRs performed were for confirmation of position. Repeated X-rays were required in some patients (see Table 1); these patients were older and tended to have a longer length of stay. A mobile CXR costs $£ 25$ in our trust, if one CXR is accepted per patient with a nasogastric tube; there was an excess of 160 images with a cost of $£ 4,000$ in the 3-month period.

Table 1 (abstract P242)

\begin{tabular}{lcccccc}
\hline $\begin{array}{l}\text { CXR for } \\
\text { NG }\end{array}$ & Patients & $\begin{array}{c}\text { Total NG } \\
\text { CXR }\end{array}$ & $\begin{array}{c}\text { Total } \\
\text { CXR }\end{array}$ & Proportion & $\begin{array}{c}\text { Mean days } \\
\text { from first to } \\
\text { last CXR }\end{array}$ & $\begin{array}{c}\text { Mean } \\
\text { age }\end{array}$ \\
\hline 1 & 47 & 47 & 321 & 0.15 & 8.45 & 67.55 \\
2 & 16 & 32 & 181 & 0.18 & 15.94 & 69.63 \\
3 & 7 & 21 & 87 & 0.24 & 17.86 & 70.00 \\
4 & 10 & 40 & 149 & 0.27 & 15.90 & 69.20 \\
5 & 3 & 15 & 52 & 0.29 & 23.33 & 66.00 \\
6 & 3 & 18 & 61 & 0.30 & 40.00 & 76.67 \\
8 & 2 & 16 & 52 & 0.31 & 39.50 & 80.00 \\
9 & 2 & 18 & 46 & 0.39 & 29.50 & 83.00 \\
\hline
\end{tabular}

Conclusion An excess of CXRs were performed for confirmation of nasogastric tube in our patient population. The recommended methods for position confirmation were reinforced amongst medical staff. The high number of repeated imaging for some patients indicates that dislodgement of tubes was also a problem. We propose that nasogastric tubes should be bridled after first dislodgement or at tracheostomy insertion to minimise dislodgement in the future.

References

1. Lorente $L$, et al:: Accidental catheter removal in critically ill patients: a prospective and observational study. Crit Care 2004, 8:R229-R233.

2. National Patient Safety Agency: Reducing Harm Caused by the Misplacement of Nasogastric Feeding Tubes. NPSA/2011/PSA002. NHS; 2011.

[http://www.nrls.npsa.nhs.uk/alerts/?entryid45=129640]

P243

Handling of dietary protein in critically ill patients

F Liebau', O Rooyackers', L Van Loon'2, J Wernerman'

${ }^{1}$ Karolinska University Hospital Huddinge, Stockholm, Sweden; ${ }^{2}$ NUtrlm,

Maastricht University, Maastricht, the Netherlands

Critical Care 2013, 17(Suppl 2):P243 (doi: 10.1186/cc12181)

Introduction Protein turnover measurements by stable isotope techniques can be applied to assess the nutritional/metabolic status in critically ill patients and their response to feeding. Because of uncertain gastrointestinal transport and uptake of nutrients, their contribution needs to be measured separately. We studied whole-body protein kinetics, with special emphasis on the contribution of dietary protein, in ICU patients and healthy controls. 
Methods Mechanically ventilated, not enterally fed ICU patients $(n=9)$ were recruited from an interdisciplinary ICU. Healthy, overnight-fasted volunteers $(n=6)$ served as reference. A primed constant i.v. infusion of ${ }^{2} \mathrm{H}$-labeled phenylalanine (Phe) and tyrosine was used to quantify whole-body protein metabolism. Patients remained on parenteral nutrition (PN) as clinically indicated; controls received PN starting 2.5 hours before starting enteral feeding. Intrinsically ${ }^{13} \mathrm{C}-\mathrm{Phe}$-labeled casein was infused for 6 hours by nasogastric tube at $0.75 \mathrm{~g}$ protein/ hour, together with maltodextrin at $2.73 \mathrm{~g} /$ hour. Protein breakdown, synthesis, net balance, and Phe splanchnic extraction were calculated before and at the end of the enteral feeding period, using equations for steady-state whole-body protein kinetics. Comparisons were made by Wilcoxon matched pairs and Mann-Whitney $U$ tests; values are reported as mean \pm SD .

Results Protein net balance was lower in patients than in the reference group at baseline $(-1.8 \pm 1.7$ vs. $0.6 \pm 0.6 \mathrm{mg} / \mathrm{kg} \mathrm{BW} /$ hour, $P=0.003)$, and after enteral feeding $(-1.1 \pm 1.5$ vs. $0.6 \pm 0.6 \mathrm{mg} / \mathrm{kg}$ BW/hour, $P=0.049$ ). Recovery of labelled Phe from enteral feeding into the systemic circulation was higher in the reference group as compared with patients $(20.3+11.2 \%$ vs. $7.0+4.8 \%, P=0.005)$. Enteral feeding did not affect protein metabolism in the reference group. In patients, protein breakdown became slightly lower during enteral feeding $(10.6 \pm 3.3$ vs. $11.2 \pm 3.3 \mathrm{mg} / \mathrm{kg}$ BW/hour, $P=0.086)$ and protein net balance became slightly higher $(-1.1 \pm 1.5$ vs. $-1.8 \pm 1.7 \mathrm{mg} / \mathrm{kg} \mathrm{BW} /$ hour, $P=0.086$ ).

Conclusion Intrinsically isotope-labelled casein can be used to quantify dietary contribution to protein metabolism in critically ill patients. Hypocaloric enteral feeding marginally improved protein balance in these patients. The low recovery of enterally administered labelled amino acid underlines the need to quantify uptake from the gastrointestinal tract when protein turnover measurements are performed in critically ill patients on enteral nutrition.

\section{P244}

Confirmation of nasogastric tube placement in critical care

M Moore, RThomson

St Georges Healthcare NHS Trust, London, UK

Critical Care 2013, 17(Suppl 2):P244 (doi: 10.1186/cc12182)

Introduction Placement of nasogastric tubes (NGTs) is commonplace in critical care. Misplacement of NGTs is rare and considered a never event [1]. Strategies to avoid never events (by confirming NGT position) include $\mathrm{pH}$ analysis of gastric secretions or chest X-ray confirmation of tube position. For this reason the authors set out to establish the efficiencies surrounding safe placement of NGTs in a 17-bed adult cardiothoracic critical care unit in a large teaching hospital.

Methods This small-scale study of 25 NGT placements during a 5-week period collated data supplied by questionnaire by healthcare workers responsible for NGT placements.

Results Analysis of Adverse Incident Reports identified no never events of misplaced NGTs within the previous 10 years. This audit revealed that the commonest type of NGT was a radio-opaque tube with stylet (corflo) (92\% of placements), with occasional use of the electromagnetic placement system (cortrak) (8\% of placements). Sizes $10(40 \%)$ and $12(56 \%)$ were most common. Tube placement was confirmed by: $\mathrm{X}$-ray (72\%); pH of aspirates (35\%); electromagnetic tube placement (one patient). The time taken from decision to place NGT to use varied (range 15 to 510 minutes). Little distinction was seen in the time taken to use and NGT confirmed by aspirate alone (205 minutes) or by X-ray (220 minutes), although the shortest interval was seen in electromagnetic NGT placement (15 minutes). The cost of NGTs confirmed by aspirate alone was low (approximately $£ 10.00$ ), higher with X-ray confirmation/electromagnetic placement (approximately $£ 45.00$ ).

Conclusion Despite the small dataset the results demonstrate a concerning delay in the application of enteral feeding and/or drug administration. Whilst reassuring in the steps taken to avoid never events, this study demonstrates that there may be delays in time-critical administration of enteral medicine or optimal nutritional practices. This study reveals a significant problem with aspirating gastric contents for $\mathrm{pH}$ testing, necessitating a large number of $\mathrm{X}$-ray position confirmations. Even if the frequency and volume of gastric aspiration were greater, there is a belief that $\mathrm{pH}$ testing may not be sufficiently accurate (since many factors alter patients' gastric $\mathrm{pH}$ ). It is possible that new technologies such as electromagnetic NGT placement may allow faster/equally safe practices. Further study including cost/benefit analysis will be needed to confirm this.

\section{Reference}

1. National Patient Safety Agency: Patient Safety Alert (5): Reducing the Harm Caused By Misplaced Nasogastric Feeding Tubes. London: NPSA; 2005 [http://www.nrls.npsa.nhs.uk/resources/?Entryld45=59794]

\section{P245}

$\mathrm{pH}$ testing to confirm nasogastric tube position on the ICU: are we wasting our time?

P Temblett, S George

Morriston ICU, Swansea, UK

Critical Care 2013, 17(Suppl 2):P245 (doi: 10.1186/cc12183)

Introduction For such a simple procedure, the insertion and position checking of nasogastric (NG) tubes can be very problematic. The UK's National Patient Safety Agency declared that 'Placement of NG tubes together with confirmation of correct placement can carry significant risks' and recommends that measuring the $\mathrm{pH}$ of NG aspirate should be the first-line method of determining correct NG position (safe range 55.5) [1].

Methods In order to assess the usefulness of $\mathrm{pH}$ testing of NG aspirates in critical care patients, a prospective survey of $\mathrm{pH}$ testing of NG tube aspirate was carried out. This was undertaken both in patients who had a newly placed NG tube and in patients who were having regular/ routine checks of their existing NG tube.

Results A total of 168 separate $\mathrm{pH}$ readings in 41 ICU patients receiving continuous enteral nutrition were recorded. In total, 137/168 patients were receiving proton pump inhibitors (PPIs). Eighteen readings were from newly placed NG tubes and 150 readings from old NG tubes. Fiftythree per cent of routine $\mathrm{pH}$ readings were falsely high; that is, $\mathrm{pH} 6$ or above despite the NG tube being in the stomach (Figure 1). Twentyeight per cent of newly placed NG tubes had falsely high $\mathrm{pH}$ readings (Figure 2).

Conclusion In this population of ICU patients, routine/daily checks of NG pH aspirate appear to be limited. This is almost certainly due to the use of continuous NG feed together with PPIs. The usefulness of $\mathrm{pH}$ testing in newly placed NG tubes, however, appears more reliable.

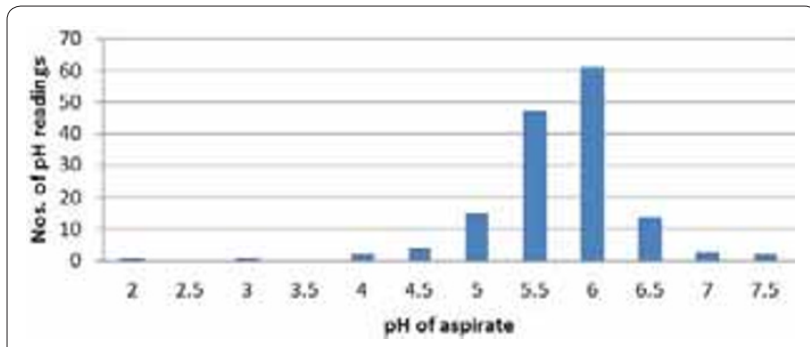

Figure 1 (abstract P245). pH values - routine checks.

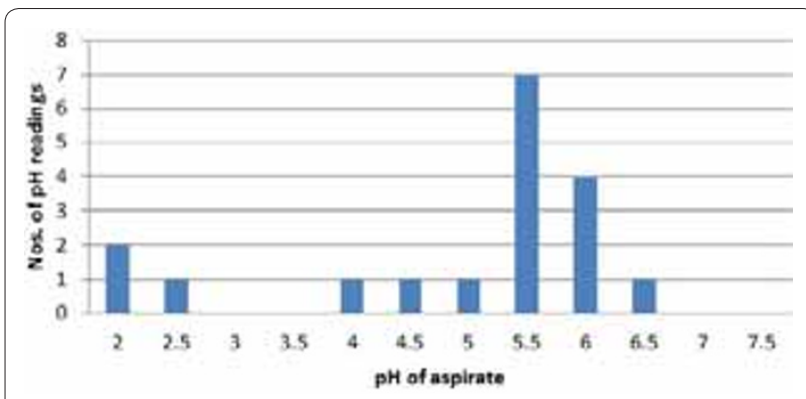

Figure 2 (abstract P245). pH values - new NG tubes. 


\section{Reference}

1. National Patient Safety Agency: Reducing the Harm Caused by Misplaced Nasogastric Feeding Tubes. NPSA/PSA001./PSA002. NPSA; 2005, 2011. [www.nrls.npsa.nhs.uk]

P246

Effect of probiotic containing lactobacillus, bifidobacterium and streptococcus thermophilus in critically ill patients

M Ebrahimi Mamaghani, S Sanaie, A Mahmoudpour, H Hamishehkar

Tabriz University of Medical Sciences, Tabriz, Iran

Critical Care 2013, 17(Suppl 2):P246 (doi: 10.1186/cc12184)

Introduction Sepsis is the most common cause of death in ICUs [1]. Destruction of intestinal barrier function and increased translocation of bacteria to systemic blood flow can lead to sepsis [2]. Probiotics may have beneficial effects in improvement of critically ill patients by modulating intestinal barrier and reduction of inflammation [3]. The aim of this trial was to determine the effect of probiotic on inflammatory biomarkers and mortality rate of sepsis in critically ill patients in the ICU.

Methods This double-blind, randomized controlled trial was conducted on 40 critically ill patients admitted to the ICU. They were randomly assigned to receive placebo or probiotic for 7 days. The APACHE score, Sequential Organ Failure Assessment (SOFA) and systemic concentrations of IL-6, procalcitonin (PCT) and protein $\mathrm{C}$ were measured before initiation of the study and on days 4 and 7. Also, 28day mortality was evaluated for each patient.

Results IL- 6 and PCT levels decreased and protein C levels increased significantly in probiotic group over the treatment period $(P<0.001)$. There was a significant difference in IL-6, PCT and protein C levels of the 7 th day between two groups ( $P=0.001,0.006$ and $<0.001$, respectively). Compared with controls, probiotic was effective in improving APACHE and SOFA scores in 7 days $(P<0.001)$. There was significant difference between the probiotic and control group in the 28-day mortality rate ( $20 \%$ vs. $55 \%$ respectively, $P=0.048$ ).

Conclusion Probiotics reduce inflammation and mortality rate in critically ill patients and might be considered as an adjunctive therapy to sepsis.

References

1. Angus DC, Linde-Zwirble WT, Lidicker J, Clermont G, Carcillo J, Pinsky MR: Epidemiology of severe sepsis in the USA: analysis of incidence, outcome, and associated costs of care. Crit Care Med 2001, 29:1303-1310.

2. Hassoun HT, Kone BC, Mercer DW: Post-injury multiple organ failure: the role of gut. Shock 2001, 15:1-10.

3. Morrow LE: Probiotics in the intensive care unit. Curr Opin Crit Care 2009, 15:144-148.

P247

Selenium levels in patients with different sources of sepsis

L French, PTemblett

Morriston Hospital, Swansea, UK

Critical Care 2013, 17(Suppl 2):P247 (doi: 10.1186/cc12185)

Introduction The aim of this study is to establish whether different types of sepsis have an impact on selenium levels. Selenium is an essential trace element involved in antioxidant and immunological reactions. Selenium levels have been shown to be low in patients with systemic inflammatory response syndrome and sepsis. Selenium replacement has been recommended in patients with sepsis $[1,2]$. Greater than 5 days of supplementation may also help to prevent the development of new infections on ICUs [3].

Methods This is a prospective survey where selenium levels were collected from patients admitted with septic shock to a tertiary ICU, for 6 months from October 2010 to March 2011.

Results Selenium levels were measured in 31 patients with septic shock. Abdominal and chest sepsis were the main sources of infection. Those with an abdominal source of sepsis had the lowest levels, as shown in Table 1. All septic shock patients who had selenium levels taken within the first 10 days of admission had subnormal levels $(<0.8 \mathrm{mg} / \mathrm{dt})$, and after 10 days had levels within the normal range, as shown in Figure 1.
Table 1 (abstract P247). Mean selenium levels in different sources of sepsis

\begin{tabular}{lcc}
\hline Source & Quantity & Level $(\mathbf{m g} / \mathbf{d l})$ \\
\hline Abdominal & 12 & 0.43 \\
Chest & 12 & 0.71 \\
Skin & 3 & 0.55 \\
CNS & 2 & 0.53 \\
GU & 2 & 0.58 \\
\hline
\end{tabular}

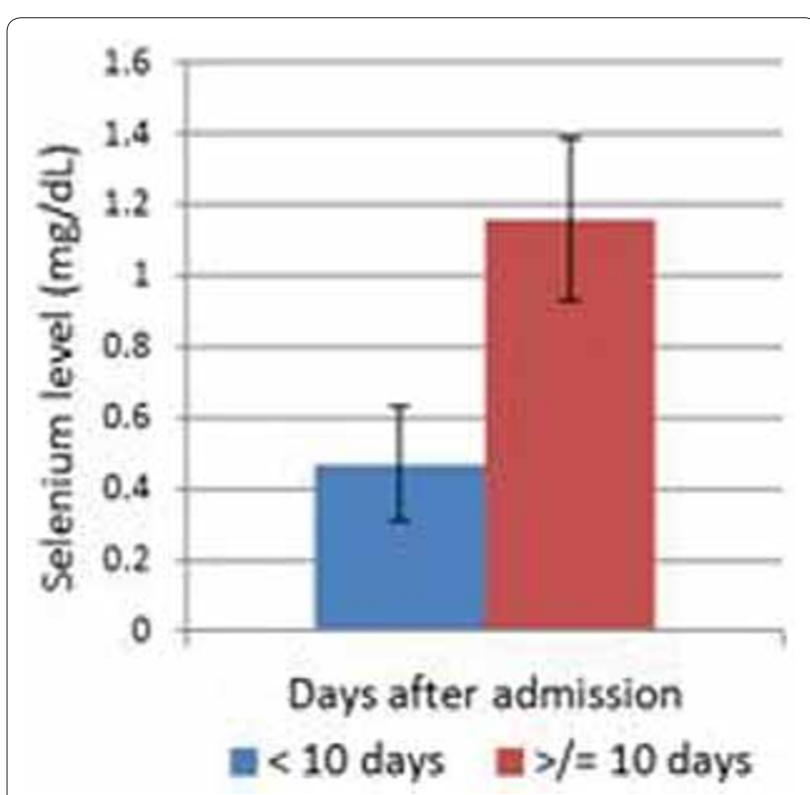

Figure 1 (abstract P247). Selenium levels before and after 10 days of admission.

Conclusion All patients admitted with early septic shock had subnormal selenium levels. Patients with an abdominal source of septic shock had the lowest levels. This survey supports previous studies indicating early supplementation may be beneficial in septic shock patients.

References

1. Berger MM: Nutr Clin Pract 2006, 21:438-449.

2. Angstwurm MW, et al:: Crit Care Med 2007, 35:118-126.

3. Andrews PJ, et al: BMJ 2011, 342:d1542.

P248

Is a combined i.v. and enteral glutamine regimen superior to a single i.v. glutamine regimen in severe thoracic trauma?

D Pavelescu, I Grintescu, I Luca-Vasiliu, L Mirea

Emergency Hospital Floreasca, Bucharest, Romania

Critical Care 2013, 17(Suppl 2):P248 (doi: 10.1186/cc12186)

Introduction Glutamine regulates many biological functions in preserving the cell, acts as a key respiratory fuel and nitrogen donor for rapidly dividing cells, and modulates the expression of many genes associated with metabolism, cell defences and repair, and cytokine production. In severe thoracic trauma, glutamine supplementation is essential because the body consumes more than it produces and glutamine effects become dependent on its route of delivery.

Methods Fifty-two patients 19 to 78 years old with surgery for severe thoracic trauma were assessed in two groups: Group A received 0.3 to $0.5 \mathrm{~g} / \mathrm{kg} /$ day i.v. glutamine $+20 \mathrm{~g}$ enteral glutamine for 7 days, supplementation to enteral nutrition; Group B receive only i.v. glutamine supplementation to enteral nutrition 0.3 to $0.5 \mathrm{~g} / \mathrm{kg} /$ day for 7 days. Weaning time, the duration of p.o. ileus, incidence and time to resolution of VAP, glycemic level and the percentage decrease of CRP at 96 hours were assessed in both groups. 


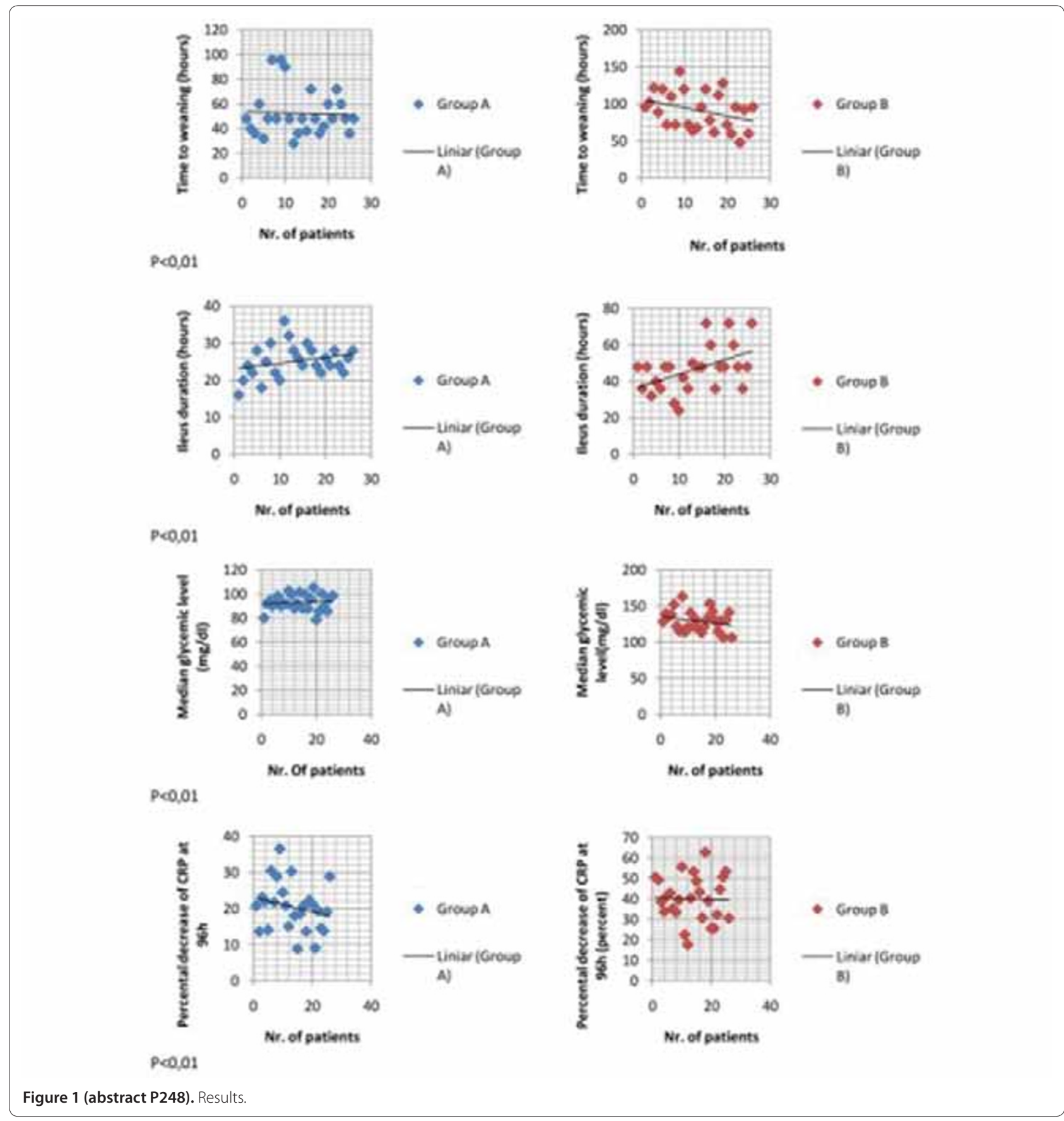

Results Weaning time and the duration of p.o. ileus were significantly lower in Group A; although the incidence of VAP is similar in both groups, the time of VAP resolution is lower, the glycemic control is better in Group A. The percentage of CRP decrease is higher in Group A. See Figure 1.

Conclusion Glutamine becomes an essential amino acid in severe thoracic trauma and when the patients are fed other than TPN (enteral, oral); although hard evidence is lacking, both administration routes may be efficient as soon as possible.

P249

Clinical effect of altered lipid emulsion composition containing fish oil in postoperative patients following abdominal surgery: a prospective, randomized, open-label, comparative, multicenter phase 3 clinical study

M Keum'1, S Hong 1 , H Han², D Yoon 3 , J Seo 4 , I Yun ${ }^{5}$

'Asan Medical Center, Seoul, South Korea;'2Seoul National University

Bundang Hospital, Seongnam, South Korea; ${ }^{3}$ Gangnam Severance Hospital, Seoul, South Korea; ${ }^{4}$ Samsung Medical Center, Seoul, South Korea; ${ }^{5}$ Konkuk

University Medical Center, Seoul, South Korea

Critical Care 2013, 17(Suppl 2):P249 (doi: 10.1186/cc12187)

Introduction Omega-3 fatty acids have been shown to decrease inflammatory responses after trauma and surgery resulting in 
reduction of morbidity and mortality in postoperative patients. The $n-6: n-3$ polyunsaturated fatty acid (PUFA) ratio in parenteral nutrition (PN) also influences the immune modulation. The aim of this study was to evaluate the optimal ratio of PUFA in postoperative patients following abdominal surgery.

Methods In a prospective, randomized, open-label, comparative, multicenter, phase 3 clinical study, we compared the safety and efficacy of a 2.1:1 ratio of n-6:n-3 fatty acid compared with 2.5:1 in postoperative patients requiring PN. Fifty-four patients were assigned to receive $\mathrm{PN}$ with Combiflex Omega peripheral ${ }^{\circledR}$ (CFO, low ratio group $n=28$ ) or SMOFKabiven peripheral ${ }^{\oplus}(\mathrm{KAB}$, high ratio group, $n=26)$ for 3 days after abdominal surgery. Safety and efficacy were monitored daily with laboratory parameters, vital signs, and adverse events from the operation day (day 0) until the end of the study (day 4).

Results Total cholesterol (TC) and low-density lipoprotein-cholesterol $(\mathrm{LDL}-\mathrm{C})$ levels were less changed significantly in the low ratio group $(3 \pm 18$ vs. $16 \pm 23 \mathrm{mg} / \mathrm{dl}, P=0.027$ for TC, $4 \pm 12$ vs. $12 \pm 18 \mathrm{mg} / \mathrm{dl}$, $P=0.026$ for LDL-C) compared with the high ratio group in postoperative patients. Other laboratory parameters and adverse events did not show statistically significant differences between the groups. See Table 1.

Table 1 (abstract P249). Lipid emulsion composition of CFO and KAB

\begin{tabular}{lcc}
\hline & CFO & KAB \\
\hline LCT $(g / l)$ & 60 & 60 \\
MCT $(g / l)$ & 50 & 60 \\
Olive oil $(g / l)$ & 50 & 50 \\
Fish oil $(g / l)$ & 40 & 30 \\
n-6:n-3 & $2.1: 1$ & $2.5: 1$ \\
\hline
\end{tabular}

Conclusion CFO containing low n-6:n-3 PUFA ratio was comparable with $K A B$ in safety and efficacy in postoperative patients requiring $P N$ and also had advantages with regard to lipid metabolism aspect.

\section{References}

1. Simopoulos AP: Biomed Pharmacother 2002, 56:365-379.

2. Heller AR, et al:: Crit Care Med 2006, 34:972-979.

P250

Influence of fat-based versus glucose-based enteral nutrition formulas on glucose homeostasis

M Wewalka, A Drolz, C Zauner

Medical University of Vienna, Austria

Critical Care 2013, 17(Suppl 2):P250 (doi: 10.1186/cc12188)

Introduction Trauma patients who receive fat-based parenteral nutrition (PEN) achieve better glucose control compared with those fed with glucose-based PEN $[1,2]$. Therefore, we determined whether fatbased enteral nutrition (EN) has the same benefit on glucose control and exogenous insulin demand in medical intensive care patients compared with glucose-based EN. Here we present preliminary data for this ongoing randomized controlled cohort study.

Methods Medical critically ill patients with need for mechanical ventilation and without contraindications for EN are included in the study. Patients are randomly assigned to receive either fat-based $(n=30)$ or glucose-based $(n=30) \mathrm{EN}$. To evaluate the individual calorie demand, indirect calorimetry is performed after an overnight fast. The determined amount of EN is administered continuously for 5 days. Glucose concentrations are measured at least three times per day and averaged. Furthermore, exogenous insulin demand per 24 hours and calorie achievement per 24 hours are evaluated daily.

Results So far, 37 patients, 16 with fat-based, 21 with glucose-based EN have been included in the study. Both groups had similar age $(62 \pm 10$ vs. $58 \pm 16$ years, $P=0.44)$, body mass index $(26.7 \pm 5.8$ vs. $28.4 \pm 4.4 \mathrm{~kg} /$ $\left.\mathrm{m}^{2}, P=0.302\right)$, SAPS II score $(62.4 \pm 12.7$ vs. $64 \pm 12.3, P=0.697)$ and fasting plasma glucose (132 $\pm 34 \mathrm{vs} .121 \pm 26 \mathrm{mg} / \mathrm{dl}, P=0.269)$. Furthermore, resting energy expenditure was similar in both groups $(1,522 \pm 365$ vs. $1,573 \pm 313 \mathrm{kcal} /$ day, $P=0.647)$. Throughout the entire study period, average blood glucose, exogenous insulin demand, and calorie achievements per day were similar between the groups (day 1: gluc: $139 \pm 30$ vs. $127 \pm 20 \mathrm{mg} / \mathrm{dl}, P=0.143$; ins: $27.8 \pm 28.4$ vs. $16.2 \pm 19.4 \mathrm{IE}, P=0.155$; EN: $52.9 \pm 25.6$ vs. $61.7 \pm 25 \%, P=0.304$; day 3: gluc: $129 \pm 17$ vs. $133 \pm 21 \mathrm{mg} / \mathrm{dl}, P=0.578$; ins: $35.1 \pm 30.9$ vs. $32.3 \pm 30.5 \mathrm{IE}, P=0.802$; EN: $78.3 \pm 28.9$ vs. $83.4 \pm 29 \%, P=0.617$; day 5 : gluc: $124 \pm 16$ vs. $132 \pm 17 \mathrm{mg} / \mathrm{dl}, P=0.276$; ins: $25.7 \pm 37$ vs. $33 \pm 26.4$ $\mathrm{IE}, P=0.562$; EN: $73.9 \pm 41.4$ vs. $87.1 \pm 23 \%, P=0.354$ ). Interestingly, the calorie achievement was not associated with insulin demand (day $1: R=0.241, P=0.156$ ) or average blood glucose (day $1: R=0.248$, $P=0.14$ ) throughout the study.

Conclusion Medical critically ill patients with fat-based or glucosebased EN achieve similar glucose control. EN was not associated with glucose concentrations or insulin demand.

References

1. Tappy L, et al:: Crit Care Med 1998, 26:860-867.

2. Huschak G, et al:: Intensive Care Med 2005, 31:1202-1208.

\section{P251}

Effect of hypocaloric versus normocaloric parenteral nutrition on whole body protein kinetics in critically ill neurosurgical patients O Rooyackers, A Berg, J Wernerman

Karolinska Institutet and University Hospital, Huddinge, Sweden

Critical Care 2013, 17(Suppl 2):P251 (doi: 10.1186/cc12189)

Introduction The optimal feeding of critically ill patients treated in the ICU is controversial. Present guidelines for protein feeding are based on weak evidence obtained with suboptimal methods. Whole body protein kinetics is an attractive technique to assess optimal protein intake by measuring the effect of protein feeding strategies on protein synthesis rates, protein degradation rates and protein balance. Here protein kinetics were measured in critically ill neurosurgical patients during hypocaloric and normocaloric parenteral nutrition.

Methods Neurosurgical patients on mechanical ventilation $(n=16)$ were studied. Energy expenditure was measured with indirect calorimetry. After that, the patients were randomized to receiving 24 hours of $50 \%$ of measured energy expenditure followed by 24 hours of $100 \%$ or $100 \%$ before $50 \%$. Whole body protein kinetics were measured during the last half hour of the feeding periods using stable isotope-labeled phenylalanine as a tracer. During a continuous infusion of labeled phenylalanine and tyrosine, plasma samples were obtained and later analyzed for the content of the labeled amino acids using mass spectrometry. Protein kinetics were calculated using standard steady-state kinetics. In addition, amino acid concentrations were analyzed by HPLC. Student's $t$ test was used for statistical analyses.

Results The patients received $0.5 \pm 0.1$ and $1.1 \pm 0.2 \mathrm{~g}$ amino acids/ $\mathrm{kg} /$ day $(P<0.001)$ on the days with 50 and $100 \%$ of measured energy expenditure respectively. Energy expenditures were $23.4 \pm 2.7$ and $24.5 \pm 2.3 \mathrm{kcal} / \mathrm{kg} /$ day $(P=0.05)$ on the 50 and $100 \%$ days respectively. Plasma amino acids concentrations were $2.8 \pm 0.5$ and $2.9 \pm 0.4 \mathrm{mM}$ $(P=0.085)$ on the 2 days respectively. Whole body protein synthesis was $12 \%$ lower when $50 \%$ of energy expenditure was given, $11.7 \pm 3.0$ versus $13.3 \pm 2.2 \mathrm{mg} / \mathrm{kg} /$ hour $(P=0.025)$, whilst protein degradation was unaltered $13.6 \pm 3.5$ versus $14.0 \pm 2.6 \mathrm{mg} / \mathrm{kg} / \mathrm{hour}(P=0.56)$. Also protein oxidation was unaltered $3.0 \pm 2.1$ versus $2.9 \pm 1.4 \mathrm{mg} / \mathrm{kg} / \mathrm{hour}$ $(P=0.85)$. This resulted in a $60 \%$ higher whole body protein balance with the normocaloric nutrition, $-1.9 \pm 2.1$ versus $-0.7 \pm 1.3 \mathrm{mg} / \mathrm{kg} /$ hour $(P=0.014)$.

Conclusion The protein kinetics measurements and the protocol used were useful to assess the efficacy of nutritional support in critically ill patients. In the critically ill neurosurgical patients treated in the ICU, hypocaloric feeding was associated with a more negative protein balance, while the amino acid oxidation was not different.

\section{P252}

Withholding parenteral nutrition for 1 week reduces ICU-acquired weakness

G Hermans, B Clerckx, TVanhullebusch, F Bruyninckx, M Casaer,

P Meersseman, D Mesotten, S Vancromphaut, P Wouters, R Gosselink, A Wilmer, G Van den Berghe

UZ Leuven, Belgium

Critical Care 2013, 17(Suppl 2):P252 (doi: 10.1186/cc12190)

Introduction ICU-acquired weakness (ICUAW) is a frequent and important complication of critical illness [1]. A large randomized 
controlled trial (EPaNIC: clinicaltrials.gov: NCT00512122) [2] showed that withholding parenteral nutrition during the first week of ICU stay whereby tolerating substantial caloric deficit (late PN) accelerated recovery and shortened weaning time as compared with early parenteral substitution for deficient enteral feeding (early PN). We examined the impact of late $\mathrm{PN}$, as compared with early $\mathrm{PN}$, on incidence and recovery of ICUAW.

Methods A preplanned subanalysis of adult patients included in the EPaNIC trial. The study was performed between October 2008 and November 2010 and included those patients who required intensive care for $\geq 8$ days as well as a computer-generated, admission categorymatched, random sample of short-stay ICU patients, the latter to correct for possible bias evoked by earlier ICU discharge in one of the two study groups. Assessors blinded for treatment allocation evaluated muscle strength clinically three times weekly from awakening onward and performed nerve conduction studies and electromyography (NCS and EMG) weekly. The primary outcome was the incidence of ICUAW, diagnosed clinically by the Medical Research Council (MRC) sum score $(<48 / 60)$ [3] at first evaluation. Secondary outcomes included ICUAW at worst and last MRC evaluation, recovery from ICUAW and incidence of abnormal findings on NCS and EMG. All analyses were performed on the total dataset and on a for-baseline characteristics propensity score-matched sample to correct for possible imbalances between the groups.

Results Clinical ICUAW evaluation was performed in 600 patients (matched $n=558$ ), electrophysiological testing in 730 (matched $n=684$ ). Late PN reduced the incidence of ICUAW at first evaluation from $43.1 \%$ to $34.4 \%, P=0.03$ (matched: early PN $41.6 \%$, late PN $33.3 \%$, $P=0.04$ ). Significantly fewer patients in the late PN group developed weakness at any time during ICU stay (late PN $37.0 \%$, early PN $46.4 \%$, $P=0.02$; matched: late PN $36.2 \%$, early PN $45.2 \%, P=0.03$ ). ICUAW may have recovered faster with late PN than with early PN $(P=0.05$, matched $P=0.06$ ). Other outcomes were not different.

Conclusion As compared with early PN, late PN reduced the incidence of ICUAW and may have accelerated recovery thereof.

References

1. Stevens et al:: Int Care Med 2007, 33:1876-1891.

2. Casaer et al:: N Eng/ J Med 2011, 365:506-516.

3. De Jonghe et al.:, JAMA 2002, 288:2859-2867.

\section{P253}

Impact of early parenteral nutrition on muscle and adipose tissue compartments during critical illness

L Langouche', MP Casaer ${ }^{2}$, W Coudyzer'2, D Vanbeckevoort²,

B De Dobbelaer², FG Güiza', PJ Wouters', D Mesotten², G Van den Berghe ${ }^{2}$

'KU Leuven, Belgium; '2University Hospitals Leuven - KU Leuven, Belgium

Critical Care 2013, 17(Suppl 2):P253 (doi: 10.1186/cc12191)

Introduction The goal of enhanced nutrition in critically ill patients is to improve outcome by reducing lean tissue wasting. However, such effect has not been proven. This study aimed to assess the effect of early administration of parenteral nutrition (PN) on muscle volume and composition by repeated quantitative computer tomography (qCT). Methods We performed a preplanned substudy of a randomized controlled trial (EPaNIC) that compared early initiation of PN when enteral nutrition was insufficient (early PN) with tolerating a pronounced nutritional deficit for 1 week in the ICU (late PN) [1]. Late PN prevented infections and accelerated recovery. We studied 15 EPaNIC study neurosurgical patients requiring prescheduled repeated follow-up CT scans and six healthy volunteers matched for age, gender and BMI. Repeated abdominal and femoral $\mathrm{qCT}$ images were obtained in a standardized manner on median ICU day 2 (IQR 2 to 3 ) and day 9 ( 8 to 10). Intramuscular, subcutaneous and visceral fat compartments were delineated manually. Muscle and adipose tissue volume and composition were quantified using standard Hounsfield Unit ranges. Results Critical illness evoked substantial loss of femoral muscle volume in 1 week, irrespective of the nutritional regimen. Early PN reduced the quality of the muscle tissue, as reflected by the attenuation, revealing increased intramuscular water/lipid content. Early PN also increased the volume of adipose tissue islets within the femoral muscle compartment. These changes in skeletal muscle integrity correlated with caloric intake. In the abdominal muscle compartments, changes were similar, albeit smaller. Femoral and abdominal subcutaneous adipose tissue compartments were unaffected by disease and nutritional strategy. Conclusion Early PN did not prevent the pronounced wasting of skeletal muscle observed over the first week of critical illness. Moreover, early PN increased the amount of adipose tissue within the muscle compartments.

Reference

1. Casaer MP, et al:: N Engl J Med 2011, 365:506-517.

\section{P254}

Withholding parenteral nutrition during the first week of critical illness increases plasma bilirubin but lowers the incidence of cholestasis and gallbladder sludge

YM Vanwijngaerden', L Langouche', M Gielen', Y Debaveye', M Casaer', C Liddle'2, S Coulter'2, R Brunner'1, P Wouters', A Wilmer', G Van den Berghe', D Mesotten

'University Hospitals KU Leuven, Belgium; ${ }^{2}$ University of Sydney, Australia Critical Care 2013, 17(Suppl 2):P254 (doi: 10.1186/cc12192)

Introduction Cholestatic liver dysfunction (CLD) during critical illness, defined by hyperbilirubinemia, often occurs and is associated with poor outcome. Parenteral nutrition (PN) is assumed to aggravate CLD. However, hyperbilirubinemia more frequently occurred when the start of PN was delayed until day 8 in the ICU (late PN) [1]. Late PN accelerated recovery as compared with early initiation of PN.

Methods This was a preplanned subanalysis of a large randomized controlled trial on early versus late initiation of PN $(n=4,640)$ [1]. Plasma total bilirubin was quantified in all patients daily while in the ICU. Liver enzymes ALT, AST, GGT and ALP were quantified twice weekly in all patients while in the ICU. In a random predefined subset of patients, circulating bile salts were also quantified with MS-HPLC at baseline and on day 3, day 5 and the last day in the ICU $(n=280)$. Gallbladder sludge was evaluated by ultrasound on ICU day 5 by blinded assessors $(n=776)$.

Results From day 1 after randomization until the end of the 7-day intervention window, plasma bilirubin was higher in the late PN than in the early PN group (all $P<0.001$ ). In the late PN group, as soon as PN was started on day 8, plasma bilirubin also fell and the two groups became comparable. Maximum levels of GGT, ALP and ALT during the ICU stay were higher in the early PN group (all $P<0.01$ ). Compared with baseline, the circulating glycine and taurine conjugated primary bile salts were elevated on day 3 , day 5 and last day of the ICU stay $(P<0.01$ for all). However, there was no difference between the two groups. More patients in the early PN than in the late PN group had gallbladder sludge on day 5 ( $45 \%$ vs. $37 \% ; P=0.04$ ).

Conclusion Tolerating substantial caloric deficit by withholding PN until day 8 of critical illness increased circulating levels of bilirubin but reduced the occurrence of gallbladder sludge and lowered GGT, ALP and ALT levels. These results suggest that hyperbilirubinemia during critical illness dies not necessarily reflect cholestasis and instead may be an adaptive response. Additional analyses on a propensity scorematched patient population are ongoing.

Reference

1. Casaer et al:: N Engl J Med 2011, 365:506-516.

\section{P255}

Impact of early parenteral nutrition on catabolism

J Gunst', I Vanhorebeek', MP Casaer', G Hermans', PJ Wouters', J Dubois², K Claes', M Schetz', G Van den Berghe'

'KU Leuven, Belgium;' Jessa Hospital, Hasselt, Belgium

Critical Care 2013, 17(Suppl 2):P255 (doi: 10.1186/cc12193)

Introduction Prolonged critically ill patients enter a state of hypercatabolism and muscle weakness, which has been associated with increased morbidity and mortality. Early, full feeding of ICU patients has been advocated to counteract catabolism. However, a large, multicenter study found that early parenteral nutrition (PN) had no significant impact on mortality and even increased dependency on intensive care with, among others, a significant prolongation of 
the duration of renal replacement therapy (RRT) [1]. The impact of the intervention on early markers of catabolism has not been investigated. Methods We studied the impact of early versus late PN on daily markers of catabolism in the ICU in the total study population and in propensity score-matched subgroups of long-stay patients. In addition, we calculated the net incorporation rate of the extra amino acids supplied by early PN.

Results Plasma urea, the urea/creatinine ratio and nitrogen excretion increased over time in the ICU. Early PN further increased these markers of catabolism, from the first day of amino acid infusion onward, and only marginally improved the nitrogen balance. Also in the group that received PN only after the first week in the ICU, ureagenesis was increased by infusing amino acids. Over the first 2 weeks, approximately two-thirds of the extra amino acids supplied by early PN were net wasted in urea. The above findings were confirmed in propensity scorematched subgroups of long-stay patients. The higher urea levels with early PN, rather than the kidney function as such, may have driven the observed longer duration of RRT, as supported by multiple regression analysis.

Conclusion The extra amino acids supplied by early PN appeared inefficient to reverse the negative nitrogen balance, not because of insufficient amino acid delivery, but rather because of insufficient incorporation with, instead, increased degradation into urea. The substantial catabolism of the extra amino acids, leading to pronounced urea generation, may have prolonged the duration of RRT in the early PN group.

Reference

1. Casaer et al:: N Engl J Med 2011, 365:506-517.

P256

Impact of early versus late parenteral nutrition on morphological and molecular markers of atrophy and autophagy in skeletal muscle of critically ill patients

I Vanhorebeek, MP Casaer, F Güiza, S Derde, I Derese, PJ Wouters,

Y Debaveye, J Gunst, G Hermans, G Van den Berghe

KU Leuven, Belgium

Critical Care 2013, 17(Suppl 2):P256 (doi: 10.1186/cc12194)

Introduction Muscle weakness of critical illness is associated with prolonged dependency on ventilatory support and delayed rehabilitation. Muscle wasting related to poor nutrition has long been considered a major determinant, whereas the importance of myofiber integrity only recently emerged [1-4]. We hypothesized that nutrient restriction early during illness aggravates atrophy while preserving myofiber integrity by activating the crucial cellular quality control pathway autophagy. The latter could be important to preserve muscle function.

Methods Critically ill patients ( $n=122$ ) were randomized to early (early$\mathrm{PN}$ ) or late (late-PN) initiation of parenteral nutrition to complete failing enteral nutrition, while maintaining normoglycemia $(80$ to $110 \mathrm{mg} /$ dl) with insulin, in the EPaNIC study [5]. Vastus lateralis biopsies were harvested after 1 week and compared with matched controls $(n=20)$. Results As compared with controls, muscle from critically ill patients showed reduced myofiber density, a shift to smaller (especially type I) myofibers, lower myosin and actin mRNA, upregulated mRNA of the ubiquitin ligases muscle-ring-finger-1 and atrogin-1, a small increase in the autophagosome formation marker LC3-II/LC3-I, and increases in the autophagic substrates ubiquitin and p62 (all $P \leq 0.006$ ). Late-PN, resulting in a larger caloric deficit than early-PN, had no substantial impact on atrophy markers. In contrast, late-PN increased LC3-II/LC3-I $(P=0.02)$, which coincided with less accumulation of ubiquitinated proteins/aggregates $(P=0.05)$. Fewer patients on late-PN developed muscle weakness as compared with early-PN ( $42 \%$ vs. $68 \%, P=0.05)$. In multivariable analysis, a lower LC3-II/LC3-I ratio $(P=0.05)$ and higher myofiber density $(P=0.04)$ were independently associated with muscle weakness.

Conclusion Early-PN did not counteract muscle atrophy whereas it suppressed autophagy and aggravated weakness. Statistically, muscle weakness was not explained by atrophy or wasting but rather by impaired autophagy and preservation of muscle density. Thus, tolerating nutrient restriction early during critical illness may preserve myofiber integrity by activating autophagy.

\section{References}

1. Debaveye, Van den Berghe: Annu Rev Nutr 2006, 26:513-538.

2. Masiero et al.: Cell Metab 2009, 10:507-515.

3. Vanhorebeek et al.: J Clin Endocrinol Metab 2011, 96:E633-E645.

4. Derde et al:: Endocrinology 2012, 153:2267-2276.

5. Casaer et al:: N Engl J Med 2011, 365:506-517.

P257

Impact of closing an emergency department on a neighbouring

teaching hospital: the concentrate effect

J Millar, R Wilson, P O'Connor, R McLaughlin

Royal Victoria Hospital, Belfast, UK

Critical Care 2013, 17(Suppl 2):P257 (doi: 10.1186/cc12195)

Introduction Closure of an acute hospitals emergency department (ED) has important ramifications for those centres expected to take up the resultant workload. The continued reconfiguration of emergency care is likely to produce an increasing number of these scenarios. Little evidence is available to support planning of such initiatives and thus the implications are difficult to anticipate. This study aims to demonstrate one hospital's experience of the rationalisation of emergency care and its effect on workload.

Methods This retrospective study was conducted in a large teaching hospital. Activity data were analysed for a 12-month period following the closure of a neighbouring ED. The results were subsequently compared against the year prior to closure. Attendance, triage data, admission rates and waiting times were compared across the two periods, as were workload data for all grades of physician. The chisquared test was used to examine differences between groups.

Results In the period studied, the gross attendance figure increased by $20,480(33.72 \%)$, whilst the admission rate rose from 22 to $27 \%$. Following closure of the neighbouring ED, the proportion of highacuity patients attending our institution increased dramatically, with the proportion of category one and two patients (Manchester Triage Scale) increasing by $8.33 \%(P=0.076)$ and $18.80 \%(P<0.001)$, respectively. Likewise, ambulance arrivals increased out of proportion to the total increase in attendances $(P=0.016)$. Admissions from the ED to the ICU increased by $63.04 \%$. Consultants workloads now include $50 \%$ more category 1 and 2 patients $(P=0.001)$.

Conclusion Reconfiguration of emergency care can have dramatic implications for existing services; these may not always be anticipated. Rationalisation of ED's may result in a concentration of high-acuity patients accompanied by a downturn in the numbers of patients whose presentations are amenable to care delivered in other settings. This abrupt change in case mix requires a re-examination of existing workforces and their seniority.

\section{P258}

Overcrowding estimation in the emergency department: is the simplest score the best?

$\checkmark$ Winkin, V Pinckaers, V D'Orio, A Ghuysen

CHU Liège, Belgium

Critical Care 2013, 17(Suppl 2):P258 (doi: 10.1186/cc12196)

Introduction Emergency department (ED) overcrowding is a major international problem with a negative impact on both patient care and providers. Among validated methods of measurement, emergency physicians have to choose between simple and complex scores [1,2]. The aim of the present study was to compare the complex National Emergency Department Overcrowding Scale (NEDOCS) with the simple occupancy rate (OR) determination. We further evaluated the correlation between these scores and a qualitative assessment of crowding.

Methods The study was conducted in two academic hospitals and one county hospital in Liège, Chênée and Verviers; each with an ED census of over 40,000 patient visits per year. Samplings occurred over a 2-week period in January 2011, with five sampling times each day.

Results ED staff considered overcrowding as a major concern in the three EDs. Median OR ranged from 68 to 100, while the NEDOCS ranged from 64.5 to 76.3 . We found a significant correlation between 
the OR and the NEDOCS (Pearson $R=0.973,0.974$ and 0.972 ), as well as between the OR, the NEDOCS and the subjective evaluation $(P=0.001)$. Conclusion Crowding evaluation in the ED requires validated, easy-touse, scores. Our study indicates that the simple OR is as accurate as the complex NEDOCS, allowing continuous crowding assessment.

References

1. Hwang U, McCarthy ML, Bernstein SL, et al:: Measures of crowding in the emergency department: a systematic review. Acad Emerg Med 2011, 18:527-538.

2. Hoot NR, Zhou C, Jones I, Aronsky D: Measuring and forecasting emergency department crowding in real time. Ann Emerg Med 2007, 49:747-755.

\section{P259}

GP-led walk-in centre in the UK: another way for urgent healthcare provision

M Arain, J Nicholl, M Campbell

The University of Sheffield, UK

Critical Care 2013, 17(Suppl 2):P259 (doi: 10.1186/cc12197)

Introduction It is evident that accident and emergency departments are overloaded with patients, which results in delays in healthcare provision [1]. A large proportion of patients consist of patients with minor illness that can be seen by a healthcare provider in a primary care setting. The aim of the study was to determine the characteristics of patients using GP walk-in services, patients' satisfaction and the effect on emergency department (ED) services.

Methods The survey was conducted in Sheffield and Rotherham walk-in centres over 3 weeks during September and October 2011. A self-reported, validated questionnaire was used to conduct survey on the patients presenting at these centres. We estimated that a sample size of around 400 patients from each centre was required to achieve statistically robust results. A post-visit, short questionnaire was also sent to those who agreed for the second questionnaire and provided contact details. ED data were also obtained from April 2008 to March 2010, 1 year before and 1 year after the opening of the GP walk-in centre. Data were entered and analysed in PASW Statistics 18. Ethical approval of the study was obtained from the NHS ethical review committee.

Results A total of 1,030 patients participated in the survey (Rotherham 501; Sheffield 529). The mean age of the participants was 32.1 years at Sheffield and 30.88 years at Rotherham. A higher proportion of users were female, around $59 \%$ at both centres. Most of the patients rated high for convenience of the centre opening hours and location (above $85 \%$, apart from the location of Sheffield centre, which was rated high by around $72 \%$ of the research participants). Overall $93 \%$ patients were satisfied with the service at Rotherham centre and around $86 \%$ at the Sheffield centre. Based on the estimation of the monthly counts of patients attending ED and the GP walk-in centre, around 14\% monthly reduction in minor attendances at ED was expected. However, ED routine data did not show any significant reduction in minor attendances as a result of the opening of the GP walk-in centre.

Conclusion These walk-in centres have been shown to increase accessibility to healthcare service through longer opening hours and walk-in facility. Although the effect on the reduction of patients' load at the ED is not visible as these centres cover a fraction of the population, the centre has a potential to divert patients from the ED.

Reference

1. Gerard K, Lattimer V: Preferences of patients for emergency services available during usual GP surgery hours: a discrete choice experiment. Fam Pract 2005, 22:28-36.

P260

Establishing a new emergency department: effects on patient flow

$\checkmark$ Rautava', TValpas', M Nurmikari², A Palomäki

'Kanta-Häme Central Hospital, Hämeenlinna, Finland: '2City of Hämeenlinna,

Finland

Critical Care 2013, 17(Suppl 2):P260 (doi: 10.1186/cc12198)

Introduction Overcrowding in emergency departments (EDs) is a widely known problem. It causes problems and delays in the ED and

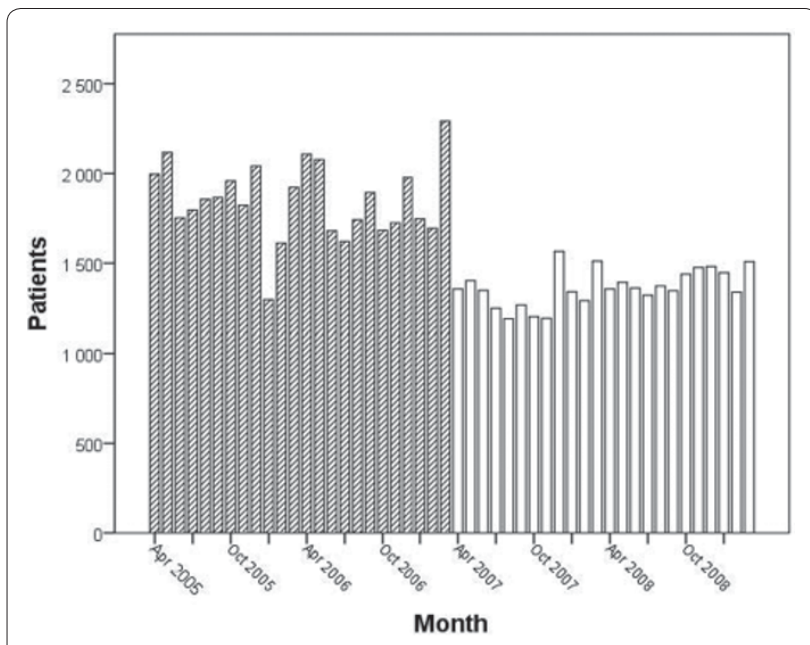

Figure 1 (abstract P260). GP patient visits before and after the reform of emergency services.

has a negative impact on patient safety [1]. The aim of this study was to analyse whether a reform of emergency care can reduce patient flow into the ED.

Methods A substantial reform of emergency care took place in the province of Kanta-Häme in Southern Finland. Three separate out-ofhours services in primary healthcare $(\mathrm{PHC})$ and one ED in the hospital were combined into one large ED in April 2007. Basic principles of the new ED were: the ED is only for those patients who are seriously ill or injured, and need immediate care; PHC (healthcare centres) take care of acute ordinary illnesses and nonserious injuries during office hours. To achieve these principles a regional five-scale triage system was planned and implemented. The information plan was established. Citizens were systematically informed about the principles of the new ED by mail, articles in the newspapers and interviews in the radio and television. The ED's Internet pages were planned and established. The number of patient visits (Hämeenlinna region) was analyzed 2 years before and after establishing the new ED.

Results During the 2-year period before the establishment of the new ED the mean number of GP patient visits was $1,845 \pm 43 /$ month. During the 2-year period after the reform the number was diminished to $1,364 \pm 21$ /month. This change was not associated with the increase of the patient visits taken care of by specialists and hospital residents. See Figure 1.

Conclusion An extensive reform of the emergency services can notably reduce patient flow into the ED.

Reference

1. Boyle A, et al:: Emerg Med Int 2012:838610. [Epub ahead of print]

\section{P261}

Prehospital EKG evaluation in Rio de Janeiro ambulances

RV Vasconcellos, FE Erthal, RV Vargas

Instituto Nacional de Cardiologia, Rio de Janeiro, Brazil

Critical Care 2013, 17(Suppl 2):P261 (doi: 10.1186/cc12199)

Introduction Rio de Janeiro's Fire Squad is responsible for EMS in the city. During 2010 we implemented 10 ambulances with EKG transmission capability in our city. Our intention was to access the prevalence of acute myocardial infarction in the prehospital setting. Methods We used the Aerotel HeartView EKG system to acquire patient examination and a blackberry phone to transmit and receive the PDF EKG trace. The PDF comes with the cardiologist's interpretation from a remote hospital, the HCOR São Paulo.

Results We realized 503 EKG examinations in total. Of these, 248 (49\%) had as the chief complaint chest pain, 101 (20\%) shortness of breath, $47(9 \%)$ syncope, $36(7 \%)$ palpitation; other complaints were $15 \%$. We detected 32 examinations (6.36\%) with ST elevation MI and 


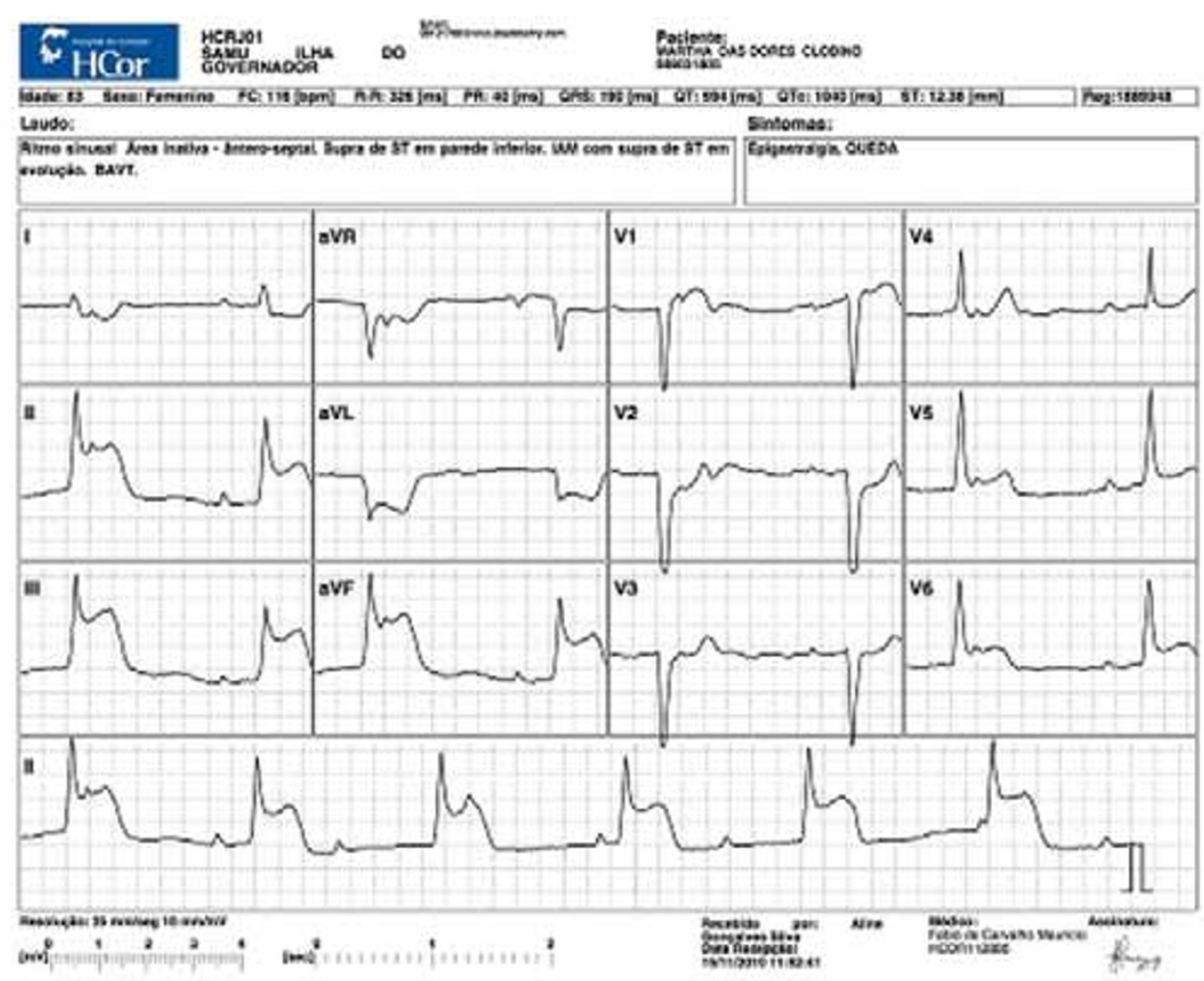

Figure 1 (abstract P261). Acute inferior MI with complete heart block.

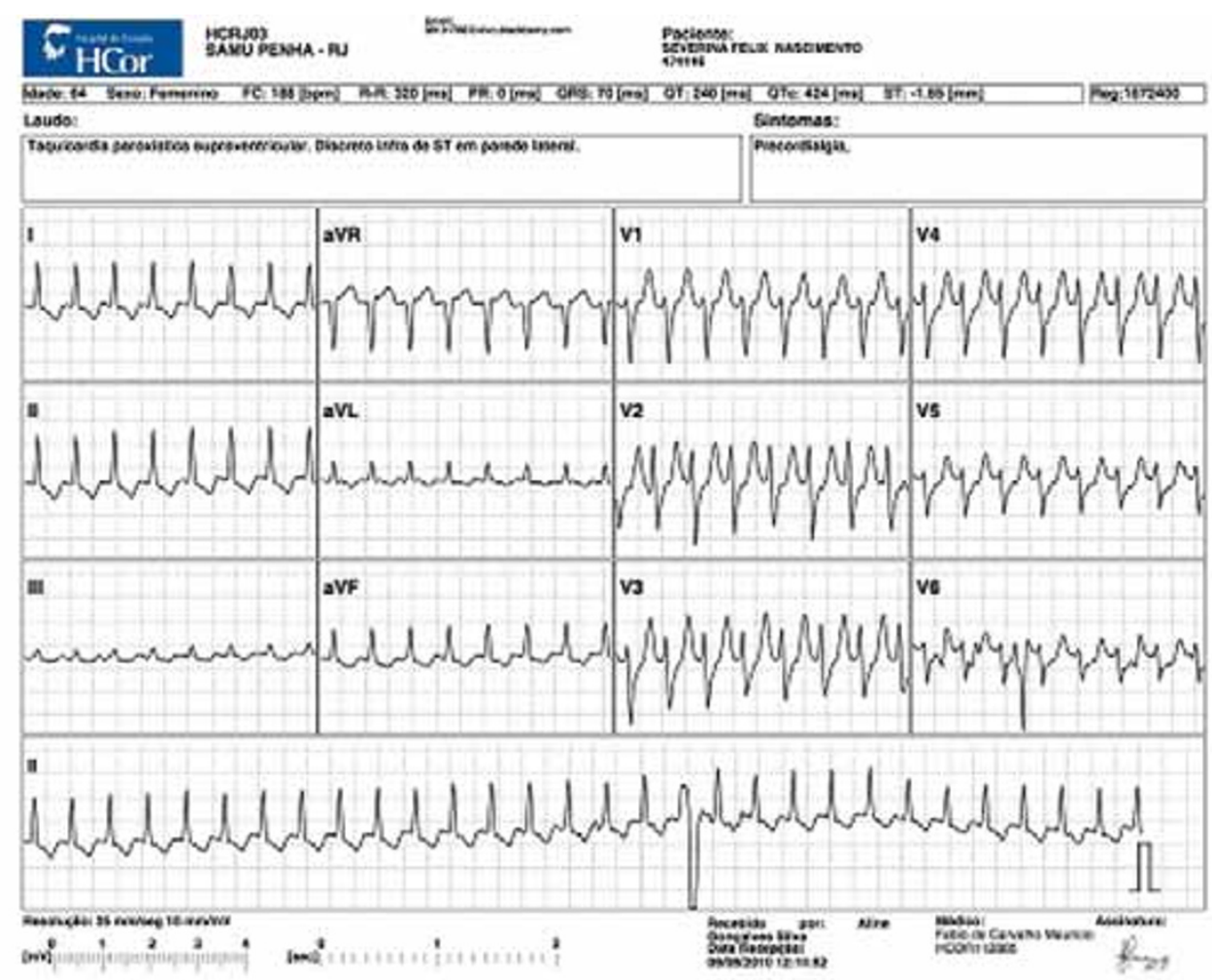

Figure 2 (abstract P261). Paroxysmal supraventricular tachycardia. 
44 examinations (8.75\%) with ST depression. Atrial fibrillation was detected in 43 examinations (8.5\%). See Figures 1 and 2.

Conclusion This experience gave us an idea of the prevalence for acute ST elevation MI in the prehospital setting, so that we can better develop our prehospital thrombolysis protocol and focus our training for cardiology care.

\section{Reference}

1. Ioannidis JP, Salem D, Chew PW, Lau J: Accuracy and clinical effect of out-ofhospital electrocardiography in the diagnosis of acute cardiac ischemia: a meta-analysis. Ann Emerg Med 2001, 37:461-470.

\section{P262}

Technique of ultrasound-guided peripheral venous access in the emergency room

MG Annetta, G Scoppettuolo, M Biancone, F Toni, M Pittiruti Catholic University, Rome, Italy

Critical Care 2013, 17(Suppl 2):P262 (doi: 10.1186/cc12200)

Introduction In emergency situations, patients may need a fast and reliable peripheral venous access, which sometimes may be difficult to obtain, because of poor visualization of the superficial veins due to edema, obesity, hypovolemia or local abnormalities. In such cases, insertion of a central line is potentially time consuming and possibly associated with complications. Furthermore, central lines inserted in emergency are known to be at high risk of infection, so guidelines recommend that they should removed within 24 to 48 hours. In this setting, ultrasound-guided placement of a peripheral venous access might be more rapid, safer and more cost-effective than a central line. Methods We have reviewed retrospectively our experience with the emergency use of $18 \mathrm{G}$ or $20 \mathrm{G}$ polyurethane catheters, 8 to $10 \mathrm{~cm}$ long, inserted by direct Seldinger technique.

Results In 1 year, 76 long peripheral catheters were inserted in emergency conditions, using ultrasound guidance. The success rate was 100\%; most lines lasted $>1$ week and were used for different purposes, including contrast medium injection.

Conclusion The direct Seldinger technique allows a rapid and safe placement of the catheter in a vein of the arm or of the forearm, even when the vessel cannot be palpated or seen, as long as it can be visualized by ultrasound and it is not deeper than $2 \mathrm{~cm}$. The long life of this type of peripheral line (up to 2 weeks) is guaranteed by the material (polyurethane being more biocompatible than Teflon) and by the length of the catheter (which reduces the risk of dislodgment). Also, these catheters are particularly cost-effective if compared with a central line or with a midline catheter, since a complete kit including catheter, $20 \mathrm{G}$ needle and $20 \mathrm{~cm}$ guidewire costs between $€ 15$ and $€ 20$.

P263

FIB the fractured femur

H Shahzad, M Majeed, D Yeo, V Gupta, U Salanke

University Hospital, Birmingham, UK

Critical Care 2013, 17(Suppl 2):P263 (doi: 10.1186/cc12201)

Introduction The lumbar plexus block provides excellent analgesia after hip and knee surgery. One approach to the three major nerves (femoral, lateral cutaneous and obturator) of the lubar plexus is the fascia iliaca compartment block, first described by Dalens and colleagues. Using this blind approach, complete nerve block in the distribution of the lumbar plexus may be achieved only in $38 \%$ of cases. Ultrasound-guided regional techniques offer a number of advantages including real-time needle guidance and direct observation of local anesthetic spread within tissue planes.

Methods We hypothesized that real-time UFIB could be successfully performed in the ED and would provide an excellent adjunct or alternative to repeat doses of IVMS for pain control in patients with HFX. The study was conducted at University Hospital, Birmingham, where we see about 90,000 patients every year. All patients with confirmed femoral (neck/shaft) fractures and pain score $>7$ were included in the study. Patients with local wounds, or suspected significant pelvic injury were excluded. A combination of $15 \mathrm{ml}$ lignocaine $1 \%$ and $15 \mathrm{ml}$ bupvivcaine $0.25 \%$ was used. VAS (0 to 10 ), pain on movement of the leg and patient satisfaction were used to assess the outcome. The assessments were made before the FIB, at 15 minutes, 30 minutes and 45 minutes.

Results A convenience sample of 19 patients was enrolled in this study. The compartment block was placed by three mergence ultrasound trainers. The mean age was 58.5 years. There were 13 females and six males. The mean pain score was 9.5 at time 0 . The mean pain score had improved to 5.5 at 15 minutes, 4.5 at 30 minutes and 3 at 45 minutes, respectively. The patient satisfaction was scored 4.5 on a scale of 1 to 5 where 5 was the most satisfied. No patient required any further analgesia up to 90 minutes and no issues were raised about the pain or discomfort upon patient transfer. We have no documented complications or side effects.

Conclusion FIB is now widely used by non-anaesthetic trainees to combat pain in preoperative care due to its safe landmarks. Our results have shown that it can be used safely and effectively for pain management in hip fracture by emergency physicians, who are trained in the technique, on the shop floor.

\section{P264}

Abdominal pain in adolescent females: a single-centre audit and review of management

F Gallagher, H Jahn, F Davies

Leicester Royal Infirmary, Leicester, UK

Critical Care 2013, 17(Suppl 2):P264 (doi: 10.1186/cc12202)

Introduction Abdominal pain in adolescent females has undergone recent changes with regards to its management under various specialities. The authors report a single-centre audit looking at the correct investigation and management of 12-year-old to 16-year-old girls with abdominal pain in the emergency department setting.

Methods A single-centre audit and retrospective analysis of patients took place using case notes and computerised records. Documentation was analysed using statistical analysis and minimum standards were set and reviewed.

Results After exclusion criteria 62 females between the ages of 12 and 16 presented to the paediatric emergency department in Leicester with abdominal pain as the predominant admission symptom during a 12-month period. Documentation of the gynaecological history was poor (menstrual history $47 \%$, sexual history $14 \%$, contraception $8 \%$ ), as was the performance of basic investigations (urine dipsticks $65 \%$, pregnancy test $42 \%$ ). Documentation was analysed with regard to discharge diagnosis. Ultrasound investigation was performed on seven of the patients but only once admitted to various specialities. No ultrasound was undertaken upon admission.

Conclusion Improvement in documentation of minimum standards for these patients is needed. A multidisciplinary care pathway could improve outcome. Consideration should be given to whether early ultrasound investigation is appropriate and there is a further need for investigation as to whether this would improve longer term outcomes.

P265

Multidisciplinary approach to improving documentation of visual acuity in patients presenting with ocular trauma

L Low, M Johnston

NHS Tayside, Dundee, UK

Critical Care 2013, 17(Suppl 2):P265 (doi: 10.1186/cc12203)

Introduction This study aimed to implement a multidisciplinary quality improvement project in Perth Royal Infirmary A\&E department to improve documentation of visual acuity (VA) in patients presenting with ocular injury.

Methods The improvement project involves a three-pronged multidisciplinary approach: ensure that equipment required for VA testing (Snellen chart and pinhole mask) was readily available; encourage VA testing at first point of contact with A\&E staff, both nursing/medical staff; and refresher online course on how to test for VA quickly and accurately, in the $6 / \mathrm{X}$ format. We compared the pre-intervention (2 September to 2 October 2012) and post-intervention (11 October to 19 November 2012) rates of VA documentation using the chi-square test. 


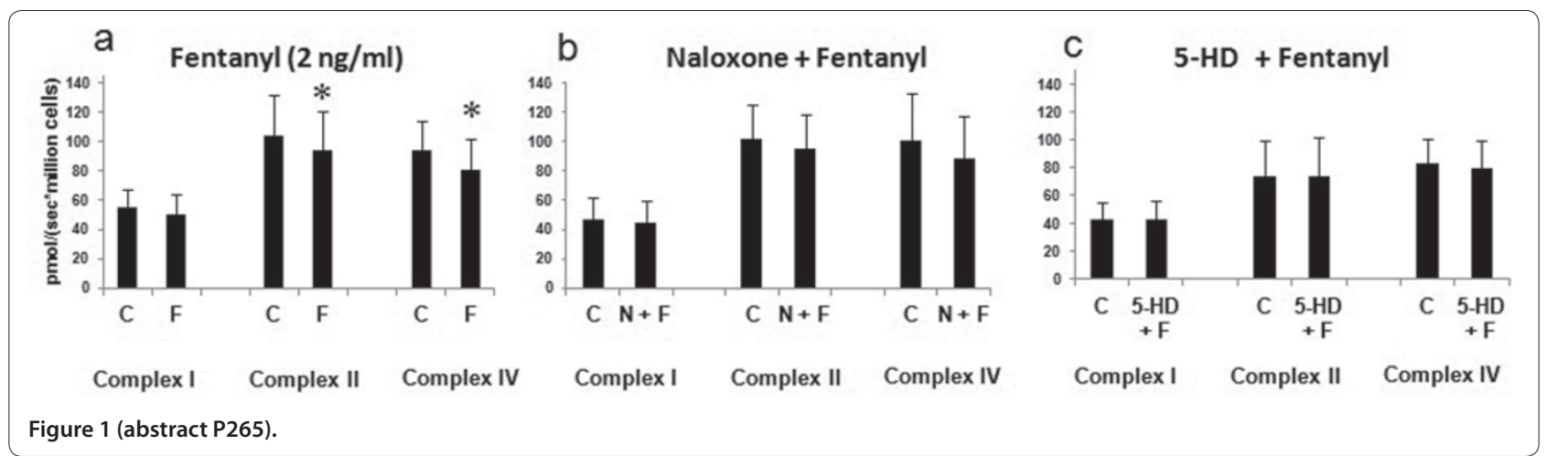

Results During the pre-intervention period, of a total of 44 patients who presented to A\&E with eye injury, only 36 patients (95\%) had their VA tested. Following intervention, there was significant improvement in VA testing, where all 43 patients presenting to A\&E with eye injuries had their VA tested $(100 \%, P=0.02)$. Documentation of VA in the correct $(6 / X)$ format increased from 82 to $84 \%$ following intervention. There was a $15 \%$ improvement in documentation of best-corrected VA, from 48 to $63 \%$ post intervention. See Figure 1 .

Conclusion Through a multidisciplinary approach, we were successful in achieving our target of $100 \%$ VA documentation rate in all patients presenting with eye injury to PRI A\&E.

\section{P266}

BiPAP for treating moderate and severe asthma exacerbations in a PED

A Williams, T Abramo

Vanderbilt University Medical Center, Nashville, TN, USA

Critical Care 2013, 17(Suppl 2):P266 (doi: 10.1186/cc12204)

Introduction BiPAP utilization for the treatment of severe refractory status asthmaticus patients has become an accepted therapy but is not well described for moderate exacerbations. We sought to analyze outcomes from our BiPAP quality database for children presenting in status asthmaticus at varying levels of severity.

Methods PED status asthmaticus patients requiring BiPAP from 1 January 2010 to 31 August 2012 had a bedside interview and documentation of information at the time therapies were given. Incomplete data were collected retrospectively. All data were stored and analyzed using a RedCap database. Subjects were stratified into severity groups based on asthma score at the time of BiPAP placement. Results There were 206 subjects in the moderate severity group and 197 in the severe group. Table 1 shows the groups were well
Table 1 (abstract P266). Comparison of children receiving BiPAP for status asthmaticus by severity of illness

\begin{tabular}{lccc}
\hline & $\begin{array}{c}\text { Moderate } \\
(\boldsymbol{n}=\mathbf{2 0 6})\end{array}$ & $\begin{array}{c}\text { Severe } \\
(\boldsymbol{n}=\mathbf{1 9 7})\end{array}$ & $\boldsymbol{P}$ value \\
\hline Age (years) & $6.8 \pm$ SD 2.8 & $7.1 \pm$ SD 4.2 & $X$ \\
Gender & $62 \%$ male & $61 \%$ male & $X$ \\
History of asthma & $92 \%$ & $87 \%$ & $X$ \\
Prior PICU admission & $21 \%$ & $32 \%$ & $X$ \\
Prior history of BiPAP use & $19 \%$ & $24 \%$ & $X$ \\
Prior history of intubation & $6 \%$ & $7 \%$ & $X$ \\
Mean triage for BiPAP start (hours) & $2.8 \pm$ SD 2.1 & $1.2 \pm$ SD 1.25 & $<0.0001$ \\
Mean time on BiPAP (hours) & $3.9 \pm$ SD 3.6 & $5.6 \pm$ SD 5.2 & $<0.0001$ \\
Median time on BiPAP (hours) & 2.7 & 3.8 & $X$ \\
Admission to PICU & $58 \%$ & $75 \%$ & $X$ \\
Total length of hospital stay & $42 \pm$ SD 26 & $50 \pm$ SD 33 & $<0.06$ \\
Serious complications & None & None & $X$ \\
72-hour returns & $n=1(0.5 \%)-$ & $n=4(2 \%)-$ & $X$ \\
& no admits & no admits & \\
\hline
\end{tabular}

matched and compares other pertinent data. Children with severe presentations were placed on BiPAP sooner $(P<0.001)$ and remained on BiPAP longer $(P<0.001)$. The moderate group had a longer wait until BiPAP placement. Tables 2 and 3 demonstrate higher initial BiPAP (IPAP/EPAP) settings with increasing age and severity. Figure 1 trends initiation and termination asthma scores stratified by severity at BiPAP

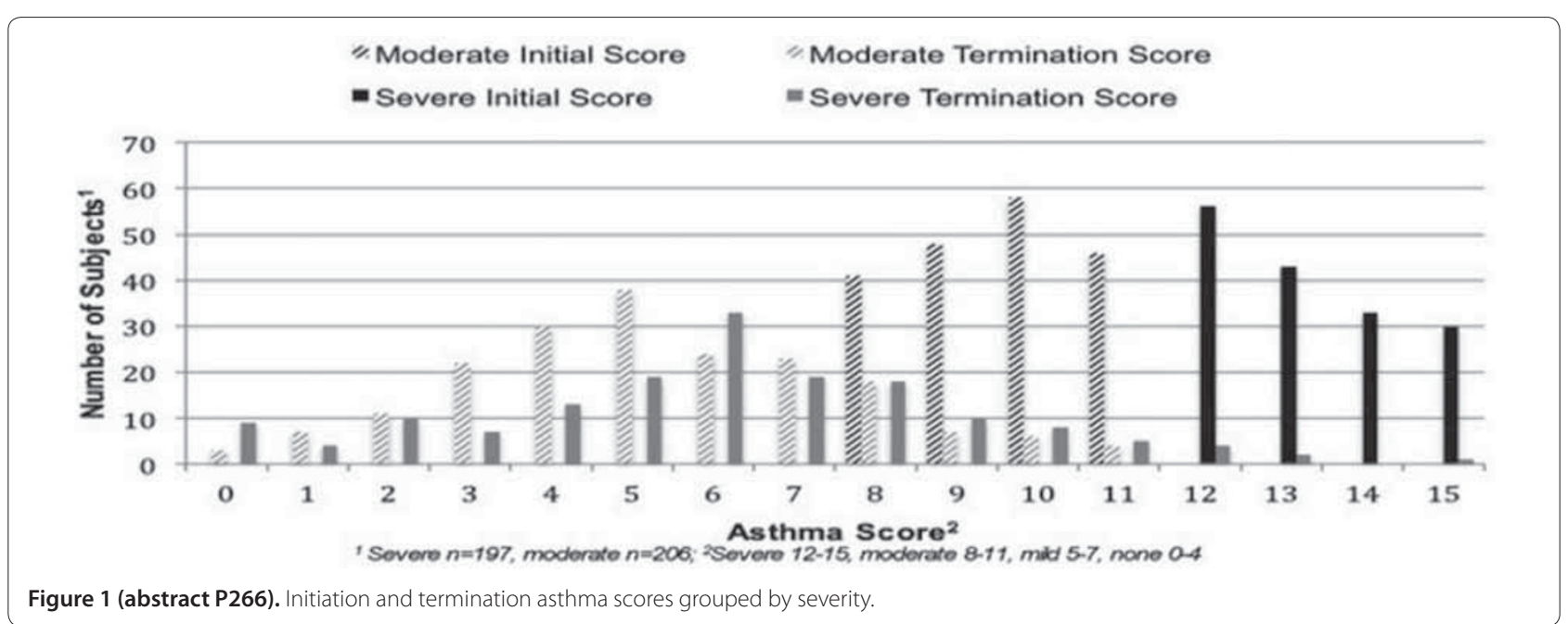


Table 2 (abstract P266). Mean settings by age: moderate group

\begin{tabular}{lcc}
\hline Age (years) & IPAP & EPAP \\
\hline$<3(n=22)$ & $12.1 \pm$ SD 2.1 & $6.4 \pm$ SD 1.3 \\
3 to $4(n=48)$ & $12.1 \pm$ SD 1.6 & $6.4 \pm$ SD 1.2 \\
5 to $6(n=51)$ & $13.0 \pm$ SD 2.3 & $6.6 \pm$ SD 1.3 \\
7 to $8(n=24)$ & $13.9 \pm$ SD 2.1 & $7.2 \pm$ SD 1.2 \\
9 to $10(n=28)$ & $13.9 \pm$ SD 3.2 & $7.4 \pm$ SD 2.0 \\
11 to $12(n=25)$ & $13.8 \pm$ SD 1.8 & $7.0 \pm$ SD 1.3 \\
13 to $14(n=10)$ & $13.8 \pm$ SD 3.2 & $7.4 \pm$ SD 1.4 \\
15 to $17(n=8)$ & $17.0 \pm$ SD 2.4 & $9.5 \pm$ SD 0.9 \\
\hline
\end{tabular}

Table 3 (abstract P266). Mean settings by age: severe group

\begin{tabular}{lcc}
\hline Age (years) & IPAP & EPAP \\
\hline$<3(n=21)$ & $12.7 \pm$ SD 2.0 & $6.8 \pm$ SD 1.3 \\
3 to $4(n=50)$ & $13.3 \pm$ SD 2.8 & $6.8 \pm$ SD 1.4 \\
5 to $6(n=26)$ & $13.9 \pm$ SD 2.2 & $7.3 \pm$ SD 1.3 \\
7 to $8(n=29)$ & $15.0 \pm$ SD 2.4 & $7.7 \pm$ SD 1.5 \\
9 to $10(n=26)$ & $15.7 \pm$ SD 3.5 & $8.0 \pm$ SD 1.8 \\
11 to $12(n=22)$ & $14.5 \pm$ SD 3.2 & $7.6 \pm$ SD 1.6 \\
13 to $14(n=9)$ & $15.3 \pm$ SD 3.2 & $8.0 \pm$ SD 1.7 \\
15 to $17(n=14)$ & $17.5 \pm$ SD 3.0 & $9.5 \pm$ SD 1.8 \\
\hline
\end{tabular}

placement. More of the severe group was admitted to the PICU and had overall longer hospitalizations $(P<0.06)$. None experienced severe complications.

Conclusion BiPAP is a beneficial therapy for children presenting to the PED with severe asthma exacerbations. It may have utility for less severe asthma exacerbations.

P267

Report of an outbreak of toxicity from a novel drug of abuse in the UK: Eric-3

C Morden, S Haig, C Kelly

Great Western Hospital, Swindon, UK

Critical Care 2013, 17(Suppl 2):P267 (doi: 10.1186/cc12205)

Introduction We present a case series of toxicity due to a novel substance in the UK: Eric-3. Novel drugs of abuse are becoming more common throughout the world, and they represent particular difficulties in their acute management. A recent report from the European Monitoring Centre for Drugs and Drug Addiction and Europol has reported 49 new psychoactive substances reported via its early warning system.

Methods This was a retrospective case-note review over a 6-month period. Patients were included if their presentation was due to recent ingestion of Eric-3. Physiological data, symptoms, outcome and destination of the patient from the emergency department were collected. Postmortem toxicological analysis was obtained for one of the two patients who died.

Results Forty-one attendances were identified from 18 patients. Two patients died and five were admitted to the ICU. Heart rate and temperature on arrival tended to be above normal (mean heart rate was $112 \mathrm{bpm}$, with an SD of 18 ; mean temperature was $37.45^{\circ} \mathrm{C}$ with an SD of 0.95$)$. In total, $63.4 \%$ of attendances included agitation and $34.1 \%$ choreiform movements. a-Methyltryptamine and 3-/4-fluoroephedrine were found in the blood of one of the patients who died.

Conclusion In this outbreak in the UK, Eric-3 gave symptoms similar to other stimulants known as legal highs, including death. It may have been a novel substance, 3-/4-fluoroephedrine. This underlines the need for prospective data collection and early national and international information sharing.

\section{References}

1. EMCDDA: Europol Annual Report on the Implementation of Council Decision 2005/387/JHA. Lisbon: EMCDDA/Europol; 2011.

2. Brandt SD, et al:: Drug Test Anal 2010, 2:377-382.

3. Sikk K, et al:: Acta Neurol Scand 2007, 115:385-389.

4. Stepens A, et al:: N Engl J Med 2008, 358:1009-1017.

5. Murphree HB, et al: Clin Pharmacol Ther 1961, 2:722-726.

P268

Abstract withdrawn

P269

Thallium group poisoning incident in Japan 2011

Y Namba, R Suzuki, J Sasaki, M Takayasu, K Watanabe, D Kenji, M Hayashi,

Y Kitamura, M Kawamo, H Masaki, E Kyuuno, M Hayashi, M Yamaguchi,

A Maeda

Showa University Fujigaoka Hospital, Yokohama, Japan

Critical Care 2013, 17(Suppl 2):P269 (doi: 10.1186/cc12207)

Introduction Thallium is an odorless, tasteless, heavy metal that has been often used for intentional poisonings. In severe patients, thallium poisoning produces neuromuscular symptoms such as extreme pain and muscle weakness.

Methods Five case reports.

Results All patients worked at a pharmaceutical factory. They joined a tea party held at their workplace at the end of April 2011. The five patients drank tea from a teapot someone had put thallium in. A few days later, they complained of femoral numbness and pain caused by pressure. About a week later, three of five patients had profound hair loss. Three weeks after the party, they came to our ER. We thought that their symptoms might be caused by some chemicals. We searched the keywords: 'lower extremity pain', 'hair loss' and 'poison' in the Internet. As a result, thallium, mercury, lead, and so forth, were suspicious metals. In those metals, thallium was most likely because it was used in their factory. We immediately examined the blood concentration of several metals and ordered iron(III)hexacyanoferrate(II) that is known as the antidote for thallium poisoning. Only thallium was positive in the blood metal concentration test. Three patients consented to oral administration of an antidote. Two patients rejected administration because their symptoms were mild and getting better. All symptoms of all patients gradually disappeared by August. We also followed up the course of blood concentration of thallium. The concentration in three patients who took the antidote was reduced more rapidly than the two patients who did not take it.

Conclusion All patients recovered without any sequelae. Three patients' hair started to grow 3 months from ingestion of thallium, and after half a year their hair was restored to their former state. We had difficulty ordering iron(III)hexacyanoferrate(II) because this is also known as an antidote for cesium. On 11 March 2011 a megathrust earthquake and tsunami hit Japan and the giant tsunami gave rise to an accident at a nuclear power generation plant. Because the rumor of radioactive substances including cesium might be spread was the talk in the city near the nuclear power plant, the authorities put the antidote under heavy supervision. We could also collect the data for the course of thallium concentration. Thallium concentration of the patients who had an antidote was reduced more rapidly but these patients had a loose stool, thought to be a side effect of this antidote.

\section{Reference}

1. Centers for Disease Control and Prevention: Thallium poisoning from eating contaminated cake - Iraq, 2008. MMWR Morb Mortal Wkly Rep 2008, 57:1015-1018

P270

Heart rate variability in children with tricyclic antidepressant intoxication

EC Dinleyici, Z Kilic, S Sahin, R Tutuncu-Toker, M Eren, ZA Yargic, P Kosger, B Ucar

Eskisehir Osmangazi University Faculty of Medicine, Eskisehir, Turkey

Critical Care 2013, 17(Suppl 2):P270 (doi: 10.1186/cc12208)

Introduction Tricyclic antidepressant (TCA) intoxication is among the most common causes of poisonings in our country. The TCA group of 
drugs affects the central nervous and cardiovascular systems, resulting in severe arrhythmia and death. Heart rate variability (HRV) analysis is a non-invasive assessment method that allows evaluation of the cardiac autonomic (sympathetic and parasympathetic) activity. The aim of this study was to evaluate HRV in children requiring ICU stay due to TCA poisoning.

Methods Twenty children with isolated TCA poisoning aged between 3 and 16 years who were hospitalized in the pediatric ICU, between March 2009 and July 2010, and 20 healthy children as a control group were enrolled. Clinical and electrocardiographic (ECG) findings were noted in the TCA poisoning group. In both groups, 24-hour time domain HRV analysis (SDNN, SDANN, SDNNi, RMSDD, NN50, and pNN50) was performed. We also recorded frequency domain analysis results at the first 5 minutes and the last 5 minutes of the 24-hour record (VLF, nLF, nHF, LF/HF ratio).

Results The average level of TCA in the study group was 1,116 \pm 635 and TCA levels were positively correlated with the duration of QRS interval $(P<0.01)$. In time-domain nonspectral evaluation, SDNN $(P<0.001)$, SDNN $(P<0.05)$, RMSDD $(P<0.01)$, and pNN50 $(P<0.01)$ were found significantly lower in the TCA intoxication group compared with the control group, while NN50 $(P<0.01)$ was significantly higher in value. The spectral analysis (frequency domain) of data recorded at first 5 minutes after intensive care admission showed that the values of the $\mathrm{nLF}(P<0.05)$ and $\mathrm{LF} / \mathrm{HF}$ ratio $(P=0.001)$ were significantly higher in the TCA intoxication group than the controls, while nHF $(P=0.001)$ values were significantly lower. The frequency domain spectral analysis of data recorded at the last 5 minutes showed a lower nHF $(P=0.001)$ in the TCA intoxication group than the controls, and the LF/HF ratio was significantly higher $(P<0.05)$ in the intoxication group. SDNN $(P<0.001)$, RMSDD $(P<0.01)$, SDNNi $(P<0.01)$, and pNN50 $(P<0.01)$ levels were higher in patients with positive ECG findings than those without positive ECG findings. The LF/HF ratio was higher in seven children with seizures $(P<0.001)$.

Conclusion Existing findings give us an idea about HRV's value to determine arrhythmia and predict convulsion risk in TCA poisonings. HRV can be used as a non-invasive method in determining the treatment and prognosis of TCA poisoning.

\section{P271}

Diagnosing anisakiasis in the emergency department

TTakabayashi, S Ishimatsu, N Otani, T Mochizuki, R Miyamichi

St. Luke's International Hospital, Tokyo, Japan

Critical Care 2013, 17(Suppl 2):P271 (doi: 10.1186/cc12209)

Introduction Humans can be incidentally parasitized by third-stage Anisakis larvae after ingestion of raw or undercooked seafood. Although the clinical symptom of anisakiasis is abdominal pain, the clinical finding is nonspecific and may be misdiagnosed as appendicitis, ileus, and so forth. Every year, $>40,000$ patients visit our ED and our hospital near the Tsukiji market (famous seafood market in Tokyo). We often observed cases of anisakiasis in patients who visited the hospital with abdominal pain as the chief complaint. Thus, we researched to determine factors useful to diagnose anisakiasis.

Methods We retrospectively reviewed data of 83 patients (58 men, 25 women) diagnosed with anisakiasis in our ED (22 July 2003 to 22 July 2012) and examined the usefulness of clinical history, blood test, diagnostic imaging, and so forth, for anisakiasis diagnosis. Diagnosis was made after (A) endoscopically proven Anisakis polypide and (B) a hematologically positive Anisakis antibody $(\lg G, \lg A)$ and CT diagnostic imaging.

Results Of the 83 patients, 39 had gastric anisakiasis and 44 had small intestinal anisakiasis. All gastric and small intestinal anisakiasis patients were diagnosed by methods $A$ and $B$, respectively. A blood test was unable to show the specific inflammatory reaction. A history of raw or undercooked seafood ingestion was noted in $95.2 \%$ (79/83) of the patients. This was observed in $100 \%$ (39/39) of the gastric anisakiasis patients and $91 \%$ (40/44) of the small intestinal anisakiasis patients. With regard to the development of symptoms, symptoms for gastric anisakiasis developed within 48 hours and reached a peak in less than 6 hours, whereas the symptoms for small intestinal anisakiasis reached a peak in 48 hours and persisted for a maximum of 5 days. Diagnostic $\mathrm{CT}$ imaging revealed that all the patients with gastric anisakiasis demonstrated edematous wall thickening of gastric mucosa, and ascites was observed in $44.4 \%(12 / 27)$ of these patients. The patients with small intestinal anisakiasis demonstrated limited edematous wall thickening of the intestinal tract, and ascites was observed in $90.9 \%$ (40/44) of these patients. Furthermore, phlegmon of mesentery fat was observed in $72.7 \%$ (32/44) of the small intestinal anisakiasis patients.

Conclusion When the cause of abdominal pain cannot be determined by initial assessment, anisakiasis should be considered, especially if the patient has a history of raw or undercooked seafood ingestion. In the $E D$, certain methods of diagnosis are evaluation of the time to develop symptoms and CT imaging, and a history of raw or undercooked seafood ingestion should be considered in the diagnosis.

References

1. Valle et al:: J Med Case Rep 2012, 6:114.

2. David Hwang et al:: Chonnam Med J 2012, 48:73-75.

\section{P272}

Implementation of a sepsis protocol in a community hospital using a Telemedicine Program

M Steinman', CA Abreu Filho', A Andrade ${ }^{2}$, R Cal', N Akamine1, J Teixeira², E Silva', A Kanamura', M Cenderoglo', C Lottenberg

'Hospital Israelita Albert Einstein, São Paulo, Brazil;'²Hospital Municipal Dr. Moysés Deutsch, São Paulo, Brazil

Critical Care 2013, 17(Suppl 2):P272 (doi: 10.1186/cc12210)

Introduction Sepsis has high incidence around the world, approximately 400,000 new cases occur annually in Brazil. In developing countries it is still an important cause of death due to poor adherence to best practice medicine protocols, mainly in community hospitals. The Brazilian mortality rate of septic shock is around $60 \%$. The aim of this study is to describe the first Brazilian initiative of implementation of a Telemedicine (TM) Project for therapeutic support of septic patients in a community hospital in São Paulo, Brazil.

Methods Since May 2012, a TM Program has been implemented at two hospitals in São Paulo - Hospital Municipal Dr. Moysés Deutsch (HMMD), a public, secondary hospital, and Hospital Israelita Albert Einstein (HIAE), a tertiary private philanthropic entity - due to a partnership with Brazilian Health Ministry. A TM Central Command was located at HIAE with Endpoint $97 \mathrm{MXP} \mathrm{Cisco}^{\oplus}$ Solution and a mobile Intern MXP ISDN/IP Cisco ${ }^{\circledR}$ for the remote hospital (HMMD) via dedicated $\mathrm{GB} / \mathrm{sec}$ connection. Imaging examinations were evaluated using PACS technology. At HMMD, the Sepsis Protocol, based on the Surviving Sepsis Campaign, was started for every recruited patient admitted to the emergency department (ED) or the ICU, and assessed by the Central Command through TM with an experienced consultant. Results Over a 6-month period, 33 patients with diagnosis of sepsis (including severe sepsis and septic shock) admitted to HMMD were evaluated by skilled doctors of HIAE via TM, and the Surviving Sepsis Campaign protocol was instituted. In total, $87.8 \%$ of the consultations originated from the ICU and $12.2 \%$ from the ED; 16 patients were male (48.4\%), mean age was 44.2 years old. TM improved diagnosis in $12.1 \%$ and influenced clinical management in $87.9 \%$ of the cases; in 11 patients (33.3\%) TM consultation led to antibiotic change. Hospital mortality rate was $33.3 \%$.

Conclusion The implementation of the TM Project at a community hospital has a major impact in the elCU and eED Sepsis Management Program, and improved compliance with recommended care bundles. Reference

1. Beale R, et al:: Promoting Global Research Excellence in Severe Sepsis (PROGRESS): lessons from an international sepsis registry. Infection 2009, 37:222-232

\section{P273}

Initial Brazilian experience of Telestroke for thrombolysis in a community hospital

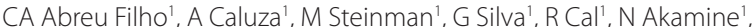

J Teixeira², A Andrade², E Silva', A Kanamura', M Cenderoglo',

C Lottenberg

${ }^{1}$ Hospital Israelita Albert Einstein, São Paulo, Brazil; ${ }^{2}$ Hospital Municipal Dr.

Moysés Deutsch, São Paulo, Brazil

Critical Care 2013, 17(Suppl 2):P273 (doi: 10.1186/cc12211) 
Introduction Stroke is the leading cause of death in Brazil, the number of stroke patients receiving thrombolysis therapy in the developing world is extremely low. Prehospital delay, financial constraints, and lack of infrastructure are the main barriers for thrombolysis therapy in developing countries. In this way, telemedicine (TM) allows knowledge transfer of medical expertise to remote community hospitals, where there is a shortage of specialized doctors. The aim of this study is to describe the first Brazilian initiative of a Telestroke Program for therapeutic support and monitoring cases of acute stroke, and venous thrombolysis of eligible cases in a community hospital in São Paulo, Brazil.

Methods Since May 2012 a TM Program has been implemented at two hospitals in São Paulo - Hospital Municipal Dr. Moysés Deutsch (HMMD), a public, secondary hospital, distant about 60 minutes from the nearest tertiary center, and Hospital Israelita Albert Einstein (HIAE), a tertiary private philanthropic entity - due to a partnership with Brazilian Health Ministry. Patients admitted to the community hospital's emergency department with acute ischemic stroke as possible diagnosis are submitted to brain imaging examinations, and considered for remote consultation. A TM Central Command was located at HIAE with Endpoint $97 \mathrm{MXP} \mathrm{Cisco}^{\circledR}$ Solution and a mobile Intern MXP ISDN/IP Cisco ${ }^{\circledR}$ for the remote hospital (HMMD). Imaging examinations were evaluated using PACS technology. At HIAE the TM Center has skilled doctors, including neurologists, available for consultation 24 hours, 7 days a week. They discussed the neurological cases via TM, and selected stroke patients for intravenous thrombolysis when timely (3 hours symptom onset).

Results HMMD receives an average of 30 cases of stroke monthly, and thrombolysis did not occur before the implementation of the TM Project, because of the lack of neurologists available to conduce the cases. After implementation of the TM Program, six cases of ischemic stroke were thrombolyzed with alteplase; only one case $(16 \%)$ progressed to death from septic shock, and one case (16\%) presented symptomatic intracranial hemorrhage.

Conclusion Thrombolysis in ischemic stroke reduces 30\% the risk of disability and $18 \%$ the mortality rate. This procedure has been only feasible to be done in the community setting because of the implementation of the TM Project, which permits the presence of a real time consultation with a specialized neurological team from a tertiary center.

P274

Analysis of emergency calls achieved in a French emergency

dispatching centre: what resources for which patients

OTilak, J Cuny, N Assez, P Goldstein, E Wiel

Lille University Hospital Center, Lille Cedex, France

Critical Care 2013, 17(Suppl 2):P274 (doi: 10.1186/cc12212)

Introduction Following patients referred by the French emergency dispatching centre (SAMU 15) in different hospital services (emergency or ICU) is not always easy. The purpose of this study was to analyze each call arriving in the emergency dispatching centre, define resources for transportation, the host hospital department, and analyze the shortterm outcome of these patients.

Methods We conducted a prospective, observational study, by collecting all data recorded at the emergency dispatching centre in a continuous 24-hour period. Each call creating a medical file was included. We identified the type of call, various administrative information, the purpose of call, resources triggered, medical advice, hospital department admission, and clinical evolution.

Results A total of 877 patients were included; $67.80 \%$ calling from home, $15.63 \%$ calling from a public place. Firemen ambulances were sent in $38.89 \%$, private ambulance in $24.65 \%$ and an emergency medical ambulance (SMUR) in $11.23 \%$. Simple medical advice without transportation was given in $13.19 \%$ of cases. In total, $22.18 \%$ of patients were entrusted to the family; $2.89 \%$ of patients refused transportation; $69.94 \%$ of triggered firemen ambulances were done by a centre 18 call, without any medical regulation by an emergency physician. A total of $68.59 \%$ patients were referred to the emergency department, $1.2 \%$ in ICU, and $1.8 \%$ in cardiac ICU. In the emergency room, $50.78 \%$ of patients received a simple medical consultation with biological analyses, and then returned home. In total, $25.50 \%$ of patients were hospitalized in a medical or surgical department, and $12.42 \%$ in the short-term hospitalised unit of the emergency department (stay duration $<24$ hours). Some $5.10 \%$ of patients worsened and were oriented in the ICU. A total $3.77 \%$ of patients in a cardiac ICU. In total, $73.84 \%$ of patients had stay duration less than 6 hours in the ED, $24.45 \%$ $<24$ hours. Forty percent of patients supported by firemen and $54 \%$ supported by private ambulance left the hospital after a single medical consultation.

Conclusion Nearly $70 \%$ of patients calling the French emergency medical dispatching centre are sent to hospital. Those transportations are supported for two-thirds of cases by a private ambulance or firemen ambulance. One out of two patients only receive a simple medical consultation in the ED, and go back home. This may concur to the deficiency of using general medicine in town. They prefer using emergency services for free. Only one patient out of four was hospitalized more than 24 hours.

\section{P275}

Reform of emergency services: immediate effects on cardiac care unit and ICU patient intake

AO Alaspää, T Valpas, V Rautava, A Palomäki

Kanta-Häme Central Hospital, Hämeenlinna, Finland

Critical Care 2013, 17(Suppl 2):P275 (doi: 10.1186/cc12213)

Introduction Early onset effective care in the emergency department (ED) has been reported to have a great influence on the intensive care patients' morbidity and mortality [1]. Little is known about the influence of the reorganisation of the ED on patient intake to the ICUs. The aim of this study was to analyse monthly intake of patients from the ED to the cardiac care unit (CCU) and ICU before and after the reform of emergency services.

Methods In Kanta-Häme Central Hospital, a new ED started on 1 April 2007. Four older emergency rooms were combined into one bigger emergency department and an observation ward was introduced with continuous follow-up of vital signs. This study is a retrospective analysis of the patient intake to the CCU and ICU 1 year before and after the reorganisation. Using as data the Finnish Intensive Care Quality Consortium (Intensium, Finland) database and the cardiac database of the hospital, patient transfer from ED to the ICU and CCU was collected and analysed. Monthly pre/post comparisons were carried out statistically by a nonparametric Wilcoxon signed-rank test.

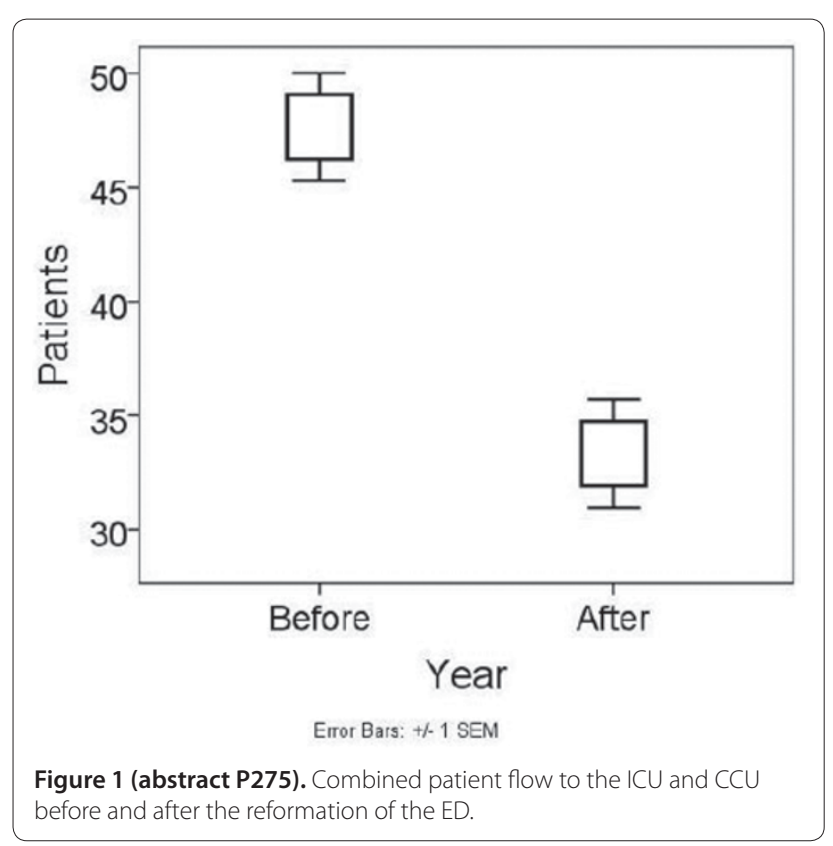


Results The total decrease in monthly patient inflow from ED to the ICU and CCU was $30.1 \%(P=0.003)$; that is, from the mean of $47.7 \pm 8.2$ to $33.3 \pm 8.3$ patients (Figure 1). The $7.1 \%$ decrease in patients taken into the ICU (12.9 \pm 4.1 to $12.0 \pm 4.6$ patients) was not statistically significant. However, the intake from the ED to the ICU decreased by $38.6 \%$ (from $34.8 \pm 6.5$ to $21.3 \pm 5.1$ patients) $(P=0.002)$.

Conclusion According to our results the reform of the ED may have a significant role in the total inflow of patients to the CCU and ICU.

\section{Reference}

1. Rivers E, et al:: NEngl J Med 2001, 345:1368-1377.

\section{P276}

Overtriage and undertriage in a prehospital system over 7 years L Carenzo, F Barra, A Messina, D Colombo, T Fontana, F Della Corte Universitè del Piemonte Orientale A. Avogadro, Novara, Italy Critical Care 2013, 17(Suppl 2):P276 (doi: 10.1186/cc12214)

Introduction The Novara 118 emergency medical system (EMS) dispatch center manages medical emergency calls coming from a region that spreads out over $1,400 \mathrm{~km}^{2}$ and includes 88 towns and a population of 385,000 people; inhabitant density is 275 inhabitants $/ \mathrm{km}^{2}$.

Methods Data collection from 1 January 2005 to 31 December 2011 was obtained (EMS software SaveOnLine ${ }^{\circledR}$ Suite 4; REGOLA s.r.l., Turin Italy). Triage used was the Medical Priority Dispatch System and severity was defined by color codes.

Results During the study period the Novara 118 dispatch center managed a total of 122,384 EMS interventions. Median (interquartile) overestimation (regardless of severity) was $10.0 \%$ (2.4 to $14.1 \%$ ) while median underestimation was $1.5 \%$ (0.8 to $2.5 \%)$. See Tables 1 and 2 .

Table 1 (abstract P276). Overtriage (\%)

\begin{tabular}{lccccccc}
\hline & \multicolumn{7}{c}{ Overtriage } \\
\cline { 2 - 8 } & $\mathbf{2 0 0 5}$ & $\mathbf{2 0 0 6}$ & $\mathbf{2 0 0 7}$ & $\mathbf{2 0 0 8}$ & $\mathbf{2 0 0 9}$ & $\mathbf{2 0 1 0}$ & $\mathbf{2 0 1 1}$ \\
\hline Other & 3.03 & 2.47 & 5.30 & 4.95 & 4.06 & 4.58 & 4.17 \\
Cardiac & 13.23 & 15.30 & 27.06 & 23.72 & 19.28 & 19.17 & 20.14 \\
Drunk & 0.00 & 0.00 & 0.00 & 0.00 & 0.00 & 0.00 & 0.00 \\
Poisoning & 10.19 & 18.92 & 17.91 & 8.47 & 8.85 & 15.20 & 11.81 \\
Oncological & 0.00 & 2.78 & 1.05 & 1.03 & 3.03 & 2.47 & 0.00 \\
Neurologic & 9.97 & 10.05 & 15.59 & 15.18 & 14.57 & 13.32 & 13.32 \\
Not identified & 13.03 & 13.15 & 11.84 & 11.07 & 10.33 & 11.79 & 7.23 \\
Mental & 1.30 & 0.41 & 2.09 & 2.34 & 2.30 & 1.27 & 2.35 \\
Respiratory & 10.67 & 10.89 & 22.09 & 18.63 & 16.29 & 18.23 & 14.12 \\
Trauma & 12.97 & 12.68 & 10.42 & 8.59 & 6.78 & 7.74 & 7.56 \\
\hline
\end{tabular}

Table 2 (abstract P276). Undertriage (\%)

\begin{tabular}{lccccccc}
\hline & \multicolumn{7}{c}{ Undertriage } \\
\cline { 2 - 8 } & $\mathbf{2 0 0 5}$ & $\mathbf{2 0 0 6}$ & $\mathbf{2 0 0 7}$ & $\mathbf{2 0 0 8}$ & $\mathbf{2 0 0 9}$ & $\mathbf{2 0 1 0}$ & $\mathbf{2 0 1 1}$ \\
\hline Other & 1.01 & 0.77 & 2.87 & 1.66 & 1.44 & 1.48 & 1.31 \\
Cardiac & 2.16 & 1.44 & 1.64 & 1.60 & 2.15 & 1.60 & 1.14 \\
Drunk & 0.00 & 1.89 & 0.00 & 0.00 & 0.00 & 0.00 & 0.00 \\
Poisoning & 8.33 & 0.00 & 0.00 & 1.69 & 0.00 & 1.60 & 0.00 \\
Oncological & 95.74 & 0.56 & 0.00 & 1.03 & 3.03 & 2.47 & 0.00 \\
Neurologic & 2.42 & 2.32 & 2.96 & 3.67 & 3.05 & 1.40 & 2.46 \\
Not identified & 1.72 & 2.13 & 1.87 & 2.20 & 2.04 & 2.74 & 3.50 \\
Mental & 1.74 & 0.83 & 1.05 & 1.17 & 0.00 & 0.42 & 0.39 \\
Respiratory & 2.30 & 4.16 & 3.76 & 3.75 & 2.57 & 2.72 & 2.46 \\
Trauma & 1.07 & 0.92 & 1.50 & 1.41 & 1.36 & 1.31 & 1.46 \\
\hline
\end{tabular}

Conclusion Overall there were no statistical differences between the observation years either for overtriage or undertriage. When observed individually, trauma showed the only significant overtriage reduction over time; there were no individual modifications in undertriage over time.

Reference

1. Neely KW, Eldurkar JA, Drake ME: Acad Emerg Med 2000, 7:174-180.

\section{P277}

Lorca's hospital evacuation and medical attention by the emergency department

L Escobar Alvaro', J Jimenez Gonzalez', A Pelaez Ballesta',

A Corbatón Anchuelo ${ }^{2}$

${ }^{1}$ Hospital Rafael Mendez, Lorca, Spain; ${ }^{2 H}$ ospital Clínico San Carlos, Madrid, Spain

Critical Care 2013, 17(Suppl 2):P277 (doi: 10.1186/cc12215)

Introduction On 11 May 2011 two moderate magnitude earthquakes hit the city of Lorca, southeast Spain. They caused 11 deaths, including two pregnant women and their babies, more than 350 injured, and moderate or severe damage to $80 \%$ of the building in the city, including Lorca's public hospital that had to be evacuated.

Methods A descriptive study of the files of Lorca's hospital and the clinical records of patients that attended our service in the 20 hours following the second earthquake on 11 May.

Results A total of 225 patients were relocated. Simultaneously 224 patients were treated by the emergency service, until the evacuation was completed and after then, from 10:00 pm to 3:00 pm the next day, 47 more in a field hospital placed just outside the hospital building. See Figures 1 and 2.

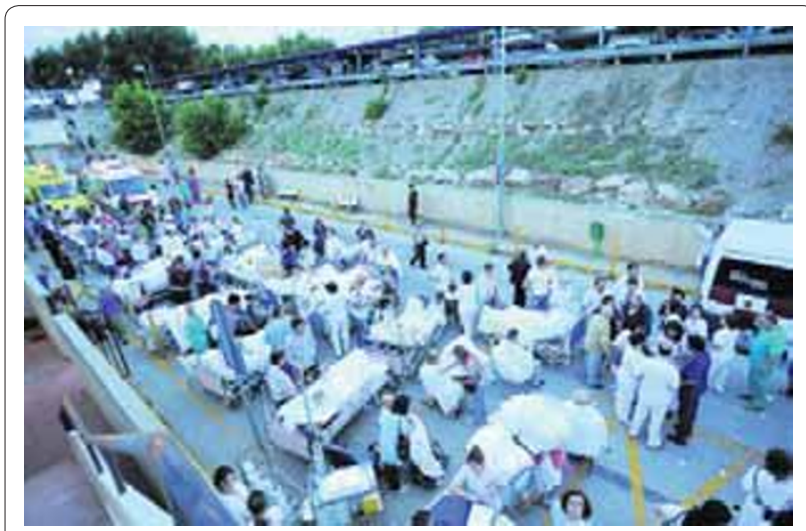

Figure 1 (abstract P277). Hospital evacuation.

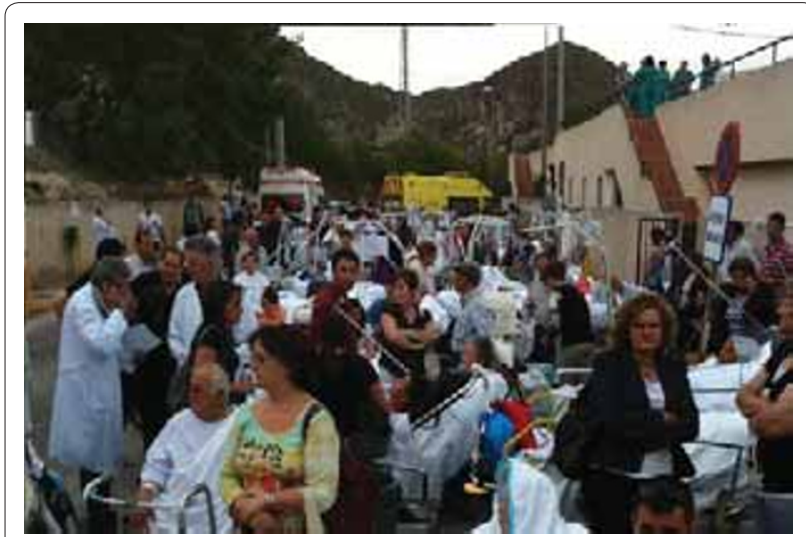

Figure 2 (abstract P277). Emergency service taking care of patients during evacuation. 
Conclusion In less than 3 hours 225 patients were evacuated and 224 were given attention by the emergency service of Lorca's hospital, with the support of personnel from other services of the hospital. The emergency service of the hospital continues to be operative in the building until evacuation is completed and in a field hospital later.

\section{P278}

War injuries and civilian accidents in Afghan children

R Kedzierewicz', P Ramiara², M Puidupin ${ }^{3}$, S Mérat ${ }^{4}$

'CMA des Alpes, Barby, France; ${ }^{2}$ HIA Sainte Anne, Toulon, France;

${ }^{3}$ HIA Desgenettes, Lyon, France: ${ }^{4}$ HIA Begin, Saint Mandé, France

Critical Care 2013, 17(Suppl 2):P278 (doi: 10.1186/cc12216)

Introduction We intended to compare paediatric traumatic injuries due to war and others related to civilian accidents (CA).

Methods We conducted an observational epidemiologic retrospective study on paediatric cases ( $<18$ years old) seen at the emergency room of Kabul International Airport NATO role 3 medical treatment facility between 2009 and 2012.

Results During 3 years, 341 children were admitted to the emergency room (ER) with a mean age of 10 years (9.49 to 10.51). Eighty-eight per cent of children (301) were traumatized, $39 \%$ of them (118) due to war injuries (WI) and 61\% (183) due to CA. Forty-three per cent of WI (gunshot wound $34 \%$, explosion $66 \%$ ) but only $19 \%$ of injuries due to CA (falls $24 \%$, transport accidents $56 \%$, burns $9 \%$, penetrating injuries $9 \%$, unknown cause $2 \%$ ) involved polytraumas defined as a New Injury Severity Score (NISS) $>15(P<0.001)$. Patients experiencing a traumatic injury related to war had lower Paediatric Trauma Scores (PTS) than those injured in a CA (PTS $=6.4$ (5.7 to 7.0) vs. PTS $=9.0$ (8.7 to 9.4 ) respectively, $P<0.00001)$, higher ISS (ISS $=16.7(13.8$ to 19.6$)$ vs. ISS $=8.9$ (7.5 to 10.2$)$ respectively, $P<0.00001)$, NISS $(P<0.0001)$, mortality rate predicted by the Trauma Injury Severity Score (TRISS, $P<0.02$ ) or A Severity Characterization of Traumas (ASCOT, $P<0.001$ ). The result is longer overall hospitalization of patients having WI $(P<0.001)$ and $a$ higher number of surgeries $(P<0.02)$. After the ER, $54 \%$ of patients with WI were hospitalized in the ICU ( $86 \%$ of them after surgery) but only $26 \%$ of patients involved in a CA ( $71 \%$ after surgery). As many patients with WI as involved in a CA $(40 \%)$ were admitted to the ward $(89 \%$ of patients with $\mathrm{WI}$ after surgery but only $63 \%$ of patients with injuries due to a (A). Thirty-three per cent of patients involved in a CA returned home and one was transferred, whereas only three patients with WI returned home after being in the ER, three patients were transferred and one died in the operating room. Observed paediatric mortality in our medical treatment facility was $2.9 \%$ (10 children out of 341 ): three children died of WI, three due to a CA and one of septic shock due to a medical cause.

Conclusion War injuries are more prone to cause polytrauma than CA. According to the PTS, ISS, NISS, TRISS and ASCOT, children experiencing WI have higher severity scores and predicted mortality rate than others, stay longer in the hospital and have more surgeries.

\section{P279}

Intensive care and disaster medicine: the role of a compendium Y Haraguchi', Y Tomoyasu', H Nishi', M Hoshino', T Tsubata², M Sakai', E Hoshino'

'Disaster Medicine Compendium Team, Japan, Tokyo, Japan; 'Keiyo Hospital,

Tokyo, Japan

Critical Care 2013, 17(Suppl 2):P279 (doi: 10.1186/cc12217)

Introduction There were several catastrophes in the last decades. The make-up of systematic measuring and life-saving medical systems, including intensive care, is thought to be an urgent and essential issue. Our efforts for establishing a disaster medicine and education system are presented.

Methods Mega-disasters or catastrophes are researched basically on actual medical experience; that is, the 9/11 attack in 2001, Hurricane Katrina in 2005, Indian Ocean earthquake with tsunami in 2004, the Chernobyl incident in 1986 as well as the Higashinihon earthquakes with tsunami, which was followed by the severest degree of nuclear disaster in 2011
Results Our research indicated that disaster medicine should be established systematically or it is necessary to compile a compendium of disaster medicine from a broad perspective or from a bird's-eye and long-term view. The Japanese version was tentatively completed with 22 volumes as of the financial year 2005, of which nearly three-quarters are written in Japanese. Although this worked partly during the aboveshown catastrophe in Japan 2011, several problems are left to be solved; that is, the insufficient operation system of the Japan DMAT or Disaster Medical Assistant Team that seemed to have caused a large number of preventable deaths.

Conclusion The large number of casualties during a major disaster is a global problem, even in the developed countries. When the role of the intensivist is reviewed, many roles were verified to be important; that is, as a leader of a medical team or triage officer as well as a professional in the field of specific intensive care. However, there are many problems to be solved in the fields of disaster medicine. In order to solve the diversification or the various medical problems, it is necessary to compile or systematize a disaster medicine of the world version. The concept of the compendium and our process of trial are shown in relation to intensive care.

\section{P280}

Factors involved in ICU mortality following medical retrieval: identifying differences between ICU survivors and nonsurvivors M Kennedy', P Visser', L Harriss ${ }^{2}$

Ambulance Victoria, Melbourne, Australia: ${ }^{2}$ Monash University, Melbourne, Australia Critical Care 2013, 17(Suppl 2):P280 (doi: 10.1186/cc12218)

Introduction The study aimed to determine factors related to ICU mortality in critically ill patients transferred by Adult Retrieval Victoria (ARV) medical staff. Patients who died in the ICU after interhospital transfer were compared with those who survived.

Methods This is a retrospective cohort study of ARV cases between 1 January 2009 and 30 June 2010. Retrieval data were linked with data from the ANZICS CORE APD (Australia and New Zealand Intensive Care Society Centre for Outcome and Resource Evaluation Adult Patient Database). Victoria Data Linkage (VDL) performed linkage of the data. Data included demographic and clinical data obtained during transfer and data related to outcome measures in the ICU.

Results Of the 601 cases transferred by ARV during the study period, 549 were eligible for linkage to 25,543 ANZICS APD case records for the same period. VDL matched 460 of these cases (83.8\%). Logistic regression analysis revealed the variables associated with mortality were advanced age $(\mathrm{OR}=1.02,95 \% \mathrm{Cl}=1.00$ to $1.04, P=0.02)$, cardiac principal referral problems $(\mathrm{OR}=1.84,95 \% \mathrm{Cl}=1.02$ to $3.32, P=0.04)$, lower mean arterial blood pressure $(\mathrm{OR}=0.97,95 \% \mathrm{Cl}=0.95$ to 0.99 , $P=0.005)$ and tachycardia (OR $=1.02,95 \% \mathrm{Cl}=1.00$ to $1.03, P=0.008)$ on arrival at the destination hospital.

Conclusion Advanced age, lower mean arterial blood pressure and tachycardia towards the completion of transfer were associated with increased ICU mortality in this population. Clinicians should be aware of the additional risk for cardiac patients.

References

1. Hill AD, Vingilis E, Martin CM, Hartford K, Speechley KN: Interhospital transfer of critically ill patients: demographic and outcomes comparison with nontransferred intensive care unit patients. J Crit Care 2007, 22:290-295.

2. Flabouris A, Hart GK, George C: Outcomes of patients admitted to tertiary intensive care units after interhospital transfer: comparison with patients admitted from emergency departments. Crit Care Resusc 2008, 10:97-105.

3. Duke GJ, Green JV: Outcome of critically ill patients undergoing interhospital transfer. Med J Aust 2001, 174:122-125.

P281

Algorithm for the resuscitation of traumatic cardiac arrest patients in a physician-staffed helicopter emergency medical service PB Sherren, C Reid, K Habig, B Burns

Greater Sydney Area HEMS, Sydney, Australia

Critical Care 2013, 17(Suppl 2):P281 (doi: 10.1186/cc12219)

Introduction Survival rates following traumatic cardiac arrest (TCA) are known to be poor but resuscitation is not universally futile [1]. 


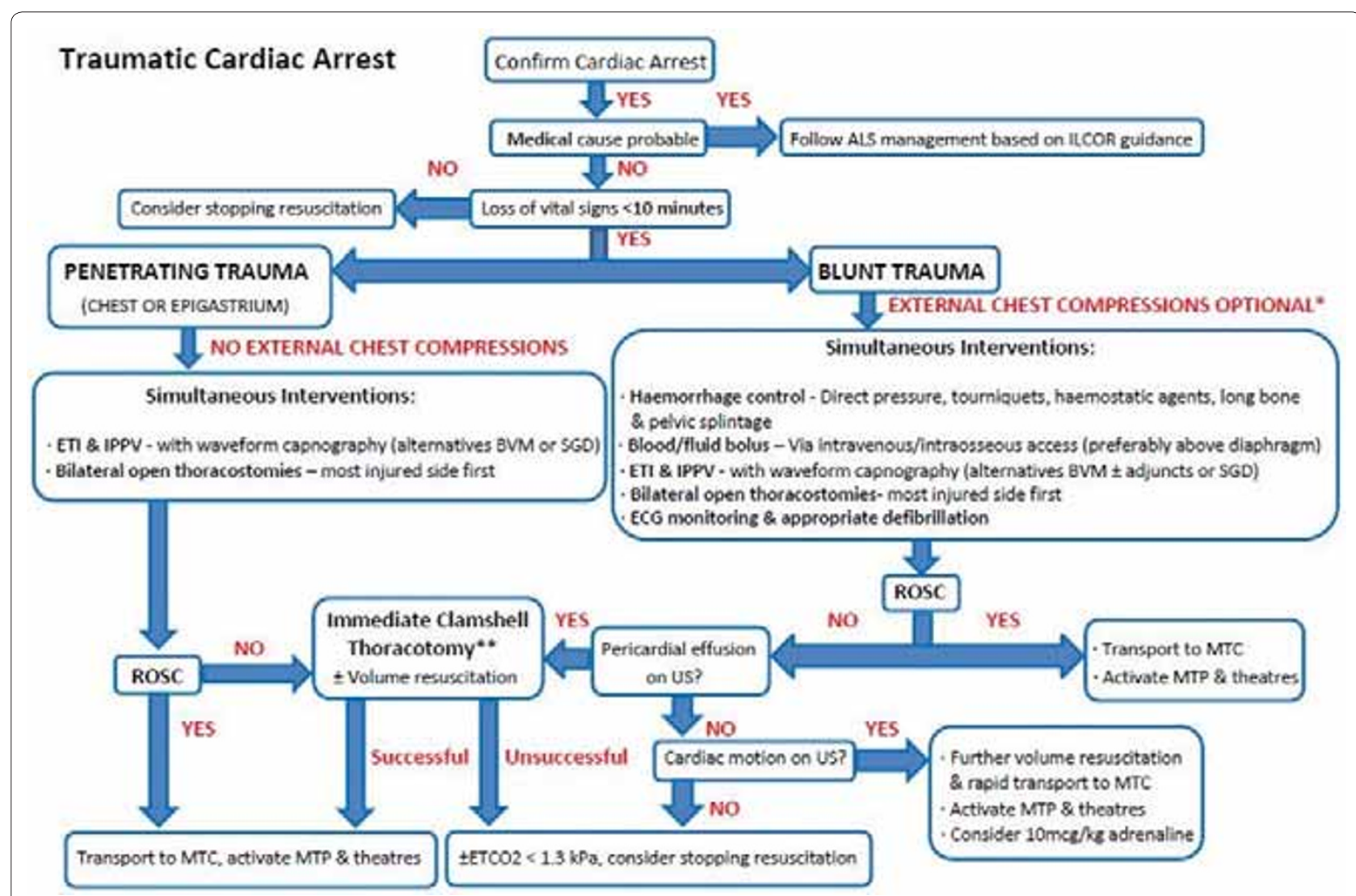

Figure 1 (abstract P281). Traumatic cardiac arrest algorithm.

There are distinct differences in the pathophysiology between medical cardiac arrests and TCA. Traumatic pathologies associated with an improved chance of successful resuscitation include hypoxia, tension pneumothorax and cardiac tamponade [1]. The authors believe a separate algorithm is required for the management of out-of-hospital TCA attended to by a highly trained physician and paramedic team. Methods A suggested algorithm for TCA was developed based on the Greater Sydney Area Helicopter Emergency Medical Service's standard operating procedures and current available evidence.

Results An algorithm for the general management of TCA can be seen in Figure 1. In TCA, priority should be given to catastrophic haemorrhage control (tourniquets, direct pressure, haemostatic agents, pelvic and long bone splintage) and volume resuscitation. Simultaneous oxygenation optimisation should occur with proactive exclusion of tension pneumothoraces with bilateral open thoracostomies. Cardiac ultrasound (US) should be used to help exclude cardiac tamponade and assist in prognostication. The US presence of true cardiac standstill versus low pressure state/pseudo-PEA, and an $\mathrm{ETCO}_{2}<1.3 \mathrm{kPa}$ carries a grave prognosis in TCA. Given the high incidence of hypovolaemia, hypoxia and obstructive shock prior to TCA, the role of adrenaline and chest compressions are limited. Figure 2 shows a suggested algorithm for the management of penetrating TCA requiring prehospital thoracotomy.

Conclusion The suggested algorithm is designed for a highly trained physician-led prehospital team and aims to maximise the number of neurologically intact survivors in out-of-hospital TCA.

\section{Reference}

1. Lockey D, et al: Ann Emerg Med 2006, 48:240-244.
P282

Are physicians required during HEMS winch rescue missions?

PB Sherren, C Hayes-Bradley, C Reid, B Burns, K Habig

Greater Sydney Area HEMS, Sydney, Australia

Critical Care 2013, 17(Suppl 2):P282 (doi: 10.1186/cc12220)

Introduction A winch-capable helicopter emergency medical service (HEMS) offers several advantages over standard rescue operations. Little is known about the benefit of physician winching in addition to a highly trained paramedic. We analysed the mission profiles and interventions performed during rescues involving the winching of a physician in the Greater Sydney Area HEMS (GSA-HEMS).

Methods All winch missions involving a physician from August 2009 to January 2012 were identified from the prospectively completed GSA-HEMS electronic database. A structured case-sheet review for a predetermined list of demographic data and physician-only interventions (POI) was conducted.

Results We identified 130 missions involving the winching of a physician, of which 120 case sheets were available for analysis. The majority of patients were traumatically injured $(90 \%)$ and male $(85 \%)$ with a median age of 37 years. Seven patients were pronounced life extinct on the scene. A total of $63 \mathrm{POI}$ were performed on 48 patients. Administration of advanced analgesia was the most common POI making up $68.3 \%$ of interventions. Patients with abnormal RTSc ${ }^{2}$ scores were more likely to receive a POI when compared with those with normal $\operatorname{RTSC}^{2}(P=0.03)$. The performance of POI had no effect on median scene times ( 45 vs. 43 minutes; $P=0.51$ ). See Tables 1 and 2.

Conclusion Our high POI rate of $40 \%$ coupled with long rescue times and the occasional severe injuries supports the argument for winching doctors. Not doing so would deny a significant proportion of patients time-critical interventions, advanced analgesia and procedural sedation. 


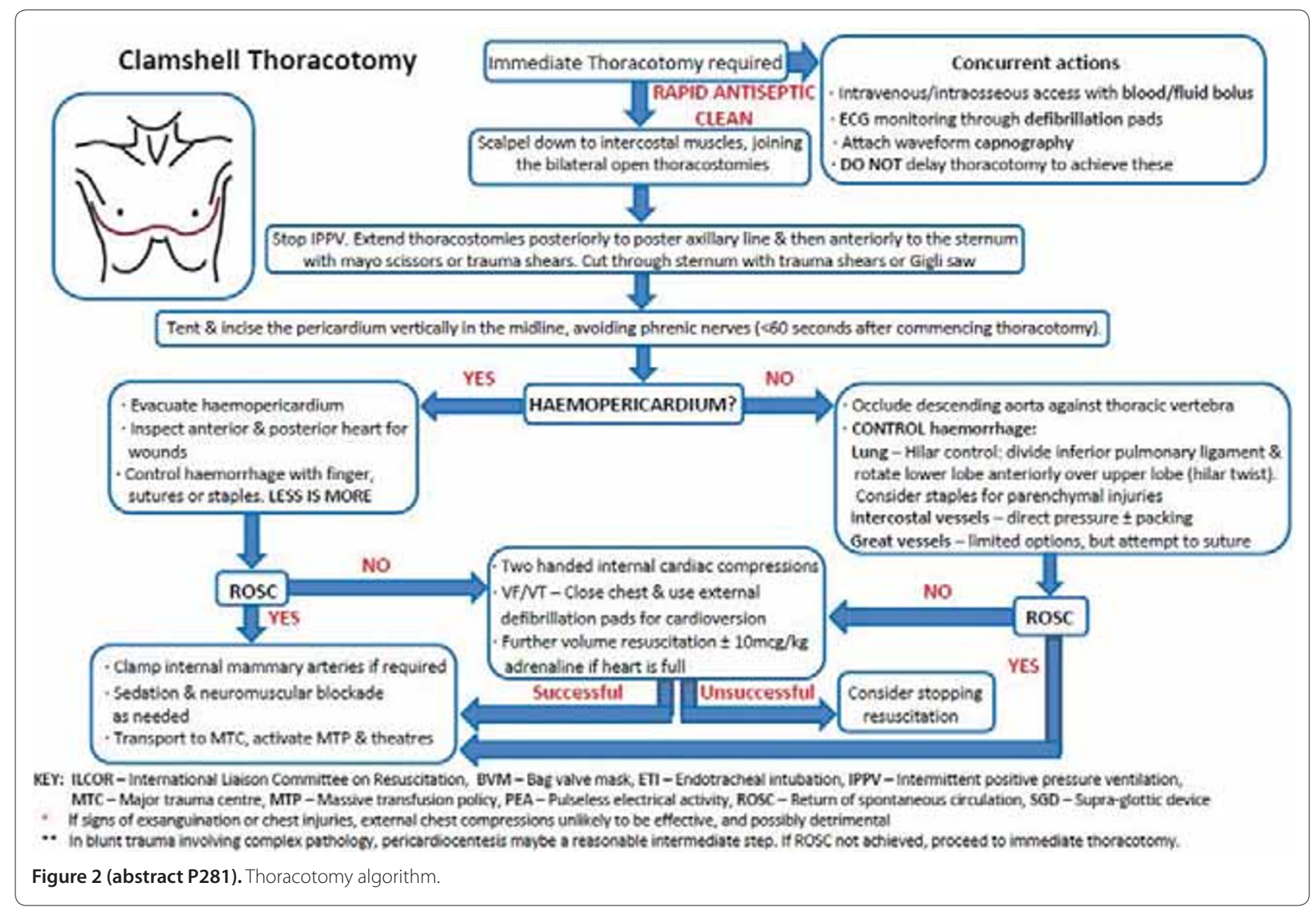

Table 1 (abstract P282). Demographic data, timings and Coded Revised Trauma Score $\left(\mathrm{RTSC}^{2}\right)$

\begin{tabular}{lc}
\hline Male & Total $(\boldsymbol{n}=\mathbf{1 2 0})$ \\
Median (IQR) age (years) & $102(85)$ \\
Received a physician-only intervention & $37(26$ to 53$)$ \\
Pronounced life extinct on arrival & $48(40)$ \\
RTSc ${ }^{2}$ & $7(5.8)$ \\
7.8408 & \\
7.0001 to 7.8407 & $104(86.7)$ \\
6.0001 to 7.0000 & $2(1.7)$ \\
5.0001 to 6.0000 & $4(3.3)$ \\
4.0001 to 5.0000 & $4(3.3)$ \\
$<4.0001$ & $0(0)$ \\
\hline
\end{tabular}

\section{P283}

Improving maintenance of critical care land and aeromedical

transfer equipment

D Ashton-Cleary, N Boyd

Royal Cornwall Hospital, Truro, UK

Critical Care 2013, 17(Suppl 2):P283 (doi: 10.1186/cc12221)

Introduction The aim was to assess the content and state of repair of equipment carried for transfer of critical care patients to other hospitals. By chance, several items of date-expired stock were identified in the
Table 2 (abstract P282). Interventions performed

\begin{tabular}{lc}
\hline Physician-only intervention & Number of interventions $(\boldsymbol{n}=\mathbf{6 3})$ \\
\hline Analgesia/procedural sedation & $42(66.7)$ \\
Intravenous ketamine & $1(1.6)$ \\
Intravenous fentanyl & $1(1.6)$ \\
Fascia iliaca compartment block & \\
Airway management & $4(6.3)$ \\
Rapid sequence induction and intubation & $1(1.6)$ \\
Surgical airway & \\
Circulatory support & $1(1.6)$ \\
Adult intraosseous access & $2(3.2)$ \\
Blood transfusion & $6(9.5)$ \\
Orthopaedic manipulation of joint/limb & $1(1.6)$ \\
Thoracostomy & $1(1.6)$ \\
Diagnostic ultrasound & $3(4.8)$ \\
Hypertonic saline administration &
\end{tabular}

Data presented as $n$ (\%).

transfer kit whilst moving a patient to a tertiary centre. This raised the possibility of a more extensive problem with the equipment bags. Due to the geographical location of our district general hospital we undertake around 70 transfers of critical care patients to other hospitals per year ( $16 \%$ by air) and it is clearly important that our equipment is well maintained for these journeys. 
Methods We maintain two identical sets of equipment (syringes, fluid, airway management items, and so forth) and drug bags to take on transfers; one equipment and one drug bag taken on each trip. The contents of all four bags were checked and itemised. By careful consideration of the aims of the bags (to provide emergency equipment and drugs for managing one patient during an en-route emergency) a new inventory was devised. Excess items were removed to lighten the bags and improve accessibility to the essential items. Expired stock was removed. A daily checking procedure and tamper-proof seals on the bags were instigated and the bags were reassessed 12 months later.

Results A total of $13.9 \%$ of drug items and $29.2 \%$ of equipment items had expired or would do so within 30 days of the initial assessment. The combined weight of one equipment and one drug bag was reduced from 14 to $9 \mathrm{~kg}$ (36\% reduction) by introducing the new inventory. At reassessment in November 2012, only 10 items of equipment (3.2\%) were expired or near to expiry and there were no expired drug items ( $4.1 \%$ near to expiry). In total, $0.3 \mathrm{~kg}$ ( 26 small items) of extraneous equipment had been added through over-restocking and was removed. Conclusion These bags are designed for a clinician to manage a patient when an emergency arises during transfer of a critical care patient. By the introduction of simple measures, the risks posed by expired items or cluttered equipment bags have almost been eradicated. Significant weight savings have been made; this offers improved ergonomics for staff and is also an important consideration for aeromedical operations. Our department was surprised to discover the extent of decline of our equipment and it may be that other departments would find themselves in a similar position. The anaesthetic registrars who routinely escort the transfer patients have a vested interest to maintain this equipment and this has secured their buy-in to the new checking procedure with clear results.

P284

Safety standards for intrahospital transfer of critical care patients

D Ashton-Cleary

Royal Cornwall Hospital, Truro, UK

Critical Care 2013, 17(Suppl 2):P284 (doi: 10.1186/cc12222)

Introduction The aim was to assess care of patients during intrahospital transfer. The UK Royal College of Anaesthetists has defined auditable standards for the care of patients and the training of escorting medical and nursing staff in this context [1].

Methods Patients in a 27-bed combined general and neurosurgical critical care unit were studied in January 2011 and May 2012. Patients undergoing radiology department imaging or intervention were identified from the electronic imaging library. Records of these transfers were sought in the critical care electronic notes and the standards of documentation graded on a five-point scale (very good, good, average, minimal, absent). Documentation of the grade and training of escorting staff was also sought. Between the two study periods, a transfer safety checklist was introduced.

Results A total of $20.9 \%$ of 143 patients underwent one or more transfers in January 2011 (40 transfers). In May 2012, 26.4\% of 151 patients underwent 57 transfers. In the first period, documentation was graded as minimal (limited to a statement that the patient had left the critical care unit) or absent in $77.5 \%$ of transfers. In the $62.5 \%$ of patients transferred whilst on invasive ventilation, $88.0 \%$ had no documentation by the doctor and in $84.0 \%$ it was not known which doctor had escorted the patient. There was only slight improvement in the second period $71.9 \%$ minimal or absent documentation, $80.0 \%$ no documentation by the doctor, $72.0 \%$ not known which doctor escorted). In the documentation available, six severe complications were noted during the second period (including episodes of severe bradycardia, hypotension and pupil dilatation).

Conclusion On average our unit conducts nearly two critical care transfers each day. Severe complications seem to complicate at least $10 \%$ of these, stressing the risk, need for good care and ongoing training. The intervention made in this audit had little impact on the standard of documentation. However, it has raised the issue within the consciousness of the staff. It is important to identify interventions that have failed to reach a gold standard to provide the impetus to seek other solutions. As a result of this study, the author has devised new hospital protocols and specific training courses to improve standards of transfer medicine locally. The study also identified our portable head CT scanner to have the potential to reduce transfers by $52 \%$ and so this has been strongly promoted.

Reference

1. Morgan G: Critical care services. In Raising the Standard: A Compendium of Audit Recipes. 2nd edition. London: Royal College of Anaesthetists; 2006.

P285

Prehospital hyperoxemia does not influence the functional neurological outcome in polytraumatized patients with traumatic head injury

V Vujanovic Popovic', T Pelcl', M Spindler', Z Klemenc Ketis², M Strnad ${ }^{1}$ 'ZD dr. Adolfa Drolca Maribor, Slovenia; 'University of Maribor, Slovenia Critical Care 2013, 17(Suppl 2):P285 (doi: 10.1186/cc12223)

Introduction The association between hyperoxemia and neurological outcome in trauma patients is not clear. We examined the association between prehospital hyperoxemia and neurological outcome in polytraumatized patients.

Methods This was a retrospective study of polytraumatized patients with traumatic head injury who were endotracheal intubated and ventilated with supplemental oxygen $(100 \%)$ in the prehospital emergency setting. Arterial partial oxygen pressure $\left(\mathrm{PaO}_{2}\right)$ was measured after arrival at the hospital trauma center. We included patients with initial $\mathrm{PaO}_{2}$ above $160 \mathrm{mmHg}$ (hyperoxemia group). The severity of the trauma was determined upon the admission to the hospital by the Injury Severity Scale (ISS) and the outcome was assessed at the discharge from the hospital using the Glasgow Coma Scale (GCS), Glasgow Outcome Scale (GOS) and Cerebral Performance Categories scale (CPC). Mann-Whitney's test was used for data analysis. Results Sixty patients were involved in the study. Forty-eight (80\%) of them were men and $86.7 \%$ sustained blunt trauma. Hyperoxemia was present in $41.6 \%$ of patients. Initial average ISS was 38, in patients with normoxemia 32.5 and in patients with hyperoxemia 35.4. Discharge GCS, GOS and CPC in the hyperoxemia group compared with the normoxemia group were 9.86 versus $9.33(P=0.503), 2.52$ versus $2.24(P=0.613)$ and 3.10 versus $3.19(P=0.936)$ with the duration of hospitalization of 26.64 days versus 27.72 days $(P=0.984)$.

Conclusion Prehospital hyperoxemia did not influence the functional neurological outcome. One of the reasons for this finding could be the short arrival time to the trauma center where repeated analyses of arterial blood gases were performed. Therefore, correction of fraction of inspired oxygen according to the arterial blood gas analysis shortens the time of hyperoxemia, thus reducing neuronal brain damage.

References

1. Beynon et al: Brain tissue oxygen monitoring and hyperoxic treatment in patients with traumatic brain injury. J Neurotrauma 2012, 29:2109-2123.

2. Brenner et al: Association between early hyperoxia and worse outcomes after traumatic brain injury. Arch Surg 2012, 16:1-5.

3. Davis et al:: Both hypoxemia and extreme hyperoxemia may be detrimental in patients with severe traumatic brain injury. J Neurotrauma 2009, 26:2217-2223.

P286

Innate immune response-mediated late increase in SuPAR in multi-trauma patients

KTimmermans, M Kox, M Vaneker, P Pickkers

Radboud University Nijmegen Medical Center, Nijmegen, the Netherlands Critical Care 2013, 17(Suppl 2):P286 (doi: 10.1186/cc12224)

Introduction The soluble form of urokinase-type plasminogen activator (suPAR) has been identified as a marker for immune activation and is demonstrated to accurately predict outcome in patients with sepsis or infectious diseases. In multi-trauma patients a considerable immunological response also occurs that is related to multiple organ failure and patient outcome. We investigated the kinetics of SUPAR, correlation with the immune response and outcome in multi-trauma patients.

Methods Blood was obtained from adult multi-trauma patients $(n=63)$ on arrival at the emergency room (ER) of the Radboud University 
Nijmegen Medical Centre and days 1, 3, 5, 7, 10 and 14 following trauma. Plasma concentrations of TNFa, IL-6, IL-10, IFNY, IL-8 and MCP- 1 were determined by Luminex, and SuPAR concentrations using ELISA. Clinical data were collected from electronic patient files. Concentrations, areas under the curve (AUC) and regression coefficients were statistically analyzed. Spearman correlation coefficients were calculated and differences between survival/nonsurvival groups were analyzed using unpaired Student $t$ tests.

Results SuPAR values at admission to the ER were higher in nonsurvivors compared with survivors $(n=16$, mean \pm SEM $4.1 \pm 0.6 \mathrm{ng} / \mathrm{ml}$ vs. $n=40$, $3.0 \pm 0.2 \mathrm{ng} / \mathrm{ml}, P=0.03$ ). SuPAR levels increased in time. An increase of suPAR did not predict or precede death, however. SuPAR AUC from ER to day 5 tended to correlate with injury severity score $(r=0.5, P=0.07)$. Plasma cytokines in the ER did not correlate with suPAR measured at the same time (for example, TNFa: $r=0.2, P=0.37$, IL-10: $r=-0.02$, $P=0.91)$, while cytokine concentrations at the ER did correlate with suPAR levels at days 3 (TNFa: $r=0.6, P<0.01, \mathrm{IL}-10: r=0.5, P=0.02$ ) and 5 (TNFa: $r=0.7, P<0.01$ ).

Conclusion Plasma concentrations of SuPAR measured at admission to the ER are associated with overall survival of multi-trauma patients. Furthermore, suPAR concentrations increased during hospital admission, with most pronounced increases found in patients that suffered more serious injury and related to the innate immune response determined in the ER. These results indicate that suPAR is an innate immune response-induced late mediator in multi-trauma patients.

\section{P287}

Single-dose estrogen infusion can amplify brain levels of Sonic hedgehog, a signal protein for neuro stem cells and repair following the indirect brain injury resulting after severe torso burns PE Pepe', JG Wigginton', JW Gatson', J Simpkins², D Maass', K AbdelFattah', AH Idris', V Warren', JP Minei

'University of Texas Southwestern Medical Center, Dallas, TX, USA; ${ }^{2}$ University of North Texas, Fort Worth, TX, USA

Critical Care 2013, 17(Suppl 2):P287 (doi: 10.1186/cc12225)

Introduction Severe burn patients are often noted to have subsequent neurocognitive problems. Experimentally, we have found striking, prolonged elevations of inflammatory markers in the brain (for example, IL-6) even when the injury occurs in a remote anatomic location. This neuroinflammatory response can also be significantly blunted by a single post-burn dose of estrogen. Sonic hedgehog (SHH), an important signaling protein found in the brain, controls and directs differentiation of neural stem cells, influencing brain regeneration and repair by generating new neurons throughout life. As estrogens not only blunt inflammation but also exert an influence on a variety of stem cells, we hypothesized that $17 \beta$-estradiol (E2) might affect levels of SHH in the post-burn rat brain.

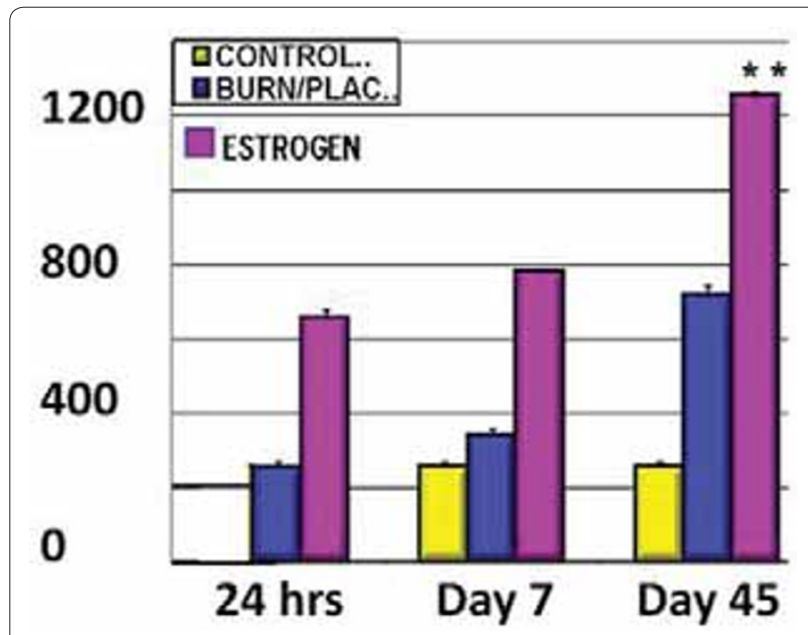

Figure 1 (abstract P287).
Methods Male rats $(n=44)$ were assigned randomly into three groups: controls/no burn ( $n=4) ;$ burn/placebo $(n=20)$; and burn/E2 $(n=20)$. Burned rats received a $40 \% 3^{\circ}$ TBSA dorsal burn, fluid resuscitation and one dose of $\mathrm{E} 2$ or placebo ( $0.5 \mathrm{mg} / \mathrm{kg}$ intraperitoneally) 15 minutes post burn. Eight animals from each of the two burn groups (burn/placebo and burn/E2) were sacrificed at 24 hours and at 7 days, respectively (sham group at 7 days only), with four each of the two burn groups sacrificed at 45 days. Brain tissue samples were analyzed by ELISA for $\mathrm{SHH}$.

Results Mean levels of SHH levels were significantly elevated within 24 hours as much as 45 days post injury in burned animals receiving the $17 \beta$-estradiol $(>1,200 \mathrm{pcg} / \mathrm{mg})$ as compared with the placebotreated burned animals $(<700 \mathrm{pg} / \mathrm{mg})$ and controls $(<300 \mathrm{pcg} / \mathrm{mg})$. See Figure 1.

Conclusion Early, single-dose estrogen administration following severe burn injury significantly elevated levels of SHH in brain tissue. This finding may represent an extremely novel and important pathway for both neuroprotection and neuroregeneration in burn patients.

\section{P288}

Feasibility and experience of using exception from informed consent in a pilot study of immediate estrogen infusion for hypotensive trauma patients

JG Wigginton', PE Pepe', V Warren', K AbdelFattah', JW Gaston', J Simpkins², JP Minei' ${ }^{1}$ D Maass' ${ }^{1}$ AH Idris'

'University of Texas Southwestern Medical Center, Dallas, TX, USA; ${ }^{2}$ University of North Texas, Fort Worth, TX, USA

Critical Care 2013, 17(Suppl 2):P288 (doi: 10.1186/cc12226)

Introduction Many proposed resuscitative therapies for cardiac arrest and trauma will require the earliest possible intervention and would occur under volatile circumstances, making true informed consent for clinical trials unfeasible. The purpose here was to report our experience using exception to informed consent during the inaugural pilot study of infusing estrogen for acute injury, the so-called RESCUE Shock study. Methods Fifty patients were enrolled in RESCUE Shock in which estrogen or placebo was infused as soon as possible in the emergency department for trauma patients with a low systolic blood pressure $(<90 \mathrm{mmHg})$ at two level I trauma centers. They were all treated with a single-dose estrogen or placebo infusion within 2 hours using exception from informed consent following US federal guidelines.

Results Investigator-initiated exception from informed consent studies is feasible, with our FDA IND approval obtained in 31 days, IRB 1 approval in 25 days, and IRB 2 approval in 24 days. Community consultation/notification was successfully accomplished with no one opting out and $47 / 50$ enrolled patients or their legal representatives were notified of participation (one died unidentified, two died with no known contact). The average number of days to verbal notification of patients or advocates was 6.55 days (range 0 to 51 days) as the study team began notification only after the patient or family was able to reasonably understand information about the study. No one decided against continued follow-up. Overall, patients and their families were very enthusiastic about participation and the data safety monitoring board had no safety concerns after reviewing all study data.

Conclusion Although delayed notice of participation occurs for many justifiable reasons, the use of exception from informed consent for novel, time-sensitive resuscitation studies is not only crucial, but can be feasible, and well accepted by patients, their advocates and communities at large.

\section{P289}

Early administration of a single dose of parenteral estrogen decreases inflammation in the heart for $\mathbf{4 5}$ days after severe burns PE Pepe, JG Wigginton, JW Gatson, AH Idris University of Texas Southwestern Medical Center, Dallas, TX, USA Critical Care 2013, 17(Suppl 2):P289 (doi: 10.1186/cc12227)

Introduction Patients with severe burn injury experience a rapid elevation in multiple circulating proinflammatory cytokines, with the levels correlating with both injury severity and outcome. In animal 
models, accumulations of these cytokines have been observed in remote organs, including the heart, brain and lungs. However, data are lacking regarding the long-term levels of cytokines in the heart following severe burn injury and also how infusion of parenteral estrogen, a powerful anti-inflammatory agent, would affect these levels. Using a rat model, we studied the effects of a full-thickness thirddegree burn on cardiac levels of IL-6 and TNFa over 45 days with and without $17 \beta$-estradiol infusion.

Methods A total of 168 male rats were assigned randomly to one of three groups: (1) Sham burn (no actual injury) group $(n=8)$; (2) Placebo (no treatment) burn group ( $n=80)$; and (3) E2 (estrogen treatment) burn group $(n=80)$. Groups 2 and 3 had 40\% TBSA third-degree dorsal burns, early fluid resuscitation and, 15 minutes post burn, $0.5 \mathrm{mg} / \mathrm{kg}$ intraperitoneal estrogen (Group 3) or placebo (Group 2). From each group of 80, eight animals were sequentially sacrificed (and cardiac tissue was sampled for IL-6, TNFa, IL-1 $\beta$ ) at one of 10 respective time points: $0,0.5,1,2,4,6,8,18,24$ hours and day 7 post burn (at day 7 only for the eight shams). The markers were measured by ELISA method. Results In the burned rats, $17 \beta$-estradiol significantly decreased the cardiac levels of TNFa at all time points through 45 days post burn, with the sham animal levels $(30 \mathrm{pg} / \mathrm{mg})$ more comparable with the estradiol group $(70 \mathrm{pg} / \mathrm{mg})$, and significantly less than the placebo animals (332 pg/mg). Similarly, IL-6 levels in the sham animals $(70 \mathrm{pg} / \mathrm{mg}$ ) were comparable with the estradiol group $(86.5 \mathrm{pg} / \mathrm{mg})$, and significantly less than the placebo group $(730 \mathrm{pg} / \mathrm{mg})$, even at 45 days post burn. Conclusion Following severe burn injury in an animal model, an early single dose of estrogen can decrease the prolonged let alone the early onset of cardiac inflammation. Based on these data, clinical studies of estrogen infusions should be seriously entertained as estrogen may not only be an inexpensive, simple adjunctive therapy in burn management, it may also obviate the need for many subsequent interventions altogether and even diminish mortality.

P290

Accuracy of FAST scan in blunt abdominal trauma in a major London trauma centre

S Fleming ${ }^{1}$, R Bird ${ }^{2}$, K Ratnasingham ${ }^{3}$, S Sarker ${ }^{3}$, M Walsh'², B Patel ${ }^{3}$

'Royal National Orthopaedic Hospital Stanmore, London, UK; 'Royal London Hospital, London, UK; ${ }^{3}$ Barts Cancer Institute, London, UK

Critical Care 2013, 17(Suppl 2):P290 (doi: 10.1186/cc12228)

Introduction Blunt abdominal trauma (BAT) is a leading cause of morbidity and mortality. Rapid diagnosis and treatment with the Advanced Trauma Life Support guidelines are vital, leading to the development of focused assessment with sonography in trauma (FAST). Methods A retrospective study carried out from January 2007 to 2008 on all patients who presented with BAT and underwent FAST scan. All patients subsequently had a CT scan within 2 hours of admission or a laparotomy within 2 days. The presence of intraperitoneal free fluid was interpreted as positive.

Results One hundred patients with BAT presented; 71 had complete data. The accuracy of FAST in BAT was 59.2\%; in these, 31 (43.7\%) were confirmed by CT and 11 (15\%) by laparotomy. There were 29 (40.8\%) inaccurate FAST scans, all confirmed by CT. FAST had a specificity of 94.7\% (95\% Cl: 0.75 to 0.99 ) and sensitivity of $46.2 \%$ (95\% Cl: 0.33 to $0.60)$. A positive predictive value of $0.96(0.81$ to 0.99$)$ and negative predictive value of 0.39 ( 0.26 to 0.54 ). Fisher's exact test shows positive FAST is significantly associated with intra-abdominal pathology $(P=0.001)$. Cohen's chance corrected agreement was 0.3. Twentyone out of 28 who underwent laparotomies had positive FAST results, indicating accuracy of $75 \%$ (95\% Cl: 57 to $87 \%$ ).

Conclusion Patients with false negative scans requiring therapeutic laparotomy is concerning. In unstable patients, FAST may help in triaging and identifying those requiring laparotomy. Negative FAST scans do not exclude abdominal injury. Further randomised control trials are recommended if the role of FAST is to be better understood. Reference

1. Smith J: Focused assessment with sonography in trauma (FAST): should its role be reconsidered? Postgrad Med J 2010, 86:285-291.
P291

Level of lipopolysaccharide-binding protein and microbial landscape with account of severity of sepsis syndromes in polytrauma

IM Ustyantseva, OI Khokhlova, OV Petukhova, YA Zhevlakova Federal Scientific Clinical Center of Miners' Health Protection, LeninskKuznetsky, Russia

Critical Care 2013, 17(Suppl 2):P291 (doi: 10.1186/cc12229)

Introduction The aim was estimation of the clinical and predictive significance of the level of lipopolysaccharide-binding protein (LBP) in blood serum and examination of microbial landscape with account of severity of sepsis syndromes in polytrauma.

Methods Clinical examination was performed, which included 99 patients with polytrauma according to sepsis syndrome: SIRS ( $n=18)$, local infection $(n=36)$, sepsis $(n=27)$, severe sepsis $(n=12)$, septic shock $(n=6)$ to the criteria of ACCP/SCCM. The microorganism identification was performed using iEMS Reader MF (Labsystems) with the La Chema multimicrotests. The content of LBP in blood serum was assessed with IMMULITE ONE (USA) with the reagents DPC (USA). Statistical analysis of the data was performed with Statistica 6.0. The numerical characteristics of variables are presented as Me (LQ to UQ). The analysis of differences was carried with the Kruskal-Wallis test for multiple comparison of independent groups, with Friedman one-way analysis of variance. The differences were statistically significant with $P<0.05$. Results In $81 \%$ of the critically ill patients with polytrauma the posttraumatic period was accompanied with development of infectious complications, Gram-negative (K. pneumoniae, Acinetobacter spp., E. coli) and Gram-positive (S. Epidermidis, S. aureus). Sepsis was diagnosed on 8 to 10 days in $45 \%$ of the patients. The significant increase of LPS-BP was found in the first 3 days of the follow-up, compared with the control values (6.7 times higher in SIRS group $\left(X^{2}(n=18, d f=3)=52.8666\right.$, $P<0.001) ; 9.9$ times higher in the group with local infection $\left(X^{2}(n=36\right.$, $\mathrm{df}=3)=91.6629, P<0.001) ; 15.2$ times higher in the sepsis group; 20.5 times higher in the severe sepsis group; 47.3 times higher in the septic shock group $\left.\left(\mathrm{X}^{2}(n=6, \mathrm{df}=3)=11.0339, P=0.0115\right)\right)$, whereas the first positive results of the microbiological examination were obtained only on 5 to 7 days. The diagnostic sensitivity of threshold concentration of LBP in blood serum $(335 \mathrm{mkg} / \mathrm{ml}$ ) was $84 \%$, diagnostic specificity was $88 \%$ (ROC curve: 0.88 ).

Conclusion The high incidence of the diagnostic levels of LBP in blood serum in patients with sepsis in the early term, before microbiological confirmation of infection, allows one to use this parameter as an early marker of development of purulent septic complications conditioned by Gram-negative microflora.

P292

Risk factors for the development of complications following blunt chest trauma: a new risk stratification tool

C Battle ${ }^{1}$, H Hutchings ${ }^{2}$, S Lovett ${ }^{2}$, P Evans ${ }^{2}$

${ }^{1}$ Morriston Hospital, ABMU Health Board, Swansea, UK; ${ }^{2}$ Swansea University, Swansea, UK

Critical Care 2013, 17(Suppl 2):P292 (doi: 10.1186/cc12230)

Introduction The aim of the study was to investigate the risk factors for the development of complications following blunt chest trauma and to develop a risk stratification tool to assist in the management of this patient group. The difficulties in the management of this patient group in the emergency department (ED) due to the development of late complications are well recognised in the literature [1].

Methods Between 2009 and 2011 a total of 276 patients were admitted to hospital from the ED of a regional trauma centre in Wales, with the primary diagnosis of blunt chest trauma. Patients with immediate lifethreatening injuries were excluded. Data were collected retrospectively and included risk factors (age, number of rib fractures, comorbidity, pre-injury anticoagulant use, smoking status, oxygen saturations and respiratory rate on initial assessment in the ED), and outcomes (mortality, any pulmonary morbidity, length of stay of 7 days or more and need for ICU admission). Development of complications was defined as the occurrence of one or more of the outcomes investigated. Multivariable logistic regression using fractional polynomials was used to identify 
risk factors and develop a risk stratification tool. The significant risk factors in the model were selected using backward elimination with the Akaike Information Criterion (AIC) at a significance level of 0.05 . The $c$ index and the Hosmer-Lemeshow $(\mathrm{H}-\mathrm{L})$ test were calculated to assess discrimination and calibration of the risk stratification tool respectively. Results A total of 161 patients out of the 276 admitted developed complications following blunt chest trauma. Implementation of backward elimination using AIC values resulted in a final model based on the significant risk factors; age, oxygen saturations, number of rib fractures, presence of chronic lung disease and pre-injury anticoagulant use (all $P<0.05$ ). The $c$ index for the tool was 0.80 and the $\mathrm{H}-\mathrm{L}$ score was $9.22(P=0.32)$, indicating good predictive capabilities of the tool.

Conclusion The results of this study highlight the risk factors for the development of complications following blunt chest trauma. A risk stratification tool has also been developed that could assist in the prediction of poor outcomes in this patient group. The next stage is to complete a prospective validation study.

Reference

1. Ahmad MA, et al:: Assessment of severity of chest trauma. Injury 2010, 41:981-983.

P293

Guidelines for chest drain insertion do not protect relevant anatomical structures

J Bowness', P Kilgour', S Whiten', I Parkin', J Mooney², P Driscoll' 'St Andrews University, St Andrews, Fife, UK; '2University of Salford, UK Critical Care 2013, 17(Suppl 2):P293 (doi: 10.1186/cc12231)

Introduction We have reported the risk of chest drain insertion inferior to the diaphragm when using current international guidelines [1]. Another complication is damage to significant peripheral nerves, such as the long thoracic nerve causing winging of the scapula [2]. We assessed these risks using: the European Trauma Course method, a patient's handbreadth below their axilla just anterior to the midaxillary line; the British Thoracic Society safe triangle [3]; and the Advanced Trauma Life Support (ATLS) course guidance [4].

Methods We used the above guidelines to place markers (representing chest drains) in the thoracic wall of 16 cadavers bilaterally (32 sides), $1 \mathrm{~cm}$ anterior to the midaxillary line. Subsequent dissection identified the course and termination of the long thoracic nerve, the site of lateral cutaneous branches of intercostal nerves, and their relation to the markers.

Results The long thoracic nerve was found in the fifth intercostal space in 16 of 32 cases, always in or posterior to the midaxillary line. Contrary to the description in Grays' Anatomy (40th edition) it terminated before the inferior border of serratus anterior. Most commonly it was found to end by branching in the fourth (right) or fifth (left) intercostal space (range third to sixth). Lateral cutaneous branches of intercostal nerves were found in the fifth intercostal space in 25 of 32 cases. Contrary to the description in Last's Anatomy (12th edition) they always passed anterior to the midaxillary line (and marker).

Conclusion Placement $1 \mathrm{~cm}$ anterior to the midaxillary line minimises risk to the long thoracic nerve and lateral cutaneous branches of intercostal nerves. We therefore conclude that not all areas of the British Thoracic Society safe triangle are indeed safe, and anteroposterior placement should follow the European Trauma Course and ATLS guidelines: just anterior to the midaxillary line (for example, $1 \mathrm{~cm}$ ).

\section{References}

1. Bowness J, et al:: It's higher than you think: chest drains and the 5th ICS. Clin Anatomy, in press.

2. Kesienne $E B$, et al:: Tube thoracostomy: complications and its management. Pulm Med 2012:256878.

3. Laws D, et al:: BTS guidelines for the insertion of a chest drain. Thorax 2003, 58:ii53-ii59.

4. American College of Surgeons Committee on Trauma: Advanced Trauma Life Support for Doctors, Course Manual. Chicago: American College of Surgeons; 2008:108.
P294

Prediction score of indications for whole body computed tomography in blunt trauma patients

H Oda, R Sasaki, A Hagiwara, A Kimura

National Center For Global Health and Medicine, Tokyo, Japan

Critical Care 2013, 17(Suppl 2):P294 (doi: 10.1186/cc12232)

Introduction Whole body computed tomography (WBCT) appears to be useful for the early detection of clinically occult injury, although its indications have been controversial. The purpose of this study was to develop a clinical prediction score to clarify the indications for blunt trauma patients with multiple injuries (MI) who require WBCT.

Methods We conducted a retrospective study of 173 patients with blunt trauma who underwent WBCT at our emergency center between June 2011 and July 2012. We chose the presence or absence of MI (Injury Severity Score $\geq 15$ ) in need of surgical intervention as the outcome variable. We used bivariate analyses to identify variables potentially predicting the presentation of Ml. The predictor variables were confirmed by multivariate logistic regression analyses. We assigned a score based on the corresponding coefficients.

Results Among the 173 patients enrolled, 53 were in the MI group. Four predictors were found to be independently significant by the logistic analysis: (1) body surface wound $\geq 3$ regions, (2) positive focused assessment with sonography for trauma, (3) white blood cell count $\geq 11,000 / \mu \mathrm{l}$, and (4) D-dimer $\geq 8 \mu \mathrm{g} / \mathrm{ml}$. Score 1 was assigned to predictor (1), score 2 was assigned to predictors (2), (3) and (4). A prediction score was calculated for each patient by adding these scores. The area under the receiver operating characteristic curve was 0.89 . No patients with a score of 1 or less had MI (Figures 1 and 2).

Conclusion In patients with a score of 1 or 0 , the presence of $\mathrm{MI}$ is less likely. These patients may not require WBCT, and selective CT scans of body parts based on clinical presentation should be considered.

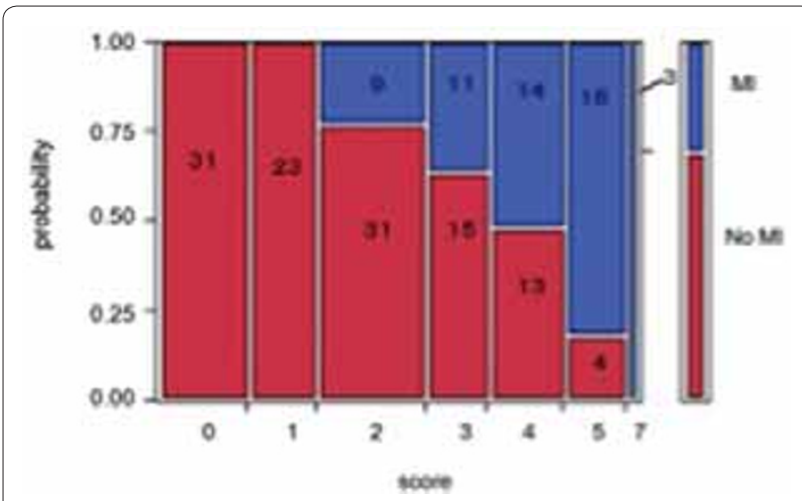

Figure 1 (abstract P294). Prediction versus MI probability (validation group).

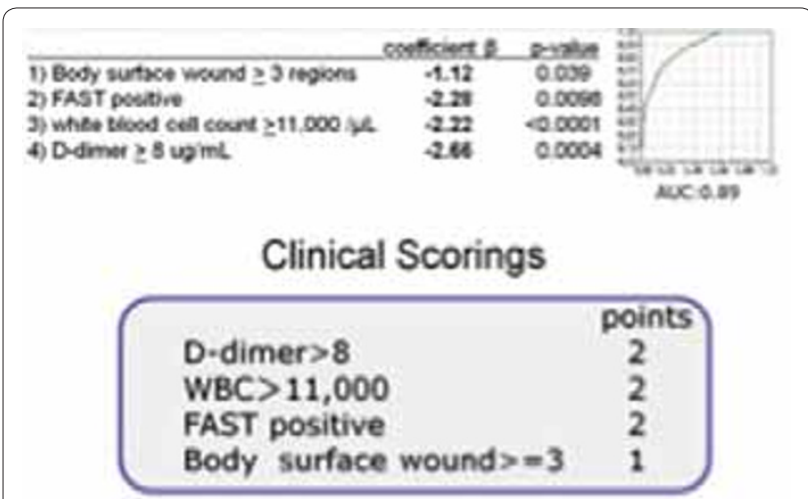

Figure 2 (abstract P294). Clinical scorings. Multivariate, logistic regression analyses. 


\section{References}

1. Huber-Wgner S, et al: Lancet 2009, 373:1455-1461.

2. Kimura A, et al:: Acad Emerg Med 2012, 19:734-735.

P295

Prehospital blood transfusion: 5-year experience of an Australian helicopter emergency medical service

PB Sherren, B Burns

Greater Sydney Area HEMS, Sydney, Australia

Critical Care 2013, 17(Suppl 2):P295 (doi: 10.1186/cc12233)

Introduction There is an emerging body of evidence suggesting that early packed red blood cell transfusion accompanied by fresh frozen plasma, while limiting crystalloids, confers a survival benefit in major trauma [1]. Prehospital blood transfusion has been infrequently described, and concerns over expense, transfusion reactions, risk of disease transmission, short shelf half-life and difficult storage have limited the interest of prehospital providers.

Methods All Greater Sydney Area HEMS (GSA-HEMS) prehospital missions involving a blood transfusion over a 66-month period were identified and reviewed. The prospectively completed GSA-HEMS electronic database was utilised to identify patients and extract data. Results We identified 158 missions involving a prehospital blood transfusion, of which 147 patient datasets were complete. The majority of patients had a blunt mechanism of injury (93.9\%) and were male $(69.3 \%)$ with a median (IQR) age of 34.5 (22 to 52 ) years (Table 1 ). The majority of patients were haemodynamically unstable, with a median (IQR) heart rate and systolic blood pressure of 115 (90 to 130) and 80 (65 to 105$) \mathrm{mmHg}$, respectively. Twenty-two patients $(15.0 \%)$ were pronounced life extinct on the scene. A total of 382 units of packed red blood cells were transfused, with a median of 3 units (range 1 to 6). No early transfusion reactions were noted. A variety of prehospital interventions accompanied the transfusions, ranging from rapid sequence intubation through to thoracotomies (Table 2).

Conclusion Despite the controversies over the role of fluids in the prehospital environment, the carriage and use of blood is both feasible and safe in a physician-led helicopter emergency medical service. Reference

1. Davenport R, et al.: J Trauma 2011, 70:90-95.

Table 1 (abstract P295). Demographics of patients receiving a prehospital blood transfusion

\begin{tabular}{lc}
\hline & Total ( $\boldsymbol{n}=147)$ \\
\hline Male (\%) & $102(69.3)$ \\
Age (years), median (IQR) & 34.5 (22 to 52) \\
Mechanisms of injury (\%) & \\
$\quad$ Motor vehicle collision & $87(59.1)$ \\
$\quad$ Motor bike collision & $20(13.6)$ \\
$\quad$ Pedestrian versus car & $9(6.1)$ \\
$\quad$ Gunshot wound/stabbing & $9(6.1)$ \\
$\quad$ Fall from a height & $5(3.4)$ \\
$\quad$ Recreational & $6(4.1)$ \\
$\quad$ Other & $11(7.5)$ \\
Number of patients trapped on arrival (\%) & $45(30.6)$ \\
Scene time (minutes), mean (SD) & $49.9(27.8)$ \\
Time from tasking to arrival at hospital (minutes), mean (SD) & $126.5(51.3)$ \\
Heart rate, median (IQR) & 115 (90 to 130) \\
Systolic blood pressure (mmHg), median (IQR) & 80 (65 to 105) \\
RTS ${ }^{2}$, median (IQR) & 5.967 (4.083 to 6.904) \\
Total number of PRBC units transfused & 382 \\
Total number of PRBC units wasted & 66 \\
Volume of crystalloid (ml), median (IQR) & 500 (0 to 1,500) \\
Pronounced life extinct on scene & $22.0)$ \\
\hline & \\
\hline & \\
& \\
&
\end{tabular}

Table 2 (abstract P295). Interventions performed

\begin{tabular}{lc}
\hline Intervention & Total $(\boldsymbol{n}=\mathbf{1 4 7})$ \\
\hline Rapid sequence intubation & $96(65.3)$ \\
Cold endotracheal intubation & $15(10.2)$ \\
Surgical airway & $1(0.7)$ \\
Thoracostomy (open or tube) & $59(40.1)$ \\
Thoracotomy & $3(2.0)$ \\
Pelvic binder or fracture splintage & $89(60.5)$ \\
Intraosseous insertion sites & $22(15.0)$ \\
$\quad$ Humerus & 19 \\
Tibia & 10 \\
Femur & 1 \\
Tourniquet application & $15(10.2)$ \\
Hypertonic saline administration & $16(10.9)$ \\
E-FAST performed & $27(18.4)$ \\
$\quad$ Negative results & 9 \\
Positive results (findings) & 18 \\
$\quad$ Abdominal free fluid & 14 \\
Pneumothorax & 2 \\
Hardiac ultrasound only; no cardiac motion & $1(0.7)$ \\
\hline
\end{tabular}

P296

Severe hyperkalemia is prevalent and can be detected by quantitative electrocardiographic findings and medication history of calcium channel blocker among the patients with symptomatic or extreme bradycardia

SB Chon

Kangwon National University Hospital, Chuncheon, South Korea

Critical Care 2013, 17(Suppl 2):P296 (doi: 10.1186/cc12234)

Introduction Severe hyperkalemia (serum potassium $\geq 6.0 \mathrm{mmol} / \mathrm{l}$ with electrocardiography (ECG) change) should be detected and treated immediately [1,2]. Among symptomatic or extreme bradycardia patients, we sought its prevalence and prediction rule by history, vital sign, and ECG $[3,4]$.

Methods A retrospective cross-sectional study was performed on patients with symptomatic (heart rate $(\mathrm{HR}) \leq 50$ /minute with dyspnea, chest pain, altered mentality, dizziness/syncope/presyncope, general weakness, oliguria, or shock) or extreme ( $\mathrm{HR} \leq 40 /$ minute) bradycardia at an ED of an academic hospital from June 2008 to March 2012. Risk factors of severe hyperkalemia were chosen by multiple logistic regression analysis among history (gender, age, comorbidity, and current medication), vital sign, and ECG (maximal precordial T wave amplitude, PR and QRS intervals). Scoring index was derived by summing up of simplified regression coefficients of independent risk factors.

Results A total of 169 cases were enrolled. Mean age was 71.2 years (SD, 12.5 years). Females numbered 87 (51.5\%). Thirty-six cases ( $21.3 \%)$ had severe hyperkalemia. Four variables were independent risk factors of severe hyperkalemia (simplified scores in parentheses): medication of calcium channel blocker (CCB: 2); maximal precordial $T \geq 8.5 \mathrm{mV}$ (2); PR (atrial fibrillation or junctional bradycardia: 1); and HR $\leq 40$ /minute (1). (Nagelkerke $R^{2}=0.503$, AUC $=0.849(95 \% \mathrm{Cl} 0.786$ to 0.899$)$.) Sensitivity and specificity reached 0.75 and 0.83 when total score was $\geq 3$. For score $\geq 4$, positive likelihood ratio reached 5.54 (sensitivity 0.50 , specificity $0.91)$.

Conclusion Severe hyperkalemia is prevalent among symptomatic or extreme bradycardia patients and could be detected immediately by a scoring index composed of quantitative ECG parameters and history of medication of $\mathrm{CCB}$.

References

1. Nyirenda MJ, et al:: Hyperkalaemia. BMJ 2009, 339:b4114

2. Alfonzo AV, et al.: Potassium disorders - clinical spectrum and emergency 
management. Resuscitation 2006, 70:10-25.

3. Neumar RW, et al:: Part 8: adult advanced cardiovascular life support: 2010 American Heart Association Guidelines for Cardiopulmonary Resuscitation and Emergency Cardiovascular Care. Circulation 2010, 122:S729-S767.

4. Severi S, et al: Heart rate response to hemodialysis-induced changes in potassium and calcium levels. J Nephrol 2001, 14:488-496.

P297

Prehospital risk stratification following out-of-hospital cardiac arrest

Y Goto', T Maeda', Y Goto²

'Kanazawa University Hospital, Kanazawa, Japan; ${ }^{Y}$ Yawata Medical Center,

Komatsu, Japan

Critical Care 2013, 17(Suppl 2):P297 (doi: 10.1186/cc12235)

Introduction Multivariate analyses have identified factors that have enabled the development of sophisticated equations and scoring models with the ability to predict outcomes following out-of-hospital cardiac arrest (OHCA). However, implementation of such outcome prediction models in research and clinical practice has been slow. The more crucial aspect of these predictions is the lack of prehospital risk stratification for OHCA patients. Prehospital risk stratification for patients after OHCA may help clinicians guide in-hospital strategies, particularly in the emergency department. The purpose of this study was to develop a simple and generally applicable prehospital riskstratification scheme for patients after OHCA.

Methods We analyzed data for 390,226 adult patients (age $\geq 18$ years) after nontraumatic OHCA, from a prospectively recorded nationwide Utstein-style Japanese database for 2005 to 2009. The endpoint was 1 -month survival with favorable neurological outcome (Cerebral Performance Category (CPC) 1 to 2).

Results Multivariate logistic regression analysis indicated the following three prehospital variables as significant high-ranking factors for predicting favorable 1-month outcomes: shockable initial rhythm (odds ratio (OR), 5.87; $95 \% \mathrm{Cl}, 5.23$ to 6.60 ), witnessed arrest (OR, 3.05; $95 \% \mathrm{Cl}, 2.83$ to 3.28 ), and age ( $\geq 18$ to $<71$ years; OR, 3.24; $95 \% \mathrm{Cl}, 2.97$ to 3.53). Using recursive partitioning analysis for development cohort data ( 2005 to $2008, n=307,896$ ), we stratified prehospital risk: if OHCA with shockable initial rhythm was witnessed, the probability of CPC 1 to 2 was $20.0 \%$ (age, $\geq 18$ to $<71$ years; grade 1 ) or $10.3 \%$ (age, $\geq 71$ years; grade 2); if OHCA with shockable initial rhythm was unwitnessed, the probability was $6.8 \%$ (age, $<81$ years; grade 3 ) or $1.8 \%$ (age, $\geq 81$ years; grade $4 a$ ); if OHCA with unshakable initial rhythm was witnessed, the probability was $1.4 \%$ (grade $4 \mathrm{~b}$ ); and if OHCA with unshakable initial rhythm was unwitnessed, the probability was $0.3 \%$ (grade 5 ). The $c$-statistics for risk stratification of the development and validation cohorts (2009 external data, $n=82,330)$ were $0.853(95 \% \mathrm{Cl}, 0.846$ to $0.859)$ and $0.875(95 \% \mathrm{Cl}, 0.865$ to 0.885$)$, respectively. The odds ratios for CPC 1 to 2 at 1 month between patients at very high and very low were $79.5(95 \% \mathrm{Cl}, 72.5$ to 87.4$)$ and $122.1(95 \% \mathrm{Cl}, 102.0$ to 147.3$)$, respectively, in these cohorts.

Conclusion Prehospital risk stratification (grade 1 to 5) of patients after OHCA using three prehospital factors (shockable initial rhythm, witnessed arrest, and age) accurately classifies the severity of OHCA and may help clinicians guide in-hospital strategies.

P298

Multidisciplinary Medical Emergency Team System increases survival in cancer patients undergoing cardiopulmonary resuscitation

L Haijar1, F Galas², S Vieira², J Almeida², E Osawa², C Park², J Fukushima², E Angelo ${ }^{2}$, A Pinheiro'2, J Auler $\mathrm{Jr}^{2}$

'Heart Institute, São Paulo, Brazil;'2 Cancer Institute of University of São Paulo, Brazil Critical Care 2013, 17(Suppl 2):P298 (doi: 10.1186/cc12236)

Introduction In the last years, ICU admission has been high in cancer patients due to increased survival related to advances in treatment. Patients with cancer reportedly have poor outcomes from cardiopulmonary resuscitation (CPR). The goal of this study was to evaluate the effectiveness of a multidisciplinary Medical Emergency Team System in the CPR outcomes in cancer patients.
Methods We performed a prospective study between January 2011 and December 2012 at a university cancer reference hospital in Brazil. Consecutive patients undergoing cardiac arrest and receiving CPR were identified, and a database was used to register baseline characteristics of patients, data for CPR and 30-day survival. During the first 12 months of the study (phase 1), patients received CPR according to the institution's protocol guided by different physicians. During the last 11 months of the study (phase 2), a multidisciplinary Medical Emergency Team System consisting of a specialized team of ICU nurses, physiotherapists and physicians using an electronic calling system was responsible for the CPR.

Results A total of 182 patients experienced in-hospital cardiac arrest and received CPR. Most patients were in hospital due to medical admission (82\%), had solid tumors $(85 \%)$, and had localized disease (60\%). Pulseless electric activity was the predominant arrest rhythm (54\%) and $45 \%$ of patients were resuscitated on the ICU. Overall 30 -day survival was $9.3 \%$. In the phase 1 of the study, 30 -day survival was $3.7 \%$, and in phase 2 , survival was $14 \%(P=0.017)$. There were no differences in the two phases of the study regarding baseline characteristics of patients, rhythm and place of CPR.

Conclusion Overall survival from CPR in cancer patients compares favorably with survival rates in noncancer patients. A multidisciplinary Medical Emergency Team System increases survival in cancer patients undergoing cardiopulmonary resuscitation.

\section{Reference}

1. Rerisfeld GM, et al:: Survival in cancer patients undergoing in-hospital cardiopulmonary resuscitation: a meta-analysis. Resuscitation 2006, 71:152-160

P299

Focused cardiac ultrasound in resuscitation

E Fish, L Fuchs, G Gulati, D Talmor, A Oren-Grinberg Beth Israel Deaconess Medical Center, Boston, MA, USA Critical Care 2013, 17(Suppl 2):P299 (doi: 10.1186/cc12237)

Introduction Focused cardiac ultrasound is recognized as a vital tool in critical care medicine. Few studies, however, have examined the utility of this modality in resuscitation. While integration of ultrasound can supplement resuscitation by potentially establishing the etiology of cardiac arrest, it must be done safely to avoid interruption of compressions. The aim of this study was to examine the integration of focused cardiac ultrasound into resuscitations in our hospital.

Methods We performed a retrospective observational study of patients undergoing resuscitation at Beth Israel Deaconess Medical Center, Boston, USA, from 2009 to 2012. Inclusion criteria were age $>17$ years

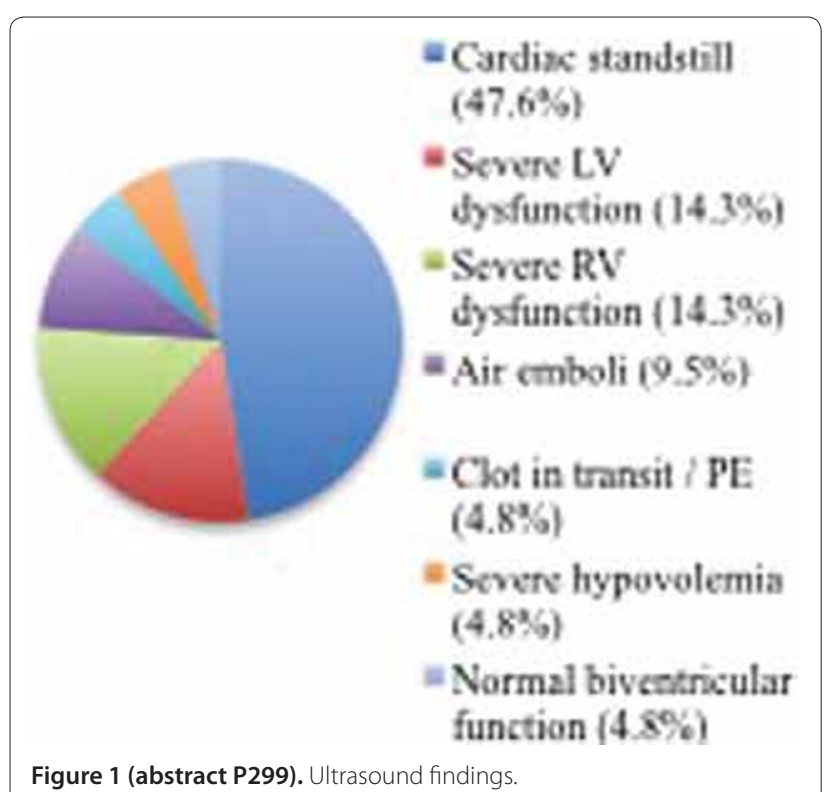


and performance of focused cardiac ultrasound during resuscitation. Recorded variables included admission data, code demographics and diagnosis, therapy outside standard ALS protocol, time to return of spontaneous circulation (ROSC), and outcomes data.

Results Of 33 eligible patients, 12 were excluded due to incomplete cardiac ultrasound reports; 21 patients were enrolled. Cohort demographics included: $57.1 \%$ male, average age 64.3 years, average BMl $28.5 \mathrm{~kg} /$ $\mathrm{m}^{2}$, average Charlson score 5.5. Resuscitations took place on the wards (52.4\%), ICU (42.9\%), or operating room (4.8\%). Most patients had an initial unshockable rhythm (71.5\%). The most common ultrasound finding was cardiac standstill (47.6\%) (Figure 1). The most common intervention as a result of the ultrasound was initiation of a pressor infusion (33.3\%), of which $71.4 \%$ were ionotropes. Additional therapies included blood transfusion $(4.8 \%)$, heparin $(9.5 \%)$, tPA $(4.8 \%)$, cardiac catheterization $(4.8 \%)$, and surgery (9.5\%). ROSC was achieved in $37.5 \%$ of patients; average time to ROSC was 13 minutes. A total $33.3 \%$ of patients who underwent ALS were alive at hospital discharge and $28.6 \%$ at 1 year.

Conclusion Focused cardiac ultrasound is a feasible adjunct to ALS resuscitation and may assist in the early identification of reversible causes of cardiac arrest. Care must be taken to ensure no interruptions to cardiac compressions are made by performance during pulse checks. Further studies are needed to examine the outcomes associated with its integration into resuscitations.

References

1. Deakin CD, et al:: Resuscitation 2010, 81:1305-1352.

2. Robson R. Resuscitation 2010, 81:1453-1454.

3. Breitkreutz R. et al:: Crit Care Med 2007, 35:S150-S161

\section{P300}

Acute intrathoracic gastric herniation as a rare cause of cardiac arrest DW Hoelen, AL Van Duijn, CL Meuwese, JP Ruurda, MA Sikma

UMC Utrecht, the Netherlands

Critical Care 2013, 17(Suppl 2):P300 (doi: 10.1186/cc12238)

Introduction In this case report, we describe a patient who presented with a cardiac arrest as a result of an obstructive shock, which progressed into cardiac arrest, caused by an acute para-esophageal gastric herniation.

Methods Our patient, with a medical history of a laparoscopic repair of a symptomatic diaphragmatic hernia 6 months prior, presented herself at the emergency department with pain in the upper abdomen and nausea. The physical examination, laboratory tests and X-ray of the thorax were normal and she was sent home. Twenty-four hours later paramedics were summoned to our patient because of increased complaints. On arrival of the paramedics she had a normal electrocardiogram (ECG) and during the transfer from her bed to the stretcher she collapsed due to pulseless electric activity (PEA), for which cardiopulmonary resuscitation was started. Sinus rhythm and output was regained after several minutes and the patient was transported to the hospital. At arrival in the hospital, the X-ray of the thorax showed an intrathoracic stomach and a significant mediastinal shift to the right. Results After emergency laparotomy, which concerned correcting the gastric herniation and resection of an ischemic part of stomach, the patient remained hemodynamically stable. Cardiac ischemia was ruled out based on ECG, laboratory findings, cardiac ultrasound and cardiac computed tomography. The ultrasound in the emergency department did show a distended right ventricle and normal left function, which disappeared later (after repositioning the stomach), which is evidence for the mediastinal shift as a cause for the PEA.

Conclusion We are the first to describe a patient requiring cardiopulmonary resuscitation for progressive obstructive shock, due to an intrathoracic stomach. Especially after a laparoscopic repair of a diaphragmatic hernia, this is a rare cause for shock and cardiac arrest, which requires a different medical approach.

\section{P301}

Implementation of dispatcher-assisted cardiopulmonary instructions using the ALERT protocol: preliminary results in Belgium

A Ghuysen', S Stipulante', M El FAssi', A Donneau'², V D'Orio', RTubes

${ }^{\prime} \mathrm{CHU}$ - Ulg Liège, Belgium: 'Liège University, Liège, Belgium

Critical Care 2013, 17(Suppl 2):P301 (doi: 10.1186/cc12239)
Introduction Early bystander cardiopulmonary resuscitation (CPR) is a key factor in improving survival from out-of-hospital cardiac arrest $(\mathrm{OOH}-\mathrm{CA})$. The ALERT algorithm, a simple and effective compression-only telephone CPR protocol, has the potential to help bystanders initiate CPR. This study evaluates the effectiveness of the implementation of this protocol in the Liege dispatching centre.

Methods We designed a before-and-after study based on a 3-month retrospective assessment of the adult victims of $\mathrm{OOH}-\mathrm{CA}$ in 2009, before the implementation of the ALERT protocol in the Liege dispatching centre, and the prospective evaluation of the same 3-month period in 2011 , immediately after the implementation of this protocol. Data were extracted from ambulance, paramedical and medical intervention teams files, as well as the audio recordings of the dispatching centre.

Results There were $233 \mathrm{OOH}-\mathrm{CAs}$ detected in the first period and 235 in the second. Victims were predominantly male $(59 \%$, both periods), aged 66 and 64 years, respectively. Callers were family members in $52 \%$ in 2009 and $64 \%$ in 2011. In 2009, only 9.9\% victims benefited from bystander $C P R$, while there were $22.5 \%$ in $2011(P<0.0002)$. Reasons for protocol underuse were: assistance not offered $(42.3 \%)$, caller remote from the victim (20.6\%) or emotionally distressed (15.5\%). Mean noflow time decreased from 253 seconds in 2009 to 168 seconds in 2011 (NS). Ten victims were admitted in ROSC to hospital in 2009 and 13 in $2011(P=0.09)$.

Conclusion Using the ALERT protocol in the Liege dispatching centre significantly improved the numbers of patients in whom bystander CPR was attempted. Dispatchers must embrace this new opportunity to help callers and be encouraged to accept the responsibility of initiating such assistance.

Reference

1. Ghuysen A, Collas D, Stipulante S, Donneau AF, Hartstein G, Hosmans T, Vantroyen B, D'Orio V: Dispatcher-assisted telephone cardiopulmonary resuscitation using a French-language compression-only protocol in volunteers with or without prior life support training: a randomized trial. Resuscitation 2011, 82:57-63.

P302

Prehospital epinephrine administration and survival among patients with unshockable initial rhythm after out-of-hospital cardiac arrest

Y Goto' ${ }^{1}$, T Maeda', Y Goto ${ }^{2}$

'Kanazawa University Hospital, Kanazawa, Japan; ${ }^{2}$ Yawata Medical Center, Komatsu, Japan

Critical Care 2013, 17(Suppl 2):P302 (doi: 10.1186/cc12240)

Introduction Epinephrine has been a cornerstone of cardiac resuscitation and advanced cardiac life support since the 1960s. However, there is little evidence from clinical trials that epinephrine administration after out-of-hospital cardiac arrest (OHCA) improves long-term survival. There would be subsets of patients for whom epinephrine administration is in fact beneficial. Our objective was to determine whether prehospital epinephrine administration would improve survival at 1 month in OHCA patients with unshockable initial rhythm.

Methods We analyzed data for 383,045 adult OHCA patients with unshockable initial rhythm, from a prospectively recorded nationwide Utstein-style Japanese database for 2007 to 2010. We divided these patients into two cohorts: prehospital epinephrine administration cohort $(n=30,237)$ and non-epinephrine administration cohort ( $n=352,808$ ). The endpoints were 1 -month survival after OHCA, prehospital return of spontaneous circulations (ROSCs), and 1-month survival with favorable neurological outcome (Cerebral Performance Category (CPC) scale, categories 1 to 2 ) at 1 month.

Results The rate of 1 -month survival was $3.72 \%$ for the epinephrine administration cohort and $2.49 \%$ for the non-epinephrine administration cohort, $17.9 \%$ versus $3.0 \%$ for prehospital ROSC, and $0.57 \%$ versus $0.77 \%$ for CPC 1 to 2 (all $P<0.0001$ ). Positive associations were observed between epinephrine administration and 1-month survival (adjusted odds ratio (aOR), $1.18 ; 95 \% \mathrm{Cl}, 1.11$ to 1.27 ), and prehospital ROSC (aOR, 5.50; $95 \% \mathrm{Cl}, 5.29$ to 5.72 ; all $P<0.0001$ ). Negative association was observed between epinephrine administration and CPC 1 to 2 (aOR, $0.56 ; 95 \% \mathrm{Cl}, 0.48$ to $0.66 ; P<0.0001$ ). Multivariate logistic analysis revealed that age ( $<66$ years; aOR, $4.31 ; 95 \% \mathrm{Cl}, 2.47$ to 8.01 ), total dose 
of epinephrine (1 mg; aOR, 3.65; 95\% Cl, 2.61 to 5.18), call-response time ( $<5$ minutes; aOR, $3.58 ; 95 \% \mathrm{Cl}, 1.98$ to 6.69$)$, witnessed arrest (aOR, $2.17 ; 95 \% \mathrm{Cl}, 1.51$ to 3.16$)$, and pulseless electrical activity as an initial rhythm (aOR, 2.02; $95 \% \mathrm{Cl}, 1.46$ to 2.80 ) were significantly associated with CPC 1 to 2 at 1 month in the epinephrine administration cohort. Conclusion In OHCA patients with unshockable initial rhythm, prehospital epinephrine administration significantly increased the rate of survival at 1 month after cardiac arrest. The best single predictor for favorable neurological outcomes at 1 month following prehospital epinephrine administration after cardiac arrest was age ( $<66$ years) followed by total dose of epinephrine $(1 \mathrm{mg})$ and then by call-response time $(<5$ minutes).

\section{P303}

Impact of termination of resuscitation for out-of-hospital

cardiopulmonary arrest in Japan

T Fukuda, N Ohashi, M Gunshin, T Matsubara, S Nakajima, Y Kitsuta,

NYahagi

Graduate School of Medicine, The University of Tokyo, Japan

Critical Care 2013, 17(Suppl 2):P303 (doi: 10.1186/cc12241)

Introduction What can one anticipate from the introduction of termination of resuscitation (TOR) for patients suffering out-of-hospital cardiopulmonary arrest (OHCA) in Japan? Irrespective of whether patients have made a living will requesting that medics do not attempt resuscitation, efforts are made to resuscitate over $90 \%$ of OHCA patients in Japan [1,2]; the number of people resuscitated exceed 120,000 every year. The 2010 American Heart Association (AHA) Guidelines for cardiopulmonary resuscitation (CPR) and emergency cardiovascular care (ECC) defined the criteria for TOR; this initiative may help reduce the number of unnecessary hospital transports by 40 to $60 \%$ and hold down medical costs [3].

Methods This was a single-center retrospective cohort study of patients who suffered OHCA and were transported to our hospital between April 2009 and March 2011. We investigated the patients' characteristics, whether they met the TOR criteria, and their outcome at the time of hospital discharge.

Results A total of 195 patients (mean age, 69 years), 67\% of whom were male, were transported to our hospital after suffering OHCA. Cardiopulmonary arrest was witnessed in 52 cases (27\%). The 2010 AHA Guidelines for CPR and ECC regarding the criteria for TOR were applied in 126 cases (65\%), of whom $113(90 \%)$ were dead on arrival, and 13 were successfully resuscitated and admitted. The outcomes for these 13 patients were as follows: 10 died in the hospital, two patients were discharged with a Glasgow Pittsburgh Cerebral Performance Category (CPC) score of 1, and one patient was transferred to another hospital with a CPC score of 3 .

Conclusion In our study, $65 \%$ of the patients who were transported to the hospital after OHCA met the criteria for TOR. Outcomes for patients who met the TOR criteria were significantly worse than those who did not meet the criteria $(2.4 \%$ vs. $14.5 \%, P<0.005)$. In Japan, efforts are made to resuscitate almost all individuals who suffer OHCA, but $75 \%$ of those patients die within a day. In light of the fact that even the medical cost for each of these patients who die within a day amounts to US\$1,500 [4], the introduction of TOR will have a particularly strong impact in Japan.

\section{References}

$$
\begin{aligned}
& \text { 1. Berdowski J, et al.: Resuscitation 2010, 81:1479-1487. } \\
& \text { 2. Fukuda T: J Jpn Assoc Acute Med 2010, 23:101-108. } \\
& \text { 3. Morrison L, et al.: N Engl J Med 2006, 355:478-487. } \\
& \text { 4. Fukuda T: J Jpn Soc Intensive Care Med 2012, 19:275. }
\end{aligned}
$$

P304

Survival from cardiopulmonary arrest after regular working hours in a tertiary-care hospital: retrospective study

A Mir, A Hussein, A Al Enezi, M Almaani, M Shah, A Shah, B Daoud King Fahd Medical City, Riyadh, Saudi Arabia

Critical Care 2013, 17(Suppl 2):P304 (doi: 10.1186/cc12242)

Introduction Detection and treatment of cardiopulmonary arrest and their antecedents may be less effective at night and weekend than weekdays because of hospital staffing and response factors [1]. Early detection and resuscitation of cardiopulmonary arrest are crucial for better clinical outcome. We conducted our study to evaluate event survival of in-hospital cardiopulmonary arrest after regular working hours in nonmonitored areas of a tertiary-care center.

Methods A retrospective chart review of all adult patients who developed in-hospital cardiopulmonary arrest between January 2010 and December 2011. Working hours are defined as 07:00 to 17:00 Saturday to Wednesday. Event survival is defined as return of spontaneous circulation (ROSC) for more than 20 minutes. Adult patients 18 years and above who suffered from in-hospital cardiopulmonary arrest were included. Patients were excluded if they had cardiopulmonary arrest in the emergency department, had implantable cardiovascular device, arrested in monitored areas or had pre-existing DNR orders. Data analysis was accomplished using SAS, version 9.3 (SAS institute, Inc., Cary, NC, USA).

Results A total of 430 cardiopulmonary arrest events occurred inhospital between January 2010 and December 2011. A total of 326 patients were excluded because 252 occurred in the ICU, 70 in the coronary care unit and four in the stork unit. In total, 104 patients were enrolled; $50.9 \%$ where female and $49.1 \%$ male. Median age was 61 years. A total $41.3 \%$ of the arrests were due to respiratory arrest and $58.7 \%$ due to cardiac arrest. Out of 104 patients who developed cardiopulmonary arrest, $47(45.19 \%)$ occurred during regular working hours, and $57(54.81 \%)$ occurred after regular working hours $(P=0.0081)$. The event survival was $87.23 \%$ during regular working hours compared with $47.37 \%$ for the patients who developed cardiopulmonary arrest after regular working hours $(P<0.0001)$. In total, $53.19 \%$ of those who developed cardiopulmonary arrest during regular working hours were discharged alive from the ICU compared with $26.32 \%$ of those who developed cardiopulmonary arrest after regular working hours.

Conclusion Event survival and survival to discharge were significantly higher in patients who developed cardiopulmonary arrest during regular working hours.

\section{Reference}

1. Peberdy MA,Ornato JP, Larkin L, et al.: Survival from in-hospital cardiac arrest during nights and weekends. JAMA 2008, 299:785-792.

\section{P305}

\section{Cognitive performance and capacity to return home following} out-of-hospital cardiac arrest

J Petrie, C Lockie, S Brett, R Stümpfle

Imperial College Healthcare NHS Trust, London, UK

Critical Care 2013, 17(Suppl 2):P305 (doi: 10.1186/cc12243)

Introduction Before the introduction of primary percutaneous coronary intervention $(\mathrm{PCl})$ and therapeutic hypothermia $(\mathrm{TH})$ to out-of-hospital cardiac arrest (OOHCA) management, survival to hospital discharge with intact neurological function was poor $[1,2]$. We aimed to quantify the survival and degree of neurological impairment in OOHCA patients admitted to our ICU since the adoption of post-OOHCA bundles.

Methods Sixty-nine consecutive OOHCA patients admitted to the ICU at Hammersmith Hospital from 1 January 2011 to 30 June 2012 were identified and reviewed from hospital databases. Cognitive status was scored using Cerebral Performance Category (CPC); 1 to 2 normal-mild and 3 to 4 moderate-severe neurological impairment. Scores were determined from ICU summaries, occupational and physiotherapy reports. Hospital discharge outcomes were determined from hospital databases.

Results TH was initiated in 93\% (64/69) of OOHCA patients and $87 \%$ (40/46) with ischaemic cardiac aetiology underwent $\mathrm{PCI}$. ICU survival was $58 \%(40 / 69) ; 65 \%(26 / 40)$ scoring CPC 1 to 2 and $35 \%$ (14/40) CPC 3 to 4 at ICU discharge. Two patients with CPC 2 improved to CPC 1 during their hospital stay. All patients with CPC 1 to 2 survived to hospital discharge; two required general rehabilitation before returning home. Only $43 \%$ (6/14) of CPC 3 to 4 patients survived to hospital discharge; none returned home. Two went into hospice care, one was repatriated to another hospital and three went to neuro-rehab. No CPC 3 to 4 patients improved CPC scores after ICU discharge. Overall hospital survival was $46 \%$. See Table 1.

Conclusion OOHCA patients admitted to our ICU had a $46 \%$ chance of surviving to hospital discharge. Most patients left hospital with good neurological status (CPC 1 to 2); moderate-severe neurological 
Table 1 (abstract P305). Discharge and neurological status of OOHCA patients

\begin{tabular}{lccccccc}
\hline & \multicolumn{3}{c}{ Discharge } & & \multicolumn{2}{c}{ Hospital discharge destination } \\
\cline { 2 - 3 } Score & ICU & Hospital & Home & & $\begin{array}{c}\text { Neuro- } \\
\text { rehab }\end{array}$ & Repatriation & Hospice \\
\hline CPC 1 & 20 & 22 & 19 & 0 & 3 & 0 \\
CPC 2 & 6 & 4 & 4 & 0 & 0 & 0 \\
CPC 3 & 4 & 3 & 0 & 3 & 0 & 0 \\
CPC 4 & 10 & 3 & 0 & & 0 & 1 & 2 \\
Total & 40 & 32 & 23 & 3 & 4 & 2 \\
\hline
\end{tabular}

disability (CPC 3 to 4) was seen in 19\%, greater than previously reported [1]. A higher proportion (35\%) of patients discharged from the ICU had moderate-severe neurological disability; most subsequently died in hospital (62\%). These figures may represent better ICU outcomes subsequent to adoption of OOHCA bundles but suggest further work is required in neuro-disabled survivors.

References

1. Cobbe SM, et al: BMJ 1999, 312:1633-1637.

2. Holzer M, et al:: N Engl J Med 2002, 346:549-556.

P306

Start value of cerebral saturation in prehospital cardiac arrest patients: does it mean something?

C Genbrugge, I Meex, S Scheyltjens, J Dens, C De Deyne

ZOL, Genk, Belgium

Critical Care 2013, 17(Suppl 2):P306 (doi: 10.1186/cc12244)

Introduction During cardiopulmonary resuscitation (CPR) monitoring possibilities are limited. Parnia and colleagues investigated the feasibility and role of near-infrared spectroscopy (NIRS) during CPR in cardiac arrest patients (CA) [1]. NIRS could have a role in predicting return of spontaneous circulation (ROSC). Recently, the Equanox ${ }^{\circledR}$ with four wavelengths sensor was validated to provide absolute data on regional cerebral saturation [2]. We measured cerebral oxygenation ( $\mathrm{rSO}_{2}$ ) during CPR with NIRS technology and analyzed the differences between initial cerebral saturations in patients achieving ROSC compared with patients without ROSC

Methods With IRB approval, $\mathrm{rSO}_{2}$ was measured with NIRS during

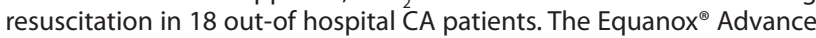
(NONIN), a NIRS monitoring device that measures absolute $\mathrm{rSO}$ values, was applied on the right side of the patient's forehead when the medical emergency team arrived in a resuscitation setting. Placement of the probe did not interfere with the advanced life support algorithm. The sensor remained on the patient's forehead during resuscitation, and if ROSC was reached the probe was removed on arrival at the emergency department. If ROSC was not achieved, the probe was removed prehospital. ROSC was defined as ROSC during more than 20 minutes. The Mann-Whitney test was utilized for comparison of survivor and nonsurvivor data. Student's $t$ test was performed to compare the initial $\mathrm{rSO}_{2}$

Results Of the 18 patients, nine patients had ROSC (survivors). The initial rhythm was the same in both groups, six patients in each group had asystole as initial rhythm. In the group of survivors were six female patients, in the nonsurvivors were two female patients. The mean age in ROSC and no-ROSC groups is respectively 75.8 years (SD \pm 12.8 ) and 69.4 years $(\mathrm{SD} \pm 22.9, P=0.48)$. The mean $\mathrm{rSO}_{2}$ at arrival of the emergency medical team was $31.56 \%$ (SD \pm 29.4 ) and $12.78 \%$ (SD $\pm 12.7)$ respectively in the ROSC group and no-ROSC group $(P=0.1)$. The mean time between collapse and start of CPR (basic life support of bystanders) was 6.9 minutes (SD \pm 8.2 ) in the no-ROSC group and 8.2 minutes (SD $\pm 7.08, P=0.69$ ) in the ROSC group.

Conclusion Initial $\mathrm{rSO}_{2}$ values in out-of hospital CA patients with ROSC showed a tendency towards higher values compared with nonsurvivors, but no significant difference could be demonstrated, probably related to the small number of patients included in this preliminary report.

References

1. Parnia et al:: Resuscitation 2012, 83:982-985.

2. MacLeod et al:: J Cardiothorac Vasc Anesth 2012, 26:1007-1014.
P307

Intensive care admission after cardiac arrest: cardiac versus noncardiac causes and consequences for treatment

T Hargreaves', H Kingston', M Crews' ${ }^{1}$, M Mogk' I Welters $^{3}$

'Royal Liverpool University Hospital, Liverpool, UK; '2Moredata GmbH, Giessen, Germany; ${ }^{3}$ Liverpool University, Liverpool, UK

Critical Care 2013, 17(Suppl 2):P307 (doi: 10.1186/cc12245)

Introduction In-hospital (IHCA) and out-of-hospital cardiac arrest $(\mathrm{OHCA})$ are associated with high mortality [1]. Studies suggest that up to $68 \%$ of OHCA is due to acute coronary syndrome, with $38 \%$ requiring percutaneous coronary intervention (PCI) [1]. However, revascularisation may not always be available or address the underlying pathology. This study aimed to establish the prevalence of different aetiologies for IHCA and OHCA, and the use of emergency treatment for these patients.

Methods A retrospective case-note review of all patients admitted between 2008 and 2011 to the ICU of an inner-city university hospital after OHCA or IHCA. Biometric data, presenting cardiac rhythm, presumed cause of arrest, management and outcomes were recorded. The Kruskal-Wallis test was used for numerical data analysis and chisquare test for categorical data.

Results Data were analysed for 64 patients - 44 (69\%) following OHCA and $20(31 \%)$ after IHCA. The median APACHE score for OHCA was 17 and for IHCA was $23.5(P=0.001)$. Hospital survival rate was $10 \%$ $(n=2)$ for IHCA and $38.6 \%(n=17)$ for OHCA $(P<0.02)$. A total of $34.1 \%$ $(n=15)$ OHCA were due to myocardial infarction (MI) compared with $10 \%(n=2)$ of IHCA $(P<0.05)$. The most prevalent aetiologies were MI $(n=17)$, hypoxia $(n=10)$, cardiac other $(n=5)$, sepsis $(n=4)$, arrhythmia $(n=3)$ and PE $(n=3)$. In two IHCA patients more than one likely cause of arrest was reported and in 19 cases no cause was identified. The presenting rhythm was ventricular fibrillation (VF) in $45.3 \%(n=29)$, pulseless electrical activity in $32.8 \%(n=21)$ and asystole in $20.3 \%$ $(n=13)$. A total of $9.4 \%(n=6)$ were thrombolysed and one $(1.6 \%)$ patient was referred for emergency $\mathrm{PCl}$.

Conclusion As previously reported [2], IHCA was associated with a worse prognosis than OHCA. The OHCA survival rate was better than reported elsewhere [3]. The percentage of IHCA attributed to MI was low. Only one OHCA patient was referred for emergency PCI. Routine coronary angiography with ad hoc PCl in VF OHCA has been associated with increased survival [4]. Greater availability of PCI post OHCA could further improve mortality in patients with a primary cardiac pathology. Further investigation should include management of noncardiogenic cardiac arrest.

References

1. Stub et al: Am J Cardiol 2011, 107:522-527.

2. Girotra et al: N Engl J Med 2012, 367:1912-1920.

3. Herlitz et al: Heart 2003, 89:25-30.

4. Cronier et al: Crit Care 2011, 15:122.

P308

Comparison of 1-year neurological outcome between intra-hospital and extra-hospital cardiac arrest survivors submitted to mild therapeutic hypothermia in a community-based setting in Brazil CA Abreu Filho', A Andrade², A Neto², S Santos², M Bracco², E Silva', A Baruzzi', J Teixeira²

'Hospital Israelita Albert Einstein, São Paulo, Brazil; ${ }^{2}$ Hospital Municipal Dr. Moysés Deutsch, São Paulo, Brazil

Critical Care 2013, 17(Suppl 2):P308 (doi: 10.1186/cc12246)

Introduction Mild therapeutic hypothermia (MTH) is the most powerful therapy to improve survival and neurologic outcome after out-ofhospital cardiac arrest. Such benefit may also occur for unconscious patients after in-hospital cardiac arrest. The aim is to compare 1-year evolution of neurological outcomes of patients treated with MTH after in-hospital versus out-of-hospital cardiac arrest.

Methods A prospective study of patients treated with MTH after cardiac arrest in a community hospital in São Paulo, Brazil. After return of spontaneous circulation, unconscious survivors received MTH using topical ice and cold saline infusions in order to achieve a 32 to $34^{\circ} \mathrm{C}$ goal temperature, within 6 hours of cardiac arrest, and maintained 
for 24 hours. Esophageal temperature was monitored; continuous intravenous sedation-analgesia was maintained for 48 hours after initiation of MTH. The Glasgow Outcome Scale (GOS) was used to analyze the neurological outcomes after hospital discharge.

Results From January 2009 to April 2012, 84 patients submitted to MTH were divided into two groups: Group 1, 54 patients presented outof-hospital cardiac arrest; and Group 2, 30 patients had intra-hospital cardiac arrest. Both groups were similar regarding to gender; Group 2 tended to be older (mean age 44.3 years vs. 33.5 years, $P=0.07$ ), and had more frequently asystole as the cardiac arrest rhythm $(45 \%$ vs. $15 \%, P=0.10$ ). Group 2 had shorter duration of resuscitation (12.3 minutes vs. 33.7 minutes, $P=0.03$ ), longer time to hypothermia initiation (309.3 minutes vs. 212.8 minutes, $P=0.04$ ), longer hospital stay after cardiac arrest (50.7 days vs. 32.4 days, $P=0.01$ ) and worse neurological outcome, characterized by the presence of GOS $\leq 3$ at 30 days $(68.4 \%$ vs. $35.1 \%, P=0.03)$. Hospital mortality was $5.5 \%$ in Group 1 and $13.3 \%$ in Group $2(P=0.21)$. The 1 -year survival rate was $85.1 \%$ in Group 1 and $83.3 \%$ in Group $2(P=0.43)$; after 1 year follow-up, GOS $\leq 3$ was present in $30.4 \%$ of Group 2 patients and in $11.1 \%$ of Group 1 patients $(P=0.04)$.

Conclusion Midterm neurological outcome of MTH after in hospital cardiac arrest seems to be not as good as after out-of-hospital cardiac arrest. Delay in hypothermia initiation, older age and associated comorbidities could explain the worse evolution of this group of patients.

\section{P309}

Primary percutaneous coronary angioplasty and therapeutic

hypothermia in out-of-hospital cardiac arrest

R Hunt, M Holl, A Bailey, P Macnaughton

Derriford Hospital, Plymouth, UK

Critical Care 2013, 17(Suppl 2):P309 (doi: 10.1186/cc12247)

Introduction The benefit of primary percutaneous intervention ( $\mathrm{PCI})$ in the management of out-of-hospital cardiac arrest (OHCA) is not clear cut [1]. It has historically been used in patients with ST elevation on post-resuscitation electrocardiogram (ECG) although this is a poor predictor of acute coronary occlusion after cardiac arrest [2]. This study investigates the benefit of $\mathrm{PCl}$ regardless of post-resuscitation ECG. Benefit is widely claimed for therapeutic hypothermia, so cooling parameters were included.

Methods We analysed all 41 consecutive adults admitted post OHCA to a university hospital ICU between January 2010 and December 2011. Patients received $\mathrm{PCl}$ regardless of ECG changes. A Cox proportional hazards model was used to determine the relationship between $\mathrm{PCl}$, cooling and survival to discharge. Routinely collected data such as demographics and details of resuscitation (OHCA Utstein data) were also included.

Results Survival to hospital discharge was $41 \%$ with $29 \%$ of survivors discharged to a neurological rehabilitation centre. Multivariate analysis using a Cox proportional hazards model showed $\mathrm{PCl}$ to be an independent predictive factor of survival, unrelated to ECG (hazards ratio, $0.0583 ; 95 \% \mathrm{Cl}, 0.0076$ to 0.4485 ). Cooling had no significant impact on patient survival. See Figure 1.

Conclusion In this small retrospective study primary $\mathrm{PCl}$ appears to be an independent predictor of survival after OHCA. This is consistent with other studies suggesting benefit for primary $\mathrm{PCl}$ regardless of the post-resuscitation ECG [3]. Cooling was not found to improve survival to discharge but further analysis is required to determine impact on neurological function.

References

1. Kern KB: Optimal treatment of patients surviving out-of-hospital cardiac arrest. JACC Cardiovasc Interv 2012, 5:597-605

2. Spaulding $C M$, et al: Immediate coronary angiography in survivors of outof-hospital cardiac arrest. N Engl J Med 1997, 336:1629-1633.

3. Dumas $F$, et al: Immediate percutaneous coronary intervention is associated with better survival after out-of-hospital cardiac arrest. Circ Cardiovasc Interv 2010, 3:200-207.

P310

Infrared pupillometry for outcome prediction after cardiac arrest and therapeutic hypothermia

T Suys, N Sala, AO Rossetti, M Oddo

Lausanne University Hospital, Lausanne, Switzerland

Critical Care 2013, 17(Suppl 2):P310 (doi: 10.1186/cc12248)

Introduction Sedation and therapeutic hypothermia (TH) modify neurological examination and alter prognostic prediction of coma after cardiac arrest (CA). Additional tools, such as EEG and evoked potentials, improve prediction of outcome in this setting, but are not widely available and require significant implementation.

Methods Using a new device for infrared pupillometry, we examined the value of quantitative pupillary light reactivity (PLR) to predict outcome in comatose post-CA patients treated with TH. Twenty-four comatose CA patients treated with $\mathrm{TH}\left(33^{\circ} \mathrm{C}, 24\right.$ hours) were prospectively studied. The percentage variation in PLR was measured during TH (12 hours from CA), using the NeuroLight Algiscan ${ }^{\circledast}$ (IDMED, Marseille, France). For each patient, three consecutive measures were performed and the best value was retained for analysis. The relationship of PLR

Table 1 (abstract P310). False-positive and false-negative rates for outcome (\% of patients)

\begin{tabular}{|c|c|c|c|c|c|}
\hline & \multicolumn{2}{|c|}{ Outcome } & \multirow[b]{2}{*}{$P$ value } & \multirow[b]{2}{*}{ FNR (\%) } & \multirow[b]{2}{*}{ FPR (\%) } \\
\hline & $\begin{array}{c}\text { Good } \\
\text { (CPC } 1 \text { to 2) } \\
\text { (\%) }\end{array}$ & $\begin{array}{c}\text { Bad } \\
\text { (CPC } 3 \text { to 5) } \\
\text { (\%) }\end{array}$ & & & \\
\hline \multicolumn{6}{|l|}{ PLR } \\
\hline$\leq 10 \%$ & 0 & 100 & $<0.0001$ & 0 & 0 \\
\hline$>10 \%$ & 100 & 0 & & & \\
\hline \multicolumn{6}{|l|}{ BRS } \\
\hline Absent & 0 & 33 & 0.02 & 0 & 28 \\
\hline Present & 39 & 28 & & & \\
\hline \multicolumn{6}{|l|}{$\mathrm{MR}$} \\
\hline Absent & 11 & 50 & 0.02 & 11 & 11 \\
\hline Present & 28 & 11 & & & \\
\hline
\end{tabular}

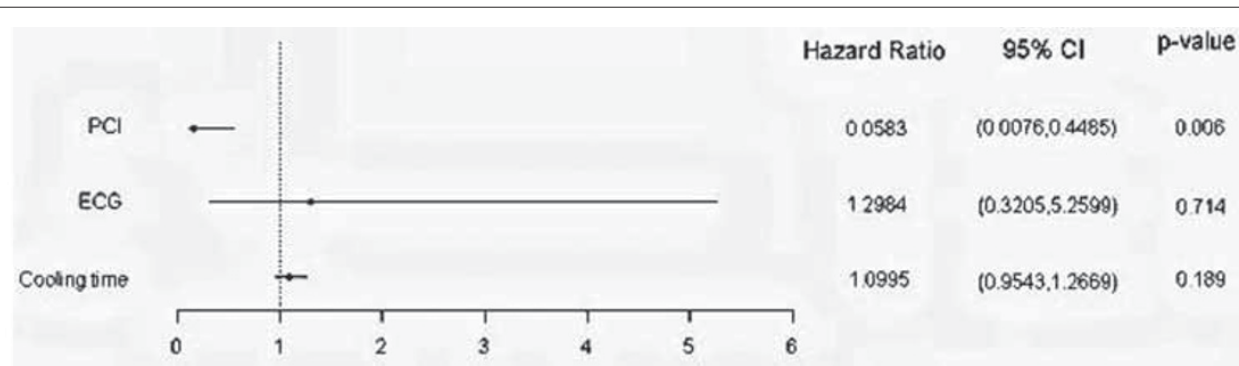

Figure 1 (abstract P309). Comparison of hazards ratios post Cox analysis. 
with survival and neurological outcome (CPC scores) at 3 months was analyzed, and the predictive value of PLR was compared with that of standard clinical examination (motor response and brainstem reflexes) performed at 48 hours from CA.

Results Quantitative PLR was strongly associated with survival (median left-eye PLR $14 \%$ (11 to $16 \%$ ) variation in survivors vs. $5.5 \%$ (4 to $8.5 \%$ ) in nonsurvivors, $P<0.0001)$ and 3 -month neurological outcome $(14 \%$ (11 to $21 \%$ ) in patients with CPC 1 to 2 vs. $5.5 \%$ (4 to $8.5 \%$ ) in those with CPC 3 to $5, P<0.0001)$. Comparable findings were obtained using right-eye PLR. A PLR $>10 \%$ was $100 \%$ predictive of patient prognosis, with false-positive and false-negative rates of $0 \%$ for outcome. Clinical examination was significantly associated with outcome; however, motor response (MR) and brainstem reflexes (BRS) yielded higher falsepositive and false-negative rates than PLR (Table 1).

Conclusion Quantitative PLR appears highly accurate and superior to standard neurological examination to predict outcome in patients with post-CA coma. Further study is warranted to confirm these promising findings.

Acknowledgements Supported by Grants from the Swiss National Science Foundation (FN 320030_138191) and the European Critical Care Research Network (ECCRN).

P311

Effect of moderate hyperventilation and induced hypertension on cerebral tissue oxygen saturation in comatose post-cardiac arrest patients treated with hypothermia

T Suys, N Sala, M Oddo

Lausanne University Hospital, Lausanne, Switzerland

Critical Care 2013, 17(Suppl 2):P311 (doi: 10.1186/cc12249)

Introduction Maintenance of adequate brain perfusion is an essential component of post-resuscitation care. Mean arterial pressure (MAP) and $\mathrm{PaCO}_{2}$ are important determinants of brain perfusion; however, no precise guidelines exist for optimal MAP and $\mathrm{PaCO}_{2}$ targets in comatose post-cardiac arrest (CA) patients.

Methods Using NIRS, we examined changes in non-invasive cerebral tissue oxygen saturation $\left(\mathrm{SctO}_{2}\right)$ following moderate hyperventilation $(\mathrm{HV})$ and induced hypertension $(\mathrm{IH})$ in comatose CA patients treated with therapeutic hypothermia $(\mathrm{TH})$. A prospective pilot study including comatose patients successfully resuscitated from out-of-hospital CA treated with $\mathrm{TH}\left(33^{\circ} \mathrm{C}\right.$ for 24 hours, using cold saline and surface cooling device), monitored for continuous SctO with the Foresight NIRS system (CAS Medical Systems, Branford, CT, USA). Moderate hyperventilation was induced for approximately 30 minutes by decreasing $\mathrm{PaCO}_{2}$ from $\sim 40$ to $\sim 30 \mathrm{mmHg}$, at stable MAP. After $\mathrm{PaCO}_{2}$ normalization, MAP was increased from $\sim 70$ to $\sim 90 \mathrm{mmHg}$ by intravenous infusion of norepinephrine, at stable $\mathrm{PaCO}_{2}$. Effects of $\mathrm{MV}$ and $\mathrm{IH}$ on $\mathrm{SctO}_{2}$ were analyzed with a paired $t$ test.

Results Ten patients (mean age, 69.5; mean time to ROSC, 19 minutes) were studied during the stable TH maintenance phase. Results are summarized in Figure 1. MV was associated with a significant reduction of $\mathrm{SctO}_{2}$ from baseline (75\% (73 to 76 ) to $69 \%$ (67.5 to 71.5$), P<0.001$ ).
No significant changes in $\mathrm{SctO}_{2}$ were found after IH (74 (72 to 76) vs. 75 (73 to 75.5$), P=0.24)$.

Conclusion Moderate HV was associated with significant reduction in cerebral saturation, whilst IH may be detrimental after $\mathrm{CA}$ and $\mathrm{TH}$, whilst increasing MAP to supranormal levels with vasopressors does not improve cerebral oxygenation. These data stress the importance of strict control of $\mathrm{PaCO}_{2}$ following $\mathrm{CA}$ and $\mathrm{TH}$ to avoid secondary cerebral ischemic insults.

Acknowledgements $\mathrm{MO}$ is supported by Grants from the Swiss National Science Foundation (FN 320030_138191) and The European Critical Care Research Network (ECCRN).

\section{P312}

Effects of viscosity on cerebral blood flow after cardiac arrest

L Bisschops, G Pop, S Teerenstra, J Van der Hoeven, C Hoedemaekers Radboud University Nijmegen Medical Centre, Nijmegen, the Netherlands Critical Care 2013, 17(Suppl 2):P312 (doi: 10.1186/cc12250)

Introduction After cardiac arrest, microcirculatory reperfusion disorders develop despite adequate cerebral perfusion pressure. Increased blood viscosity strongly hampers the microcirculation, resulting in plugging of the capillary bed, arteriovenous shunting and diminished tissue perfusion. The aim of the present study was to assess blood viscosity in relation to cerebral blood flow in patients after cardiac arrest.

Methods We performed an observational study in 10 comatose patients after cardiac arrest. Patients were treated with hypothermia for 24 hours. Blood viscosity was measured ex vivo using a Contraves LS300 viscometer. Mean flow velocity in the middle cerebral artery (MFVMCA) was measured by transcranial Doppler (TCD) at the same time points.

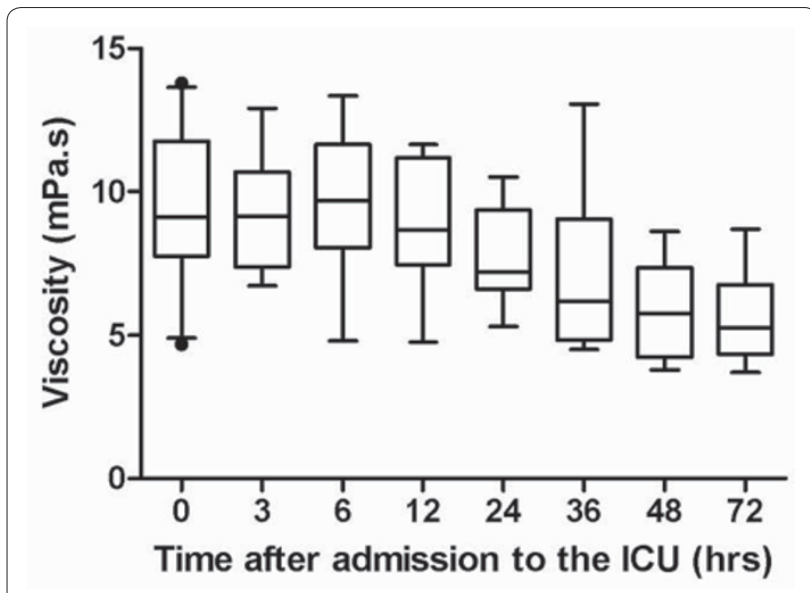

Figure 1 (abstract P312).
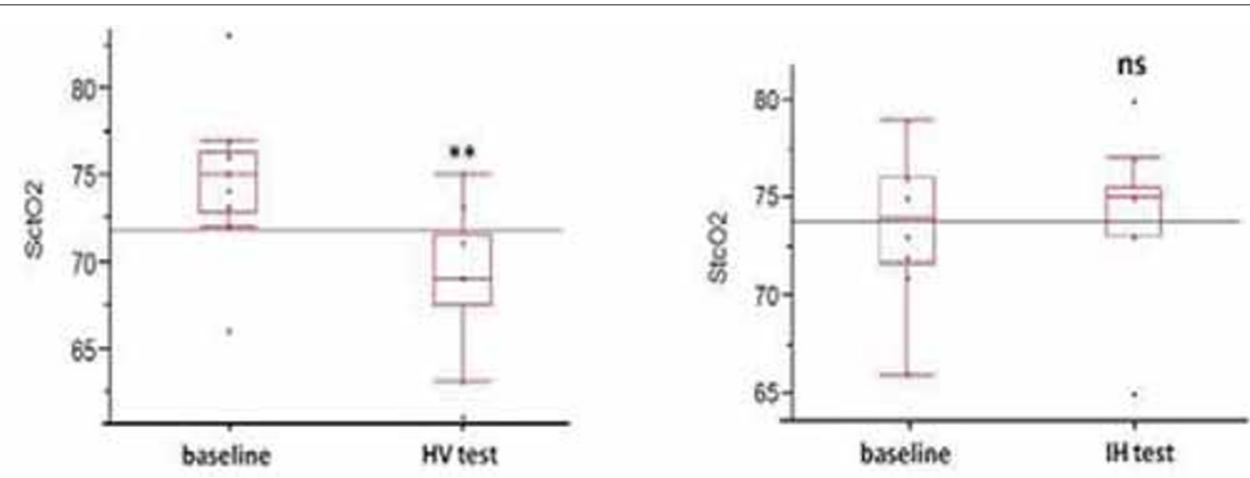

Figure 1 (abstract P311). Changes in $\mathrm{SctO}_{2}$ after moderate $\mathrm{HV}$ and $\mathrm{IH}$ tests ( $n=10$ patients). 
Results The median viscosity on admission was 9.12 (8.19 to 11.19) $\mathrm{mPa}$. second, and remained stable at 3 and 6 hours after admission. From 6 hours after admission, viscosity decreased significantly to 3.66 (3.12 to 4.04) $\mathrm{mPa}$.second $(P<0.001)$. Median MFVMCA was low $(27.0$ (23.8 to 30.5$) \mathrm{cm} /$ second) on admission, and significantly increased to 63.0 (51.0 to 80.0$) \mathrm{cm} / \mathrm{second}$ at 72 hours $(P<0.001)$. There was a significant association between viscosity and the MFVMCA $(P=0.0019)$. See Figure 1.

Conclusion Viscosity decreases in the first 3 days after cardiac arrest and is strongly associated with an increase in cerebral blood flow. Since viscosity is a major determinant of cerebral blood flow, repeated measurements may guide therapy to help restore cerebral oxygenation after cardiac arrest.

P313

Therapeutic mild hypothermia after cardiac arrest in shockable and nonshockable rhythms: does it improve both survival and neurological outcome?

A Gupta

Fortis Escorts Heart Institute, New Delhi, India

Critical Care 2013, 17(Suppl 2):P313 (doi: 10.1186/cc12251)

Introduction Although therapeutic mild hypothermia (TMH) after resuscitation from cardiac arrest (CA) has been postulated and studied to be associated with good outcome of the patients, there is no dearth of data that does not favour TMH. Our aim was to find out whether TMH is associated with good outcome after CA in shockable rhythm (SR) compared with nonshockable rhythm (NR), in terms of survival as well as neurological outcome.

Methods We reviewed medical records of all CA patients (in-hospital or out-of-hospital arrest) in whom cardiopulmonary resuscitation (CPR) was performed at our hospital from 1 February 2011 to 31 January 2012 (12 months). The following information was collected: first documented rhythm, whether TMH done or not, and two outcome measures including survival to hospital discharge and neurological outcome at the time of hospital discharge. A measure of good neurological outcome was Cerebral Performance Category score 1 or 2 (CPC, fivepoint scale; $1=$ good cerebral performance to $5=$ brain death). Then we quantified the association of TMH with SR as well as NR for both the parameters of outcome - that is, survival to hospital discharge and good neurological outcome - by logistic regression analysis.

Results We had 297 CA patients (168 SR, 129 NR) in whom CPR was done. Return of spontaneous circulation was achieved in 90 patients. TMH was induced in 57 patients (33 SR, 24 NR). Survival to hospital discharge was observed in 27 patients (18/33 (54.5\%) SR, 9/24 (37.5\%) NR), out of which 18 patients (10/33 (30\%) SR, 8/24 (33\%) NR) had good neurological outcome. On analysis, TMH was found to be associated with increased odds of survival to hospital discharge (although statistically not significant) in SR patients compared with NR patients (odds ratio $(\mathrm{OR})=2.00 ; 95 \% \mathrm{Cl}=0.68$ to $5.85 ; P=0.2837$ ), but it was not associated with any better neurological outcome in terms of CPC score in patients presenting with $\mathrm{SR}$ rather than $\mathrm{NR}(\mathrm{OR}=0.87 ; 95 \% \mathrm{Cl}=0.28$ to $2.68 ; P=1.0000$ ). Rather, the odds for good neurological outcome were more in favour of NR (pulseless electrical activity/asystole).

Conclusion Although TMH might be associated with better survival chances in patients presenting with SR, neurological outcome was no better (rather worse) in this group of patients when compared with patients with NR as the first documented rhythm.

P314

Agreement between temperature measurements during cooling and warming through the esophagus

EB Kulstad', DM Courtney ${ }^{2}$, P Shanley ${ }^{3}$, A Metzger ${ }^{3}$, T Matsuura ${ }^{3}$, J Rees ${ }^{4}$, K Lurie $^{3}$, S McKnite ${ }^{5}$

'Advocate Christ Medical Center, Oak Lawn, IL, USA; ${ }^{2}$ Northwestern University,

Chicago, IL, USA; ${ }^{3}$ University of Minnesota Medical Center, Minneapolis, MN,

USA: ${ }^{4}$ Advanced Circulatory Systems, Minneapolis, MN, USA: ${ }^{5}$ Minneapolis

Medical Research Foundation, Minneapolis, MN, USA

Critical Care 2013, 17(Suppl 2):P314 (doi: 10.1186/cc12252)

Introduction Measurement of temperature during treatment with therapeutic hypothermia can yield variable values depending on where temperature is measured. Measurements of temperature can be made rectally, intravascularly, vaginally, or from the bladder, but agreement between these sites is often uncertain. We measured temperature at multiple sites during experimental induction and reversal of hypothermia in swine with a novel esophageal cooling device, hypothesizing that agreement between sites would fall within standard acceptance criteria (average difference \pm 1.96 standard deviation of the difference) in Bland-Altman analyses.

Methods Five female Yorkshire swine (mean weight $65 \mathrm{~kg}$ ) were anesthetized and cooled for 24 hours, then gradually rewarmed, with a novel esophageal heat transfer device powered by an external chiller (Gaymar MediTherm III). Swine temperature was measured intravascularly, rectally, and either vaginally or in the bladder. Comparisons between temperature readings were then made via Bland-Altman plots.

Results Agreement between the different sources of temperature measurement was generally high, with less than $10 \%$ outliers beyond \pm 1.96 SD. The best agreement was seen between intravascular temperature measurement and bladder temperature measurement $\left(\mathrm{SD}=0.14^{\circ} \mathrm{C}\right)$, with vaginal measurements showing less agreement $\left(\mathrm{SD}=0.23^{\circ} \mathrm{C}\right)$, and rectal measurements showing the least $\left(\mathrm{SD}=0.31^{\circ} \mathrm{C}\right)$ with intravascular.

Conclusion Bladder temperature measurements demonstrated the best agreement with intravascular temperature measurements during cooling with an esophageal heat transfer device; however, reasonable agreement was demonstrated with rectal and vaginal temperature measurements, suggesting that these sites are also acceptable for use. Reference

1. Arrich J, Holzer M, Herkner H, Mullner M: Hypothermia for neuroprotection in adults after cardiopulmonary resuscitation. Cochrane Database Syst Rev 2009, 4:CD004128.

P315

Association between oxygenation and 6-month mortality during post-cardiac arrest care

M Skrifvars ${ }^{1}$, JVaahersalo ${ }^{1}$, M Reinikainen², S Bendel', J Kurola ${ }^{3}$, M Tiainen $^{1}$ R Raj', V Pettilä', TVarpula', FinnResusci Study Group

'Helsinki University Hospital, HUS, Finland; '2North Karelia Central Hosital,

Joensuu, Finland: ${ }^{3}$ Kuopio University Hospital, Kuopio, Finland

Critical Care 2013, 17(Suppl 2):P315 (doi: 10.1186/cc12253)

Introduction Optimal oxygenation level during post-cardiac arrest (PCA) care is currently undefined, and studies have suggested harm from hyperoxia exposure [1]. We aimed to assess the optimal oxygenation level and possible associations of time-weighted exposure to hyperoxia on outcome in patients during PCA care.

Methods We conducted a prospective observational cohort study in 21 ICUs in Finland between 2009 and 2010. The Utstein Guidelines were used for collecting resuscitation and PCA care data, such as initial rhythm and delay to return of spontaneous circulation (ROSC). Measured arterial blood oxygen values during the first 24 hours from admission to the ICU were divided into the following predefined oxygenation categories: low $(<10 \mathrm{kPa})$, normal (10 to $19 \mathrm{kPa})$, intermediate (20 to $29 \mathrm{kPa}$ ), and high ( $>30 \mathrm{kPa}$ ). Exposure to hyperoxia was defined as $\mathrm{paO}_{2}$ levels higher than $40 \mathrm{kPa}$ [1]. Time spent in different oxygenation categories and the highest, lowest and median oxygen values during the first 24 hours were calculated and included in separate multivariate regression models along with age, delay to ROSC, initial rhythm and the use of therapeutic hypothermia for the prediction of 6-month mortality.

Results A total of 489 patients were included. The average number of $\mathrm{paO}_{2}$ measurements during the first 24 hours was eight per patient. A total of $6 \%$ of patients experienced paO, values higher than $40 \mathrm{kPa}$ at any time during the first 24 hours. Average times spent in each time oxygenation category during the first 24 hours were as follows: low $14 \%$, normal $69 \%$, intermediate $14 \%$, and high $2 \%$ of the time. Survivors spent less time in the low band $(P=0.029)$ and more time in the intermediate band $(P=0.029)$ compared with nonsurvivors. The median $\mathrm{paO}_{2}$ during the first 24 hours was higher in survivors than in nonsurvivors ( $15 \mathrm{kPa}$ vs. $14 \mathrm{kPa}, P=0.016$ ) but there was no difference in lowest (11 kPa vs. $10 \mathrm{kPa}, P=0.162$ ) or the highest $\mathrm{paO}_{2}$ values 
(22 kPa vs. $20 \mathrm{kPa}, P=0.054$ ). In separate multivariate models neither time spent in the low or the intermediate categories, or the median, highest or lowest $\mathrm{paO}_{2}$ was found to correlate with mortality.

Conclusion In this multicentre observational study we were unable to define an optimal oxygenation level during PCA care, but hypoxia seemed to be more harmful than hyperoxia. Exposure to hyperoxia was less common than in previous trials, and we were unable to confirm previous findings indicating an association with mortality.

\section{Reference}

1. Kilgannon JH, et al:. JAMA 2010, 303:2165-2171.

\section{P316}

Neuron-specific enolase and bispectral index/suppression ratio for prognostication after cardiac arrest

M Van Laer', K Deschilder², P Lormans², J Gillet', W Stockman²

'UZ Leuven, Belgium; ${ }^{2 H}$ HRM, Roeselare, Belgium

Critical Care 2013, 17(Suppl 2):P316 (doi: 10.1186/cc12254)

Introduction Neuron-specific enolase (NSE) values $>33 \mu \mathrm{g} / \mathrm{ml}$ [1] and low bispectral index (BIS) [2] values correlate with bad outcome after cardiac arrest (CA).

Methods In this nonblinded prospective study, we observed all CA patients from February 2011 until September 2012 surviving at least 24 hours. NSE was measured between 24 and 72 hours after CA. From October 2011 onward, we recorded BIS and suppression ratio (SR) values as soon as possible after arrival in the ICU. Patients treated with therapeutic hypothermia $(\mathrm{TH})\left(33^{\circ} \mathrm{C}\right.$ for 24 hours) received cisatracurium. Cerebral Performance Category (CPC) [3] 1 and 2 were considered good outcome, CPC 3 to 5 bad outcome and were recorded after 3 months. Statistical analysis was performed using SPSS statistics 19.

Results NSE $>45$ occurred in $24 / 68$ patients (35.3\%) and invariably correlates with bad outcome. The positive predictive value (PPV) NSE $>45$ for bad outcome is $100 \%$. No patient in this group ever had a GCS $\geq 12$. NSE $>33$ and $<45$ occurred in $16 / 68$ patients (23.5\%). Thirteen out of 16 patients (81.2\%) had bad outcome. However, $7 / 16$ patients (43.8\%) woke up at some time (GCS $\geq 12$ ). NSE $<33$ occurred in $28 / 68$ patients $(41.2 \%), 17 / 28$ patients $(60.7 \%)$ had good outcome and $23 / 28$ patients (88.4\%) had GCS $\geq 12$ at some time. The PPV NSE $<33$ for good outcome is $60.7 \%$. The BIS and SR were measured in only 28 patients. Initial BIS $\leq 10$ occurred in $13 / 28$ patients $(46.4 \%)$ and correlates with bad outcome in $12 / 13$ patients $(92.3 \%)$. BIS $>30$ occurred in nine patients, 6/9 (66.7\%) had good outcome. Initial SR $\geq 75$ occurred in $11 / 28$ patients $(39.3 \%)$ and invariably correlates with bad outcome. NSE $>25$ and SR $>60$ occurred in $15 / 28$ patients (53.6\%) and invariably correlates with bad outcome.

Conclusion NSE > 45 uniformly correlates with bad outcome after CA. However, we urge caution for the use of intermediate values (33 to 45). In preliminary data, we report that SR $>75$ might correlate with bad outcome and that combining NSE and SR might improve the predictive value. Also, low NSE and good initial BIS values correlate with preserved cerebral potential and should encourage the clinician.

\section{References}

1. Zandbergen et al: Neurology 2006, 66:62-68.

2. Leary et al:: Resuscitation 2010, 81:1133-1137.

3. Ajam et al:: Scand J Trauma Resusc Emerg Med 2011, 19:38.

P317

Motor scores, therapeutic hypothermia and neurological outcome after cardiac arrest

M Davidson

Plymouth Hospitals NHS Trust, Plymouth, UK

Critical Care 2013, 17(Suppl 2):P317 (doi: 10.1186/cc12255)

Introduction Accurate prediction of neurological outcome after cardiac arrest is desirable to prevent inappropriate withdrawal of lifesustaining therapy in patients who could have a good neurological outcome, and to limit active treatment in patients whose ultimate neurological outcomes are poor. Established guidelines to predict neurological outcome after cardiac arrest were developed before the widespread use of therapeutic hypothermia. The American Association of Neurology guidelines [1] currently recommend that absent or extensor motor scores on day 3 post arrest are reliable indicators or poor neurological outcome with a false positive rate of 0 to $3 \%$.

Methods A review of existing literature was undertaken to examine whether the utility of motor scores to predict poor neurological outcome is influenced by the use of therapeutic hypothermia.

Results Six studies were identified [2-7] that investigated the use of motor scores on day 3 post cardiac arrest in patients who had received therapeutic hypothermia. False positive rates (defined as 1 - specificity) for predicting poor neurological outcome were calculable in five of the six studies [2-6] and were $14 \%, 24 \%, 11 \%, 25 \%$ and $12 \%$ respectively. In all studies the FPR for motor scores of extension or worse were significantly higher than the $0 \%(0$ to $3 \% 95 \% \mathrm{Cls}$ ) in the AAN guidelines. Conclusion Motor scores at day 3 post cardiac arrest of extension or worse do not reliably predict poor neurological outcome when therapeutic hypothermia has been used. Clinical neurological findings may not be valid predictors of poor neurological outcome after therapeutic hypothermia.

References

1. Wijdicks et al.: Neurology 2006, 67:203-210.

2. Al Thenayan et al: Neurology 2008, 71:1535-1537.

3. Rossetti et al:: Ann Neurol 2010, 67:301-307.

4. Bisschops et al: Resuscitation 2011, 82:696-701.

5. Cronberg et al:: Neurology 2011, 77:623-630

6. Samaniego et al:: Neurocrit Care 2011, 15:113-119.

7. Fugate et al:: Ann Neurol 2010, 68:907-914.

\section{P318}

Differences of calcium binding protein immunoreactivities in the young hippocampal CA1 region from the adult following transient ischemic damage

CW Park, HY Lee, JH Cho, MH Won

Kangwon National University, Chuncheonsi, South Korea

Critical Care 2013, 17(Suppl 2):P318 (doi: 10.1186/cc12256)

Introduction It has been reported that the young are much more resistant to transient cerebral ischemia than the adult.

Methods In the present study, we compared the chronological changes of calcium binding proteins (CBPs) (calbindin 28k (CB-D 28k), calretinin (CR) and parvalbumin (PV)) immunoreactivities and levels in the hippocampal CA1 region of the young gerbil with those in the adult following 5 minutes of transient cerebral ischemia induced by the occlusion of both the common carotid arteries.

Results In the present study, we examined that about $90 \%$ of CA1 pyramidal cells in the adult gerbil hippocampus died at 4 days post ischemia; however, in the young hippocampus, about $56 \%$ of them died at 7 days post ischemia. We compared immunoreactivities and levels of CBPs, such as CB-D 28k, CR and PV. The immunoreactivities and protein levels of all the CBPs in the young sham were higher than those in the adult sham. In the adult, the immunoreactivities and protein levels of all the CBPs were markedly decreased at 4 days post ischemia; however, in the young, they were apparently maintained. At 7 days post ischemia, they were decreased in the young; however, they were much higher than those in the adult.

Conclusion In brief, the immunoreactivities and levels of CBPs were not decreased in the ischemic $C A 1$ region of the young 4 days after transient cerebral ischemia. This finding indicates that the longer maintenance of CBPs may contribute to a less and more delayed neuronal death/ damage in the young.

P319

Early hypothermia improves survival and reduces the rise of serum biomarkers after traumatic brain injury in swine

M Kumar, AD Goldberg, M Kashiouris, L Keenan, A Rabinstein

Mayo Clinic, Rochester, MN, USA

Critical Care 2013, 17(Suppl 2):P319 (doi: 10.1186/cc12257)

Introduction Poor outcomes in clinical trials on the use of therapeutic hypothermia following traumatic brain injury (TBI) may be due to the 
delay in reaching target temperature [1]. We hypothesize that early and rapid induction of hypothermia will mitigate neuronal injury and improve survival in a swine model of TBI.

Methods Twenty domestic cross-bred pigs ( 34 to $35 \mathrm{~kg}$ ) were subjected to a 5 ATM $(100 \mathrm{~ms})$ lateral fluid percussion TBI. The brain temperature and ICP were measured using Camino ${ }^{\oplus}$. Serum biomarkers for neuronal injury - S-100ß, neuron-specific enolase, glial fibrillary acid protein (GFAP), and neurofilaments heavy chain - were measured daily using enzyme-linked immunosorbent assay. Twelve of the injured animals were rapidly cooled to $32^{\circ} \mathrm{C}$ within 90 minutes of the injury using a transpulmonary hypothermia technique [2]. Hypothermia was maintained for 48 hours. Eight injured control animals were maintained at $37^{\circ} \mathrm{C}$. In both groups, anesthesia (isoflurane $1 \%$ ) was discontinued and the animals were weaned off the ventilator after 48 hours. Five days post injury, the surviving animals were euthanized and necropsied. The data were analyzed using a log-rank (Mantel-Cox) test, and ANOVA.

Results Ten of the 12 hypothermia and four of the eight normothermia animals survived to the end of the 5 -day study $\left(x^{2}=2.597, \mathrm{df}=1\right.$, $P=0.1071)$. Although the probability of type I error between survival curves was $11 \%$, the study was clinically significant and showed a clear trend toward improved survival with hypothermia. The intracranial pressures were significantly $(P<0.05)$ lower in the hypothermia group. Both interventions - that is, general anesthesia and hypothermia mitigated the rise of serum biomarkers following TBI. However, the suppression of biomarkers was sustained during the recovery period only in the hypothermia group. With the exception of the GFAP levels, the curves of all biomarkers were significantly different between the groups.

Conclusion Our preliminary findings show early initiation, rapid induction, and prolonged maintenance (48 hours) of cerebral hypothermia to lower intracranial pressure, blunt the rise in serum biomarkers, and improve survival following TBI.

\section{References}

1. Clifton GL, et al: Lancet Neurol 2011, 10:131-139.

2. Kumar M.e et al: Anesth Analg 2012, 114:S-160.

P320

Epidemiology of moderate and severe traumatic brain injury in Cairo University Hospital in $\mathbf{2 0 1 0}$

T Montaser', A Hassan ${ }^{2}$

'Shobra General Hospital, Cairo, Egypt; ${ }^{2}$ King Khaled University Hospital

Riyadh, Saudi Arabia

Critical Care 2013, 17(Suppl 2):P320 (doi: 10.1186/cc12258)

Introduction Traumatic brain injury (TBI) is a contributing factor to approximately one-third of all injury-related deaths in the USA annually. Updated statistical records for TBI in Egypt are lacking. The current research is aiming to estimate the prevalence of TBI in Egypt in order to develop a comprehensive TBI prevention program.

Methods A 1-year period (one calendar month every quarter of 2010) descriptive epidemiological study of moderate and severe TBI cases admitted to the emergency department, Cairo main university hospital. The data collection sheet included personal data (age, sex and residency), incident-related data (cause, nature and time of injury) and both clinical and radiological findings.
Results Table 1 shows the magnitude of the problem, highlighting the leading causes of TBI in Egypt in 2010. Male sex was predominantly affected, $79 \%$ of cases. Moderate and severe injuries account for $17.2 \%$ of all TBI presented cases.

Conclusion TBI is a serious public health problem in Egypt. Further data interpretation over wider periods of time should be conducted for better understanding of TBI prevalence is highly recommended to develop effective injury prevention programs.

\section{P321}

Alcohol intoxication impedes the recognition of traumatic brain injury in the prehospital setting and may worsen 6-month outcome R Raj, I Siironen, R Kivisaari, M Kuisma, M Skrifvars Helsinki University Central Hospital, Helsinki, Finland Critical Care 2013, 17(Suppl 2):P321 (doi: 10.1186/cc12259)

Introduction Transport directly to a neurosurgical trauma center has shown to reduce mortality in patients with traumatic brain injury (TBI). We hypothesized that alcohol intoxication may impede prehospital recognition of $\mathrm{TBI}$, resulting in transportation to a non-neurosurgical hospital.

Methods A retrospective analysis of TBI patients admitted to a designated neurosurgical trauma center's ICU in 2009/10 and primarily treated by the Emergency Medical Service (EMS). Patients were divided into two categories based either direct or indirect trauma transfer by the EMS. Directly transferred patients are directly transported to the neurosurgical trauma center from the injury scene while indirectly transported patients are initially transported to another nonneurosurgical hospital before re-transfer to the trauma center. Data from patient journals and EMS forms were extracted. The blood alcohol level (BAL) was measured by the EMS using an alcohol breath-test. Logistic regression modeling was used to identify variables present at scene associated with transport destination.

Results Totally 470 patients met the inclusion criteria; $60 \%$ were transported directly and $40 \%$ indirectly. In the direct group $15 \%$ of patients had a positive BAL, compared with $26 \%$ for those indirectly transported. In the logistic regression model, factors associated with direct transport were: $\mathrm{BAL} \geq 2.3 \%$ ( $\mathrm{OR}: 0.06, \mathrm{Cl}: 0.01$ to 0.36 ), male gender (OR: 0.35 ; $\mathrm{Cl}: 0.16$ to 0.76 ), GCS 13 to 15 (OR: $0.28 ; \mathrm{Cl}$ 0.10 to 0.74 ), high-energy trauma (OR: $9.42 ; \mathrm{Cl} 2.15$ to 41.20 ), major extracranial injury (OR: 7.92; $\mathrm{Cl} 2.57$ to 24.41), EMS physician telephone consultation (OR: $6.02 ; \mathrm{Cl} 2.51$ to 14.11 ) or presence on scene (OR: 8.63; $\mathrm{Cl} 3.50$ to 21.26 ) and incident at a public place outside (OR: 3.05 ; $\mathrm{Cl}$ : 1.34 to 6.4 ) and inside (OR: 2.92; Cl: 1.07 to 8.01 ). Median time delay to trauma center admission was 1:07 hours (IQR: 0.52 to $1: 28$ ) for directly transported patients and 4:06 (IQR: 2.54 to 5:43) for those indirectly transported $(P<0.001)$. There was a clear trend towards poorer neurological outcome for patients with delayed trauma center admission in univariate analysis $(P=0.001)$.

Conclusion Heavily alcohol intoxicated TBI patients are commonly initially transported to a non-neurosurgical trauma center and this may worsen 6-month neurological outcome.

References

1. Hartl R, et al: J Trauma 2006, 60:1250-1256.

2. Shahin H, et al:: J Trauma 2010, 69:1176-1181.

Table 1 (abstract P320). Causes of TBI in different age groups in Egypt

\begin{tabular}{lccccccc}
\hline Age (years) & $\begin{array}{c}\text { NO }=844 / \\
\text { 4 months }\end{array}$ & FFH & MVC & $\begin{array}{c}\text { Abuse/ } \\
\text { neglect }\end{array}$ & $\begin{array}{c}\text { Violence/ } \\
\text { assaults }\end{array}$ & $\begin{array}{c}\text { Struck by/ } \\
\text { against }\end{array}$ & $\begin{array}{c}\text { Other causes/ } \\
\text { not known }\end{array}$ \\
\hline 1 to 5 & $54(6 \%)$ & $20(37 \%)$ & $10(19 \%)$ & $8(15 \%)$ & - & $8(15 \%)$ & $8(14 \%)$ \\
6 to 18 & $91(11 \%)$ & $28(31 \%)$ & $20(22 \%)$ & $5(6 \%)$ & $6(5 \%)$ & $22(25 \%)$ & $10(11 \%)$ \\
19 to 29 & $150(18 \%)$ & $49(33 \%)$ & $45(30 \%)$ & - & $14(9 \%)$ & $18(12 \%)$ & $24(16 \%)$ \\
30 to 55 & $381(45 \%)$ & $114(30 \%)$ & $134(35 \%)$ & - & $19(5 \%)$ & $50(13 \%)$ & $64(17 \%)$ \\
Above 55 & $168(20 \%)$ & $47(28 \%)$ & $41(24 \%)$ & $11(7 \%)$ & $5(3 \%)$ & $25(15 \%)$ & $39(23 \%)$ \\
\hline
\end{tabular}


P322

Predictive ability of the IMPACT-TBI prognostic model is improved by adding markers of coagulation

R Raj, J Siironen, R Kivisaari, L Handolin, J Hernesniemi, M Skrifvars

Helsinki University Central Hospital, Helsinki, Finland

Critical Care 2013, 17(Suppl 2):P322 (doi: 10.1186/cc12260)

Introduction One of the most used prognostic models for traumatic brain injury is the IMPACT-TBI model, which predicts 6-month mortality and unfavorable outcome. Our aim was to study whether adding markers of coagulation improves the model's predictive power when accounting for extracranial injury.

Methods Patients with a TBI admitted to a designated trauma center in 2009/10 were screened retrospectively and included according to the IMPACT study criteria. The predictive outcome was calculated for included patients using the full IMPACT-TBI model. To assess coagulopathy and extracranial injury we used the prothrombin time percentage (PT), platelet count $\left(10^{9}\right)$, and injury severity score (ISS). PT, platelets and ISS were added to the IMPACT model, creating new models. The predictive power of the new models was tested by comparing their area under the receiver operating characteristic curve (AUC) with the original model.

Results Totally 342 were included. Six-month mortality was $32 \%$ and unfavorable outcome $57 \%$. The IMPACT model's predictive power for mortality and neurological outcome; AUC: $0.85, \mathrm{Cl}: 0.81$ to 0.89 ; and AUC: $0.81, \mathrm{Cl}: 0.76$ to 0.86 . Nonsurvivors and patients with an unfavorable outcome had lower platelets (159; 165 vs. 191; 198), PT (76; 77 vs. $86 ; 90)$ and higher ISS $(50 ; 44 \%$ ISS $>25$ vs. $34 ; 32 \%$ ISS $>25)$ compared with survivors and favorable outcome $(P<0.05)$. Addition of PT but not ISS nor platelets resulted in a significant improvement of the IMPACT model's predictive power for 6 -month mortality; AUC: $0.87, \mathrm{Cl}$ : 0.81 to $0.89, P=0.017$ (Figure 1). In multivariate analysis PT remained an independent significant predictor of outcome when adjusting for IMPACT prognosis and ISS (OR: 0.985, Cl: 0.975 to 0.996 ).

Conclusion Addition of PT to the IMPACT model improves the predictive power for 6-month mortality but not neurological outcome when adjusting for ISS.

References

1. Steyerberg EW, et al:: PLoS Med 2008, 5:e165.

2. Marmarou A, et al.: J Neurotrauma 2007, 24:239-250.

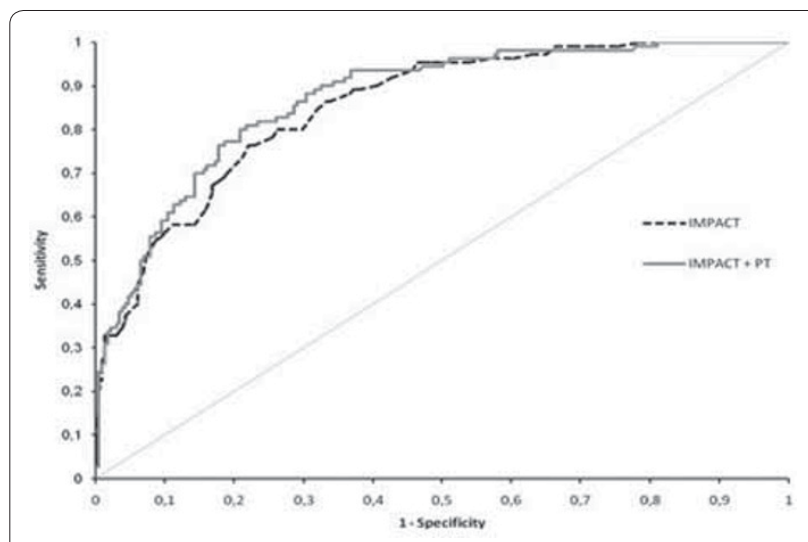

Figure 1 (abstract P322).

\section{P323}

Nonischemic endogenous lactate production in humans with severe traumatic brain injury

N Sala', T Suys', JB Zerlauth', J Bloch'1, P Magistretti², R Meuli', M Oddo 'Lausanne University Hospital, Lausanne, Switzerland; 'EPFL, Lausanne,

Switzerland

Critical Care 2013, 17(Suppl 2):P323 (doi: 10.1186/cc12261)

Introduction Evidence suggest that endogenous lactate, produced by aerobic glycolysis, is an important substrate for neurons, particularly in conditions of increased energy demand. This study aimed to examine brain lactate metabolism in patients with severe traumatic brain injury (STBI).

Methods A prospective cohort of STBI patients monitored with cerebral microdialysis (CMD) and brain tissue oxygen ( $\mathrm{PbtO}_{2}$ ) was studied. Brain lactate metabolism was assessed by quantification of elevated CMD lactate samples ( $>4 \mathrm{mmol} / \mathrm{l})$. These were matched to pyruvate and $\mathrm{PbtO}_{2}$, and dichotomized as hyperglycolytic (CMD pyruvate $>119 \mu \mathrm{mol} / \mathrm{l}$ ) versus nonhyperglycolytic or as hypoxic $\left(\mathrm{PbtO}_{2}\right.$ $<20 \mathrm{mmHg}$ ) versus nonhypoxic. Data were expressed as percentages per patient. Global brain perfusion (categorized as oligemic, normal or hyperemic) was assessed with CT perfusion (CTP).

Results Twenty-four patients (total 1,782 CMD samples) were studied. Samples with elevated CMD lactate were frequently observed $(41 \pm 8 \%$ SEM of individual samples). Brain lactate elevations were predominantly hyperglycolytic $(73 \pm 8.2 \%)$, whilst only $14 \pm 6.3 \%$ of them were hypoxic. Trends over time of both lactate patterns are shown in Figure 1. On CTP ( $n=17$; average 48 hours from TBI) hyperglycolytic lactate was always associated with normal or hyperemic CTP, whilst hypoxic lactate was associated with oligemic CTP (Table 1).

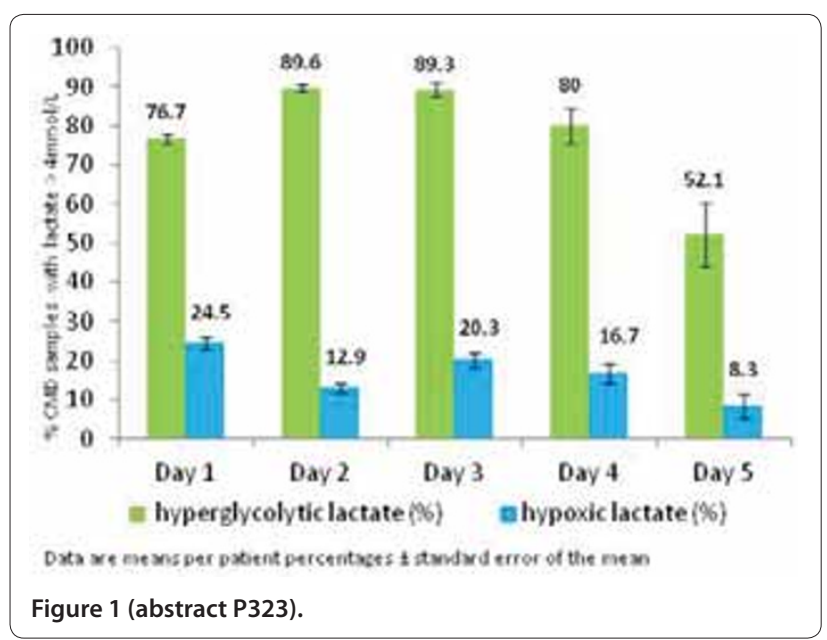

Table 1 (abstract P323). Lactate elevations and brain perfusion

\begin{tabular}{lccc}
\hline & Oligemia (\%) & Normal (\%) & Hyperemia (\%) \\
\hline HG lactate & 0 & 58 & 42 \\
HX lactate & 100 & 0 & 0 \\
\hline
\end{tabular}

HG, hyperglycolytic; HX, hypoxic.

Conclusion Our findings suggest predominant nonischemic lactate release after TBI and identify, for the first time, an association between cerebral hyperglycolytic lactate production and normal to supranormal brain perfusion. Our data support the concept that lactate may be used as energy substrate by the injured human brain.

Acknowledgement Supported by the Swiss National Science Foundation.

\section{P324}

Japan Coma Scale used in the prehospital setting can predict clinical outcome in severe pediatric trauma

TYagi, N Saito, Y Hara, H Hisashi Matumoto, K Mashiko Nippon Medical School Chiba Hokusoh Hospital, Inzai/Chiba, Japan

Critical Care 2013, 17(Suppl 2):P324 (doi: 10.1186/cc12262)

Introduction In the prehospital setting, it is difficult to use the Glasgow Coma Scale (GCS) to evaluate the consciousness state using in pediatric patients with severe trauma. The Japan Coma Scale (JCS) is a consciousness scale used widely in Japan and, with its four grades, is simpler and quicker to use than the GCS. This study examined whether 
the JCS can predict clinical traumatic brain injury (TBI) and outcome in pediatric patients aged 3 to 15 years in the prehospital setting. Methods This retrospective study analyzed data from the Japan Trauma Data Bank. Registered pediatric patients aged 3 to 15 years with severe trauma (maximum Abbreviated Injury Scale score $\geq 3$ or Injury Severity Score $\geq 9$ ) were divided into four groups according to JCS score in the prehospital setting (Grade 0: alert, Grade 1: possible eye-opening, not lucid, Grade 2: possible eye-opening upon stimulation, Grade 3: no eye-opening and coma). TBI was defined as maximum head AIS $\geq 3$.

Results A total of 1,562 patients were included (Grade 0: 673, Grade 1: 410, Grade 2: 230, Grade 3: 249). Victims of blunt trauma accounted for $98.1 \%$. Median age was 9 (interquartile range: 7 to 12) years, median ISS was 16 (9 to 21). There was strong agreement between the JCS in prehospital setting and GCS scores on arrival at hospital $(r=-0.745$, $P<0.001)$. Multivariate analysis adjusted for age and ISS revealed that the JCS was independently associated with TBI (odds ratio (OR): 2.5 ; $95 \% \mathrm{Cl}: 2.1$ to $2.8, P<0.001$ ) and hospital mortality (OR: 3.8; $95 \% \mathrm{Cl} 2.4$ to $6.0, P<0.001)$. See Table 1 .

Table 1 (abstract P324)

\begin{tabular}{lcc}
\hline & TBI, $\boldsymbol{n}(\%)$ & Mortality (\%) \\
\hline JCS grade 0 & $213(31.6)$ & 0.1 \\
JCS grade 1 & $225(62.2)$ & 0.7 \\
JCS grade 2 & $184(80.0)$ & 2.6 \\
JCS grade 3 & $230(92.4)$ & 15.7 \\
\hline
\end{tabular}

Conclusion There was strong association between JCS score and clinical outcome in pediatric patients with severe trauma. The results support the use of the JCS in the prehospital transport destination criteria for children.

\section{P325}

Effect of positive end-expiratory pressure on ultrasound

measurement of optical sheath nerve diameter: preliminary study

TAslanidis, E Anastasiou, E Geka, E Efthimiou, A Myrou, A Kontos,

D Papadopoulos, E Boultoukas, M Giannakou-Peftoulidou

A.H.E.P.A. University Hospital, Thessaloniki, Greece

Critical Care 2013, 17(Suppl 2):P325 (doi: 10.1186/cc12263)

Introduction Non-invasive ocular ultrasonography has recently been proposed to detect elevated intracranial pressure (ICP). On the other hand, the effect of positive end-expiratory pressure (PEEP) on ICP is well documented. The aim of the present ongoing study is to record the effect of changes of airway pressures on optical sheath nerve diameter (OSND).
Methods In this prospective observational study, measurements of OSND were carried out in 21 patients of a polyvalent 10-bed adult ICU before and 2 minutes after changes of PEEP from a baseline of 5 with $1 \mathrm{cmH}_{2} \mathrm{O}$ increments, to a maximum of 12 . Two measurements were performed with a $7.5 \mathrm{MHz}$ probe (MicroMaxx; Fujifilm Sonosite Inc., USA) in each eye before and after PEEP changes. Demographic data (APACHE II score, age, sex, diagnosis) Ppeak and Pm were also recorded. Exclusion criteria were intracranial pathology, ocular neuritis and trauma. Normality with P-P plots and the Kolmogorov-Smirnov test were calculated followed by bivariate and single linear regression analysis (IBM SPPS $v 17$, significance $P<0.05$ ).

Results Seventy-two measurements were included for further analysis. Mean \pm SD values were: PEEP $(7.9 \pm 2.3)$, Ppeak $(24.35 \pm 5)$, Pm $(12.03 \pm 3.16)$, OSNDright $(4.03 \pm 0.79)$, OSNDleft $(4.1 \pm 0.79), \triangle$ OSND $(0.24 \pm 0.2)$, while values for age and APACHE II score were $62 \pm 4$ years and $14.4 \pm 1.4$ respectively. PEEP and OSND seem to have a moderate relation (tendency equation: $\mathrm{OSND}=2.454+0.581 \times \mathrm{PEEP}$ ) compared with the weaker effect of Ppeak and Pm (Table 1).

Table 1 (abstract P325). Correlations $\left(r^{2}\right)$ between airway pressures and OSND

\begin{tabular}{lcc}
\hline & OSNDright & OSNDleft \\
\hline PEEP & $0.581^{*}$ & $0.571^{*}$ \\
Ppeak & $0.467^{*}$ & $0.448^{*}$ \\
Pm & $0.491^{* *}$ & $0.467^{* *}$ \\
\hline
\end{tabular}

${ }^{*} P<0.01,{ }^{* *} P<0.05$.

Conclusion Our study identified a moderate relation between PEEP and OSND and a weaker one between Ppeak, Pm and OSND. Thus, in selected cases OSND could serve as a bedside marker of effect of airway pressure to ICP. Yet, larger studies are needed to come to a safer conclusion.

Reference

1. Geeraerts T, et al:: Int Care Med 2008, 34:2062-2067.

P326

National survey of current protocols and management of the traumatic brain injury patients in UK ICUs

B Lewinsohn, S Panchatsharam, S Wijayatilake, A Billini, G Delacedra, R Jain, J Khan, R Shetty, A Lewinsohn

Queens Hospital, Romford, UK

Critical Care 2013, 17(Suppl 2):P326 (doi: 10.1186/cc12264)

Introduction Following primary neurological insult, initial management of traumatic brain-injured (TBI) patients has a clearly defined pathway [1]. However, after arrival at tertiary centers, further management is not

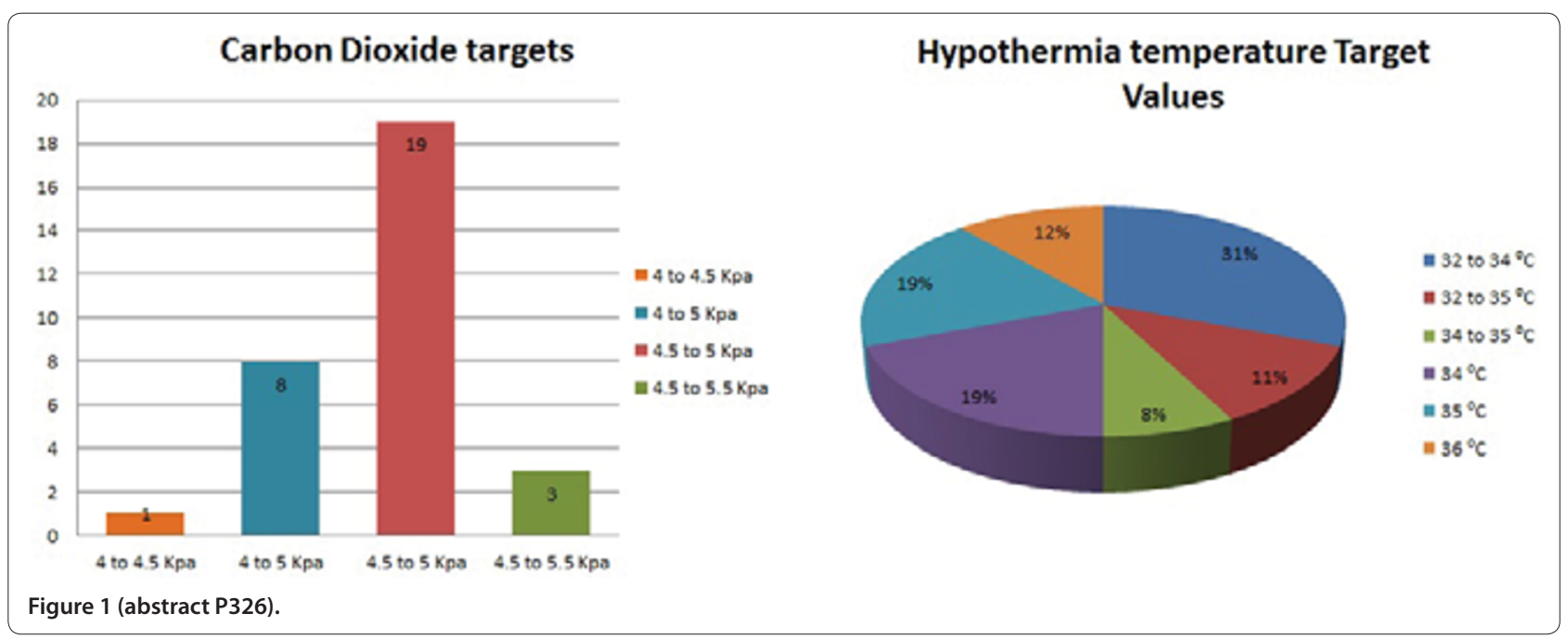


standardized. Intracranial hypertension (ICH), systemic hypotension, hypoxia, hyperpyrexia and hypocapnia have all been shown to independently increase mortality [2]. Despite numerous studies, there is currently no level 1 evidence to support any specific management [3]. Our objective was to provide an overview of the current clinical management protocols in the UK.

Methods Thirty-one ICUs managing patients with severe TBI were identified from the RAIN (Risk Adjustment In Neurocritical care) study, and a telephone survey was conducted.

Results A total $97 \%$ of units used a cerebral perfusion pressure protocol for the initial management, with $83 \%$ targeting pressures of 60 to $70 \mathrm{mmHg}$ and $17 \%$ aimed for $>70 \mathrm{mmHg}$. Ninety-one percent of units monitored $\mathrm{CO}_{2}$ routinely with $61 \%$ targeting $\mathrm{CO}_{2}$ of 4.5 to $5 \mathrm{kPa}$ (Figure 1). Regarding osmotherapy, mannitol was still the preferred agent, with $48 \%$ of units using it as first line; $32 \%$ used hypertonic saline, while $20 \%$ of units used either depending on clinicians' preference. Sixteen percent questioned were currently enrolled on the Eurotherm hypothermia trial, while $16 \%$ never used hypothermia and one unit used prophylactic hypothermia routinely. The remaining $65 \%$ of units used hypothermia only to manage refractory $\mathrm{ICH}$.

Conclusion There is no clear consensus on the initial targets used. The surviving sepsis campaign showed that protocol-led care can reduce mortality [4]. Perhaps it is time for a similar approach to be adopted, with specialists coming to together to review the evidence and formulate guidelines that can then be tested.

References

1. NICE: Head Injury; Triage, Assessment, Investigation and Early Management of Head Injury. London: National Collaborating Centre for Acute Care:

September 2007.

2. Wijayatilake et al:: Updates in the management of intracranial pressure in traumatic brain injury. Curr Opin Anaesthesiol 2012, 25:540-547.

3. Guidelines for the Management of Severe Traumatic Brain Injury. 3rd edition. New York: Brain Trauma Foundation; 2007.

4. Barochia et al:: Bundled care for septic shock. Crit Care Med 2010, 38:668-678.

P327

Low-frequency autoregulation index for calculation of optimal cerebral perfusion pressure in severe traumatic brain injury

F Guiza', G Meyfroidt' ${ }^{1}$, M Schuhmann², G Van den Berghe', I Piper ${ }^{3}$, B Depreitere

'University Hospitals Leuven, Belgium; ${ }^{2}$ University Hospital Tübingen

Germany; 3 Southern General Hospital, Glasgow, UK

Critical Care 2013, 17(Suppl 2):P327 (doi: 10.1186/cc12265)

Introduction Traumatic brain injury (TBI) is a major cause of permanent disability and death in young patients. Controversy exists regarding the optimal cerebral perfusion pressure (CPP) required in TBI management. A tool for monitoring autoregulation and determining an optimal CPP is the pressure reactivity index (PRx), defined as a moving correlation coefficient between the mean arterial blood pressure (MAP) and intracranial pressure (ICP) at a frequency of at least $60 \mathrm{~Hz}$. This requirement of high frequency has constrained its use to a few academic centers. An association was shown between outcome and continuous optimal CPP based on 4 hours of PRx [1]. We present a novel low-frequency autoregulation index (LAx), based on correlations between ICP and MAP at a standard minute-by-minute time resolution. Methods A total of 182 patients from the Brain-IT [2] multicentre European database had registered outcome and ICP and MAP for the first 48 ICU hours. Twenty-one TBI patients admitted to the university hospitals of Leuven, Belgium and Tubingen, Germany were continuously monitored using ICM+ software (Cambridge Enterprise) allowing for continuous PRx calculation. Autoregulation indices versus CPP plots for PRx and LAx were computed to determine optimal CPP every minute during the first 48 ICU hours [1].

Results On the Brain-IT database, LAx resulted in an optimal CPP for $90 \%$ of the first 48 hours. Table 1 shows recommendations with respect to outcome. In the Leuven-Tübingen database, PRx and LAx resulted in $44 \%$ and $92 \%$ recommendations respectively. The average difference between methods was $5.26 \mathrm{mmHg}$.

Conclusion The differences in optimal CPPs derived from PRx and LAx were not clinically significant. LAx allowed for recommendations to be
Table 1 (abstract P327)

\begin{tabular}{lccc}
\hline & Nonsurvivors & Survivors & P value \\
\hline Time within CPPopt-\% & $18.3(15.5 ; 24.4)$ & $24.8(19.7 ; 28.7)$ & 0.0004 \\
|CPP-CPPopt $(\mathrm{mmHg})$ & $7.05(4.98 ; 9.73)$ & $5.37(4.49 ; 6.82)$ & 0.0024 \\
\hline
\end{tabular}

computed for longer periods. Significantly better outcome (Table 1) was observed in patients for whom optimal CPP derived from LAx was maintained.

References

1. Aries M, et al.: Crit Care Med 2012, 40:2456-2463.

2. Piper I, et al:: Acta Neurochirurgica 2003, 145:615-628.

P328

Cerebral $\mathrm{rSO}_{2}$ monitoring in pediatric altered mental status patients

T Abramo, I Kane

Vanderbilt School of Medicine, Nashville, TN, USA

Critical Care 2013, 17(Suppl 2):P328 (doi: 10.1186/cc12266)

Introduction Pediatric patients with altered mental status (AMS) present with poor histories resulting in delayed testing and potential poor outcomes. Non-invasive detection for altered cerebral physiology related to TBI would improve resuscitation and outcome. Cerebral $\mathrm{rSO}_{2}\left(\mathrm{r}_{\mathrm{c}} \mathrm{SO}_{2}\right)$ studies demonstrate its utility in certain neurological emergencies.

Methods A retrospective analysis of $\mathrm{r} \mathrm{SO}_{2}$ utility in $\mathrm{AMS}$. $\mathrm{rCSO}$ data were collected every 30 seconds for AMS patients who had a head CT. Patients with a negative head CT were compared with those with an abnormal head CT. ROC analysis was performed to find the AUC for each summary statistic and performance characteristics. Subgroup analysis was done to determine whether $\mathrm{r} \mathrm{SO}_{2}$ predicted injury and location.

Results $\mathrm{r}_{\mathrm{c}} \mathrm{SO}_{2}$ readings across $5,15,20$, and 30 minutes were stable (Figure 1). $\mathrm{r}_{\mathrm{SO}}$ readings with one or both sides $<50 \%$ or a wide difference between $L$ and $R$ cerebrum was predictive of an abnormal CT scan. A mean difference of 4.2 was $82 \%$ sensitive for detecting a CT lesion with $62 \%$ specificity, $88 \%$ PPV, and $52 \%$ NPV; a mean difference of 12.2 was $100 \%$ specific for an abnormal head CT. Lower mean $\mathrm{r}_{\mathrm{SO}}$ readings localized to the $\mathrm{CT}$ pathology side, and higher $\mathrm{r}_{\mathrm{C}} \mathrm{SO}_{2}$ readings trend toward the EDH group.

Conclusion Cerebral $\mathrm{rCSO}_{2}$ monitoring can non-invasively detect altered cerebral physiology and pathology related to TBI as the cause for pediatric altered mental status. The utility of $\mathrm{r} \mathrm{SO}_{2}$ monitoring has shown its potential for localizing and characterizing intracranial lesions among these altered children. Further studies utilizing $\mathrm{r}_{\mathrm{SO}_{2}}$ monitoring as an adjunct tool in pediatric altered mental status evaluation and management are ongoing.

\section{P329}

Intravenous paracetamol for fever control in acute brain-injured patients: cerebral and hemodynamic effects

E Picetti ${ }^{1}$, I Rossi ${ }^{1}$, P Ceccarelli' ${ }^{1}$, S Risolo ${ }^{1}$, P Schiavi ${ }^{2}$, V Donelli', A Crocamo', M Antonini ${ }^{1}$, M Caspani $^{1}$

'IServizio Anestesia e Rianimazione, Azienda Ospedaliero-Universitaria di

Parma, Italy; ${ }^{2}$ Neurochirurgia e Neurotraumatologia, Azienda OspedalieroUniversitaria di Parma, Italy

Critical Care 2013, 17(Suppl 2):P329 (doi: 10.1186/cc12267)

Introduction Fever is a dangerous secondary insult for the injured brain [1]. We investigated the cerebral and hemodynamic effects of intravenous (i.v.) paracetamol administration for the control of fever in neurointensive care unit (NICU) patients.

Methods The i.v. paracetamol (1 $\mathrm{g}$ in 15 minutes) was administered to NICU patients with a body temperature (Temp.) $>37.5^{\circ} \mathrm{C}$. Its effects on mean arterial pressure (MAP), heart rate (HR), intracranial pressure (ICP), cerebral perfusion pressure (CPP), jugular venous oxygen saturation $\left(\mathrm{SjVO}_{2}\right)$ and Temp. were recorded at the start of paracetamol infusion (T0) and after 30 (T30), 60 (T60) and 120 (T120) minutes. Interventions for the maintenance of CPP $>60 \mathrm{mmHg}$ or ICP $<20 \mathrm{mmHg}$ were recorded. 

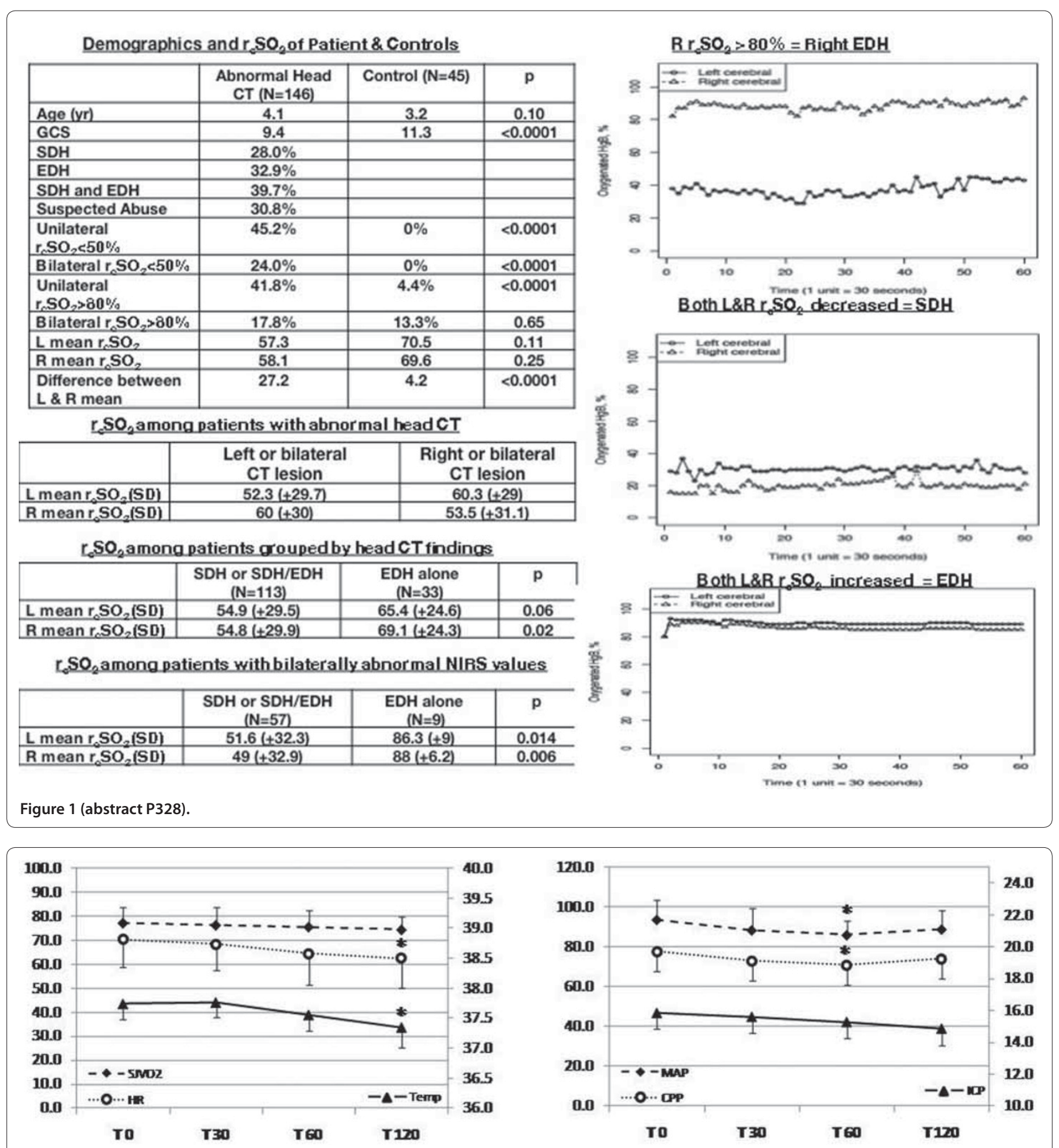

Figure 1 (abstract P329). ${ }^{*} P<0.05$ versus T0.

Results Fifteen NICU patients (nine subarachnoid hemorrhage, five traumatic brain injury, mean age $54.9 \pm 16.8$, seven (50\%) males, median GCS 7) were prospectively studied. We analyzed the administration of one dose of paracetamol for each patient (total 14 cases). After infusion of paracetamol we found a decrease of Temp. (from $37.8 \pm 0.3$ to $37.4 \pm 0.4^{\circ} \mathrm{C}, P<0.001$ ), MAP (from $94.7 \pm 9.9$ to $86.1 \pm 6.7 \mathrm{mmHg}$, $P=0.008$ ), CPP (from $79.6 \pm 13.1$ to $70.8 \pm 7.6 \mathrm{mmHg}, P=0.011$ ) and HR (from $71.5 \pm 14.9$ to $63.8 \pm 16.3 \mathrm{bpm}, P<0.001$ ) with respect to the starting value (ANOVA for repeated measures), whereas ICP and $\mathrm{SjVO}_{2}$ remained unchanged (Figure 1). In five cases norepinephrine infusion was started for CPP $<60 \mathrm{mmHg}$. In another two cases, for the same reason, the norepinephrine dosage was augmented. The proportion of patients who had infusion of norepinephrine increased from $42.8 \%$ at T0 to $78.6 \%$ at T120 ( $P=0.02$, chi-square for trends).

Conclusion Use of i.v. paracetamol is effective in the maintenance of normothermia in acute brain-injured patients. However, adverse hemodynamic effects, which could represent a secondary insult for the injured brain, must be rapidly recognized and treated.

Reference

1. Greer DM, Funk SE, Reaven NL, Ouzounelli M, Uman GC: Impact of fever on outcome in patients with stroke and neurologic injury. Stroke 2008, 39:3029-3035. 
P330

Retrospective analysis of the hemodynamic effects of induction of barbiturate coma in patients with refractory elevated intracranial pressure

S D'Hollander1', M Vander Laenen', JB Gillet², C De Deyne', R Heylen', W Boer ${ }^{1}, \mathrm{~F}$ Jans

'ZOL Genk, Belgium; 2UZ Leuven, Belgium

Critical Care 2013, 17(Suppl 2):P330 (doi: 10.1186/cc12268)

Introduction Standard clinical practice in patients with elevated intracranial pressure (ICP) is to keep cerebral perfusion pressure (CPP) at (or above) $60 \mathrm{mmHg}$ : we performed this retrospective study to analyze whether induction of barbiturate coma impedes compliance to this target CPP value. It has been repetitively shown that, although proven as an efficient therapy for refractory elevated ICP, barbiturate coma may cause a decrease in mean arterial pressure (MAP) and CPP [1].

Methods All patients that had received sodium thiopental in our ICU during 2011 were identified and their medical and nursing records were retrospectively analyzed. The effect of administration of sodium thiopental (loading dose 3 to $5 \mathrm{mg} / \mathrm{kg}$, followed by a continuous infusion adjusted to obtain an EEG burst suppression ratio between 40 and $80 \%$ ) on MAP, ICP and CPP was evaluated. Values are reported as mean \pm SD. The changes over time in ICP, MAP and CPP are reported as means with their $95 \% \mathrm{Cl}$.

Results In 2011, a total of 20 patients were treated in our ICU with barbiturate coma for refractory elevated ICP. In five patients, systemic hypothermia was simultaneously induced. These patients were excluded from further analysis. Six patients died during their stay in the ICU and nine patients could be discharged to the neurosurgical ward. The mean peak ICP value before induction of barbiturate coma was $26 \pm 3 \mathrm{mmHg}$, and the ICP value 6 hours later was $20 \pm 6 \mathrm{mmHg}$. The MAP at those same time points was $91 \pm 12 \mathrm{mmHg}$ and $81 \pm 11 \mathrm{mmHg}$, respectively. The CPP, calculated from both previous values, was $66 \pm 13 \mathrm{mmHg}$ and $61 \pm 11 \mathrm{mmHg}$, respectively. At both time points, the dose of noradrenaline was comparable $(10.1 \pm 9.6 \mu \mathrm{g} /$ minute and $11.5 \pm 13.2 \mu \mathrm{g} /$ minute, respectively). Analysis of the change in ICP, MAP and CPP over the 6-hour period showed a significant decrease in ICP of $-5.8 \mathrm{mmHg}(-9.3 ;-2.3)$ (mean, $95 \% \mathrm{Cl}$ ). The MAP significantly decreased by $-10.4 \mathrm{mmHg}(-19.1 ;-1.7)$. The decrease in CPP was not significant $(-4.6 \mathrm{mmHg}(-14.2 ; 5.0))$

Conclusion This retrospective study indicates that inducing a thiopental coma in patients with refractory elevated ICP effectively reduces ICP. Although the concomitant reduction in MAP was significant, the resulting decrease in CPP was small and nonsignificant. Moreover, the target CPP of $60 \mathrm{mmHg}$ was maintained after induction of barbiturate coma.

Reference

1. Haddad et al: Scand J Trauma Resusc Emerg Med 2012, 20:12-27.

\section{P33'}

Hospital readmissions following traumatic brain injury

A Boutin, K Francisque, L Moore, F Lauzier, X Neveu, A Turgeon

Université Laval, Québec, Canada

Critical Care 2013, 17(Suppl 2):P331 (doi: 10.1186/cc12269)

Introduction Evaluating resource utilization is paramount in critically ill patients with traumatic brain injury (TBI), but little is known on readmissions after hospital discharge. We evaluated rates and determinants of unplanned readmission following TBI.

Methods We conducted a multicenter retrospective cohort study from April 1998 to March 2009. Data were obtained from a Canadian provincial trauma system, based on mandatory contribution from 59 trauma centres, and a hospital discharge database. Patients aged $\geq 16$ years with TBI (ICD-9 or ICD-10 codes of 850-854 and S06, respectively) were included. Patients who died during the index hospitalization, who lived outside the province, who could not be linked with the hospital discharge database were excluded. We collected baseline and trauma characteristics, hospital admissions in the 12 months preceding index admission, and readmissions in the 12 following months. Primary outcome was unplanned readmission 30 days, 3 months and 6 months post discharge. We evaluated sociodemographic and clinical factors associated with readmissions using a logistic regression model.

Results Among 18,342 adult patients with TBI identified in the registry, 14,777 patients were included among which 2,363 had severe, 1,106 moderate and 11,308 mild traumatic brain injury. Most patients were young (mean age: $52 \pm 23$ years) and had no comorbidity (73.6\%). Overall, 1,032 patients $(7.0 \%)$ were readmitted within 30 days, $12.7 \%$ within 3 months and $17.6 \%$ within 6 months. At 30 days post discharge, $311(30.1 \%)$ were readmitted for a complication. The median length of stay was 8 days (Q1 to Q3: 3 to 20). More than 10\% of patients aged $\geq 75$ years with $\geq 1$ comorbidity or with $\geq 1$ admission prior to index hospitalisation were readmitted. The severity of the TBI was not an independent predictor of readmission. Age, highest AIS, number of comorbidities, number of admissions prior to index hospitalization, level of index trauma center and discharge destination were associated with readmissions on multivariate analysis.

Conclusion Readmissions in the months following TBI are frequent, but were not found to be associated with the TBI severity. Further studies evaluating reasons for readmission are warranted in order to develop strategies to prevent such events.

\section{P332}

Risk factors of pituitary disorders following traumatic brain injury

F Lauzier ${ }^{1}$, O Lachance', I Cote ${ }^{1}$, B Senay ${ }^{1}$, P Archambault ${ }^{1}$, F Lamontagne ${ }^{2}$ A Boutin', M Shemilt', L Moore', F Bernard ${ }^{3}, C_{\text {C Gagnon'1, D Cook }}^{4}$,

AF Turgeon

'Universite Laval, Quebec, Canada; ${ }^{2}$ Universite de Sherbrooke, Canada; ${ }^{3}$ Universite de Montreal, Canada: ${ }^{4}$ McMaster University, Hamilton, Canada Critical Care 2013, 17(Suppl 2):P332 (doi: 10.1186/cc12270)

Introduction Pituitary disorders following traumatic brain injury (TBI) are frequent, but their determinants are poorly understood. We performed a systematic review to assess the risk factors of TBIassociated pituitary disorders.

Methods We searched MEDLINE, Embase, Scopus, The Cochrane Library, BIOSIS, and Trip Database, and references of narrative reviews for cohort, cross-sectional and case-control studies enrolling at least five adults with $T B I$ in whom $\geq 1$ pituitary axis was tested and one potential predictor reported. Two independent investigators selected citations, extracted data and assessed the risk of bias. We pooled the data from all studies assessing a specific predictor, regardless of the pituitary axis being evaluated. When more than one pituitary axis was assessed, we used the data related to hypopituitarism or the data from the most defective axis. When a pituitary axis was evaluated several times, we used assessment farthest from the injury. A meta-analysis was performed using random effect models and $l^{2}$ was used to evaluate heterogeneity. Studies were considered at low risk of bias if the authors defined inclusion/exclusion criteria, did not use voluntary sampling, and tested $>90 \%$ of patients with proper detailed diagnostic criteria. Results Among 13,559 citations, we included 26 studies (1,708 patients). Increased age was associated with pituitary disorders (weighted mean difference $=3.2,95 \% \mathrm{Cl}=0.3$ to $6.1,19$ studies, 1,057 patients, $R^{2}=59 \%$ ). This finding was no longer significant when only considering studies with low risk of bias. TBI severity was associated with an increased risk of developing pituitary disorders $(R R=1.49,1.24$ to $1.77, R=17 \%$ for moderate/severe vs. mild TBI; $R R=1.78,1.09$ to $2.91, R^{2}=80 \%$ for severe vs. $\mathrm{mild} /$ moderate TBI), while sex was not (RR for male $=1.05,0.98$ to $1.13,15$ studies, 870 patients, $P^{2}=0 \%$ ). Among CT scan findings, only skull fractures were associated with pituitary disorders $(R R=1.75,1.13$ to 2.70 , six studies, 345 patients, $R^{2}=47 \%$ ). An insufficient number of studies with low risk of bias assessing the association between GCS, CT scan findings and pituitary disorders was retrieved to perform meta-analysis.

Conclusion Age, TBI severity and skull fractures are associated with an increased risk of pituitary disorders. Further studies are necessary to identify additional factors that will help developing targeted screening strategies. 
P333

Vasopressin reduces the cumulative epinephrine dose in hypothermic swine with traumatic brain injury

AD Goldberg, MG Kashiouris, L Keenan, A Rabinstein, M Kumar

Mayo Clinic, Rochester, MN, USA

Critical Care 2013, 17(Suppl 2):P333 (doi: 10.1186/cc12271)

Introduction Prevention of secondary neurologic injuries is paramount for improved neurologic outcomes after traumatic brain injury (TBI). Evidence suggests that although therapeutic hypothermia (TH) lowers intracranial pressure and attenuates secondary cerebral insults after TBI [1], it also induces hypotension. Brief episodes of mild hypotension in brain-injured patients can trigger secondary injuries, which have been associated with increased mortality in patients with TBI [2]. Vasopressin mitigates hypotension in septic shock and improves coronary perfusion in hypothermic cardiac arrest models [3]. We hypothesized that a lowdose vasopressin infusion may reduce the cumulative epinephrine dose in hypothermic, brain-injured swine.

Methods Six domestic cross-bred pigs were subjected to epinephrine infusion after general anesthesia, standardized TBI and transpulmonary hypothermia $\left(32^{\circ} \mathrm{C}\right.$ for 48 hours). All animals received the same care, aiming for a mean arterial pressure $>60 \mathrm{mmHg}$. At hour 24, animals received additional vasopressin infusion at 0.04 units/minute. We measured the cumulative epinephrine dose for each animal pre and post vasopressin infusion (Figure 1) and performed a twosample Wilcoxon rank-sum test, comparing the median cumulative epinephrine doses in the two groups.

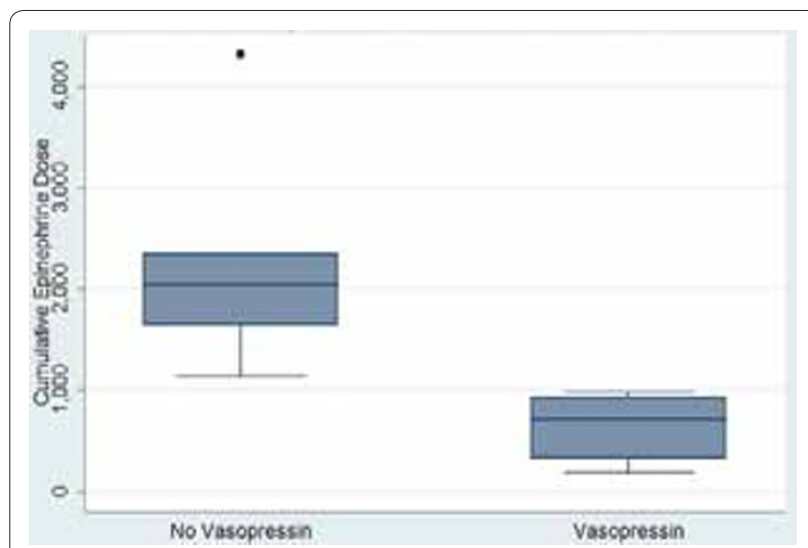

Figure 1 (abstract P333). Effect of vasopressin on cumulative amount of epinephrine administered.

Results The median cumulative epinephrine dose in the animals that received the vasopressin infusion was $715 \mathrm{mg}$ with a 25th to 75th interquartile range (IQR) of 320 to $930 \mathrm{mg}$. The median cumulative epinephrine dose in the control group was $2,044 \mathrm{mg}$ (IQR 1,640 to $2,344 \mathrm{mg})$. This was statistically significant $(P=0.003)$, based on the Wilcoxon rank-sum test.

Conclusion A low-dose infusion of vasopressin can significantly reduce vasopressor requirements and improves hemodynamics in hypothermic, brain-injured swine. This hemodynamic stability may improve neurological outcomes.

\section{References}

1. Polderman KH, et al: Intensive Care Med 2002, 28:1563-1573.

2. Fearnside MR, et al: Br J Neurosurg 1993, 7:267-279.

3. Voelckel WG, et al: Anesth Analg 2000, 91:627-634.

P334

Long-term functional outcome in adults with severe TBI: a meta-analysis

M Asselin', Y Lachance', G Lalonde', A Boutin', M Shemilt', L Moore', F Lauzier' R Zarychanski ${ }^{2}$, P Archambault', F Lamontagne', D Fergusson ${ }^{3}$, A Turgeon

'Université Laval, Québec, Canada; ${ }^{2}$ University of Manitoba, Winnipeg,

Canada: ${ }^{3}$ University of Ottawa, Canada

Critical Care 2013, 17(Suppl 2):P334 (doi: 10.1186/cc12272)
Introduction Severe traumatic brain injury (TBI) is a major cause of death and of severe neurologic sequelae. Long-term functional outcome of $\mathrm{TBI}$ and its best timing of assessment are not well understood, and may be evaluated too prematurely in clinical studies because of resources required to do so without too much missing data. Hence, we conducted a systematic review of studies in severe TBI patients to evaluate the long-term functional outcome. We hypothesized that functional impact measured by the Glasgow Outcome Scale (GOS), or the extended version (GOSe), may plateau after several months in patients with severe TBI.

Methods We performed a systematic review of randomized controlled trials and cohort studies (prospective and retrospective) in patients with severe TBI. We searched MEDLINE, Embase, Cochrane Central, BIOSIS, CINAHL and Trip Database from their inception to December 2011. References of included studies were searched for additional studies. Two reviewers independently determined study eligibility and collected data. The primary outcome measure was the proportion of unfavourable functional outcome (GOS 1 to 3 or GOSe 1 to 4 ) at 6 to 12 months, 12 to 18 months, 18 to 24 months and more than 24 months after severe TBI. We calculated Freeman Tukey-type arcsine squareroot transformations and pooled data using random-effect models. Heterogeneity was assessed with the $R^{2}$ test and sensitivity analyses were based on a priori hypotheses.

Results In total, 4,432 studies were assessed for eligibility; 209 studies $(n=31,540)$ were included. In the 188 studies using the GOS, a poor functional outcome was observed in $56.6 \%(95 \% \mathrm{Cl}=54.0$ to $59.1 \%$, $\left.P^{2}=91 \%\right), 51.9 \%\left(95 \% \mathrm{Cl}=38.0\right.$ to $\left.59.0 \%, P^{2}=84 \%\right), 57.0 \%(95 \% \mathrm{Cl}=48.2$ to $\left.55.5 \%, P^{2}=93 \%\right)$ and $56.9 \%\left(95 \% \mathrm{Cl}=48.2\right.$ to $\left.65.1 \%, P^{2}=93 \%\right)$ of patients at 6 to 12 months, 12 to 18 months, 18 to 24 months and beyond 24 months, respectively. In the 18 studies using GOSe, a poor functional outcome was observed in $62.9 \%$ of patients at 6 to 12 months $\left(95 \% \mathrm{Cl}=55.9\right.$ to $\left.69.2 \%, R^{2}=90 \%\right)$ and $54.6 \%$ at 12 to 18 months $(95 \% \mathrm{Cl}=43.2$ to $65.8 \%, R=90 \%)$. Heterogeneity was present in most analyses and was not entirely explained by the planned sensitivity analyses.

Conclusion Considering that the incidence of patients with an unfavourable outcome remained constant at different assessments, a follow-up of severe TBI patients longer than 12 months does not provide incremental information. Functional outcomes measured longer than 12 months after the injury may not be warranted in clinical studies.

P335

Monitoring of severe traumatic brain injury patients in UK ICUs: a national survey

S Panchatsharam, B Lewinsohn, G De La Cerda, D Wijayatilake

Queen's Hospital, Romford, UK

Critical Care 2013, 17(Suppl 2):P335 (doi: 10.1186/cc12273)

Introduction Prevention of secondary brain injury is the cornerstone in the management of patients with severe traumatic brain injury (TBI) and raised intracranial pressure (ICP). Although a variety of monitoring methods are available, due to lack of strong evidence their use varies considerably [1]. The objective of this survey was to provide an overview of the current practice in monitoring of patients with severe TBI in all neuro-ICUs across the UK.

Methods The ICUs managing adult patients with severe TBI were identified from the RAIN (Risk Adjustment In Neurocritical care) study sites, and the Society of British Neurosurgeons. Thirty-one centers were identified, and a telephonic survey was conducted by the investigators. Data were collected using a single-page questionnaire containing 18 questions.

Results All 31 (100\%) units used ICP monitoring and parenchymal ICP bolt was the most widely used method (77\%). Thirty (96.7\%) units used a cerebral perfusion pressure protocol to guide therapy. Twenty-eight (90.3\%) units used continuous capnography. Twelve (38.7\%) units used transcranial Doppler. Eight (25.8\%) units used partial pressure of oxygen in brain tissue. Four (12.9\%) units used microdialysis. Only one unit used jugular bulb oximetry. None of the units used near-infrared spectrometry or optic nerve sheath diameter. Eight units (25.8\%) used a cerebral function monitor and seven (22.5\%) units used the bispectral index for guiding depth of sedation. 
Conclusion This survey shows that there is no clear consensus on what types of monitoring should be used to guide management of patients with TBI. A CPP protocol, based on measurement of ICP, is the most widely used method, although a recent randomized control trial did not show any benefit in outcome [2]. Other invasive monitoring methods, although they may help in individualized care, are still not yet popular to due lack of strong evidence. Larger multicentre portfolio studies are needed to establish their benefits.

\section{References}

1. Wijayatilake et al:: Updates in the management of intracranial pressure in traumatic brain injury. Curr Opin Anaesthesiol 2012, 25:540-547.

2. Chesnut et al:: A trial of intracranial-pressure monitoring in traumatic brain injury. N Eng/ J Med 2012, 367:2471-2481.

\section{P336}

Survival analysis after head injury: is a normal INR reassuring?

K Leclerc-Gagne

Hôpital Sacré-Coeur de Montréal, Canada

Critical Care 2013, 17(Suppl 2):P336 (doi: 10.1186/cc12274)

Introduction Intracranial bleeding after head injury is an important issue in the emergency department (ED), and elevated INR increases this risk [1]. A retrospective study showed an association between different levels of normal INRs $(<1.6)$ and elevated risk of intracranial bleeding, with a significant elevation of this risk over an INR threshold of 1.3 [2]. The objective of the study was to evaluate clinical impact of an INR within the normal range in patients with head trauma. We compared mortality between patients with INR $<1.3$ and those with INR $\geq 1.3$ to $<1.6$.

Methods A post hoc analysis of prospective data collected from 3,356 patients seen in a tertiary-care ED from March 2008 to February 2011. We included patients aged 16 years old and over with an INR $<1.6$ and a head CT performed within 24 hours of the trauma. We followed these patients until December 2012, performed a chi-square test between mortality of the two groups and calculated the hazard ratio (HR) from survival analysis using Cox regression.

Results Patient mean age was 55.1 years (SD \pm 23 ), $65 \%$ were men and mean follow-up duration was 3.1 years $(S D \pm 0.8)$. A total of 115 patients (15.9\%) died during follow-up: 16 (36.4\%) in the group with INR $\geq 1.3$ and $99(14.6 \%)$ in the group with INR $<1.3(P<0.001)$. Results showed a

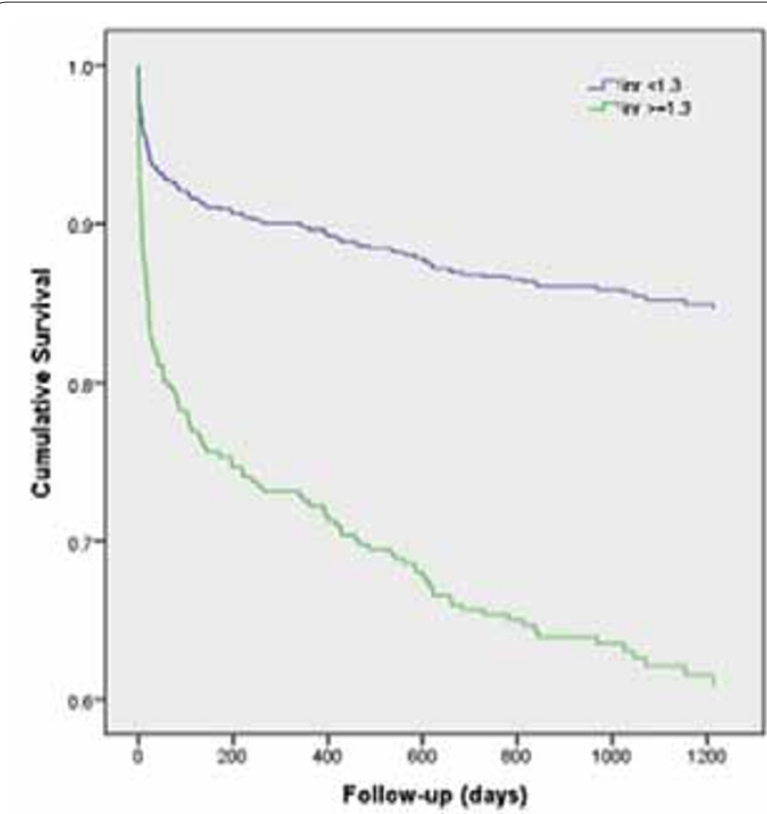

Figure 1 (abstract P336). Survival analysis of patients with INR $<1.3$ and with INR $\geq 1.3$ significantly higher risk of death in the group with INR $\geq 1.3$ : $\mathrm{HR}=2.99$ $(95 \% \mathrm{Cl}=1.8$ to 5.1$) ; P<0.001$ (Figure 1 ).

Conclusion In patients with head injury and normal INR $(<1.6)$, there is an association between an INR $\geq 1.3$ and higher risk of death. Therefore, it would be useful to request an INR in patients presenting with a head injury when bleeding is suspected, even in the absence of anticoagulant.

\section{References}

1. Lingsma HF, et al: Early prognosis in traumatic brain injury: from prophecies to predictions. Lancet Neurol 2010, 9:543-554.

2. Chauny JM, et al: Non Elevated INR and Post Traumatic Intracranial Bleeding. Day of Research in Neurotraumatology. Montreal: Hôpital du Sacré-Cœur; 22 June 2012.

\section{P337}

One-year extended Glasgow Outcome Scale and hospital mortality predictors in patients with severe traumatic brain injury in Brazil RTuron', FR Ferreira', D Prado', P Kurtz², M Damasceno

${ }^{1}$ Hospital de Clínicas de Niteroi, Brazil;, ${ }^{2}$ Clinica São Vicente, Rio de Janeiro, Brazil

Critical Care 2013, 17(Suppl 2):P337 (doi: 10.1186/cc12275)

Introduction Traumatic brain injury (TBI) is a leading cause of death and disability worldwide. Because mortality seriously underestimates the impact of TBI, an outcome tool including vegetative state and severe disability should be used. Our purpose was to study the longterm outcome of a small cohort of severe TBI patients in Brazil.

Methods This was a retrospective analysis of a prospectively collected database. We included 34 consecutive adult patients admitted with severe TBI to a tertiary private hospital in Rio de Janeiro, Brazil between 2009 and 2011. We analyzed data on demographics, admission, clinical scores, imaging and complications, as well as hospital mortality and 1 -year extended Glasgow Outcome Scale (eGOS) of all patients.

Results We analyzed 33 patients with severe TBI. Mean age was $36( \pm 20)$ years and $79 \%$ were male. All patients were mechanically ventilated and $70 \%$ underwent ICP monitoring. Overall hospital mortality was $36 \%$ and $60 \%$ had unfavorable outcome (eGOS $<4$ ) after 1 year. In univariate analysis, higher APACHE II scores, the presence of midriasis and intracranial hypertension were more frequent in nonsurvivors compared with survivors. Furthermore, older age, higher APACHE ॥I score and worse GCS on admission were associated with unfavorable outcome after 1 year, as measured by eGOS $<4$.

Conclusion Our descriptive results suggest a significant burden of severe TBI in a small cohort of young patients. Moreover, severity of the primary disease, age and pupil reflexes, as well as intracranial hypertension seemed to be associated with worse neurological outcome.

P338

Early respiratory deterioration predicts poor outcome after aneurysmal subarachnoid hemorrhage

E Van Lummel, L Vergouw, M Van der Jagt

Erasmus MC, Rotterdam, the Netherlands

Critical Care 2013, 17(Suppl 2):P338 (doi: 10.1186/cc12276)

Introduction Pulmonary complications are frequently occurring medical complications after aneurysmal subarachnoid hemorrhage (aSAH) [1]. Early respiratory deterioration (ERD) may be associated with delayed cerebral ischemia (DCl) or outcome and would then be a potential target for therapeutic interventions. We investigated whether respiratory deterioration within the first 72 hours after admission predicted $\mathrm{DCl}$ or poor outcome.

Methods We conducted a retrospective study in 137 consecutively admitted patients with aSAH, admitted between October 2007 and October 2011 to the ICU of a university hospital. ERD was defined as increased need for ventilatory support the second or third day after admission (Table 1). Elective intubation for a surgical procedure was not included as ERD. Inclusion criteria were availability of detailed information on respiratory status and level of support, admission within 48 hours after hemorrhage and age $\geq 18$ years. Multivariable 
Table 1 (abstract P338). Categories of respiratory support

\begin{tabular}{ll}
\hline Category & $\begin{array}{l}\text { Level of respiratory support } \\
\text { (compared with first admission day) }\end{array}$ \\
\hline 1 & Up to $5 \mathrm{IO}_{2}$ \\
2 & $>5 \mathrm{IO}_{2}$ by nasal canulla or Venturi mask \\
3 & Nonrebreathing mask or non-invasive positive-pressure \\
& ventilation \\
4 & Invasive mechanical ventilation \\
When mechanically & Decreased $\mathrm{PaO}_{2} / \mathrm{FiO}_{2}$ ratio \\
terminated & \\
\hline
\end{tabular}

survival analysis was used to investigate associations of $\mathrm{DCl}$, death and Glasgow Outcome Scale (GOS) with ERD adjusted for condition on admission, Hijdra score, treatment of ruptured aneurysm and pulmonary comorbidity. GOS was assessed at 3 to 6 months after the bleed. DCI was defined as described recently [2].

Results Mean age of the patients was $55.9( \pm 12.7)$ and $63.5 \%$ was female. A total $46.7 \%$ of the patients developed DCl. Mortality was $25.6 \%$. Forty percent of the patients were classified as having ERD. ERD was not associated with $\mathrm{DCl}$ (adjusted $\mathrm{HR}=1.48 ; 95 \% \mathrm{Cl}=0.88$ to 2.49 ; $P=0.14)$. ERD showed a trend towards an association with mortality (adjusted $\mathrm{HR}=2.22 ; 95 \% \mathrm{Cl}=0.96$ to $5.14 ; P=0.06$; additionally adjusted for age, and rebleed). A clear association was found between absence of ERD and functional outcome with ordinal logistic regression analysis ( 0.98 point increase in GOS score at 3 to 6 months; $95 \% \mathrm{Cl}=0.14$ to 1.82 ; $P=0.02$; additionally adjusted for age and rebleed).

Conclusion ERD within 72 hours after admission is associated with increased risk of poor functional outcome after aSAH, but not DCI. Further investigations are required to assess whether prevention of ERD may improve outcome.

References

1. Friedman JA, et al:: Neurosurgery 2003, 52:1025-1031.

2. Vergouwen MD, et al: Stroke 2010, 41:2391-2395.

P339

Evaluation of proximal-distal velocity gradient in spastic middle cerebral artery after aneurismal subarachnoid hemorrhage

M Gotti', F Stretti', S Pifferi', V Conte', M Zara', N Stocchetti ${ }^{2}$

'Fondazione IRCCS Ca' Granda-Ospedale Maggiore Policlinico, Milan, Italy; ${ }^{2}$ Fondazione IRCCS Ca' Granda-Ospedale Maggiore Policlinico, Milan University, Milan, Italy

Critical Care 2013, 17(Suppl 2):P339 (doi: 10.1186/cc12277)

Introduction Delayed cerebral ischemia (DCl) worsens neurological outcome in patients with aneurysmal subarachnoid hemorrhage (aSAH). DCl pathogenesis is multifactorial and not completely understood, but vasospasm plays a central role. Transcranial colorcoded duplex sonography (TCCDS) is a non-invasive bedside tool to explore cerebral vessels, but specificity is still suboptimal. The aim of this study was to evaluate the proximal-distal gradient of mean cerebral blood flow velocity (CBF-V) in middle cerebral artery (MCA) as a possible indicator of critical vasospasm.

Methods Consecutive aSAH patients (WFNS 1 to 5 , age 18 to 80 years) admitted to NeurolCU between November 2011 and September 2012 were included in this study. TCCDS was used to assess CBF-V in MCA of both sides: we defined TCCDS vasospasm as CBF-V $>120 \mathrm{~cm} / \mathrm{second}$. Magnetic resonance angiography (MRA, 3D TOF HR) was performed to evaluate vasospasm at early ( $<3$ days) and delayed ( 7 to 10 days) time points. When patients underwent MRA, in the same clinical conditions, we recorded CBF-V with TCCDS at two levels: at the origin (proximal CBF-V) and at the end (distal CBF-V) of the MCA. Then we calculated the absolute value of proximal-distal CBF-V gradient for MCA of both sides. We defined clinical vasospasm as the appearance of a new neurological deficit confirmed by imaging. The relationships between the absolute value of TCCDS CBF-V gradient and (1) TCCDS vasospasm, (2) MRA vasospasm and (3) clinical vasospasm were explored.
Results We included 26 consecutive aSAH patients (WFNS 1 to 5 , age $57 \pm 12$ years). The absolute value of MCA CBF-V gradient was higher in: (1) vessels affected by TCCDS vasospasm when compared with vessels not affected $(63 \mathrm{~cm} /$ second, IR 23 to 85 and $15 \mathrm{~cm} /$ second IR 7 to 23 respectively, $P<0.001$ ); (2) vessels affected by MRA vasospasm when compared with vessels not affected $(43 \mathrm{~cm} / \mathrm{second}$, IR 18 to 78 and $13 \mathrm{~cm} /$ second IR 6 to $22, P<0.001) ;$ and (3) in patients who developed clinical vasospasm than in patients who did not $(56 \mathrm{~cm} / \mathrm{second}$ IR 11 to 124 and $16 \mathrm{~cm} /$ second IR 8 to 30 respectively, $P<0.01)$. Considering only the subset of MCAs affected by MRA vasospasm, the CBF-V gradient was higher when clinical vasospasm was also present $(75 \mathrm{~cm} /$ second IR 56 to 124 and $23 \mathrm{~cm} / \mathrm{second}$ IR 17 to 30 respectively, $P<0.05)$. Conclusion Proximal-distal CBF-V gradient of MCA might be a reliable indicator of critical vasospasm but further studies are warranted to define threshold values and specificity in aSAH patients.

\section{P340}

Microparticles in aneurismal subarachnoid hemorrhage: role in acute and delayed cerebral ischemia

V Conte', A Artoni', S Magnoni', M Leoni', L Capuano', V Civelli',

N Stocchetti ${ }^{2}$

'Fondazione IRCCS Ca'Granda-Ospedale Maggiore Policlinico, Milan, Italy; ${ }^{2}$ Fondazione IRCCS Ca' Granda-Ospedale Maggiore Policlinico, Milan University, Milan, Italy

Critical Care 2013, 17(Suppl 2):P340 (doi: 10.1186/cc12278)

Introduction Microthrombosis has been demonstrated in early and delayed cerebral ischemia after aneurysmal subarachnoid hemorrhage (aSAH). Markers of coagulation activation as microparticles (MPs) are an established risk factor for thrombosis. Our hypothesis was that levels of microparticles might correlate with aSAH severity, early cerebral ischemia $(\mathrm{ECl})$ and delayed cerebral ischemia (DCl).

Methods Consecutive aSAH patients (age 18 to 80) admitted to our department between November 2011 and September 2012 were enrolled in the study. Total MPs (anxV+), platelet MPs (PLT-MPs, anx+l CD41+), tissue factor MPs (TF-MPs, anx+/CD142+), and endothelial MPs (E-MPs, anx+/CD144+) were assessed in venous blood samples. Measurement of MPs levels were obtained at early $(<3$ days) and delayed phase ( 7 to 10 days). Multiparameter MRI (T2; FLAIR; T1; AngioRM 3D TOF $\mathrm{HR}$; DWI) was performed to evaluate ischemic damage and vasospasm at the same time points. Qualitative evaluation of severity of ischemic damage was performed on DWI sequences. Levels of MPs were evaluated comparing different groups in terms of SAH severity (World Federation of Neurological Surgeons score or WFNS), ECI severity and occurrence of delayed complications (vasospasm and DCI).

Results Twenty-five patients (age $57 \pm 12$ years, WFNS 1 to 5 ) were included in the analysis. Overall increased levels of MPs were observed at early phase after aSAH when compared with controls. Contrarily to our hypothesis, in the early phase no significant differences of MP level were observed in WFNS 4 to $5(n=9)$ when compared with WFNS 1 to $2(n=16)$ patients. Interestingly, patients with most severe $\mathrm{ECl}$ and $\mathrm{DCl}$ showed the highest values of MPs, but this trend did not reach statistical significance, probably because of the small number of patients included in the study.

Conclusion Microparticles and microthrombosis may increase the severity of early and delayed ischemic damage after aSAH.

\section{P341}

Intracranial pressure after subarachnoid hemorrhage

A Lombardo' ${ }^{1}$ T Zoerle', ER Zanier ${ }^{2}$, L Longhi', N Stocchetti ${ }^{1}$

'Fondazione IRCCS Ca' Granda-Ospedale Maggiore Policlinico, University of

Milan, Italy; ${ }^{2}$ Mario Negri Institute, Milan, Italy

Critical Care 2013, 17(Suppl 2):P341 (doi: 10.1186/cc12279)

Introduction Elevated intracranial pressure (ICP) may have deleterious effects on cerebral metabolism and mortality after aneurysmal subarachnoid hemorrhage $(\mathrm{SAH})[1,2]$, but its relevance has not yet been well explored. Aims of this study are to track ICP changes after $\mathrm{SAH}$, to identify clinical factors associated with it and to explore the relationship between ICP and outcome. 
Methods A total of 116 consecutive SAH patients with ICP monitoring were enrolled. Episodes of ICP $>20 \mathrm{mmHg}$ for at least 5 minutes and the mean ICP value for every 12-hour interval were analyzed. The highest mean ICP collected in every patient was identified. ICP values were analyzed in relation to clinical and CT findings; 6-month outcome and ICU mortality were also introduced in multivariable logistic models. Results Eighty-one percent of patients had at least one episode of elevated ICP and $36 \%$ had a highest mean ICP $>20 \mathrm{mmHg}$. The number of patients with highest mean ICP $>20 \mathrm{mmHg}$ or with episodes of HICP was maximum at day 3 after SAH and decreased only after day 7 . Neurological status, aneurysmal rebleeding, amount of blood on $\mathrm{CT}$ and $\mathrm{CT}$ ischemic lesion occurred within 72 hours from SAH were significantly related to highest mean ICP $>20 \mathrm{mmHg}$ in a multivariable model. Patients with highest mean ICP $>20 \mathrm{mmHg}$ showed significantly higher mortality in ICU. However, ICP is not an independent predictor of 6 months unfavorable outcome.

Conclusion Elevated intracranial pressure is a common complication in the first week after SAH. It is associated with early brain injury severity and ICU mortality.

References

1. Heuer $\mathrm{GG}$, et al:: Relationship between intracranial pressure and other clinical variables in patients with aneurismal subarachnoid hemorrhage. J Neurosurg 2004, 101:408-416.

2. Nagel A, et al:. Relevance of intracranial hypertension for cerebral metabolism in aneurismal subarachnoid hemorrhage. Clinical article. $J$ Neurosurg 2009, 111:94-101.

\section{P342}

Prevalence of pituitary disorders associated with aneurysmal subarachnoid hemorrhage

F Lauzier, I Cote, A Bureau, M Shemilt, A Boutin, L Moore, C Gagnon, AF Turgeon

Universite Laval, Quebec, Canada

Critical Care 2013, 17(Suppl 2):P342 (doi: 10.1186/cc12280)

Introduction Pituitary disorders are an often-neglected consequence of aneurysmal subarachnoid hemorrhage (aSAH). We systematically reviewed their prevalence, aiming particularly at studies with low risk of bias.

Methods We searched Embase, MEDLINE, The Cochrane Library, Trip Database, references of included studies and narrative reviews. We included cohort studies, cross-sectional studies and RCTs published in any language that tested the integrity of $\geq 1$ pituitary axis in adults with aSAH. Studies including more than $50 \%$ of non-aneurismal SAH were excluded. Studies were considered at low risk of bias if the authors defined inclusion/exclusion criteria, avoided voluntary sampling, and tested $>90 \%$ of included patients with proper detailed diagnostic criteria. Studies testing all pituitary axes were considered as evaluating hypopituitarism, which was defined as the dysfunction of $\geq 1$ axis. We used a Freeman Tukey-type arcsine square-root transformation and pooled prevalences using the DerSimonian-Laird random-effect method. We determined the degree of heterogeneity with $R^{2}$ values.

Results Among 12,363 citations, we included 28 studies $(1,628$ patients). Patients were mostly female (64\%) aged $50.5 \pm 5.4$. Sixteen studies reported the severity of aSAH, 14 reported the procedure for securing the aneurysm and 13 reported the location of aneurysm. Overall, hypopituitarism was observed in $53.4 \%$ of patients at shortterm ( $<3$ months), $36.5 \%$ at mid-term (3 to 12 months) and $34.2 \%$ at long-term (>12 months) (Table 1). There was an insufficient number of studies with low risk of bias to perform sensitivity analyses according to study quality.

Conclusion The exact prevalence of pituitary disorders following aSAH remains uncertain, mainly due to high heterogeneity and the small number of studies with low risk of bias. However, the prevalence seems to decrease during the recovery phase. The prevalence, risk factors and clinical significance of pituitary disorders in aSAH will require further rigorous evaluation.

P343

Correlation between the timing of endovascular coiling or microsurgical clipping and long-term outcomes of patients after aneurysmal subarachnoid haemorrhage

N Ojukwu, R Jain, S Wijayatilake, A Bellini, R Shetty, J Khan, G De la Cerda

Queen's Hospital, London, UK

Critical Care 2013, 17(Suppl 2):P343 (doi: 10.1186/cc12281)

Introduction Aneurysmal subarachnoid haemorrhage (aSAH) is associated with a high morbidity and mortality. Although UK Anaesthesia Guidelines advocate early coiling or clipping of the aneurysm within the first 72 hours of admission for all grades of aSAH, the optimal timing of treatment and whether this is linked with better neurological long-term outcome are a subject of debate [1]. We aimed to investigate whether the timing of the occlusion of the aneurysm translates into better outcome.

Methods A retrospective analysis of prospective collected data in a tertiary neuroscience centre from January to September 2012. All

Table 1 (abstract P342)

\begin{tabular}{|c|c|c|c|c|c|c|}
\hline & Hypopituitarism & GH & АCTH & TSH & Gonadal & ADH \\
\hline \multicolumn{7}{|l|}{ Short term } \\
\hline All studies & $\mathbf{5 3 . 4 \%}(20.5$ to 84.7$)$ & $\mathbf{3 0 . 4 \%}(11.5$ to 53.2$)$ & $13.2 \%$ (4.4 to 25.1$)$ & $\mathbf{4 9 . 2 \%}(0.1$ to 17.7$)$ & $26.1 \%$ (8.8 to 48.0$)$ & $6.3 \%(2.7$ to 11.0$)$ \\
\hline$n$ (patients) & $5(235)$ & $8(346)$ & $13(537)$ & $7(327)$ & $8(351)$ & $3(481)$ \\
\hline$r^{2}$ & $96.0 \%$ & $93.5 \%$ & $90.2 \%$ & $89.1 \%$ & $93.0 \%$ & $61.1 \%$ \\
\hline Studies of low risk of bias (patients) & $2(56)$ & - & - & $2(56)$ & $1(26)$ & - \\
\hline \multicolumn{7}{|l|}{ Mid-term } \\
\hline All studies & $36.5 \%$ (13.6 to 62.8$)$ & 7.3\% (0.6 to 18.3$)$ & $\mathbf{8 . 9 \%}(1.3$ to 21.0$)$ & $\mathbf{8 . 8 \%}(2.4$ to 18.0$)$ & $3.9 \%(0.0$ to 11.9$)$ & $3.7 \%$ (0.6 to 13.5$)$ \\
\hline$n$ (patients) & $4(109)$ & $6(158)$ & $7(208)$ & $5(176)$ & $6(178)$ & $2(56)$ \\
\hline$r^{2}$ & $85.7 \%$ & $70.9 \%$ & $79.4 \%$ & $59.4 \%$ & $66.8 \%$ & $29.0 \%$ \\
\hline Studies with low risk of bias (patients) & $1(40)$ & - & - & $1(40)$ & - & - \\
\hline \multicolumn{7}{|l|}{ Long-term } \\
\hline All studies & $34.2 \%$ (18.7 to 51.4$)$ & $17.1 \%$ (9.4 to 26.4$)$ & $11.2 \%$ (4.8 to 19.4 ) & $\mathbf{2 . 3} \%$ (0.4 to 5.3$)$ & $3.2 \%(0.7$ to 6.8$)$ & $0 \%(0$ to 1.5$)$ \\
\hline$n$ (patients) & $12(486)$ & $13(531)$ & $13(531)$ & $12(486)$ & $13(351)$ & $3(142)$ \\
\hline$r^{2}$ & $92.5 \%$ & $82.9 \%$ & $82.9 \%$ & $49.0 \%$ & $62.9 \%$ & $0 \%$ \\
\hline Studies with low risk of bias (patients) & $2(40)$ & $2(40)$ & $2(40)$ & $2(40)$ & $1(10)$ & - \\
\hline
\end{tabular}




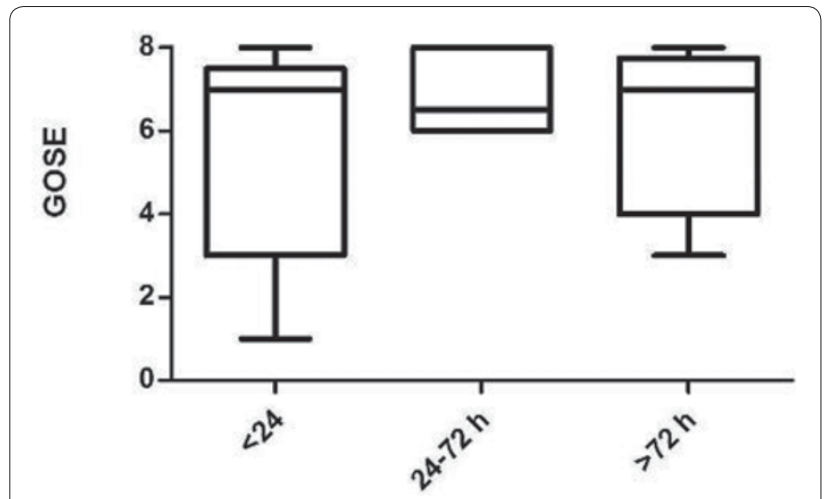

Timing of coiling or clipping

Figure 1 (abstract P343). Time of coiling/clipping versus GOSE boxplot.

patients were managed according to the local Guidelines for the management of aSAH. Outcome was assessed at 3 months using the extended Glasgow Outcome Scale (GOSE) defining good recovery as a GOSE $\geq 7$ and poor outcome as GOSE $\leq 6$.

Results A total of 28 patients were included within the study period. Three patients were not expected to survive the first 24 hours and were not included in the study. Seventeen patients were classified as good grade aSAH (WFNS I to III) and eight as poor grade (IV to V). Twenty-two patients underwent successful coiling while the other three required clipping due to unsuccessful coiling. We did not find any correlation between the timing of coiling/clipping and the 3-month GOSE (Figure 1). A total $44 \%$ of the patients had a poor 3-month GOSE while $56 \%$ had a good long-term functional outcome. The overall mortality rate was $21 \%$.

Conclusion Overall mortality in patients with aSAH is low when aneurysm is treated early post rupture of aneurysm. We did not find any correlation between the timing of occlusion of aneurysm and the 3-month functional outcome.

Reference

1. van der Schaaf I, et al: Stroke 2006, 37:572-573.

P344

Time course of neuropsychological functioning after aneurysmal subarachnoid hemorrhage

B Zarino', G Bertani', V Conte', S Magnoni', A Di Cristofori', N Stocchetti

'Fondazione IRCCS Ca'Granda-Ospedale Maggiore Policlinico, Milan,

Italy; ${ }^{2}$ Fondazione IRCCS Ca' Granda-Ospedale Maggiore Policlinico, Milan

University, Milan, Italy

Critical Care 2013, 17(Suppl 2):P344 (doi: 10.1186/cc12282)

Introduction Early and delayed cognitive dysfunctions are an understudied issue after aneurysmal subarachnoid hemorrhage (aSAH), irrespective of neurological outcome. The aim of this study was to describe early and delayed changes of cognitive functions, activities of everyday life, and quality of life in aSAH patients.

Methods Consecutive aSAH patients admitted to our ICU between November 2011 and September 2012 were prospectively studied. Patients underwent neuropsychological evaluation at early $(<3$ days, 10 days) and delayed time points ( 1 month, 3 months). Patients were tested for language, verbal fluency, short-term and long-term memory, attention, executive functions, praxis, and neglect. Impairments in activities of everyday life were assessed using the Activities of Daily Living scale and the Instrumental Activities of Daily Living scale. The SF-36 was used to assess the quality of life at 3 months. Since complications of aneurysm treatment in addition to aSAH severity may significantly affect cognitive status, patients were evaluated according to the World Federation of Neurological Surgeons score after treatment (WFNSpt).
Results All WFNSpt 1 to 2 patients completed neuropsychological tests at each time point. WFNSpt 3 and WFNS 4 patients were testable in $80 \%$ and $50 \%$ of the cases respectively at early time points. WFNS 5 patients were not testable at any time point. In all testable patients, cognitive functions were severely impaired at early time points. At 3 months in WFNS 1 to 3 a good recovery of language deficits while only a partial recovery of attention, memory and executive functions were observed; at the same time point $70 \%$ of WFNS 4 patients became testable, but they had a worse recovery of all cognitive functions. At 1 month after SAH less than $30 \%$ of patients return to work, at 3 months approximately $50 \%$. Despite a good recovery of everyday life activities at 3 months, for all patients quality of life was lower than a normal population.

Conclusion Cognitive dysfunction has different time courses after aSAH: significant deficits in different cognitive domains, worse quality of life and difficulties in return to work persist in more than $50 \%$ of patients at 3 months following SAH.

\section{P345}

Neuroprotective effect of a new synthetic aspirin-decursinol

adduct in a rat model of ischemic stroke

YH Lee, CW Park, JH Cho, MH Won

Kangwon National University, Chuncheonsi, South Korea

Critical Care 2013, 17(Suppl 2):P345 (doi: 10.1186/cc12283)

Introduction Stroke is a major cause of death. This study investigated the preventative effect of a new synthetic drug on brain function in experimentally induced ischemic stroke.

Methods Male Sprague-Dawley rats were administered aspirin (ASA), decursinol (DA) or ASA-DA before and after ischemic insults. Brain and neuronal damage were examined by TTC staining, PEP-CT, NeuN immunohistochemistry and F-J B histofluorescence. Gliosis was also observed by GFAP and iba- 1 immunohistochemistry.

Results Pretreatment with $20 \mathrm{mg} / \mathrm{kg}$, but not $10 \mathrm{mg} / \mathrm{kg}$, of ASA-DA protected against ischemic neuronal death and damage, and its neuroprotective effect was much more pronounced than that of ASA or DA alone. In addition, treatment with $20 \mathrm{mg} / \mathrm{kg}$ ASA-DA reduced the ischemia-induced activation of astrocytes and microglia.

Conclusion Our findings indicate that ASA-DA, a new synthetic drug, prevents against transient focal cerebral ischemia, which provides a resource for the development of its clinical application for stroke.

\section{P346}

Prevalence of acute critical neurological disease in children: a global epidemiological assessment (PANGEA)

E Fink', R Tasker', J Beca ${ }^{3}$, MJ Bell', RS Clark', J Hutchison',

M Vavilala', RS Watson', L Weissfeld', PM Kochanek', DC Angus?', PANGEA Investigators

'Children's Hospital of Pittsburgh, PA, USA; ${ }^{2}$ Children's Hospital Boston, MA, USA; ${ }^{3}$ Starship Children's Hospital, Auckland, New Zealand; ${ }^{4}$ The Hospital for Sick Children, Toronto, Canada; '5 Seattle Children's Hospital, Seattle, WA, USA; ${ }^{6}$ University of Pittsburgh, PA, USA; 'University of Pittsburgh Medical Center, Pittsburgh, PA, USA

Critical Care 2013, 17(Suppl 2):P346 (doi: 10.1186/cc12284)

Introduction Acute neurological injury is a leading cause of morbidity and mortality in children. Global prevalence and regional disparities of etiology, interventions, and outcomes are unknown. The aim of this point-prevalence study was to measure the burden of pediatric neurological injury and to describe variations in interventions and outcomes in ICUs.

Methods One hundred and three ICUs on six continents enrolled subjects on 4 specific days in a 1-year period. Included subjects were between ages 7 days and 17 years who were diagnosed with acute traumatic brain injury, stroke, cardiac arrest, central nervous system infection or inflammation, status epilepticus, spinal cord lesion, hydrocephalus, or brain mass. Sites completed a secure web-based case report form that included subject and hospital demographics, details about the neurological disease, interventions, length of stay, and Pediatric Cerebral Performance Category (PCPC) score (good outcome $=$ PCPC 1 to 3 ) and mortality at hospital discharge. 
Results Of 3,113 subjects screened, 1,009 (32\%) met enrollment criteria. The mean number of subjects enrolled per site for each study day was 2.4. Most sites were dedicated pediatric ICUs with a mean number of 22 ICU beds (range 3 to 72). ICUs had resources to invasively monitor intracranial pressure (93\%), continuous electroencephalography (14\%), invasive and non-invasive brain tissue oxygenation (14\% and $57 \%$ ), and somatosensory evoked potentials (39\%). There were on average 11 ICU faculty and six fellows per site, and nearly one-half reported a neurocritical care ICU team. Subjects were 58\% male and 52\% white, and $60 \%$ had normal pre-admission PCPC scores (85\%). Status epilepticus and cardiac arrest (both 21\%) had the highest prevalence. Sixty-one per cent of subjects were mechanically ventilated during ICU admission. ICU length of stay was a mean 29 days (median 43 days) and hospital LOS was a mean 43 days (median 61 days). Survival at hospital discharge was $87 \%$ with $58 \%$ of subjects discharged home and $17 \%$ to inpatient rehabilitation.

Conclusion Acute neurological disease is a significant pediatric health issue. These data suggest a vital need for increased research and healthcare resources to assist in the challenge of improving outcomes for these children.

\section{P347}

Survival analysis of patients taking dabigatran who consulted an emergency department for acute bleeding: a first alarm OA Arlet, EN Notebaert, JP Paquet, RD Daoust, MV Vincent, JC Chauny Hôpital du Sacré-Coeur de Montréal, Université de Montréal, Canada Critical Care 2013, 17(Suppl 2):P347 (doi: 10.1186/cc12285)

Introduction The newly approved oral anticoagulant dabigatran has no effective antidote. We therefore suspected an overall increase in mortality in patients presenting to the emergency department (ED) with a bleeding complication on dabigatran compared with warfarin or aspirin.

Methods We conducted a post hoc analysis on a database of all patients admitted to a tertiary-care ED with any kind of bleeding or suspicion of one from March 2011 to August 2012 who were taking dabigatran, warfarin, or aspirin. The primary endpoint was long-term survival. Patients were censored at death or at the end of the study period (7 December 2012). We performed a Cox proportional hazard model, controlled for age, to calculate the hazard ratio (HR) for dabigatran versus warfarin and one for warfarin versus aspirin. Statistical significance was set at $\alpha=0.05$ and results are presented with $95 \% \mathrm{Cl}$. Results In total, 943 patients met the inclusion criteria with a mean follow-up period of 1 year. The mean age was 74.3 years and $50.4 \%$ were men. A total of 108 deaths $(11.5 \%)$ were recorded within the follow-up period; eight (25\%) for dabigatran compared with 44 (12.6\%) for warfarin and $56(9.9 \%)$ for aspirin. The mortality risk for patients on dabigatran was significantly higher than for patients on warfarin: $\mathrm{HR}=2.1$ (95\% Cl: 1.0 to 4.5), $P=0.05$ after controlling for age. Aspirin had a lower (but not statistically significant) mortality risk compared with warfarin; $\mathrm{HR}=0.75(95 \% \mathrm{Cl}: 0.50$ to 1.14$), P=0.18$ after controlling for age.

Conclusion The results showed higher overall mortality in patients who presented to the ED with a bleeding complication and were taking dabigatran compared with warfarin or aspirin. Physicians should be aware of the potential higher mortality with dabigatran over warfarin when treating a bleeding patient. However, this was a singlecentre retrospective analysis with a small number of patients taking dabigatran $(n=32)$, and further studies are needed to corroborate the results.

\section{P348}

Bedside anti-Xa measurement for therapeutic assessment of a prophylactic anticoagulation regimen

B Dewulfi', I Herck², F De Somer², K Francois ${ }^{2}$, J Decruyenaere

'AZ Sint-Jan, Brugge, Belgium; '2 University Hospital, Gent, Belgium

Critical Care 2013, 17(Suppl 2):P348 (doi: 10.1186/cc12286)

Introduction Dose adjustments of low molecular weight heparin (LMWH) based on daily anti-Xa measurement by chromogenic assay remain controversial in daily clinical practice. One of the major obstacles is the cost of such a test. An affordable and reliable bedside test could change practice to an individual tailored dosing of LMWH. The aim of our study was to evaluate whether a prophylactic dose regimen of $40 \mathrm{mg}$ enoxaparine in cardiac surgical patients increases the anti-Xa activity to the level necessary for efficient prevention of a thromboembolic event [1]. Secondarily we tested whether there was a reliable correlation between a bedside anti-Xa measurement compared with a two-stage chromogenic assay at the laboratory [2].

Methods This was an open, single-centre, prospective, nonrandomized clinical trial at a university hospital. All patients that needed prophylactic dosing of enoxaparine after cardiac surgery were duly informed and after giving written consent we included 44 patients with a mean Euroscore of 1.66. The demographic specifications, medical and surgical history of all patients were collected. Anti-Xa activity was measured at three different points in time. We determined baseline, peak and trough anti-Xa activity: preoperatively, and respectively 4 hours after the third dose of enoxaparine and 30 minutes before the fourth dose. Each measurement was done with both techniques, the two-stage chromogenic assay at the laboratory (Biophen ${ }^{\oplus}$ ) and the bedside assay (Hemochron ${ }^{\circledR} \mathrm{Jr}$ ).

Results Our dose regimen of enoxaparine achieved in one-half of the included patients a sufficient anti-Xa activity for prevention of thromboembolic events. One-half of the patients with insufficient antiXa activity had a body mass index over $30 \mathrm{~kg} / \mathrm{m}^{2}$. Comparison of the bedside assay with the two-stage chromogenic assay by means of the Pearson's correlation coefficient showed correlation of the two tests if no variables were taken into account. In the Bland-Altman analysis we could not confirm this correlation.

Conclusion The bedside anti-Xa activity assay with a Hemochron device tends to show some correlation with the two-stage chromogenic assay, but insufficient to be used as an alternative, in this small but uniform patient population. Use of a standard dosing protocol for enoxaparine administration is prone for underdosage in post-cardiac surgery patients and may increase postoperative morbidity.

References

1. Ribic C, et al: J Crit Care 2009, 24:197-205

2. Gehrie E, et al:: Am J Hematol 2012, 87:194-196.

P349

Effective dosage of enoxaparin for intensive care patients

S Robinson, A Zincuk, U Larsen, P Toft

Odense University Hospital, Odense, Denmark

Critical Care 2013, 17(Suppl 2):P349 (doi: 10.1186/cc12287)

Introduction We hypothesized that higher doses of enoxaparin would improve thromboprophylaxis without increasing the risk of bleeding. Critically ill patients are predisposed to venous thromboembolism, leading to increased risk of adverse outcome [1]. Peak anti-factor Xa (anti-Xa) levels of 0.1 to $0.4 \mathrm{IU} / \mathrm{ml}, 4$ hours post administration of enoxaparin, reflect adequate thromboprophylaxis for medico-surgical patients.

Methods The sample population consisted of 78 patients, randomized to receive subcutaneous (s.c.) enoxaparin: $40 \mathrm{mg} \times 1$ (control group), versus $30 \mathrm{mg} \times 2,40 \mathrm{mg} \times 2$ or $1 \mathrm{mg} / \mathrm{kg} \times 1$ (test groups) for a period of 3 days. Anti-Xa activity was measured at baseline, and at 4, 12, 16 and 24 hours post administration on each day. Patients did not differ significantly between groups.

Results On day 1 of administration, doses of $40 \mathrm{mg} \times 1$ and $40 \mathrm{mg}$ $\times 2$ yielded similar mean peak anti-Xa of $0.20 \mathrm{IU} / \mathrm{ml}$ and $0.17 \mathrm{IU} / \mathrm{ml}$ respectively, while a dose of $30 \mathrm{mg} \times 2$ resulted in subtherapeutic levels of anti-Xa $(0.08 \mathrm{IU} / \mathrm{ml})$. Patients receiving $1 \mathrm{mg} / \mathrm{kg}$ enoxaparin achieved near-steady-state levels from day 1 with mean peak anti-Xa levels of $0.34 \mathrm{IU} / \mathrm{ml}$. Steady-state anti-Xa was achieved for all doses of enoxaparin at day 3. At steady state, mean peak anti-Xa levels of $0.13 \mathrm{lU} /$ $\mathrm{ml}$ and $0.15 \mathrm{IU} / \mathrm{ml}$ were achieved with doses of $40 \mathrm{mg} \times 1$ and $30 \mathrm{mg} \times 2$ respectively. This increased significantly to $0.33 \mathrm{IU} / \mathrm{ml}$ and $0.40 \mathrm{IU} / \mathrm{ml}$ for doses of $40 \mathrm{mg} \times 2$ and $1 \mathrm{mg} / \mathrm{kg}$ enoxaparin respectively $(P=0.0000)$ (Figure 1). A dose of $1 \mathrm{mg} / \mathrm{kg}$ enoxaparin yielded therapeutic anti-Xa levels for over $80 \%$ of the study period. There were no adverse effects. 


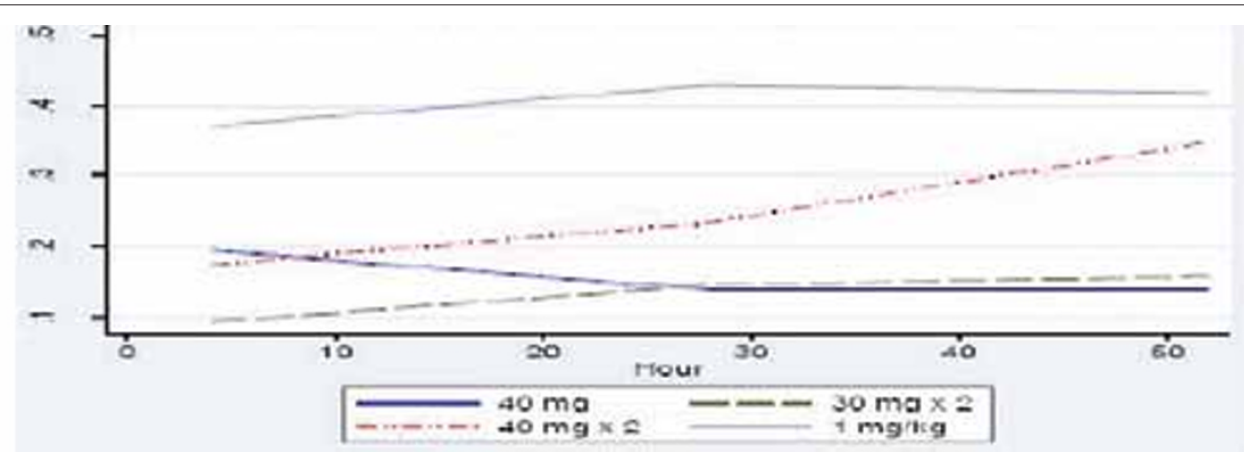

Figure 1 (abstract P349). Peak anti-Xa over 3 days with varying doses of enoxaparin.

Conclusion Current standard s.c. doses of $40 \mathrm{mg} \times 1$ enoxaparin (Europe) or $30 \mathrm{mg} \times 2$ (North America) yield levels of anti-Xa thought to be subtherapeutic for critically ill patients. A weight-based dose yielded the best anti-Xa levels, allowed the establishment of near-steady-state levels from the first day of enoxaparin administration, and may thus be more appropriate, convenient, and effective. A new study using $1 \mathrm{mg} /$ $\mathrm{kg}$ enoxaparin s.c. with clinical endpoints has recently been approved by the Danish Ministry of Health.

Reference

1. Cook D, et al: N Engl J Med 2011, 364:1305-1314.

P350

Abstract withdrawn

P351

Anti-Xa activities following 5,000 IU and 7,500 IU of s.c. dalteparin in critically ill patients and 5,000 IU in medical patients: a prospective randomized study

GH Heinz', U Priglinger ${ }^{2}$, I Pabinger ${ }^{3}$

'University Clinic of Internal Medicine II, Vienna, Austria; ${ }^{2}$ University Clinic of Emergency Medicine, Vienna, Austria; ${ }^{3}$ University Clinic of Internal Medicine I, Vienna, Austria

Critical Care 2013, 17(Suppl 2):P351 (doi: 10.1186/cc12289)

Introduction Unfractionated heparin is preferred over LMWH in ICU patients but LMWH is used more frequently in many European ICUs. Thromboprophylaxis with standard doses of nadroparin and enoxaparin has been shown to result in significantly lower anti-Xa in ICU patients when compared with medical patients [1,2].

Methods ICU patients (SAPS $44 \pm 16, \mathrm{MV}, n=44$; pressors $n=32$ ) received 7,500 IU (Group 1, $n=25$ ) or 5,000 IU dalteparin s.c. (Group 2, $n=29$ ). Twenty-nine medical patients receiving 5,000 IU dalteparin served as controls (Group 3).

Results Group 2 had significantly lower areas under the Xa curve (AUC) compared with Groups 1 and 3 (Table 1). Differences were not significant between Groups 1 and 3. Peak anti-Xa activities ( $C_{\max }$-anti-Xa) were delayed ( $\mathrm{t}_{\max }$-anti-Xa) in Group 2 compared with Groups 1 and 3 (Table 1).

Conclusion In ICU patients a s.c. dose of 5,000 IU dalteparin results in significantly lower Xa activities when compared with normal ward patients. A s.c. dose of 7,500 IU dalteparin in ICU patients resulted in kinetics and peak anti-Xa activities comparable with medical patients receiving 5,000 IU dalteparin.

References

1. Dörffler-Melly J, deJonge $E$, dePont $A C$, et al:: Bioavailability of subcutaneous low-molecular-weight heparin to patients on vasopressors. Lancet 2002, 359:849-850.

2. Priglinger U, Delle Karth G, Geppert A, et al.: Prophylactic anticoagulation with enoxaparin: is the subcoutaneous route appropriate in the critically ill? Crit Care Med 2003, 31:1405-1409.

P352

Randomized double-blind placebo-controlled PK/PD study on the effects of a single intravenous dose of the anti-hepcidin Spiegelmer NOX-H94 on serum iron during experimental human endotoxemia LVan Eijk', DW Swinkels', A John', F Schwoebel'2, F Fliegert², L Summo²,

S Vauleon'2, JM Laarakkers'? ${ }^{1}$ K Riecke², P Pickkers'

'Radboud University Nijmegen Medical Centre, Nijmegen, the Netherlands;

${ }^{2}$ NOXXON Pharma AG, Berlin, Germany

Critical Care 2013, 17(Suppl 2):P352 (doi: 10.1186/cc12290)

Introduction Anemia is very frequently encountered on the ICU. Increased hepcidin production is one of the cornerstones of the pathophysiology of anemia of inflammation. The first-in-class hepcidin antagonist NOX-H94, a PEGylated anti-hepcidin L-RNA oligonucleotide, is in development for targeted treatment of anemia of inflammation. We investigated whether NOX-H94 prevents the inflammation-induced serum iron decrease during experimental human endotoxemia.

Methods A randomized, double-blind, placebo-controlled trial in 24 healthy young men. At T = 0 hours, $2 \mathrm{ng} / \mathrm{kg} E$. coli endotoxin was administered intravenously (i.v.), followed by $1.2 \mathrm{mg} / \mathrm{kg} \mathrm{NOX-H94} \mathrm{or}$ placebo i.v. at $\mathrm{T}=0.5$ hours. Blood was drawn serially after endotoxin administration for measurements of inflammatory parameters,

Table 1 (abstract P351). Pharmacokinetics

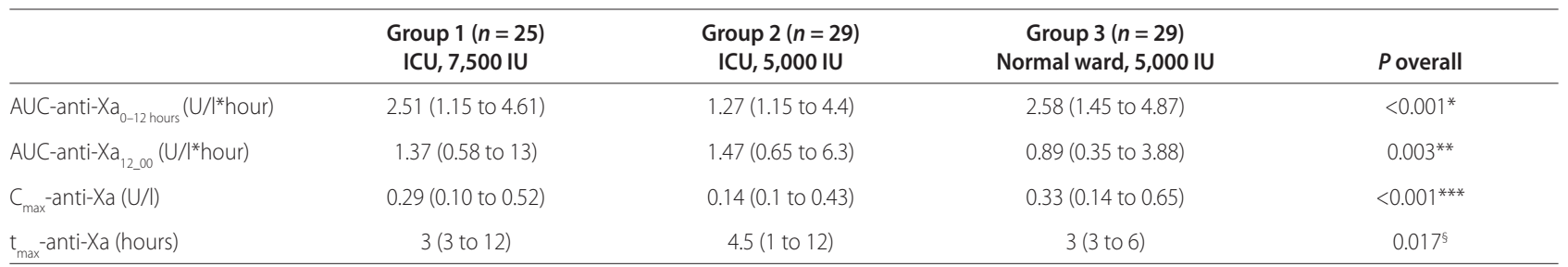

$C_{\text {max }}$ peak anti-Xa level; $t_{\max }$ time of anti-Xa peak. Medians and range. ${ }^{*}$ Group 1 versus $2, P=0.001$; Group 1 versus $3, P=0.51 .{ }^{* *}$ Group 1 versus $2, P=0.96 ;$ Group 1 versus 3, $P=0.045$; Group 2 versus 3, $P=0.13$. ${ }^{* *}$ Group 1 versus $2, P=0.001$; Group 1 versus 3, $P=0.29$. ${ }^{\circledR}$ Group 1 versus 3, $P=0.09 ;$ Group 1 versus 2, $P=0.8 ;$ Group 2 versus $3, P=0.018$. 
cytokines, NOX-H94 pharmacokinetics, total hepcidin-25, and iron parameters. The difference of serum iron change from baseline at $T=9$ hours was defined as the primary endpoint.

Results Endotoxin administration led to flu-like symptoms. Inflammatory parameters (CRP, body temperature, leucocytes, and plasma levels of TNFa, IL-6, IL-10, and IL-1RA) peaked markedly and similarly in both treatment groups. NOX-H94 was well tolerated. Plasma concentrations peaked at $0.7 \pm 0.4$ hours after the start of administration, after which they declined according to a two-compartment model, with a $\mathrm{T}_{1 / 2}$ of $22.5 \pm 4.28$ hours. In the placebo group, serum iron increased from $19.0 \pm 7.6 \mu \mathrm{g} / \mathrm{l}$ at baseline to a peak at $\mathrm{T}=3$ hours, returned close to baseline at $T=6$ hours and decreased under the baseline concentration at $\mathrm{T}=9$ hours, reaching its lowest point at $\mathrm{T}=12$ hours. In the NOX-H94 group, serum iron concentrations rose until $T=6$ hours and then slowly declined until $T=24$ hours. From 6 to 12 hours post LPS, the serum iron concentrations in NOX-H94-treated subjects were significantly higher than in placebo-treated subjects $(P<0.0001$, ANCOVA).

Conclusion Experimental human endotoxemia induces a robust inflammatory response and a subsequent decrease in serum iron. Treatment with NOX-H94 had no effect on innate immunity, but effectively prevented the inflammation-induced drop in serum iron concentrations. These findings demonstrate the clinical potential of the anti-hepcidin drug NOX-H94 for further development to treat patients with anemia of inflammation.

\section{P353}

Haemoglobin concentration on admission to intensive care influences hospital mortality rates and length of stay: a retrospective study

M Adams, P Dean, P Doherty, S Noble, A Mackay

Victoria Infirmary, Glasgow, UK

Critical Care 2013, 17(Suppl 2):P353 (doi: 10.1186/cc12291)

Introduction The association between haemoglobin concentrations and mortality has been studied in patients with various comorbidities $[1,2]$. This study aims to determine the association between haemoglobin levels on admission to intensive care and patient length of stay and mortality.

Methods A retrospective collection of data from patient admissions to a single five-bed ICU over a 15-year period identified 5,826 patients between April 1994 and November 2012. Patients were split into groups according to haemoglobin concentration on admission. The data were analysed to determine whether there was any relationship between haemoglobin concentration at ICU admission and any of our outcome measures (unit and hospital mortality, unit and hospital length of stay). Results Patients with haemoglobin concentrations $\leq 10 \mathrm{~g} / \mathrm{dl}$ and $>10.1 \mathrm{~g} / \mathrm{dl}$ were used in mortality comparisons. Patients with a haemoglobin concentration $\leq 10 \mathrm{~g} / \mathrm{dl}$ had an increase in ICU mortality compared with those with haemoglobin levels $>10 \mathrm{~g} / \mathrm{dl}(\mathrm{OR}=1.36,95 \%$ $\mathrm{Cl}=1.30$ to $1.71, P<0.0001)$. A similar difference was seen with hospital mortality $(\leq 10 \mathrm{~g} / \mathrm{dl} 37.7 \% \mathrm{vs}$. $>10 \mathrm{~g} / \mathrm{dl} 27.3 \%, P<0.0001)$. Unit length of stay was significantly longer in patients with admission $\mathrm{Hb} \leq 10 \mathrm{~g} /$ dl (5.34 days) compared with an admission $\mathrm{Hb}>10 \mathrm{~g} / \mathrm{dl}$ (4.08 days), $P<0.0001$. Hospital length of stay was also significantly longer in patients with $\mathrm{Hb} \leq 10 \mathrm{~g} / \mathrm{dl}$ versus $\mathrm{Hb}>10 \mathrm{~g} / \mathrm{dl}$ (21.6 days vs. 15.5 days, $P<0.0001)$. There was seen to be an inverse correlation between haemoglobin concentration and patient age $(r=-0.174 ; P<0.0001)$.

Conclusion Haemoglobin concentrations $\leq 10 \mathrm{~g} / \mathrm{dl}$ on admission to the ICU are associated with an increase in ICU mortality, hospital mortality, unit length of stay and hospital length of stay when compared with patients admitted with haemoglobin concentrations $>10 \mathrm{~g} / \mathrm{dl}$.

\section{References}

1. Zhao Y, et al:: Crit Care 2007, 11:P234

2. Sekhon MS, et al:: Crit Care 2012, 16:R128.

\section{P354}

Influence of epidural anesthesia on the hemocoagulation disorders and quantity of septic complications in patients with acute necrotizing pancreatitis

O Tarabrin, S Shcherbakov, A Tkachenko, O Kushnir, I Grychushenko

Odessa National Medical University, Odessa, Ukraine

Critical Care 2013, 17(Suppl 2):P354 (doi: 10.1186/cc12292)
Introduction According to many authors, acute necrotizing pancreatitis (ANP) still remains one of the difficult problems of abdominal surgery. The complexity of the pathogenesis of the disease, features of the pancreas pathomorphology, abdominal hypertension, and high mortality (30 to 70\%) necessitate a search for new ways to treat this disease.

Methods The study was conducted in 44 patients with ANP, who were divided into two groups according to type of analgesia: epidural or opioids. Patients from the first group $(n=23)$ had epidural analgesia by ropivacaine 6 to $14 \mathrm{mg} /$ hour during 7 to 10 days, and from the second group $(n=21)$ opioid analgesia by trimeperidine $20 \mathrm{mg}$ three times a day during the same period. We monitored the level of septic and thrombohemorrhagic complications by clinical and instrumental data, during the month after treatment starting. The hemostatic system was evaluated using indicators of hemoviscoelastography (Mednord-01M analyzer).

Results It was found that all patients with ANP initially have hypercoagulation and fibrinolysis inhibition. Levels of hemostatic disorders correlate with the level of septic complications, treatment in the ICU, and mortality. In the first group we noted a deep vein thrombosis, two pneumonia, seven pseudopancreatic cysts and abscesses, two deaths and time of stay in the ICU as 15.4 days. In the second group: three cases of deep vein thrombosis, four pneumonia, 10 pseudopancreatic cysts and abscesses, two episodes of gastroduodenal bleeding, five deaths and time of stay in the ICU as 27.8 days.

Conclusion Using epidural anesthesia in patients with ANP reduced the number of septic complications on $36.6 \%$, and reduced the mortality rate from $23.8 \%$ (second group) to $8.7 \%$ (first group). We think that violations of blood coagulation and microcirculation are the basis for ischemia, necrosis in tissues and septic complications. Epidural analgesia is an effective method to decrease the level of septic and thrombohemorrhagic complications and mortality in ANP patients.

P355

Hemoviscoelastography: a new method of hemostasis monitoring

O Tarabrin, S Galich, D Gavrychenko, G Mazurenko, A Suhanov

Odessa National Medical University, Odessa, Ukraine

Critical Care 2013, 17(Suppl 2):P355 (doi: 10.1186/cc12293)

Introduction Deep vein thrombosis of lower extremities and pulmonary embolism occupy an important place in the structure of postoperative morbidity and mortality.

Methods After ethics approval and informed consent, we studied the functional state of hemostasis in a group of 57 healthy volunteers, who were not receiving drugs affecting coagulation, and 43 patients with postphlebothrombotic syndrome (PPTS). In the PPTS patients we conducted baseline studies of coagulation state and daily monitoring of dynamic changes in the functional state of hemostasis, a comparative evaluation of performance low-frequency piezoelectric vibration hemoviscoelastography (LPVH) and platelet aggregation test (PAT), standard coagulation tests (SCT), and thromboelastogram (TEG). Results We found that LPVH correlated with SCT, PAT and TEG. However, our proposed method is more voluminous: indexes ICC (the intensity of the contact phase of coagulation), $\mathrm{t} 1$ (the time for the contact phase of coagulation), and $\mathrm{AO}$ (initial rate of aggregation of blood) are consistent with PAT; indexes ICD (the intensity of coagulation drive), CTA (a constant thrombin activity) and CP (the clot intensity of the polymerization) are consistent with SCT and TEG. In addition, the advantage of this method is to determine the intensity of fibrinolysis with the indicator IRIS (the intensity of the retraction and clot lysis).

Conclusion LPVH allows one to make a total assessment of all parts of hemostasis: from initial viscosity and platelet aggregation to coagulation and lysis of clots, as well as their interaction. These figures are objective and informative, as evidenced by close correlation with the performance of traditional coagulation methods.

\section{P356}

Thromboelastographic monitoring of fondaparinux in surgical patients B Martinez', R Giacomello', R Paniccia

'University Hospital of Udine, Italy; 'University of Florence, Italy Critical Care 2013, 17(Suppl 2):P356 (doi: 10.1186/cc12294)

Introduction Fondaparinux is currently the first therapeutic choice in several clinical settings such as deep venous thrombosis (DVT) 
prophylaxis in orthopedics or in cases of acute coronary syndromes. The main drawback of FOND is that routine monitoring is not currently available. This could be a problem during the management of critical and surgical patients, especially in cases of old patients and renal failure. The aim of this study is to evaluate the ability of thromboelastography (TEG) to determine the level of anticoagulation due to FOND in a surgical population.

Methods We prospectively analyzed all patients to whom elective major orthopedic surgery was consecutively performed in a 2-month period. All the patients received FOND $2.5 \mathrm{mg}$ in the postoperative period according to ACCP 2012 Guidelines. Native and heparinase (hep) TEG (Haemoscope Corporation, Niles, IL, USA) tests activated with kaolin were performed using whole blood citrated samples at four times: T0, before FOND administration; T1, 2 hours after administration; T2, 17 hours after administration (half-life); T3, 24 hours after administration. The following native and hep TEG parameters were analyzed: reaction time (R), a angle, maximum amplitude (MA) and coagulation index $(\mathrm{Cl})$. These parameters were compared with levels of anti-Xa. Unvariate analysis and Spearman's test were applied to our data.

Results Eighteen patients were analyzed. Ten patients met the inclusion criteria. The mean $\mathrm{R}$ value increased from T1 to T3. The mean $R$ parameter was in the normal range at any phase of the study and there was no significant differences between the $\mathrm{R}$ mean value at the different phases. The lowest value of $\mathrm{R}$ was at $\mathrm{T} 1$, which coincides with plasmatic peak concentration of FOND. This value did not correlate with anti-Xa mean value at $\mathrm{T} 1$, which showed the highest value at that time. There was significant difference between the mean native and hep $\mathrm{R}$ value only at T2 $(P<0.05)$, native and hep a angle at T3, MA and MA hep at T2 $(P<0.01)$ and $\mathrm{Cl}$ and $\mathrm{Cl}$ hep at T3 $(P<0.02)$. Only the parameter MA had significant variation over time $(P<0.02)$.

Conclusion $\mathrm{R}$ represents the time necessary to thrombin formation and in the presence of FOND we hypothesized a prolonged $\mathrm{R}$ time. In our population, TEG performed with citrated kaolin-activated whole blood was not able to detect prophylatic doses of FOND in every phase. On the contrary, levels of anti-Xa were able to reveal the exact pharmacokinetics of the drug. Further studies including a large number of patients are necessary.

References

1. Falck-Ytter Y, et al: Chest 2012, 141:2

2. FUTURA/OASIS Trial Group, Steg PG, Jolly SS, Mehta SR, et al:: Low-dose vs standard dose unfractioned heparin for percutaneous coronary intervention in acute coronary syndromes treated with fondaparinux: the FUTURA/OASIS randomized trial. JAMA 2010, 304:1339-1349.

P357

Urokinase-induced fibrinolysis in thromboelastography (UKIF-TEG) to assess fibrinolysis in critically ill patients

L Zacchetti, M Panigada, M Cressoni, T Marchesi, L Gattinoni

Fondazione IRCCS Ca' Granda Ospedale Policlinico Milano, Milan, Italy

Critical Care 2013, 17(Suppl 2):P357 (doi: 10.1186/cc12295)

Introduction Coagulopathy, particularly a trend toward hypercoagulability and hypofibrinolysis, is common in critically ill patients and correlates with worse outcome. Available laboratory coagulation tests to assess fibrinolysis are expensive and time demanding. We investigated whether a modified thromboelastography with the plasminogen activator urokinase (UKIF-TEG) [1] may be able to evaluate fibrinolysis in a population of critically ill patients.

Methods UKIF-TEG was performed as follows: first urokinase was added to citrate blood to give final concentrations of $160 \mathrm{UI} / \mathrm{ml}$, then thromboelastography (TEG) analysis was started after kaolin activation and recalcification with calcium chloride. Basal TEG (no addition of urokinase) was also performed. Fibrinolysis was determined by the loss of clot strength after the maximal amplitude (MA), and recorded as Ly30 (percentage lysis at 30 minutes after MA) and as Ly60 (percentage lysis at 60 minutes after MA).

Results UKIF-TEG was performed on 17 healthy volunteers and 18 critically ill patients. Ly 60 was predicted by Ly 30 according to an exponential function, so we used Ly30 as an indicator of clot lysis. Basal TEG showed increased coagulability and a trend toward less fibrinolysis in critically ill patients compared with healthy volunteers (reaction time

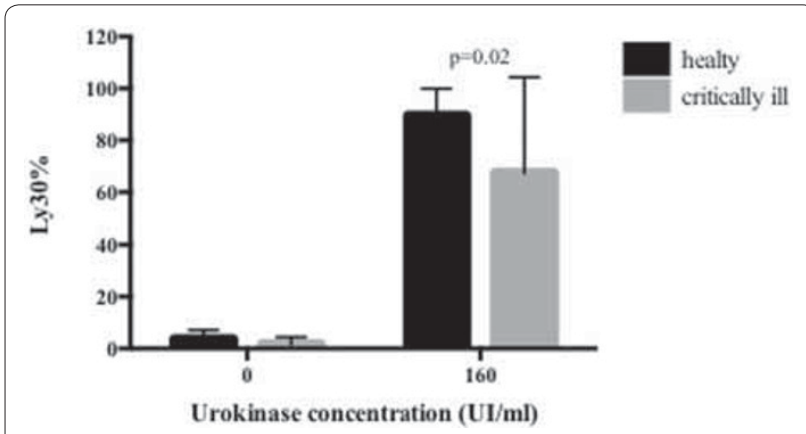

Figure 1 (abstract P357).

$8.7 \pm 3.4$ minutes vs. $12.2 \pm 1.9$ minutes, $P<0.001 ; a$-angle $59.7 \pm 9.4$ vs. $47.2 \pm 11.8, P<0.01)$. This reduction of fibrinolysis was more evident at a urokinase concentration of $160 \mathrm{UI} / \mathrm{ml}$ (Figure 1).

Conclusion UKIF-TEG could be a feasible point-of-care method to evaluate fibrinolysis in critically ill patients.

Reference

1. Gallimore MJ, et al:. J Thromb Haemost 2005, 3:2506-2513.

P358

Bleeding complications after central line insertions and the relevance of pre-procedure coagulation tests and blood component therapy

TK Kander, US Schött

Lund University, Lund, Sweden

Critical Care 2013, 17(Suppl 2):P358 (doi: 10.1186/cc12296)

Introduction Bleeding complications in association with insertion of central venous catheters are reported in 0.5 to $1.6 \%$ of cases, are rarely fatal, but sometimes require intervention. The aim of this study was to map pre-procedural variables at the insertion of a central venous catheter and to investigate whether any independent variable could be identified as an independent risk factor for bleeding complications associated with insertion of central venous catheters.

Methods In this retrospective study, we investigated 1,737 consecutive insertions of central venous catheters on 1,444 patients in a large university hospital during 2009 to 2010. Pre-procedural coagulation status, blood component use, type of catheter, insertion site, and complications during insertion were recorded and compared with bleeding complications documented in electronic charts.

Results No serious bleeding complications were recorded in connection with the insertions of central venous catheters. Sixteen out of 1,769 (0.9\%) insertions caused grade 2 bleeding, being defined as a bleeding requiring prolonged compression at the insertion site. Insertion of a large-bore central dialysis catheter was found to be an independent risk factor for bleeding complications. Neither conventional coagulation tests nor accidental arterial puncture or the number of needle passes could predict bleeding complications in this study.

Conclusion This retrospective study shows that serious bleeding complications in association with central line insertion are uncommon and that insertion of dialysis catheter is an independent risk factor for mild bleeding complications. Coagulopathy, measured with conventional coagulation tests, is not an independent risk factor for bleeding complication at insertion of central venous catheters.

\section{P359}

Efficacy of tranexamic acid in decreasing blood loss during cesarean section

V Zaporozhan, O Tarabrin, D Gavrychenko, G Mazurenko, O Saleh,

I Lyoshenko

Odessa National Medical University, Odessa, Ukraine

Critical Care 2013, 17(Suppl 2):P359 (doi: 10.1186/cc12297)

Introduction Despite significant progress in obstetric care, the problem of bleeding during labor remains unsolved. Annually in the world, 125,000 women die from obstetric hemorrhage. 
Methods We performed a randomized, double-blind study in 37 patients who underwent cesarean section. Patients were divided into two groups: the first group $(n=19)$ received preoperative (30 minutes before operation) tranexamic acid $10 \mathrm{mg} / \mathrm{kg}$; the second group ( $n=18$ ) received preoperative placebo. The condition of hemostasis was monitored by haemoviscoelastography.

Results All patients included in the study before surgery had moderate hypercoagulation and normal fibrinolysis: increasing the intensity of clot formation (ICF) to $11.4 \%$ compared with normal rates; the intensity of the retraction and clot lysis (IRCL) was $16.45 \pm 1.40$ in both groups. At the start of the operation in patients (Group 1), ICF decreased by 9.7\% $(P<0.05)$, and IRCL decreased by $27.6 \%(P<0.05)$ compared with preoperatively. In Group 2, there was ICF decrease by $8.8 \%(P<0.05)$, and IRCL increase by $11.4 \%(P<0.05)$ compared with preoperatively. At the end of the operation, the condition of hemostasis in both groups came almost to the same value - moderate hypocoagulation, depressed fibrinolysis. In both groups there were no thrombotic complications. Intraoperative blood loss in the first group was $300 \pm 40.5$ and in the second was $500 \pm 60.6$.

Conclusion Using of tranexamic acid before surgery significantly reduces intraoperative blood loss by $60 \%$, without thrombotic complications.

P360

In vitro correction of hypothermic and dilutive crystalloid and colloid rotational thromboelastography-monitored coagulopathy with fibrinogen and factor XIII

D Winstedt, OThomas, US Schött

Lund University, Lund, Sweden

Critical Care 2013, 17(Suppl 2):P360 (doi: 10.1186/cc12298)

Introduction Rotational thromboelastography (ROTEM) can detect dilutive and hypothermic effects on coagulation and evaluate corrective treatments. The aim of this in vitro study was to study whether fibrinogen concentrate alone or combined with factor XIII could reverse colloid-induced and crystalloid-induced coagulopathies in the presence and absence of hypothermia.

Methods Citrated venous blood from 10 healthy volunteers was diluted by $33 \%$ using 130/0.42 hydroxyethyl starch or Ringer's acetate. ROTEM was used to evaluate the effect of addition of either fibrinogen concentrate corresponding to $4 \mathrm{~g} / 70 \mathrm{~kg}$, or this fibrinogen dose combined with factor XIII equivalent to $20 \mathrm{IU} / \mathrm{kg}$. Blood was analyzed at 33 or $37^{\circ} \mathrm{C}$ with ROTEM ExTEM and FibTEM reagents.

Results A significant dilutive response was shown in both groups: hypocoagulation was greater in the starch group. Hypothermia lengthened the following: ExTEM clotting time (CT), clot formation time and a angle; FibTEM maximal clot formation (MCF). Irrespective of temperature, fibrinogen overcorrected Ringer's acetate's effects on all ROTEM parameters and partially reversed starch's effects on EXTEM CT and FibTEM MCF. FibTEM demonstrated that factor XIII provided an additional procoagulative effect in the Ringer's acetate group at both temperatures but not the starch group. The only ExTEM parameter to be improved by addition of factor XIII was MCF at $33^{\circ} \mathrm{C}$.

Conclusion ROTEM shows that fibrinogen concentrate can reverse dilutive coagulation defects induced by colloid and crystalloid at both 33 and $37^{\circ} \mathrm{C}$. Some additional reversal was provided by factor XIII: higher doses of both fibrinogen and factor XIII may counteract starch's effects on clot structure.

P361

Dose-response of fibrinogen and factor XIII concentrate for

correcting albumin-induced coagulopathy

US Schött, DW Winstedt, JH Hanna

Lund University, Lund, Sweden

Critical Care 2013, 17(Suppl 2):P361 (doi: 10.1186/cc12299)

Introduction Natural colloid albumin induces a lesser degree of dilutional coagulopathy than synthetic colloids. Fibrinogen concentrate has emerged as a promising strategy to treat coagulopathy, and factor XIII (FXIII) works synergistically with fibrinogen to correct coagulopathy following haemodilution with crystalloids. Objectives were to examine the ability of fibrinogen and FXIII concentrates to reverse albumininduced dilutional coagulopathy.
Methods High and low concentrations of both fibrinogen and FXIII were used to reverse coagulopathy induced by $1: 1$ dilution in vitro with $5 \%$ albumin of blood samples from healthy volunteers, monitored by rotational thromboelastometry (ROTEM).

Results Haemodilution with albumin significantly attenuated EXTEM maximum clot firmness (MCF), a angle (AA), clotting time (CT) and clot formation time (CFT), and FIBTEM MCF $(P<0.001)$. Following haemodilution, both doses of fibrinogen significantly corrected all ROTEM parameters $(P \leq 0.02)$, except the lower dose did not correct AA. Compared with the lower dose, the higher dose of fibrinogen significantly improved FIBTEM MCF and EXTEM MCF, AA and CFT $(P<0.001)$. The lower dose of FXIII did not significantly correct any of the ROTEM parameters, and the high dose only improved EXTEM CT $(P=0.004)$. All combinations of high/low concentrations of fibrinogen/ FXIII significantly improved all ROTEM parameters examined $(P \leq 0.001)$. Fibrinogen concentration generally had a greater effect on each parameter than did FXIII concentration; the best correction of ROTEM parameters was achieved with high-dose fibrinogen concentrate and either low-dose or high-dose FXIII.

Conclusion Fibrinogen concentrate successfully corrected initiation, propagation and clot firmness deficits induced by haemodilution with albumin, and FXIII synergistically improved fibrin-based clot strength. Reference

1. Anesth Analg 2008, 106:1360-1365.

P362

Intraoperative cell savage for life-threatening haemorrhagic caesarean section

AS Ducloy', C Barre-Drouard², E Jaillette², S Depret-Mosser², B Wibaut², BVallet ${ }^{2}, S_{\text {Susen }}^{2}$

'Hôpital Jeanne de Flandre, Lille, France; ${ }^{2}$ Centre hospitalier regional

universitaire Lille, France

Critical Care 2013, 17(Suppl 2):P362 (doi: 10.1186/cc12300)

Introduction Postpartum hemorrhage (PPH) remains the leading cause of maternal mortality. After IOCS introduction in our north of France tertiary-care obstetric unit, the consecutive cohort of severe PPH was collected prospectively and compared regarding the use of IOCS or not.

Methods A case-control prospective open-label study comparing the management and outcome of a consecutive cohort of 324 severe PPHs in elective and emergency hemorrhagic caesarean sections (CS) over 3 years.

Results IOCS was used in 70 severe PPHs and 254 severe PPH controls were managed without IOCS. Placenta accreta can be selected as the best indication for $\mathrm{RBC}$ restitution. In the 1,500 to $3,000 \mathrm{ml} \mathrm{PPH}$, allogeneic transfusion was decreased in the IOCS group: 17.6 versus $56.3 \%(P=0.006)$; PRBC: 0 (0 to 3$)$ versus 3 (0 to 6$)(P=0.045)$. IOCS spared 87 blood bank PRBC $(17,374 \mathrm{ml})$; that is, $24.2 \%$ of the total transfusion need. No amniotic fluid embolism has been observed in the group with IOCS whereas one case appeared in the control group without IOCS.

Conclusion Regarding the literature [1-4] and our study, IOCS could be used safely in PPH during CS. A leukocyte filter for retransfusion has been recommended and Rhesus isoimmunization must be precluded and monitored by repeated fetal RBC testing.

References

1. Rainaldi et al:: Br J Anaesth 1998, 80:195-198

2. Bernstein et al: Anesth Analg 1997, 85:831-833.

3. Catling et al:: Int J Obstet Anesth 1999, 8:79-84.

4. Waters et al: Anesthesiology 2000, 92:1531-1536.

P363

Does protamine infusion reduce postoperative blood loss and

heparin rebound in cardiac surgery patients? A retrospective

analysis of 240 patients on the cardiac ICU

E Helme, H Meeran, N Al-Subaie

St George's Hospital, London, UK

Critical Care 2013, 17(Suppl 2):P363 (doi: 10.1186/cc12301)

Introduction Cardiac surgery is associated with significant blood loss. Teoh and colleagues demonstrated a reduction in postoperative 
bleeding with the use of a protamine infusion and an abolishment of heparin rebound [1]. The aim of this study was to see whether the use of postoperative protamine infusions in our cardiac ITU was associated with a reduction in heparin rebound and blood loss.

Methods Data from 240 cardiac surgery patients were retrospectively analysed. Of these, 157 had routine management with a bolus of protamine to correct the activated clotting time and then expectant management of subsequent bleeding, and 47 had the same but also a protamine infusion of 10 to $80 \mathrm{mg} /$ hour for between 3 and 8 hours postoperatively. Blood loss was measured at 1, 6, 12 and 24 hours. In all, excessive bleeding was investigated using thromboelastography (TEG). Rebound heparinisation was determined by a ratio of R-times (heparinase/plain) $<0.8$. The Mann-Whitney $\mathrm{U}$ test and the chi-squared test were used to assess statistical significance.

Results There was no significant difference in blood loss between the two groups. Blood loss at 1 hour in the infusion and non-infusion group was 145 and $88 \mathrm{ml}$, respectively $(P=0.06)$; at 6 hours: 450 and $392 \mathrm{ml}$ $(P=0.5)$; at 12 hours: 620 and $595 \mathrm{ml}(P=0.62)$; and at 24 hours: 971 and $872 \mathrm{ml}(P=0.12)$. There was also no significant difference in those getting heparin rebound with $40 \%$ in the infusion group and $47 \%$ in the non-infusion group $(P=0.54)$.

Conclusion Unlike Teoh and colleagues [1], we did not find an advantage in using protamine infusions. That there were still cases of heparin rebound in the infusion group suggests that the infusion was not as effective as expected and/or the dose was inadequate. However, previous studies assessed heparin rebound using isolated clotting parameters $[1,2]$. Here, we used TEG. As TEG measures the thrombodynamic properties of whole blood coagulation, perhaps it is a more reliable indicator of heparin activity? As a retrospective study, there are limitations; namely, the nonstandardised management of the patients and the potential bias in the anaesthetists' selection of patients for an infusion. This group may be inherently higher risk for bleeding. However, heparin rebound is common and protamine is a simple, relatively safe and low-cost intervention compared with transfusion and so further study is needed.

References

1. Teoh K, et al:: J Thorac Cardiovasc Surg 2004, 128:211-219.

2. Teoh K, et al: Circulation 1993, 88:420-425.

P364

Transfusion Requirements in Surgical Oncology Patients (TRISOP): a randomized, controlled trial

J Almeida', F Galas', E Osawa', J Fukushima', S Moulin', C Park',

E Almeida', S Vieira', J Vincent², A Rhodes', M Balzan', J Inacio',

H Palomba', R Nakamura', F Bergamin', A Sandrini', U Ribeiro Jr',

J Auler Jr', L Hajjar'

'Instituto do Cancer do Estado de São Paulo, Brazil:'2Erasme Hospital,

Université libre de Bruxelles, Brussels, Belgium; ${ }^{3}$ St George's Healthcare NHS

Trust and St George's University of London, UK

Critical Care 2013, 17(Suppl 2):P364 (doi: 10.1186/cc12302)

Introduction The purpose of this study was to evaluate whether a restrictive strategy of red blood cell (RBC) transfusion was superior to a liberal one for reducing mortality and severe clinical complications among patients undergoing major cancer surgery.

Methods The trial was designed as a phase III, randomized, controlled, parallel-group, superiority trial. The inclusion criteria were adult patients with cancer who were undergoing major abdominal surgery requiring postoperative care in an ICU. The patients were randomly allocated to treatment with either a liberal RBC transfusion strategy (transfusion when hemoglobin levels decreased below $9 \mathrm{~g} / \mathrm{dl}$ ) or a restrictive RBC transfusion strategy (transfusion when hemoglobin levels decreased below $7 \mathrm{~g} / \mathrm{dl}$ ). The primary outcome was a composite endpoint of death or severe complications. The patients were monitored for 30 days.

Results A total of 1,521 patients were screened for eligibility and 248 met the inclusion criteria. After exclusions for medical reasons or a lack of consent, 198 patients were included in final analysis, with 101 allocated to the restrictive group and 97 to the liberal group. The primary composite endpoint - all-cause mortality, cardiovascular complications, acute respiratory distress syndrome, acute kidney injury requiring renal replacement therapy, septic shock or reoperation at 30 days - occurred in $19.6 \%$ of the patients in the liberal strategy group and in $35.6 \%$ in the restrictive group $(P=0.012)$. The liberal strategy group had a significantly lower 30-day mortality rate as compared with the restrictive group $(8.2 \%(95 \% \mathrm{Cl}, 4.2$ to $15.4 \%)$ vs. $22.8 \%(95 \% \mathrm{Cl}, 15.7$ to $31.9 \%)$, respectively, $P=0.005)$. The occurrence of cardiovascular complications was lower in the liberal group than in the restrictive group (5.2\% (95\% Cl, 2.2 to $11.5 \%$ ) vs. $13.9 \%$ ( $95 \% \mathrm{Cl}, 8.4$ to $21.9 \%)$, respectively, $P=0.038$ ). The restrictive strategy group had a higher $60-$ day mortality rate as compared with the liberal group $(23.8 \%(95 \% \mathrm{Cl}$, 16.5 to $32.9 \%$ ) vs. $11.3 \%(95 \% \mathrm{Cl}, 6.5$ to $9.2 \%)$, respectively, $P=0.022)$. Conclusion The liberal RBC transfusion strategy with a hemoglobin trigger of $9 \mathrm{~g} / \mathrm{dl}$ was associated with fewer major postoperative complications in patients undergoing major cancer surgery compared with the restrictive strategy.

Acknowledgement ClinicalTrials.gov NCT 01560494.

\section{P365}

Red blood cell transfusion is a predictor of mortality and morbidity in children undergoing cardiac surgery

C Colognesi, R Maia, L Hajjar, F Bergamin, J Fukushima, E Osawa,

J Almeida, L Camara, S Zeferino, V Guimaraes, F Galas

Heart Institute, São Paulo, Brazil

Critical Care 2013, 17(Suppl 2):P365 (doi: 10.1186/cc12303)

Introduction Red blood cell (RBC) transfusion is associated with morbidity and mortality in critically ill patients. Congenital cardiac surgeries are associated with high rates of bleeding and consequently with high rates of allogeneic transfusion. We aimed to evaluate the association of transfusion with worse outcomes in children undergoing cardiac surgery.

Methods We performed a prospective cohort study of 205 patients undergoing cardiac surgery for congenital heart disease. We recorded baseline characteristics, RACHS-1 score, intraoperative data, transfusion requirement and severe postoperative complications as need for reoperation, acute kidney injury, arrhythmia, severe sepsis, septic shock, bleeding, stroke, and death during 30 days. We performed univariate analysis using baseline, intraoperative and postoperative variables. Selected variables $(P<0.10)$ were included in a forward stepwise multiple logistic regression model to identify predictive factors of a combined endpoint including 30-day mortality and severe complications.

Results One hundred and thirty-six patients (66.3\%) were exposed to RBC transfusion. In the intraoperative room, $63.4 \%$ of patients received at least one RBC unit, and in the ICU, $11.2 \%$ of children were transfused. From all patients, $66(32.1 \%)$ presented the combined endpoint. Patients with complications had higher RACHS-1 score, were younger (69 months ( 0 to 137 ) vs. 73 months ( 37 to 138 ), $P<0.001$ ), had a lower weight (13 kg (3 to 23 ) vs. $20 \mathrm{~kg}$ (12 to 36$), P<0.001$ ), a longer time of surgery (475 minutes ( 410 to 540 ) vs. 353 (275 to 433 ), $P<0.001$ ), a longer duration of cardiopulmonary bypass ( 205 minutes (175 to 235) vs. 106 minutes (73 to 123), $P=0.003$ ), a lower $\mathrm{SVO}_{2}$ at the end of surgery (59\% (IQR 39 to 80 ) vs. $78 \%$ (71 to 83 ), $P<0.001)$, a higher arterial lactate at the end of surgery $(6.9 \mathrm{mmol} / \mathrm{l}(4.3$ to 9.2$)$ vs. $2.7 \mathrm{mmol} / \mathrm{l}$ (73 to 123), $P=0.003$ ), a lower intraoperative hematocrit $(26.2 \pm 5.6 \%$ vs. $29.5 \pm 6 \%(P<0.001))$ and a lower hematocrit at the end of surgery $(33.4 \pm 6.7 \%$ vs. $36.9 \pm 6.9 \%(P<0.001))$ as compared with patients without complications. Patients with complications were more exposed to RBC transfusion in the intraoperative room $(75 \%$ vs. $57 \%$, $P=0.011)$ and in the ICU (21\% vs. $6.4 \%, P=0.002)$. In an adjusted model of logistic regression, $\mathrm{RBC}$ transfusion is an independent risk factor of combined endpoint (OR 4.25 ( $95 \% \mathrm{Cl}, 1.359$ to 13.328), $P=0.013$ ).

Conclusion Blood transfusion after pediatric cardiac surgery is a risk factor for worse outcome including 30-day mortality. Avoiding blood transfusion must be a goal of best postoperative care. 
P366

Red blood cell transfusion results in worse outcomes in patients with left ventricular dysfunction undergoing on-pump cardiac surgery

R Miadda, C Colognesi, L Hajjar, M Sundin, L Camara, S Zeferino, F Bergamin, A Leme, V Guimaraes, F Galas

Heart Institute, São Paulo, Brazil

Critical Care 2013, 17(Suppl 2):P366 (doi: 10.1186/cc12304)

Introduction Cardiac surgeries are associated with high rates of bleeding and consequently with high rates of allogeneic transfusion. Patients with left ventricular dysfunction usually present a higher incidence of complications after cardiac surgery, including low output syndrome and organ dysfunction. It is a matter of controversy whether this subgroup of patients need higher hemoglobin levels to optimize oxygen delivery. We aimed to evaluate the association of transfusion with worse outcomes in patients with left ventricular dysfunction.

Methods A prospective cohort study was conducted in 215 patients undergoing cardiac surgery, presenting ejection fraction $<35 \%$. We recorded baseline characteristics, intraoperative data, transfusion requirement and severe postoperative complications as need for reoperation, acute kidney injury, arrhythmia, severe sepsis, septic shock, bleeding, stroke, and death during 30 days. We performed univariate analysis using baseline, intraoperative and postoperative variables associated with severe postoperative complications. Selected variables $(P<0.10)$ were included in a forward stepwise multiple logistic regression model to identify predictive factors of a combined endpoint including 30-day mortality and severe complications.

Results Two hundred and fifteen patients were included in the study. The mean ejection fraction was 31\%. From all patients, 129 (54.4\%) presented the combined endpoint. The incidence of RBC transfusion was higher in the group with complications compared with the group without complications both intraoperative and postoperative, $P<0.001$. Patients with complications were older, had lower initial hemoglobin levels and had higher EuroSCORE. At multivariate analysis, age $(\mathrm{OR}=1.07,95 \% \mathrm{Cl}=1.013$ to $1.137, P=0.016)$ and perioperative $\mathrm{RBC}$ transfusion ( $\mathrm{OR}=7.78,95 \% \mathrm{Cl}=2.13$ to $28.41, P=0.002$ ) were independent predictors of severe postoperative complications after cardiac surgery.

Conclusion In patients with left ventricular dysfunction, red blood transfusion after cardiac surgery is a risk factor for worse outcome including 30-day mortality.

\section{P367}

Defining the need for a massive transfusion in severe blunt traumatic patients

T Umemura, H Ishikura, Y Nakamura, K Hoshino, T Nishida, T Kamitani Fukuoka University, Fukuoka, Japan

Critical Care 2013, 17(Suppl 2):P367 (doi: 10.1186/cc12305)

Introduction We do not have enough criteria to make a judgment of the need for a massive transfusion (MT) in severe blunt traumatic patients. As a scoring system to predict the need for a MT, we usually use the Assessment Blood Consumption score (ABCs) and/or the Trauma-Associated Severe Hemorrhage score (TASHs). However, for these scoring systems, the procedure is slightly complicated. The aim of this study was to establish a predictor of a MT using coagulation or fibrinolysis markers.

Methods A retrospective analysis of MT was conducted in patients with severe blunt traumatic injury, which was defined as Injury Severity Score (ISS) of 16 or more admitted to the ICU between 1 June 2009 and 31 December 2010. Blood samples were collected from patients immediately after arriving at our level I trauma center. We defined the patients who received more than 10 unit packed red blood cells (PRBCs) within the first 24 hours as a MT group and who received less than 9 units PRBCs as a non-MT group. After the demographic data, number of units of PRBCs and the need for massive transfusions were recorded and analyzed in each groups, we compared data between two groups.

Results There were 114 patients who met the inclusion criteria. Fifty patients received blood transfusions (43.9\%; 50/114). There were 27 patients in the MT group (23.7\%; 27/114) and 87 in the non-MT group. The MT group was significantly higher in the ratio of females $(P<0.001)$, ISS $(P<0.01)$, PT-INR $(P<0.001)$, APTT $(P<0.05), \mathrm{ABCs}(P<0.001)$ and TASHs $(P<0.001)$ than in the on-MT group. On the other hand, the MT group was significantly lower in $\operatorname{Ps}(P<0.05)$ and fibrinogen level $(P<0.001)$ than the non-MT group. In the receiver operating characteristics (ROC) analysis, the area under the curve (AUC) to distinguish a MT was the highest for TASHs $(0.831, P<0.001)$, followed by fibrinogen $(0.758, P<0.001)$, and $A B C s(0.732, P<0.001)$. Fibrinogen was only a predictor of a MT without a scoring system such as $A B C$ s and TASHs, and the optimal cutoff value was $205 \mathrm{mg} / \mathrm{dl}$.

Conclusion We found that the level of fibrinogen was the most valuable predictor of a MT in the coagulation or fibrinolysis markers. It is certain that the level of fibrinogen at admission was not as useful as the TASHs about predicting a MT in this study. Whereas the scoring systems require the assessment of several factors, the measurement of fibrinogen is simple, easy and quick. We strongly suggest that the level of fibrinogen will be a useful predictor of a MT at in severe blunt traumatic patients.

References

1. Timothy C, et al.: J Trauma 2009, 66:346-352.

2. Yücel N, et al.: J Trauma 2006, 60:1228-1236.

3. Sørensen B, et al: Semin Thromb Hemost 2012, 38:268-273.

P368

Association between red blood cell transfusions and clinical outcome in critically ill children

P Demaret ${ }^{1}$, M Tucci ${ }^{2}$, T Ducruet ${ }^{2}$, H Trottier $^{2}$, J Lacroix ${ }^{2}$

'CHC - Clinique de l'Espérance, Montegnée, Belgium; 'CHU Sainte-Justine,

Montreal, Canada

Critical Care 2013, 17(Suppl 2):P368 (doi: 10.1186/cc12306)

Introduction Red blood cell (RBC) transfusions are frequent in critically ill children. Their benefits are clear in several situations. However, issues surrounding their safety have emerged in the past decades. It is important to identify the potential complications associated with RBC transfusions, in order to evaluate their risk-benefit ratio better

Methods A single-center prospective observational study of all children admitted to the pediatric intensive care unit (PICU) over a 1 -year period. The variables possibly related to RBC transfusions were identified before the study was initiated, and their presence was assessed daily for each child. In transfused cases (TCs), a variable was considered as a possible outcome related to the transfusion only if it was observed after the first transfusion.

Results During the study period, 913 admissions were documented, 843 of which were included in the study. Among them, 144 (17\%) were transfused. When comparing TCs with nontransfused cases (NTCs), the odds ratio (OR) of new or progressive multiple organ dysfunction syndrome (NPMODS) was $2.39(95 \% \mathrm{Cl}=1.58$ to $3.62, P<0.001)$. This association remained statistically significant in the multivariate analysis for children with admission PRISM score $\leq 5(\mathrm{OR}=2.41,95 \% \mathrm{Cl}=1.08$ to $5.37, P=0.032)$. TCs were ventilated longer than NTCs ( $14.1 \pm 32.6$ days vs. $4.3 \pm 9.6$ days, $P<0.001)$. This difference was still significant after adjustment using a Cox model. Moreover, we observed an adjusted dose-effect relationship between RBC transfusions and length of mechanical ventilation. The PICU length of stay was significantly increased for TCs $(12.4 \pm 26.2$ days vs. $4.9 \pm 10.2$ days, $P<0.001)$, even after multivariate adjustment (hazard ratio of PICU discharge for TCs: $0.61,95 \% \mathrm{Cl}=0.5$ to $0.74, P<0.001$ ). We also observed an adjusted dose-effect relationship between RBC transfusions and PICU length of stay. The paired analysis for comparison of pre-transfusion and posttransfusion values showed that the arterial partial pressure in oxygen was significantly reduced after the first transfusion (mean difference $42.8 \mathrm{mmHg}, 95 \% \mathrm{Cl}=27.2$ to $58.3, P<0.001$ ). The paired analysis also showed an increased proportion of renal replacement therapy, while the proportions of sepsis, severe sepsis and septic shock did not differ. Conclusion RBC transfusions were associated with prolonged mechanical ventilation and prolonged PICU stay. The risk of NPMODS was increased in some transfused children. Moreover, our study questions the ability of stored RBCs to improve oxygenation in critically ill children. These results should help to improve transfusion practice in the PICU. 
P369

Microcirculation and blood transfusions during sepsis: leukoreduced (LR) versus non-LR red blood cells

A Donati', E Damiani', R Domizi', C Scorcella', A Carsetti', S Tondi', R Castagnani', N Mininno', P Pelaia', C Ince ${ }^{2}$

'Università Politecnica delle Marche, Ancona, Italy; ${ }^{2}$ Academic Medical Center, Amsterdam, the Netherlands

Critical Care 2013, 17(Suppl 2):P369 (doi: 10.1186/cc12307)

Introduction Microcirculatory alterations during sepsis impair tissue oxygenation, which may be further worsened by anemia. Blood transfusions proved not to restore $\mathrm{O}_{2}$ delivery during sepsis [1]. The impact of storage lesions and/or leukocyte-derived mediators in red blood cell (RBC) units has not yet been clarified [2]. We compared the effects of leukoreduced (LR) versus nonLR packed RBCs on microcirculation and tissue oxygenation during sepsis.

Methods A prospective randomized study. Twenty patients with either sepsis, severe sepsis or septic shock requiring RBC transfusion randomly received nonLR (Group 1, $n=10$ ) or LR (Group 2,n=10) fresh RBCs ( $<10$ days old). Before and 1 hour after transfusion, microvascular

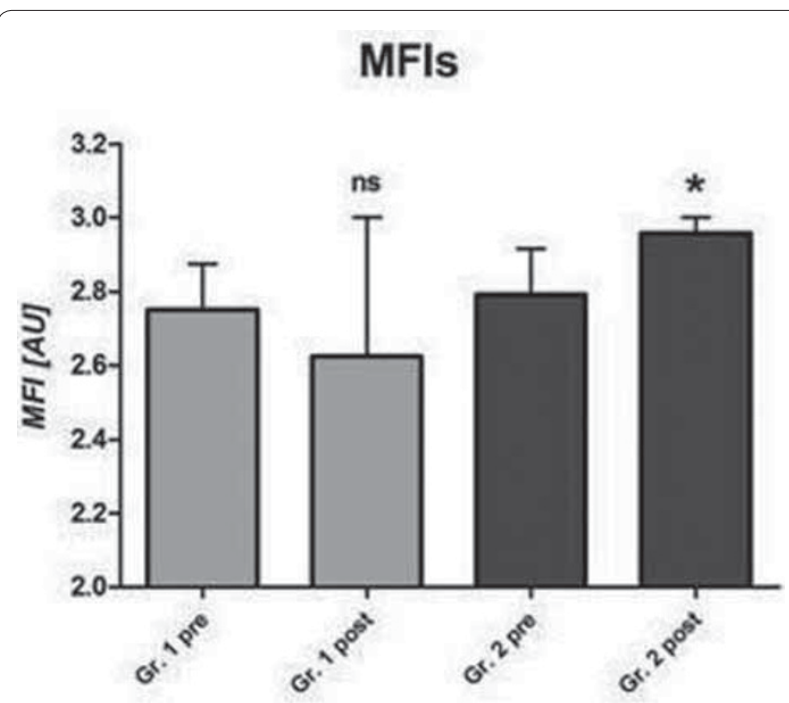

Figure 1 (abstract P369). Blood transfusion and microvascular flow.

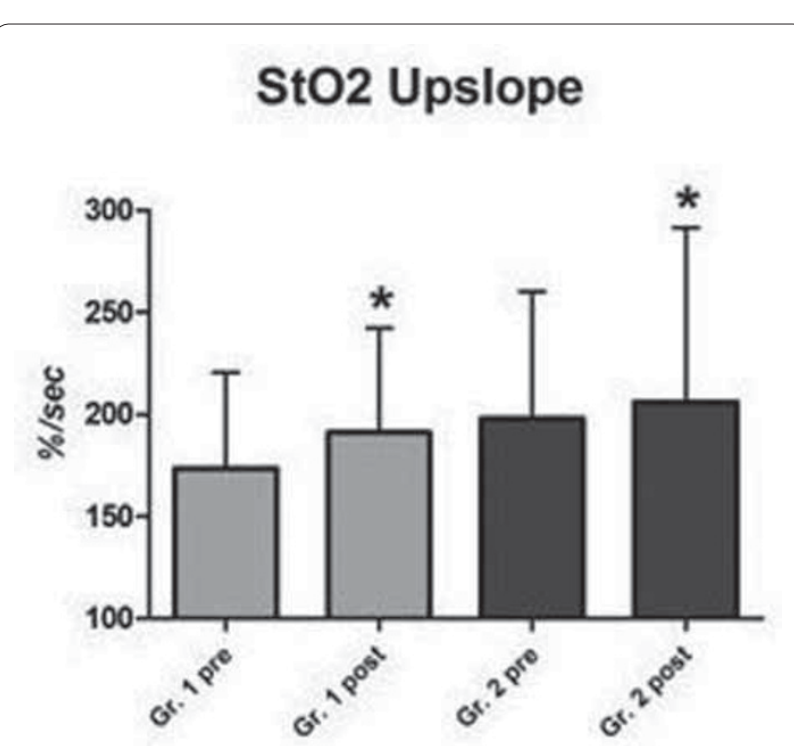

Figure 2 (abstract P369). Blood transfusion and microvascular reactivity density and flow were assessed with sidestream dark-field imaging sublingually. Thenar tissue $\mathrm{O}_{2}$ saturation $\left(\mathrm{StO}_{2}\right)$ was measured using near-infrared spectroscopy and a vascular occlusion test was performed.

Results The De Backer score $(P=0.02)$, total vessel density $(P=0.08)$, perfused vessel density $(P=0.04)$, proportion of perfused vessels $(P=0.01)$, and microvascular flow index $(P=0.04$, Figure 1$)$ increased only in Group 2. The $\mathrm{StO}_{2}$ upslope (Figure 2) during reperfusion increased in both groups $(P<0.05)$. In Group 1 the baseline $\mathrm{StO}_{2}$ and $\mathrm{StO}_{2}$ downslope during ischemia increased, probably reflecting a lower $\mathrm{O}_{2}$ consumption.

Conclusion Unlike nonLR RBCs, the transfusion of fresh LR RBCs seems to improve microvascular perfusion and might help to restore tissue oxygenation during sepsis.

References

1. Marik PE, et al.: JAMA 1993, 269:3024-3029.

2. Sparrow RL: Blood Transfus 2010, 8(Suppl 3):s26-s30.

\section{P370}

Massive blood transfusion for obstetric haemorrhage

S Simmons', WE Pollock', L Phillips'2, S McDonald ${ }^{3}$

${ }^{1}$ Mercy Hospital for Women, Heidelburg, Australia; ${ }^{2}$ Monash University,

Prahran, Australia; ${ }^{3}$ La Trobe University, Bundoora, Australia

Critical Care 2013, 17(Suppl 2):P370 (doi: 10.1186/cc12308)

Introduction Obstetric haemorrhage remains a leading cause of maternal mortality and severe morbidity. Cardiovascular and haemostatic physiology alters in pregnancy and massive transfusion protocols have been implemented for obstetric haemorrhage based on limited evidence. The objective of this study was to examine the pattern and rate of blood products used in massive transfusion for obstetric haemorrhage in a tertiary obstetric hospital.

Methods Massive transfusion was defined as 5 or more units of red blood cells within 4 hours in accordance with the Australian Massive Transfusion Registry definition. Following ethics approval, all cases filling this criterion were identified in the hospital's birthing and blood bank systems. Data were extracted from the medical histories and analysed using SPSS. $P<0.05$ was considered statistically significant. Results Twenty-eight women in three years (2009 to 2011) underwent a massive transfusion for obstetric haemorrhage, with nine receiving more than 10 units of RBCs in 24 hours. Eleven (39\%) were admitted to the ICU and 11 underwent a hysterectomy, of which six were admitted to the ICU. The median estimated blood loss was 4,335 ml (IQR 3,000 to 5,000 ). Median blood product delivery was RBC 8 units (IQR 6 to 13); FFP 4 units (IQR 4 to 8); platelets 4 units (IQR 0 to 8 ) and cryoprecipitate 3 units (IQR 0 to 10). One-half of the women received the first four units of RBCs in less than 34 minutes. Other blood products were started a median of 49 minutes, 44 minutes and 75 minutes after the RBC transfusion commenced, respectively. Eight women had a fibrinogen level $<0.8 \mathrm{~g} / \mathrm{l}$ on the initial coagulation test during the haemorrhage. The remaining 20 women had a median fibrinogen level of $3.7 \mathrm{~g} / \mathrm{l}(\mathrm{IQR}$ 3.15 to 4.9). There was no difference in the transfusion of $\operatorname{RBCs}(P=0.20)$, FFPs $(P=0.96)$ and platelets $(P=0.48)$ in women who showed an initial low fibrinogen and those who did not, although there was a difference in the number of units of cryoprecipitate $(P<0.05)$. The median lowest $\mathrm{Hb}$ during the haemorrhage was $66 \mathrm{~g} / \mathrm{l}$ (IQR 51 to 80 ) and median discharge $\mathrm{Hb}$ was $103 \mathrm{~g} / \mathrm{l}$ (IQR 96 to 113). No blood product reaction was noted and there was one death.

Conclusion Massive transfusion for obstetric haemorrhage involved rapid blood product administration with no consistent pattern in the ratio of products administered.

\section{P371}

Blood transfusion practices in the ICU of a level 1 trauma centre and tertiary cardiac unit

Y Mustafa, B Pouchet

University Hospitals Birmingham NHS Foundation Trust, Birmingham, UK Critical Care 2013, 17(Suppl 2):P371 (doi: 10.1186/cc12309)

Introduction Blood transfusions are associated with longer ICU and hospital inpatient durations, and an increase in mortality [1]. This study was undertaken to investigate whether the practice of packed red cell 
(PRC) transfusions in the ICU was in accordance with the best clinical evidence. A number of studies, most notably the TRICC study [2], have shown that indications for ICU blood transfusions are a haemoglobin $(\mathrm{Hb})$ level of $<7 \mathrm{~g} / \mathrm{dl}$ or evidence of acute haemorrhage [3]. These criteria were therefore employed.

Methods This study prospectively examined episodes of PRC unit transfusions over a 2-month period in the ICU of a large level 1 trauma centre and a tertiary cardiac unit. The number of PRC units transfused in each episode was recorded by nurses, along with the proposed indication and concurrent $\mathrm{Hb}$ level. The data were analysed to assess the number of transfusions administered contrary to the guidelines, along with the average $\mathrm{Hb}$ level at which a PRC unit was transfused and the average number of units administered per episode.

Results A total 175 units of PRC were transfused in the ICU, over 105 episodes during the 2-month period (excluding immediately postoperative transfusions). Ninety-four units (53.7\%) administered in 64 transfusion episodes (61.0\%) occurred contrary to the guidelines. In $89.3 \%$ of these cases the recorded reason for transfusion was an apparently low $\mathrm{Hb}$ level. The median (IQR (range)) $\mathrm{Hb}$ level at which patients were transfused: within guidelines was $6.9 \mathrm{~g} / \mathrm{dl}$ ( 6.6 to $7.7(4.8$ to 13.9)); within guidelines, excluding cases of acute blood loss, was $6.6 \mathrm{~g} / \mathrm{dl}$ ( 6.5 to 6.8 (5.5 to 6.9$)$ ); and outside the guidelines was $7.7 \mathrm{~g} / \mathrm{dl}$ (7.4 to 8.2 (7.0 to 9.7)). One unit of PRC was transfused in 57 episodes (54.3\%), 2 units of PRC were transfused in 36 episodes (34.3\%), and 3 to 6 units were transfused in 12 episodes (11.4\%), with two-thirds of the latter due to acute haemorrhage.

Conclusion Our results indicate a liberal transfusion threshold currently exists in the ICU. Patients are frequently receiving excessive PRC transfusions for $\mathrm{Hb}$ levels above the recommended concentration. In the 2-month study period, these were associated with a cost of approximately $£ 12,220$. We recommend increased staff awareness of the guidelines to reduce the number of unnecessary transfusions. This would decrease exposure of ICU patients to unnecessary risks of blood transfusion, reduce cost of treatment and help to preserve a valuable resource.

References

1. Corwin HL, et al: Crit Care Med 2004, 32:39-52.

2. Hebert PC: Br J Anaesth 1998, 81(Suppl 1):25-33.

3. Napolitano et al:: Crit Care Med 2009, 37:3124-3157.

P372

C1 inhibitor attenuates pulmonary inflammation in an in vivo model of transfusion-related acute lung injury

M Müller', PRTuinman', G Jongsma', AM Tuip-de Boer', L Boon²,

SS Zeerleder', NP Juffermans

'Academic Medical Center, Amsterdam, the Netherlands; 'Bioceros, Utrecht,

the Netherlands

Critical Care 2013, 17(Suppl 2):P372 (doi: 10.1186/cc12310)

Introduction Transfusion-related acute lung injury (TRALI) has a high incidence in critically ill and surgical patients and contributes to adverse outcome, while specific therapy is absent. Recently it was demonstrated that complement activation plays a pivotal role in TRALI. We aimed to determine whether a $\mathrm{C} 1$ inhibitor is beneficial in a two-hit mouse model of antibody-mediated TRALI.

Methods BALB/c mice were primed with lipopolysaccharide (LPS, from E. coli $0111: \mathrm{B} 4)$ that was administered intraperitoneally in a dose of $0.1 \mathrm{mg} / \mathrm{kg}$, after which TRALI was induced by injecting MHC-I antibody against $\mathrm{H} 2 \mathrm{Kd}(\mathrm{lgG} 2 \mathrm{a}, \mathrm{k})$ at a dose of $2 \mathrm{mg} / \mathrm{kg}$. Mice infused with PBS or LPS served as controls. Concomitantly, mice infused with the MHC-I antibody were treated with $\mathrm{C} 1$ inhibitor (Cetor ${ }^{\oplus}$; Sanquin, Amsterdam, the Netherlands) in a dose of $400 \mathrm{lU} / \mathrm{kg}$ intravenously. After infusion, mice were mechanically ventilated with a lung-protective pressurecontrolled mode for 2 hours and then sacrificed, after which a bronchoalveolar lavage (BAL) was done. Statistics were analyzed by one-way ANOVA, values expressed as mean and standard deviation. Results Injection of LPS and MHC-I antibodies resulted in TRALI, indicated by increased levels of protein in the BAL fluid, wet/dry ratios and levels of KC, MIP-2 and IL-6. C1 inhibitor Cetor ${ }^{\oplus}$ significantly reduced total protein in BAL fluid from $792(169)$ to $421(230) \mu \mathrm{g} / \mathrm{ml}$ $(P<0.001)$ and tended to reduce the wet/dry ratio from $5.3 \pm 0.3$ to
$4.9 \pm 0.5(P=0.09)$. Cetor ${ }^{\circledast}$ also reduced BALF levels of MIP-2 from 214 (54) to $127(22) \mathrm{pg} / \mathrm{ml}(P<0.01)$. KC and IL-6 levels were not affected. Conclusion In a model of antibody-mediated TRALI, C1 inhibitor attenuated pulmonary inflammation. $\mathrm{C} 1$ inhibition may be a potential beneficial intervention in TRALI.

P373

Incidence, risk factors and outcome of TRALI after cardiac surgery

A Dijkhuizen, R De Bruin, S Arbous

Leiden University Medical Center, Leiden, the Netherlands

Critical Care 2013, 17(Suppl 2):P373 (doi: 10.1186/cc12311)

Introduction Transfusion-related acute lung injury (TRALI) is a syndrome that presents as a sudden onset of respiratory distress 6 hours after transfusion of blood products. The diagnosis is based on clinical and radiographic findings. Particularly at risk for TRALI are cardiac surgery patients. However, specific patient risk factors and data on outcome are largely unknown. The aim of this study was to investigate incidence, risk factors and outcome of TRALI in cardiac surgical patients on cardiopulmonary bypass.

Methods All thoracic surgery patients from a university hospital in the Netherlands of 18 years and older admitted to the ICU from January 2009 until December 2011 were screened. Included patients were observed during surgery and the first 24 hours on the ICU for the onset of possible TRALI. The Canadian Consensus Conference TRALI definition was used. Two independent physicians blinded to the predictor variables scored the chest radiographs for the onset of bilateral interstitial abnormalities on $2 \mathrm{~K}$ monitors. When interpretation differed, chest radiographs were reviewed by a third physician to achieve consensus. The European System for Cardiac Operative Risk Evaluation (EURO score) and the American Association of Anesthesiology (ASA) were scored before surgery. By calculating the Acute Physiology and Chronic Health Evaluation (APACHE) II and IV scores the severity of illness was determined on arrival in the ICU.

Results In total, 1,787 cardiac surgical patients were included. A total of 19 (1.1\%) patients developed TRALI within 24 hours following surgery. Patients developing TRALI were older compared with patients not developing TRALI, mean age respectively 71 and 65 years $(P=0.035)$. Furthermore, patients developing TRALI had higher APACHE II, APACHE IV, EURO and ASA score $(P=0.000, P=0.000, P=0.000$ and $P=0.37)$. Compared with patients not developing TRALI, patients developing TRALI had a longer cardiopulmonary bypass time. Of the 19 patients developing TRALI, four patients (21.1\%) died during ICU stay, in total six patients (31.6\%) died during hospital stay. Patients developing TRALI were ventilated longer and had a longer ICU and hospital stay.

Conclusion Older age, higher APACHE II, IV, EURO and ASA scores and a longer time on cardiopulmonary bypass and ventilation time are risk factors for the development of TRALI. The ICU and hospital stay are longer, and the prognosis is bad for patients developing TRALI.

Reference

1. Vlaar APJ, et al:: Crit Care Med 2010, 38:771-778.

\section{P374}

Albumin-induced coagulopathy is less severe and more

effectively reversed with fibrinogen concentrate than is synthetic colloid-induced coagulopathy

DW Winstedt, JH Hanna, US Schött

Lund University, Lund, Sweden

Critical Care 2013, 17(Suppl 2):P374 (doi: 10.1186/cc12312)

Introduction Volume resuscitation is essential to restore normovolemia during hemorrhagic shock, burns and sepsis. However, synthetic colloids cause dilutional coagulopathy. The aims were to determine whether the natural colloid albumin induces a lesser degree of coagulopathy compared with synthetic colloids, and the comparative effectiveness of fibrinogen concentrate to reverse coagulopathy following dilution with these solutions.

Methods Rotational thromboelastometry-based tests were used to examine coagulation parameters in samples from 10 healthy volunteers, in undiluted blood and samples diluted 1:1 with saline, 
Ringer's acetate, hydroxyethyl starch (HES) 130/0.4, buffered HES $130 / 0.4,3 \%$ dextran $60,6 \%$ dextran 70 or $5 \%$ albumin. Samples were analyzed before and after addition of $2 \mathrm{mg} / \mathrm{ml}$ fibrinogen concentrate. Results EXTEM maximum clot firmness (MCF), clot formation time (CFT) and a angle (AA) decreased significantly upon dilution with all colloid solutions $(P<0.001)$, although a significantly greater coagulopathic effect was seen for samples diluted with synthetic colloid solutions versus albumin $(P \leq 0.001)$. A significant reduction in the platelet component of clot strength (EXTEM MCF - FIBTEM MCF) was seen for samples diluted with synthetic colloids $(P<0.001)$ but not albumin $(P=0.10)$. Following addition of fibrinogen, FIBTEM MCF, EXTEM MCF and EXTEM AA were significantly higher, and EXTEM CFT was significantly shorter in samples diluted with albumin versus those treated with HES or dextran $(P \leq 0.001)$.

Conclusion Hemodilution using albumin induced a lesser degree of coagulopathy compared with the synthetic colloids HES and dextran. In addition, albumin-induced coagulopathy was more effectively reversed following addition of fibrinogen concentrate compared with coagulopathy induced by synthetic colloids.

P375

Network meta-analysis of clinical trials of fluid treatments in sepsis demonstrates improved survival with albumin compared with crystalloid and hydroxyethyl starch

M Bansal', A Farrugia², G Martin ${ }^{3}$

'Plasma Protein Therapeutics Association, Annapolis, MD, USA; ${ }^{2}$ University of Western Australia, Crawley, Australia; ${ }^{3}$ Emory University School of Medicine, Atlanta, GA, USA

Critical Care 2013, 17(Suppl 2):P375 (doi: 10.1186/cc12313)

Introduction Fluid resuscitation is widely practiced in treating sepsis. Comparative assessment of the different fluid modalities is hampered by a paucity of direct trials. We present a network meta-analysis for assessing the relative effectiveness of two fluid treatments in sepsis when they have not been compared directly in a randomized trial but have each been compared with a common treatment.

Methods A systematic review of trials sepsis yielded 13 trials for assessment in network meta-analysis. The indirect comparison between albumin, HES and crystalloid was conducted using Bayesian methods for binomial likelihood, fixed-effects network meta-analysis with a Monte Carlo Gibbs sampling method. Studies in septic patients with crystalloid as a reference treatment compared with any formulation of the colloid treatments albumin or HES were included, as were direct head-to-head trials between the two colloids.

Results Odds ratios between the different treatments were obtained (Figure 1). Ranking the interventions [1] demonstrated that albumin ranked highest in lowering mortality at a $96.4 \%$ probability compared with $3.6 \%$ and $0.01 \%$ for crystalloid and HES, respectively.

Conclusion Albumin as a fluid therapy in sepsis is associated with the lowest mortality of the three modalities studied.

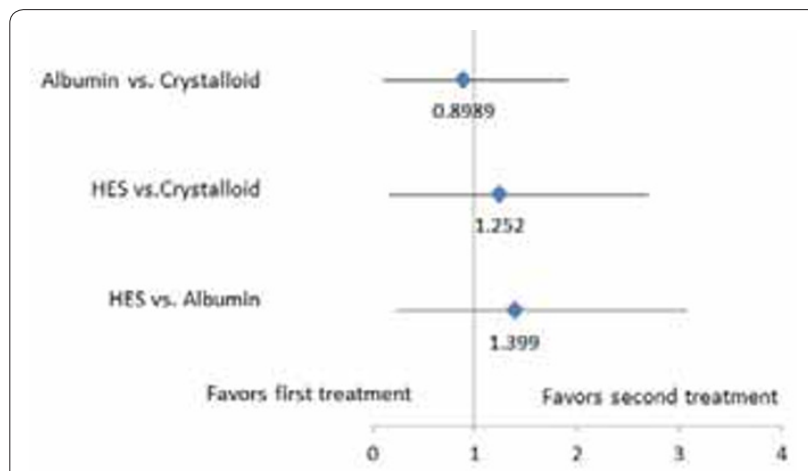

Figure 1 (abstract P375). Forest plot of results of Bayesian fixed-effect network meta-analysis of mortality.

\section{Reference}

1. Salanti G, Ades AE, loannidis JP: Graphical methods and numerical summaries for presenting results from multiple-treatment meta-analysis: an overview and tutorial. J Clin Epidemiol 2011, 64:163-171.

P376

Assessing the economic impact of catheter-related bloodstream infections when switching from open to closed system delivery of human albumin solutions in ICUs

F Axelsen', P Riss²

'Baxter Healthcare, Zurich, Switzerland; ${ }^{2}$ Medical University of Vienna, Austria Critical Care 2013, 17(Suppl 2):P376 (doi: 10.1186/cc12314)

Introduction Hospital-acquired infections create a significant burden to the healthcare system. To understand the economic impact, a model was developed to assess the cost of differences in catheter-related bloodstream infections (BSI) in ICUs based on either closed or open albumin systems.

Methods A model was developed with the aim of showing the economic consequences of differences between intravenous (i.v.) infusions of albumin via open or closed infusion containers. The model took a healthcare perspective in the UK. The impact of central venous catheter-related BSI was evaluated. The model took differences in $\mathrm{BSI}$ rates and the associated cost consequences for the hospital into account. The model bases on the evidence that closed system delivery helps to reduce the risk of external contamination, which decreases the BSI rate and reduces overall mortality [1-3]. We assessed the total costs using public list prices and literature. The model assumes that infection rates for using open and closed albumin delivery systems are similar to any other closed or open nonalbumin i.v. fluid delivery system used in the ICU. The model looks at reduction in central-line-associated BSI and the potential cost saving of this reduction.

Results The economic model shows the potential cost saving by switching from open to closed albumin administration systems. It is measured in occurrence of BSI in the ICU, estimated occurrence of BSI in the albumin population and incidence of overall mortality in the ICU and cost of BSI per albumin patient. Finally, the model describes the potential cost savings by switching to a closed system. Using closed albumin delivery systems may overall provide a cost saving for the hospital and healthcare sector. Assuming 800 patients treated in an ICU per year with all in all $4,800 \mathrm{ml}$ albumin, the total annual cost saving would be $£ 24,000$ switching from open to closed albumin infusions.

Conclusion Switching from an open to a closed albumin infusion system may result in a reduction in the risk of external contamination, resulting in less infection and may provide a cost saving for the healthcare system.

References

1. Maki et al: Infect Control Hosp Epidemiol 2011, 32:50-58.

2. Tacconelli et al:. J Hosp Infection 2009, 72:97-103.

3. Franzetti et al.: Epidemiol Infect 2009, 137:1041-1048.

\section{P377}

Comparing the hemodynamic effects of six different colloids

N Kteniadakis, V Grosomanidis, E Oloktsidou, B Fyntanidou, C Nouris,

K Kotzampasi, D Vasilakos

Aristotle Medical School, Thessaloniki, Greece

Critical Care 2013, 17(Suppl 2):P377 (doi: 10.1186/cc12315)

Introduction Despite the fact that colloids are widely used, doubt still remains as to which colloid is the best and what is the ideal volume of administration. The aim of our study was to compare the hemodynamic effects of six different colloids.

Methods A total of 180 patients were enrolled in our study undergoing general surgery procedures, who were assigned to six groups $(A, B, C, D$, $\mathrm{E}, \mathrm{F})$. After fasting fluid replacement and induction of anesthesia, $500 \mathrm{ml}$ Gelofusine, Haemaccel, Voluven, HES 6\%, HES 10\% and Hemohes were administered to each group respectively. An oesophageal Doppler monitor probe was inserted into the patients for measuring stroke volume (SV) and cardiac output (CO). Standard monitoring included $\mathrm{ECG}, \mathrm{IBP}, \mathrm{ETCO}_{2}$ and $\mathrm{SpO}_{2}$, and heart rate (HR). Systemic arterial pressure 
(SAP), SV and CO were recorded directly before the administration of any colloid (T0) and every 5 minutes for the next 1 hour (T1 to T12). Kolmogorov-Smirnov was used to test normal distribution of data and ANOVA was used for the statistical analysis. $P<0.05$ was considered statistically significant.

Results Demographic data and ASA classification did not differ statistically significant among the six groups of the study. CO, SV, HR and SAP did not show any statistically significant evolution compared with their baseline value during the study period. Moreover, there were no statistically significant differences among the six study groups with regard to any of the recorded parameters.

Conclusion According to our results, volume replacement with the six colloids tested in our study did not result in any hemodynamic response. Within comparison of these six colloids did not reveal any statistically significant difference in any of the parameters recorded according to our protocol.

\section{P378}

Strong ion difference and arterial bicarbonate concentration as cornerstones of the impact of fluid therapy on acid-base balance D Ottolina, M Ferrari, L Zazzeron, E Scotti, M Stanziano, C Rovati,

C Marenghi, L Gattinoni, P Caironi

Fondazione IRCCS Ca'Granda-Ospedale Maggiore Policlinico Università

degli Studi di Milano, Milan, Italy

Critical Care 2013, 17(Suppl 2):P378 (doi: 10.1186/cc12316)

Introduction The biochemical characteristics of infused fluids may be important in regulating acid-base balance, by modifying plasmatic volume and strong ion difference. In vitro and animal studies $[1,2]$ have shown that volume and strong ion difference of infused fluids (SIDin) as well as the arterial baseline bicarbonate concentration $\left(\mathrm{HCO}_{3}-\mathrm{a}\right)$ influence acid-base variations. Our aim was to verify these changes in critically ill patients after surgery.

Methods An electronic-dedicated database was created to retrospectively collect volume, type of fluids infused and plasmatic acidbase balance variations in postoperative ICU patients from admission to 9:00 am of the day after. SIDin was calculated as the average SID of all fluids infused during the whole study period (crystalloids, colloids and blood products). Arterial base excess variation ( $\triangle \mathrm{BEa}$ ) was computed as the difference between values at 9:00 am on the day after and those at entry. We report data from all patients admitted in 2006 and 2007 (650 patients)

Results Nine patients not receiving intravenous infusions were excluded. The remaining population was divided into three groups according to SIDin distribution (Group 1, $18 \pm 12$; Group 2, $47 \pm 6$; Group 3, $55 \pm 0 \mathrm{mEq} / \mathrm{l})$. We observed a progressive increment in $\triangle \mathrm{BEa}$ between the groups $(1.1 \pm 2.0$ vs. $2.8 \pm 2.9$ vs. $3.0 \pm 2.8 \mathrm{mmol} / \mathrm{l}$, $P<0.001)$. We further subdivided each group by the median value of baseline $\mathrm{HCO}_{3}$-a (24.3 (22.3 to 26.1$\left.) \mathrm{mmol} / \mathrm{l}\right)$ and we analyzed the $\triangle \mathrm{BEa}$ : we observed a greater increase in patients with lower baseline $\mathrm{HCO}_{3}$-a (Group $1,1.8 \pm 2.9$ vs. $0.2 \pm 2.6, \mathrm{mmol} / \mathrm{l}, P<0.001$; Group 2, $4.0 \pm 2.7$ vs. $1.5 \pm 2.6, \mathrm{mmol} / \mathrm{l}, P<0.001$; Group $3,4.4 \pm 2.8$ vs. $1.7 \pm 2.0 \mathrm{mmol} / \mathrm{l}$, $P<0.001$ ), as compared with those with higher baseline levels. When the study population was divided into quartiles of the difference between SIDin and $\mathrm{HCO}_{3}-\mathrm{a}, \triangle \mathrm{BEa}$ appeared to increase with the rise of such difference $(P<0.001)$.

Conclusion SIDin affects the acid-base status per se and in relationship with $\mathrm{HCO}_{3}-\mathrm{a}$. We verified this hypothesis in critically ill patients, highlighting the importance of the difference between SIDin and $\mathrm{HCO}_{3}-\mathrm{a}$, which better describes and predicts the acid-base modifications to fluid therapy.

\section{References}

1. Carlesso $\mathrm{E}$, et al: The rule regulating $\mathrm{pH}$ changes during crystalloid infusion. Intensive Care Med 2011, 37:461-468

2. Langer T, et al: In vivo conditioning of acid-base equilibrium by crystalloid solutions: an experimental study on pigs. Intensive Care Med 2012 38:686-693.
P379

Microcirculatory response to fluid challenge: should we prefer balanced colloids to rebalance tissue perfusion?

A Donati, E Damiani, R Domizi, C Scorcella, A Carsetti, S Tondi, R Castagnani, N Mininno, V Monaldi, P Pelaia

Università Politecnica delle Marche, Ancona, Italy

Critical Care 2013, 17(Suppl 2):P379 (doi: 10.1186/cc12317)

Introduction Fluid resuscitation should improve tissue oxygenation in hypovolemia, besides restoring macrohemodynamic stability [1]. We evaluated the microvascular response to fluid challenge with different colloid solutions and its relation to macrohemodynamics.

Methods An observational study of patients receiving a fluid challenge ( $500 \mathrm{ml}$ colloids in 30 minutes) according to the attending physician's decision. Before and after the infusion, sublingual microcirculation was evaluated with sidestream dark-field imaging (Microscan; Microvision Medical, Amsterdam, the Netherlands). Microvascular flow and density were assessed for small vessels [2]. The cardiac index $(\mathrm{Cl})$, intrathoracic blood volume index (ITBVI) and extravascular lung water index (ELWI) were measured in seven patients with PiCCO2 (Pulsion Medical System, Munich, Germany).

Results Ten patients (two sepsis, four trauma, three intracranial bleeding, one post surgery) received either saline-based hydroxyethyl starch (HES) 130/0.4 (Amidolite ${ }^{\oplus}$; B.BraunSpA; $n=5$ ) or balanced HES 130/0.42 (Tetraspan ${ }^{\oplus} ;$ B.BraunSpA; $\left.n=5\right)$. The $\mathrm{Cl}(P=0.02)$ and ITBVI $(P=0.07)$ tended to increase, the EVLWI did not change. Microvascular flow and density improved in the whole sample. No correlation was found between macro-circulatory and micro-circulatory parameters. Balanced HES led to a greater increase in capillary density than $\mathrm{NaCl}$ HES (Figure 1).

Conclusion Balanced HES may be more efficacious than saline-based HES in recruiting the microcirculation, thereby improving tissue $\mathrm{O}_{2}$ delivery.

References

1. Futier et al: Crit Care 2011, 15:R214

2. De Backer et al: Crit Care 2007, 11:R101.

P380

Procedural sedation in the emergency department

MS Shah ${ }^{1}$, FS Shah², KP Pope ${ }^{3}$, AS Abbas $^{4}$

'Luton and Dunstable Hospital, London, UK; 'irmingham Hospitals NHS

Trust, Birmingham, UK: ${ }^{3}$ Chesterfield NHS Trust, Chesterfield, UK: ${ }^{4}$ Colchester

Hospital, Colchester, UK

Critical Care 2013, 17(Suppl 2):P380 (doi: 10.1186/cc12318)

Introduction Are safety guidelines being followed when administering procedural sedation in the emergency department? Between November 2004 and November 2008, the NPSA received 498 alerts of patients being given the wrong dose of midazolam for procedural sedation [1]. In the first 5 years of midazolam use there were 86 deaths, most related to procedural sedation [2].

Methods We searched through the controlled drugs book in resuscitation over a 2-month period and found a list of patients who had received midazolam or fentanyl. From this, we could make a search for the relevant $A$ and $E$ notes for these patients. From these notes, we looked for (see shorthand in Table 1): verbal consent documentation (consent), past medical history recorded (pmhx), safe initial dose of midazolam (midaz), pre-procedure monitoring (pre), post-procedure monitoring (post), and monitoring for 1 hour before discharge $(1 \mathrm{hr})$. Following introduction of a reminder in the controlled drugs book/ sedation room and staff education, the case notes were analysed over another 2-month period (24 sets of notes) to assess practise against safety guidelines.

Results See Table 1 (key for shorthand in Methods).

Conclusion The re-audit notices within the procedural sedation room and controlled drug book front cover served as a reminder of good practise. The visibility of this reminder (within the CD book) helped ensure better adherence to the audit standard. This reminder will now be kept within the $C D$ book.

References

1. [http://www.npsa.nhs.uk/corporate/news/

reducing-risk-of-midazolam-overdose-in-adults] 


\section{Perfused Boundary Region}

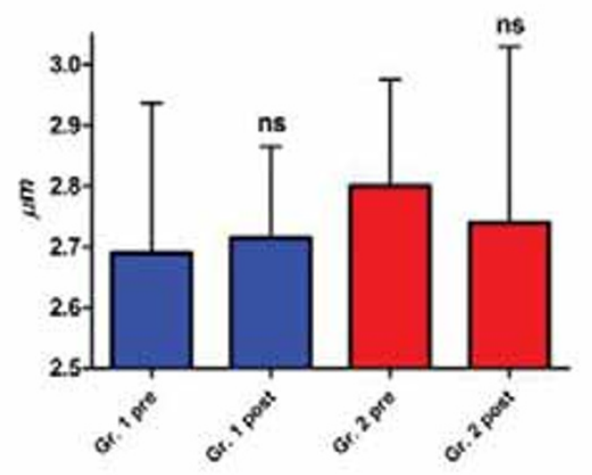

Heparan Sulfate

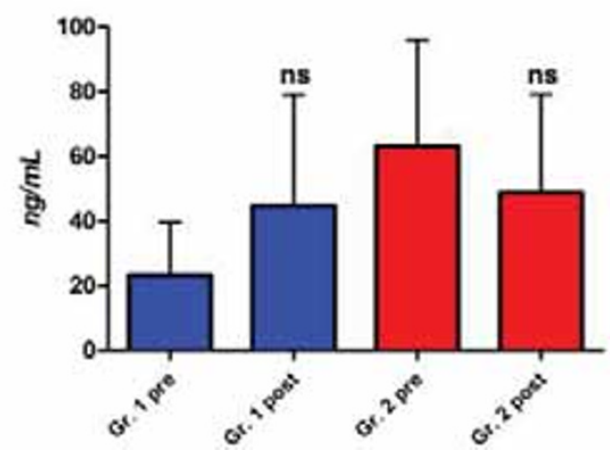

\section{Syndecan-1 (CD138)}

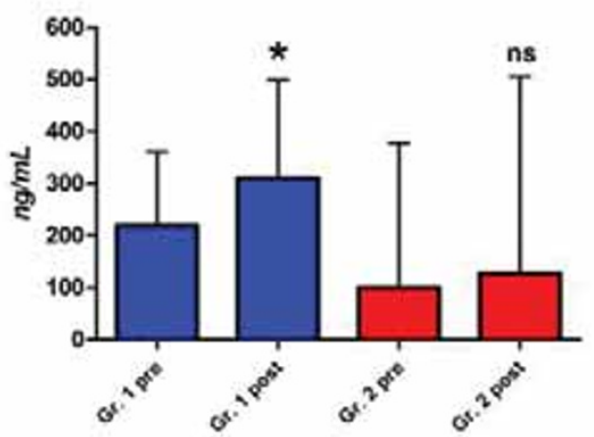

CORRELATION between PBR and HEPARAN SULFATE

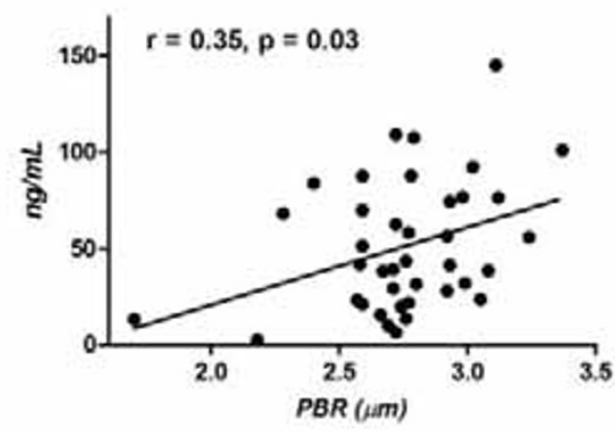

Figure 1 (abstract P379). Microvascular response to fluid challenge: effects of different colloids.

Table 1 (abstract P380). Before and after education programme and reminder in the controlled drugs book

\begin{tabular}{|c|c|c|c|c|}
\hline & \multicolumn{2}{|c|}{ Before (\%) } & \multicolumn{2}{|c|}{ After (\%) } \\
\hline & Yes & No & Yes & No \\
\hline Consent & 35 & 65 & 50 & 50 \\
\hline Pmhx & 55 & 45 & 67 & 33 \\
\hline Midaz & 58 & 42 & 88 & 12 \\
\hline Pre & 25 & 75 & 54 & 46 \\
\hline Post & 10 & 90 & 71 & 29 \\
\hline $1 \mathrm{hr}$ & 55 & 45 & 54 & 46 \\
\hline
\end{tabular}

2. Epstein B: Data Retrieval Unit HFD-737. Department of Health and Human Services, Office of Epidemiology and Biostatistics, Center for Drug Evaluation and Research; 27 June 1989.

P381

Impact of an age, kidney and liver function adjusted sedation protocol in critically ill patients

S Haddad, H Tamim, C Gonzales, A Rishu, A Deeb, Y Arabi

King Abdulaziz Medical City, Riyadh, Saudi Arabia

Critical Care 2013, 17(Suppl 2):P381 (doi: 10.1186/cc12319)

Introduction Daily sedation interruption and protocol implementation have been recommended to reduce excessive sedation; however, their use has been inconsistent. We hypothesized that the use of an age, kidney and liver function adjusted sedation protocol would be associated with reduced doses and improved outcomes compared with a standard protocol.

Methods This was a prospective cohort study comparing 3 months of a standard protocol (control group) with 3 months of an adjusted protocol (intervention group). In the adjusted protocol, patients were divided into three categories: category 1 (age $<60$ years, and normal kidney and liver function), category 2 (age $=60$ to 70 years, or moderate kidney or liver function impairment), and category 3 (age >70 years, or severe kidney or liver function impairment). The upper limits of analgesics and sedatives doses were determined by age, and kidney and liver function, being lowest in category 3 , and lower in category 2 than category 1 . All consecutive adults mechanically ventilated patients who required infusion of analgesics and/or sedatives for $>24$ hours were included in the study. We compared the main outcomes of both groups including average daily doses of analgesics and sedatives; average Sedation-Agitation Scale (SAS), pain and GCS scores; mechanical ventilation duration (MVD); sedation-related complications during ICU stay; ICU and hospital length of stay (LOS), and ICU and hospital mortality.

Results Two hundred and four patients were included in the study (control group $=105$; adjusted protocol group $=99$ ). There was no difference in baseline characteristics between the two groups. The adjusted protocol group, compared with the control group, received significantly lower average daily doses of fentanyl $(2,162 \pm 2,110 \mu \mathrm{g}$ vs. $3,650 \pm 3,253 \mu \mathrm{g}, P=0.0001)$, nonsignificant lower average daily doses of midazolam and dexmedetomidine, and a trend toward higher average daily doses of propofol. Pain score was higher in the adjusted protocol group $(0.98 \pm 0.72$ vs. $0.16 \pm 0.35, P<0.0001)$ with no difference in SAS or GCS scores. Sedation-related complications during ICU stay were not different between the two groups; however, agitation $(S A S=5)$ was less frequent in the adjusted protocol group ( $3 \%$ vs. $30 \%, P<0.0001$ ). ICU mortality was significantly lower in the 
adjusted protocol group ( $18 \%$ vs. $36 \%, P=0.004)$ with no significant differences in MVD, ICU and hospital LOS, and hospital mortality. Conclusion The use of an age, kidney and liver function adjusted sedation protocol is associated with lower doses of analgesics and sedatives, lower risk of agitation and lower ICU mortality.

P382

Risk and benefit of protocol sedation and daily interruption of sedation for mechanically ventilated patients: a systematic review K Hosokawa' ${ }^{1}$ M Egi $^{2}$, M Nishimura ${ }^{3}$

${ }^{1}$ Kyoto Prefectural Yosanoumi Hospital, Kyoto, Japan; ${ }^{2}$ Okayama University Hospital, Okayama, Japan; ${ }^{3}$ Tokushima University Hospital, Tokushima, Japan Critical Care 2013, 17(Suppl 2):P382 (doi: 10.1186/cc12320)

Introduction Protocol-directed sedation (PS) and daily interruption of sedation (DIS) are two major strategies to optimize sedation depth for mechanically ventilated patients. To clarify the clinical risk and benefits of PS and DIS, we performed a systematic review of randomized controlled trials (RCTs) in previous literature.

Methods We searched the MEDLINE database from January 1990 to October 2012 for studies on PS and DIS in critically ill patients. English-language manuscripts were included when they assessed the impact of PS or DIS on outcomes in critically ill patients requiring mechanical ventilation for $>48$ hours. PS is defined as sedation practices implemented by ICU staff using a sedation scale and a written protocol. DIS is defined as strategies with cessation of the continuous sedatives.

Results We retrieved 902 citations. Among them, nine RCTs fulfilled inclusion criteria. PS was compared with the standard practice in one RCT, DIS with the standard practice in four, PS with DIS in two, and PS with PS plus DIS (PS/DIS) in two. PS or DIS reduced the duration of mechanical ventilation by $28.9 \%$ and ICU stay by $27.8 \%$ compared with the standard practice. Neither the duration of mechanical ventilation nor ICU stay differed among DIS, PS and PS/DIS. Mortality did not differ among the three strategies. DIS increased the daily dose of sedatives and the workload of nurses in one recent RCT.

Conclusion PS or DIS decreased the duration of mechanical ventilation and ICU stay. PS seems to be superior to DIS based on the doses of sedatives and the workload of nurses.

P383

Chemical and physical compatibility of continuous intravenous drug infusion combinations used in paediatric intensive care C Cole', A Fox' ${ }^{1}$, M Van Der Merwe'2, L Dickson², K Ball², A Bevan'1, A Hocking', J Pappachan

'University Hospitals Southampton, UK; ${ }^{2}$ University of Portsmouth, UK Critical Care 2013, 17(Suppl 2):P383 (doi: 10.1186/cc12321)

Introduction The aim of this research was to provide clinically relevant evidence for Y-site compatibility of drug infusion combinations used in the PICU. Pharmacists and clinicians regularly have to interpret limited published data, particularly when more than two drugs are Y-sited. The risk of potential incompatibility must be balanced against that of additional line insertion.

Methods A full 28-factorial design (total 256 combinations) was used to investigate chemical and physical compatibility of five drugs (clonidine, morphine, ketamine, midazolam and furosemide). The drugs were studied at their highest commonly infused concentrations and exposed to three variations in environmental conditions (diluent: sodium chloride $0.9 \%$ or glucose $10 \%$; temperature 25 or $37^{\circ} \mathrm{C}$; and normal room lighting or blue light phototherapy). Chemical stability was assessed using HPLC; $>10 \%$ reduction in concentration indicated incompatibility. Physical incompatibility was confirmed by precipitation, $\mathrm{pH}$ or colour change.

Results Environmental conditions had no effect on the drug mixtures. The precipitation observed in incompatible combinations was due to either a change in $\mathrm{pH}$, or with ketamine the presence of benzethonium chloride. Of 31 possible drug combinations, 12 were incompatible. A further three combinations were incompatible at extreme $\mathrm{pH}$, or were of concern and so should be avoided. The incompatible formulations all contained furosemide. All combinations of the sedative agents studied were chemically and physically compatible.

Conclusion This work provides evidence for Y-site compatibility of morphine, midazolam, clonidine and ketamine in any combination, which will potentially reduce the need for extra intravenous lines. Furosemide is incompatible with any of these sedative drugs and must be infused via a separate line. These results will aid clinical decisionmaking and help satisfy the requirements of recent UK Department of Health legislation relating to the mixing of medicines.

\section{Reference}

1. Mixing of Medicines Prior to Administration in Clinical Practice: Medical and Non-medical Prescribing [http://www.dh.gov.uk/en/

Publicationsandstatistics/Publications/PublicationsPolicyAndGuidance/ DH_116361

P384

Abstract withdrawn

\section{P385}

Benzodiazepines induce hyperglycemia in rats by affecting peripheral disposal of glucose

VS Salice, FV Valenza, MP Pizzocri, LV Valenti, GC Chevallard, MU Umbrello, SG Gatti, SF Fargion, Gl lapichno, LG Gattinoni

Università degli Studi Milano, Milan, Italy

Critical Care 2013, 17(Suppl 2):P385 (doi: 10.1186/cc12323)

Introduction In light of the interest in the relationship between glycemia control in critically ill subjects and outcome, we set up a study to investigate whether benzodiazepine, commonly used in anesthesia and ICUs, interferes with glucose metabolism and to explore the mechanism.

Methods A total of 40 sedated and paralyzed Sprague-Dawley rats $(301 \pm 55 \mathrm{~g})$ were investigated in four consecutive studies. (1) To investigate the effects of diazepam on blood glucose, 15 rats were randomly assigned to intraperitoneal anesthesia with tiopenthal $80 \mathrm{mg} / \mathrm{kg}$ (DZPO), tiopenthal $40 \mathrm{mg} / \mathrm{kg}$ + diazepam $5 \mathrm{mg} / \mathrm{kg}$ (DPZ5) or tiopenthal $40 \mathrm{mg} / \mathrm{kg}$ + diazepam $15 \mathrm{mg} / \mathrm{kg}$ (DZP15). Blood levels of glucose (GEM premier 3000; IL) were measured at time intervals over 2 hours. (2) Ten animals randomized to DZP0 or DZP5 underwent an intravenous glucose tolerance test with glucose bolus $(0.5 \mathrm{~g} / \mathrm{kg})$. Acute insulin response, the mean value of blood insulin (Insulin ELISA kit; Millipore) from 2 to 10 minutes after glucose bolus, was measured as index of insulin secretion. (3) A hyperinsulinemic euglycemic clamp obtained by a continuous intravenous infusion of insulin (130 mUl/ $\mathrm{kg} /$ minute) was run in 10 animals randomized to DZP0 or DZP5 and the glucose infusion rate (GIR, $\mathrm{mg} / \mathrm{kg} /$ minute) was assessed [1]. (4) The effect of midazolam on blood glucose was tested in five additional animals (M5: tiophental $40 \mathrm{mg} / \mathrm{kg}+$ midazolam $5 \mathrm{mg} / \mathrm{kg}$ ). Data are presented as mean \pm SEM. Statistical analysis (ANOVA, $t$ test) was conducted with Sigma Stat 3.1 (Systat Software).

Results (1) Diazepam was associated with higher levels of blood glucose in a dose-response fashion: DZP0 $128 \pm 7 \mathrm{mg} / \mathrm{dl}$, DZP5 $166 \pm 7.3$, DZP15 $197 \pm 7(P<0.05)$. (2) The acute insulin response to intravenous glucose tolerance test was similar in DZP0 and DZP5 (DZP0 $3.97 \pm 0.42 \mathrm{ng} / \mathrm{ml}$, DZP5 $3.68 \pm 0.44, P=0.68$ ), while blood glucose levels were different (DZP0 $192 \pm 5 \mathrm{mg} / \mathrm{dl}$, DZP5 $217 \pm 5$, $P<0.05)$. (3) During hyperinsulinemic euglycemic clamp, blood glucose levels were similar $(109 \pm 3 \mathrm{mg} / \mathrm{dl}, P=0.2)$, but the DZP5 group showed a trend through lower values of GIR (DZPO $30.8 \pm 2 \mathrm{mg} /$ $\mathrm{kg} /$ minute, DZP5 $24.7 \pm 2, P=0.08$ ). (4) Infusion of midazolam was associated with higher blood glucose levels (DZP0 $128 \pm 5 \mathrm{mg} / \mathrm{dl}, \mathrm{M} 5$ $151 \pm 6, P<0.05$ )

Conclusion Both diazepam and midazolam significantly alter plasma glucose levels in rats. Peripheral disposal of glucose rather than altered pancreas secretion of insulin explains the benzodiazepine-associated hyperglycemia.

Reference

1. DeFronzo: Am J Physiol 1979, 273:E214-E223. 
P386

Prolonged sedation in ARDS patients with inhaled anesthetics: our experience

S Redaelli, P Mangili, V Ormas, S Sosio, L Peluso, F Ponzoni, N Patroniti, A Pesenti

University of Milan-Bicocca, Monza, Italy

Critical Care 2013, 17(Suppl 2):P386 (doi: 10.1186/cc12324)

Introduction We report our experience in the use of isoflurane for prolonged sedation in severe ARDS patients. Prolonged sedation in the ICU may be difficult because of tolerance, drug dependence and withdrawal, drug interactions and side effects. Inhaled anesthetics have been proposed for sedation in ventilator-dependent ICU patients. AnaConDa is a device that allows a safety and easy administration of inhaled anesthetics in the ICU.

Methods From January 2009 to June 2012, 15 patients were sedated with isoflurane by means of the AnaConDa device. We consider administration of isoflurane as a washout period from common sedative drugs in patients with (at least one of): high sedative drug dosage (propofol $\geq 300 \mathrm{mg} / \mathrm{hour}$ or midazolam $\geq 8 \mathrm{mg} / \mathrm{hour}$ ) to reach the target Richmond Agitation Sedation Score (RASS) or inadequate paralysis; two or more hypnotic drugs to reach the target RASS (propofol, midazolam, hydroxyzine, haloperidol, diazepam, quetiapine); and hypertriglyceridemia. During isoflurane administration previous hypnotic drugs were interrupted. We retrospectively collected data before, during and after administration of isoflurane: hemodynamic parameters, renal and hepatic function, level of sedation (RASS) and sedative drug dosage. All data are reported as mean \pm standard deviation, otherwise as median (minimum to maximum).

Results Mean age was $43 \pm 12$ years and SAPS II was $35.7 \pm 11 ; 13$ patients were treated with ECMO for severe ARDS and four had a history of drug abuse; median ICU length of stay was 41 (15 to 127) days and they were ventilated for 41 (12 to 114) days. Due to severe critical illness, target RASS was -4 for all patients, most of which were also paralysed. Isoflurane was administered in nine patients because of a high level of common sedative drugs, in five patients due to the use of two or more hypnotic drugs and in one patient because of hypertrigliceridemia. Isoflurane administration lasted $5.6 \pm 1.8$ days. During isoflurane administration no alteration in renal function or hemodynamic instability was recorded. After the isoflurane washout period we observed a reduction in sedative drug dosage in 10 patients while two patients were quickly weaned from mechanical ventilation and the target RASS raised to 0 . In two patients isoflurane was precautionarily interrupted because of concomitant alteration of liver function and suspected seizures respectively.

Conclusion Inhaled anesthetics could be successfully used in the ICU especially in case of an inadequate sedation plan; for example, in patients with a history of drug abuse or young severe ARDS patients that required deep sedation and paralysis for a long period.
P387

Involvement of mitochondrial ATP-sensitive $\mathrm{K}^{+}$channels in fentanylinduced mitochondrial dysfunction of cultured human hepatocytes S Djafarzadeh, M Vuda, J Takala, SM Jakob Bern University Hospital (Inselspital) and University of Bern, Switzerland Critical Care 2013, 17(Suppl 2):P387 (doi: 10.1186/cc12325)

Introduction Pharmacological agents used to treat critically ill patients may alter mitochondrial function. The aim of the present study was to investigate whether fentanyl, a commonly used analgesic drug, interacts with hepatic mitochondrial function.

Methods The human hepatoma cell line HepG2 was exposed to fentanyl at $0.5,2$ or $10 \mathrm{ng} / \mathrm{ml}$ for 1 hour, or pretreated with naloxone (an opioid receptor antagonist) at $200 \mathrm{ng} / \mathrm{ml}$ or 5 -hydroxydecanoate (5-HD; a specific inhibitor of mitochondrial ATP-sensitive $\mathrm{K}^{+}$(KATP) channels) at $50 \mu \mathrm{M}$ for 30 minutes, followed by incubation with fentanyl at $2 \mathrm{ng} / \mathrm{ml}$ for an additional hour. The mitochondrial complex I-dependent, II-dependent and IV-dependent oxygen consumption rates of the permeabilized cells were measured using a high-resolution oxygraph (Oxygraph-2k; Oroboros Instruments, Innsbruck, Austria). The respiratory electron transfer capacity of intact cells was evaluated using FCCP (carbonyl cyanide $p$-trifluoromethoxyphenylhydrazone) to obtain the maximum flux.

Results Incubation of HepG2 cells with fentanyl (1 hour, $2 \mathrm{ng} / \mathrm{ml}$ ) induced a reduction in complex II-dependent and IV-dependent respiration (Figure 1). Cells pretreated with 5-HD before the addition of fentanyl exhibited no significant changes in complex activities in comparison with controls. Pretreatment with naloxone tended to abolish the fentanyl-induced mitochondrial dysfunction. Treatment with fentanyl led to a reduction in cellular ATP content $(0.24 \pm 0.06$ in controls vs. $0.17 \pm 0.14 \mu \mathrm{mol} / \mathrm{mg}$ cellular protein in stimulated cells; $P=0.02$ ). We did not observe any difference in basal or FCCP-uncoupled respiration rates of cells treated with fentanyl at $2 \mathrm{ng} / \mathrm{ml}$ compared with controls (data not shown).

Conclusion Fentanyl reduces cultured human hepatocyte mitochondrial respiration by a mechanism that is blocked by a KATP channel antagonist. In contrast, antagonism with naloxone does not seem to completely abolish the effect of fentanyl.

Acknowledgement This study was supported by the Swiss National Science Foundation (grant number 32003B_127619).

P388

Effects of clonidine on microcirculatory alterations during endotoxemia

K Schmidt, C Philipsenburg, A Zivkovic, S Hofer

Universitätsklinikum Heidelberg, Germany

Critical Care 2013, 17(Suppl 2):P388 (doi: 10.1186/cc12326)

Introduction Endothelial dysfunction during endotoxemia is responsible for the functional breakdown of microvascular perfusion and microvessel permeability. The cholinergic anti-inflammatory pathway (CAP) is a neurophysiological mechanism that regulates the inflammatory response by inhibiting proinflammatory cytokine synthesis, thereby preventing tissue damage. Endotoxemia-induced

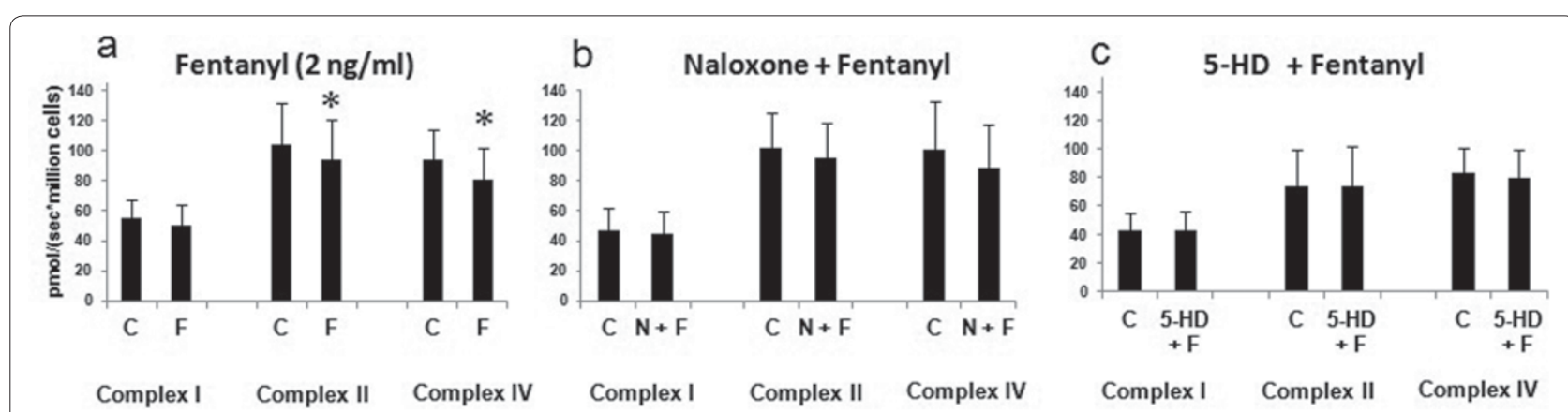

Figure 1 (abstract P387). Cellular oxygen consumption after incubation with fentanyl, naloxone or 5-HD. 
microcirculatory dysfunction can be reduced by cholinergic CAP activation. Clonidine improves survival in experimental sepsis [1] by reducing the sympathetic tone, resulting in the parasympatheticmediated CAP activation. The aim of this study was to determine the effects of clonidine on microcirculatory alterations during endotoxemia. Methods Using fluorescent intravital microscopy, we determined the venular wall shear rate, macromolecular efflux and leukocyte adhesion in mesenteric postcapillary venules of male Wistar rats. Endotoxemia was induced over 120 minutes by intravenous infusion of lipopolysaccharide (LPS). Control groups received an equivalent volume of saline. Clonidine $10 \mu \mathrm{g} / \mathrm{kg}$ was applied as i.v. bolus in treatment groups. Animals received either (i) saline alone, (ii) clonidine 10 minutes prior to saline administration, (iii) clonidine 45 minutes prior to LPS administration, (iv) clonidine 10 minutes prior to LPS administration, (v) clonidine 30 minutes after LPS administration or (vi) LPS alone.

Results All LPS groups (iii to vi) showed a significantly reduced venular wall shear rate compared with the saline group after 120 minutes. There were no significant differences between the numbers of adhering leukocytes in the clonidine/LPS groups (iii, iv, v) and the LPS group after 120 minutes. Macromolecular efflux significantly increased in all groups over the time period of 120 minutes. After 120 minutes there was no difference between the LPS group and the clonidine 10 minutes prior to LPS administration group (iv) whereas all other groups (i, ii, iii, v) showed a significantly reduced macromolecular efflux compared with the LPS group.

Conclusion Clonidine has no positive effect on microhemodynamic alterations and leukocyte-endothelial interaction during endotoxemia. The reduction of capillary leakage in clonidine-treated groups depends on the time interval relative to the initiation of endotoxemia. Endothelial permeability and leukocyte activation are regulated by different pathways when stimulated by clonidine during endotoxemia. We conclude that clonidine might have an important time-dependent anti-inflammatory and protective effect on endothelial activation during inflammation.

Reference

1. Hofer S, et al:: Crit Care 2009, 13:R11.

\section{P389}

Conjunctival administration of S+ketamine in rabbits

L Hess', J Malek', A Kurzova², Z Simunkova', M Votava ${ }^{2}$

'Institute for Clinical and Experimental Medicine, Praque, Czech Republic

${ }^{2} 3$ rd Medical Faculty of Charles University, Prague, Czech Republic

Critical Care 2013, 17(Suppl 2):P389 (doi: 10.1186/cc12327)

Introduction Delivering analgesics via conjunctival application could provide rapid and convenient pain relief in disaster medicine. There are sporadic reports from the USA concerning inhalation administration of aerosol with various drugs producing a wide variety of effects from anxiolysis, sedation, and loss of aggressiveness to immobilisation. We attempted to determine in an animal experiment whether conjunctival administration of S+ketamine could produce significant effect without side effects.

Methods After ethic committee approval, 10 rabbits were administered conjunctival S+ketamine $2.5 \mathrm{mg} / \mathrm{kg}$. Measured parameters were $\mathrm{SpO}_{2^{\prime}}$ blood pressure (BP) and heart rate (HR) before administration and in 1-minute intervals and immobilisation time (loss of righting reflex). The measurements were performed for 20 minutes. Conjunctival irritation was measured 1, 10 and 20 minutes after administration according to modified technical standard EN IS O 10993-10. ANOVA test was used for statistical analysis of hemodynamic parameters.

Results The immobilisation time was $207 \pm 60$ seconds. There were no changes in cardiorespiratory parameters (initial, 10 and 20 minutes after administration: HR $255.5 \pm 24.7,265.8 \pm 26.0$ and $267.8 \pm 20.4$, systolic BP $114.3 \pm 8.6,108.2 \pm 10.7$ and $113.9 \pm 11.9 \mathrm{mmHg}$, diastolic BP $66.8 \pm 11.6,68.4 \pm 10.6$ and $69.7 \pm 9.3 \mathrm{mmHg}$ and $\mathrm{SpO}_{2} 99.2 \pm 1.0$, $98.2 \pm 0.6$ and $98.7 \pm 0.5$ ). These results are in contrast to conjunctival administration of sufentanil $5 \mu \mathrm{g} / \mathrm{kg}$ that caused a significant decrease of $\mathrm{SpO}_{2}$ and $\mathrm{HR}$ [1]. We can speculate that the reason for stability of cardiorespiratory parameters was due to the sympathoadrenergic effect of ketamine or due to the method of administration. There were no signs of conjunctival irritation in any animal ( $\mathrm{S}+$ ketamine is a preservative-free solution).

Conclusion Conjunctival S+ketamine $2.5 \mathrm{mg} / \mathrm{kg}$ in rabbits produced rapid onset without changes in cardiorespiratory parameters and without signs of irritation of the eye. The results of our project warrant further research to increase the variety of drugs and methods of their administration for anxiolysis, sedation and analgesia in disaster medicine.

Acknowledgement The study was supported by scientific grant IGA NT 11284.

Reference

1. Malek J, et al:: Conjunctival administration of sufentanil in rabbits. Eur J Pain 2011, Suppl 5:212.

\section{P390}

Use of propofol for procedural sedation reduces length of stay in the emergency department

J Millar, F Adamson, P O'Connor, R Wilson, E Ferrie, R McLaughlin

Royal Victoria Hospital, Belfast, UK

Critical Care 2013, 17(Suppl 2):P390 (doi: 10.1186/cc12328)

Introduction Procedural sedation is used in the emergency department (ED) to facilitate short but painful interventions. Many patients are suitable for discharge after completion. Ideally, the agent used to achieve sedation should not have a prolonged effect, allowing safe discharge in the shortest time frame. We hypothesised that propofol, with its short onset and offset, may reduce length of stay (LOS) in comparison with traditional benzodiazepines.

Methods Data from a prospective registry were analysed for the period 1 August 2011 to 31 January 2012. Patients who underwent procedural sedation and who were discharged from the ED were identified. Individuals were grouped as having received propofol, midazolam or a combination of the two. All were discharged when fully alert and able to eat and drink. Demographic details and the type of procedure undertaken were extracted. ANOVA was performed to identify differences in the length of stay between groups, in addition to descriptive analysis.

Results During the study period 75 patients underwent procedural sedation and were discharged from the ED. The median age was 40 years and $57 \%$ were male. The commonest procedure performed was shoulder reduction (52\%). In the propofol group $(n=20)$ the mean LOS was 100 minutes compared with 165 minutes in those receiving midazolam $(n=40)$ and 141 minutes in those receiving a combination $(n=15), P=0.004$. There was no difference in adverse events between groups. See Figure 1.

Conclusion Propofol is increasingly used in EDs for procedural sedation due to its short duration of action. This study suggests that a shorter duration of action and faster recovery may result in a reduced LOS in the ED.

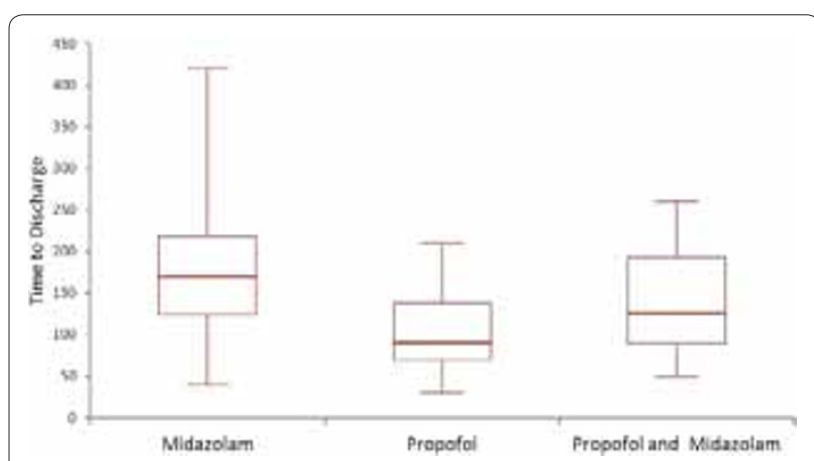

Figure 1 (abstract P390). Length of stay (minutes) in the ED. 
P391

Pre-PRIS? Prospective monitoring for early markers of propofol infusion syndrome

M Stovell, S Smith, M Udberg, W Loh, P Nair

Walton Centre Neurology \& Neurosurgery, Liverpool, UK

Critical Care 2013, 17(Suppl 2):P391 (doi: 10.1186/cc12329)

Introduction The use of propofol for sedation in intensive care has been associated with the propofol infusion syndrome (PRIS) characterised by cardiac dysfunction, metabolic acidosis, renal failure, rhabdomyolysis and hyperlipidaemia. We prospectively monitor biochemical markers that we believe demonstrate early signs of this dangerous, often fatal syndrome. When this Pre-PRIS state is identified, propofol is withdrawn whilst the syndrome is still reversible.

Methods We prospectively audited our monitoring of these markers over a 4-month period in propofol-sedated patients: propofol infusion rate, creatine kinase $(\mathrm{CK})$, triglycerides $(\mathrm{TG})$, creatinine, lactate, $\mathrm{pH}$ and base deficit. We defined the criteria for Pre-PRIS as requiring a CK $\geq 320 \mathrm{mmol} / \mathrm{l}$ that had doubled from its base level and a rise in TG $\geq 1.7 \mathrm{IU} / \mathrm{l}$; both that followed a trend with propofol dose.

Results Data were collected from 54 adults; 50 received an infusion for $\geq 24$ hours. We noted a significant CK rise in $11(22 \%)$ of our patients that could be attributed to propofol alone. They all had raised TG $(\geq 1.7 \mathrm{IU} / \mathrm{l})$; six $(12 \%)$ were markedly raised $(\geq 3.4 \mathrm{IU} / \mathrm{l})$. No patients became significantly acidotic, developed cardiac arrhythmias or renal failure as a result of propofol. In the 11 patients who met our criteria, highest daily propofol dose varied from 1.5 to $4 \mathrm{mg} / \mathrm{kg} / \mathrm{hour}$. Pre-PRIS developed from between 2 and 6 days of infusion. The mean propofol dose in patients displaying Pre-PRIS was $2.2 \mathrm{mg} / \mathrm{kg} / \mathrm{hour}$ whilst in those not meeting our criteria it was $2.0 \mathrm{mg} / \mathrm{kg} /$ hour. No patients developed sequelae of full PRIS. See Table 1.

Table 1 (abstract P391)

\begin{tabular}{lccc}
\hline & Total & Trauma & Nontrauma \\
\hline$\geq 24$ hours infusion & 50 & 34 & 16 \\
$\uparrow C K+\uparrow T G \geq 3.4$ & 6 & 6 & 0 \\
$\uparrow C K+\uparrow T G \geq 1.7$ & 5 & 3 & 2 \\
\hline
\end{tabular}

Conclusion We propose that a paired rise in CK and TG that can be attributed to propofol alone represents a Pre-PRIS state that is at risk of developing into full PRIS. We noted this in $22 \%$ of our patients, all on modest doses of propofol. It is unclear what proportion of patients will develop the full syndrome as it is not ethically possible to continue propofol in this situation. We advocate daily monitoring of CK and TG to identify Pre-PRIS so that propofol can be reduced or substituted to avoid the morbidity and mortality of the full syndrome.

P392

Safety profile of 1,008 cases of propofol sedation in the emergency department

S Bradburn, G Lloyd, B Newstead

Royal Devon and Exeter Hospital, Exeter, UK

Critical Care 2013, 17(Suppl 2):P392 (doi: 10.1186/cc12330)

Introduction Until recently there were no guidelines for the reporting of adverse events (AEs) during procedural sedation [1,2]. A consensus document released in 2012 by the world SIVA International Sedation Task Force proposed a benchmark for defining AEs [3]. We analysed 1,008 cases of procedural sedation in the emergency department. Methods The study is based on 1,008 patients who received procedural sedation with propofol in the emergency department between December 2006 and March 2012. Patients were selected and sedated to a strict protocol by ED consultant staff. We applied the AE tool by performing a search through patient records, discussion with consultants performing the sedation and consensus opinion.

Results From 1,008 cases we identified 11 sentinel (six of hypotension, five cases of hypoxia), 34 moderate, 25 minor and three minimal risk adverse events.
Conclusion The study shows a $1 \%$ adverse event rate. This supports use of propofol sedation by emergency physicians but within the limits of a strict governance framework. Our safety analysis using the World SIVA adverse events tool provides a reference point for further studies.

References

1. Green SM, Yealy DM: Procedural sedation goes Utstein: the Quebec guidelines. Ann Emerg Med 2009, 53:436-438

2. Green SM, Krauss B: Barriers to propofol use in emergency medicine. Ann Emerg Med 2008, 52:392-398.

3. Mason KP, Green SM, Piacevoli Q: International Sedation Task Force: Adverse event reporting tool to standardize the reporting and tracking of adverse events during procedural sedation: a consensus document from the World SIVA International Sedation Task Force. Br J Anaesth 2012, 108:13-20.

\section{P393}

Use of physical restraint in Dutch ICUs: prevalence and motives

RJ Raijmakers', RL Vroegop', H Tekatli', M Van den Boogaard²,

AW Van der Kooi ${ }^{1}$, AJ Slooter ${ }^{1}$

'University Medical Center Utrecht, the Netherlands; ${ }^{2}$ Radboud University

Nijmegen Medical Centre, Nijmegen, the Netherlands

Critical Care 2013, 17(Suppl 2):P393 (doi: 10.1186/cc12331)

Introduction Physical restraints are used to facilitate essential care and prevent secondary injuries. However, physical restraint may be regarded as humiliating. It may lead to local injury and increase the risk of delirium and post-traumatic stress syndrome. Research on physical restraint is scarce. The aim of this study is to investigate the scope of physical restraint use.

Methods Twenty-one ICUs ranging from local hospitals to academic centres were each visited twice and 327 patients were included. We recorded characteristics of restrained patients, motives and awareness of nurses and physicians.

Results Physical restraint was applied in 74 (23\%) patients, ranging from 0 to $54 \%$ in different hospitals. Frequent motives for restraint use were 'possible threat to airway' (36\%) and 'pulling lines/probes' (31\%). Restrained subjects more often had a positive CAM-ICU (34\% vs. $16 \%, P<0.001)$, could less frequently verbally communicate $(14 \%$ vs. $49 \%, P<0.001)$, and received more often antipsychotics (49\% vs. $28 \%, P<0.001)$, or benzodiazepines ( $55 \%$ vs. $36 \%, P=0.003)$. The use of physical restraint was registered in the patient's files in $48 \%$ of cases. Of the 310 interviewed nurses, 258 (83\%) were familiar with a physical restraint protocol and $89(29 \%)$ used it in any situation. Thirty percent of the 60 interviewed physicians were aware of the physical restraint status of their patients.

Conclusion Physical restraint is frequently used in Dutch ICUs, but the frequency differs strongly between different ICUs. Attending physicians are often not aware of physical restraint use.

P394

Factors influencing the use of physical restraints in Canadian ICUs B Sneyers ${ }^{1}$, E Luk², M Perreault ${ }^{3}$, D Williamson ${ }^{3}$, L Rose ${ }^{2}$, S Mehta $^{4}$, L Burry ${ }^{4}$ 'Université Catholique de Louvain, Brussels, Belgium; ' Lawrence S. Bloomberg Faculty of Nursing, University of Toronto, Ontario, Canada; ${ }^{3}$ Université de Montréal, Canada; ${ }^{4}$ Mount Sinai Hospital, University of Toronto, Ontario, Canada

Critical Care 2013, 17(Suppl 2):P394 (doi: 10.1186/cc12332)

Introduction Physical restraint (PR) use in critically ill patients has been associated with delirium, unplanned extubation, prolonged ICU length of stay, and post-traumatic stress disorder. Our objectives were to define prevalence of PR use, and to examine patient, treatment, or institutional factors associated with their use in Canadian ICUs.

Methods We conducted an analysis of a database of sedative, analgesic, antipsychotic and neuromuscular blocker prescribing practices and PR use in 711 mechanically ventilated (MV) adults in 51 Canadian ICUs (3,619 patient-days). Data were collected during a 2-week period (2008 to 2009). Logistic and Poisson regression analysis were used to identify factors associated with PR use and number of days of use respectively. Results PR was used on 1 or more days in $52.6 \%$ (374/711) of patients. For patients restrained, the mean number of days restrained was 
$4.1 \pm 4.0$ and the mean number of restraint applications per patient was $1.2 \pm 0.5$. Treatment characteristics associated with PR use were: daily benzodiazepine dose (OR $=1.01,95 \% \mathrm{Cl}: 1.00$ to 1.01$)$, daily opiate dose $(\mathrm{OR}=1.00,95 \% \mathrm{Cl}: 1.00$ to 1.01$)$, daily sedation interruption (OR $=1.84$, $95 \% \mathrm{Cl}: 1.24$ to 2.72 ), use of antipsychotics (OR $=3.42,95 \% \mathrm{Cl}: 1.98$ to 5.91 ) and agitation (Sedation-Agitation Scale (SAS) $>4, \mathrm{OR}=2.55,95 \%$ Cl: 1.20 to 5.44). Additionally, number of days of PR use was increased according to the following: daily sedation interruption (IRR $=4.52$, $95 \% \mathrm{Cl}: 2.09$ to 9.75 ), use of antipsychotics (IRR $=18.65,95 \% \mathrm{Cl}: 7.95$ to 43.77 ), the presence of a heavily sedated score (SAS $<3$, IRR $=2.57$, $95 \%$ Cl: 1.06 to 6.24$)$ or the presence of an adverse event, such as selfextubation (IRR $=10.46,95 \% \mathrm{Cl}: 2.55$ to 42.92 ). Hospital characteristics associated with PR use included the proportion of MV patients in the ICU. University hospitals were associated with reduced PR use, compared with community hospitals ( $\mathrm{OR}=0.272,95 \% \mathrm{Cl}: 0.15$ to 0.50$)$. Nonmodifiable patient characteristics (age, sex, APACHE score, patient category, prior substance abuse, prior psychotropic medication, preexisting psychiatric condition or dementia) were not associated with PR use.

Conclusion We identified multiple factors associated with PR use and number of days of PR use. Particularly among those related to treatment, strategies such as daily sedation interruption or use of antipsychotics are associated with PR use and increasing the number of days of use.

P395

Current practices in ICU delirium management: a prospective multicenter study in the Netherlands

ZTrogrlic, E Ista, A Slooter, J Bakker, M Van der Jagt, on behalf of iDECePTIvE Study Group

Erasmus MC University Medical Center, Rotterdam, the Netherlands Critical Care 2013, 17(Suppl 2):P395 (doi: 10.1186/cc12333)

Introduction As part of a multicenter prospective study on barriers and facilitators for implementation of protocolled care for delirious critically ill patients, we aimed to describe current practices in delirium management in a representative cohort of ICU patients.

Methods We included consecutively admitted patients during a 4-month period in a prospective multicenter study in six ICUs (one academic, teaching and nonteaching hospitals) in the southwest of the Netherlands. We assessed: percentage of patients screened for delirium with validated screening tools; pharmacological treatment with haloperidol or other antipsychotic drugs; psychohygiene (use of hearing aids and or glasses, preventing sleep disturbances); and access to early mobilization and physiotherapy. Delirium was pragmatically defined as administration of haloperidol and/or delirium reported by physicians or ICU nurses in patient records, as assessed by a designated research nurse. Differences between centers were tested with nonparametric tests.

Results We assessed 1,576 patients, corresponding with 8,150 ICU treatment days with a median length of stay of 3 days (IQR 2 to 5 ). The mean age of the patients was 62 years $(S D=16)$ and $58 \%$ were male. Delirium occurred in $23 \%(356 / 1,576)$ of patients with a median duration of 3 days (IQR 2 to 7 ) and ranged from 11 to $40 \%$ for each ICU. Delirium assessment with the CAM-ICU at any point during ICU stay was performed in $38 \%$ of all patients. Screening with CAM-ICU was applied in three ICUs, in 29 to $96 \%$ of the patients in these centers. Of 3,564 documented screening days with the CAM-ICU, it was positive in 1,459 (41\%); in only $120(8 \%)$ of these CAM-ICU-positive days there was a documented action or treatment started for delirium. However, patients still received haloperidol on $52 \%(n=766)$ of all CAM-ICUpositive days. Patients received benzodiazepines in 49\% $(n=1,141)$ of patient sedation days. Delirium preventive interventions were physiotherapy (19\% of 8,150 ICU days), mobilization (10\%), glasses use $(2.6 \%)$ and hearing aid use $(0.3 \%)$. Presence of hearing or visual impairment at admission was not documented in $65 \%$ of patients.

Conclusion Daily screening for ICU delirium with a validated screening instrument is applied in less than one-half of the time in critically ill patients and management of delirium is often not guided by this screening. Haloperidol was used as the first-choice medication. Measures aimed at delirium prevention (psychohygiene and early mobilization) were carried only in a small minority or were not documented. To implement protocolled delirium care in the region at study, a multifaceted tailored implementation program is needed.

\section{P396}

Intraoperative dexamethasone and delirium after cardiac surgery: a randomized clinical trial

AC Sauër, AJ Slooter, DS Veldhuijzen, MM Van Eijk, D Van Dijk

University Medical Center Utrecht, the Netherlands

Critical Care 2013, 17(Suppl 2):P396 (doi: 10.1186/cc12334)

Introduction The objective of this study is to investigate the effect of intraoperative administration of dexamethasone versus placebo on the incidence of delirium in the first four postoperative days after cardiac surgery.

Methods Within the context of the large multicenter Dexamethasone for Cardiac Surgery (DECS) trial [1] for which patients were randomized to $1 \mathrm{mg} / \mathrm{kg}$ dexamethasone or placebo at induction of anesthesia, a monocenter substudy was conducted. The primary outcome of this study was the incidence of delirium in the first four postoperative days. Secondary outcomes were duration of delirium, use of restrictive measures and sedative, antipsychotic and analgesic requirements. Delirium was assessed daily by trained research personnel, using the Richmond Agitation Sedation Scale and the Confusion Assessment Method. Medical, nursing and medication charts were evaluated for signs of delirium and use of prespecified medication. Analysis was by intention to treat.

Results Of 768 eligible patients, complete data on delirium could be collected in 738 patients. The incidence of delirium was $14.2 \%$ in the dexamethasone group and $14.9 \%$ in the placebo group (odds ratio $=0.95,95 \% \mathrm{Cl}=0.63$ to 1.43 ). No significant difference was found on the duration of delirium between the intervention (median $=2$ days, interquartile range 1 to 3 days) and placebo (median $=2$ days, interquartile range 1 to 2 days) group $(P=0.45)$. The use of restrictive measures and administration of sedatives, haloperidol, benzodiazepine and opiates were comparable between both groups.

Conclusion Intraoperative injection of dexamethasone seems not to affect the incidence or duration of delirium in the first 4 days after cardiac surgery, suggesting this regimen is safe to use in the operative setting with respect to psychiatric adverse events.

Reference

1. Dieleman JM, et al: JAMA 2012, 308:1761-1767.

\section{P397}

Current perspectives, beliefs and practices concerning delirium in critically ill patients: a multicenter survey among Dutch healthcare professionals

ZTrogrlic, M Van der Jagt, P Van der Voort, J Bakker, E Ista, on behalf of iDECePTIvE Study Group

Erasmus MC University Medical Center, Rotterdam, the Netherlands

Critical Care 2013, 17(Suppl 2):P397 (doi: 10.1186/cc12335)

Introduction The beliefs, knowledge and practices regarding ICU delirium among ICU professionals may vary. This may interfere with the implementation of the Dutch ICU Delirium guideline. We aimed to get insight into potential barriers and facilitators for delirium guideline implementation that may help to find an effective implementation strategy.

Methods An online survey was sent to healthcare professionals from the six participating ICUs. Respondents included ICU physicians, nurses and delirium experts (psychiatrists, neurologists, geriatricians, nurse experts). The survey consisted of statements on beliefs, knowledge and practices towards ICU delirium. Agreement with statements by more than $75 \%$ of respondents were regarded as facilitating items and agreement lower than $50 \%$ as barriers for implementing protocolled care.

Results Of the 565 surveys distributed, 360 were completed (63.7\%). The majority of respondents were ICU nurses (79\%). Delirium was considered a major problem $(83 \%)$ that requires adequate treatment $(99 \%)$ and is underdiagnosed (81\%). Respondents considered that 
routine screening of delirium can improve prognosis (95\%). However, only a minority (20\%) answered that delirium is preventable. Only $39 \%$ of the respondents had received any training about delirium in the previous 3 years and $77 \%$ of them found training useful. The mean delirium knowledge score was 6.6 out of $10(S D=1.54)$. When all groups were mutually compared, nurses scored lower than delirium experts (ANOVA, $P=0.013)$. The respondents $(58 \% ; n=210)$ from three ICUs indicated that CAM-ICU assessment was department policy. However, $50 \%(n=106)$ of these respondents felt unfamiliar with CAMICU and only $47 \%(n=99)$ of them indicated that a positive CAM-ICU was used for treatment decisions. Haloperidol was the first-choice pharmacological treatment. Only $21 \%$ of all respondents knew that a national ICU delirium guideline existed, but in-depth knowledge was generally low.

Conclusion Our survey showed that healthcare professionals considered delirium an important but underdiagnosed form of organ failure. In contrast, screening tools for delirium are scarcely used, knowledge can be improved and protocolled treatment based on positive screening is often lacking. These results suggest that the focus of implementation of ICU delirium management should not be on motivational aspects, but on knowledge improvements, training in screening tools and implementation of treatment and prevention protocols.

\section{P398}

International validation of the delirium prediction model for ICU patients (PREDELIRIC): a multicenter observational study

$M$ van den Boogaard ${ }^{1}$, L Schoonhoven ${ }^{2}$, E Maseda $^{3}$, C Plowright $^{4}$,

C Jones $^{5}$, A Luetz ${ }^{6}$, PV Sackey ${ }^{7}$, P Jorens ${ }^{8}$, LM Aitken ${ }^{9}$, F van Haren ${ }^{10}$ JG van der Hoeven', P Pickkers

'Radboud University Nijmegen Medical Centre, Nijmegen, the Netherlands; ${ }^{2}$ Radboud University Nijmegen Medical Centre, Scientific Institute for Quality of Healthcare, Nijmegen, the Netherlands: ${ }^{3}$ Hospital Universitario La Paz, Madrid, Spain: ${ }^{4}$ Medway Maritime Hospital, Kent, UK: ${ }^{5}$ Whiston Hospital, Prescot, UK; ${ }^{6}$ Charité - Universitaetsmedizin Berlin, Germany; ${ }^{7}$ Karolinska University Hospital, Stockholm, Sweden; ${ }^{8}$ University of Antwerpen, Belgium; ${ }^{9}$ Princess Alexandra Hospital, Woolloongabba, Australia; ${ }^{10} \mathrm{Canberra} \mathrm{Hospital,}$ Canberra, Australia

Critical Care 2013, 17(Suppl 2):P398 (doi: 10.1186/cc12336)

Introduction Delirium is a serious and frequent disorder in ICU patients. The aim of this study was to internationally validate the existing Prediction of Delirium ICU (PREDELIRIC) model to predict delirium in ICU patients.

Methods A prospective multicenter cohort study was performed in eight ICUs in six countries (Table 1). The 10 predictors (age, APACHE II score, urgent and admission category, infection, coma, sedation, morphine use, urea level, metabolic acidosis) were collected within 24 hours after ICU admission. CAM-ICU was used to identify ICU delirium.

Results A total of 2,852 adult ICU patients were screened and 1,824 $(64 \%)$ were included. Main exclusion reasons were length of stay $<1$ day (19.1\%) and sustained coma (4.1\%). Mean \pm CAM-ICU compliance

Table 1 (abstract P398). PREDELIRIC AUROC of different centers

\begin{tabular}{lccc}
\hline & Delirium (\%) & AUROC & $\mathbf{9 5 \% ~ C l}$ \\
\hline Belgium (\%) & $86(15.2)$ & 0.79 & 0.74 to 0.84 \\
Germany (\%) & $60(26.9)$ & 0.85 & 0.80 to 0.91 \\
Spain (\%) & $23(18.0)$ & 0.88 & 0.81 to 0.94 \\
Sweden (\%) & $30(39.0)$ & 0.71 & 0.60 to 0.83 \\
Australia Brisbane (\%) & $42(12.8)$ & 0.81 & 0.75 to 0.88 \\
Australia Canberra (\%) & $23(11.8)$ & 0.80 & 0.72 to 0.89 \\
UK_Prescot (\%) & $73(30.8)$ & 0.65 & 0.57 to 0.73 \\
UK_Kent (\%) & $26(36.6)$ & 0.76 & 0.65 to 0.88 \\
PREDELIRIC overall & $\mathbf{3 6 3 ( 1 9 . 9 )}$ & $\mathbf{0 . 7 7}$ & $\mathbf{0 . 7 4}$ to 0.79 \\
\hline
\end{tabular}

was $82 \pm 16 \%$ and inter-rater reliability $0.87 \pm 0.17$. Overall delirium incidence was $19.9 \%$ (range 27.2\%). Despite significant differences between centres on all 10 predictors, the overall area under the receiver operating characteristic curve (AUROC) of the eight centers was 0.77 ( $95 \%$ Cl: 0.74 to 0.79 ).

Conclusion The overall predictive value of the PREDELIRIC model was good, indicating that the prediction model can now be used internationally.

\section{P399}

Delirium in ICU patients is associated with increased temperature variability

AW Van der Kooi, TH Kappen, RJ Raijmakers, IJ Zaal, AJ Slooter

University Medical Center Utrecht, the Netherlands

Critical Care 2013, 17(Suppl 2):P399 (doi: 10.1186/cc12337)

Introduction Delirium is an acute disturbance of consciousness and cognition. It is a common disorder in the ICU and associated with impaired long-term outcome [1,2]. Despite its frequency and impact, delirium is poorly recognized by ICU physicians and nurses using delirium screening tools [3]. A completely new approach to detect delirium is to use monitoring of physiological alterations. Temperature variability, a measure for temperature regulation, could be an interesting parameter for monitoring of ICU delirium, but this has never been investigated before. The aim of this study was to investigate whether temperature variability is affected during ICU delirium.

Methods We included patients in whom days with delirium could be compared with days without delirium, based on the Confusion Assessment Method for the ICU and inspection of medical records. Patients with conditions affecting thermal regulation, including infectious diseases, and those receiving therapies affecting body temperature were excluded. Twenty-four ICU patients were included after screening 334 delirious ICU patients. Daily temperature variability was determined by computing the mean absolute second derivative of the temperature signal. Per patient, temperature variability during delirious days was compared with nondelirium days using a Wilcoxon signed-rank test. With a linear mixed model, differences between delirium and nondelirium days with regard to temperature variability were analysed adjusted for daily mean Richmond Agitation and Sedation Scale scores, daily maximum Sequential Organ Failure Assessment score, and within-patient correlation.

Results Temperature variability was increased during delirium days compared with days without delirium (mean difference $=-0.007,95 \%$ $\mathrm{Cl}=-0.004 ;-0.011, P<0.001)$. Adjusting for confounders did not alter our findings (adjusted mean difference $=-0.005,95 \% \mathrm{Cl}=-0.008$; $-0.002, P<0.001)$.

Conclusion Temperature variability is increased during delirium in ICU patients, which reflects the encephalopathy that underlies delirium. Opportunities for delirium monitoring using temperature variability should be further explored. Particularly, in combination with electroencephalography it could provide the input for an objective tool to monitor delirium.

References

1. Pisani MA, et al:: Am J Respir Crit Care Med 2009, 180:1092-1097.

2. Ely EW, et al.: JAMA 2004, 291:1753-1762.

3. van Eijk MM, et al:: Am J Respir Crit Care Med 2011, 184:340-344.

P400

Long-term outcome of delirium in critically ill patients

A Wolters, D Van Dijk, O Cremer, D De Lange, A Slooter

UMC Utrecht, the Netherlands

Critical Care 2013, 17(Suppl 2):P400 (doi: 10.1186/cc12338)

Introduction In ICU patients, little research has been performed on the relationship between delirium and long-term outcome, including health-related quality of life (HRQoL), cognitive functioning and mortality. In addition, results seem to be inconsistent. Furthermore, in studies that reported increased mortality in delirious patients, no proper adjustments were made for severity of illness during ICU admission. This study was conducted to investigate the association 
between ICU delirium and long-term HRQoL, cognitive functioning and mortality. The hypothesis was that delirious patients have worse long-term outcome in comparison with nondelirious patients.

Methods A prospective observational cohort study was conducted. A median of 12 months after ICU discharge, questionnaires were sent to all survivors. HRQoL and cognitive functioning were measured with the EuroQol-6D. Age, gender and severity of illness were considered relevant covariates. Severity of illness was estimated using the APACHE IV score and the maximal SOFA score during admission. HRQoL was investigated with linear regression analysis, cognitive functioning using logistic regression and mortality with Cox regression analysis. Results The patient population consisted of 690 patients admitted to the ICU, subdivided into delirious $(n=257)$ and nondelirious patients ( $n=433)$. During follow-up, $181(26 \%)$ patients died. The response rate of the questionnaire was $70.6 \%$. After adjusting for the predefined covariates, delirium was significantly associated with a lower HRQoL (adjusted $\beta$ : $-0.137 ; 95 \% \mathrm{Cl}=-0.140$ to -0.005 ) and more mild and severe cognitive impairment (adjusted odds ratio: respectively: $2.3 ; 95 \%$ $\mathrm{Cl}=1.3$ to 4.2 and $5.8 ; 95 \% \mathrm{Cl}=1.3$ to 15.2 ). No significant association between delirium and long-term mortality was found (adjusted hazard ratio: $1.0 ; 95 \% \mathrm{Cl}=0.7$ to 1.4 ).

Conclusion Delirium during ICU admission was associated with lower $\mathrm{HRQ}$ oL and worse cognitive functioning, 1 year after discharge. Furthermore, delirium on the ICU was not associated with long-term mortality after adjusting for relevant covariates, including severity of illness during ICU admission.

\section{P401}

Gastrointestinal symptoms in the ICU: a comparison of primary and secondary acute gastrointestinal injury

A Reintam Blaser, J Starkopf

University of Tartu, Tartu University Hospital, Tartu, Estonia

Critical Care 2013, 17(Suppl 2):P401 (doi: 10.1186/cc12339)

Introduction We aimed to clarify the differences between primary and secondary acute $\mathrm{Gl}$ injury.

Methods A total of 2,690 consecutive adult patients were retrospectively studied during their first week in the ICU. Pathology in the $\mathrm{Gl}$ system or laparotomy defined the primary GI insult. If Gl symptoms developed without primary GI insult it was considered secondary Gl injury. Absent bowel sounds (BS), vomiting/regurgitation, diarrhoea, bowel distension, Gl bleeding, and high gastric residuals (GRV $>1,000 \mathrm{ml} / 24$ hours) were recorded daily.

Results In total, 2,690 patients (60.4\% male), median age 59 years (range 16 to 92), were studied. Eighty-four per cent of them were ventilated, $72 \%$ received vasopressor/inotrope. Median (IQR) APACHE II score was 14 (9 to 21) and SOFA on the first day was 6 (3 to 10). A total $35.5 \%$ had primary GI pathology. During the first week $34 \%$ of patients had absent BS, 38\% vomiting/regurgitation, 9\% diarrhoea, $7 \%$ bowel distension, $6 \%$ high GRV and $5 \%$ Gl bleeding. All symptoms except diarrhoea occurred more often $(<0.001)$ in patients with primary $\mathrm{Gl}$ insult. Eighty-five per cent of patients with primary $\mathrm{Gl}$ insult versus $46 \%$ without developed at least one $\mathrm{Gl}$ symptom. The incidence of GI symptoms was significantly higher in nonsurvivors. ICU mortality was lower in patients with primary than secondary $\mathrm{Gl}$ injury $(43.6 \%$ vs. $61.2 \%, P=0.046)$. Nonsurvivors without primary $\mathrm{Gl}$ insult developed GI symptoms later (Figure 1).

Conclusion Primary and secondary acute GI injury have different incidence, dynamics and outcome.

\section{P402}

Effects of proton pump inhibitor versus $\mathrm{H} 2$-receptor antagonist stress ulcer prophylaxis on ventilator-associated pneumonia: a pilot study

JF Fogas, KK Kiss, FG Gyura, ZT Tóbiás, ZM Molnár

University of Szeged, Hungary

Critical Care 2013, 17(Suppl 2):P402 (doi: 10.1186/cc12340)

Introduction Ventilator-associated pneumonia (VAP) is the most frequent ICU-acquired infection among patients receiving mechanical

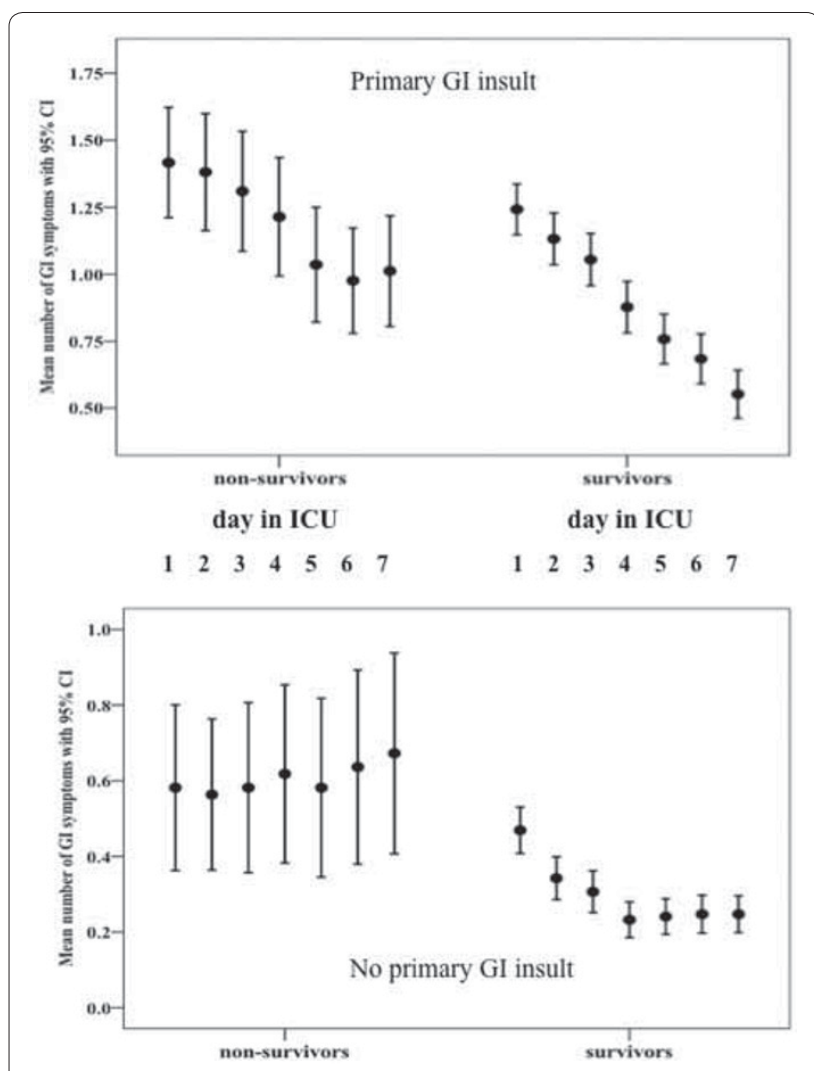

Figure 1 (abstract P401). Daily dynamics of the number of Gl symptoms. $P<0.001$ between survivors and nonsurvivors in all time points.

ventilation with relative risk of 9 to $27 \%$ and with mortality of 25 to $50 \%[1,2]$. One of the promoting factors of VAP is the increased $\mathrm{pH}$ of the gastric acid, which occurs when H2-receptor antagonists (H2RA) or proton pump inhibitors (PPI) are used for stress ulcer prophylaxis. The aim of this study was to investigate effects of PPI versus H2RA on the occurrence of VAP and, as second endpoint, the occurrence of gastrointestinal bleeding (GIB).

Methods After ethics committee approval mechanically ventilated ( $>48$ hours) adults were recruited in a prospective randomized controlled, double-blind clinical trial and randomised into PPI or H2RA groups. Patients with admission diagnosis of pneumonia or other acute inflammatory pulmonary disease, severe sepsis/septic shock, and/or regular PPI/H2RA users were excluded. The diagnostic criteria for VAP were: leukocytosis, increased PCT level, fever, purulent tracheal secretion, positive microbiological result of tracheal sample, and new/ increased infiltrate on chest X-ray. Data are presented as median (interquartile range, IQR). For statistical analysis Mann-Whitney $\mathrm{U}$ test and chi-square tests were used.

Results Out of 94 patients recruited 14 were excluded due to early weaning/death $(n=9)$ or protocol violation $(n=6)$. The remaining 79 patients were analysed (PPI, $n=38$; H2RA, $n=41$ ). There was no significant difference $(P>0.05)$ between the groups regarding demographics: age 67 (56 to 77 ) versus 72 (58 to 79 ) years; male/ female: $23 / 15$ versus $25 / 16$; length of mechanical ventilation: 5 ( 3 to 9) versus 5 ( 2 to 8 ) days; APACHE II score: 28 ( 22 to 32 ) versus 26 ( 21 to 36) (PPI vs. H2RA, respectively). There was no significant difference in the number of cases with VAP in the PPI versus H2RA groups: 9 (24\%) versus $10(24 \%)$. None of the patients developed Gl bleeding during their stay on the ICU.

Conclusion The results of this pilot study suggest that there may be no difference in the incidence of VAP and Gl bleeding if stress ulcer prophylaxis is performed by H2RA or PPI. As the latter is more expensive, its use as first choice in critical care should be questioned. 
However, the completion of the study on the planned 198 patients is required to come to the final conclusions.

\section{References}

1. Efrati S, et al: J Clin Monit Comput 2010, 24:161-168.

2. Torres A, et al: Intensive Care Med 2009, 35:9-29.

P403

Early diagnosis of liver failure in septic patients using the maximal liver function capacity test (LiMAx test): comparison with conventional methods

M Kaffarnik, M Stockmann, J Lock

Charite Berlin Campus Virchow, Berlin, Germany

Critical Care 2013, 17(Suppl 2):P403 (doi: 10.1186/cc12341)

Introduction Patients with bacterial sepsis often suffer from multiorgan failure. No reliable parameter exists for the exact estimation of the liver function. The indocyaningreen test (ICG test) showed a correlation between liver function and mortality rate. The new maximal liver function capacity test (LiMAx test) is a real-time method to investigate the liver function.

Methods Thirty septic patients were prospectively included in the study. The LiMAx test was performed on days 0, 2, 5 and 10 after sepsis onset and was compared with ICG test and liver-specific laboratory parameters. Primary endpoint was the mortality rate after 90 days. Secondary endpoint was the comparison with the ICG test.

Results The LiMAx test showed low results initially with increasing values on days 5 and 10 . The 90-day mortality rate in patients with a low LiMAx test on day $2(<100 \mu \mathrm{g} / \mathrm{kg} / \mathrm{hour})$ was significantly higher than in patients with a LiMAx test $>100 \mu \mathrm{g} / \mathrm{kg} /$ hour. The LiMAx test was comparable with the ICG test. Patients with indication for renal replacement therapy during hospital treatment showed significantly lower LiMAx values than patients without dialysis. See Figure 1.

Conclusion With the LiMAx test we can detect a liver dysfunction in septic patients early on days 0 to 2 . The LiMAx test is equal with the ICG test and a result of $<100 \mu \mathrm{g} / \mathrm{kg} / \mathrm{hour}$ on day 2 suggests a low probability of survival in septic patients.

\section{References}

1. Stockmann et al: New liver function test for prediction of postoperative outcome in liver surgery. HPB 2010, 12:139.

2. Lock et al: Early diagnosis of primary nonfunction and indication for reoperation after liver transplantation. Liver Transp/ 2010, 16:172.

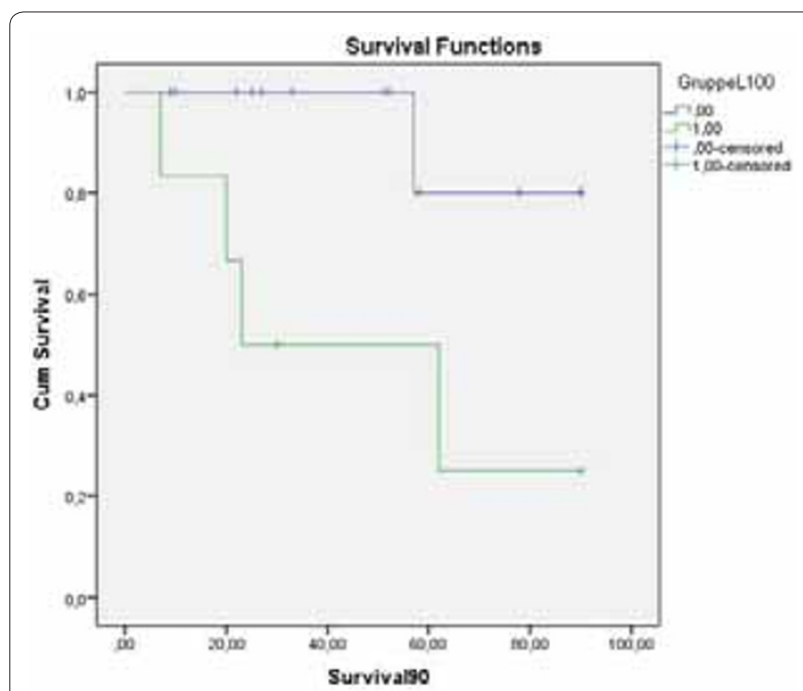

Figure 1 (abstract P403). Kaplan-Meier survival curve of patients with high and low LiMAx results.
P404

Impact of liver resection on portal venous pressure and renal function

P Biesenbach, T Grünberger, B Payer, A Ferlitsch, W Druml, E Fleischmann, F Luf, J Mühlbacher

Medical University of Vienna, Austria

Critical Care 2013, 17(Suppl 2):P404 (doi: 10.1186/cc12342)

Introduction Liver dysfunction - in correlation to the severity of functional impairment - but also any increase in portal pressure per se (hepatorenal reflex) can induce alterations in renal function and ultimately result in hepatorenal syndrome (HRS). In this prospective investigation we determined the impact of liver resection on portal venous pressure by measuring the hepatic venous pressure gradient (HVPG), on concentrations of vasoactive peptides and on renal function. Methods Twenty patients (mean age 66.3 years) undergoing elective liver resection surgery because of malignant tumor were assessed and grouped according to resection size: (Group 1) hemihepatectomy, $n=13$ versus (Group 2) segmentectomy, $n=7$. HPVG was measured before and after resection by canulation of a hepatic vein under fluoroscopic guidance, liver function was assessed by the indocyanine green plasma disappearance rate (ICG-PDR).

Results HVPG increased in Group 1 from 3.7 to $5.4 \mathrm{mmHg}(P<0.05)$ and decreased in Group 2 (4.8 to $4.3 \mathrm{mmHg}, P=\mathrm{NS}$ ) (Table 1). Liver function as assessed by ICG-PDR decreased in Group 1 by day 1 (18.5 to 15.3, $P<0.05)$ and remained stable in Group $2(25.5$ to $26.8, P=N S)$. Renin, aldosterone, $\mathrm{ADH}$, adrenaline, noradrenaline and dopamine increased significantly $(P<0.05)$ in Group 1 during operation. Group 2 showed a significant rise only in $\mathrm{ADH}$ and dopamine. Acute kidney injury occurred in five of 13 patients in Group $1(P<0.05)$.

Table 1 (abstract P404)

\begin{tabular}{ccc}
\hline & Group 1 & Group 2 \\
\hline Renin & 36.4 & 7.9 \\
PostOP & $189.7^{*}$ & 30.5 \\
Day 1 & 258 & 31.3 \\
ADH3.49 & 2.59 & \\
PostOP & $16.11^{*}$ & $10.8^{*}$ \\
Day 1 & 10.8 & 2.39 \\
Creatine & 0.8 & 0.83 \\
Day 1 & $1.25^{*}$ & 0.91 \\
\hline
\end{tabular}

* $P<0.05$ compared with preOP value.

Conclusion Depending on resection size liver resection acutely increases portal venous pressure and induces neurohumoral activation resulting in compromised renal function and increased risk of developing AKI.

P405

Value of the preoperative Model for End-stage Liver Disease score in predicting postoperative outcome in living donor liver transplantation recipients

Y Nassar, K Omar, A Battah, A Mwafy

Cairo University, Giza, Egypt

Critical Care 2013, 17(Suppl 2):P405 (doi: 10.1186/cc12343)

Introduction Partial liver grafting predisposes recipients to a unique set of potential technical and anatomic complications that are not prevalent in whole deceased donor grafts. We aimed to assess the preoperative Model for End-stage Liver Disease (MELD) score, postoperative 30-day clinical and laboratory follow-up, and detection of the value of MELD score in predicting 30-day postoperative outcome of LDLT recipients.

Methods A prospective multicenter registry involving 142 patients who underwent LDLT from October 2004 to December 2010. Preoperative 
MELD score was calculated: $(0.378 \times \log$ (bilirubin) $(\mathrm{mg} / \mathrm{dl})+1.120 \times \log$ $($ INR $)+0.957 \times \log$ (creatinine) $(\mathrm{mg} / \mathrm{dl})+0.643) \times 10$ then rounded to the nearest integer. Postoperative daily recording of all the clinical and laboratory data was done for a period of 30 days.

Results The survival rate recorded in our study was $86.62 \%$. The most frequent complications were renal complications (86.6\%), pulmonary complications (73.9\%), neurological complications (14\%), cardiovascular complications (12.6\%), infectious complications (13.3\%), intra-abdominal infections (10.5\%) and immunosuppressant toxicity (7.7\%). MELD score proved to be a good predictor of perioperative outcome, patients with MELD score $(>12)$ was associated with less favorable outcome, accuracy was (80.3\%).

Conclusion MELD score $>12$ was associated with less favorable outcome with an accuracy of $80.3 \%$.

P406

Does just fungal evidence increase the mortality of critically ill

patients with liver cirrhosis? A retrospective analysis

T Lahmer, U Mayr, M Messer, B Saugel, C Schultheiss, R Schmid, W Huber

University Hospital Munich, Germany

Critical Care 2013, 17(Suppl 2):P406 (doi: 10.1186/cc12344)

Introduction Patients with liver cirrhosis (LC) are susceptible to a variety of complications. Especially, infection disorders can reduce their life expectancy markedly. The aim of this retrospective analysis was to detect the incidence and prognosis in patients with LC and fungal evidence (FE).

Methods A 3-year retrospective analysis of ICU patients with LC. Patients with LC with/without FE were compared with a matched control group based on comparable age and APACHE II score. Specimens were collected based on clinical and/or laboratory (infection signs) reasons and were analyzed by microbiological standard methods.

Results A total of 101 patients with LC were enrolled in this analysis. Basic data for both groups: Child-Pugh Score: $4^{*} A, 18^{*} B, 79^{*} \mathrm{C}$; reason for ICU admission: $28^{*}$ hepatorenal syndrome, 18*SBP, $20^{*} \mathrm{Gl}$ bleeding, $19^{*}$ sepsis, $16^{*}$ pneumonia; etiology of LC: $83^{*}$ alcoholic, $8^{*} \mathrm{NASH}, 8^{*}$ viral (hepatitis B/C), 2*PSC. Fifty-five (54\%) patients with LC had FE (mean age: $57.6 \pm 14$ years, sex M/F: 40/15, APACHE II score $27 \pm 2$ ). Compared with the 46 patients with LC but without FE (mean age: $59.2 \pm 12$ years, sex M/F: $35 / 11$, APACHE II score $23 \pm 4$ ), these patients have a significantly higher APACHE II score $(27 \pm 2$ vs. $23 \pm 4 ; P<0.001)$ and significantly higher mortality $(43 / 55$ (78\%) vs. $15 / 46(33 \%) ; P<0.001)$. Multivariate analysis showed that $\mathrm{FE}$ is associated with a higher mortality $(P<0.001)$ but is not associated with age, sex or CHILD score. Seventeen (31\%) of the 55 patients in the control group had FE (screening patients $n=147$, mean age $65.8 \pm 12$ years, sex M/F: 35/20, APACHE II score $26.5 \pm 3$, reason for ICU admission: 19* pneumonia, $16^{*}$ sepsis, $9 *$ intracerebral haemorrhage, $7^{*}$ acute abdomen, $4^{*}$ lymphatic disorder). The mortality rate was significantly lower $(27 / 55(49 \%)$ vs. $43 / 55(78 \%) ; P=0.003)$ as compared with patients with LC and FE. The main localisation of fungal was in both groups the lung (46 vs. 14), followed by urine (18 vs. 2), blood culture (6 vs. 1 ) and ascites (4 vs. 0). Predominantly candida species were detected (especially C. albicans/glabrata), five patients in the LC group had aspergillus fumigatus. In LC patients, FE could be detected in many cases in several compartments.

Conclusion ICU patients with LC have a high incidence of FE. The mortality rate is therefore significantly higher as compared with patients with LC without FE and also as compared with a control group without LC. Therefore early antimycotic treatment should be considered.

\section{P407}

Predictors of persistent organ failure in ICU patients with severe acute pancreatitis

I Aleksandrova, M Ilynskiy, S Rei, V Kiselev, G Berdnikov, L Marchenkova Hospital Research Institute for Emergency Medicine N.V. Sklifosovsky, Moscow, Russia

Critical Care 2013, 17(Suppl 2):P407 (doi: 10.1186/cc12345)

Introduction Severe acute pancreatitis (SAP) requiring admission to an ICU is associated with high mortality (hospital mortality reached 42\%) and long lengths of stay [1]. Survival among patients with predicted SAP at admission has been shown to correlate with the duration of organ failure (OF) [2]. The systemic determinant of severity in a new classification of acute pancreatitis (AP) is also based on identification of patients with transient or persistent OF [3].

Methods The aim of the study was to retrospectively determine the predictors of early persistent OF in ICU patients with SAP. The analysis involved 152 patients. The median time interval between the onset of AP and admission was $24(9 ; 48)$ hours. The patients were divided into two groups: the first group $(n=46)$ had transient OF and the second group $(n=106)$ had persistent OF. The ability of the APACHE II score, total SOFA score and number of organ/system failure to discriminate transient from persistent OF was explored with receiver operating characteristic (ROC) curves.

Results Hospital mortality was significantly higher in the second group as compared with the first group ( $45 \%$ vs. $7 \%, P=0.000$ ); while infectious complications were $39 \%$ versus $11 \%(P=0.001)$ and median lengths of ICU stay were $8(5 ; 18)$ days for the second group and $6(4 ; 7)$ days for the first group $(P=0.001)$. Optimum cutoff levels (by ROC curve analysis) were APACHE II score $\geq 12$ (sensitivity $0.790 ; 1$ - specificity 0.119 ), total SOFA score $\geq 4$ (sensitivity $0.829 ; 1$ - specificity 0.190 ), and failure $\geq 2$ organs/systems (sensitivity $0.819 ; 1-$ specificity 0.214 ). See Table 1.

Table 1 (abstract P407)

\begin{tabular}{lccc}
\hline Variable & AUC & Standard error & $95 \% \mathrm{Cl}$ \\
\hline APACHE II score & 0.934 & 0.023 & 0.889 to 0.980 \\
SOFA score & 0.908 & 0.026 & 0.857 to 0.960 \\
Number OF & 0.901 & 0.026 & 0.849 to 0.952
\end{tabular}

AUC, areas under the curves.

Conclusion Persistent OF in ICU patients with SAP is associated with the highest risk of hospital mortality. Early identification of patients with a high likelihood of persistent OF (APACHE II score $\geq 12$, total SOFA score $\geq 4$, failure $\geq 2$ organs/systems) is an important goal in determining optimal management of ICU patients with SAP.

References

1. Harrison DA, et al:: Crit Care 2007, 11(Suppl 1):S1

2. Johnson CD, et al: Gut 2004, 53:1340-1344.

3. Dellinger EP, et al:: Ann Surg 2012. [Epub ahead of print]

P408

Increased plasma levels of thrombopoietin in patients with severe acute pancreatitis

L Pigozzi', O Bosco', B Vizio², M Loiacono', M Lucchiari', G Mengozzi', C Moiraghi', G Montrucchio², E Lupia'

'Città della Salute e della Scienza Hospital, Torino, Italy; ${ }^{2}$ University of Torino, Italy

Critical Care 2013, 17(Suppl 2):P408 (doi: 10.1186/cc12346)

Introduction The aim of this study was to evaluate the accuracy of thrombopoietin (TPO) plasma levels as a biomarker of clinical severity in patients with acute pancreatitis (AP). TPO is a humoral growth factor that stimulates megakaryocyte proliferation and differentiation [1]. Furthermore, it favors platelet aggregation and polymorphonuclear leukocyte activation [2]. Elevated plasmatic concentrations of TPO have been shown in patients with critical diseases, including ACS, burn injury and sepsis [2]. In particular, clinical severity is the major determinant of elevated TPO levels in patients with sepsis [3]. AP is a relatively common disease whose diagnosis and treatment are often difficult, especially in the clinical setting of the emergency department (ED). About $20 \%$ of patients with AP develop a severe form of the disease. In order to early identify those patients affected by severe AP, several biomarkers have been studied. No data regarding TPO plasma levels in patients with AP are currently available.

Methods We enrolled patients with AP at the moment of the first clinical evaluation in the ED. AP patients were classified as having mild or severe forms of AP on the basis of the APACHE II score $(\geq 8)$. TPO concentrations were determined by ELISA. 


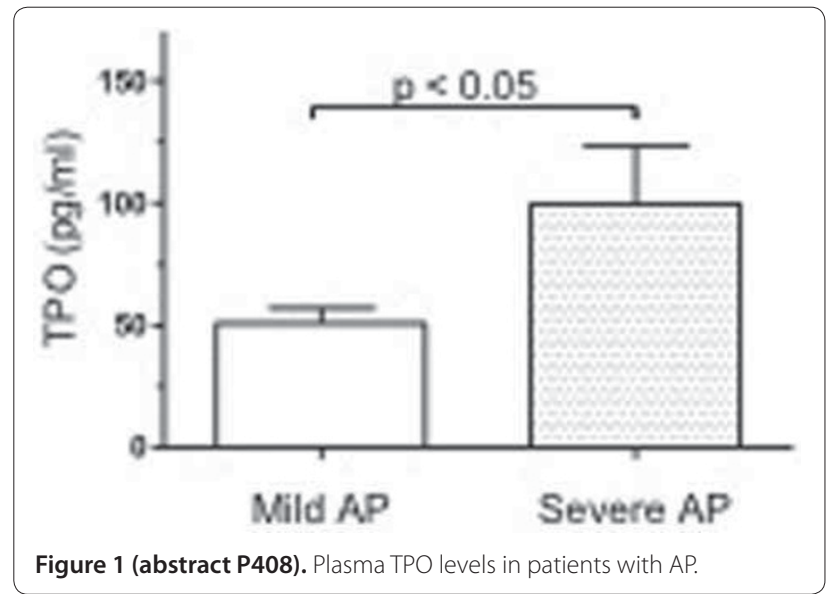

Results We studied 41 patients with AP (17 severe and 24 mild pancreatitis). No differences for gender and age were detected between patients with mild and severe disease. TPO plasma levels were significantly higher in patients with severe AP $(99.33 \pm 23.68 \mathrm{pg} /$ $\mathrm{ml}$ ) than in those with mild AP $(50.81 \pm 6.73 \mathrm{pg} / \mathrm{ml})$ (Figure 1). The ROC curve led us to calculate a cutoff value of $51.55 \mathrm{pg} / \mathrm{ml}$. This value identified correctly 12 out of the 17 patients affected by severe AP. Conclusion TPO may be proposed as biomarker of clinical severity in patients with AP at the time of first evaluation in the ED. Further studies, involving larger numbers of patients, are needed in order to validate these preliminary data.

References

1. Kaushansky K: J Thromb Haemost 2003, 1:1587-1592.

2. Lupia E, et al:: Mediators Inflamm 2012, in press.

3. Zakynthinos SG, et al:: Crit Care Med 2004, 32:1004-1010.

\section{P409}

Severe renal dysfunction measured by pRIFLE in children is an independent factor associated with death and not predicted by PIM2

J Piva', P Lago ${ }^{2}$, G Pedro 3 , F Cabral ${ }^{3}$

'UFRGS, Porto Alegre, Brazil; ${ }^{2}$ Hospital de Clinicas de Porto Alegre, Brazil;

${ }^{3}$ PUCRS, Porto Alegre, Brazil

Critical Care 2013, 17(Suppl 2):P409 (doi: 10.1186/cc12347)

Introduction The objective was to evaluate the effect of the renal dysfunction measured by pRIFLE on clinical outcome in children admitted to the pediatric intensive care unit (PICU).

Methods A retrospective cohort involving all children admitted between August 2009 and July 2010 to a Brazilian PICU located in a university-affiliated hospital. Patients were classified according to the pRIFLE at admission as well the maximum pRIFLE during the PICU stay. The endpoints were: length of stay (LOS) in the PICU, LOS on mechanical ventilation, mortality rate quoted by predicted mortality of the Pediatric Index of Mortality (PIM2).

Results In total, 375 children were included in the study. A total 169 (45\%) of them presented renal dysfunction according to the pRIFLE. Renal injury and renal failure were associated with higher LOS in the $\mathrm{PICU}$ and length of mechanical ventilation. The observed and predicted mortality by PIM2 did not show differences. However, the more severe renal presentation (Injury and Failure) was associated with higher mortality rate and was not predicted by PIM2 ( $8 \%$ vs. $11 \%, P<0.01)$.

Conclusion Renal dysfunction measured by pRIFLE is a very common finding (45\%) among pediatric patients admitted to the PICU. The more severe renal affection is associated with higher mortality, length of MV and length of PICU stay.

\section{References}

1. Picca S, Picardo S: Acute kidney injury in an infant after cardiopulmonary bypass. Semin Nephrol 2008, 28:470-476.

2. Hassinger $A B$, Becker CL: Predictive power of serum cistatin C. Pediatr Crit Care Med 2012, 13:435-440
P410

Thorough evaluation for the new acute kidney injury criteria by Kidney Disease Improving Global Outcomes

J Izawa, S Uchino, T Fujii, T Arii, T Fukushima, S Kawano

Jikei University School of Medicine, Tokyo, Japan

Critical Care 2013, 17(Suppl 2):P410 (doi: 10.1186/cc12348)

Introduction Two previous classifications of acute kidney injury (AKI) that are known as RIFLE criteria and AKIN criteria have shown that AKI is associated with increased morbidity and mortality. Differences in predicting ability for prognosis, however, have been reported. In 2012, Kidney Disease Improving Global Outcomes (KDIGO) created the new AKI criteria, combining RIFLE and AKIN criteria. However, such a combination might cause inconsistency among each definition in the criteria. We have investigated all of the definitions in the new KDIGO criteria in detail.

Methods This is a retrospective historical cohort study including adult patients admitted to the ICU (Jikei University, Tokyo, Japan) between January 2010 and October 2011. Patients undergoing chronic dialysis were excluded. KDIGO criteria were applied to all patients to diagnose AKI. Hospital mortality of patients with AKI diagnosed by the 10 definitions in the criteria was compared.

Results A total of 2,399 patients were evaluated. AKI occurred in $26.6 \%$ with standard definition of KDIGO; $18.0 \%$ with creatinine criteria alone; $15.5 \%$ with urine output alone. By multivariable analysis, each AKI stage was associated with hospital mortality: $5.6 \%$, odds ratio 2.95 , for Stage $1 ; 10.1 \%$, odds ratio 5.52 , for Stage $2 ; 30.2 \%$, odds ratio 21.30 , for Stage 3. Crude hospital mortality stratified by the 10 definitions showed increasing trends with stage progression. Mortality of the three definitions in Stage 1 was from $4.2 \%$ to $8.8 \%$. Stage 2 had two definitions and their mortality was $12.1 \%$ and $12.9 \%$. Stage 3 had five definitions and their mortality was from $20.5 \%$ to $55.6 \%$.

Conclusion AKI defined by the new KDIGO criteria was associated with increased hospital mortality. In addition, definitions in the KDIGO criteria seem to be appropriate because of clear relations between mortality and stage progression.

P411

Urinary neutrophil gelatinase-associated lipocalin as an early predictor of acute kidney injury in cardiac surgery patients

T García Rodríguez San Miguel

Hospital Santa Creu i Sant Pau, Barcelona, Spain

Critical Care 2013, 17(Suppl 2):P411 (doi: 10.1186/cc12349)

Introduction To evaluate whether urinary neutrophil gelatinaseassociated lipocalin (UNGAL) detects acute kidney injury (AKI) earlier than the estimated glomerular filtration rate (eGFR) in cardiac surgery patients.

Methods Two-hundred and seventy-four adult patients undergoing cardiac surgery were consecutively included from February to December 2011. Exclusion criteria were absence of diuresis due to end-stage renal disease or chronic renal failure and a previous cardiac catheterism with i.v. contrast use the week before surgery. Four serial blood and urine samples immediately before (PRE) and after (POST) surgery, and 1 day (1d) and 2 days (2d) after surgery were obtained. UNGAL was measured in an Architect 6200 (Abbott Diagnostics). AKIN criteria were used to diagnose AKI. The study was approved by the local ethics committee and all patients gave informed consent. Delta UNGAL was defined as the difference between the PRE and the POSTs concentrations.

Results One-hundred and eighty-one patients (66.1\%) were men; mean age was $68.2 \pm 12.2$ years. Valve replacement was performed in 123 , coronary artery bypass graft (CABG) in 81 , valve surgery + CABG in 48 , cardiac transplant in five, aorta aneurism surgery in nine, and other procedures in eight patients. ICU and hospital stays were $6.7 \pm 8.1$ and $15.7 \pm 13.9$ days, respectively. Renal replacement therapy (RRT) was required in 16 patients (5.8\%) within 48 hours of ICU stay and in 28 patients $(10.2 \%)$ within 4 weeks. Mortality at 28 days was $2.9 \%$. Eighty-six patients (31.4\%) were diagnosed with AKI within 48 hours of surgery. Area under the ROC curve of POST UNGAL for AKI diagnosis was $0.72(0.66$ to 0.79$)(P<0.0001)$ at an optimal cutoff value of $180 \mu \mathrm{g} / \mathrm{l}$, 
with $78.7 \%$ specificity, $64 \%$ sensitivity and $74.1 \%$ accuracy. UNGAL advanced diagnosis of AKI in 44 patients (51.2\%), whereas diagnosis was achieved at the same time as AKI criteria in 11 patients; AKI criteria outperformed UNGAL in only $36 \%$ of cases. Accordingly, UNGAL was useful to diagnose postoperative AKI in $63.9 \%$ of cases. Median delta uNGAL was 12.5 (from -1.9 to 71.1 ) and 154.5 (from 16.6 to 484.5 ) $\mu \mathrm{g} / \mathrm{l}$ in non-AKI and AKI patients, respectively $(P<0.0001)$ and its area under the ROC curve for AKI prediction was $0.70(0.63$ to 0.77$)(P<0.0001)$.

Conclusion Compared with AKIN criteria, a urinary NGAL concentration $>180 \mu \mathrm{g} / \mathrm{l}$ anticipates AKI diagnosis in more than $50 \%$ of cardiac surgery patients in the first 24 to 48 hours after intervention.

\section{P412}

Atrial natriuretic peptide attenuates metabolic acidosis and inflammation of the kidney, lung and heart in a rat model of renal ischemia-reperfusion injury

M Khin Hnin Si, C Mitaka, M Tulafu, S Abe, S Ikeda

Tokyo Medical and Dental University Graduate School, Tokyo, Japan

Critical Care 2013, 17(Suppl 2):P412 (doi: 10.1186/cc12350)

Introduction Renal ischemia-reperfusion injury (IRI) is a common cause of acute kidney injury and occurs in various clinical conditions including shock and cardiovascular surgery. Renal IRI releases proinflammatory cytokines within the kidney. Atrial natriuretic peptide (ANP) has natriuretic, diuretic and anti-inflammatory effects [1] and plays an important role of regulating blood pressure and volume homeostasis. The hypothesis was that renal IRI induces inflammation not only in the kidney but also in remote organs such as the lung and heart and ANP attenuates renal injury and inflammation in the kidney, lung and heart.

Methods Male Sprague-Dawley rats were anesthetized with pentobarbital. Tracheostomy was performed and rats were ventilated at VT $10 \mathrm{ml} / \mathrm{kg}$ with $5 \mathrm{cmH}_{2} \mathrm{O}$ PEEP. The right carotid artery was catheterized for blood sampling and continuous blood pressure measurements. The right femoral vein was catheterized for infusion of saline or ANP. Rats were divided into three groups; IRI group $(n=10)$, left renal pedicle was clamped for 30 minutes; IRI+ANP group $(n=10)$, left renal pedicle was clamped for 30 minutes, ANP ( $0.2 \mu \mathrm{g} / \mathrm{kg} /$ minute, for 3 hours 25 minutes) was started 5 minutes after clamp; and Sham group $(n=6)$, the shamoperated rats. Hemodynamics, arterial blood gas, and plasma lactate levels were measured at baseline and at 1 hour, 2 hours and 3 hours after declamp. The mRNA expression of IL-6 in the kidney, lung, and heart were measured. The kidney, lung and heart were immunostained to examine the localization of IL- 6 and NF-KB and assigned an expression score. The wet/dry ratio of the lung was also measured.

Results Renal IRI induced metabolic acidosis, pulmonary edema, mRNA expression of IL- 6 in the kidney, lung and heart. Renal IRI increased immunohistochemical localization of IL- 6 in the proximal convoluted tubule of the left kidney and NF-KB in the bronchial epithelial cells of the lung. ANP attenuated metabolic acidosis, pulmonary edema and expression of IL-6 mRNA in the kidney, heart, and lung. ANP decreased immunohistochemical localization of IL-6 in the left kidney and NF-KB in the lung.

Conclusion These findings suggested that inflammation within the kidney after renal IRI was extended into the lung and heart. ANP attenuated metabolic acidosis and inflammation in the kidney, lung and heart in a rat model of renal IRI. ANP may attenuate organ crosstalk between the kidney, lung and heart.

Reference

1. Vollmar AM: The role of atrial natriuretic peptide in the immune system. Peptide 2005, 26:1086-1094.

P413

Perioperative sodium bicarbonate to prevent acute kidney injury after cardiac surgery: a multicenter double-blind randomized controlled trial

A Haase-Fielitz', M Haase', M Plass², P Murray³, M Bailey4, R Bellomo5, S Bagshaw ${ }^{6}$

'Otto von-Guericke University, Magdeburg, Germany: 'German Heart Center, Berlin, Germany; ${ }^{3}$ University College, Dublin, Ireland; ${ }^{4}$ ANZIC, Melbourne, Australia; ${ }^{5}$ Austin Hospital, Melbourne, Australia; ${ }^{6}$ University of Alberta, Canada Critical Care 2013, 17(Suppl 2):P413 (doi: 10.1186/cc12351)

Introduction Evidence suggests a nephroprotective effect of urinary alkalinization in patients at risk of acute kidney injury (AKI).

Methods In a multicenter, double-blind, RCT we enrolled 350 adult cardiac surgery patients. At induction of anesthesia, patients received either 24 hours of intravenous infusion of sodium bicarbonate $(5.1 \mathrm{mmol} / \mathrm{kg})$ or sodium chloride $(5.1 \mathrm{mmol} / \mathrm{kg})$. The primary endpoint was the proportion of patients developing AKI.

Results Sodium bicarbonate increased urinary pH (from 6.0 to 7.5, $P<0.001)$. More patients in the bicarbonate group (83/174 (47.7\%)) developed AKI compared with control (64/176 (36.4\%), OR = 1.60 ( $95 \% \mathrm{Cl}, 1.04$ to 2.45 ); unadjusted $P=0.032$ ). A greater postoperative

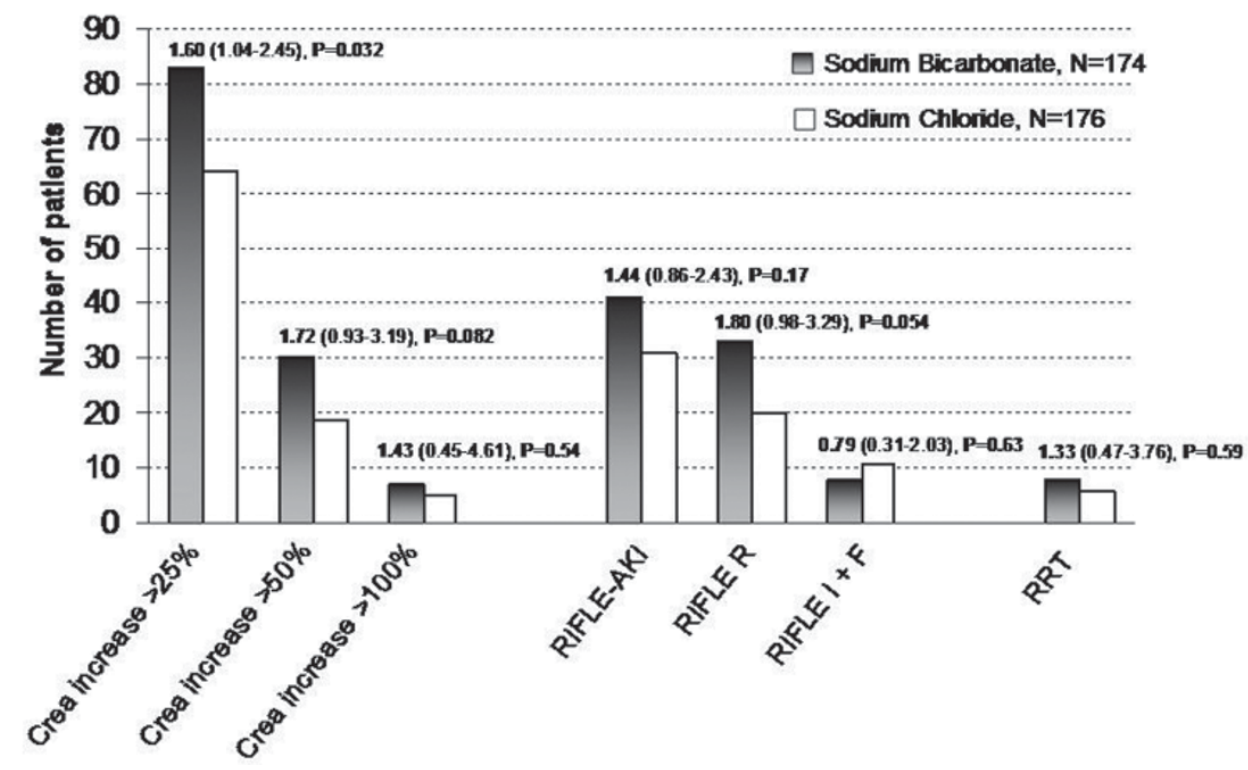

Figure 1 (abstract P413). 
increase in urinary NGAL in patients receiving bicarbonate infusion was observed compared with control $(P=0.011)$. The incidence of postoperative RRT was similar but hospital mortality was increased in patients treated with bicarbonate compared with chloride $(11 / 174$ (6.3\%) vs. $3 / 176(1.7 \%)$, OR 3.89 (1.07 to 14.2$), P=0.031$ ). See Figure 1. Conclusion On this basis of our findings we do not recommend the use of perioperative infusions of sodium bicarbonate to reduce the incidence or severity of AKI in this patient group.

P414

Tubular damage biomarkers linked to inflammation or iron metabolism predict acute kidney injury

A Haase-Fielitz', S Westphal', R Bellomo², P Devarajan³, M Westerman ${ }^{4}$, P Mertens', M Haase

'Otto von-Guericke University, Magdeburg, Germany; ${ }^{2}$ Austin Hospital,

Melbourne, Australia; ${ }^{3}$ Cincinnati Children's Hospital, Cincinnati, OH, USA;

${ }^{4}$ Intrinsic LifeSciences, La Jolla, CA, USA

Critical Care 2013, 17(Suppl 2):P414 (doi: 10.1186/cc12352)

Introduction Here, we compare the value of acute tubular damage markers measured early in the course after cardiac surgery for prediction of postoperative AKI compared with conventional markers used in clinical routine for risk assessment of acute renal function loss. Methods One hundred adult patients undergoing cardiac surgery in the control arm of a RCT (NCT00672334) were analyzed. We quantified the following biomarkers in urine: NGAL, hepcidin, midkine, IL-6, $a_{-}-$ microglobulin; in plasma: NGAL, hepcidin, haptoglobin, CK, CKMB, CRP, leukocytes, lactate, urea and creatinine.

Results Preoperatively, no biomarker predicted AKI. At ICU arrival, four urinary tubular damage markers showed good discriminatory ability (AUC $\geq 0.80$ ) for subsequent development or absence of AKI (Figure 1). An excellent predictive value was found for UNGAL/uhepcidin ratio (AUC 0.90, Figure 1). This ratio combines an AKI prediction marker (NGAL) and a marker of protection from AKI (hepcidin), potentiating their individual discriminatory values. Contrarily, at ICU admission, none of the plasma biomarkers was a good early AKI predictor with AUC-ROC $\geq 0.80$.

Conclusion Several urinary markers of acute tubular damage predict AKI after cardiac surgery and the biologically plausible combination of NGAL and hepcidin provides excellent AKI prediction.

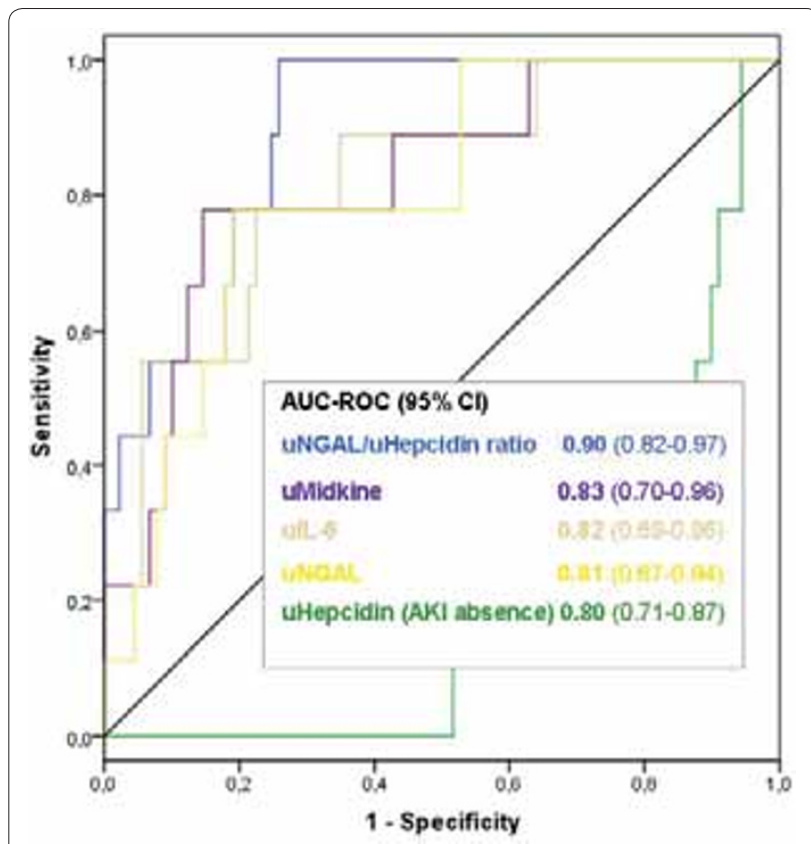

Figure 1 (abstract P414)
P415

Renal response and acid-base balance alterations during furosemide administration

L Zazzeron, D Ottolina, E Scotti, M Ferrari, M Stanziano, C Rovati, F Zadek, C Marenghi, L Gattinoni, P Caironi

Fondazione IRCCS Ca'Granda - Ospedale Maggiore Policlinico, Milan, Italy

Critical Care 2013, 17(Suppl 2):P415 (doi: 10.1186/cc12353)

Introduction Furosemide is one of the most employed diuretics in the ICU for its ability to induce negative water balance. However, one common side effect is metabolic alkalosis [1]. We aimed to describe the time course of urinary excretion and changes in plasmatic acid-base balance in response to the administration of furosemide.

Methods We connected the urinary catheter of 39 ICU patients to a quasi-continuous urine analyzer (Kidney INstant monitorinG ${ }^{\circledast}$ ), allowing measurement of $\mathrm{pH}(\mathrm{pHU})$, sodium, chloride, potassium and ammonium concentrations $(\mathrm{Na}+\mathrm{U}, \mathrm{Cl}-\mathrm{U}, \mathrm{K}+\mathrm{U}, \mathrm{NH} 4+\mathrm{U})$ every 10 minutes. The study period lasted 3 hours after a single intravenous bolus of furosemide (time 0 ). In 13 patients receiving two or more administrations over a longer period (46 (26 to 49 ) hours), according to clinical needs, we reviewed data on fluid therapy, hemodynamics and acid-base balance from the beginning to the end of the observation. Results Ten minutes after furosemide administration, $\mathrm{Na}+\mathrm{U}$ and $\mathrm{Cl}-\mathrm{U}$ rose from $65 \pm 6$ to $140 \pm 5$ and from $109 \pm 6$ to $150 \pm 5 \mathrm{mEq} / \mathrm{l}$ respectively, while $\mathrm{K}+\mathrm{U}$ fell from $60 \pm 5$ to $39 \pm 4 \mathrm{mEq} / \mathrm{l}(P<0.001$ for all electrolytes vs. time 0 ) with a consequent increase in urinary anion gap $(A G U=\mathrm{Na}+\mathrm{U}+\mathrm{Cl}-\mathrm{U}-\mathrm{K}+\mathrm{U})$. Urinary output increased from $10(5$ to 19 ) to 53 (29 to 71$) \mathrm{ml} / 10$ minutes $(P<0.05)$. After the first hour $\mathrm{Cl}-\mathrm{U}$ remained higher than $\mathrm{Na}+\mathrm{U}$, which progressively decreased, leading to a reduction in $\mathrm{AGU}$ and $\mathrm{pHU}$ over time. In parallel, a progressive increment in $\mathrm{NH} 4+\mathrm{U}$ was observed. In patients receiving more than one administration we observed an increase in arterial base excess $(1.8 \pm 0.8$ vs. $5.0 \pm 0.6 \mathrm{mmol} / \mathrm{l}, P<0.001)$ and plasmatic strong ion difference (SIDpl) (31 (30 to 33 ) vs. 35 (34 to 36 ) $\mathrm{mEq} / \mathrm{l}, P=0.01$ ) during the study period. These changes were due to a decrease in plasmatic $\mathrm{Cl}^{-}$concentration ( $\left.109.0 \pm 1.1 \mathrm{vs.} 106.6 \pm 0.9 \mathrm{mEq} / \mathrm{l}, P=0.009\right)$. Plasmatic sodium and potassium concentrations did not change. In these patients, considering the total amount of administered fluids and urine, a negative water and chloride balance was observed $(-460 \pm 403 \mathrm{ml}$ and $-48 \pm 48 \mathrm{mEq}$, respectively).

Conclusion Furosemide acts immediately after administration, causing a rise in urinary output, $\mathrm{Na}+\mathrm{U}$ and $\mathrm{Cl}-\mathrm{U}$ concentrations. Loop-diureticinduced metabolic alkalosis may be due to an increased urinary chloride loss and the associated increase in SIDpl.

Reference

1. Wilcox CS: Metabolic and adverse effects of diuretics. Semin Nephrol 1999, 19:557-568.

P416

Red cell distribution width is not a predictor of mortality in acute kidney injury

P Purvis ${ }^{1}$, J Kinsella', R Docking ${ }^{2}$

'University of Glasgow, UK; ${ }^{2}$ Glasgow Royal Infirmary, Glasgow, UK

Critical Care 2013, 17(Suppl 2):P416 (doi: 10.1186/cc12354)

Introduction Given the significant morbidity and mortality associated with acute kidney injury (AKI), there is a need to find factors to help aid decision-making regarding levels of therapeutic support. As a prognostic biomarker, the red cell distribution width (RDW) has attracted interest in the setting of critical care when added to existing scoring systems [1]. By examining RDW in a previously studied AKI cohort, we aimed to evaluate the utility of this routine blood test.

Methods A cohort of 209 mixed critical care patients who received renal replacement therapy for AKI had their demographic and biochemical data retrieved from electronic databases. Outcomes were gathered for ICU and hospital mortality. Incomplete datasets were discarded, leading to 153 complete sets. RDW data were taken from the first sample after admission to the ICU, as were all other biochemical values apart from pre-RRT creatinine and potassium. Overall cohort characteristics were gathered, and two groups were created: those with a RDW value within normal range $(\leq 14.5 \%)$ and those with a greater than normal value 
(>14.5\%). We then further subgrouped RDW to assess the correlation between rising levels and ICU mortality.

Results A total $77.1 \%$ of our cohort had a RDW greater than the normal laboratory range at time of ICU admission. Key baseline characteristics (age, APACHE II score, length of stay, ICU mortality) did not differ significantly between patients with normal and abnormal RDW. When subgroup analysis was performed, no statistically significant correlation between rising RDW and ICU mortality was found (Spearman correlation $=0.426, P=0.233$ ).

Conclusion In this cohort of critically ill patients with AKI, RDW was not found to be a predictor of mortality. Our results contradict those of recent studies [1,2]. However, both groups of RDW patients in our study suffered a higher ICU mortality than in other studies. To further explain these findings, we intend to perform multivariate logistic regression analysis and assess the effect of social deprivation on RDW.

\section{References}

1. Hunziker et al:: Red cell distribution width improves the simplified acute physiology score for risk prediction in unselected critically ill patients. Crit Care 2012, 16:R89.

2. Oh et al: Red blood cell distribution width is an independent predictor of mortality in acute kidney injury patients treated with continuous renal replacement therapy. Nephrol Dial Transplant 2012, 27:589-594.

P417

Value of Tc-99m DMSA renal scan in assessing the prognosis and outcome of acute renal failure due to sepsis

S Fawzi, K Mashour, A Amin, H Gamal

Cairo University, Cairo - Manial, Egypt

Critical Care 2013, 17(Suppl 2):P417 (doi: 10.1186/cc12355)

Introduction Acute renal failure (ARF) is a common complication in patients admitted to the ICU. Sepsis is also a well-known risk factor for the development of ARF. The combination of ARF and severe sepsis was reported to carry a mortality up to $70 \%$ whereas the mortality of ARF alone is 40 to $45 \%$. The aim of the study is to evaluate the role of renal perfusion scanning in detecting the prognosis and outcome of patients with acute renal failure due to sepsis.

Methods Forty patients with acute renal failure due to sepsis, aged between 15 and 74 years, were enrolled in the study. They were monitored for their ICU prognosis and outcome after doing renal perfusion scanning. All patients were subjected to routine ICU and laboratory investigations including APACHE II and SOFA score.

Results Thirty patients had normal renal scan and 10 patients had abnormal renal scan. The mortality percentage was higher among abnormal renal scan cases (three out of 10,30\%) compared with cases with normal renal scan (seven out of $30,23.3 \%$ ) with nonsignificant $P$ value: 0.6 . The median length of stay/day in ICU was longer among nonsurvivors than survivors $15.5 \pm 10,11.5 \pm 8, P$ value: 0.058 (approaching significance). APACHE II score was higher in nonsurvivors than survivors $23.9 \pm 3.2,19.6 \pm 4.2, P$ value: 0.0001 . The percentage of mortality among cases that needed mechanical ventilation was higher (nine out of $16,56.3 \%$ ) compared with mortality cases that did not need mechanical ventilation (one out of $24,4.2 \%$ with $P$ value: 0.0001 ). Conclusion ARF may exert an independent adverse effect on outcome in septic and septic shock patients. It is also a risk factor for mortality. Tc-99m DMSA scanning is useful for detecting renal dysfunction and help to predict the outcome and prognosis.

Reference

1. Ympa YP, et al:: Am J Med 2005, 118:827-832.

P418

Estimated glomerular filtration rate based on hospital discharge creatinine may significantly overestimate renal function and underestimate chronic kidney disease in survivors of critical illness I Kolic, J Purdell-Lewis, CJ Kirwan, JR Prowle

Royal London Hospital, Barts Health NHS Trust, London, UK Critical Care 2013, 17(Suppl 2):P418 (doi: 10.1186/cc12356)

Introduction Acute kidney injury (AKI) complicates over 50\% of ICU admissions. Episodes of AKI are a major risk factor for development or progression of chronic kidney disease (CKD); however, methods of estimated glomerular filtration rate (eGFR) may be poorly calibrated

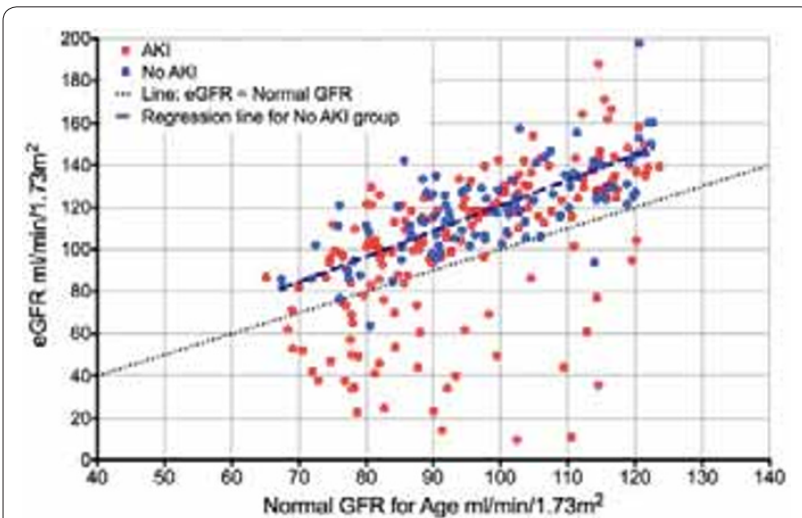

Figure 1 (abstract P418). eGFR at hospital discharge versus expected normal GFR for age in 280 ICU survivors.

to survivors of critical illness who may have reduced muscle mass. We hypothesized that eGFR may underestimate rates and severity of CKD in ICU survivors.

Methods A retrospective observational study of renal function in all patients admitted to a London teaching hospital ICU for $\geq 5$ days and surviving to hospital discharge in 2011. We excluded cases with current or new diagnosis of end-stage renal disease or renal transplant. We assessed AKI in ICU by KDIGO 1 criteria and hospital discharge eGFR by the CKD-EPI equation. For comparison we assumed a normal GFR in a healthy individual as $120 \mathrm{ml} /$ minute $/ 1.73 \mathrm{~m}^{2}$ at age 20 decreasing by 0.8 per year over age 20 .

Results We identified 282 patients, 180 of whom had AKI. Median age was 50 and $68 \%$ were male. Median hospital discharge serum creatinine was $57 \mu \mathrm{mol} / \mathrm{l}$ (range 16 to 654), median eGFR was significantly higher than predicted normal GFR for age at 115 versus predicted $95(P<0.001$, median difference 16). In patients who had not had AKI discharge the eGFR was 119 versus normal predicted $98(P<0.001$, median difference 19), suggesting that eGFR could be overestimating true GFR in our population by at least a factor of 1.23 (Figure 1). Applying this correction factor to eGFRs of patients who had recovered from AKI resulted in $44 \%$ more diagnoses of CKD (eGFR <60) at hospital discharge ( $36 \mathrm{vs.} 25)$.

Conclusion eGFR may overestimate renal function in survivors of critical illness confounding identification of CKD in this at-risk population. Prospective studies with measurement of actual GFR are required to assess the burden of CKD in survivors of critical illness.

\section{P419}

Varying models of intra-abdominal hypertension and their effect on renal function in a porcine model

RWise', R Rodseth', I De Laet², L Luis', L Correa-Martin, M Garcia ${ }^{4}$

G Castellanos ${ }^{5}, \mathrm{M} \mathrm{Malbrain}^{2}$

'University of Kwazulu Natal, Durban, South Africa; ${ }^{2}$ ZNA Stuivenberg,

Antwerp, Belgium; ${ }^{3}$ Neuron NPh, S.A., Granada, Spain; ${ }^{4}$ Jesús Usón Minimally

Invasive Surgery Centre, Cacères, Spain; ${ }^{5}$ Virgen de la Arrixaca' University

Hospital, Murcia, Spain

Critical Care 2013, 17(Suppl 2):P419 (doi: 10.1186/cc12357)

Introduction Intra-abdominal hypertension (IAH) is an independent predictor of renal impairment and mortality [1]. Organ dysfunction caused by the pressure effect of IAH is well understood, but how this is modified in the presence of bowel obstruction is unclear. The aim of this study was to determine how different IAH models cause renal dysfunction in a pig model.

Methods Twenty-four pigs were divided into three groups; a control group $(n=5)$, a pneumoperitoneum (Pn) $(n=10)$, and an intestinal occlusion (Oc) model $(n=10)$. IAP was maintained for 3 hours at $20 \mathrm{mmHg}$ during which time creatinine, urea, urine output, potassium, and glomerular filtration pressure (GFP) were measured. Statistical analysis was performed using repeated-measures ANOVA.

Results Over the first 3 hours there was a statistically significant difference between the control group and both IAH models for 
potassium $(P=0.002)$, urea $(P=0.045)$, creatinine $(P=0.012)$, GFP $(P<0.001)$, and urine output $(P=0.003)$. Over the full 5 -hour period there was a statistically significant difference in the potassium measurement between the $\mathrm{Pn}$ and the Oc model $(P=0.01)$. The other parameters did not show a significant difference: urea $(P=0.171)$, creatinine $(P=0.074)$, GFP $(P=0.141)$, and urine output $(P=0.242)$.

Conclusion As expected the IAH models resulted in significantly worse renal function after 3 hours. This early renal dysfunction may be as a result of an early inflammatory process that has been associated with the pathophysiology of acute kidney injury. Potassium was significantly elevated in the Pn group as compared with the Oc group. Early changes in potassium levels with IAH may be a marker of early renal dysfunction and the usefulness of other renal biomarkers, such as NGAL, prompts further investigation.

Reference

1. Malbrain ML, et al: Incidence and prognosis of intraabdominal hypertension in a mixed population of critically ill patients: a multiplecenter epidemiological study. Crit Care Med 2005, 33:315-322.

P420

Renal resistive index can predict response to furosemide in septic

patients with oliguria

I Gornik, A Godan, V Gasparovic

University Hospital Centre Zagreb, Croatia

Critical Care 2013, 17(Suppl 2):P420 (doi: 10.1186/cc12358)

Introduction Oliguria is common in septic patients and is frequently therapeutically addressed with loop diuretics; that is, furosemide. Diuretic treatment in shock and hypovolemia is not rational, but can be tried in oliguric patients with normovolemia or hypervolemia and without hypotension. In such patients it still does not always increase dieresis and can also be harmful. The resistive index is a measure of pulsatile blood flow that reflects the resistance to blood flow caused by the microvascular bed distal to the site of measurement. It can reflect functional status of the tissue distal to the point of measurement. We investigated whether measuring the renal resistive index (RI) could be helpful in determining which patients will respond to furosemide treatment.

Methods We included medical ICU patients with sepsis and oliguria (urine output $<1 \mathrm{ml} / \mathrm{kg} /$ hour) who were prescribed i.v. furosemide. Patients with known chronic renal failure, hypovolemia (CVP $<10 \mathrm{mmHg}$ ) or severe hypotension (MAP $<80 \mathrm{mmHg}$ ) were excluded. Resistive index ( 1 - (end diastolic velocity / maximum systolic velocity) $\times 100$ ) was measured in at least three segmental arteries of both kidneys, the average of all measurements was reported as the result. Repeated assessments were viewed as independent if separated by more than 24 hours. Furosemide was given intravenously in the dose of $40 \mathrm{mg}$ after RI measurement. Positive response to furosemide was defined as doubling of hourly dieresis or achieving urine output $>1.5 \mathrm{ml} / \mathrm{kg} / \mathrm{hour}$ after drug administration.

Results We included 47 patients with a total of 59 measurements. In 28 cases patients had positive response to furosemide. Median RI in responders was 0.67 (range 0.55 to 0.78 ) and in nonresponders 0.79 (range 0.58 to 0.81 ); $P=0.027$. Construction of receiver operating characteristic curve showed $83 \%$ sensitivity and $81 \%$ specificity for the cutoff RI 0.73 . No other measured patient characteristic was found to be predictive of response to diuretic treatment.

Conclusion Our results show that the RI could be used to guide diuretic treatment in nonhypovolemic, nonhypotensive septic patients. Further studies are needed to confirm those preliminary results.

\section{P421}

Urinary chitinase 3-like 1, a novel biomarker for acute kidney injury

in adult cardiac intensive care patients: a pilot study

J De Loor', E Hoste², I Herck², K Francois², L De Crop², C Clauwaert², K Demeyere', E Meyer'

${ }^{1}$ Faculteit diergeneeskunde, Merelbeke, Belgium; '2UZ Gent, Belgium

Critical Care 2013, 17(Suppl 2):P421 (doi: 10.1186/cc12359)

Introduction As a proof of concept, the potential added value of chitinase 3-like 1 (CHI3L1) as a more early and specific diagnostic parameter for acute kidney injury (AKI) was investigated in adult ICU patients that underwent elective cardiac surgery.
Methods A prospective single-center cohort study started in May 2012 in an academic cardiac surgery ICU. Blood and urine were collected preoperatively (baseline), at ICU admission and at several time points until 48 hours after ICU admission. Patients of age $<18$ years were excluded, as well as patients with AKI based on the KDIGO sCr and urine output criteria, patients with chronic kidney disease stage 5 and/or with recent renal transplantation ( $\leq 3$ months before cardiac surgery). $\mathrm{AKI}$ was defined on $\mathrm{KDIGO} \mathrm{sCr}$ criteria only. Baseline $\mathrm{s} C \mathrm{r}$ was assessed as the $\mathrm{s} \mathrm{Cr}$ concentration measured before start of cardiac surgery. Urinary CHI3L1 was normalized to urinary creatinine. For both serum and urine CHI3L1, we measured changes from baseline. Data are expressed as median (interquartile range), and number (\%).

Results Data for 17 patients (nine males, eight females) were generated for interim analysis. Five patients (29.4\%), of which four were females, developed AKI KDIGO class 1. AKI patients were older compared with non-AKI patients (82.0 years (77.5 to 83.5 ) vs. 66.5 (60.3 to 75.8 ); $P=0.002)$. The baseline $\mathrm{s} C r$ did not differ significantly between the AKI (1.12 mg/dl (0.80 to 1.15$)$ ) and non-AKI $(0.90 \mathrm{mg} / \mathrm{dl}$ (0.86 to 1.01$)$; $P=0.383$ ) group. Baseline serum CHI3L1 was highly variable in both AKI and non-AKI patients. Moreover, no trend towards a higher serum $\mathrm{CHI}$ L1 level in AKI versus non-AKI patients was observed either at baseline or during the study follow-up. In contrast, the increase in the urinary CHI3L1 to urinary creatinine ratio from baseline was higher in the AKI versus non-AKI group at time points 4 hours and 6 hours after admission to the ICU ( $2.00 \mathrm{ng} / \mathrm{mg} \mathrm{Cr}$ (1.38 to 3.03 ) vs. 0.26 (0.05 to 0.88 ); $P=0.002$, resp. 2.47 (1.09 to 7.26) vs. $0.23(-0.18$ to 0.74$) ; P=0.006)$ and, more importantly, occurred before the diagnosis of $\mathrm{AKI}$ by $\mathrm{sCr}$ ( $>16$ hours after ICU admission).

Conclusion This interim analysis of an ongoing pilot study, with 300 ICU patients in three cohorts (general ICU, pediatric and adult cardiac surgery ICU), shows promising results for urinary $\mathrm{CHI} 3 \mathrm{~L} 1(\mathrm{ng} / \mathrm{mg} \mathrm{Cr})$ as an early diagnostic biomarker for AKI in adult ICU patients that undergo elective cardiac surgery.

P422

Use, type and duration of cardiopulmonary bypass are related to acute kidney injury occurrence and urinary NGAL concentrations

T García Rodríguez San Miguel

Hospital Santa Creu i Sant Pau, Barcelona, Spain

Critical Care 2013, 17(Suppl 2):P422 (doi: 10.1186/cc12360)

Introduction To analyze whether variables related to cardiopulmonary bypass (CPB) influence acute kidney injury (AKI) occurrence and urinary neutrophil gelatinase-associated lipocalin (UNGAL) in cardiac surgery patients.

Methods A total of 274 adult cardiac surgery patients were consecutively included from February to December 2011. Exclusion criteria were absence of diuresis due to end-stage renal disease or chronic renal failure and cardiac catheterism with i.v. contrast in the week before surgery. $C P B$, when performed, was used as standard $C P B$ (SCPB) or MiniCPB. We obtained four serial blood and urine samples, immediately before (PRE) and after (POST) surgery, and 1 day (1d) and 2 days (2d) after surgery. UNGAL was measured by Architect 6200 (Abbott Diagnostics). AKIN criteria were used to diagnose AKI. The study was approved by the local ethics committee and all patients gave informed consent.

Results One hundred and eighty-one patients (66.1\%) were men; mean age was $68.2 \pm 12.2$ years. ICU and hospital stays were $6.7 \pm 8.1$ and $15.7 \pm 13.9$ days, respectively. Twenty-eight-day mortality was $2.9 \%$. Eighty-six patients (31.4\%) were diagnosed with AKI within 48 hours after surgery. In total, 219 patients required CPB (195 SCPB, 24 MiniCPB) and 55 did not (no-CPB). Seven no-CPB patients (12.7\%) developed AKI and their median UNGAL POST was 330 (42.6 to 489.9$) \mu \mathrm{g} / \mathrm{l}$ compared with $13.6(6.9$ to 38.3$) \mu \mathrm{g} / \mathrm{l}$ in the 48 patients without $A K I(P<0.0001)$. Of the 195 patients undergoing SCPB, 76 (38.9\%) developed AKI and 119 did not; POST uNGAL was 204 (34.8 to 575.7$) \mu \mathrm{g} / \mathrm{l}$ and 44.5 (13.2 to $175.8) \mu \mathrm{g} / \mathrm{l}(P<0.0001)$, respectively. In the 24 patients under MiniCPB, POST uNGAL was 113 (58.8 to 211.8$) \mu \mathrm{g} / \mathrm{l}$ in those (three patients, $12.5 \%$ ) developing AKI and 19.1 (9.2 to 41.8$) \mu \mathrm{g} / \mathrm{l}$ in those without AKI $(P=0.01)$. Aortic clamp time $(r=0.31, P<0.0001)$ and cardiopulmonary bypass time $(r=0.30, P<0.0001)$ correlated with POST UNGAL concentrations. 
Incidence of AKI in patients without CPB or with MiniCPB was identical and significantly lower $(P<0.0001)$ than in SCPB patients.

Conclusion The incidence of AKI was higher in patients undergoing SCPB. Aortic clamp and cardiopulmonary bypass time correlated with the POST UNGAL concentration. The higher values found in patients without AKI undergoing SCPB suggest that a subclinical AKI, only detectable by UNGAL, could exist in this group.

P423

Symmetrical dimethylarginine is a more sensitive biomarker of renal dysfunction than creatinine

JJ Dixon', K Lane', RN Dalton², IA MacPhee', BJ Philips'

'Acute Kidney Injury Research Group, St George's Hospital and University of London, UK; ${ }^{2}$ King's College, University of London, UK

Critical Care 2013, 17(Suppl 2):P423 (doi: 10.1186/cc12361)

Introduction Current definitions of acute kidney injury (AKI) are based upon changes in serum creatinine $(\mathrm{SCr})$ concentration and urine output: both have limitations in patients with AKI. Diagnosis may be delayed if using these criteria alone. We have previously validated a method of measuring the glomerular filtration rate (GFR) intended for use in patients with AKI (a continuous infusion of very low dose iohexol; CIVLDI). In this study we compare the performance of symmetrical dimethylarginine (SDMA) against SCr and CIVILDI. SDMA is the structural isomer of the endogenous nitric oxide inhibitor asymmetrical dimethylarginine. SDMA increases in parallel with $\mathrm{SCr}$. Despite this, no formula exists to estimate GFR from SDMA concentration. Tubular secretion of SCr may be as high as 40\%. SDMA may be a more sensitive biomarker of renal dysfunction than $\mathrm{SCr}$; however, the tubular reabsorption of SDMA is unknown. The aims were to compare the performance of SDMA against SCr and accurately measured GFR, and to derive a formula to estimate GFR from SDMA concentration.

Methods Seventeen volunteers had GFR measured twice via measuring the clearance of a $5 \mathrm{ml}$ i.v. bolus of iohexol, or CIVLDI. Serum and urine iohexol, $\mathrm{Cr}$ and SDMA were measured by high-performance liquid chromatography/tandem mass spectrometry (HPLC-ms/ms) at 10 time points. Fractional excretion of SDMA and $\mathrm{SCr}$ were calculated using iohexol as the denominator. SDMA was plotted against measured GFR, and estimated GFR equations were derived from linear, quadratic and third-order polynomial plots.

Results Mean GFR measured by single injection was $78.7 \pm 28.5 \mathrm{ml} /$ minute $/ 1.73 \mathrm{~m}^{2}$, and $78.9 \pm 28.6 \mathrm{ml} /$ minute $/ 1.73 \mathrm{~m}^{2}$ when measured by CIVLDI $(P=0.82)$. Mean SDMA concentration was similar on both occasions ( $641 \pm 38$ vs. $623 \pm 22 \mathrm{nmol} / / ; P=0.68$ ). Tubular reabsorption of SDMA was lower in subjects with GFR $<60 \mathrm{ml} /$ minute $/ 1.73 \mathrm{~m}^{2}$ $\left(12 \pm 8 \%\right.$ vs. $18 \pm 8 \%$ in subjects with GFR $>60 \mathrm{ml} /$ minute $/ 1.73 \mathrm{~m}^{2}$; $P=0.0002)$, and tubular secretion of SCR was higher in subjects with GFR $<60 \mathrm{ml} /$ minute $/ 1.73 \mathrm{~m}^{2}$ (29\% vs. $\left.23 \% ; P<0.0001\right)$. The third-order polynomial equation $(r=0.93)$ estimated GFR better than quadratic $(r=0.92)$ and linear $(r=0.87)$ equations. Bland-Altman comparison revealed no bias when the third-order equation was used (precision: $\pm 20 \mathrm{ml} /$ minute $/ 1.73 \mathrm{~m}^{2}$ ).

Conclusion SDMA appears to be an accurate and precise estimate of GFR and a more sensitive biomarker of renal dysfunction than $\mathrm{SCr}$. We predict SDMA will perform better than $\mathrm{SCr}$ as a biomarker of AKI. This forms the basis of a future study.

P424

Prognostic value of neutrophil gelatinase-associated lipocalin and transpulmonary thermodilution-derived parameters within 48 hours after admission

W Huber, J Rauch, B Saugel, S Mair, M Messer, T Lahmer, C Schultheiss, P Luppa, RM Schmid

Klinikum Rechts der Isar, Technical University of Munich, Il. Medizinische Klinik,

Munich, Germany

Critical Care 2013, 17(Suppl 2):P424 (doi: 10.1186/cc12362)

Introduction Outcome of ICU patients is predicted by multifactorial scores such as APACHE II and SOFA. Furthermore, markers of single organ failure such as the extravascular lung water index (EVLWI) and several biomarkers of acute kidney injury (AKI) have been associated with mortality. Regarding a delayed increase in creatinine, other biomarkers of AKI including neutrophil gelatinase-associated lipocalin (NGAL) have been suggested. It was the aim of our study to compare the predictive capabilities of NGAL and transpulmonary thermodilution (TPTD)-derived parameters within 48 hours after ICU admission.

Methods Urinary NGAL, serum creatinine and BUN as well as TPTDderived parameters were measured 0 hours, 12 hours, 24 hours and 48 hours after ICU admission. Primary endpoint: prediction of ICU mortality. Secondary endpoint: requirement of renal replacement therapy (RRT). Statistics: ROC-AUC. IBM SPSS 20.

Results There were 91 patients (34 female, 57 male), $65 \pm 14$ years, APACHE II score $20.6 \pm 8.3$, SOFA score $9.3 \pm 4.2$. Etiology: 10 ARDS, 36 sepsis, 22 cirrhosis, six pancreatitis, eight cardiogenic shock, two CNS-affections, seven various. Mortality was best predicted by SOFA (ROC-AUC: $0.747 ; P<0.001$ ) and APACHE II (AUC: 0.705; $P<0.001$ ). Among admission parameters of renal function only NGAL significantly predicted mortality (AUC: 0.647; $P=0.023$ ), whereas creatinine $(P=0.290)$ and BUN $(P=0.067)$ were not predictive. ROC-AUCs for NGAL further increased after 12 hours $(0.659), 24$ hours $(0.691 ; P=0.01)$ and 48 hours $(0.728 ; P=0.004)$. NGAL on admission also predicted requirement of RRT during the ICU stay (AUC: $0.678 ; P=0.015$ ), which was also predicted by creatinine (AUC 0.688; $P=0.010$ ) and BUN (AUC: $0.649 ; P=0.043$ ). Among baseline TPTD parameters, only the pulmonary vascular permeability index (PVPI) significantly predicted mortality (AUC: $0.700 ; P=0.007$ ). The EVLWI (AUC 0.628; $P=0.083$ ) slightly failed significance. Cardiac index, global end-diastolic volume index and heart rate were not predictive. Furthermore, PVPI was the only TPTD-derived parameter to predict requirement of RRT (AUC: $0.693 ; P=0.009$ )

Conclusion Urinary NGAL on admission significantly predicts mortality, whereas creatinine and BUN were not predictive. The predictive capabilities of NGAL further increased after 12 hours, 24 hours and 48 hours, with the ROC-AUC of NGAL 48 hours exceeding the AUC of the APACHE II score. NGAL on admission also predicts requirement of RRT. Among TPTD-derived parameters, PVPI on admission is significantly associated with mortality and requirement of RRT.

\section{P425}

Assessment of neutrophil gelatinase-associated lipocalin as a biomarker of acute kidney injury in acute heart failure patients H Michalopoulou, H Michalopoulou, P Stamatis, J Xenogiannis, D Stamatis Metaxa Hospital, Athens, Greece

Critical Care 2013, 17(Suppl 2):P425 (doi: 10.1186/cc12363)

Introduction Growing evidence hints that bidirectional interaction between heart failure and kidney disease and renal insufficiency is a strong predictor of mortality as well as causally linked to the progression of heart failure. Neutrophil gelatinase-associated lipocalin (NGAL) is an early predictor of acute kidney injury (AKI). We evaluated the impact of NGAL on morbidity and mortality in patients with acute heart failure.

Methods Seventy-six patients presenting with symptoms consistent with acute heart failure (median age 72 years, $56 \%$ male) were enrolled. Plasma NGAL levels were measured by an ELISA at admission and compared with the glomerular filtration rate (eGFR) and B-natriuretic peptide (BNP) levels. The primary outcome was AKI development defined by RIFLE criteria (fall in GFR $>25 \%$ or creatinine rise $\geq 50 \%$ from baseline, or a fall in urine output $<0.5 \mathrm{ml} / \mathrm{kg} /$ hour) and secondary outcomes were duration of hospital stay and in-hospital mortality. Results AKI developed in 11 patients (14.4\%). The subgroup that during hospitalization developed renal dysfunction had increased NGAL values with respect to patients with preserved renal function $(146 \pm 42$ vs. $84 \pm 24 \mathrm{ng} / \mathrm{ml}, P<0.05)$. NGAL significantly associated with admission eGFR $(r=0.72, P<0.01)$ and BNP levels $(r=0.80, P<0.01)$. For prediction of AKI, NGAL $>140 \mathrm{ng} / \mathrm{ml}$ has been related to renal insufficiency with sensitivity of $83 \%$ and specificity of $74 \%$ with area under the receiver operator curve of 0.70 (0.58 to 0.82 ). In the follow-up setting, NGAL was associated with recurrent hospital admission for heart failure (HR: 3.1, $\mathrm{Cl}: 1.5$ to 6.2 ) and cardiac death (HR: $2.2, \mathrm{Cl}: 1.8$ to 4.5 ).

Conclusion NGAL is emerging as a promising biomarker of AKI in the setting of acute heart failure and elevated NGAL levels indicate a poor prognosis in this population regarding morbidity and mortality. 
P426

Neutrophil gelatinase-associated lipocalin predicts postoperative fluid overload after cardiac surgery

M Haase', P Devarajan'2, P Michael', R Bellomo ${ }^{4}$, A Haase-Fielitz'

'Otto von-Guericke University, Magdeburg, Germany; ${ }^{2}$ incinnati Children's Hospital Medical Center, Cincinnati, OH, USA; ${ }^{3}$ German Heart Center, Berlin, Germany; ${ }^{4}$ Austin Hospital, Melbourne, Australia

Critical Care 2013, 17(Suppl 2):P426 (doi: 10.1186/cc12364)

Introduction Neutrophil gelatinase-associated lipocalin (NGAL), measured early after cardiac surgery, has been demonstrated to predict postoperative acute kidney injury (AKI). Fluid overload potentially masks a subsequent acute renal function loss through dilution of serum creatinine and maintenance of urine output just above AKIdefining criteria.

Methods We investigated the early postoperative value of NGAL versus that of simultaneously measured serum creatinine to predict subsequent fluid overload. We studied 100 adult cardiac surgery patients in the control arm of a RCT (NCT00672334). Severe postoperative fluid overload was defined as positive fluid balance $>10 \%$ of preoperative body weight within 48 hours after surgery.

Results Severe postoperative fluid overload was present in $5 \%$ of patients with a mean positive fluid balance of $15.8 \pm 9.5 \mathrm{I}$. At ICU admission, urine NGAL predicted severe fluid overload (AUC-ROC 0.82 $(95 \% \mathrm{Cl}=0.70$ to 0.94$)$ ) (Figure 1 ) and mortality (AUC $0.88(0.78$ to 0.97$)$ ). Serum creatinine measured at the same time did not predict severe fluid overload (AUC 0.52 (0.26 to 0.79 )) or mortality (AUC 0.61 (0.16 to 0.99)).

Conclusion Early NGAL-guided adjustments to fluid management may reduce organ edema after cardiac surgery. Findings should be validated in larger cohorts.

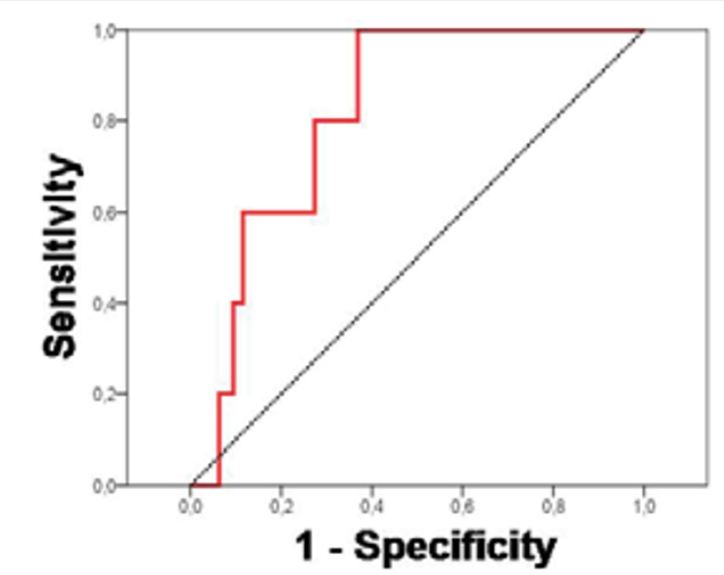

Figure 1 (abstract P426). NGAL at ICU admission predicts fluid overload of $>10 \%$ of body weight.
P427

Survivors of acute kidney injury requiring renal replacement therapy rarely receive follow-up: identification of an unmet need

CJ Kirwan, R Taylor, JR Prowle

The Royal London Hospital, Barts Health NHS Trust, London, UK

Critical Care 2013, 17(Suppl 2):P427 (doi: 10.1186/cc12365)

Introduction Acute kidney injury (AKI) occurs in more than $50 \%$ of ICU admissions, requiring renal replacement therapy (RRT) in around $10 \%$ of cases. There is now increasing evidence that AKI is a risk factor for the development and progression of chronic kidney disease (CKD); however, when AKI occurs as a complication of critical illness appropriate follow-up may be neglected. Accordingly, we reviewed the follow-up of renal function in all patients who received RRT on our ICU and survived to hospital discharge.

Methods A retrospective audit of patients who received RRT in a central London adult critical care unit during 2011.

Results Of 921 patients admitted, 203 received RRT with 109 surviving to hospital discharge. We excluded 52 patients who had end-stage renal disease, renal transplant or known glomerular disease. Of the remaining $57 \mathrm{AKI}$ patients, median age was 60 (range: 18 to 77) and $37(65 \%)$ were male. Median discharge creatinine was $74.5 \mu \mathrm{mol} / \mathrm{l}$ (27 to 662). Forty-two (74\%) were offered follow-up, but in only six cases $(11 \%)$ was this to nephrology services. Twenty-eight attended follow-up (five to nephrology) at a median time of 6 weeks; however, creatinine was measured at in only 14 and in six of these it had risen (by median $16.5 \mu \mathrm{mol} / \mathrm{l})$. In addition, 14 patients had creatinine measured 3 to 6 months post discharge and in eight it had risen (by median $31.5 \mu \mathrm{mol} / \mathrm{l})$.

Conclusion Follow-up of patients who received RRT for AKI in the ICU was poor and they were rarely referred to nephrologists. Where renal function was measured after discharge, there was evidence of progressive renal dysfunction; however, renal function was often not assessed. We propose an algorithm for clinicians to guide follow-up. See Figure 1.

\section{P428}

Aetiology and outcomes for dialysis-dependent acute kidney injury patients on the ICU

M Hameed', P Carmichael ${ }^{2}$

'Salford Royal NHS Foundation Trust, Salford, UK; ${ }^{2}$ The Royal Wolverhampton Hopsitals NHS Trust, Wolverhampton, UK

Critical Care 2013, 17(Suppl 2):P428 (doi: 10.1186/cc12366)

Introduction AKI is a common occurrence in sick hospitalized patients, in particular those admitted to intensive care. Published data suggest that 4 to $5 \%$ of all critically ill patients develop severe AKI and require initiation of renal replacement therapy (RRT) [1,2]. Such patients have high mortality rates often exceeding $60 \%$ [2]. We aimed to review the outcomes of patients admitted to the ICU and required renal replacement therapy for AKI. We examined whether aetiology of AKI, comorbidity burden, hospital length of stay and treatment in ICU had any significant association with survival in the study cohort.

Methods During 2009, 56 patients were identified to have received RRT with AKI who were admitted to the ICU at the Royal Wolverhampton Hospitals NHS Trust. Computerised and paper-based case records were examined for these patients to collect the data. AKIN classification was used to classify the severity of AKI.

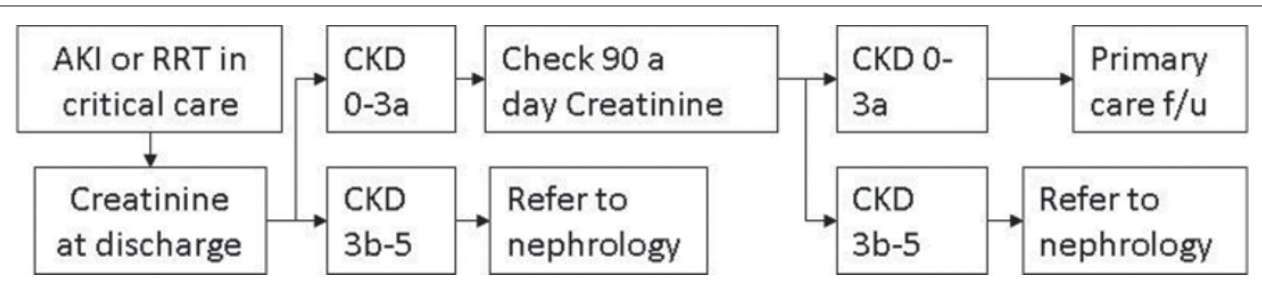

Figure 1 (abstract P427). A suggested follow-up pathway for AKI survivors. 
Results Median age at admission was 66 years ( 27 to 85 ) with 29 males and 27 females. Thirty-one (55.4\%) patients had sepsis and 20 (35.7\%) patients had ATN as the main cause of AKI. Thirty-two patients (57\%) had three organ failures at the time of commencement of RRT. Fortysix patients $(82.1 \%)$ received haemofiltration only. Thirty-two $(57 \%)$ patients died, with more than $80 \%$ of these occurring in the ITU. There was no significant difference in survival when compared with duration of haemofiltration, length of stay, number of organs failed and number of comorbidities. However, significantly more patients that died had AKI due to sepsis $(P=0.003)$ or if they received mechanical ventilation $(P=0.48)$ or inotropes $(0.04)$. Of the 27 patients who survived until discharge from hospital, 18 (66.7\%) had normal renal function, eight (29.6\%) had AKIN stage I and only one patient required maintenance haemodialysis.

Conclusion Individuals who develop dialysis-dependent AKI in the ICU setting in general terms either die or recover. Sepsis is the most common association with death. The need for mechanical ventilation and inotropic therapy are both associated with increased incidence of death.

\section{References}

1. Metnitz PG, et al: Effect of acute renal failure requiring renal replacement therapy on outcome in critically ill patients. Crit Care Med 2002, 30:2051-2058.

2. Uchino $S$, et al:: Acute renal failure in critically ill patients: a multinational, multicenter study. JAMA 2005, 294:813-818.

P429

Efficiency of substitutive renal therapy in the complex intensive care of multiple organ failure in patients with polytrauma S Kravtsov, A Shatalin, V Agadzhanyan, D Skopintsev

Federal State Budgetary Medical Prophylactic Institution 'Scientific Clinical Center of the Miners' Health Protection', Leninsk-Kuznetsky, Russia Critical Care 2013, 17(Suppl 2):P429 (doi: 10.1186/cc12367)

Introduction This study was to evaluate the efficiency of the early start of intermittent substitutive renal therapy in patients with polytrauma complicated by multiple organ failure syndrome.

Methods Forty-two patients with polytrauma complicated by multiple organ failure syndrome were included in the study. The age of the patients was from 20 to 60 years ( $38.3 \pm 1.6$ years average). All patients were divided into two equal groups. In the control group (CG) the criteria for the start of the substitutive renal therapy were: hyperkalemia $\geq 6 \mathrm{mmol} / \mathrm{l}$, plasma creatinine $\geq 280 \mu \mathrm{mol} / \mathrm{l}$, diuresis $\leq 20 \mathrm{ml} /$ hour. In the investigation group (IG) there were subtests to carry out the substitutive renal therapy, allowing one to start it in the earlier period of the multiple organ failure progression. These are increase of $\mathrm{Na}^{+}>150 \mathrm{mmol} / \mathrm{l}$, osmolarity $>300 \mathrm{mOsm} / \mathrm{l}$, elevation of the plasma toxicity according to the average molecule concentration $\geq 1.0$, diuresis decrease $\leq 40 \mathrm{ml} /$ hour. These were examined: lethality, quantity of the substitutive renal therapy procedures, mechanical lung ventilation duration (MLV), intensive care and hospital duration. The substitutive renal therapy was carried out by AK-200-Ultra apparatus (Gambro, Sweden). The statistical analysis was realized using Statistica 6.1 and the Mann-Whitney $U$ test.

Results The average quantity of the substitutive renal therapy procedures in the CG was $13.4 \pm 0.7$, in the IG it was $8 \pm 0.6(P<0.05)$. The recuperation of the renal excretory functions was on $19 \pm 1$ day in 12 patients of the $C G$, and on $11 \pm 1.3$ day in the IG, from the moment of substitutive renal therapy start $(P<0.05)$. Lethality in the $C G$ was $43 \%$ (nine patients), and in the IG it was $29 \%$ (six patients, $P<0.05$ ). The duration of the MLV in the CG and IG was $21 \pm 1.2$ days and $16 \pm 1.2$, respectively $(P<0.05)$. In the IG the duration of the ICU was lower by $23 \%$, hospitality duration was lower by $17 \%(P<0.05)$.

Conclusion The efficiency of the substitutive renal therapy depends directly on the hydroelectrolytic and metabolic changes and toxicosis degrees in the polytrauma complicated by multiple organ failure syndrome. The early start of the dialysis methods treatment allows one to achieve the earlier recuperation of the renal functions and to decrease the lethality level by $14 \%$.
P430

Can treatment with the molecular adsorbent recirculation system be the solution for type-1 hepatorenal syndrome?

L Lavayssiere

University Hospital, Toulouse, France

Critical Care 2013, 17(Suppl 2):P430 (doi: 10.1186/cc12368)

Introduction The aim of this retrospective study was to determine whether the molecular adsorbent recirculation system (MARS) can improve renal function in HSR1 patients.

Methods Thirty-two patients with chronic liver disease and HRS1 were treated by MARS sessions that were performed every other day. The endpoint was renal function improvement by 28 days after diagnosis of HRS1 that was defined as a serum creatinine level $<133 \mu \mathrm{mol} / \mathrm{l}$. Partial renal recovery was defined as a $10 \%$ decrease in baseline serum creatinine level.

Results The mean number of MARS sessions required by each patient was $3.5 \pm 1.5$. The median time between admission and the start of MARS therapy was 3 ( 0 to 15 ) days. Of the total patients, 13 (40\%) had improved renal function. Among these, nine (28\%) had complete renal recovery. Among the patients that survived, only $40 \%(6 / 15)$ had improved renal function. When MARS was started, $53 \%$ of patients had failed renal failure according to RIFLE criteria. Seven patients received a liver transplant after diagnosis of HRS. Of these, four had complete or partial recovery after transplantation (57\%) versus nine of the 25 patients who did not undergo liver transplantation $(36 \%), P=$ NS. The 28-day survival rate was $47 \%$.

Conclusion MARS therapy improved renal function in only very few patients with HRS1. Even if liver transplantation is the best option, MARS could be used as a bridge until transplantation. Some authors argue that prolonged duration of dialysis ( $>1$ month) before transplantation may result in chronic renal failure probably because there are two etiologies of AKI in end-stage liver disease: acute tubular necrosis (ATN) and real functional renal failure. ATN before liver transplantation has been identified as a risk factor for patients' mortality and chronic renal failure at 1 and 5 years post liver transplantation. Further prospective controlled studies including large number of patients are required.

References

1. Nadim MK, Genyk YS, Tokin C, et al:. Impact of etiology of acute kidney injury on outcomes following liver transplantation: acute tubular necrosis versus hepatorenal syndrome. Liver Transp/ 2012, 18:539-548.

2. Cholongitas E, Senzolo M, Patch D, Shaw S, O'Beirne J, Burroughs AK:

Cirrhotics admitted to intensive care unit: the impact of acute renal failure on mortality. Eur I Gastroenterol Hepatol 2009, 21:744-750.

\section{P431}

Effect of continuous high-volume hemofiltration on patients with acute respiratory distress syndrome

A Kuzovlev' ${ }^{1}$ ETishkov² O Bukaev²

${ }^{\prime}$ V.A. Negovsky Scientific Research Insitute of General Reanimatology RAMS, Moscow, Russia; ${ }^{2}$ A.I. Evdokimov Moscow State University of Medicine and Dentistry, Moscow, Russia

Critical Care 2013, 17(Suppl 2):P431 (doi: 10.1186/cc12369)

Introduction The aim of this study was to investigate the effects of intermittent venovenous high-volume hemofiltration (IHVHF) on extravascular lung water, respiratory function and cytokine removal in patients with acute respiratory distress syndrome (ARDS) associated with postoperative multiple organ dysfunction syndrome in severe abdominal infection patients.

Methods Thirty patients with severe abdominal infection and ARDS were randomized into the IHVHF group $(n=15)$ and control group $(n=15)$. All patients were mechanically ventilated. The IHVHF technique with a rate of 100 to $120 \mathrm{ml} / \mathrm{kg} / \mathrm{hour}$ during 6 to 8 hours was used. The PiCCOplus system was used to monitor cardiac output (CO), extravascular lung water index (EVLWI) and intrathoracic blood volume index (ITBVI). Arterial partial pressure of oxygen $\left(\mathrm{PaO}_{2}\right)$, arterial partial pressure of carbon dioxide $\left(\mathrm{PaCO}_{2}\right)$, oxygenation index $\left(\mathrm{PaO}_{2} / \mathrm{FiO}_{2}\right)$ and dynamic lung compliance (Cdyn), oxygen delivery and oxygen consumption were measured. Serum concentrations of TNFa, IL-6, IL-8 
and lactate were measured by ELISA method. The APACHE II scores before and after IHVHF therapy were detected.

Results All indexes in the control group did not show any significant improvement before and after treatment $(P<0.05)$. There was a significant decrease of serum concentrations of TNFa, IL- 6, IL- 8 and lactate, increase of CO and decrease of EVLWI 24 hours after IHVHF $(P<0.05)$. The indexes of oxygen delivery, consumption, $\mathrm{PaO}_{2^{\prime}}$ and $\mathrm{Cdyn}$ improved significantly, $\mathrm{PaO}_{2} / \mathrm{FiO}_{2}$ increased markedly compared with those before IHVHF and the control group. Significant differences were detected in all of the hemodynamics and oxygen indexes $(P<0.05)$.

Conclusion IHVHF presents with a significant beneficial effect on respiratory function in patients with ARDS as a result of removal of cytokines, decrease in EVLWI, improvement of hemodynamic parameters, and correction of disturbances in oxygen balance. IHVHF adjuvant treatment for ARDS reduces pulmonary edema, improves $\mathrm{PaO}_{2} / \mathrm{FiO}_{2}$ and $\mathrm{Cdyn}$ and mechanical ventilation parameters. IHVHF may be a promising treatment for ARDS in postoperative multiple organ dysfunction syndrome in severe abdominal infection patients. Reference

1. Honoré P, et al: Anestesiol Reanimatol 2008, 6:4-11.

P432

Dialyzer clearance of myoglobin with middle molecule filter and low blood flow CVVHD in patients with rhabdomyolysis-associated acute kidney injury

I Leppanen', T Ahonen', J Tenhunen²

'Tampere University Hospital, Tampere, Finland; ${ }^{2}$ Uppsala University Hospital, Uppsala, Sweden

Critical Care 2013, 17(Suppl 2):P432 (doi: 10.1186/cc12370)

Introduction Rhabdomyolysis-associated acute kidney injury can be treated with various modes of renal replacement therapy (RRT) $[1,2]$. We have used continuous venoveno hemodialysis (CVVHD) with middle molecule filter and low extracorporeal blood flow (100 to $180 \mathrm{ml} /$ minute) to avoid albumin loss and hemodynamic instability. Methods We treated nine patients suffering from rhabdomyolysis and acute kidney injury with various causes. We used a CVVHD mode with low (100 to $180 \mathrm{ml} /$ minute) blood flow and a middle molecule filter (Ultraflux EMiC2; $1.8 \mathrm{~m}^{2}$ surface area, polysulfone membrane, cutoff at $40 \mathrm{kDa}$, blood filling volume $130 \mathrm{ml}$, blood flow range 100 to $350 \mathrm{ml} /$ minute, maximum dialysate flow $1,000 \mathrm{ml} /$ minute). Myoglobin and albumin concentrations were measured from prefilter and postfilter samples and dialyzer clearances were calculated (CLdial $=$ blood flow $\times$ Cpre - Cpost/Cpre + UFXCpre/Cpost). Measurements were taken at 0,15 minutes, 30 minutes, 4 hours, 12 hours, 24 hours, 36 hours and 48 hours from the start of the CRRT or until CRRT was no longer needed. Results The baseline and prefilter plasma albumin concentrations varied from 8 to $29 \mathrm{~g} / \mathrm{l}$. Postfilter and post-treatment albumin concentrations remained comparable. Prefilter concentrations of myoglobin (17.8 kDa) varied from 96,109 to $747 \mu \mathrm{g} / \mathrm{l}$ and the dialyzer clearance of myoglobin from 50.6 to $0 \mathrm{ml} /$ minute. The mean dialyzer clearance was $23.1 \mathrm{ml} /$ minute and the median clearance $22.2 \mathrm{ml} /$ minute. Maximal changes between corresponding prefilter and postfilter samples were: absolute concentration change $27,091 \mu \mathrm{g} / \mathrm{l}$, percentage $34 \%$, dialyzer clearance $50.6 \mathrm{ml} /$ minute. Clearances were achieved with low extracorporeal blood flow between 100 and $180 \mathrm{ml} /$ minute, most commonly $120 \mathrm{ml} /$ minute. Ultrafiltration (UF) was used only at five of the 56 time points calculated, because of hemodynamic instability. All patients required either vasopressor or vasopressor and inotrope support. The highest dialyzer clearance of myoglobin ( $50.6 \mathrm{ml} /$ minute) was measured at the prefilter myoglobin of $29,266 \mu \mathrm{g} / \mathrm{l}$, blood flow $180 \mathrm{ml} / \mathrm{ml}$ and no UF.

Conclusion In a rhabdomyolysis-associated kidney injury a middle molecule filter and a low blood flow CVVHD offer a safe and effective treatment choice for patients requiring vasopressor or vasopressor and inotrope for hemodynamic support.

References

1. Albert C, et al:: Crit Care Resusc 2012, 14:159-162.

2. Sorrentino SA, et al:: Crit Care Med 2011, 39:184-186.
P433

Daily fluid balance predicts hospital mortality in critically ill patients receiving continuous renal replacement therapy

M Kashiouris, A Akhoundi, S Chaudhary, V Velagapudi, A Goldberg,

K Kashani

Mayo Clinic, Rochester, MN, USA

Critical Care 2013, 17(Suppl 2):P433 (doi: 10.1186/cc12371)

Introduction It has been suggested that fluid balance is a biomarker in critically ill patients [1]. There is a paucity of randomized trials examining the effect of daily fluid balance on outcomes in patients on continuous renal replacement therapy (CRRT). The RENAL trial did not find mortality difference with higher CRRT dose [2], but did not investigate the effect of daily fluid balance on patient outcomes. A post hoc analysis suggested survival benefit in patients with negative fluid balance [3]. In this study, we hypothesize that daily fluid balance is an independent predictor of mortality in critically ill patients.

Methods We conducted a retrospective cohort study in eight ICUs of a tertiary academic center. We constructed a robust clustered linear regression model of daily fluid balance and all-cause hospital mortality among 595 critically ill patients receiving CRRT. We adjusted the model for the Charlson comorbidity score, the daily SOFA scores in the first week after initiation of CRRT as well the type of ICU.

Results After adjusting for the type of ICU and the daily severity of illness, patients who died had on average $779 \mathrm{ml}$ higher daily fluid balance compared with patients who survived $(P<0.001,95 \%$ $\mathrm{Cl}=385$ to $1,173 \mathrm{ml}$, Figure 1). Severity of illness predicted daily fluid accumulation; each additional point of the SOFA score predicted an additional $57 \mathrm{ml}$ of extra daily fluid $(P=0.002)$.

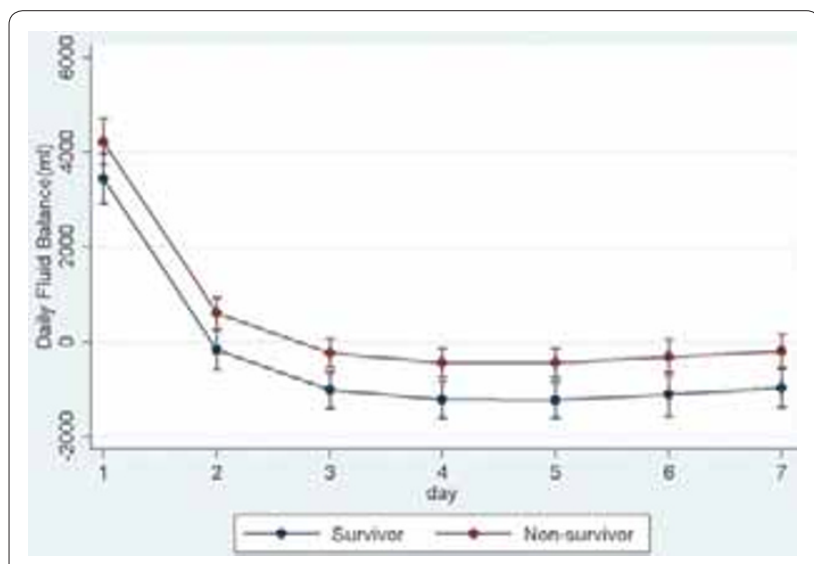

Figure 1 (abstract P433). Daily fluid balance and 95\% Cls among survivors and nonsurvivors.

Conclusion Among critically ill patients who are receiving CRRT, daily fluid balance is an independent predictor of mortality, even after adjusting for the daily severity of illness and comorbidities.

References

1. Bagshaw SM, et al:: Crit Care 2008, 12:169.

2. Bellomo R, et al:: NEngl J Med 2009, 361:1627.

3. Bellomo R, et al.: Crit Care Med 2012, 40:1753-1760.

P434

Fluid balance and renal outcomes in patients requiring renal replacement therapy in the ICU

JA Silversides, R Kuint, R Pinto, R Wald, M Hladunewich, NK Adhikari

University of Toronto, Canada

Critical Care 2013, 17(Suppl 2):P434 (doi: 10.1186/cc12372)

Introduction Fluid overload is associated with mortality in critically ill patients with acute kidney injury (AKI) [1-3]. We explored this relationship in patients with AKI who received renal replacement therapy (RRT) in the ICU to investigate the relationship between fluid 
balance and intradialytic hypotension with mortality and recovery of renal function.

Methods We conducted a retrospective cohort study among patients aged $\geq 16$ years who had RRT initiated and continued for $\geq 2$ days in a level 2 or 3 ICU at two academic centres, and had fluid balance data available. Patients with end-stage kidney disease, within 1 year of a renal transplant or who had RRT initiated to treat a toxic ingestion were excluded. We used multivariable logistic regression to determine the relationship between mean daily fluid balance over the first 7 days following RRT initiation and the outcomes of mortality and RRT dependence in survivors.

Results A total of 522 patients were included (319 male, mean age 64 years); 264 (50.6\%) died in hospital. Survivors and those who died were similar with respect to age, weight and incidence of heart failure, liver cirrhosis and primary renal diagnoses. Independent risk factors for hospital mortality were higher Sequential Organ Failure Assessment score at RRT initiation (odds ratio $(\mathrm{OR})=1.18,95 \%$ confidence interval $=1.11$ to 1.25$)$, higher Charlson comorbidity index $(\mathrm{OR}=1.22$, 1.09 to 1.37 ), lower baseline creatinine $(\mathrm{OR}=0.984$ per $10 \mu \mathrm{mol} / \mathrm{l}$ 0.970 to 0.998 ), lower minimum mean arterial pressure (MAP) on day 1 ( $\mathrm{OR}=0.81$ per $10 \mathrm{mmHg}, 0.66$ to 0.95$)$ and more positive fluid balance for the first 7 days (OR 1.03 per $100 \mathrm{ml}, 1.02$ to 1.05). Of 172 hospital survivors, 57 (33.1\%) were RRT dependent at discharge. Although univariable analysis suggested lower SOFA scores and higher baseline serum creatinine levels in those who remained RRT dependent, no factor was independently associated with RRT dependence at discharge in a multivariate model.

Conclusion In this cohort of patients with AKI requiring RRT, a more positive fluid balance over 1 week and lower initial minimum MAP were associated with mortality. Among survivors, a less positive fluid balance was not associated with increased risk of RRT dependence at discharge, suggesting that conservative fluid management does not significantly attenuate renal recovery.

\section{References}

Bouchard J, et al: Kidney Int 2009, 76:422-427.

2. Grams ME, et al.: Clin J Am Soc Nephro/ 2011, 6:966-973.

3. Payen D, et al:: Crit Care 2008, 12:R74.

P435

Pharmacokinetics and pharmacodynamics of esomeprazole and ranitidine during continuous venovenous hemodiafiltration V Fuhrmann ${ }^{1}$, P Schenk ${ }^{2}$, W Jaeger ${ }^{3}$, M Miksits ${ }^{3}$, R Kitzberger ${ }^{4}$, U Holzinger ${ }^{1}$, $\mathrm{CMadl}^{\mathrm{s}}$

'Medical University Vienna, Austria; 'Landesklinikum Thermenregion

Hochegg, Grimmenstein, Austria; ${ }^{3}$ University Vienna, Austria; ${ }^{4}$ Kaiser Franz Josef Krankenhaus, Vienna, Austria: ${ }^{5}$ Krankenanstalt Rudolfstiftung Wien, Vienna, Austria

Critical Care 2013, 17(Suppl 2):P435 (doi: 10.1186/cc12373)

Introduction Esomeprazole, a proton pump inhibitor, and ranitidine, a histamine-2 receptor antagonist are frequently used in intensive care patients requiring stress ulcer prophylaxis. Continuous venovenous hemodiafiltration (CVVHDF) is an important extracorporeal renal replacement therapy in critically ill patients suffering from multiple organ failure. This study investigates the pharmacokinetics and pharmacodynamics of esomeprazole and ranitidine in anuric critically ill patients undergoing CVVHDF.

Methods Esomeprazole $40 \mathrm{mg}$ was administered intravenously once daily in nine intensive care patients and ranitidine $50 \mathrm{mg}$ was administered intravenously three times daily in nine intensive care patients with acute renal failure undergoing CVVHDF who required stress ulcer prophylaxis. The concentration of esomeprazole and ranitidine in serum and ultradiafiltrate was determined by highperformance liquid chromatography. The intragastric $\mathrm{pH}$ was obtained via intragastric $\mathrm{pH}$-metry.

Results The mean peak prefilter concentration, $\mathrm{AUC}_{0-24^{\prime}}$ volume of distribution, half-life, total clearance and hemodiafiltration clearance of esomeprazole were $6.9 \mu \mathrm{mol} / \mathrm{l}, 30.6 \mu \mathrm{mol}$. hour/l, $53.3 \mathrm{l}, 2.0$ hours, $1.4 \mathrm{l} /$ hour and $0.2 \mathrm{l} /$ hour, respectively. In the ranitidine group the mean peak prefilter concentration, $\mathrm{AUC}_{0-8^{\prime}}$ volume of distribution, half-life, total clearance and hemodiafiltration clearance were $5.5 \mu \mathrm{mol} / \mathrm{l}, 12.0 \mu \mathrm{mol}$. hour/l, $91.4 \mathrm{I}, 14.4$ hours, $5.8 \mathrm{I}$ and $1.3 \mathrm{I}$, respectively. Median time of intragastric $\mathrm{pH}>5$ and median intragastric $\mathrm{pH}$ decreased significantly in the ranitidine group over time $(P<0.05)$ but not in the esomeprazole group.

Conclusion Although pharmacokinetics of esomeprazole and ranitidine are only little influenced by CVVHDF, we found a significant decrease of intragastric $\mathrm{pH}$ in patients treated with ranitidine. Esomeprazole may be superior for long-term stress ulcer prophylaxis in critically ill patients undergoing CVVHDF.

\section{P436}

Pharmacokinetics of gentamicin and vancomycin during continuous venovenous hemofiltration in critically ill septic patients with acute kidney injury

N Petejova', J Duricova', A Martinek', J Zahalkova

'University Hospital, Ostrava, Czech Republic; ${ }^{2}$ Hospital, Sternberk, Czech

Republic

Critical Care 2013, 17(Suppl 2):P436 (doi: 10.1186/cc12374)

Introduction Acute kidney injury (AKI) is a common complication of critical illness and sepsis [1]. Dosing of antibacterial agents in septic patients is complicated by altered pharmacokinetics due to both acute renal failure and critical illness [2]. Current dosing regimens for administration of gentamicin and vancomycin to septic patients with $\mathrm{AKI}$ on continuous venovenous hemofiltration $(\mathrm{CVVH})$ at a filtration rate of $45 \mathrm{ml} / \mathrm{kg} / \mathrm{hour}$ are missing.

Methods Seventeen septic patients with AKI treated with vancomycin and seven patients with gentamicin on CVVH were included. In the vancomycin group, patients received the first dose of $1.0 \mathrm{~g}$ intravenously followed by $1.0 \mathrm{~g} / 12$ hours if not adjusted. In the gentamicin group, patients received a loading dose of $240 \mathrm{mg}$ followed by a maintenance dose every 24 hours. The vancomycin maintenance dose was optimized to achieve $A \mathrm{C}_{0-24} / \mathrm{MIC} \geq 400(\mathrm{Cmin}>10 \mathrm{mg} / \mathrm{l})$, gentamicin target was $\mathrm{Cmax} / \mathrm{MIC}$ of 8 to 10 . Maintenance doses were adjusted according to drug level simulation using a pharmacokinetic programme.

Results The median vancomycin total clearance (Cltot) was 0.89 and $0.55 \mathrm{ml} / \mathrm{minute} / \mathrm{kg}$ on the first and second day of the study. CRRT clearance accounted for about 50 to $60 \%$ of vancomycin Cltot found in a population with normal renal function $(0.97 \mathrm{ml} /$ minute $/ \mathrm{kg})$. Vancomycin serum concentrations after the first dose were below the required target of $10 \mathrm{mg} / \mathrm{l}$ as early as 6 hours in 10 patients. AUC $/$ MIC $\geq 400$ ratio was achieved in $67 \%$ of patients on the first day. The median gentamicin Cltot was 0.68 and $0.79 \mathrm{ml} /$ minute $/ \mathrm{kg}$ on the first and second day of the study. CRRT clearance accounted for about $50 \%$ of gentamicin Cltot found in a population without renal impairment $(0.73 \mathrm{ml} / \mathrm{minute} / \mathrm{kg})$. The target Cmax/MIC ratio was achieved in $78 \%$ of patients after the first dose.

Conclusion CVVH at a filtration rate of $45 \mathrm{ml} / \mathrm{kg} /$ hour leads to high removal of both antibiotics. Due to rapid change in patient's clinical status it was impossible to predict a fixed dosage regimen. We recommend administration of unreduced loading dose and: blood sampling as early as 6 hours after first vancomycin dose; blood sampling 30 to 60 minutes after gentamicin administration and before the next dose; and the maintenance dose should be based on druglevel monitoring.

References

1. Uchino S, et al.: JAMA 2005, 294:813-818.

2. Roberts JA, et al.: Crit Care Med 2009, 37:840-851.

\section{P437}

Effects of two different regional citrate anticoagulation CVVH protocols on acid-base status and phosphate supplementation S Morabito', V Pistolesi', V Pistolesi', L Tritapepe ${ }^{2}$, L Tritapepe ${ }^{2}$, EV Vitaliano ${ }^{3}$, EV Vitaliano ${ }^{3}$, L Zeppilli', L Zeppilli', F Polistena', F Polistena', E Fiaccadori', E Fiaccadori', A Pierucci', A Pierucci

'Umberto I, Policlinico di Roma, 'Sapienza' University, Rome, Italy; 'Umberto I, Policlinico di Roma, Rome, Italy; ${ }^{3}$ Pertini Hospital, Rome, Italy; ${ }^{*}$ University of Parma Medical School, Parma, Italy

Critical Care 2013, 17(Suppl 2):P437 (doi: 10.1186/cc12375)

Introduction Regional citrate anticoagulation (RCA) is increasingly used in high bleeding risk patients undergoing CRRT. Regardless of anticoagulation protocol, hypophosphatemia occurs frequently in 
CRRT. The aim was to evaluate the effects on electrolyte and acid-base status of a new RCA-CVVH protocol using an $18 \mathrm{mmol} / \mathrm{l}$ citrate solution combined with a phosphate-containing replacement fluid, compared with a previously adopted RCA-CVVH protocol combining a $12 \mathrm{mmol} / \mathrm{l}$ citrate solution with a conventional replacement fluid.

Methods Until September 2011, RCA-CVVH was routinely performed in our centre with a $12 \mathrm{mmol} / \mathrm{l}$ citrate solution and a postdilution replacement fluid with bicarbonate $\left(\mathrm{HCO}_{3}{ }^{-} 32, \mathrm{Ca}^{2+} 1.75, \mathrm{Mg}^{2+} 0.5, \mathrm{~K}^{+}\right.$ $2 \mathrm{mmol} / \mathrm{l}$ ) (protocol A). In cases of metabolic acidosis, not related to inappropriate citrate metabolism and persisting after optimization of RCA-CVVH parameter setting, bicarbonate infusion was scheduled. Starting from September 2011, in order to optimize buffer balance and to reduce the need for phosphate supplementation, a new $\mathrm{RCA}-\mathrm{CVVH}$ protocol has been designed using an $18 \mathrm{mmol} / \mathrm{l}$ citrate solution combined with a recently introduced phosphate-containing replacement fluid with bicarbonate $\left(\mathrm{HCO}_{3}{ }^{-} 30\right.$, phosphate $1.2, \mathrm{Ca}^{2+} 1.25$, $\mathrm{Mg}^{2+}$ 0.6, $\mathrm{K}^{+} 4 \mathrm{mmol} / \mathrm{l}$ ) (protocol B).

Results In 30 cardiac surgery patients with AKI, acid-base status and electrolytes have been evaluated comparing protocol A (20 patients, running time 5,283 hours) versus protocol B (10 patients, 1,170 hours) (median (IQR)): $\mathrm{pH} 7.40$ (7.36 to 7.44 ) versus 7.43 (7.41 to 7.47$)(P<0.0001)$, bicarbonate 22.1 (20.9 to 23.5$)$ versus 24.4 (23.2 to 25.6$) \mathrm{mmol} / \mathrm{l}(P<0.0001)$, base excess $-3.1(-4.6$ to -1.15$)$ versus 0 $(-1.5$ to 1.1$)(P<0.0001)$, systemic $\mathrm{Ca}^{2+} 1.16(1.1$ to 1.23$)$ versus 1.14 (1.08 to 1.19$) \mathrm{mmol} / \mathrm{l}(P<0.0001)$, phosphate $0.7(0.5$ to 1$)$ versus 1.1 (0.9 to 1.4$) \mathrm{mmol} / \mathrm{l}(P<0.0001)$. Protocol $\mathrm{A}$ required bicarbonate infusion in $90 \%$ of patients $(6 \pm 6.4 \mathrm{mmol} / \mathrm{hour})$ and sodium phosphate supplementation in all cases $(4.1 \pm 2.4 \mathrm{~g} /$ day $)$. A lower amount of phosphate supplementation ( $0.9 \pm 2 \mathrm{~g} /$ day) was needed in $30 \%$ of patients undergoing protocol $B$ while bicarbonate infusion was never required. Filter life was comparable $(51.8 \pm 36.5$ vs. $46.8 \pm 30.3$ hours, $P=\mathrm{NS}$ ).

Conclusion Protocol B provided a buffer balance more positive than protocol $A$ and allowed one to adequately control acid-base status without additional bicarbonate infusion and in the absence of alkalosis, despite the use of a standard bicarbonate concentration replacement solution. Furthermore, the combination of a phosphate-containing replacement fluid appeared effective to prevent hypophosphatemia.

P438

Abstract withdrawn

\section{P439}

Muscle wasting in the ICU can be reliably monitored using ultrasound

H Jørgensen', B Nedergaard², T Gilsaa'

'Lillebaelt Hospital, Kolding, Denmark; ${ }^{2}$ Odense University Hospital,

Svendborg, Denmark

Critical Care 2013, 17(Suppl 2):P439 (doi: 10.1186/cc12377)

Introduction The aim of this study was to establish the intraobserver and interobserver variation of ultrasonographic measurements of the rectus femoris muscle cross-section area (RF-CSA). Muscle wasting is frequent in the ICU, affecting more than one-half of the patients with severe sepsis [1]. Muscle mass reduces rapidly, and 15 to $20 \%$ is lost within the first week [1]. To monitor muscle mass, ultrasound has the benefits of being both readily available in the ICU and non-invasive. Ultrasonographic measurement of RF-CSA has an almost perfect correlation with MRI (mean interclass correlation (ICC) $=0.999)$ [2] and $\mathrm{RF}-\mathrm{CSA}$ is linearly related to maximum voluntary contraction strength in both healthy subjects and COPD patients $(r=0.78)$ [3].

Methods The study had two purposes: to determine the intraobserver variation for RF-CSA by one observer scanning 15 healthy adult volunteers three times each at 2-day intervals; and to determine the interobserver variation for RF-CSA by two observers each scanning 15 adult ICU patients on the same day. Patients were in a supine position, legs in passive extension. The transducer was placed perpendicular to the long axis of the right thigh over the RF, two-thirds of the distance from the anterior superior iliac spine to the superior patellar border [1]. RF-CSA was calculated by planimetry. At each scan, three measurements were made. For intraobserver variation, the $3 \times 3$ scans were analyzed using the interclass correlation coefficient. For interobserver variation, the three measurements from each observer were averaged and compared using Bland-Altman statistics.

Results Intraobserver variation: 15 healthy adults, age $39.6 \pm 2.4$ years, weight $66.8 \pm 2.3 \mathrm{~kg}$, sex three male/12 female. ICC: 0.996 ( $95 \%$ Cl: 0.990 to 0.998$)$. Interobserver variation: 15 ICU patients, age: $77 \pm 8.3$ years, weight: $71.3 \pm 9.1 \mathrm{~kg}$, sex nine male/six female. Bland-Altman: bias: $-0.07 \mathrm{~cm}^{2}, 95 \%$ limits of agreement -0.188 to $0.048 \mathrm{~cm}^{2}$.

Conclusion Ultrasonographic measurement of RF-CSA is easily learned and quickly performed. It has a very low intraobserver and interobserver variation and can be recommended as a reliable method for monitoring muscle wasting in the ICU.

References

1. Parry SM, et al:: BMJ Open 2012, e001891. doi:10.1136/bmjopen

2. Reeves ND, et al.: Eur J Appl Physiol 2004, 91:116-118.

3. Seymour JM, et al:: Thorax 2009, 64:418-423.

\section{P440}

Critical illness induces nutrient-independent adipogenesis and accumulation of alternatively activated tissue macrophages

M Marques, S Vander Perre, A Aertgeerts, S Derde, F Guiza, M Casaer, $G$ Hermans, G Van den Berghe, L Langouche

KU Leuven, Belgium

Critical Care 2013, 17(Suppl 2):P440 (doi: 10.1186/cc12378)

Introduction In artificially fed critically ill patients, adipose tissue reveals an increased number of small adipocytes and accumulation of M2-type macrophages [1]. We hypothesized that nutrient-independent factors of critical illness explain these findings, and also that M2macrophage accumulation during critical illness may not be limited to adipose tissue.

Methods We performed a randomized investigation in a septic mouse model of critical illness and a study of ICU patient biopsies. In the critically ill mouse, we compared the effect of parenteral nutrition ( $n=13)$ with fasting $(n=11)$ on body composition, adipocyte cell size, and macrophage accumulation in adipose tissue, liver and lung. Fed healthy control mice $(n=11)$ were studied for comparison. In vivo adipose tissue was harvested after 1 week of illness from human patients $(n=40)$ who participated in a RCT on early parenteral nutrition versus tolerating nutrient restriction [2], adipose tissue morphology was characterized and compared with healthy controls $(n=13)$.

Results Irrespective of nutritional intake, critically ill mice lost body weight, total fat and fat-free mass. Part of the fat loss was explained by reduced ectopic fat accumulation. Adipocyte cell number and the adipogenic markers peroxisome proliferator-activated receptor $\gamma$ and CCAT/enhancer binding-protein $\beta$ increased with illness, again irrespective of nutritional intake. Macrophage accumulation with predominant M2-phenotype was observed in adipose tissue, liver and lungs of critically ill mice, further accentuated by fasting in visceral tissues. Macrophage M2-markers correlated with chemoattractant factor expression in all studied tissues. In human subcutaneous adipose tissue biopsies of critically ill patients, increased adipogenic markers and M2 macrophage accumulation were present irrespective of nutritional intake.

Conclusion Adipogenesis and accumulation of M2-macrophages are hallmarks of critical illness, irrespective of nutritional management in humans and mice. Critical illness evokes macrophage polarization to the M2-state not only in adipose tissue but also in liver and lungs, which is further accentuated by fasting.

References

1. Langouche L, et al:: Am J Respir Crit Care Med 2010, 182:507-516.

2. Casaer MP, et al: NEngl J Med 2011, 365:506-517. 
P442

Hypocalcemia during magnesium therapy for obstetric ICU admissions

PD Levin, A Szalat, M Vizana, CL Sprung, R Zaguri, Y Haviv

Hadassah Hebrew University Medical Center, Jerusalem, Israel

Critical Care 2013, 17(Suppl 2):P442 (doi: 10.1186/cc12380)

Introduction Intravenous magnesium sulfate is commonly used in obstetric patients with pre-eclampsia. Following a case of acute symptomatic hypocalcemia we retrospectively examined a cohort of patients to investigate the frequency of hypocalcemia.

Methods Obstetric patients were identified from the ICU admissions database and divided into two groups - those treated with magnesium (for suspected pre-eclampsia) and those admitted for other obstetric indications (postpartum hemorrhage, infection, etc.). The baseline calcium values were compared, as well as the lowest and discharge values. Albumin and magnesium values were also compared. All comparisons used Student's $t$ test.

Results Data were collected on 88 parturients admitted over 2 years including $40(45 \%)$ who received magnesium and $48(55 \%)$ who did not. Magnesium-treated women were younger (age: $31 \pm 7$ vs. $36 \pm 5$ years, $P=0.02)$. The baseline calcium concentrations were similar for the two groups ( $2.2 \pm 0.2$ vs. $2.2 \pm 0.1 \mathrm{mmol} / \mathrm{l}, P=0.85)$. Patients receiving magnesium had significantly higher magnesium concentrations $(2.1 \pm 0.4$ vs. $0.7 \pm 0.2 \mathrm{mmol} / \mathrm{l}, P<0.001)$, and significantly lower calcium concentrations during therapy $(1.6 \pm 0.3$ vs. $1.9 \pm 0.3 \mathrm{mmol} / \mathrm{l}$, $P<0.001$ ). At discharge, the calcium levels were closer (magnesium treated $1.9 \pm 0.2$ vs. untreated $2.1 \pm 0.1 \mathrm{mmol} / \mathrm{l}, P=0.02$ ). The albumin concentrations did not differ between the two groups (magnesium treated $27 \pm 13$ vs. nontreated $33 \pm 23 \mathrm{~g} / \mathrm{l}, P=0.134$ ). Normal values: calcium 2.15 to $2.55 \mathrm{mmol} / \mathrm{l}$, magnesium 0.7 to $0.95 \mathrm{mmol} / \mathrm{l}$, albumin 35 to $50 \mathrm{~g} / \mathrm{l}$.

Conclusion Magnesium therapy was associated with hypocalcemia. Potential causative mechanisms include a renal excretion interaction and magnesium-induced suppression of parathyroid hormone secretion. Physicians should be aware of the potential for symptomatic hypocalcemia during magnesium therapy.

P443

Hyponatremia and intensive care outcome

P Dean, R Docking, D Govenden, A Davidson, A Mackay

Victoria Infirmary, Glasgow, UK

Critical Care 2013, 17(Suppl 2):P443 (doi: 10.1186/cc12381)

Introduction Disorders of sodium $\left(\mathrm{Na}^{+}\right)$and water homeostasis are common in hospitalised patients. Hyponatremia in particular has been associated with worse hospital outcome and length of stay [1]. We aimed to define the incidence of hyponatremia (serum $\mathrm{Na}^{+}$ $\leq 134 \mathrm{mmol} / \mathrm{l}$ ) in our intensive care population and to determine whether it was associated with ICU outcome or length of stay.

Methods Demographics, APACHE II score, outcome data and admission sodium were retrieved from the Ward Watcher system in the Victoria Infirmary ICU for 2,440 consecutive admissions from January 2005 to present. We divided patients into three groups depending on serum $\mathrm{Na}^{+}$( $\leq 134 \mathrm{mmol} / \mathrm{l}, 135$ to $\left.144 \mathrm{mmol} / \mathrm{l}, \geq 145 \mathrm{mmol} / \mathrm{l}\right)$ and compared APACHE II score, length of stay and ICU outcome between patients with a low versus a normal serum $\mathrm{Na}^{+}$. Data were analysed using the chi-squared test, Student's $t$ test and the Mann-Whitney test where appropriate.

Results Of the 2,440 patients studied, 1,993 had APACHE II data and serum $\mathrm{Na}^{+}$recorded and so were included for analysis. In total, 453 patients $(22.7 \%)$ had a serum $\mathrm{Na}^{+} \leq 134 \mathrm{mmol} / \mathrm{l}$ and 1,388 patients (67.1\%) had a serum $\mathrm{Na}^{+}$of 135 to $144 \mathrm{mmol} / \mathrm{l}$. Patients with a low $\mathrm{Na}^{+}$ had a higher mortality $(\mathrm{OR}=1.48,95 \% \mathrm{Cl}=1.16$ to $1.90, P<0.001)$, a higher APACHE II score (22 vs. $19, P<0.001)$ and higher mean age ( 60 years vs. 58 years, $P<0.001$ ) than patients with a normal serum $\mathrm{Na}^{+}$. Mean length of stay of patients with low serum $\mathrm{Na}^{+}$was also longer (5.1 days vs. 4.6 days) although this was not statistically significant $(P=0.09)$.

Conclusion In summary, hyponatremia is a useful index of severity of illness in our ICU population. Whether this is a direct adverse effect of low serum sodium levels, or if hyponatremia is simply a marker for 'sicker' patients, is not known.

Reference

1. Asadollahi et al:: Hyponatraemia as a risk factor for hospital mortality. QJM 2006, 99:877-880.

P444

Strong ion gap can be accurately estimated with a simple bedside equation

L Busse, L Chawla, R Panchamia, D Choi, E Nobakht, E Brasha-Mitchell, M Seneff

George Washington University Medical Center, Washington, DC, USA

Critical Care 2013, 17(Suppl 2):P444 (doi: 10.1186/cc12382)

Introduction The anion gap (AG) is used routinely in the assessment of metabolic acidosis, but can be misleading in patients with hypoalbuminemia and other disorders commonly encountered in intensive care. This approach to acid-base analysis relies on assessment of $\mathrm{pH}, \mathrm{pCO}_{2}$, sodium, bicarbonate and chloride, and can lead to underestimation or overestimation of the true electrochemical status of a patient, as it does not include important ions such as lactate, calcium, magnesium, and albumin. The strong ion gap (SIG) is an alternative to the AG and is based upon Stewart's physical chemistry approach. However, the SIG is cumbersome to calculate. As such, a number of shortcut equations have been developed in an effort to approximate the SIG. We sought to compare three such equations, the Kellum corrected anion gap (KellAGc), the Moviat equation, and EZSIG, in an effort to evaluate precision and accuracy [1-3].

Methods We conducted a retrospective chart review of consecutive patients admitted to the ICU of George Washington University Medical Center from September 2010 to March 2011. Of the 1,516 patients screened, 200 met inclusion criteria, which included availability of all laboratory components to calculate the SIG, obtained within 1 hour of each other. Demographic data and serum values for $\mathrm{pH}, \mathrm{pCO}_{2}$, albumin, lactate, sodium, potassium, chloride, bicarbonate, magnesium, phosphate, and calcium were collected. The AG, SIG, KellAGc, EZSIG, and Moviat equations were subsequently calculated and compared using Pearson correlation and Bland-Altman analysis.

Results The mean SIG was $3.25 \pm 3.5$. Mean values for KellAGc, Moviat, and EZSIG were $4.5 \pm 5.0,1.77 \pm 2.2$, and $3.6 \pm 3.7$, respectively. Pearson correlation coefficients for KellAGc, Moviat, and EZSIG when compared with the SIG were $r=0.77, P=0.0001 ; r=0.88, P=0.001$; and $r=0.89$, $P=0.001$, respectively. In Bland-Altman analysis, the mean bias for the test equations versus the SIG were: KellAGc (1.25), Moviat (-1.48), and EZSIG (0.40).

Conclusion While all three equations correlated highly with the SIG, the EZSIG and Moviat outperformed the KellAGc in Pearson and BlandAltman analysis. The EZSIG had a smaller bias than the Moviat equation and a slightly better correlation ( 0.89 vs. 0.88 ). In the assessment of critically ill patients, EZSIG is a candidate scanning equation for the measurement of the SIG when all SIG components are not available.

References

1. Kellum JA: Crit Care 2005, 9:500-507.

2. Moviat M, et al: Crit Care 2003, 7:R41-R45

3. Busse L, et al:: Chest J 2011, 140(4_MeetingAbstracts):1012A-1012A.

P445

Relative adrenal insufficiency in burns

A Mokline, L Gharsallah, I Rahmani, H Oueslati, B Gasri, S Tlaili,

R Hammouda, A Ksontini, A Ghanem, AA Messadi

Burn and Trauma Center, Tunis, Tunisia

Critical Care 2013, 17(Suppl 2):P445 (doi: 10.1186/cc12383)

Introduction Relative adrenal insufficiency (RAI) is an uncommon disorder among burn patients, which can often go unrecognized. However, there are no or almost no data about the incidence of this disease in burns. The goal of the current study is to evaluate the incidence of RAl in burn patients during the acute phase.

Methods A prospective study, approved by our Institutional Ethics Committee, was conducted in a 20-bed adult burn ICU at a 
university-affiliated teaching hospital in Tunis. Patients admitted within the first 24 hours post burn with greater than $10 \%$ total body surface area (TBSA) burned were enrolled in this study from 1 January 2009 to 30 June 2010. Exclusion criteria were pregnancy, history of adrenal insufficiency, or steroid therapy within 6 months prior to burns. A short corticotrophin test $(250 \mu \mathrm{g})$ was performed, and cortisol levels were measured at baseline (CS T0) and 60 minutes post test. Adrenal insufficiency was defined by a response $\leq 9 \mu \mathrm{g} / \mathrm{dl}$. Relative adrenal insufficiency was further defined by a baseline cortisol $>20 \mu \mathrm{g} / \mathrm{dl}$. Results Patients were assigned into two groups: G1 (RAl, $n=7$ ) and G2 (absence Al, $n=11$ ). Comparative study of the two groups shows the results presented in Table 1.

Table 1 (abstract P445). Characteristics of the two groups

\begin{tabular}{lccc}
\hline & G1 & G2 & P value \\
\hline Age & $38 \pm 13$ & $34 \pm 12$ & NS \\
TBSA & $57.8 \pm 30$ & $26 \pm 22$ & 0.019 \\
UBS & $158 \pm 100$ & $70 \pm 99$ & NS \\
ABSI & $8 \pm 3$ & $6 \pm 3$ & NS \\
CST0 & $37 \pm 11$ & $19 \pm 14$ & 0.01 \\
DCS & $2.2 \pm 1.8$ & $19 \pm 6$ & $<0.001$ \\
Shock & $6(86 \%)$ & $4(36 \%)$ & 0.04 \\
\hline
\end{tabular}

Conclusion RAI is common in severely burned patients during the acute phase, and is associated with shock. Further prospective controlled studies will be necessary to establish risk factors of RAI in severely burned patients and its impact on their prognosis.

P446

Albumin-adjusted calcium concentration should not be used to identify hypocalcaemia in critical illness

T Steele', R Kolamunnage-Dona', C Downey², C Toh', I Welters

'University of Liverpool, UK; 'Royal Liverpool University Hospital, Liverpool, UK

Critical Care 2013, 17(Suppl 2):P446 (doi: 10.1186/cc12384)

Introduction Hypocalcaemia is common in critical illness and accurate assessment is crucial. Small studies have shown that albumin-adjusted calcium (adjCa) does not accurately predict the ionised calcium (iCa) concentration in critically ill patients, yet adjCa continues to be widely used [1]. We investigated the reliability of using adjCa to identify hypocalcaemia in a large, diverse population requiring intensive care. Methods $\mathrm{In}$ a retrospective study of patients admitted to the ICUs of a tertiary care hospital between January 2008 and 2012, iCa and pH were extracted from routine blood gas results and total calcium, albumin and phosphate from routine biochemistry results. adjCa was calculated using a formula derived from and validated on the local population [2]. Sensitivity, specificity, positive and negative predictive values (PPV and NPV) and area under the curve (AUC) of adjCa for predicting hypocalcaemia (iCa $<1.1 \mathrm{mmol} / \mathrm{l})$ were calculated.

Results Data from 1,038 patients were included. Admission iCa was available for 976 (94\%) patients. A total of 539 (55\%) were hypocalcaemic (iCa $<1.1 \mathrm{mmol} / \mathrm{l})$. adjCa was available for 1,031 (99\%), with 602 (58\%) classified as hypocalcaemic (adjCa $<2.2 \mathrm{mmol} / \mathrm{l})$. adjCa $<2.2 \mathrm{mmol} / \mathrm{l}$ had sensitivity of $78 \%$ and specificity of $63 \%$ for detecting iCa $<1.1 \mathrm{mmol} / \mathrm{l}$. The best previously published formula had an AUC of $0.81(95 \% \mathrm{Cl}=0.78$ to 0.83$)$, compared with an AUC of 0.78 (0.75 to 0.81$)$ for the locally validated formula [1]. It was hypothesized that albumin, $\mathrm{pH}$ or phosphate derangements could affect the proportion of albuminbound calcium and the performance of adjustment formulae. Albumin concentration was not correlated with the difference between adjCa and iCa. Weak but statistically significant correlations were found for $\mathrm{pH}$ $(r=0.164 ; P<0.001)$ and phosphate $(r=0.135 ; P<0.001)$. The sensitivity and specificity of adjCa $<2.2 \mathrm{mmol} / \mathrm{l}$ for detecting hypocalcaemia did not improve when looking at patients with normal pH (sensitivity $60.4 \%$; specificity $69 \%$ ) or phosphate (32.2\%; $78.9 \%)$.

Conclusion adjCa is a relatively poor predictor of iCa in critically ill patients. This cannot be explained by abnormalities of albumin, phosphate or $\mathrm{pH}$. With direct iCa measurements now readily available on ward-based analysers, adjCa should not be used to determine calcium status in critical illness.

References

1. Slomp J, et al:: Albumin-adjusted calcium is not suitable for diagnosis of hyper- and hypocalcemia in the critically ill. Crit Care Med 2003, 31:1389-1393.

2. James $M$, et al.: Derivation and internal validation of an equation for albumin-adjusted calcium. BMC Clin Pathol 2008, 8:12.

P447

Vitamin D status and overall and sepsis mortality in critically ill patients

P Zajic, C Schnedl, TValentin, A Grisold, A Holl, S Farzi, S Fruhwald, H Dobnig, TR Pieber, K Amrein

Medical University of Graz, Austria

Critical Care 2013, 17(Suppl 2):P447 (doi: 10.1186/cc12385)

Introduction Vitamin D status may affect hospital mortality and infectious complications in critical illness.

Methods A total of 655 mixed critically ill patients with available 25(OH)D levels hospitalized between 2008 and 2010 were included. Cox regression analysis adjusted for SAPS 2, age and gender was performed. 25(OH)D levels were categorized by month-specific tertiles (high, intermediate, low) to reflect seasonal variation. Primary endpoint was the correlation between $25(\mathrm{OH}) \mathrm{D}$ and hospital mortality. Secondary endpoints were sepsis mortality and blood culture positivity.

Results Hospital mortality was significantly higher in patients in the low $(H R=1.98)$ and the mid-range tertile $(H R=1.88)$ compared with the highest tertile. Vitamin D levels were significantly lower in patients with lethal sepsis compared with other causes of death $(12.3 \pm 5.0$ vs. $18.2 \pm 11.2, P=0.02$ ). Blood culture positivity rates did not differ between the groups ( $23.0 \%$ vs. $26.8 \%$ vs. $17.3 \%, P=0.361)$. See Table 1 .

Table 1 (abstract P447)

\begin{tabular}{lcccc}
\hline Tertile & $\begin{array}{c}\text { Low } \\
(\boldsymbol{n}=\mathbf{2 1 6})\end{array}$ & $\begin{array}{c}\text { Intermediate } \\
(\boldsymbol{n}=\mathbf{2 1 9})\end{array}$ & $\begin{array}{c}\text { High } \\
(\boldsymbol{n}=\mathbf{2 2 0})\end{array}$ & P value \\
\hline $25(\mathrm{OH}) \mathrm{D}(\mathrm{ng} / \mathrm{ml})$ & $10.4( \pm 3.6)$ & $17.6( \pm 5.1)$ & $30.7( \pm 11.4)$ & $<0.001$ \\
SAPS 2 & $32( \pm 15)$ & $28( \pm 16)$ & $28( \pm 16)$ & 0.007 \\
Hospital mortality & $58(26.9 \%)$ & $48(21.2 \%)$ & $29(13.2 \%)$ & 0.002 \\
Lethal sepsis & $11(5.1 \%)$ & $9(4.1 \%)$ & $0(0.0 \%)$ & 0.005 \\
\hline
\end{tabular}

Conclusion Low 25(OH)D status is predictive of all-cause and sepsis mortality in the critically ill. Interventional studies are needed to investigate the effect of vitamin D on mortality and sepsis incidence and outcomes.

\section{P448}

Etiology of low testosterone levels in male patients with severe sepsis requiring mechanical ventilation

A Bech, HVan Leeuwen, $\mathrm{H}$ De Boer

Rijnstate Hospital, Arnhem, the Netherlands

Critical Care 2013, 17(Suppl 2):P448 (doi: 10.1186/cc12386)

Introduction Low testosterone levels are frequently found in critically ill male patients. The etiology and clinical significance is still poorly understood. In the present study we have investigated the kinetics and pathophysiology of altered gonadal hormone synthesis in male patients with severe sepsis and respiratory failure.

Methods All male patients with severe sepsis and respiratory failure who were admitted to the ICU of a large teaching hospital in the Netherlands between September 2011 and June 2012 were included. Steroid hormone levels were measured on days 1, 3 and 7 .

Results In total, 18 patients were included. The mean age was $69 \pm 2$ years, mean weight $76 \pm 2 \mathrm{~kg}$, APACHE II score $23 \pm 2$ and most patients suffered from pneumosepsis. On the first day of intubation, total and free testosterone levels were extremely low in most patients and remained low during the first week (Figure 1). 17 $\beta$-Estradiol levels were elevated on day 1 and decreased during the first week. LH and 

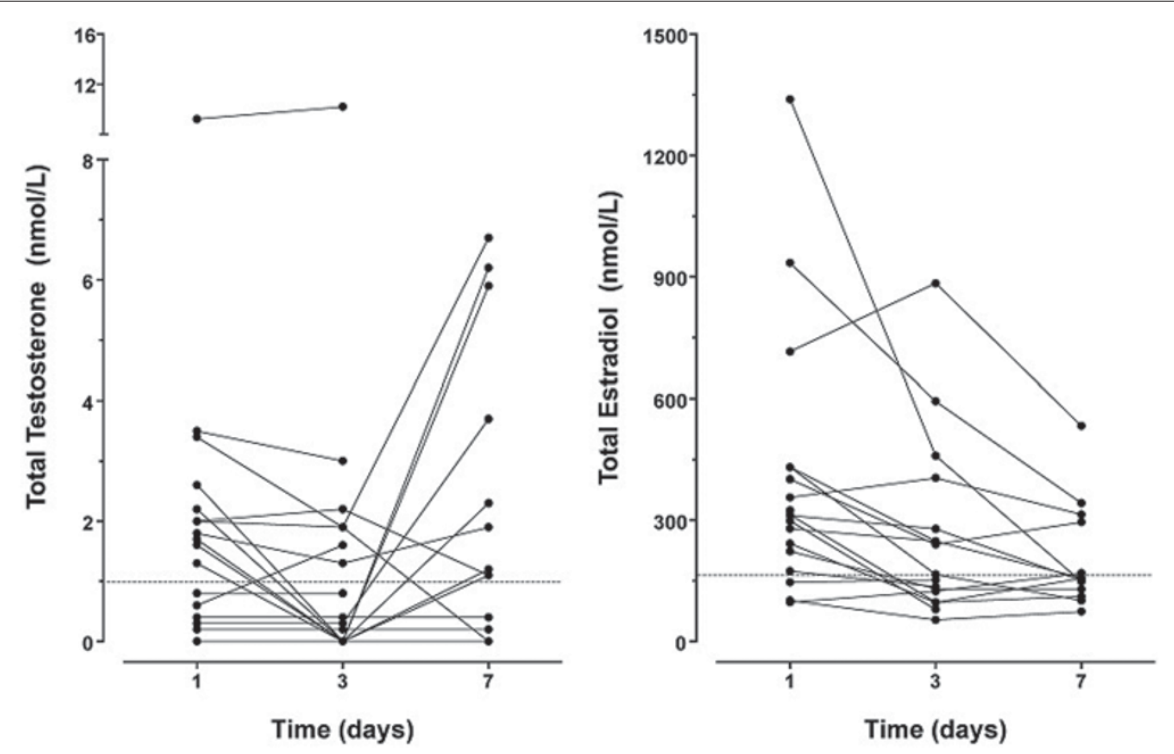

Figure 1 (abstract P448).

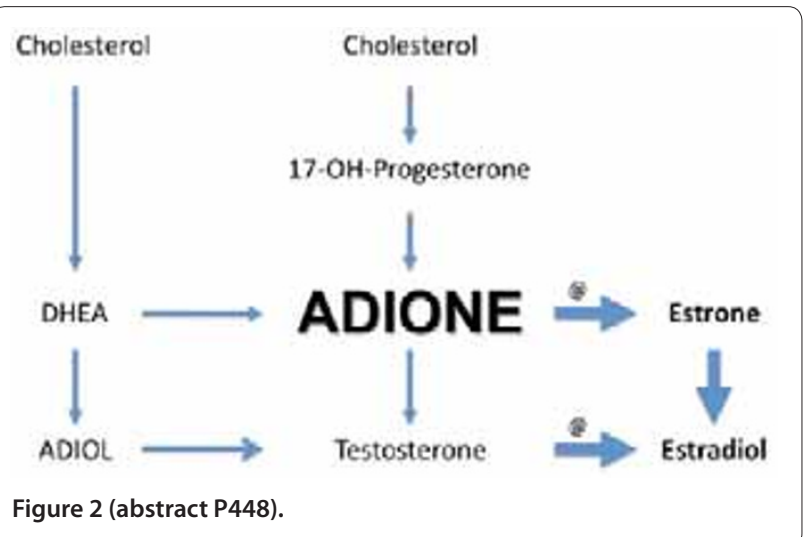

FSH levels were inappropriately low. All lipoprotein fractions and their apo-proteins were reduced as well as 17-OH-progesterone, DHEA and DHEAS. In contrast, androstenedione (adione) levels were elevated. This suggests preferential and stimulated synthesis of androstenedione (Figure 2). The high 17 $\beta$-estradiol levels indicate that androstenedione is shunted into the estrogen pathway, a process that requires high aromatase activity. The high estradiol/total testosterone ratio supports this conclusion.

Conclusion Hyperestrogenic hypotestosteronemia is a frequent finding in the acute phase of severe sepsis in male patients with respiratory failure. It is suggested to be caused by decreased androgen production and shunting of androgen to estrogen synthesis as a result of increased aromatase activity. The clinical relevance of gonadal hormone substitution needs further study.

P449

Efficacy of calcium replacement in hypocalcaemia

H Chatha, K Sim

Whiston Hospital, Liverpool, UK

Critical Care 2013, 17(Suppl 2):P449 (doi: 10.1186/cc12387)

Introduction The aim of the study is to examine the efficacy of calcium replacement in patients with hypocalcaemia in the ICU. Hypocalcaemia affects between 15 and $50 \%$ of admissions to the ICU [1].

Methods Adjusted calcium results $<2.0 \mathrm{mmol} / \mathrm{l}$ were followed up from admission for 5 days or until discharge or death. On each day the adjusted calcium result and the calcium replacement were documented. Adequate replacement is defined as $40 \mathrm{ml}$ of $10 \%$ calcium gluconate if the adjusted calcium is $<2.0 \mathrm{mmol} / \mathrm{l}$ and $20 \mathrm{ml}$ of $10 \%$ calcium gluconate if the adjusted calcium result is between 2.0 and $2.15 \mathrm{mmol} / \mathrm{l}$.

Results Eighty-eight adjusted calcium results were $<2.0 \mathrm{mmol} / \mathrm{l}$. In 74 results adequate replacement was given and the average increase in calcium was $0.2 \mathrm{mmol} / \mathrm{l}$. This led to an increase in the adjusted calcium to $>2.15 \mathrm{mmol} / \mathrm{l}$ in $36 \%$ of results and improvement to $>2.0 \mathrm{mmol} / \mathrm{l}$ in $89 \%$ of results. In 14 results no replacement was given and the average increase in calcium was $0.07 \mathrm{mmol} / \mathrm{l}$, which equated to $57 \%$ of calcium results remaining $<2.0 \mathrm{mmol} / \mathrm{l}$. Unpaired $t$ test showed a $P$ value of 0.0006 . A total of 150 adjusted calcium results were between 2.0 and $2.15 \mathrm{mmol} / \mathrm{l}$. In 111 results adequate replacement was given and the average increase was $0.006 \mathrm{mmol} / \mathrm{l}$. The adjusted calcium fell to $<2.0 \mathrm{mmol} / \mathrm{l}$ in $26 \%$ of results and normalised to $>2.15 \mathrm{mmol} / \mathrm{l}$ in $23 \%$. Where the adjusted calcium fell despite replacement, $51 \%$ of results were below $2.05 \mathrm{mmol} / \mathrm{l}$. In 39 results no replacement was given, leading to an average fall in calcium of $0.03 \mathrm{mmol} / \mathrm{l}$. Thirtythree percent of calcium results fell to $<2.0 \mathrm{mmol} / \mathrm{l}$ and $20 \%$ of results normalised. Unpaired $t$ test showed a $P$ value of 0.07 .

Conclusion Calcium replacement with $40 \mathrm{ml}$ of $10 \%$ calcium gluconate is effective when the adjusted calcium is $<2.0 \mathrm{mmol} / \mathrm{l}$. Replacement with $20 \mathrm{ml}$ of $10 \%$ calcium gluconate when the adjusted calcium is between 2.0 and $2.15 \mathrm{mmol} / \mathrm{l}$ is not much more effective than no replacement. This suggests that either replacement is not needed or there is under-replacement in mild hypocalcaemia. Adjusted calcium may be giving falsely low calcium results in mild hypocalcaemia. The measurement of ionised calcium is a possible solution $[2,3]$.

\section{References}

1. Zaloga GP: Crit Care Med 1992, 20:251-262.

2. Slomp J, et al:: Crit Care Med 2003, 31:1389-1393.

3. Byrnes MC, et al:: Am J Surg 2005, 189:310-314.

\section{P450}

Endogenous melatonin in critically ill patients

VS Salice, IG Galluccio, BS Salihovic, IP Piva, FM Marazzo, CV Villa,

MT Taverna, MU Umbrello, GM Mistraletti, Gl lapichino Università degli Studi Milano, Milan, Italy

Critical Care 2013, 17(Suppl 2):P450 (doi: 10.1186/cc12388)

Introduction Melatonin could have a meaningful role in critically ill patients, because of its immunomodulatory, antioxidant and sleep regulation properties; it is reduced in critical illness. The purpose of 


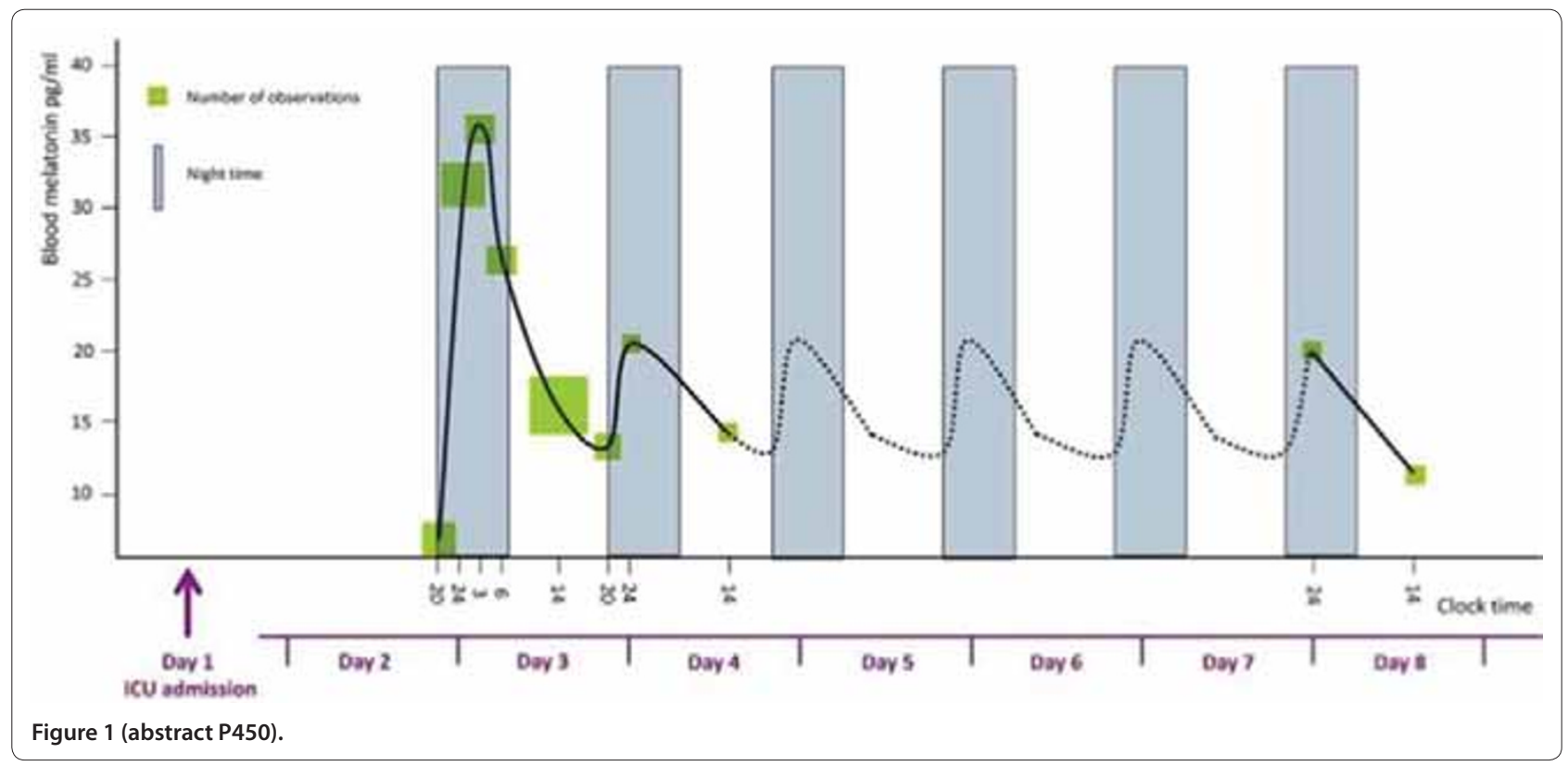

this study is to describe the endogenous blood melatonin values in ICU patients and their correlation with clinical parameters.

Methods Seventy-three high-risk critically ill patients mechanically ventilated for $>48$ hours were enrolled. Blood samples for melatonin assay were collected between the 3rd and the 8th day of the ICU stay. Melatonin was determined by radioimmunoassay and ELISA. The peak and the area under the curve (AUC) calculated for each patient were correlated with the clinical parameters using the regression for quantiles test.

Results Endogenous melatonin was found lower in critically ill patients compared with healthy subjects (Figure 1), although it showed a great individual variability and it generally maintained a night-time increase. In the univariate analysis the peak was found related to: blood creatinine $(P=0.034)$; patients in coma $(P=0.024)$; hospital mortality $(P=0.016)$. The AUC was found related to: SAPS II $(P=0.047)$; creatinine $(P<0.001)$; AST $(P<0.001)$; ALT $(P<0.001)$; hospital mortality $(P<0.022)$. Peak and AUC were found higher in nonsurvivor patients.

Conclusion In accord with previous studies, the endogenous blood melatonin was found reduced in ICU patients. The higher melatonin peak in renal failure may be due to an increased distribution volume; greater AUC in patients with liver failure could be due to a less efficient removal of the hormone from the systemic circulation. The finding of increased peak and AUC in nonsurvivor patients could be due to a hormonal response increased by the body stress reaction, potentially similar to cortisol [1], or to a higher production of a physiological antioxidant [2] with a decreased ability to use it.

References

1. Venkatesh B, et al.: Best Practice Res Clin End Met 2011, 25:719-733.

2. Flaring UB, et al:: Intensive Care Med 2005, 31:1072-1078.

\section{P451}

Succinate ameliorates mitochondrial oxygen consumption of metformin-intoxicated human platelets

A Protti', M Monti', A Lecchi', A Artoni', N Greppi', L Gattinoni ${ }^{1}$

'Fondazione IRCCS Ca' Granda Ospedale Maggiore Policlinico, Milan, Italy;

¿2Università degli Studi di Milano, Milan, Italy

Critical Care 2013, 17(Suppl 2):P451 (doi: 10.1186/cc12389)

Introduction Metformin intoxication inhibits mitochondrial complex I and oxygen consumption $\left(\mathrm{VO}_{2}\right)$. Succinate bypasses complex I by donating electrons to complex II. The aim of this study was to clarify whether succinate ameliorates mitochondrial $\mathrm{VO}_{2}$ of metforminintoxicated human platelets.

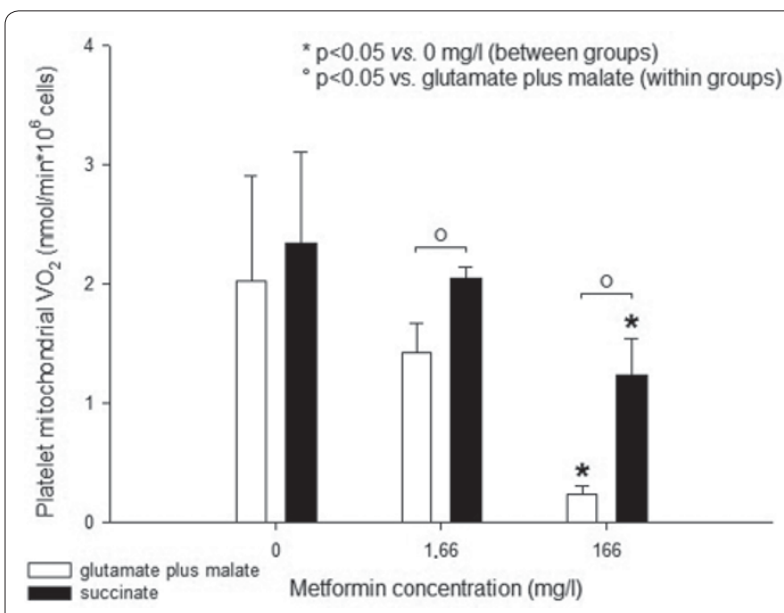

Figure 1 (abstract P451). Mitochondrial oxygen use of human platelets incubated with metformin.

Methods Platelet-rich-plasma was incubated for 72 hours with metformin at a final concentration of $0 \mathrm{mg} / \mathrm{l}$ (control), $1.66 \mathrm{mg} / \mathrm{l}$ (therapeutic dose) or $166 \mathrm{mg} / \mathrm{l}$ (toxic dose). Platelet $\mathrm{VO}_{2}$ was then measured with a Clark-type electrode, in the presence of glutamate plus malate (complex I electron donors) (final concentration: $20 \mathrm{mmol} / \mathrm{l}$ for both) or succinate (complex II electron donor) $(30 \mathrm{mmol} / \mathrm{l})$, before and after adding cyanide $(40 \mathrm{mmol} / \mathrm{l})$. Mitochondrial (cyanide-sensitive) and extra-mitochondrial (cyanide-insensitive) $\mathrm{VO}_{2}$ were corrected for platelet count.

Results The main results, from four preliminary experiments, are shown in Figure 1. In the presence of glutamate plus malate, only platelets incubated with a high dose of metformin had a mitochondrial $\mathrm{VO}_{2}$ significantly lower than controls. In the presence of succinate, mitochondrial $\mathrm{VO}_{2}$ of controls did not change significantly whereas that of platelets incubated with metformin did. The effect of succinate tended to become larger as the dose of metformin was increased from 0 up to $166 \mathrm{mg} / \mathrm{l}\left(0.3 \pm 0.2\right.$ vs. $0.6 \pm 0.3$ vs. $1.0 \pm 0.3 \mathrm{nmol} / \mathrm{minute}^{*} 10^{6}$ cells) $(P=0.068)$. Even so, mitochondrial $\mathrm{VO}_{2}$ of platelets incubated with the highest dose of metformin did not return to the levels of controls. Extra-mitochondrial $\mathrm{VO}_{2}$ was always the same. 
Conclusion Succinate ameliorates (but does not return to normal) mitochondrial $\mathrm{VO}_{2}$ of human platelets incubated with a toxic dose of metformin.

\section{P452}

Does chronic metformin therapy offer cardiomyoprotection in coronary artery bypass grafting surgery patients? A case-control study

A Delaporte, JP Hacquebard, P Origer, I Pastijn, M Maatouk, O Germay,

E Stevens, P Kapessidou, A Roman

CHU Saint-Pierre, Brussels, Belgium

Critical Care 2013, 17(Suppl 2):P452 (doi: 10.1186/cc12390)

Introduction Metformin, widely used as an antidiabetic drug, activates the AMP activated protein kinase, a key regulator of the metabolism providing protection under fuel deficiency. Chronic metformin therapy has been shown in long-term follow-up clinical studies to reduce cardiovascular mortality [1]. In animal experiments, acute metformin pretreatment has been shown to reduce ischemia-reperfusion injury on cardiomyocytes [2]. We want to evaluate whether outcomes are affected in coronary artery bypass grafting (CABG) surgery.

Methods We performed a retrospective case-control study on 341 patients admitted exclusively for CABG surgery with cardiopulmonary bypass between January 2006 and April 2012. Eighty-one patients chronically on metformin were matched 1:3 with nonmetformintreated ones using gender, age, body mass index and number of arterial bypass grafts. Global mortality, ICU-free 28 postoperative days and creatinine kinase MB (CKMB) at postoperative baseline, 8 and 18 hours post baseline were compared.

Results Metformin-treated patient mortality was not significantly lower than in nonmetformin (1.2\% vs. 3.1\%, $P=0.24$, Fischer exact test). ICU-free 28 postoperative days were higher in the metformin group (median 23.4 days; $95 \% \mathrm{Cl}=22.5$ to 24.3 ) than in the nonmetformin group (median 22.9 days; $95 \% \mathrm{Cl} 22.3$ to $23.5, P<0.04$, Wilcoxon test). Median CKMB levels at postoperative baseline were 21 versus $26 \mathrm{ng} / \mathrm{ml}$ $(P<0.003)$, at 8 hours were 22 versus $53 \mathrm{ng} / \mathrm{ml}(P<0.004)$ and 18 hours post baseline were 21.5 versus $27.5 \mathrm{ng} / \mathrm{ml}(P<0.012)$. See Table 1 .

Table 1 (abstract P452). Case-control analysis

\begin{tabular}{lccc}
\hline & Metformin & Nonmetformin & $\begin{array}{c}\text { Statistics, } \\
\boldsymbol{P} \text { value }\end{array}$ \\
\hline Mortality & $1 / 81$ & $8 / 260$ & 0.24 \\
28 days free ICU (days) & 23.4 & 22.9 & $<0.04$ \\
CKMBT 0 hours $(\mathrm{ng} / \mathrm{ml})$ & 21 & 26 & $<0.003$ \\
CKMB T+8 hours $(\mathrm{ng} / \mathrm{ml})$ & 22 & 53 & $<0.004$ \\
CKMB +18 hours $(\mathrm{ng} / \mathrm{ml})$ & 21.5 & 27.5 & $<0.012$ \\
\hline
\end{tabular}

Conclusion These data suggest that chronic preoperative treatment with metformin is associated with lower CKMB levels at postsurgery baseline, 8 and 18 hours post baseline with a shorter ICU stay in patients receiving $C A B G$ surgery under extracorporeal circulation.

References

1. Roussel R, et al:: Arch Intern Med 2010, 170:1892-1899.

2. Gundewar S, et al:: Circ Res 2009, 104:403-411.

P453

Metformin-associated lactic acidosis: risk factors and prognostic

factors

V Di Falco, A Milano, M Battilana, F Araosta, A Grosso, D Albanese,

F Petrini, L Di Liberato

ASL Chieti SS. Annunziata, Chieti, Italy

Critical Care 2013, 17(Suppl 2):P453 (doi: 10.1186/cc12391)

Introduction Metformin, an oral hypoglycemic drug, belongs to the biguanide class and is now generally accepted as first-line treatment in type 2 diabetes mellitus, especially in overweight patients [1]. In some predisposing conditions, the use of metformin may result in metforminassociated lactic acidosis (MALA), a rare adverse event associated with a high mortality rate [2]. The aim of this study is to assess risk factors and prognostic factors in patients with MALA.

Methods We conducted a retrospective study of patients with MALA admitted to the ICU of ASL 2 Chieti between 1 January 2008 and 30 September 2012. The eligibility criteria were: diagnosis of diabetes mellitus type 2 , treatment with oral hypoglycemic drugs containing metformin, increased anion gap metabolic acidosis $\left(\mathrm{pH}<7.35, \mathrm{HCO}_{3}^{-}\right.$ $<22 \mathrm{mmol} / \mathrm{l}$, lactate $>5 \mathrm{mmol} / \mathrm{l})$. For each patient we evaluated: sex, age, home care, SAPS II score [3], blood tests.

Results Ten patients were selected, five males and five females, with a mean age of 72.2. On admission, nine of 10 patients had a framework of general illness and acute renal failure; one patient appeared with a probable acute abdomen not subsequently confirmed on CT. Eight patients had a state of dehydration resulting from gastroenteritis, diarrhea, fever; two patients had taken NSAIDs in the days prior to hospitalization. No patient, in these conditions, has discontinued treatment with metformin. All patients showed a critical clinical framework with a SAPS II mean score of 77.5. Cardiovascular and respiratory support was required in all cases. The hemogas analysis showed that patients had a severe metabolic acidosis (mean $\mathrm{pH}=6.96$ ) with increased plasma lactate (mean lactate $=15.93 \mathrm{mmol} / \mathrm{l}$ ). Prothrombin activity was normal in eight of 10 patients. The overall mortality was $70 \%$.

Conclusion Patient education on correct use of metformin is essential to prevent MALA, specially in those clinical conditions of increased risk (for example, acute renal failure), as recommended by the AIFA 2011 guidelines [4]. In our study, a higher plasma concentration of lactate represents the main negative prognostic factor, as pointed out by other studies [5]. The prothrombin activity, which is considered to be a decisive prognostic factor in the study of Peters and colleagues [6], was not impaired in patients with poor outcome.

References

1. UK Prospective Diabetes Study Group: Lancet 1998, 352:854-865.

2. Lalau JD: Drug Saf 2010, 33:727-740.

3. Le Gall JR, et al: JAMA 1993, 270:2957-2963.

4. Italian Medicines Agency 2011 [http://www.agenziafarmaco.gov.it/it/content/ raccomandazioni-sull\%E2\%80\%99utilizzo-dei-medicinali-base-di-metforminanella-gestione-del-diabete-m]

5. Dell'Aglio DM, et al: Ann Emerg Med 2009, 54:818-823.

6. Peters N, et al: Crit Care 2008, 12:R149.

\section{P454}

Multiscale modeling of acute insulin resistance in critical care

A Pritchard-Bell', G Clermont², B Yegneswaran², R Parker'

'University of Pittsburgh, PA, USA; ${ }^{2}$ University of Pittsburgh Medical Center,

Pittsburgh, PA, USA

Critical Care 2013, 17(Suppl 2):P454 (doi: 10.1186/cc12392)

Introduction Stress hyperglycemia in the critically ill is a complex process in which insulin signaling is systematically hijacked to provide energy substrate for metabolic priorities such as cell healing or infection containment. Fluctuating levels of plasma glucose are associated with increased mortality in the ICU [1]. We develop a multiscale mathematical model that can characterize the severity of stress hyperglycemia based on a fundamental understanding of the signaling molecules involved.

Methods Insulin resistance following insult has been shown to be driven primarily by the immune response via the cytokine IL- 6 [2]. We created a multiscale mathematical model that links circulating glucose and insulin concentration dynamics from the extended minimal model [3] to a cellular insulin response model [4] that captures insulinmediated glucose uptake in an insulin-responsive cell.

Results Inhibitory dynamics driven by IL- 6 were incorporated into the cellular model to attenuate an insulin signaling intermediate (insulin receptor substrate 1) according to the proposed biological mechanisms. The percentage reduction in glucose uptake as a function of IL-6 concentration was fit to data from patients who underwent elective abdominal surgery [2], shown in Figure 1. The overall multiscale model captures decreased insulin signaling as a result of increased IL- 6 levels and the subsequent hyperglycemia that may ensue. 


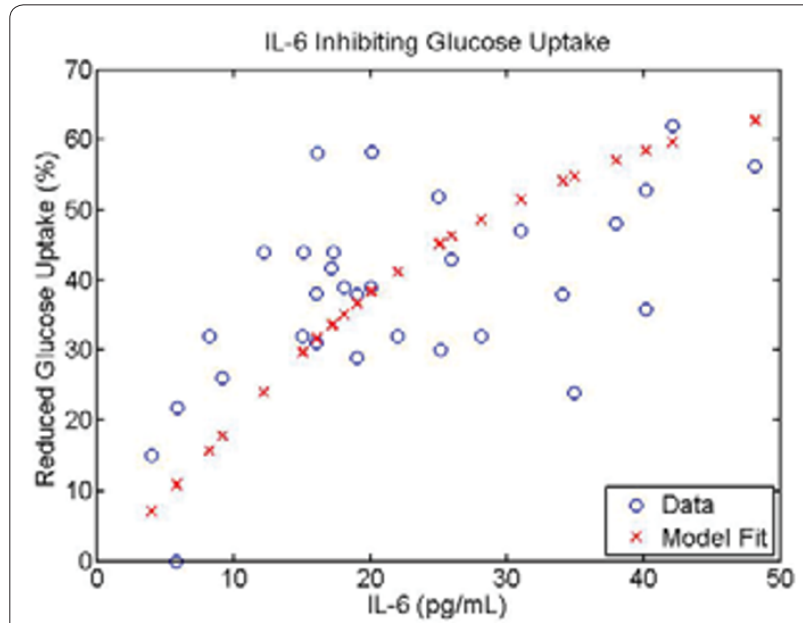

Figure 1 (abstract P454). Reduced glucose uptake driven by increased plasma IL-6 levels.

Conclusion A multiscale model has been developed to describe the inhibitory effects of IL-6 on insulin-mediated glucose uptake. Cellular inhibitory dynamics were shown to capture reduced insulin sensitivity on the macroscale, which could then be used to characterize insulin sensitivity and to provide insulin treatment advice to reduce glucose variability.

References

1. Van den Berghe G: NEngl J Med 2001, 345:1359-1367.

2. Thorell A: Clin Nutr 1996, 15:75-79.

3. Roy A: Diabetes Technol Ther 2006, 8:617-626

4. Sedaghat A: Am J Physiol Endocrinol Metab 2002, 283:E1084-E1 101.

\section{P455}

Impact of a real-time electronic persistent hyperglycemia alert on glucose control in the ICU

K Colpaert, S Oeyen, T van den Driessche, C Danneels, J Decruyenaere Ghent University Hospital, Ghent, Belgium

Critical Care 2013, 17(Suppl 2):P455 (doi: 10.1186/cc12393)

Introduction Hyperglycemia is frequently encountered in critically ill patients, and associated with adverse outcome. Improvement of glucose protocol adherence may be accomplished using electronic alerts. We configured a non-intrusive real-time electronic alert, called a GLYC sniffer, as part of our Intensive Care Information System (ICIS) that continuously evaluates the occurrence of persistent hyperglycemia and hypoglycemia. The GLYC sniffer is configured in such a way to give an alert: if two consecutive glucose values are $>150 \mathrm{mg} / \mathrm{dl}$, with a minimum interval of 60 minutes; or if a glucose value is $<80 \mathrm{mg} / \mathrm{dl}$. We wanted to evaluate whether the GLYC sniffer would improve the glucose control.

Methods A single-center, prospective intervention study during a 6 -month period in our 22-bed surgical ICU. Two study phases were compared: a 3-month pre-alert phase with no alerting to the nurses, and a 3-month intervention phase where the GLYC sniffer was alerting through the Clinical Notification System of our ICIS.

Results A total of 652 different patients having a total of 699 admission episodes was recorded during the study period. There were no significant differences between the two study groups regarding baseline demographic data, first glucose value upon admission. A total of 2,335 GLYC sniffer alerts were recorded during the whole study period: $84.3 \%$ persistent hyperglycemia alerts $(1,021$ in the pre-alert group, 948 in the alert group), and $15.7 \%$ hypoglycemia alerts (139 vs. 227). A significantly lower percentage of glucose values in the alert group were hyperglycemic $(19.5 \%$ vs. $26.5 \%, P<0.001)$. The proportion of persistent hyperglycemic values (i.e. consecutive glucose values exceeding the limit of $150 \mathrm{mg} / \mathrm{dl}$ ) was significantly lower in the alert group $(9.9 \%$ vs. $15.4 \%, P<0.001)$. The patients in the alert group spend significantly more time within the set target glucose interval of 80 to $150 \mathrm{mg} / \mathrm{dl}(82.3 \%$ vs. $75.0 \%, P=0.009)$. A significantly lower proportion of patients experienced a new-onset hypoglycemic event ( $<80 \mathrm{mg} / \mathrm{dl}$ ) in the alert group ( $19.3 \%$ vs. $26.2 \%, P=0.04$ ).

Conclusion A real-time electronic persistent glycemia sniffer resulted in a significantly higher proportion of normoglycemia, without increasing the variability. Furthermore, hypoglycemic events occurred less frequently, and were resolved more timely. Smart alerting is able to improve quality of care, while diminishing the problem of alert fatigue.

\section{P456}

Individualized targeted glucose control to avoid hypoglycemia

SP Gawel', G Clermont², T Ho', BM Newman ³, B Yegneswaran², RS Parker' 'University of Pittsburgh, PA, USA; '2University of Pittsburgh Medical Center, Pittsburgh, PA, USA; 3 lowa State University, Ames, IA, USA

Critical Care 2013, 17(Suppl 2):P456 (doi: 10.1186/cc12394)

Introduction Hyperglycemia and hypoglycemia have been linked to worse outcomes in critically ill patients. While there is controversy as to the optimal tightness of glucose control in critically ill patients, there is agreement that an upper limit to safe glucose levels exists and that avoiding hypoglycemic episodes should be prioritized. Our algorithm can assist clinicians in maintaining blood glucose ([Gbl]) within a desired target range while avoiding hypoglycemia.

Methods Our model predictive control (MPC) algorithm uses insulin and glucose as control inputs and a linearized model of glucoseinsulin-fatty acid interactions. To allow the controller model to learn from data, a moving horizon estimation (MHE) technique tailored the tissue sensitivity to insulin to individual responses. Patient data ([Gbl] measurements, insulin and nutritional infusion rates) were from the HIDENIC database at the University of Pittsburgh Medical Center. [Gbl] measurements, typically hourly, were interpolated to impute a measurement every 5 minutes. The model captured patient [Gbl] via nonlinear least squares by adjusting insulin sensitivity (SI) and endogenous glucose production (EGP0). The resulting virtual patient (VP) is used to evaluate the performance of the MPC-MHE algorithm. Results MPC controller performance on one VP is shown in Figure 1. Across a population of $10 \mathrm{VPs}$, the average [Gbl] under MPC is $6.31 \mathrm{mmol} / \mathrm{l}$, the average minimum is $4.62 \mathrm{mmol} / \mathrm{l}$, the population individual minimum is $3.49 \mathrm{mmol} / \mathrm{l}$ and the average absolute average residual error is $0.83 \mathrm{mmol} / \mathrm{l}$ from a $5.6 \mathrm{mmol} / \mathrm{l}$ target. With standard intervention, the $10 \mathrm{VPs}$ have an average [Gbl] of $9.32 \mathrm{mmol} / \mathrm{l}$, an average minimum [Gbl] of $3.77 \mathrm{mmol} / \mathrm{l}$, and a population minimum [Gbl] of $2.78 \mathrm{mmol} / \mathrm{l}$. Algorithm performance deteriorates significantly if the imputed sampling time exceeds 30 minutes, underlining the importance of dynamic variations in insulin sensitivity in this population.

Conclusion The MPC-MHE algorithm achieves targeted glucose control in response to changing patient dynamics and multiple measured disturbances for a pilot population of 10 VPs. Furthermore, the MHE scheme updates patient parameters in real time in response to changing patient dynamics.

P457

Hyperglycemia in intensive care identifies patients at high risk of developing diabetes: preliminary data of a multicentre study E Lukic, I Gornik, M Grgic-Medic, D Milicic, V Vegar, V Ivancan, M Peric,

$\checkmark$ Gasparovic, I Pavlic Renar

University Hospital Centre Zagreb, Croatia

Critical Care 2013, 17(Suppl 2):P457 (doi: 10.1186/cc12395)

Introduction A recent study showed that hyperglycaemia (blood glucose $\geq 7.8 \mathrm{mmol} / \mathrm{l})$ in nondiabetic patients hospitalised in a medical ICU is associated with increased risk of diabetes [1]. We investigated a large mixed ICU population to confirm these results.

Methods This study retrospectively included patients with negative history of diabetes admitted to ICUs during the year 2007. We excluded patients receiving steroids, with newly diagnosed diabetes and those with end-stage disease. Patients were followed-up 5 years after index 


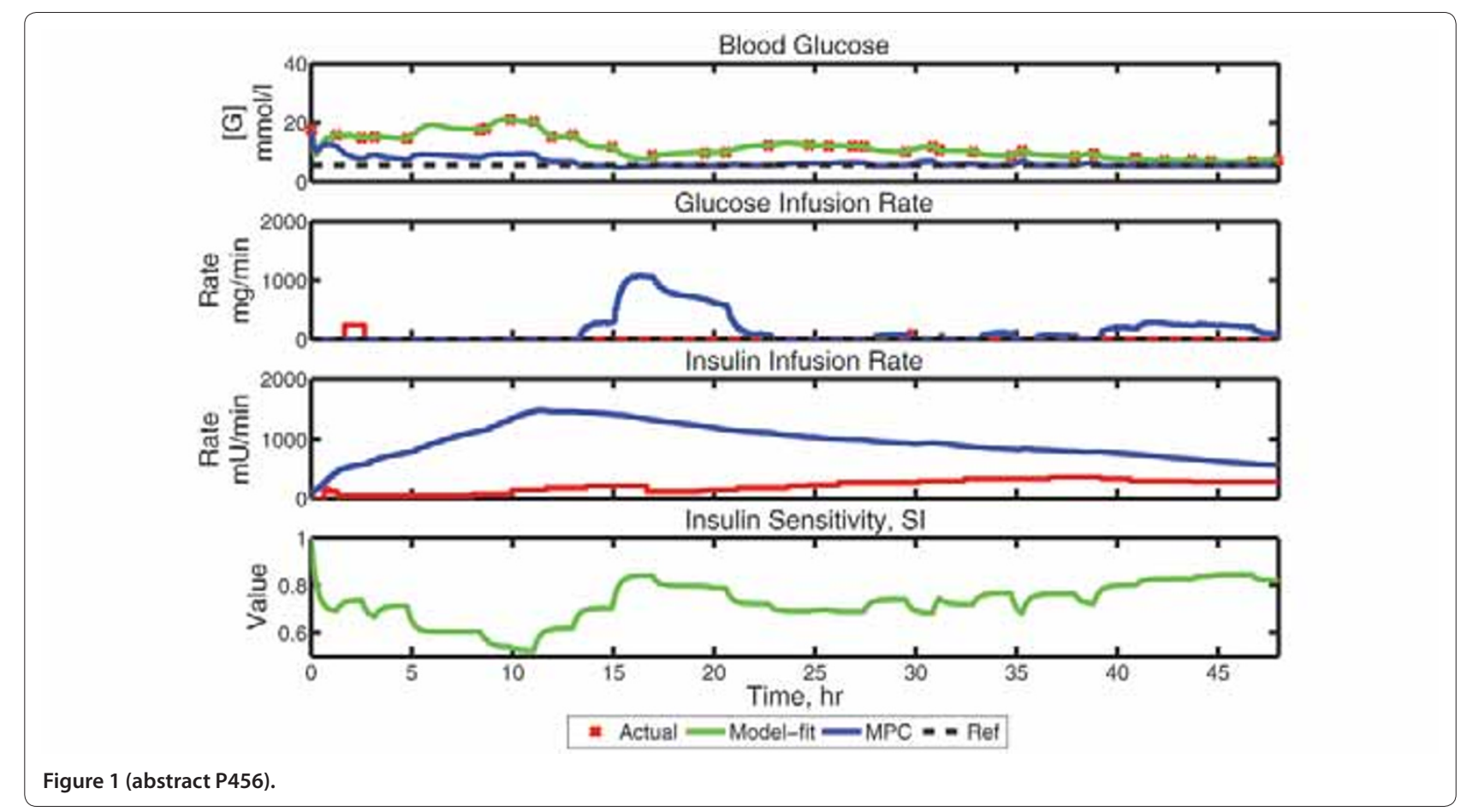

admission. Diagnosis of diabetes within 6 months from the index admission was presumed as revealing DM at inclusion, which excluded the patient. Patients who were taking glucocorticoids during the followup period were excluded. The rest of the patients, if consented, were assessed for their diabetic status with a single $\mathrm{HbA} 1 \mathrm{c}$ measurement.

Results A total of 2,696 patients were included in the study: 1,441 medical and 1,255 surgical, hyperglycaemia $(\geq 7.8 \mathrm{mmol} / \mathrm{l})$ was registered in 1,419 (52\%). During the 5 years from index admission 409 died and 38 were taking glucocorticoids, and 10 refused participation. Diabetes was diagnosed in 189 patients during the 6 months after index admission. The remaining cohort of 2,320 patients had 1,169 patients who were hyperglycaemic during hospitalisation. Diabetes was already diagnosed in 132 patients, of whom 112 were hyperglycaemic in the ICU; prediabetes was already diagnosed in 121 patients, of whom 83 had ICU hyperglycaemia. Since 125 patients (55 hyperglycaemic) refused $\mathrm{HbA} 1 \mathrm{c}$ testing, it was performed on 1,852 patients and revealed diabetes in 136 patients (107 had hyperglycaemia) and prediabetes in 103 patients (75 were hyperglycaemic in ICU). Overall, patients who had hyperglycaemia had $19.8 \%$ cumulative 5 -year incidence of diabetes and $14.3 \%$ of prediabetes. Nonhyperglycaemic ICU patients had cumulative incidences of $4.5 \%$ and $6.6 \%$, respectively. Relative risk for developing diabetes during 5 years after ICU admission is 4.2 (95\% $\mathrm{Cl}=3.2$ to 5.9 ) for patients who did have hyperglycaemia.

Conclusion The results of this study confirm in a large mixed medical/ surgical ICU population that hyperglycaemia occurring in severe illness or the perioperative period is associated with increased risk of developing diabetes. Suggesting lifestyle changes to reduce the risk and regular follow-up should be implemented at least for these patients.

\section{Reference}

1. Gornik I, et al:: A prospective observational study of the relationship of critical illness associated hyperglycaemia in medical ICU patients and subsequent development of type 2 diabetes. Crit Care 14:R130.

P458

Association between average glucose levels and hospital mortality among critically ill patients

B Yegneswaran', R Parket ${ }^{2}$, S Gawel ${ }^{2}$, A Pritchard-Bell ${ }^{2}$, H Ho$^{2}$, G Clermont ${ }^{2}$ 'UPMC Hamot, Erie, PA, USA; '2 University of Pittsburgh, PA, USA

Critical Care 2013, 17(Suppl 2):P458 (doi: 10.1186/cc12396)
Introduction The objective of the study was to determine the association between average glucose level (AVG) and hospital outcomes in critically ill patients.

Methods We performed a chart review of the MIMICII and HIDENIC databases, prospective cohorts of over 32,000 and 46,000 patients admitted to the ICUs at Beth Israel Deaconess Medical Center between 2001 and 2007 and the ICUs at University of Pittsburgh Medical Center between 1991 and 2008, respectively. All admissions of patients $\geq 18$ years without the diagnosis of DKA and NKHS were included. Diabetics were identified from ICD-9 documentation. Propensity score for death (pDead) was computed from either SAP1 (MIMICII) or APACHE III (HIDENIC) to assess the risk of death. Hypoglycemia was defined as AVG $\leq 60 \mathrm{mg} / \mathrm{dl}$. AVG was computed as the area under the glucose curve throughout ICU admission. Mortality was examined within bins (each bin is categorized by a $10 \mathrm{mg} / \mathrm{dl}$ increase in AVG) and was compared between adjacent categories using a chi-square test. The same method was repeated among diabetics, nondiabetics, patients with lower ( $p$ Dead greater than median) and higher (pDead lower than

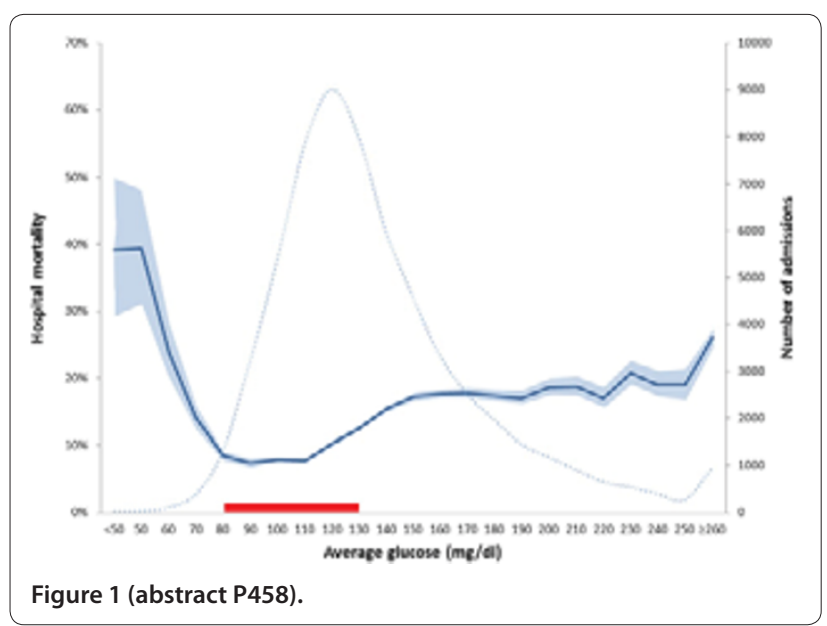


median) risk of death. Statistical significance was noted following a Bonferroni correction.

Results MIMICII database: 21,209 admissions met inclusion criteria. Mean age: $62.5 \pm 16.4$ years; female: 9,070 (42.8\%); diabetes: 5,496 (25.9\%); mean admission SAP1 score: $13.8 \pm 5.2$; mean LOS: 9 days (IQR 6.15); hospital mortality: $10.3 \%$. HIDENIC database: 38,872 admissions met inclusion criteria. Mean age: $59.4 \pm 17.3$ years; female: 16,675 (42.9\%); diabetes: 11,326 (29.1\%); mean admission APACHE III score: $80.2 \pm 14$; mean LOS: 11 days (IQR 7.22); hospital mortality: 14.3\%. In both databases, association between average glucose and hospital mortality followed a bathtub shape with mortality nadir between 80 and $130 \mathrm{mg} / \mathrm{dl}$. No mortality difference was observed between adjacent AVG glucose bins in the bottom of the bathtub (Figure 1). This relationship was preserved in nondiabetics, and for patients with higher risk of death.

Conclusion Low and high average glucose are associated with mortality.

\section{P459}

Continuous glucose monitoring in critically ill adults: comparison of two different calibration protocols

L Leelarathna', S English², HThabit', K Caldwell', J Allen',

K Kumareswaran'1, M Wilinska', M Nodale', J Mangat'1, M Evans',

R Burnstein ${ }^{2}$, R Hovorka ${ }^{1}$

'University of Cambridge, UK: ${ }^{2}$ Addenbrookes Hospital, Cambridge, UK

Critical Care 2013, 17(Suppl 2):P459 (doi: 10.1186/cc12397)

Introduction We evaluated the clinical and numerical accuracy of the Freestyle Navigator continuous glucose monitoring (CGM) system, in critically ill adults using two different methods of calibration. Studies looking at intensive glucose control have yielded conflicting results with increased rates of iatrogenic hypoglycemia. Availability of accurate real-time glucose information may improve safety and efficacy of glucose control in the critical care unit.

Methods In a randomized prospective trial, paired CGM and reference glucose (hourly arterial blood gas (ABG)) were collected from 24 adults with critical illness (age $60 \pm 14$ years, BMI $29.6 \pm 9.3 \mathrm{~kg} / \mathrm{m}^{2}$, APACHE score range 6 to 19) and hyperglycemia (glucose $\geq 10 \mathrm{mmol} / \mathrm{l}$ or treated with intravenous insulin), over 48 hours. In 12 subjects, CGM was forcecalibrated at variable 1 to 6 hourly intervals using ABG glucose (FC arm). In the other 12 subjects, the sensor was calibrated according to the manufacturer's instructions (1, 2, 10, 24 hours after insertion) using arterial blood and built-in glucometer (MC arm).

Results Two groups had similar characteristics at baseline. A total of 1,060 CGM/ABG pairs were analyzed and reference glucose ranged from 4.3 to $18.8 \mathrm{mmol} / \mathrm{l}$. Median (IQR) absolute relative deviation was lower in the FC arm $(7.0 \%(3.5,13.0)$ vs. $12.8 \%(6.3,21.8)$, FC vs. MC, $P<0.001)$. Similarly, the percentage of points in the Clarke error grid zone A points meeting ISO criteria were higher with $\mathrm{FC}(87.8 \%$ vs. $70.2 \%)$. Sensor bias (median (IQR)) was significantly lower in the FC $\operatorname{arm}(-0.1(-0.7,0.4) \mathrm{mmol} / \mathrm{l}$ vs. $-1.1(-2.3,-0.1) \mathrm{mmol} / \mathrm{l}, P<0.001)$. The median (IQR) interval between calibrations in FC arm was 169 (122, 213) minutes.

Conclusion CGM accuracy in the MC arm was comparable with accuracy in subjects with type 1 diabetes. Further significant improvements to CGM accuracy in critical care are possible by increasing the frequency of calibrations. Such accurate CGM may provide valuable information to guide insulin therapy in critically ill subjects.

\section{References}

1. Weinstein RL, et al:: Diabetes Care 2007, 30:1125-1130.

2. Kovatchev B, et al: Diabetes Care 2008, 31:1160-1164.

\section{P460}

Root cause analysis of hypoglycemic events in critically ill patients A McDonald, J Krinsley

Stamford Hospital, Stamford, CT, USA

Critical Care 2013, 17(Suppl 2):P460 (doi: 10.1186/cc12398)

Introduction Hypoglycemia $(\mathrm{H})$ is a complication of intensive insulin (IN) therapy of critically ill patients and is independently associated with increased risk of mortality (M). Heightened attention to identified risk factors (RF) and causes of hypoglycemic events (HE) may lead to improvement in glycemic control. A limited literature describes RF for $\mathrm{H}$ but a detailed root cause analysis (RCA) of individual $\mathrm{HE}$ has not been published.

Methods This is a retrospective analysis of prospectively collected data including 835 patients admitted consecutively between 1 February 2012 and 31 October 2012 to the 16-bed medical-surgical ICU of a university-affiliated teaching hospital. The blood glucose (BG, $\mathrm{mg} / \mathrm{dl}$ ) target was 90 to 120 and the monitoring frequency was every 3 hours, or every 1 hour when the patient received i.v. IN infusion. HE data were collected by the bedside nurse, using a cut off of $\mathrm{BG}<60$, and information was abstracted from the unit's database. RF included: shock (SH), renal insufficiency (RI), hepatic failure (HF), mechanical ventilation (MV), and diabetes mellitus (DM). Attributable causes included: IN treatment, improper monitoring frequency (MF), interruption of feeding (FE), and spontaneous (SP). We measured rates of severe $(<40)$ and mild $(40$ to 69) $\mathrm{H}$, and compared them as well as percentage of values in the optimum range (70 to 139$)$ and hyperglycemic range (>139) with data from the preceding 3 months (PRE).

Results Sixty-three (7.5\%) patients sustained a total of $79 \mathrm{HE}$. They were older, more likely to be diabetic, and had higher APACHE IV predicted ( $37.3 \%$ vs. $15.4 \%, P<0.0001)$ and observed $(25.4 \%$ vs. $8.6 \%, P<0.0001)$ $M$ than those without H. RF: SH 24.1\%; RI 24.1\%; HF 8.9\%; MV 46.8\%; DM 36.5\%. Attributable causes: IN 58.2\%; MF 8.9\%; FE 6.3\%; SP 36.7\%. BG $<40,40$ to 69 and $>139$ decreased $78.6 \%, 39.0 \%$ and $9.3 \%(P=0.0006$, $P<0.0001, P<0.0001)$. BG 70 to 139 increased $5.5 \%(P<0.0001)$. In total, $4.2 \%$ and $22.4 \%$ of PRE patients sustained severe and mild $\mathrm{H}$. In total, $0.7 \%$ and $14.1 \%$ of RCA patients sustained severe and mild $\mathrm{H}$ $(P=0.0029, P=0.0003)$.

Conclusion IN treatment was associated with barely more than one-half of HE in this population of intensively monitored patients. Implementation of an initiative mandating RCA of every $\mathrm{HE}$, including identification of RF and attributable causes, was associated with a significant improvement in glycemic control.

\section{P461}

Continuous arterial and venous glucose monitoring by quenched chemical fluorescence in ICU patients after cardiac surgery

S Bird', L Macken', O Flower', F Bass', N Hammond', S Webb', N Kennedy', A Baker1, E Yarad', C Chau'², M Librande², P Strasma², S Finfer

'Royal North Shore Hospital, Sydney, Australia; ${ }^{2}$ Glumetrics, Irvine, CA, USA Critical Care 2013, 17(Suppl 2):P461 (doi: 10.1186/cc12399)

Introduction Continuous glucose monitoring (CGM) in ICUs has the potential to improve patient safety outcomes. The GluCath Intravascular CGM System uses a novel quenched chemical fluorescence sensing mechanism to measure glucose concentration in venous or arterial blood (BG). This is the first report of this system deployed for 48 hours in both arteries and veins of ICU patients.

Methods This ongoing clinical study evaluates up to two sensors per subject in 20 patients undergoing cardiac surgery. An arterial sensor is deployed via a standard $20 \mathrm{G}$ radial artery catheter inserted for routine care and an optional venous sensor is deployed percutaneously in an upper arm vein. Data are presented from the first five patients. Outcome measures are qualitative (ease of use, workflow fit) and quantitative (accuracy vs. reference analyzer). Sensors were inserted shortly after ICU admission, with ultrasound guidance for venous sensor insertion. In vivo calibration was performed at 1 and 2 hours, then each morning. Glucose values were recorded every 10 seconds by the system. Hourly arterial reference samples were analyzed via Radiometer ABL 800 Flex Blood Gas Analyzer (BGA).

Results Arterial sensors were successfully deployed in all five patients and did not interfere with clinical care, blood pressure monitoring or sampling. One arterial catheter failed resulting in sensor removal at 36 hours. The venous sensor was deployed in three patients, but removed from two patients due to thrombosis identified during surveillance ultrasound examinations. A total of 202 reference BG samples ranging from 5.3 to $11.3 \mathrm{mmol} / \mathrm{l}$ were collected. Precision between arterial and venous sensors $(n=2)$ was $9.3 \% \mathrm{CV}$. Arterial sensor accuracy compared with BGA was 5.5\% MARD. One hundred 


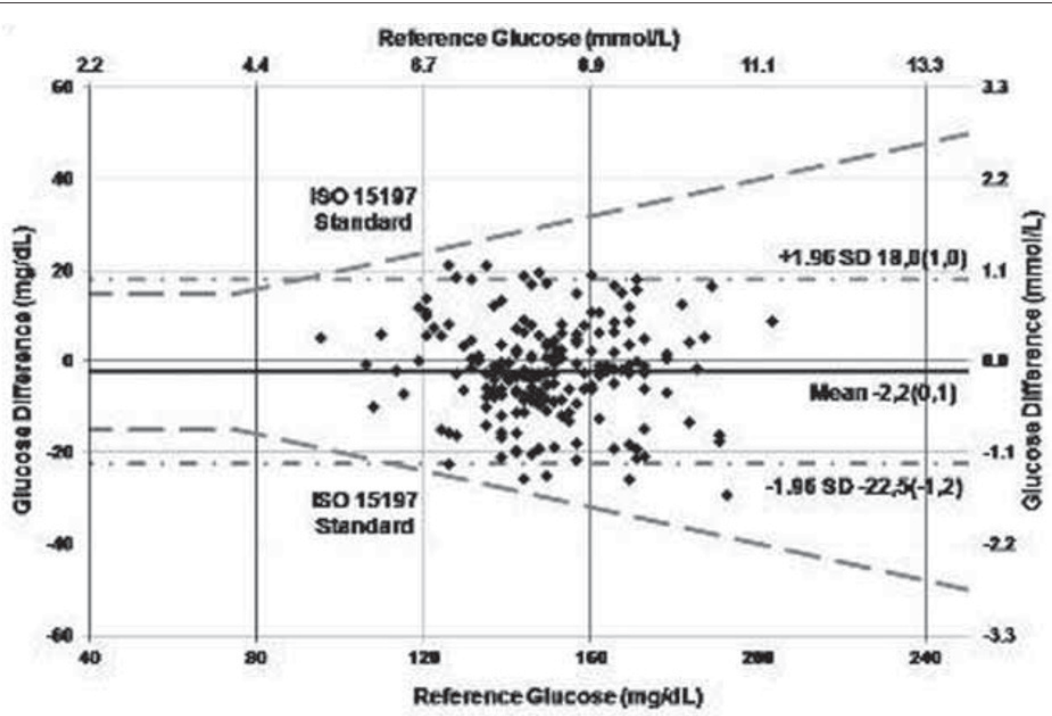

Figure 1 (abstract P461). Arterial sensor ISO-modified Bland-Altman plot.

percent (202/202) of arterial sensor measurements met ISO 15197 criteria (within $=/-20 \%$ of reference measurements if $B G \geq 4.2 \mathrm{mmol} /$; Figure 1).

Conclusion The GluCath System measured glucose concentration continuously in cardiac surgery ICU patients. Arterial catheter deployment did not appear to compromise line function or patient care. Percutaneous venous deployment was feasible, but may be associated with risk of local venous thrombosis.

\section{P462}

Performance of the Medtronic Sentrino ${ }^{\circledR}$ continuous glucose management system in the cardiac ICU

M Kosiborod', R Gottlieb², J Sekella², D Peterman', A Grodzinsky', P Kennedy', M Borkon

'Saint Luke's Mid America Heart Institute, Kansas City, MO, USA; '2Medtronic Minimed, Northridge, CA, USA

Critical Care 2013, 17(Suppl 2):P462 (doi: 10.1186/cc12400)

Introduction Blood glucose (BG) control reduces morbidity and length of stay, and is standard practice in patients undergoing cardiac surgery [1]. However, maintaining BG in the target range, while avoiding hypoglycemia, is challenging. Continuous glucose monitoring (CGM) is a promising technology that may help address these challenges. We investigated the performance and safety of Medtronic Sentrino ${ }^{\oplus}$, a newly developed CGM for critically ill adults, in the cardiac ICU.

Methods Adult patients with actual or planned cardiac ICU admission at a single tertiary center were approached for participation and signed consent. Other inclusion criteria were treatment with i.v. insulin (target BG $<140 \mathrm{mg} / \mathrm{dl}$ ) and life expectancy $>96$ hours. After initiation of i.v. insulin, Sentrino ${ }^{\oplus}$ subcutaneous glucose sensors were inserted into patients' anterior thighs with planned study participation of 72 to 96 hours. Reference BG was collected according to ICU protocol, obtained from central venous catheter and analyzed with bedside blood gas analyzer (BGA; i-STAT ${ }^{\oplus}$, Abbott, USA). Sensor glucose (SG) results were displayed, and its predictive alerts and alarms fully enabled. Additional reference BGs were obtained during alarms and calibration. All treatment decisions were based on BGA data, not on SG values.

Results A total of 21 patients were enrolled; all successfully completed the study. Mean age was 65 years, $38 \%$ were women, $67 \%$ had diabetes. Types of surgery were CABG (38\%), valve replacement (29\%), combined CABG and valve (19\%) and cardiac transplant (14\%). SG was displayed $95 \%$ of the time during the study, and 864 paired BG-SG points were used for analysis. Overall mean absolute relative difference (MARD) was $12.2 \%$. No differences in CGM system accuracy were seen within subgroups of low versus high Society of Thoracic Surgeons (STS) score (MARD $12.1 \%$ and $10.6 \%$ for STS $>8 \%$ vs. $\leq 8 \%$, respectively) or hemodynamic status (MARD $12.0 \%$ and $12.4 \%$ for compromised vs. stable hemodynamics). Consensus grid analysis showed $>99 \%$ of SG values within $A / B$ zones, and $0 \%$ in $D / E$ zones. No device or study-related adverse events were reported. In total, $80 \%$ and $100 \%$ of clinicians found Sentrino ${ }^{\oplus}$ easy to use after one and two patients, respectively.

Conclusion The Sentrino ${ }^{\circledR}$ CGM system demonstrated good analytic and clinically relevant accuracy, excellent reliability and safety in critically ill cardiac patients; and was easy to use and integrate in the cardiac ICU. Future studies are needed to determine whether CGM can improve BG control and reduce hypoglycemia in this patient group. Reference

1. Lazar HL, et al:: Ann Thorac Surg 2009, 87:663-669.

\section{P463}

Intensive insulin therapy in critically ill children: impact on blood glucose dynamics and its relation with mortality

M Van Tornout, M Gielen, T Van Herpe, D Vlasselaers, L Desmet,

I Vanhorebeek, G Van den Berghe, D Mesotten

University Hospitals Leuven, Belgium

Critical Care 2013, 17(Suppl 2):P463 (doi: 10.1186/cc12401)

Introduction A large RCT showed that tight glucose control (TGC), targeting age-adjusted normal fasting blood glucose levels with insulin infusion, decreased morbidity and mortality in critically children [1]. However, the incidence of hypoglycemia increased substantially in the TGC group. We aimed to assess the effect of TGC on the three domains of blood glucose dynamics (hyperglycemia, hypoglycemia and blood glucose variability) and their independent association with mortality in the pediatric ICU.

Methods This is a preplanned substudy of a published RCT in one 10-bed pediatric ICU. Seven hundred patients (age 1 to 16 years), admitted to the PICU between October 2004 and December 2007, were randomized to either TGC ( 50 to $80 \mathrm{mg} / \mathrm{dl}$ in infants, 70 to $100 \mathrm{mg} / \mathrm{dl}$ in children) or to the usual care tolerating hyperglycemia up to $215 \mathrm{mg} /$ $\mathrm{dl}$ (UC). Patients with at least two arterial blood glucose measurements were included (UC $n=349 ; \operatorname{TGC} n=348$ ).

Results Mean blood glucose levels were lowered from $143 \pm 34 \mathrm{mg} / \mathrm{dl}$ in the UC group to $104 \pm 24 \mathrm{mg} / \mathrm{dl}(P<0.0001)$. The median number of samples per patient did not differ between UC (21 (13 to 48)) and TGC (22 (15 to 49)). TGC lowered the hyperglycemic index, a marker of 
hyperglycemia over time, from median 44 (IQR 29 to 66) $\mathrm{mg} / \mathrm{dl}$ in UC to 12 (7 to 23 ) $\mathrm{mg} / \mathrm{dl}$, as well as the glycemic penalty index, an aggregate measure of dysglycemia, from median 44 ( 32 to 58 ) in UC to 22 (16 to 30) (both $P<0.0001$ ). Despite frequent hypoglycemia $<40 \mathrm{mg} / \mathrm{dl}$ (1.4\% in UC, $25.0 \%$ in TGC, $P<0.0001)$, TGC did not result in increased blood glucose variability, as reflected by the standard deviation of the blood glucose time series (TGC: 36 (24 to 50) mg/dl, UC 34 (26 to 48$) \mathrm{mg} / \mathrm{dl}, P=0.82$ ). In multivariable logistic regression analysis, adjusting for baseline risk factors (age, severity of illness, malignancy, type of admission), hypoglycemia ( $\mathrm{OR}=1.6,95 \% \mathrm{Cl}=0.19$ to 2.22 ) and blood glucose variability $(\mathrm{OR}=1.0,95 \% \mathrm{Cl}=0.97$ to 1.02 , per $\mathrm{mg} / \mathrm{dl}$ ) were not associated with PICU mortality. In contrast, hyperglycemic index $(\mathrm{OR}=1.02,95 \% \mathrm{Cl}=1.01$ to 1.04 , per $\mathrm{mg} / \mathrm{dl})$ was independently associated with mortality and its lowering statistically explained the outcome benefit of the intervention.

Conclusion TGC substantially reduced the hyperglycemic index, at the expense of increased hypoglycemia incidence, but without affecting blood glucose variability. Statistical analysis suggested strictly avoiding hyperglycemia in order to bring about outcome benefit with insulin in critically ill children.

Reference

1. Vlasselaers D, et al.: Lancet 2009, 373:547-556

P464

Implementation of evidence-based protocols improves survival: a 15-year surgical ICU experience in 10,172 patients

SA Nasraway

Tufts Medical Center, Boston, MA, USA

Critical Care 2013, 17(Suppl 2):P464 (doi: 10.1186/cc12402)

Introduction There has been enormous interest in measuring ICU performance in terms of mortality and resource use, owing to increased public and health insurer scrutiny. We elected to describe the performance of our surgical ICU, using a standardized mortality ratio (SMR = observed / predicted mortality).

Methods The primary cohort was all patients admitted to the surgical ICU from March 2010 through February 2012 and related outcomes. The change in SMR was longitudinally determined from the latest 15year study period (1997 to 2011) comprised of 10,172 patients.

Results There were 1,799 ICU admissions in the primary cohort. Hospital mortality, observed and predicted by APACHE IV, was measured. Crude hospital mortality was $8.4 \%$. The hospital SMR (observed / predicted mortality) was 0.58 ( $95 \% \mathrm{Cl}: 0.49$ to 0.65 ). The SMR decreased by $20 \%$ from 0.73 to 0.58 over the 15 -year study period, an absolute $1 \%$ per year decrease $(P=0.039 ; 95 \% \mathrm{Cl}:-0.02$ to -0.002$)$, as shown in Figure 1 .

Conclusion Mortality was less than predicted and steadily declined during the previous 15 years. The SMR can be used to track success when new quality measures are introduced or changes in the delivery of care are made. ICUs should report their standardized mortality ratios to evaluate performance.

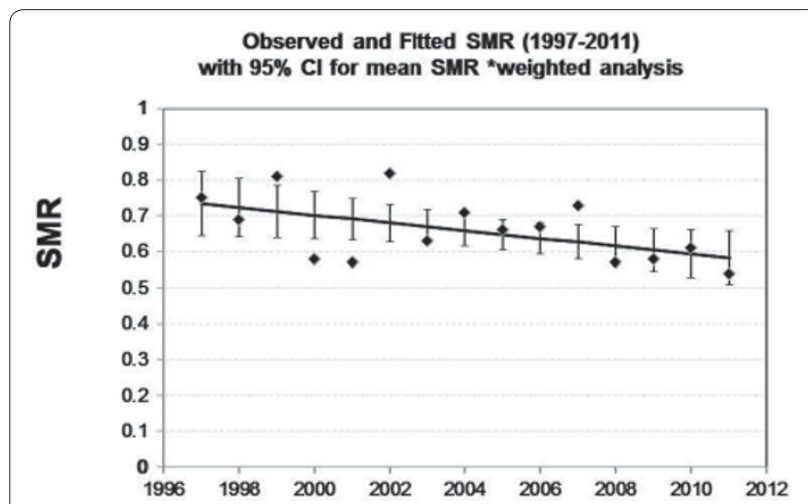

Figure 1 (abstract P464). Tufts surgical ICU, 15-year outcome standardized hospital mortality ratio.
P465

Decubitus ulcers are associated with prolonged length of stay in critically ill patients

WT McGee', BH Nathanson², E Lederman', TL Higgins

'Baystate Medical Center, Springfield, MA, USA; ${ }^{2}$ OptiStatim LLC, Longmeadow, MA, USA

Critical Care 2013, 17(Suppl 2):P465 (doi: 10.1186/cc12403)

Introduction How decubitus ulcers (DU) diagnosed on admission to the ICU affect resource use is unknown. We hypothesize that this easily identifiable clinical finding would be associated with longer hospitalizations.

Methods Clinical assessment was used to identify patients with DU on admission to the ICU. The association of DU on hospital and ICU length of stay (LOS) was assessed with generalized linear models that adjusted for ICU admission source, age, APACHE IV score, diabetes, sepsis, hepatic failure, dialysis and mechanical ventilation.

Results DU was present on admission in 180 of 2,723 (6.6\%) during a 19-month period in 2011 to 2012 at a single teaching hospital in New England. Patients with decubiti were sicker on admission (mean (SD) APACHE IV 80.2 (28.2) vs. $58.7(30.7), P<0.001)$, more likely to be age 65 or older $(58.9 \%$ vs. $41.8 \%, P<0.001)$ and require more organ supportive therapies (Table 1). DU patients had a higher in-hospital mortality rate $(32.2 \%$ vs. $18.3 \%, P<0.001)$ and longer mean ICU and hospital LOS (4.9 vs. 3.3 days and 15.6 vs. 10.5 days, respectively $(P<0.001)$ ). After multivariate adjustment, DU patients stayed 3.2 days, $95 \% \mathrm{Cl}(1.6$, 4.8) longer in the hospital than non-DU patients and stayed 2.5 days longer, $95 \% \mathrm{Cl}(0.7,0.43)$ among survivors. DU patients had longer ICU stays $(1.3$ days, $95 \% \mathrm{Cl}(0.3,1.3))$ in general but were statistically similar among survivors.

Table 1 (abstract P465)

\begin{tabular}{lccc}
\hline & $\begin{array}{c}\text { Admitted } \\
\text { with } \\
\text { decubitus } \\
(\boldsymbol{n}=\mathbf{1 8 0})\end{array}$ & $\begin{array}{c}\text { Admitted } \\
\text { without } \\
\text { decubitus } \\
(\boldsymbol{n}=\mathbf{2}, 543)\end{array}$ & $\begin{array}{c}\boldsymbol{P} \\
\text { value }\end{array}$ \\
\hline Variable & $106(58.9 \%)$ & $1,062(41.8 \%)$ & $<0.001$ \\
\hline Age 65 or older & $80.2(28.2 \%)$ & $58.7(30.7)$ & $<0.001$ \\
APACHE IV score on admission & $53(29.4 \%)$ & $420(16.5 \%)$ & $<0.001$ \\
Diabetes & $16(8.9 \%)$ & $58(2.3 \%)$ & $<0.001$ \\
Dialysis & $3(1.7 \%)$ & $56(2.2 \%)$ & 1.000 \\
Hepatic failure & $123(68.3 \%)$ & $1,457(57.3 \%)$ & \\
Mechanically ventilated during & & &
\end{tabular}

hospitalization

Admission source to the ICU

\begin{tabular}{lccc} 
Direct admit & $10(5.6 \%)$ & $154(6.1 \%)$ & \\
ER & $84(46.7 \%)$ & $1,206(47.4 \%)$ & \\
Floor & $25(13.9 \%)$ & $214(8.4 \%)$ & 0.017 \\
OR & $19(10.6 \%)$ & $459(18.1 \%)$ & \\
Other location & $42(23.3 \%)$ & $510(20.1 \%)$ & \\
tcomes & & & \\
ICU LOS (days) & $4.9(4.9)$ & $3.3(4.7)$ & $<0.001$ \\
Hospital LOS (days) & $15.6(20.7)$ & $10.5(11.1)$ & $<0.001$ \\
ICU mortality & $30(16.7 \%)$ & $308(12.1 \%)$ & 0.073 \\
Hospital mortality & $58(32.2 \%)$ & $464(18.3 \%)$ & $<0.001$ \\
Readmission rate for & 20 out of 112 & 173 out of 2,079 & 0.002 \\
survivors & $(16.4 \%)$ & $(8.3 \%)$ & \\
\hline
\end{tabular}

Conclusion The presence of decubiti on admission to the ICU is associated with longer hospitalizations even after adjusting for age, acuity, and organ supportive therapies. DU on admission to ICU provide a unique, unambiguous marker of increased resource utilization. 
P466

HIV patients admitted to an ICU of a university hospital experience of 15 years: 1995 to 2009

A Agrifoglio, M Arce, M Hernández, P Millan, C Guallar, I Pozuelo,

M Jiménez

Hospital Universitario La Paz, Madrid, Spain

Critical Care 2013, 17(Suppl 2):P466 (doi: 10.1186/cc12404)

Introduction HIV infection is a major public health problem in the world. The use of prophylaxis against opportunist infection and the introduction of HAART in 1996 increased life expectancies. The therapeutic use of ICU resources for HIV patients has been controversial, questioning the admission of these patients especially in advanced stages of the disease, given the poor prognosis. The aim of this study was to determine the experience of the past 15 years in relation to the income of these patients in an ICU.

Methods A retrospective case series consisting of patients with diagnosis of HIV infection (known or unknown) admitted between January 1995 and December 2009. We collected demographic and epidemiological data, process of acquisition of the disease, infection status: known or unknown patient infected, whether or not receiving antiretroviral therapy and whether it was effective (undetectable viral load at the time of admission), APACHE II, cause of admission, need for mechanical ventilation (MV), pathology related or unrelated to HIV infection and ICU mortality.

Results During this period 12,607 patients were admitted to the ICU, 188 (1.5\%) HIV-positive. Mean APACHE II score 17.6, median age 39 years, $73 \%$ men and $90 \%$ Spanish nationality. Principal risk behavior: addiction drugs injection (67\%). Seventeen percent of patients did not know who was infected with HIV at the time of admission to the ICU. Fifty-three percent were not receiving HAART. Of the 88 patients treated, $93 \%$ were receiving HAART (effective in $88 \%$ of cases). Sixty percent of the patients came from the emergency department of the hospital. Main admission diagnoses: acute respiratory failure caused by infection (Streptococcus pneumoniae and Pneumocystis jirovecii), neurological disorders (coma for illicit drugs and psychotropic) and septic shock. Seventy percent required MV. Of patients whose HIV infection was not known, $93.5 \%$ were admitted for related pathology. In patients of known infection, the pathology associated with HIV was $30 \%$. Average length of stay 9 was days. ICU mortality was 35\%. Most frequent causes of death: septic shock and multiple organ failure.

Conclusion Depending on the patient and the cause of admission, ICU admission may represent an excellent opportunity as a screening method to determine HIV status. Given the greater efficacy of HAART at present, most patients with medical or surgical conditions unrelated to HIV infection will be eligible to join the ICU. People with HIV can and should benefit from using reasonable and individualized care in an ICU. References

1. Huang L, et al:: N Engl J Med 2006, 355:173-181.

2. Akgun K, et al: I Intensive Care Med 2011, 26:151-164.

\section{P467}

Prevalence and prognosis of organ dysfunctions of AIDS critically ill patients

AJ Japiassu, RA Amâncio, D Medeiros, EM Mesquita, EM Motta, FA Bozza Oswaldo Cruz Foundation, Rio de Janeiro, Brazil Critical Care 2013, 17(Suppl 2):P467 (doi: 10.1186/cc12405)

Introduction The aim was to analyze the prognosis of AIDS patients with organ dysfunctions at ICU admission.

Methods A prospective cohort study, including all patients with HIV/ AIDS diagnosis, who were admitted to a specialized ICU from November 2009 until May 2012. Patients with less than 24 hours of ICU stay were excluded. Demographics and nutritional status were collected. The organ dysfunctions were classified according to the SOFA score, and categorized as absent ( 0 SOFA point), mild ( 1 to 2 points) and severe ( 3 to 4 points). We expressed numeric variables as median and interquartile interval $(25 \%$ to $75 \%)$. We performed a multivariate analysis of possible variables associated with hospital mortality $(P<0.2)$, and we explored the 7-day, 28-day and 60-day survival of patients with and without independent risk factors.
Results We included 139 patients with HIV/AIDS admitted to the ICU. Median age was 40 (31 to 48 ) years and $71 \%$ were male. Severe malnutrition was common (34\%). The CD4 cell count was 84 (25 to 274) cells $/ \mathrm{mm}^{3}$ and viral load was 17,733 (67 to 174,214$)$ copies $/ \mathrm{ml} ; 57 \%$ had at least one opportunistic infection; $55 \%$ had used antiretroviral therapy previous to ICU admission. Mechanical ventilation was used by $46 \%$ of patients and hospital mortality was $42 \%$. Total SOFA score was 5 ( 2 to 9) points. Cardiovascular dysfunction was the most common on the first day of stay ( $51 \%)$, followed by respiratory (42\%), neurological (40\%), renal (35\%), hematological (27\%) and hepatic (17\%). Cardiovascular and renal dysfunctions presented with higher rate of severe dysfunction (30\% and 15\%, respectively). Rates of neurological $(P=0.002)$, renal $(P=0.009)$ and hematological $(P=0.003)$ dysfunctions were higher in nonsurvivors. Age, CD4 cell count, malnutrition, and opportunistic infections were included in the multivariate analysis. Neurological dysfunction was the independent risk factor for hospital mortality (odds 3.2 (1.4 to 7.2)). The presence of neurological dysfunction was dichotomized: associated or not with primary neurological diagnosis; survival was lower in the patients with neurological dysfunction and without primary neurological diagnosis (log-rank test 0.001 in the 7-day and 0.02 in the 28-day analysis). Sixty-day survival was similar in primary and secondary neurological dysfunction, but it remained lower than in patients without neurological impairment.

Conclusion Neurological dysfunction was independently associated with hospital survival, mainly in those AIDS critically patients without primary neuropathy.

\section{P468}

Mortality predictors in septic HIV patients in the ICU

P Vidal-Cortés', P Lameiro-Flores', A Aller-Fernández ${ }^{2}$, M Mourelo-Fariña², P Fernández-Ugidos', R Gómez-López', M Alves-Pérez', E Rodríguez-García ${ }^{1} \mathrm{CH} \cup$ Ourense, Spain: ${ }^{2} \mathrm{CH} \cup$ A Coruña, Spain

Critical Care 2013, 17(Suppl 2):P468 (doi: 10.1186/cc12406)

Introduction Sepsis in HIV patients carries a high mortality. Our purpose is to identify mortality predictors in this population.

Methods A retrospective study of HIV patients admitted to our ICU between January 2005 and December 2009. We identify septic patients and analyzed demographic factors, etiology, organ failure and outcome. We used logistic regression to calculate the relative mortality risk for each qualitative variable and the beta $(\beta)$ coefficient for quantitative variables.

Results Sixty-two septic patients were admitted to our ICU during the study period $(71 \%$ men, mean age: $40.59 \pm 8.12)$. A total of $56.5 \%$ came from the ED, mean hospital stay before ICU admission: $6.14 \pm 10.96$ days. A total of $66.1 \%$ had a history of intravenous drug use, other comorbidities: COPD (9.7\%), cirrhosis $(8.1 \%)$, solid or hematologic malignancy (12.9\%). A total of $40.3 \%$ were under HAART. Mean CD4 count at admission: $219.62 \pm 353.93$ cells $/ \mathrm{mm}^{3}$. Mean viral load: $4.57 \pm 3.25$ log. Mean albumin levels: $2.3 \pm 0.53 \mathrm{~g} / \mathrm{dl}$. APACHE II: $21.98 \pm 7.97$. The lung was the most frequent focus of infection (65.6\%) followed by the CNS. The most common pathogen was S. pneumoniae (28.8\%), followed by P. jirovecii (13.6\%). In total, $62.9 \%$ needed vasopressors, $79 \%$ mechanical ventilation and $19.4 \%$ renal replacement. Mean ICU and hospital length of stay: $10.43 \pm 10.52$ and $34.76 \pm 29.64$ days. ICU mortality: $33.9 \%$; hospital mortality: $41.9 \%$. ICU mortality increases for each day of hospital stay before ICU admission ( $\beta=1.062,95 \% \mathrm{Cl}=1.009$ to $1.118, P=0.022$ ), each point of: cardiac frequency at admission $(\beta=1.059,95 \% \mathrm{Cl}=1.024$ to $1.096, P=0.001)$, $\mathrm{FiO}_{2}(\beta=1.034,95 \% \mathrm{Cl}=1.011$ to $1.057, P=0.003), \mathrm{pCO}_{2}$ (per $\mathrm{mmHg}$ ) $(\beta=1.052,95 \% \mathrm{Cl}=1.007$ to $1.098, P=0.023)$; and decreases for each point of: albumin ( $\mathrm{g} / \mathrm{dl})(\beta=-0.307,95 \% \mathrm{Cl}=0.095$ to $0.986, P=0.047)$, mean arterial pressure $(\mathrm{mmHg})(\beta=0.951,95 \% \mathrm{Cl}=0.904$ to 0.999 , $P=0.048), \mathrm{pH}(\beta=0.002,95 \% \mathrm{Cl}=0.0$ to $0.172, P=0.006)$, first ICU 24-hour diuresis (ml) $(\beta=0.999,95 \% \mathrm{Cl}=0.998$ to $1.000, P=0.004)$ and base excess ( $\beta=0.912,95 \% \mathrm{Cl}=0.836$ to $0.995, P=0.039$ ). Mortality increases threefold $(\beta=3.134,95 \% \mathrm{Cl}=1.051$ to $9.345, P=0.04)$ if patients do not come from the ED, almost 10 -fold if the patients need vasopressors for more than 24 hours $(\beta=9.975,95 \% \mathrm{Cl}=2.054$ to $48.451, P=0.004$ ) and fivefold if patients need renal replacement $(\beta=5.692,95 \% \mathrm{Cl}=1.466$ to $22.099, P=0.012)$. 
Conclusion Neither immune status-related variables nor comorbidity or infection focus are mortality predictors. Poor nutritional status, delayed ICU admission, shock or renal failure increase the ICU relative mortality risk. Tachycardia, hypotension, hypercapnia, acidosis, and oliguria in the first ICU 24 hours increase significantly ICU mortality. Mechanical ventilation is not a mortality predictor.

\section{P469}

Outcome of intensive care treatment for lung cancer patients

L Hajjar', F Galas², D Goto², A Tavares², M Santos², A Freitas², T Oliveira²,

P Tonini², L Cavichio², M Bazan², J Almeida², M Cavalcante'2, J Fukushima²,

S Vieira², E Osawa²

${ }^{1}$ Heart Institute, São Paulo, Brazil; ${ }^{2}$ Cancer Institute of University of São Paulo,

Brazil

Critical Care 2013, 17(Suppl 2):P469 (doi: 10.1186/cc12407)

Introduction Patients with lung cancer commonly require the ICU for a variety of acute illnesses related to the underlying malignancy, treatment, or comorbid conditions. ICU admission of patients with nonresectable lung cancer has been criticized based on the high mortality rate in this population. However, recent advances in critical care may have changed this scenario. The aim of this study was to identify factors associated with hospital mortality in this group of patients.

Methods A retrospective study was conducted in consecutive medical and surgical patients with lung cancer admitted to a university hospital ICU in São Paulo, between 2007 and 2012. A univariate analysis was performed to identify associated variables with hospital mortality. Selected variables were included in the multivariate model.

Results From 597 patients included in the study, 477 were medical admissions (79.8\%) and 120 were surgical admissions (20.2\%). Four hundred and twenty (70\%) patients had metastasis, 222 patients (37\%) required the ICU because of respiratory failure and 121 (20\%) because of septic shock. The ICU and hospital mortality rates were $53.9 \%$ and $64 \%$, respectively. In the univariate analysis, variables associated with hospital mortality were diagnosis of nonsmall-cell lung cancer, higher Charlson morbidity index, medical admission, active neoplasm, vasopressor need at admission to and at 24 hours of ICU, acute renal failure at admission, non-invasive ventilation or mechanical ventilation need at admission to and at 24 hours of ICU and a higher admission arterial lactate. By multivariate analysis, risk factors of hospital mortality were diagnosis of nonsmall-cell lung cancer $(\mathrm{OR}=3.32 ; 95 \% \mathrm{Cl}, 2.08$ to $5.29, P<0.001)$, medical admission $(\mathrm{OR}=2.89 ; 95 \% \mathrm{Cl}, 1.75$ to 4.77 , $P<0.001)$, acute renal failure at admission $(\mathrm{OR}=4.09 ; 95 \% \mathrm{Cl}, 1.49$ to $11.21, P<0.001)$, non-invasive ventilation at 24 hours of ICU (OR $=2.50$; $95 \% \mathrm{Cl}, 1.48$ to $4.23, P=0.001)$ and mechanical ventilation at 24 hours of ICU (OR $=3.68 ; 95 \% \mathrm{Cl}, 1.87$ to $7.26, P<0.001$ ).

Conclusion Hospital survival in patients with lung cancer requiring ICU admission was 36\%. Our results provide evidence that ICU management may be appropriate in patients with nonresectable lung cancer and appoint risk factors for mortality, helping to better triage cancer patients who will benefit from ICU care.

\section{P470}

Outcome of older patients with cancer requiring ICU admission

L Hajjar1, M Franca Cavalcante², F Galas², JT Fukushima², M R Sundin²,

F Bergamin², C Park', H Palomba², D M Goto², A Tavares², M Santos²,

A Freitas ${ }^{2}$, PTonini², L Cavichio², J P Almeida², M Bazan²

'Heart Institute, São Paulo, Brazil;'² Cancer Institute of University of São Paulo, Brazil

Critical Care 2013, 17(Suppl 2):P470 (doi: 10.1186/cc12408)

Introduction Because the prognosis of older patients with cancer may be poor compared with younger patients, it remains controversial whether they benefit from ICU treatment. The objective of this study was to identify factors associated with hospital mortality in older patients with cancer requiring the ICU.
Methods A retrospective study was conducted in consecutive medical and surgical older patients with cancer admitted to a university hospital ICU in São Paulo, between 2007 and 2012. Univariate and multivariate analysis were performed to identify associated and independent factors related to hospital mortality.

Results From 8,976 patients with cancer requiring ICU at the period, 600 patients were 80 years old or higher. Most patients were male (53\%), had solid neoplasm (86\%), were from medical admission (54\%) and $37 \%$ had metastatic disease. The mean age was 84 years $( \pm 3)$. The ICU and hospital mortality rates were $30 \%$ and $44 \%$, respectively. In the univariate analysis, variables associated with hospital mortality were diagnosis of lung cancer, medical admission, active neoplasm, vasopressor need at 24 hours of ICU, acute renal failure at admission, mechanical ventilation need at admission to and at 24 hours of ICU and a higher admission arterial lactate. By multivariate analysis, risk factors of hospital mortality were diagnosis of lung cancer $(\mathrm{OR}=2.32 ; 95 \% \mathrm{Cl}$, 1.08 to $3.29, P<0.001)$, medical admission $(\mathrm{OR}=6.473 ; 95 \% \mathrm{Cl}, 2.76$ to $15.15, P<0.001)$, acute renal failure at admission $(\mathrm{OR}=3.09 ; 95 \% \mathrm{Cl}, 1.49$ to $9.21, P<0.001)$, mechanical ventilation at 24 hours of ICU $(O R=5.68$; $95 \% \mathrm{Cl}, 2.87$ to $7.26, P<0.001)$ and lactate levels at admission $(\mathrm{OR}=1.02$; $95 \% \mathrm{Cl}, 1.006$ to $1.051, P<0.001)$.

Conclusion Hospital survival in older patients with cancer requiring ICU admission is acceptable. Our results provide evidence that ICU management may be appropriate in older patients with cancer and appoint risk factors for mortality, helping to better triage cancer patients who will benefit from ICU care.

\section{P471}

Identifying risk factors for readmission to the ICU: a qualitative approach

DG Strange, KTjelle, A Lindhardt, K Jensen

Bispebjerg Hospital, Copenhagen NV, Denmark

Critical Care 2013, 17(Suppl 2):P471 (doi: 10.1186/cc12409)

Introduction Readmission to the ICU within 48 hours is an indicator of quality of intensive care and is associated with an increase in mortality. During the last years several groups have published data based on multivariate logistic regression analysis to describe characteristics of patients who needed readmission to the ICU. Older age, comorbid conditions and severity of illness (APACHE score) have been among the strongest predictors for readmission. In our ICU most patients are in the groups formerly identified as risk groups, which means that stratification and prediction of readmission is difficult. Because of the unusual high severity of acute and pre-existing illnesses we could not find a data match on comparable patient groups. To investigate whether we could reduce our rate of readmission we therefore decided to perform a qualitative investigation to identify risk factors related to readmission. After identification of the risk factors we will take actions to optimize care and perform ongoing control of the implemented actions to secure that they decreases the rate of readmission.

Methods Retrospective data on patients readmitted to the ICU within 48 hours during an 8-month period (November 2011 to June 2012) were drawn from the Critical Information System (CIS) at ICU ZIT, Bispebjerg Hospital, Denmark. ZIT is a multidisciplinary unit with 10 beds and 500 to $600 \mathrm{admissions/year} \mathrm{and} \mathrm{a} \mathrm{median} \mathrm{SAPS} \mathrm{II} \mathrm{score} \mathrm{of} \mathrm{47.} \mathrm{A} \mathrm{group} \mathrm{of}$ consultants, junior doctors and nurses from the ICU and the ward each read the patient files with focus on pattern recognition and suggested trigger points to focus on. Data on trigger points were then drawn from the CIS system and re-evaluated. Finally, five key points were identified and serves as basis for future actions.

Results In a qualitative analysis, readmissions to the ICU are related to the following five key points - discharge outside day hours, lack of infection control, stay in ICU $<2$ days, lung physiotherapy ordinated but not effectuated, and several minor organ dysfunctions (atrial fibrillation and acute kidney injury). Age, diagnosis, SAPS II score or ventilator treatment during intensive care was not different in patients with successful discharge and patients readmitted in this group of patients. Conclusion It is possible and suitable to identify key points for future efforts in a given subgroup of patients using a systematic qualitative approach. 
P472

Clinical and laboratory postoperative impact on outcome of living donor liver transplantation recipients

Y Nassar, K Omar, A Battah, A Mwafy

Cairo University, Giza, Egypt

Critical Care 2013, 17(Suppl 2):P472 (doi: 10.1186/cc12410)

Introduction Adult recipients of living donor liver transplantation have a wide range of postoperative medical comorbidities altering various clinical and laboratory parameters. We aimed to assess 30day postoperative clinical and laboratory changes and their value in predicting outcome of LDLT recipients.

Methods A prospective registry involving patients who underwent LDLT in the period from October 2004 to December 2010. Postoperative 30day clinical and laboratory data included: clinical $\mathrm{BP}, \mathrm{HR}$, temperature, ICU stay, liver functions AST, ALT, GGT, ALP, TotPtn, Alb, TBil, DBil, INR, PTT, CRP, kidney functions $\mathrm{Crea}, \mathrm{Na}, \mathrm{K}$, blood picture $\mathrm{Hb}$, $\mathrm{Hct}$, TLC and Plt count.

Results Our study involved 142 recipients, 124 (87\%) males and 18 (13\%) females, who underwent living donor liver transplantation; mean age was $53 \pm 14.8$ years, ranging from 24 to 66 years; 29 cases (20.43\%) at Kasr Al Aini University Hospital and 113 cases (79.57\%) at the International Medical Center. The 30-day mortality rate was $13.38 \%$. Clinical and laboratory parameters showed gradual resolution towards normal values in survivors or deterioration in nonsurvivors, allowing one to predict mortality at various cutoff points. Clinical parameters: HR $>94$ cutoff (Acc 76.8\%, Sens $89.5 \%$, Spec 62.6\%, PPV 27, NPV 97) and ICU stay $>8$ days (Acc 76.2\%, Sens 75.3\%, Spec 95.7\%, PPV 95.7, NPV 35.3). Laboratory parameters: INR $>3.06$ cutoff (Acc $87.5 \%$, Sens $92.9 \%$, Spec 78.6\%, PPV 96.3, NPV 64.7), followed by PTT $>53.3$ (Acc 79.2\%, Sens $78.6 \%$, Spec 75\%, PPV 93.6, NPV 42.9), Na $>142.9$ (Acc 72.9\%, Sens $80 \%$, Spec 72.3\%, PPV 26.7, NPV 96.6), TBil >15 (Acc 71.3\%, Sens 95.5\%, Spec 50\%, PPV 92.4, NPV 63.6), ALT > 379 (Acc 67.5\%, Sens 72.2\%, Spec 73.8\%, PPV 28.9, NPV 94.7), TProt $<4.6$ (Acc 63.5\%, Sens 55.6\%, Spec 73.3\%, PPV 24.4, NPV 91.4), AST > 145 (Acc 57.4\%, Sens 50\%, Spec $84.4 \%$, PPV 32.1, NPV 92), Hct $<24.4$ (Acc $54.2 \%$, Sens $47.4 \%$, Spec $81.5 \%$, PPV 34.6, NPV 88.2), CRP > 12 (Acc 51.6\%, Sens 43.7\%, Spec 73.3\%, PPV 90.5, NPV 17.7), GGT $>22$ (Acc $51.4 \%$, Sens $83.3 \%$, Spec 36.9\%, PPV 16.3, NPV 93.7).

Conclusion Predictors of mortality were INR $>3.06$ (accuracy $87.5 \%$ ) followed by PTT $>53.3$ (79.2\%), HR $>94$ (76.8\%), ICU stay $>8$ days (76.2\%), Na $>142.9$ (72.9\%), TBil $>15$ (71.3\%), ALT >379 (67.5\%), TProt $<4.6(63.5 \%)$, AST $>145$ (57.4\%), Hct <24.4 (54.2\%), CRP >12 (Acc 51.6\%) and GGT $>22$ (51.4\%).

P473

Are APACHE II scores better predictors of mortality than routine laboratory values?

Z Baykara, H Özocak, A Kuş, Z Arslan, B Yüksel, C Aksu, M Ertagın, M Solak, KToker

University of Kocaeli, Turkey

Critical Care 2013, 17(Suppl 2):P473 (doi: 10.1186/cc12411)

Introduction Even though more accurate and updated versions such as APACHE III and IV have been developed, APACHE II has remained the most widely used severity scoring system in the ICU [1]. In the present study, we aimed to develop a model mainly based on the laboratory data available upon admission to predict mortality and to compare its performance with that of APACHE II in a mixed ICU in Turkey.

Methods A total of 645 adult patients who were over 18 years old and who stayed in the ICU for more than 24 hours were included in this study. Using stepwise multivariate logistic regression analysis, a mortality prediction model was developed based on the laboratory data, diagnostic category and age. The adjusted probability of death, according to the diagnostic category of the APACHE II score (adj-APACHE II), was calculated. The ability of the laboratory model and the adjAPACHE II model to discriminate between survivors and nonsurvivors was assessed using receiver operating characteristic (ROC) curves. The calibration of the laboratory model and the adj-APACHE II model was assessed by the Hosmer and Lemeshow goodness-of-fit test.

Results The areas under the ROC curves of the laboratory models and the adj-APACHE II scores for the prediction of mortality were $0.80(95 \%$ $\mathrm{Cl}: 0.76$ to 0.85$)$ and 0.78 ( $95 \% \mathrm{Cl}: 0.74$ to 0.83$)$, respectively $(P>0.05, z$ statistic). The Hosmer-Lemeshow statistic had a chi-squared value of
$2.01(P=0.98)$ for the laboratory model and $13.2(P=0.10)$ for the adjAPACHE II model.

Conclusion If the aim is to predict mortality as accurately as APACHE II, the mortality prediction model based mainly on routine admission laboratory tests can achieve this using computer technology, without labor-related costs, as soon as the patient is admitted to the ICU. However, it is necessary to perform multicenter validation studies. Reference

1. Breslow MJ, Badawi O: Severity scoring in the critically ill: part 1 interpretation and accuracy of outcome prediction scoring systems. Chest 2012, 141:245-252.

\section{P474}

Complementarity of prognosis models SAPS 3 and EuroSCORE in cardiac surgery

MJ Delgado-Amaya', E Curiel-Balsera', MD Arias-Verdu', E Castillo-Lorente², FJ Carrero-Gómez ${ }^{3}$, E Aguayo-DeHoyos ${ }^{4}$,

A Herruzo-Avilés

'Hospital Regional Carlos Haya, Málaga, Spain; ${ }^{2}$ Complejo Hospitalario de Jaén, Spain ${ }^{3}$ Puerta del Mar Hospital, Cádiz, Spain; ${ }^{4}$ Virgen de las Nieves Hospital, Granada, Spain; ${ }^{5}$ Virgen del Rocio Hospital, Seville, Spain Critical Care 2013, 17(Suppl 2):P474 (doi: 10.1186/cc12412)

Introduction The aim of this study is to evaluate the performance of SAPS 3 and EuroSCORE and whether there is complementarity between them in ICU patients admitted after cardiac surgery.

Methods An observational, prospective and multicenter study of patients included in the ARIAM registry of adult cardiac surgery. We analyzed clinical variables, surgery data and postoperative complications, outcomes and scores (SAPS 3 and EuroSCORE). Discrimination was assessed using the area under the ROC curve. With the standardized mortality ratio (SMR) we evaluated the agreement between predicted and observed mortality. We used multiple logistic regression for multivariate analysis.

Results A total of 5,642 patients were included, admitted to any five hospitals, consecutively between 2008 and 2012. Mean age was $63.62 \pm 12.80$ years. Surgery room mortality was $1.4 \%$, ICU mortality was $7.9 \%$ and in-hospital mortality was $11.2 \%$. SAPS 3 was calculated in 5,383 patients. SAPS 3 was $40.91 \pm 10.46$, and the expected rate of mortality was $11.09 \%$ (with the southwestern Europe equation), with an observed hospital mortality of $9.6 \%, \mathrm{SMR}=0.87$ ( 0.79 to 0.94$)$. With the general equation, the expected mortality was $10.47 \%$, SMR $=0.917$ ( 0.843 to 0.996 ). Discrimination of SAPS 3 was evaluated using the area under the ROC curve, it was 0.77 ( 0.75 to 0.79 ). The 30 -day mortality was $9.6 \%$, with an expected 30-day mortality predicted by EuroSCORE of $7.86 \%(\mathrm{SMR}=1.22 ; \mathrm{Cl}=1.12$ to 1.32$)$. The area under the $\mathrm{ROC}$ curve was 0.734 for EuroSCORE ( 0.712 to 0.755 ). Multivariate analysis with logistic regression showed complementarity between SAPS 3 and logistic EuroSCORE (vers. I). There is a relationship between hospital mortality and the probability of dying predicted by SAPS 3 (general equation), OR 1.05 (1.04 to 1.06), and logistic EuroSCORE (vers. I), OR 1.024 (1.015 to 1.032). The model with both variables has a similar discrimination to SAPS 3 alone. The area under the ROC curve of the combined model was 0.778 (0.758 to 0.799 ) and 0.77 (0.75 to 0.79 ) using SAPS 3 only. Conclusion There is complementarity between the SAPS 3 model and EuroSCORE. However, a joint model with both prognosis scores has no appreciable improvement in discrimination, compared with the SAPS 3 model. In our study, SAPS 3 shows very useful to rule out that the superior observed mortality (compared with the mortality predicted by EuroSCORE) is not attributable to inadequate care of patients in the ICU, the observed mortality being even slightly below the predicted mortality for patients undergoing cardiac surgery at the reference hospitals in which the SAPS 3 system was developed.

\section{P475}

Comparing intensive care treated primary, secondary and tertiary sepsis with respect to length of stay, organ failure and mortality: a pilot study

S Castegren', M Jonasson', J Sjölin², M Castegren

'Centre for Clinical Research Sörmland, Uppsala University, Uppsala, Sweden;

${ }^{2}$ Medical Sciences, Uppsala University, Uppsala, Sweden

Critical Care 2013, 17(Suppl 2):P475 (doi: 10.1186/cc12413) 
Introduction It may be hypothesized that a patient's inflammatory status might affect the clinical picture and outcome of a subsequent septic insult. To test this hypothesis, patients were categorized according to previous inflammatory activity and analyzed with respect to clinical picture and outcome in a retrospective study.

Methods The Swedish ICU registry was used to identify all adult patients diagnosed with severe sepsis or septic shock in a single Swedish ICU during 2006 to 2011. The charts were reviewed and patients categorized into four groups based on whether the patient had suffered systemic inflammatory-inducing insults previous to the septic episode. The patients were categorized as: (A) primary sepsis, no recent ( $<30$ days) trauma/infection, ongoing systemic inflammatory or immunosuppressive disease or malnutrition; (B) secondary sepsis, recent ( $<7$ days) trauma/infection or systemic inflammatory disease, but no immunosuppressive disease or malnutrition; (C) tertiary sepsis, $>1$ fulfilled criteria of secondary sepsis during the last 10 days or immunosuppressive disease or malnutrition; and (D) patients not attributable to A to C. After the categorization, physiological and laboratory parameters during the ICU stay as well as therapy and outcome were extracted from the charts. Only significant differences $(P<0.05)$ are presented; Mann-Whitney and chi-squared tests were used.

Results A total of 273 charts were reviewed. In total, 242 were categorized into A $(n=126), \mathrm{B}(n=79)$ or $\mathrm{C}(n=37)$. $\mathrm{D}(n=31)$ consisted mainly of patients with hematological malignancies $(n=12)$ and patients with chemotherapy or immunosuppressive treatment $(n=14)$. The groups differed in length of stay with $A<B<C$. During the first 3 days the SOFA score was higher in $A$ compared with $C$ and in $B$ compared with $C$. The duration of antibiotic therapy was longer in both $B$ and $C$ compared with $A$. There were no differences in 28-day mortality (A: 34/126 $=27 \%$, B: 30/79 $=38 \%, C: 15 / 37=40 \%$ ); however, the proportions of patients dying between days 8 and 28 were higher in $B(14 / 63=22 \%)$ and $C(7 / 29=24 \%)$ compared with $A(5 / 95=5 \%)$.

Conclusion In this retrospective material it was possible to categorize $88.6 \%$ of all patients as having primary, secondary or tertiary sepsis. The categories differed in clinical picture at presentation as well as in outcome. A prospective study is warranted to validate the results of this study.

P476

Survival of older patients after critical care

D Ashton-Cleary, R Sinclair

Royal Cornwall Hospital, Truro, UK

Critical Care 2013, 17(Suppl 2):P476 (doi: 10.1186/cc12414)

Introduction We aimed to assess the survival of older patients after admission to critical care compared with the population in general.

Methods We reviewed admission data for a 12-bed critical care unit in a district general hospital in the UK between 2001 and 2010. We calculated critical care and hospital discharge survival rates and studied the trend in these through the decade. We searched hospital records to establish whether these patients were still alive at November 2012 to determine life-expectancy (minimum 22-month follow-up on all patients). This was compared with UK Office of National Statistics data for the local population [1]. Discharge destination (care facility or home address) was recorded as a surrogate marker of change in quality of life post discharge.

Results A total of 481 patients aged 80 or over were admitted between 1 January 2001 and 31 December 2010, representing 9.6\% of the admissions during this time with minimal annual change. Admission age ranged from 80 to 96 years (median 83 ) with a median APACHE II score of 20 ( 6 to 48 , IQR 16 to 25 ). In total, $318(66.1 \%)$ survived to critical care discharge and 256 (53.2\%) survived to hospital discharge; 228 $(89.0 \%)$ went home rather than to a care facility. Those under 80 years demonstrated $80.6 \%$ critical care survival, $65.5 \%$ hospital survival and a median APACHE II score of 17 ( 0 to 51, IQR 13 to 23). Eighty patients died within the 22-month follow-up period with an average life expectancy of 7.4 months. None reached life expectancy (average curtailment of 5.6 years compared with age-matched and sex-matched population statistics). Neither of these figures changed significantly through the decade. However, 10-year follow-up on 2001/2002 admissions showed that, despite this early mortality effect, the average post-discharge lifespan was 5.1 years with 2.2 years shortfall in life expectancy.

Conclusion Older people represent a growing proportion of the population although their representation in the critical care population remained constant in this 10-year study. These patients had a slightly higher median APACHE II score and $14.5 \%$ greater critical care mortality than the younger patients. The majority of survivors were able to go home; however, 31\% died within 22 months with significant life expectancy curtailment, surviving on average only 7.4 months after discharge; this has not changed in the last 10 years. Those who survived this initial period (69\%) had a much better outlook. This information may be vital to patients and physicians when discussing admission to critical care.

Reference

1. Office of National Statistics Population Data [http://www.ons.gov.uk/ons/ taxonomy/index.html?Nscl=Population]

\section{P477}

Eldicus scores for admission to our ICU: a retrospective study

G Zani, B Gemelli, S Vitali, C Villani, R Ragazzi, S Spadaro, CA Volta, R Alvisi University of Ferrara, Italy

Critical Care 2013, 17(Suppl 2):P477 (doi: 10.1186/cc12415)

Introduction The indication for ICU admission is poorly defined and identifying patients who may benefit from it is extremely difficult. As reported in literature, the Eldicus scores require further validation in centers in different countries and with different patient populations [1]. Previous studies have demonstrated that patients with intermediate severities of illness are those that benefit from ICU treatment; instead, patients who are too well or too sick should not be admitted. There are many unmeasurable factors that influence the triage decision and can influence the subsequent outcome. The Eldicus scores could be the first triage decision rule. The aim of this study was to confirm the ability of the Eldicus scores to discriminate the survival status in our general ICU. Methods We retrospectively enrolled all patients $>18$ years admitted to the Ferrara ICU from January to June 2012. Patients' data were recorded using Prosafe international data collection software and consulting the individual medical records. Data were chosen based on variables used in the Eldicus scores. As indicated in the Eldicus algorithm, for each patient the initial refusal score was calculated, to evaluate the too severe patients. Then we counted the final triage score, to discover whether some patients were too healthy to be admitted to the ICU. Correlation between the severity of the scores and patients' outcome was estimated using the Wilcoxon's rank-sum test.

Results Ninety-eight patients were finally enrolled. Data are presented as median \pm SD. The SAPS II score was $33 \pm 13$ and was similar to those reported in the literature [1]. The Karnofsky performance status scale was $69 \pm 20$. The Eldicus initial score was $56 \pm 30$. The final score was $70 \pm 48$. Seventy-eight percent of the analyzed patients were alive at hospital discharge. A significant correlation between the severity scores and in-hospital outcome was found $(P<0.001)$. Limitations of our study were the low sample size and the fact that patients not admitted to the ICU were not considered.

Conclusion The Eldicus scores appear to be useful in identifying patients that may benefit more from ICU admission. Further studies should confirm the ability of these scores in helping identify those patients who really benefit from ICU admission.

Reference

1. Sprung CL, et al:: Crit Care Med 2012, 40:125-131.

\section{P478}

Developing a screening instrument for predicting psychological morbidity after critical illness

A Schandl', M Bottai', E Hellgren', Ö Sundin ${ }^{3}$, P Sackey'

'Karolinska University Hospital Solna, Stockholm, Sweden; 'Karolinska Institutet, Stockholm, Sweden: ${ }^{3}$ Mid Sweden University, Östersund, Sweden Critical Care 2013, 17(Suppl 2):P478 (doi: 10.1186/cc12416)

Introduction The purpose was to develop a predictive screening instrument for use at ICU discharge to identify patients at risk for posttraumatic stress, anxiety or depression. 
Methods Potential risk factors for psychological problems were prospectively collected at ICU discharge. Two months after ICU discharge 252 ICU survivors received the questionnaires PostTraumatic Stress Symptom scale-10 (PTSS-10) and Hospital Anxiety and Depression Scale (HADS) to estimate the degree of post-traumatic stress, anxiety and depression.

Results Of the 150 responders, $31 \%$ had adverse psychological outcome, defined as PTSS- $10>35$ and/or HADS subscales $\geq 8$. After analysis, six predictors with weighted risk scores were included in the screening instrument: major pre-existing disease, being a parent to children younger than 18 years of age, previous psychological problems, in-ICU agitation, being unemployed or sick-listed at ICU admission and appearing depressed in the ICU. Each predictor corresponded to a given risk score. The total risk score, the sum of individual risk scores, was related to the probability for adverse psychological outcome in the individual patient. The predictive accuracy of the screening instrument, as assessed with area under the receiver operating curve, was 0.77 . When categorizing patients in three risk probability groups - low ( 0 to $29 \%$ ), moderate (30 to $59 \%$ ) and high (60 to $100 \%$ ) risk - the actual prevalence of adverse psychological outcome in respective groups was $12 \%, 50 \%$ and $63 \%$.

Conclusion The preliminary screening instrument may aid ICU clinicians in identifying patients at risk for adverse psychological outcome after critical illness. Prior to wider clinical use, external validation is needed.

\section{P479}

Prediction of sequences of organ dysfunction in critical patients studied with Bayesian analysis

M Sandri', P Berchialla², D Gregori³, L Gottin', V Sweiger', E Polati', RA De Blasi ${ }^{4}$

'University of Verona, Italy; ${ }^{2}$ University of Torino, Italy; ${ }^{3}$ University of Padova, Italy; ${ }^{4}$ University of Rome 'La Sapienza', Rome, Italy

Critical Care 2013, 17(Suppl 2):P479 (doi: 10.1186/cc12417)

Introduction The multiorgan dysfunction syndrome (MODS) is a dynamic process involving simultaneously or consecutively two or more organ systems [1]. The organ dysfunction's degree can be assessed by three severity scores (SOFA [2], MODS [3], LODS [4]), but they have some limitations: they do not allow the evaluation of the clinical course of a patient, they are not reliable in populations different from the reference one, and they do not support clinicians' decisions. Because MODS implies a systemic inflammatory reaction leading to microcirculatory dysfunction, our hypothesis was that organ failures follow a predictable sequence of appearance. Our aims were to verify the presence of more likely organ failure sequences and to assess an online method to predict the evolution of MODS in a patient. The high mortality and morbidity rate of MODS in ICUs can in fact be reduced only by a prompt and well-timed treatment [5].

Methods We selected 73 patients consecutively admitted to the ICU of Sant'Andrea Hospital from January to June 2012. The inclusion criteria were at least two organ systems with SOFA $\geq 2$, ICU length of stay $>48$ hours. For each patient we calculated the SOFA since the beginning of the inclusion criteria and daily for 8 days. For the statistical analysis we used Dynamic Bayesian Networks (DBNs) [6]. DBNs were applied to model SOFA changes in order to identify the most probable sequences of organs failures in a patient who experienced a first known failure.

Results We created a DBN for the analysis of MODS studying the relations between organ failures at different times. The DBN was made so that each organ failure is dependent on the previous one. We also considered a corrective factor to take account that not all patients completed the observation. Using software (GeNie) we obtained the probabilities of the organ failure sequences.

Conclusion The use of DBNs, although with our limited set of data, allowed us to identify the most likely organ dysfunction sequences associated with a first known one. Capability to predict these sequences in a patient makes DBNs a promising prognostic tool for physicians in order to treat patients in a timely manner, or to test a treatment efficacy. References

1. Levy MM, et al:: 2001 SCCM/ESICM/ACCP/ATS/SIS International Sepsis Definitions Conference. Intensive Care Med 2003, 29:530-538.

2. Vincent $\mathrm{JL}$, et al:: The SOFA (Sepsis-Related Organ Failure Assessment) score to describe organ dysfunction/failure. On behalf of the Working Group on
Sepsis-Related Problems of the European Society of Intensive Care Medicine. Intensive Care Med 1996, 22:707-710.

3. Marshall JC, et al: Multiple organ dysfunction score: a reliable descriptor of a complex clinical outcome. Crit Care Med 1995, 23:1638-1652.

4. Le Gall JR, et al.: The logistic organ dysfunction system: a new way to assess organ dysfunction in the intensive care unit. JAMA 1996, 276:802-810.

5. Kilica YA, et al:: Visualizing multiple organ failure: a method for analyzing temporal and dynamic relations between failing systems and interventions. Crit Care 2007, 11:417.

6. Murphy K, et al:: Modelling Gene Expression Data using Dynamic Bayesian Networks. Technical report. University of California; 1999.

\section{P480}

Comparison of IGS 2 score and a new neural network biomarker score performance in predicting 1-year mortality

C Romdhani, I Labbene, M Belhadj Amor, Z Hajjej, A Massoudi, M Ferjani Military Hospital of Tunis, Tunisia

Critical Care 2013, 17(Suppl 2):P480 (doi: 10.1186/cc12418)

Introduction The aim of this study was to compare the IGS 2 score with a predictive model built using clinical characteristics and biomarkers associated with 1-year mortality from procalcitonin, C-reactive protein, troponin I (Tnl) and NT-Pro-BNP.

Methods We conducted a prospective observational cohort study in a polyvalent ICU. We calculated the IGS 2 severity score in the first 24 hours. We dosed NT-Pro-BNP, Tnl, procalcitonin and C-reactive protein at admission to the ICU.To test the biomarker model, the patient database was divided into training (70\%) and test (30\%) databases. A neural network model was trained from variables associated with 1-year mortality. All statistics were done using R v.2.15.1. We used the pROC package to plot and compare ROC curves.

Results Lower age, NT-Pro-BNP and Tnl were significantly associated with 1-year survival. We built a neural network model using the training database. We used the test database to compare our model with the IGS 2 score. The difference between the AUC of the biomarker score and the IGS 2 score was statistically significant (Figure 1).

Conclusion Our new neural network model built using age, NT-PROBNP and Tnl succeeded the internal-validation processes and predicts 1 -year mortality better than IGS 2.

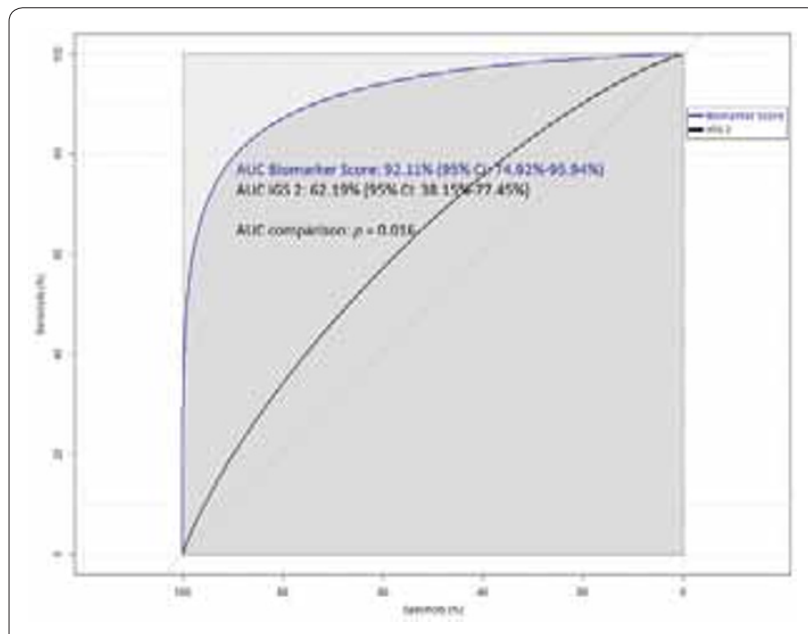

Figure 1 (abstract P480). Comparison of area under ROC curves (AUC) of the biomarker score and IGS 2.

\section{P481}

Significance of preadmission quality of life for mortality in the ICU: a prospective cohort study

RB Bukan', A Moeller', M Henning', K Mortensen², T Klausen', TWaldau'

'Herlev University Hospital, Herlev, Denmark; 'University of Copenhagen, Denmark

Critical Care 2013, 17(Suppl 2):P481 (doi: 10.1186/cc12419) 
Introduction Assessing whether a critically ill patient should be admitted to an ICU remains difficult and mortality amongst ICU patients is high. To render intensive care with no prospect of success is an immense emotional burden for both patient and relatives, and a great socioeconomic burden for society as well. Therefore, validated strategies that can help identify patients who will benefit from intensive care are in demand. This study seeks to investigate whether preadmission quality of life can act as a predictor of mortality amongst patients admitted to the ICU.

Methods All patients ( $>18$ years) admitted to the ICU for more than 24 hours are included. In order to assess preadmission quality of life, the patient or close relatives complete the Short-Form 36 (SF-36) within 72 hours after ICU admission. Mortality is evaluated from ICU admission until 30 days hereafter. Logistic regression and receiver operating characteristic analyses are employed to assess predictive value for mortality using five models: SF-36 Physical Component Summary (PCS) and APACHE II (model A), SF-36 PCS (model B), SF-36 General Health (GH) and APACHE II (model C), SF-36 GH (model D), and APACHE II (model E). Classification tables are composed in order to assess sensitivity, specificity, positive and negative predictive values and likelihood ratios.

Results Preliminary results, based upon data from 175 included patients, reveal an ICU mortality of $12.6 \%$ and 30 -day mortality of $22.9 \%$. No patients were lost to follow-up. When the Physical Component of SF-36 (PCS) was used as an estimate of preadmission quality of life, the area under the curve for model $B(A U C=0.80$ ) was comparable with model $E$ (AUC $=0.81)$, and better than model A (AUC $=0.85)$. The General Health item of SF-36 (GH), used as an estimate of preadmission quality of life, gave an AUC $=0.76$ (model D). All models were controlled for sex and age.

Conclusion Preliminary results indicate that the SF-36 GH and the SF-36 PCS are as good as APACHE II to predict mortality 30 days after ICU admission. However, in order to conclude whether preadmission quality of life can contribute to triage, by successfully identifying patients suitable for intensive care, final analyses, due in 2013, are awaited. These results will clarify whether future randomized controlled trials, in which preadmission quality of life acts as a supplement to triage, are justifiable.

\section{P482}

Predicting physical function and mental health in trauma intensive care patients 2 years after hospitalisation

L Aitken', B Macfarlane', W Chaboyer', M Schuetz', C Joyce', AG Barnett ${ }^{3}$

'Princess Alexandra Hospital, Brisbane, Australia; ${ }^{2}$ Griffith University,

GoldCoast, Australia: ${ }^{3}$ Queensland University of Technology, Brisbane,

Australia

Critical Care 2013, 17(Suppl 2):P482 (doi: 10.1186/cc12420)

Introduction Long-term compromise after traumatic injury is significant; however, few modifiable factors that influence outcome have been identified. The aim of this study was to identify acute and early post-acute predictors of long-term recovery amenable to change through intervention.

Methods Adults ( $>17$ years) admitted to the ICU, Princess Alexandra Hospital, Australia following injury were prospectively followed. Data were collected on demographics, pre-injury health, injury characteristics and acute care factors. Psychosocial measures (selfefficacy (SE), illness perception (IP), post-traumatic stress disorder (PTSD) symptoms and psychological distress) and health status (SF-36) were collected via questionnaire 1, 6, 12, and 24 months post injury. Outcomes of interest were the Physical Function (PF) and Mental Health $(\mathrm{MH})$ subscales of the SF-36. Regression models were used to estimate predictors of physical function and mental health over a 2-year period. A subject-specific intercept in a mixed model was used to account for repeated data from participants over time.

Results Participants $(n=123)$ were young (median 37, IQR 22 to 55 years), predominantly male (83\%) and spent on average 3 days in the ICU and 3 weeks in hospital. Response rates were over $55 \%$ at each follow-up, with responders similar to nonresponders except for being generally older. PF and MH scores improved over time, although the averages remained below the Australian norms at 24 months.
Predictors of PF included IP $(\beta=-1.5,95 \% \mathrm{Cl}=-3.1$ to $-1.1, P<0.01), \mathrm{SE}$ $(\beta=1.8,95 \% \mathrm{Cl}=1.3$ to $2.6, P<0.01)$, hospital length of stay $(\beta=-1.7$, $95 \% \mathrm{Cl}=-2.0$ to $-0.8, P<0.01)$, never having been married $(\beta=1.8,95 \%$ $\mathrm{Cl}=0.3$ to $5.5, P=0.03)$, and having injury insurance $(\beta=-2.7,95 \%$ $\mathrm{Cl}=-6.9$ to $-1.9, P<0.01)$. Predictors of MH included PTSD symptoms $(\beta=-2.4,95 \% \mathrm{Cl}-3.4$ to $-1.4, P<0.01)$, psychological distress $(\beta=-6.9$, $95 \% \mathrm{Cl}=-8.9$ to $-5.2, P<0.01)$, SE $(\beta=0.6,95 \% \mathrm{Cl}=0.2$ to $1.1, P<0.01)$, and unemployment $(\beta=-2.3,95 \% \mathrm{Cl}=-5.0$ to $-0.2, P=0.04)$.

Conclusion Trauma ICU patients experience compromised physical function and mental health 24 months after injury. Psychological distress, self-efficacy and illness perception influence outcomes and are potentially amenable to change in response to interventions initiated during hospital stay.

P483

Do ICU patients' discharge characteristics change with Swiss

Diagnosis Related Groups?

G Rauchenstein-Ziltener, CA Rüst, P Fodor, S Blumenthal

Tiemli City Hospital, Zurich, Switzerland

Critical Care 2013, 17(Suppl 2):P483 (doi: 10.1186/cc12421)

Introduction Swiss Diagnosis Related Groups (SwissDRG) have been effective since 1 January 2012. The influence of this new system on patients' discharge characteristics from a large ICU is not known. With the introduction of the DRG we expect patients to be discharged after a shorter length of stay on the ICU and with higher severity of illness. Methods The ICU of the City Hospital Triemli in Zurich has an interdisciplinary organization with surgical and internal medical patients, with a maximum occupancy of 18 beds and a center function for the surrounding hospitals. In this ongoing prospective observational study, we collect and analyze the anonymized data of all patients discharged from our ICU prior to and after the introduction of the SwissDRG. The primary endpoint was the length of stay on the ICU in hours. The secondary endpoints were the severity of illness of the patients at the time of discharge, detected by the scoring system SAPS II as well as measured by the number of readmissions to the ICU. Initially all patients were analyzed and in a second step only patients within percentiles 6 to 94 were considered. We also analyzed the subgroups of patients referred internally, patients sent back to referring hospital and patients regionalized to a homebase hospital. The statistics have been done with SPSS and $P<0.05$ was considered significant.

Results We present the results of an 18-month period, 9 months prior to and 9 months after the introduction of the SwissDRG. Data of 1,491 and 1,492 patients were analyzed, respectively. When all patients were included, we found prior to and after the introduction of the DRGs a comparable length of stay on the ICU (mean \pm SD of $52.1 \pm 2.2$ hours vs. $50.82 \pm 2.2$ hours), no difference in the severity of illness at discharge detected by the SAPS II (mean \pm SD of $27.9 \pm 0.3$ vs. $28.4 \pm 0.3$ ) and the number of readmissions (91 vs. 92). There was also no significant difference when only percentiles 6 to 94 were included or when the three subgroups were analyzed.

Conclusion Up to now, the introduction of the SwissDRG has no influence on patients' discharge characteristics from a large ICU. Data assessment will continue and further data analysis has to be performed.

P484

Do Diagnosis Related Groups change admission practice to a large Swiss ICU?

K Homburg, CA Rüst, P Fodor, S Blumenthal

Triemli City Hospital, Zurich, Switzerland

Critical Care 2013, 17(Suppl 2):P484 (doi: 10.1186/cc12422)

Introduction The new Swiss financing system by means of Diagnosis Related Groups (DRG), effective 1 January 2012, forms the basis of a performance-based system to reimburse general hospital services. There are only few data on the influence of DRG on ICU patients $[1,2]$. We expect that the introduction of the DRG in Switzerland will change the number of admissions from external hospitals to a large ICU with a centre function and will influence the severity of disease of the admitted patients. 
Methods The ICU of the Triemli City Hospital in Zurich has an interdisciplinary organisation with surgical and internal medical patients, with a maximum occupancy of 18 beds and a centre function for the surrounding hospitals of the region. In this prospective ongoing observational study, we collect and analyse the anonymised data of all patients admitted to our ICU from an external hospital during 12 months prior to (1 January to 31 December 2011) and after (1 January to 31 December 2012) the introduction of the DRG in Switzerland. Exclusion criteria are admissions by the emergency department, self-assignments into the hospital and internal relocations. The primary endpoint is the number of admissions from an external hospital to our ICU. Secondary endpoints are the severity of the disease of the admitted patients, detected by the scoring systems SAPS II and APACHE II as well as the length of stay in external hospitals before admission. The statistical analysis is descriptive.

Results We present the preliminary data for 10 months (in each case January to October) before and after the introduction of the DRG. We observed an increase of $9.2 \%$ (391 vs. 427 patients) of admissions to our ICU after the introduction of the DRG. The severity of disease determined by the SAPS II score is unchanged (mean 26.7 vs. 26.0 points, $P=0.466)$. The severity of disease determined by the APACHE II score is significantly lower (15.4 vs. 14 points, $P=0.017$ ). We also noted that after the introduction of the DRG the patients were earlier transferred from an external hospital to our ICU (mean time until transfer 29.9 vs. 18.7 hours), but this value was not significant $(P=0.55)$. Conclusion Up to now the introduction of the DRG in Switzerland has had a complex influence on the number and the kind of patients admitted from an external hospital to an ICU with centre function. Data assessment and analysis will continue.

References

1. Ahmad M, et al:: Chest 1988, 93:176-179.

2. Thomas F, et al:: Chest 1987, 91:418-423.

P485

Variable cost of a Greek ICU

ETsigou', D Karabatsou', E Boutzouka', V Psallida', G Baltopoulos',

MTsironi ${ }^{2}$

'A. Anargiroi Hospital, Athens, Greece; ${ }^{2}$ University of Peloponnisos, Sparti, Greece

Critical Care 2013, 17(Suppl 2):P485 (doi: 10.1186/cc12423)

Introduction The ICU consumes almost $20 \%$ of total hospital resources, despite accounting for less than $10 \%$ of hospital beds. Medical cost can be divided into a fixed part, consisting of personnel and accommodation expenses, and a variable part, determined by the needs of each patient (medications, consumables, examinations). In order to rationalize medical cost, estimation of expenses must be real and individualized. The aim of this study was to assess the variable cost of critically ill patients in a new, seven-bed, adult, general ICU, using bottom-up costing methodology.

Methods All 138 patients who were treated during 2011, and stayed for at least 24 hours, were included in the study. Data were retrospectively collected from patient records and included demographics, cause of admission, APACHE II score at admission, length of stay (LOS) and outcome. Cost was recorded for every day and for every patient, based on a hospital-specific cost catalogue and on national agreements. Data are presented as mean \pm SEM. Analysis of data was carried out using Graph Pad Prism 5.0., applying Student's $t$ test.

Results The age of participants ( 84 men and 54 women, 71 medical and 67 surgical) was $68.75 \pm 1.18$, APACHE II score was $18.64 \pm 0.61$, LOS was $18.46 \pm 2.54$, and mortality was $19 \%$. The majority of the patients (61\%) were mechanically ventilated. The total days of ICU stay were 2,548 and the total variable cost was $€ 1,460,465$ ( $€ 573.18$ per patient and per day). Cost per patient was subdivided as follows: medication (including drugs, fluids, blood products, nutrition): $56.49 \%$, examinations (including laboratory, microbiological assays and diagnostic procedures): $22.23 \%$, consumables: $21.26 \%$. As for medication cost, the largest part was comprised of antibiotics (45.69\%), followed by blood products (17.61\%) and cardiovascular drugs (12.39\%). Costs for medical patients were significantly higher than those for surgical patients $(P<0.0001)$.

Conclusion The total average cost per patient and per day was found to be $€ 573.18$. Medication expenditure was responsible for the highest variable cost of intensive care treatment. Among drugs, antibiotics accounted for the largest part. A medical cause of admission was correlated with higher costs.

References

1. McLaughlin AM, et al: Intensive Care Med 2009, 35:2135-2140.

2. Rossi C, et al: Intensive Care Med 2006, 32:545-552.

P486

Audit of the medical staffing of and referrals to a mixed adult ICU in a teaching hospital

A Needham, T Lyndham, D O'Neil, J Greiff

University Hospitals of Leicester, UK

Critical Care 2013, 17(Suppl 2):P486 (doi: 10.1186/cc12424)

Introduction Leicester Royal Infirmary is a (funded) 15-bed ICU, annual admission 1,102 patients per year (ICNARC data 2011 to 2012). It houses a broad range of specialty, has over 1,000 beds and an emergency department (ED) responsible for 297 admissions over the 2011 to 2012 period. Unit guidelines state that a doctor should be immediately available to the unit at all times, ideally physically present on the unit. This audit looked in detail at each occasion a junior doctor left the unit, the timings, reason, and outcome of each episode.

Methods Between 17 October 2011 and 14 November 2011, and between 2 July 2012 and 30 July 2012, junior doctors on the ICU completed a data-capture form after each occasion that they left the unit for a clinical need. Data collected were matched to the objectives. The 2011 audit results were communicated at the University Hospitals of Leicester Trust ICU Audit Meeting. A system was introduced so that daytime referrals were directed to the ICU consultant. The impact of this intervention was assessed by the 2012 re-audit.

Results There were 105 occasions when a junior doctor left the unit in 2011 , totalling 118 hours and 38 minutes ( 5 days), a continuation of the upward trend from the previous 2 years. In 2012 this reduced to 47 occasions totalling 40 hours and 55 minutes. Most attended referrals were during the daytime, 54\% in 2011 and $68 \%$ in 2012. The majority of referrals originated from the $\mathrm{ED} /$ medicine, combined numbers $58 \%$ in 2011 and 38\% in 2012. In 2011 only 30\% of ED referrals had been discussed with a consultant of the referring team and only $32 \%$ of medical referrals. Just two of the 14 inappropriate ED referrals were discussed with the referring consultant, and none of the six inappropriate medical referrals were discussed with a medical consultant. In 2012 just $33 \%$ of ED/medical referrals had prior discussion with a consultant from the referring team. At the point of leaving, the ICU was left without a doctor on 22 occasions in 2011 and six occasions in the 2012 audit. Most occasions occurred at either night shift/weekend (86\% in 2011/100\% in 2012). Referral resulted in a patient being admitted to the ICU on 20 occasions in 2011 and 12 in 2012.

Conclusion The latest audit follows introduction of a referral system directly to the ICU consultant and may account for the reduction in numbers of referrals attended by junior doctors. ED/medicine persist as the main source of referral to the ICU. Discussion with the referring team consultant may reduce inappropriate referrals. ICU staffing should not be reduced.

\section{P487}

Impact of the time elapsed between ICU request and actual admission on mortality and length of stay GM Filho', TA Silva', AR Santana', FB Soares', LJ Almeida', LG Godoy'? TA Rodrigues', MO Maia'2, JA Neto², AP Amorim³, EB Moura², FF Amorim 'Escola Superior de Ciências da Saúde, Brasília, Brazil:'2Unidade de Terapia Intensiva Adulto do Hospital Santa Luzia, Brasilia, Brazil; ${ }^{3}$ Liga Academica de Medicina Intensiva do Distrito Federal (LIGAMI), Brasilia, Brazil Critical Care 2013, 17(Suppl 2):P487 (doi: 10.1186/cc12425)

Introduction Measures to ensure an appropriate early treatment for critically ill patients result in significant decreases in mortality $[1,2]$. This study aims to evaluate the impact of the time elapsed from request until admission to the ICU on mortality and ICU length of stay (LOS). Methods A retrospective cohort study performed on patients in the ICU of Hospital Regional de Samambaia over a period of 4 years, from January 2008 to December 2011. The patients were allocated into two groups: patients who waited longer than 6 hours, long waiting period 
(LWP, $n=300$ ); and patients whose waiting time was equal to or less than that period, short waiting period (SWP, $n=113$ ).

Results In total, 413 patients were included, 300 of which belonged to the LWP group (65.4\%). For the entire cohort, the mean APACHE II score was $19 \pm 7$, the mean age was $52 \pm 22$ years, and 211 patients were male (51.1\%). The LWP group did not show difference in the APACHE II score $(19 \pm 7$ vs. $18 \pm 8, P=0.13)$, but was older $(55 \pm 20$ vs. $49 \pm 23, P=0.01)$. LWP also had a higher incidence of primary bloodstream infection $(23.8 \%$ vs. $10.4 \%, P=0.01)$ and catheter-associated urinary tract infection $(10.2 \%$ vs. $1.9 \%, P=0.01$ ). LWP patients had higher mortality ( $37.8 \%$ vs. $25.9 \%$, $P=0.02$ ) and longer ICU LOS ( $21 \pm 47$ vs. $14 \pm 18$ days, $P=0.01)$. Relative risk for death in the LWP was 1.74 (95\% Cl: 1.11 to 2.72 ).

Conclusion Despite showing no significant differences on APACHE II scores from the SWP group, patients from the LWP group presented greater incidence of primary bloodstream infection, catheterassociated urinary tract infection, higher mortality outcomes and longer ICU LOS.

\section{References}

1. Amorim FF, et al: I Clin Med Res 2012, 4:410-414.

2. Rivers E, et al:: NEngl J Med 2001, 345:1368-1377.

P488

Medical problems: review from the major nuclear incidents

Y Haraguchi', Y Tomoyasu' ${ }^{2}$, H Nishi ${ }^{3}$, M Hoshino ${ }^{4}$, M Sakai ${ }^{5}$, E Hoshino ${ }^{6}$

'Disaster Medicine Compendium Team, Japan, Tokyo, Japan;' 2Shirahigebashi Hospital, Tokyo, Japan; ${ }^{3}$ Tokyo Disaster Medical Center, Tokyo, Japan: ${ }^{4}$ Shisei

Hospital, Saitama, Japan; ${ }^{5}$ Tokyo Women's Medical College, Tokyo, Japan;

${ }^{6}$ Chiba Nursing Association, Chiba, Japan

Critical Care 2013, 17(Suppl 2):P488 (doi: 10.1186/cc12426)

Introduction Intensivists are expected to have many roles during and after a major disaster/catastrophe; that is, triage, intensive care, education for people, and so forth. The roles of intensivists against special disaster or nuclear disaster are studied based on actual experiences.

Methods Several disasters are studied. The Fukushima Daiichi Nuclear plant explosion after the Higashinihon earthquake 2011 was medically reviewed based on the total 30-day stay on-site in addition to several days around the site. The Chernobyl incident 1986 was inspected 15 years after the incident. Other nuclear disasters are included.

Results Many serious problems were revealed in the medical teams, which are as follows: inappropriate basic preparedness against large special disasters, including nuclear disaster; lack of appropriate education and training for medical teams against nuclear disaster that is, most members of Japan DMAT or the disaster medical assistance team are still laypersons; incorrect standard/rules of Japan DMAT, which were excessively focused upon cure of the usual type of injury and planned short period or nearly 48 hours, which should be abandoned; and insufficient consideration to the weak/vulnerable people or CWAP, children, (pregnant) women, aged people, and the poor people/sicker patients. Many of them died because of an insufficient emergency transportation system from their contaminated houses or hospital. Conclusion In order to cope with the special disasters, such as NBC or nuclear, biological and chemical disaster, it is insufficient to take makeshift measures or use cheap tricks. Working out the systematization of disaster medicine, based upon the academic viewpoints and philosophy/reliability, is essential to protect the people and the nation.

\section{P489}

Variation in acute care burden and supply across diverse urban settings

S Murthy', S Austin ${ }^{2}$, H Wunsch ${ }^{3}$, NK Adhikari ${ }^{4}$, V Karir ${ }^{2}$, K Rowan ${ }^{5}$,

ST Jacob ${ }^{6}$, J Salluh', F Bozza ${ }^{8}$, B Du ${ }^{9}$, Y An ${ }^{10}$, B Lee ${ }^{2}$, FWu$^{2}$, C Oppong ${ }^{11}$

R Venkataraman ${ }^{12}, V_{\text {Velayutham }}^{13}, \mathrm{D} \mathrm{Angus}^{2}$

'Hospital for Sick Children, Toronto, Canada; ' ${ }^{\prime}$ University of Pittsburgh, PA, USA; ${ }^{3}$ Columbia University, New York, NY, USA; ${ }^{4}$ University of Toronto, Canada; 5 Intensive Care National Audit \& Research Centre, London, UK: ${ }^{U}$ University of Washington, Seattle, WA, USA: ${ }^{7} D^{\prime} O R$ Institute for Research and Education, Rio de Janeiro, Brazil: ${ }^{8}$ Oswaldo Cruz Foundation, Rio de Janeiro, Brazil,; 9 Peking Union Medical College Hospital, Beijing, China: ${ }^{10}$ Peking University People's Hospital, Beijing, China; "Komfo Anokye Teaching Hospital, Kumasi, Ghana; "2 Apollo Hospital, Chennai, India; ${ }^{13}$ Stanley Medical College Hospital, Chennai, India

Critical Care 2013, 17(Suppl 2):P489 (doi: 10.1186/cc12427)
Introduction The World Bank has warned that the rapid growth of the world's urban population can only be accommodated safely if cities adequately develop key infrastructure, such as the provision of acute care resources. Yet, even basic descriptive information on urban acute care supply and demand is extremely limited. We therefore conducted a pilot assessment across seven diverse urban settings across the world. Methods We selected a convenience sample of seven large cities with varying geographical and socioeconomic characteristics: Boston, Paris, Bogota, Recife, Liaocheng, Chennai, and Kumasi. To estimate acute care supply, we developed an instrument to collect data on acute and critical care infrastructure. We collected data from municipal authorities and local research collaborators. We expressed the burden of acute disease as the number of deaths due to acute illnesses, estimated from the 2008 Global Burden of Disease Study. Results were expressed as acute care supply and acute deaths per 100,000 population and acute care supply per 100 acute deaths.

Results The supply of hospital beds varied from 72.4/100,000 population in Kumasi to $245.8 / 100,000$ in Boston. ICU beds with capacity for invasive mechanical ventilation and intensive nursing services ranged from $0.4 / 100,000$ in Kumasi to $19 / 100,000$ population in Boston. The number of ambulances varied 70 -fold between cities. The gap between cities widened when demand was estimated based on disease burden, with a 70-fold difference between cities in ICU beds/acute deaths. In general, most of the data were unavailable from municipal authorities.

Conclusion The provision of acute care services, a key aspect of urban infrastructure, varied substantially across the seven diverse urban settings we studied. Furthermore, the local municipal authorities generally appeared to have little knowledge of their acute care infrastructure, with implications for future planning and development.

P490

Differences in severity and resource utilization for medical and

surgical ICU patients

BH Nathanson', WT McGee², E Lederman², TL Higgins²

'OptiStatim LLC, Longmeadow, MA, USA; ${ }^{2}$ Baystate Medical Center,

Springfield, MA, USA

Critical Care 2013, 17(Suppl 2):P490 (doi: 10.1186/cc12428)

Introduction Medical and surgical patients use the ICU differently. Resources may not always be allocated by severity of illness, but by custom or habit, particularly if different groups administer bed control and triage. Specialty-specific differences may exist even when a single team controls triage. Variability in resource utilization has important implications for cost-containment and triage.

Methods Patients admitted to a single, closed medical/surgical ICU with full-time intensivists and unified triage control in a large, university-affiliated hospital were evaluated during 2011 to 2012. Patients who died in the ICU were excluded. The day of discharge (D/C) and severity using APACHE IV and its related Acute Physiology Score

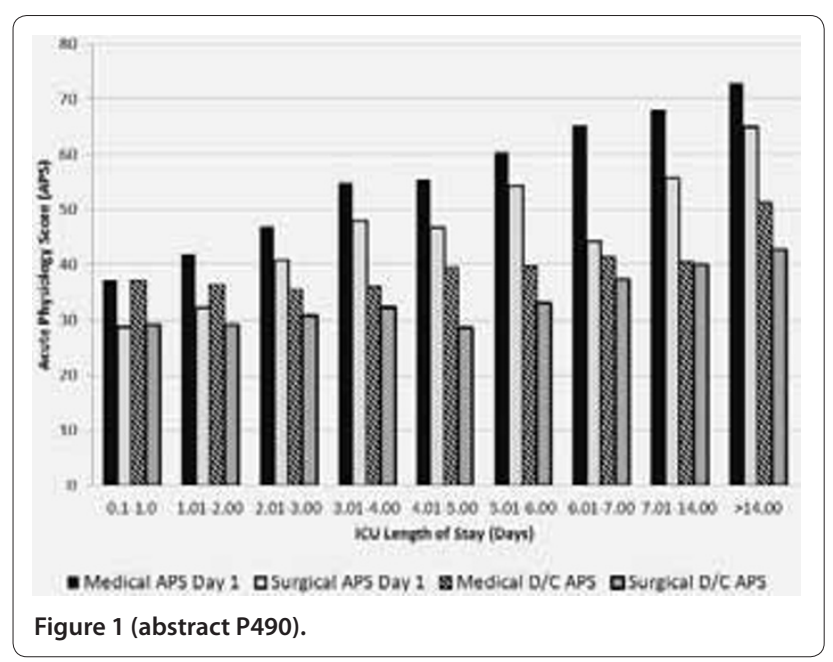


(APS) component were calculated daily for the first 7 days. Trend was assessed across days by Cuzick's test.

Results A total of 719 surgical and 925 medical patients met inclusion criteria. In total, $20.2 \%$ of surgical and $21.3 \%$ of medical patients had an ICU LOS $\angle 1 ; P=0.58$. Admission severity was correlated with length of stay, $P=0.014$ for both medical and surgical patients. Medical patients are sicker on admission and $D / C$ from the ICU than surgical patients $(P<0.05)$ (Figure 1).

Conclusion ICU utilization differed by patient type even with unified triage control within a single unit. Surgical patients were less severely ill on admission to and D/C from the ICU. A significant percentage of medical and surgical patients are $\mathrm{D} / \mathrm{C}$ within 1 day and may be more efficiently served in a less resource-intensive environment. The reasons for the differences in ICU utilization for surgical versus medical patients require clarification and may have implications for both resource utilization and cost.

P491

Educational effectiveness of introductory training for the Rapid

Response System in Japan

T Kodama', M Nakagawa², E Kawamoto ${ }^{3}$, S Fujiwara ${ }^{4}$, H Imai ${ }^{3}$, S Fujitani ${ }^{5}$,

K Atagi $i^{6}$

'The University of Texas Southwestern Medical Center at Dallas, TX, USA;

${ }^{2}$ Social Insurance Kinan Hospital, Tanabe-City, Japan; ${ }^{3}$ Mie University Hospital,

Tsu-City, Japan; ${ }^{4} \mathrm{NHO}$ Ureshino Medical Center, Ureshino-City, Japan;

${ }^{5}$ Tokyobay Urayasulchikawa Medical Center, Urayasu-City, Japan; ${ }^{6}$ Osaka City

General Hospital, Osaka-City, Japan

Critical Care 2013, 17(Suppl 2):P491 (doi: 10.1186/cc12429)

Introduction Interest in safety and clinical outcomes of inpatients has been growing in Japan, because the 100,000 Lives Campaign was introduced under the Japanese Patient Safety Act in 2008. In this act, an introduction of the Rapid Response System (RRS) was one of the mainstreams to inpatients' care. However, many Japanese healthcare providers cannot understand how to achieve the introduction of the RRS, because there are few who have knowledge of the system. Therefore, we developed a new introductory training course for the RRS. The educational effectiveness was analyzed through the surveillance questionnaires after the course.

Methods The educational program includes a lecture series concerning the outline and management methods, introduction of facilities that have already deployed, small group discussions, and teaching methods-of-training for the medical emergency team using a simulator. Evaluation was made in the five-point scale by 82 participants $(58$ physicians, 16 nurses and eight other professions) throughout seven courses. The questionnaires are: A. understanding of RRS, B. knowledge acquisition about patient safety, C. expectation for decreasing the cardiopulmonary arrest by RRS, and D. expectation for decreasing the psychological burden by RRS.

Results Seventy-three participants (89.0\%) answered the questionnaires. The numbers of participants who scored more than four points were as follows: A. was 71 (97.2\%), B. was 70 (95.9\%), C. was 64 (87.7\%), and D. was $68(93.2 \%)$, respectively. The majority of participants obtained the correct knowledge, and had a solid understanding for the RRS. It was evident that providing abundant material and didactic lectures traced from the introduction to management, and collecting and resolving the questions, promoted comprehension. However, there is a limitation of whether or not the participants introduce the RRS into their own institutions. It is essential to improve the course and continue to support the activities of the participants.

Conclusion Our training course may promote the introduction and dissemination of the RRS in Japan.

References

1. Leape LL, et al: JAMA 2005, 293:2384-2390,

2. Jones D, et al: N Engl J Med 2011, 365:139-146.

P492

Standards of referrals and admissions of critically ill patients to the ICU

$\mathrm{ANg}$

Glasgow Victoria Infirmary Hospital, Glasgow, UK

Critical Care 2013, 17(Suppl 2):P492 (doi: 10.1186/cc12430)
Introduction The management of emergency medical admissions has been a subject of recent clinical incidents. There was a high percentage of patients that were referred to the ICU by staff in training, and $21 \%$ of referrals were made by junior doctors. Consultant physicians had no knowledge of the case in $57 \%$ of referrals.

Methods A prospective study of 21 cases of referrals and admissions to the ICU was conducted at the Glasgow Victoria Infirmary Hospital from 8 to 21 September 2012. A questionnaire was produced relating to the referrals, admissions, seniority involvement, cause of referral, and time of patient review by the ICU consultant after ICU admission. They were distributed to specialist registrars and the ICU consultants. All data were electronically recorded into an Excel database. Questionnaires that were not completely filled were further investigated using patient clinical notes and contact with medical staff. Information that may identify a patient or clinician was removed from the questionnaire for confidentiality purposes.

Results Twenty-one complete questionnaires were collected. Fiftyseven percent (12/21) of cases involved admission to the ICU. Nine percent of the cases involved contacting either a specialist registrar or ICU consultant intensivist for assistance in practical procedures. Of the patients admitted to the ICU, 33\% (4/12) were from medical wards, $33 \%$ were admitted from A\&E. Consultants were the most common professionals who referred patients to critical care $(48 \% ; 10 / 21)$. Fourteen percent of cases (3/21) involved the referral of patients into ICU by a junior doctor, but only one of the referrals was accepted by the ICU intensivist. Consultants referred or were aware of the referral in $71 \%(15 / 21)$ of cases. Of admissions, $58 \%(7 / 12)$ were accepted by the ICU consultant and the remaining by the specialist registrars. All accepted were acknowledged by the ICU consultant. After admission all of the patients were reviewed by the ICU consultant and the time of review after admission was on average 1 hour 23 minutes ( 25 minutes to 3 hours 45 minutes)

Conclusion There is still an issue with junior doctors referring patients to the ICU without the acknowledgement of consultant physicians, resulting in unnecessary admissions and decreased time that ICU trainees spend in the ICU. There are more appropriate ICU admissions when there is involvement with seniority. Contact with ICU staff to perform practical procedures outside the ICU and not about admissions should be explored further.

Reference

1. Gillbe C, Gunning K: Standards for Consultant Staffing of Intensive Care Units. ICS \& IBTICM Standards. Intensive Care Society; 2006.

\section{P493}

Knowledge of confidentiality, consent and information disclosure is not affected by position or experience in adult critical care

M Lowings', A Molokhia ${ }^{2}$, C Anderson ${ }^{3}$, S Cowman ${ }^{4}$, T Dixson ${ }^{5}$, KEl-Boghdadly ${ }^{6}$, L Williams ${ }^{2}$

'St Thomas' Hospital, London, UK; 'Lewisham University NHS Trust, London, UK: ${ }^{3}$ King's College Hospital, London, UK: ${ }^{4}$ Great Ormond Street Hospital, London, UK; ${ }^{5}$ Queen Elizabeth Hospital, Woolwich, UK; ${ }^{6}$ Princess Royal University Hospital, Bromley, UK

Critical Care 2013, 17(Suppl 2):P493 (doi: 10.1186/cc12431)

Introduction Teaching of medical ethical issues including confidentiality and consent have long been a small part of the medical curriculum. These issues are more complex in an ICU where patients may lack capacity. Documents such as Good Medical Practice 1995, Confidentiality 2009 and the Mental Capacity Act 2005 give guidance to medical professionals in these matters in the UK.

Methods A questionnaire was distributed amongst staff in four ICUs in South London. Results were analysed according to level of experience and background (medical/nursing or allied health professional (AHP)).

Results Of 225 questionnaires distributed, the response rate was $66 \%$ (31\% doctors, $56 \%$ nurses and $13 \%$ AHP). Staff with either less than 1 year experience or greater than 10 years experience had the greatest exposure to the Mental Capacity Act and Data Protection Act, suggesting a gap in knowledge in staff with intermediate experience. Knowledge of the Caldicott principles were unaffected by experience, with many experienced respondents having 'No Idea'. The majority of respondents (unaffected by experience) felt that 
when giving information to relatives face to face, relatives should be kept fully informed. When giving information over the telephone, most doctors felt the response should be tailored to the knowledge of the person being spoken to whilst nurses were split between tailoring the response, giving full information, setting up a password system and not giving any information at all. Most respondents felt date of birth and hospital number constituted 'Patient Identifiable Information'. However, experienced staff did not appreciate the importance of unusual diagnosis and clinical photographs as also being able to identify patients. Similarly, the majority knew that the patient themselves identified the 'Next of Kin' but 7\% (unaffected by experience) felt this was decided by the family and felt the family could decide on resuscitation status. When consent is required for an elective procedure in a patient who lacks capacity, doctors tended to have a better understanding of the need to delay the procedure where possible than nurses, the majority of which felt this could be decided by the next of kin or two consultant doctors. Most doctors felt that 'Acting in the Patient's Best Interests' would mean doing what would give the patient the best outcome rather than doing what the patient would have wanted (unaffected by experience). The majority of staff, on answering this questionnaire, felt that they lacked sufficient knowledge on the subject and most felt annual reminders would be useful.

Conclusion The ICU is an environment where issues of consent, confidentiality and disclosure of information occur daily. Staff feel they lack knowledge in these areas that is unaffected by their experience. We need to ensure that all staff have the necessary knowledge to deal with these situations.

P494

Survey of alcohol-related admissions to critical care and the resource and financial implications

M Slattery, P Temblett, M Heatley, N Singatullina, B Jones ABMU University Trust, Swansea, UK

Critical Care 2013, 17(Suppl 2):P494 (doi: 10.1186/cc12432)

Introduction Alcohol-related hospital and ICU admissions are known to have a huge impact on healthcare resources in the UK. Excessive use of alcohol is independently associated with sepsis, septic shock and hospital mortality among ICU patients. This study assesses the relationship between alcohol abuse and intensive care resource utilisation in a mixed medical, surgical and neurosurgical ICU.

Methods A prospective survey of emergency alcohol-related admissions over a 1-year period was undertaken at a tertiary university adult general and neurosurgical ICU. All patients were screened for acute and chronic alcohol abuse on admission. Acute alcohol abuse was defined as being intoxicated with alcohol at the time of admission and chronic alcohol abuse was defined as chronic alcohol use exceeding recommended UK national guidelines on consumption. The amount of alcohol consumption was obtained, diagnosis on admission, ICU and hospital mortality, length of stay, and total cost were recorded. All patients were screened for alcohol-related comorbidities. Comparative retrospective data were obtained for the same time period for nonalcohol-related emergency ICU admissions. Data were analyzed using SPSS.

Results In total, $7.7 \%$ of patients were admitted with a history of acute/chronic alcohol excess. Sixty-seven per cent of alcoholrelated admissions were due to acute alcohol excess. Neurosurgical patients admitted due to alcohol excess had higher ITU mortality than nonalcohol-related neurosurgical patients: $32.1 \%$ versus $14.39 \%$ $(P=0.02)$, respectively. Ninety-three per cent of alcohol-related neurosurgical admissions were caused by acute alcohol intoxication. The intensive care cost was significantly higher for alcohol-related ( $£ 12,396$ per patient) compared with nonalcohol-related neurosurgical admissions ( $£ 7,284$ per patient). Of the medical patients admitted, $60 \%$ of these admissions were due to acute alcohol excess. The cost of intensive care treatment was lower for alcohol-related medical admissions.

Conclusion This is one of the largest studies of alcohol-related admissions to critical care. Our survey confirms that alcohol-related admissions to the ICU are commonplace; however, our frequency is significantly less than previously reported. Our study reveals interspecialty variations in demographic data, APACHE II scores, mortality and cost of admission. Neurosurgical alcohol-related admissions bear higher mortality and result in greater resource utilisation relative to nonalcohol-related neurosurgical admissions. Alcohol continues to burden both our patients and critical care.

\section{P495}

Association of sleep disordered breathing with clinical trajectories in patients undergoing cardiac surgery J Roggenbach, B Tan, E Von der Leyen, A Weymann, M Karck, E Martin, SHofer

University of Heidelberg, Germany

Critical Care 2013, 17(Suppl 2):P495 (doi: 10.1186/cc12433)

Introduction The prevalence of sleep disordered breathing (SDB) is presumably high among individuals with cardiac diseases [1], nonetheless SDB remains predominantly undiagnosed. However, unrecognized SDB might have relevant impact on the postoperative course of patients undergoing cardiac surgery [2].

Methods Polygraphic recordings of 181 patients, without previous diagnosis of SDB, undergoing standard cardiac surgical procedures with extracorporeal circulation were obtained during a preoperative night. The apnea-hypopnea index (AHI - the number of apneas, hypopneas per hour recorded) was determined and compared with clinical characteristics and postoperative course.

Results The prevalence of SDB was considerably high among all examined patients. Median AHI was 20.8 (interquartile range, 10.6 to 36.4). Preoperative AHI was $>30$ in $32 \%$ of all examined individuals. During the first three postoperative days, preoperative $\mathrm{AHI}>30$ was associated with a prolonged weaning time, a reduced oxygenation index (arterial $\mathrm{pO}_{2} / \mathrm{FiO}_{2}$ ), an impaired kidney function, an augmented inflammatory response and an overall increased length of stay in the ICU. The observed association of high preoperative $\mathrm{AHI}$ values with postoperative clinical characteristics remained statistically significant throughout the first three postoperative days.

Conclusion Undiagnosed SDB is highly prevalent among cardiac surgical patients. Clinical trajectories of individuals with severe SDB are described by a prolonged recovery of pulmonary function, delayed weaning and a pronounced inflammatory response after surgery. Screening for SDB might identify patients that are susceptible for a complicated postoperative course.

References

1. Schiza et al:. I Clin Sleep Med 2012, 8:21-26.

2. Kaw et al.: Br J Anaesth 2012, 109:897-906.

P496

Can massage therapy help cardiac patients? A literature review plus a case study

A Fenlon

St Georges NHS Trust, London, UK

Critical Care 2013, 17(Suppl 2):P496 (doi: 10.1186/cc12434)

Introduction A literature review was performed to assess whether massage benefits patients postoperatively following coronary bypass grafts (CABG) and or valve replacement/repair. A case study on a patient who had suffered a hypoxic brain post cardiac arrest was conducted. Methods A review on MEDLINE and Cochrane using search terms massage, cardiac and ICU identified nine research papers on the benefits of massage postoperatively for the aforementioned patient group. Other papers were listed but unrelated to cardiac surgery. None of the nine papers identified for this review were ICU specific in the title but the ICU was mentioned in the main text body. For the purpose of this review the selected papers are researching the effects of massage on physiological parameters, anxiety, pain, calm and perceived stress indicators in the CABG and/or valve repair/replacement. Out of these nine papers, one is British (2002). Five are American (2006 to 2012), two are Brazilian (2010) and one is an Indian paper (2010). All papers are randomised control trials (RCTs). Papers written prior to 1999 were excluded from this literature review. 
Results Research from 1999 states there are methodological errors in prior papers and very few large-scale studies prior to this date, destabilising the validity and reliability of research from the papers written before 1999. The later research suggests that any change in the measured physiological parameters of blood pressure, heart rate and respiratory rate are insignificant $(P>0.05)$. Pain, anxiety, rest and a calm score perform better with $P>4.71$ overall. Another American study showed that the length of stay is reduced if a patient receives healing touch postoperatively. However, in one RCT pain and anxiety increases and in some case $\mathrm{SpO}_{2}$ decreases. A case study was chosen by the author and the results support the benefits of massage with the cardiac patient group.

Conclusion There appears to be some benefits but larger-scale studies are required within this and other ICUs in Britain. The author will be inquiring at her workplace whether further studies can be performed and whether she can initiate the research. She will identify further case studies and trial massage on select patients. The author recommends training for nurses interested in massage therapy so a bigger caseload can be identified.

\section{P497}

Ischemic stroke in different ICU types

M Damasceno, F Rodriguez, RTuron

Hospital de Clinicas de Niterói, Rio de Janeiro, Brazil

Critical Care 2013, 17(Suppl 2):P497 (doi: 10.1186/cc12435)

Introduction In our hospital there are a general intensive care unit (GICU) and a neurointensive care unit (NICU). Despite the preference for the NICU, in both there are admissions for ischemic stroke. There are different staff for each ICU, with the same physician's leadership. We have decided to evaluate the performance of both ICUs, analysing whether there are differences in results as some authors publish best results in specialized ICUs.

Methods Using prospectively collected data, we undertook a retrospective evaluation of all patients admitted to the GICU and NICU of our hospital with the diagnosis of ischemic stroke, from December 2010 to November 2012. In both ICUs there are intensivists, but in the $\mathrm{NICU}$ the intensivists have special expertise in neuroscience. Data were collected from Epimedmonitor.

Results A total of 3,854 admissions were registered in the period in both ICUs, with 257 (6.7\%) being by ischemic stroke - 49 in GICU and 208 in NICU. Mean age of patients: 73 years in GICU and 70.1 years in NICU. Admissions from emergency unit: 44 (89.8\%) GICU, 181 (87\%) NICU. Mean SAPS 3 score: 50.7 (29 to 71 ) GICU, 50.6 (29 to 98) NICU. Patients admitted with infection: $6(12.2 \%) \mathrm{GICU}, 10(4.81 \%)$ NICU. Mean Charlson comorbidity index points: 1.61 (median 1.0) GICU, 1.1 (median 1.0) NICU. Mean length of ICU stay: 4.8 days (median 3 ) GICU, 4.3 days (median 3) NICU. Mean length of hospital stay: 12.2 days (median 8) GICU, 14.5 days (median 8) NICU. Predicted hospital mortality (mean + SD): $20.07 \% \pm 13.48 \mathrm{GICU}, 20.4 \% \pm 13.83 \mathrm{NICU}$. Hospital mortality rate: $12.5 \%$ GICU, $5.39 \%$ NICU. Observed-to-expected (O/E) mortality ratios: $0.62 \mathrm{GICU}, 0.26 \mathrm{NICU}$.

Conclusion Despite the similar proportions numbers for patients in both ICUs, the mortality rate and the O/E mortality ratio for ischemic stroke were higher in patients of the GICU when compared with the NICU.

References

1. Varelas PN, et al: Neurocrit Care 2008, 9:293-299.

2. Berchad EM, et al: Neurocrit Care 2008, 9:287-292.

\section{P498}

Prevention of ventilator-associated pneumonia in the surgical ICU at Siriraj Hospital

CH Pisitsak, O Chaiwat

Siriraj Hospital, Bangkok, Thailand

Critical Care 2013, 17(Suppl 2):P498 (doi: 10.1186/cc12436)

Introduction VAP has continued to be a major cause of morbidity and mortality in critically ill patients in Thailand for decades. Previous research found that the implementation of VAP care bundles and the educational program can reduce VAP incidence in the ICU [1]. In this research we aimed to observe the reduction of VAP incidence after the implementation of VAP care bundles to ICU medical personnel.

Methods Inclusion criteria: all adult surgical patients ( $>18$ years old) who are on ventilatory support in the surgical ICU at Siriraj Hospital. There are two groups, divided into pre-educational group (group I) and post-educational group (group II) ( $n=220 /$ group). We also observed the adherence rate to VAP care bundles according to the educational program. The pretest and post-test to determine the efficacy of the educational program were done. The VAP care bundles consisted of weaning according to weaning protocol, sedation vacation, headof-bed elevation, measurement of cuff pressures four times/day, $2 \%$ chlorhexidine use for mouth care and emptying of ventilator circuit condensate.

Results There were 45.38 and 25.25 episodes of VAP per 1,000 ventilatordays in group I and group II, respectively $(P=0.020)$. The incidence of VAP was $21.82 \%$ in group I and $9.09 \%$ in group II $(P=0.000)$. There was significant reduction in the length of ventilatory support per person (group I $=2$, group $I I=1$ (median), $P=0.013,95 \% \mathrm{Cl}=0.319$ to 0.936 ) and mortality rate (group $\mathrm{I}=15.5 \%$, group $\mathrm{II}=8.2 \%, P=0.017$ ). There was no significant difference in $\mathrm{LOI}, \mathrm{LOH}$ and ATB cost. The pretest scores were 15.53 and 17.53 on average from 40 medical personnel in group I and group II, respectively $(P=0.000)$. The head-of-bed elevation adherence rate was improved after the educational program (group $I=50.1 \%$, group $\|=70.36 \%, P=0.017$ ). But the adherence to other bundles was not improved. See Tables 1 and 2 .

Table 1 (abstract P498). Impact of the educational program on outcomes

\begin{tabular}{lccc}
\hline Outcome & Pre-education & Post-education & $P$ value \\
\hline VAP & 45.38 & 25.25 & 0.020 \\
Ventday & 2 & 1 & 0.013 \\
LOI & 3 & 3 & 0.054 \\
LOH & 17 & 17 & 0.960 \\
ATB cost & $2,842.50$ & $2,098.00$ & 0.994 \\
\% death & 15.5 & 8.2 & 0.017 \\
\hline
\end{tabular}

Table 2 (abstract P498). Adherence rate to VAP care bundles

\begin{tabular}{lccc}
\hline Bundle & Pre-education & Post-education & $\boldsymbol{P}$ value \\
\hline Wean & 95.04 & 96.08 & 0.723 \\
Sedate & 92.26 & 95.28 & 0.467 \\
HOB & 50.10 & 70.36 & 0.017 \\
Cuff pressure & 87.29 & 80.78 & 0.278 \\
M care & 89.60 & 80.86 & 0.204 \\
Drain & 95.42 & 99.42 & 0.130
\end{tabular}

Conclusion The educational program and the implementation of VAP care bundles can reduce the incidence of VAP, length of ventilatory support and mortality rate in the ICU.

\section{Reference}

1. Apisarnthanarak A, Pinitchai U, Thongphubeth K, Yeukyen C, Warren DK, Zack $J \mathrm{E}$, et al: Effectiveness of an educational program to reduce ventilatorassociated pneumonia in a tertiary care center in Thailand: a 4-year study. Clin Infect Dis 2007, 45:704-711.

P499

Development of a severe influenza critical care curriculum and training materials for resource-limited settings JV Diaz', P Lister ${ }^{2}$, JR Ortiz ${ }^{3}$, NK Adhikari ${ }^{4}$, N Adhikari ${ }^{1}$ World Health Organization, San Francisco, CA, USA; ${ }^{2}$ Great Ormond Street Hospital, London, UK; ${ }^{3}$ University of Washington, Seattle, WA, USA; ${ }^{4}$ Sunnybrook Health Sciences Centre and University of Toronto, Canada; ${ }^{5}$ Health Sciences Centre and University of Toronto, Canada Critical Care 2013, 17(Suppl 2):P499 (doi: 10.1186/cc12437) 
Introduction The $2009 \mathrm{H} 1 \mathrm{~N} 1$ pandemic caused surges of severely ill patients with viral pneumonia requiring ventilation and particularly affected high-dependency medical services such as ICUs in settings that lack sufficient personnel and resources to provide optimal care. Ministries of Health of several countries asked the World Health Organization (WHO) for clinical management guidance.

Methods We developed (2009 to 2011) and piloted (2011 to 2012) curriculum and training materials targeted at clinicians without formal critical care training who care for adult and paediatric patients with severe acute respiratory infections in ICUs in resource-limited settings. Results With contributions from 37 global experts, we developed a 3-day course including 14 learning units in early recognition, pathophysiology, oxygen therapy, influenza diagnostics, infection control, resuscitation of septic shock, antimicrobial therapy, monitoring, lungprotective ventilation for acute respiratory distress syndrome, sedation, weaning, preventive care, quality improvement, and ethics. Teaching techniques are appropriate for adult learners and include short slideshow-based lectures, interactive small-group role-play sessions and a toolkit with practical resources such as checklists. With funding from the WHO and local Ministries of Health, the training course was piloted among 67 clinician participants in the Caribbean and Indonesia (29 and 38, respectively) and taught by external critical care specialists. Participants' daily evaluations rated all units as at least very good. Test scores to assess participant critical care knowledge improved significantly from before the training to immediately after (Caribbean, 58 to $80 \%$; Indonesia, 56 to $75 \%$; $P<0.001$ for both).

Conclusion It was feasible to develop and deliver an advanced critical care curriculum and related training materials in a short, interactive workshop for noncritical care trained clinicians in resource-limited settings. However, the small-scale programme was labour and time intensive. Widespread dissemination of these materials requires identification of target countries, engagement of Ministries of Health, and secure funding. Longer-term evaluation is necessary to determine whether such programmes improve processes of care and clinical outcomes for critically ill patients.

\section{P500}

Quality assurance in severe sepsis: an individualised audit/feedback system results in substantial improvements at a UK teaching hospital

M Simmonds, E Blyth, W Robson, M Chikhani

Nottingham University Hospitals NHS Trust, Nottingham, UK

Critical Care 2013, 17(Suppl 2):P500 (doi: 10.1186/cc12438)

Introduction Following our study of severe sepsis care across three centres [1], we aimed to introduce a rapid feedback mechanism into our rolling audit programme. Whilst previous audits raised awareness of severe sepsis, only whole organisation performance was reported and no feedback was given to individual clinicians. It is recognised that such feedback loops can improve clinical practice [2].

Methods Patients admitted to critical care (58 beds, four units) with a primary admission diagnosis of infection were screened for severe sepsis. Pre-ICU care was then audited against the Surviving Sepsis Guidelines [3]. Time zero is defined as when criteria for severe sepsis were first met. An individualised traffic-light report was then generated and emailed to the patient's consultant and other stakeholders involved in care (Figure 1). We aimed to report cases within 7 days of critical care admission. A cumulative report is generated monthly to track organisation-wide performance.

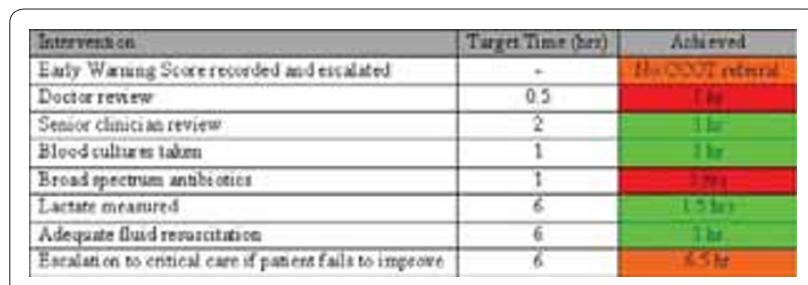

Figure 1 (abstract P500). Example report.

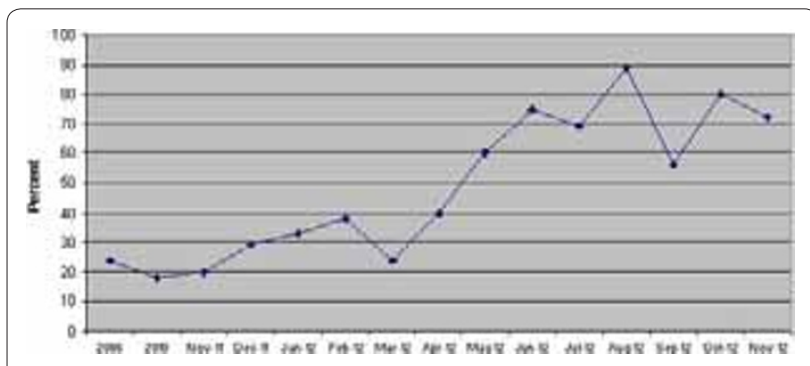

Figure 2 (abstract P500). Compliance with pre-ICU resuscitation bundle.

Results Since November 2011, 153 cases of severe sepsis have been audited and reported back to clinicians. Compliance with antibiotics in $<1$ hour has risen from 35 to $75 \%$ and compliance with the preICU elements of the resuscitation bundle has risen from 20 to $70 \%$ (Figure 2). Feedback from clinicians has been encouraging as our reports highlight both positive and negative examples of practice.

Conclusion Individualised feedback on sepsis care has led to substantial improvements in guideline compliance. This concept could be translated to other time-dependent patient pathways.

References

1. Simmonds MJR, Chikhani M, Smith P, et al:: Multi-departmental system analysis is needed for evaluation of severe sepsis care: a multi-centre study [abstract]. Presented at the BMJ International Forum on Quality and Safety in Healthcare; 2011; Amsterdam.

2. Jamtvedt $\mathrm{G}$, Young JM, Kristoffersen DT, et al.: Audit and feedback: effects on professional practice and health care outcomes. Cochrane Database Syst Rev 2006, 2:CD000259.

3. Dellinger RP, Levy MM, Carlet JM, et al: Surviving Sepsis Campaign: international guidelines for management of severe sepsis and septic shock: 2008. Crit Care Med 2008, 36:296-327.

P501

Safety climate perception for staff nurses in an ICU

RL Rivera-Romero, M Torres-Campos, MJ Delgado-Amaya, E Curiel-Balsera, JG Quesada-García

Hospital Regional Carlos Haya, Málaga, Spain

Critical Care 2013, 17(Suppl 2):P501 (doi: 10.1186/cc12439)

Introduction When we talk about safety culture, we speak of being aware that things can go wrong. We must be able to recognize mistakes and learn from them, sharing that information fairly and impartially to try to prevent its recurrence. Organizations such as the Agency for Healthcare Research and Quality (AHRQ) have developed tools to help organizations measure their safety culture and there is little information about our country.

Methods A descriptive survey study. We sent the Spanish version of the questionnaire on patient safety culture (AHRQ) to the nursing staff of a polyvalent ICU of 42 beds in a tertiary hospital.

Results The questionnaire was sent to 179 nurses, receiving correctly answered 88 surveys (response rate of $49.16 \%$ ). On a scale of 0 to 10, 6.97 points was obtained to estimate the safety climate for staff respondents. The item best scored was teamwork in the unit (65.9\%). Detected as a fortress, 'communication between nurses at shift changes' (76.1\% positive responses). The worst rating was obtained in the section on human resources, followed by management support in the field of patient safety.

Conclusion The perception of safety culture in an ICU by nursing staff is far from optimal levels. The team work dimension was identified as the most valued by workers, with the transmission of information on shift changes the most valued item. 
P502

Physico-technical medicine: teaching technical skills to the intensivist

J Seifried, S Richter, J Guttmann, S Schumann

University Medical Center Freiburg, Germany

Critical Care 2013, 17(Suppl 2):P502 (doi: 10.1186/cc12440)

Introduction The requirements for the intensivist in handling medical technology are constantly growing. It appears necessary to acquire technological competences particularly within the fields of medical technology and physics. In the master's degree program 'MasterOnline Physico-Technical Medicine', such technical authority is conveyed. To cope with the intensive vocational situation of the physician, this study course follows the blended-learning concept; that is, it is conceived as an online study course with small portions of intermittent presence phases. Within the first year, technical basic skills such as 'measurement technique', 'informatics', and 'advanced physics' are covered. Subsequently, two of various advanced courses in different fields of medical technology ('technology in intensive care medicine', 'technology in surgery', 'technical cardiology', 'radiology', and other) are selected.

Methods In a survey, we evaluated the study course. Therefore, a questionnaire was distributed among all students including the topics course contents, learning materials, time management, supervision, and overall impression. The students were asked to score their agreement to the statements 'content is well structured', 'content extent is appropriate' and 'content is relevant for medical purposes' on a scale ranging from 1 (fully disagree) to 5 (fully agree).

Results The students participated actively in this study course with highest motivation and large commitment. The students' workload was in the targeted range of about 10 hours/week. Content structure was scored with $4.3 \pm 0.1$, content extent with $4.1 \pm 0.2$ and medical relevance with $4.3 \pm 0.2$

Conclusion The blended-learning concept fulfills the requirements for occupation-accompanying continued medical education, since it offers the possibility to study self-employed accessing text documents, lecture recordings, and electronic lectures and to convert in concentrated presence phases this knowledge into practical exercises.

\section{P503}

First-hour protocol clarifies resource management and nursing staff education in the ICU

M Arbelius-IItanen, R Siren, J Heinilä, P Korhonen, T Sutinen, V Jalkanen,

TAhonen, S Karlsson

Tampere University Hospital, Tampere, Finland

Critical Care 2013, 17(Suppl 2):P503 (doi: 10.1186/cc12441)

Introduction The first-hour protocol determines the patient-specific resources for the start of an ICU stay [1]. Staff resources are decided through triage. Task charts direct the start of intensive care. Our primary goal is to improve patient care.

Methods A triage method (red, yellow, green) is used to manage ICU resources according to the severity of illness. For example, one doctor and one nurse would admit a stable (green) patient coming from the operating room for postoperative ICU care. A patient in septic shock with multiple organ disorder (red), on the other hand, would be admitted by a team of two doctors and three nurses. Each staff member has a task chart in a checking-list format. Also, an admission chart is used to improve data collection. The use of the protocol started as a pilot study in early 2012. Simulation education for staff members started in August 2012 and has included video recordings and debriefing of each simulated ICU admission. Primary goal-directed therapy goals have been mean arterial pressure (MAP $>65 \mathrm{mmHg}$ ), $\mathrm{SpO}_{2}>94 \%$, timing of the laboratory tests, start of antibiotics, and blood glucose level 6 to $8 \mathrm{mmol} / \mathrm{l}$. Quality indicators have been followed from the data provided by The Finnish Intensive Care Consortium. Questionnaires for the staff members have been used to evaluate opinions about the firsthour protocol.

Results According to the questionnaire replies, $80 \%$ ( $n=64 / 80$ ) of our nurses estimate that the first-hour protocol has improved the starting process of our patients'intensive care. Twenty percent $(n=16 / 80)$ of the nurses considered that the protocol has no effect, and none thought it to be adverse for patient care. Corresponding numbers for our ICU doctors were $87 \%$ (beneficial $n=13 / 15$ ), 13\% (no effect $n=2 / 15$ ) and $0 \%$ (adverse). Furthermore, $82.5 \%(n=66 / 80$ ) of the nurses replied that education of new nurse staff members has improved because of the first-hour protocol. A total of $17.5 \%(n=14 / 80)$ thought there has been no effect, and none considered the protocol harmful for education. For ICU doctors the protocol did not bring either clear educational advantages or disadvantages. The variable life-adjusted display curves (The Finnish Intensive Care Consortium) have shown improvement in our patient care after the implementation of the first-hour protocol. However, we cannot determine whether it is a significant factor in our intensive care results.

Conclusion The first-hour protocol has helped us in resource management, start of the patients' intensive care and education of nursing staff.

Reference

1. Brown R, et al: Aust Crit Care 2012, 25:178-187.

P504

Influence of critical care nurse education and work environment on outcomes of mechanically ventilated older adults

DM Kelly', LH Aiken²

'University of Pittsburgh, PA, USA; ${ }^{2}$ University of Pennsylvania, Philadelphia, $P A, U S A$

Critical Care 2013, 17(Suppl 2):P504 (doi: 10.1186/cc12442)

Introduction Demand for critical care services is increasing yet a comprehensive understanding of how critical care nurses - the largest group of ICU direct care providers - impact outcomes remains unclear. The purpose of this study was to determine how critical care nurse education (hospital proportion of bachelor's prepared ICU nurses) and ICU work environment influenced 30-day mortality of mechanically ventilated older adults.

Methods A multi-state cross-sectional nurse survey was linked to hospital administrative data and Medicare claims (2006 to 2008). The final sample included 55,159 mechanically ventilated older adults in 303 hospitals. Logistic regression modeling was employed to jointly assess the relationship of critical care nurse education, work environment and staffing on 30-day mortality while adjusting for hospital and patient characteristics and accounting for clustering.

Results A $10 \%$ increase in the proportion of ICU nurses with a bachelor's degree or higher was associated with $2 \%$ lower odds of death while controlling for patient and hospital characteristics. Patients cared for in better work environments experienced $11 \%$ lower odds of riskadjusted death than those cared for in poorer ICU work environments. Conclusion Patients cared for in hospitals with a greater proportion of bachelor's prepared ICU nurses and in better ICU work environments experienced significantly lower odds of death. As the demand for critical care services increases, attention to the education level of ICU nurses and ICU work environment may be warranted to optimize currently available resources and potentially yield better outcomes.

\section{P505}

Activity and case-mix changes in a medical ICU after the geographical transfer of a third-level university hospital

JC Cebrián, FM Monsalve, JB Bonastre

Hospital Universitario y Politecnico La Fe, Valencia, Spain

Critical Care 2013, 17(Suppl 2):P505 (doi: 10.1186/cc12443)

Introduction Information about big hospital geographical transfer is scarce in the medical literature. On 20 February 2011 our hospital (in fact, a big university complex) was transferred from their previous location in the north-center of our city towards a new southern peripheral, geographical location. This transfer has been done without any changes in assisted population or nursing/medical staff. The only change was a slight increase in bed number (21 to 24). Our aim is to analyze changes in activity indexes (length of stay, occupancy rate, and so forth) and case mix (origin, previous quality of life and NYHA score, main diagnostic groups, severity scores, in-ICU and in-hospital mortality). 
Methods To compare our number of admissions, related activity and case-mix indicators 1 year before and after the geographical change was done. We analyzed our whole number of patients admitted to the ICU. We used the chi-square test for categorical variables and one-way analysis of variance for quantitative data. Minitab and Statbas statistical programs were used. We plotted activity data using the BarberJohnson 1 diagram.

Results A total of 2,774 cases (63\% males; mean age 61 years) were admitted to our ICU during the period (1 year before and after the transfer). No differences between both groups were founded in demographic data, Knaus score and NYHA status. Regarding their origin, we found more patients admitted from other hospital centers (20 vs. $29 \%$; $P<0.001$ ). APACHE II score increased from 17.24 to $19.08 \%$ $(P<0.001)$ and a slight increase change in SAPS 3 score was also found (52.29 to $53.75 ; P<0.01)$. Our in-ICU mortality remains lower $(15.5$ to $15.6 \%)$ whereas observed mortality decreased (22.37 to $19.88 \%$; $P<0.001)$. An increase in our neurologic patients has been the most consistent change regarding diagnostic groups. The activity indexes show a slightly decrease in occupancy rate (79.2 vs. 76.8).

Conclusion According to the previous data our ICU seems to perform better in the new location with a decrease in the standardized mortality rate. On the other hand, we are admitting more patients transferred from other hospitals. A better occupancy rate was found.

Reference

1. Yates J: Hospital Beds: A Problem for Diagnostic and Management. Heinemann Medical Books; 1982

\section{P506}

Are general wards sufficiently staffed to care for level 1 patients?

G Rajendran, CTjen, S Hutchinson, S Fletcher

Norfolk \& Norwich University Hospital, Norwich, UK

Critical Care 2013, 17(Suppl 2):P506 (doi: 10.1186/cc12444)

Introduction There are several definitions of level 1 (L1) care, all refer to a group at risk of clinical deterioration on the ward [1-3]. There is evidence that ward patients who become acutely unwell often receive suboptimal care [4]. A regional study commissioned by Norfolk, Suffolk \& Cambridgeshire Critical Care Network (NSCCCN) found that a majority of ward patients may be of $L 1$ dependency and death rates appear to be correlated with $\mathrm{L} 1$ status. We aim to examine the relationship between the ward distribution of illness acuity, staffing and patient outcome. Methods Data were collected as part of NSCCCN's observational prevalence study in 2010. Ward surveys included acuity of illness, staffing levels and skill mix. Secondary data were obtained from the Patient Administration System. Emergency, oncology, paediatric and maternity units were excluded.

Results Complete datasets were obtained from 1,402 patients in 22 wards in our university hospital over two seasons. This constitutes $98.3 \%$ of inpatients from those wards. The mean ward occupancy rate was $94 \%$ (10th to 90 th percentile: $85 \%$ to $100 \%$ ). At least one L1 acuity criterion was scored by $898(64 \%)$ patients, with $25 \%$ from geriatrics followed by orthopaedics (17\%) and general surgery (10\%). Each ward had an average of eight qualified nursing staff (range: 4 to 12) equating to an average staff:patient ratio (SPr) of 0.253 . There was no correlation between ward occupancy and nursing staff (Pearson correlation, corr: 0.55 ), nor between prevalence of $L 1$ criteria and staffing (corr: 0.34 ). The admission rate to intensive care was noted to be higher if the patients were nursed in a ward with lower than average SPr compared with higher $\operatorname{SPr}(2.7 \%$ vs. $1.2 \%, P=0.058$ Fisher's exact), but this was not statistically significant. Senior nursing (Band 6) staff were part of the skill mix on only nine of 44 ward surveys.

Conclusion Better outcome with improved SPr may be unsurprising, although if proven conclusively would significantly inform workforce planning. Lack of correlation between staffing levels and occupancy or acuity is also interesting given that we know L1 criteria are associated with worse outcome.

\section{References}

1. Levels of Critical Care for Adult Patients. Intensive Care Society: 2002

[http://www.ics.ac.uk/professional/standards_safety_quality/standards_and_ guidelines/levels_of_critical_care_for_adult_patients]

2. Acutely III Patients in Hospital. NICE Guideline 50. NICE; 2007. [http://www.nice.org.uk/cg50]

3. AUKUH Acuity/Dependency Tool. Association of UK University Hospitals; 2007. [http://www.aukuh.org.uk/index.php/affiliate-groups/directors-of-nursing/ patient-care-portfolio]

4. An Acute Problem? NCEPOD; 2005.

[http://www.ncepod.org.uk/2005report/index.html]

\section{P507}

\section{Sleep quality among ICU workers}

G Burghi', I Serkochian'1, B Frache², P Alzugaray², K Goinheix',

M Rodriguez Verde ${ }^{3}, \mathrm{H}_{\text {Bagnulo', E Azoulay }}$

'Hospital Maciel, Montevideo, Uruguay; ${ }^{2}$ Sanatorio Americano, Montevideo,

Uruguay; ${ }^{3}$ Hospital de Paysandú, Uruguay; ${ }^{4}$ Hôpital Saint Louis, Paris, France

Critical Care 2013, 17(Suppl 2):P507 (doi: 10.1186/cc12445)

Introduction Prolonged shifts, workload, stress, and different conflicts are associated with burnout, loss of psychological wellbeing, and probably with an inadequate sleep quality (ISQ). This relevant disturbance leads to deterioration of the work performance, may impair quality of care provided to patients and increases the incidence of serious adverse events. The objective was to determine the prevalence of ISQ and sleepiness among Uruguayan ICU workers, and to evaluate risk factors associated with ISQ.

Methods A survey was conducted in six Uruguayan ICUs. The sleep quality was evaluated on the basis of the Pittsburgh score (PS), and the sleepiness was identified by the Epworth scale. ISQ was defined as PS greater than 5 points and sleepiness by an Epworth scale higher than 6 points. ICU's, patient's, and clinician's characteristics were assessed for their association with the prevalence of ISQ. All variables with $P<0.2$ in univariate analysis were included in a model of ordinal regression. $P<0.05$ was considered statistically significant.

Results The survey was completed by 129 ICU workers. The global prevalence of ISQ in ICU was $67.4 \%$. ISQ was observed in $45 \%$ of physicians and $82 \%$ of nurses and nurses assistant $(P<0.001)$. Sleep medication was used by $13.3 \%$ of the ICU team. Univariate analysis showed that ISQ was significantly associated with sex $(73 \%$ vs. $43 \%, P=0.03$ in women and men, respectively), marital status (84\% vs. $61 \%, P=0.01$ in single and couple workers, respectively), more than 60 hours working in the last week $(76 \%$ vs. $61 \%, P=0.07)$ and less than 6 sleeping hours ( $95 \%$ vs. $54 \%, P<0.0001$ ). Multivariable analysis demonstrated that a sleep duration less than 6 hours was independently associated with ISQ $(\mathrm{OR}=24.5 ; 95 \% \mathrm{Cl}=5.2$ to 115.8 ; $P<0.0001)$. Furthermore, pathologic sleepiness was present in $59.3 \%$ of ICU workers. Sleepiness was independently associated with use of sleep medication $(\mathrm{OR}=5.9 ; 95 \% \mathrm{Cl}=1.2$ to $28.5 ; P=0.025)$.

Conclusion The prevalence of ISQ and sleepiness is very high among ICU workers. Those disturbances are independently associated with a sleep duration less than 6 hours, and sleep medication use, respectively. These results highlights that strategies to decrease ISQ and sleepiness in ICU clinicians are urgently needed to improve work performance, improve quality of care provided and prevent adverse events.

\section{P508}

Work-related stress amongst doctors in intensive care, anaesthetics, accident and emergency and general medicine

Il Tuthill, MS Ahmed, G Mathew, AC Bolton, AA Molokhia

University Hospital Lewisham, London, UK

Critical Care 2013, 17(Suppl 2):P508 (doi: 10.1186/cc12446)

Introduction Work-related stress is a potential problem among doctors and is associated with anxiety, depression, reduced job satisfaction, days off work, errors and near misses [1]. To compare stress levels between different groups of doctors and identify causes of stress, we conducted a survey at University Hospital Lewisham using the UK Health and Safety Executive's Management Standards (HSEMS). HSEMS is a validated tool developed to identify work conditions that warrant interventions to reduce stress levels across organisations [2].

Methods We conducted an anonymous survey of doctors working in anaesthetics, intensive care, general medicine and accident and emergency (A\&E) departments over 6 weeks using the HSEMS 
questionnaire. We also surveyed awareness of the Trust's stress management services and whether staff had a designated supervisor or mentor. Results were analysed using the HSEMS Analysis Tool, which rates stressors with a score from 1 to 5 (5 represents the lowest amount of stress). We compared the Trust's results against HSEMS national standards.

Results Seventy-two doctors completed the survey. Lowest stress levels were found in doctors working in intensive care $(n=12$, mean 3.63, SD 0.39). This was followed by medicine $(n=26$, mean 3.55 , SD 0.47), anaesthetics ( $n=27$, mean 3.40, SD 0.44), and A\&E ( $n=7$, mean 3.11, SD 0.65), which had the highest stress levels. There was no significant difference in stress levels between different grades of doctors. When compared with HSEMS targets, staff relationships and peer support exceeded national standards. However, management of organisational change and demands at work need improvement. The majority of doctors (82\%) had no idea what stress management services were provided by the Trust. Seventy-nine per cent of doctors had an allocated supervisor or mentor, $91 \%$ of those felt able to approach their supervisor.

Conclusion These survey results provide reassurance that stress levels in intensive care compare well, despite critically unwell patients and higher mortality rates. We identified areas that need improvement within the Trust and will present these results to all relevant departments. With the support of hospital management we will initiate HSEMS-validated measures to reduce stress.

\section{References}

1. Kerr et al.: HSE management standards and stress-related work outcomes. Occup Med (Lond) 2009, 59:574-579.

2. Health and Safety Executive Management Standards [http://www.hse.gov. uk/stress/standards/index.htm]

P509

Audit of obstetric critical care admissions in a high-risk population K El-Boghdadly', J Aron², DN Onwochei'

'Princess Royal University Hospital, London, UK; ${ }^{2}$ University Hospital Lewisham, London, UK

Critical Care 2013, 17(Suppl 2):P509 (doi: 10.1186/cc12447)

Introduction South-east London (SEL) presents unique challenges to healthcare providers due to its diverse demographic. The high levels of poverty, immigration and psychiatric illness impact delivery of obstetric care. These were identified as risk factors for poor outcome in the latest CMACE report [1]. The Intensive Care National Audit and Research Centre (ICNARC) produced data on obstetric critical care admissions in 2007 [2]. We reviewed the obstetric critical care admissions in three SEL hospitals and compared this with the national average determined in the ICNARC and CMACE data.

Methods All critical care admissions in three high-risk obstetric units in SEL (1 August 2009 to 31 July 2011) were screened for patients who were currently or recently pregnant. We compared local results with national data by ICNARC and CMACE.

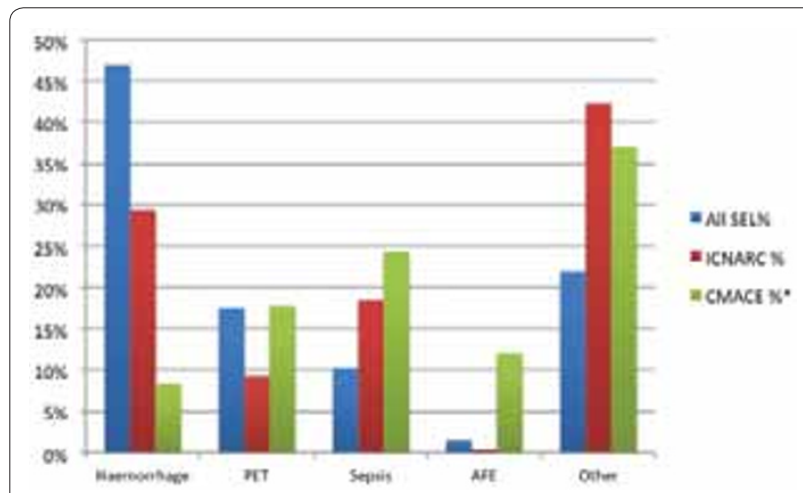

Figure 1 (abstract P509). Causes for critical care admission in SEL, CMACE and ICNARC.
Results There were 68 obstetric critical care admissions in the SEL hospitals within the audited time frame. The mean age was 30.05 in ICNARC data compared with 33.93 in SEL. Average APACHE II scores were lower in SEL compared with the ICNARC data, but length of stay was greater in SEL (2.72 days) compared with ICNARC (1.5 days). Haemorrhage was the most common reason for admission in SEL, whilst sepsis was the leading cause of death according to the latest CMACE report (Figure 1).

Conclusion Data from national audits may guide protocol, but services must be tailored to local circumstances. SEL has unique population characteristics and obstetric critical care admissions differ significantly from national statistics; in particular, haemorrhage is over-represented in our region. Critical care services were generally required for a short period of time; during this period, routine postpartum care may be omitted as treatment priorities differ. Dedicated critical care services on the labour ward may be a way to combine postnatal care with transient high-dependency requirements. This may enhance patient experience and prove cost-effective.

References

1. Centre for Maternal and Child Enquiries: Saving mothers'lives: reviewing maternal deaths to make motherhood safer: 2006-08. The Eighth Report on Confidential Enquiries into Maternal Deaths in the United Kingdom. BJOG 2011, 118(Suppl 1):1-203.

2. Female Admissions (Aged 16-50 Years) to Adult, General Critical Care Units in England, Wales and Northern Ireland, Reported as 'Currently Pregnant' or 'Recently Pregnant' [http://www.oaa-anaes.ac.uk/assets/_ managed/editor/File/Reports/ICNARC_obs_report_Oct2009.pdf]

P510

Influence of adverse drug events on the mortality and the length of hospital stay in ICUs in Japan: the JADE Study

Y Ohta', M Sakuma', D Bates ${ }^{2}$, T Morimoto'

'Kinki University, Osaka, Japan; ${ }^{2}$ Harvard University, Boston, MA, USA

Critical Care 2013, 17(Suppl 2):P510 (doi: 10.1186/cc12448)

Introduction Adverse drug events (ADEs) are associated with a substantial increase in morbidity and mortality in any setting. Because patients in ICUs were critically ill with complex diseases and varied organ dysfunction, the incidence of ADEs on such patients is much more crucial than the counterparts. We thus assessed the nature of ADEs and their influence in ICUs.

Methods We conducted a prospective cohort study at ICUs at three large tertiary-care hospitals in Japan. Trained research nurses reviewed all medical charts, incident reports and reconciliations from the pharmacy to identify suspected ADEs as well as the background of patients. ADEs are any injuries that result from the use of a drug. After suspected ADEs are collected by research nurses, physician reviewers independently evaluated them and classified them as ADEs or rule violations. We used the validated methodology [1].

Results We included 459 patients with 3,231 patient-days. The median age was 70 years and the median length of stay was 3 days. In total, 70 patients $(15 \%)$ had at least one ADE during their stay in the ICU. The median ICU stay in patients who had ADEs was 14 days while 2 days in patients who had no ADEs $(P<0.0001)$. The median length of the ADE onset days since admission was 3 days. Regarding the mortality, 73 patients (16\%) were dead during their ICU stay: 12 deaths (17\%) in patients who had ADEs and three of 12 deaths were caused by an $A D E$, and 61 deaths $(16 \%)$ in counterparts $(P=0.8)$. There were no significant differences of patients' characteristics between patients with ADEs and without ADEs (Table 1).

Conclusion ADEs were associated with longer stay and caused a part of death in ICU (4\%) although they did not increase the mortality. Because the characteristics of patients were not associated with ADEs, early detection and intervention for ADEs could be important to improve the morbidity and reduce the death caused by ADEs in ICUs.

Reference

1. Morimoto T, et al:: Qual Saf Health Care 2004, 13:306-314 
Table 1 (abstract P510). Patient characteristics

\begin{tabular}{|c|c|c|c|}
\hline Characteristic & $\begin{array}{l}\text { Patients } \\
\text { with } \\
\text { ADEs } \\
(n=70)\end{array}$ & $\begin{array}{l}\text { Patients } \\
\text { without } \\
\text { ADEs } \\
(n=389)\end{array}$ & $P$ value \\
\hline Age $\geq 65$ years, $n(\%)$ & $49(70)$ & $227(58)$ & 0.08 \\
\hline Male, $n(\%)$ & $40(57)$ & $250(64)$ & 0.2 \\
\hline \multicolumn{4}{|l|}{ Department } \\
\hline Medicine & $35(50)$ & $228(59)$ & \\
\hline Surgery & $35(50)$ & $161(41)$ & 0.2 \\
\hline Unconsciousness, GCS $\leq 8$ & $13(19)$ & $91(23)$ & 0.4 \\
\hline Heart failure, NYHA = 4 & $37(53)$ & $216(56)$ & 0.7 \\
\hline Respiratory failure & $44(62)$ & $262(67)$ & 0.5 \\
\hline Kidney failure & $6(9)$ & $58(15)$ & 0.2 \\
\hline \multicolumn{4}{|c|}{ Clinical laboratory measurements, median (25\%, 75\%) } \\
\hline Blood urea nitrogen (mg/dl) & $21(15,39)$ & $19(14,31)$ & 0.2 \\
\hline Creatinine (mg/dl) & $0.8(0.5,1.5)$ & $0.9(0.7,1.7)$ & 0.8 \\
\hline Aspartate aminotransferase (IU/I) & $27(20,47)$ & $30(22,48)$ & 0.5 \\
\hline Alanine transaminase (IU/I) & $20(13,42)$ & $20(12,33)$ & 1.0 \\
\hline Total protein (g/dl) & $6.7(5.9,7.4)$ & $6.6(5.9,7.3)$ & 0.8 \\
\hline $\begin{array}{l}\text { Number of medications on admission } \geq 4 \text {, } \\
n(\%)\end{array}$ & $38(54)$ & $223(57)$ & 0.6 \\
\hline $\begin{array}{l}\text { Number of medications on admission, } \\
\text { median }(25 \%, 75 \%)\end{array}$ & $5(3,7)$ & $5(4,8)$ & 0.7 \\
\hline
\end{tabular}

GCS, Glasgow Common Scale; NYHA, New York Heart Association.

\section{P511}

Attitude and knowledge of intensive care staff concerning donation in Hungary: it is the first step to change

A Smudla', S Mihály², J Fazakas'

'Semmelweis University, Budapest, Hungary; ${ }^{2}$ Hungarian National Blood

Transfusion Service, Budapest, Hungary

Critical Care 2013, 17(Suppl 2):P511 (doi: 10.1186/cc12449)

Introduction In Hungary, despite the high level of social support, the number of organ recoveries from deceased donors has not changed significantly. The donation activity shows a positive relationship with the level of education of staff in ICUs as well as with their attitude towards transplantation. The aim of this cross-sectional study is to estimate the attitude and knowledge of intensive care specialists and nurses as regard donation and transplantation.

Methods The self-completed questionnaire that consisted of 20 items was completed at the Congress of Hungarian Society of Anesthesiology and Intensive Therapy in 2011. Besides the epidemiological data, the intensive care specialists $(n=179)$ and nurses $(n=103)$ were asked about donation activity, participation in an organ donation course, selfreported knowledge of joining Eurotransplant, donor management, legislation, and transplantation. The data were analyzed by SPSS 17.0. Results A total of $53.6 \%$ of physicians and $16.7 \%$ of nurses attended an earlier organ donation course $(P<0.01)$. The average age of those who participated in training was significantly higher among doctors $(P<0.01)$. Fifty-nine percent of doctors and $65.1 \%$ of nurses did not even want to participate in such training. Donation activity was higher among staff who joined training $(P<0.01)$. Independently from accepting the presumed consent legislation (91.1\%), 66\% of physicians agreed with the hospital practice that requests the adult donor's relatives to consent to organ recovery. This standpoint did not depend on donation activity, participation in an organ donation course, opinion about legislation and the nature of staff. A total $95.4 \%$ of participants consented to their organ retrieval after death. The staff who participated in an organ donation course had more knowledge regarding the law and ethics of donation $(P<0.01)$, donor management $(P<0.01)$, living and deceased donor transplantation $(P<0.01)$ and joining Eurotransplant $(P<0.01)$. Older professionals had more information about all fields $(P<0.01)$. Nurses had less knowledge concerning donor management $(P<0.01)$, law and ethics $(P<0.01)$ and deceased donor transplantation $(P<0.01)$ than doctors.

Conclusion Education about organ donation needs to be part of specialist training of intensive care staff, and refresher courses every fifth year as well. The course should include knowledge regarding brain death, donor management and communication with family. This is the first step to improve the number of transplantations.

Reference

1. Roels L, et al:: Transplant Int 2010, 23:842-850.

P512

Specialist nurse for organ donation in an emergency department

will increase organ donation

T Neill, J Millar, P O'Connor, P Glover

Royal Victoria Hospital, Belfast, UK

Critical Care 2013, 17(Suppl 2):P512 (doi: 10.1186/cc12450)

Introduction In the UK, three people die each day awaiting transplantation, due to the unavailability of donor organs. Traditionally, donor identification has been restricted to the ICU. However, following the UK Organ Donation Taskforce report in 2008 [1], a number of emergency departments (EDs) have been working with specialist nurses for organ donation (SN:OD) to identify potential donors and approach their families for consent in the ED. We present our initial experience after the introduction of a SN:OD to an Irish teaching hospital's ED.

Methods We conducted a retrospective review of deaths in our ED during a 28-month period. For those who died in the ED, case notes were reviewed to identify those suitable for organ donation. Referral and donation rates were compared in two cohorts, pre and post introduction of a SN:OD. Fisher's exact test was used to assess differences between groups.

Results Ninety-one deaths occurred in the study period. Following introduction of the SN:OD, referrals increased from zero to eight. Of the eight referred, three received consent and were transferred to the $I C U$, two of whom became successful donors. The number of missed potential donors fell from six to one $(P=0.009)$.

Conclusion Introduction of a SN:OD and a clinical pathway has led to the identification of previously missed potential organ donors in the ED. Several patients have subsequently been admitted to critical care solely to facilitate organ donation.

Reference

1. UK Organ Donation Taskforce: Organs for Transplants: A Report from the Organ Donation Taskforce. Department of Health; 2008.

P513

Sepsis in HIV patients admitted to the ICU

P Vidal-Cortés ${ }^{1}$ P Lameiro-Flores' 1 M Mourelo-Fariña ${ }^{2}$. A Aller-Fernández ${ }^{2}$ R Gómez-López', P Fernández-Ugidos', M Alves-Pérez', E Rodríguez-García 'CHU Ourense, Spain: ${ }^{2} \mathrm{CHU}$ A Coruña, Spain

Critical Care 2013, 17(Suppl 2):P513 (doi: 10.1186/cc12451)

Introduction Our objective was to analyze septic HIV patients admitted to intensive care.

Methods A retrospective study of HIV patients admitted to our ICU between January 2005 and December 2009. We identify patients admitted to the ICU with sepsis and analyze demographic factors, etiology, organ failure and outcome, and we compare immune status, frequency of organ failure and outcome between these patients and those admitted for other reasons. We use Student's $t$ test to compare quantitative variables, and the chi-square test for qualitative data.

Results A total of 104 HIV patients were admitted to our ICU during the study period, 62 with sepsis ( $71 \%$ men, mean age: $40.59 \pm 8.12$ ). Of sepsis patients, $56.5 \%$ were admitted from the ER and $38.7 \%$ from a medical ward; $66.1 \%$ had history of intravenous drugs use, other comorbidities: COPD $(9.7 \%)$, cirrhosis $(8.1 \%)$, solid or hematologic malignancy $(12.9 \%) ; 8.1 \%$ patients were unaware of their condition 
(2.4\% in non-infected patients, $P=0.223$ ) and $40.3 \%$ were under HAART (64.3\% in patients admitted without infection, $P=0.016)$. Mean CD4 count at admission: $219.62 \pm 353.93$ cells $/ \mathrm{mm}^{3}$ (vs. $370.22 \pm 362.56$, $P=0.048$ ). Mean viral load: $4.57 \pm 3.25 \log$ (vs. $2.257 \pm 1.96, P=0.001$ ). Of sepsis patients, $62.1 \%$ were on their CD4 nadir (vs. $34.5 \%$ in nonseptic patients, $P=0.009$ ). Mean albumin levels were $2.3 \pm 0.53 \mathrm{~g} /$ dl (vs. $2.92 \pm 0.94, P<0.001$ ). APACHE II at admission was $21.98 \pm 7.97$ (vs. $18.15 \pm 8.47, P=0.046$ ). At admission, $52.8 \%$ were on severe sepsis and $44.3 \%$ on septic shock. The lung was the most frequent source of infection (65.6\%) followed by CNS (16.4\%), UTI (4.9\%) and IE (4.9\%). The most common pathogen isolated on these patients was $S$. pneumoniae (28.8\%), followed by P. jirovecii (13.6\%), toxoplasma (8.5\%), E. coli (5.1\%) and $H$. influenzae (5.1\%). In total, $62.9 \%$ needed vasopressors (vs. $28.6 \%$ in non-infected patients, $P=0.001$ ), $79 \%$ mechanical ventilation (vs. $42.85 \%, P<0.001)$ and $19.4 \%$ renal replacement $(9.5 \%$ in no septic patients, $P=0.173$ ). Mean ICU and hospital LOS was $10.43 \pm 10.52$ and $34.76 \pm 29.64$ days in septic patients versus $6.04 \pm 8.45(P=0.026)$ and $20.54 \pm 27.93$ days $(P=0.016)$. ICU mortality: $33.9 \%$ (19\% in nonseptic patients, $P=0.098$ ), hospital mortality: $41.9 \%$ (vs. $23.8 \%, P=0.057$ ).

Conclusion Sepsis is a common reason for admission to the ICU in HIV patients and is accompanied by high mortality. Pneumonia is the most frequent source of infection. Septic patients are less frequently under HAART and have a worse inmune status (lower CD4 count and higher viral load). Despite a higher APACHE II, and a higher need for hemodynamic and respiratory support, there is no statistically significant difference in ICU and hospital mortality between septic and nonseptic patients.

\section{P514}

Mortality related to respiratory failure among pediatric hematology patients requiring intensive care

H Okuno, K Atagi, H Shimaoka

Osaka City General Hospital, Osaka, Japan

Critical Care 2013, 17(Suppl 2):P514 (doi: 10.1186/cc12452)

Introduction Although recent reports show an improvement in outcomes for pediatric hematology patients requiring intensive care [1,2], respiratory failure remains one of the major risks of pediatric mortality. This study was conducted to assess our hypothesis that mortality associated with respiratory failure is higher than that for other organ failures in pediatric hematology patients admitted to our ICU.

Methods A retrospective study analyzed children with hematological disorders admitted to our ICU between April 2005 and June 2012. All of the included children required emergency admission and invasive mechanical ventilation. Those who did not need intubation, or required intubation only for therapeutic intervention and died within 24 hours of ICU admission were excluded. The survival group was defined as patients who were discharged from the ICU, and the nonsurvival group was defined as those who died in the ICU or within 7 days after discharge from the ICU. The PELOD score and PIM-II were applied as morbidity scoring systems

Results Twenty-seven patients, including 18 males and nine females, with a median age of 6.1 years (range, 0.2 to 16.6 years) were analyzed. Sixteen patients had leukemia, five had hemophagocytic syndrome, six had solid tumors. The average predicted mortality rate was $31.3 \%$ in PIM-II. The survival group included 15 patients $(56 \%)$ and the nonsurvival group included 12 patients (44\%). When the survival group was compared with the nonsurvival group, there were no significant differences in the systolic blood pressure $(101.3 \pm 13.9 \mathrm{mmHg}$ vs. $92.8 \pm 25.4 \mathrm{mmHg} ; P=0.15)$, the proportion of patients requiring continuous renal replacement therapy ( $33.3 \%$ vs. $50.0 \% ; P=0.30)$, and PELOD score $(15.5 \pm 10.4$ vs. $21.8 \pm 15.4 ; P=0.22)$. In the nonsurvival group, the PIM-II was higher than that in the survival group $(27.9 \pm 10.4$ vs. $35.7 \pm 9.0 ; P=0.06)$; the $\mathrm{PaO}_{2} / \mathrm{FiO}_{2}(272.5 \pm 136.7$ vs. $153.3 \pm 123.3$; $P=0.03)$ and oxygenation index $(6.7 \pm 8.1$ vs. $14.1 \pm 9.5 ; P=0.04)$ were significantly worse in the nonsurvival group than in the survival group. Conclusion The data show that respiratory failure is more strongly associated with mortality than other organ failures in pediatric hematology patients requiring intensive care. These results also suggest that mechanical ventilation intervention in patients with respiratory failure must occur earlier to improve the outcomes for these patients.

\section{References}

1. Tamburro RF, et al.: Pediatr Crit Care Med 2008, 9:270-277.

2. Chima RS, et al:: Pediatr Crit Care Med 2012, 13:e336-e342.

\section{P515}

Survival of critically ill patients with haematological malignancies compared with patients without haematological malignancy

R Pugh', P Hampshire2, P Hajimichael $^{3}$

'Glan Clwyd Hospital, Rhyl, UK' 'Royal Liverpool University Hospital, Liverpool,

UK: ${ }^{3}$ Christie Hospital, Manchester, UK

Critical Care 2013, 17(Suppl 2):P515 (doi: 10.1186/cc12453)

Introduction Critically ill patients with haematological malignancies (HM) have high hospital mortality [1]. Severity of illness scores may underestimate mortality in such patients [2].

Methods Data collection was conducted at three hospitals from 2008 to 2011. Patients with any active HM condition were matched with two control patients at two hospitals and with one control at Christie Hospital. Control patients had the same APACHE II (within 2 points) and admission diagnosis, but no HM. Readmissions and planned surgical cases were excluded.

Results A total of 163 patients with HM were compared with 237 control patients. Seventy-four admissions with HM were identified at two hospitals, and each was matched with two control patients. Eightynine admissions with HM from Christie Hospital were identified. These were matched with 89 controls. Patients with HM spent significantly longer in hospital before ICU admission (Table 1). Unit and hospital mortality rates were not statistically different between patients with HM and without HM (Table 2).

Table 1 (abstract P515). Patient characteristics

\begin{tabular}{lccc}
\hline & Haematology & Controls & $P$ value \\
\hline Age, mean (SD) & $56.5(15.1)$ & $57.1(15.7)$ & 0.717 \\
APACHE II, mean (SD) & $22.7(6.1)$ & $23.1(6.0)$ & 0.484 \\
ICU LOS (median) & $3.2(1.8$ to 6.2$)$ & $3.1(1.3$ to 8.4$)$ & 0.948 \\
LOS prior (median) & $6.5(2$ to 17$)$ & $2.0(0$ to 7$)$ & $<0.0001$ \\
\hline
\end{tabular}

Table 2 (abstract P515). Unit and hospital mortality

\begin{tabular}{lccc}
\hline & $\begin{array}{c}\text { Patients } \\
(\boldsymbol{n})\end{array}$ & $\begin{array}{c}\text { ICU mortality, } \\
\boldsymbol{n}(\%)\end{array}$ & $\begin{array}{c}\text { Hospital mortality, } \\
\boldsymbol{n}(\%)\end{array}$ \\
\hline Haematology & 163 & $52(31.9 \%)$ & $81(49.7 \%)$ \\
Controls & 237 & $73(30.8 \%)$ & $99(41.8 \%)$ \\
$P$ value & & 0.827 & 0.126 \\
\hline
\end{tabular}

Conclusion Unit mortality of critically ill patients with HM was similar to those without HM. Hospital mortality in patients with $\mathrm{HM}$ was higher than those without $\mathrm{HM}$, although not statistically significant. Severity of illness at presentation to critical care is the main determinant of outcome in patients with HM.

References

1. Hampshire PA, et al:: Crit Care 2009, 13:R137

2. Massion PB, et al:: Crit Care Med 2002, 30:2260-2270.

P516

Outcome of critically ill patients with haematological malignancy admitted to the ICU as an emergency

T Lofaro', S Easdale², S Rowe ${ }^{2}$, M Ostermann², R Carr ${ }^{2}$

'Queen Elisabeth Hospital, Woolwich, UK; ${ }^{2}$ Guys' and St Thomas' Hospital,

London, UK

Critical Care 2013, 17(Suppl 2):P516 (doi: 10.1186/cc12454)

Introduction ICU admission policies regarding patients with haematological malignancy still vary despite data showing an acceptable prognosis. Our aim was to review ICU and 6-month outcomes in this 
group when requiring emergency admission to the ICU in a tertiary cancer centre.

Methods A retrospective review of medical notes between 2004 and 2012.

Results A total of 249 patients were admitted, of whom 54 had more than one admission. There were 310 episodes in total. Leukaemia $n=85$; lymphoma $n=90$; myeloma $n=36$. We compared the characteristics of those who survived ICU admission with those who failed to survive to discharge from ICU. The two populations were similar (age 51 vs. 57; males $59 \%$ vs. $57 \%$ ). Those who survived had a lower APACHE II score on admission (19 vs. $23 ; P<0.001$ ), lower mean organ failure scores (1 vs. $2 ; P<0.05)$, lower requirements of inotropes ( $26 \%$ vs. $50 \%$; $P=0.001)$, ventilation ( $31 \%$ vs. $64 \% ; P=0.001)$ and filtration $(11 \%$ vs. $26 \%$; $P=0.004)$. There was no difference in the prevalence of sepsis at the time of admission (64\% vs. $70 \%)$. Both groups included patients with prior bone marrow transplant ( $38 \%$ vs. $40 \%$ ). Of note, ICU and 6-month survival were $27 \%$ and $50 \%$, respectively. These values are lower than those reported in the literature to date.

Conclusion ICU and 6-month mortalities were $27 \%$ and $50 \%$, respectively. Patients with haematological malignancy stand to benefit from intensive care, and should be offered admission based on clinical need.

References

1. Cuthberson et al:: The outcome of haematological malignancy in Scottish intensive care units. J Intensive Care Soc 2008, 9:135-140.

2. Evison JM, et al:: Intensive care unit admission in patients with haematological disease: incidence, outcome and prognostic factors. Swiss Med Wkly 2001, 131:681-688.

3. Beed et al:: Intensive care management of patients with haematological malignancy. Conti Edu An Crit Care Pain 2010, 10:167-171.

4. Kleber et al:: Comorbidity as a prognostic variable in multiple myeloma: comparative evaluation of common comorbidity scores and use of a novel MM-comorbidity score. Blood Cancer J 2011, 1:e35.

5. McGrath S, et al:: ICU and 6-month outcome of oncology patients in the intensive care unit. QJM 2010, 103:397-403.

P517

Effect of organizational structure of the ICU on the prognosis: open format versus semi-closed format

TTaniguchi, M Okajima

Kanazawa University Hospital, Kanazawa, Japan

Critical Care 2013, 17(Suppl 2):P517 (doi: 10.1186/cc12455)

Introduction In the year 2009, the organizational structure of the ICU in the Kanazawa University Hospital changed from an open to a semiclosed format ICU. The objective of this study was to evaluate the effect of this organizational change on outcome in high-risk surgical patients. Methods The medical records of all consecutive high-risk surgical patients admitted to the ICU from 2006 to 2009 (open format, $n=1,598$ ) and from 2009 to 2012 (semi-closed format, $n=1,521$ ) were reviewed. Parameters studied were mortality and ICU length of stay.

Results Mortality of ICU patients was $9.9 \%$ in the open format group and $6.6 \%$ in the closed format group $(P<0.05)$. The average length of hospital stay was 4.9 days in the open format group and 4.8 days in the closed format group.

Conclusion Our results suggest that a semi-closed format is a more favorable setting than an open format to improve mortality in the ICU and to warrant safe outcome in this patient group.

P518

Use of defaults on an electronic prescribing tool influences the type of fluid received by patients

L Herbert, C Bordeaux, M Thomas, L Burrows, J Bewley, T Gould

Bristol Royal Infirmary, Bristol, UK

Critical Care 2013, 17(Suppl 2):P518 (doi: 10.1186/cc12456)

Introduction Many evidence-based interventions are not delivered to patients [1]. This may not be due to a clinician's intentional decisions. The aim of this project was to compare the use of starch before and after removing it as an option from an e-prescribing template.

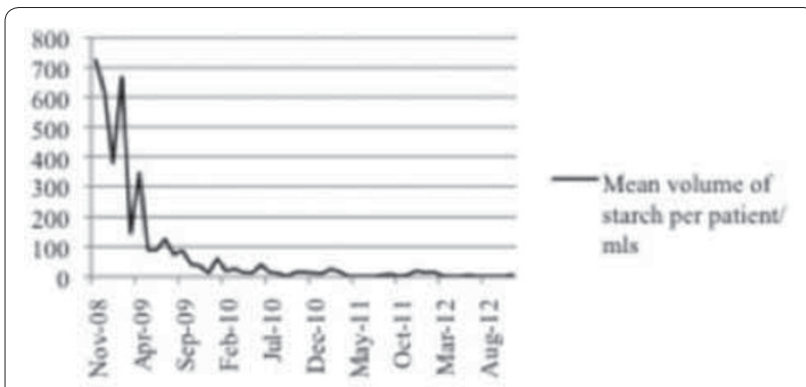

Figure 1 (abstract P518). Starch administration between November 2008 and November 2012

Methods Our e-prescribing software enables users to prescribe intravenous fluids from a series of menus. One of these is a template that has several fluids available to use as a bolus when instructed by a clinician. We removed starch as an option from the template in April 2009. Starch could still be prescribed elsewhere on the prescribing system. Data on the use of starch from November 2008 to November 2012 were analysed as the mean volume of starch infused per patient per month. The mean of each set of parameters was then compared using a Student's $t$ test.

Results The mean volume of starch per patient administered before and after electronic prescription options were altered was $480 \mathrm{ml}$ and $21 \mathrm{ml}$, respectively $(P=0.004)$. See Figure 1 .

Conclusion Despite clinicians intending to reduce the use of starch it was still regularly administered on our ICU. The removal of a default prescribing option dramatically reduced the volume of starch used whilst not restricting the ability to make a conscious choice to prescribe it. Adjusting default options has potential to influence clinical decisions and ensure more reliable, evidence-based care.

Reference

1. Schultz MJ, et al:. J Crit Care 2005, 20:199-204.

P519

Automated, electronic monitoring for early detection of sepsis

S Rauch', M Fischer', C Martin², A Sablotzki ${ }^{3}$

'Alb Fils Kliniken, Göppingen, Germany; ${ }^{2} F a$. Löser, Leipzig, Germany; ${ }^{3}$ Klinikum Sankt Georg, Leipzig, Germany

Critical Care 2013, 17(Suppl 2):P519 (doi: 10.1186/cc12457)

Introduction Early detection of sepsis is important for a sufficient treatment to reduce mortality. We hypothesized that using modified systemic inflammatory response syndrome criteria over 1 hour using an electronic software program facilitates the clinical diagnosis of sepsis. Methods After IRB approval and informed consent we enrolled in this prospective, observational, single-center study 1,119 consecutive patients (age $68.6 \pm 16.4$, female/male 476/649) admitted over a 6-month period to a surgical ICU. A total 149 of them met modified systemic inflammatory response criteria. Patients were monitored by an electronic software program using live data from the laboratory and bedside monitors to detect modified systemic inflammatory response syndrome criteria persisting over 1 hour. The physicians were blinded to the software program alerts that notified in real time when modified systemic inflammatory response syndrome criteria were detected and persisted over 1 hour, but did not provide treatment recommendations. Results There was a total of 149 modified systemic inflammatory response syndrome criteria alerts. Seventy-four were confirmed as true sepsis cases by physicians. The overall incidence of sepsis was $7 \%$. Patients were categorized into length of stay $<24$ hours, 24 to 96 hours and $>96$ hours. The overall sensitivity of our system for detecting sepsis was $68 \%$ and the specificity was $91 \%$. The positive predictive value is $34 \%$ and the negative predictive value is $98 \%$.

Conclusion Real-time alerts using an automated, electronic monitoring of modified systemic inflammatory response syndrome criteria facilitate the clinical diagnosis of sepsis. 
P520

Admission to the ICU overnight: is it really a bad thing?

M Adams, P Dean, K MacDowall, P Stenhouse, A Mackay

Victoria Infirmary, Glasgow, UK

Critical Care 2013, 17(Suppl 2):P520 (doi: 10.1186/cc12458)

Introduction Admission to hospital overnight has been shown to increase mortality and decrease hospital length of stay [1]. The objective of this study was to determine whether this relationship is valid in patients admitted to our ICU, and whether length of stay was affected.

Methods A retrospective data collection identified 5,827 patients admitted to a five-bed ICU from April 1994 to November 2012. Data regarding patient age, sex, APACHE II score and ICU admission date and time were collected along with the length of stay in the unit and hospital. Definitions of day and night were set to local ICU standards of 9:00 am to 8:00 pm. Patients were then separated into two groups and analysed using Analyse-It software for Excel.

Results Crude ICU and hospital mortality rates in patients admitted during the day and overnight were examined. There was no significant difference in unit mortality (day $22.3 \%$ vs. night $22.7 \%, O R=1.02,95 \%$ $\mathrm{Cl}=0.91$ to $1.16, P=0.718$ ) or hospital mortality (day $30.7 \%$ vs. night $29.1 \%, \mathrm{OR}=0.93,95 \% \mathrm{Cl}=0.83$ to $1.04, P=0.203$ ). The mean unit length of stay showed no difference in patients admitted during daytime compared with those admitted overnight (4.27 days vs. 4.09 days, $P=0.162$ ). The mean hospital length of stay was decreased in patients admitted during daytime compared with patients admitted overnight (19.3 days vs. 21.7 days, $P=0.004$ ). The average age of patients was less in those admitted out of hours (night 56.5 years vs. day 59.2 years, $P=<.0001$ ). There was no significant difference in APACHE II scores of patients between the groups (day $19 \mathrm{vs.} \mathrm{night} 19, P=0.580$ ).

Conclusion There is no significant difference between the mortality of patients admitted overnight and patients admitted during the day to our unit. The hospital length of stay is increased in patients who are admitted overnight to intensive care; however, ICU length of stay is not affected. Adjustment for other confounders such as current bed occupancy and staffing ratios during the entire patient stay may help to understand the differences seen in the hospital length of stay. Reference

1. Kuiisten HA, et al: Intensive Care Med 2010, 36:1765-1771.

\section{P521}

Assessing the quality of interdisciplinary rounds

EC Ten Have, JE Tulleken

Universitair Medical Center Groningen, the Netherlands

Critical Care 2013, 17(Suppl 2):P521 (doi: 10.1186/cc12459)

Introduction Interdisciplinary rounds (IDRs) in the ICU are increasingly recommended to support quality improvement and to reduce conflicts, but uncertainty exists about assessing the quality of IDRs. We developed, tested, and applied a scoring instrument to assess the quality of IDRs in ICUs.

Methods A literature search was performed to identify criteria for instruments about assessing team processes in the ICU. Then, 10 videotaped patient presentations led by different intensivists were analyzed by Delphi rounds. Appropriate and inappropriate behaviors were highlighted. The IDR-Assessment Scale was developed and statistically tested. The inter-rater reliability was evaluated by rating nine randomly selected videotaped patient presentations by three raters. Finally, the scale was applied to 98 videotaped patient presentations during 22 IDRs in three ICUs for adults in two hospitals in Groningen.

Results The IDR-Assessment Scale had 19 quality indicators, subdivided into two domains: Patient Plan of Care, and Process. The domain Patient Plan of Care reflects the technical performance from the initial identification of a goal to the evaluative phase. The domain Process reflects the team processes that are important to ensure that the appropriate plan of care is agreed, understood, and executed as planned by all care providers. Indicators were essential or supportive. The inter-rater reliability of nine videotaped patient presentations among three raters was satisfactory $(\kappa=0.85)$. The overall item score correlations between three raters were excellent $(r=0.80$ to 0.94). Internal consistency in 98 videotaped patient presentations was acceptable $(\alpha=0.78)$. Application to 22 IDRs led by 14 different intensivists in three ICUs in two hospitals demonstrated that indicators could be unambiguously rated. The staff and management of all three ICUs that were rated had considered their IDRs to be adequately performed, and they were surprised by these study results.

Conclusion This study showed that the quality of IDRs can be reliably assessed for patient plan of care and process. The IDR-Assessment Scale had satisfactory inter-rater reliability, excellent overall item score correlations, and acceptable internal consistency. Our instrument may provide feedback for ICU professionals and managers to develop adjustments in quality of care. Testing the IDR-Assessment Scale in other ICUs may be required to establish general applicability.

Reference

1. Reader TW, et al: Developing a team performance framework for the intensive care unit. Crit Care Med 2009, 37:1787-1793.

\section{P522}

Leadership training and quality improvement of interdisciplinary rounds in the ICU

EC Ten Have, JE Tulleken

Universitair Medical Center Groningen, the Netherlands

Critical Care 2013, 17(Suppl 2):P522 (doi: 10.1186/cc12460)

Introduction The development of patient-centered care by interdisciplinary teams in the ICU has focused attention on leadership behavior. The purpose of this intervention study was to measure the effect of leadership training on the quality of performed interdisciplinary rounds (IDRs) in the ICU.

Methods In this nonrandomized intervention study, participants included nine intensive care medicine fellow trainees (intervention group) and 10 experienced intensivists (control group). Participants in the intervention and control groups previously were untrained in leading IDRs in the ICUs. After each participant led an IDR that was videotaped, the fellow trainees participated in a 1-day leadership training, which was consistent with principles of adult learning and behavioral modeling. After training, each fellow trainee led another IDR that was videotaped. Quality of the performed IDRs was measured by review of videotapes of the 19 IDRs lead by 19 intensivists, including 198 patient discussions subdivided into four ICUs, and assessment with the IDR-Assessment Scale.

Results Comparison of the intervention versus control groups shows that the intervention group has more yes scores on the IDR-Assessment Scale than the control group. This difference was significant in 12 of the, in total, 19 quality indicators.

Conclusion Quality of leadership will be reliably trained and measured in the context of IDRs in ICUs. Training in a simulation environment, with real-life IDR scenarios including conflicting situations, and workplacebased feedback in the preparation and feedback phases, appears to be effective to train leadership behaviour.

References

1. Ten Have ECM, et al:: Assessing the quality of interdisciplinary rounds in the intensive care unit. J Crit Care 2013, in press.

2. Miller A, et al:: Uni- and interdisciplinary effects on round and handover content in intensive care units. Hum Factors 2009, 51:339-353.

3. Pronovost PJ, et al.: Perspective: physician leadership in quality. Acad Med 2009, 84:1651-1656.

\section{P523}

Let's go round again! Quality improvement through intentional rounding

P Doyle, F Cox, R Tollyfield, A Seraj

Harefield Hospital, Harefield, UK

Critical Care 2013, 17(Suppl 2):P523 (doi: 10.1186/cc12461)

Introduction Harefield Hospital is a 150-bed cardiothoracic tertiary referral centre with transplantation, artificial heart, ECMO and primary angioplasty services. Our 35-bed critical care department consists of 18 intensive therapy unit, seven recovery and 10 high-dependency 
beds. Intentional rounds or proactive patient rounds were recognised by the Royal College of Physicians and the Royal College of Nursing [1] as structured, evidence-based processes for nurses to carry out regular checks with individual patients at set intervals. The senior nursing team decided to adapt this initiative to the intensive care setting in order to address clinical challenges and provide guidance for shift leaders to focus on key elements of care.

Methods Our intentional rounds, performed once per shift (twice daily), include two components. First, pressure area care - this component involves the shift leader checking whether key elements of pressure sore prevention have been performed. These include completion of the Waterlow risk assessment tool [2], noting the frequency of repositioning, use of lateral positioning and pressure-relieving pads. Second, renal replacement therapy rates - this element was identified as an area for focus after we established that our haemofiltration fluid use per hour of therapy was twice that of a near identical clinical setting. This pattern continued even after adopting similar therapy guidelines. The shift leader was guided to check whether therapy rates had been adjusted in line with latest biochemical results.

Results The incidence of pressure ulcers in the 4 months since the initiative began has averaged 2.25 per month compared with 7.8 per month prior to commencement of intentional rounding. Added to the rounding tool at the end of September 2012, RRT rates in the preceding 4 months averaged $31.5 \mathrm{ml} / \mathrm{kg} /$ hour over 24 hours, an $11.9 \%$ reduction from the previous average of $35.75 \mathrm{ml} / \mathrm{kg} /$ hour. If the pattern of RRT was to continue, this could equate to a cost saving of $U K £ 40,000$ per annum.

Conclusion The use of a modified targeted intentional rounding tool by the nursing shift leader can help ensure that best practice guidelines are adhered to. This strategy can improve patient outcomes and provide potentially significant fiscal benefits.

References

1. RCP, RCN: Ward Rounds in Medicine. Principles for Best Practice. London: Royal College of Physicians, Royal College of Nursing; 2012.

2. Waterlow J: The importance of accurate risk assessment and appropriate intervention in tissue viability. Br J Nurs 2009, 18:1090.

\section{P524}

Prospective observational study of handover in a medical ICU

A Mukhopadhyay', B Leong', A Lua², R Aroos², J Wong ${ }^{2}, \mathrm{~N} \mathrm{Koh}^{2}$, N Goh², $\mathrm{KSee}^{1}$, J Phua ${ }^{1}$, Y Kowitlawakul $^{3}$

'National University Health System, Singapore; ${ }^{2}$ National University of Singapore, Singapore; ${ }^{3}$ Alice Lee School of Nursing, National University of

Singapore, Singapore

Critical Care 2013, 17(Suppl 2):P524 (doi: 10.1186/cc12462)

Introduction Handovers are often associated with poor communication. ICU patients with multiple complex problems are ideal to study naturally occurring handovers. However, few studies have been conducted in the ICU.

Methods We conducted questionnaires of physicians and nurses involved and observed handovers in real time of medical ICU patients over 1 month.

Results We interviewed 580 of 672 physicians and nurses involved $(86.3 \%)$ and observed 90 real-time handovers (45 patients, $26.8 \%$ ) of 168 patients. Mean duration of handover was 391.3 ( \pm 263.6$)$ seconds, $78.5 \%$ were face to face and $1.26( \pm 1.75)$ distractions per handover were noted, person-to-person calling being the commonest mode of distraction (46.7\%). Nurses received training during induction in significantly higher numbers, covered allied specialties more and reviewed the patients early (all $P<0.05$ ). Perception of the relative importance of different components of the handover varied significantly between donors, recipients, physicians and nurses. Both physicians and nurses seldom (39.7\%) reviewed the available electronic past medical records of the patient before handover, which in addition to training in handover and overall confidence level in the management following handover are significantly associated with better satisfaction in univariate analysis; only the confidence level in patient management remained significant after multivariate analysis. However, agreement between donor and recipient on overall satisfaction was poor $(P>0.05)$. Nursing handovers were significantly longer than physicians'
$(572.08 \pm 214.68$ vs. $168.6 \pm 97.27$ seconds, $P<0.001)$ but are also associated with higher distractions particularly during evening shifts. Conclusion A higher percentage of nurses received handover training; nursing handovers are longer and more inclusive of other components of patient management; perceived importance of components of handover varies among healthcare professionals; distractions are common during handovers and associated with longer duration, by nurses and in the evening shifts; and higher confidence level in patient's management following the handover is associated with better satisfaction.

References

1. Ye K, et al: Handover in the emergency department: deficiencies and adverse effects. Emerg Med Australas 2007, 19:433-441

2. Apker J, et al.: Communicating in the 'gray zone': perceptions about emergency physician hospitalist handoffs and patient safety. Acad Emerg Med 2007, 14:884-894.

P525

Impact of knowledge transfer through the implementation of a telemedicine program in a community hospital in Brazil

CA Abreu Filho', M Steinman', A Andrade'2, R Cal', N Akamine', J Teixeira², E Silva', A Kanamura', M Cenderoglo', C Lottenberg

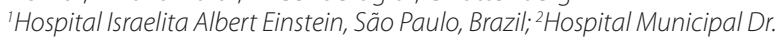
Moysés Deutsch, São Paulo, Brazil

Critical Care 2013, 17(Suppl 2):P525 (doi: 10.1186/cc12463)

Introduction Emergency survival rates vary significantly according to the quality of care, which depends on human and technological resources. Emergency and critical care medicine physicians must make fast decisions; the presence of experienced consultants improves survival. In developing countries, there is a shortage of skilled doctors. The aim is to describe the first Brazilian initiative of realtime teleconferencing telemedicine (TM) providing 24/7 emergency department (ED) and ICU coverage.

Methods Since May 2012 a TM program has been implemented at two hospitals in São Paulo, Brazil - Hospital Municipal Dr. Moysés Deutsch (HMMD), a public, secondary hospital, and Hospital Israelita Albert Einstein (HIAE), a tertiary private philanthropic entity - due to a partnership with the Brazilian Health Ministry. TM Central Command was located at HIAE with Endpoint $97 \mathrm{MXP} \mathrm{Cisco}^{\circledR}$ Solution and a mobile Intern MXP ISDN/IP Cisco ${ }^{\circledR}$ for the remote hospital (HMMD) via dedicated $\mathrm{GB} / \mathrm{sec}$ connection. Imaging examinations were evaluated using PACS technology. Every recruited patient was assessed by the Central Command through TM with an experienced consultant.

Results Over a 6-month period, 131 teleconsultations (114 patients) were done. Mean age was 50.1 years, $57.1 \%$ was male and mean APACHE II score was 24.3. A total of $64.8 \%$ originated from the ICU and $35.2 \%$ from the ED. Main consultation diagnoses were sepsis (31.3\%); stroke (29.8\%); survival from cardiac arrest (6.1\%); trauma (6.1\%); and acute myocardial infarction (5.3\%). TM improved diagnosis in $14.5 \%$ and influenced the clinical management in $85.5 \%$ of the consultations. Invasive procedures were indicated in $61.1 \%$. Life-saving procedures were TM related in seven patients $(6.1 \%)$ : stroke thrombolysis $(n=6)$ and limb amputation $(n=1)$. Seven patients $(6.1 \%)$ were transferred and submitted to surgical procedures (heart surgery $(n=2)$, neurosurgery $(n=4)$ and liver transplantation $(n=1))$. The majority of the patients remained at HMMD and were discharged.

Conclusion ATM program is feasible to be implemented in a community hospital. The major benefit is expertise medical transfer from the tertiary hospital to the community setting, improving diagnosis and management of critical care patients, and avoiding routine transfer to a major urban center.

P526

Utilization of telemedicine to improve burn care in a developing

country

J Knittel, G Fuzaylov

Massachusetts General Hospital, Boston, MA, USA

Critical Care 2013, 17(Suppl 2):P526 (doi: 10.1186/cc12464)

Introduction Our objective is to present our experience from Shriner's Hospital and Massachusetts General Hospital in Boston, MA, USA in 
using telemedicine to provide acute burn and critical care consultation on pediatric and adult burn patients in Lviv, Ukraine, as well as in triage and transport of critically ill patients from Lviv to a tertiary-care facility in the USA for further management.

Methods Using a new telemedicine learning center established at City Hospital \#8 in Lviv, Ukraine, consultations regarding acutely injured burn victims occurred between physicians in Ukraine and physicians at Shriners Hospital and Massachusetts General Hospital in Boston. After the initial presentation, each patient was reviewed on a daily basis by physicians in Boston. Skype, an Internet-based communication tool, was used in communication with the Burn Center in Lviv. Radiographic images were scanned and digitalized using an electronic scanner, and JPEG image compression was used to facilitate the transmission of radiographic images and patient charts. Informed consent and HIPPA guidelines were followed in transmitting any patient-related information.

Results Since 2011 we have provided consultation on 14 patients in Lviv, Ukraine, ranging in age from 15 months to 63 years. Each patient had an average of six consultations. We present two of these cases as examples of the capabilities of our telemedicine program. The first case involved a 15-month-old female with 40\% TBSA from scald injury, where telemedicine was instrumental in the primary assessment as well as to arrange a direct assessment from a nearby burn surgeon. The second case resulted from a house fire with multiple casualties, where physicians in Boston were able to utilize telemedicine to guide the initial resuscitation and airway management of three critically burned children, as well as to arrange for transport of one of the victims, an 11-year-old male with $87 \%$ TBSA, from Ukraine to the USA for acute management. Multiple difficulties were overcome in implementing the system between the two countries including: time zone differences, language barrier, and different approaches to patient care.

Conclusion We have established a telemedicine program linking physicians in Boston, MA, USA with City Hospital \#8 in Lviv, Ukraine to improve care in pediatric and adult burn patients. Our program has provided consultation on 14 patients since 2011, and it highlights the capabilities of telemedicine for acute consultation as well as triage and transport of critically ill patients to tertiary-care facilities.

\section{P527}

Different aspects of therapy limitation: a comparative study of the nurse's view

O Szűcs', G Élő', L Szabó', C Varga², J Gál', L Zubek'

'Semmelweis University, Budapest, Hungary; ZUniversity of Kaposvár, Hungary Critical Care 2013, 17(Suppl 2):P527 (doi: 10.1186/cc12465)

Introduction During the last few years the frequency of end-oflife decisions (EOLD) significantly increased in ICUs. The method of nurse involvement in making EOLD is different worldwide [1,2]. The purpose of this study was to analyze opinions of nurses about therapy restriction. We have examined with a multicenter study the opinions of the medical stuff about end-of-life care in Hungarian ICUs.

Methods We performed a questionnaire evaluation among physicians and nurses of ICUs about influencing factors of therapy restriction, the method of the decision-making process, and the frequency of different EOLD. The questionnaire, containing 21 questions, was delivered electronically to Hungarian ICUs, and then we analyzed the responses anonymously. The retrieved 302 answers (191 physicians, 102 nurses) were analysed using a nonparametric Student's test.

Results A total $71 \%$ of the nurse responders work in university clinics, $2 \%$ in regional centrum, $24 \%$ in municipal hospital, $3 \%$ in other ICUs. The nurses found both human (2.72/5 vs. $1.98 / 5)$ and material $(2.81 / 5$ vs. 2.12/5) resources more restrictive factors during patient admission than physicians $(P=0.025, P=0.0024)$. Nurses working in municipal hospital were more strongly influenced by lack of material and human resources $(3.34 / 5,3.3 / 5)$ than nurses working in university clinics $(2.2 / 5$, $2.43 / 5$ ) $, P=0.01, P=0.025$. Younger nurses (working between 6 and 10 years) were more interested in the patient's or surrogate's wishes than older nurses (working more than 10 years). Religion did not influence patient admission and forego therapy; however, religious nurses compared with atheists and nonpracticing believers preferred to prolong therapy against the patient's will $(P=0.04)$. Nurses felt that physicians slightly involved them in the end-of-life decision-making process $(2.1 / 5$ vs. $2.4 / 5 P=0.0001)$.

Conclusion We found that the workplace, level of medical attendance, godliness, work experience, and position in medical staff strongly influenced making EOLD. While limitation of the therapy should be team work, nurses felt their opinions were hardly taken into consideration, although nurses seemed to be more realistic in the decision-making process.

References

1. Prendergast et al:: A national survey of end-of-life care for critically ill patients. Am J Respir Crit Care Med 1998, 158:1165-1167.

2. Banbenishty et al:: Nurse involvement in end-of-life decision making: the ETHICUS Study. Intensive Care Med 2006, 32:129-132.

P528

Alternative to improve palliative care for all patients and families in critical care units: development and preliminary evaluation following MRC guidance of the King's Psychosocial, Assessment and Care tool

I Higginson, C Rumble, J Koffman, P Hopkins, S Heenen, W Prentice,

R Burman, S Leonard, O Dampier, J Noble, M Morgan, C Shipman

King's Health Partners AHSC, London, UK

Critical Care 2013, 17(Suppl 2):P528 (doi: 10.1186/cc12466)

Introduction More than one in five people admitted to an ICU will die there. Research has highlighted concerns about support for patients and families and decision-making in this context [1,2]. Here, we describe the development and evaluation of a tool to improve palliative care in a 32-bed general ICU in a central London teaching hospital.

Methods Medical Research Council guidance for complex interventions Phase 0 to I comprised literature review, theoretical modelling, observation and qualitative interviews and focus groups with staff and families exploring concerns and views of interventions identified in the literature review. Phase II comprised intervention development, implementation and evaluation of tool feasibility and effects using staff survey, observation, audit of records and relative survey.

Results Phase I: 47 staff and 24 family members were interviewed. The short time between decisions for treatment withdrawal and death, plus concerns for support management, communication and decision-making, highlighted a need to ensure excellent psychosocial assessment for all. Phase II: as part of integrated care guidelines, we developed the King's Psychosocial Assessment and Care tool (K-PACE). K-PACE is used for all patients entering the ICU, completed within 24 hours of admission. It contains psychosocial assessment of the family and patient needs, and identifies key individuals for contact. Educational training was supported by K-PACE and was implemented in two waves. Post-implementation survey of 95 ICU staff found that most $(80 \%)$ were aware of K-PACE. Eighty-two per cent of nurses but only $17 \%$ of doctors had completed the tool. In total, $158 / 213$ (74\%) family members responded to the survey (additionally three patients responded). There were high levels of satisfaction for symptom control and psychosocial care but concerns continued regarding explanation of treatment and care.

Conclusion K-PACE is a feasible tool to improve the palliative care of patients and their families in the ICU. Further refinement is needed and planned, with consideration of roll-out into the wider medical centre. References

1. Azoulay $E$, et al:: Half the families of ICU patients experience inadequate communication with physicians. Crit Care Med 2000, 28:3044-3049.

2. Asch DA, et al:: Conflicts between physicians' practices and patients' wishes. Am J Respir Crit Care Med 1995, 151:288-292.

P529

End-of life thoughts in the ICU: results of a survey

A Yaguchi, M Namiki, N Saito, R Nagai, M Takeda, T Harada, R Moroi

Tokyo Women's Medical University, Tokyo, Japan

Critical Care 2013, 17(Suppl 2):P529 (doi: 10.1186/cc12467)

Introduction The decision of terminal care in the ICU is a very tough issue because the law, ethics, traditions and futility should 
be concerned involving the family's will. Especially, stopping or withdrawing therapy is a quite difficult operation in Japan because of legal issues. Our hypothesis is that some difference exists in thoughts between physicians and nurses for terminal patients in the ICU. The aim of this study is to know their real thoughts.

Methods A questionnaire survey was performed on physicians and nurses in our medico-surgical ICU. The questionnaire consists of 11 questions with five optional answers related to the thoughts of participants about treatment of hopeless or brain death patients. Concretely, the questions were; whether to withhold therapy or not, whether to accept to withdraw therapy or not and with family's will, whether to accept to immediately stop therapy and with family's will, whether to positively or not donate organs from a brain death patient, necessity of ICU care for brain death patients, and feeling guilty and stress for stopping or withdrawing therapy. The optional answer has five gradations from 'Yes' to 'No' for all questions. The participants were asked to answer the questionnaire by expressing themselves without regarding legal issues or the consensus. It was guaranteed to be anonymous for them in the data analysis. The answers were compared between physicians and nurses. The Mann-Whitney $U$ test was used for statistical analysis. $P<0.05$ was considered statistically significant.

Results There were in total 52 participants (response rate $98.1 \%$ ) with 20 physicians and 32 nurses. Withdrawing therapy was significantly accepted in nurses than in physicians ( $83 \%$ vs. $55 \%, P=0.039$ ), when the family well understood. Withholding therapy should not be operated for brain death patients for physicians (65\%), while it seemed a difficult judgement for nurses $(23 \%, P=0.021)$. ICU care for brain death patients is less necessary for physicians than nurses $(80 \%$ vs. $53 \%, P=0.016)$. There were no significant differences in other questions between physician and nurses such as feeling guilty or stress for stopping or withdrawing therapy.

Conclusion Some of end-of-life thoughts in the ICU showed differences between physicians and nurses.

P530

End-of-life decisions in Slovenian ICUs: a cross-sectional survey

S Grosek', M Orazem², M Kanic ${ }^{2}$, G Vidmar ${ }^{3}$, U Groselj

'University Medical Centre Ljubljana, Slovenia; ' University of Ljubljana,

Slovenia; ${ }^{3}$ University Rehabilitation Institute, Republic of Slovenia, Ljubljana,

Slovenia

Critical Care 2013, 17(Suppl 2):P530 (doi: 10.1186/cc12468)

Introduction The purpose of our study was to assess the attitudes of Slovenian intensivists towards end-of-life (EOL) decision-making and to analyze the decision-making process in their clinical practice.

Methods A cross-sectional survey among Slovenian intensivists and intensive care medicine residents from 35 different ICUs was performed using a questionnaire containing 43 questions about views on EOL decision-making. Fisher's exact test and the Fisher-Freeman-Halton test were applied to cross-tabulated data; significance level was set at $P$ $\leq 0.001$ due to the large number of tested hypotheses.

Results The response rate was $72.1 \%$ (267 questionnaires were returned out of 370 distributed), which represented roughly the same percentage of all Slovenian intensivists. Termination of futile treatment was assessed as ethically acceptable $(P<0.001)$. The statement that there is no ethical distinction between withholding and withdrawing of treatment could not be confirmed (the answers 'there is a difference' and 'undecided' were less frequent, but not statistically significant; $P=0.216$ ). A do-not-resuscitate order (DNR) was used more often than other withholding treatment limitations $(P<0.001)$. A DNR was used most frequently in internal medicine ICUs $(P<0.001$; compared with paediatric and surgical ICUs). Withdrawal of inotropes or antibiotics was used more often than withdrawal of mechanical ventilation or extubation $(66.7 \%$ vs. $12.0 \% ; P<0.001)$. Withdrawal of mechanical ventilation or extubation was more often used in the paediatric ICUs $(21.7 \%)$ as compared with the internal medicine ICUs (19.6\%) and the surgical ICUs $(3 \%)(P<0.001)$. Over two-thirds $(70.6 \%)$ of intensivists were against termination of hydration, which would be more often used in the internal medicine ICUs $(P<0.001)$. Thirty-one percent of intensivists used written DNR orders.

Conclusion Termination of futile treatment was found to be ethically acceptable for Slovenian intensivists, although they were not convinced that withholding and withdrawing of treatment were ethically equal. A DNR would be used most often. Withdrawal of inotropes or antibiotics would be used more often than withdrawal of mechanical ventilation or extubation. Termination of artificial hydration would be rarely used in practice.

P531

Attitudes of intensivists in the UK to withdrawal of futile therapy

M Mariyaselvam, M Irvine, J Carter, M Blunt, P Young

Queen Elizabeth Hospital, Kings Lynn, UK

Critical Care 2013, 17(Suppl 2):P531 (doi: 10.1186/cc12469)

Introduction We aimed to determine the current practice and attitudes of consultants in intensive care medicine when withdrawing futile life-sustaining therapy. Published guidelines suggest variation in withdrawal of futile life-sustaining therapy and are therefore not prescriptive [1]. Although there is an awareness of differing practices, the extent of these variations is not established.

Methods We surveyed a convenience sample of delegates at the Intensive Care Society (UK) State of the Art Meeting (2012) on attitudes and practice regarding withdrawal practice. Anonymised data were collected using surveymonkey.com.

Results Of 457 consultant attendees from the UK, 149 completed the survey (33\%). For $58 \%$ of consultants there was no formal institutional protocol for withdrawal of futile therapy. When deciding to withdraw therapy, $57 \%$ of consultants routinely seek and document a second opinion. Regarding donation after cardiac death (DCD), 93\% of consultants were happy to delay withdrawal to facilitate successful donation, $85 \%$ have already done so in their practice and $14 \%$ routinely withdraw therapy in theatres rather than on the ICU. Even if it would impact on the care of other patients, $48 \%$ would delay withdrawal of therapy to facilitate DCD. For patients accepted for DCD, 36\% think that some intensivists withdraw more aggressively (in essence, hasten death) in the hope of improving the likelihood of a successful organ donation and $29 \%$ have felt pressurised to withdraw therapy more quickly than their usual practice. Furthermore, 45\% experienced pressure to refer a patient for DCD when it they felt it was not appropriate.

Conclusion This survey confirms variation in the practice and attitudes to withdrawal of futile therapy amongst UK consultant intensivists. Formal protocols were frequently unavailable to guide withdrawal and second opinions were often not sought. Nearly one-half of the intensivists delay withdrawal to facilitate donation, even if this may impact on the care of other patients. Many intensivists have felt pressure to refer for donation when they feel this is inappropriate and there is a perception that some intensivists may withdraw care more aggressively in those who are accepted for DCD to improve the likelihood of a successful donation. This survey may help inform debate in this ethically challenging area.

Reference

1. Organ Donation after Circulatory Death

[http://www.ics.ac.uk/professional/standards_and_guidelines/dcd]

\section{P532}

Parent's perception on end-of-life care in Brazil

P Lago, J Piva, G Halal, M Halal

Hospital de Clinicas de Porto Alegre, Brazil

Critical Care 2013, 17(Suppl 2):P532 (doi: 10.1186/cc12470)

Introduction The aim of the study is evaluate the perceptions of parents of children who died in two Brazilian pediatric ICUs.

Methods An exploratory-descriptive study with a qualitative approach in the PICU of Hospital São Lucas and Hospital de Clinicas de Porto Alegre involving 15 parents of children who died. Data collection was performed through three steps: (a) the researchers contacted the parents through a telephone call to invite them to attend the hospitals; (b) at the hospital, the doctors who assisted the children clarified doubts about the therapy offered; and (c) an interview was carried out by two researchers not involved in the care. Data analysis was performed using the technique of thematic content analysis. 
Results and conclusion The research shows that the difficulty of communication is a factor that impacts negatively on the grieving process. Moreover, it stresses the importance for parents to rediscuss the moment of their child's death with health professionals.

\section{References}

1. Meyer E, Ritholz M, Burns J, Truog R: Improving the quality of end-of-life care in the pediatric intensive care unit: parents priorities and recommendations. Pediatrics 2006, 3:469-457.

2. Meyer E: On speaking less and listening more during end-of-life conferences. Crit Care 2004, 32:1609-1611.

3. Piva, JP, Lago P, Othero J, Garcia PC: Evaluating end of life in ten Brazilian pediatric and adults intensive care units. J Med Ethics 2010, 36:344-348.

4. Meert, Ket al:: Parents' perspectives on physician-parent communication near the time of a child's death in the pediatric intensive care unit. Pediatr Crit Care 2008, 9:2-7.

P533

Getting critical care patients home for end-of-life care

LS Nielsen

Kolding Sygehus, Kolding, Denmark

Critical Care 2013, 17(Suppl 2):P533 (doi: 10.1186/cc12471)

Introduction Despite our efforts in making patients healthy and going home, critical illness has a mortality, in Danish ICUs, of 10 to $12 \%$. Approximately $90 \%$ of deaths in ICUs happen after life-sustaining therapy has been withheld or withdrawn. Although trying to provide patients and family with what we suppose is a good death, most patients would prefer dying at home, and sometimes patients and family ask for the possibility of doing that. The last 2 years we transferred seven patients from our unit to end-of-life care in their own home.

Methods After making the decision of withholding or withdrawing intensive care therapy, the care of the patient changes from an active, medical, technological treatment to relief and care. In that period we determine whether the patient's condition is stable enough to go home. We try to find out if it is a wish for the patient to go home, and if the family has resources to take care of the patient at home. If that is the case, we start planning care at home, arrange for transportation, and contact the primary care physician and nurse. Due to the patient's condition on the day of transferring, we plan following the patient by either a nurse or a doctor.

Results Seven terminally ill patients wishing to go home for dying were transferred home. Diagnoses varied: end-stage lung disease, cancer, surgical complications. Ages ranged from 68 to 84 years. All patients survived transport home, and time at home varied from a few hours to 4 days. Later contacts with patients' families indicated that both patient and family were grateful, and that they did not experience the patient having pain or dyspnea at home.

Conclusion Sending critically ill patients home to die is not common. Anyhow, our experiences doing that are only positive. Terminally ill patients, awake to make a decision of their own, and in a condition making it possible, should have the choice to go home to die, with our help in logistics, planning and transportation.

Reference

1. Lusardi $P$, et al:: The going home initiative: getting critical care patients home with hospice. Crit Care Nurse 2011, 31:46-57.

P534

Project PIX (Post Intensive care eXercise): impact on physical fitness and focus group analysis of quality of life following exercise rehabilitation

J Goodman'1, W Walker², J Wright' ${ }^{1}$, G Danjoux', S Howell ${ }^{3}$, D Martin', SBonner ${ }^{1}$

James Cook University Hospital, Middlesbrough, UK; ${ }^{2}$ West Park Hospital, Darlington, UK; ${ }^{3}$ University of Leeds, UK; ${ }^{4}$ Teeside University, Middlesbrough, UK

Critical Care 2013, 17(Suppl 2):P534 (doi: 10.1186/cc12472)

Introduction This study assessed the effect of a hospital-based aerobic exercise programme on physical fitness and health-related quality of life (HRQoL) for survivors of ICU admission. Including the qualitative arm of the study, we examined the patient experience after critical illness, their views of the exercise programme and the effects on their HRQoL.

Methods A randomised controlled trial was undertaken in adult survivors of ICU admission. They were allocated to receive an 8-week in-hospital supervised aerobic programme consisting of two cycle ergometry and one unsupervised session per week (exercise group) or no exercise (control group). Primary outcomes were the anaerobic threshold (in $\mathrm{ml} \mathrm{O}_{2} / \mathrm{kg}$ mass/minute), physical function and mental health scores (SF-36 questionnaire), measured at weeks 9 and 26. Participants were then allocated to focus groups where the interpretation of experiences was compared with outcomes from the PIX study.

Results Fifty-nine patients were recruited to the study. The anaerobic threshold increased at week 9 in the exercise group by a clinically and statistically significant amount of $2 \mathrm{ml} \mathrm{O} / \mathrm{kg}$ mass/minute $(90 \% \mathrm{Cl}, 1$ to $3 \mathrm{ml} / \mathrm{kg} /$ minute). There was further improvement in fitness levels in both groups by week 26 (although no significant difference between groups). No significant difference in HRQOL measures between groups was demonstrated; however, the exercise group did show an improvement in their mental health scores. The focus groups centred on feelings of isolation, abandonment, vulnerability, dependency and reduced physical activity post hospital discharge. Many reported a lack of social inclusion as they did not have the energy or confidence to venture outside. However, those in the exercise group felt that the rehabilitation programme was motivating, built up confidence, improved fitness, helped social interaction and gave them a sense of achievement.

Conclusion The 8-week exercise intervention resulted in statistically significant improvements in fitness at 9 weeks while focus group participants highlighted the positive effects of the exercise intervention leading to enhanced energy levels, motivation and achievement. Psychological benefits of the exercise programme are apparent from the focus group, emphasising the important link between physical and mental health.

\section{P535}

Feasibility of neuromuscular electrical stimulation in critically ill patients

J Segers, G Hermans, F Bruyninckx, G Meyfroidt, D Langer, R Gosselink UZ/KU Leuven, Belgium

Critical Care 2013, 17(Suppl 2):P535 (doi: 10.1186/cc12473)

Introduction Survivors of critical illness often have a prolonged stay on the ICU. These patients may suffer from ICU-acquired weakness. It has been shown that reduction in muscle mass and muscle strength occurs early after admission to the ICU. However, in the very early stage on the ICU, patients are often sedated and not able to participate in any active mobilizations. Therefore the use of neuromuscular electrical stimulation (NMES) is becoming a treatment of interest in the ICU. The aim was to study the feasibility and safety of NMES in a surgical and medical ICU of a large, tertiary referral university hospital.

Methods Fifty patients with an expected prolonged stay on the ICU of 5 more days (judged on day 3) with no trauma or neurological disease were included. They then received daily a NMES session (DUO 500; Gymna, Belgium) for 25 minutes on the quadriceps bilaterally during their entire stay on the ICU. The main outcome was the ability to produce a contraction of the quadriceps through NMES. The muscle contraction was quantified on a 5-point scale: 1 (no contraction palpable and visible) up to 5 (contraction very well palpable and visible). Patients were classified as responders when an adequate muscle bulk was obtained in $\geq 75 \%$ of the sessions. The potential factors associated with the feasibility were: gender, age, body mass index (BMI), diagnosis of sepsis, Barthel index prior to admission to the hospital, APACHE II score, Glasgow Coma Scale (GCS), five questions for adequacy, stimulus intensity and leg edema. A multiple regression analysis was performed to identify the factors determining whether or not a contraction could be expected in a patient. Safety of NMES was assessed through heart rate, blood pressure, oxygen saturation and respiratory rate.

Results $\ln 48 \%$ of the patients we were able to achieve adequate muscle contractions in more than $75 \%$ of the sessions. GCS $(P=0.047)$, edema 
$(P=0.001)$ and sepsis $(P=0.010)$ were significantly different between responders and nonresponders. Responders had a lower mean GCS ( $7 \pm 3$ vs. $9 \pm 3$ ), lower amount of edema and were less likely to have had sepsis. In a multiple regression analysis, sepsis, edema, BMI and age explained $51 \%$ of the variance. As for safety, none of the parameters changed significantly.

Conclusion In patients with a better neurological condition, sepsis and/ or leg edema it was more difficult to obtain an adequate quadriceps contraction with NMES. NMES is safe to apply on the ICU.

\section{P536}

Follow-up and counseling service in trauma patients: needs and

goals - a preliminary study

DL Grieco, M Biancone, SL Cutuli, G Pintaudi, MT Santantonio,

ES Tanzarella, F Toni, AG De Belvis, MG Bocci, C Sandroni, M Antonelli Catholic University School of Medicine, Rome, Italy

Critical Care 2013, 17(Suppl 2):P536 (doi: 10.1186/cc12474)

Introduction Trauma is the most common cause of morbidity in young people. It has a high social impact both because of the high cost of the acute treatments and because of the physical and psychological consequences that it may cause. A prospective, observational, singlecenter study on quality of life 12 to 24 months after trauma was carried out. The aim of the study is to evaluate life quality after trauma and to identify the most important needs of the patients, in order to improve the level of care after an ICU stay and to implement a faster and more effective reintegration into the active and productive society.

Methods All patients admitted to the 18-bed ICU of our university hospital between 5 May 2010 and 28 February 2011 because of a trauma who stayed in the ICU for at least 48 hours were eligible for the study. Patients were contacted and invited to attend a multidisciplinary interview. Data concerning family, working and economic status, main disabilities and medical needs were collected. The SF12 questionnaire was administered to define physical component summary (PCS) and mental component summary (MCS) scores. SPSS 20.0 was used to perform statistical analysis.

Results Sixty-two patients matched inclusion criteria and were contacted. Thirty-three patients $(90.9 \%$ males; age $38.7 \pm 14.3$ years; mean SAPS II $33.75 \pm 11.79$ ) were interviewed. Mean ICU stay was $25.7 \pm 15.3$ days. Fifteen patients $(45.5 \%)$ had undergone temporary tracheostomy. Twenty-six patients were employed at the moment of trauma: of these, $11(42.3 \%)$ stated to be jobless and one was pensioned at follow-up. In the whole cohort, the unemployment rate increased from 12.1 to $45.6 \%$. Mean PCS was $43.59 \pm 6.43$, mean MCS was $41.04 \pm 7.69$. At follow-up, the 14 employed patients had a lower MCS versus the 19 jobless patients ( $37.94 \pm 7.14$ vs. $43.33 \pm 7.44$ $P=0.04$ ), while no differences concerning PCS were found. The three patients that were living on their own when the trauma occurred showed, at follow-up, a lower mean MCS score towards the rest of the cohort $(32.2 \pm 0.47$ vs. $41.93 \pm 7.51 ; P<0.001)$.

Conclusion Trauma is associated with an increase of the unemployment rate in young people. Patients that are employed at follow-up seemed to have a worse mental health status towards the unemployed ones. Conversely, the employed patients did not show any trend to a lower physical health status. Thus a counseling service that provides psychological care would be useful to implement more effective work reintegration for trauma patients.

\section{P537}

Post-traumatic stress disorder - are we doing all we can? A UK national-based survey

XWatson

Ashford and St Peter's Hospital, Surrey, UK

Critical Care 2013, 17(Suppl 2):P537 (doi: 10.1186/cc12475)

Introduction Post-traumatic stress disorder (PTSD) is a well-recognised complication in patients discharged from the ICU. ICU clinics have been recommended to treat physical and psychosocial problems post discharge, with guidelines issued by the Department of Health UK [1]. Recent evidence has advocated the use of patient diaries to reduce the incidence of new-onset PTSD [2]. Family support groups may also play a pivotal role [3]. We performed a national survey via the Intensive Care Society (ICS) to determine the provision of follow-up clinics, patient diary services and family support groups.

Methods An online structured questionnaire was sent via the ICS to all ICU linkmen at 124 hospitals in the UK. Responses were received from 77 ICUs, a $62 \%$ response rate.

Results Out of 77 ICUs, 37 (48\%) run a follow-up clinic. The majority of clinics (51\%) only invite patients who have been admitted for more than 3 to 4 days. Only 10 clinics (30\%) receive funding from the ICU budget or PCT, with the majority (67\%) receiving no funding at all. Only $44 \%$ (34) of ICUs use patient diaries, mostly as a nonfunded service (68\%). Additionally, $91 \%$ of ICUs do not run family support groups; from the minority that do, these are mostly held quarterly and are largely not funded (55\%).

Conclusion This survey demonstrates that the provision of ICU clinics in the UK is not well established, with only $48 \%$ currently providing a regular service. Currently $67 \%$ of clinics are not funded and further resources should be employed so this service becomes an integral part of the ICU pathway. Despite recent evidence demonstrating that diaries reduce new-onset PTSD, only $44 \%$ of ICUs in our study provide this service. Ninety-one per cent of ICUs do not provide family support groups; similarly, it appears that financial constraints are the limiting factor.

References

1. DH and Modernisation Agency: The National Outreach Report. London: NHS Modernisation Agency; 2003.

2. Jones C, et al:: Crit Care Med 2010, 14:168

3. Orcutt TA: Nurs Crit Care 2010, 5:33-37.

P538

Implementation of an ICU follow-up clinic: outcomes and patient satisfaction after 1 year

G De la Cerda

Queens Hospital, London, UK

Critical Care 2013, 17(Suppl 2):P538 (doi: 10.1186/cc12476)

Introduction The aim was to analyse the outcomes and patient satisfaction of a recently implemented ICU follow-up clinic. These clinics are National Institute for Clinical Excellence recommended [1].

Methods A retrospective analysis of prospective collected data from January to December 2012. The clinic is run monthly by an ICU consultant and a critical care outreach sister. Criteria to be invited to the clinic are mechanical ventilation $\geq 3$ days. Patients filled an anonymous satisfaction survey after the clinic.

Results Our attendance rate is $50 \%$ (26 patients), which is similar to other series reported in the literature. Those patients who attended the clinic required a longer length of mechanical ventilation (5.3 days vs. 7.1) and a longer length of stay in the ICU (7.6 vs. 13) and in hospital (14 vs. 28). We identified a wide range of physical and nonphysical morbidities on these patients (Figure 1). We referred them to the appropriate specialities. Patients were very satisfied with this new service (Figure 2).

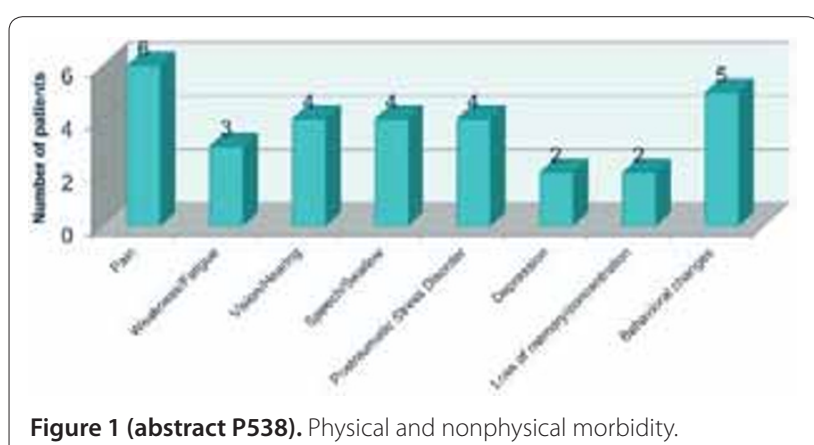




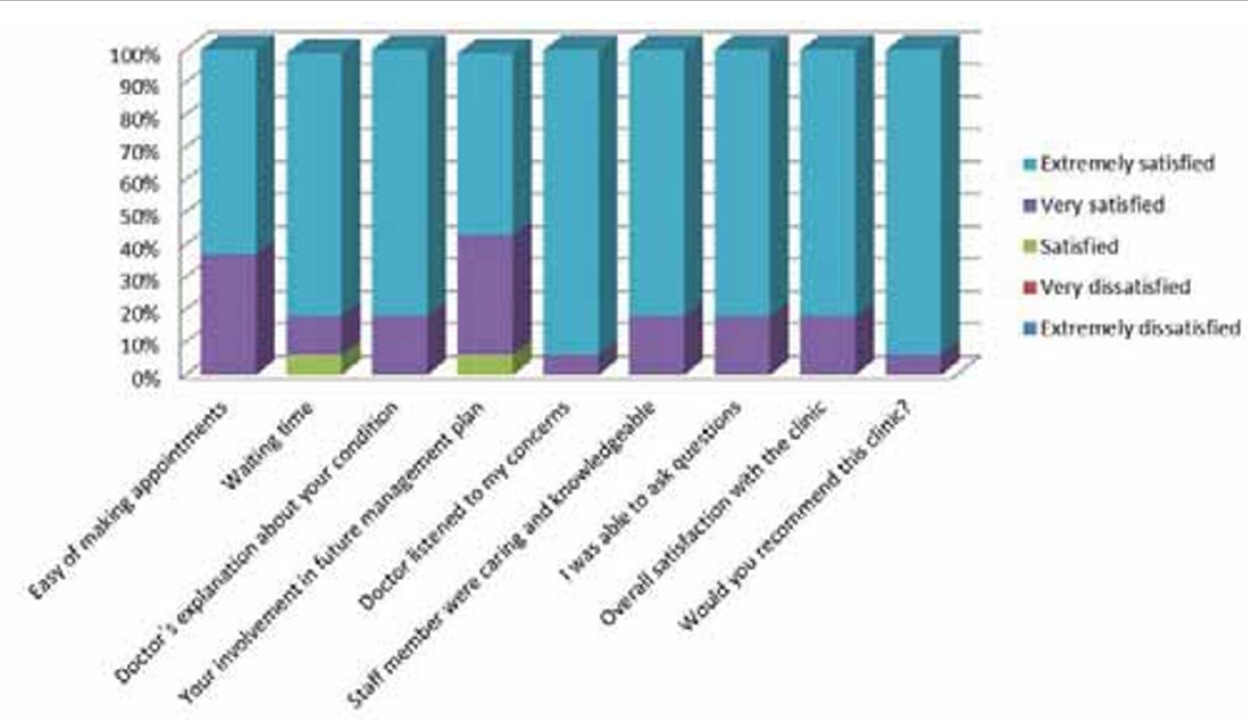

Figure 2 (abstract P538). Patient satisfaction survey.

Conclusion Our follow-up clinic has enabled us to identify a wide range of complications related to ICU admission and coordinate their future management. This clinic improved patient satisfaction.

Reference

1. Rehabilitation after Critical IIIness [www.nice.org.uk/CG83]

\section{P539}

Acute exercise response in the critically ill

N Collings ${ }^{1}$, R Young $^{2}$

'University Hospital Southampton, UK; ${ }^{2}$ Sheffield Hallam University, Sheffield, UK

Critical Care 2013, 17(Suppl 2):P539 (doi: 10.1186/cc12477)

Introduction This study aims to quantify the acute exercise response to early passive and active activities in order to inform exercise prescription when designing rehabilitation programmes for the critically ill. Critical care survival is often associated with a poor functional outcome [1], with recent investigations presenting the case for early rehabilitation in order to optimise functional recovery [2]. There, remains, however, a scarcity of research investigating the immediate response to exercise and subsequent exercise prescription, in the acute phase following critical illness.

Methods This study is a prospective randomised controlled trial with a repeated-measures crossover design. Eligible participants, requiring mechanical ventilation for 4 or more days, completed two exercise activities routinely used in early critical care rehabilitation, a passive chair transfer (PCT) and active sitting on the edge of the bed (SOEOB). The oxygen consumption and cardiovascular parameters were measured to quantify and compare the exercise response between the two activities.

Results Data are presented as the median (interquartile range). Data for five patients have been collected, aged 68 years (23), with an ITU stay of

Table 1 (abstract P539)

\begin{tabular}{lcc}
\hline Parameter & PCT $(\boldsymbol{n}=\mathbf{5})$ & SOEOB $(\boldsymbol{n}=\mathbf{5})$ \\
\hline Oxygen consumption (ml/minute) & $270(46.5)$ & $334(121)$ \\
Carbon dioxide production (ml/minute) & $142(45.5)$ & $185(121)$ \\
Heart rate (bpm) & $92(24)$ & $77.5(29)$ \\
Mean arterial pressure (mmHg) & $100.3(20)$ & $97.3(13)$ \\
Minute ventilation (I/minute) & $11.0(3.5)$ & $12.1(3.4)$ \\
\hline
\end{tabular}

15 days (10.5) and duration of mechanical ventilation 8 days (12), at the point of intervention. Exercise response results are reported (Table 1). Conclusion Intensive care patients with prolonged mechanical ventilation demonstrate a higher rate of oxygen consumption when actively sitting on the edge of the bed, compared with a passive chair transfer. This may have important consequences for early mobilisation of the critically ill.

References

1. Unroe et al:: Ann Intern Med 2010, 153:167-175.

2. Thomas: Phys Ther Rev 2011, 16:46-57.

\section{P540}

Randomized, controlled pilot study of early rehabilitation strategies in acute respiratory failure

D Files, P Morris, S Shrestha, S Dhar, M Young, J Hauser, E Chmelo,

C Thompson, L Dixon, K Murphy, B Nicklas, M Berry

Wake Forest University School of Medicine, Winston Salem, NC, USA

Critical Care 2013, 17(Suppl 2):P540 (doi: 10.1186/cc12478)

Introduction Optimal patient evaluations of ICU rehabilitation therapy remain unclear.

Methods One hundred ICU patients with acute respiratory failure were randomized to receive early rehabilitation (ER) or usual-care (UC). Cohort $1(n=50)$ received ER as one physical therapy (PT) session/day versus UC; Cohort $2(n=50)$ received ER as 2 PT/day with the second session resistance training, versus UC. UC was without ER. Blood was drawn for cytokines through day 7. Cohort 2 underwent strength and physical functional assessments using the Short Physical Performance Battery (SPPB), a valid and reliable measure of physical function consisting of walking speed, balance, and repeated chair stands. It is a well-studied composite measure in older persons, but has not been used in ICU survivors. Small changes of 0.5 to 0.6 points in the SPPB have been shown to be clinically meaningful.

Results Baseline parameters were similar between groups. Median days from enrollment to first PT were 4 (IQR 1 to 7.25). Deaths occurred in eight UC subjects and four in ER $(P=0.22)$. For both arms, ventilator days, ICU days and hospital days were not statistically different. ER had ventilator-free days of $22,95 \% \mathrm{Cl}=19.9$ to 24.6 , where UC had 22.3 days, 19.9 to $24.6, P=0.99$. ICU-free days for $E R$ was $21,95 \% \mathrm{Cl}=19.1$ to 23.6 , and that for UC was $21.0,18.8$ to $23.2, P=0.84$. Similarly, hospital days for $E R$ was $16.7,95 \% \mathrm{Cl}=11.8$ to 21.4 , and for UC was $18.2,13.8$ to 22.7 , $P=0.45$. TNF, IL-6 and IL-8 through day 7 were not different between groups. Despite similar baseline acuity and inflammatory profiles, Cohort 2 ER group strength scores were numerically but not statistically higher. Grip strength, as a percentage of predicted for ER was 66 versus 
39 for UC, $P=0.06$. Dynamometry for ER was 211 versus $181 \mathrm{lbs}$ for UC, $P=0.124$. Although the difference in SPPB values for ER versus UC (5 vs. $3, P=0.172$ ) was not statistically different, it was greater than the minimal clinically significant difference. There were no differences in adverse events.

Conclusion In this pilot study, early ICU rehabilitation was safe, and was associated with numerically although not statistically shorter hospital stay, greater strength and improved functional scores. Particularly, the SPPB demonstrated discriminatory ability in groups of ICU survivors with low physical function. Future early ICU rehabilitation studies should consider ICU survivor assessments using the SPPB due to its ease, reproducibility and discriminatory ability following ICU and hospital discharge.

Acknowledgement Supported by NIH grants 1R011186-01 and P30 AG21332.

P541

Early results of a 6-week exercise programme in post-ICU patients

C Battle', K James' ${ }^{1}$, P Temblett ${ }^{1}$, H Hutching $^{2}$

'Morriston Hospital, ABMU Health Board, Swansea, UK; ${ }^{2}$ Swansea University, Swansea, UK

Critical Care 2013, 17(Suppl 2):P541 (doi: 10.1186/cc12479)

Introduction The aim of this study was to investigate the effect of a 6-week exercise programme on outcomes in post-ICU patients. With improvements in intensive care medicine, increasing numbers of patients are surviving catastrophic illness [1]. Severe weakness is common in patients with prolonged critical illness and results in considerable morbidity, mortality and healthcare costs [2]. The NICE83 guidelines Rehabilitation in Critical Care recommend follow-up for post-ICU patients and that further research is needed in this field [3].

Methods Patients who have been discharged home from hospital following an ICU stay of 48 hours or more were recruited to the study. Patients were only excluded if they were not considered safe for exercise. Baseline measurements were completed prior to stratified (age, gender, APACHE II score) random allocation to either the exercise or control group. Outcome measures included cardiopulmonary fitness (6-minute walk test), balance (Berg Balance Scale), grip strength (JAMAR grip dynamometer) and hospital anxiety and depression (HAD score). The exercise group completed a 6-week supervised exercise programme, twice a week for up to 1 hour. In the seventh week, all patients repeated the baseline measurements. An unpaired Student's $t$ test was used to compare any differences between the control and exercise groups.

Results At baseline measurements, there were no statistical differences in age, gender, length of stays or APACHE II scores between the two groups. Results indicate that the exercise group $(n=10)$ had significantly greater improvements in cardiopulmonary fitness $(P<0.001)$ and balance $(P<0.05)$ compared with the control group $(n=10)$. Greater improvements were also evident in anxiety, depression and grip strength in the exercise group, although not statistically significant.

Conclusion This pilot study highlights that a 6-week supervised exercise programme can significantly improve cardiopulmonary fitness and balance in post-ICU patients. Further recruitment to the study and 6-month/1-year follow-up is needed.

References

1. Baker C, et al: Physical rehabilitation following critical illness. J Intensive Care Soc 2008, 9:166-169.

2. Jones $C$, et al: Long term outcome from critical illness. Anaesth Intensive Care Med 2006, 7:155-156.

3. CG83 Critical Illness Rehabilitation: Guideline 2009 [www.nice.org.uk/CG83]

P542

Recognition of the primary stressors affecting intensive care patients: a systematic review

S Birch, S Elliot

Leeds Teaching Hospitals, Leeds University Teaching Hospitals Trust, Leeds, UK Critical Care 2013, 17(Suppl 2):P542 (doi: 10.1186/cc12480)

Introduction Intensive care patients suffer psychological and physiological distress that may have debilitating and long-lasting effects [1-3]. Healthcare professionals are in a position to help avoid or alleviate this stress [4]. To action this it is important to identify the main stressors from the patient's perspective. A systematic review was performed to provide a list of what patients consider stressors in intensive care. These were then ranked in order to provide an identification tool that can be used to shape appropriate care.

Methods A systematic review was performed using MEDLINE, CINAHL, Psych INFO and Academic Search Complete. Grey literature was included and searches were not restricted to type of intensive care or country. Criteria were used to filter those articles that identified the patients' views of their stressor, not the patient experience. Eligible articles were critiqued using the Critical Appraisal Skills Programme for qualitative studies [5] and brought together using a narrative synthesis. All of the reviewed studies used a questionnaire as a means to identify what elements on the intensive care patients found stressful. A list of the top-10 stressors could then be expressed for each study and compared. From this information, a set of guidelines for best practice were devised.

Results A total 1,424 articles were systematically assessed for suitability and applicability. Of these, 14 articles remained eligible for review. The stressors were ranked by their frequency in the individual studies' top10 lists. In rank order: 1, Tubes. 2, Pain. 3, Sleep Difficulties. 4, Thirst. 5, Lack of Patient Understanding. Overall, stressors were found to be similar throughout all of the studies.

Conclusion The review identified a list of the most pertinent common stressors. Awareness of these and ranking in priority may enable plans of care to be instigated to effectively alleviate patient stress.

References

1. Jones C, et al: Clin Intensive Care 1998, 9:199-205.

2. Scragg P, et al:: Anaesthesia 2001, 56:9-14.

3. Perrins J, et al:: Intensive Crit Care Nurs 1998, 14:108-116.

4. Ballard KS: Issues Ment Health Nurs 1981, 3:89-108.

5. Public Health Resource Unit: Critical Appraisal Skills Programme. 10 Questions to Help You Make Sense of Qualitative Research. [http://www.medev.ac.uk/ static/uploads/workshop_resources/166/166_Qualitative_Appraisal_Tool.pdf]

\section{P543}

Quality of care in the ICU from the perspective of relatives

M Van Mol', E Bakker', A Rensen ${ }^{3}$, I Menheere ${ }^{3}$, L Verharen $^{3}$

'Erasmus MC, Rotterdam, the Netherlands; ${ }^{2} \mathrm{OU}$, Heerlen, the Netherlands:

${ }^{3} \mathrm{HAN}$, Nijmegen, the Netherlands

Critical Care 2013, 17(Suppl 2):P543 (doi: 10.1186/cc12481)

Introduction This study describes the development and validation of the Consumer Quality Index Relatives in ICUs (CQI 'R-ICU'), which aims to measure the satisfaction of relatives and to identify aspect of care that need improvement in the ICU in a reliable and valid way. According to the quality standards of the Dutch Society of Intensive Care, every ICU needs to record the satisfaction of relatives [1]. At this moment there is insufficient insight into the quality of care offered to relatives on the ICU because an evidence-based Dutch measurement instrument is missing.

Methods The CQI 'R-ICU' has been developed based on a scientific and standardised method [2]. A mixed design method is used, consisting of qualitative and quantitative survey studies. Factor analyses are carried out to determine the underlying structure of the newly developed questionnaire. Multiple regression analysis is used to explore the relationship between demographic variables and the perceived quality of care.

Results In six hospitals the COI 'R-ICU' is sent to relatives after receiving informed consent $(n=441), 55.1 \%$ of the respondents are the patient's partner. Respondents seem to be most satisfied with the presence of a professional at first entrance to the ICU. The highest need for improvement scores relate to information about meals, parking and other disciplines (for example, social worker, spiritual worker or psychologist). Factor analysis shows that quality of care is determined by four clusters of items: Support, Communication, General Information and Organisation. The reliability of the CQI 'R-ICU' is sufficiently high, only Communication and Support are significant predictors of total quality judgement of relatives (adj. $R^{2}=0.74$ ). In addition, there is a significant difference in mean total quality judgement between the six hospitals as well as between the four wards within Erasmus MC. None 
of the demographic variables such as sex, age, education, race and length of stay had an effect on perceived quality of care.

Conclusion The CQI 'R-ICU' turned out to be a valid, reliable, sensitive and feasible instrument. Large-scale implementation is recommended. Acknowledgements Thanks to researchers of Ziekenhuis Gelderse Vallei, Kennemer Gasthuis, Catharinaziekenhuis, Ziekenhuis Rivierenland and Scheperziekenhuis.

\section{References}

1. Vos et al:: Quality measurement at intensive care units: which indicators should we use? J Crit Care 2007, 22:267-274.

2. Sixma et al:: Handboek CQI Ontwikkeling: richtlijnen en voorschriften voor de ontwikkeling van een CQI meetinstrument. Utrecht: Nivel; 2008.

P544

Family satisfaction in the ICU: enhancing patient experience

E Kursumovic, J Bilinska, A Molokhia

University Hospital Lewisham, London, UK

Critical Care 2013, 17(Suppl 2):P544 (doi: 10.1186/cc12482)

Introduction Following recommendations made by the Scottish Intensive Care Society [1], we have introduced an annual Family Satisfaction Survey in the intensive care unit (FS-ICU), in anticipation that this will become an integral part of how we measure, improve and strive to advance patient care in the future. We aim to recognise factors impacting patient care and highlight areas for improvement. Improving understanding of patients' needs is currently a UK national goal for CQUIN 2011/2012. An estimated $£ 6.9$ billion per year can be saved by reaching these goals [2] and a positive patient experience results in improved long-term outcomes and shorter hospital stays [2]. Methods We performed a study to assess Family Satisfaction over a 10week period in a 16-bed critical care unit (CCU). A modified version of an FS-ICU published in US and Canadian studies [3] was distributed to up to two family members per patient. Five-point Likert scale responses were linearly transformed to give percentage scores. Higher values represented a greater degree of satisfaction.

Results We received and analysed 32 completed surveys. Seventyeight per cent of relatives reported that the treatment of the patient was excellent, including symptom control such as pain, breathlessness and agitation $(72 \%, 69 \%, 100 \%$, respectively). Ninety-one per cent felt the care and frequency of communication provided by nurses was excellent or very good, compared with $81 \%$ by doctors. Only $9 \%$ of family members were offered spiritual support during their CCU experience. Forty-seven per cent felt they would have liked more involvement in the decision-making process.

Conclusion The vast majority of relatives rated their overall experience on CCU as excellent or very good. The study highlighted two main areas for improvement: provision of spiritual support as well as family and patient involvement in the decision-making process. We will arrange multi-disciplinary teaching sessions focusing on the positive impact, and therefore importance of patient satisfaction. A poster providing information about available spiritual support will be displayed around our CCU. Further studies are required to evaluate these measures.

\section{References}

1. Quality Indicators for Critical Care in Scotland [http://www.sicsag.scot.nhs. uk/SICSQIG-report-2012-120209.pdf]

2. Using the Commissioning for Quality and Innovation (CQUIN) Payment Framework - Guidance on National Goals for 2011/12 [http://www.dh.gov. uk/prod_consum_dh/groups/dh_digitalassets/@dh/@en/@ps/documents/ digitalasset/dh_123009.pdf]

3. Wall et al: Refinement, scoring, and validation of the Family Satisfaction in the Intensive Care Unit (FS-ICU) survey. Crit Care Med 2007, 35:271-279.
P545

Family satisfaction with patient care in ICUs of nonacademic Brazilian public hospitals

J Othero, AB Cavalcanti, JC Mouro, K Normilio-da-Silva, R Pusch, F Moreira, AA Kodama, O Berwanger

Hospital do Coração - HCor, São Paulo, Brazil

Critical Care 2013, 17(Suppl 2):P545 (doi: 10.1186/cc12483)

Introduction We are conducting a cluster randomized trial with two parallel arms to evaluate strategies to improve family satisfaction with the care that themselves and their critically ill relatives receive in the ICUs of nonacademic Brazilian public hospitals. Here we report the results of the baseline phase of this trial.

Methods In this baseline phase, we interviewed the family member most closely involved with the care of critically ill patients who stayed in the ICU for at least 72 hours. We applied a form with 24 questions divided into four domains: overall ICU experience, communication, decision-making, and questions related to end-of-life care for patients who died in the ICU. Each question scored from 0 (very poor) to 100 (excellent). The form was adapted from the Family Satisfaction with Care in the ICU (FS-ICU 24). As many questions assessed the quality of intensivist care or communication, the interview was applied by a psychologist or a nurse.

Results Families of 564 patients were interviewed. A total 45/564 (8.3\%) died in the ICU. Most respondents were satisfied with overall ICU experience (mean \pm SD score $85.5 \pm 11.9$ ). However, family satisfaction with communication $(67.8 \pm 18.0)$ and decision-making $(69.5 \pm 21.1)$ resulted in somewhat lower scores. Most families of patients who died in the ICU (38/45 (82.6\%)) considered that their relative's life was neither extended nor shortened unnecessarily. Also, most of the families believed that their relative did not suffer or suffered little in the ICU (37/46 (80.4\%)) and felt supported by the healthcare team (40/46 (87.0\%)).

Conclusion Most families were satisfied with the care themselves and their critically ill relatives received in the ICU. Also, most relatives of patients who died in the ICU felt that end-of-life care was adequate. Although we believe there is much room for improvement in communication, decision-making and support critically ill patients and their families, as their baseline satisfaction with patient care is quite high, it may be hard to demonstrate substantial improvement after interventions.

\section{P546}

Correction: Urinary neutrophil gelatinase-associated lipocalin as an early predictor of acute kidney injury in cardiac surgery patients T García ${ }^{*}$, AJ Betbesé1, M García², M Argilaga², A Castellvín,

J Ordonez-Llanos ${ }^{3}$

'Intensive Care, Hospital de la Santa Creu i Sant Pau, Barcelone, Spain;

${ }^{2}$ Anethesiology Department, Hospital de la Santa Creu i Sant Pau, Barcelone, Spain: ${ }^{3}$ Biochemistry Department, Hospital de la Santa Creu i Sant Pau,

Barcelone, Spain

Critical Care 2013, 17(Suppl 2):P546 (doi: 10.1186/cc12604)

Correction After the publication of this abstract [1], we found that not all authors were listed. A full author list is provided here. We regret any inconvenience this error may have caused.

\section{Reference}

1. García Rodríguez San Miguel T: Urinary neutrophil gelatinase-associated lipocalin as an early predictor of acute kidney injury in cardiac surgery patients. Critical Care 2013, 17(Suppl 2):P411 
P547

Correction: The use, type and duration of cardiopulmonary bypass are related to acute kidney injury occurrence and urinary NGAL concentrations.

T García'*, AJ Betbesé2, M García², V Baños², R Bonet³, J Ordonez-Llanos ${ }^{3}$ 'Intensive Care, Hospital de la Santa Creu i Sant Pau, Barcelone, Spain;

${ }^{2}$ Anethesiology Department, Hospital de la Santa Creu i Sant Pau, Barcelone, Spain; ${ }^{3}$ Biochemistry Department, Hospital de la Santa Creu i Sant Pau,

Barcelone, Spain

Critical Care 2013, 17(Suppl 2):P547 (doi: 10.1186/cc12604)

Correction After the publication of this abstract [1], we found that not all authors were listed. A full author list is provided here. We regret any inconvenience this error may have caused.

Reference

1. García Rodríguez San Miguel T: Use, type and duration of cardiopulmonary bypass are related to acute kidney injury occurrence and urinary NGAL concentrations. Critical Care 2013, 17(Suppl 2):P422

P548

Correction: Current practices in ICU delirium management: a prospective multicenter study in the Netherlands

ZTrogrlic', E Ista' , A Slooter ${ }^{2}$, H Ponssen ${ }^{3}$, J Schoonderbeek ${ }^{4}$, F Schreiner ${ }^{5}$, S Verbrugge ${ }^{6}$, S Duran $^{7}$, J Bakker ${ }^{1}, \mathrm{M}$ van der Jagt ${ }^{1}$

'Erasmus University Medical Center, Department of Intensive Care, Rotterdam, The Netherlands; ${ }^{2}$ University Medical Center Utrecht, Department of Intensive Care, The Netherlands; ${ }^{3}$ Department of Intensive Care, Albert Schweitzer Hospital, Dordrecht, The Netherlands; ${ }^{4}$ Department of Intensive Care, Ikazia Hospital, Rotterdam, The Netherlands; ${ }^{5}$ Department of intensive care, IJsselland Hospital, Rotterdam, The Netherlands; ${ }^{6}$ Department of Intensive Care, Sint Franciscus Gasthuis, Rotterdam, The Netherlands; ' Department of Intensive Care, Maasstad Ziekenhuis, Rotterdam, The Netherlands.

Critical Care 2013, 17(Suppl 2):P548 (doi: 10.1186/cc12703)

Correction After the publication of this abstract [1], we found that not all authors were listed. A full author list is provided here. We regret any inconvenience this error may have caused.

\section{Reference}

1. Z Trogrlic, E Ista, A Slooter, J Bakker, M Van der Jagt, of iDECePTIvE Study Group: Current practices in ICU delirium management: a prospective multicenter study in the Netherlands. Critical Care 2013, 17(Suppl 2):P395.
P549

Correction: Current perspectives, beliefs and practices concerning delirium in critically ill patients: a multicenter survey among Dutch healthcare professionals

ZTrogrlic', M van der Jagt', P van der Voort'2, H Ponssen³ , J Schoonderbeek', F Schreiner ${ }^{5}$, S Verbrugge ${ }^{6}$, S Duran 7 , J Bakker ${ }^{1}$, E Ista $^{1}$

'Erasmus University Medical Center, Department of Intensive Care, Rotterdam, The Netherlands; ${ }^{2}$ Onze Lieve Vrouwe Gasthuis (OLVG), Department of Intensive Care, Amsterdam, The Netherlands; ${ }^{3}$ Department of Intensive Care, Albert Schweitzer Hospital, Dordrecht, The Netherlands; ${ }^{4}$ Department of Intensive Care, Ikazia Hospital, Rotterdam, The Netherlands; ${ }^{5}$ Department of intensive care, IJsselland Hospital, Rotterdam, The Netherlands; ${ }^{6}$ Department of Intensive Care, Sint Franciscus Gasthuis, Rotterdam, The Netherlands; ${ }^{7}$ Department of Intensive Care, Maasstad Ziekenhuis, Rotterdam, The Netherlands.

Critical Care 2013, 17(Suppl 2):P549 (doi: 10.1186/cc12704)

Correction After the publication of this abstract [1], we found that not all authors were listed. A full author list is provided here. We regret any inconvenience this error may have caused.

\section{Reference}

1. Z Trogrlic, M Van der Jagt, P Van der Voort, J Bakker, E Ista, of iDECePTIvE Study Group: Current perspectives, beliefs and practices concerning delirium in critically ill patients: a multicenter survey among Dutch healthcare professionals. Critical Care 2013, 17(Suppl 2):P397. 